
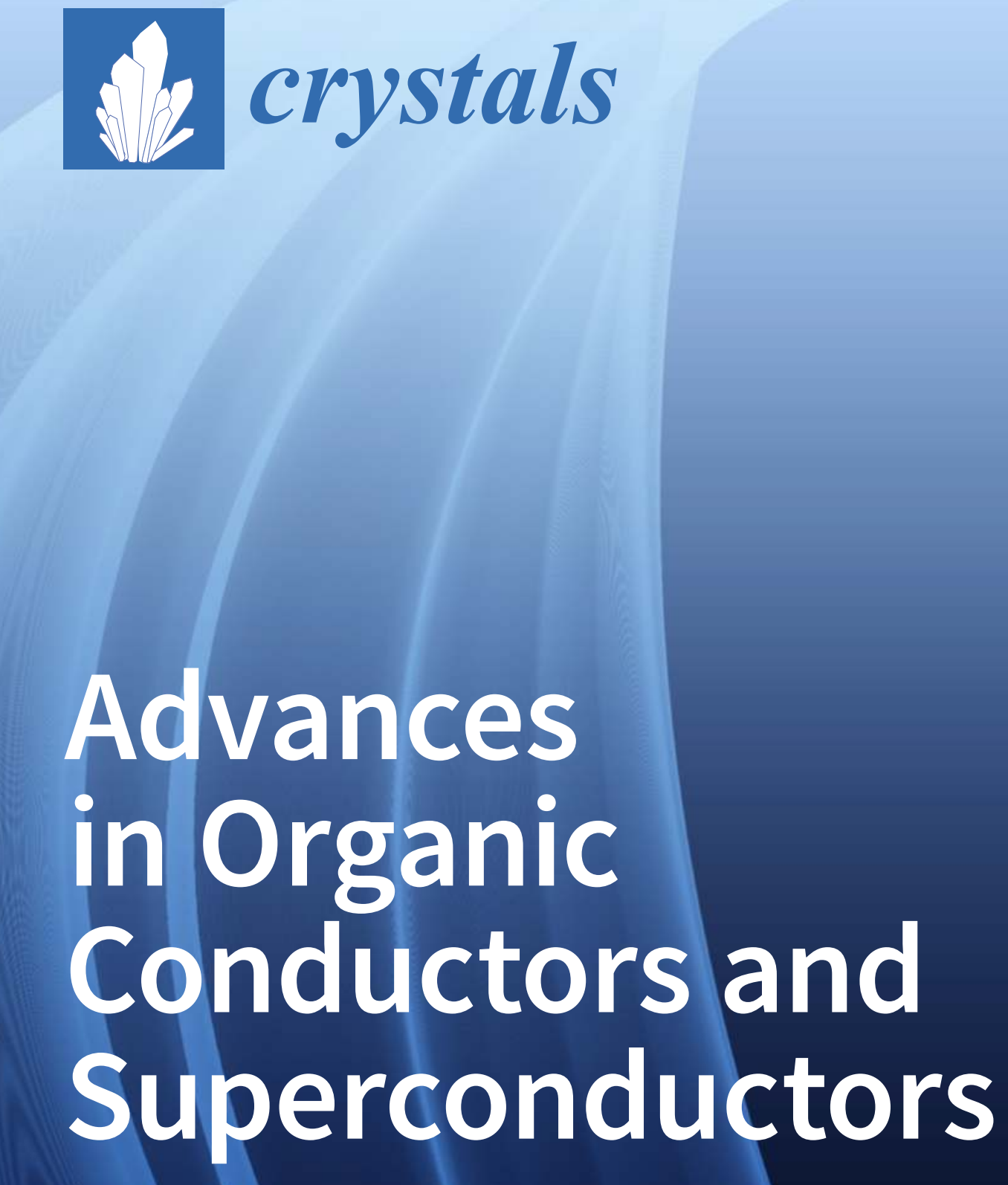

Edited by

Martin Dressel

Printed Edition of the Special Issue Published in Crystals 


\section{Advances in Organic Conductors and Superconductors}





\section{Advances in Organic Conductors and Superconductors}

Special Issue Editor

Martin Dressel 
Special Issue Editor

Martin Dressel

Universität Stuttgart

Germany

\section{Editorial Office}

MDPI

St. Alban-Anlage 66

Basel, Switzerland

This is a reprint of articles from the Special Issue published online in the open access journal Journal of Composites Science (ISSN 2504-477X) from 2017 to 2018 (available at: http://www.mdpi. com/journal/crystals/special_issues/Superconductors)

For citation purposes, cite each article independently as indicated on the article page online and as indicated below:

LastName, A.A.; LastName, B.B.; LastName, C.C. Article Title. Journal Name Year, Article Number, Page Range.

ISBN 978-3-03897-180-1 (Pbk)

ISBN 978-3-03897-181-8 (PDF)

Articles in this volume are Open Access and distributed under the Creative Commons Attribution (CC BY) license, which allows users to download, copy and build upon published articles even for commercial purposes, as long as the author and publisher are properly credited, which ensures maximum dissemination and a wider impact of our publications. The book taken as a whole is (C) 2018 MDPI, Basel, Switzerland, distributed under the terms and conditions of the Creative Commons license CC BY-NC-ND (http:/ / creativecommons.org/licenses/by-nc-nd/4.0/). 


\section{Contents}

About the Special Issue Editor $\ldots \ldots \ldots \ldots \ldots \ldots \ldots \ldots$ vii

\section{Martin Dressel}

Advances in Organic Conductors and Superconductors

Reprinted from: Crystals 2018, 8, 332, doi: $10.3390 /$ cryst8090332 . . . . . . . . . . . . . .

Jochen Wosnitza

Spatially Nonuniform Superconductivity in Quasi-Two-Dimensional Organic

Charge-Transfer Salts

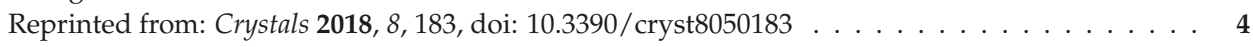

\section{Chales C Agosta}

Inhomogeneous Superconductivity in Organic and Related Superconductors

Reprinted from: Crystals 2018, 8, 285, doi: 10.3390 /cryst8070285 . . . . . . . . . . . . . . . 17

Akihiro Otsuka, Dmitri V. Konarev, Rimma N. Lyubovskaya, Salavat S. Khasanov, Mitsuhiko Maesato, Yukihiro Yoshida and Gunzi Saito

Design of Spin-Frustrated Monomer-Type $\mathrm{C}_{60}{ }^{\bullet-}$ Mott Insulator

Reprinted from: Crystals 2018, 8, 115, doi: 10.3390/cryst8030115

\section{Kohei Ueda, Takao Tsumuraya and Reizo Kato}

Temperature Dependence of Crystal Structures and Band Parameters in Quantum Spin Liquid $\beta^{\prime}$-EtMe $35 \mathrm{Sb}\left[\mathrm{Pd}(\mathrm{dmit})_{2}\right]_{2}$ and Related Materials

Reprinted from: Crystals 2018, 8, 138, doi: 10.3390/cryst8030138

Pascale Foury-Leylekian, Vita Ilakovac-Casses, Victor Balédent, Pierre Fertey, Alla Arakcheeva, Ognjen Milat, Denis Petermann, Gilles Guillier, Kazuya Miyagawa, Kazushi Kanoda, Pere Alemany, Enric Canadell, Silvia Tomic and Jean-Paul Pouget (BEDT-TTF $)_{2} \mathrm{Cu}_{2}(\mathrm{CN})_{3}$ Spin Liquid: Beyond the Average Structure

Reprinted from: Crystals 2018, 8, 158, doi: 10.3390/cryst8040158

Rudra Sekhar Manna, Steffi Hartmann, Elena Gati, John A. Schlueter, Mariano de Souza and Michael Lang

Low-Temperature Lattice Effects in the Spin-Liquid Candidate $\kappa$-(BEDT-TTF $)_{2} \mathrm{Cu}_{2}(\mathrm{CN})_{3}$

Reprinted from: Crystals 2018, 8, 87, doi: 10.3390/cryst8020087 . . . . . . . . . . . . . 107

Marko Pinterić, David Rivas Góngora, Željko Rapljenović, Tomislav Ivek, Matija Čulo, Bojana Korin-Hamzić, Ognjen Milat, Branko Gumhalter, Predrag Lazić, Miriam Sanz Alonso, Weiwu Li, Andrej Pustogow, Guilherme Gorgen Lesseux, Martin Dressel and Silvia Tomić

Electrodynamics in Organic Dimer Insulators Close to Mott Critical Point

Reprinted from: Crystals 2018, 8, 190, doi: 10.3390/cryst8050190 . . . . . . . . . . . . . 11

Jens Müller and Tatjana Thomas

Low-Frequency Dynamics of Strongly Correlated Electrons in (BEDT-TTF $)_{2} \mathrm{X}$ Studied by Fluctuation Spectroscopy

Reprinted from: Crystals 2018, 8, 166, doi: 10.3390/cryst8040166 . . . . . . . . . . . . . . 134 
Yasuhiro Nakazawa, Shusaku Imajo, Yuki Matsumura, Satoshi Yamashita and Hiroki Akutsu

Thermodynamic Picture of Dimer-Mott Organic Superconductors Revealed by Heat Capacity Measurements with External and Chemical Pressure Control

Reprinted from: Crystals 2018, 8, 143, doi: 10.3390/cryst8040143

Elena Gati, Ulrich Tutsch, Ammar Naji, Markus Garst, Sebastian Köhler, Harald Schubert, Takahiko Sasaki and Michael Lang

Effects of Disorder on the Pressure-Induced Mott Transition in $\kappa$-(BEDT-TTF) ${ }_{2} \mathrm{Cu}\left[\mathrm{N}(\mathrm{CN})_{2}\right] \mathrm{Cl}$

Reprinted from: Crystals 2018, 8, 38, doi: 10.3390/cryst8010038 . . . . . . . . . . . . . . . 187

Streit Cunningham, Elena I. Zhilyaeva, Svetlana A. Torunova, Rimma N. Lyubovskaya, John A. Schlueter and Natalia Drichko

Raman Scattering as a Probe of the Magnetic State of BEDT-TTF Based Mott Insulators

Reprinted from: Crystals 2018, 8, 233, doi: 10.3390/cryst8060233 . . . . . . . . . . . . . . . 207

Rafaela A. L. Silva, Isabel C. Santos, Sandra Rabaça, Elsa B. Lopes, Vasco Gama, Manuel Almeida and Dulce Belo

Synthesis and Characterization of Charge Transfer Salts Based on $\left[\mathrm{M}(\mathrm{dcdmp})_{2}\right](\mathrm{M}=\mathrm{Au}, \mathrm{Cu}$ and Ni) with TTF Type Donors

Reprinted from: Crystals 2018, 8, 141, doi: 10.3390/cryst8030141 . . . . . . . . . . . . . . 218

Tatiana G. Prokhorova, Eduard B. Yagubskii, Leokadiya V. Zorina, Sergey V. Simonov, Vladimir N. Zverev, Rimma P. Shibaeva and Lev I. Buravov

Specific Structural Disorder in an Anion Layer and Its Influence on Conducting Properties of New Crystals of the (BEDT-TTF $)_{4} \mathrm{~A}^{+}\left[\mathrm{M}^{3+}(\mathrm{ox})_{3}\right] \mathrm{G}$ Family, Where $\mathrm{G}$ Is 2-Halopyridine; $\mathrm{M}$ Is $\mathrm{Cr}$, $\mathrm{Ga} ; \mathrm{A}^{+}$Is $\left[\mathrm{K}_{0.8}\left(\mathrm{H}_{3} \mathrm{O}\right)_{0.2}\right]^{+}$

Reprinted from: Crystals 2018, 8, 92, doi: 10.3390/cryst8020092 _ . . . . . . . . . . . . . 234

Sandra Rabaça, Sandrina Oliveira, Vasco Gama, Isabel C. Santos, Gonçalo Oliveira, Elsa B. Lopes, Enric Canadell and Manuel Almeida

$\beta^{\prime \prime}$-(CNB-EDT-TTF) $)_{4} \mathrm{BF}_{4}$; Anion Disorder Effects in Bilayer Molecular Metals

Reprinted from: Crystals 2018, 8, 142, doi: 10.3390/cryst8040142 . . . . . . . . . . . . . . 248

Daigo Ohki, Genki Matsuno, Yukiko Omori and Akito Kobayashi

Optical Conductivity in a Two-Dimensional Extended Hubbard Model for an Organic Dirac

Electron System $\alpha$-(BEDT-TTF $)_{2} \mathrm{I}_{3}$

Reprinted from: Crystals 2018, 8, 137, doi: 10.3390/cryst8030137 . . . . . . . . . . . . . . 257

Naoya Tajima

Effects of Carrier Doping on the Transport in the Dirac Electron System $\alpha$-(BEDT-TTF) ${ }_{2} \mathrm{I}_{3}$ under

High Pressure

Reprinted from: Crystals 2018, 8, 126, doi: 10.3390/cryst8030126 . . . . . . . . . . . 265

Tobias Peterseim and Martin Dressel

Light-Induced Current Oscillations in the Charge-Ordered State of (TMTTF $)_{2} \mathrm{SbF}_{6}$

Reprinted from: Crystals 2017, 7, 278, doi: 10.3390/cryst7090278 . . . . . . . . . . . . . 282

Roland Rösslhuber, Eva Rose, Tomislav Ivek, Andrej Pustogow, Thomas Breier, Michael Geiger, Karl Schrem, Gabriele Untereiner and Martin Dressel

Structural and Electronic Properties of (TMTTF) ${ }_{2} X$ Salts with Tetrahedral Anions

Reprinted from: Crystals 2018, 8, 121, doi: 10.3390/cryst8030121 . . . . . . . . . . . . . 302 


\section{About the Special Issue Editor}

Martin Dressel received his Ph.D. degree from the Universität Göttingen, Germany, in 1989, for studying magnetic-transport in organic conductors and superconductors by microwaves. Since 1989, he has held positions at the Laser-Laboratory Göttingen, Germany, the University of British Columbia, Vancouver, Canada, and the University of California, Los Angeles, CA, USA. He received his Habilitation from the technical Universität Darmstadt, Germany, in 1996, and then, joined the Center of Electronic Correlations and Magnetism, Universität Ausgburg, Germany. Since 1998, he is Head of the 1. Physikalisches Institut, Universität Stuttgart, Germany. His research interests include the electronic and magnetic properties of low-dimensional electron systems. The group is renowned for its investigations on the electrodynamics of electronically correlated matter, in particular organic conductors. 



\title{
Editorial \\ Advances in Organic Conductors and Superconductors
}

\author{
Martin Dressel \\ 1. Physikalisches Institut, Universität Stuttgart, Pfaffenwaldring 57, D-70569 Stuttgart, Germany; \\ dressel@pi1.physik.uni-stuttgart.de; Tel.: +49-711-685-64946
}

Received: 18 August 2018; Accepted: 20 August 2018; Published: 21 August 2018

Crystalline conductors and superconductors based on organic molecules are a rapidly progressing field of solid-state science, involving chemists, and experimental and theoretical physicists from all around the world. In focus are solids with electronic properties governed by delocalized $\pi$-electrons. Although carbon-based materials of various shades have gained enormous interest in recent years, charge transfer salts are still paradigmatic in this field. Progress in molecular design is achieved via tiny but ingenious modifications, as well as by fundamentally different approaches. The wealth of exciting physical phenomena is unprecedented and could not have been imagined when the field took off almost half a century ago.

Organic low-dimensional conductors are prime examples of Luttinger liquids; they exhibit a tendency toward Fermi surface instabilities, but can also be tuned across a dimensionality-driven phase diagram like no other system. Superconductivity comes at the border to ordered phases in the spin and charge sectors, and, at high fields, the Fulde-Ferrell-Larkin-Ovchinnikov (FFLO) state is well established. The interplay between charge and magnetic order is still under debate, but electronic ferroelectricity is well established. After decades of intense research, the spin liquid state was first discovered in organic conductors when the amount of geometrical frustration and electronic correlations is just right. They drive the metal and superconductor into an insulating Mott state, solely via electron-electron interactions. However, what do we know about the effect of disorder? Can we tune the electronic properties by pressure, by light, or by field? Research is still addressing basic questions, but devices are not out of reach. These are currently open questions, as well as hot and timely topics.

Although the superconducting transition temperature remains rather moderate for organic materials, the questions addressed are of fundamental importance, and for several topics molecular conductors are by far the best model system for detailed investigations. The spatial modulation of the superconducting order parameter in the Fulde-Ferrell Larkin-Ovchinikov state is certainly one of them [1,2]. The conceptional paper by Otsuka et al. reviews current ideas on the design of a spin-frustrated Mott insulator and points towards future developments in quantum spin liquids [3]. No question, this exciting research topic has placed organic crystals again at the forefront of solid-state physics. The class of $\beta^{\prime}-\mathrm{Et}_{x} \mathrm{Me}_{4-x} \mathrm{Z}\left[\mathrm{Pd}(\mathrm{dmit})_{2}\right]_{2}$ with $\mathrm{Z}=\mathrm{P}, \mathrm{As}, \mathrm{Sb}$ and $x=0,1,2$ is a topic of the paper from Ueda et al. [4]. A completely new view on the structural properties of $\kappa$-(BEDT-TTF $)_{2} \mathrm{Cu}_{2}(\mathrm{CN})_{3}$ is given by Foury-Leylekian and collaborators [5], who found that the compound contains two non-equivalent dimers in the unit cell and charge disproportionation between the dimers. Here, simple facts, assumed to be settled for decades, were revisited, such as the symmetry of a crystal; this laid new ground for the re-interpretation of numerous experimental observations. The low-temperature anomaly observed in the quantum spin liquid compound $\kappa$-(BEDT-TTF $)_{2} \mathrm{Cu}_{2}(\mathrm{CN})_{3}$, however, remains an open issue [6]. In any case, the electrodynamic properties of these organic dimer Mott insulators appear to be fairly well understood by now $[7,8]$. The Mott transition observed in the two-dimensional organic $\kappa$-phase BEDT-TTF salts is the focus of the detailed pressure-dependent measurements of the heat capacity [9] and thermal expansion [10]. 
Magnetic excitations were investigated by Raman scattering, suggesting intra-dimer charge degree of freedom for some salts [11]. The combination of a correlation-driven Mott transition and a disorder-driven Anderson transition has become more of an issue in recent years. The contribution of Rabaça et al. and Prokhorova et al. considers structural disorder [12-14]. The existence of Dirac-like electrons in $\alpha$-(BEDT-TTF $)_{2} \mathrm{I}_{3}$ is another topic that has attracted interest for more than a decade, from a theoretical side [15] as well as from the side of applications [16]. Pressure-dependent experiments are also important for the one-dimensional TMTTF salts, in order to study the charge and anion ordering $[17,18]$.

In 2012, Reizo Kato edited a seminal collection of 45 contributions on various aspects of "Molecular Conductors" as a Special Issue of Crystals, which has been well received by the community. The present Special Issue on "Advances in Organic Conductors and Superconductors" may become a status report summarizing the progress achieved in the last five years.

\section{Referencens}

1. Wosnitza, J. Spatially Nonuniform Superconductivity in Quasi-Two-Dimensional Organic Charge-Transfer Salts. Crystals 2018, 8, 183. [CrossRef]

2. Agosta, C.C. Inhomogeneous Superconductivity in Organic and Related Superconductors. Crystals 2018, 8285. [CrossRef]

3. Otsuka, A.; Konarev, D.; Lyubovskaya, R.; Khasanov, S.; Maesato, M.; Yoshida, Y.; Saito, G. Design of Spin-Frustrated Monomer-Type $\mathrm{C}_{60}^{\bullet-}$ Mott Insulator. Crystals 2018, 8, 115. [CrossRef]

4. Ueda, K.; Tsumuraya, T.; Kato, R. Temperature Dependence of Crystal Structures and Band Parameters in Quantum Spin Liquid $\beta^{\prime}$-EtMe $\mathrm{Sb}_{3}\left[\mathrm{Pd}(\mathrm{dmit})_{2}\right]_{2}$ and Related Materials. Crystals 2018, 8, 138. [CrossRef]

5. Foury-Leylekian, P.; Ilakovac, V.; Balédent, V.; Fertey, P.; Arakcheeva, A.; Milat, O.; Petermann, D.; Guillier, G.; Miyagawa, K.; Kanoda, K.; et al. (BEDT-TTF $)_{2} \mathrm{Cu}_{2}(\mathrm{CN})_{3}$ Spin Liquid: Beyond the Average Structure. Crystals 2018, 8, 158. [CrossRef]

6. Manna, R.; Hartmann, S.; Gati, E.; Schlueter, J.; de Souza, M.; Lang, M. Low-Temperature Lattice Effects in the Spin-Liquid Candidate $\kappa$-(BEDT-TTF $)_{2} \mathrm{Cu}_{2}(\mathrm{CN})_{3}$. Crystals 2018, 8, 87. [CrossRef]

7. Pinterić, M.; Rivas Góngora, D.; Rapljenović, V.; Ivek, T.; Čulo, M.; Korin-Hamzić, B.; Milat, O.; Gumhalter, B.; Lazić, P.; Sanz Alonso, M.; et al. Electrodynamics in Organic Dimer Insulators Close to Mott Critical Point. Crystals 2018, 8, 190. [CrossRef]

8. Müller, J.; Thomas, T. Low-Frequency Dynamics of Strongly Correlated Electrons in (BEDT-TTF) ${ }_{2} X$ Studied by Fluctuation Spectroscopy. Crystals 2018, 8, 166. [CrossRef]

9. Nakazawa, Y.; Imajo, S.; Matsumura, Y.; Yamashita, S.; Akutsu, H. Thermodynamic Picture of Dimer-Mott Organic Superconductors Revealed by Heat Capacity Measurements with External and Chemical Pressure Control. Crystals 2018, 8, 143. [CrossRef]

10. Gati, E.; Tutsch, U.; Naji, A.; Garst, M.; Köhler, S.; Schubert, H.; Sasaki, T.; Lang, M. Effects of Disorder on the Pressure-Induced Mott Transition in $\kappa-(\mathrm{BEDT}-\mathrm{TTF})_{2} \mathrm{Cu}\left[\mathrm{N}(\mathrm{CN})_{2}\right] \mathrm{Cl}$. Crystals 2018, 8, 38. [CrossRef]

11. Hassan, N.; Cunningham, S.; Zhilyaeva, E.; Torunova, S.; Lyubovskaya, R.; Schlueter, J.; Drichko, N. Raman Scattering as a Probe of the Magnetic State of BEDT-TTF Based Mott Insulators. Crystals 2018, 8, 233. [CrossRef]

12. Silva, R.; Santos, I.; Rabaça, S.; Lopes, E.; Gama, V.; Almeida, M.; Belo, D. Synthesis and Characterization of Charge Transfer Salts Based on $\left[\mathrm{M}(\mathrm{dcdmp})_{2}\right](\mathrm{M}=\mathrm{Au}, \mathrm{Cu}$ and Ni) with TTF Type Donors. Crystals 2018, 8, 141. [CrossRef]

13. Prokhorova, T.; Yagubskii, E.; Zorina, L.; Simonov, S.; Zverev, V.; Shibaeva, R.; Buravov, L. Specific Structural Disorder in an Anion Layer and Its Influence on Conducting Properties of New Crystals of the (BEDT-TTF $)_{4} \mathrm{~A}^{+}\left[\mathrm{M}^{3+}(\mathrm{ox})_{3}\right] \mathrm{G}$ Family, Where G Is 2-Halopyridine; $\mathrm{M}$ Is $\mathrm{Cr}$, Ga; $\mathrm{A}^{+}$Is $\left[\mathrm{K}_{0.8}\left(\mathrm{H}_{3} \mathrm{O}\right)_{0.2}\right]^{+}$. Crystals 2018, 8, 92. [CrossRef]

14. Rabaça, S.; Oliveira, S.; Gama, V.; Santos, I.; Oliveira, G.; Lopes, E.; Canadell, E.; Almeida, M. $\beta^{\prime \prime}$-(CNB-EDT-TTF $)_{4} \mathrm{BF}_{4}$; Anion Disorder Effects in Bilayer Molecular Metals. Crystals 2018, 8, 142. [CrossRef]

15. Ohki, D.; Matsuno, G.; Omori, Y.; Kobayashi, A. Optical Conductivity in a Two-Dimensional Extended Hubbard Model for an Organic Dirac Electron System $\alpha$-(BEDT-TTF) ${ }_{2} \mathrm{I}_{3}$. Crystals 2018, 8, 137. [CrossRef] 
16. Tajima, N. Effects of Carrier Doping on the Transport in the Dirac Electron System $\alpha$-(BEDT-TTF) ${ }_{2} \mathrm{I}_{3}$ under High Pressure. Crystals 2018, 8, 126. [CrossRef]

17. Peterseim, T.; Dressel, M. Light-Induced Current Oscillations in the Charge-Ordered State of (TMTTF $)_{2} \mathrm{SbF}_{6}$. Crystals 2017, 7, 278. [CrossRef]

18. Rösslhuber, R.; Rose, E.; Ivek, T.; Pustogow, A.; Breier, T.; Geiger, M.; Schrem, K.; Untereiner, G.; Dressel, M. Structural and Electronic Properties of (TMTTF) ${ }_{2} X$ Salts with Tetrahedral Anions. Crystals 2018, 8, 121. [CrossRef]

(C) 2018 by the authors. Licensee MDPI, Basel, Switzerland. This article is an open access article distributed under the terms and conditions of the Creative Commons Attribution (CC BY) license (http://creativecommons.org/licenses/by/4.0/). 


\title{
Article \\ Spatially Nonuniform Superconductivity in Quasi-Two-Dimensional Organic Charge-Transfer Salts
}

\author{
Jochen Wosnitza \\ Hochfeld-Magnetlabor Dresden (HLD-EMFL), Helmholtz-Zentrum Dresden-Rossendorf, \\ 01328 Dresden, Germany; j.wosnitza@hzdr.de
}

Received: 14 February 2018; Accepted: 20 April 2018; Published: 24 April 2018

\begin{abstract}
In the following, a brief overview on the recently found robust experimental evidence for the existence of the Fulde-Ferrell-Larkin-Ovchinnikov (FFLO) state in layered organic superconductors is given. These electronically quasi-two-dimensional (2D) clean-limit superconductors are ideally suited for observing FFLO states. Applying a magnetic field parallel to the layers suppresses orbital effects and superconductivity is observed beyond the Pauli paramagnetic limit. Both, thermodynamic as well as microscopic experimental data show the existence of an additional high-field low-temperature superconducting state having a one-dimensionally modulated order parameter.
\end{abstract}

Keywords: organic superconductors; FFLO state; specific heat; NMR

\section{Introduction}

In most cases, superconductivity is destroyed at high magnetic fields. For spin-singlet pairing, the highest field where this phase change can occur is at the Pauli paramagnetic limit when the Zeeman energy of the itinerant electrons becomes larger than the condensation energy [1,2]. There may, however, exist superconductivity even beyond the Pauli limit with a spatially modulated superconducting order parameter. This was predicted already in 1964, independently by Fulde and Ferrell [3] as well as Larkin and Ovchinnikov [4]. Such now-called FFLO (or LOFF) states can appear in superconductors with very large orbital critical field, $B_{\text {orb }}$, that is, when the usually dominant orbital pair breaking is of minor importance. Only then, superconductivity is eventually suppressed at the Pauli limit $B_{P}=\Delta_{0} /\left(\sqrt{2} \mu_{B}\right)$, where $\mu_{B}$ is the Bohr magneton and $\Delta_{0}$ the zero-temperature superconducting energy gap [1,2]. According to Gruenberg and Gunther, the Maki parameter [5], $\sqrt{2} B_{\text {orb }} / B_{P}$, has to be larger than 1.8 for the possible occurrence of an FFLO state [6]. Further to that, the mean free path of the charge carriers needs to be larger than the coherence length, i.e., clean-limit superconductors are needed [7-9]. For the latter point, however, some theory works suggest that FFLO phases may survive even with some disorder [10-13]. (For related experimental work see [14-16].)

When applying a magnetic field, the Fermi surface splits into two with spin-up and spin-down electrons. If this splitting is not too large, that is for fields below $B_{P}$, still the usual BCS-like pairing with opposite spin and momentum is energetically favourable (left part of Figure 1). Beyond the Pauli limit, however, pairing with finite total momentum, $q$, becomes the more stable ground state (right part of Figure 1), until the normal state finally is reached at a higher field beyond $B_{P}$. In the most simple of the possible FFLO states, the Cooper-pair wave function oscillates with $q \propto B$, leading to an equally oscillating Cooper-pair density also in real space (for possible more elaborated FFLO phases see $[8,9]$ ). By that, part of the superconducting volume becomes normal, namely at the wave-function zeros where the excess spins accumulate, allowing the superconducting state to survive even beyond the Pauli limit. 

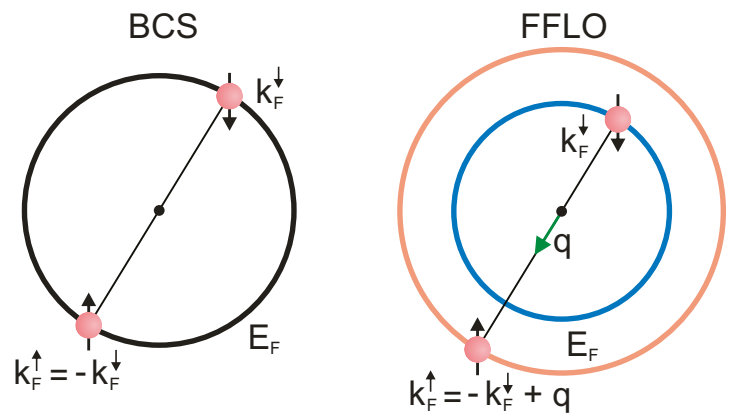

Figure 1. Schematic sketch of (left) the usual BCS pairing state with zero resulting momentum and spin and (right) the FFLO pairing state with a finite center-of-mass momentum, $q$. The circles represent the Fermi surfaces for spin-up and spin-down electrons.

Nonuniform FFLO states can appear only at temperatures much lower than the superconducting zero-field transition at $T_{\mathcal{C}}$, namely below $T^{\star}=0.56 T_{\mathcal{C}}$ [5]. For temperatures between $T^{\star}$ and $T_{\mathcal{C}}$, only the uniform superconducting state can exist below the upper critical field $B_{c 2}$. Below $T^{\star}$, marking a tricritical point, the FFLO phase may emerge. Here, a first-order phase transition may occur, whereas above $T^{\star}$ the phase transition to the normal state is second order. For three-dimensional (3D) superconductors the stability region for FFLO states is rather limited but enlarges considerably for electronically highly anisotropic materials [9]. This stability region depends on the dimensionality and becomes much larger for lower dimensions (see e.g., $[8,9,17])$. Indeed, Figure 2 shows the calculated phase diagram for a two-dimensional (2D) superconductor with isotropic in-plane Fermi velocity [9]. For a more general in-plane anisotropy the FFLO-phase region becomes even larger [18].

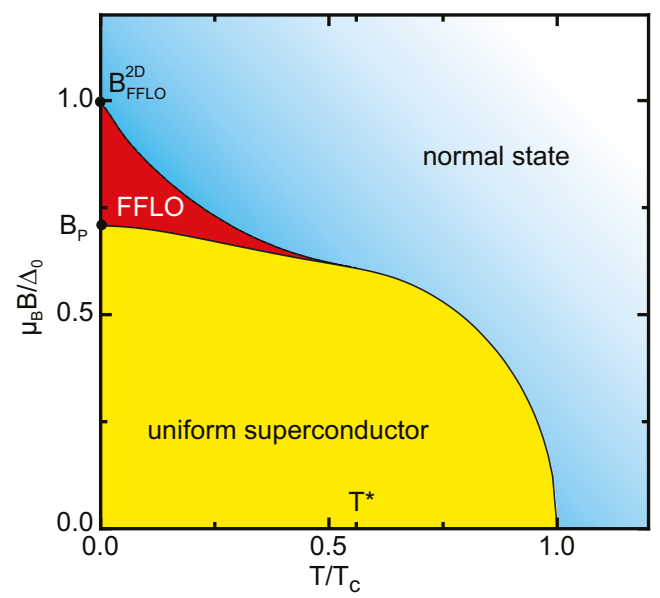

Figure 2. Calculated phase diagram of a Pauli-limited two-dimensional superconductor. Above $T^{\star}=0.56 T_{c}$ only the uniform superconducting (yellow region) or, above $B_{c 2}$, the normal state (blue) can exist. Below $T^{\star}$, the FFLO state (red) may evolve up to $\mu_{B} B_{\mathrm{FFLO}}^{2 \mathrm{D}}=\Delta_{0}$.

It is worthwhile to mention that the notion of FFLO states is of much broader importance than for superconductivity in condensed matter. These exotic states are predicted to exist in general in polarized, spin-imbalanced Fermi systems, such as ultra-cold atomic gases [19], nuclear matter, and dense quark matter [20]. Although there have been some reports on the observation of FFLO states in cold atomic gases [21,22] these results are questioned and solid evidence for FFLO states has, so far, only been 
found in condensed-matter systems, such as the organic superconductors discussed in the following chapter. Other candidate materials, such as heavy-fermion and iron-based superconductors, are briefly mentioned in Section 3.

\section{FFLO Evidence in Layered Organic Superconductors}

For FFLO states to occur certain restrictions apply. As mentioned, the superconductor has to be strongly Pauli limited, with a Maki parameter larger than 1.8, and needs to be in the clean limit. Further, the stability region of FFLO states is rather limited in 3D but is large and experimentally more easily accessible in 2D electron systems. Prime candidate materials are, therefore, quasi-2D organic superconductors, for instance, with the molecular building blocks bisethylenedithio-tetrathiafulvalene (BEDT-TTF or ET for short) or bisethylenedithio-tetraselenafulvalene (BETS). Although there exist controversies on the nature of the superconducting state, with nuclear magnetic resonance (NMR) results evidencing node-like $d$-wave symmetries $[23,24]$ and, on the other side, specific-heat data showing an s-wave-like fully gapped order parameter [25], the singlet nature of the pairing, important here, is proven by above NMR data. A more detailed discussion on the nature of the superconducting state can be found in [26]. In any case, the organic materials are well-studied, high-quality crystals with nearly ideal 2D electronic structure and strong type-II spin-singlet superconductors, usually in the clean limit with long mean free paths [26-30]. When aligning the magnetic field parallel to the highly conducting layers, orbital pair breaking is largely reduced and Pauli limitation becomes dominant.

There indeed have been a number of studies for such field orientation reporting evidence for the existence of FFLO states in these 2D organic superconductors. These claims were mainly based on transport data, such as on thermal-conductivity [31], interplane-resistance [32,33], and penetration-depth measurements [34-36]. Clear thermodynamic and microscopic evidence for a high-field FFLO phase was missing until 2011.

\subsection{Thermodynamic Data and Phase Diagrams}

The first thermodynamic evidence for the appearance of an FFLO state was found for the organic superconductor $\kappa$-(BEDT-TTF $)_{2} \mathrm{Cu}(\mathrm{NCS})_{2}$ by use of specific-heat measurements [37]. Figure 3 shows the obtained data for in-plane field alignment after subtracting the phonon part of the specific heat. The latter was extracted by measuring the normal-state specific heat separately in a field of $14 \mathrm{~T}$ applied perpendicular to the layers. At $T_{c}=9.1 \mathrm{~K}$, a clear $\lambda$-like anomaly is resolved which reduces in size and shifts to lower temperatures with increasing magnetic field. Above $21 \mathrm{~T}$, however, the anomaly sharpens and a second transition appears at somewhat lower temperatures in fields up to $23 \mathrm{~T}$. This latter anomaly showed a hysteresis in the specific-heat data evidencing the first-order nature of the transition [37].

This second anomaly was not observed in later work that measured the penetration depth using a tunnel-diode-oscillator technique [36], magnetic torque [38], or magnetic-field-dependent calorimetry [39]. A possibly reason for this discrepancy could have been a small misalignment of the investigated crystal in the work reported in Ref. [37]. The measurements were performed on a fixed platform in a 28-T magnet at the Grenoble High Magnetic Field Laboratory (LNCMI-Grenoble). Although the crystal was aligned in the best possible way, an out-of-plane orientation of the magnetic field on the sub-degree level cannot be excluded. Indeed, as was shown later, rotating the magnetic field by a small amount (of the order of 1 degree) immediately suppresses the FFLO state. Detailed angular-dependent specific-heat studies of another organic superconductor, $\beta^{\prime \prime}$-(BEDT-TTF) ${ }_{2} \mathrm{SF}_{5} \mathrm{CH}_{2} \mathrm{CF}_{2} \mathrm{SO}_{3}$ discussed further below, showed that a second anomaly just below $B_{c 2}$ appears when the magnetic field is aligned by about $0.2-0.3$ degree off the in-plane orientation $[40,41]$. This makes it rather likely that the sharp first-order transition shown in Figure 3 for $\kappa$-(BEDT-TTF $)_{2} \mathrm{Cu}(\mathrm{NCS})_{2}$ is an artifact due to a small out-of-plane component of the magnetic field. 


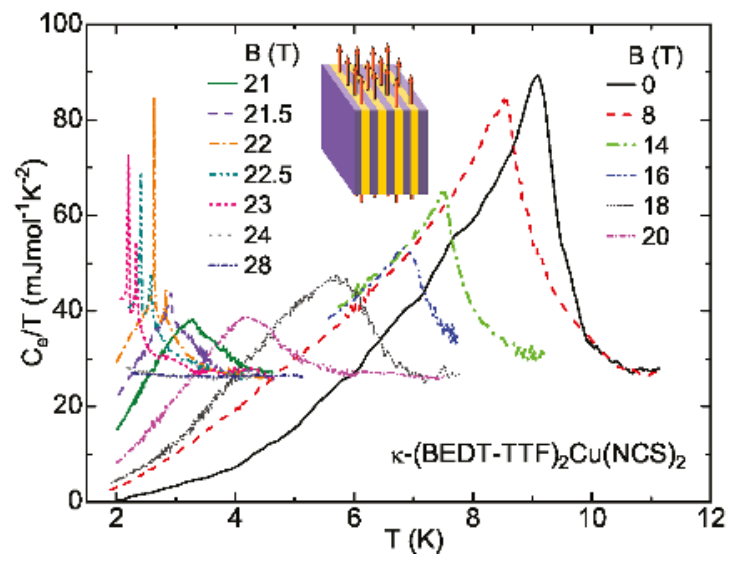

Figure 3. Electronic part of the specific heat of $\kappa$-(BEDT-TTF $)_{2} \mathrm{Cu}(\mathrm{NCS})_{2}$, divided by temperature, vs. temperature in parallel magnetic fields (inset) up to $28 \mathrm{~T}$.

The $B_{c 2}$ data extracted from the specific heat (Figure 3) are shown as black circles in Figure 4. From the specific-heat data, it is possible to determine the Maki parameter. The orbital critical field can be estimated from the initial critical-field slope at $T_{c}$, yielding $d B_{c 2} / d T \approx 20 \mathrm{~T} / \mathrm{K}$ using the data points at 0 and $8 \mathrm{~T}$, and the Werthamer-Helfand-Hohenberg (WHH) extrapolation [42], $B_{\text {orb }}=0.7 T_{c} d B_{c 2} / d T \approx 130 \mathrm{~T}$. The Pauli limit can reliably be estimated from the jump height and temperature dependence of the specific-heat anomaly resulting in the strong-coupling value $\Delta_{0} / k_{B} T_{c} \approx 2.4$ [43] and, finally, in $B_{P}=22(1) \mathrm{T}$. Together with $B_{\text {orb }} \approx 130 \mathrm{~T}$, this leads to a Maki parameter of about 8.4, comfortably larger than the 1.8 required for FFLO states to occur.

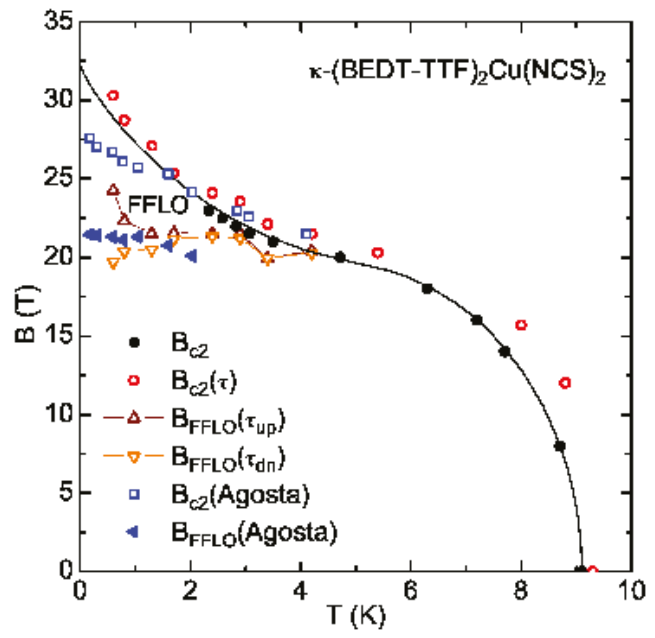

Figure 4. Superconducting phase diagram of $k$-(BEDT-TTF $)_{2} \mathrm{Cu}(\mathrm{NCS})_{2}$ as obtained from the specific-heat data shown in Figure 3 (black circles), torque, $\tau$, data (open circles and triangles) [38], as well as data taken from the work of Agosta et al. (blue squares and triangles) [39]. The solid line is the calculated $B_{c 2}$ for an isotropic in-plane Fermi velocity.

The solid line in Figure 4 represents $B_{c 2}$, the calculated phase transition from the normal to the superconducting state for a 2D superconductor with isotropic in-plane Fermi surface, as shown in 
Figure 2. Very good agreement with the specific-heat data is found. The phase diagram in Figure 4 further includes data from magnetic-torque measurements, where a rotator made possible precise alignment of the magnetic field parallel to the layers [38]. These data overestimate somewhat the more reliable specific-heat $B_{c 2}$ values. The torque measurements, however, done with sweeping the magnetic field, allowed to resolve the hysteretic phase transition from the uniform to the FFLO superconducting state (open triangles).

In a very recent ac-specific-heat and magnetocaloric-effect measurement the phase transition from the uniform to the FFLO phase was verified thermodynamically by performing field sweeps (closed blue triangles in Figure 4) [39]. At low temperatures, the $B_{c 2}$ values in this experiment (open blue squares) lie clearly above the Pauli limit, again consistent with the existence of the FFLO phase, however, they fall somewhat below the torque data and the calculated line. Here, more work is necessary for a more reliable determination of the $B_{c 2}$ line at lowest temperature.

There exist a large number of quasi-2D organic superconductors $[27,28]$ most of which are candidates for showing FFLO states. However, for practical reasons, $T_{\mathcal{c}}$ should be in a range, so that not too low temperatures or too high magnetic fields are needed to reach these states. Further, high-quality crystals of sufficient size are required for resolving the phase transitions in bulk experiments. These conditions are favorably fulfilled in the electronically nearly ideal 2D material $\beta^{\prime \prime}$-(BEDT-TTF) $)_{2} \mathrm{SF}_{5} \mathrm{CH}_{2} \mathrm{CF}_{2} \mathrm{SO}_{3}[44-46]$.

For this superconductor, specific heat [40] showed a strong upward curvature of the superconducting phase boundary above $B_{P}$, consistent with the existence of an FFLO state, even though a transition from the uniform to the FFLO phase was not directly observed. The electronic part of the specific heat divided by temperature is shown in the relevant temperature range in Figure 5. Here, 10-T specific-heat data in perpendicular field were used to subtract the phonon contribution. From an equal-entropy construction of the zero-field data, the bulk $T_{\mathcal{C}}=4.3 \mathrm{~K}$ can be determined. From the idealized jump at $T_{\mathcal{C}}$ and the temperature dependence of the specific heat the zero-temperature energy gap $\Delta_{0} / k_{B} T_{C}=2.18$, evidencing a moderately enhanced coupling strength, and the Pauli limit $B_{P}=9.73(3) \mathrm{T}$ is found [40]. When applying magnetic fields by use of a rotator precisely aligned parallel to the conducting layers, the specific-heat anomaly shifts to lower temperatures and remains resolvable at least up to $11 \mathrm{~T}$ (Figure 5). As evident from the data, superconductivity persists clearly beyond the Pauli limit.

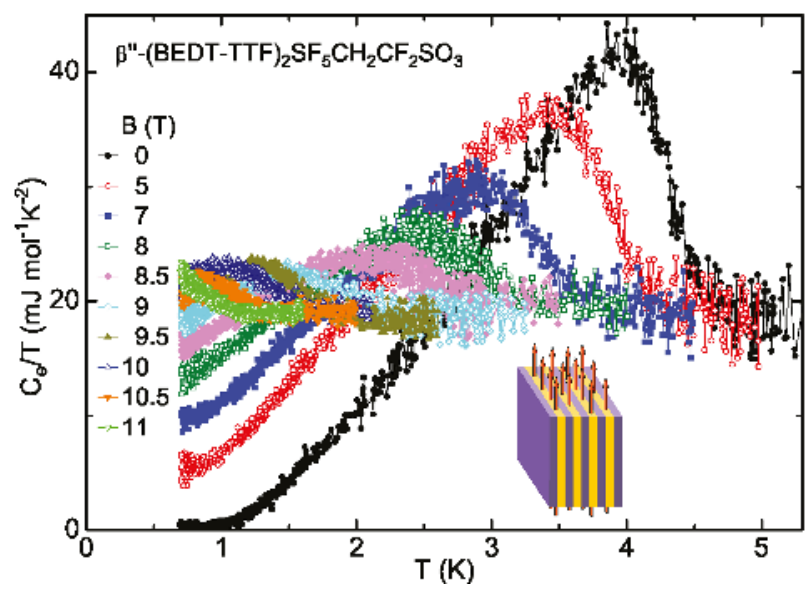

Figure 5. Temperature dependence of the electronic part of the specific heat, divided by temperature, of $\beta^{\prime \prime}$-(BEDT-TTF $)_{2} \mathrm{SF}_{5} \mathrm{CH}_{2} \mathrm{CF}_{2} \mathrm{SO}_{3}$ in various parallel magnetic fields up to $11 \mathrm{~T}$.

The superconducting phase diagram determined from these specific-heat measurements is shown in Figure 6. Again, the initial slope of the critical field at $T_{\mathcal{C}}$ (approximately $25 \mathrm{~T} / \mathrm{K}$ ) allows to estimate 
$B_{\text {orb }} \approx 75 \mathrm{~T}$, that is much larger than $B_{P}$ and results in a Maki parameter close to 11 . The data show the typical behavior of a Pauli-limited superconductor. The very steep slope of the $B_{c 2}$ line near $T_{c}$ rapidly becomes shallow at higher magnetic fields when the Pauli limit is approached. The dotted line in Figure 6 depicts the estimated $B_{c 2}$ line without the appearance of an FFLO state. Obviously, the specific-heat data lie clearly above this estimate with a changed curvature of the $B_{c 2}$ line: Strongly suggesting the emergence of an FFLO state.

The relative increase of the upper critical field, $B_{c 2}$, at low temperatures is clearly larger than for $\kappa$-(BEDT-TTF $)_{2} \mathrm{Cu}(\mathrm{NCS})_{2}$. The blue dashed line in Figure 6 is equivalent to the solid line shown in Figure 4, just normalized for $T_{c}$ and $B_{P}$. As mentioned, this theory line was calculated assuming an isotropic in-plane Fermi velocity. Earlier investigations of the Fermi-surface topology of $\beta^{\prime \prime}$-(BEDT-TTF $)_{2} \mathrm{SF}_{5} \mathrm{CH}_{2} \mathrm{CF}_{2} \mathrm{SO}_{3}$, however, showed that the electronically $2 \mathrm{D}$ material has as well a pronounced anisotropy of the Fermi surface within the planes (inset of Figure 6) [47]. The experimental aspect ratio of the in-plane Fermi-surface ellipse is 9. Calculating the critical-field line for the same Fermi-velocity ratio yields the dotted line in Figure 6. The experimental data lie just between this line and the calculations for the isotropic in-plane case. For the aspect ratio 2.25, excellent agreement with the experimental data is obtained (solid line in Figure 6). In view of the rather simple mean-field theory used for the calculations, neglecting any kind of fluctuations, the description of the experimental data is superb $[18,48]$.

It is worthwhile to mention that the phase diagram of $\beta^{\prime \prime}$-(BEDT-TTF) ${ }_{2} \mathrm{SF}_{5} \mathrm{CH}_{2} \mathrm{CF}_{2} \mathrm{SO}_{3}$ is independent of in-plane orientation of the magnetic field, in contrast to out-of-plane rotations. When measuring the specific heat for three different in-plane field alignments, the extracted $B_{c 2}$ lines fall on top of each other within error bars [18]. This suggests negligible orbital effects [49] and a pinning of the FFLO modulation vector $q$ at some optimum position, most probably parallel to the short axis of the Fermi-surface ellipse depicted in the inset of Figure 6.

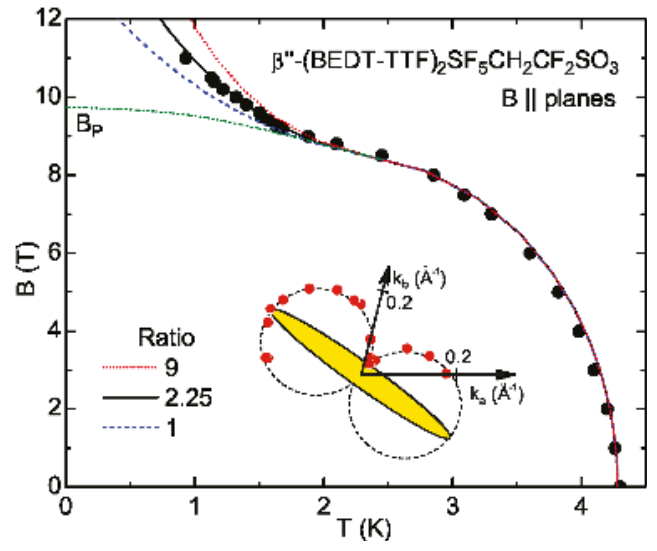

Figure 6. Phase diagram of $\beta^{\prime \prime}$-(BEDT-TTF) ${ }_{2} \mathrm{SF}_{5} \mathrm{CH}_{2} \mathrm{CF}_{2} \mathrm{SO}_{3}$ as extracted from the data in Figure 5. Three lines show the calculated $B_{c 2}$ for different Fermi-velocity ratios of elliptical Fermi surfaces. The dotted line is a rough extrapolation of the high-temperature data towards the Pauli limit at $T=0$, estimating the transition from the uniform to the FFLO superconductivity. The inset depicts the measured in-plane Fermi surface [47].

\subsection{Microscopic Evidence}

The thermodynamic measurements discussed above give strong evidence in favor for the existence of FFLO states, but they cannot supply microscopic insight into the spatially modulated order parameter. This was finally provided by sophisticated ${ }^{13} \mathrm{C}$ NMR experiments. First, such measurements were performed for $\kappa$-(BEDT-TTF $)_{2} \mathrm{Cu}(\mathrm{NCS})_{2}$ in resistive magnets of the high-field laboratories in 
Tallahassee [50] and, later, in Grenoble [51]. The NMR spectra taken in Tallahassee at $0.35 \mathrm{~K}$ showed, up to about $21.3 \mathrm{~T}$, only a gradual field-dependent broadening. Beyond this field, i.e., above the estimated Pauli limit, the spectra broaden considerably until, at about $25 \mathrm{~T}$, the linewidth of the spectra is indistinguishable from that of the normal state [50]. The abrupt change of the NMR spectra around the Pauli limit is taken as evidence for a Zeeman-driven phase transition into another superconducting state, giving further evidence for the FFLO state.

In the other mentioned NMR work by the group in Grenoble, a very strong enhancement of the spin-lattice relaxation rate, $T_{1}^{-1}$, was found when going from the normal-conducting to the FFLO state as a function of temperature [51]. A similar enhancement was found when going from the uniform superconducting to the FFLO state as a function of magnetic field. This was attributed to excess low-energy density of states due to Andreev bound states formed near the nodes of the FFLO order parameter and was taken as a direct evidence for a modulated FFLO order parameter [51]. Figure 7 shows the field evolution of the NMR relaxation rate divided by temperature, $\left(T_{1} T\right)^{-1}$, at various temperatures (see the Supplemental Information in Ref. [52]). At 1.1 K, similar as reported in Ref. [51], a large enhancement of $\left(T_{1} T\right)^{-1}$, compared to the normal-state value, is seen when entering the FFLO state above and around the zero-temperature Pauli limit (dashed vertical line in Figure 7). However, at $0.35 \mathrm{~K}$, a temperature much lower than investigated in [51], no enhancement in $\left(T_{1} T\right)^{-1}$ is found. This may be explained by an Andreev bound-state energy larger than the thermal energy and a concomitant reduced influence of these states on the relaxation rate.

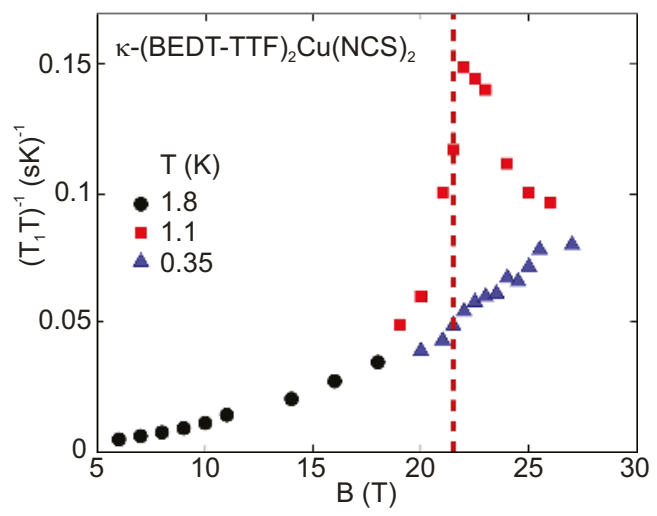

Figure 7. Field dependence of the spin-lattice relaxation rate, $T_{1}^{-1}$, divided by temperature of $\kappa$-(BEDT-TTF $)_{2} \mathrm{Cu}(\mathrm{NCS})_{2}$. The data taken at $1.1 \mathrm{~K}$ exhibit an increase much beyond the normal-state value in the region where the FFLO phase occurs, whereas the effect is quenched at the lower temperature of $0.35 \mathrm{~K}$. The dashed vertical line depicts the Pauli limit.

For $\beta^{\prime \prime}$-(BEDT-TTF) ${ }_{2} \mathrm{SF}_{5} \mathrm{CH}_{2} \mathrm{CF}_{2} \mathrm{SO}_{3}$, more detailed NMR studies are possible due to the field-temperature range easier accessible with superconducting magnets and available cryostat systems. For the measurements, as before, ${ }^{13} \mathrm{C}$ isotopes were introduced on the two central carbon sites of the BEDT-TTF molecules [52]. These sites are the most sensitive to the conducting electrons in the 2D BEDT-TTF-based materials. In [52], NMR spectra were taken for a number of accurately in-plane aligned magnetic fields at 0.13 and $1.7 \mathrm{~K}$. Figure 8 a shows the field-dependent shift of the NMR spectra, or more precisely the first moments of the NMR spectral positions, at these temperatures. At low fields, below about $9 \mathrm{~T}$ in the Shubnikov phase, only a moderate increase of the shift due to orbital contributions is visible. In the normal state, above $10 \mathrm{~T}$ at $1.7 \mathrm{~K}$, the NMR line shift is constant and caused by the hyperfine fields associated with the paramagnetic spin susceptibility. Between these two limits, the hyperfine fields at the ${ }^{13} \mathrm{C}$ sites increase steeply evidencing the appearance of the nonuniform electron polarization expected for the FFLO state. These line shifts are accompanied 
by considerable line broadenings of the NMR spectra (see [18,52]). The onsets of the rapid line-shift increase fits favorably to the dotted line plotted in Figure 6. The NMR data at $1.7 \mathrm{~K}$ indicate that the FFLO state appears at 9 and is present at $9.5 \mathrm{~T}$, whereas at $0.13 \mathrm{~K}$, the nonuniform superconductivity evolves above $9 \mathrm{~T}$ and remains up to the highest measured field of $11.9 \mathrm{~T}$, in agreement with the specific-heat data.

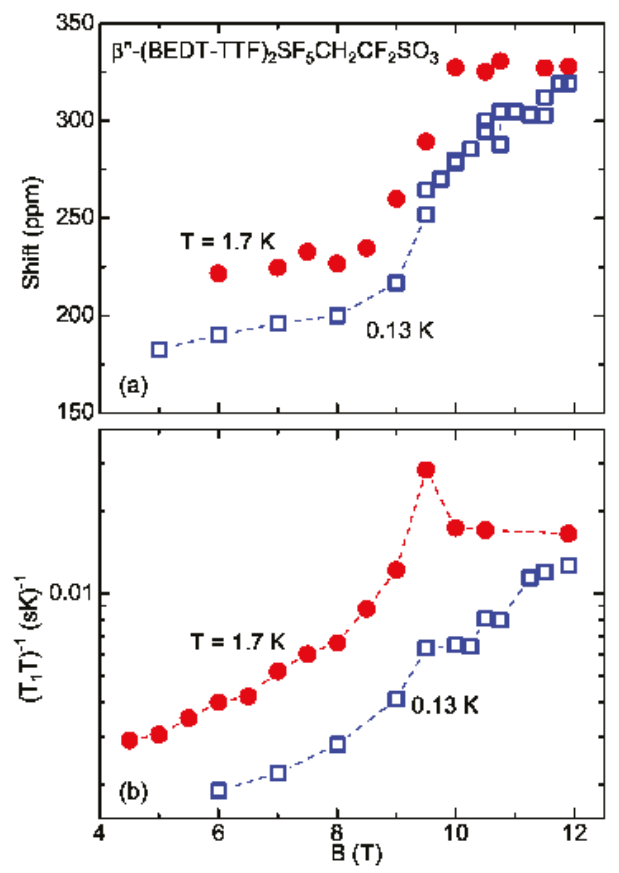

Figure 8. (a) Field dependence of the ${ }^{13} \mathrm{C}$ NMR shift in $\beta^{\prime \prime}$-(BEDT-TTF) ${ }_{2} \mathrm{SF}_{5} \mathrm{CH}_{2} \mathrm{CF}_{2} \mathrm{SO}_{3}$. (b) Spin-lattice relaxation rate divided by temperature, $\left(T_{1} T\right)^{-1}$, vs magnetic field. The data taken at $T=1.7 \mathrm{~K}$ show an increase at $9.3 \mathrm{~T}$ greater than the normal-state value, similar as visible in Figure 7 for $\kappa$-(BEDT-TTF $)_{2} \mathrm{Cu}(\mathrm{NCS})_{2}$ and discussed in connection with the occurrence of the FFLO phase. The dashed lines connecting the data points are guides to the eye.

Complementary field-dependent data of the spin-lattice relaxation rate are shown in Figure 8b. Similar as for $\kappa$-(BEDT-TTF) ${ }_{2} \mathrm{Cu}(\mathrm{NCS})_{2}$ (Figure 7 ), a clear enhancement of $\left(T_{1} T\right)^{-1}$ appears at $1.7 \mathrm{~K}$ indicating as well the existence of the FFLO state even at this higher temperature. At $0.13 \mathrm{~K}$, no enhancement in $\left(T_{1} T\right)^{-1}$ is detected, equally consistent for both investigated organic superconductors. As mentioned, this may be a signature of Andreev bound states in the FFLO phase. However, whether this assignment is correct needs further proof.

In the FFLO state, several types of periodic structures of the order parameter, one or two dimensional, may occur [8,9,53-55]. Accordingly, nonuniform electron-spin polarizations appear that lead to spatially oscillating local fields at the ${ }^{13} \mathrm{C}$ nuclei sites and corresponding characteristic broadenings of the NMR spectra. Assuming the simplest modulation of the order parameter with a single- $q$ (i.e., one-wavelength) sinusoidal oscillation, the NMR line broadens to a double-horn structure, in case the original NMR line is not already too wide due to other sample-inherent local-field inhomogeneities [18]. In the present case, the two inequivalent BEDT-TTF molecules per unit cell with two ${ }^{13} \mathrm{C}$ each lead to four main NMR lines (ignoring dipole-dipole splitting) that can be well resolved in the normal state [52]. 
The blue line in Figure 9 shows a measured NMR spectrum for $\beta^{\prime \prime}-(\mathrm{BEDT}-\mathrm{TTF})_{2} \mathrm{SF}_{5} \mathrm{CH}_{2} \mathrm{CF}_{2} \mathrm{SO}_{3}$ at $0.13 \mathrm{~K}$ and $10.25 \mathrm{~T}$. This spectrum can be simulated using the mentioned simple $1 \mathrm{D}$ sinusoidal modulation of the spin polarization (green line in Figure 9). Thereby, the different orbital parts and hyperfine couplings of the four independent, Gaussian-broadened main NMR lines (red and orange lines) have been taken into account (see Ref. [52] for details). The simulated total NMR line nicely agrees with the measured data. Indeed, assuming a 2D order-parameter modulation for the NMR-line simulation fails to describe the measured data. The NMR spectra, therefore, give clear microscopic evidence for a spatially single- $q 1 \mathrm{D}$ modulated nonuniform superconducting state, fully in line with the long-sought FFLO prediction.

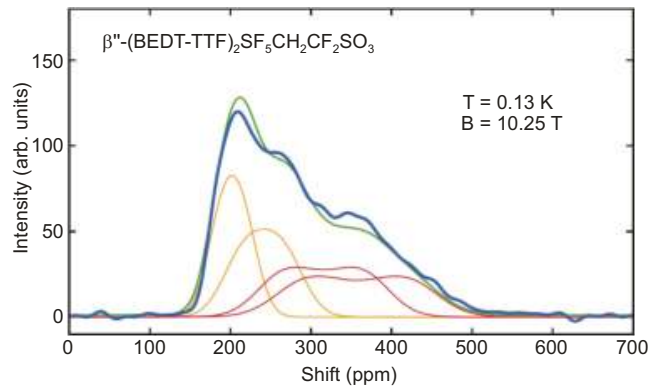

Figure 9. Experimental NMR spectrum (blue) of $\beta^{\prime \prime}$-(BEDT-TTF) ${ }_{2} \mathrm{SF}_{5} \mathrm{CH}_{2} \mathrm{CF}_{2} \mathrm{SO}_{3}$ at $0.13 \mathrm{~K}$ and $10.25 \mathrm{~T}$ compared with that generated by assuming a 1D sinusoidal modulation of the spin polarization (green). The orange and red lines depict the four independent contributions.

\section{Other FFLO Candidate Materials}

The question arises as to whether FFLO states are unique for 2D organic superconductors. Indeed, the conditions to observe FFLO states are ideal in these molecular conductors, namely high crystal quality with long mean-free paths, very large orbital critical fields with corresponding large Maki parameters for in-plane aligned magnetic fields, and a rather extended stability region of the FFLO phases in the $B-T$ phase diagram due to the two dimensionality. However, other material classes as well fulfil these conditions and FFLO states are to be expected.

Heavy-fermion materials, for example, are good candidates for showing FFLO states: The orbital critical fields are often high and may exceed the Pauli limit due to the large effective masses and anisotropies favor the occurrence of nonuniform pairing. Indeed, in the heavy-fermion superconductor $\mathrm{CeCoIn}_{5}$ clear thermodynamic and microscopic evidence for the existence of an additional superconducting state at low temperatures and high magnetic fields was found [56-58]. Initially, this state was suggested to be a realization of a FFLO state. This, however, was revised a few years later when neutron-scattering data revealed clearly the existence of incommensurate antiferromagnetic order just inside this superconducting state. Thereby, the incommensurable antiferromagnetic wave vector $Q$ was proven to be field independent [59]. This now-called $Q$ phase certainly is a unique and fascinating state of matter, but clearly not a realization of the originally proposed FFLO states [60-63]. Whether it is a more complicated version of such a state [64] or is of completely different origin is still the subject of intense research.

Recently, specific-heat and magnetic-torque experiments revealed compelling evidence for the existence of a superconducting high-field low-temperature state above the Pauli limit in $\mathrm{KFe}_{2} \mathrm{As}_{2}$ [65]. The anisotropic iron-pnictide superconductors, therefore, appear to be the second material class in which thermodynamic data strongly suggest the existence of an FFLO state. Further work is needed to establish this finding with additional evidence. 


\section{Conclusions and Outlook}

More than 50 years after the theoretical work of Fulde-Ferrell and Larkin-Ovchinnikov, there is now solid evidence for the realization of FFLO states in organic superconductors. Various experiments have provided strong support for this notion. In particular, specific-heat and NMR experiments helped to determine the superconducting phase diagram and to gain microscopic insight into the nonuniform modulation of the order parameter. A 1D sinusoidally oscillating spin polarization can favorably explain the NMR spectra. Further experiments that would allow one to directly determine the modulation wave vector, such as neutron scattering or scanning tunneling microscopy, would be highly desirable. In addition, there are predictions for the existence of a quantum critical point at the transition from the FFLO to the normal state [66] for which experimental evidence would be needed. Finally, first indication for the existence of an FFLO state was found very recently in an iron-pnictide superconductor widening the possibility to further study the nature of these nonuniform superconducting states in different material classes.

Acknowledgments: Contributions to this work by B. Bergk, R. Beyer, S. E. Brown, A. Demuer, E. L. Green, G. Koutroulakis, H. Kühne, R. Lortz, S. Molatta, Y. Nakazawa, J. A. Schlueter, I. Sheikin, S. Yasin, H. H. Wang, Y. Wang, and G. Zwicknagl are gratefully acknowledged. Part of the work was supported by HLD at HZDR, member of the European Magnetic Field Laboratory (EMFL).

Conflicts of Interest: The author declares no conflict of interest.

\section{References}

1. Clogston, A.M. Upper limit for the critical field in hard superconductors. Phys. Rev. Lett. 1962, 9, $266-267$. [CrossRef]

2. Chandrasekhar, B.S. A note on the maximum critical field of high-field superconductors. Appl. Phys. Lett. 1962, 1, 7-8. [CrossRef]

3. Fulde, P.; Ferrell, R.A. Superconductivity in a strong spin exchange field. Phys. Rev. 1964, 135, A550-A563. [CrossRef]

4. Larkin, A.I.; Ovchinnikov, Y.N. Inhomogeneous state of superconductors. Sov. Phys. JETP 1965, 20, 762-768.

5. Maki, K.; Tsuneto, T. Pauli Paramagnetism and Superconducting State. Prog. Theor. Phys. 1964, 31, 945-956. [CrossRef]

6. Gruenberg, L.W.; Gunther, L. Fulde-Ferrell effect in type-II superconductors. Phys. Rev. Lett. 1966, 16, 996-998. [CrossRef]

7. Aslamazov, L.G. Influence of impurities on the existence of an imhomogeneous state in a ferromagnetic superconductor. Sov. Phys. JETP 1969, 28, 773-775.

8. Matsuda, Y.; Shimahara, H. Fulde-Ferrell-Larkin-Ovchinnikov state in heavy fermion superconductors. J. Phys. Soc. Jpn. 2007, 76, 051005. [CrossRef]

9. Zwicknagl, G.; Wosnitza, J. Breaking translational invariance by population imbalance: The Fulde-Ferrell-LarkinOvchinnikov states. In BCS: 50 years; Cooper, L.N.; Feldman, D., Eds.; World Scientific: Singapore, 2011; pp. 337-371.

10. Wang, Q.; Hu, C.-R.; Ting, C.-S. Impurity-induced configuration-transition in the Fulde-Ferrell-Larkin-Ovchinnikov state of a $d$-wave superconductor. Phys. Rev. B 2007, 75, 184515. [CrossRef]

11. Cui, Q.; Yang, K. Fulde-Ferrell-Larkin-Ovchinnikov state in disordered s-wave superconductors. Phys. Rev. B 2008, 78, 054501. [CrossRef]

12. Ptok, A. The Fulde-Ferrell-Larkin-Ovchinnikov Superconductivity in Disordered Systems. Acta Phys. Pol. A 2010, 118, 420-422. [CrossRef]

13. Ptok, A.; Kapcia, K.J. Probe-type of superconductivity by impurity in materials with short coherence length: The $s$-wave and $\eta$-wave phases study. Supercond. Sci. Technol. 2015, 28, 045022. [CrossRef]

14. Tokiwa, Y.; Movshovich, R.; Ronning, F.; Bauer, E.D.; Papin, P.; Bianchi, A.D.; Rauscher, J.F.; Kauzlarich, S.M.; Fisk, Z. Anisotropic Effect of Cd and Hg Doping on the Pauli Limited Superconductor CeCoIn 5 . Phys. Rev. Lett. 2008, 101, 037001. [CrossRef] 
15. Movshovich, R.; Tokiwa, Y.; Kurita, N.; Ronning, F.; Bauer, E.D.; Bianchi, A.; Papin, P.; Fisk, Z. Possible Fulde-Ferrel-Larkin-Ovchinnikov Inhomogeneous Superconducting State in CeCoIn 5 : Cd- and Hg-doping Studies. J. Supercond. Nov. Magn. 2009, 22, 291-293. [CrossRef]

16. Tokiwa, Y.; Movshovich, R.; Ronning, F.; Bauer, E.D.; Bianchi, A.D.; Fisk, Z.; Thompson, J.D. Anomalous effect of doping on the superconducting state of CeCoIn 5 in high magnetic fields. Phys. Rev. B 2010, 82, 220502. [CrossRef]

17. Ptok, A. The influence of the dimensionality of the system on the realization of unconventional Fulde-Ferrell-Larkin-Ovchinnikov pairing in ultra-cold Fermi gases. J. Phys. Condens. Matter 2017, $29,475901$. [CrossRef] [PubMed]

18. Wosnitza, J. FFLO states in layered organic superconductors. Ann. Phys. 2018, 530, 1700282. [CrossRef]

19. Sheehy, D.E.; Radzihovsky, L. BEC-BCS crossover, phase transitions and phase separation in polarized resonantly-paired superfluids. Ann. Phys. 2007, 322, 1790-1924. [CrossRef]

20. Casalbuoni, R.; Nardulli, G. Inhomogeneous superconductivity in condensed matter and QCD. Rev. Mod. Phys. 2004, 76, 263-320. [CrossRef]

21. Zwierlein, M.W.; Schirotzek, A.; Schunck, C.H.; Ketterle, W. Fermionic superfluidity with imbalanced spin populations. Science 2006, 311, 492. [CrossRef] [PubMed]

22. Partridge, G.B.; Li, W.; Kamar, R.I.; Liao, Y.; Hulet, R.G. Pairing and phase separation in a polarized Fermi gas. Science 2006, 311, 503-505. [CrossRef] [PubMed]

23. Soto, S.M.D.; Slichter, C.P.; Kini, A.M.; Wang, H.H.; Geiser, U.; Williams, J.M. ${ }^{13}$ C NMR studies of the normal and superconducting states of the organic superconductor $\kappa-(\mathrm{ET})_{2} \mathrm{Cu}\left[\mathrm{N}(\mathrm{CN})_{2}\right] \mathrm{Br}$. Phys. Rev. B 1995, 52, 10364-10368. [CrossRef]

24. Mayaffre, H.; Wzietek, P.; Jérome, D.; Lenoir, C.; Batail, P. Superconducting State of $\kappa-(\mathrm{ET})_{2} \mathrm{Cu}\left[\mathrm{N}(\mathrm{CN})_{2}\right] \mathrm{Br}$ Studied by ${ }^{13} \mathrm{C}$ NMR: Evidence for Vortex-Core-Induced Nuclear Relaxation and Unconventional Pairing. Phys. Rev. Lett. 1995, 75, 4122-4125. [CrossRef] [PubMed]

25. Elsinger, H.; Wosnitza, J.; Wanka, S.; Hagel, J.; Schweitzer, D.; Strunz, W. $\kappa-(B E D T-T T F){ }_{2} \mathrm{Cu}\left[\mathrm{N}(\mathrm{CN})_{2}\right] \mathrm{Br}$ : A Fully Gapped Strong-Coupling Superconductor. Phys. Rev. Lett. 2000, 84, 6098-6101. [CrossRef]

26. Wosnitza, J. Superconductivity in Layered Organic Metals. Crystals 2012, 2, 248-265. [CrossRef]

27. Lang, M.; Müller, J. Organic Superconductors. In Superconductivity; Bennemann, K.H., Ketterson J.B., Eds.; Springer: Berlin/Heidelberg, Germany, 2008; Volume II, pp. 1155-1223.

28. Ishiguro, T.; Yamaji, K.; Saito G. Organic Superconductors; Springer: Berlin/Heidelberg, Germany, 1998.

29. Wosnitza, J. Superconducting properties of quasi-two-dimensional organic metals. Physics C 1999, 317-318, 98-107. [CrossRef]

30. Wosnitza, J. Perspectives on the Nature of Superconductivity in Organic Metals. J. Low Temp. Phys. 1999, 117, 1701-1710. [CrossRef]

31. Tanatar, M.; Ishiguro, T.; Tanaka, H.; Kobayashi, H. Magnetic field-temperature phase diagram of the quasi-two-dimensional organic superconductor $\lambda$-(BETS) ${ }_{2} 2 \mathrm{GaCl}_{4}$ studied via thermal conductivity. Phys. Rev. B 2002, 66, 134503. [CrossRef]

32. Uji, S.; Terashima, T.; Nishimura, M.; Takahide, Y.; Konoike, T.; Enomoto, K.; Cui, H.; Kobayashi, H.; Kobayashi, A.; Tanaka, H.; et al. Vortex Dynamics and the Fulde-Ferrell-Larkin-Ovchinnikov State in a Magnetic-Field-Induced Organic Superconductor. Phys. Rev. Lett. 2006, 97, 157001. [CrossRef] [PubMed]

33. Uji, S.; Iida, Y.; Sugiura, S.; Isono, T.; Sugii, K.; Kikugawa, N.; Terashima, T.; Yasuzuka, S.; Akutsu, H.; Nakazawa, Y.; et al. Fulde-Ferrell-Larkin-Ovchinnikov superconductivity in the layered organic superconductor $\beta^{\prime \prime}$-(BEDT-TTF $)_{4}\left[\left(\mathrm{H}_{3} \mathrm{O}\right) \mathrm{Ga}\left(\mathrm{C}_{2} \mathrm{O}_{4}\right)_{3}\right] \mathrm{C}_{6} \mathrm{H}_{5} \mathrm{NO}_{2}$. Phys. Rev. B 2018, 97, 144505. [CrossRef]

34. Cho, K.; Smith, B.E.; Coniglio, W.A.; Winter, L.E.; Agosta, C.C.; Schlueter, J.A. Upper critical field in the organic superconductor $\beta^{\prime \prime}-(\mathrm{ET})_{2} \mathrm{SF}_{5} \mathrm{CH}_{2} \mathrm{CF}_{2} \mathrm{SO}_{3}$ : Possibility of Fulde-Ferrell-Larkin-Ovchinnikov state. Phys. Rev. B 2009, 79, 220507(R). [CrossRef]

35. Coniglio, W.A.; Winter, L.E.; Cho, K.; Agosta, C.C.; Fravel, B; Montgomery, L.K. Superconducting phase diagram and FFLO signature in $\lambda$-(BETS ${ }_{2} \mathrm{GaCl}_{4}$ from rf penetration depth measurements. Phys. Rev. B 2011, 83, 224507. [CrossRef]

36. Agosta, C.C.; Jin, J.; Coniglio, W.A.; Smith, B.E.; Cho, K.; Stroe, I.; Martin, C.; Tozer, S.W.; Murphy, T.P.; Palm, E.C.; et al. Experimental and semiempirical method to determine the Pauli-limiting field in quasi-two-dimensional superconductors as applied to $\kappa$-(BEDT-TTF $)_{2} \mathrm{Cu}(\mathrm{NCS})_{2}$ : Strong evidence of a FFLO state. Phys. Rev. B 2012, 85, 214514. [CrossRef] 
37. Lortz, R.; Wang, Y.; Demuer, A.; Böttger, P.H.M.; Bergk, B.; Zwicknagl, G.; Nakazawa, Y.; Wosnitza, J. Calorimetric Evidence for a Fulde-Ferrell-Larkin-Ovchinnikov Superconducting State in the Layered Organic Superconductor $\kappa$-(BEDT-TTF) ${ }_{2} \mathrm{Cu}(\mathrm{NCS})_{2}$. Phys. Rev. Lett. 2007, 99, 187002. [CrossRef] [PubMed]

38. Bergk, B.; Demuer, A.; Sheikin, I.; Wang, Y.; Wosnitza, J.; Nakazawa, Y.; Lortz, R. Magnetic torque evidence for the Fulde-Ferrell-Larkin-Ovchinnikov state in the layered organic superconductor $\kappa$-(BEDT-TTF $)_{2} \mathrm{Cu}(\mathrm{NCS})_{2}$. Phys. Rev. B 2011, 83, 064506. [CrossRef]

39. Agosta, C.C.; Fortune, N.A.; Hannahs, S.T.; Gu, S.; Liang, L.; Park, J.; Schlueter, J.A. Calorimetric Measurements of Magnetic-Field-Induced Inhomogeneous Superconductivity Above the Paramagnetic Limit. Phys. Rev. Lett. 2017, 118, 267001. [CrossRef]

40. Beyer, R.; Bergk, B.; Yasin, S.; Schlueter, J.A.; Wosnitza, J. Angle-Dependent Evolution of the Fulde-Ferrell-Larkin-Ovchinnikov State in an Organic Superconductor. Phys. Rev. Lett. 2012, 109, 027003. [CrossRef] [PubMed]

41. Beyer R.; Wosnitza, J. Emerging evidence for FFLO states in layered organic superconductors. Low Temp. Phys. 2013, 39, 293-300. [CrossRef]

42. Werthamer, N.R.; Helfand, E.; Hohenberg, P.C. Temperature and Purity Dependence of the Superconducting Critical Field, $H_{c 2}$. III. Electron Spin and Spin-Orbit Effects. Phys. Rev. 1966, 147, 295-302. [CrossRef]

43. Wosnitza, J.; Wanka, S.; Hagel, J.; Reibelt, M.; Schweitzer, D.; Schlueter, J.A. Thermodynamic properties of quasi-two-dimensional organic superconductors. Synth. Met. 2003, 133-134, 201-203. [CrossRef]

44. Wosnitza, J.; Wanka, S.; Hagel, J.; Balthes, E.; Harrison, N.; Schlueter, J.A.; Kini, A.M.; Geiser, U.; Mohtasham, J.; Winter, R.W.; et al. Two-dimensional Fermi liquid with fixed chemical potential. Phys. Rev. B 2000, 61, 7383-7387. [CrossRef]

45. Wosnitza, J.; Hagel, J.; Qualls, J.S.; Brooks, J.S.; Balthes, E.; Schweitzer, D.; Schlueter, J.A.; Geiser, U.; Mohtasham, J.; Winter, R.W.; et al. Coherent versus incoherent interlayer transport in layered metals. Phys. Rev. B 2002, 65, 180506(R). [CrossRef]

46. Wosnitza, J. Quasi-Two-Dimensional Organic Superconductors. J. Low Temp. Phys. 2007, 146, 641-667. [CrossRef]

47. Wosnitza, J.; Wanka, S.; Qualls, J.S.; Brooks, J.S.; Mielke, C.H.; Harrison, N.; Schlueter, J.A.; Williams, J.M.; Nixon, P.G.; Winter, R.W.; et al. Fermiology of the Organic Superconductor $\beta^{\prime \prime}$-(BEDT-TTF) ${ }_{2} \mathrm{SF}_{5} \mathrm{CH}_{2} \mathrm{CF}_{2} \mathrm{SO}_{3}$. Synth. Met. 1999, 103, 2000-2001. [CrossRef]

48. Zwicknagl, G. TU Braunschweig, Braunschweig, Germany. Private Communication, 2017.

49. Croitoru, M.D.; Houzet, M.; Buzdin, A.I. In-Plane Magnetic Field Anisotropy of the Fulde-Ferrell-Larkin-Ovchinnikov State in Layered Superconductors. Phys. Rev. Lett. 2012, 108, 207005. [CrossRef]

50. Wright, J.A.; Green, E.; Kuhns, P.; Reyes, A.; Brooks, J.; Schlueter, J.; Kato, R.; Yamamoto, H.; Kobayashi, M.; Brown, S.E. Zeeman-Driven Phase Transition within the Superconducting State of $\kappa$-(BEDT-TTF $)_{2} \mathrm{Cu}(\mathrm{NCS})_{2}$. Phys. Rev. Lett. 2011, 107, 087002. [CrossRef]

51. Mayaffre, H.; Krämer, S.; Horvatić, M.; Berthier, C.; Miyagawa, K.; Kanoda, K.; Mitrovic, V.F. Evidence of Andreev bound states as a hallmark of the FFLO phase in $\kappa$-(BEDT-TTF $)_{2} \mathrm{Cu}(\mathrm{NCS})_{2}$. Nat. Phys. 2014, 10, 928-932. [CrossRef]

52. Koutroulakis, G.; Kühne, H.; Schlueter, J.A.; Wosnitza, J.; Brown, S.E. Microscopic Study of the Fulde-Ferrell-LarkinOvchinnikov State in an All-Organic Superconductor. Phys. Rev. Lett. 2016, 116, 067003. [CrossRef]

53. Shimahara, H. Structure of the Fulde-Ferrell-Larkin-Ovchinnikov State in Two-Dimensional Superconductors. J. Phys. Soc. Jpn. 1998, 67, 736-739. [CrossRef]

54. Wang, Q.; Chen, H.-Y.; Hu, C.-R.; Ting, C.S. Local Tunneling Spectroscopy as a Signature of the Fulde-Ferrell-Larkin-Ovchinnikov State in s- and d-Wave Superconductors. Phys. Rev. Lett. 2006, 96, 117006. [CrossRef]

55. Zhou, T.; Ting, S. Phase diagram and local tunneling spectroscopy of the Fulde-Ferrell-Larkin-Ovchinnikov states of a two-dimensional square-lattice d-wave superconductor. Phys. Rev. B 2009, 80, 224515. [CrossRef]

56. Bianchi, A.; Movshovich, R.; Capan, C.; Pagliuso, P.G.; Sarrao, J.L. Possible Fulde-Ferrell-Larkin-Ovchinnikov Superconducting State in CeCoIn 5 . Phys. Rev. Lett. 2003, 91, 187004. [CrossRef] [PubMed]

57. Radovan, H.A.; Fortune, N.A.; Murphy, T.P.; Hannahs, S.T.; Palm, E.C.; Tozer, S.W.; Hall, D. Magnetic enhancement of superconductivity from electron spin domains. Nature 2003, 425, 51-54. [CrossRef] [PubMed] 
58. Kakuyanagi, K.; Saitoh, M.; Kumagai, K.; Takashima, S.; Nohara, M.; Takagi, H.; Matsuda, Y. Texture in the Superconducting Order Parameter of $\mathrm{CeCoIn}_{5}$ Revealed by Nuclear Magnetic Resonance. Phys. Rev. Lett. 2005, 94, 047602. [CrossRef]

59. Kenzelmann, M.; Strässle, T.; Niedermayer, C.; Sigrist, M.; Padmanabhan, B.; Zolliker, M.; Bianchi, A.D.; Movshovich, R.; Bauer, E.D.; Sarrao, J.L.; et al. Coupled Superconducting and Magnetic Order in CeCoIn 5 . Science 2008, 321, 1652-1654. [CrossRef] [PubMed]

60. Kenzelmann, M.; Gerber, S.; Egetenmeyer, N.; Gavilano, J.L.; Strässle, T.; Bianchi, A.D.; Ressouche, E.; Movshovich, R.; Bauer, E.D.; Sarrao, J.L.; et al. Evidence for a Magnetically Driven Superconducting $Q$ Phase of CeCoIn 5 . Phys. Rev. Lett. 2010, 104, 127001. [CrossRef] [PubMed]

61. Raymond, S.; Lapertot, G. Ising Incommensurate Spin Resonance of CeCoIn 5 : A Dynamical Precursor of the Q Phase. Phys. Rev. Lett. 2015, 115, 037001. [CrossRef] [PubMed]

62. Mierzejewski, M.; Ptok, A.; Maśka, M.M. Mutual enhancement of magnetism and Fulde-Ferrell-Larkin-Ovchinnikov superconductivity in CeCoIn 5 . Phys. Rev. B 2009, 80, 174525. [CrossRef]

63. Ptok, A.; Maśka, M.M.; Mierzejewski, M. Coexistence of superconductivity and incommensurate magnetic order. Phys. Rev. B 2011, 84, 094526. [CrossRef]

64. Hosoya, K.; Ikeda, R. Possible triplet superconducting order in a magnetic superconducting phase induced by paramagnetic pair breaking. Phys. Rev. B 2017, 95, 224513. [CrossRef]

65. Cho, C.; Yang, J.H.; Yuan, N.F.Q.; Shen, J.; Wolf, T.; Lortz R. Thermodynamic Evidence for the Fulde-Ferrell-Larkin-Ovchinnikov State in the $\mathrm{KFe}_{2} \mathrm{As}_{2}$ Superconductor. Phys. Rev. Lett. 2017, 119, 217002. [CrossRef] [PubMed]

66. Piazza, F.; Zwerger, W.; Strack, P. FFLO strange metal and quantum criticality in two dimensions: Theory and application to organic superconductors. Phys. Rev. B 2016, 93, 085112. [CrossRef]

(c) 2018 by the author. Licensee MDPI, Basel, Switzerland. This article is an open access article distributed under the terms and conditions of the Creative Commons Attribution (CC BY) license (http:/ / creativecommons.org/licenses/by/4.0/). 
Article

\title{
Inhomogeneous Superconductivity in Organic and Related Superconductors
}

\author{
Charles C. Agosta \\ Department of Physics, Clark University, 950 Main Street, Worcester, MA 01610, USA; cagosta@clarku.edu \\ Received: 16 May 2018; Accepted: 24 June 2018; Published: 11 July 2018
}

\begin{abstract}
Evidence of inhomogeneous superconductivity, in this case superconductivity with a spatially modulated superconducting order parameter, has now been found in many materials and by many measurement methods. Although the evidence is strong, it is circumstantial in the organic superconductors, scant in the pnictides, and complex in the heavy Fermions. However, it is clear some form of exotic superconductivity exists at high fields and low temperatures in many electronically anisotropic superconductors. The evidence is reviewed in this article, and examples of similar measurements are compared across different families of superconductors. An effort is made to find a consistent way to measure the superconducting energy gap across all materials, and use this value to predict the Clogston-Chandrasakhar paramagnetic limit $H_{P}$. Methods for predicting the existence of inhomogeneous superconductivity are shown to work for the organic superconductors, and then used to suggest new materials to study.
\end{abstract}

Keywords: organic superconductors; inhomogeneous superconductors; FFLO; quasi 2D materials

\section{Introduction}

It is becoming evident that an exotic superconducting phase, associated with inhomogeneous superconductivity, has been realized in a number of organic conductors, and possibly in a pnictide superconductor as well. In this chapter, we will compare experimental evidence that supports the discovery of Fulde, Ferrell, Larkin, Ovchinnikov (FFLO) states, and examine methods to search for new materials where a FFLO state could be stabilized.

The most compelling evidence for the FFLO state comes from systematic measurements of quasi-two-dimensional organic superconductors that suggest that an inhomogeneous superconducting state can be stabilized if a magnetic field is applied precisely parallel to the conducting layers. This exotic superconducting state, a tunable mixture of a spatially modulated superconducting order parameter and a magnetic lattice created by the remaining unpaired electrons, was predicted over 50 years ago, and is called the FFLO state after the authors Fulde, Ferrell, Larkin, and Ovchinnikov, who first predicted it $[1,2]$. The topology of the FFLO state enhances the stability of the superconducting state, and allows superconductivity to survive at higher magnetic fields than what is predicted from the size of the superconducting energy gap. It only exists when orbital effects, manifested as vortices in superconductors, can be suppressed, and an advantage of anisotropic superconductors is that they can be studied with or without the effects of vortices, by aligning the magnetic field perpendicular or parallel to the conducting planes, respectively. The FFLO state is highly tunable via temperature, the direction and strength of the magnetic field, and pressure. It is sensitive to impurity scattering and a magnetic scattering process called spin-orbit scattering, an effect that magnetically spin flips itinerant electrons. These attributes make it a rich system for the study of correlated electrons, and, in particular, superconductivity. Many of the details of the FFLO state will be discussed below with references. The discussion will focus on organic superconductors where the evidence for the FFLO state is more compelling, although pnictides and heavy Fermions will be addressed briefly. 
A sampling of the best recent evidence for the FFLO state comes from the following measurements of organic superconductors:

- $\quad \kappa$-(BEDT-TTF $)_{2} \mathrm{Cu}(\mathrm{NCS})_{2}$, specific heat [3], torque magnetometry [4,5], rf penetration depth [6], resistivity [5], NMR [7,8],

- $\lambda$-(BETS $)_{2} \mathrm{GaCl}_{4}$ thermal conductivity [9], rf penetration depth [10],

- $\quad \beta^{\prime \prime}-(\mathrm{ET})_{2} \mathrm{SF}_{5} \mathrm{CH}_{2} \mathrm{CF}_{2} \mathrm{SO}_{3}$ rf penetration depth [11], NMR [12].

For the rest of this manuscript, we will use these abbreviations for the following compounds:

- $\quad \kappa-(\mathrm{BEDT}-\mathrm{TTF})_{2} \mathrm{Cu}(\mathrm{NCS})_{2}: \kappa \mathrm{ET}-\mathrm{CuNCS}$,

- $\lambda$-(BETS $)_{2} \mathrm{GaCl}_{4}: \lambda \mathrm{BETS}-\mathrm{GaCl}$,

- $\quad \beta^{\prime \prime}-(\mathrm{ET})_{2} \mathrm{SF}_{5} \mathrm{CH}_{2} \mathrm{CF}_{2} \mathrm{SO}_{3}: \beta^{\prime \prime} \mathrm{ET}-\mathrm{SF} 5$,

- $\quad \kappa-(\mathrm{ET})_{2} \mathrm{Cu}\left[\mathrm{N}(\mathrm{CN})_{2}\right] \mathrm{Br}: \kappa \mathrm{ET}-\mathrm{Br}$.

Given the number of materials now showing evidence of the FFLO state, and the wide variety of measurement techniques available to investigate the details of the FFLO state, the prospect of learning new fundamental facts about the microscopic mechanisms of superconductivity is compelling. For example, as detailed measurements of the FFLO state have become available, comparison between theory, particularly early theory [13-19], and experiments (see references throughout the article) are finally possible. Certain results have been consistent with theory, such as the first order nature of the vortex state to the FFLO state phase line [3,20], but other aspects of the FFLO state, such as the onset temperature, $T^{*}$, are not consistent with prevailing theory [17]. After a brief description of inhomogeneous superconductivity, the results of experiments that have been done to date will be reviewed to see how well the results match each other, and with what is expected from the theory of inhomogeneous superconductivity. In addition, tests will be described to determine if a superconductor is a candidate for inhomogeneous superconductivity. This analysis will begin to determine if the proposed FFLO states that have been discovered are universal, or specific to each material.

These studies are important for the basic and applied understanding of materials. As an example, superconductivity is sometimes related to the existence of a quantum critical point, and a quantum critical point may be an important signature of the FFLO state [21]. The FFLO state has also been studied, at least theoretically, in cold atom systems, and attempts have been made to create model systems to study FFLO interactions with highly tunable parameters [22-27]. On the more practical side, the understanding of correlated electron states in new materials is not only the basis of understanding phenomena such as superconductivity, but it is also the starting point for developing new electronic devices, many which will be based on magnetic properties. The FFLO superconducting state will further the understanding of the relationship between superconductivity and magnetism. Magnetism is now recognized as one of the key drivers of superconductivity, but the exact nature of the mechanism that lets magnetism drive superconductivity is not known (see, for instance, these reviews [28,29]). The interplay of magnetism and superconductivity is critical to the understanding of most superconductors of interest, including metallic, cuprate, and iron based pnictide materials.

\subsection{Inhomogeneous Superconductivity}

In order to understand the genesis of inhomogeneous superconductivity, it is important to understand what suppresses superconductivity. In most cases, superconductivity is destroyed in an external magnetic field due to vortices-non-superconducting regions containing a magnetic field line shielded by circulating Cooper pairs-which increase in density as the magnetic field is increased and ultimately displace the superconducting phase. Chandrasekhar [30] and, independently, Clogston [31] were the first to recognize that, if the formation of vortices could be suppressed, superconductivity could persist only to a magnetic field limit determined by the Pauli paramagnetism of the electrons 
denoted as $H_{P}$, and properly called the Chandrasekhar-Clogston Pauli paramagnetic limit [32]. We will refer to this level of magnetic field as the paramagnetic limit for short.

At this magnetic field, the energy to flip an electron spin between the up and down states, the Zeeman energy $\mu_{b} H$ exceeds the binding energy of the Cooper pairs, destroying the pairs. Soon after the Chandrasekhar and Clogston papers, it was found that the upper critical field of a superconductor in the paramagnetic limit changes from a continuous to a first-order transition below $t=T / T_{c} \sim 0.5$ [33,34]. After further study, it was proposed that, if Pauli paramagnetism was the dominant cause for limiting superconductivity and the superconductor was clean $(r>1$, where $r=\ell / \xi$ and $\ell$ is the mean free path of the quasiparticles and $\xi$ is the superconducting coherence length), a new kind of superconducting state could be stabilized at a magnetic field above $H_{P}$. This new superconducting state is the FFLO state and is characterized by Cooper pairs with non-zero momentum, and a spatially modulated order parameter $[1,2]$.

A diagram of this modified electron pairing is shown in Figure 1, reproduced from a recent article by Wosnitza [35]. A momentum $\mathbf{q}$ is added to one of the $\mathbf{k}$ vectors (electrons) to compensate for the energy difference in a magnetic field between the up and down spins, which creates an electron with non-zero momentum, but a Cooper pair with a center of mass momentum of zero. As described in the figure caption, in real material, more complex diagrams could exist. The Fermi surface could create a preferential direction for $\mathbf{q}$, creating a modulated order parameter in one dimension, or a set of $\mathbf{q}$ vectors creating a two- or three-dimensional modulation of the order parameter $[15,16,20]$. In particular, it is shown that, in higher magnetic fields, more complex combinations of $\mathbf{q}$-vectors form more complicated, higher dimensional order parameter patterns. In Figure 2, a cartoon taken from Agosta et al. [3], shows a simple real space model of a FFLO state with the q-vector aligned with the applied magnetic field. In a real, highly anisotropic organic superconductor as suggested by the discussion of Figure 1, the q-vector could also align in another direction in the conducting plane, for example perpendicular to the magnetic field, as determined by the anisotropic Fermi surface and as depicted in Figure $5 \mathrm{~b}$ from Mayaffre et al. [8]. The influence of the Fermi surface on the stability and the details of a FFLO state are discussed in a number of papers [36-40], including how an elliptical Fermi surface can improve the stability of the FFLO state [35]. Other symmetry breaking properties of the material such as the crystal anisotropies or the symmetry of the Cooper pair wave function could also influence the $\mathbf{q}$-vector, as described in many studies [13,15,16,20,38,41,42]. As suggested above, the greatest influence on $\mathbf{q}$ in the organic superconductors is probably the shape of the Fermi surface, particularly if the closed pockets are highly elliptical [35].
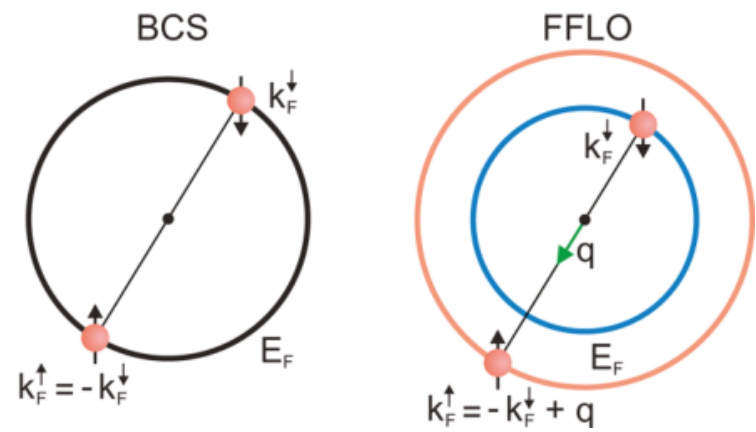

Figure 1. Color online. From Wosnitza [35] On the left, the traditional BCS Cooper pair is represented. On the right, the energy of the up and down electrons have been shifted by the magnetic field. A momentum $\mathbf{q}$ can be added to one of the electrons to create a zero momentum center of mass Cooper pair. In the diagram, the initial momentum of the electrons is isotropic. In real material, the shape of the Fermi surface could create more complex diagrams. 


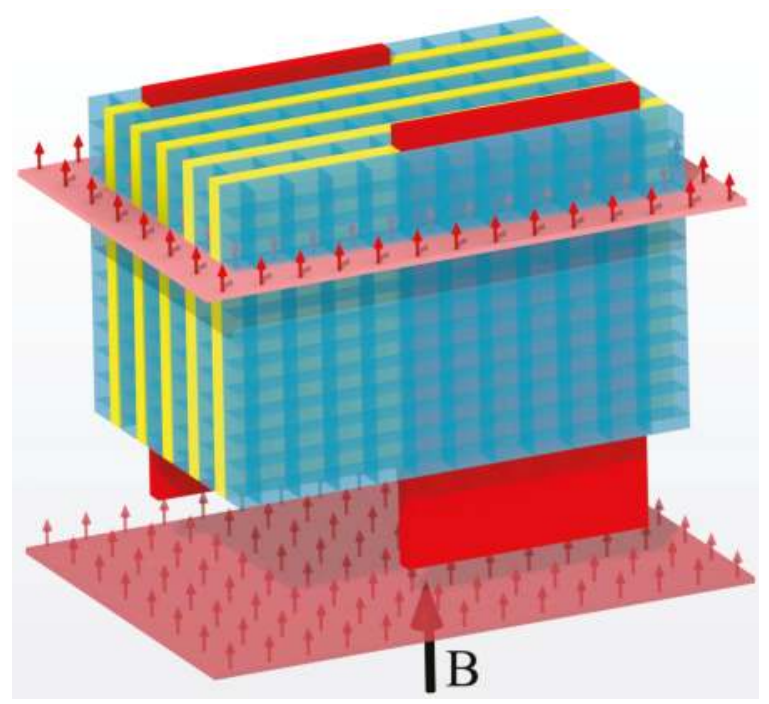

Figure 2. Color online, from [3]. Cartoon of the FFLO state showing the nodes in the order parameter as horizontal planes where we estimate the spin-polarization to be $\approx 10 \%$ at $25 \mathrm{~T}$ in the low temperature limit. In the diagram the black arrow labeled $\mathbf{B}$ represents the applied magnetic field, and the red arrows represent the net spin polarization. Although the diagram is schematic, all of the lengths are to scale; small boxes represent the unit cells of $\kappa$ ET-CuNCS, yellow slabs represent the least conducting layers of the crystal, and red rectangles represent Josephson core-less vortices at about the right distance apart in a $25 \mathrm{~T}$ field. The full height of the crystal is $\approx 20 \mathrm{~nm}$.

As another example of the effect of the crystal anisotropy, observe that the vortices depicted in Figure 2 are highly elongated Josephson vortices. This is due to the difficulty of driving super-currents perpendicular to the conducting planes in a highly anisotropic superconductor such as the crystalline organics where the layers are Josephson coupled. The perpendicular current density is limited to the value of the Josephson tunneling current density, and the vortex needs to elongate to find enough surface area to have equal current along the superconducting planes as there exists across the quasi-insulating layers, to form the vortex. The existence of inhomogeneous superconductivity in highly 2D Josephson coupled layered superconductors was treated early on, before the discovery of organic superconductors, by Bulaevskii [43]. There is no doubt that Josephson coupling exists in organic conductors, as it has been measured indirectly [44,45] as well as being imaged directly [46]. Furthermore, in a recent calculation, it is shown that, in these highly anisotropic organic superconductors, orbital currents are confined to just one layer [47].

\subsection{Critical Parameters}

Ideally, one should be able to measure a few critical parameters that will indicate if a material is in the paramagnetic limit at low temperatures or, in more interesting cases, has a FFLO or related inhomogeneous superconducting state. One of two parameters that are useful for this purpose is the Maki parameter

$$
\alpha_{M}=\sqrt{2} H_{o r b}^{0} / H_{P}
$$

where

$$
H_{o r b}^{0}=\left.0.7 T_{c} \frac{d H_{c 2}}{d T}\right|_{T_{c}}
$$


the orbital critical field at zero temperature [48], is the critical field as if there were no spin-paramagnetic pair breaking, just vortices. This formula was derived for 3D isotropic superconductors, and, for 2D materials, the more complicated results in Klemm et al. [32] or Schneider et al. [49] should be used; however for a simple comparison of the anisotropy between materials, the $H_{c 2}$ slope near $T_{\mathcal{c}}$ is valid. In addition, many superconductors of recent interest are two gap, or multiband superconductors, and additional theories exist for those materials [42]. Therefore, a large Maki parameter $\alpha_{M}$ suggests that the orbital pair breaking happens at a higher field than $H_{P}$, and Pauli paramagnetism will dominate, favoring inhomogeneous superconductivity. The other parameter $r$, defined above, is a measure of how clean the system is. It measures the ability for the material to support an extended wave function, necessary for the long range order of the FFLO state [39], although there are claims that clean ordered systems are not absolutely necessary [50]. These parameters come from routine measurements. In the ratio $r=\ell / \xi, \ell$ comes from the scattering time, which is related to the Dingle temperature, $T_{D}$, and the Fermi velocity, $v_{F}=\sqrt{2 E_{F} / m^{*}}$, where $E_{F}$ is the Fermi energy and $m^{*}$ is the effective mass. The Fermi energy, effective mass, and $T_{D}$, can all be measured via Shubnikov-de Haas (SdH) or de Haas-van Alphen oscillations. The other necessary parameter for finding $r$ is $\xi$, which is found from the measurement of the superconducting critical field versus temperature and the formula $H_{c 2}=\Phi_{0} / 2 \pi \mu_{0} \tilde{\xi}^{2}$, or estimated from the size of the superconducting energy gap, $\Delta$, and the formula $\xi=\hbar v_{F} / 2 \Delta$. Note that, for the same Fermi energy, a higher effective mass results in a smaller $v_{F}$ and a smaller $\xi$, and hence a higher $H_{\text {orb }}^{0}$. For this reason, heavy fermion superconductors should favor the FFLO state. In general, $H_{o r b}^{0}$ comes from critical field measurements and $H_{P}$ can be found by analyzing specific heat measurements and determining the superconducting energy gap, as is described in detail below.

\section{Materials and Methods}

Most of the discussion in this article will focus on the crystalline organic conductors. Although the search for inhomogeneous superconductivity has spanned many years, and began in low dimensional single layers of superconducting materials [51], the first credible evidence was from experiments on heavy Fermions such as $\mathrm{UPd}_{2} \mathrm{Al}_{3}, \mathrm{CeRu}_{2}[52,53]$, and CeCoIn 5 [54,55]. These first claims were later found to be incorrect as discussed in Section 3.1. The crystalline organics then became the prime candidates for stabilizing inhomogeneous superconductivity because they are highly anisotropic layered materials, in some cases with incoherent transport between the layers [56-58]. Their high anisotropy allows vortices to hide between the most conducting layers if a magnetic field is aligned precisely parallel to the layers. In this orientation, the vortices have a diminished influence on the superconductivity $[59,60]$. As explained above, the vortices become Josephson vortices, weak interlayer vortices that can slide in and out of the material in the spaces between the most conducting layers. A vivid illustration of the disappearance of the influence of the vortices is shown in Figure 3. A number of features indicated in the figure such as the critical field, $H_{c 2}$, the vortex melting transition $\left(H_{m}\right)$ - the kink below $H_{c 2}$, the irreversibility transition $\left(H_{i r r}\right)$-the end of the hysteresis, and, at the lowest temperatures flux jumps, can be seen in most of the traces. The last three features, which all depend directly on vortices, are completely absent when the sample layers are parallel (black trace) to the magnetic field. In this orientation, when Jopsephson vortices are confined to the least conducting layers, $H_{o r b}^{0}$ is essentially infinite and superconductivity is destroyed by Pauli paramagnetism, resulting in high values of $\alpha_{m}$. Crystalline organic superconductors also can have large mean free paths, $\ell$, if they are synthesized carefully. Measurements of $\ell$ range from 10 to over $100 \mathrm{~nm}$ [61]. Given a high value of $\alpha_{m}$ and $r$, inhomogeneous superconductivity is likely to exist at the right combination of temperature and magnetic field.

A number of different methods have been used to study the FFLO state. A key indication of the existence of the FFLO state is the identification of the phase line separating the traditional vortex superconducting state from the inhomogeneous superconducting state. Given that these are both superconducting states, electrical resistance is always zero and rarely a useful measurement. We have 
used rf penetration depth via the tunnel diode oscillator (TDO) method because it is a very sensitive measurement [62] and rather simple and robust, allowing us to use it in dc and pulsed fields [10,63], down to temperatures below $65 \mathrm{mK}$ [64] and in pressure cells [65]. Specific heat is much more difficult to measure, but detailed thermodynamic information is critical to understand the nature of phase transitions [3,55]. NMR is one of the few microscopic probes that have been used to study inhomogeneous superconductivity $[7,8,12]$, and results from an NMR experiment will be discussed in Section 3. Other methods such as magnetic torque [4,5] and thermal conductivity [9] have also been used to locate the phase transitions into and out of the FFLO state.

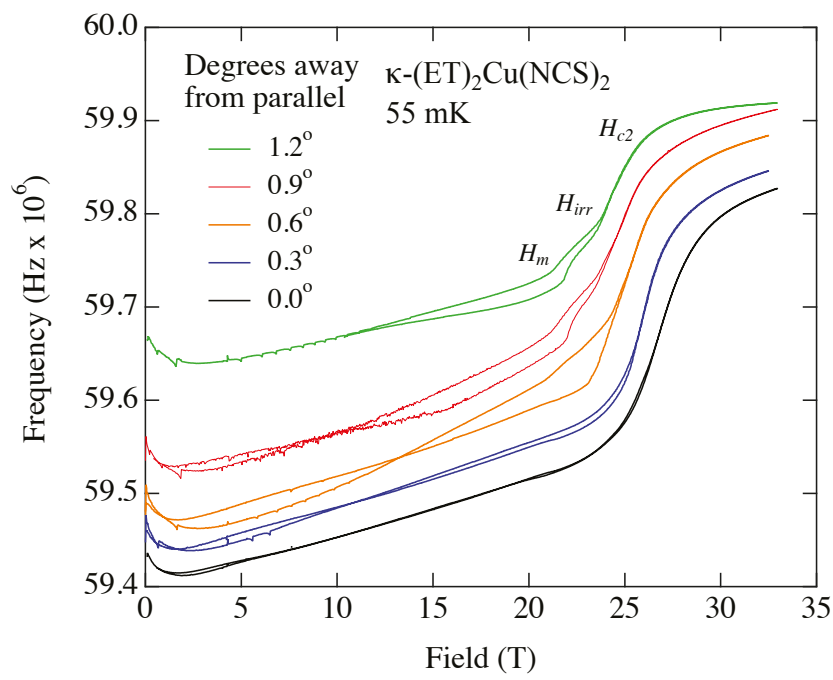

Figure 3. The angle dependence of the penetration depth near the parallel orientation for $\kappa$ ET-CuNCS. The lowest trace is exactly parallel, or $90^{\circ}$ in our absolute coordinates. The other traces are in order of increasing angle. The traces are vertically shifted to aid visualization. It is truly remarkable how all the vortex details are absent at the exactly parallel orientation.

\subsection{The Paramagnetic Limit}

A universal property of the FFLO state is that Cooper pairs are broken apart by Pauli paramagnetism as opposed to the formation of vortices. As we described above and as Clogston and Chadrasakhar $[30,31]$ showed, the ultimate critical magnetic field for a superconductor should occur when the Zeeman energy is greater than the superconducting energy gap. In the simplest case, the Zeeman energy is $\mu_{B} H$, and the BCS energy gap is $1.746 K_{B} T_{c}$. Of course, most superconductors of current interest are not accurately described by BCS theory, so we use a semi empirical method to find $H_{P}$. As we showed in a previous paper [6], following Clogston [31], we can find the critical magnetic field associated with the quenching of superconductivity by estimating the superconducting energy gap by analyzing specific heat data and setting this energy equal to the gain in free energy in a metal with susceptibility $\chi_{e}$. More specifically, we equate the superconducting condensation energy

$$
U_{c}=1 / 2 N\left(E_{f}\right) \Delta(0)^{2},
$$

where $N\left(E_{f}\right)$ is the density of states at the Fermi energy and $\Delta(0)$ is the superconducting energy gap at zero temperature, with

$$
\Delta F=1 / 2 \mu_{0} \chi_{e} H_{P}^{2}
$$


the magnetic energy of a metal with susceptibility $\chi_{e}$. The susceptibility $\chi_{e}$ can be expressed as $\mu_{B}^{2} N\left(E_{f}\right)$, but it is important to notice that $\mu_{0} H^{2}$ already has the units of energy density, so $\chi_{e}$ must be dimensionless. The expression $\mu_{B}^{2} N\left(E_{f}\right)$ has dimensions of $J / T^{2} m^{3}$, exactly the inverse of $\mu_{0}$. Therefore, we substitute $\mu_{0} \chi_{e}$ into Equation (4) and after equating $U_{c}=\Delta F$ and noticing that the density of states cancels out, we end up with the common result

$$
B_{P}=\frac{\Delta}{\sqrt{2} \mu_{B}}
$$

after using the relation that $B=\mu_{0} H$ and knowing that $\mathrm{B}$ is what we measure in the laboratory. This is the result of a direct comparison of the energy needed to break a Cooper pair with the energy needed to flip a electron spin. Orlando et al. [66] added a correction to Formula (5) of $1 / \sqrt{1+\lambda}$ where $\lambda$ is the electron-phonon interaction parameter, in order to account for many body effects. This factor was corrected by Schossmann and Carbotte [67] to not have the squareroot in the denominator. McKenzie adds a practical version of this correction to Equation (5) calling it $g^{*} / g$ [68], which also takes into account the effects on $g$, the gyromagnetic ratio. The result is

$$
B_{P}=\left(\frac{g}{g^{*}}\right) \frac{\Delta}{\sqrt{2} \mu_{B}} .
$$

The ratio $\mathrm{g}^{*} / \mathrm{g}$ can be found from specific heat and susceptibility measurements, or from spin-splitting of quantum oscillations, a measurement that is common in our laboratory. There is a table with $g^{*} / g$ found by both methods in McKenzie's paper on the arXiv [69]. Despite knowing that $B_{P}$ is really the more useful parameter in this calculation, we will continue to use $H_{P}$ as the designation of the Chandrasekhar-Clogston Pauli paramagnetic limit as is common in most articles.

\subsection{Specific Heat, Energy Gap, and Other Parameters}

We have found the superconducting energy gap $\Delta$ by fitting specific heat data in the superconducting state to the Alpha Model, a semi empirical model loosely based on BCS theory, and created by Padamsee et al [70]. Our most recent fits have been done using the more recent version by Johnston [71], which allows direct integration of the specific heat data to find the size of the gap. In this model, the ratio $\alpha=\Delta / k_{B} T_{C}$, is a free parameter rather than fixing $\alpha$ at the BCS value of 1.764, although the gap in the model has the same temperature dependence as the BCS model. The calculation is based on the universal expression of the entropy, $S=k_{B} \sum f \ln (f)$, where $f$ is the Fermi distribution function. The sum can be turned into an integral over the energy range of the quasiparticles, and for a given $\alpha, T_{\mathcal{C}}$, and $\gamma$, the entropy is calculated as a function of temperature. The calculated specific heat can then be found by taking the derivative of the entropy with respect to the temperature. In the Johnston version, the derivative of the entropy integral is done analytically and new integral results that can be used to find the specific heat directly.

We have fit the specific heat of many of the superconductors that we have studied to find the optimal $\alpha$, and hence the energy gap $\Delta$. One of the additional complications of fitting the specific heat to the Alpha Model is that the fit is sensitive to symmetry of the superconducting order parameter. The original versions of both Padamsee and Johnston use s-wave pairing. We modified the integral expressions for d-wave symmetry. In most cases, we fit the data using both forms of the equation, and pick the best fit to determine $\alpha$, and ideally determine the pairing symmetry, a question which is still not completely settled in the organics. We find d-wave as the best fit for $\kappa$ ET-CuNCS as did Taylor et al. [72], although we find s-wave as the best fit for the other organics, which is contrary to some of the other evidence in, for example, the $\kappa$ ET-Br material [73]. Part of the challenge in organics is that the lattice is soft, so the phonon contribution to the specific heat continues to low temperatures. One way to subtract the phonon contribution is to measure the specific heat at a magnetic field large enough to quench the superconductivity and subtract that curve from the specific heat measured 
with no magnetic field. With this method the phonon contribution is subtracted, but the electronic linear term is also subtracted and mixed with the phonon terms. The linear term determines the constant $\gamma$, which is a measure of the electronic density of states. The ability to determine $\gamma$ is critical to getting a good result for the $\alpha$ fits. It is unclear what our conclusion should be for the pairing symmetry, particularly if we believe that all the organics have the same pairing. We have also noticed that the shape of the fits are not perfect with s or d-wave pairing, which possibly suggests that the pairing has some other symmetry such as $\mathrm{s}+\mathrm{d}$, or anisotropic s-wave, a notion supported by some experiments [74] and recent theory [75]. At this point in time, we will take the best fits, s-wave or $\mathrm{d}$-wave and use that value of $\alpha$ for each superconductor. Once we have $\alpha$, the value of $H_{P}$ is then calculated from Equation (6). $H_{P}$ is important for the study of the FFLO state because it determines the magnetic field that separates the vortex state of superconductivity from the FFLO state. Given that the superconducting energy gap does not change much in the lower half of the temperature range of the superconducting phase diagram, $H_{P}$ and hence the vortex state-FFLO state phase line should be virtually temperature independent [76].

The results of fitting for $\alpha$ and the corresponding energy gap $H_{P}$ are shown for a number of materials in Table 1. We have also collected a number of other useful parameters for the study of inhomogeneous superconductors. Most of the compounds are from the family of organic crystalline superconductors. We have also added the heavy fermion $\mathrm{CeCoIn}_{5}$ and the pnictides $\mathrm{KFe}_{2} \mathrm{As}_{2}$ and LiFeP as examples of other materials where the FFLO state has been claimed to be found.

Table 1. Parameters that are useful in the study of inhomogeneous superconductivity. The ratio $\alpha$ is determined from the specific heat data in the reference next to $\alpha$. The other parameters are calculated from values in the table or references. The $T_{C}$ values come from the specific heat data referenced for $\alpha$.

\begin{tabular}{ccccccccc}
\hline Material & $\alpha$ & $\boldsymbol{g}^{*} / g$ & $\boldsymbol{T}_{\boldsymbol{c}}(\mathrm{K})$ & $\boldsymbol{H}_{\boldsymbol{p}}(\mathrm{T})$ & $\boldsymbol{\alpha}_{\boldsymbol{M}}$ & $\boldsymbol{H}_{\boldsymbol{o r b}}^{0}(\mathrm{~T})$ & $\xi(\AA)$ & $\ell(\AA)$ \\
\hline$\kappa-(\mathrm{BEDT}-\mathrm{TTF})_{2} \mathrm{Cu}\left(\mathrm{NCS}_{2}\right.$ & $3.0[72]$ & $1.26[69]$ & 9.6 & 21.6 & 4.9 & $130[35]$ & 13 & $900[77]$ \\
$\beta^{\prime \prime}-(\mathrm{BEDT}-\mathrm{TTF})_{2} \mathrm{SF}_{5} \mathrm{CH}_{2} \mathrm{CF}_{2} \mathrm{SO}_{3}$ & $1.94[78]$ & $1.0[69]$ & 4.5 & 9.2 & 3.9 & $75[35]$ & 21 & 520 \\
$\alpha-(\mathrm{ET})_{2} \mathrm{NH}_{4} \mathrm{Hg}(\mathrm{SCN})_{4}$ & $1.76[79]$ & $0.86[69]$ & 0.96 & 2.1 & 5.5 & $8.1[76]$ & 53 & $681[76]$ \\
$\lambda-(\mathrm{BETS})_{2} \mathrm{GaCl}_{4}$ & $1.83[80]$ & $1.0[69]$ & 4.3 & 8.3 & 3.9 & $23.1[10]$ & 31.5 & $170[56]$ \\
$\kappa-(\mathrm{ET})_{2} \mathrm{Cu}\left[\mathrm{N}(\mathrm{CN})_{2}\right] \mathrm{Br}$ & $2.77[72]$ & $1.4[69]$ & 11.5 & 23.8 & 9.6 & $161[81]$ & 12 & $260[82]$ \\
$\mathrm{CeCoIn}$ & $3.03[83,84]$ & $0.73[83]$ & 2.16 & 9.44 & 6.5 & $43.5[85]$ & 23 & 810 \\
$\mathrm{KFe}_{2} \mathrm{As}_{2}$ & $1.75[86]$ & $1.3[87]$ & 3.14 & 4.84 & 2.9 & 9.9 & 48 & $1770[88]$ \\
$\mathrm{LiFeP}$ & $1.89[89]$ & 1.0 & 17.6 & 34.9 & 2.1 & $51[90]$ & 21 & $5500[90]$ \\
\hline
\end{tabular}

As mentioned earlier, to form an FFLO state, $H_{\text {orb }}^{0}$ needs to be higher than $H_{P}$, as determined by $\alpha_{M}$. It was determined theoretically that, above the critical value of $\alpha_{M}=1.8$, the FFLO state could be stablilzed [91] in a clean material $(r>2)$. The search for the FFLO state involves careful measurements of some parameter that can be probed in the superconducting state around the value of $H_{P}$, such as specific heat, penetration depth or NMR, in order to find evidence of a phase transition.

\section{Results and Discussion}

The material with the most compelling data that suggests the existence of the FFLO state is $\kappa$ ET-CuNCS. Evidence for the FFLO state in this material has been found with numerous measurement techniques, including NMR $[7,8]$, rf penetration depth $[6,92]$, magnetic torque $[4,5]$, specific heat $[3,93]$ and transport [5]. The phase diagram of this material depicting examples of these measurements is shown in Figure 4. The phase diagram is certainly suggestive of a FFLO state. Two telling characteristics of the phase line separating the vortex state from the FFLO state are that it occurs at the paramagnetic limit, $H_{P}$, that was calculated with the specific heat data via the Alpha Model, and also that the phase line has near zero slope, consistent with the superconducting energy gap, which is almost temperature independent below $T_{c} / 2$. 


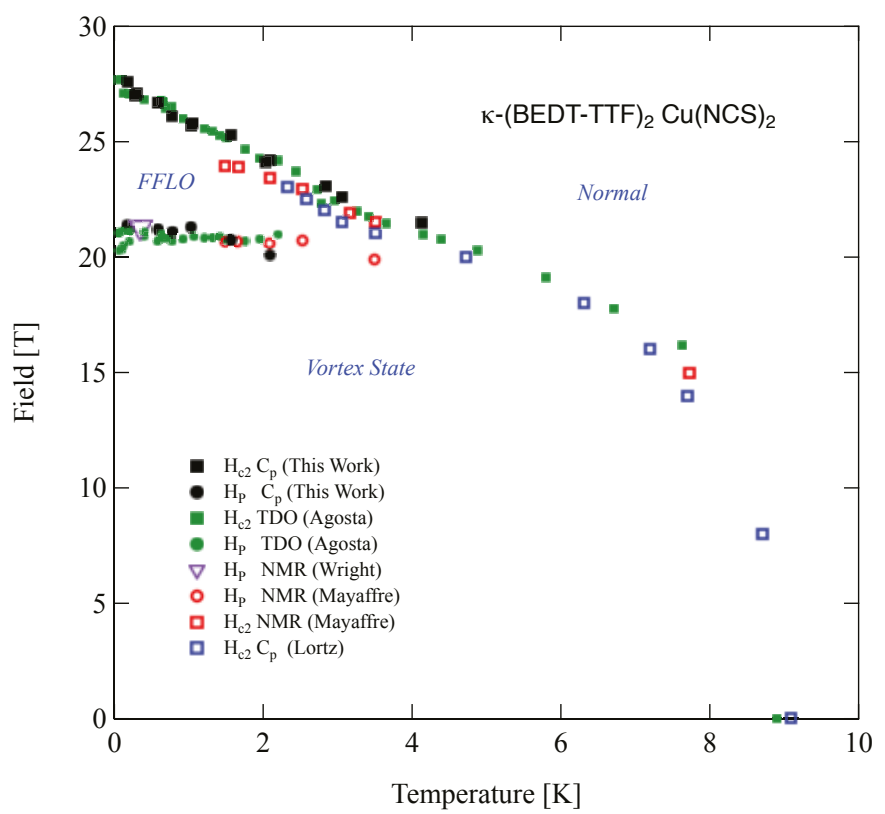

Figure 4. Color online. From Agosta et al. [3] Phase diagram of $\kappa$ ET-CuNCS for parallel magnetic field $(\theta=0)$. Solid black circles represent calorimetric observations of the phase transitions between the lower and higher field superconducting phases at $H_{P}$, and squares, the normal and superconducting state at $H_{c 2}(T)$. Points from an earlier calorimetric determination of $H_{c 2}(T)$ [93] are shown as open blue squares. Also included are determinations of both the $H_{c 2}$ and $H_{p}$ phase boundaries by means of rf penetration measurements (green) [6] and NMR measurements [7,8] (open purple and red symbols, respectively).

Also noteworthy is that the $H_{P}$ phase line is a first order transition based on hysteresis in the specific heat as the transition is crossed in up and down sweeps of the magnetic field [3]. An example of the hysteresis in the specific heat is shown in Figure 5a along with a TDO measurement that shows the same hysteresis. The TDO measurement is a much simpler measurement, and the idea that it also can provide evidence of a first order transition is useful for future experiments. The magneto-caloric measurements, shown in Figure 5b, also provided the important evidence that the higher field state has a greater entropy than the vortex state, consistent with the fact that the FFLO state is less ordered than the uniform superconducting state, because of the unpaired electrons $[3,94,95]$. Another piece of information that can be gathered from the phase diagram is that the enhancement of the ultimate critical field $H_{c 2}$ is $\approx 1.4 H_{P}$, consistent with predictions [20,36] for a 2D material. One difference that can be found between this data and the theory is that $T^{*}$, the place where the FFLO phase starts is at $T_{c} / 3$, much lower than $\approx T_{\mathcal{c}} / 2$ as most theories predict.

Although this is the best example of the FFLO state, it is worth comparing these results to other materials where credible FFLO states exist. In Figure 6, we show superimposed phase diagrams of three organic conductors where there is evidence for the FFLO state, each normalized by their own experimentally determined $H_{P}$ and $T_{c}$. In this figure, the three materials scale relatively well, although the upper critical field for $\lambda \mathrm{BETS}-\mathrm{GaCl}$ is not quite as high as the others. This would suggest that it is not as two-dimensional as the other superconductors [20], however, we have used the vortex-FFLO phase line as the $H_{P}$ for this figure. It is also possible that impurities or spin-orbit scattering could have raised the value of $H_{P}[32,48]$. 

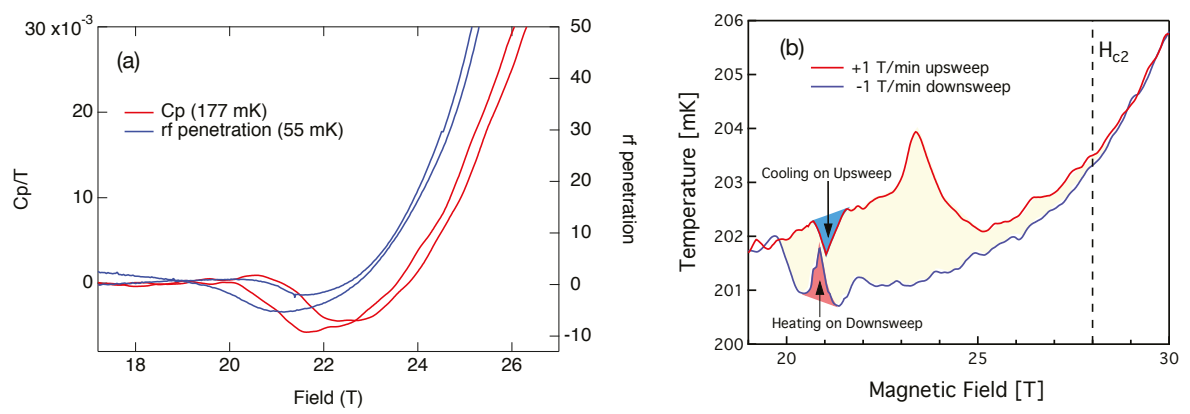

Figure 5. Color online. (a) specific heat and TDO up and down field traces as the measurements cross the vortex-FFLO phase transition showing the hysteresis. This is direct evidence of a first order transition; (b) from Agosta et al. [3], the magneto caloric effect, which shows the direction of the flow of latent heat as the vortex-FFLO phase line is crossed. This information can be used to show that the higher field state, in this case the FFLO state, is of higher entropy than the vortex state, consistent with what is expected for this phase transition $[3,94,95]$.

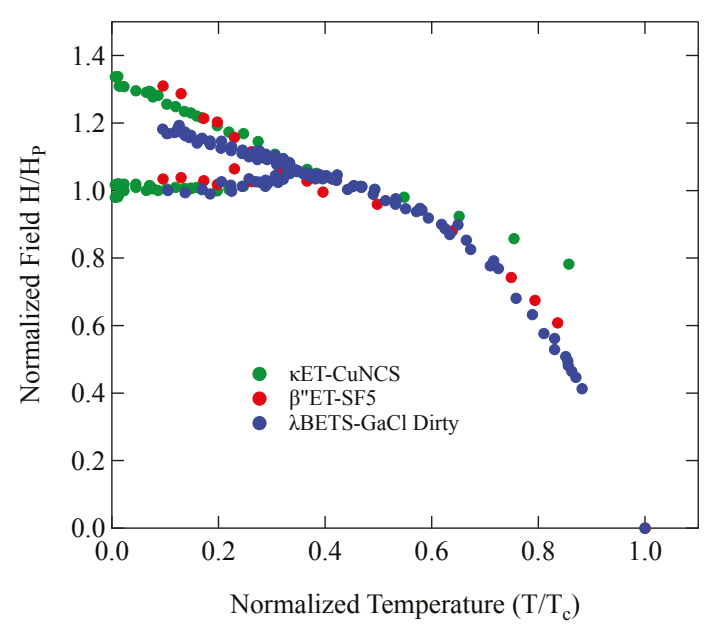

Figure 6. Color online. Phase diagram of $\kappa$ ET-CuNCS, $\lambda$ BETS-GaCl and $\beta " E T-S F 5$ each normalized using the phase line between the vortex and FFLO states. The BETS does not seem to have as much enhancement of the upper critical field as the other two superconductors, which scale identically.

To probe these ideas, we have preliminary data for cleaner samples of BETS, synthesized by Kobayashi, and the data suggest that the scattering details may be important. In Figure 7, we show preliminary new data on an absolute scale with the previous data. The cleaner samples have a lower $H_{P}$, but surprisingly an identical $H_{c 2}$. The drop in the value of $H_{P}$ is expected if it is dependent on similar pair breaking mechanisms as $H_{c 2}$ [32,48]. In particular, less spin-orbit scattering should lower the critical field. The surprise is that the upper critical field for the FFLO state, where the material crosses into the normal state, is robust with respect to the degree of scattering, unlike $H_{P}$, the upper critical field for the vortex state. In the traditional superconducting state, $\mathrm{H}_{c 2}$ is very sensitive to scattering. This may be understandable in the context of $H_{c 2}$ as calculated by $[32,48]$ where similar changes in the $H_{c 2}$ phase line are found for greater orbital effects (vortices) or more spin orbit scattering. However, spin-orbit scattering can directly modify $H_{P}$, but vortex effects will not. In addition, if orbital (vortex) effects are suppressed, as they are in the highly anisotropic organics, $H_{c 2}$ must be the result 
of the magnetic energy in the system, which is more immune to scattering events than orbital effects. Therefore, the vortex FFLO phase line should follow $H_{P}$, which is dependent on the energy gap and spin orbit scattering, and $\mathrm{H}_{c 2}$ will be insensitive to scattering. In many of these materials, it may be important to always adjust $H_{P}$ to account for the degree of scattering and spin-orbit scattering to properly interpret the results.

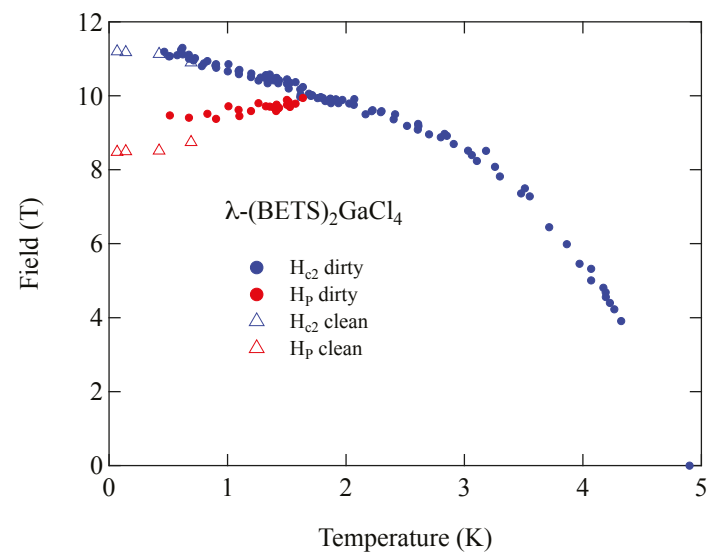

Figure 7. We obtained new samples from Kobayashi that are higher quality samples (more pure) based on Shubnikov-de Haas oscillations. The higher quality samples show on an absolute scale that the upper critical field of the clean and dirty samples is the same, but $H_{P}$ is very sensitive to impurities. The new data was taken in a dilution refrigerator and we are looking forward to extending the data to higher temperatures in the near future.

One of the more promising measurements showing further evidence for the FFLO state was made with NMR by Koutroulakis et al. [12] on the crystalline organic $\beta$ "ET-SF5. In this measurement, the line shape of carbon atoms in the FFLO phase was measured and compared to a calculated line shape. The signal comes from the substitution of four inequivalent carbon atoms in the ET molecule with ${ }^{13} \mathrm{C}$ to have an active NMR target. The data is compared to a calculation based on the sum of the four inequivalent line-shapes from the four inequivalent carbon atoms modified by the effects of a one-dimensional q-vector. It is not yet clear if the features of the line-shape are unique enough to offer proof that the FFLO state is the cause of the line-shape, but it is more compelling that any other microscopic measurement so far. The data is reproduced in Figure 8.

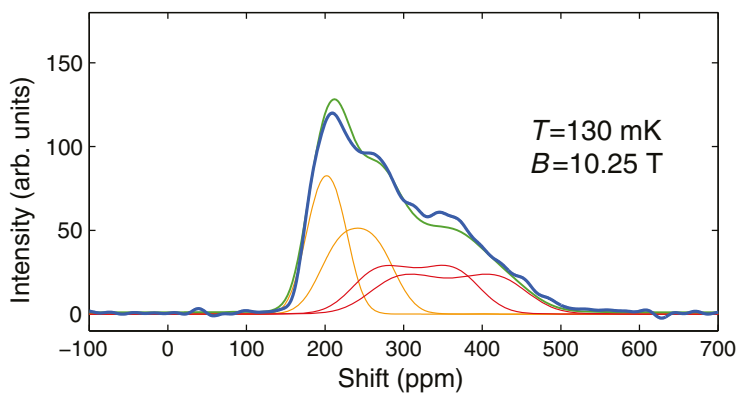

Figure 8. Color online. Example of spectrum simulation compared to recorded spectrum (blue) from Koutroulakis et al. [12]. The simulation is a sum (green) of four Gaussian-broadened contributions (red, orange) arising from a single-Q sinusoidal modulation of the SC order parameter. 


\subsection{Other Materials}

Two other materials outside of the class of organic conductors are worth mentioning: the heavy Fermion superconductor $\mathrm{CeCoIn}_{5}$ and the pnictide superconductor $\mathrm{KFe}_{2} \mathrm{As}_{2}$. An interesting discussion of the FFLO state in heavy fermion and pnictide superconductors was recently published that supports the existence of inhomogeneous superconductivity in these classes of materials [37]. The heavy fermion superconductor $\mathrm{CeCoIn}_{5}$ was one of the first materials where there was thought to be credible experimental evidence for the FFLO state. This evidence was in the form of calorimetric observations of a bulk field induced phase transition between two superconducting phases [54,55]. This transition was initially attributed to FFLO superconductivity but is now known to correspond to the onset of spin-density wave (SDW) ordering within the superconducting state [95-99] and is called the Q state. The Q state is the coexistence of a SDW and a superconducting state, although it is not clear if the superconducting state is a FFLO state or a uniform state. The q-vector measured by neutron scattering suggests a modulated state, but it does not change wavelength as a function of magnetic field as is expected for the FFLO state [97]. Details of the SDW ordering observed by NMR have led to suggestions of a lower field FFLO transition [100] and/or coupling of a FFLO phase to this SDW transition [100-102], but there is no clear thermodynamic evidence for these proposals [95,103,104]. The possibility remains of a more complex coupling of the SDW transition to a modified FFLO phase or pair density wave (PDW) [100,104]. One way to gain more insight into the nature of the Q phase is to scale the $\mathrm{CeCoIn}_{5}$ data on the same phase diagram with an organic superconductor as seen in Figure 9. In this figure, the TDO data from ETCuNCS is used as a baseline, and the CeCoIn $\mathrm{data}^{-}$ is scaled using $H_{P}$ from Table 1 . We note that $\alpha$ for CeCoIn 5 was calculated based on two sets of specific heat data $[83,84]$ with nearly identical results. The parameter $g * / g$ was more difficult to determine, and for this study we relied on $\gamma$ and $\chi$ measurements [83] averaged over temperatures below $T_{\mathcal{C}}$ and used Wilson's ratio [69]. What is surprising is that although the vortex-FFLO phase line found by specific heat and torque does not line up with $H_{P}$, and it also wouldn't even have the right slope for the $H_{P}$ line, the phase line found by TDO rf penetration measurements [64] matches the value of $H_{P}$ as calculated from the specific heat. Adding to this evidence for a phase line at 9.2 T, Koutroulakis et al. [100] identified a phase they call the exotic superconducting state, or ESC that starts at a minimum magnetic field of $9.2 \mathrm{~T}$. It may be worth looking at the CeCoIn 5 specific heat data to see if there is any indication of this lower phase line. In any case, it can be seen that the Q state, as it is called, is not a simple FFLO state, or at least not the same as found in the organic and pnictide superconductors.

The pnictides are not nearly as anisotropic as the organics [90], but their high electron masses and tunable anisotropy suggest that they may be candidates for inhomogeneous superconductivity $[105,106]$. A very recent result claims the possible existence of a FFLO state in the two band pnictide superconductor $\mathrm{KFe}_{2} \mathrm{As}_{2}$ [107] based on specific heat and magnetic torque measurements. Using the values of $\alpha$ from the two band alpha model in this $\mathrm{KFe}_{2} \mathrm{As}_{2}$ paper and others [86,108], we calculated $H_{P}$ between 4.45 and $5.23 \mathrm{~T}$ (the result in Table 1 is the average). For convenience, we used the value from Figure 4 in Cho et al. [107] to scale their phase diagram and superimpose it on the phase diagram from the organic conductors. The result is in Figure 10. This diagram and the $\mathrm{KFe}_{2} \mathrm{As}_{2}$ specific heat data raise a number of questions about the claim of the FFLO state in this material.

The enhancement of the critical field over $H_{P}$ in $\kappa$ ET-CuNCS is 1.35 , and in $\mathrm{KFe}_{2} \mathrm{As}_{2}$ it is 1.24 if you extrapolate to zero temperature. This is consistent with the difference in anisotropy. According to Matsuda [20], the enhancement in an ideal 2D and 3D system should be 1.4 and 1.2 respectively. The ratio of parallel and perpendicular critical fields at zero temperature is one indication of the anisotropy of a material, and this value is $\sim 4-5$ for both $\mathrm{KFe}_{2} \mathrm{As}_{2}$ [86] and for $\kappa \mathrm{ET}-\mathrm{CuNCS}$, yet this is misleading because $\kappa$ ET-CuNCS is strongly paramagnetically limited. Using the initial slopes of the critical field lines in different orientations as described in Section 1.2, Equation (2) determines $H_{o r b}^{0}$. $H_{o r b}^{0}$ is a much better measure of the superconducting anisotropy because it is based on the anisotropy of the vortices, and hence the superconducting coherence lengths, $\xi$. This anisotropy measurement is reliable as long 
as the diameter of the vortices is larger than the interlayer spacing, a limit that is always true close to $T_{C}$ where $\xi$ diverges. Using these slopes, the anisotropy of $\mathrm{KFe}_{2} \mathrm{As}_{2}$ is still 4.5 but for $\kappa$ ET-CuNCS the anisotropy is $\approx 21$, if the slope of $H_{c 2}$ is properly measured [93]. Thus, this anisotropy explains the difference in critical field enhancement, but the anisotropy of $\mathrm{KFe}_{2} \mathrm{As}_{2}$ is inconsistent with the onset of the FFLO state in a multiband superconductor. According to Gurevich [42], the lower anisotropy of the pnictides should result in the FFLO state starting at $t<0.5$, below the results found by Cho et al., and certainly below the results of the highly anisotropic organic conductors. Zocco et al. [88] has a higher temperature onset of the FFLO state according to their calculations, but a much smaller critical field enhancement than the data.

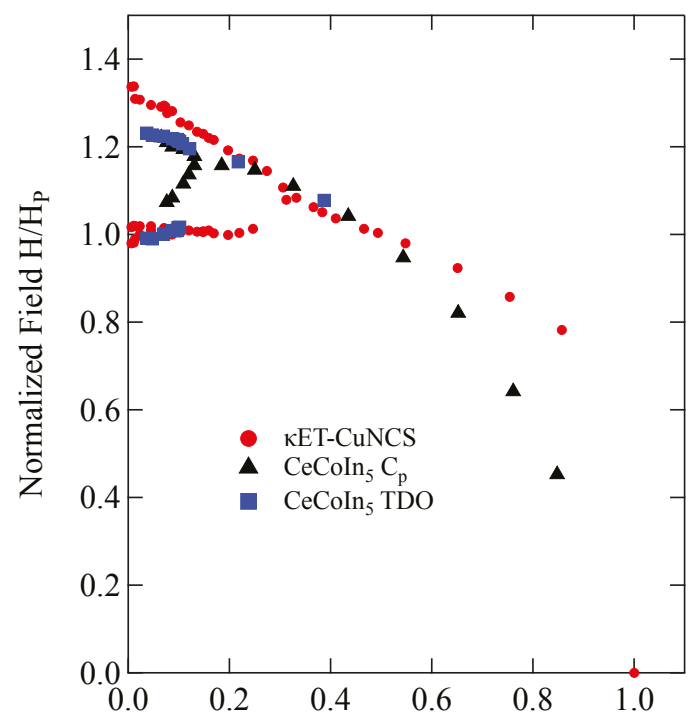

Normalized Temperature $\left(\mathrm{T} / \mathrm{T}_{\mathrm{c}}\right)$

Figure 9. Color online. Phase diagram of $\mathrm{CeCoIn}_{5}$ with $\kappa$ ET-CuNCS as our baseline FFLO phase diagram. If the magnetic field is normalized by $H_{P}$ as calculated by Equation (6), the TDO measurement [64] (blue squares) finds a phase line at $H_{P}$. NMR experiments [100] confirm a change of the material properties at this field value too, suggesting that there may be a FFLO type transition at the calculated $H_{P}$. The more vertical phase line discovered by specific heat [54] (black triangles) at about $t=0.1$ could be related to the SDW.

The second quantitative difference between the organic phase diagrams and the $\mathrm{KFe}_{2} \mathrm{As}_{2}$ phase diagram is the slope of the vortex state-FFLO state phase line at $H_{P}$. As expected, to first order, this line should have no slope if $H_{P}$ is proportional to the energy gap, given that the BCS superconducting energy gap is almost constant for $t<0.4$. Within experimental error, this is almost the case for $\kappa$ ET-CuNCS. Doing a fit to our largest set of data points, from the TDO measurements, we find a slope of $<0.05 \mathrm{~T} / \mathrm{K}$. The slope of this phase line for $\mathrm{KFe}_{2} \mathrm{As}_{2}$ is $0.3 \mathrm{~T} / \mathrm{K}$. The increased slope could be due to the presence of vortices, as expected for the pnictides, which are less anisotropic than the organic superconductors. Another interpretation is that this line is really $H_{c 2}$ and the bump in specific heat at a higher field is not due to the FFLO state. Given the form of the specific heat data in Agosta et al. [3] and the calculations of Ptok [106], a small jump in specific heat at $H_{c 2}$ followed by a large jump in specific heat at the FFLO to uniform superconductivity phase line does not make sense. In Ptok, these jumps are calculated as at least similar in size, and given that $H_{c 2}$ is a transition into a bulk 
superconducting state, with the formation of Cooper pairs throughout the sample, the resulting drop in entropy should produce a robust specific heat peak at $H_{c 2}$, and as calculated in Ptok and in many publications for single band superconductors [109]. Furthermore, without more angular data to see if the specific heat signature of the FFLO state slowly changes when the sample is rotated with respect to the magnetic field, the data is less compelling. Many of the other studies of $\mathrm{KFe}_{2} \mathrm{As}_{2}$ that have been done, as referenced in the above paragraph, and in particular the one by Zocco et al., do not see any indication of a FFLO state. There still is hope to find indications of the FFLO state in $\mathrm{KFe}_{2} \mathrm{As}_{2}$ and other pnictides, but the present claim in Cho et al. although exciting, needs more evidence.

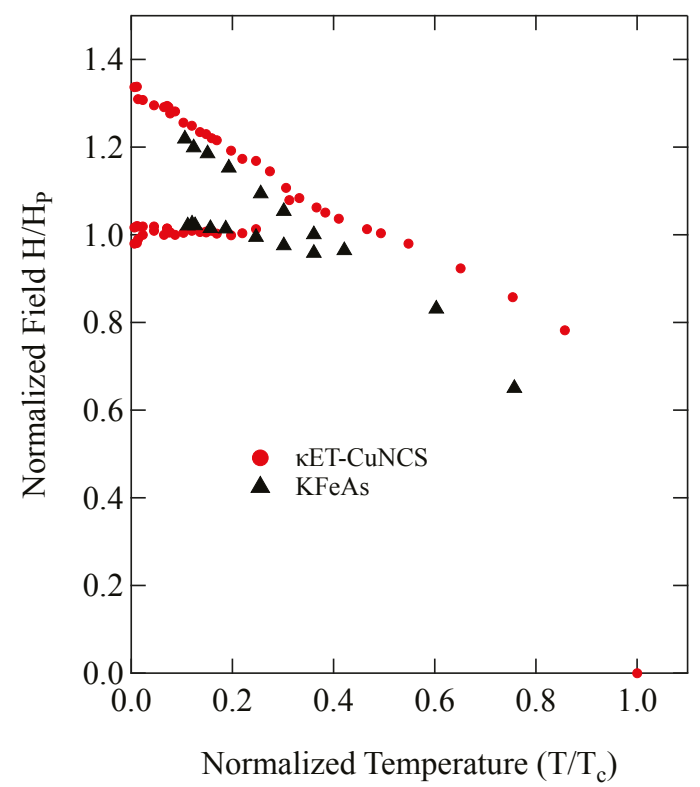

Figure 10. Color online. The phase diagram of $\kappa$ ET-CuNCS superimposed with the $\mathrm{KFe}_{2} \mathrm{As}_{2}$ data from Cho et al. [107], normalized to $T_{C}$ and $H_{P}$ for each sample respectively. We note the two main differences, the slope of the vortex-FFLO transition, and the overall enhancement of the FFLO state over $H_{P}$.

\subsection{New Materials}

There have been predictions of the FFLO state in LiFeP [90], and this is another good example of a material where it is difficult to predict if the FFLO state exists. LiFeP as many of the pnictides, has two superconducting energy gaps, and it is unclear, when there are multiple gaps, which one dominates. It is reasonable to think that the largest gap, with the largest $T_{C}$ will dominate. This is because above $H_{P}$ for the largest gap, all Cooper pairs will be subject to pair breaking via the Zeeman energy. The data claiming inhomogeneous superconductivity in $\mathrm{KFe}_{2} \mathrm{As}_{2}$ [107], if it is correct, shows the $H_{P}$ phase line at the value of the higher gap energy consistent with the argument above. For this reason, the parameters for both of the pnictides in Table 1 correspond to the higher energy of the two gaps found in each compound. The energy gaps and ratio $\alpha$ for these materials were not calculated from our version of the alpha model but came from the papers referenced in the table, and use a two gap model. It is also important to note that $g^{*} / g$ for LiFeP is not known, so it was set to equal one. Given what is known, the value of $H_{P}$ in $\mathrm{LiFeP}$ is above $\mathrm{H}_{c 2}$ and no FFLO state should exist. It is possible that a weak variation of the FFLO state could exist when the magnetic field was greater than 
$H_{P}$ corresponding to the lower energy gap, but it is difficult to search for evidence of such a weak FFLO state, if it exists at all.

\section{Conclusions}

It is clear that an exotic superconducting state exists in the low temperature high field quadrant of the superconducting phase diagram in quasi 2D anisotropic organic superconductors, and possibly heavy fermion and pnictide superconductors. So far, the data that have been collected on the organics is consistent with inhomogeneous superconductivity. Microscopic probes such as NMR will help determine if these are truly FFLO states, but spatial probes such as STM, neutrons, or X-rays will eventually be the way to measure the $\mathbf{q}$-vectors, and unambiguously identify these exotic correlated electron states. There are still many methods available for the organics that can be used to expand the parameter phase space, including pressure, defects, and chemical substitution. It has been shown that $\mathrm{CeCoIn}_{5}$ is more complex than the other materials, and the specific heat data needs a second look. It also may be a good candidate for STM. Other classes of superconductors such as the pnictides are promising new materials for understanding inhomogeneous superconductivity. Finally, a topic not covered in this review, and closely related theoretically [110,111] and experimentally [112] to this subject is the study of inhomogeneous superconductivity in quasi 1D materials. It will be insightful to discuss 1D and 2D materials together in the future.

Acknowledgments: The author thanks Andrei Lebed for helpful discussions. A portion of this work was performed at the National High Magnetic Field Laboratory, which is supported by National Science Foundation Cooperative Agreement No. DMR-11157490 and the state of Florida.

Conflicts of Interest: The author declares no conflict of interest.

\section{References}

1. Fulde, P.; Ferrell, R.A. Superconductivity in a Strong Spin-Exchange Field. Phys. Rev. 1964, 135, A550-A563, doi:10.1103/PhysRev.135.A550.

2. Larkin, A.I.; Ovchinnikov, Y.N. Inhomogeneous State of Superconductors. Sov. Phys. JETP 1965, 20, 762-769.

3. Agosta, C.C.; Fortune, N.A.; Hannahs, S.T.; Gu, S.; Liang, L.; Park, J.H.; Schleuter, J.A. Calorimetric Measurements of Magnetic-Field-Induced Inhomogeneous Superconductivity Above the Paramagnetic Limit. Phys. Rev. Lett. 2017, 118, 267001, doi:10.1103/PhysRevLett.118.267001.

4. Bergk, B.; Demuer, A.; Sheikin, I.; Wang, Y.; Wosnitza, J.; Nakazawa, Y.; Lortz, R. Magnetic torque evidence for the Fulde-Ferrell-Larkin-Ovchinnikov state in the layered organic superconductor $\kappa$-(BEDT-TTF ${ }_{2} \mathrm{Cu}(\mathrm{NCS})_{2}$. Phys. Rev. B 2011, 83, 064506.

5. Tsuchiya, S.; Yamada, J.i.; Sugii, K.; Graf, D.; Brooks, J.S.; Terashima, T.; Uji, S. Phase Boundary in a Superconducting State of $\kappa$-(BEDT-TTF $)_{2} \mathrm{Cu}(\mathrm{NCS})_{2}$ : Evidence of the Fulde-Ferrell-Larkin-Ovchinnikov Phase. J. Phys. Soc. Jpn. 2015, 84, 034703, doi:10.7566/JPSJ.84.034703.

6. Agosta, C.C.; Jin, J.; Coniglio, W.A.; Smith, B.E.; Cho, K.; Stroe, I.; Martin, C.; Tozer, S.W.; Murphy, T.P.; Palm, E.C.; et al. Experimental and semiempirical method to determine the Pauli-limiting field in quasi-twodimensional superconductors as applied to $\kappa$-(BEDT-TTF $)_{2} \mathrm{Cu}(\mathrm{NCS})_{2}$ : Strong evidence of a FFLO state. Phys. Rev. B 2012, 85, 214514.

7. Wright, J.A.; Green, E.; Kuhns, P.; Reyes, A.; Brooks, J.; Schlueter, J.; Kato, R.; Yamamoto, H.; Kobayashi, M.; Brown, S.E. Zeeman-Driven Phase Transition within the Superconducting State of $\kappa$-(BEDT-TTF $)_{2} \mathrm{Cu}(\mathrm{NCS})_{2}$. Phys. Rev. Lett. 2011, 107, 087002, doi:10.1103/PhysRevLett.107.087002.

8. Mayaffre, H.; Kramer, S.; Horvatić, M.; Berthier, C.; Miyagawa, K.; Kanoda, K.; Mitrović, V.F. Evidence of Andreev bound states as a hallmark of the FFLO phase in $\kappa$-(BEDT-TTF) ${ }_{2} \mathrm{Cu}(\mathrm{NCS})_{2}$. Nat. Phys. 2014, 10, 928-932, doi:10.1038/nphys3121.

9. Tanatar, M.A.; Ishiguro, T.; Tanaka, H.; Kobayashi, H. Magnetic field-temperature phase diagram of the quasitwo-dimensional organic superconductor $\lambda$-(BETS) ${ }_{2} \mathrm{GaCl}_{4}$ studied via thermal conductivity. Phys. Rev. B $2002,66,134503$. 
10. Coniglio, W.A.; Winter, L.E.; Cho, K.; Agosta, C.C.; Fravel, B.; Montgomery, L.K. Superconducting phase diagram and FFLO signature in $\lambda$-(BETS $)_{2} \mathrm{GaCl}_{4}$ from rf penetration depth measurements. Phys. Rev. B 2011, 83, 224507, doi:10.1103/PhysRevB.83.224507.

11. Cho, K.; Smith, B.E.; Coniglio, W.A.; Winter, L.E.; Agosta, C.C.; Schlueter, J.A. Upper critical field in the organic superconductor $\beta^{\prime \prime}-(\mathrm{ET})_{2} \mathrm{SF}_{5} \mathrm{CH}_{2} \mathrm{CF}_{2} \mathrm{SO}_{3}$ : Possibility of Fulde-Ferrell-Larkin-Ovchinnikov state. Phys. Rev. B 2009, 79, 220507, doi:10.1103/PhysRevB.79.220507.

12. Koutroulakis, G.; Kuhne, H.; Schlueter, J.A.; Wosnitza, J.; Brown, S.E. Microscopic Study of the Fulde-FerrellLarkin-Ovchinnikov State in an All-Organic Superconductor. Phys. Rev. Lett. 2016, 116, 067003, doi:10.1103/PhysRevLett.116.067003.

13. Burkhardt, H.; Rainer, D. Fulde-Ferrell-Larkin-Ovchinnikov state in layered superconductors. Ann. Phys. 1994, 506, 181-194.

14. Buzdin, A.I.; Kachkachi, H. Generalized Ginzburg-Landau theory for nonuniform FFLO superconductors. Phys. Lett. A 1997, 225, 341-348, doi:10.1016/S0375-9601(96)00894-8.

15. Houzet, M.; Buzdin, A. Influence of the paramagnetic effect on the vortex lattice in 2D superconductors. EPL (Europhys. Lett.) 2000, 50, 375.

16. Klein, U.; Rainer, D.; Shimahara, H. Interplay of Fulde-Ferrell-Larkin-Ovchinnikov and Vortex States in Two-Dimensional Superconductors. J. Low Temp. Phys. 2000, 118, 91-104.

17. Shimahara, H. Fulde-Ferrell state in quasi-two-dimensional superconductors. Phys. Rev. B 1994, 50, 12760, doi:10.1103/PhysRevB.50.12760.

18. Agterberg, D.F.; Yang, K. The effect of impurities on Fulde-Ferrell-Larkin-Ovchinnikov superconductors. J. Phys. Cond. Matt. 2001, 13, 9259-9270, doi:10.1088/0953-8984/13/41/315.

19. Houzet, M.; Meurdesoif, Y.; Coste, O.; Buzdin, A. Structure of the non-uniform Fulde-Ferrell-LarkinOvchinnikov state in 3D superconductors. Phys. C Superconduct. 1999, 316, 89-96.

20. Matsuda, Y.; Shimahara, H. Fulde-Ferrell-Larkin-Ovchinnikov State in Heavy Fermion Superconductors. J. Phys. Soc. Jpn. 2007, 76, 051005.

21. Piazza, F.; Zwerger, W.; Strack, P. FFLO strange metal and quantum criticality in two dimensions: Theory and application to organic superconductors. Phys. Rev. B 2016, 93, 085112, doi:10.1103/PhysRevB.93.085112.

22. Kinnunen, J.J.; Baarsma, J.E.; Martikainen, J.P.; Törmä, P. The Fulde-Ferrell-Larkin-Ovchinnikov state for ultracold fermions in lattice and harmonic potentials: A review. Rep. Prog. Phys. 2018, 81, 046401, doi:10.1088/1361-6633/aaa4ad.

23. Zwierlein, M.W.; Schirotzek, A.; Schunck, C.H.; Ketterle, W. Fermionic Superfluidity with Imbalanced Spin Populations. Science 2006, 311, 492-496, doi:10.1126/science.1122318.

24. Liao, Y.A.; Rittner, A.S.C.; Paprotta, T.; Li, W.; Partridge, G.B.; Hulet, R.G.; Baur, S.K.; Mueller, E.J. Spin-imbalance in a one-dimensional Fermi gas. Nature 2010, 467, 567-569.

25. Heidrich-Meisner, F.; Orso, G.; Feiguin, A.E. Phase separation of trapped spin-imbalanced Fermi gases in one-dimensional optical lattices. Phys. Rev. A 2010, 81, 1136-1137, doi:10.1103/PhysRevA.81.053602.

26. Olsen, B.A.; Revelle, M.C.; Fry, J.A.; Sheehy, D.E.; Hulet, R.G. Phase diagram of a strongly interacting spinimbalanced Fermi gas. Phys. Rev. A 2015, 92, 063616, doi:10.1103/PhysRevA.92.063616.

27. Korolyuk, A.; Massel, F.; Törmä, P. Probing the Fulde-Ferrell-Larkin-Ovchinnikov Phase by Double Occupancy Modulation Spectroscopy. Phys. Rev. Lett. 2010, 104, 236402, doi:10.1103/PhysRevLett.104.236402.

28. Paglione, J.; Greene, R.L. High-temperature superconductivity in iron-based materials. Nat. Phys. 2010, 6, 645-658, doi:10.1038/nphys1759.

29. Stewart, G.R. Unconventional superconductivity. Adv. Phys. 2017, 66, 75-196, doi:10.1080/00018732.2017.1331615.

30. Chandrasekhar, B.S. A Note on the Maximum Critical Field of High-Field Superconductors. Appl. Phys. Lett. 1962, 1, 7, doi:10.1063/1.1777362.

31. Clogston, A.M. Upper Limit for the Critical Field in Hard Superconductors. Phys. Rev. Lett. 1962, 9, $266-267$. doi:10.1103/PhysRevLett.9.266.

32. Klemm, R.A.; Luther, A.; Beasley, M.R. Theory of the upper critical field in layered superconductors. Phys. Rev. B 1975, 12, 877-891, doi:10.1103/PhysRevB.12.877.

33. Saint-James, D.; Sarma, G.; Thomas, E.J. Type II Superconductivity; Saint-James, D., Sarma, G., Thomas, E.J., Eds.; International Series of Monographs on Natural Philosophy; Pergamon Press: Oxford, NY, USA, 1969; Volume 17. 
34. Casalbuoni, R.; Nardulli, G. Inhomogeneous superconductivity in condensed matter and QCD. Rev. Mod. Phys. 2004, 76, 263-320, doi:10.1103/RevModPhys.76.263.

35. Wosnitza, J. FFLO States in Layered Organic Superconductors. Annalen der Physik 2018, 530, 1700282, doi:10.1002/andp.201700282.

36. Zwicknagl, G.; Wosnitza, J. Breaking Translational Invariance By Population Imbalance: The Fulde-FerrellLarkin-Ovchinnikov States. Int. J. Mod. Phys. B 2010, 24, 3915-3949, doi:10.1142/S0217979210056396.

37. Ptok, A.; Kapcia, K.J.; Piekarz, P.; Oleś, A.M. The ab initio study of unconventional superconductivity in CeCoIn5 and FeSe. New J. Phys. 2017, 19, 063039, doi:10.1088/1367-2630/aa6d9d.

38. Denisov, D.; Buzdin, A.; Shimahara, H. Types of Fulde-Ferrell-Larkin-Ovchinnikov states induced by anisotropy effects. Phys. Rev. B 2009, 79, 064506.

39. Croitoru, M.D.; Buzdin, A.I. Resonance in-plane magnetic field effect as a means to reveal the Fulde-Ferrell-Larkin-Ovchinnikov state in layered superconductors. Phys. Rev. B 2012, 86, 064507, doi:10.1103/PhysRevB.86.064507.

40. Ptok, A. The influence of the dimensionality of the system on the realization of unconventional FuldeFerrell-Larkin-Ovchinnikov pairing in ultra-cold Fermi gases. J. Phys. Condens. Matter 2017, 29, 475901, doi:10.1088/1361-648X/aa928d.

41. Croitoru, M.D.; Houzet, M.; Buzdin, A.I. In-Plane Magnetic Field Anisotropy of the Fulde-FerrellLarkin-Ovchinnikov State in Layered Superconductors. Phys. Rev. Lett. 2012, 108, 207005.

42. Gurevich, A. Upper critical field and the Fulde-Ferrel-Larkin-Ovchinnikov transition in multiband superconductors. Phys. Rev. B 2010, 82, 184504, doi:10.1103/PhysRevB.82.184504.

43. Bulaevskii, L.N. Inhomogeneous state and the anisotropy of the upper critical field in layered superconductors with Josephson layer interaction. Zhurnal Eksperimentalnoi i Teoreticheskoi Fiziki 1974, $38,1278-1288$.

44. Mola, M.M.; King, J.T.; McRaven, C.P.; Hill, S.; Qualls, J.S.; Brooks, J.S. Josephson plasma resonance in א-(BEDT-TTF $)_{2} \mathrm{Cu}(\mathrm{NCS})_{2}$. Phys. Rev. B 2000, 62, 5965-5970, doi:10.1103/PhysRevB.62.5965.

45. Hill, S.; Mola, M.M.; Qualls, J.S. Interlayer electrodynamics in the organic superconductor $\kappa$-(BEDT$\mathrm{TTF})_{2} \mathrm{Cu}(\mathrm{NCS})_{2}(\mathrm{BEDT}-\mathrm{TTF}=$ bis-ethylenedithio-tetrathiafulvalene): Evidence for a transformation within the vortex state. J. Phys. Condens. Matter 2002, 14, 6701.

46. Kirtley, J.R.; Moler, K.A.; Schlueter, J.A.; Williams, J.M. Inhomogeneous interlayer Josephson coupling in $\kappa$-(BEDT-TTF $)_{2} \mathrm{Cu}(\mathrm{NCS})_{2}$. J. Phys. Condens. Matter 1999, 11, 2007-2016.

47. Lebed, A.G. Orbital effect for the Fulde-Ferrell-Larkin-Ovchinnikov phase in a quasi-two-dimensional superconductor in a parallel magnetic field. Phys. Rev. B 2018, 97, 144504, doi:10.1103/PhysRevB.97.144504.

48. Werthamer, N.R.; Helfand, E.; Hohenberg, P.C. Temperature and Purity Dependence of the Superconducting Critical Field, $H_{c 2}$. III. Electron Spin and Spin-Orbit Effects. Phys. Rev. 1966, 147, 295-302, doi:10.1103/PhysRev.147.295.

49. Schneider, T.; Schmidt, A. Dimensional crossover in the upper critical field of layered superconductors. Phys. Rev. B 1993, 47, 5915-5921, doi:10.1103/PhysRevB.47.5915.

50. Ptok, A. The Fulde-Ferrell-Larkin-Ovchinnikov Superconductivity in Disordered Systems. Acta Phys. Pol. A 2010, 118, 420-422, doi:10.12693/aphyspola.118.420.

51. Tedrow, P.M.; Meservey, R. Spin-Paramagnetic Effects in Superconducting Aluminum Films. Phys. Rev. B 1973, 8, 5098-5108, doi:10.1103/PhysRevB.8.5098.

52. Gloos, K.; Modler, R.; Schimanski, H.; Bredl, C.D.; Geibel, C.; Steglich, F.; Buzdin, A.I.; Sato, N.; Komatsubara, T. Possible formation of a nonuniform superconducting state in the heavy-fermion compound $\mathrm{UPd}_{2} \mathrm{Al}_{3}$. Phys. Rev. Lett. 1993, 70, 501-504, doi:10.1103/PhysRevLett.70.501.

53. Tachiki, M.; Takahashi, S.; Gegenwart, P.; Weiden, M.; Lang, M.; Geibel, C.; Steglich, F.; Modler, R.; Paulsen, C.; Ōnuki, Y. Generalized Fulde-Ferrell-Larkin-Ovchinnikov state in heavy-fermion and intermediate-valence systems. Z. Phys. B Condens. Matter 1997, 100,369-380, doi:10.1007/s002570050135.

54. Radovan, H.A.; Fortune, N.A.; Murphy, T.P.; Hannahs, S.T.; Palm, E.C.; Tozer, S.W.; Hall, D. Magnetic enhancement of superconductivity from electron spin domains. Nature 2003, 425, 51-55. doi:10.1038/nature01842.

55. Bianchi, A.; Movshovich, R.; Capan, C.; Pagliuso, P.G.; Sarrao, J.L. Possible Fulde-Ferrell-Larkin-Ovchinnikov Superconducting State in CeCoIn. Phys. Rev. Lett. 2003, 91, 187004, doi:10.1103/physrevlett.91.187004. 
56. Mielke, C.; Singleton, J.; Nam, M.S.; Harrison, N.; Agosta, C.C.; Fravel, B.; Montgomery, L.K. Superconducting properties and Fermi-surface topology of the quasi-two-dimensional organic superconductor $\lambda$-(BETS) ${ }_{2} \mathrm{GaCl}_{4}$ (BETS=; bis(ethylene-dithio)tetraselenafulvalene). J. Phys. Condens. Matter 2001, 13, 8325-8345.

57. Singleton, J.; Goddard, P.; Ardavan, A.; Harrison, N.; Blundell, S.; Schlueter, J.; Kini, A. Test for interlayer coherence in a quasi-two-dimensional superconductor. Phys. Rev. Lett. 2002, 88, 037001.

58. Singleton, J.; Goddard, P.A.; Ardavan, A.; Coldea, A.I.; Blundell, S.J.; McDonald, R.D.; Tozer, S.; Schlueter, J.A. Persistence to High Temperatures of Interlayer Coherence in an Organic Superconductor. Phys. Rev. Lett. 2007, 99, 027004, doi:10.1103/PhysRevLett.99.027004.

59. Mansky, P.A.; Chaikin, P.M.; Haddon, R.C. Vortex lock-in state in a layered superconductor. Phys. Rev. Lett. 1993, 70, 1323-1326, doi:10.1103/PhysRevLett.70.1323.

60. Mansky, P.A.; Chaikin, P.M.; Haddon, R.C. Evidence for Josephson vortices in $\kappa$-(BEDT-TTF) ${ }_{2} \mathrm{Cu}(\mathrm{NCS})_{2}$. Phys. Rev. B 1994, 50, 15929-15944, doi:10.1103/PhysRevB.50.15929.

61. Singleton, J.; Mielke, C. Quasi-two-dimensional organic superconductors: A review. Contemp. Phys. 2002, 43, 63-96.

62. Agosta, C.; Martin, C.; Radovan, H.; Palm, E.; Murphy, T.; Tozer, S.; Cooley, J.; Schlueter, J.; Petrovic, C. Penetration depth studies of organic and heavy fermion superconductors in the Pauli paramagnetic limit. J. Phys. Chem. Solids 2006, 67, 586-589.

63. Coffey, T.; Bayindir, Z.; DeCarolis, J.F.; Bennett, M.; Esper, G.; Agosta, C.C. Measuring radio frequency properties of materials in pulsed magnetic fields with a tunnel diode oscillator. Rev. Sci. Inst. 2000, 71, 4600-4606, doi:10.1063/1.1321301.

64. Martin, C.; Agosta, C.C.; Tozer, S.W.; Radovan, H.A.; Palm, E.C.; Murphy, T.P.; Sarrao, J.L. Evidence for the Fulde-Ferrell-Larkin-Ovchinnikov state in $\mathrm{CeCoIn}_{5}$ from penetration depth measurements. Phys. Rev. B 2005, 71, 020503, doi:10.1103/PhysRevB.71.020503.

65. Martin, C.; Agosta, C.; Tozer, S.; Radovan, H.; Kinoshota, T.; Tokumoto, M. Critical Field and Shubnikov-de Haas Oscillations of $\kappa$-(BEDT-TTF $)_{2} \mathrm{Cu}(\mathrm{NCS})_{2}$ under Pressure. J. Low Temp. Phys. 2005, 138, 1025-1037.

66. Orlando, T.P.; McNiff, E.J., Jr.; Foner, S.; Beasley, M.R. Critical fields, Pauli paramagnetic limiting, and material parameters of $\mathrm{Nb}_{3} \mathrm{Sn}$ and $\mathrm{V}_{3}$ Si. Phys. Rev. B 1979, 19, 4545-4561.

67. Schossmann, M.; Carbotte, J. Pauli limiting of the upper critical magnetic field. Phys. Rev. B 1989, 39, 4210-4216, doi:10.1103/PhysRevB.39.4210.

68. Zuo, F.; Brooks, J.S.; McKenzie, R.H.; Schlueter, J.A.; Williams, J.M. Paramagnetic limiting of the upper critical field of the layered organic superconductor $\kappa$-(BEDT-TTF $)_{2} \mathrm{Cu}(\mathrm{SCN})_{2}$. Phys. Rev. B 2000, 61, 750-755, doi:10.1103/PhysRevB.61.750.

69. McKenzie, R.H. Wilson's ratio and the spin splitting of magnetic oscillations in quasi-two-dimensional metals. arXiv 1999, arXiv.9905044.

70. Padamsee, H.; Neighbor, J.; Shiffman, C. Quasiparticle phenomenology for thermodynamics of strongcoupling superconductors. J. Low Temp. Phys. 1973, 12, 387-411.

71. Johnston, D.C. Elaboration of the $\alpha$-model derived from the BCS theory of superconductivity. Superconduct. Sci. Technol. 2013, 26, 115011.

72. Taylor, O.J.; Carrington, A.; Schlueter, J.A. Specific-Heat Measurements of the Gap Structure of the Organic Superconductors $\kappa-(\mathrm{BEDT}-\mathrm{TTF})_{2}-\mathrm{Cu}\left[\mathrm{N}(\mathrm{CN})_{2}\right] \mathrm{Br}$ and $\kappa-(\mathrm{ET})_{2} \mathrm{Cu}(\mathrm{NCS})_{2}$. Phys. Rev. Lett. 2007, 99, 057001, doi:10.1103/PhysRevLett.99.057001.

73. Nakazawa, Y.; Kanoda, K. Low-temperature specific heat of $\kappa$-(BEDT-TTF $)_{2} \mathrm{Cu}\left[\mathrm{N}(\mathrm{CN})_{2}\right] \mathrm{Br}$ in the superconducting state. Phys. Rev. B 1997, 55, R8670-R8673, doi:10.1103/PhysRevB.55.R8670.

74. Analytis, J.G.; Ardavan, A.; Blundell, S.J.; Owen, R.L.; Garman, E.F.; Jeynes, C.; Powell, B.J. Effect of Irradiation-Induced Disorder on the Conductivity and Critical Temperature of the Organic Superconductor $\kappa$-(BEDT-TTF) ${ }_{2} \mathrm{Cu}(\mathrm{NCS})_{2}$ ]. Phys. Rev. Lett. 2006, 96, 177002, doi:10.1103/PhysRevLett.96.177002.

75. Zantout, K.; Altmeyer, M.; Backes, S.; Valentí, R. Superconductivity in correlated BEDT-TTF molecular conductors: Critical temperatures and gap symmetries. Phys. Rev. B 2018, 97, 014530, doi:10.1103/PhysRevB.97.014530.

76. Coffey, T.; Martin, C.; Agosta, C.C.; Kinoshota, T.; Tokumoto, M. Bulk two-dimensional Pauli-limited superconductor. Phys. Rev. B 2010, 82, 212502, doi:10.1103/PhysRevB.82.212502.

77. Mihut, I.; Agosta, C.; Martin, C.; Mielke, C.; Coffey, T. Incoherent Bragg reflection and Fermi-surface hot spots in a quasi-two-dimensional metal. Phys. Rev. B 2006, 73, 125118, doi:10.1103/PhysRevB.73.125118. 
78. Wanka, S.; Hagel, J.; Beckmann, D.; Wosnitza, J.; Schlueter, J.A.; Williams, J.M.; Nixon, P.G.; Winter, R.W.; Gard, G.L. Specific heat and critical fields of the organic superconductor $\beta^{\prime \prime}$-(BEDT-TTF) ${ }_{2} \mathrm{SF}_{5} \mathrm{CH}_{2} \mathrm{CF}_{2} \mathrm{SO}_{3}$. Phys. Rev. B 1998, 57, 3084-3088, doi:10.1103/PhysRevB.57.3084.

79. Elsinger, H.; Wosnitza, J.; Wanka, S.; Hagel, J.; Schweitzer, D.; Strunz, W. $\kappa$-(BEDT-TTF $)_{2}-\mathrm{Cu}\left[\mathrm{N}(\mathrm{CN})_{2}\right] \mathrm{Br}$ : A Fully Gapped Strong-Coupling Superconductor, Phys. Rev. Lett. 2000, 84, 6098-6101. doi:10.1103/PhysRevLett.84.6098.

80. Imajo, S.; Kanda, N.; Yamashita, S.; Akutsu, H.; Nakazawa, Y.; Kumagai, H.; Kobayashi, T.; Kawamoto, A. Thermodynamic Evidence of d-Wave Superconductivity of the Organic Superconductor $\lambda$-(BETS) ${ }_{2} \mathrm{GaCl}_{4}$. J. Phys. Soc. Jpn. 2016, 85, 043705, doi:10.7566/JPSJ.85.043705.

81. Kwok, W.; Welp, U.; Carlson, K.; Crabtree, G.; Vandervoort, K.; Wang, H.; Kini, A.; Williams, J.; Stupka, D.; Montgomery, L. Unusual behavior in the upper critical magnetic fields of the ambient-pressure organic superconductor $\kappa-(\mathrm{BEDT}-\mathrm{TTF})_{2} \mathrm{Cu}\left[\mathrm{N}(\mathrm{CN})_{2}\right] \mathrm{Br}$ [where BEDT-TTF represents bis (ethylenedithio) tetrathiofulvalene]. Phys. Rev. B 1990, 42, 8686-8689.

82. Mielke, C.; Harrison, N.; Rickel, D.; Lacerda, A.; Vestal, R.; Montgomery, L. Fermi-surface topology of $\kappa-(B E D T-T T F)_{2} \mathrm{Cu}\left[\mathrm{N}(\mathrm{CN})_{2}\right] \mathrm{Br}$ at ambient pressure. Phys. Rev. B 1997, 56, 4309-4312.

83. Petrovic, C.; Pagliuso, P.G.; Hundley, M.F.; Movshovich, R.; Sarrao, J.L.; Thompson, J.D.; Fisk, Z.; Monthoux, P. Heavy-fermion superconductivity in CeCoIn 5 at 2.3 K. J. Phys. Condens. Matter 2001, 13, L337.

84. Ikeda, S.; Shishido, H.; Nakashima, M.; Settai, R.; Aoki, D.; Haga, Y.; Harima, H.; Aoki, Y.; Namiki, T.; Sato, H.; et al. Unconventional Superconductivity in CeCoIn5 Studied by the Specific Heat and Magnetization Measurements. J. Phys. Soc. Jpn. 2001, 70, 2248-2251, doi:10.1143/JPSJ.70.2248.

85. Murphy, T.P.; Hall, D.; Palm, E.C.; Tozer, S.W.; Petrovic, C.; Fisk, Z.; Goodrich, R.G.; Pagliuso, P.G.; Sarrao, J.L.; Thompson, J.D. Anomalous superconductivity and field-induced magnetism in CeCoIn 5 . Phys. Rev. B 2002, 65, doi:10.1103/PhysRevB.65.100514.

86. Abdel-Hafiez, M.; Aswartham, S.; Wurmehl, S.; Grinenko, V.; Hess, C.; Drechsler, S.L.; Johnston, S.; Wolter, A.U.B.; Büchner, B.; Rosner, H.; et al. Publisher's Note: Specific heat and upper critical fields in $\mathrm{KFe}_{2} \mathrm{As}_{2}$ single crystals. Phys. Rev. B 2012, 85, 179901.

87. Hardy, F.; Böhmer, A.E.; Aoki, D.; Burger, P.; Wolf, T.; Schweiss, P.; Heid, R.; Adelmann, P.; Yao, Y.X.; Kotliar, G.; et al. Evidence of Strong Correlations and Coherence-Incoherence Crossover in the Iron Pnictide Superconductor $\mathrm{KFe}_{2} \mathrm{As}_{2}$. Phys. Rev. Lett. 2013, 111, 027002, doi:10.1103/PhysRevLett.111.027002.

88. Zocco, D.A.; Grube, K.; Eilers, F.; Wolf, T.; Löhneysen, H.v. Pauli-Limited Multiband Superconductivity in $\mathrm{KFe}_{2} \mathrm{As}_{2}$. Phys. Rev. Lett. 2013, 111, 057007, doi:10.1103/PhysRevLett.111.057007.

89. Kim, H.; Tanatar, M.A.; Song, Y.J.; Kwon, Y.S.; Prozorov, R. Nodeless two-gap superconducting state in single crystals of the stoichiometric iron pnictide LiFeAs. Phys. Rev. B 2011, 83, 100502, doi:10.1103/PhysRevB.83.100502.

90. Cho, K.; Kim, H.; Tanatar, M.; Song, Y.; Kwon, Y.; Coniglio, W.; Agosta, C.; Gurevich, A.; Prozorov, R. Anisotropic upper critical field and possible Fulde-Ferrel-Larkin-Ovchinnikov state in the stoichiometric pnictide superconductor LiFeAs. Phys. Rev. B 2011, 83, 060502, doi:10.1103/PhysRevB.83.060502.

91. Gruenberg, L.W.; Gunther, L. Fulde-Ferrell Effect in Type-II Superconductors. Phys. Rev. Lett. 1966, 16, 996-998, doi:10.1103/PhysRevLett.16.996.

92. Singleton, J.; Symington, J.A.; Nam, M.S.; Ardavan, A.; Kurmoo, M.; Day, P. Observation of the Fulde-Ferrell-Larkin-Ovchinnikov state in the quasi-two-dimensional organic superconductor $\kappa$-(BEDT$\mathrm{TTF})_{2} \mathrm{Cu}(\mathrm{NCS})_{2}$ (BEDT-TTF $=$ bis(ethylene-dithio)tetrathiafulvalene). J Phys. Condens. Matter 2000, 12, L641-L648.

93. Lortz, R.; Wang, Y.; Demuer, A.; Böttger, P.H.M.; Bergk, B.; Zwicknagl, G.; Nakazawa, Y.; Wosnitza, J. Calorimetric Evidence for a Fulde-Ferrell-Larkin-Ovchinnikov Superconducting State in the Layered Organic Superconductor $\kappa$-(BEDT-TTF) ${ }_{2} \mathrm{Cu}(\mathrm{NCS})_{2}$. Phys. Rev. Lett. 2007, 99, 187002, doi:10.1103/PhysRevLett.99.187002.

94. Cai, Z.; Wang, Y.; Wu, C. Stable Fulde-Ferrell-Larkin-Ovchinnikov pairing states in two-dimensional and three-dimensional optical lattices. Phys. Rev. A 2011, 83, 063621.

95. Tokiwa, Y.; Bauer, E.D.; Gegenwart, P. Quasiparticle Entropy in the High-Field Superconducting Phase of CeCoIn 5 . Phys. Rev. Lett. 2012, 109, 116402.

96. Young, B.L.; Urbano, R.R.; Curro, N.J.; Thompson, J.D.; Sarrao, J.L.; Vorontsov, A.B.; Graf, M.J. Microscopic Evidence for Field-Induced Magnetism in CeCoIn 5 . Phys. Rev. Lett. 2007, 98, 036402. doi:10.1103/PhysRevLett.98.036402. 
97. Kenzelmann, M.; Strässle, T.; Niedermayer, C.; Sigrist, M.; Padmanabhan, B.; Zolliker, M.; Bianchi, A.D.; Movshovich, R.; Bauer, E.D.; Sarrao, J.L.; et al. Coupled Superconducting and Magnetic Order in CeCoIn 5 . Science 2008, 321, 1652-1654, doi:10.1126/science.1161818.

98. Kenzelmann, M.; Gerber, S.; Egetenmeyer, N.; Gavilano, J.L.; Strässle, T.; Bianchi, A.D.; Ressouche, E.; Movshovich, R.; Bauer, E.D.; Sarrao, J.L.; et al. Evidence for a Magnetically Driven Superconducting Q Phase of CeCoIn 5 . Phys. Rev. Lett. 2010, 104, 127001, doi:10.1103/PhysRevLett.104.127001.

99. Raymond, S.; Ramos, S.M.; Aoki, D.; Knebel, G.; Mineev, V.P.; Lapertot, G. Magnetic Order in Ce 0.95Nd 0.05CoIn 5: The Q-Phase at Zero Magnetic Field. J. Phys. Soc. Jpn. 2014, 83, 013707, doi:10.7566/JPSJ.83.013707.

100. Koutroulakis, G.; Stewart, M.D.J.; Mitrovic, V.F.; Horvatic, M.; Berthier, C.; Lapertot, G.; Flouquet, J. Field Evolution of Coexisting Superconducting and Magnetic Orders in CeCoIn 5 . Phys. Rev. Lett. 2010, 104, 087001, doi:10.1103/PhysRevLett.104.087001.

101. Kumagai, K.; Shishido, H.; Shibauchi, T.; Matsuda, Y. Evolution of Paramagnetic Quasiparticle Excitations Emerged in the High-Field Superconducting Phase of CeCoIn 5 . Phys. Rev. Lett. 2011, 106, 137004, doi:10.1103/PhysRevLett.106.137004.

102. Hatakeyama, Y.; Ikeda, R. Antiferromagnetic order oriented by Fulde-Ferrell-Larkin-Ovchinnikov superconducting order. Phys. Rev. B 2015, 91, 094504, doi:10.1103/physrevb.91.094504.

103. Fortune, N.A.; Hannahs, S.T. Top-loading small-sample calorimeters for measurements as a function of magnetic field angle. J. Phys. Conf. Ser. 2014, 568, 032008, doi:10.1088/1742-6596/568/3/032008.

104. Kim, D.Y.; Lin, S.Z.; Weickert, F.; Kenzelmann, M.; Bauer, E.D.; Ronning, F.; Thompson, J.D.; Movshovich, R. Intertwined Orders in Heavy-Fermion Superconductor CeCoIn 5 . Phys. Rev. X 2016, 6, 041059, doi:10.1103/PhysRevX.6.041059.

105. Ptok, A.; Crivelli, D. The Fulde-Ferrell-Larkin-Ovchinnikov State in Pnictides. J. Low Temp. Phys. 2013, 172, 226-233, doi:10.1007/s10909-013-0871-0.

106. Ptok, A. Multiple phase transitions in Pauli-limited iron-based superconductors. J. Phys. Condens. Matter 2015, 27, 482001, doi:10.1088/0953-8984/27/48/482001.

107. Cho, C.W.; Yang, J.H.; Yuan, N.F.Q.; Shen, J.; Wolf, T.; Lortz, R. Thermodynamic Evidence for the Fulde-Ferrell-Larkin-Ovchinnikov State in the $\mathrm{KFe}_{2} \mathrm{As}_{2}$ Superconductor. Phys. Rev. Lett. 2017, 119, 593-595, doi:10.1103/PhysRevLett.119.217002.

108. Kittaka, S.; Aoki, Y.; Kase, N.; Sakakibara, T.; Saito, T.; Fukazawa, H.; Kohori, Y.; Kihou, K.; Lee, C.H.; Iyo, A.; et al. Thermodynamic Study of Nodal Structure and Multiband Superconductivity of $\mathrm{KFe}_{2} \mathrm{As}_{2}$. J. Phys. Soc. Jpn. 2014, 83, 013704, doi:10.7566/JPSJ.83.013704.

109. Poole, C.; Farach, H.; Creswick, R.; Prozorov, R. Superconductivity; Elsevier Science: New York, NY, USA, 2014.

110. Buzdin, A.I.; Fiz, V.T. Phase diagrams of electronic and superconducting transitions to soliton lattice states. Sov. Phys. JETP 1983, 58, 428, doi:10.1007/BF02880301.

111. Lebed, A.G.; Wu, S. Larkin-Ovchinnikov-Fulde-Ferrell phase in the superconductor $(\mathrm{TMTSF})_{2} \mathrm{ClO}_{4}$ : Theory versus experiment. Phys. Rev. B 2010, 82, 172504, doi:10.1103/PhysRevB.82.172504.

112. Lee, I.J.; Brown, S.E.; Naughton, M.J. Unconventional Superconductivity in a Quasi-One-Dimensional System (TMTSF) 2X. J. Phys. Soc. Jpn. 2006, 75, 051011, doi:10.1143/JPSJ.75.051011.

(C) 2018 by the author. Licensee MDPI, Basel, Switzerland. This article is an open access article distributed under the terms and conditions of the Creative Commons Attribution (CC BY) license (http:/ / creativecommons.org/licenses/by/4.0/). 


\title{
Design of Spin-Frustrated Monomer-Type $\mathrm{C}_{60}{ }^{\bullet-}$ Mott Insulator
}

\author{
Akihiro Otsuka ${ }^{1,2, *}$, Dmitri V. Konarev ${ }^{3, *}$, Rimma N. Lyubovskaya ${ }^{3}$, Salavat S. Khasanov ${ }^{4}$, \\ Mitsuhiko Maesato ${ }^{2}$, Yukihiro Yoshida ${ }^{2,5}$ and Gunzi Saito ${ }^{5,6, *}$ \\ 1 Research Center for Low Temperature and Materials Sciences, Kyoto University, Sakyo-ku, \\ Kyoto 606-8501, Japan \\ 2 Department of Chemistry, Graduate School of Science, Kyoto University, Sakyo-ku, Kyoto 606-8502, Japan; \\ maesato@kuchem.kyoto-u.ac.jp (M.M.); yoshiday@ssc.kuchem.kyoto-u.ac.jp (Y.Y.) \\ 3 Institute of Problems of Chemical Physics RAS, Chernogolovka, Moscow Region 142432, Russia; \\ lyurn@icp.ac.ru \\ 4 Institute of Solid State Physics RAS, Chernogolovka, Moscow Region 142432, Russia; khasanov@issp.ac.ru \\ 5 Department of Agriculture, Meijo University, 1-501 Shiogamaguchi, Tempaku-ku, Nagoya 468-8502, Japan \\ 6 Toyota Physical and Chemical Research Institute, Nagakute, Aichi 480-1192, Japan \\ * Correspondence: otsuka@kuchem.kyoto-u.ac.jp (A.O.); konarev3@yandex.ru (D.V.K.); \\ gsaito@meijo-u.ac.jp (G.S.); Tel.: +81-75-753-4062 (A.O. \& D.V.K. \& G.S.)
}

Received: 30 January 2018; Accepted: 25 February 2018; Published: 28 February 2018

\begin{abstract}
Spin-frustrated monomer-type Mott insulator $\mathrm{C}_{60} 0^{--}$solids are discussed in this review article. For the $\mathrm{C}_{60}{ }^{\circ-}$ solids, the interfullerene center-to-center distance $(r)$ is the key parameter that controls the competition between covalent bond-formation, itinerancy, and spin frustration. Eight $\mathrm{C}_{60}{ }^{\bullet-}$ salts with various compositions and dimensionalities are reviewed. In all of these $\mathrm{C}_{60}{ }^{\bullet-}$ salts except one, neither bond-formation nor long-range magnetic ordering was observed down to low temperatures. A plot of Weiss temperature $\left(\left|\Theta_{\mathrm{CW}}\right|\right)$ against $r$ shows that $\left|\Theta_{\mathrm{CW}}\right|$ grows rapidly below $r=10.0 \AA$.
\end{abstract}

Keywords: spin frustration; Mott insulators; key-keyhole relation; quantum spin liquid

\section{Introduction: Quantum Spin Liquid State}

Strong geometrical spin frustration suppresses the classical long-range magnetic ordering of the Néel state, and allows the novel quantum states such as the quantum spin liquid (QSL) state for two-dimensional (2D) $S=1 / 2$ antiferromagnets ( $\mathrm{AFs}$ ), as proposed by Anderson [1,2]. The QSL phase is a quantum-disordered insulating phase, which has been theoretically predicted to have a ground state with many degenerate states [3], and, hence, to exhibit large spin entropy, even at $0 \mathrm{~K}$. To obtain spin-frustrated materials, the geometries of spin lattice subject to contradictory constraints are crucial. The spin lattices-triangle (Figure 1a), kagome (Figure 1b), zigzag (Figure 1c), tetrahedron (Figure 1d), honeycomb (Figure 1e), and hyperkagome (Figure 1f)—have been discussed with the basic building block being the triangle [4]. The magnetic exchange interaction $J$ of spins is proportional to the square of transfer interaction $t$ (Equation (1)), where $U$ is the on-site Coulomb repulsion energy,

$$
J \sim 4 t^{2} / U
$$

A small distance between two spins ( $r$ ) induces a large $t$ and eventually, a metallic band with itinerant electrons is formed. Equation (1) suggests that the QSL state may be adjacent to such an itinerant (metallic or superconducting (SC)) phase. When the distance between the spin sites in the triangular spin lattice becomes extremely large, all of the spins may act as Curie spins. With decreasing distance between the spins, spins feel frustration to each other and form a QSL or a compromised 
spin configurations which do not have spin frustration with geometry of spin lattice of $120^{\circ}$ (triangle, Figure 1g), $109^{\circ}$ (tetrahedron, Figure 1h), or collinear AF (AFC) (Figure 1i) structures [5-11]. When the triangular spin lattice is distorted, the AF phase may get preferentially stabilized. Therefore, the QSL, $\mathrm{AF}, 120^{\circ}$ structure (Figure $1 \mathrm{~g}$ ), AFC, and metallic (or SC) phases compete with each other in a triangular spin lattice based on the parameters $r, t, W, U, J$, outer stimuli (temperature $T$, pressure $P$, magnetic field $H$, etc.), and the geometry of the spin lattice, where $W$ is the bandwidth.
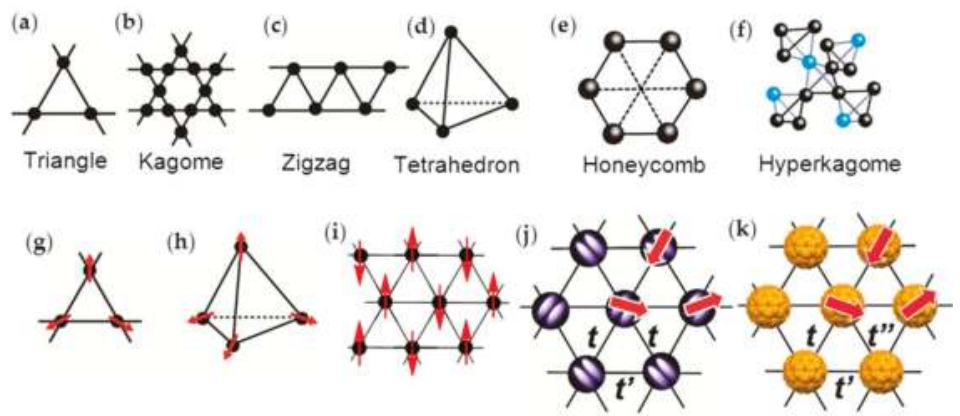

Figure 1. Geometries of spin lattices having strong spin-frustration (a-f), non-frustrated spin-configurations (g) $120^{\circ}$, (h) $109^{\circ}$, and (i) collinear AF (AFC): (j,k) are schematic view of triangular spin lattice of dimer-type Mott insulator of $\kappa-(E T)_{2} X(j)$ and monomer-type Mott insulator of $\mathrm{C}_{60} \bullet^{-}(\mathbf{k})$. Purple ellipsoid in (j) is an ET molecule and the black circle represents one spin site $(E T)_{2}$. In (j), $t$ and $t^{\prime}$ are interdimer transfer interactions between parallel dimers and perpendicular dimers, respectively, and $t^{\prime} / t$ represents the shape of the isosceles triangular spin lattice. In $(\mathbf{k}), t, t^{\prime}$, and $t^{\prime \prime}$ are interfullerene transfer interactions. Red arrows indicate spins. $(\mathbf{j}, \mathbf{k})$ were reproduced from [12].

The Curie-Weiss temperature $\Theta_{\mathrm{CW}}$ given by,

$$
\Theta_{\mathrm{CW}}=2 z S(S+1) J / 3 k_{\mathrm{B}}
$$

is a parameter showing the easiness to access the QSL state, where, $z, S$, and $k_{\mathrm{B}}$ are the number of the nearest neighbour sites, spin quantum number, and Boltzmann constant, respectively. The frustration index $f$ defined by Equation (3),

$$
f=-\Theta_{\mathrm{CW}} / T_{\mathrm{m}}
$$

was proposed by Ramirez as a measure of the spin frustration $[13,14]$. Strong spin-frustrated systems are thought to be those associated with $f>10$. In Equation (3), $T_{\mathrm{m}}$ is the temperature at which magnetic ordering occurs.

The easiest way to design spin-frustrated systems is to arrange magnetic transition metals in a triangular or kagome geometry. Numerous inorganic compounds have been examined based on this idea. A few examples are $\mathrm{VX}_{2}(\mathrm{X}=\mathrm{Cl}, \mathrm{Br})$ [15], $\mathrm{ABO}_{2}$ [16-21] $(\mathrm{A}=$ monocation, $\mathrm{B}=$ trivalent transition metal ions: $\mathrm{CuFeO}_{2}$ [16-18], $\mathrm{LiNiO}_{2}$ [19], $\mathrm{NaTiO}_{2}$, [19], $\mathrm{LiCrO}_{2}$ [20-22]), $\mathrm{ABX}_{3}[23,24]$ ( $\mathrm{X}=$ halogenide ions: $\mathrm{CsCoCl}_{3}$ [23], $\mathrm{CsMnBr}_{3}$ [24]), and large clusters $\left(\mathrm{Mo}_{72} \mathrm{Fe}_{30}, \mathrm{Mo}_{72} \mathrm{~V}_{20}\right)$ [25] for the triangular lattice, and $\mathrm{KFe}_{3}\left(\mathrm{SO}_{4}\right)_{2}(\mathrm{OH})_{6}$ [26-28] and $\mathrm{Rb}_{2} \mathrm{SnCu}_{3} \mathrm{~F}_{12}$ [29] for the kagome lattice. Some typical magnetically frustrated inorganic systems of transition metal oxides studied prior 2000 have been summarized by Ramirez [13] and Greedan [4].

However, QSL systems are scarce in materials with spin quantum numbers $S>1 / 2$, even in the triangular spin lattices and kagome lattices with $f \geq 10^{2}$ [30-32]. Even for the triangular $S=1 / 2$ spin systems with large $\left|\Theta_{\mathrm{CW}}\right|$ and $f$, no QSL materials have been prepared due to the difficulty in maintaining the precise geometry of spin-frustrated lattices at low temperatures [33-35]. 
The first real QSL candidate was a charge-transfer (CT) salt of a dimer-type Mott insulator $\kappa-(\mathrm{ET})_{2} \mathrm{Cu}_{2}(\mathrm{CN})_{3}[36]$, where ET is bis(ethylenedithio)tetrathiafulvalene (chemicals in this review are shown in Figure 2) and $\left[\mathrm{Cu}_{2}(\mathrm{CN})_{3}{ }^{-}\right]_{\infty}$ is a diamagnetic polymeric anion. The planar tridentate coordination of diamagnetic $\mathrm{Cu}(\mathrm{I})$ ions in $\left[\mathrm{Cu}_{2}(\mathrm{CN})_{3}{ }^{-}\right]_{\infty}$ is the main driving force for the twodimensional (2D) triangular magnetic lattice that is composed of partially charged $(\mathrm{ET})_{2}{ }^{\bullet+}$, since $\left[\mathrm{Cu}_{2}(\mathrm{CN})_{3}{ }^{-}\right]_{\infty}$ has openings, and the arrangement of the anion openings is triangular due to the planar tridentate coordination of $\mathrm{Cu}(\mathrm{I})$ ions. The geometrical fit between a spin-site $(\mathrm{ET})_{2}{ }^{\bullet+}$ and the anion opening results in a triangular magnetic lattice (Figure 1j) according to a key-keyhole relation, where the key is the spin-site, $(\mathrm{ET})_{2}{ }^{\bullet+}$, and the keyhole is the anion opening.

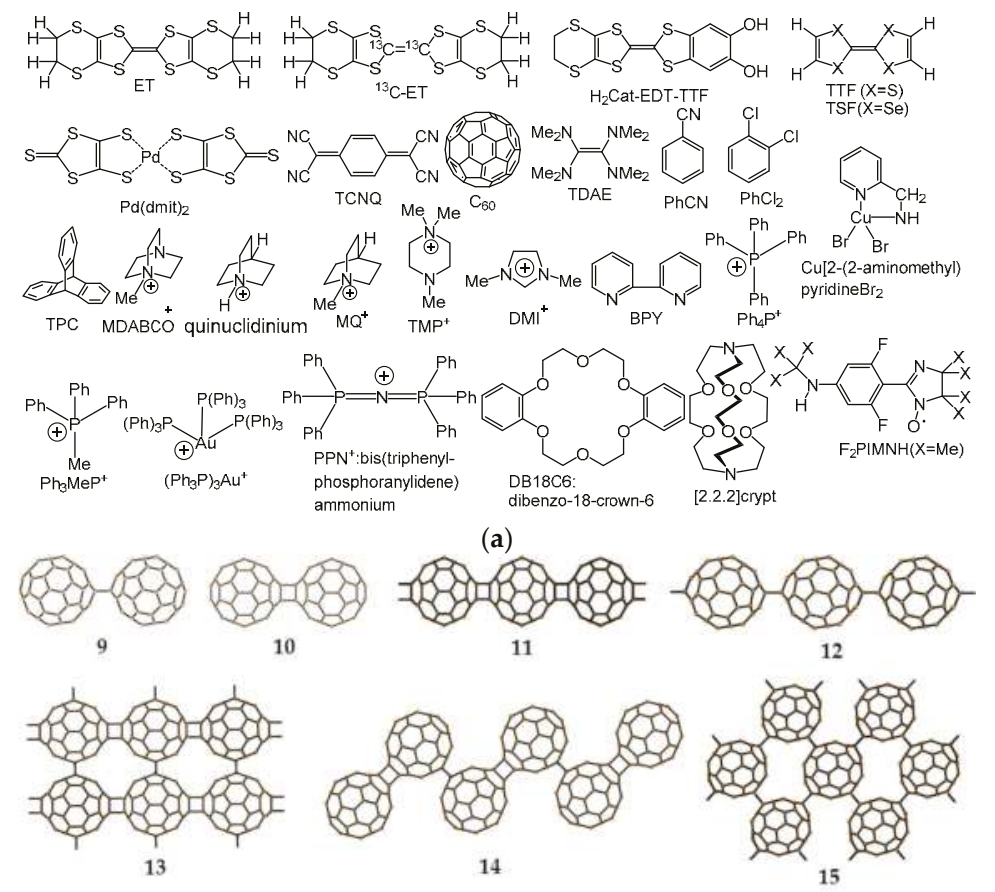

(b)

Figure 2. (a) Chemicals in this review. The abbreviations are, TDAE: tetrakis(dimethylamino)ethylene, TPC: triptycene, $\mathrm{MDABCO}^{+}: \mathrm{N}$-methyldiazabicyclooctane cation, $\mathrm{MQ}^{+}: N$-methylquinuclidinium cation, $\mathrm{DMI}^{+}: N, N^{\prime}$-dimethylimidazolium cation, $\mathrm{TMP}^{+}: N, N, N^{\prime}$-trimethylpiperazinium cation, BPY: 2,2'-bipyridine, $\mathrm{PhCN}$ : benzonitrile ( $\mathrm{Ph}=$ phenyl group), $\mathrm{PhCl}_{2}$ : o-dichlorobenzene, $\mathrm{Ph}_{4} \mathrm{P}^{+}$: tetraphenylphosphonium cation, $\mathrm{Ph}_{3} \mathrm{MeP}^{+}$: methyltriphenylphosphonium cation, $\left(\mathrm{Ph}_{3} \mathrm{P}\right)_{3} \mathrm{Au}^{+}$: tris(triphenyl) phosphine $\mathrm{Au}$ cation, $\mathrm{PPN}^{+}$: bis(triphenylphosphoranylidene)ammonium cation, DB18C6: dibenzo-18-crown-6, and [2.2.2]crypt: 4,7,13,16,21,24-hexaoxa-1,10-diazabicyclo-[8.8.8] hexacosane; (b) Some examples of fullerene dimers and polymers. $\mathrm{C}_{60} \sigma$-dimer (9) [37], $\mathrm{C}_{60} \pi$-dimer (10) [38], $\left(\mathrm{C}_{60}{ }^{-}\right)_{\mathrm{n}}$ polymer in $\mathrm{MC}_{60}(\mathrm{M}=\mathrm{K}, \mathrm{Rb}$, and $\mathrm{Cs})(\mathbf{1 1})[39,40]$, linear polymer of $\left(\mathrm{C}_{60}{ }^{3-}\right)_{\mathrm{n}}$ trianion in $\mathrm{Na}_{2} \mathrm{RbC}_{60}$ (12) [41], polymeric layer of $\left(\mathrm{C}_{60}{ }^{4-}\right)_{\mathrm{n}}$ tetraanion in $\mathrm{Li}_{4} \mathrm{C}_{60}$ (13) [42], zigzag polymer of $\left(\mathrm{C}_{60}{ }^{2+}\right)_{\mathrm{n}}$ dication in $\left(\mathrm{C}_{60}{ }^{2+}\right)\left(\mathrm{AsF}_{6}{ }^{-}\right)_{2}$ (14) [43], polymeric layer of $\left(\mathrm{C}_{60}{ }^{4-}\right)_{\mathrm{n}}$ tetraanion in $\mathrm{Na}_{4} \mathrm{C}_{60}$ (15) [44].

The features of the Mott insulator $\kappa-(\mathrm{ET})_{2} \mathrm{Cu}_{2}(\mathrm{CN})_{3}$ are: (1) it has a nearly equilateral triangular lattice $\left(t^{\prime} / t=1.09\right)$ with very strong electron correlation $(U / W=0.93)$, (2) the QSL state is experimentally 
confirmed down to $20 \mathrm{mK}[36,45,46]$, (3) the anisotropic SC state resides directly next to the QSL state without passing through the spin ordered AF state under pressure [47-49], (4) the transition from the QSL state to the metallic state shows positive pressure dependence, indicating that the residual spin entropy is in the QSL state [47-50], and (5) ${ }^{13} \mathrm{C}$ NMR measurements under hydrostatic pressure on the salt of ET with ${ }^{13} \mathrm{C}$ enriched at the central $\mathrm{C}=\mathrm{C}$ bond $\left({ }^{13} \mathrm{C}\right.$-ET $)$ indicate $d$-wave SC symmetry [51]. Thus, a competition among the localized (and frustrated), itinerant, and exotic pairing of spins is manifested in this salt [36,45-53]. We have proposed designing principles for a QSL candidate residing next to the $\mathrm{SC}$ state based on the crystal, electronic, and spin structures for selected $\kappa-(\mathrm{ET})_{2} \mathrm{X}(\mathrm{X}=$ anion) including $\mathrm{X}=\mathrm{Cu}_{2}(\mathrm{CN})_{3}$ as follows [54].

The requirements for a QSL state next to an itinerant state for $\kappa-(\mathrm{ET})_{2} \mathrm{X}$ are:

(1) the system has a low spin state $(S=1 / 2)$,

(2) the system should be a Mott insulator in ambient conditions,

(3) its Mott insulating state has both a partial CT state close to the itinerant region and a small Mott gap,

(4) the spin lattice should have a geometry that affords a strong geometrical frustration, i.e., $t^{\prime} / t \sim 1$ for a triangular spin lattice,

(5) a high $\left|\Theta_{\mathrm{CW}}\right|$ or high $|J|$ value to observe the QSL state at the experimentally available temperatures, and

(6) the material must maintain weak energy dispersion along the weakest direction for the magnetic interactions of the 2D system, i.e., negligibly weak magnetic interaction perpendicular to the $2 \mathrm{D}$ magnetic layer in order to keep the geometry of spin-frustrated spin lattice down to low temperatures.

Since the discovery of the QSL state in $\kappa-(\mathrm{ET})_{2} \mathrm{Cu}_{2}(\mathrm{CN})_{3}\left(\left|\Theta_{\mathrm{CW}}\right|=375 \mathrm{~K},|J| / k_{\mathrm{B}}=250 \mathrm{~K}\right.$, $f>1.3 \times 10^{4}$ ), several materials based on the triangular, kagome, honeycomb, and hyperkagome spin lattices have been reported to have such a spin state [55-57]. Some organic and inorganic compounds with sufficiently large $\left|\Theta_{\mathrm{CW}}\right|,|J|$, and $f$ examined on single crystals include $\kappa-\mathrm{H}_{3}(\mathrm{Cat}-$ EDT-TTF) ${ }_{2}$ (triangle, $\mathrm{H}_{2}$ Cat-EDT-TTF: catechol-fused ethylenedithio-TTF, $\left|\Theta_{\mathrm{CW}}\right|=120-150 \mathrm{~K}$, $\left.|J| / \mathrm{k}_{\mathrm{B}}=80-100 \mathrm{~K}, f>2.4 \times 10^{3}\right)[58]$, a CT solid of (ethyltrimethylantimonate) $\left[\mathrm{Pd}(\mathrm{dmit})_{2}\right]_{2}$ (triangle, dmit: 4,5-dimercapto-1,3-dithiole-2-thione, $\left|\Theta_{\mathrm{CW}}\right|=325-375 \mathrm{~K},|\mathrm{~J}| / k_{\mathrm{B}}=220-250 \mathrm{~K}$, $\left.f>1.6 \times 10^{4}\right)$ [59], $\mathrm{ZnCu}_{3}(\mathrm{OH})_{6} \mathrm{Cl}_{2}$ (kagome, $\left.\left|\Theta_{\mathrm{CW}}\right|=314 \mathrm{~K},|J| / k_{\mathrm{B}}=180 \mathrm{~K}, f>5.1 \times 10^{3}\right)[60-62]$, $\left[\mathrm{NH}_{4}\right]_{2}\left(\mathrm{C}_{7} \mathrm{H}_{14} \mathrm{~N}\right)\left[\mathrm{V}_{7} \mathrm{O}_{6} \mathrm{~F}_{18}\right]$ (kagome, $\left.\mid \Theta_{\mathrm{CW}} \mathrm{I}=81 \mathrm{~K}, f \geq 2.0 \times 10^{3}\right)$ [63] where $\left(\mathrm{C}_{7} \mathrm{H}_{14} \mathrm{~N}\right)$ is quinuclidinium ion, and $\left[\left(\mathrm{C}_{2} \mathrm{H}_{5}\right)_{3} \mathrm{NH}\right]_{2} \mathrm{Cu}_{2}$ (oxalate) $)_{3}$ (hyperhoneycomb, $\left|\Theta_{\mathrm{CW}}\right|=180 \mathrm{~K}$, $f>3 \times 10^{3}$ ) [64].

Another organic QSL system of a dimer-type Mott insulator $\kappa-(\mathrm{ET})_{2} \mathrm{Ag}_{2}(\mathrm{CN})_{3}\left(t^{\prime} / t=0.97\right)$ was very recently developed by us $\left(\left|\Theta_{\mathrm{CW}}\right|=263 \mathrm{~K},|J| / k_{\mathrm{B}}=175 \mathrm{~K}, f>2.1 \times 10^{3}\right)$ having a different key-keyhole relation from that of $\kappa-(\mathrm{ET})_{2} \mathrm{Cu}_{2}(\mathrm{CN})_{3}$ resulting in both a more robust QSL state and a higher critical temperature $\left(T_{\mathrm{C}}\right)$ of $\mathrm{SC}$ than the $\mathrm{X}=\mathrm{Cu}$ salt $[54,65]$, with similar relaxor ferroelectric response $[66,67]$. Among these QSL systems, only dimer-type ET salts $\kappa-(\mathrm{ET})_{2} \mathrm{Cu}_{2}(\mathrm{CN})_{3}$ and $\kappa-(\mathrm{ET})_{2} \mathrm{Ag}_{2}(\mathrm{CN})_{3}$ manifest competition among the itinerancy of electrons (metal), pairing of two electrons (SC), and localization (Mott insulator and QSL).

In order to have such competition between localized and itinerant spins in a monomer-type Mott insulator, the best candidate will be the $\mathrm{C}_{60} \mathrm{CT}$ materials having triangular or hexagonal packing of $\mathrm{C}_{60}{ }^{\bullet-}$ molecules (Figure $1 \mathrm{k}$ ) among several kinds of such monomer-type Mott insulators based on TTF, TSF, ET, TCNQ, etc. [67-69], where TTF, TSF, and TCNQ are well known donor or acceptor molecules in the CT salts [70]. In these monomer-type Mott insulators, the upper-HOMO band for the CT solid of $\mathrm{D}^{\bullet+} \mathrm{X}^{-}$(D: TTF, TSF, ET) and the lower-LUMO band for the CT solid of $\mathrm{M}^{+} \mathrm{A}^{\bullet-}(\mathrm{A}$ : TCNQ) are completely filled, where HOMO and LUMO are the highest occupied and lowest unoccupied molecular orbitals, respectively. The Mott gap being large makes it difficult for the competition between localization and itinerancy in the solids, thus requiring partial CT state or dimer-type Mott insulating 
state to satisfy the requirement 3 above mentioned. While for the $\mathrm{C}_{60} \mathrm{CT}$ solids, a partial CT state such as $\mathrm{ET}^{1 / 2+}$ is not necessary and the completely ionized $\mathrm{C}_{60}{ }^{\bullet-}$ molecules are able to afford the itinerant state owing to the triply degenerate LUMO $t_{1 \mathrm{u}}$ orbital, e.g., the compound $\mathrm{CsC}_{60}$ quenched in liquid $\mathrm{N}_{2}$ is reported to exhibit metallic behavior down to low temperatures [71,72].

In this review, the preparation, crystal and electronic structures, and the physical properties of spin- frustrated monomer-type Mott insulators of $\mathrm{C}_{60}{ }^{-}-\mathrm{CT}$ solids, namely $\left(\mathrm{MDABCO}^{+}\right)\left(\mathrm{C}_{60}{ }^{\bullet-}\right)(\mathbf{1})$, $\left(\mathrm{Ph}_{3} \mathrm{MeP}^{+}\right)\left(\mathrm{C}_{60}{ }^{\bullet-}\right)(2),\left(\mathrm{TPC}^{0}\right)\left(\mathrm{MDABCO}^{+}\right)\left(\mathrm{C}_{60}{ }^{--}\right)(3),\left(\mathrm{TPC}^{0}\right)\left(\mathrm{MQ}^{+}\right)\left(\mathrm{C}_{60}{ }^{\bullet-}\right)(4),\left(\mathrm{PhCN}^{0}\right)\left(\mathrm{TMP}^{+}\right)\left(\mathrm{C}_{60}{ }^{\bullet-}\right)$ (5), $\left.\left(\mathrm{PhCN}^{0}\right)\left(\mathrm{Ph}_{3} \mathrm{MeP}^{+}\right)\left(\mathrm{C}_{60}{ }^{\bullet-}\right)(6),\left(\mathrm{PhCl}_{2}^{0}\right)\left\{\left(\mathrm{Ph}_{3} \mathrm{P}\right)_{3} \mathrm{Au}^{+}\right)\right\}_{2}\left(\mathrm{C}_{60}{ }^{\bullet-}\right)_{2}\left(\mathrm{C}_{60}\right)(7)$, and $\left(\mathrm{DMI}^{+}\right)_{3}\left(\mathrm{C}_{60}{ }^{\bullet-}\right)\left(\mathrm{I}^{-}\right)_{2}$ (8), and the design of the QSL systems will be discussed.

\section{Characteristic Features of $\mathrm{C}_{60}$ : Superconductors and Other Functions for $\mathrm{C}_{60}$ Charge-Transfer Materials}

Superconductivity (SC) is one of the most remarkable features of $\mathrm{C}_{60} \mathrm{CT}$ materials. An icosahedral $\mathrm{C}_{60}$ molecule with $I_{\mathrm{h}}$ symmetry has triply degenerate LUMO and LUMO +1 orbitals with $t_{1 \mathrm{u}}$ and $t_{1 \mathrm{~g}}$ symmetries, respectively. Such multiple degeneracy $(N)$ contributes to the relaxation of the Mott criterion [73,74], i.e., $U / W \sim \sqrt{ } N$ or an upper limit of $U / W \sim 2.5$, and the enhancement of the density of states at the Fermi level $\left(D\left(\varepsilon_{\mathrm{F}}\right)\right)$ inducing high $T_{\mathrm{C}}$ for SC [75], when $\mathrm{C}_{60}$ is placed in the highly symmetric, i.e., cubic crystal field. Typical SC materials are represented as $\mathrm{A}_{3} \mathrm{C}_{60}$ (A: alkali metal), e.g., $\mathrm{Rb}_{3} \mathrm{C}_{60}$ $\left(T_{\mathrm{C}}=29 \mathrm{~K}\right.$ [76]), $\mathrm{Rb}_{2} \mathrm{CsC}_{60}\left(T_{\mathrm{c}}=31 \mathrm{~K}\right.$ [77]), and $\mathrm{RbCs}_{2} \mathrm{C}_{60}\left(T_{\mathrm{C}}=33 \mathrm{~K}\right.$ [77]), with a face-centered cubic (fcc) structure. The structural and physical properties of $\mathrm{A}_{3} \mathrm{C}_{60}$ and the related fullerene compounds were reviewed $[73,78-80]$. The critical temperature $T_{\mathrm{C}}$ varies monotonously with the lattice constant, independent of the type of the alkali dopant [77,81]. Thus far, about 40 SC materials have been synthesized with the highest $T_{\mathrm{c}}$ of $33 \mathrm{~K}\left(\mathrm{RbCs}_{2} \mathrm{C}_{60}\right)$ at normal pressure and $38 \mathrm{~K}$ ( $\mathrm{A} 15$ or body-centered cubic (bcc) $\mathrm{Cs}_{3} \mathrm{C}_{60}$ ) under a pressure of approximately $0.7 \mathrm{Gpa}$ [82]. The fcc phase of $\mathrm{Cs}_{3} \mathrm{C}_{60}$ also shows $\mathrm{SC}\left(T_{\mathrm{C}}=35 \mathrm{~K}\right)$ under an applied hydrostatic pressure of approximately $0.8 \mathrm{Gpa}$ [83].

The critical temperature $T_{\mathrm{c}}$ decreases as the valence state $(n)$ of $\mathrm{C}_{60}$ deviates from $n=-3$ or lowering the symmetry of the crystal (non-cubic) such as $\mathrm{Yb}_{2.75} \mathrm{C}_{60}(n=-5.5$, orthorhombic, $\left.T_{\mathrm{c}}=6 \mathrm{~K}\right)$ [84], $\mathrm{Sm}_{2.75} \mathrm{C}_{60}\left(n=-5.5\right.$, orthorhombic, $\left.T_{\mathrm{c}}=8 \mathrm{~K}\right)$ [85], $\mathrm{Ba}_{4} \mathrm{C}_{60}(n=-8$, body-centered orthorhombic (bco), $\left.T_{\mathrm{c}}=6.7 \mathrm{~K}\right)$ [86,87], $\mathrm{Sr}_{4} \mathrm{C}_{60}\left(n=-8\right.$, bco, $\left.T_{\mathrm{c}}=4.4 \mathrm{~K}\right)[86]$ and $\mathrm{K}_{3} \mathrm{Ba}_{3} \mathrm{C}_{60}(n=-9$, bcc, $T_{\mathrm{c}}=5.6 \mathrm{~K}$ ) [88]. $\mathrm{Eu}_{6} \mathrm{C}_{60}$ with bcc packing undergoes a ferromagnetic transition at $12 \mathrm{~K}$, owing from $\mathrm{Eu}^{2+}$ cations with $S=7 / 2$ spin [89]. $\mathrm{Ce}_{x} \mathrm{C}_{60}$ shows the coexistence of $\mathrm{SC}$ and ferromagnetism below $13.5 \mathrm{~K}$, although its crystal structure and composition are currently not clear [90].

$\mathrm{A}_{3} \mathrm{C}_{60}$ SCs show a dome-shaped curve of normalized $T_{\mathrm{c}}$ versus lattice volume $[82,83,91]$, which may be a hallmark of the competition between electron-phonon attractive and electron-electron repulsive interactions. The observation of a Hebel-Slichter peak in the relaxation rate just below $T_{\mathrm{C}}$ in NMR and $\mu$ SR indicate a BCS-type isotopic gap [92,93].

Besides SCs, $\left(\mathrm{TDAE}^{+}\right)\left(\mathrm{C}_{60}{ }^{\bullet-}\right)$ is a soft ferromagnet with a Curie temperature of $16.1 \mathrm{~K}$, in which only spins on $\mathrm{C}_{60}{ }^{\bullet-}$ contribute to the ferromagnetism, where TDAE is tetrakis(dimethylamino) ethylene [94].

\section{Requirements for Spin-Frustrated Spin Lattice of $\mathrm{C}_{60}{ }^{\bullet-}$}

\subsection{Competition among Bond-Formation, Itinerancy, Localization, and Frustration in Fulleride Solids}

It can be easily seen that the close packing of $\mathrm{C}_{60} \bullet-$ leads to triangular (Figure $1 \mathrm{k}$ ) or honeycomb (Figure 1e) spin lattices. In the $\mathrm{C}_{60}$ system, there is another factor, namely, the bond-formation between $\mathrm{C}_{60}{ }^{\bullet-}$ molecules, which leads to competition among itinerancy, localization, and frustration. Figure $3 \mathrm{a}, \mathrm{b}$ summarize the electronic competition for bond-formation, such as dimers and polymers (Figure $2 \mathrm{~b}$ ) (top panel of Figure 3a), itinerancy (monomeric $\mathrm{C}_{60}$ metallic solids, middle panel in Figure 3a), and localization (monomeric AF solids, bottom panel in Figure 3a) for 27 situations in $\mathrm{C}_{60} \mathrm{CT}$ solids as a function of center-to-center interfullerene distance $r$, as reported in the 
literatures [37,38,44,79,95-98]. The van der Waals (vdW) diameter [99] of $\mathrm{C}_{60}$ is $10.18 \AA$ indicated by green dotted line in Figure 3a,b.

When the interfullerene distance of the $\mathrm{C}_{60}$ solids is small $(r<9.4 \AA$, which is indicated by blue dotted line in Figure $3 a, b)$, the $\mathrm{C}_{60}{ }^{\circ-}$ molecules tend to form dimers (9 and 10 in Figure 2b) [37,38] or polymers (11-15 in Figure 2b) by single or double bonds. Polymer $\mathbf{1 1}$ with $\mathrm{K}^{+}$is a 3D metal [39], while those with $\mathrm{Rb}^{+}$and $\mathrm{Cs}^{+}$are one-dimensional (1D) metals and become spin-density-wave insulators at low temperatures $[40,100]$. Single carbon-carbon bonded polymer 12 in $\mathrm{Na}_{2} \mathrm{CsC}_{60}$ seems to be an $\mathrm{SC}$ with $T_{\mathrm{c}}$ about $3 \mathrm{~K}$ lower than that for the starting non-polymeric phase. Analogous lowering of $T_{\mathrm{c}}$ at the formation of polymer $\mathbf{1 2}$ in $\mathrm{Na}_{2} \mathrm{RbC}_{60}$ leads to very low $T_{\mathrm{c}}$ or the absence of SC [41,101].

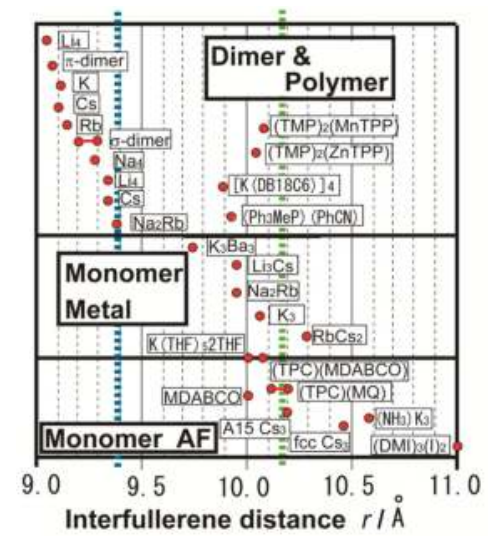

(a)

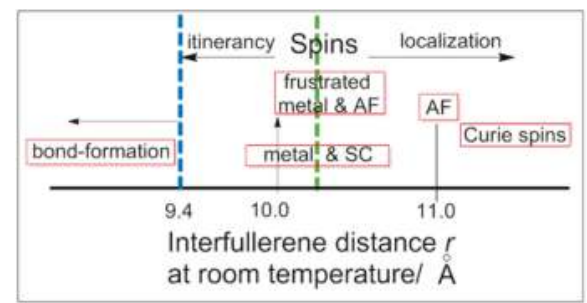

(b)

Figure 3. (a,b) Bond formation (dimer and polymer), monomer metal, and monomer AF regions of $\mathrm{C}_{60}{ }^{n-}$ with the center-to-center interfullerene distance $r$ at RT except [K(DB18C6) $]_{4}\left(\mathrm{C}_{60}\right)_{5} \cdot 12 \mathrm{THF}$ $\left(225 \mathrm{~K}\right.$ indicated as $\left.[\mathrm{K}(\mathrm{DB} 18 \mathrm{C} 6)]_{4}\right),\left\{\left(\mathrm{TMP}^{+}\right)_{2} \mathrm{M}^{\mathrm{II}} \mathrm{TPP}\right\}\left(\mathrm{C}_{60}{ }^{\bullet-}\right)_{2}\left(\mathrm{PhCN}_{2}\left(\mathrm{PhCl}_{2}\right)_{2}(270 \mathrm{~K}\right.$ indicated as $(\mathrm{TMP})_{2}(\mathrm{MnTPP})$ and $250 \mathrm{~K}$ indicated as $(\mathrm{TMP})_{2}(\mathrm{ZnTPP}), \mathrm{K}\left(\mathrm{C}_{60}{ }^{\bullet-}\right)(\mathrm{THF})_{5} \cdot 2 \mathrm{THF}(260 \mathrm{~K}$ indicated as $\left.\mathrm{K}(\mathrm{THF})_{5} 2 \mathrm{THF}\right)$ and $(\mathrm{MDABCO})\left(\mathrm{C}_{60}{ }^{\bullet-}\right)(250 \mathrm{~K}$ indicated as $\mathrm{MDABCO})$. Cation or donor species are shown in boxes and Figure $2 \mathrm{a}$. $\mathrm{Li}_{4}$ and $\mathrm{Cs}$ are plotted twice according to different interfullerene distances in the $2 \mathrm{D}$ polymer and in different phases (polymer or dimer), respectively. $\mathrm{K}(\mathrm{THF})_{5} 2 \mathrm{THF}$ and (TPC)(MDABCO) are located on the borderline between monomer metal and monomer AF. Blue dotted line and green one indicate $r=9.4 \AA$ and van der Waals diameter of $\mathrm{C}_{60}(10.18 \AA)$, respectively.

The compound $\mathbf{1 3}$ is an insulator with high ionic conductivity [42], 14 is a diamagnetic semiconducting polymer [43], and $\mathbf{1 5}$ is a highly correlated metal [43].

Even when $r>9.4 \AA, \mathrm{C}_{60}{ }^{\bullet}-$ bond-formation proceeds for the cation species, which are not able to prevent bond-formation. The $\mathrm{C}_{60}{ }^{\bullet-}$ molecules in $[\mathrm{K}(\mathrm{DB} 18 \mathrm{C} 6)]_{4}\left(\mathrm{C}_{60}\right)_{5} 12 \mathrm{THF}$, where DB18C6 is dibenzo-18-crown-6 ether and THF is tetrahydrofuran, dimerize at $r=9.89 \AA$ (at $225 \mathrm{~K}$ ), but remain as a monomer at $r=10.1 \AA$ [102]. For $\left(\mathrm{MQ}^{+}\right)\left(\mathrm{Co}^{\mathrm{II}} \mathrm{OEP}\right)\left(\mathrm{C}_{60}{ }^{\bullet-}\right)\left(\mathrm{PhCl}_{2}\right)$, where OEP is octaethylporphyrin, no bond-formation between $\mathrm{C}_{60}{ }^{\circ}-$ molecules $(r=9.88 \AA$ at $100 \mathrm{~K})$ is observed owing to the steric protection by the coordination of $\mathrm{C}_{60}{ }^{--}$with $\mathrm{Co}^{\mathrm{II}}$, that gives rise to a diamagnetic state [103]. However, for $\left\{\left(\mathrm{TMP}^{+}\right)_{2} \mathrm{M}^{\mathrm{II}} \mathrm{TPP}\right\}\left(\mathrm{C}_{60}{ }^{\bullet-}\right)_{2}(\mathrm{PhCN})_{2}\left(\mathrm{PhCl}_{2}\right)_{2}(\mathrm{M}=\mathrm{Zn}, \mathrm{Mn}$, TPP: tetraphenylporphyrin), where $\mathrm{M}^{\mathrm{II}} \mathrm{TPP}$ is weakly coordinated with $\mathrm{TMP}^{+}$, but, no coordination of $\mathrm{C}_{60}{ }^{-}-$with $\mathrm{M}^{\mathrm{II}}$, with $r=10.04 \AA$ $(\mathrm{M}=\mathrm{Zn}$ at $250 \mathrm{~K})$ and $r=10.08 \AA(\mathrm{M}=\mathrm{Mn}$ at $270 \mathrm{~K})$, singly bonded $\left(\mathrm{C}_{60}{ }^{-}\right)_{2}$ dimers form at $100 \mathrm{~K}$ [104]. Structural analysis of $\left(\mathrm{Ph}_{3} \mathrm{MeP}^{+}\right)\left(\mathrm{C}_{60}{ }^{-}-\right)(\mathrm{PhCN})$ indicates that the lowest value of $r$ is $9.92 \AA$ at room temperature (RT) for monomer state, and singly bonded $\left(\mathrm{C}_{60}{ }^{-}\right)_{2}$ dimers appear when the temperature is reduced to $120 \mathrm{~K}$ for $r=9.28 \AA$ [105]. $\left(\mathrm{DMI}^{+}\right)_{2}\left(\mathrm{C}_{60}{ }^{\bullet-}\right)\left\{\mathrm{Cd}(\right.$ diethyldithiocarbamate $\left.) \mathrm{I}^{-}\right\}(r=10.03 \AA$ at $250 \mathrm{~K}$ ) shows reduced-temperature dependent bond-formation, such as (1) monomeric $\mathrm{C}_{60}{ }^{\bullet-}$ upon 
instant quenching below $95 \mathrm{~K},(2)$ a mixture of dimerized $\left(\mathrm{C}_{60}{ }^{-}\right)_{2}$ and monomeric $\mathrm{C}_{60}{ }^{\bullet-}$, and (3) stable

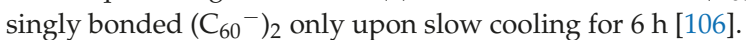

As can be seen in Figure $3 a, C_{60}$ compounds having an interfullerene distance in the range $9.4 \AA<$ $r<10.0 \AA$ at RT have not been extensively explored. It is highly plausible that the shorter interfullerene distance that is less than $9.7 \AA$ instantaneously leads to bond-formation resulting in $r \leq 9.4 \AA$.

Above results indicate that, even at $r \sim 9.9-10.1 \AA, \mathrm{C}_{60}{ }^{\bullet-}$ anion molecules dimerize, when the $\mathrm{C}_{60}{ }^{\bullet-}$ molecules are not properly protected against bond-formation.

An isolated fulleride anion structure has been observed for the interfullerene distance $r \geq 12 \AA$ by using both bulky cation molecules $\left(\mathrm{Ph}_{4} \mathrm{P}^{+}, \mathrm{PPN}^{+},\left[\mathrm{Ru}(\mathrm{BPY})_{3}\right]^{2+}\right)$ [107-116], and fulleride anions with a charge of -2 or -3 and alkali metal cations, which coordinate with THF, crown ether or cryptand molecules [113-116]. The preparative methods of these compounds were reviewed [95].

When $r$ is greater than $9.73 \AA$ and less than $12 \AA$, the "bond-formation", "monomer metal", and "monomer AF" in Figure 3a compete with each other. The "monomer metal" is observed for many $\mathrm{C}_{60}{ }^{\mathrm{n}-}$ solids, e.g., $\mathrm{K}_{3} \mathrm{Ba}_{3} \mathrm{C}_{60}$ (bcc, $r=9.74 \AA$ ) [88], $\mathrm{Li}_{3} \mathrm{CsC}_{60}$ (fcc, $r=9.98 \AA$ ) [117], $\mathrm{Na}_{2} \mathrm{RbC}_{60}$ (fcc, $r=9.96 \AA$ ) [118], $\mathrm{K}_{3} \mathrm{C}_{60}$ (fcc, $r=10.07 \AA$ ) [119], and $\mathrm{RbCs}_{2} \mathrm{C}_{60}$ (fcc, $r=10.19 \AA$ ) [77]. The "monomer $\mathrm{AF}^{\prime \prime}$ state is observed for a wide range of $r$. The A15 $\mathrm{Cs}_{3} \mathrm{C}_{60}(r=10.20 \AA)$ solid shows AF ordering below $T_{\mathrm{N}}=46 \mathrm{~K}\left(\Theta_{\mathrm{CW}}=-68 \mathrm{~K}\right)$ [120], and the fcc phase $(r=10.44 \AA)$ also shows AF ordering at $2.2 \mathrm{~K}$ $\left(\Theta_{\mathrm{CW}}=-105 \mathrm{~K}\right)$ at normal pressure $[83,121,122]$, where $T_{\mathrm{N}}$ is Néel temperature. The intercalation of $\mathrm{NH}_{3}$ molecules (e.g., $\left(\mathrm{NH}_{3}\right) \mathrm{K}_{3} \mathrm{C}_{60}, r=10.57 \AA$ at $\mathrm{RT}$ ) results in a phase transition from a cubic to orthorhombic lattice structure accompanied by the appearance of AF ordering instead of SC [123] and exhibits an SC state under pressure $\left(T_{\mathrm{C}}=28 \mathrm{~K}\right.$ at $\left.15 \mathrm{kbar}\right)$ [124].

Figure 3 will be a guiding map for the search for $\mathrm{C}_{60}$ functional materials. A phase diagram of $\mathrm{C}_{60}{ }^{3-}$ solids indicates that the SC state is in the vicinity of spin ordered AF states while the critical temperature $T_{C}$ decreases as approaching to the AF phase $[82,120,125]$. Therefore, the $\mathrm{C}_{60}$ system is a potential candidate for the QSL state to be close to the metallic, SC, and bond-formed states.

The $\mathrm{C}_{60}{ }^{--}$molecules in both the $\mathrm{A} 15$ and fcc phases of $\mathrm{Cs}_{3} \mathrm{C}_{60}$ are not in the high-spin state $(S=3 / 2)$, which prefers spin-ordering, but are in the low-spin states $(S=1 / 2)$ due to the splitting of the $t_{1 \mathrm{u}}$-orbitals by the Jahn-Teller effect. The triangular spin lattices of fcc have strong spin frustration with strong AF interactions characterized by its frustration index $f \sim 48$ for $\mathrm{Cs}_{3} \mathrm{C}_{60}$. However, the $\mathrm{Cs}_{3} \mathrm{C}_{60}$ solid in the fcc structure prefers the AF state as compared to the QSL state. A large frustration index $f$ and a low-spin state are necessary for QSL state formation, however, the geometry of the triangular spin lattice is also important. For example, $\kappa-(\mathrm{ET})_{2} \mathrm{X}$ with a nearly equilateral triangular spin lattice $\left(t^{\prime} / t=0.97-1.09\right.$ for $\mathrm{X}=\mathrm{Ag}_{2}(\mathrm{CN})_{3}$ and $\left.\mathrm{Cu}_{2}(\mathrm{CN})_{3}\right)$ exhibits the QSL state [36,54], while the distorted triangular spin lattice (e.g., $t^{\prime} / t=0.715$ for $X=\mathrm{Cu}\left[\mathrm{N}(\mathrm{CN})_{2}\right] \mathrm{Cl}[126,127], t^{\prime} / t=1.79$ for $\mathrm{X}=\mathrm{CF}_{3} \mathrm{SO}_{3}$ ) [128] exhibits an AF ordered spin state. Similarly a spin-frustrated system in which $t^{\prime} / t$ deviates from unity shows $\mathrm{AF}$ ordering, i.e., $\mathrm{Cs}_{2} \mathrm{CuCl}_{4}\left(t^{\prime} / t=1.71\right)$ with $T_{\mathrm{N}}=0.69 \mathrm{~K}$ [129].

The fundamental and simple prerequisites for the formation of QSL $\mathrm{C}_{60}$ system are:

(I) existence of $\mathrm{C}_{60}{ }^{\bullet-}$ or low-spin state of $\mathrm{C}_{60}{ }^{3-}(S=1 / 2)$,

(II) no polymerization between $\mathrm{C}_{60}$ molecules,

(III) triangular or hexagonal packing of $\mathrm{C}_{60}$ with equal interfullerene distance $r$, or $t^{\prime} / t \sim 1$, and

(IV) strong AF interactions.

It is believed that smaller $r$ values lead to stronger AF interactions. The critical $r$ between the "itinerant", "localized spin", and "bond-formation" has not been elucidated yet, and the competition strongly depends on the environment of the $\mathrm{C}_{60}$ molecules, pattern, and dimensionality of packing of $\mathrm{C}_{60}$ molecules, charge of $\mathrm{C}_{60}$ molecules, etc.

\subsection{Charged State of $\mathrm{C}_{60}$ and Effective On-Site Coulomb Repulsion}

The $\mathrm{C}_{60}{ }^{n-}$ species $(n=0,1,2,3)$ are well discriminated by IR and UV-Vis-NIR spectra [78-80,95]. The on-site Coulomb energy of free $C_{60}$ molecule $\left(U_{0}\right)$ was calculated by DFT as 2.7-3.1 eV [130-132]. 
The effective $U\left(U_{\text {eff }}=U_{0}-V, V\right.$ is nearest neighbour Coulomb repulsion energy) values in solids are found to be $0.8-1.3 \mathrm{eV}$ [130-132], when an electron is added to a $\mathrm{C}_{60}$ molecule surrounded by other $\mathrm{C}_{60}$ molecules in the fcc lattice, which is due to polarization by the charged $\mathrm{C}_{60}$ molecules. While, Auger spectroscopy yields $U_{\text {eff }}$ in the range $1.4-1.6 \mathrm{eV}[133,134]$. The first CT band of non-metallic $\mathrm{M}^{+}\left(\mathrm{C}_{60}{ }^{\circ-}\right)$ solid corresponds to $U_{\text {eff. }}$ With organic cation or supramolecular cation in this paper, the IR and NIR absorption spectra of CT solids in $\mathrm{KBr}$ show CT band peaks at 0.25 and $0.68 \mathrm{eV}$ for $\left(\mathrm{TPC}^{0}\right)\left(\mathrm{MDABCO}^{+}\right)\left(\mathrm{C}_{60}{ }^{\bullet-}\right)$ [12], $0.69 \mathrm{eV}$ for $\left(\mathrm{TDAE}^{+}\right)\left(\mathrm{C}_{60^{\bullet}}{ }^{-}\right)$[12], $0.71 \mathrm{eV}$ for $\left(\mathrm{TPC}^{0}\right)\left(\mathrm{MQ}^{+}\right)\left(\mathrm{C}_{60}{ }^{\bullet}\right)$ [12], $0.74 \mathrm{eV}$ for $\left(\mathrm{PhCN}^{0}\right)\left(\mathrm{Ph}^{3} \mathrm{MeP}^{+}\right)\left(\mathrm{C}_{60}{ }^{-}-\right)$[105], and $0.77 \mathrm{eV}$ for $\left(\mathrm{PhCN}^{0}\right)\left(\mathrm{TMP}^{+}\right)\left(\mathrm{C}_{60}{ }^{-}\right)$[12]. The lowest band of $0.25 \mathrm{eV}$ in $\left(\mathrm{TPC}^{0}\right)\left(\mathrm{MDABCO}^{+}\right)\left(\mathrm{C}_{60}{ }^{-}-\right)$is ascribed to the intraband absorption due to the metallic nature. The estimated $U_{\text {eff }}\left(U_{\text {eff }}=0.68-0.77 \mathrm{eV}\right)$ should be smaller than those for (alkali metal $\left.^{+}\right)\left(\mathrm{C}_{60}{ }^{-}\right)$owing to the high polarizability of organic cation molecules, which is similar to that proposed for TCNQ CT solids [135].

The effect of orbital degeneracy on the Mott-Hubbard criterion leads to a conclusion that the Mott transition takes place at $U / W=\sqrt{ } 3$ or an upper limit of $U / W \sim 2.5$, attributed to triple degeneracy or negligible splitting of $t_{1 \mathrm{u}}$ orbitals [73,94]. Therefore, this relaxed Mott criterion is effective as long as the splitting of $t_{1 \mathrm{u}}$ orbitals by Jahn-Teller distortion is not large enough, and forms one LUMO band. The upper limit of $U / W$ requires $W$ to be $0.27 \mathrm{eV}$, by considering the lowest value of $U_{\text {eff }}$ (calculated value $-0.68 \mathrm{eV}$ ), which is about $2-3$ times the calculated $W$ value $(0.10-0.15 \mathrm{eV}$ for $\left.\left(\mathrm{TPC}^{0}\right)\left(\mathrm{MDABCO}^{+}\right)\left(\mathrm{C}_{60}{ }^{-}-\right)\right)$by the AM1 method [12]. A preliminary DFT calculation for this CT solid indicates a total $W$ of about $0.48 \mathrm{eV}$ at $160 \mathrm{~K}$ [12], which is still smaller than the estimated $U_{\text {eff }}$ and is not able to account for the metallic nature. Since the calculated $W$ values in these systems are not reliable at present, only the ratio of overlap integrals or transfer interactions will be discussed in the following.

\subsection{Packing of $C_{60}$ and Magnetic Interactions in Fulleride Solids}

So far, no structure-magnetic property relationship, especially concerning the geometrical spin frustration and the QSL state has been studied for the triangular or hexagonally packed $\mathrm{C}_{60}{ }^{\circ-}$ solids. Hexagonal packing of $\mathrm{C}_{60}{ }^{\bullet-}$ has been suggested for (tetramethylammonium $)\left(\mathrm{C}_{60}{ }^{\bullet}-\right) \cdot 1.5 \mathrm{THF}$ polycrystals based on the postulated structure, from both the calculation of total energy for various arrangements of the component molecules and the observed powder diffraction pattern [136]. The estimated $r$ was $10.13 \AA$. The conductivity of the pellet sample was $10^{-2} \mathrm{~S} \cdot \mathrm{cm}^{-1}$ and the effective magnetic moment of the complex was $\sim 1.75 \mu_{\mathrm{B}}$ in good agreement with the value for the system containing one $S=1 / 2$ spin per formula unit. However, the susceptibility did not indicate AF interactions. $\left(\mathrm{Na}^{+}\right)\left(\mathrm{C}_{60^{-}}{ }^{-}\right)(\mathrm{THF})_{5}$ polycrystals [137] showed a Curie-Weiss behavior above $200 \mathrm{~K}$ $\left(\mu=1.70 \mu_{\mathrm{B}}, \Theta_{\mathrm{CW}}=-58 \mathrm{~K}\right.$ ) suggesting triangular or hexagonal packing of $\mathrm{C}_{60^{\circ}}{ }^{--}$. The $\chi$ (magnetic susceptibility) value decreased sharply below $180 \mathrm{~K}\left(\mu=\right.$ ca. $\left.0.8 \mu_{\mathrm{B}}\right)$, probably owing to the dimerization of $\mathrm{C}_{60}{ }^{\bullet-}$, though no crystal structure above $200 \mathrm{~K}$ was found.

Recent work on needle-like single crystal (up to $6 \mathrm{~mm}$ ) of $\left(\mathrm{K}^{+}\right)\left(\mathrm{C}_{60}{ }^{\bullet-}\right)(\mathrm{THF})_{5}(\mathrm{THF})_{2}$ revealed that $\mathrm{K}^{+}$cation coordinate with five THF molecules (by $\mathrm{K} \cdots \mathrm{O}$ contacts) to form bulky cationic building units of $\left[\mathrm{K}(\mathrm{THF})_{5}\right]^{+}$, which separates $\mathrm{C}_{60}{ }^{\circ-}$ corrugated layers with a distorted square arrangement [138]. At $\mathrm{RT}, \mathrm{C}_{60}{ }^{\circ}-$ molecules display rotational disorder, and ordering takes place below $240 \mathrm{~K}$ with a simultaneous appearance of a Dysonian EPR line down to $40 \mathrm{~K}$. In the non-conductive region in the $240-295 \mathrm{~K}$ range, Curie-Weiss behavior $\left(\Theta_{\mathrm{CW}}=-54 \mathrm{~K}\right)$ was observed with $r=\sim 10.01 \AA$ at $260 \mathrm{~K}$. A metallic nature in the conductive region between $240 \mathrm{~K}$ and $40 \mathrm{~K}$ was suggested by the observation of the Dysonian EPR line shape, though the Dysonian line shape has been observed not only in metallic materials, but also in semiconductors with very high conductivity [139]. It should be emphasized that "localization" or "itinerancy" of spins in $\mathrm{C}_{60}$ solids is strongly associated with the rotational disorder and ordering of $\mathrm{C}_{60}$ molecules.

Crystals of $[\mathrm{K}([2.2 .2] \mathrm{crypt})]_{2}\left(\mathrm{C}_{60}{ }^{2-}\right) \cdot 4$ (toluene) exhibit a $2 \mathrm{D}$ distorted hexagonal layer of $\mathrm{C}_{60}{ }^{2-}$ dianion molecules separated by layers of $[\mathrm{K}([2.2 .2] \text { crypt })]^{+}$cations [115]. The shortest interfullerene distance of $13.77 \AA$ is too long owing to the large size of cation to have appropriate intermolecular 
interactions for the spin-frustrated system, regardless of the magnitude of effective spin of the dianion system.

\subsection{Requirements for Triangular or Hexagonal Packing of $\mathrm{C}_{60}{ }^{\bullet-}$ by Key-Keyhole Relation: $2 \mathrm{D}$ or $3 D$ Polycationic Template}

The donor molecules that can reduce fullerene moieties are sparse because of the weak electron-accepting ability of fullerene moiety [140]. Simple use of very strong donor species, such as alkali metal has not led to $2 \mathrm{D}$ triangular or hexagonal packing of $\mathrm{C}_{60}{ }^{\circ-}$. A strategy to obtain new versatile ionic complexes, including polymerized fullerenes has been developed by Konarev, et al. [12,37,38,95,103-106,141-149], which can lead to multi-component CT complexes composed of small-sized strong donor molecules including alkali metals $\left(\mathrm{D}_{2}\right)$, which are able to ionize fullerene, and a structure-forming neutral molecule including solvent molecules $\left(\mathrm{D}_{1}\right)$, e.g., $\left\{\left[\left(\mathrm{D}_{1}{ }^{0}\right)\left(\mathrm{D}_{2}{ }^{+}\right)\right]\left(\right.\right.$fullerene $\left.\left.{ }^{-}\right)\right\}$. A variety of single crystals of $\left[\left(\mathrm{D}_{1}^{0}\right)\left(\mathrm{D}_{2}^{+}\right)\right]\left(\mathrm{C}_{60}{ }^{-}-\right)$with versatile packing patterns of $\mathrm{C}_{60}{ }^{\circ-}$ molecules have been prepared according to this concept, which satisfies requirement (I) described in Section 3.1.

The distance between the cation species in the supramolecule formed between $D_{1}$ and $D_{2}$ molecules $\left[\left(\mathrm{D}_{1}{ }^{0}\right)\left(\mathrm{D}_{2}{ }^{+}\right)\right]$is the essential parameter to control both the bond-formation and the AF interaction among $\mathrm{C}_{60}{ }^{\bullet-}$ molecules. Therefore, the $r$ value is the key to satisfy requirements (II) and (IV). When the supramolecular assemblies of $\mathrm{D}_{1}$ and $\mathrm{D}_{2}$ molecules have a periodic cationic site with sufficient space to hold one $\mathrm{C}_{60}{ }^{\circ-}$ molecule, $\mathrm{C}_{60} 0^{--}$molecules (spin site = key) will be arranged according to the pattern of the cationic parts of supramolecular assemblies $\left[\left(\mathrm{D}_{1}^{0}\right)\left(\mathrm{D}_{2}{ }^{+}\right)\right](=$keyhole $)$. This is the key-keyhole relation between $\mathrm{C}_{60^{-}}{ }^{-}$molecules and polycationic template $\left[\left(\mathrm{D}_{1}{ }^{0}\right)\left(\mathrm{D}_{2}{ }^{+}\right)\right]$. To construct triangular or hexagonal packing of $\mathrm{C}_{60}{ }^{--}$, the template $\left[\left(\mathrm{D}_{1}{ }^{0}\right)\left(\mathrm{D}_{2}{ }^{+}\right)\right]$should have a triangular or hexagonal pattern of cationic sites. It was observed that the threefold symmetry of $\mathrm{D}_{1}$ and $\mathrm{D}_{2}$ molecules satisfies requirement (III) $[12,146]$. Higher than threefold symmetry would be satisfactory. However, the design principle to satisfy the requirement for $t^{\prime} / t \sim 1$ has not been developed yet for multi-component solid $\left[\left(\mathrm{D}_{1}^{0}\right)\left(\mathrm{D}_{2}{ }^{+}\right)\right]\left(\mathrm{C}_{60}{ }^{\bullet-}\right)$ and two-component solid $\left(\mathrm{D}^{+}\right)\left(\mathrm{C}_{60^{\bullet}}{ }^{-}\right)$.

A good chemical choice of $\mathrm{D}_{1}$ and/or $\mathrm{D}_{2}$ concerning the donor strength, size, shape, and symmetry satisfies all of the requirements, (I)-(III). Among the multi-component complexes $\left\{\left[\left(\mathrm{D}_{1}{ }^{0}\right)\left(\mathrm{D}_{2}{ }^{+}\right)\right]\left(\mathrm{C}_{60} 0^{-}\right)\right\}, \mathrm{C}_{60^{\bullet}}{ }^{-}$molecules form $2 \mathrm{D}$ hexagonal packing (triangular spin lattice, Figure $1 \mathrm{k}$ ) in $\left(\mathrm{TPC}^{0}\right)\left(\mathrm{MDABCO}^{+}\right)\left(\mathrm{C}_{60}{ }^{-}\right)(3)$ and $\left(\mathrm{TPC}^{0}\right)\left(\mathrm{MQ}^{+}\right)\left(\mathrm{C}_{60^{\circ}}{ }^{-}\right)(4)$ and three-dimensional (3D) one in $\left(\mathrm{DMI}^{+}\right)_{3}\left(\mathrm{C}_{60}{ }^{--}\right)\left(\mathrm{I}^{-}\right)_{2}(8)[12,145,146]$. The coexistence of monomer metallic and monomer AF phases was observed in $3(r=10.07 \AA$ at RT). Insulators 4 and 8 are AF insulators with $2 \mathrm{D}$ and 3D packing of $\mathrm{C}_{60}{ }^{\circ-}$, respectively, with $r=10.12-10.18 \AA$ at $250 \mathrm{~K}$ and $10.06-10.12 \AA$ at $100 \mathrm{~K}$ for 4 and 11.05 and $13.36 \AA$ at $100 \mathrm{~K}$ for 8 . Even in two-component materials, $\left(\mathrm{D}^{+}\right)\left(\mathrm{C}_{60^{-}}{ }^{-}\right)$, the bond-formation sometimes is prevented when $r$ is large enough and the spins on $\mathrm{C}_{60}{ }^{-}{ }^{-}$interact antiferromagnetically. In $\left(\mathrm{MDABCO}^{+}\right)\left(\mathrm{C}_{60^{\circ}}{ }^{-}\right)(\mathbf{1}), 3 \mathrm{D}$ close packing of $\mathrm{C}_{60^{\circ}}{ }^{--}$and high $\left|\Theta_{\mathrm{CW}}\right|$ were realized and the geometry of the spin lattice is 3D distorted bipyramid with $r=10.01-10.11 \AA$ at $250 \mathrm{~K}$ and 9.91-10.12 $\AA$ at $100 \mathrm{~K}$ [147]. The solid $\left(\mathrm{Ph}_{3} \mathrm{MeP}^{+}\right)\left(\mathrm{C}_{60}{ }^{\bullet-}\right)(2)$ has double chains of $\mathrm{C}_{60}{ }^{\bullet-}$ with zigzag spin lattice having weak interchain interactions with $r=10.08-10.10 \AA$ at $100 \mathrm{~K}$ [105].

\section{Key-Keyhole Relations in $\mathrm{C}_{60^{\circ}}{ }^{-}$Charge-Transfer Solids}

\subsection{Three-Component Materials}

4.1.1. Coexistence of Itinerant and Frustrated Spins in $2 \mathrm{D}$ Hexagonal Packing of $\mathrm{C}_{60}{ }^{\bullet-}$ in $\left(\mathrm{TPC}^{0}\right)\left(\mathrm{MDABCO}^{+}\right)\left(\mathrm{C}_{60}{ }^{\bullet-}\right)$

Single crystals of $\left(\mathrm{TPC}^{0}\right)\left(\mathrm{MDABCO}^{+}\right)\left(\mathrm{C}_{60}{ }^{-}\right)$(3) were obtained by the diffusion method. $\mathrm{C}_{60}$, reductant $\mathrm{CH}_{3} \mathrm{CH}_{2} \mathrm{SNa}$, and MDABCO-I were stirred in a $\mathrm{PhCl}_{2} / \mathrm{PhCN}$ mixture. TPC was dissolved in the obtained solution and $n$-hexane was layered. The diffusion was carried out over a period of two months to give black hexagonal prisms on the walls of the tube of sizes up to $0.5 \times 2 \times 2 \mathrm{~mm}^{3}$ (Figure 4c) [146]. 
The formation of 3 can be well interpreted by two kinds of key-keyhole relation (Figure 4). In the first step, three TPC molecules $\left(\mathrm{D}_{1}\right)$ surround $\mathrm{MDABCO}^{+}$molecule $\left(\mathrm{D}_{2}{ }^{+}\right)$, both molecules have threefold symmetry, to form cationic supramolecular unit (Supramolecule 1: $\left[\left(\mathrm{TPC}^{0}\right)_{3}\left(\mathrm{MDABCO}^{+}\right)\right]$) as shown in Figure 4(a-1).

A $\mathrm{C}_{60}{ }^{\bullet-}$ molecule fit into the concave of the supramolecular unit to form a unit of \{Supramolecule 2: $\left.\left[\left(\mathrm{TPC}^{0}\right)_{3}\left(\mathrm{MDABCO}^{+}\right)\right]\left(\mathrm{C}_{60}{ }^{\bullet-}\right)\right\}$ according to the second key-keyhole relation (Figure 4(b-1)). The Supramolecule 2 units assemble to form 2D layer of hexagonally packed $\mathrm{C}_{60}{ }^{\bullet-}$ in the $a b$ plane $\left(\left[\left(\mathrm{TPC}^{0}\right)\left(\mathrm{MDABCO}^{+}\right)\left(\mathrm{C}_{60}{ }^{\bullet-}\right)\right]_{\infty}\right.$, Figure $\left.4(\mathrm{~b}-2)\right)$. In another view, $\mathrm{C}_{60}{ }^{\bullet-}$ molecules were assembled according to the $2 \mathrm{D}$ sheet of the polycationic template $\left[\left(\mathrm{TPC}^{0}\right)\left(\mathrm{MDABCO}^{+}\right)\right]_{\infty}$ shown in Figure 4(a-2) to form crystal 3. Such packing of $\mathrm{C}_{60}{ }^{\bullet-}$ was generated on one side of the polycationic template and is denoted as Layer $A$.

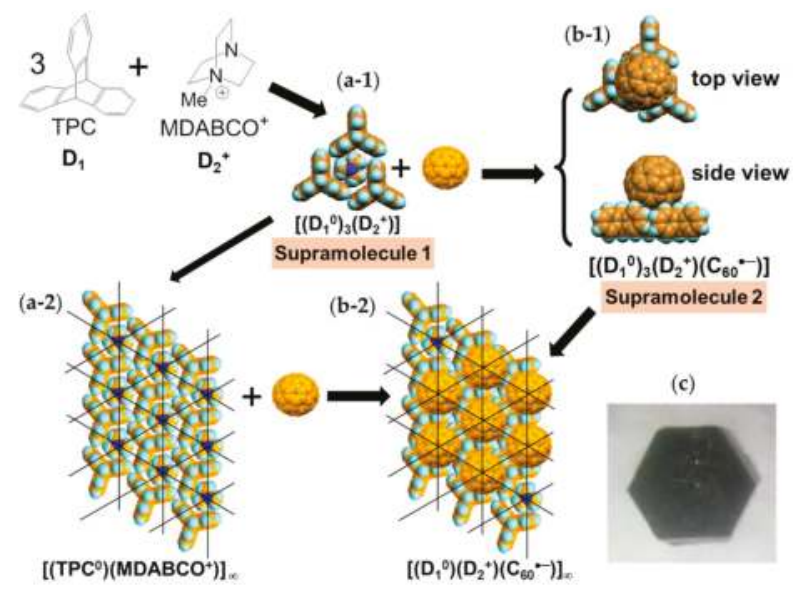

Figure 4. The formation of two-dimensional (2D) $\mathrm{C}_{60}{ }^{\bullet-}$ layer (Layer $A$ ) on the polycationic template of $\left[\left(\mathrm{TPC}^{0}\right)\left(\mathrm{MDABCO}^{+}\right)\right]_{\infty}$ : Three TPC $\left(\mathrm{D}_{1}\right)$ and one $\mathrm{MDABCO}^{+}\left(\mathrm{D}_{2}^{+}\right)$molecules form the first supramolecular unit ((a-1): Supramolecule $\left.1\left[\left(\mathrm{D}_{1}^{0}\right)_{3}\left(\mathrm{D}_{2}{ }^{+}\right)\right]\right)$, and $\mathrm{C}_{60}{ }^{\bullet-}$ molecule fits into the concave to form the second supramolecular unit ((b-1): Supramolecule $\left.2\left[\left(\mathrm{D}_{1}^{0}\right)_{3}\left(\mathrm{D}_{2}{ }^{+}\right)\right]\left(\mathrm{C}_{60}{ }^{\bullet-}\right)\right)$. They assemble to form a $2 \mathrm{D}$ layer (Layer $A$ ) of hexagonal packing of $\mathrm{C}_{60}{ }^{\bullet-}\left((\mathbf{b}-\mathbf{2})\right.$ : $\left.\left[\left(\mathrm{TPC}^{0}\right)\left(\mathrm{MDABCO}^{+}\right)\left(\mathrm{C}_{60}{ }^{\bullet-}\right)\right]_{\infty}\right)$. In another view, the 2D arrangement of the first supramolecular units (a-2), where $\mathrm{N}$ atoms of MDABCO agglomerate at the crossing points of lines, works as a cationic template to arrange $\mathrm{C}_{60}{ }^{\bullet-}$ molecules at the crossing points to form hexagonal layer of $\mathrm{C}_{60}{ }^{\bullet-}$ (Layer $A$ ) (b-2). (N: blue, H: pale blue, C: yellow). The thin lines in (a-2,b-2) are a guide to the eye. (c) Single crystal showing the $a b$ face.

Figure 5 shows the size of $\mathrm{MDABCO}^{+}$(Figure 5a,b), the height of TPC (Figure 5c), and some parts of Supramolecule 1 (Figure $5 \mathrm{~d}$ ) at $200 \mathrm{~K}$. The neighboring TPC molecules are separated by $9.82 \AA$, so that it is able to prevent the bond-formation between $\mathrm{C}_{60}{ }^{\bullet-}$ molecules when they pack on the template in a hexagonal lattice structure, where the intermolecular distance of $\mathrm{MDABCO}^{+}$molecules corresponds to $\mathrm{N} \cdots \mathrm{N}$ (blue points in Figure 4(a-2)) with separation distance of $9.99 \AA$. The MDABCO ${ }^{+}$ molecule is thicker $(7.27 \AA)$ than the thickness of the holder, which is composed of three TPC molecules (7.05 $\AA$ ). Consequently, one side of Supramolecule 1 has a concave shape where $\mathrm{N}$ atoms are centered and the $\mathrm{Me}$ of $\mathrm{N}-\mathrm{Me}^{+}$group of $\mathrm{MDABCO}^{+}$extrudes from the holder on the opposite side. Therefore, the $\mathrm{C}_{60}{ }^{\bullet-}$ hexagonal layer on the top of the polycationic template in Figure 4(a-2), namely Layer $A$ (Figure $6 \mathrm{a}, \mathrm{b}$ ) has different steric and electronic environment than the $\mathrm{C}_{60}{ }^{\bullet-}$ hexagonal layer at the bottom of the polycationic template (Layer B, Figure 6a,c).

Figure 7 demonstrates the key-keyhole relation for layer $B$. Three $\mathrm{TPC}^{0}$ and three MDABCO${ }^{+}$ molecules constitute Supramolecule 3, $\left[\mathrm{TPC}^{0}\right]_{3}\left[\mathrm{MDABCO}^{+}\right]_{3}$ (Figure $7 \mathrm{a}$ ), according to the first 
key-keyhole relation. Here, six methyl groups of six $\mathrm{MDABCO}^{+}$molecules, three from the top $\left(\mathrm{TPC}^{0}\right)\left(\mathrm{MDABCO}^{+}\right)$layer and three from the bottom layer, constitute an octopore to hold one $\mathrm{C}_{60}{ }^{\bullet-}$ molecule to afford $\left\{\left(\mathrm{TPC}^{0}\right)_{6}\left(\mathrm{MDABCO}^{+}\right)_{6}\left(\mathrm{C}_{60^{\bullet}}{ }^{-}\right)\right\}$, according to the second key-keyhole relation.

Figure $7 \mathrm{~b}$ shows one Supramolecule $4,\left(\mathrm{TPC}^{0}\right)_{3}\left(\mathrm{MDABCO}^{+}\right)_{3}\left(\mathrm{C}_{60}{ }^{\bullet-}\right)$. The $2 \mathrm{D}$ assembly of Supramolecule 4 generates the hexagonally packed $\mathrm{C}_{60}{ }^{\circ-}$ Layer $B$ (Figure $7 \mathrm{c}$ ), which corresponds to Figure 6c.

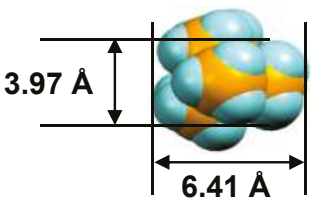

(a)

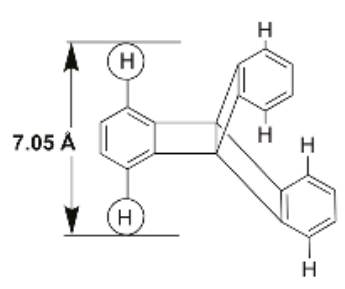

(c)

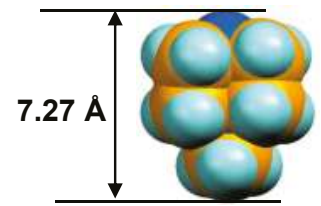

(b)

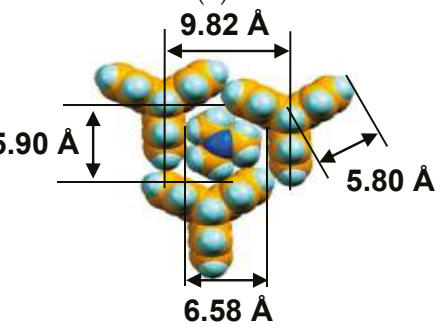

(d)

Figure 5. Size of the component molecules and parts of Supramolecule $1\left(\mathrm{TPC}^{0}\right)\left(\mathrm{MDABCO}^{+}\right)$, at $200 \mathrm{~K}$. $\mathrm{C}$ : yellow, H: white-blue, $\mathrm{N}$ : blue: (a,b) Molecular size of $\mathrm{MDABCO}^{+}$molecule; (c) The height of TPC molecule (Schematic figure); (d) Supramolecule 1 made of three TPC $^{0}$ molecules and one $\mathrm{MDABCO}^{+}$molecule.

(a)

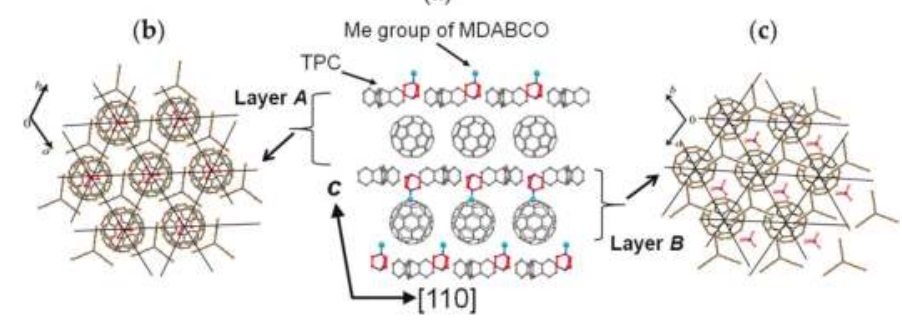

Figure 6. Crystal structure of $\left(\mathrm{TPC}^{0}\right)\left(\mathrm{MDABCO}^{+}\right)\left(\mathrm{C}_{60}{ }^{-}\right)$at $160 \mathrm{~K}$ : The $\left(\mathrm{TPC}^{0}\right)\left(\mathrm{MDABCO}^{+}\right)$layer and $\mathrm{C}_{60}{ }^{\bullet-}$ layer stack along the $c$ axis with the sequence of the Layer $A$ of $\mathrm{C}_{60}{ }^{\bullet-} /\left(\mathrm{TPC}^{0}\right)\left(\mathrm{MDABCO}^{+}\right)$ layer/Layer $B$ of $\mathrm{C}_{60}{ }^{\bullet-}$. (a) $\mathrm{C}_{60}{ }^{\bullet-}$ molecules in Layer $A$ are arranged between the $\mathrm{N}$ atoms of MDABCO (drawn in red and $\mathrm{N}$ atoms and methyl groups are drawn in dark and bright blue, respectively) when viewed along the $c$ axis. The methyl groups of MDABCO molecules are arranged towards the $\mathrm{C}_{60}$ molecules in Layer $B$ and outline an octopore around $C_{60}$. Configuration of the molecules in the slabs $A(\mathbf{b})$ and $B(\mathbf{c})$. The thin lines show the geometry (triangular lattice) connected between the centers of $\mathrm{C}_{60}$ molecules.

At $300 \mathrm{~K}, \mathrm{C}_{60}{ }^{\bullet-}$ molecules are ordered in Layer $A\left(r=10.07 \AA\right.$, overlap integral $s=1.91 \times 10^{-3}$, Figure 8a), while $\mathrm{C}_{60}{ }^{\circ-}$ molecules in Layer $B$ are disordered. At the same temperature, half of the $\mathrm{MDABCO}^{+}$cations are disordered between three orientations that are linked by their rotation about the lattice threefold axis. On Layer $A$, the $r$ value decreases monotonously to $9.97 \AA$ at $183 \mathrm{~K}$ at which 
temperature a transition from rhombohedral to triclinic occurs. Assuming linear shrinkage of the interfullerene distance along the $a$ axis below $160 \mathrm{~K}, r=9.54 \AA$ is evaluated at around $4 \mathrm{~K}$, where no dimerization was experimentally detected. The calculated Fermi surfaces of both $\mathrm{C}_{60}{ }^{\bullet-}$ layers have a closed 2D pocket at $\Gamma$ point and suggest 2D metallic nature in the $a b$ plane (Figure $8 \mathrm{~b}$ ).

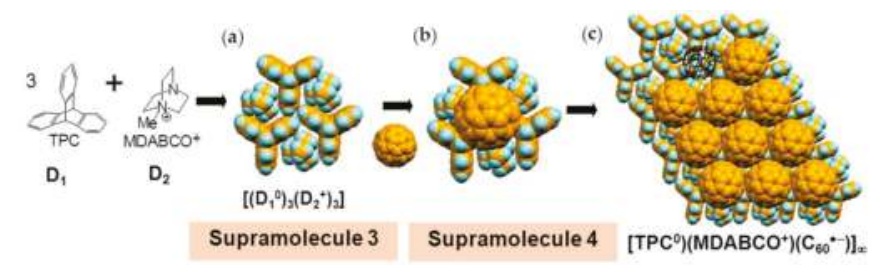

Figure 7. Formation of a $2 \mathrm{D} \mathrm{C}_{60}{ }^{\bullet-}$ layer (layer $B$ ) on the polycationic template of $\left[\left(\mathrm{TPC}^{0}\right)\right.$ $\left.\left(\mathrm{MDABCO}^{+}\right)\right]_{\infty}$ : (a) Three $\operatorname{TPC}^{0}\left(\mathrm{D}_{1}\right)$ and three $\mathrm{MDABCO}^{+}\left(\mathrm{D}_{2}{ }^{+}\right)$molecules form Supramolecule 3 $\left[\left(\mathrm{D}_{1}^{0}\right)_{3}\left(\mathrm{D}_{2}{ }^{+}\right)_{3}\right] ;$ (b) $\mathrm{A} \mathrm{C}_{60}{ }^{\bullet-}$ molecule fits into the concave to form Supramolecule $4\left\{\left[\left(\mathrm{D}_{1}^{0}\right)_{3}\left(\mathrm{D}_{2}{ }^{+}\right)_{3}\right]\right.$ $\mathrm{C}_{60}{ }^{\bullet-}$; ; (c) 2D layer (Layer $B$ ) of hexagonal packing of $\mathrm{C}_{60}{ }^{\bullet-}$ by assembly of Supramolecule 4 .

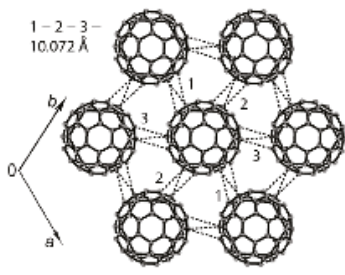

(a)

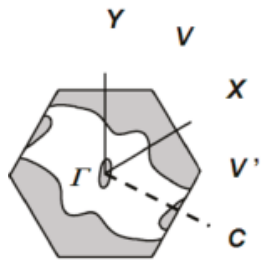

(c)

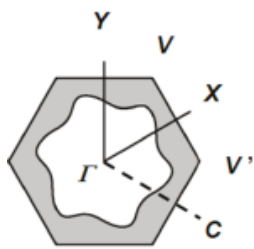

(b)

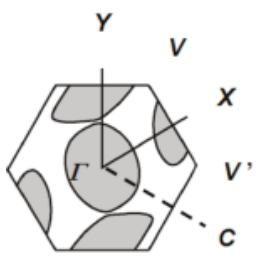

(d)

Figure 8. (a) View of the $a b$ plane of fullerene Layer $A$ in $\left(\mathrm{TPC}^{0}\right)\left(\mathrm{MDABCO}^{+}\right)\left(\mathrm{C}_{60}{ }^{--}\right)$at $300 \mathrm{~K}$. The van der Waals C ...C contacts shorter than $3.42 \AA$ are shown by dashed lines. Numbers 1-3 indicate the $r$ values; (b) The calculated Fermi surface of Layer $A$ at $200 \mathrm{~K}$, (c) that of Layers $A$ at $160 \mathrm{~K}$, and (d) that of Layer $B$ at $160 \mathrm{~K}$. (a) was reproduced from [12] and (b-d) were from [146].

With decreasing temperature, ordering of the orientations of $\mathrm{C}_{60}{ }^{\bullet-}$ and $\mathrm{MDABCO}^{+}$started below $200 \mathrm{~K}$. A complete ordering of all three of the component molecules was found in the crystal structure at $160 \mathrm{~K}$ (Figure 6). These observations suggest that the orientation disorder of $\mathrm{C}_{60}{ }^{\bullet-}$ is closely linked with that of $\mathrm{MDABCO}^{+}$. The calculated overlap integrals at $160 \mathrm{~K}$ are $s=2.57 \times 10^{-3}(/ / a), 2.03 \times 10^{-3}$ $(/ / b)$, and $2.76 \times 10^{-3}(/ / a+b)$ for Layer $A$ and $2.45 \times 10^{-3}(/ / a), 2.21 \times 10^{-3}(/ / b)$, and $1.61 \times 10^{-3}$ $(/ / a+b)$ for Layer $B$. The calculated bandwidth $W$ is $0.103 \mathrm{eV}$ at $300 \mathrm{~K}$ for Layer $A, 0.150 \mathrm{eV}$ and $0.133 \mathrm{eV}$ for Layers $A$ and $B$ at $160 \mathrm{~K}$, respectively. The calculated anisotropy of the transfer interactions $t_{\mathrm{a}}: t_{\mathrm{b}}: t_{\mathrm{a}+\mathrm{b}}=1: 1: 1$ for Layer $A$ above $183 \mathrm{~K}$ changed to $t_{\mathrm{a}}: t_{\mathrm{b}}: t_{\mathrm{a}+\mathrm{b}}=1.27: 1: 1.36$ for Layer $A$ and $t_{\mathrm{a}}: t_{\mathrm{b}}: t_{\mathrm{a}+\mathrm{b}}$ $=1.52: 1.37: 1$ for Layer $B$ at $160 \mathrm{~K}$. The ratio of the triangular spin lattice is defined as $2 t_{\mathrm{a}} /\left(t_{\mathrm{b}}+t_{\mathrm{a}+\mathrm{b}}\right)$, $2 t_{\mathrm{b}} /\left(t_{\mathrm{a}}+t_{\mathrm{a}+\mathrm{b}}\right)$, and $2 t_{\mathrm{a}+\mathrm{b}} /\left(t_{\mathrm{a}}+t_{\mathrm{b}}\right)$. However, the last two definitions provide inadequate $t^{\prime} / t$ values of $0.60-0.76$ for layer $A$ and $0.54-0.69$ for Layer $B$ at $160 \mathrm{~K}$ that suggests a much enhanced $2 \mathrm{D}$ nature 
than that at RT. So using the ratio $t^{\prime} / t=2 t_{\mathrm{a}} /\left(t_{\mathrm{b}}+t_{\mathrm{a}+\mathrm{b}}\right)$, the calculated anisotropy is $1.00(300 \mathrm{~K})$ and $1.07(160 \mathrm{~K})$ for Layer $A$ and $0.99(185 \mathrm{~K})$ and $1.28(160 \mathrm{~K})$ for Layer $B$. The spin frustration of this spin lattice is comparable to that of $\kappa-(\mathrm{ET})_{2} \mathrm{Cu}_{2}(\mathrm{CN})_{3}\left(t^{\prime} / t=1.09\right.$ at $\mathrm{RT}, 1.07$ at $\left.100 \mathrm{~K}\right)$ [54] and stronger than a Mott insulator $\kappa-(\mathrm{ET})_{2} \mathrm{~B}(\mathrm{CN})_{4}[150]\left(t^{\prime} / t=1.42\right.$ at $\mathrm{RT}, 1.61$ at $100 \mathrm{~K}$, ground state is valence-bond solid). However, the increase of $W$ together with the ordering of $\mathrm{C}_{60}{ }^{\bullet-}$ molecules in Layer $B$ below $200 \mathrm{~K}$ gave rise to a superior itinerancy (metallic state) than localization (QSL state) for this salt.

The resistivity measurements carried out using the four probe method indicate that salt 3 is metallic within the $a b$ plane from $360\left(1.8 \mathrm{~S} \cdot \mathrm{cm}^{-1}\right)$ to $200 \mathrm{~K}\left(14 \mathrm{~S} \cdot \mathrm{cm}^{-1}\right)$, after which a rapid enhancement of the metallic nature occurs from $200 \mathrm{~K}$ to $185 \mathrm{~K}\left(33 \mathrm{~S} \cdot \mathrm{cm}^{-1}\right)$ (Figure 9a).

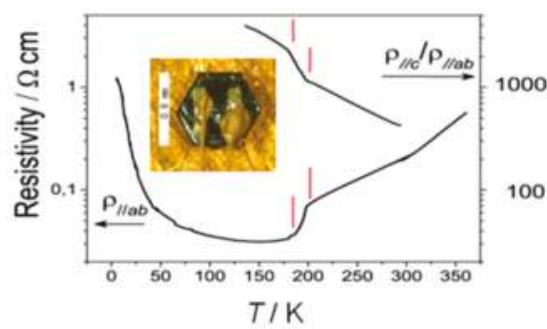

(a)

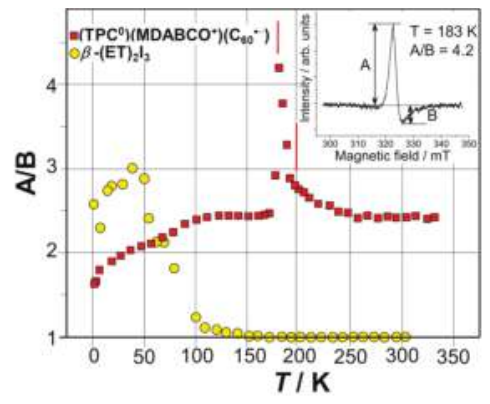

(c)

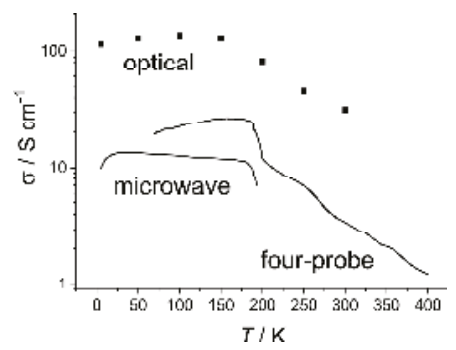

(b)

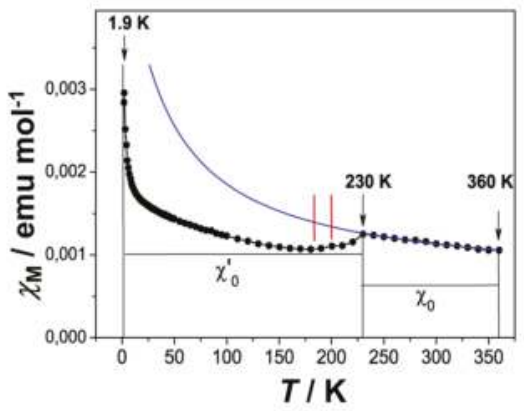

(d)

Figure 9. (a) Temperature dependent resistivity in the $a b$ plane and the ratio $\sigma_{/ / c} / \sigma_{/ / a b}$ of $\left(\mathrm{TPC}^{0}\right.$ ) $\left(\mathrm{MDABCO}^{+}\right)\left(\mathrm{C}_{60}{ }^{\circ-}\right)$ by the four probe method (inset: single crystal with gold wires and paste) (from [146]); (b) Temperature dependence of conductivity by the four probe method, microwave and optical conductivity (from [12]); (c) Temperature dependent EPR peak ratio A/B for the Dysonian line from the single crystal (inset: the spectrum at $183 \mathrm{~K}$ ) (from [146]) is compared with that of a superconductor $\beta$-(ET) $\mathrm{I}_{3}\left(T_{\mathrm{c}}=1.5 \mathrm{~K}\right)$; (d) Temperature dependent molar magnetic susceptibility $\left(\chi_{\mathrm{M}}\right)$ (closed circles, and blue curve fits in $\chi_{0}+C /\left(T-\Theta_{\mathrm{CW}}\right)\left(\chi_{0}=6.5 \times 10^{-4} \mathrm{emu} \cdot \mathrm{mol}^{-1}, \mathrm{C}=\right.$ $0.160 \mathrm{emu} \cdot \mathrm{K} \cdot \mathrm{mol}^{-1}$, and $\left.\Theta_{\mathrm{CW}}=-31 \mathrm{~K}\right)$. Red bars for $(\mathbf{a}, \mathrm{c}, \mathrm{d})$ show temperature interval from 185 to $200 \mathrm{~K}$ (from [146]).

The temperature range of this anomaly between $200 \mathrm{~K}$ and $185 \mathrm{~K}$, indicated by two red lines, coincides well with that of an ordering of $\mathrm{C}_{60}{ }^{\bullet-}$ in Layer $B$, showing that the ordered $\mathrm{C}_{60}{ }^{\bullet-}$ radical anions in Layer $B$ start to participate in the metallic transport below $200 \mathrm{~K}$. The resistivity could not be measured correctly below $70 \mathrm{~K}$ because of a large increase in the contact resistance, due to its air-sensitivity, even though it was measured in an inert atmosphere. The contactless microwave and optical measurements revealed that the conductivity increased down to $100-25 \mathrm{~K}$ and the metallic state is preserved down to $5 \mathrm{~K}$ (Figure 9b) [12]. The optical conductivity is nearly flat below $150 \mathrm{~K}$ down to $5 \mathrm{~K}$. 
The asymmetry ratio of the Dysonian EPR line shape between the maximum and minimum of the absorption derivative (A/B, Figure 9c inset) of 3 is compared with that of $\beta$-(ET) ${ }_{2} \mathrm{I}_{3}$ (Figure 9c) [151], which is highly metallic and shows SC with $T_{\mathrm{C}}=1.5 \mathrm{~K}$ [152]. For $\beta$ - $(\mathrm{ET})_{2} \mathrm{I}_{3}$, the EPR signal is Lorentzian $(\mathrm{A} / \mathrm{B}=1)$ at $\mathrm{RT}$ and the peak ratio $\mathrm{A} / \mathrm{B}$ increases below $130 \mathrm{~K}$ to about $\mathrm{A} / \mathrm{B}=3.0$ (at approximately $50 \mathrm{~K}$ ) followed by a gradual decrease down to $5 \mathrm{~K}$. In comparison, the A/B values of 3 are considerably large even at $\mathrm{RT}(\mathrm{A} / \mathrm{B}=2.4)$. It exhibits an abrupt increase below $200 \mathrm{~K}$ reaching a maximum of 4.2 at $183 \mathrm{~K}$, which coincides well with the rapid conductivity increase, then falls to $2.3-2.4$ below $183 \mathrm{~K}$. The A/B ratio slowly decreases below $100 \mathrm{~K}$, but the Dysonian shape is observed even at $4 \mathrm{~K}(\mathrm{~A} / \mathrm{B}=$ $1.64)$, thus confirming the existence of a highly conducting state down to $4 \mathrm{~K}$.

At $230-330 \mathrm{~K}$, molar magnetic susceptibility $\left(\chi_{\mathrm{M}}\right)$ can be fitted by a combination of the Pauli and Curie-Weiss terms: $\chi_{\mathrm{M}}=\chi_{0}+C /\left(T-\Theta_{\mathrm{CW}}\right)$ with a constant $\chi_{0}=6.5 \times 10^{-4} \mathrm{emu} \cdot \mathrm{mol}^{-1}$, $C=0.160 \mathrm{emu} \cdot \mathrm{K} \cdot \mathrm{mol}^{-1}$, and $\Theta_{\mathrm{CW}}=-31 \mathrm{~K}$ (blue curve in Figure 9d). $C$ of $0.160 \mathrm{emu} \cdot \mathrm{K} \cdot \mathrm{mol}^{-1}$ corresponds to the contribution of about $43 \%$ of the spins from the total amount of $\mathrm{C}_{60}$ $\left(C=0.374 \mathrm{emu} \cdot \mathrm{K} \cdot \mathrm{mol}^{-1}\right.$ for $100 \%$ of spins). Consequently, the spins in one layer (Layer B) are treated as localized ones and they interact antiferromagnetically with $\Theta_{C W}$ of $-31 \mathrm{~K}$. A reversible decrease in $\chi_{\mathrm{M}}$ is observed at $200-230 \mathrm{~K}$. Below $200 \mathrm{~K}$, the temperature-independent susceptibility $\left(\chi^{\prime}{ }_{0}\right)$ of about $10.0 \times 10^{-4} \mathrm{emu} \cdot \mathrm{mol}^{-1}$ is attributed to the Pauli paramagnetic contribution, implying a metallic state down to $1.9 \mathrm{~K}$. The scenario is that ordering of both $\mathrm{C}_{60}{ }^{\circ-}$ in layer $B$ and $\mathrm{MDABCO}^{+}$triggered a transition from a non-metallic and antiferromagnetically frustrated state to a metallic state for spins of $\mathrm{C}_{60} 0^{--}$in Layer $B$, while ordered $\mathrm{C}_{60}{ }^{-}-$in Layer $A$ kept its $2 \mathrm{D}$ itinerancy over the entire temperature range. The strong coupling between the ordering of $C_{60}$ and physical properties is intriguing and was previously observed in some fullerene salts [153-156].

Salt 3 is the first 2D monomer-type $\mathrm{C}_{60^{\circ}}{ }^{--}$organic metal composed of only light elements $(\mathrm{C}, \mathrm{H}$, $\mathrm{N})$. Even though rapidly cooled $\mathrm{AC}_{60}{ }^{\bullet-}(\mathrm{A}=\mathrm{Cs}$ and $\mathrm{Rb})$ were reported to be monomer-type metals below $150 \mathrm{~K}$ and $125 \mathrm{~K}$, respectively, definitive information is needed concerning the stoichiometry, metallic behavior, dimensionality, and crystal structure to confirm a monomer-type metal $[71,72,157]$.

Summarizing the information concerning the geometry of spin lattice of 3 , the $\mathrm{C}_{60}{ }^{\bullet-}$ molecules form hexagonal stacking according to the geometry of cationic template $\left(\mathrm{TPC}^{0}\right)\left(\mathrm{MDABCO}^{+}\right)$, both of the component molecules have threefold symmetry, by key-keyhole relation. Layer $B$ has $t^{\prime} / t=0.99(185 \mathrm{~K})$, which indicates strong spin frustration and is close to those of QSL candidates $\kappa-(E T)_{2} \mathrm{M}_{2}(\mathrm{CN})_{3}(\mathrm{M}=\mathrm{Cu}$; $\left.t^{\prime} / t=1.09, \mathrm{Ag} ; t^{\prime} / t=0.97\right),\left|\Theta_{\mathrm{CW}}\right|=31 \mathrm{~K}$ is estimated in the range of $260-300 \mathrm{~K}$, and $r=10.07 \AA$ (RT). No dimerization of $\mathrm{C}_{60}{ }^{\circ}{ }^{-}$occurred down to $1.9 \mathrm{~K}$.

\subsubsection{Only Frustrated Spins in $2 \mathrm{D}$ Hexagonal Packing of $\mathrm{C}_{60}{ }^{\bullet-}$ in $\left(\mathrm{TPC}^{0}\right)\left(\mathrm{MQ}^{+}\right)\left(\mathrm{C}_{60}{ }^{\bullet-}\right)$}

By a using $\mathrm{MQ}^{+}$instead of $\mathrm{MDABCO}^{+}$, where $\mathrm{MQ}^{+}$is $N$-methylquinuclidinium cation, both of which have threefold symmetry, an $\mathrm{AF}$ insulator $\left(\mathrm{TPC}^{0}\right)\left(\mathrm{MQ}^{+}\right)\left(\mathrm{C}_{60}{ }^{-}-\right)(4)$ was obtained [12]. The $\mathrm{C}_{60}$ molecule, a 10-fold molar excess of $\mathrm{CH}_{3} \mathrm{CH}_{2} \mathrm{SNa}$, and a 5 -fold molar excess of MQ.I were reacted in a $\mathrm{PhCl}_{2} / \mathrm{PhCN}$ mixture. Into a filtered solution, TPC was dissolved and filtered. $n$-Hexane was layered over the obtained solution. Black hexagonal prisms up to $0.2 \times 0.5 \times 0.5 \mathrm{~mm}^{3}$ were harvested after two months.

A major difference between $\mathrm{MQ}^{+}$(Figure $10 \mathrm{a}-\mathrm{c}$ ) and $\mathrm{MDABCO}^{+}$is that the vertical size of $\mathrm{MQ}^{+}$ $(7.87 \AA)$ is larger than that of $\mathrm{MDABCO}^{+}$cation $(7.27 \AA)$ since the carbon atom with hydrogen in $\mathrm{MQ}^{+}$ instead of uncharged nitrogen atom in $\mathrm{MDABCO}^{+}$that is caused some kind of distortion in the layered packing of $\mathrm{C}_{60}$ molecules. The size of $\mathrm{MQ}^{+}$is $5.86 \AA \times 6.40 \AA$ (Figure $10 \mathrm{c}$ ), which is very close to that of $\mathrm{MDABCO}^{+}$. Figure $10 \mathrm{~d}$,e show the sizes of the fundamental units at $250 \mathrm{~K}$. Similar to the $\mathrm{MDABCO}^{+}$ salt, three TCP and one $\mathrm{MQ}^{+}$molecules form Supramolecule $5 ;\left(\mathrm{TPC}^{0}\right)_{3}\left(\mathrm{MQ}^{+}\right)$, with a periodicity of TPC molecules of an average of $10.15 \AA$ (Figure 10d) and periodicity of $\mathrm{N}$-site in $\mathrm{MQ}^{+}$molecules of an average of $10.04 \AA$ (Figure 10e).

Crystal 4 has lower symmetry (triclinic unit cell) than 3 at RT. Similar to 3, crystal 4 has layered packing in which hexagonal fullerene layers alternate with the $\left(\mathrm{TPC}^{0}\right)\left(\mathrm{MQ}^{+}\right)$layers along the $c$ axis 
(Figure 11a) in the sequence of $\left(\mathrm{TPC}^{0}\right)\left(\mathrm{MQ}^{+}\right)$layer $/ \mathrm{C}_{60}{ }^{\bullet-}$ Layer $A /\left(\mathrm{TPC}^{0}\right)\left(\mathrm{MQ}^{+}\right)$layer $/ \mathrm{C}_{60}{ }^{\bullet-}$ Layer $B$. Figure $11 \mathrm{~b}, \mathrm{c}$ show the view along the $c$ axis for Layer $A$ and Layer $B$, respectively.

The key-keyhole relation between $\mathrm{C}_{60}$ in Layer $A$ and $\left(\mathrm{TPC}^{0}\right)\left(\mathrm{MQ}^{+}\right)$is presented in Figure 12. Three TPC molecules and one $\mathrm{MQ}^{+}$form Supramolecule 5, $\left(\mathrm{TPC}^{0}\right)_{3}\left(\mathrm{MQ}^{+}\right)$, via the first key-keyhole relation. Then, $\mathrm{C}_{60}{ }^{\bullet-}$ molecule fits into the concave of Supramolecule 5 to give Supramolecule 6 , $\left[\left(\mathrm{TPC}^{0}\right)_{3}\left(\mathrm{MQ}^{+}\right)\right]\left(\mathrm{C}_{60}{ }^{\bullet-}\right)$, similar to the case of $\left\{\left[\left(\mathrm{TPC}^{0}\right)_{3}\left(\mathrm{MDABCO}^{+}\right)\right]\left(\mathrm{C}_{60}{ }^{\bullet-}\right)\right\}$ (Figure 4(b-1)) by the second key-keyhole relation. The Supramolecule 6 units assembled to form (Supramolecule 6$)_{\infty}$ generating Layer $A$ of $\left(\mathrm{TPC}^{0}\right)\left(\mathrm{MQ}^{+}\right)\left(\mathrm{C}_{60}{ }^{\bullet-}\right)$ (Figures $11 \mathrm{~b}$ and $\left.12 \mathrm{~d}\right)$.

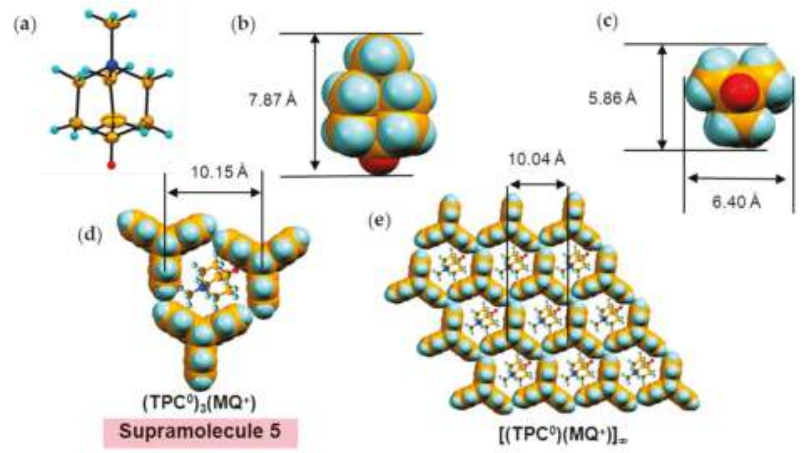

Figure 10. (a-c) Shape and size of cation molecule $\mathrm{MQ}^{+}$in $\left(\mathrm{TPC}^{0}\right)\left(\mathrm{MQ}^{+}\right)\left(\mathrm{C}_{60}{ }^{-}\right)$at $250 \mathrm{~K}$. The $\mathrm{H}$ atom attached to one $\mathrm{N}$ atom is shown in red; (d) The distance of the neighboring TPC molecules in Supramolecule $5\left(\mathrm{TPC}^{0}\right)_{3}\left(\mathrm{MQ}^{+}\right)$; (e) Intermolecular distance between neighboring $\mathrm{MQ}^{+}$molecules $(\mathrm{N} \cdots \mathrm{N} \text { distance) in the polycationic template of (Supramolecule } 5)_{\infty}$.

(b)

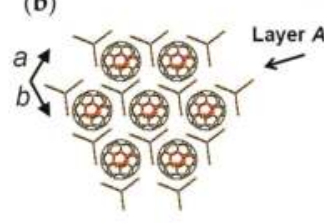

(a)

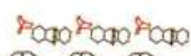

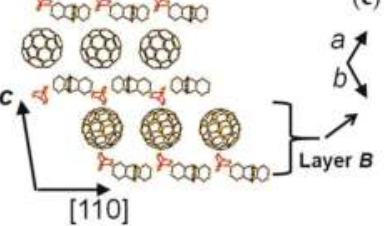

(c)

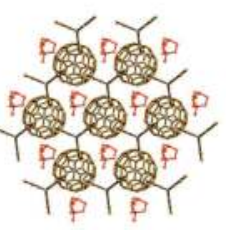

Figure 11. (a) Crystal structure $(250 \mathrm{~K})$ projected along the $[11(-) 0]$ axis. The $\left(\mathrm{TPC}^{0}\right)\left(\mathrm{MQ}^{+}\right)$layer and $\mathrm{C}_{60}{ }^{\bullet-}$ layer stack along the $c$ axis with the sequence of $\left(\mathrm{TPC}^{0}\right)\left(\mathrm{MQ}^{+}\right)$layer $/ \mathrm{C}_{60}{ }^{\bullet-}$ Layer $A /\left(\mathrm{TPC}^{0}\right)\left(\mathrm{MQ}^{+}\right)$layer $/ \mathrm{C}_{60}{ }^{\bullet-}$ Layer $B$. The $\mathrm{MQ}^{+}$cations are shown in major occupied orientation in red color; (b,c) Projected views perpendicular to the layers, $A$ layer slab (b) and B layer slab (c) [12].

From a different point of view, $\mathrm{C}_{60}{ }^{\bullet-}$ molecules fit into the concaves in the polycationic template of layered unit of $\left[\left(\mathrm{TPC}^{0}\right)\left(\mathrm{MQ}^{+}\right)\right]_{\infty}$ (Figure 12c) to form Layer $A$ in 4 (Figure 12d). Figure 12e,f illustrate how one $\mathrm{C}_{60}{ }^{\bullet-}$ molecule in Layer $A$ is embedded between two layers of $\left(\mathrm{TPC}^{0}\right)\left(\mathrm{MQ}^{+}\right)$where the TPC molecules in the upper and lower layers are shown in different colors. $\mathrm{C}_{60}{ }^{\bullet-}$ molecules in Layer $A$ are well fitted in the TPC hole formed by the six TPC molecules in the upper and lower $\left(\mathrm{TPC}^{0}\right)\left(\mathrm{MQ}^{+}\right)$ layers (Figure 12e). In Supramolecule $5\left[\left(\mathrm{TPC}^{0}\right)_{3}\left(\mathrm{MQ}^{+}\right)\right]$(Figure 10d), the extra hydrogen atom (red circle in Figure 10a) in $\mathrm{MQ}^{+}$prevents the $\mathrm{MQ}^{+}$cation from arranging vertically relative to the fullerene layers resulting in a lowered crystal symmetry. Figure $13 \mathrm{~g}$, h show the calculated Fermi surfaces by the tight-binding method combined with the semiempirical (AM1) molecular orbital calculations based on crystal structures at $250 \mathrm{~K}$ and $100 \mathrm{~K}$, respectively. The band calculation of Layer $A$ at $250 \mathrm{~K}$ indicates 
that the salt has 2D Fermi surfaces. However, due to the doubling of the unit cell along the $b$ axis, a semi-metallic Fermi surface was estimated at $100 \mathrm{~K}$.

Surprisingly, Layer $B$ also has a hexagonal packing of $\mathrm{C}_{60}{ }^{\bullet-}$ molecules in spite of the use of longer cation molecule $\mathrm{MQ}^{+}$by $0.60 \AA$ than $\mathrm{MDABCO}^{+}$. Figure 13 shows the formation of Layer $B$ similar to that in Figure 7. Figure 13a shows Supramolecule 7 by the first key-keyhole relation made of three TPC and three $\mathrm{MQ}^{+}$molecules. The $\mathrm{C}_{60}$ molecule fit into the hollow site where three $\mathrm{MQ}^{+}$molecules formed that corresponds to the crossing points of black lines in Figure 13c to form Supramolecule 8 $\left[\left(\mathrm{TPC}^{0}\right)_{3}\left(\mathrm{MQ}^{+}\right)_{3}\left(\mathrm{C}_{60}{ }^{--}\right)\right]$(Figure 13b). This is the second key-keyhole relation.

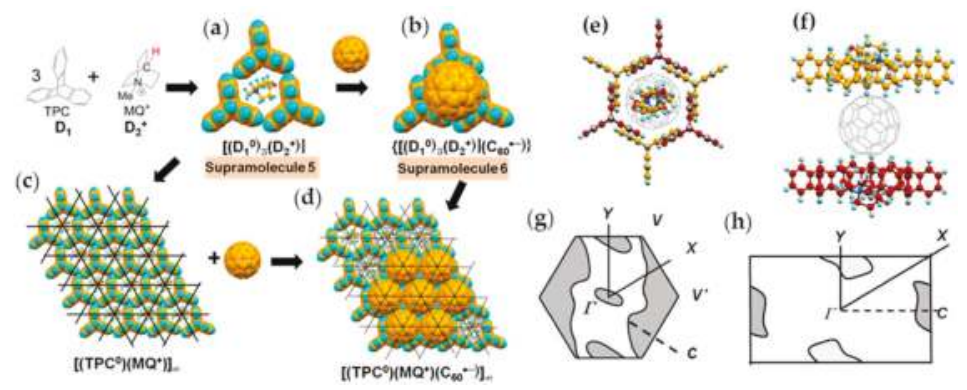

Figure 12. Key-keyhole relation for the Layer $A$ in $\left(\mathrm{TPC}^{0}\right)\left(\mathrm{MQ}^{+}\right)\left(\mathrm{C}_{60^{\circ}}{ }^{-}\right)$: Asymmetric placing of $\mathrm{MQ}^{+}$ in the $\left(\mathrm{TPC}^{0}\right)_{3}$ hole to form Supramolecule 5 by first key-keyhole relation (a) and docking of $\mathrm{C}_{60}{ }^{\circ-}$ into the pit of Supramolecule 5 gives Supramolecule 6 (second key-keyhole relation) (b); (c) A layer of $\left[\left(\mathrm{TPC}^{0}\right)\left(\mathrm{MQ}^{+}\right)\right]_{\infty}$ composed of Supramolecule 5; (d) A layer of $\left[\left(\mathrm{TPC}^{0}\right)\left(\mathrm{MQ}^{+}\right)\right]_{\infty}$ and $\mathrm{C}_{60} 0^{\bullet}$ - molecules are assembled by fitting the $\mathrm{C}_{60}$ molecules into the concaves in the layer of $\left[\left(\mathrm{TPC}^{0}\right)\left(\mathrm{MQ}^{+}\right)\right]_{\infty}$ to form the Layer $A$ of $\left(\mathrm{TPC}^{0}\right)\left(\mathrm{MQ}^{+}\right)\left(\mathrm{C}_{60}{ }^{-}-\right.$). Black lines are guides to the eye. $\mathrm{C}_{60}$ molecules are arranged at the crossing points of black lines; (e,f) show the relation among the $\mathrm{C}_{60}$ molecule in Layer $A$ and upper and lower layers of $\left(\mathrm{TPC}^{0}\right)\left(\mathrm{MQ}^{+}\right)$. Upper and lower layers of $\left(\mathrm{TPC}^{0}\right)\left(\mathrm{MQ}^{+}\right)$are drawn in different colors: top down view (e) and side view (f); (g,h) Calculated Fermi surface of $\mathrm{C}_{60}$ assemble in Layer $A$ at $250 \mathrm{~K}(\mathrm{~g})$ and $100 \mathrm{~K}(\mathbf{h})[12]$.

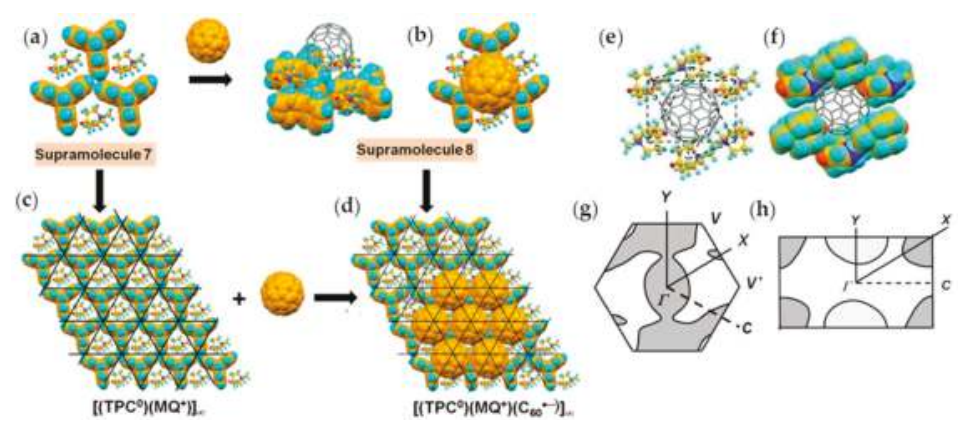

Figure 13. Schematic key-keyhole relation to form Layer $B$ of $\left(\mathrm{TPC}^{0}\right)\left(\mathrm{MQ}^{+}\right)\left(\mathrm{C}_{60}{ }^{--}\right)$: (a) Supramolecule 7: an assembly of three TPC and three $\mathrm{MQ}^{+}$molecules for Layer $B$ by the first key-keyhole relation; (b) $\mathrm{C}_{60}{ }^{\bullet-}$ molecule fit into Supramolecule 7 to form Supramolecule $8\left[\left(\mathrm{TPC}^{0}\right)_{3}\left(\mathrm{MQ}^{+}\right)_{3}\left(\mathrm{C}_{60}{ }^{\bullet-}\right)\right]$ in the Layer B; (c) Polycationic template of 2D layer $\left(\mathrm{TPC}^{0}\right)\left(\mathrm{MQ}^{+}\right)$composed of Supramolecule 7; (d) $\mathrm{C}_{60}{ }^{\bullet-}$ molecules are assembled by fitting the $\mathrm{C}_{60}$ molecules into the concaves in the layer of $\left(\mathrm{TPC}^{0}\right)\left(\mathrm{MQ}^{+}\right)$to form the Layer $B$ of $\left(\mathrm{TPC}^{0}\right)\left(\mathrm{MQ}^{+}\right)\left(\mathrm{C}_{60}{ }^{--}\right)$. Dark thin lines are guides to the eye; $(\mathbf{e}, \mathbf{f})$ Six $\mathrm{MQ}^{+}$molecules from the adjacent $\left(\mathrm{TPC}^{0}\right)\left(\mathrm{MQ}^{+}\right)$layers form octopores for $\mathrm{C}_{60}{ }^{--}$in Layer $B ;(\mathbf{g}, \mathbf{h})$ Calculated Fermi surface of $C_{60}$ assembly in Layer $B$ at $250 \mathrm{~K}$ (g) and $100 \mathrm{~K}$ (h) [12]. 
The $2 \mathrm{D}$ assembly of supramolecular units $\left[\left(\mathrm{TPC}^{0}\right)_{3}\left(\mathrm{MQ}^{+}\right)_{3}\left(\mathrm{C}_{60^{-}}{ }^{-}\right)\right]$leads to Layer $B$ on the $\left(\mathrm{TPC}^{0}\right)\left(\mathrm{MQ}^{+}\right)$layer (Figure 13d). From another view, the $\mathrm{C}_{60}{ }^{\circ-}$ molecules assemble using polycationic template of 2D assembly of the first supramolecular units (Figure 13c) and leads to Layer $B$ on the $\left(\mathrm{TPC}^{0}\right)\left(\mathrm{MQ}^{+}\right)$layer (Figure 13d).

In Layer $B, \mathrm{C}_{60}{ }^{--}$molecules are sandwiched between $2 \mathrm{D}$ layer of $\left(\mathrm{TPC}^{0}\right)\left(\mathrm{MQ}^{+}\right)$formed by Supramolecule 7 (Figure 13e). Here, the $\mathrm{C}_{60}{ }^{\circ-}$ molecules are more in contact with $\mathrm{MQ}^{+}$than with the TPC molecules. The intermolecular interactions between $\mathrm{C}_{60}{ }^{\circ-}$ and $\mathrm{MQ}^{+}$are dominant factors that determine the packing of $\left(\mathrm{TPC}^{0}\right)\left(\mathrm{MQ}^{+}\right)$and $\mathrm{C}_{60}{ }^{\circ-} \cdot \mathrm{C}_{60} 0^{-}$molecules in Layer $B$, which are allocated at each hollow site where the six $\mathrm{MQ}^{+}$cation molecules combine: three $\mathrm{MQ}^{+}$molecules in one $\left(\mathrm{TPC}^{0}\right)\left(\mathrm{MQ}^{+}\right)$layer and three $\mathrm{MQ}^{+}$in the neighboring $\left(\mathrm{TPC}^{0}\right)\left(\mathrm{MQ}^{+}\right)$layer constitute an octopore for $\mathrm{C}_{60}{ }^{\bullet-}$ molecule in Layer B (Figure 13e,f). The fusion of the assemblies of $\left(\mathrm{TPC}^{0}\right)\left(\mathrm{MQ}^{+}\right)$and $\mathrm{C}_{60} 0^{\bullet-}$ molecules while maintaining the relation of octopores in Figure 13e,f leads to the packing pattern of $\mathrm{C}_{60^{\circ}}{ }^{-}$in Layer $B$ in the $a b$ plane (Figure 13d). Similar to Layer $A$, the band calculation of Layer $B$ indicates that the salt has $2 \mathrm{D}$ Fermi surfaces at $250 \mathrm{~K}$ and a semi-metallic Fermi surface at $100 \mathrm{~K}$ (Figure 13g,h).

The $\mathrm{MQ}^{+}$cations are disordered between two orientations at $250 \mathrm{~K}$ and even $100 \mathrm{~K}$. In spite of the disorder of $\mathrm{MQ}^{+}$, the fullerene anions are ordered in 4 at both temperatures. The center-to-center interfullerene distances of $10.12,10.16$, and $10.18 \AA$ at $250 \mathrm{~K}$ (Figure 14a,b) are noticeably larger than the distance of $10.07 \AA$ in 3 at $300 \mathrm{~K}$. Owing to the increased interfullerene distance in 4 , the average overlap integrals are smaller than those of Layer $A$ in 3 , giving rise to localized nature of spins (Mott insulator) in $\mathbf{4}$ with distorted triangular spin lattice.

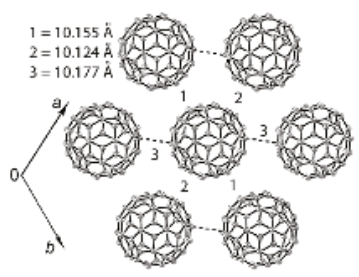

(a)

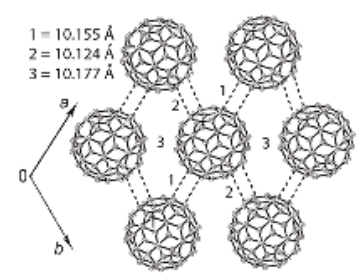

(b)

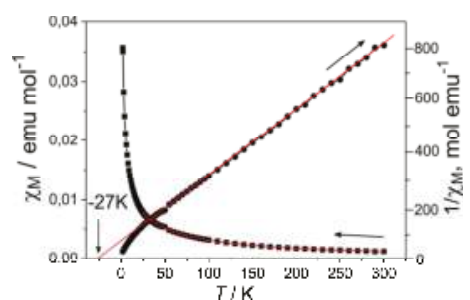

(c)

Figure 14. Packing of $\mathrm{C}_{60}{ }^{\bullet-}$ at $250 \mathrm{~K}$ and magnetic behavior of $\left(\mathrm{TPC}^{0}\right)\left(\mathrm{MQ}^{+}\right)\left(\mathrm{C}_{60}{ }^{\bullet-}\right)$ [12]: (a) Packing pattern of $\mathrm{C}_{60}$ in Layer $A$ in the $a b$ plane; (b) Packing pattern of $C_{60}$ in Layer $B$ in the $a b$ plane. Van der Waals C ...C contacts shorter than $3.42 \AA$ are shown by dashed lines. Numbers $1-3$ indicate the center-to-center distances between $\mathrm{C}_{60}{ }^{-}$; (c) Temperature dependence of molar magnetic susceptibility and reciprocal molar magnetic susceptibility of $\left(\mathrm{TPC}^{0}\right)\left(\mathrm{MQ}^{+}\right)\left(\mathrm{C}_{60}{ }^{--}\right)$. Red curve shows the fitting of the molar magnetic susceptibility data in the $50-300 \mathrm{~K}$ by the Curie-Weiss law with Weiss temperature of $-27 \mathrm{~K}$.

The calculated overlap integrals at $100 \mathrm{~K}$ are $s=0.78 \times 10^{-3}(/ / a), 1.82 \times 10^{-3}(/ / b)$, and 2.24 $\times 10^{-3}(/ / a+b)$ for Layer $A$ and $2.81 \times 10^{-3}(/ / a), 1.97 \times 10^{-3}(/ / b)$, and $1.51 \times 10^{-3}(/ / a+b)$ for Layer B. The calculated bandwidths are 0.103 (0.112) and $0.097(0.113) \mathrm{eV}$ for Layer $A$ and Layer $B$ at $250 \mathrm{~K}(100 \mathrm{~K})$, respectively. Similar to 3 , the ratios $2 t_{\mathrm{b}} /\left(t_{\mathrm{a}}+t_{\mathrm{a}+\mathrm{b}}\right)$ and $2\left(t_{\mathrm{a}}+t_{\mathrm{b}}\right) /\left(t_{\mathrm{a}}+t_{\mathrm{b}}\right)$ are 0.85 and 1.17 for Layer $A$ and 0.91 and 0.64 for Layer $B$ at $100 \mathrm{~K}$. Since the calculated Fermi surface shows 1D properties, it is more appropriate to use $2 t_{\mathrm{a}} /\left(t_{\mathrm{a}}+t_{\mathrm{a}+\mathrm{b}}\right)$ instead of $2 t_{\mathrm{b}} /\left(t_{\mathrm{a}}+t_{\mathrm{a}+\mathrm{b}}\right)$ and $2 t_{\mathrm{a}+\mathrm{b}} /\left(t_{\mathrm{a}}+t_{\mathrm{b}}\right)$. The calculated anisotropy of the transfer interactions at $250 \mathrm{~K}$ is $t_{\mathrm{a}}: t_{\mathrm{b}}: t_{\mathrm{a}+\mathrm{b}}=1.04: 1: 1\left(t^{\prime} / t=1.04\right)$ and 1:1.40:1.23 $\left(t^{\prime} / t=0.76\right)$ for Layer $A$ and Layer $B$, respectively. The anisotropy changed to 1:0.90:1.11 $\left(t^{\prime} / t=0.99\right)$ for Layer $A$ and 1:0.70:0.54 $\left(t^{\prime} / t=1.61\right)$ for Layer $B$ at $100 \mathrm{~K}$. The anisotropy of Layer $A$ is close to that of $\kappa-(\mathrm{ET})_{2} \mathrm{Cu}_{2}(\mathrm{CN})_{3}$, and the geometrical spin frustration is comparable to that of 3 . 
The relatively large distances between $\mathrm{C}_{60}{ }^{\circ-}$ prevent their dimerization but allow for the manifestation of a magnetic interaction between them. Reciprocal molar magnetic susceptibility is described well by the Curie-Weiss law in the $30-300 \mathrm{~K}$ range with negative Weiss temperature of $\Theta_{\mathrm{CW}}=-27 \mathrm{~K}$ (Figure 14c), indicating AF interaction of spins in the fullerene layers. The $\left|\Theta_{\mathrm{CW}}\right|$ is small, owing to the weaker AF interactions than that in 3 because of the larger interfullerene distance. In spite of the strong AF interaction of spins, magnetic ordering is not observed down to $1.9 \mathrm{~K}$ in this distorted triangular spin lattice system $(f>14)$. The resistivity measurements were impossible owing to small size of the crystals.

In summary, concerning the geometry of spin lattice of 4 , the $\mathrm{C}_{60}{ }^{\bullet-}$ molecules form hexagonal stacking according to the geometry of cationic template $\left(\mathrm{TPC}^{0}\right)\left(\mathrm{MQ}^{+}\right)$by key-keyhole relation similar to that for 3 . There are two fulleride layers and both have distorted hexagonal arrangement of $\mathrm{C}_{60}{ }^{\bullet-}$ with $t^{\prime} / t=0.99$ for Layer $A$ and $t^{\prime} / t=1.61$ for Layer B. Owing to large $r(10.12-10.18 \AA$ at $250 \mathrm{~K})$, small $\left|\Theta_{\mathrm{CW}}\right|(27 \mathrm{~K})$ is estimated in the range of $30-300 \mathrm{~K}$. No dimerization of $\mathrm{C}_{60} 0^{\circ}$ occurred down to $1.9 \mathrm{~K}$ $(f>14)$.

4.1.3. Distorted Hexagonal Packing of $\mathrm{C}_{60}{ }^{\bullet}-$ in $\left(\mathrm{PhCN}^{0}\right)\left(\mathrm{TMP}^{+}\right)\left(\mathrm{C}_{60}{ }^{--}\right),\left(\mathrm{PhCN}^{0}\right)\left(\mathrm{Ph}_{3} \mathrm{MeP}^{+}\right)\left(\mathrm{C}_{60}{ }^{\bullet-}\right)$, and $\left(\mathrm{PhCl}_{2}^{0}\right)\left[\left(\mathrm{Ph}_{3} \mathrm{P}\right)_{3} \mathrm{Au}^{+}\right]_{2}\left(\mathrm{C}_{60}{ }^{-}\right)_{2}\left(\mathrm{C}_{60}\right)$

\section{$\left(\mathrm{PhCN}^{0}\right)\left(\mathrm{TMP}^{+}\right)\left(\mathrm{C}_{60}{ }^{\bullet-}\right)$}

Single crystals of the three-component salt $\left(\mathrm{PhCN}^{0}\right)\left(\mathrm{TMP}^{+}\right)\left(\mathrm{C}_{60}{ }^{--}\right)(5)$ were unintentionally obtained, where $\mathrm{TMP}^{+}$is $N, N, N^{\prime}$-trimethylpiperazinium cation $\left(\mathrm{D}_{2}{ }^{+}\right)$and solvent molecule $\mathrm{PhCN}$ is neutral $\left(\mathrm{D}_{1}{ }^{0}\right)$ [148]. The crystal structure was solved at $120 \mathrm{~K}$ and $90 \mathrm{~K}$. The $\mathrm{C}_{60}$ molecules are disordered at $120 \mathrm{~K}$ and ordered at $90 \mathrm{~K}$ with trebling of the unit cell, which made the calculation of band parameters difficult.

The $2 \mathrm{D} \mathrm{C}_{60}{ }^{--}$hexagonal layer in 5 is sandwiched between the layers composed of $\left(\mathrm{PhCN}^{0}\right)\left(\mathrm{TMP}^{+}\right)$ (Figure 15a). Since the components molecules $\mathrm{D}_{1}$ and $\mathrm{D}_{2}$ do not have threefold symmetry, the $\mathrm{C}_{60}{ }^{\circ-}$ layer shows distorted hexagonal packing (Figure 15b). Further, the $\mathrm{TMP}^{+}$cations form pairs and are deeply embedded in the $\mathrm{C}_{60} 0^{--}$layers to deform the $\mathrm{C}_{60}{ }^{\circ-}$ packing and are found near the center of the hexagonally arranged $\mathrm{C}_{60}{ }^{\circ-}$ molecules. Methyl groups of $\mathrm{TMP}^{+}$and $\mathrm{PhCN}$ molecules work to prevent the close approach of $\mathrm{C}_{60} 0^{\bullet-}$ molecules and no bond-formation between $\mathrm{C}_{60}{ }^{\bullet}$ - was detected down to $2 \mathrm{~K}$. The shortest $r$ value along the interlayer direction is $10.39 \AA$ at $120 \mathrm{~K}$, indicating weak interlayer interactions, where the spin lattice should be 2D.

Only three types of interfullerene interactions with different $r(9.90,10.20$, and $10.43 \AA$ at $120 \mathrm{~K})$ are essential within the $C_{60}$ layer and the ratio of the transfer interactions are $t_{1}: t_{2}: t_{3}=0.9: 1: 0.5$ for the major $\mathrm{C}_{60}$ orientation. In spite of the hexagonal environment of the fullerenes in the layers, vdW C ... $\mathrm{C}$ contacts are formed with only three fullerene neighbors. Therefore, the geometry of model spin lattice is not triangular owing to weak magnetic interaction shown by dashed green lines (for $r=10.43 \AA$, $s_{3}=0.66 \times 10^{-3}$ ) in Figure 15c. Blue (for $r=9.90 \AA, s_{1}=1.25 \times 10^{-3}$ ) and red (for $r=10.20 \AA, s_{2}=1.41$ $\times 10^{-3}$ ) lines represent the main interactions $J_{1}: J_{2}: J_{3}=0.79: 1: 0.22$. Even though the $r$ value for blue line is much shorter than that of the red line, $s_{2}$ is larger than $s_{1}$ owing to more favorable orientation of $\mathrm{C}_{60}$ for $s_{2}$. The main magnetic interactions indicated by red lines extend along the $b$ axis, and such 1D zigzag magnetic chains are connected by magnetic interactions along the $c$ axis by blue lines. The unit of the spin lattice is edge-shared hexagonal, which is composed of four red lines and two blue lines (Figure 15c) and forms a 2D layer.

The temperature dependence of the reciprocal magnetic susceptibility for salt $\mathbf{5}$ is linear in the $70-300 \mathrm{~K}$ range with $\Theta_{\mathrm{CW}}=-11 \mathrm{~K}\left(f=5.5\right.$, no dimerization of $\mathrm{C}_{60}{ }^{\bullet-}$ occurred $)$. The resistivity at $\mathrm{RT}$ is $\rho=7 \times 10^{7} \Omega \cdot \mathrm{cm}$. 


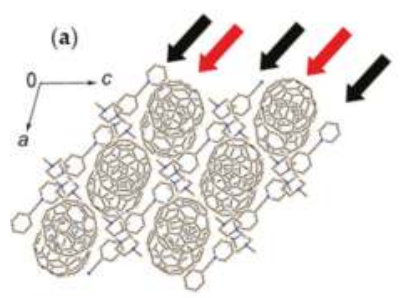

(c)

(b)
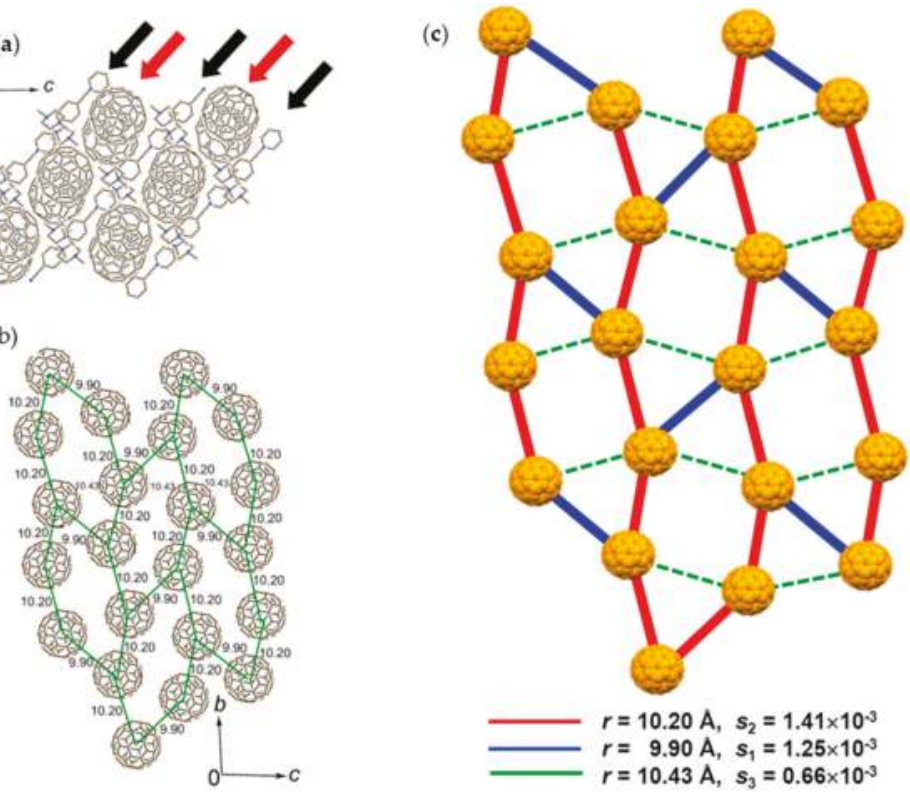

Figure 15. (a) Crystal structure of $\left(\mathrm{PhCN}^{0}\right)\left(\mathrm{TMP}^{+}\right)\left(\mathrm{C}_{60}{ }^{\bullet-}\right)$ at $90 \mathrm{~K}$ viewed along the $b$ axis. Black and red arrows indicate the layer of $\left(\mathrm{PhCN}^{0}\right)\left(\mathrm{TMP}^{+}\right)$and $\left(\mathrm{C}_{60}{ }^{\bullet-}\right)$, respectively; (b) View of the $\mathrm{C}_{60}$ layer at $120 \mathrm{~K}$ showing the $r \leq 10.20 \AA$ by green lines; (c) Possible spin lattice geometry corresponding to (b) with overlap integrals; red line: $s_{2}=1.41 \times 10^{-3}(r=10.20 \AA)$, blue line: $s_{1}=1.25 \times 10^{-3}(r=9.90 \AA)$ and green line: $s_{3}=0.66 \times 10^{-3}(r=10.43 \AA)$ giving $J_{1}: J_{2}: J_{3}=0.79: 1: 0.22$ for the major $\mathrm{C}_{60}$ orientation. Only one $\mathrm{C}_{60}{ }^{\bullet-}$ orientation is shown in (c). (a,b) were reproduced from [148].

$\left(\mathrm{PhCN}^{0}\right)\left(\mathrm{Ph}_{3} \mathrm{MeP}^{+}\right)\left(\mathrm{C}_{60} 0^{-}\right)$

Insoluble precipitates obtained by the reduction of $\mathrm{C}_{60}$ with sodium fluorenone ketyl in $\mathrm{PhCl}_{2}$ in the presence of $\mathrm{Ph}_{3} \mathrm{PMeBr}$ were dissolved by the addition of $\mathrm{PhCN}$. $n$-Hexane was layered on the filtered solution to grow single crystals of $\left(\mathrm{PhCN}^{0}\right)\left(\mathrm{Ph}_{3} \mathrm{MeP}^{+}\right)\left(\mathrm{C}_{60}{ }^{\bullet-}\right)(6)$ where $\mathrm{Ph}_{3} \mathrm{MeP}^{+}$has a threefold symmetry [105]. The crystal structure at $250 \mathrm{~K}$ (Figure 16) shows hexagonal packing of $\mathrm{C}_{60}{ }^{\bullet-}$ and supramolecules $\left(\mathrm{PhCN}^{0}\right)\left(\mathrm{Ph}_{3} \mathrm{PMe}^{+}\right)$are located in the centers of fullerene hexagon.

Hence, $\mathrm{C}_{60}{ }^{--}$has only three negatively charged fullerene neighbors similar to that observed in 5 (Figure 15b). The somewhat low value of $r=9.92,9.96$, and $10.07 \AA$ may result in higher spin frustration than for 5 . The lowest interlayer $r$ is $10.15 \AA$ suggesting strong 2D nature within the $\mathrm{C}_{60}$ layer in Figure 16. The $\mathrm{C}_{60}{ }^{--}$molecules $\mathrm{a}_{1}-\mathrm{a}_{4}$ in Figure 16 form a flat layer, where the overlap integrals are $s\left(\mathrm{a}_{1}-\mathrm{a}_{2}\right)=2.14 \times 10^{-3}, s\left(\mathrm{a}_{1}-\mathrm{a}_{3}\right)=2.29 \times 10^{-3}, s\left(\mathrm{a}_{1}-\mathrm{a}_{4}\right)=0.70 \times 10^{-3}$, and $s\left(\mathrm{a}_{2}-\mathrm{a}_{3}\right) \sim s\left(\mathrm{a}_{3}-\mathrm{a}_{4}\right)$ $\sim s\left(\mathrm{a}_{2}-\mathrm{a}_{4}\right) \sim s\left(\mathrm{a}_{1}-\mathrm{a}_{1^{\prime}}\right)=0$. The spin lattice geometry (Figure 16b) is approximated as the 1D nonuniform zigzag chain along the $b$ axis with alternating red and blue lines and the lines are connected by weak magnetic interactions by green lines (ratio of $J$ values $=1: 0.87: 0.09$ ). Salt $\mathbf{6}$ shows much stronger 1D properties than those of salt 5 .

The EPR intensity decreases from 295-220 K, smoothly followed by a rapid decrease due to reversible dimerization of $\mathrm{C}_{60} 0^{-}$below $220 \mathrm{~K}$. Upon cooling down to $120 \mathrm{~K}$, the $\mathrm{C}_{60}{ }^{\bullet}$ - radical anion pairs, which has $r=9.92 \AA$ at $250 \mathrm{~K}$, form singly bonded $\left(\mathrm{C}_{60}{ }^{-}\right)_{2}$ dimers with $r=9.28 \AA$. Therefore, no $\Theta_{\mathrm{CW}}$ value is determined in this system. The steric protection to avoid the bond-formation is not sufficient in this solid. The solvent free crystal $\left(\mathrm{Ph}_{3} \mathrm{MeP}^{+}\right)\left(\mathrm{C}_{60}{ }^{--}\right)(2)$ exhibits completely different structural and physical properties (vide infra) without the dimerization down to $1.9 \mathrm{~K}$. 


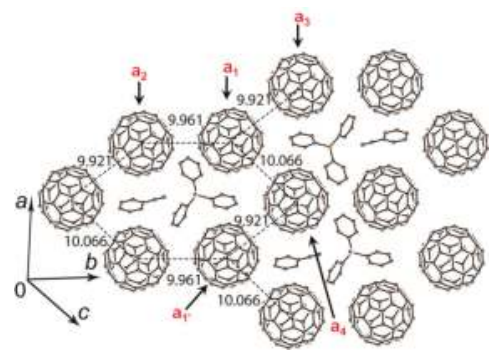

(a)

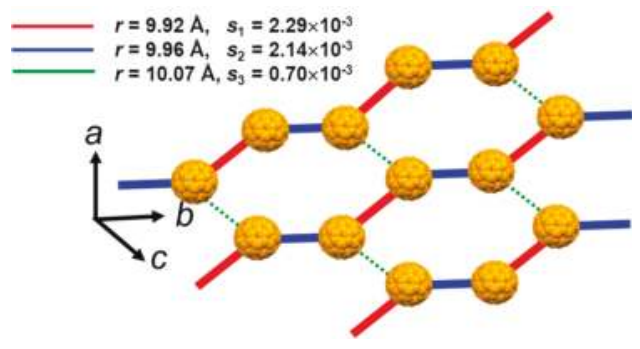

(b)

Figure 16. (a) Crystal structure of $\left(\mathrm{PhCN}^{0}\right)\left(\mathrm{Ph}_{3} \mathrm{MeP}^{+}\right)\left(\mathrm{C}_{60}{ }^{--}\right)$at $250 \mathrm{~K}$. Four $\mathrm{C}_{60}{ }^{\bullet-}$ molecules $\left(\mathbf{a}_{1}-\mathbf{a}_{4}\right)$ form a flat layer [105]; (b) Schematic of possible spin lattice with magnetic interactions with overlap integrals $s_{1}=2.29 \times 10^{-3}($ red, $r=9.921 \AA)>s_{2}=2.14 \times 10^{-3}$ (blue, $r=9.961 \AA$ ) $>s_{3}=0.70 \times 10^{-3}$ (green, $r=10.066 \AA$ ), giving $J_{1}: J_{2}: J_{3}=1: 0.87: 0.09$. Only one major orientation is shown for $\mathrm{C}_{60}{ }^{\bullet-}$ in $(\mathbf{a}, \mathbf{b}$ ).

$\left(\mathrm{PhCl}_{2}{ }^{0}\right)\left\{\left(\mathrm{Ph}_{3} \mathrm{P}\right)_{3} \mathrm{Au}^{+}\right\}_{2}\left(\mathrm{C}_{60} 0^{--}\right)_{2}\left(\mathrm{C}_{60}\right)$

By using a very bulky cation $\left(\mathrm{Ph}_{3} \mathrm{P}\right)_{3} \mathrm{Au}^{+}$with $\mathrm{C}_{3 \mathrm{v}}$ symmetry, single crystals of $\left(\mathrm{PhCl}_{2}{ }^{0}\right)$ $\left\{\left(\mathrm{Ph}_{3} \mathrm{P}\right)_{3} \mathrm{Au}^{+}\right\}_{2}\left(\mathrm{C}_{60}{ }^{\bullet-}\right)_{2}\left(\mathrm{C}_{60}\right)$ (7) with a highly symmetric trigonal lattice were obtained, in which the supramolecule $\left\{\left(\mathrm{PhCl}_{2}{ }^{0}\right)\left[\left(\mathrm{Ph}_{3} \mathrm{P}\right)_{3} \mathrm{Au}^{+}\right]_{2}\right\}$ is a cationic template that accommodates $\mathrm{C}_{60}$ molecules hexagonally [149]. The crystal structure was solved for a crystal slowly cooled down to $100 \mathrm{~K}$.

Hexagonal corrugated $\mathrm{C}_{60}$ layer is sandwiched between the layers of $\left\{\left(\mathrm{PhCl}_{2}{ }^{0}\right)\left[\left(\mathrm{Ph}_{3} \mathrm{P}\right)_{3} \mathrm{Au}^{+}\right]_{2}\right\}$ along the $c$ axis (Figure 17a).

Fullerenes and $\mathrm{PhCl}_{2}$ molecules located on the $C_{3 \mathrm{v}}$ symmetry axes are statistically disordered between three orientations. The $\left(\mathrm{Ph}_{3} \mathrm{P}\right)_{3} \mathrm{Au}^{+}$cations are ordered and located on the $C_{3 \mathrm{v}}$ symmetry axis. The $\left(\mathrm{Ph}_{3} \mathrm{P}\right)_{3} \mathrm{Au}^{+}$cations are too large in size to fit into the size of a $\mathrm{C}_{60}$ molecule, but the size of supramolecule $\left\{\left(\mathrm{PhCl}_{2}{ }^{0}\right)\left[\left(\mathrm{Ph}_{3} \mathrm{P}\right)_{3} \mathrm{Au}^{+}\right]_{2}\right\}$ approximately corresponds to that of three $\mathrm{C}_{60}$ molecules. The $\mathrm{C}_{60}$ layer and $\left\{\left(\mathrm{PhCl}_{2}{ }^{0}\right)\left[\left(\mathrm{Ph}_{3} \mathrm{P}\right)_{3} \mathrm{Au}^{+}\right]_{2}\right\}$ layer, which is indicated by black arrows in Figure $17 \mathrm{~b}$, alternate along the $c$ axis. $\mathrm{C}_{60}$ molecules move from the layers toward planar $\mathrm{PhCl}_{2}$ molecules to form strongly corrugated $\mathrm{C}_{60}$ layers.

The fullerene layer consists of different charged $\mathrm{C}_{60}$ molecules with -1 and 0 denoted as $I$ and II, respectively, in Figure 17a-c). Interestingly, $\mathrm{C}_{60}{ }^{\circ-}$ molecules are sandwiched between a $\left(\mathrm{Ph}_{3} \mathrm{P}\right)_{3} \mathrm{Au}^{+}$ cation molecule and a $\mathrm{PhCl}_{2}$ molecule while $\mathrm{C}_{60}{ }^{0}$ molecules are sandwiched between two $\left(\mathrm{Ph}_{3} \mathrm{P}\right)_{3} \mathrm{Au}^{+}$ molecules along the $c$ axis, as shown in Figure $17 \mathrm{~b}$. Negatively charged and neutral $\mathrm{C}_{60}$ molecules are closely packed within hexagonal layers with $r(I \cdots I I)=10.02 \AA$, while between $\mathrm{C}_{60^{\circ}}{ }^{-}$, it is long with $r(I \cdots I)=10.37 \AA$ due to corrugation. The magnetic interactions between $\mathrm{C}_{60}{ }^{\circ-}$ molecules in the neighboring fullerene layers are expected to be small based on its $r$ value $(r \sim 13.9 \AA)$. As a result, the magnetic interactions are $2 \mathrm{D}$. Each $\mathrm{C}_{60}{ }^{\circ-}$ has only three negatively charged fullerene neighbors within a fullerene layer, namely $\mathrm{C}_{60}{ }^{\circ-}$ molecule $\mathrm{a}_{1}$ is surrounded by $\mathrm{C}_{60} 0^{-}$- molecules $\mathrm{a}_{2}-\mathrm{a}_{4}$ in Figure 17c. They form distorted tetrahedral spin lattice composed of $\mathrm{C}_{60} 0^{-{ }^{-}}$molecules $\mathrm{a}_{1}-\mathrm{a}_{4}$ (red lines in Figure 17d). The $\mathrm{a}_{1}$ molecule projects out of the $\mathrm{a}_{2}-\mathrm{a}_{4}$ plane in Figure $17 \mathrm{~d}$ by only $3.03 \AA$. The tetrahedral units are arranged in the $2 \mathrm{D}$ plane by apex-sharing. The overlap integrals have not been obtained due to severe disorder of fullerene molecules. Owing to the very large center-to-center distance between the $\mathrm{C}_{60} 0^{\circ}$ molecules, the $\mathrm{AF}$ interaction is weak $\left(\Theta_{\mathrm{CW}}=-5 \mathrm{~K}, f=2.6\right)$. In order to enhance the magnetic interactions, smaller sized cationic supramolecules than $\left[\left(\mathrm{PhCl}_{2}{ }^{0}\right)\left\{\left(\mathrm{Ph}_{3} \mathrm{P}\right)_{3} \mathrm{Au}^{+}\right\}_{2}\right]$ well matched with two $\mathrm{C}_{60}$ molecules would be preferable. The resistivity at RT is approximately $\rho=4$ $\times 10^{5} \Omega \cdot \mathrm{cm}$. EPR measurements confirmed no dimerization of $\mathrm{C}_{60} 0^{-}$down to $4.2 \mathrm{~K}$. 


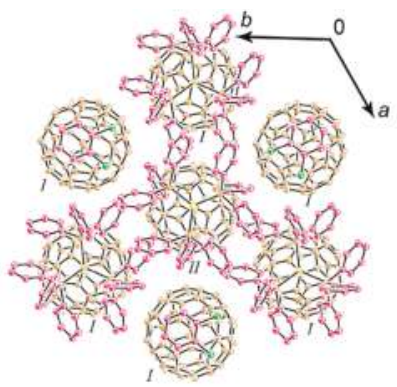

(a)

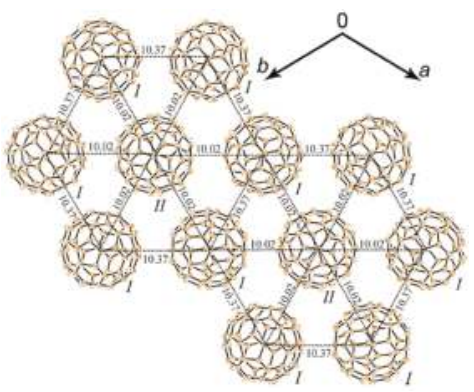

(c)

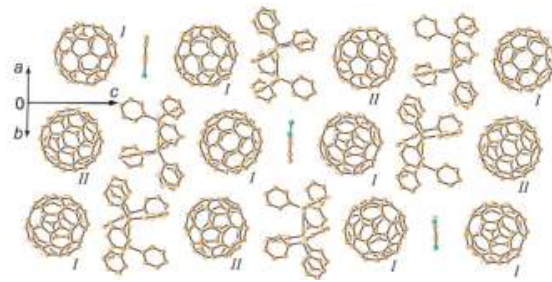

(b)

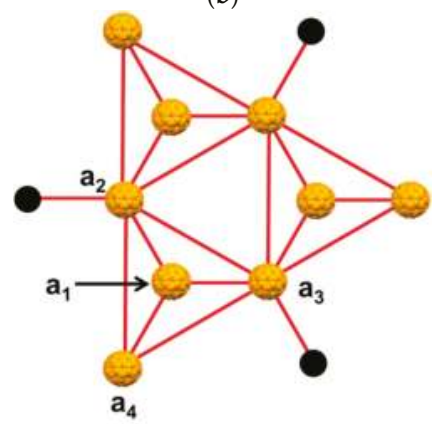

(d)

Figure 17. (a) Crystal structure of $\left(\mathrm{PhCl}_{2}^{0}\right)\left[\left(\mathrm{Ph}_{3} \mathrm{P}\right)_{3} \mathrm{Au}^{+}\right]_{2}\left(\mathrm{C}_{60}{ }^{\bullet-}\right)_{2}\left(\mathrm{C}_{60}\right)$ at $100 \mathrm{~K}$ viewed along the $c$ axis (in the cationic supramolecule, $\mathrm{C}$ : red-violet, $\mathrm{Cl}$ : green, $\mathrm{P}$ : orange, Au: yellow). Only one of three orientations is shown for $\mathrm{C}_{60}$ and $\mathrm{PhCl}_{2}$ molecules. I: $\mathrm{C}_{60}{ }^{\bullet-}, \mathrm{II}: \mathrm{C}_{60}{ }^{0}$; (b) View along hexagonal $\mathrm{C}_{60}$ layers and the diagonal of the $a b$ plane. Black arrows indicate the $\left(\mathrm{PhCl}_{2}{ }^{0}\right)\left[\left(\mathrm{Ph}_{3} \mathrm{P}\right)_{3} \mathrm{Au}^{+}\right]_{2}$ supramolecule layer $\left(r_{\mathrm{a}}=10.37 \AA, r_{\mathrm{b}} \sim 13.92 \AA\right)$; (c) View of the $\mathrm{C}_{60}$ layer at $100 \mathrm{~K}$ for $\left(\mathrm{PhCl}_{2}{ }^{0}\right)\left[\left(\mathrm{Ph}_{3} \mathrm{P}\right)_{3} \mathrm{Au}^{+}\right]_{2}\left(\mathrm{C}_{60}{ }^{\bullet-}\right)_{2}\left(\mathrm{C}_{60}\right)$ showing $r$ in $\AA$. Black dashed lines link $\mathrm{C}_{60}$ centers. Four $\mathrm{C}_{60}{ }^{\bullet-}$ molecules $\left(\mathrm{a}_{1}-\mathrm{a}_{4}\right)$ form tetrahedral spin lattice with $r=10.37 \AA$ and the $\mathrm{a}_{1}$ molecule projects out of the a2-4 plane by $3.03 \AA$; (d) Possible spin lattice geometry (red lines). Only one orientation of $\mathrm{C}_{60}{ }^{\bullet-}$ is shown. Black ball is $\mathrm{C}_{60}{ }^{\bullet-}$. (a-c) were reproduced from [149].

\subsubsection{Frustrated Spins in 3D Hexagonal Packing of $\mathrm{C}_{60}{ }^{\bullet-}$ in $\left(\mathrm{DMI}^{+}\right)_{3}\left(\mathrm{C}_{60} 0^{--}\right)\left(\mathrm{I}^{-}\right)_{2}$}

Hexagonal packing of $\mathrm{C}_{60^{\bullet-}}$ molecules is also formed in $\left(\mathrm{DMI}^{+}\right)_{3}\left(\mathrm{C}_{60^{\circ-}}\right)\left(\mathrm{I}^{-}\right)_{2}(\mathbf{8})$ [145], where cationic template is delivered by a three-dimensional (3D) network of $\left[\left(\mathrm{DMI}^{+}\right)_{3}\left(\mathrm{I}^{-}\right)_{2}\right]($ Figure 18), where $\mathrm{DMI}^{+}$is $N, N^{\prime}$-dimethylimidazolium cation with no threefold symmetry. This system is not within the $\left(\mathrm{D}_{1}^{0}\right)\left(\mathrm{D}_{2}{ }^{+}\right)\left(\mathrm{C}_{60}{ }^{--}\right)$scheme, but the supramolecular cationic template $\left[\left(\mathrm{DMI}^{+}\right)_{3}\left(\mathrm{I}^{-}\right)_{2}\right]$ has threefold symmetry.

The single crystals were obtained by a diffusion method. $\mathrm{C}_{60}$, an excess of DMI-I and reductant $\mathrm{CH}_{3} \mathrm{CH}_{2} \mathrm{SNa}$ were stirred in a $\mathrm{PhCl}_{2} / \mathrm{PhCN}$ mixture. The mixture was cooled and $n$-hexane was layered over the solution. The diffusion was carried out during one month to give the single crystals on the wall of the tube with the size of $1 \times 1 \times 0.5 \mathrm{~mm}^{3}$.

The 3D network of $\left[\left(\mathrm{DMI}^{+}\right)_{3}\left(\mathrm{I}^{-}\right)_{2}\right]$ is held together by the hydrogen $(\mathrm{H})$-bonds between the $\mathrm{H}$ atoms of $\mathrm{DMI}^{+}$cation (Figure 18a) and $\mathrm{I}^{-}$anion. The crystal structures at $100 \mathrm{~K}$ are shown in Figure 18b,c. The $\mathrm{H}$ atoms at 3- and 4-positions of $\mathrm{DMI}^{+}$have $\mathrm{H}$-bonds with one kind of $\mathrm{I}^{-}$anion, denoted as $\mathrm{I}_{B}{ }^{-}$. One $\mathrm{I}_{B}{ }^{-}$anion is surrounded by six DMI ${ }^{+}$molecules; namely, $\mathrm{I}_{B}{ }^{-}$anion in red color in Figure 18b,c has H-bonds with six $\mathrm{H}$ atoms at 4-position (three up and three down along the $c$ axis, see Figure 18c). The supramolecular unit composed of three $\mathrm{DMI}^{+}$molecules and two $\mathrm{I}_{\mathrm{B}}{ }^{-}$anion molecules; Supramolecule $9\left[\left(\mathrm{DMI}^{+}\right)_{3}\left(\mathrm{I}_{\mathrm{B}}{ }^{-}\right)_{2}\right]$, which corresponds to the unit encircled by 
magenta dotted circle in Figure $18 b, c$, has a similar threefold symmetry like TPC molecule as shown in Figure 18d. Three Supramolecular 9 units encircle one $\mathrm{C}_{60} 0^{--}$molecules to form Supramolecule 11 $\left[\left(\mathrm{DMI}^{+}\right)_{3}\left(\mathrm{I}_{\mathrm{B}}{ }^{-}\right)_{2}\right]_{3}\left(\mathrm{C}_{60}{ }^{\bullet-}\right)$ which is schematically seen in Figure $18 \mathrm{f}$ where magenta figure $\mathrm{Y}$ represents Supramolecule 9 and $\mathrm{B}$ represents an $\mathrm{I}_{\mathrm{B}}{ }^{-}$anion.

(a)

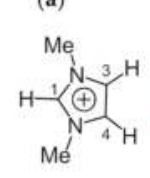

(b)

(f)

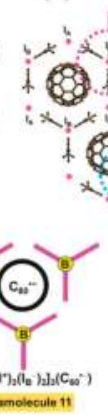

(d)

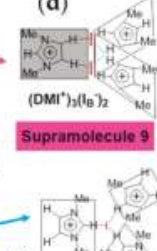

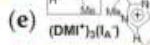
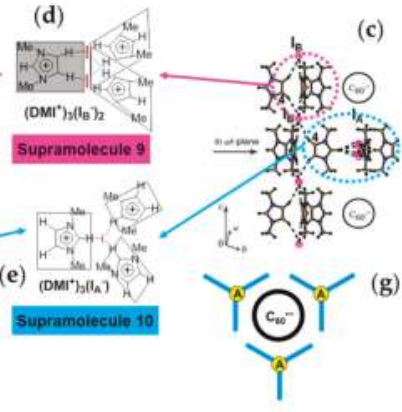

Supramaibcule 12

(h-3) (h-2)

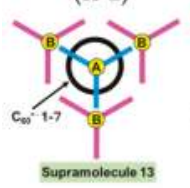

$(\mathrm{h}-4)$

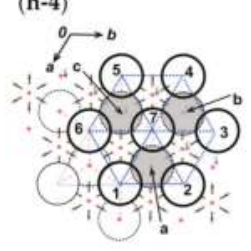

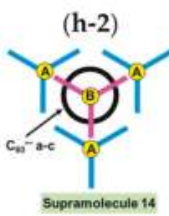

ans

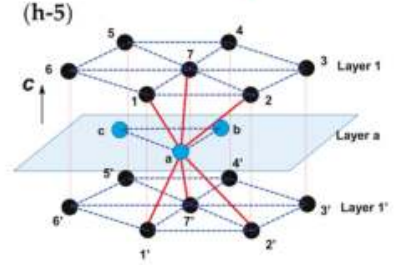

Figure 18. (a) DMI ${ }^{+}$molecule; (b) View of the honeycomb $\mathrm{DMI}^{+} \mathrm{I}^{-}$layer in the $a b$ plane of $\left(\mathrm{DMI}^{+}\right)_{3}$ $\left(\mathrm{C}_{60^{\circ}}{ }^{-}\right)\left(\mathrm{I}^{-}\right)_{2}$ at $100 \mathrm{~K}$; (c) The formation of the H-bonds (dashed lines) between $\mathrm{H}$ atoms of $\mathrm{DMI}^{+}$and $\mathrm{I}^{-}\left(\mathrm{I}_{\mathrm{A}}\right.$ and $\left.\mathrm{I}_{\mathrm{B}}\right)$ in the $a b$ plane and along the $c$ axis. The positions of $\mathrm{C}_{60^{\circ}}{ }^{-}$are shown by black circles; (d) The supramolecular unit $\left(\mathrm{DMI}^{+}\right)_{3}\left(\mathrm{I}_{\mathrm{B}}{ }^{-}\right)_{2}$ (Supramolecule 9) within the $a b$ plane which is indicated by magenta circle in $(\mathbf{b}, \mathbf{c})$ and has a threefold symmetry like TPC; (e) The supramolecular unit $\left(\mathrm{DMI}^{+}\right)_{3}\left(\mathrm{I}_{\mathrm{A}}^{-}\right)$(Supramolecule 10) within the $a b$ plane which is indicated by blue circle in $(\mathbf{b}, \mathbf{c})$ and has a threefold symmetry like TPC; (f) Three units of Supramolecule 9 (magenta figure $\mathrm{Y}$ ) form a wall to enclose one $\mathrm{C}_{60}{ }^{\circ}$ to form Supramolecule 11, where B represents an $\mathrm{I}_{\mathrm{B}}{ }^{-}$anion; (g) Three units of Supramolecule 10 (blue figure $\mathrm{Y}$ ) form a wall to enclose one $\mathrm{C}_{60}{ }^{\circ-}$ (Supramolecule 12), where $A$ represents an $\mathrm{I}_{\mathrm{A}}{ }^{-}$anion; (h-1-h-4) Supramolecules 11 and 12 are capped along the $c$ axis by Supramolecules 10 and $\mathbf{9}$, respectively, to form Supramolecule $13\left[\left(\mathrm{DMI}^{+}\right)_{3}\left(\mathrm{I}^{-}\right)_{3}\right]_{3}\left[\left(\mathrm{DMI}^{+}\right)_{3}\left(\mathrm{I}^{-}\right)\right]\left(\mathrm{C}_{60}{ }^{-}\right)$(h-1) and Supramolecule 14 $\left[\left(\mathrm{DMI}^{+}\right)_{3}\left(\mathrm{I}^{-}\right)\right]_{3}\left[\left(\mathrm{DMI}^{+}\right)_{3}\left(\mathrm{I}^{-}\right)_{3}\right]\left(\mathrm{C}_{60}{ }^{\bullet-}\right)(\mathbf{h}-2)$. Their combination leads to a crystal of $\left(\mathrm{DMI}^{+}\right)_{3}\left(\mathrm{C}_{60}{ }^{\bullet-}\right)\left(\mathrm{I}^{-}\right)_{2}$. Packing of units of Supramolecule 13 (including $\mathrm{C}_{60^{\circ}}{ }^{-}$molecules labeled 1, 2, and 7) above the unit of Supramolecule 14 (including $\mathrm{C}_{60} 0^{-}$molecule labeled a) leads to a crystal (h-3); (h-4) A schematic figure showing a layer (Layer 1) of hexagonal packing of $\mathrm{C}_{60} 0^{--}$molecules (labeled as 1-7) above a layer (Layer a) of the hexagonal packing of $\mathrm{C}_{60} 0^{\circ-}$ molecules (labeled as a-c) viewed along the $c$ axis; (h-5) shows alternating stacking of Layer 1, Layer a, and Layer $1^{\prime}$ along the $c$ axis with $r=11.05 \AA$ (red line) and $13.36 \AA ̊$ (blue dotted line). 
$\mathrm{H}$ atoms at 1-position have contacts with different kind of $\mathrm{I}^{-}$anions $\left(\mathrm{I}_{\mathrm{A}}^{-}\right)$, and three $\mathrm{DMI}^{+}$ molecules and one $\mathrm{I}_{\mathrm{A}}^{-}$anion molecule form another supramolecular unit $\left[\left(\mathrm{DMI}^{+}\right)_{3}\left(\mathrm{I}_{\mathrm{A}}{ }^{-}\right)\right]$in the $a b$ plane encircled by blue circle (Supramolecule 10, Figure 18e) in Figure 18b,c, which has also threefold symmetry like TPC. Similar to Supramolecule 11, one $\mathrm{C}_{60}{ }^{--}$molecule is surrounded by three Supramolecular 10 units $\left[\left(\mathrm{DMI}^{+}\right)_{3}\left(\mathrm{I}_{\mathrm{A}}{ }^{-}\right)\right]$in the $a b$ plane to form Supramolecule 12, where blue figure $\mathrm{Y}$ represents Supramolecule 10 and A represents an $\mathrm{I}_{\mathrm{A}}{ }^{-}$anion (Figure 18g).

The top and bottom of Supramolecule 11 are capped by Supramolecule 10 along the $c$ axis, yielding Supramolecule 13, [(Supramolecule 9) ${ }_{3}($ Supramolecule 10$)\left(\mathrm{C}_{60}{ }^{\bullet-}\right)$ ], as schematically shown in Figure 18(h-1), where $\mathrm{C}_{60^{\circ}}{ }^{-}$molecules labeled 1-7 form one layer (Layer 1). Similar capping occurs for Supramolecule 12 by Supramolecule 9 to form Supramolecule 14, [(Supramolecule 9) (Supramolecule 10) ${ }_{3}\left(\mathrm{C}_{60}{ }^{\circ-}\right)$ ], in Figure $18(\mathrm{~h}-2)$, where $\mathrm{C}_{60^{\circ}}{ }^{--}$molecules labeled a-c form one layer (Layer a). Two kinds of Supramolecule units $\mathbf{1 3}$ and $\mathbf{1 4}$ stack alternately along the $c$ axis to give the single crystal of 8 . Figure 18(h-3) schematically illustrates the molecular packing. In the crystal viewed along the $c$ axis (Figure 18(h-4,h-5)), four $\mathrm{C}_{60}{ }^{\circ-}$ molecules, namely three molecules from Layer 1 (drawn in black in Figure 18(h-5)) and one molecule from Layer a (drawn in blue in Figure 18(h-5)) form tetrahedral $[(1,2,7, \mathrm{a}),(3,4,7, \mathrm{~b})$ and $(5,6,7, \mathrm{c})]$ structure. The labeling of $\mathrm{C}_{60}{ }^{\bullet-}$ molecules is the same as that in Figure 18(h-1-h-4)) Center-to-center distances between $\mathrm{C}_{60}{ }^{\circ-}$ of $13.36 \AA$ are marked by blue dotted lines and those of $11.05 \AA$ (between blue and black circles) are marked by red lines in Figure 18(h-5).

As a result, geometry of the unit of model spin lattice is not triangular, but distorted tetragonal, i.e., tetrahedron with $t^{\prime} / t \sim 0$. The AF spin configuration is expected within a 1D Mott insulating $\mathrm{C}_{60}{ }^{\bullet-}$ chain of black-blue-black-blue- fullerenes along the $c$ axis, while within a layer of blue or black fullerenes in the $a b$ plane parallel spin configurations are expected. Such spin units form a column along the $c$ axis and the columns are arranged in the $b c$ plane (Figure 19a). The other spin unit composed of $\mathrm{C}_{60}{ }^{\circ-}$ molecules $[(3,4,7, \mathrm{~b})$ and $(5,6,7, \mathrm{c})]$ has the same distorted tetrahedral geometry and form similar 2D packing to that in Figure 19a. These 2D spin sheets are connected, for example, through 7 and $7^{\prime}$ to form 3D network of magnetic interactions. It is emphasized here that according to the 3D template network composed of $\mathrm{DMI}^{+}$and $\mathrm{I}^{-}$molecules, the $\mathrm{C}_{60}{ }^{\circ-}$ radical anions provide the $2 \mathrm{H}$-hexagonal 3D packing by a 3D key-keyhole relation between cation $\left[\left(\mathrm{DMI}^{+}\right)_{3}\left(\mathrm{I}^{-}\right)_{2}\right]^{+}$and anion $\mathrm{C}_{60}{ }^{\bullet-}$ molecules.

The geometry of the spin lattice of 8 is expected to be 3D apex sharing triangular bipyramid based on the $r$ values. We calculated overlap integrals based on the two main orientations of $\mathrm{C}_{60}$ molecules at $100 \mathrm{~K}$ and the mean square of each is $s=0.12 \times 10^{-3}$. Based on the overlap integrals, the model spin lattice is shown in Figure 19b, which are arranged in the $a b$ plane to form 3D apex sharing triangular bipyramid.

The effective magnetic moment of 8 is $1.64 \mu_{\mathrm{B}}$, slightly smaller than the value of $1.73 \mu_{\mathrm{B}}$ for the system containing one $S=1 / 2$ spin per formula unit. The Curie-Weiss temperature of $-9.6 \mathrm{~K}$ in the 40-300 K range indicates AF coupling of spins (Figure 19d). Effective magnetic moment also decreases below about $50 \mathrm{~K}$ (Figure 19e) due to AF coupling of spins. Because of the large interfullerene distance, $11.05 \AA$ at $100 \mathrm{~K}$, and small overlap integrals, the AF interaction is not strong. The long-range magnetic ordering is not observed down to $1.9 \mathrm{~K}$.

In summary, concerning the geometry of the spin lattice of $\mathbf{8}$, the cationic supramolecule $\left[\left(\mathrm{DMI}^{+}\right)_{3}\left(\mathrm{I}^{-}\right)_{2}\right]$ forms threefold assemblies that act as a template for the $\mathrm{C}_{60}{ }^{\bullet-}$ molecules to achieve hexagonal stacking of $\left(\mathrm{C}_{60}{ }^{\circ}-\right)$. The overlap integrals lead to a 3D apex sharing triangular bipyramid with $r=11.05 \AA$ at $100 \mathrm{~K}$. Owing to a large $r$, a small $\left|\Theta_{\mathrm{CW}}\right|(9.6 \mathrm{~K})$ is estimated in the range of 50-300 K. No dimerization of $\mathrm{C}_{60^{\circ}}{ }^{-}$takes place down to $1.9 \mathrm{~K}(f \sim 5)$. 


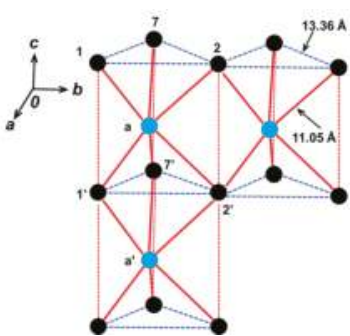

(a)

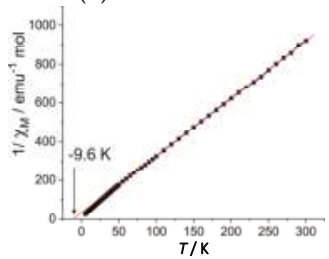

(d)
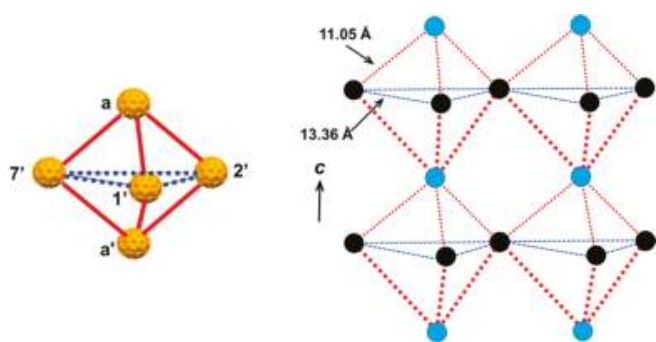

(c)

(b)

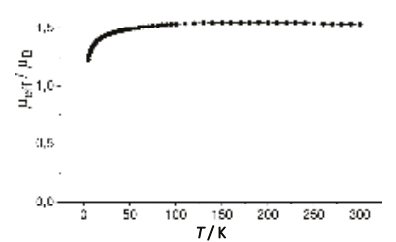

(e)

Figure 19. (a) Schematic figure of $2 \mathrm{H}$-hexagonal packing of $\mathrm{C}_{60} 0^{\bullet-}$ molecules in $\left(\mathrm{DMI}^{+}\right)_{3}\left(\mathrm{C}_{60}{ }^{\bullet-}\right)\left(\mathrm{I}^{-}\right)_{2}$ at $100 \mathrm{~K}$ showing apex-sharing bipyramid; (b) Model spin unit of triangular bipyramid with only one $\mathrm{C}_{60}{ }^{\bullet-}$ orientation. Labeling of $\mathrm{C}_{60}$ molecules correspond to those in Figure 18; (c) Model spin lattice based on overlap integrals; (d) Temperature dependence of reciprocal molar magnetic susceptibility of $\left(\mathrm{DMI}^{+}\right)_{3}\left(\mathrm{C}_{60}{ }^{\bullet-}\right)\left(\mathrm{I}^{-}\right)_{2}$. Red curve shows the fitting of the molar magnetic susceptibility data in the $50-300 \mathrm{~K}$ by the Curie-Weiss law with Weiss temperature of -9.6 K [145]; (e) Temperature dependence of magnetic moment of $\left(\mathrm{DMI}^{+}\right)_{3}\left(\mathrm{C}_{60^{\bullet}}{ }^{-}\right)\left(\mathrm{I}^{-}\right)_{2}$.

\subsection{Two-Component Materials}

\subsubsection{Frustrated Spins in 3D Corrugated Packing of $\mathrm{C}_{60^{\circ}}{ }^{--}$in $\left(\mathrm{MDABCO}^{+}\right)\left(\mathrm{C}_{60^{\circ}}{ }^{-}\right)$3D Spin Lattice}

3D close packing of $\mathrm{C}_{60^{\circ}}{ }^{-}$and high $\left|\Theta_{\mathrm{CW}}\right|$ are realized in the two-component CT solid of $\left(\mathrm{MDABCO}^{+}\right)\left(\mathrm{C}_{60}{ }^{--}\right)(\mathbf{1})$ where cation molecules $\mathrm{MDABCO}^{+}$show threefold symmetry [147]. Black block single crystals of $\mathbf{1}\left(\sim 0.4 \times 0.4 \times 0.5 \mathrm{~mm}^{3}\right)$ were obtained by the reduction of $\mathrm{C}_{60}$ with slight excess of strong reductant sodium fluorenone ketyl in $\mathrm{PhCl}_{2}$ in the presence of stoichiometric amount of MDABCO-I. Then, $n$-hexane was layered to precipitate $\mathrm{CT}$ solids. The crystal structures were solved at $250 \mathrm{~K}$ and $100 \mathrm{~K}$. At $250 \mathrm{~K}$, there is one independent $\mathrm{C}_{60}{ }^{\circ-}$ and one ordered $\mathrm{MDABCO}^{+}$molecules. The ordering of $\mathrm{C}_{60} 0^{-}$is observed below $160 \mathrm{~K}$, with a trebling of the unit cell $b$ axis.

The $\mathrm{C}_{60} 0^{-}$radical anions form square corrugated fullerene layers in the $b c$ plane and the $\mathrm{C}_{60}$ layers alternate with the $\mathrm{MDABCO}^{+}$cations along the $a$ direction (Figure $20 \mathrm{a}$ at $100 \mathrm{~K}$ ). 2D C60 layer in the $b c$ plane is corrugated along the $c$ axis (Figure $20 \mathrm{~b}$ at $100 \mathrm{~K}$ ). The corrugation of $\mathrm{C}_{60}$ layer is clearly shown in Figure 20c (at $100 \mathrm{~K}$ ) and $\mathrm{C}_{60}$ columns extend along the $b$ axis.

$\mathrm{A} \mathrm{C}_{60}{ }^{--}$molecule (drawn in red in Figure 20d at $250 \mathrm{~K}$, labeled as (A)) is surrounded by eight neighboring $\mathrm{C}_{60}{ }^{\circ-}$ molecules labeled as (1), (2), and (3)). There are three kinds of small $r$ values: 10.01 Å with molecule (3), $10.08 \AA$ with molecule (2), and $10.11 \AA$ A with molecule (1) at $250 \mathrm{~K}$. Four $\mathrm{C}_{60} 0^{\bullet-}$ molecules, all of them are molecules (2) among the eight neighbors, are located in the $b c$ plane to form

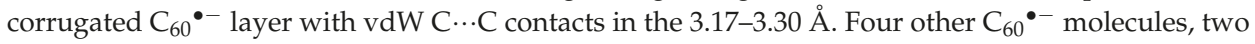
(1) and two (3), are located in the adjacent fullerene layers without short vdW C...C contacts, though the $r$ between molecules (A) and (3) is shorter than that between molecules (A) and (2).

The ordering of fullerenes is observed below $160 \mathrm{~K}$ with trebling of the unit cell $b$ axis. As a result, the packing of $\mathrm{C}_{60} 0^{-}$molecules becomes denser and strongly anisotropic; namely $r$ changes from (A) - (2) $=10.08 \AA$ at $250 \mathrm{~K}$ to $(10.01,10.04,10.05 \AA)$ at $100 \mathrm{~K}$ in the $b c$ plane. Similarly, (A) - (3) and (1) - (2) 
reduce to 9.91 and $9.96 \AA$, but (A) - (1) becomes a little longer, $10.12 \AA$. Additional vdW interfullerene C...C contacts are formed for each $\mathrm{C}_{60}{ }^{\bullet-}$ within the $b c$ plane and between fullerene layers to account for the total number of such contacts as 18 for each $\mathrm{C}_{60}{ }^{\circ-}$ at $100 \mathrm{~K}$.

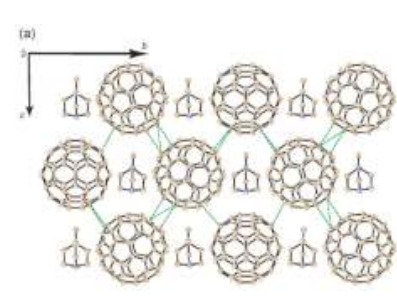

(a)

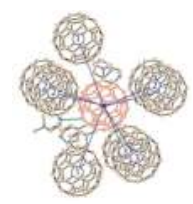

(d)

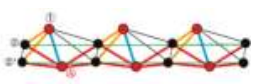

$(\mathbf{e - 4 )}$

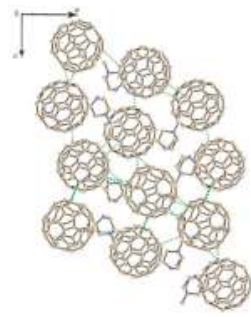

(b)

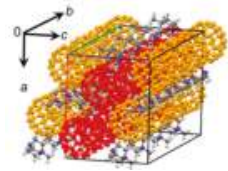

(c-1)

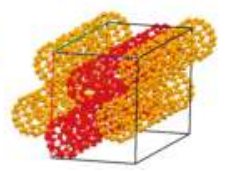

$(\mathrm{c}-2)$

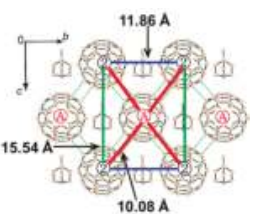

$(\mathrm{e}-1)$

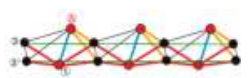

$(\mathrm{e}-5)$

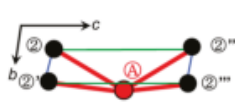

$(\mathrm{e}-2)$

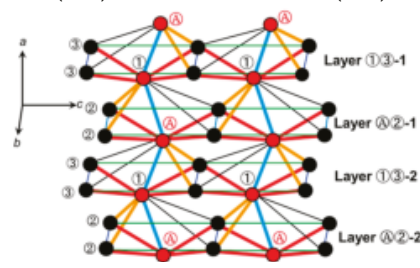

(e-6)

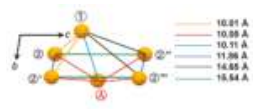

$(\mathrm{e}-3)$

Figure 20. (a) View of crystal structure of $\left(\mathrm{MDABCO}^{+}\right)\left(\mathrm{C}_{60}{ }^{\bullet-}\right)$ along the $a$ axis at $100 \mathrm{~K}$; (b) View along the $b$ axis at $100 \mathrm{~K}$. The van der Waals contacts between fullerenes are shown in green dashed lines in (a,b); (c) $\mathrm{C}_{60}{ }^{--}$molecules stack along the $b$ axis to form columns as shown with (c-1) or without $\mathrm{MDABCO}^{+}$(c-2, for simplicity). Yellow and red $\mathrm{C}_{60}{ }^{\bullet-}$ columns are arranged alternately along the $c$ axis to form a corrugated sheet in the $b c$ plane; (d) Environment of one $\mathrm{C}_{60}{ }^{\bullet-}$ radical anion (shown by red color) from eight neighboring $\mathrm{C}_{60}{ }^{\circ-}$ and five $\mathrm{MDABCO}^{+}$cations at $250 \mathrm{~K}$. Center-to-center distances between red fullerene (A) and surrounding $\mathrm{C}_{60}{ }^{\bullet-}$ (marked by numbers 1-3): $\mathrm{A}-1$ : 10.107, A-2: 10.081 and A-3: $10.008 \AA$. Van der Waals contacts between $\mathrm{C}_{60}{ }^{\bullet-}$ and nitrogen atom of the $\mathrm{MDABCO}^{+}$cation are shown by green dashed lines. Only the major orientation of $\mathrm{C}_{60}{ }^{\bullet-}$ is shown; (e) (e-1) Corrugated layer composed of molecules A and 2 at $250 \mathrm{~K}$; (e-2) Geometry of a unit of possible spin lattice made of A and 2; (e-3) Schematic of bipyramidal spin lattice composed of molecules A, 1, and 2 ; (e-4) The unit shown by (e-3) forms 2D layer by sharing an edge. Only the ribbon extending along the $c$ axis is shown; (e-5) The other unit of bipyramidal spin lattice composed of $1, \mathrm{~A}$, and 3 forms equivalent 2D layer; (e-6) The model geometry of the possible spin lattice obtained by sharing apexes of the layer in (e-4,e-5) along the $a$ axis to form a 3D distorted bipyramidal spin lattice (a,b,d), (e-1) from [147]).

Since the structure at $100 \mathrm{~K}$ is complicated by the appearance of one and a half independent molecules both for $\mathrm{C}_{60}{ }^{\bullet-}$ and $\mathrm{MDABCO}^{+}$molecules, at first the schematic of a possible spin lattice is examined based on the crystal structure at $250 \mathrm{~K}$. Figure $20(\mathrm{e}-1)$ shows the packing of $\mathrm{C}_{60}{ }^{\bullet-}$ viewed along the a axis, that corresponds to Figure $20 \mathrm{a}$ at $100 \mathrm{~K}$. The square corrugated $\mathrm{C}_{60}{ }^{\bullet-}$ packing composed of $\mathrm{C}_{60}{ }^{\bullet-}$ molecules (one (A) and four (2)) form a pyramidal shape with $\left.r(A)-(2)\right)=10.08 \AA$, 
which is schematically shown in Figure 20(e-2). The other combination of $\mathrm{C}_{60}{ }^{\bullet-}$ molecules (1) and (3) form the equivalent pyramid and these two pyramids have short contacts with $r$ values of $10.01 \AA$ for 1-2 and $10.11 \AA$ for (1)-(A). As a consequence, the unit of intermolecular interactions is approximated as a distorted bipyramid, as shown in Figure 20(e-3). The plane of (2)-(2) - (2) ${ }^{\prime \prime}-(2)^{\prime \prime \prime}$ bisects the bond (1)-(A) by $6.91 \AA$ and $3.20 \AA$. The distorted bipyramids are connected to each other by sharing edge in the $b c$ plane to form a 2D sheet. Figure 20(e-4) shows the part of the sheet extending along the $c$ axis. The other unit of bipyramidal spin lattice composed of $\mathrm{C}_{60}{ }^{\bullet-}$ molecules (1), (A), and (3) forms equivalent 2D layer with different orientation (Figure 20(e-5)). The model geometry of the possible spin lattice of 1 is obtained by sharing apexes of the layers in Figure 20(e-4) and Figure 20(e-5) along the $a$ axis to form 3D distorted bipyramidal spin lattice in which corrugated layers alternate as Layer (A) (2)/ Layer (1) (3) along the $a$ axis (Figure 20(e-6)).

At low temperatures, the $\mathrm{C}_{60}{ }^{\bullet-}$ environment is strongly anisotropic in terms of $r$ and overlap integrals s. The $r$ values inside corrugated Layer (A)(2) or Layer (1) (3), namely bonds (A)- (2) and (1)-, show shrinkage by $0.3-0.7 \%$, while those bonds that are connecting neighboring corrugated layers, namely bonds (1)-(2) and (A)- (3), show larger shrinkage by $0.5-1.0 \%$. Therefore, though the corrugated square nature shown by Figure 20(e-2) is important to account for the spin interactions, the spin interactions between corrugated layers along the $a$ axis becomes more significant at low temperatures. The model spin lattice geometry keeps the distorted bipyramidal one down to low temperatures. No dimerization was detected down to $1.9 \mathrm{~K}$.

The temperature dependence of the molar magnetic susceptibility $\chi_{\mathrm{M}}$ of $\mathbf{1}$ showed a maximum at $46 \mathrm{~K}$, followed by a decrease but then an increase below $10 \mathrm{~K}$ owing to the Curie impurity of about $2.7 \%$ of total amount of $\mathrm{C}_{60}{ }^{\bullet-}$. Figure 21 shows the temperature dependence of $\chi_{\mathrm{M}}$ and $1 / \chi_{\mathrm{M}}$ after the correction of Curie impurity. The magnetic susceptibility clearly indicates a characteristic peak near $50 \mathrm{~K}$. Such peak in $\chi_{\mathrm{M}}$ has been usually detected in the low-D (1D-2D) Mott insulators with strong spin frustration, such as $\kappa-(\mathrm{ET})_{2} \mathrm{X}\left(\mathrm{X}=\mathrm{Cu}_{2}(\mathrm{CN})_{3}, \mathrm{Ag}_{2}(\mathrm{CN})_{3}, \mathrm{~B}(\mathrm{CN})_{4}\right.$, $\left.\mathrm{CF}_{3} \mathrm{SO}_{3}\right)[36,45-51,54,65,66,128,150,158]$. The $\Theta_{\mathrm{CW}}$ temperature of $-118 \mathrm{~K}$ was derived in the $70-300 \mathrm{~K}$ range (Figure $21 \mathrm{~b}$ ). The temperature dependence of $\chi_{\mathrm{M}}$ was fitted by the Heisenberg model for square $2 \mathrm{D}$ AF coupling of spins [159] to give $J / k_{\mathrm{B}}=-25.3 \mathrm{~K}$. The long-range magnetic ordering is not observed down to $1.9 \mathrm{~K}(f>62)$.

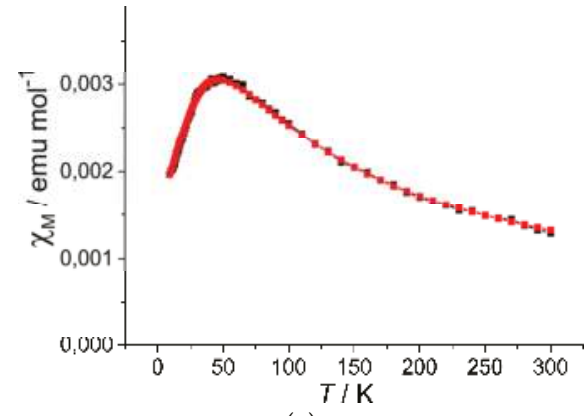

(a)

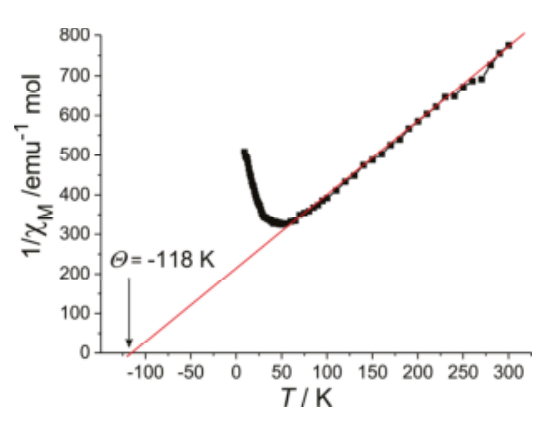

(b)

Figure 21. Temperature dependence of molar magnetic susceptibility $\chi_{\mathrm{M}}(\mathbf{a})$ and $1 / \chi_{\mathrm{M}}(\mathbf{b})$ of $\left(\mathrm{MDABCO}^{+}\right)\left(\mathrm{C}_{60^{\circ}}{ }^{-}\right)$after correction of Curie impurity. Red curve in (a) is the fit by the Heisenberg model for square $2 \mathrm{D}$ AF coupling of spins with $J / k_{\mathrm{B}}=-25.3 \mathrm{~K}$. Red line in $(\mathbf{b})$ is the Curie-Weiss fit with $\Theta_{\mathrm{CW}}=-118 \mathrm{~K}$ [147].

The overlap integrals between $\mathrm{C}_{60}{ }^{\bullet-}$ radical anions based on the real crystal structures are helpful to understand the situation of intermolecular interaction. At $250 \mathrm{~K}, \mathbf{1}$ contains only one crystallographically independent $\mathrm{C}_{60}{ }^{\bullet-}$ and one $\mathrm{MDABCO}^{+}$, however the $\mathrm{C}_{60}{ }^{\bullet-}$ radical anions are 
disordered between three orientations with 49.4/25.3/25.3 (\%) occupancies. On the other hand, 1 contains one and a half crystallographically independent $\mathrm{C}_{60} 0^{--}$and $\mathrm{MDABCO}^{+}$at $100 \mathrm{~K}$. Half of $\mathrm{C}_{60}{ }^{\circ-}$ is well ordered at $100 \mathrm{~K}$, while another $\mathrm{C}_{60}{ }^{\circ-}$ is rotationally disordered between two orientations with $91.3 / 8.7$ (\%) occupancies. Although it is not reasonable to discuss the overlap integrals at $250 \mathrm{~K}$ due to the severe orientational disorder, we can calculate the overlap integrals in $\mathbf{1}$ at $100 \mathrm{~K}$, assuming that all of the $\mathrm{C}_{60}{ }^{--}$are well ordered ignoring the minor orientation $(8.7 \%)$ in one of the two kinds of $\mathrm{C}_{60} 0^{-}$.

Figure 22 shows the network between the two kinds of $\mathrm{C}_{60}{ }^{--}$radical anions at $100 \mathrm{~K}$ that are connected by several magnitudes of overlap integrals. The dominant interaction (red line) is $4.74 \times 10^{-3}$ between the molecules 1 with $9.96 \AA$ distance that forms a pair of $\mathrm{C}_{60}{ }^{\bullet-}$ between the adjacent layers. The second largest interaction (purple line) is $2.66 \times 10^{-3}$ between the molecules 2 with $10.14 \AA$ uniform distance that extends linearly along the $a$ axis. The third one (black line) is $1.96 \times 10^{-3}$ between the molecules 1 with $10.05 \AA$ distance which forms zig-zag path ways along the $c$ axis. Note that the shortest distance of $9.91 \AA$ (orange line) between the different kinds of molecules 1 and 2 resulted in only the fifth largest interaction of $1.25 \times 10^{-3}$. As demonstrated also in 2 and 5 , the magnitude of overlap integrals between $\mathrm{C}_{60}{ }^{\circ-}$ anion radicals does not necessarily scale with the closeness between them. The relative orientation of the molecular orbitals in the nearest neighbors as well as the center-to-center distances between them plays an important role to characterize the molecular interactions in the crystal.

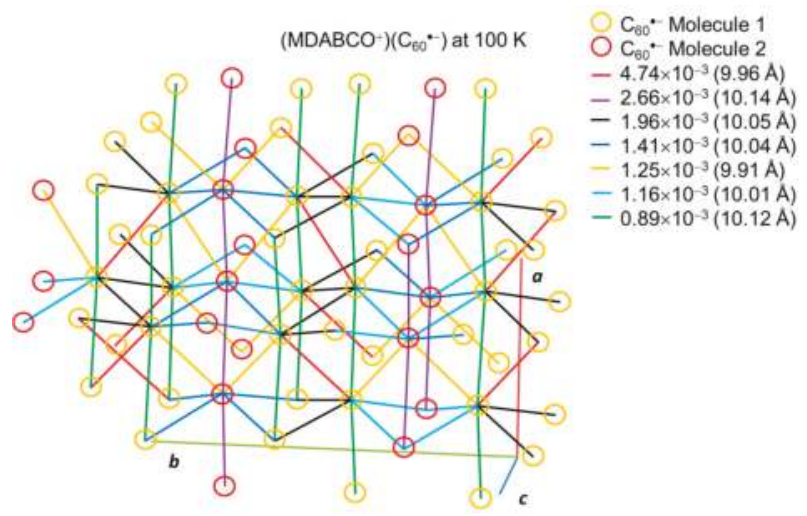

Figure 22. Overlap integrals between $\mathrm{C}_{60}{ }^{\bullet-}$ anion radicals in $\left(\mathrm{MDABCO}^{+}\right)\left(\mathrm{C}_{60}{ }^{\bullet-}\right)$ at $100 \mathrm{~K}$. One (molecule 1) and a half (molecule 2) of $\mathrm{C}_{60}{ }^{\bullet-}$ spheres are crystallograpically independent.

The small size of the $\mathrm{MDABCO}^{+}$cations with threefold symmetry and the absence of solvent molecules induced a densely packed $3 \mathrm{D}$ bipyramidal $\mathrm{C}_{60} 0^{--}$packing in $\mathbf{1}$ resulting in strong $\mathrm{AF}$ interactions. However, the strong anisotropic packing of $\mathrm{C}_{60} 0^{--}$may reduce the $\mathrm{AF}$ interaction considerably. The key-keyhole relation between $\mathrm{MDABCO}^{+}$and $\mathrm{C}_{60}{ }^{--}$is not clear in $\mathbf{1}$, however, it should be emphasized that $\mathrm{MDABCO}^{+}$cations work to prevent the bond-formation even though the $r$ values became small (1)-(2) $=9.91 \AA$, (A)-(3) $=9.96 \AA$ at $100 \mathrm{~K}$ ).

4.2.2. Frustrated Spins in Double Chains of Triangles from $\mathrm{C}_{60^{\bullet}}{ }^{--}$in $\left(\mathrm{Ph}_{3} \mathrm{MeP}^{+}\right)\left(\mathrm{C}_{60}{ }^{\bullet-}\right)$ : Weakly Coupled Zigzag Chains

Single crystals of $\left(\mathrm{Ph}_{3} \mathrm{MeP}^{+}\right)\left(\mathrm{C}_{60}{ }^{\bullet-}\right)(2)$, where $\mathrm{Ph}_{3} \mathrm{MeP}^{+}$is a triphenylmethylphosphonium cation with threefold symmetry, were prepared by the reduction of $\mathrm{C}_{60}$ by $\left(\mathrm{Ph}_{3} \mathrm{MeP}^{+}\right)($vanadyl(IV) phthalocyanine) in $\mathrm{PhCl}_{2}$ and slow mixing of the obtained $\mathrm{PhCl}_{2}$ solution in $n$-hexane [105]. 
The crystal structure determined at $100 \mathrm{~K}$ indicates nearly ordered state of $\mathrm{C}_{60}{ }^{\bullet-}$ molecules. Crystal 2 involves a nearly isolated double chain with nearly equivalent fullerene triangles with small $r$ values $10.08 \AA, 10.103 \AA$, and $10.103 \AA$ at $100 \mathrm{~K}$ (Figure 23a). The overlap integral is $s_{2}=1.19 \times 10^{-3}$ for $r=10.079 \AA$ while that with longer $r=10.103 \AA$ has larger $s_{1}=1.79 \times 10^{-3}$ owing to favorable orientation of $\mathrm{C}_{60}{ }^{\circ-}$ molecules. Even though the double chain can be characterized as a zigzag chain, the chains are not isolated but are coupled weakly with a separation of $r=10.309 \AA\left(s_{3}=0.58 \times 10^{-3}\right)$ within the $b c$ plane (Figure 23b). Therefore, the possible spin lattice of 2 consists of pseudo 3D weakly coupled zigzag chains (Figure 23c) with $t^{\prime} / t=1.50$ and $J_{1}: J_{2}: J_{3}=1: 0.44: 0.10$.

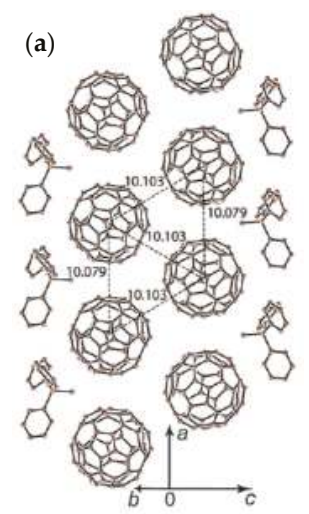

(c)

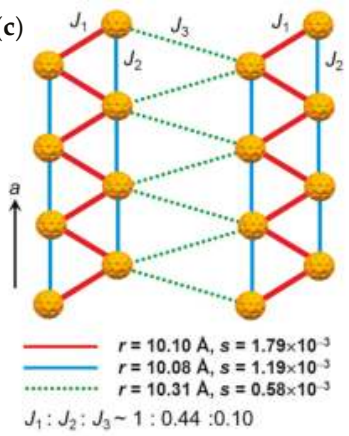

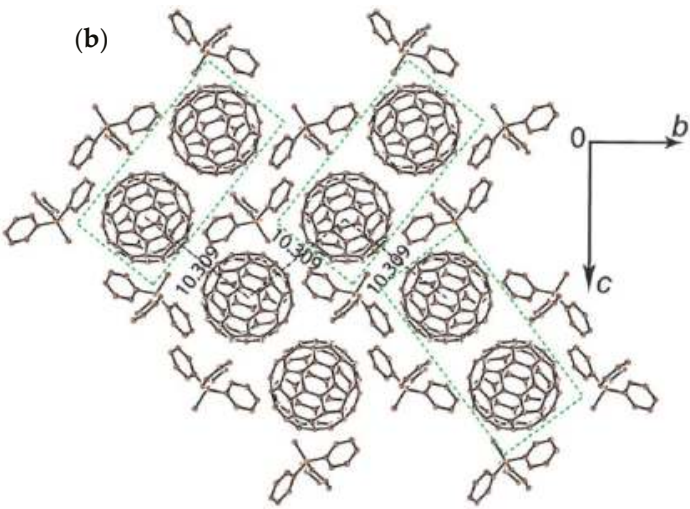

(d)

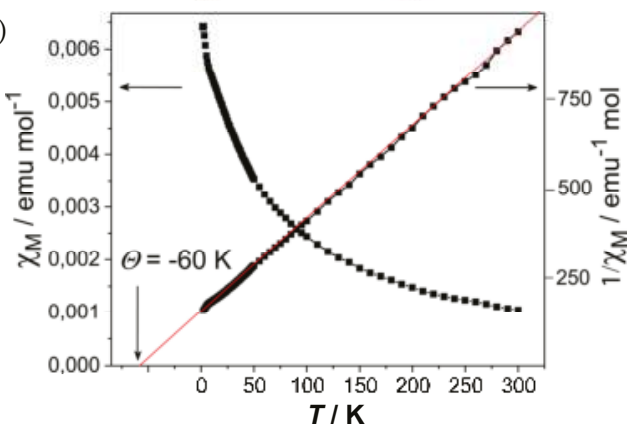

Figure 23. (a) Crystal structure of $\left(\mathrm{Ph}_{3} \mathrm{MeP}^{+}\right)\left(\mathrm{C}_{60}{ }^{\bullet-}\right)$ showing the double chains containing triangles from $\mathrm{C}_{60}{ }^{\bullet-}$; (b) view along the $a$ axis showing the arrangement of double chains in the $b c$ plane; (c) Schematic of possible zigzag spin lattice with weak interchain interactions $\left(J_{1}: J_{2}: J_{3}=1: 0.44: 0.10\right)$ together with $r$ and $s$; (d) Temperature dependence of molar magnetic susceptibility $\chi_{\mathrm{M}}$ and $1 / \chi_{\mathrm{M}}$ after correction of Curie impurity. Red curve in (d) is the Curie-Weiss fit with $\Theta_{\mathrm{CW}}=-60 \mathrm{~K}$. (a,b,d) were reproduced from [105].

Several zigzag-chain spin systems, which are the simplest frustrated magnet and treated by $J_{1}-J_{2}$ model or zigzag chain model, have been developed, such as $\mathrm{CaV}_{2} \mathrm{O}_{4}\left(S=1, \mathrm{~V}^{3+}, T_{\mathrm{N}}=69 \mathrm{~K}\right)$ [160], $\mathrm{Cu}$ [2-(2-aminomethyl)pyridine] $\mathrm{Br}_{2}\left(S=1 / 2, \mathrm{Cu}^{2+}\right)[161,162],(\mathrm{VO})\left(\mu_{3}-\mathrm{MoO}_{4}\right)(\mathrm{BPY})\left(S=1 / 2, \mathrm{~V}^{4+}\right)[163,164]$, and $\mathrm{F}_{2} \mathrm{PIMNH}$ [165]. A theoretical study predicted that the $S=1 / 2$ zigzag chain has a gapless phase for $J_{1} / J_{2}$ $<0.241$ [166,167], as exemplified for $\mathrm{Cu}$ [2-(2-aminomethyl)pyridine] $\mathrm{Br}_{2}\left(J_{1} / J_{2}=0.2, J_{1} / k_{\mathrm{B}}=8.5 \mathrm{~K}\right)[162]$ and $(\mathrm{VO})\left(\mu_{3}-\mathrm{MoO}_{4}\right)(\mathrm{BPY})\left(J^{\prime} / J=0.2, J_{1} / k_{\mathrm{B}}=51 \mathrm{~K}\right)$ [164]. It is known that the interchain magnetic interactions are critical for the spin-ladder system either to manifest a Néel ordered or disordered spin-frustrated state, and the critical value is reported to be $J / J^{\prime}=0.11$, where $J$ and $J^{\prime}$ are intraladder and interladder interactions, respectively [168]. Even though the actual $J_{3}$ values for the above zigzag systems were not estimated in these reports, it is likely that the $J_{3}$ values are very small according to their crystal structures. If we are able 
to expand the separation between the zigzag chains for 2 using more bulky cation molecules than $\mathrm{Ph}_{3} \mathrm{MeP}^{+}$, we may have a real zigzag system of $\mathrm{C}_{60} 0^{\bullet-}$.

The temperature dependence of the molar magnetic susceptibility $\chi_{\mathrm{M}}$ shows an increase below $10 \mathrm{~K}$, owing to the Curie impurity of about $1.2 \%$ of total amount of $\mathrm{C}_{60}{ }^{\bullet-}$. Figure $23 \mathrm{~d}$ showed the temperature dependence of $\chi_{\mathrm{M}}$ and $1 / \chi_{\mathrm{M}}$ after the correction of Curie impurity. No peak of $\chi_{\mathrm{M}}$ was observed even though 2 has strong spin frustration. The magnetic behavior is described well by the Curie-Weiss law in the 30-300 K range with $\Theta_{\mathrm{CW}}=-60 \mathrm{~K}$, and no AF ordering was observed down to $1.9 \mathrm{~K}(f>30)$. Between $\mathrm{C}_{60}{ }^{\bullet-}$ molecules arranged along the $a$ axis, Me groups of $\mathrm{Ph}_{3} \mathrm{MeP}^{+}$cation molecules penetrate and prevent the dimerization even at $r=10.08-10.10 \AA$.

\section{Summary}

Geometrical spin frustration is discussed for monomer-type Mott insulators of $\mathrm{C}_{60} \mathrm{CT}$ solids. When compared with the ET QSL system, bond-formation between $\mathrm{C}_{60}$ molecules and disorder of $\mathrm{C}_{60}$ molecule additionally participate in the competition among the itinerancy, localization, and spin frustration. The donor ability, size, shape, and symmetry of donor molecules in multi-component approach provide suitable geometrical space and spatial regulation for $\mathrm{C}_{60}{ }^{\bullet-}$ molecules by forming versatile supramolecules through the key-keyhole relation. A hexagonal packing of $\mathrm{C}_{60}{ }^{\bullet-}$ is achieved by the multi-component concept using cations $\left(\mathrm{MDABCO}^{+}\right.$or $\mathrm{MQ}^{+}$) and structure defining molecule (TPC) with threefold symmetry. $\mathrm{C}_{60}{ }^{\bullet-}$ molecules are packed according to the pattern of the polycationic supramolecular template of $\left[\left(\mathrm{TPC}^{0}\right)\left(\mathrm{MDABCO}^{+}\right)\right]$or $\left[\left(\mathrm{TPC}^{0}\right)\left(\mathrm{MQ}^{+}\right)\right]$. $\left(\mathrm{TPC}^{0}\right)\left(\mathrm{MDABCO}^{+}\right)\left(\mathrm{C}_{60}{ }^{\bullet-}\right)$ has uniform close packed hexagonal layers of two types, with an ordered $\mathrm{C}_{60}{ }^{\bullet-}$ layer (Layer $A$ ) and disordered $\mathrm{C}_{60}{ }^{\bullet-}$ layer (Layer $B$ ). The Layer $A$ with $t^{\prime} / t=1.00$ at $300 \mathrm{~K}$ shows $2 \mathrm{D}$ metallic conductivity, whereas AF interaction of spins is observed in nonmetallic Layer $B$ above $200 \mathrm{~K}$. This AF layer has monomer-type Mott insulating state with $t^{\prime} / t=0.99($ at $185 \mathrm{~K})$ and $\Theta_{\mathrm{CW}}=-31 \mathrm{~K}$. The disordered layer becomes metallic below $200 \mathrm{~K}$ through the ordering of $\mathrm{C}_{60}{ }^{\bullet-}$. $\left(\mathrm{TPC}^{0}\right)\left(\mathrm{MQ}^{+}\right)\left(\mathrm{C}_{60}{ }^{-}\right)$also has a 2D hexagonal packing of $\mathrm{C}_{60}{ }^{\bullet-}$ and shows relatively longer interfullerene distances (10.12-10.18 at $250 \mathrm{~K})$ than that in $\left(\mathrm{TPC}^{0}\right)\left(\mathrm{MDABCO}^{+}\right)\left(\mathrm{C}_{60}{ }^{-}-\right)(10.07 \AA$ at $300 \mathrm{~K}) .\left(\mathrm{TPC}^{0}\right)\left(\mathrm{MQ}^{+}\right)\left(\mathrm{C}_{60}{ }^{-}\right)$has a monomer-type Mott insulating state with $t^{\prime} / t=0.99$ (Layer $A$ at $100 \mathrm{~K}$ ), $t^{\prime} / t=1.61$ (Layer $B$ at $100 \mathrm{~K}$ ), and $\Theta_{\mathrm{CW}}=-27 \mathrm{~K}(f \sim 14)$. Solvent molecules participate in the formation of three-component $\mathrm{C}_{60}$ solids to achieve $\mathrm{C}_{60}$ hexagonal packing in $\left(\mathrm{PhCN}^{0}\right)\left(\mathrm{TMP}^{+}\right)\left(\mathrm{C}_{60}{ }^{\bullet-}\right)$ and $\left(\mathrm{PhCN}^{0}\right)\left(\mathrm{Ph}_{3} \mathrm{MeP}^{+}\right)\left(\mathrm{C}_{60}{ }^{\bullet-}\right)$. The former has a distorted edge-shared honeycomb $2 \mathrm{D}$ spin lattice with $J_{1}: J_{2}: J_{3}=0.79: 1: 0.22$ and $\Theta_{\mathrm{CW}}=-11 \mathrm{~K}(f \sim 5)$, and the latter has a non-uniform $1 \mathrm{D}$ zigzag chain of $\mathrm{C}_{60}{ }^{\bullet-}$ as an effective spin geometry and forms singly bonded dimers that are below $220 \mathrm{~K} .\left(\mathrm{PhCl}_{2}{ }^{0}\right)\left[\left(\mathrm{Ph}_{3} \mathrm{P}\right)_{3} \mathrm{Au}^{+}\right]_{2}\left(\mathrm{C}_{60}{ }^{\bullet-}\right)_{2}\left(\mathrm{C}_{60}{ }^{0}\right)$ has a charge-disproportionated hexagonal 2D layer and the $\mathrm{C}_{60}{ }^{\bullet-}$ molecules form an apex-sharing tetrahedral spin lattice with large $r=10.37 \AA$ at $100 \mathrm{~K}$ and small $\Theta_{\mathrm{CW}}=-5(f \sim 3)$. 3D hexagonal packing is realized in $\left(\mathrm{DMI}^{+}\right)_{3}\left(\mathrm{C}_{60}{ }^{\bullet-}\right)\left(\mathrm{I}^{-}\right)_{2}$ in which $\mathrm{H}$-bonds between $\mathrm{DMI}^{+}$and $\mathrm{I}^{-}$formed a polycationic template $\left[\left(\mathrm{DMI}^{+}\right)_{3}\left(\mathrm{I}^{-}\right)_{2}\right]$ with threefold symmetry. A possible geometry of spin lattice is an apex-sharing bipyramid $\left(t^{\prime} / t=0\right)$ and the long interfullerene distance of $11.05 \AA$ at $100 \mathrm{~K}$ resulted in weak magnetic interaction $\Theta_{\mathrm{CW}}=-9.6 \mathrm{~K}(f \sim 5)$. For two-component systems, a triangular unit of $\mathrm{C}_{60}{ }^{\bullet-}$ is observed in $\left(\mathrm{MDABCO}^{+}\right)\left(\mathrm{C}_{60}{ }^{\bullet-}\right)$ and $\left(\mathrm{Ph}_{3} \mathrm{MeP}^{+}\right)\left(\mathrm{C}_{60}{ }^{\bullet-}\right)$ both cation molecules have threefold symmetry. 3D close packing of $\mathrm{C}_{60}{ }^{\bullet-}$ was observed in $\left(\mathrm{MDABCO}^{+}\right)\left(\mathrm{C}_{60}{ }^{\bullet-}\right)$ with a possible geometry of spin lattice of deformed 3D edge-shared bipyramid. The AF interaction with high $\Theta_{\mathrm{CW}}$ of $-118 \mathrm{~K}(f \sim 62)$ was observed though the bipyramidal geometry becomes distorted at low temperatures. $\left(\mathrm{Ph}_{3} \mathrm{MeP}^{+}\right)\left(\mathrm{C}_{60}{ }^{\bullet-}\right)$ has double chains that are composed of a triangular arrangement of $\mathrm{C}_{60}{ }^{\bullet-}$, resulting in weakly coupled zigzag chains with $t^{\prime} / t=1.50, J_{1}: J_{2}: J_{3}=1: 0.44: 0.10$, and $\Theta_{\mathrm{CW}}=-60 \mathrm{~K}(f>30)$.

Even though the overlap integrals between $\mathrm{C}_{60}$ molecules depend on the molecular orientation of $\mathrm{C}_{60}$, the center-to-center distance between $\mathrm{C}_{60}{ }^{\bullet-}$ molecules $r$ is the key parameter that determines the competition among the bond-formation, itinerancy, and spin frustration. Figure 24 shows the relation between $\left|\Theta_{\mathrm{CW}}\right|$ values and interfullerene distances $r$ in this study. In the following, the summary and the perspective developed are presented. 
1. $\left|\Theta_{\mathrm{CW}}\right|$ seems to increase rapidly when $r<10 \AA$ and magnetic dimensionality is 3D. Such low values of $r$ were realized for two-component CT solids with cation molecules of small size with threefold symmetry. However, it is difficult to find a good key-keyhole relation to provide uniform triangular or hexagonal packing of $\mathrm{C}_{60}{ }^{\bullet-}$ for the two-component case. Furthermore, single crystals of $\mathrm{CT}$ solids were not always obtainable. For example, a quinuclidinium cation, which is smaller than $\mathrm{MDABCO}^{+}$and $\mathrm{MQ}^{+}$, gave no CT solids so far.

2. The cationic supramolecular template with threefold symmetry leads to uniform triangular or hexagonal packing of $\mathrm{C}_{60}{ }^{\bullet-}$ for three-component case based on the key-keyhole relation. For three-component case, it is critical to decrease the $r$ value and increase the magnetic dimensionality.

In all of these monomer-type $\mathrm{C}_{60^{\circ}}{ }^{-}$Mott insulator except $\mathbf{6}$, no long-range magnetic ordering nor bond-formation were observed down to low temperatures. However, their $\left|\Theta_{\mathrm{CW}}\right|$ values in the range 10-118 K, are not large enough to detect QSL state at experimentally available temperatures. This is apparent from the comparison with the $\left|\Theta_{\mathrm{CW}}\right|$ values of the QSL systems obtained; $\left|\Theta_{\mathrm{CW}}\right|$ $=180,263,314,375$, and $325-375 \mathrm{~K}$ for $\left[\left(\mathrm{C}_{2} \mathrm{H}_{5}\right)_{3} \mathrm{NH}\right]_{2} \mathrm{Cu}_{2}$ (oxalate) $)_{3}, \kappa-(\mathrm{ET})_{2} \mathrm{Ag}_{2}(\mathrm{CN})_{3}, \mathrm{ZnCu}_{3}(\mathrm{OH})_{6} \mathrm{Cl}_{2}$, $\kappa-(\mathrm{ET})_{2} \mathrm{Cu}_{2}(\mathrm{CN})_{3}$, and $\mathrm{EtMe}_{3} \mathrm{Sb}\left[\mathrm{Pd}(\mathrm{dmit})_{2}\right]$, respectively $[36,59-62,64,65]$. It is important to explore a $\mathrm{C}_{60}{ }^{\bullet-}$ Mott insulator with $r=9.4-10 \AA$ to detect a QSL state neighboring metallic and SC states, as a decrease in $r$ increases $|t|,|J|$, and $\left|\Theta_{\mathrm{CW}}\right|$ values drastically according to their relations (Equations (1) and (2)).

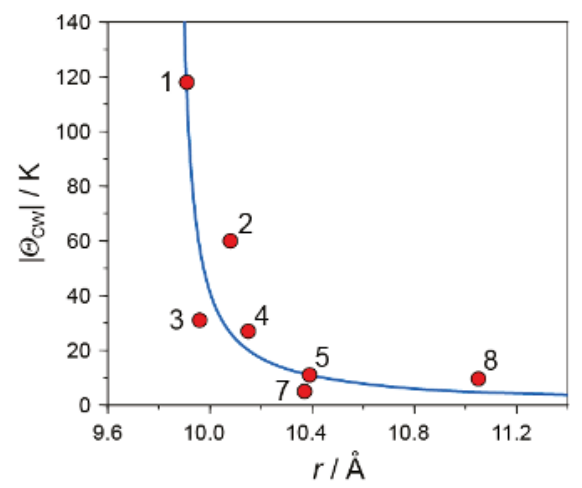

Figure 24. Relation between Curie-Weiss temperature $\left|\Theta_{\mathrm{CW}}\right|$ and interfullerene center-to-center distance $r$ for $\mathrm{C}_{60}{ }^{\bullet-} \mathrm{CT}$ solids in this study. 1: $\left(\mathrm{MDABCO}^{+}\right)\left(\mathrm{C}_{60}{ }^{\bullet-}\right)\left(\left|\Theta_{\mathrm{CW}}\right|=118 \mathrm{~K}, 3 \mathrm{D}, r=9.91 \AA\right.$ at $100 \mathrm{~K}), 2:\left(\mathrm{Ph}_{3} \mathrm{MeP}^{+}\right)\left(\mathrm{C}_{60}{ }^{\bullet-}\right)\left(\left|\Theta_{\mathrm{CW}}\right|=60 \mathrm{~K}\right.$, pseudo $3 \mathrm{D}$, coupled double chains, $r=10.08 \AA$ at $100 \mathrm{~K}), 3$ : $\left(\mathrm{TPC}^{0}\right)\left(\mathrm{MDABCO}^{+}\right)\left(\mathrm{C}_{60}{ }^{\bullet-}\right)\left(\left|\Theta_{\mathrm{CW}}\right|=31 \mathrm{~K}, 2 \mathrm{D}\right.$ layer $r=10.06 \AA$ at $300 \mathrm{~K}, 9.97 \AA$ at $\left.185 \mathrm{~K}\right), 4$ : $\left(\mathrm{TPC}^{0}\right)\left(\mathrm{MQ}^{+}\right)\left(\mathrm{C}_{60}{ }^{\bullet-}\right)\left(\left|\Theta_{\mathrm{CW}}\right|=27 \mathrm{~K}, 2 \mathrm{D}\right.$ layer, $r=10.12-10.18 \AA$ at $\left.250 \mathrm{~K}\right), 5:\left(\mathrm{PhCN}^{0}\right)\left(\mathrm{TMP}^{+}\right)\left(\mathrm{C}_{60}{ }^{\bullet}\right)$ $\left(\left|\Theta_{\mathrm{CW}}\right|=11 \mathrm{~K}, 2 \mathrm{D}\right.$ hexagon, $r=10.39 \AA$ at $\left.120 \mathrm{~K}\right), 7:\left\{\left(\mathrm{Ph}_{3} \mathrm{P}\right)_{3} \mathrm{Au}^{+}\right\}_{2}\left(\mathrm{C}_{60}{ }^{\bullet-}\right)_{2}\left(\mathrm{C}_{60}\right)\left(\mathrm{PhCl}_{2}\right)\left(\left|\Theta_{\mathrm{CW}}\right|=5 \mathrm{~K}\right.$, 2D tetragonal, $r=10.37 \AA$ at $100 \mathrm{~K}), 8$ : $\left(\mathrm{DMI}^{+}\right)_{3}\left(\mathrm{C}_{60}{ }^{-}-\right)\left(\mathrm{I}^{-}\right)_{2}\left(\left|\Theta_{\mathrm{CW}}\right|=9.6 \mathrm{~K}, 3 \mathrm{D}\right.$ triangular bipyramid, $r=11.05 \AA$ at $100 \mathrm{~K})$. The blue line is a guide to the eye.

Acknowledgments: This work was supported by Russian Science Foundation RSF-18-13-00292 and JSPS KAKENHI Grant Number JP23225005 "Development of multi-electronic-functions based on spin triangular lattice".

Conflicts of Interest: The authors declare no conflict of interest. 


\section{References}

1. Anderson, P.W. Resonating valence bonds: A new kind of insulator? Mater. Res. Bull. 1973, 8, 153-160. [CrossRef]

2. Fazekas, P.; Anderson, P.W. On the ground state properties of the anisotropic triangular antiferromagnet. Philos. Mag. 1974, 30, 423-440. [CrossRef]

3. Wannier, G.H. Antiferromagnetism. The triangular ising net. Phys. Rev. 1950, 79, 357-364. [CrossRef]

4. Greedan, J.E. Geometrically frustrated magnetic materials. J. Mater. Chem. 2001, 11, 37-53. [CrossRef]

5. Anderson, P.W. Ordering and antiferromagnetism in ferrites. Phys. Rev. 1956, 102, 1008-1013. [CrossRef]

6. Hirakawa, K.; Yoshizawa, H.; Ubukoshi, K. Magnetic and neutron scattering study of one-dimensional heisenberg antiferromagnet $\mathrm{CsVCl}_{3}$. J. Phys. Soc. Jpn. 1982, 51, 1119-1122. [CrossRef]

7. Ferey, G.; De Pape, R.; Leblanc, M.; Pannetier, J. Ordered magnetic frustration: VIII. Crystal and magnetic structures of the pyrochlore form of iron trifluoride between 2.5 and $25 \mathrm{~K}$ from powder neutron diffraction. Comparison with the other varieties of $\mathrm{FeF}_{3}$. Rev. Chim. Miner. 1986, 23, 474-484.

8. Huse, D.; Elser, V. Simple variational wave functions for two-dimensional heisenberg spin-1/2 antiferromagnets. Phys. Rev. Lett. 1988, 60, 2531-2534. [CrossRef] [PubMed]

9. Tocchio, L.F.; Gros, C.; Valentif, R.; Becca, F. One-dimensional spin liquid, collinear, and spiral phases from uncoupled chains to the triangular lattice. Phys. Rev. B 2014, 89, 235107. [CrossRef]

10. Yamada, A. Magnetic properties and Mott transition in the Hubbard model on the anisotropic triangular lattice. Phys. Rev. B 2014, 89, 195108. [CrossRef]

11. Yamada, A. Magnetic properties and Mott transition of the Hubbard model for weakly coupled chains on the anisotropic triangular lattice. Phys. Rev. B 2014, 90, 235138. [CrossRef]

12. Konarev, D.V.; Khasanov, S.S.; Otsuka, A.; Maesato, M.; Uruichi, M.; Yakushi, K.; Shevchun, A.F.; Yamochi, H.; Saito, G.; Lyubovskaya, R.N. Metallic and Mott insulating spin-frustrated antiferromagnetic states in ionic fullerene complexes with a two-dimensional hexagonal $\mathrm{C}_{60}{ }^{-}$packing motif. Chem. Eur. J. 2014, 20, 7268-7277. [CrossRef] [PubMed]

13. Ramirez, A.P. strongly geometrically frustrated magnets. Annu. Rev. Mater. Sci. 1994, 24, 453-480. [CrossRef]

14. Schiffer, P.; Ramirez, A.P. Recent experimental progress in the study of geometrical magnetic frustration. Comments Condens. Matter Phys. 1996, 18, 21-50.

15. Hirakawa, K.; Kadowaki, H.; Ubukoshi, K. Study of frustration effects in two-dimensional triangular lattice antiferromagnets-neutron powder diffraction study of $\mathrm{VX}_{2}, \mathrm{X} \equiv \mathrm{Cl}, \mathrm{Br}$ and I. J. Phys. Soc. Jpn. 1983, 52, 1814-1824. [CrossRef]

16. Ajiro, Y.; Asano, T.; Takagi, T.; Mekata, M.; Katori, H.A.; Goto, T. High-field magnetization process in the triangular lattice antiferromagnet $\mathrm{CuFeO}_{2}$ up to 100 T. Physica B 1994, 201, 71-74. [CrossRef]

17. Kimura, T.; Lashley, J.C.; Ramirez, A.P. Inversion-symmetry breaking in the noncollinear magnetic phase of the triangular-lattice antiferromagnet $\mathrm{CuFeO}_{2}$. Phys. Rev. B 2006, 73, 220401. [CrossRef]

18. Mitamura, H.; Mitsuda, S.; Kanetsuki, S.; Katori, H.A.; Sakakibara, T.; Kindo, K. Dielectric polarization measurements on the antiferromagnetic triangular lattice system $\mathrm{CuFeO}_{2}$ in pulsed high magnetic fields. J. Phys. Soc. Jpn. 2007, 76, 094709. [CrossRef]

19. Hirakawa, K.; Kadowaki, H.; Ubukoshi, K. Experimental studies of triangular lattice antiferromagnets with $S=1 / 2: \mathrm{NaTiO}_{2}$ and $\mathrm{LiNiO}_{2}$. J. Phys. Soc. Jpn. 1985, 54, 3526-3536. [CrossRef]

20. Tauber, A.; Moller, W.M.; Banks, E. Magnetic ordering in $\mathrm{LiCr}_{1-\mathrm{x}} \mathrm{Fe}_{\mathrm{x}} \mathrm{O}_{2}$. J. Solid State Chem. 1972, 4, $138-152$. [CrossRef]

21. Ajiro, Y.; Kikuchi, H.; Sugiyama, S.; Nakashima, T.; Shamoto, S.; Nakayama, N.; Kiyama, M.; Yamamoto, N.; Oka, Y. $\mathrm{Z}_{2}$ Vortex-induced broadening of the EPR linewidth in the two-dimensional triangular lattice antiferromagnets, $\mathrm{HCrO}_{2}$ and $\mathrm{LiCrO}_{2}$. J. Phys. Soc. Jpn. 1988, 57, 2268-2271. [CrossRef]

22. Moreno, N.O.; Israel, C.; Pagliuso, P.G.; Garcia-Adeva, A.J.; Rettori, C.; Sarrao, J.L.; Thompson, J.D.; Oseroff, S.B. Magnetic properties of the frustrated antiferromagnet $\mathrm{LiCrO}_{2}$. J. Mag. Mag. Mater. 2004, 272-276, e1023-e1024. [CrossRef]

23. Mekata, M. Antiferro-ferrimagnatic transition in triangular ising lattice. J. Phys. Soc. Jpn. 1977, 42, 76-82. [CrossRef] 
24. Plakhty, V.P.; Kulda, J.; Visser, D.; Moskvin, E.V.; Wosnitza, J. Chiral critical exponents of the triangular-lattice antiferromagnet $\mathrm{CsMnBr}_{3}$ as determined by polarized neutron scattering. Phys. Rev. Lett. 2000, 85, 3942-3945. [CrossRef] [PubMed]

25. Müller, A.; Kögerler, P.; Dress, A.W.M. Giant metal-oxide-based spheres and their topology: From pentagonal building blocks to keplerates and unusual spin systems. Coord. Chem. Rev. 2001, 222, 193-218. [CrossRef]

26. Takano, M.; Shinjo, T.; Kiyama, M.; Takada, T. Magnetic properties of jarosites, $\mathrm{RFe}_{3}(\mathrm{OH})_{6}\left(\mathrm{SO}_{4}\right)_{2}\left(\mathrm{R}=\mathrm{NH}_{4}\right.$, Na or K). J. Phys. Soc. Jpn. 1968, 25, 902. [CrossRef]

27. Townsend, M.G.; Longworth, G.; Roudaut, E. Triangular-spin, kagome plane in jarosites. Phys. Rev. B 1986, 33, 4919-4926. [CrossRef]

28. Maegawa, S.; Nishiyama, M.; Tanaka, N.; Oyamada, A.; Takano, M. Observation of successive phase transitions in kagomé lattice antiferromagnets $\mathrm{RFe}_{3}(\mathrm{OH})_{6}\left(\mathrm{SO}_{4}\right)_{2}\left[\mathrm{R}=\mathrm{NH}_{4}, \mathrm{Na}, \mathrm{K}\right]$. J. Phys. Soc. Jpn. 1996, 65, 2776-2778. [CrossRef]

29. Matan, K.; Ono, T.; Fukumoto, Y.; Sato, T.J.; Yamaura, J.; Yano, M.; Morita, K.; Tanaka, H. Pinwheel valence-bond solid and triplet excitations in the two-dimensional deformed kagome lattice. Nat. Phys. 2010, 6, 865-869. [CrossRef]

30. Nakatsuji, S.; Nambu, Y.; Tonomura, H.; Sakai, O.; Jonas, S.; Broholm, C.; Tsunetsugu, H.; Qiu, Y.; Maeno, Y. Spin Disorder on a triangular lattice. Science 2005, 309, 1697-1700. [CrossRef] [PubMed]

31. Manson, J.L.; Ressouche, E.; Miller, J.S. Spin Frustration in $\mathrm{M}^{\mathrm{II}}\left[\mathrm{C}(\mathrm{CN})_{3}\right]_{2}(\mathrm{M}=\mathrm{V}, \mathrm{Cr})$. A magnetism and neutron diffraction study. Inorg. Chem. 2000, 39, 1135-1141. [CrossRef] [PubMed]

32. Ramirez, A.P.; Espinosa, G.P.; Cooper, A.S. Strong Frustration and dilution-enhanced order in a quasi-2D spin glass. Phys. Rev. Lett. 1990, 64, 2070-2073. [CrossRef] [PubMed]

33. Okamoto, Y.; Nohara, M.; Aruga-Katori, H.; Takagi, H. Spin-liquid state in the $S=1 / 2$ hyperkagome antiferromagnet $\mathrm{Na}_{4} \mathrm{Ir}_{3} \mathrm{O}_{8}$. Phys. Rev. Lett. 2007, 99, 137207. [CrossRef] [PubMed]

34. Okamoto, Y.; Yoshida, H.; Hiroi, Z. Vesignieite $\mathrm{BaCu}_{3} \mathrm{~V}_{2} \mathrm{O}_{8}(\mathrm{OH})_{2}$ as a candidate spin- $1 / 2$ kagome antiferromagnet. J. Phys. Soc. Jpn. 2009, 78, 033701. [CrossRef]

35. Yoshida, H.; Yamaura, J.; Isobe, M.; Okamoto, Y.; Nilsen, G.J.; Hiroi, Z. Orbital switching in a frustrated magnet. Nat. Commun. 2012, 3, 860. [CrossRef] [PubMed]

36. Shimizu, Y.; Miyagawa, K.; Kanoda, K.; Maesato, M.; Saito, G. Spin liquid state in an organic mott insulator with a triangular lattice. Phys. Rev. Lett. 2003, 91, 107001. [CrossRef] [PubMed]

37. Konarev, D.V.; Khasanov, S.S.; Otsuka, A.; Saito, G. The Reversible Formation of a single-bonded $\left(\mathrm{C}_{60}{ }^{-}\right)_{2}$ dimer in ionic charge transfer complex: $\mathrm{Cp}_{2}{ }_{2} \mathrm{Cr} \cdot \mathrm{C}_{60}\left(\mathrm{C}_{6} \mathrm{H}_{4} \mathrm{Cl}_{2}\right)_{2}$. The molecular structure of $\left(\mathrm{C}_{60}{ }^{-}\right)_{2}$. J. Am. Chem. Soc. 2002, 124, 8520-8521. [CrossRef] [PubMed]

38. Konarev, D.V.; Khasanov, S.S.; Otsuka, A.; Saito, G.; Lyubovskaya, R.N. Negatively charged $\pi-\left(\mathrm{C}_{60}{ }^{-}\right)_{2}$ Dimer with biradical state at room temperature. J. Am. Chem. Soc. 2006, 128, 9292-9293. [CrossRef] [PubMed]

39. Stephens, P.W.; Bortel, G.; Faigel, G.; Tegze, M.; Jánossy, A.; Pekker, S.; Oszlanyi, G.; Forró, L. Polymeric fullerene chains in $\mathrm{RbC}_{60}$ and $\mathrm{KC}_{60}$. Nature 1994, 370, 636-639. [CrossRef]

40. Bommeli, F.; Degiorgi, L.; Wachter, P.; Legeza, Ö.; Jánossy, A.; Oszlanyi, G.; Chauvet, O.; Forro, L. Metallic conductivity and metal-insulator transition in $\left(A \mathrm{C}_{60}\right)_{n}(A=\mathrm{K}, \mathrm{Rb}$, and $\mathrm{Cs})$ linear polymer fullerides. Phys. Rev. B 1995, 51, 14794-14797. [CrossRef]

41. Bendele, G.M.; Stephens, P.W.; Prassides, K.; Vavekis, K.; Kortados, K.; Tanigaki, K. Effect of charge state on polymeric bonding geometry: The ground state of $\mathrm{Na}_{2} \mathrm{RbC}_{60}$. Phys. Rev. Lett. 1998, 80, 736-739. [CrossRef]

42. Margadonna, S.; Pontiroli, D.; Belli, M.; Shiroka, T.; Riccò, M.; Brunelli, M. $\mathrm{Li}_{4} \mathrm{C}_{60}$ : A polymeric fulleride with a two-dimensional architecture and mixed interfullerene bonding motifs. J. Am. Chem. Soc. 2004, 126, 15032-15033. [CrossRef] [PubMed]

43. Riccò, R.; Pontiroli, D.; Mazzani, M.; Gianferrari, F.; Pagliari, M.; Goffredi, A.; Brunelli, M.; Zandomeneghi, G.; Meier, B.H.; Shiroka, T. Fullerenium salts: A new class of $\mathrm{C}_{60}$-based compounds. J. Am. Chem. Soc. 2010, 132, 2064-2068. [CrossRef] [PubMed]

44. Oszlányi, G.; Baumgartner, G.; Faigel, L.; Forró, L. $\mathrm{Na}_{4} \mathrm{C}_{60}$ : An alkali intercalated two-dimensional polymer. Phys. Rev. Lett. 1997, 78, 4438-4441. [CrossRef]

45. Ohira, S.; Shimizu, Y.; Kanoda, K.; Saito, G. Spin liquid state in $\kappa-(\mathrm{BEDT}-\mathrm{TTF})_{2} \mathrm{Cu}_{2}(\mathrm{CN})_{3}$ studied by muon spin relaxation method. J. Low Temp. Phys. 2006, 142, 153-158. [CrossRef] 
46. Pratt, F.L.; Baker, P.J.; Blundell, S.J.; Lancaster, T.; Ohira-Kawamura, S.; Baines, C.; Shimizu, Y.; Kanoda, K.; Watanabe, I.; Saito, G. Magnetic and non-magnetic phases of a quantum spin liquid. Nature 2011, 471, 612-616. [CrossRef] [PubMed]

47. Shimizu, Y.; Maesato, M.; Saito, G.; Drozdova, O.; Ouahab, L. Transport properties of a Mott insulator $\kappa-(\mathrm{ET})_{2} \mathrm{Cu}_{2}(\mathrm{CN})_{3}$ under the uniaxial strain. Synth. Met. 2003, 133, 225-226. [CrossRef]

48. Saito, G.; Maesato, M. Organic superconductors. Mol. Cryst. Liq. Cryst. 2006, 455, 31-46. [CrossRef]

49. Shimizu, Y.; Maesato, M.; Saito, G. Uniaxial strain effects on mott and superconducting transitions in $\kappa-(\mathrm{ET})_{2} \mathrm{Cu}_{2}(\mathrm{CN})_{3}$. J. Phys. Soc. Jpn. 2011, 80, 074702. [CrossRef]

50. Kurosaki, Y.; Shimizu, Y.; Miyagawa, K.; Kanoda, K.; Saito, G. Mott transition from a spin liquid to a fermi liquid in the spin-frustrated organic conductor $\kappa-(\mathrm{ET})_{2} \mathrm{Cu}_{2}(\mathrm{CN})_{3}$. Phys. Rev. Lett. 2005, 95, 177001. [CrossRef] [PubMed]

51. Shimizu, Y.; Kasahara, H.; Furuta, T.; Miyagawa, K.; Kanoda, K.; Maesato, M.; Saito, G. Pressure-induced superconductivity and Mott transition in spin-liquid $\kappa-(\mathrm{ET})_{2} \mathrm{Cu}_{2}(\mathrm{CN})_{3}$ probed by ${ }^{13} \mathrm{C}$ NMR. Phys. Rev. $B$ 2010, 81, 224508. [CrossRef]

52. Saito, G.; Yoshida, Y. Development of conductive organic molecular assemblies: Organic metals, superconductors, and exotic functional materials. Bull. Chem. Soc. Jpn. 2007, 80,1-137. [CrossRef]

53. Saito, G.; Yoshida, Y. Organic superconductors. Chem. Rec. 2011, 11, 124-145. [CrossRef] [PubMed]

54. Hiramatsu, T.; Yoshida, Y.; Saito, G.; Otsuka, A.; Yamochi, H.; Maesato, M.; Shimizu, Y.; Ito, H.; Kishida, H. Quantum spin liquid: Design of a quantum spin liquid next to a superconducting state based on a dimer-type ET Mott insulator. J. Mater. Chem. C 2015, 3, 1378-1388. [CrossRef]

55. Balents, L. Spin liquids in frustrated magnets. Nature 2010, 464, 199-208. [CrossRef] [PubMed]

56. Norman, M.R. The Challenge of unconventional superconductivity. Science 2011, 332, 196-200. [CrossRef] [PubMed]

57. Powell, B.J.; McKenzie, R.H. Quantum frustration in organic Mott insulators: From spin liquids to unconventional superconductors. Rep. Prog. Phys. 2011, 74, 056501. [CrossRef]

58. Isono, T.; Kamo, H.; Ueda, A.; Takahashi, K.; Kimata, M.; Tajima, H.; Tsuchiya, S.; Terashima, T.; Uji, S.; Mori, H. Gapless quantum spin liquid in an organic spin-1/2 triangular-lattice $\kappa-\mathrm{H}_{3}$ (Cat-EDT-TTF)2. Phys. Rev. Lett. 2014, 112, 177201. [CrossRef] [PubMed]

59. Itou, T.; Oyamada, A.; Maegawa, S.; Tamura, M.; Kato, R. Spin-liquid state in an organic spin-1/2 system on a triangular lattice, $\mathrm{EtMe}_{3} \mathrm{Sb}\left[\mathrm{Pd}(\mathrm{dmit})_{2}\right]_{2}$. J. Phys. Condens. Matter 2007, 19, 145247. [CrossRef]

60. Shores, M.P.; Nytko, E.A.; Bartlett, B.M.; Nocera, D.G. A structurally perfect $S=1 / 2$ kagomé antiferromagnet. J. Am. Chem. Soc. 2005, 127, 13462-13463. [CrossRef] [PubMed]

61. Mandels, P.; Bert, F. Quantum kagome antiferromagnet $\mathrm{ZnCu}_{3}(\mathrm{OH})_{6} \mathrm{Cl}_{2}$. J. Phys. Soc. Jpn. 2010, 79, 011001. [CrossRef]

62. Han, T.-H.; Helton, J.S.; Chu, S.; Nocera, D.G.; Rodriguez-Rivera, J.A.; Broholm, C.; Lee, Y.S. Fractionalized excitations in the spin-liquid state of a kagome-lattice antiferromagnet. Nature 2012, 492, 406-410. [CrossRef] [PubMed]

63. Clark, L.; Orain, J.C.; Bert, F.; De Vries, M.A.; Aidoudi, F.H.; Morris, R.E.; Lightfoot, P.; Lord, J.S.; Telling, M.T.F.; Bonville, P.; et al. Gapless spin liquid ground State in the $S=1 / 2$ vanadium oxyfluoride kagome antiferromagnet $\left[\mathrm{NH}_{4}\right]_{2}\left[\mathrm{C}_{7} \mathrm{H}_{14} \mathrm{~N}\right]\left[\mathrm{V}_{7} \mathrm{O}_{6} \mathrm{~F}_{18}\right]$. Phys. Rev. Lett. 2013, 110, 207208. [CrossRef] [PubMed]

64. Zhang, B.; Baker, P.J.; Zhang, Y.; Wang, D.; Wang, Z.; Su, S.; Zhu, D.; Pratt, F.L. Quantum spin liquid from a three-dimensional copper-oxalate framework. J. Am. Chem. Soc. 2018, 140, 122-125. [CrossRef] [PubMed]

65. Shimizu, Y.; Hiramatsu, T.; Maesato, M.; Otsuka, A.; Yamochi, H.; Ono, A.; Itoh, M.; Yoshida, M.; Takigawa, M.; Yoshida, Y.; et al. Pressure-tuned exchange coupling of a quantum spin liquid in the molecular triangular lattice $\kappa-(\mathrm{ET})_{2} \mathrm{Ag}_{2}(\mathrm{CN})_{3}$. Phys. Rev. Lett. 2016, 117, 107203. [CrossRef] [PubMed]

66. Abdel-Jawad, M.; Terasaki, I.; Sasaki, T.; Yoneyama, N.; Kobayashi, N.; Uesu, Y.; Hotta, C. Anomalous dielectric response in the dimer Mott insulator $\kappa$-(BEDT-TTF $)_{2} \mathrm{Cu}_{2}(\mathrm{CN})_{3}$. Phys. Rev. B 2010, 82, 125119. [CrossRef]

67. Hiramatsu, H.; Yoshida, Y.; Saito, G.; Otsuka, A.; Yamochi, H.; Shimizu, Y.; Hattori, Y.; Nakamura, Y.; Kishida, H.; Ito, H.; et al. Spin frustration in antiperovskite systems: $\left(\mathrm{TTF}^{\bullet+} \text { or } \mathrm{TSF}^{\bullet+}\right)_{3}\left[\left(\mathrm{Mo}_{6} \mathrm{X}_{14}\right)^{2-} \mathrm{Y}^{-}\right]$. J. Mater. Chem. C 2015, 3, 11046-11054. [CrossRef]

68. Bray, J.W.; Interrante, L.V.; Jacobs, I.S.; Bonner, J.C. The Spin-Peierls Transition. In Extended Linear Chain Compounds: Volume 3; Miller, J.S., Ed.; Plenum Press: New York, NY, USA, 1983; pp. 353-415. 
69. Batail, P.; Livage, C.; Parkin, S.S.P.; Coulon, C.; Martin, J.D.; Canadell, E. Antiperovskite structure with ternary tetrathiafulvalenium salts: construction, distortion, and antiferromagnetic ordering. Angew. Chem. Int. Ed. Engl. 1991, 30, 1498-1500. [CrossRef]

70. Conwell, E. (Ed.) Semiconductors and Semimetals: Volume 27, Highly Conducting Quasi-One-Dimensional Organic Crystals; Academic Press: Boston, MA, USA, 1988.

71. Kosaka, M.; Tanigaki, K.; Tanaka, T.; Atake, T.; Lappas, A.; Prassides, K. Conducting phase of rapidly cooled $A \mathrm{C}_{60}(A=\mathrm{Cs}$ and $\mathrm{Rb})$. Phys. Rev. B 1995, 51, 12018-12021. [CrossRef]

72. Brouet, V.; Alloul, H.; Forró, L. Coexistence of spin singlets and metallic behavior in simple cubic $\mathrm{CsC}_{60}$. Phys. Rev. B 2002, 66, 155123. [CrossRef]

73. Gunnarsson, O.; Koch, E.; Martin, R.M. Mott transition in degenerate Hubbard models: Application to doped fullerenes. Phys. Rev. B 1996, 54, R11026-R11029. [CrossRef]

74. Gunnarsson, O. Superconductivity in fullerides. Rev. Mod. Phys. 1997, 69, 575-606. [CrossRef]

75. Tinkham, M. Introduction to Superconductivity, 2nd ed.; McGraw-Hill: New York, NY, USA, 1996.

76. Rosseinsky, M.J.; Ramirez, A.P.; Glarum, S.H.; Murphy, D.W.; Haddon, R.C.; Hebard, A.F.; Palstra, T.T.M.; Kortan, A.R.; Zahurak, S.M.; Makhija, A.V. Superconductivity at $28 \mathrm{~K}$ in $\mathrm{Rb}_{\mathrm{x}} \mathrm{C}_{60}$. Phys. Rev. Lett. 1991, 66, 2830-2832. [CrossRef] [PubMed]

77. Tanigaki, K.; Ebbesen, T.W.; Saito, S.; Mizuki, J.; Tsai, J.S.; Kubo, Y.; Kuroshima, S. Superconductivity at 33 K in $\mathrm{Cs}_{x} \mathrm{Rb}_{y} \mathrm{C}_{60}$. Nature 1991, 352, 222-223. [CrossRef]

78. Rosseinsky, M.J. Recent developments in the chemistry and physics of metal fullerides. Chem. Mater. 1998, 10, 2665-2685. [CrossRef]

79. Andreoni, W. (Ed.) The Physics of Fullerene-Based and Fullerene-Related Materials; Springer: Dordrecht, The Netherlands, 2000.

80. Reed, C.A.; Bolskar, R.D. Discrete fulleride anions and fullerenium cations. Chem. Rev. 2000, 100, 1075-1120. [CrossRef] [PubMed]

81. Fleming, R.M.; Ramirez, A.P.; Rosseinsky, M.J.; Murphy, D.W.; Haddon, R.C.; Zahurak, S.M.; Makhija, A.V. Relation of structure and superconducting transition temperatures in $\mathrm{A}_{3} \mathrm{C}_{60}$. Nature 1991, 352, 787-788. [CrossRef]

82. Ganin, A.Y.; Takabayashi, Y.; Khimyak, Y.Z.; Margadonna, S.; Tamai, A.; Rosseinsky, M.J.; Prassides, K. Bulk superconductivity at $38 \mathrm{~K}$ in a molecular system. Nat. Mater. 2008, 7, 367-371. [CrossRef] [PubMed]

83. Ganin, A.Y.; Takabayashi, Y.; Jeglič, Y.; Arčon, D.; Potočnik, A.; Baker, P.J.; Ohishi, Y.; McDonald, M.T.; Tzirakis, M.D.; McLennan, A.; et al. Polymorphism control of superconductivity and magnetism in $\mathrm{Cs}_{3} \mathrm{C}_{60}$ close to the Mott transition. Nature 2010, 466, 221-225. [CrossRef] [PubMed]

84. Özdaş, E.; Kortan, A.R.; Kopylov, N.; Ramirez, A.P.; Siegrist, T.; Rabe, K.M.; Bair, H.E.; Schuppler, S.; Citrin, P.H. Superconductivity and cation-vacancy ordering in the rare-earth fulleride $\mathrm{Yb}_{2.75} \mathrm{C}_{60}$. Nature 1995, 375, 126-129. [CrossRef]

85. Chen, X.H.; Roth, G. Superconductivity at $8 \mathrm{~K}$ in samarium-doped C60. Phys. Rev. B 1995, 52, 15534-15536. [CrossRef]

86. Brown, C.M.; Taga, S.; Gogia, B.; Kordatos, K.; Margadonna, S.; Prassides, K.; Iwasa, Y.; Tanigaki, K.; Fitch, A.N.; Pattison, P. Structural and electronic properties of the noncubic superconducting fullerides $A^{\prime}{ }_{4} \mathrm{C}_{60}\left(A^{\prime}=\mathrm{Ba}, \mathrm{Sr}\right)$. Phys. Rev. Lett. 1999, 83, 2258-2261. [CrossRef]

87. Baenitz, M.; Heinze, M.; Lüders, K.; Werner, H.; Schlögl, R.; Weiden, M.; Sparn, G.; Steglich, F. Superconductivity of Ba doped $\mathrm{C}_{60}$-Susceptibility results and upper critical field. Solid State Commun. 1995, 96, 539-544. [CrossRef]

88. Iwasa, Y.; Hayashi, H.; Furudate, T.; Mitani, T. Superconductivity in $\mathrm{K}_{3} \mathrm{Ba}_{3} \mathrm{C}_{60}$. Phys. Rev. B 1996, 54, 14960-14962. [CrossRef]

89. Ksari-Habiles, Y.; Claves, D.; Chouteau, G.; Touzain, P.; Jeandey, C.; Oddou, J.L.; Stepanov, A. Superexchange and magnetic relaxation in novel Eu-DOPED C 60 phases. J. Phys. Chem. Solids 1997, 58, 1771-1778. [CrossRef]

90. Maruyama, Y.; Motohashi, S.; Sakai, N.; Watanabe, K.; Suzuki, K.; Ogata, H.; Kubozono, Y. Possible competition of superconductivity and ferromagnetism in $\mathrm{Ce}_{x} \mathrm{C}_{60}$ compounds. Solid State Commun. 2002, 123, 229-233. [CrossRef]

91. Zadik, R.H.; Takabayashi, Y.; Klupp, G.; Colman, R.H.; Ganin, A.Y.; Potočnik, A.; Jeglič, P.; Arčon, D.; Matus, P.; Kamarás, K.; et al. Optimized unconventional superconductivity in a molecular Jahn-Teller metal. Sci. Adv. 2015, 1, e1500059. [CrossRef] [PubMed] 
92. Sasaki, S.; Matsuda, A.; Chu, C.W. Fermi-liquid behavior and BCS s-wave pairing of $\mathrm{K}_{3} \mathrm{C}_{60}$ observed by ${ }^{13}$ C-NMR. J. Phys. Soc. Jpn. 1994, 63, 1670-1673. [CrossRef]

93. Kiefl, R.F.; MacFarlane, W.A.; Chow, K.H.; Dunsiger, S.; Duty, T.L.; Johnston, T.M.S.; Schneider, J.W.; Sonier, J.; Brard, L.; Strongin, R.M.; et al. Coherence Peak and Superconducting energy gap in $\mathrm{Rb}_{3} \mathrm{C}_{60}$ observed by muon spin relaxation. Phys. Rev. Lett. 1993, 70, 3987-3990. [CrossRef] [PubMed]

94. Allemand, P.-M.; Khemani, K.C.; Koch, A.; Wudl, F.; Holczer, K.; Donovan, S.; Grüner, G.; Thompson, J.D. Organic Molecular Soft ferromagnetism in a fullerene $\mathrm{C}_{60}$. Science 1991, 253, 301-303. [CrossRef] [PubMed]

95. Konerav, D.V.; Lyubovskaya, R.N. Molecular design, study of the structures and properties of ionic fullerene compounds. Rus. Chem. Rev. 2012, 81, 336-366. [CrossRef]

96. Prassides, K. Polymer and Dimer Phases in Doped Fullerenes. In The Physics of Fullerene-Based and FullereneRelated Materials; Andreoni, W., Ed.; Kluwer Academic Publishers: Boston, MA, USA, 2000; pp. 175-202.

97. Eklund, P.C.; Rao, A.M. (Eds.) Fullerene Polymers and Fullerene Polymer Composites; Springer: Berlin, Germany, 2000.

98. Rosseinsky, M.J. Fullerene intercalation chemistry. J. Mater. Chem. 1995, 5, 1497-1513. [CrossRef]

99. Bondi, A. Van der waals volumes and radii. J. Phys. Chem. 1964, 68, 441-451. [CrossRef]

100. Brouet, V.; Alloul, H.; Yoshinari, Y.; Forro, L. NMR Evidence for $1 \mathrm{D}$ Antiferromagnetic Properties in $\mathrm{Cs}_{1} \mathrm{C}_{60}$ and $\mathrm{Rb}_{1} \mathrm{C}_{60}$ Polymers. Phys. Rev. Lett. 1996, 76, 3638-3641. [CrossRef] [PubMed]

101. Schirber, J.E.; Morosin, B.; Kwei, G.H.; Yildirim, T.; Fischer, J.E.; Jorgensen, J.D. Superconductivity in the polymeric phase of $\mathrm{Na}_{2} \mathrm{CsC}_{60}$. Physica C 2001, 353, 207-212. [CrossRef]

102. Kozhemyakina, N.V.; Amsharov, K.Y.; Nuss, J.; Jansen, M. Synthesis and Structure analysis of $(\mathrm{K}[\mathrm{DB} 18 \mathrm{C} 6])_{4}\left(\mathrm{C}_{60}\right)_{5} \cdot 12 \mathrm{THF}$ containing $\mathrm{C}_{60}$ in three different bonding states. Chem. Eur. J. 2011, 17, 1798-1805. [CrossRef] [PubMed]

103. Konarev, D.V.; Khasanov, S.S.; Saito, G.; Lyubovskaya, R.N. Design of molecular and ionic complexes of fullerene $\mathrm{C}_{60}$ with metal(II) octaethylporphyrins, $\mathrm{M}^{\mathrm{II}} \mathrm{OEP}(\mathrm{M}=\mathrm{Zn}, \mathrm{Co}, \mathrm{Fe}$, and $\mathrm{Mn})$ containing coordination $\mathrm{M}-\mathrm{N}($ ligand $)$ and $\mathrm{M}-\mathrm{C}\left(\mathrm{C}_{60}{ }^{-}\right)$bonds. Cryst. Growth Des. 2009, 9, 1170-1181. [CrossRef]

104. Konarev, D.V.; Khasanov, S.S.; Mukhamadieva, G.R.; Zorina, L.V.; Otsuka, A.; Yamochi, H.; Saito, G.; Lyubovskaya, R.N. Magnetic and structural transitions at dimerization of $\mathrm{C}_{60}{ }^{\bullet-}$ in ionic fullerene complexes with metalloporphyrins: $\left\{\left(\mathrm{TMP}^{+}\right)_{2} \cdot \mathrm{MITPP}\right\} \cdot\left(\mathrm{C}_{60}{ }^{-}\right)_{2} \cdot\left(\mathrm{C}_{6} \mathrm{H}_{4} \mathrm{Cl}_{2}\right)_{2} \cdot\left(\mathrm{C}_{6} \mathrm{H}_{5} \mathrm{CN}\right)_{2}(\mathrm{M}=\mathrm{Zn}$ and Mn $)$. Inorg. Chem. 2010, 49, 3881-3887. [CrossRef] [PubMed]

105. Konarev, D.V.; Khasanov, S.S.; Kuzmin, A.V.; Otsuka, A.; Yamochi, H.; Saito, G.; Lyubovskaya, R.N. Effective magnetic coupling with strong spin frustration in $\left(\mathrm{Ph}_{3} \mathrm{MeP}^{+}\right)\left(\mathrm{C}_{60}{ }^{\bullet-}\right)$ and reversible $\mathrm{C}_{60}{ }^{\bullet-}$ dimerization in $\left(\mathrm{Ph}_{3} \mathrm{MeP}^{+}\right)\left(\mathrm{C}_{60}{ }^{--}\right) \cdot \mathrm{C}_{6} \mathrm{H}_{5} \mathrm{CN}$. Effect of solvent on structure and properties. New J. Chem. 2016, 40, 2792-2798. [CrossRef]

106. Konarev, D.V.; Khasanov, S.S.; Otsuka, A.; Yamochi, H.; Saito, G.; Lyubovskaya, R.N. Effect of the cooling

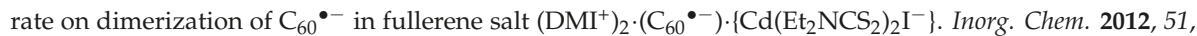
3420-3426. [CrossRef] [PubMed]

107. Pénicaud, A.; Peréz-Benítez, A.; Gleason, V.R.; Muñoz, P.E.; Escudero, R. Electrocrystallizing $\mathrm{C}_{60}$ : Synthesis, single crystal X-ray structure, and magnetic (ESR, SQUID) characterization of $\left[\left(\mathrm{C}_{6} \mathrm{H}_{5}\right)_{4} \mathrm{P}\right]_{2}\left[\mathrm{C}_{60}\right][\mathrm{I}]_{x}$. J. Am. Chem. Soc. 1993, 115, 10392-10393. [CrossRef]

108. Bilow, U.; Jansen, M. Electrocrystallisation and crystal structure determination of $\mathrm{Ph}_{4} \mathrm{PC}_{60} \cdot \mathrm{Ph}_{4} \mathrm{PCl}$. J. Chem. Soc. Chem. Commun. 1994, 403-404. [CrossRef]

109. Bilow, U.; Jansen, M. Electrocrystallization of fullerides. Z. Anorg. Allg. Chem. 1995, 621, 982-986. [CrossRef]

110. Gritsenko, V.V.; Dyachenko, O.A.; Shilov, G.V.; Spitsyna, N.G.; Yagubskii, E.B. Crystal and molecular structures of new fullerides, $\left(\mathrm{Ph}_{4} \mathrm{P}\right)_{2} \mathrm{C}_{60} \mathrm{Hal}\left(\mathrm{Hal}=\mathrm{Br}\right.$ or I) and $\left(\mathrm{Ph}_{4} \mathrm{As}\right)_{2} \mathrm{C}_{60} \mathrm{Cl}$. Rus. Chem. Bull. 1997, 46, 1878-1882. [CrossRef]

111. Kobayashi, H.; Moriyama, H.; Kobayashi, A.; Watanabe, T. Synthesis and characterization of some fulleride compounds by electrocrystallization of $C_{60}$. Synth. Met. 1995, 70, 1451-1452. [CrossRef]

112. Hong, J.; Shores, M.P.; Elliott, C.M. Establishment of structure-conductivity relationship for Tris $\left(2,2^{\prime}-\right.$ bipyridine) ruthenium ionic $\mathrm{C}_{60}$ salts. Inorg. Chem. 2010, 49, 11378-11385. [CrossRef] [PubMed]

113. Fässler, T.F.; Hoffmann, R.; Hoffmann, S.; Wörle, M. Triple-decker type coordination of a fullerene trianion in $\left[\mathrm{K}([18] \text { crown-6) }]_{3}\left[\eta^{6}, \eta^{6}-\mathrm{C}_{60}\right]\left(\eta^{3}-\mathrm{C}_{6} \mathrm{H}_{5} \mathrm{CH}_{3}\right)_{2}\right.$ - Single crystal structure and magnetic properties. Angew. Chem. Int. Ed. 2000, 39, 2091-2094. [CrossRef] 
114. Boeddinghaus, M.B.; Salzinger, M.; Fässler, T.F. Synthesis, X-ray single-crystal structure determination, and magnetic properties of $\left[\mathrm{Rb}(\text { benzo[18]crown-6) }]^{+}\right.$salts containing well-ordered fulleride trianions $\mathrm{C}_{60}{ }^{3-}$. Chem. Eur. J. 2009, 15, 3261-3267. [CrossRef] [PubMed]

115. Fässler, T.F.; Spiekermann, A.; Spahr, M.E.; Nesper, R. Unprecedented layered structure of a fulleride: synthesis, structure, and magnetic properties of a potassium-containing salt with a $\mathrm{C}_{60}{ }^{2-}$ counterion. Angew. Chem. Int. Ed. Engl. 1997, 36, 486-488. [CrossRef]

116. Janiak, C.; Mühle, S.; Hemling, H.; Köhler, K. The solid-state structure of $\mathrm{K}_{3} \mathrm{C}_{60}(\mathrm{THF})_{14}$. Polyhedron 1996, 15 , 1559-1563. [CrossRef]

117. Kosaka, M.; Tanigaki, K.; Prassides, K.; Margadonna, S.; Lappas, A.; Brown, C.M.; Fitch, A.N. Superconductivity in $\mathrm{Li}_{x} \mathrm{CsC}_{60}$ fullerides. Phys. Rev. B 1999, 59, R6628-R6630. [CrossRef]

118. Tanigaki, K.; Prassides, K. Conducting and superconducting properties of alkali-metal $\mathrm{C}_{60}$ fullerides. J. Mater. Chem. 1995, 5, 1515-1527. [CrossRef]

119. Tanigaki, K.; Hirosawa, I.; Ebbesen, T.W.; Mizuki, J.; Shimakawa, Y.; Kubo, Y.; Tsai, J.S.; Kuroshima, S. Superconductivity in sodium- and lithium-containing alkali-metal fullerides. Nature 1992, 356, 419-421. [CrossRef]

120. Takabayashi, Y.; Ganin, A.Y.; Jeglič, P.; Arčon, D.; Takano, T.; Iwasa, Y.; Ohishi, Y.; Takata, M.; Takeshita, N.; Prassides, K.; et al. The disorder-free Non-BCS superconductor $\mathrm{Cs}_{3} \mathrm{C}_{60}$ emerges from an antiferromagnetic insulator parent state. Science 2009, 323, 1585-1590. [CrossRef] [PubMed]

121. Suzuki, Y.; Shibasaki, S.; Kubozono, Y.; Kambe, T. Antiferromagnetic resonance in the Mott insulator fcc-Cs ${ }_{3} \mathrm{C}_{60}$. J. Phys. Condens. Matter 2013, 25, 366001. [CrossRef] [PubMed]

122. Ihara, Y.; Alloul, H.; Wzietek, P.; Pontiroli, D.; Mazzani, M.; Riccò, M. NMR Study of the Mott transitions to superconductivity in the two $\mathrm{Cs}_{3} \mathrm{C}_{60}$ Phases. Phys. Rev. Lett. 2010, 104, 256402. [CrossRef] [PubMed]

123. Takenobu, T.; Muro, T.; Iwasa, Y.; Mitani, T. Antiferromagnetism and phase diagram in ammoniated alkali fulleride salts. Phys. Rev. Lett. 2000, 85, 381-384. [CrossRef] [PubMed]

124. Zhou, O.; Palstra, T.T.M.; Iwasa, Y.; Fleming, R.M.; Hebard, A.F.; Sulewski, P.E.; Murphy, D.W.; Zegarski, B.R. Structural and electronic properties of $\left(\mathrm{NH}_{3}\right)_{x} \mathrm{~K}_{3} \mathrm{C}_{60}$. Phys. Rev. B 1995, 52, 483-489. [CrossRef]

125. Iwasa, Y.; Takenobu, T. Superconductivity, Mott-Hubbard states, and molecular orbital order in intercalated fullerides. J. Phys.: Condens. Matter 2003, 15, R495-R519. [CrossRef]

126. Welp, U.; Fleshler, S.; Kwok, W.K.; Crabtree, G.W.; Carlson, K.D.; Wang, H.H.; Geiser, U.; Williams, J.M.; Hitsman, V.M. Weak Ferromagnetism in $\kappa-(\mathrm{ET})_{2} \mathrm{Cu}\left[\mathrm{N}(\mathrm{CN})_{2}\right] \mathrm{Cl}$, where (ET) is Bis(ethylenedithio) tetrathiafulvalene. Phys. Rev. Lett. 1992, 69, 840-843. [CrossRef] [PubMed]

127. Miyagawa, K.; Kawamoto, A.; Nakazawa, Y.; Kanoda, K. antiferromagnetic ordering and spin structure in the organic conductor, $\kappa-(\mathrm{BEDT}-\mathrm{TTF})_{2} \mathrm{Cu}\left[\mathrm{N}(\mathrm{CN})_{2}\right] \mathrm{Cl}$. Phys. Rev. Lett. 1995, 75, 1174-1177. [CrossRef] [PubMed]

128. Ito, H.; Asai, T.; Shimizu, Y.; Hayama, H.; Yoshida, Y.; Saito, G. Pressure-induced superconductivity in the antiferromagnet $\kappa$-(ET) ${ }_{2} \mathrm{CF}_{3} \mathrm{SO}_{3}$ with quasi-one-dimensional triangular spin lattice. Phys. Rev. B 2016, 94 , 020503. [CrossRef]

129. Coldea, R.; Tennant, D.A.; Tsvelik, A.M.; Tylczynski, Z. Experimental realization of a 2D fractional quantum spin liquid. Phys. Rev. Lett. 2001, 86, 1335-1338. [CrossRef] [PubMed]

130. Antropov, P.V.; Gunnarsson, O.; Jepsen, O. Coulomb integrals and model Hamiltonians for $\mathrm{C}_{60}$. Phys. Rev. B 1992, 46, 13647-13650. [CrossRef]

131. Pederson, M.R.; Quong, A.A. Polarizabilities, charge states, and vibrational modes of isolated fullerene molecules. Phys. Rev. B 1992, 46, 13584-13591. [CrossRef]

132. Martin, R.L.; Ritchie, J.P. Coulomb and exchange interactions in $\mathrm{C}_{60}{ }^{n-}$. Phys. Rev. B 1993, 48, 4845-4849. [CrossRef]

133. Brühwiler, P.A.; Maxwell, A.J.; Nilsson, A.; Mårtensson, N.; Gunnarsson, O. Auger and photoelectron study of the Hubbard $U$ in $\mathrm{C}_{60}, \mathrm{~K}_{3} \mathrm{C}_{60}$, and $\mathrm{K}_{6} \mathrm{C}_{60}$. Phys. Rev. B 1993, 48, 18296-18299. [CrossRef]

134. Lof, R.W.; van Veenendaal, M.A.; Koopmans, B.; Jonkman, H.T.; Sawatzky, G.A. Band gap, excitons, and coulomb interaction in solid $C_{60}$. Phys. Rev. Lett. 1992, 68, 3924-3927. [CrossRef] [PubMed]

135. LeBlanc, O.H., Jr. On the Electrical conductivities of tetracyanoquinodimethan anion-radical salt. J. Chem. Phys. 1965, 42, 4307-4308. [CrossRef] 
136. Douthwaite, R.E.; Green, M.A.; Green, M.L.H.; Rosseinsky, M.J. Synthesis, reactivity, structure and electronic properties of $\left[\mathrm{N}\left(\mathrm{CH}_{3}\right)_{4}\right] \mathrm{C}_{60} \cdot 1.5$ thf: Fullerides with simple hexagonal packing. J. Mater. Chem. 1996, 6, 1913-1920. [CrossRef]

137. Douthwaite, R.E.; Brough, A.R.; Green, M.L.H. Synthesis and characterisation of $\mathrm{NaC}_{60} \cdot 5$ thf. J. Chem. Soc. Chem. Commun. 1994, 3, 267-268. [CrossRef]

138. Kromer, A.; Wedig, U.; Roduner, E.; Jansen, M.; Amsharov, K.Y. Counterintuitive anisotropy of electron transport properties in $\mathrm{KC}_{60}(\mathrm{THF})_{5}$.2THF fulleride. Angew. Chem. Int. Ed. 2013, 52, 12610-12614. [CrossRef] [PubMed]

139. Kodera, H. Dyson effect in the electron spin resonance of phosphorus doped silicon. J. Phys. Soc. Jpn. 1970, 28, 89-98. [CrossRef]

140. Saito, G.; Teramoto, T.; Otsuka, A.; Sugita, Y.; Ban, T.; Kusunoki, M.; Sakaguchi, K. Preparation and ionicity of $\mathrm{C}_{60}$ charge transfer complexes. Synth. Met. 1994, 64, 359-368. [CrossRef]

141. Konarev, D.V.; Khasanov, S.S.; Vorontsov, I.I.; Saito, G.; Antipin, M.Y.; Otsuka, A.; Lyubovskaya, R.N. The formation of a single-bonded $\left(\mathrm{C}_{70}{ }^{-}\right)_{2}$ dimer in a new ionic multicomponent complex of cyclotriveratrylene: $\left(\mathrm{Cs}^{+}\right)_{2}\left(\mathrm{C}_{70}{ }^{-}\right)_{2} \cdot \mathrm{CTV} \cdot(\mathrm{DMF})_{7}\left(\mathrm{C}_{6} \mathrm{H}_{6}\right)_{0.75}$. Chem. Commun. 2002, 21, 2548-2549. [CrossRef]

142. Konarev, D.V.; Khasanov, S.S.; Otsuka, A.; Yoshida, Y.; Saito, G. Synthesis and crystal structure of ionic multicomponent complex: $\left\{\left[\mathrm{Cr}^{\mathrm{I}}(\mathrm{PhH})_{2}\right]^{\bullet+}\right\}_{2}\left[\mathrm{Co}^{\Pi \mathrm{TPP}}\left(\mathrm{C}_{60}(\mathrm{CN})_{2}\right)\right]^{-}\left[\mathrm{C}_{60}(\mathrm{CN})_{2}\right]^{\bullet-} \cdot 3\left(o-\mathrm{C}_{6} \mathrm{H}_{4} \mathrm{Cl}_{2}\right)$ containing $\mathrm{C}_{60}(\mathrm{CN})_{2}{ }^{\bullet-}$ radical anion and $\sigma$-bonded diamagnetic $\mathrm{Co}^{\mathrm{II}} \mathrm{TPP}\left(\mathrm{C}_{60}(\mathrm{CN})_{2}\right)^{-}$anion. J. Am. Chem. Soc. 2002, 124, 7648-7649. [CrossRef] [PubMed]

143. Konarev, D.V.; Khasanov, S.S.; Saito, G.; Otsuka, A.; Yoshida, Y.; Lyubovskaya, R.N. Formation of single-bonded $\left(\mathrm{C}_{60}{ }^{-}\right)_{2}$ and $\left(\mathrm{C}_{70}{ }^{-}\right)_{2}$ dimers in crystalline ionic complexes of fullerenes. J. Am. Chem. Soc. 2003, 125, 10074-10083. [CrossRef] [PubMed]

144. Konarev, D.V.; Neretin, I.S.; Saito, G.; Slovokhotov, Y.L.; Otsuka, A.; Lyubovskaya, R.N. Multicomponent ionic complexes of cobalt(ii) tetraphenylporphyrin with $\mathrm{c}_{60}$ fullerides-Transition from the $\sigma$-bonded $\left[\left(\mathrm{Co}^{\mathrm{II}} \mathrm{TPP}\right) \cdot\left(\mathrm{C}_{60}{ }^{-}\right)\right]$anion to nonbonded $\mathrm{Co}^{\mathrm{II}} \mathrm{TPP}$ and $\mathrm{C}_{60}{ }^{\bullet-}$ components. Eur. J. Inorg. Chem. 2004, 2004, 1794-1798. [CrossRef]

145. Konarev, D.V.; Khasanov, S.S.; Otsuka, A.; Saito, G.; Lyubovskaya, R.N. Ionic fullerene complex $\left(\mathrm{DMI}^{+}\right)_{3} \cdot\left(\mathrm{C}_{60}{ }^{\bullet-}\right) \cdot\left(\mathrm{I}^{-}\right)_{2}$ with $2 \mathrm{H}$-hexagonal fullerene packing and 3-D DMI ${ }^{+} \mathrm{I}^{-}$network. CrystEngComm 2009, 11, 811-816. [CrossRef]

146. Konarev, D.V.; Khasanov, S.S.; Otsuka, A.; Maesato, M.; Saito, G.; Lyubovskaya, R.N. A two-dimensional organic metal based on fullerene. Angew. Chem. Int. Ed. 2010, 49, 4829-4832. [CrossRef] [PubMed]

147. Konarev, D.V.; Khasanov, S.S.; Otsuka, A.; Yamochi, H.; Saito, G.; Lyubovskaya, R.N. Strong Antiferromagnetic coupling of spins in the $\left(\mathrm{MDABCO}^{+}\right)\left(\mathrm{C}_{60}{ }^{\bullet-}\right)$ salt with $3 \mathrm{D}$ close packing of the $\mathrm{C}_{60}{ }^{\bullet-}$ radical anions $\left(\mathrm{MDABCO}^{+}\right.$: N-Methyldiazabicyclooctanium Cation). Chem. Asian J. 2014, 9, 1629-1635. [CrossRef] [PubMed]

148. Konarev, D.V.; Kuzmin, A.V.; Khasanov, S.S.; Ishikawa, M.; Otsuka, A.; Yamochi, H.; Saito, G.; Lyubovskaya, R.N. Structure and magnetic properties of the ionic fullerene salt $\left(\mathrm{TMP}^{+}\right) \cdot\left(\mathrm{C}_{60}{ }^{\bullet-}\right) \cdot \mathrm{C}_{6} \mathrm{H}_{5} \mathrm{CN}$ containing layers of monomeric $\mathrm{C}_{60}{ }^{\bullet-}$ radical anions. New J. Chem. 2013, 37, 2521-2527. [CrossRef]

149. Konarev, D.V.; Khasanov, S.S.; Otsuka, A.; Ishikawa, M.; Yamochi, H.; Saito, G.; Lyubovskaya, R.N. Formation of hexagonal fullerene layers from neutral and negatively charged fullerenes in $\left\{\left(\mathrm{Ph}_{3} \mathrm{P}\right)_{3} \mathrm{Au}^{+}\right\}_{2}\left(\mathrm{C}_{60}{ }^{\bullet-}\right)_{2}$ $\left(\mathrm{C}_{60}\right) \cdot \mathrm{C}_{6} \mathrm{H}_{4} \mathrm{Cl}_{2}$ containing gold cations with the $\mathrm{C}_{3 v}$ symmetry. Inorg. Chem. 2014, 53, 6850-6855. [CrossRef] [PubMed]

150. Yoshida, Y.; Ito, H.; Maesato, M.; Shimizu, Y.; Hayama, H.; Hiramatsu, T.; Nakamura, Y.; Kishida, H.; Koretsune, T.; Hotta, C.; et al. Spin-disordered quantum phases in a quasi-one-dimensional triangular lattice. Nat. Phys. 2015, 11, 679-683. [CrossRef]

151. Sugano, T.; Saito, G.; Kinoshita, M. Conduction-electron-spin resonance in organic conductors: $\alpha$ and $\beta$ phases of di[bis(ethylenedithiolo)tetrathiafulvalene]triiodide [(BEDT-TTF) ${ }_{2} \mathrm{I}_{3}$ ]. Phys. Rev. B 1986, 34, 117-125. [CrossRef]

152. Yagubskii, E.B.; Shchegolev, I.F.; Laukhin, V.N.; Kononovich, P.A.; Karstovnik, M.V.; Zvarykina, A.V.; Buravov, L.I. Normal-pressure superconductivity in an organic metal (BEDT-TTF) ${ }_{2} \mathrm{I}_{3}$ [bis (ethylene dithiolo) tetrathiof ulvalene triiodide]. JETP Lett. 1984, 39, 12-16.

153. Yildirim, T.; Fischer, J.E.; Harris, A.B.; Stephens, P.W.; Liu, D.; Brard, L.; Strongin, R.M.; Smith, A.B. Orientational phase transition in $\mathrm{Na}_{x} \mathrm{C}_{60}(1<x<3)$. Phys. Rev. Lett. 1993, 71, 1383-1386. [PubMed] 
154. Prassides, K.; Christides, C.; Thomas, I.M.; Mizuki, J.; Tanigaki, K.; Hirosawa, I.; Ebbesen, T.W. Crystal structure, bonding, and phase transition of the superconducting $\mathrm{Na}_{2} \mathrm{CsC}_{60}$ fulleride. Science 1994, 263, 950-954. [CrossRef] [PubMed]

155. Mihailovic, D.; Arcon, D.; Verturini, P.; Blinc, R.; Omerzu, A.; Cevc, P. Orientational and magnetic ordering of buckyballs in TDAE- $\mathrm{C}_{60}$. Science 1995, 268, 400-402. [CrossRef] [PubMed]

156. Akada, M.; Yamamoto, T.; Kumashiro, R.; Hojyo, A.; Matsui, H.; Toyota, N.; Lu, J.P.; Tanigaki, K. Erectric transport and modulated density of states in rotational order and disorder in $\mathrm{Na}_{2} \mathrm{CsC}_{60}$. In Multifunctional Conducting Molecular Materials; Saito, G., Wudl, F., Haddon, R.C., Tanigaki, K., Enoki, T., Katz, H.E., Maesato, M., Eds.; RSC Publishing: Cambridge, UK, 2007; pp. 191-197.

157. Brouet, V.; Alloul, H.; Quéré, F.; Baumgartner, G.; Folló, L. Detection by NMR of a "Local Spin Gap" in quenched $\mathrm{CsC}_{60}$. Phys. Rev. Lett. 1999, 82, 2131-2134. [CrossRef]

158. Pinterić, M.; Lazić, P.; Pustogow, A.; Ivek, T.; Kuveždić, M.; Milat, O.; Gumhalter, B.; Basletić, M.; Čulo, M.; Korin-Hamzić, B.; et al. Anion effects on electronic structure and electrodynamic properties of the Mott insulator $\kappa$-(BEDT-TTF $)_{2} \mathrm{Ag}_{2}(\mathrm{CN})_{3}$. Phys. Rev. B 2016, 94, 161105. [CrossRef]

159. Lines, M.E. The quadratic-layer antiferromagnet. J. Phys. Chem. Solids 1970, 31, 101-116. [CrossRef]

160. Zong, X.; Suh, B.J.; Niazi, A.; Yan, J.Q.; Schlagel, D.L.; Lograsso, T.A.; Johnston, D.C. ${ }^{17} \mathrm{O}$ and ${ }^{51}$ V NMR for the zigzag spin- 1 chain compound $\mathrm{CaV}_{2} \mathrm{O}_{4}$. Phys. Rev. B 2008, 77, 014412. [CrossRef]

161. Helis, H.M.; Goodman, W.H.; Wilson, R.B.; Morgan, J.A.; Hodgson, D.J. A novel halogen-bridged system: Synthesis and structures of dibromo[2-(2-aminomethyl)pyridine]copper(II) and dibromo(2-methyl1,2-diaminopropane)copper(II). Inorg. Chem. 1977, 16, 2412-2416. [CrossRef]

162. Kikuchi, H.; Nagasawa, H.; Ajiro, Y.; Asano, T.; Goto, T. Susceptibility and high-field magnetization of one-dimensional $S=1 / 2$ Heisenberg antiferromagnet with next-nearest exchange interaction. Physica B 2000, 284-288, 1631-1632. [CrossRef]

163. Khan, M.I.; Giri, S.; Ayesh, S.; Doedens, R.J. Synthesis and characterization of a new hybrid chain derived from oxovanadyl and molybdate moieties [ $\mathrm{V}^{\mathrm{IV}} \mathrm{O}\left(\mu_{3}-\mathrm{MoO}_{4}\right)\left(2,2^{\prime}\right.$-bpy)]: Topological equivalence of $\left\{\mathrm{MoO}_{4}\right\}$ and $\left\{\mathrm{SO}_{4}\right\}$ motifs. Inorg. Chem. Commun. 2004, 7, 721-724. [CrossRef]

164. Kikuchi, H.; Ishikawa, Y.; Fujii, Y.; Matsuo, A.; Kindo, K. Magnetic properties of $S=1 / 2 J_{1}-J_{2}$ one-dimensional magnets, $\mathrm{VO}\left(\mathrm{XO}_{4}\right)\left(2,2^{\prime}\right.$-bpy $)(\mathrm{X}=\mathrm{S}, \mathrm{Mo}$; bpy = bipyridine). J. Phys. Conf. Ser. 2014, 568, 042017. [CrossRef]

165. Hosokoshi, Y.; Katoh, K.; Inoue, K.; Goto, T. Construction of a quantum-spin system of $S=1 / 2$ antiferromagnetic chain with the next-nearest-neighbor interactions. J. Phys. Soc. Jpn. 1999, 68, 2910-2913. [CrossRef]

166. Nomura, K.; Okamoto, K. Phase diagram of $S=1 / 2$ antiferromagnetic $X X Z$ chain with next-nearest-neighbor interactions. J. Phys. Soc. Jpn. 1993, 62, 1123-1126. [CrossRef]

167. Maeshima, N.; Okunishi, K. Antiferromagnetic zigzag spin chain in magnetic fields at finite temperatures. Phys. Rev. B 2000, 62, 934-939. [CrossRef]

168. Troyer, M.; Zhitomirsky, M.E.; Ueda, K. Nearly critical ground state of $\mathrm{LaCuO}_{2.5}$. Phys. Rev. B 1997, 55, R6117-R6120. [CrossRef]

(C) 2018 by the authors. Licensee MDPI, Basel, Switzerland. This article is an open access article distributed under the terms and conditions of the Creative Commons Attribution (CC BY) license (http:/ / creativecommons.org/licenses/by/4.0/). 
Article

\title{
Temperature Dependence of Crystal Structures and Band Parameters in Quantum Spin Liquid $\beta^{\prime}-\mathrm{EtMe}_{3} \mathrm{Sb}\left[\mathrm{Pd}(\mathrm{dmit})_{2}\right]_{2}$ and Related Materials
}

\author{
Kohei Ueda ${ }^{1, *}$, Takao Tsumuraya ${ }^{1,2}$ and Reizo Kato ${ }^{1}$ \\ 1 Condensed Molecular Materials Laboratory, RIKEN, 2-1, Hirosawa, Wako-shi, Saitama 351-0198, Japan; \\ tsumu@kumamoto-u.ac.jp (T.T.); reizo@riken.jp (R.K.) \\ 2 Computational Materials Science Unit, National Institute for Materials Science, Tsukuba-shi, \\ Ibaraki 305-0047, Japan \\ * Correspondence: ueda.kohei@rs.tus.ac.jp; Tel.: +81-4-7122-1158
}

Received: 27 February 2018; Accepted: 15 March 2018; Published: 17 March 2018

\begin{abstract}
In an isostructural series of anion radical salts $\beta^{\prime}-\left(\mathrm{Me}_{4-\mathrm{x}} \mathrm{Et}_{\mathrm{x}} \mathrm{Z}\right)\left[\mathrm{Pd}(\mathrm{dmit})_{2}\right]_{2}(\mathrm{Z}=\mathrm{P}, \mathrm{As}, \mathrm{Sb}$; $\mathrm{x}=0,1,2),\left[\mathrm{Pd}(\mathrm{dmit})_{2}\right]_{2}{ }^{-}$units form a two-dimensional Mott insulator layer with a quasi-isosceles triangular lattice. The anisotropy of the triangular lattice is characterized by a ratio of interdimer transfer integrals, $t^{\prime} / t$. The crystal structures of $\mathrm{EtMe}_{3} \mathrm{Sb}, \mathrm{Me}_{4} \mathrm{Sb}, \mathrm{Me}_{4} \mathrm{As}$, and $\mathrm{Et}_{2} \mathrm{Me}_{2} \mathrm{As}$ salts were determined in the range of 5-295 K by the single crystal X-ray diffraction technique. Interdimer transfer integrals, Fermi surface, and band structures at low temperatures were calculated by the tight binding method and the first-principles density-functional theory (DFT) method based on experimentally obtained crystal structures. Interdimer transfer integrals increased with lowering temperature. At $5 \mathrm{~K}$, the ratio $t^{\prime} / t$ decreased by about $15 \%$ from the room temperature value in every salt. The relationship between the transfer integrals and interdimer $\mathrm{S} \cdots \mathrm{S}$ distances indicated that the change of the $t^{\prime} / t$ value with temperature was due to a thermal contraction, rather than the arch-shaped molecular distortion of the $\mathrm{Pd}(\mathrm{dmit})_{2}$ molecule associated with the cation dependence of $t^{\prime} / t$.
\end{abstract}

Keywords: molecular conductor; quantum spin liquid; geometrical frustration; crystal structure; band calculation

\section{Introduction}

The metal dithiolene complex $\mathrm{Pd}(\mathrm{dmit})_{2}$ (dmit: 1,3-dithiole-2-thione-4,5-dithiolate, Figure 1a) forms various anion radical salts with closed shell monovalent cations: (Cation) $\left[\mathrm{Pd}(\mathrm{dmit})_{2}\right]_{2}[1-4]$. Many of them are categorized to a two-dimensional (2D) Mott insulator with localized $S=1 / 2$ spin on a strongly dimerized unit, $\left[\mathrm{Pd}(\mathrm{dmit})_{2}\right]_{2}{ }^{-}$. In a series of isostructural salts $\beta^{\prime}-\left(\mathrm{Me}_{4-\mathrm{x}} \mathrm{Et} \mathrm{x}_{\mathrm{x}} \mathrm{Z}\right)\left[\mathrm{Pd}(\mathrm{dmit})_{2}\right]_{2}$ $(\mathrm{Z}=\mathrm{P}, \mathrm{As}, \mathrm{Sb} ; \mathrm{x}=0,1,2)$ with the space group of $\mathrm{C} 2 / \mathrm{c}$, cation and anion layers are repeated alternately (Figure 1c). The unit cell contains two crystallographically equivalent anion layers interrelated by the glide plane (Figure 1d). Within the anion layer, the dimer units form a triangular lattice that can induce geometrical frustration (Figure 1f). The cation is located on the two-fold axis and $\mathrm{EtMe}_{3} \mathrm{Z}$-type cations without the two-fold symmetry show two possible orientations with the occupancy of $50 \%$ for each orientation.

The electronic structure of molecular conductors is simple and can be explained by tight-binding approximation $[1,3-6]$. We can describe the band structure of the $\beta^{\prime}-\mathrm{Pd}(\mathrm{dmit})_{2}$ salts using a dimer model where electrons are considered to move from dimer to dimer $[1,3,4]$. The metal dithiolene complex is characterized by a small energy gap between the Highest Occupied Molecular Orbital (HOMO) and the Lowest Unoccupied Molecular Orbital (LUMO; $\Delta \varepsilon$ in Figure 1b). In the dimer, each 
HOMO and LUMO in the monomer forms a bonding pair and an anti-bonding pair with a large energy gap (dimerization gap, $\Delta \varepsilon_{1}$ and $\Delta \varepsilon_{2}$ ). The dimerization gap is governed by the intradimer transfer integral $T_{\mathrm{A}}$ (Figure 1e). When the dimerization gaps are large enough, the energy level of the anti-bonding HOMO pair can be located higher than that of the bonding LUMO pair (Figure 1b) [7]. Since the dimer unit has one negative charge, the anti-bonding HOMO pair has one unpaired electron. When each energy level forms an energy band in the crystal, the conduction band originates from the HOMO of the monomer. The conduction band is two-dimensional, half-filled, and narrow. Therefore, most of the $\beta^{\prime}-\mathrm{Pd}(\mathrm{dmit})_{2}$ salts are Mott insulators at ambient pressure. The interdimer transfer integrals, $t_{\mathrm{B}}, t_{\mathrm{S}}$, and $t_{\mathrm{r}}$ (Figure 1f) were obtained by the following equations using the intermolecular transfer integrals, $T_{\mathrm{B}}, T_{\mathrm{r}}, T_{\mathrm{p}}$, and $T_{\mathrm{q}}: t_{\mathrm{B}}=T_{\mathrm{B}} / 2, t_{\mathrm{s}}=\left(T_{\mathrm{p}}+2 T_{\mathrm{q}}\right) / 2$, and $t_{\mathrm{r}}=T_{\mathrm{r}} / 2$ [3]. In the $\beta^{\prime}-\mathrm{Pd}(\mathrm{dmit})_{2}$ salts, the $t_{\mathrm{B}}$ and $t_{\mathrm{S}}$ values are nearly the same and the $t_{\mathrm{r}}$ is smaller, thus, the dimer units form an isosceles triangular lattice characterized by $t^{\prime} / t$ where $t=\left(t_{\mathrm{B}}+t_{\mathrm{S}}\right) / 2$ and $t^{\prime}=t_{\mathrm{r}}$.

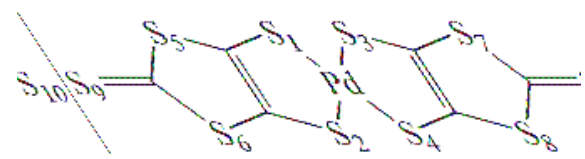

(a)

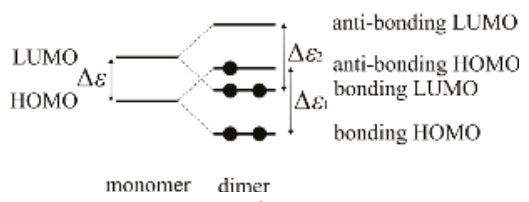

(b)

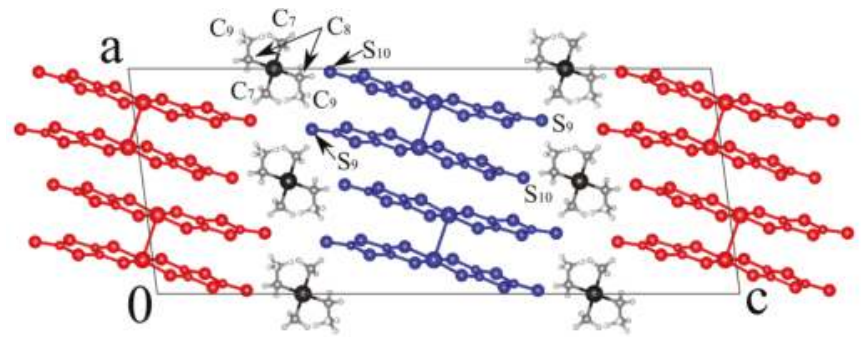

(c)

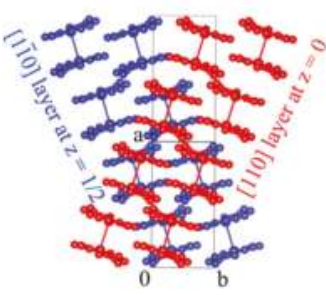

(d)

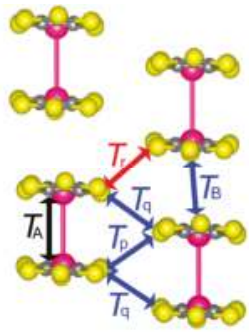

(e)

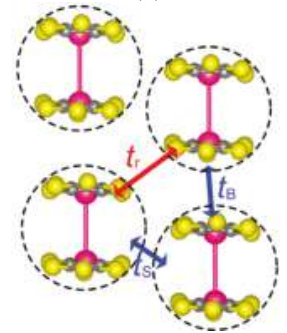

(f)

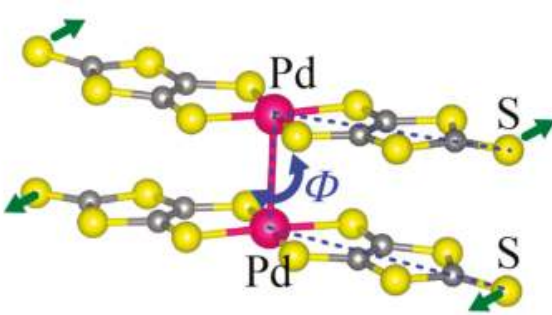

(g)

Figure 1. (a) Molecular structure of $\mathrm{Pd}(\mathrm{dmit})_{2}$. (b) Schematic illustration of orbital levels for monomer and dimer. (c) and (d) Crystal structure of $\beta^{\prime}$-EtMe $\mathrm{Sb}_{3}\left[\mathrm{Pd}(\mathrm{dmit})_{2}\right]_{2}$. End-on projections of the anion layer with notations of (e) intermolecular transfer integrals and (f) interdimer transfer integrals. (g) An arch-shaped distortion of the Pd(dmit) 2 molecule. The torsion angle of S-Pd-Pd-S, $\Phi$, characterizes the degree of the distortion.

Temperature dependence of the magnetic susceptibility for the $\beta^{\prime}-\mathrm{Pd}(\mathrm{dmit})_{2}$ salts can be described by the model of a spin-1/2 Heisenberg antiferromagnet on the triangular lattice with $J=200-300 \mathrm{~K}$ [8]. The magnetic ground state of the $\beta^{\prime}-\mathrm{Pd}(\mathrm{dmit})_{2}$ salts can be classified into three types depending on the counter cation: antiferromagnetic long-range order (AFLO), quantum spin liquid (QSL), and charge ordered nonmagnetic state (CO) [8-16]. The CO phase transition in the $\mathrm{Et}_{2} \mathrm{Me}_{2} \mathrm{Sb}$ salt is accompanied 
by a large structural transition [15], where the coupling between the lattice and the electron plays an important role. The difference in the ground state can be understood by the anisotropy of the isosceles triangular lattice $t^{\prime} / t$ that can be tuned by the choice of counter cation [1-4]. The QSL state, where spin fluctuation prevents the spin ordering/freezing and provides liquid-like properties among the spins even at zero temperature, is realized in the $\mathrm{EtMe}_{3} \mathrm{Sb}$ salt with $t^{\prime} / t=0.91$ (at room temperature). The AFLO phase is observed in salts with smaller $t^{\prime} / t$, namely closer to the square lattice. Moreover, the Néel temperature $T_{\mathrm{N}}$ becomes higher with decreasing $t^{\prime} / t[1,2,4,8]$. The transfer integrals between dimers are governed mostly by overlaps between $\pi$-electrons on sulfur atoms. The cation dependence of $t^{\prime} / t$ is not simply explained by the cation size, but by a cation-dependent distortion of the $\mathrm{Pd}(\mathrm{dmit})_{2}$ molecule [3]. In the dimer unit located at the center of symmetry, the $\mathrm{Pd}(\mathrm{dmit})_{2}$ molecules are not completely eclipsed and the $\mathrm{Pd}(\mathrm{dmit})_{2}$ molecule is slightly arched within the molecular plane. The degree of the arch-shaped distortion is described by the torsion angle of S-Pd-Pd-S, $\Phi$, as shown in Figure 1g. The $\Phi$ value shows a systematic change depending on the counter cation (from $-1.1^{\circ}$ for $\mathrm{Me}_{4} \mathrm{P}^{+}$to $+2.2^{\circ}$ for $\mathrm{Et}_{2} \mathrm{Me}_{2} \mathrm{Sb}^{+}$) and linearly correlates to the three interdimer transfer integrals, $t_{\mathrm{B}}, t_{\mathrm{S}}$, and $t_{\mathrm{r}}$.

The triangular lattice of the dimer units is not a result of the crystal symmetry but is formed through the combination of two larger interdimer transfer integrals along the side-by-side $\left(t_{\mathrm{S}}\right)$ and stacking $\left(t_{\mathrm{B}}\right)$ directions and the one along the diagonal direction $\left(t_{\mathrm{r}}\right)$. A classical antiferromagnetic ordered structure, where two adjacent spins form an antiparallel configuration, is constricted on a triangular lattice. Such geometrical frustration becomes stronger near the regular triangular lattice, namely $t^{\prime} / t=1$. The QSL state is realized in some molecular conductors with a slightly distorted triangular lattice $[13,14,17,18]$ including the $\mathrm{EtMe}_{3} \mathrm{Sb}$ salt. The reason why the QSL is observed in the distorted triangular lattice system in molecular conductors remains an open question. Since molecular conductors are characterized by a soft crystal lattice, intermolecular distances and molecular arrangement can be largely affected by temperature. Therefore, interdimer transfer integrals and the anisotropy of the triangular lattice $t^{\prime} / t$ also change with temperature. In the early stage, crystal structures of some $\beta^{\prime}-\mathrm{Pd}(\mathrm{dmit})_{2}$ salts were determined at $8 \mathrm{~K}$ [19]. As for the $\mathrm{EtMe}_{3} \mathrm{Sb}$ salt with the QSL ground state, the detailed temperature dependence of the geometry of the triangular lattice and interdimer transfer integrals remain unknown, whereas some calculations of the DFT-derived interdimer transfer integrals for previous structural data at $4.5 \mathrm{~K}$ have been reported [20,21]. The knowledge of crystal and electronic structures at low temperatures is indispensable for understanding the nature of QSL.

In this study, we investigated low-temperature crystal structures of the QSL material, the $\mathrm{EtMe}_{3} \mathrm{Sb}$ salt, and the $\mathrm{Me}_{4} \mathrm{Sb}, \mathrm{Et}_{2} \mathrm{Me}_{2} \mathrm{As}$, and $\mathrm{Me}_{4} \mathrm{As}$ salts with the AFLO ground state $\left(T_{\mathrm{N}}=12-35 \mathrm{~K}\right)$ as a reference by using the single crystal $\mathrm{X}$-ray diffraction technique down to $5 \mathrm{~K}$ from room temperature. Based on the structural data, we calculated low-temperature electronic structures with the tight binding method [5,6] and the first principles method [22-25]. We also discuss the temperature dependence of structural and band parameters including the anisotropy of the triangular lattice $t^{\prime} / t$.

\section{Materials and Methods}

Single crystals of all salts were grown by aerial oxidation of (Cation $)_{2}\left[\mathrm{Pd}(\mathrm{dmit})_{2}\right]$ in acetone containing acetic acid [3]. Single crystal x-ray diffraction data were collected by a Rigaku UltraX-6-E Imaging Plate system with monochromated Mo K $\alpha$ radiation (Rigaku, Akishima-shi, Japan). Low temperature experiments were carried out in the cryostat, cooled by a GM refrigerator, with a carbon fiber window down to $5 \mathrm{~K}$. Initial structures were determined by the direct method (SHELXS or SHELXD) [26], and then were refined on $F^{2}$ by the full-matrix least-squares method (SHELXL) [26]. Atomic displacements of all non- $\mathrm{H}$ atoms were determined with the anisotropic model. The $\mathrm{H}$ atom coordinates were placed on calculated positions and refined with the riding model. The ethyl group in the $\mathrm{EtMe}_{3} \mathrm{Sb}$ cation was treated by taking two equivalent positions around the two-fold axis. 
Intermolecular transfer integrals were calculated by using extended Hückel calculations with semi-empirical parameters (shown in Table 1) based on the obtained crystal structures.

The first-principles density-functional theory (DFT) calculations were performed using the full potential linearized augmented plane wave (FLAPW) method as implemented in the QMD-FLAPW12 code [22,23]. Self-consistent-field (SCF) calculations were performed with the scalar-relativistic scheme and the improved tetrahedron integration method [24] up to $7 \times 7 \times 2 \mathbf{k}$-mesh points in the Brillouin zone (BZ). The exchange correlation contribution to the potential was included using the generalized gradient approximation (GGA) by Perdew-Burke-Ernzerhof [25]. We used a plane-wave cutoff of $|\mathbf{k}+\mathbf{G}|<4.5$ a.u. ${ }^{-1}(\sim 20 \mathrm{Ry})$ and 11.6 a.u..$^{-1}(\sim 135 \mathrm{Ry})$ for LAPW basis functions, and charge density and potential, respectively. Muffin-tin sphere radii were assumed to be $1.48 \AA$ for $\mathrm{Sb}, 1.27 \AA$ for $\mathrm{Pd}, 0.35 \AA$ for $\mathrm{H}, 0.65 \AA$ for $\mathrm{C}$, and $0.90 \AA$ for $\mathrm{S}$. For the calculations of the Fermi surface, a band structure for $28 \times 28 \times 4 \mathbf{k}$-mesh points (1097 k-points in the irreducible wedge of BZ) was fitted with symmetrized star functions by a spline method. In the symmetry group of $C 2 / c$, cations were located on the two-fold axis, but the EtMe 3 Z-type cation did not have a two-fold symmetry. In this case, the cation was described by a superposition of two possible orientations with an occupancy of 0.5 for each orientation. In this study, the $\mathrm{EtMe}_{3} \mathrm{Sb}$ cation was replaced by the $\mathrm{Et}_{2} \mathrm{Me}_{2} \mathrm{Sb}$ cation to perform the DFT calculations.

Table 1. Semi-empirical parameters for Slater-type atomic orbitals.

\begin{tabular}{ccccc}
\hline Atom & Orbital & $\boldsymbol{I}_{\mathbf{p}}(\mathbf{R y d})$ & $\zeta_{\mathbf{1}}$ & $\zeta_{\mathbf{2}}$ \\
\hline $\mathrm{C}$ & $2 \mathrm{~s}$ & 1.573 & 1.625 & \\
& $2 \mathrm{p}$ & 0.838 & 1.625 & \\
$\mathrm{~S}$ & $3 \mathrm{~s}$ & 1.620 & 2.122 & \\
& $3 \mathrm{p}$ & 0.770 & 1.827 & \\
$\mathrm{Pd}$ & $5 \mathrm{~s}$ & 0.676 & 2.19 & \\
& $5 \mathrm{p}$ & 0.390 & 2.15 & 2.613 \\
& $4 \mathrm{~d}$ & 0.949 & 5.98 & $(0.6372) *$ \\
\hline
\end{tabular}

${ }^{*}$ Contraction coefficients used in the double- $\zeta$ expansion.

\section{Results and Discussions}

\subsection{Temperature Dependence of Band Parameters}

The $\mathrm{EtMe}_{3} \mathrm{Sb}$ salt showed no structural transition down to $5 \mathrm{~K}$, that is, the space group and the essential molecular arrangement remained unchanged in the experimental temperature range. From room temperature to $5 \mathrm{~K}$, the unit cell volume underwent a reduction by $3.2 \%$. The temperature dependence of lattice parameters of the $\mathrm{EtMe}_{3} \mathrm{Sb}$ salt is shown in Figure S1. Crystal structures of the $\mathrm{EtMe}_{3} \mathrm{Sb}$ salt were determined with the $R$ values of $5-9 \%$ at each temperature: $5.6 \%$ at $295 \mathrm{~K}, 5.7 \%$ at $232 \mathrm{~K}, 7.0 \%$ at $161 \mathrm{~K}, 6.4 \%$ at $101 \mathrm{~K}, 7.6 \%$ at $53 \mathrm{~K}, 8.1 \%$ at $21 \mathrm{~K}$, and $8.9 \%$ at $5 \mathrm{~K}$. Figure 2 shows the temperature dependence of the intermolecular and interdimer transfer integrals for the $\mathrm{EtMe}_{3} \mathrm{Sb}$ salt calculated by the extended Hückel method using obtained crystal structures. Every transfer integral increased almost linearly with lowering temperature. The $t_{\mathrm{B}}, t_{\mathrm{S}}$, and $t_{\mathrm{r}}$ values changed similarly to each other, keeping the triangular lattice down to the lowest temperature. In contrast, the reduction of the cell volume by the cation effect resulted in a decrease of the $t_{\mathrm{r}}$ value [3]. This suggested that the temperature effect on the interdimer transfer integrals differed from the cation effect. Among these three interdimer transfer integrals, the temperature dependence of $t_{\mathrm{r}}$ was more moderate than those of others, and thus $t^{\prime} / t$ decreased by about $16 \%$ from the room temperature to $5 \mathrm{~K}$ (Figure $3 \mathrm{a}$ ). That is, the triangular lattice in the $\mathrm{EtMe}_{3} \mathrm{Sb}$ salt became anisotropic with lowering temperature. 


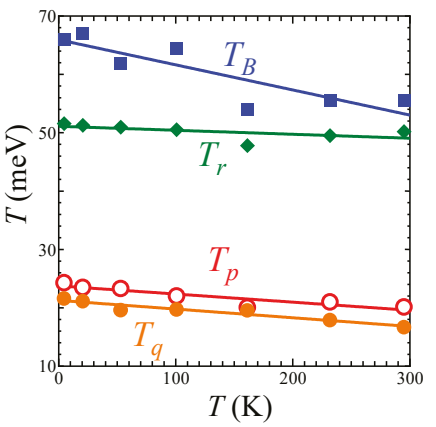

(a)

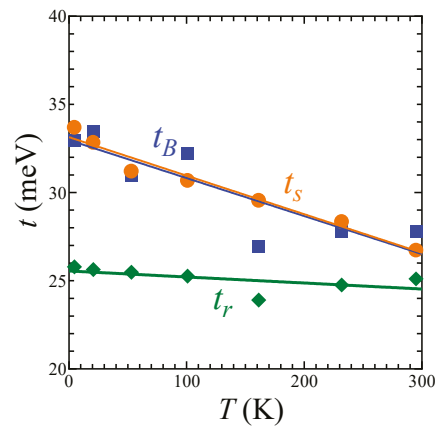

(b)

Figure 2. Temperature dependence of (a) intermolecular transfer integrals, $T_{\mathrm{B}}, T_{\mathrm{r}}, T_{\mathrm{p}}$, and $T_{\mathrm{q}}$ and (b) interdimer transfer integrals, $t_{\mathrm{B}}, t_{\mathrm{s}}$, and $t_{\mathrm{r}}$, in the $\mathrm{EtMe}_{3} \mathrm{Sb}$ salt.

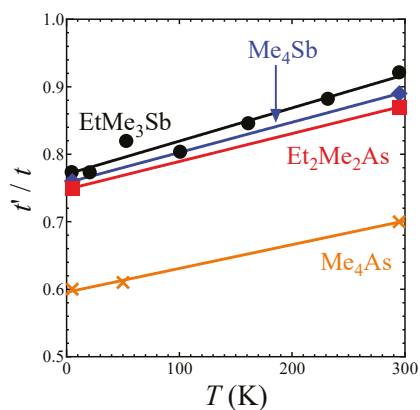

(a)

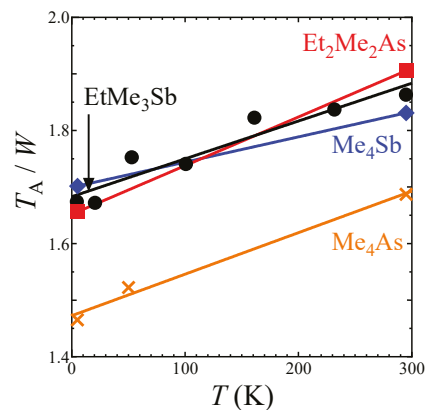

(b)

Figure 3. Temperature dependence of (a) $t^{\prime} / t$ and (b) $T_{\mathrm{A}} / W$ in the $\mathrm{Me}_{4} \mathrm{Sb}, \mathrm{Me}_{4} \mathrm{As}, \mathrm{Et}_{2} \mathrm{Me}_{2} \mathrm{As}$, and $\mathrm{EtMe}_{3} \mathrm{Sb}$ salts.

Intermolecular and interdimer transfer integrals for all salts are shown in Tables S1 and S2. Other salts with the AFLO ground state also showed no structural transition down to $5 \mathrm{~K}$. The crystal structures of these salts were determined with $R$ values of $4-7 \%$ at each temperature: $5.3 \%$ at $295 \mathrm{~K}$ and $6.0 \%$ at $5 \mathrm{~K}$ for the $\mathrm{Me}_{4} \mathrm{Sb}$ salt; $5.9 \%$ at $295 \mathrm{~K}, 4.0 \%$ at $53 \mathrm{~K}$, and $5.7 \%$ at $5 \mathrm{~K}$ for the $\mathrm{Me}_{4} \mathrm{As}$ salt; and $5.7 \%$ at $295 \mathrm{~K}$ and $7.1 \%$ at $5 \mathrm{~K}$ for the $\mathrm{Et}_{2} \mathrm{Me}_{2}$ As salt. Temperature dependences of the $t_{\mathrm{B}}, t_{\mathrm{S}}$, and $t_{\mathrm{r}}$ values in these salts were similar to the case of the $\mathrm{EtMe}_{3} \mathrm{Sb}$ salt, and thus, the $t^{\prime} / t$ value of each salt decreased by about $15 \%$ from room temperature to $5 \mathrm{~K}$ (Figure 3a). This was in contrast to the case of another triangular lattice system based on $\mathrm{k}-(\mathrm{ET})_{2} \mathrm{X}\left(\mathrm{ET}=\right.$ bis(ethylenedithio)tetrathiafulvalene, $\mathrm{X}=\mathrm{Cu}_{2}(\mathrm{CN})_{3}$, $\left.\mathrm{B}(\mathrm{CN})_{4}, \mathrm{Cu}\left[\mathrm{N}(\mathrm{NCS})_{2}\right]\right)$, where the $t^{\prime} / t$ values increased with lowering temperatures [27].

The intradimer transfer integral $T_{\mathrm{A}}$ is associated with the effective on-site Coulomb energy on the dimer unit as a rough approximation [28]. The ratio $T_{\mathrm{A}} / W$, where $W$ is a bandwidth, gives an indication of the strength of the correlation effect. For all salts, the ratio decreased almost linearly with lowering temperature (Figure $3 b$ ), which suggests that the correlation effect in the $\beta^{\prime}-\mathrm{Pd}(\mathrm{dmit})_{2}$ salts weakened with lowering temperature.

Figure 4 shows the calculated Fermi surface of the $\mathrm{EtMe}_{3} \mathrm{Sb}$ salt obtained by the tight-binding method (dimer model) using interdimer transfer integrals determined in this study at $5 \mathrm{~K}, 101 \mathrm{~K}$, and $295 \mathrm{~K}$ [1]. This also shows the enhancement of the anisotropy of the triangular lattice in the low temperature region. Since the unit cell contains two crystallographically equivalent anion layers with different stacking directions [110] and $[1 \overline{1} 0]$ (Figure 1d), the Fermi surface consists of two cylinders associated with these 
two anion layers. The Fermi surface at $295 \mathrm{~K}$ shows a nearly isotropic 2D nature represented by mutually superposed cylinders. As the anisotropy of the triangular lattice is enhanced due to the difference in the temperature dependence of transfer integrals $\left(\left|\mathrm{d} t_{\mathrm{S}} / \mathrm{d} T\right| \sim\left|\mathrm{d} t_{\mathrm{B}} / \mathrm{d} T\right|>\mid \mathrm{d} t_{\mathrm{r}} / \mathrm{d} T\right) \mid$, the Fermi surface becomes anisotropic with lowering temperatures as seen from a decrease in the overlapping portion of the Fermi surface.

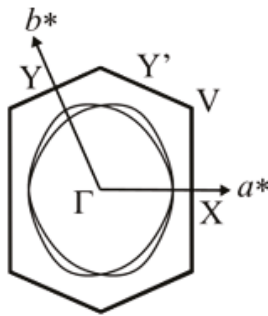

(a)

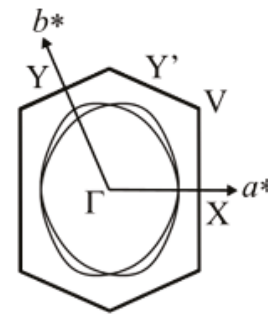

(b)

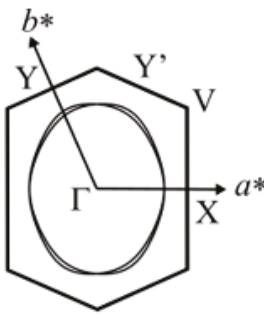

(c)

Figure 4. Fermi surface of the $\mathrm{EtMe}_{3} \mathrm{Sb}$ salt calculated by the extended Hückel and tight binding methods at $5 \mathrm{~K}(\mathbf{a}), 101 \mathrm{~K}(\mathbf{b})$, and $295 \mathrm{~K}(\mathbf{c})$. The $C$-centered monoclinic cell is reduced to the primitive one as $a_{\mathrm{p}}=(\boldsymbol{a}+\boldsymbol{b}) / 2, \boldsymbol{b}_{\mathrm{p}}=\boldsymbol{b}, c_{\mathrm{p}}=\boldsymbol{c}$.

We also performed the first-principles DFT calculations. The calculated Fermi surface and band structure are shown in Figure 5. Calculated band structures and band widths at 5 K, $101 \mathrm{~K}$, and $295 \mathrm{~K}$ are shown in Figure S2 and Table S3. Concerning the Fermi surface, both calculation methods provided similar temperature dependence. This indicated that the dimer model using only HOMO-HOMO interactions could be used to describe the low-temperature electric structure of the EtMe $\mathrm{Sb}_{3}$ salt around the Fermi level. On the other hand, since it is difficult to estimate the energy gap between HOMO and LUMO correctly with the extended Hückel method, the DFT calculations provided useful information on the arrangement of the HOMO and LUMO bands. In the tight binding model, the temperature-dependent interrelation between the HOMO and LUMO bands was explained by the dimerization gap (associated with the intradimer transfer integrals) and the band width (associated with the interdimer transfer integrals). The stronger dimerization enlarged the intradimer transfer integrals and made the anti-bonding HOMO band and the bonding LUMO band separate from each other. On the other hand, enlargement of the interdimer transfer integrals widened each band, leading to the band overlapping. The extended Hückel calculations indicated that the lowering of temperature induced the enlargement of both intra- and interdimer transfer integrals, which provided contrary effects on the band overlap. The first-principles DFT calculation showed that the Fermi level crossed the anti-bonding HOMO band that was separated from other bands at $295 \mathrm{~K}$ (Figure 5d). This conduction band becomes wider with lowering temperatures but remains isolated from the other bands even at the lowest temperature $(5 \mathrm{~K})$. That is, no band overlap occurred and the conduction band remained half-filled down to the lowest temperature. This indicated that the dimer model using only HOMO-HOMO interactions still worked to describe the electronic structure near the Fermi level of this system in the low-temperature region.

The DFT calculations provided other useful information. Figure 5e shows the partial density of states (PDOS) in the conduction band for the d-orbitals of Pd, the p-orbitals of $\mathrm{S}$, and the p-orbitals of $\mathrm{C}$ in the dmit ligand at $5 \mathrm{~K}$. This indicated that the p-orbitals of $\mathrm{S}$ played a dominant role in the band formation around the Fermi level. We also mention the possibility that the cations directly affected the electronic structure around the Fermi level. The energy levels of s- and p-orbitals of the $\mathrm{C}$ atoms in the $\mathrm{EtMe}_{3} \mathrm{Sb}$ cation were separated from the Fermi level by more than $3 \mathrm{eV}$. The PDOS of these $\mathrm{C}$ orbitals in the $\mathrm{EtMe}_{3} \mathrm{Sb}$ cation was less than 0.015 /eV in Figure 5e. Therefore, it is quite unlikely that the cation orbitals hybridize to the conduction band. 


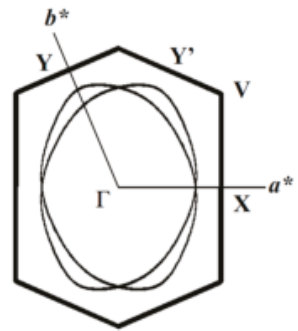

(a

(d)

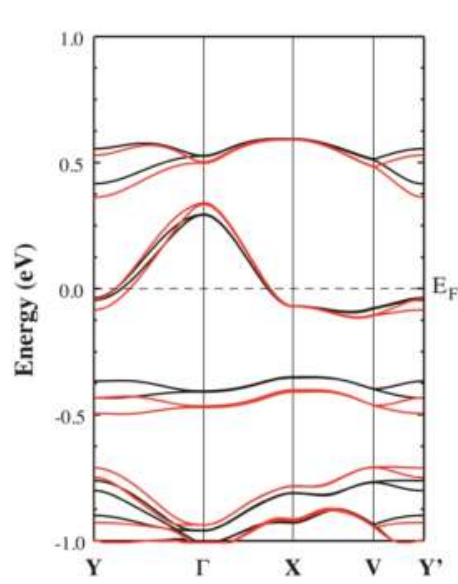

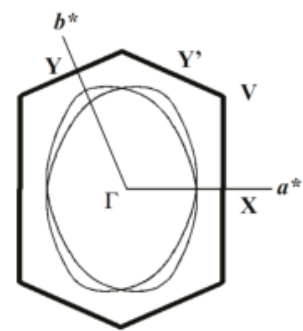

(b)

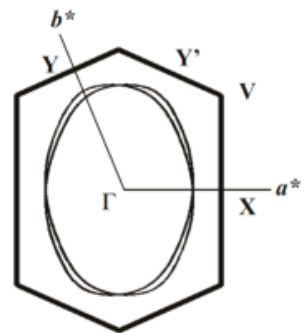

(c)

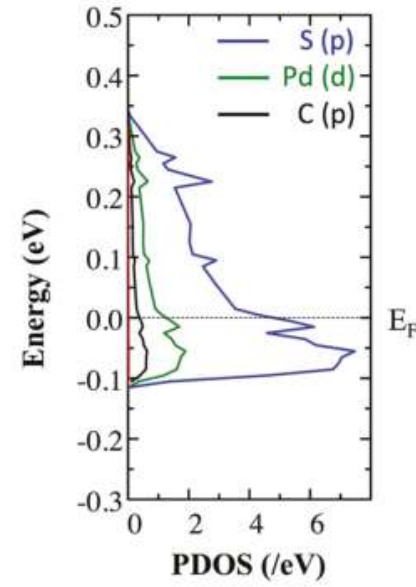

(e)

Figure 5. Electronic structure of the $\mathrm{EtMe}_{3} \mathrm{Sb}$ salt calculated by the first-principles DFT method. Fermi surface at $5 \mathrm{~K}(\mathbf{a}), 101 \mathrm{~K}(\mathbf{b})$, and $295 \mathrm{~K}$ (c). (d) Band dispersion at $5 \mathrm{~K}$ (red) and $295 \mathrm{~K}$ (black). (e) Partial density of states (PDOS) at $5 \mathrm{~K}$ in the conduction bands for the d-orbitals of Pd (green), the p-orbitals of $\mathrm{S}$ (blue), the p-orbitals of $\mathrm{C}$ in the dmit ligand (black), and the s- and p-orbitals of $\mathrm{C}$ in the $\mathrm{EtMe}_{3} \mathrm{Sb}$ cation (red). The blue and black lines each show the summation of the PDOS of all the $\mathrm{S}$ and $\mathrm{C}$ atoms belonging to the dmit ligand.

\subsection{Temperature Dependence of Structural Parameters}

We now discuss the temperature dependence of structural parameters associated with the transfer integrals in the $\mathrm{EtMe}_{3} \mathrm{Sb}$ salt. As above-mentioned, the p-orbital in $\mathrm{S}$ governs the transfer integrals between HOMOs. Figure $6 \mathrm{a}, \mathrm{b}$ show the temperature dependence of intradimer $\mathrm{S} \cdots \mathrm{S}$ distances $\left(d_{\mathrm{A} 1}-d_{\mathrm{A} 5}\right)$, and the arch-shaped distortion angle $\Phi$ that characterizes the dimer structure. The $S \cdots S$ distances linearly decreased with lowering temperature. The $\Phi$ value did not show any significant changes. Figure $6 \mathrm{c}$ indicates a linear relation between the intradimer transfer integral $T_{\mathrm{A}}$ and $S \cdots S$ distances $d_{\mathrm{AX}}(\mathrm{X}=1-5)$, which suggests that $T_{\mathrm{A}}$ is governed simply by the intradimer $\mathrm{S} \cdots \mathrm{S}$ distances. The arch-shaped molecular distortion plays an important role in the cation dependence of interdimer transfer integrals, $t_{\mathrm{B}}, t_{\mathrm{S}}$, and $t_{\mathrm{r}}[3]$. The linear relationship between $\Phi$ and interdimer transfer integrals was reported for all salts. Since $\Phi$ is independent of temperature, the temperature dependence of interdimer transfer integrals should originate in other mechanisms. 


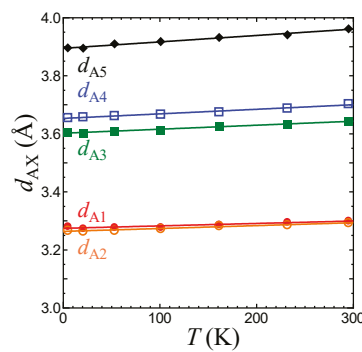

(a)

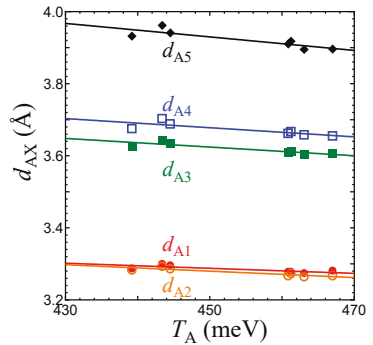

(c)

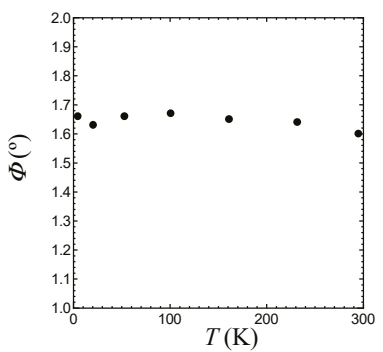

(b)

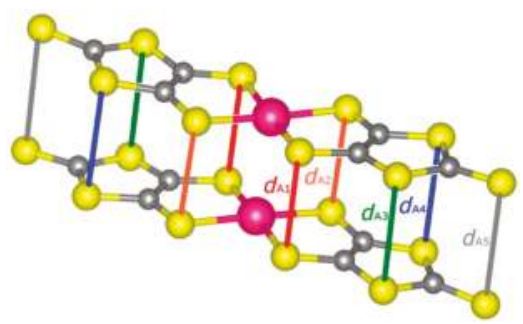

Figure 6. Temperature dependence of (a) intradimer S ..S distances, $d_{\mathrm{AX}}(\mathrm{X}=1-5)$ and (b) $\Phi$, and (c) relationship between $T_{\mathrm{A}}$ and $d_{\mathrm{AX}}$ in the $\mathrm{EtMe}_{3} \mathrm{Sb}$ salt.

To consider the origin of the temperature dependence of the interdimer transfer integrals, $t_{\mathrm{B}}, t_{\mathrm{S}}$, and $t_{\mathrm{r}}$ in the EtMe 3 Sb salt, we examined the intermolecular $\mathrm{S} \cdots \mathrm{S}$ distances, $d_{\mathrm{BX}}, d_{\mathrm{rX}}, d_{\mathrm{pX}}$, and $d_{\mathrm{qX}}$ that contribute to the intermolecular transfer integrals, $T_{\mathrm{B}}, T_{\mathrm{r}}, T_{\mathrm{p}}$, and $T_{\mathrm{q}}$, respectively (Figure 7). Considering that the van der Waals radius of $S$ is $1.85 \AA, T_{B}$ is associated with longer $S$. . S distances $\left(d_{\mathrm{BX}}\right)$ when compared with other intermolecular transfer integrals. Nevertheless, $T_{\mathrm{B}}$ is the largest transfer integral among these four transfer integrals, because $T_{\mathrm{B}}$ consists of mainly p $\sigma \cdots$ p $\sigma$ contacts that give larger overlap integrals. On the other hand, all S. . S distances that contribute to $T_{\mathrm{r}}\left(d_{\mathrm{rX}}\right)$ are shorter than the van der Waals distance. Since $T_{\mathrm{r}}$ includes the $\mathrm{p} \pi \cdots \mathrm{p} \pi$ character as well as the p $\sigma \cdots p \sigma$ character, $T_{\mathrm{r}}$ is the second largest. $T_{\mathrm{p}}$ and $T_{\mathrm{q}}$ are associated with long and short $\mathrm{S} \cdots \mathrm{S}$ distances and exhibit both $p \sigma \cdots p \sigma$ and $p \pi \cdots p \pi$ characters. Therefore, they are smaller than $T_{B}$ and $T_{\mathrm{r}}$. The interdimer transfer integral $t_{\mathrm{S}}$, however, is expressed as a summation of $T_{\mathrm{q}}$ and $T_{\mathrm{p}}$, $t_{\mathrm{S}}=\left(2 T_{\mathrm{q}}+T_{\mathrm{p}}\right) / 2$, and thus $t_{\mathrm{S}}$ is comparable to $t_{\mathrm{B}}\left(=T_{\mathrm{B}} / 2\right)$. As a result, the triangular lattice with the relation of $t_{\mathrm{B}} \sim t_{\mathrm{S}}>t_{\mathrm{r}}$ is formed in this system.

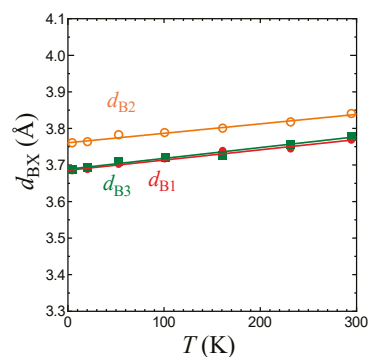

(a-1)

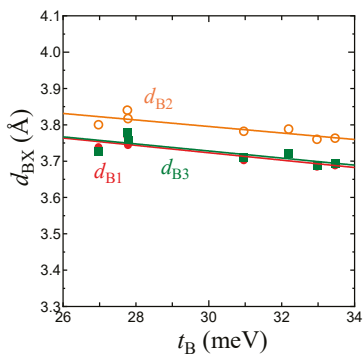

(a-2)

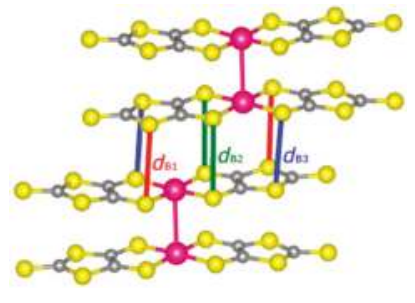

Figure 7. Cont. 


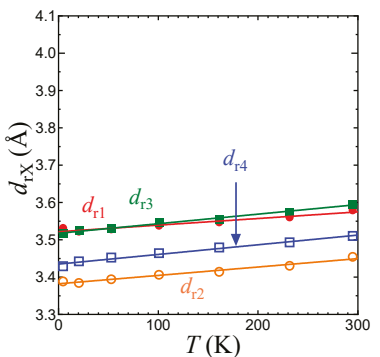

(b-1)

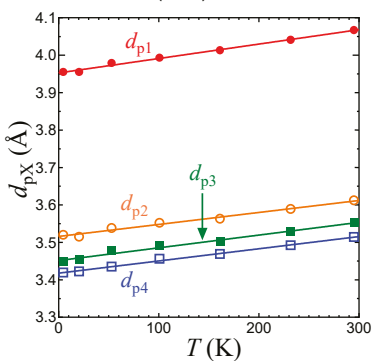

(c-1)

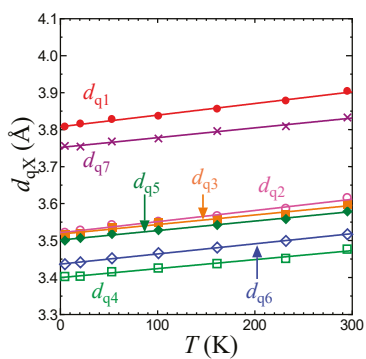

(d-1)

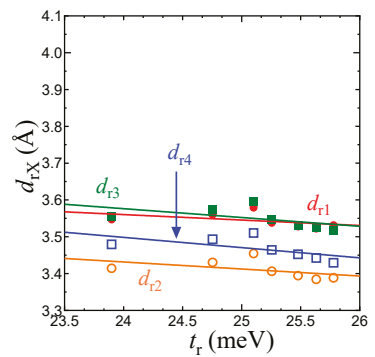

(b-2)

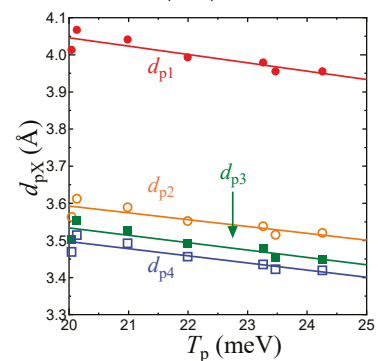

(c-2)

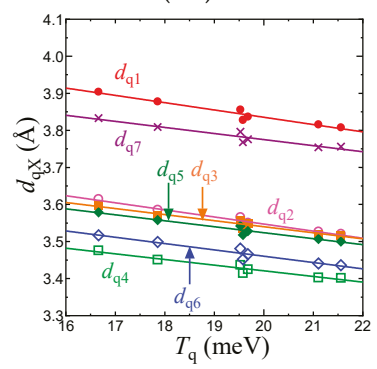

(d-2)
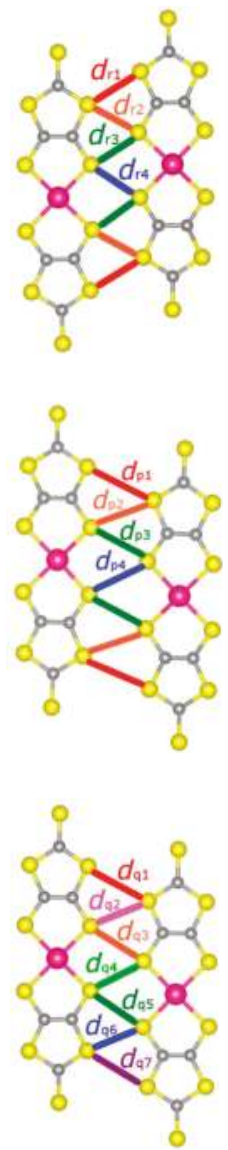

Figure 7. Temperature dependence of intermolecular $S \cdots S$ distances associated with $T_{B}(\mathbf{a}-\mathbf{1}), T_{\mathrm{r}}(\mathbf{b}-\mathbf{1})$, $T_{\mathrm{p}}(\mathbf{c}-\mathbf{1})$, and $T_{\mathrm{q}}(\mathbf{d}-\mathbf{1})$ in the $\mathrm{EtMe}_{3} \mathrm{Sb}$ salt. Relationships between the $\mathrm{S} \cdots \mathrm{S}$ distances and intermolecular transfer integrals, $T_{\mathrm{B}}(\mathbf{a}-2), T_{\mathrm{r}}(\mathbf{b}-2), T_{\mathrm{p}}(\mathbf{c}-2)$, and $T_{\mathrm{q}}(\mathbf{d}-2)$ in the $\mathrm{EtMe}_{3} \mathrm{Sb}$ salt.

With lowering temperature, all intermolecular S...S distances were reduced linearly (Figure 7). From $295 \mathrm{~K}$ to $5 \mathrm{~K}$, the amount of the reduction was about $0.1 \AA$ for all cases, suggesting an almost isotropic thermal contraction of the anion layer. For each transfer integral, there existed a linear relationship with the corresponding S...S distances (Figure 7). Such linear relations for all the intermolecular S...S distances and the intermolecular transfer integrals were not observed in the case of cation dependence [3]. This also suggests that the temperature dependence of the transfer integrals was not due to the arch-shape distortion.

Each average of $d_{\mathrm{BX}}, d_{\mathrm{pX}}, d_{\mathrm{qX}}$, and $d_{\mathrm{rX}}$ (represented as $\bar{d}_{\mathrm{B}}, \bar{d}_{\mathrm{p}}, \bar{d}_{\mathrm{q}}$, and $\bar{d}_{\mathrm{r}}$ ) decreased by $2.2 \%, 2.7 \%$, $2.3 \%$, and $1.6 \%$, respectively, from room temperature to $5 \mathrm{~K}$. The smallest variation of $\bar{d}_{\text {r corresponded }}$ to a very moderate temperature dependence of $t_{\mathrm{r}}$. Although the reduction of $\bar{d}_{\mathrm{B}}$ was not so large, the enlargement of $T_{\mathrm{B}}$ and thus $t_{\mathrm{B}}$ was more intensive than those of other transfer integrals due to its p $\sigma \cdots$ p $\sigma$ character. The variation of $t_{\mathrm{S}}$, which is the summation of three transfer integrals, was as large as that of $t_{\mathrm{B}}$. Consequently, the relationship between the variations, $\left|\mathrm{d} t_{\mathrm{S}} / \mathrm{d} T\right| \sim\left|\mathrm{d} t_{\mathrm{B}} / \mathrm{d} T\right|>\left|\mathrm{d} t_{\mathrm{r}} / \mathrm{d} T\right|$, led to the decrease of $t^{\prime} / t$ in the low temperature region. 
Let us move on to the origin of the temperature dependence of $T_{\mathrm{A}} / W$ (the indication of the electron correlation). The average of the intradimer S $\cdots$ S distances $d_{\mathrm{Ax}}\left(\bar{d}_{\mathrm{A}}\right)$ decreased by about $1.1 \%$ from room temperature to $5 \mathrm{~K}$. This variation was about half as large as those of the interdimer S. . S distances, $\bar{d}_{\mathrm{B}}, \bar{d}_{\mathrm{p}}$, and $\bar{d}_{\mathrm{q}}$. Since the $\mathrm{Pd}(\mathrm{dmit})_{2}$ molecules are tightly dimerized, the intradimer S. . S distances are supposed to change differently from the interdimer S $\cdots S$ distances with lowering temperature. On the other hand, the band width is associated with the interdimer transfer integrals. From room temperature to $5 \mathrm{~K}$, the $T_{\mathrm{A}}$ value increased by $5 \%$, while the band width $W$ increased by $17 \%$, which resulted in the decrease of $T_{\mathrm{A}} / W$. Strong dimerization is a common feature of the $\beta^{\prime}-\mathrm{Pd}(\mathrm{dmit})_{2}$ salts, thus, each salt showed a similar temperature dependence of the $T_{\mathrm{A}} / W$ value.

Considering the temperature dependence of the structural parameters that related to the band parameters in the $\mathrm{EtMe}_{3} \mathrm{Sb}$ salt, we concluded that the temperature dependence of the anisotropy of the triangular lattice $\left(t^{\prime} / t\right)$, one of the most important parameters in this frustrated Mott system, primarily originated from the almost isotropic thermal contraction of the anion layer. This is a common feature of the $\beta^{\prime}-\mathrm{Pd}(\mathrm{dmit})_{2}$ salts. In this frustrated Mott system with the triangular lattice of the strongly dimerized units, the electronic structure became anisotropic and the electron correlation was reduced with lowering temperatures.

\subsection{Cation-Anion Interactions}

In the $\beta^{\prime}-\mathrm{Pd}(\mathrm{dmit})_{2}$ salts, the counter cation strongly affects the anisotropy of the triangular lattice [3]. In addition, it has been suggested that the cation-anion interaction plays an important role in realizing the QSL state in the K-type ET salts [29-31]. Therefore, we discuss the cation-anion interaction in the $\beta^{\prime}-\mathrm{Pd}(\mathrm{dmit})_{2}$ salts, especially the $\mathrm{S} \cdot \mathrm{H}-\mathrm{C}$ short contacts between the terminal thioketone group of the $\mathrm{Pd}(\mathrm{dmit})_{2}$ molecule and the hydrogen atom in the cation. In this study, the torsion angles of the $-\mathrm{CH}_{3}$ unit were refined with the AFIX 137 command in the refinement process with SHELX. Refined torsion angles are those in the staggered conformation except for in the $\mathrm{EtMe}_{3} \mathrm{Sb}$ salt at $5 \mathrm{~K}$ (vide infra). The number of short S . H H-C contacts ( $<3.0 \AA$ : sum of the van der Waals radii of $S$ atom and $\mathrm{H}$ atom) in the $\mathrm{Me}_{4} \mathrm{As}, \mathrm{Me}_{4} \mathrm{Sb}, \mathrm{Et}_{2} \mathrm{Me}_{2} \mathrm{As}$, and $\mathrm{EtMe}_{3} \mathrm{Sb}$ salts are summarized in Table 2. Figure 8 shows the temperature dependence of the short $\mathrm{S} \cdots \mathrm{H}-\mathrm{C}$ contacts in the EtMe $\mathrm{Sb}_{3}$ salt. Two S $\cdots \mathrm{H}-\mathrm{C}_{9}$ contacts were much shorter at $5 \mathrm{~K}$ than at other temperatures. These very short contacts are caused by an anomalous torsion angle of the $-\mathrm{CH}_{3}$ unit. The refined torsion angle of this $-\mathrm{CH}_{3}$ unit at $5 \mathrm{~K}$ was about $30^{\circ}$, which was far from the angle in the staggered conformation.

Table 2. The number of short S...H-C contacts between the cation and terminal thioketone of the $\mathrm{Pd}(\mathrm{dmit})_{2}$ molecule at $295 \mathrm{~K}$ and $5 \mathrm{~K}$.

\begin{tabular}{|c|c|c|c|c|c|c|c|c|}
\hline \multirow[b]{2}{*}{ S $\cdots \mathrm{H}$ distance: $d(\mathrm{~S} \cdots \mathrm{H}-\mathrm{C}) / \AA$} & \multicolumn{2}{|c|}{$\mathrm{Me}_{4} \mathrm{As}$} & \multicolumn{2}{|c|}{$\mathrm{Me}_{4} \mathrm{Sb}$} & \multicolumn{2}{|c|}{$\mathrm{Et}_{2} \mathrm{Me}_{2} \mathrm{As}$} & \multicolumn{2}{|c|}{$\mathrm{EtMe}_{3} \mathrm{Sb}^{*}$} \\
\hline & $295 \mathrm{~K}$ & $5 \mathrm{~K}$ & $295 \mathrm{~K}$ & $5 \mathrm{~K}$ & $295 \mathrm{~K}$ & $5 \mathrm{~K}$ & $295 \mathrm{~K}$ & $5 \mathrm{~K}$ \\
\hline $2.95-3.00$ & 2 & 4 & & 4 & 12 & 8 & 4 & 2 \\
\hline $2.90-2.95$ & 2 & & 2 & 2 & & 2 & & 6 \\
\hline $2.85-2.90$ & & & & & & 4 & & \\
\hline $2.80-2.85$ & 2 & 2 & 2 & 2 & & & 1 & 2 \\
\hline $2.75-2.80$ & & & & & & & 1 & 1 \\
\hline $2.70-2.75$ & & & & & & & 1 & \\
\hline $2.65-2.70$ & & & & & & & & \\
\hline $2.60-2.65$ & & & & & & & & 1 \\
\hline $2.55-2.60$ & & & & & & & & \\
\hline $2.50-2.55$ & & & & & & & & 1 \\
\hline
\end{tabular}

* The number of the short S $\cdots \mathrm{H}-\mathrm{C}_{9}$ contacts in the $\mathrm{EtMe}_{3} \mathrm{Sb}$ salt was counted as half of that in Figure 9, considering the occupancy factor of 0.5 for the ethyl group. 


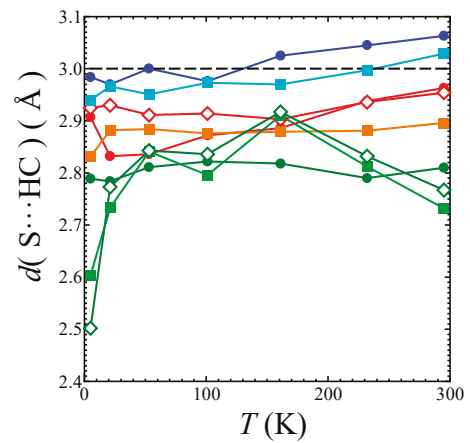

Figure 8. Temperature dependence of short S $\cdots \mathrm{H}-\mathrm{C}$ distances $d(\mathrm{~S} \cdots \mathrm{H}-\mathrm{C})$ in the $\mathrm{EtMe}_{3} \mathrm{Sb}$ salt: the black dashed line shows the sum of the van der Waals radii of $\mathrm{S}$ atom and $\mathrm{H}$ atom. The red filled circles, orange filled squares, and red open diamonds mark the $\mathrm{S} \cdots \mathrm{H}-\mathrm{C}_{7}$ contacts. The blue filled circles and light blue filled squares mark the $\mathrm{S} \cdots \mathrm{H}-\mathrm{C}_{8}$ contacts. The green filled circles, spring green filled squares, and green open diamonds mark the $\mathrm{S} \cdots \mathrm{H}-\mathrm{C}_{9}$ contacts. See also Figures $1 \mathrm{c}$ and 9.

The $\mathrm{S} \cdots \mathrm{H}-\mathrm{C}$ contacts in the EtMe $\mathrm{Sb}_{3} \mathrm{Sb}$ salt are shown in Figure 9. Two Pd(dmit $)_{2}$ molecules in the dimer are related by the inversion center, and there are two crystallographically independent terminal $S$ atoms $\left(S_{9}\right.$ and $\left.S_{10}\right)$. These $S$ atoms contact three cations (labeled as Figure $\left.9 a-c\right)$ through eight $\mathrm{S} \cdots \mathrm{H}-\mathrm{C}$ contacts (three $\mathrm{S} \cdots \mathrm{H}-\mathrm{C}_{7}$ contacts, I-7, II-7, and III-7; two S $\cdots \mathrm{H}-\mathrm{C}_{8}$ contacts, I-8 and II-8; three S $\cdots$ H-C $C_{9}$ contacts, I-9, II-9, and III-9 in Figure 9) in the EtMe $\mathrm{E}_{3} \mathrm{Sb}$ salt. Cation $a$ contacts two terminal $S$ atoms (Figure 9), and each of the cations $b$ and $c$ contact one $S$ atom. Such features of the cation-anion contacts are commonly observed in the $\beta^{\prime}-\mathrm{Pd}(\mathrm{dmit})_{2}$ salts.

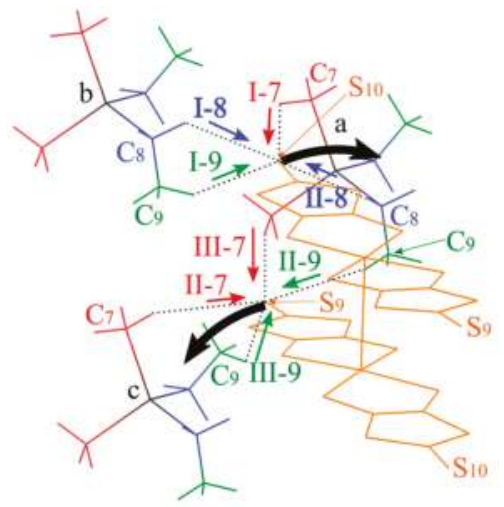

(a)

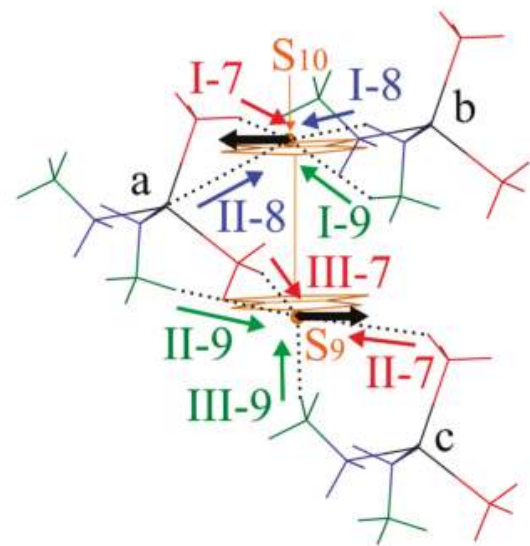

(b)

Figure 9. Cont. 


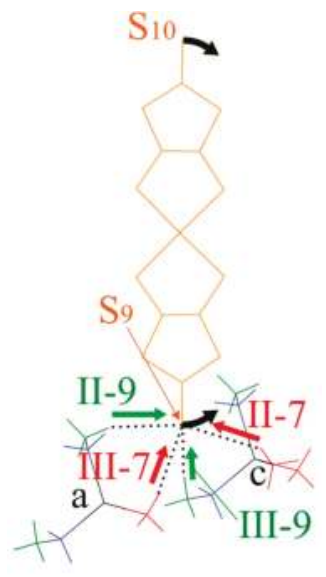

(c-1)

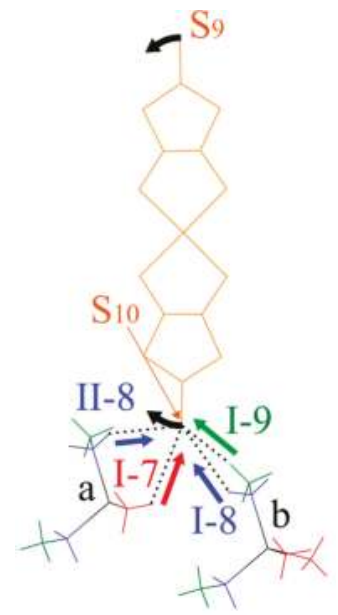

(c-2)

Figure 9. Schematic illustration of the short S . H-C contacts. (a) Oblique view. (b) End-on projections of the dimer arrangement. (c) Views along the vector connecting the two Pd atoms in the dimer, (c-1) shows the short S $\cdots$ H-C contacts between the $\mathrm{S}_{9}$ atom in the lower $\mathrm{Pd}(\mathrm{dmit})_{2}$ molecule in (b) and cations $a$ and $c$, (c-2) shows the S $\cdots$ H-C short contacts between the $\mathrm{S}_{10}$ atom in the upper $\mathrm{Pd}(\mathrm{dmit})_{2}$ molecule in (b) and cations $a$ and $b$ : black arrows indicate the direction of the arch-shaped distortion with a positive $\Phi$ value. The red, green and blue arrows indicate the directions of repulsive S $\cdots \mathrm{H}-\mathrm{C} 7$, S. . H-C8, and S. . H-C9 interactions, respectively.

We discuss how the $\mathrm{S} \cdots \mathrm{H}-\mathrm{C}$ contacts affect the arch-shaped distortion of the $\mathrm{Pd}(\mathrm{dmit})_{2}$ molecule by considering a contact angle: the $\mathrm{S} \cdots \mathrm{H}-\mathrm{C}$ interaction that can affect the $\Phi$ angle should have a component parallel to the displacement of the $\mathrm{S}$ atom in the distortion. The S $\cdots \mathrm{H}-\mathrm{C}_{9}$ contacts associated with the ethyl group are rather short in the $\mathrm{EtMe}_{3} \mathrm{Sb}$ salt. These tight cation-anion interactions would dominate the arch-shape distortion in the $\beta^{\prime}-\mathrm{Pd}(\mathrm{dmit})_{2}$ salts containing the ethyl groups. When the $\mathrm{S} \cdots \mathrm{H}-\mathrm{C}$ interaction was repulsive/attractive, two $\mathrm{S} \cdots \mathrm{H}-\mathrm{C}_{9}$ contacts (I-9 and II-9 in Figure 9) effectively made the $\Phi$ angle large/small. As shown in Figure 9b,c-1, the III-9 contact was almost perpendicular to the displacement of the $S$ atom in the arch-shaped distortion, thus, the effect of this contact on the $\Phi$ angle was negligible. In the $\mathrm{Et}_{x} \mathrm{Me}_{4-x} \mathrm{Z}$ salts, the $\Phi$ angle became larger by increasing the number of the ethyl group $x$ [3]. Accordingly, the $\mathrm{S} \cdots \mathrm{H}-\mathrm{C}_{9}$ contact angle and the $x$ dependence of $\Phi$ indicated that the $\mathrm{S} \cdots \mathrm{H}-\mathrm{C}_{9}$ contacts appeared, effectively, repulsive.

In the $\mathrm{Me}_{4} \mathrm{P}$ and $\mathrm{Me}_{4}$ As salts where the cation did not contain the ethyl groups, the $\Phi$ angle was negative. Assuming that the $\mathrm{S} \cdots \mathrm{H}-\mathrm{C}_{7}$ and $\mathrm{S} \cdots \mathrm{H}-\mathrm{C}_{8}$ interactions were also repulsive, the I-7, II-7, and II- 8 contacts decreased the $\Phi$ angle and the III-7 and I- 8 contacts increased the $\Phi$ angle (Figure 9). The II-7 and III-7 contacts pushed the $\mathrm{S}_{9}$ atom, and they acted on the $\Phi$ angle oppositely. Thus, the distortion through the $\mathrm{S}_{9}$ atom was determined by the competition of these two contacts in the $\mathrm{Me}_{4} \mathrm{Z}$ salts. The II-7 contact was nearly along the displacement of the $S$ atom, whereas the III-7 contact had less components parallel to the displacement. This difference in the contact angle was expected to provide the negative $\Phi$ value in the $\mathrm{Me}_{4} \mathrm{P}$ and $\mathrm{Me}_{4} \mathrm{As}$ salts. A similar mechanism involving the I-7, I-8. and II- 8 contacts could be applied to the distortion through the $S_{10}$. Note that the I-8 contact that increased the $\Phi$ angle was longer and the II- 8 contact that decreased the $\Phi$ value was shorter in the $\mathrm{Me}_{4}$ As salt than in the $\mathrm{Me}_{4} \mathrm{Sb}$ salt. These structural features were consistent with the smaller $\Phi$ value in the $\mathrm{Me}_{4}$ As salt than that in the $\mathrm{Me}_{4} \mathrm{Sb}$ salt [3]. 


\section{Conclusions}

The crystal structures of quasi-triangular frustrated $S=1 / 2$ system, the $\beta^{\prime}-\mathrm{Pd}(\mathrm{dmit})_{2}$ salts, were determined in the range of 5-295 K by the single crystal X-ray diffraction technique. Band parameters, Fermi surface, and band structures were calculated by the tight binding calculation based on the extended Hückel method and the first-principles DFT calculation using the obtained crystal structures. In the QSL material, the EtMe 3 Sb salt, every interdimer transfer integral, $t_{\mathrm{B}}, t_{\mathrm{S}}$, and $t_{\mathrm{r}}$ lineally increased with lowering temperatures. The change of $t_{\mathrm{r}}$ was more moderate than those of $t_{\mathrm{B}}$ and $t_{\mathrm{S}}$, thus the anisotropy of the triangular lattice was enhanced by lowering temperatures. Interdimer transfer integrals in the $\mathrm{Me}_{4} \mathrm{Sb}, \mathrm{Me}_{4} \mathrm{As}$ and $\mathrm{Et}_{2} \mathrm{Me}_{2} \mathrm{As}$ salts also increased with lowering temperature, and the triangular lattice became anisotropic in the low temperature region. It was quite interesting that the QSL state in the $\mathrm{EtMe}_{3} \mathrm{Sb}$ salt was realized in the rather anisotropic triangular lattice system where the spin frustration would be reduced. Recently, vibrational spectroscopy has revealed that charge and lattice fluctuations associated with the formation of closed shell tetramers and octamers can suppress the magnetic order [32].

At each temperature, the Fermi surface calculated by the tight binding method reproduced the results of the first-principles DFT calculation. The conduction band, which originated from the anti-bonding HOMO pair, remained isolated from other bands and half-filled even at $5 \mathrm{~K}$. These results indicated that the dimer picture based on only HOMO-HOMO interactions was still available down to the low temperature region.

Since the p-orbitals of the S atom mainly contributed to the formation of the conduction band, the intermolecular S ..S contacts were closely related to the band parameters. In the cation effect on the intermolecular transfer integrals, the arch-shaped distortion of the $\mathrm{Pd}(\mathrm{dmit})_{2}$ molecule tuned the intermolecular S. . S contacts. In the temperature effect, however, the S-Pd-Pd-S torsion angle, $\Phi$, which characterizes the arch-shaped molecular distortion, was almost temperature-independent, and the intermolecular transfer integrals were linearly correlated to the intermolecular S . S S distances. All these results suggested that the temperature dependence of the band parameters was mainly associated with the thermal contraction of the $\mathrm{Pd}(\mathrm{dmit})_{2}$ layer.

The cation-anion interactions through the short S . . H-C contacts acted repulsively and induced the arch-shaped distortion. This was categorized as the steric effect, and we did not detect any electronic effect of the cation-anion interactions. In this sense, it should be noted that the electronic fluctuations by the rotations of the methyl and ethyl groups in the cation did not directly induce the enhancement of the dielectric constant in the $\mathrm{EtMe}_{3} \mathrm{Sb}$ salt, which was revealed by the recent NMR measurements [33].

Supplementary Materials: The following are available online at http://www.mdpi.com/2073-4352/8/3/138/s1. Figure S1: Temperature dependence of lattice constants in the $\mathrm{EtMe}_{3} \mathrm{Sb}$ salt; Figure S2: Band structures of the $\mathrm{EtMe}_{3} \mathrm{Sb}$ salt calculated with the first-principles DFT method; Table S1: Intermolecular transfer integrals in (Cation) $\left[\mathrm{Pd}(\mathrm{dmit})_{2}\right]_{2}$ calculated by using the extended Hückel method; Table S2: Interdimer transfer integrals, $t^{\prime} / t$, and band widths in (Cation) $\left[\mathrm{Pd}(\mathrm{dmit})_{2}\right]_{2}$ calculated by using the extended Hückel method; Table S3: Band width of the conduction band, $W$, in the $\mathrm{EtMe}_{3} \mathrm{Sb}$ salt calculated with the first-principles DFT method.

Acknowledgments: This work was supported by the JSPS KAKENHI Grants, JP16H06346 and JP16K17756. The first-principles calculations were performed by the RIKEN supercomputer Hokusai GreatWave and NIMS Numerical Materials Simulator.

Author Contributions: All authors conceived and designed the experiments; Kohei Ueda performed crystal structure analyses; Kohei Ueda and Reizo Kato performed the extended Hückel and the tight binding calculations; Takao Tsumuraya performed the first-principles DFT calculations; Reizo Kato contributed reagents/materials; Kohei Ueda and Reizo Kato wrote the paper with inputs from all authors.

Conflicts of Interest: The authors declare no conflict of interest.

\section{References}

1. Kato, R. Conducting Metal Dithiolene Complexes: Structural and Electronic Properties. Chem. Rev. 2004, 104, 5319-5346. [CrossRef] [PubMed] 
2. Kanoda, K.; Kato, R. Mott Physics in Organic Conductors with Triangular Lattices. Ann. Rev. Condens. Matter Phys. 2011, 2, 167-188. [CrossRef]

3. Kato, R.; Hengbo, C. Cation Dependence of Crystal Structure and Band Parameters in a Series of Molecular Conductors, $\beta^{\prime}$-(Cation) $\left[\mathrm{Pd}(\mathrm{dmit})_{2}\right]_{2}$ (dmit = 1,3-dithiole-2-thione-4,5-dithiolate). Crystals 2012, 2, 861-874. [CrossRef]

4. Kato, R. Development of $\pi$-Electron Systems Based on [M(dmit $\left.)_{2}\right](\mathrm{M}=\mathrm{Ni}$ and Pd; dmit: 1,3-dithiole-2thione-4,5-dithiolate) Anion Radicals. Bull. Chem. Soc. Jpn. 2014, 87, 355-374. [CrossRef]

5. Kato, R.; Liu, Y.-L.; Hosokoshi, Y.; Aonuma, S.; Sawa, H. Se-Substitution and Cation Effects on the High-Pressure Molecular Superconductor, $\beta-\mathrm{Me}_{4} \mathrm{~N}\left[\mathrm{Pd}(\mathrm{dmit})_{2}\right]_{2}-\mathrm{A}$ Unique Two-Band System. Mol. Cryst. Liq. Cryst. 1997, 296, 217-244. [CrossRef]

6. Mori, T.; Kobayashi, A.; Sasaki, Y.; Kobayashi, H.; Saito, G.; Inokuchi, H. The Intermolecular Interaction of Tetrathiafulvalene and Bis(ethylenedithio)tetrathiafulvalene in Organic Metals. Calculation of Orbital Overlaps and Models of Energy-band Structures. Bull. Chem. Soc. Jpn. 1984, 57, 627-633. [CrossRef]

7. Canadell, E.; Rachidi, I.E.-I.; Ravy, S.; Pouget, J.-P.; Brossard, L.; Legros, J.-P. On the band electronic structure of $\mathrm{X}\left[\mathrm{M}(\mathrm{dmit})_{2}\right]_{2}\left(\mathrm{X}=\mathrm{TTF},\left(\mathrm{CH}_{3}\right)_{4} \mathrm{~N} ; \mathrm{M}=\mathrm{Ni}, \mathrm{Pd}\right)$ molecular conductors and superconductors. J. Phys. 1989, 50, 2967-2981. [CrossRef]

8. Tamura, M.; Kato, R. Magnetic Susceptibility of $\beta^{\prime}-\left[\mathrm{Pd}(\mathrm{dmit})_{2}\right]$ salts (dmit = 1,3-dithiol-2-thione-4,5-dithiolate, $\mathrm{C}_{3} \mathrm{~S}_{5}$ ): Evidence for Frustration in Spin-1/2 Heisenberg Antiferromagnets on a Triangular Lattice. J. Phys. Condens. Matter 2002, 14, L729-L734. [CrossRef]

9. Nakamura, T.; Takahashi, T.; Aonuma, S.; Kato, R. EPR Investigation of the Electronic States in $\beta^{\prime}$-type $\left[\mathrm{Pd}(\mathrm{dmit})_{2}\right]_{2}$ Compounds (where dmit is 2-thioxo-1,3-dithiole-4,5-dithiolate). J. Mater. Chem. 2001, 11, 2159-2162. [CrossRef]

10. Otsuka, K.; Iikubo, H.; Kogure, T.; Takano, Y.; Hiraki, K.I.; Takahashi, T.; Cui, H.; Kato, R. Antiferromagnetic Ordering in Quasi-Triangular Localized Spin System, $\beta^{\prime}-\mathrm{Et}_{2} \mathrm{Me}_{2} \mathrm{P}\left[\mathrm{Pd}(\mathrm{dmit})_{2}\right]_{2}$, Studied by ${ }^{13} \mathrm{C}$ NMR. J. Phys. Soc. Jpn. 2014, 83, 054712. [CrossRef]

11. Ohira, S.; Tamura, M.; Kato, R.; Watanabe, I.; Iwasaki, M. Muon Spin Relaxation Study of the Magnetic Transition in a Two-Dimensional Distorted Triangular Lattice $\beta^{\prime}-\left(\mathrm{CH}_{3}\right)_{4} \mathrm{P}\left[\mathrm{Pd}(\mathrm{dmit})_{2}\right]_{2}$. Phys. Rev. B 2004, 70, 220404. [CrossRef]

12. Ohira, S.; Tamura, M.; Kato, R.; Iwasaki, M. Magnetic Order and Charge Separation in 2D Distorted Triangular Lattice Systems $\beta^{\prime}-X\left[P d(d m i t)_{2}\right]_{2}$. Phys. B 2006, 374-375, 122-125. [CrossRef]

13. Itou, T.; Oyamada, A.; Maegawa, S.; Tamura, M.; Kato, R. Quantum Spin Liquid in the Spin-1/2 Triangular Antiferromagnet $\mathrm{EtMe}_{3} \mathrm{Sb}\left[\mathrm{Pd}(\mathrm{dmit})_{2}\right]_{2}$. Phys. Rev. B 2008, 77, 104413. [CrossRef]

14. Itou, T.; Oyamada, A.; Maegawa, S.; Kato, R. Instability of a Quantum Spin Liquid in an Organic Triangular-Lattice Antiferromagnet. Nat. Phys. 2010, 6, 673-676. [CrossRef]

15. Nakao, A.; Kato, R. Structural Study of Low Temperature Charge-Separated Phases of Pd(dmit) $)_{2}$-Based Molecular Conductors. J. Phys. Soc. Jpn. 2005, 74, 2754-2763. [CrossRef]

16. Tamura, M.; Takenaka, K.; Takagi, H.; Sugai, S.; Tajima, A.; Kato, R. Spectroscopic evidence for the low-temperature charge-separated state of [Pd(dmit) ${ }_{2}$ ] salts. Chem. Phys. Lett. 2005, 411, 133-137. [CrossRef]

17. Shimizu, Y.; Miyagawa, K.; Kanoda, K.; Maesato, M.; Saito, G. Spin Liquid State in an Organic Mott Insulator with a Triangular Lattice. Phys. Rev. Lett. 2003, 91, 107001. [CrossRef] [PubMed]

18. Isono, T.; Kamo, H.; Ueda, A.; Takahashi, K.; Kimata, M.; Tajima, H.; Tsuchiya, S.; Terashima, T.; Uji, S.; Mori, H. Gapless Quantum Spin Liquid in an Organic Spin-1/2 Triangular-Lattice $\kappa-\mathrm{H}_{3}(\text { Cat-EDT-TTF })_{2}$. Phys. Rev. Lett. 2014, 112, 177201. [CrossRef] [PubMed]

19. Rouzière, S.; Yamaura, J.-I.; Kato, R. Low-temperature structural studies of molecular $\beta^{\prime}-\mathrm{Pd}(\mathrm{dmit})_{2}$ conductors. Phys. Rev. B 1999, 60, 3113-3119. [CrossRef]

20. Tsumuraya, T.; Seo, H.; Tsuchiizu, M.; Kato, R.; Miyazaki, T. Cation Dependence of the Electronic States in Molecular Triangular Lattice System $\beta^{\prime}-\mathrm{X}\left[\mathrm{Pd}(\mathrm{dmit})_{2}\right]_{2}$ : A First-Principles Study. J. Phys. Soc. Jpn. 2013, 82, 033709. [CrossRef]

21. Nakamura, K.; Yoshimoto, Y.; Imada, M. Ab initio two-dimensional multiband low-energy models of $\mathrm{EtMe}_{3} \mathrm{Sb}\left[\mathrm{Pd}(\mathrm{dmit})_{2}\right]_{2}$ and $\kappa-(\mathrm{BEDT}-\mathrm{TTF})_{2} \mathrm{Cu}(\mathrm{NCS})_{2}$ with comparisons to single-band models. Phys. Rev. $B$ 2012, 86, 205117. [CrossRef] 
22. Wimmer, E.; Krakauer, H.; Weinert, M.; Freeman, A.J. Full-potential self-consistent linearized-augmentedplane-wave method for calculating the electronic structure of molecules and surfaces: $\mathrm{O}_{2}$ molecule. Phys. Rev. B 1981, 24, 864-875. [CrossRef]

23. Weinert, M. Solution of Poisson's equation: Beyond Ewald-type methods. J. Math. Phys. 1981, 22, 2433-2439. [CrossRef]

24. Blöchl, P.E.; Jepsen, O.; Andersen, O.K. Improved tetrahedron method for Brillouin-zone integrations. Phys. Rev. B 1994, 49, 16223-16234. [CrossRef]

25. Perdew, J.P.; Burke, K.; Ernzerhof, M. Generalized Gradient Approximation Made Simple. Phys. Rev. Lett. 1996, 77, 3865-3868. [CrossRef] [PubMed]

26. Sheldrick, G.M. A short history of SHELX. Acta Crystallogr. Sect. A Found. Crystallogr. 2008, 64, 112-122. [CrossRef] [PubMed]

27. Yoshida, Y.; Ito, H.; Maesato, M.; Shimizu, Y.; Hayama, H.; Hiramatus, T.; Nakamura, Y.; Kishida, H.; Koretsune, T.; Hotta, C.; et al. Spin-disordered quantum phases in a quasi-one-dimensional triangular lattice. Nat. Phys. 2015, 11, 679-683. [CrossRef]

28. Tamura, M.; Kato, R. Effective on-site repulsion in molecular conductors with dimeric structure: Is the transfer integral a good measure of correlation. J. Phys. Soc. Jpn. 2004, 73, 3108-3110. [CrossRef]

29. Pinterić, M.; Čulo, M.; Milat, O.; Basletić, M.; Korin-Hamzić, B.; Tafra, E.; Hamzić, A.; Ivek, T.; Peterseim, T.; Miyagawa, K.; et al. Anisotropic charge dynamics in the quantum spin-liquid candidate א-(BEDT-TTF $)_{2} \mathrm{Cu}_{2}(\mathrm{CN})_{3}$. Phys. Rev. B 2014, 90, 195139. [CrossRef]

30. Dressel, M.; Lazić, P.; Pustogow, A.; Zhukova, E.; Gorshunov, B.; Schlueter, J.A.; Milat, O.; Gumhalter, B.; Tomić, $\mathrm{S}$. Lattice vibrations of the charge-transfer salt $\kappa-(\mathrm{BEDT}-\mathrm{TTF})_{2} \mathrm{Cu}_{2}(\mathrm{CN})_{3}$ : Comprehensive explanation of the electrodynamic response in a spin-liquid compound. Phys. Rev. B 2016, 93, 081201. [CrossRef]

31. Pinterić, M.; Lazić, P.; Pustogow, A.; Ivek, T.; Kuveždić, M.; Milat, O.; Gumhalter, B.; Basletić, M.; Čulo, M.; Korin-Hamzić, B.; et al. Anion effects on electronic structure and electrodynamic properties of the Mott insulator k-(BEDT-TTF) ${ }_{2} \mathrm{Ag}_{2}(\mathrm{CN})_{3}$. Phys. Rev. B 2016, 94, 161105. [CrossRef]

32. Yamamoto, T.; Fujimoto, T.; Naito, T.; Nakazawa, Y.; Tamura, M.; Yakushi, K.; Ikemoto, Y.; Moriwaki, T.; Kato, R. Charge and Lattice Fluctuations in Molecule-Based Spin Liquids. Sci. Rep. 2017, 7, 12930. [CrossRef] [PubMed]

33. Fujiyama, S.; Kato, R. Algebraic Charge Dynamics of the Quantum Spin Liquid $\left.\beta^{\prime}-\mathrm{EtMe} \mathrm{Sb}_{3} \mathrm{Pd}(\mathrm{dmit})_{2}\right]_{2}$. Phys. Rev. B 2018, 97, 035131. [CrossRef]

(C) 2018 by the authors. Licensee MDPI, Basel, Switzerland. This article is an open access article distributed under the terms and conditions of the Creative Commons Attribution (CC BY) license (http:/ / creativecommons.org/licenses/by/4.0/). 


\title{
$(\mathrm{BEDT}-\mathrm{TTF})_{2} \mathrm{Cu}_{2}(\mathrm{CN})_{3}$ Spin Liquid: Beyond the Average Structure
}

\author{
Pascale Foury-Leylekian ${ }^{1, *}$, Vita Ilakovac-Casses ${ }^{2,3}$, Victor Balédent ${ }^{1}$, Pierre Fertey ${ }^{4}$, \\ Alla Arakcheeva ${ }^{5}$, Ognjen Milat ${ }^{6}$, Denis Petermann ${ }^{1}$, Gilles Guillier ${ }^{1}$, Kazuya Miyagawa ${ }^{7}$, \\ Kazushi Kanoda ${ }^{7}$, Pere Alemany ${ }^{8}$, Enric Canadell ${ }^{9}$, Silvia Tomic ${ }^{6}$ and Jean-Paul Pouget ${ }^{1}$ \\ 1 Laboratory of Solid State Physics, CNRS UMR 8502, Univ. Paris Sud, Paris Saclay University, \\ 91405 Orsay CEDEX, France; victor.baledent@u-psud.fr (V.B.); denis.petermann@u-psud.fr (D.P.); \\ guillier@lps.u-psud.fr (G.G.); pouget@lps.u-psud.fr (J.-P.P.) \\ 2 UPMC, LCP-MR, Sorbonne University, CNRS UMR 7614, F-75252 Paris, France; \\ vita.ilakovac-casses@upmc.fr \\ 3 Department of Physcs, Cergy-Pontoise University, F-95031 Cergy-Pontoise, France \\ 4 Synchrotron SOLEIL, L'Orme des Merisiers, Saint-Aubin, B.P. 48, F-91192 Gif-sur-Yvette, France; \\ pierre.fertey@synchrotron-soleil.fr \\ 5 Laboratory of Condensed Matter, EPFL, CH-1015 Lausanne, Switzerland; allaarakcheeva@gmail.com \\ 6 Institute of Physics, Bijenička Cesta 46, HR-10000 Zagreb, Croatia; milat@ifs.hr (O.M.); stomic@ifs.hr (S.T.) \\ 7 Department of Applied Physics, University of Tokyo, Tokyo 113-8656, Japan; \\ kazuya@ap.t.u-tokyo.ac.jp (K.M.); kanoda@ap.t.u-tokyo.ac.jp (K.K.) \\ 8 Department of Materials Science and Chemical Physics and Institute of Theoretical and Computational \\ Chemistry (IQTCUB), University of Barcelona, Martí i Franquès 1, 08028 Barcelona, Spain; \\ p.alemany@ub.edu \\ 9 Institute of Materials Science of Barcelona (ICMAB-CSIC), Campus de la UAB, 08193 Bellaterra, Spain; \\ canadell@icmab.es \\ * Correspondence: pascale.foury@u-psud.fr; Tel.: +33-169-156-055
}

Received: 27 February 2018; Accepted: 30 March 2018; Published: 4 April 2018

\begin{abstract}
We present here the first accurate determination of the exact structure of k-(BEDT-TTF $)_{2} \mathrm{Cu}_{2}(\mathrm{CN})_{3}$. Not only did we show that the room temperature structure used over the last twenty years was incorrect, but we were also able to correctly and precisely determine it. The results of our work provide evidence that the structure presents a triclinic symmetry with two non-equivalent dimers in the unit cell, which implies a charge disproportionation between the dimers. However, structural refinement shows that the charge disproportionation is quite weak at room temperature.
\end{abstract}

Keywords: mott transition; molecular spin liquids; geometrical frustration; $\mathrm{X}$-ray diffraction

\section{Introduction}

One of the richest subjects of study in condensed matter over the last 30 years concerns Mott insulators with strong magnetic interactions and antiferromagnetic ground state in whose proximity unconventional superconductivity can emerge. A more fascinating and fertile situation occurs when spin frustration comes into play [1]. In such a case, long-range magnetic order can be easily suppressed by quantum fluctuations, and a quantum spin liquid (QSL) ground state is expected [2]. The strong magnetic frustration is typically achieved for localized $S=1 / 2$ spins antiferromagnetically (AFM) coupled on a perfect triangular lattice [3]. However, the experimental realization of such a system turned out to be rare, and few inorganic QSLs with a triangular network of spins have been discovered [4-7]. Recently, two families of layered (2D) organic salts- $k$ phases of the BEDT-TTF donors, $\mathrm{k}-(\mathrm{BEDT}-\mathrm{TTF})_{2} \mathrm{X}$, and $\beta$ phases of the $\mathrm{Pd}(\mathrm{dmit})_{2}$ acceptor, $\beta-\mathrm{Y}\left[\mathrm{Pd}(\mathrm{dmit})_{2}\right]_{2}$ - have been 
found to present a quasi-ideal triangular lattice of $S=1 / 2$ spins [8,9]. Here, BEDT-TTF stands for bis-ethylene-dithio-tetrathiafulvalene, and dmit is dimercaptoisotrithione; $X$ and $Y$ are, respectively, anionic and cationic species. In $\mathrm{k}-(\mathrm{BEDT}-\mathrm{TTF})_{2} \mathrm{X}$ salts built with two dimers of BEDTT-TTF and two anions $\mathrm{X}$ per unit cell, half an electron per BEDT-TTF is transferred on average to the anion layer, leaving one hole per BEDT-TTF dimer and thus one $S=1 / 2$ spin. A $2 \mathrm{D}$ array of inter-dimer transfer integrals $\left(\mathrm{t}_{0}, \mathrm{t}_{0}{ }^{\prime}\right)$ connects the spins $1 / 2$ in a quasi-isotropic triangular lattice with a ratio $\mathrm{t}_{0} / \mathrm{t}_{0}{ }^{\prime}$, which is in the range 0.6-1.1 [10-12]. Of particular interest is the $\mathrm{k}-(\mathrm{BEDT}-\mathrm{TTF})_{2} \mathrm{Cu}_{2}(\mathrm{CN})_{3}$ compound (here after named $\mathrm{k}-\mathrm{ET}-\mathrm{Cu}$ ) in which the $\mathrm{t}_{0} / \mathrm{t}_{0}{ }^{\prime}$ ratio and thus $\mathrm{J} / \mathrm{J}$ ' is very close to 1 and therefore presents a case of a strong degree of frustration. This property, in the presence of a strong AFM interaction between spins where J $250 \mathrm{~K}$, has been considered as a necessary ingredient for QSL [13,14]. Indeed, NMR [13] and heat capacity [15] measurements performed a decade ago have provided evidence of a spin-liquid ground state in $\mathrm{k}$-ET-Cu.

However, this salt is also a $\frac{1}{4}$ filled $2 \mathrm{D}$ electron gas with strong electron-electron interactions: the onsite Coulomb repulsion $U$ is comparable to the bandwidth $U / W \approx 1.8[10,16]$. It can thus stabilize various competing electronic phases [17], which might strongly impact the QSL. In particular, one expects bond order waves (BOWs) where holes are localized on intermolecular bonds competing with charge order (CO) states, where the charge occupancy of the molecules is modulated. Due to the strong dimerization of the donor layer, $\mathrm{k}$-ET-Cu has one hole with spin $1 / 2$ localized per dimer (i.e., on the intra-dimer bond) and is thus generally regarded as a Mott-Hubbard localized system. In addition, the possible emergence of a (polar) CO state in $\mathrm{k}$-ET-Cu is an important issue that should not be neglected since ferroelectricity induced by an ordering of electric dipoles on the BEDT-TTF molecular dimers has been suggested to drive the spin-order in the Mott-Hubbard phase of $\mathrm{K}$-(BEDT-TTF $)_{2} \mathrm{Cu}(\mathrm{SCN})_{2} \mathrm{Cl}[18]$. The coupling of the electric dipoles with the spins could be a possible mechanism for the stabilization of QSL [19-24].

It is now established that $\mathrm{k}$-ET-Cu presents a very rich pressure-temperature phase diagram. At ambient pressure, this salt exhibits a thermodynamic phase transition around $6 \mathrm{~K}$ [25] with significant lattice [25] and sound velocity [26] anomalies. Interestingly, a recent resonant inelastic X-ray scattering study has been able to probe in the anion layer the vibration spectra of $\mathrm{N}$ atoms sizably coupled to the donors (see below) and to measure the electron-phonon coupling [27]. Under a low pressure of $0.4 \mathrm{GPa}$, the system becomes metallic and superconducting [28].

In $\mathrm{k}$-(BEDT-TTF $)_{2} \mathrm{X}$ compounds, which all exhibit such a subtle coupling between spin, charge, and lattice degrees of freedom, it is obvious that the detailed structure and its thermal evolution has a strong influence on the spin coupling and Mott behavior. Moreover, the crystallographic structure of $\mathrm{k}$-ET-Cu is not obvious because this salt contains various intrinsic disorders within the anionic layer as well as in the molecular stack. The room temperature structure of $\mathrm{k}$-ET-Cu has been intensively studied [29-33]. More recent studies of the thermal evolution of the structure have also been published [11,12,34].

In all of these previous works, the structure is refined in the monoclinic and centrosymmetric $\mathrm{P} 21 / \mathrm{c}$ space group. The structure consists of $(b, c)$ layers of strongly dimerized BEDT-TTF molecules, with each dimer oriented approximately perpendicularly to its nearest neighbors. The four molecules of the unit cell are equivalent by the symmetry elements of the space group. BEDT-TTF layers alternate along the a direction with $\mathrm{Cu}_{2} \mathrm{CN}_{3}$ anionic layers. The anionic layer is composed of $\mathrm{CuCN}$ polymeric-like chains running along the $\mathbf{b}$ axis. There are two chains per unit cell related by the inversion center. The chains are connected by $\mathrm{C}-\mathrm{N}$ groups (hereafter labeled $\mathrm{C} 2-\mathrm{N} 2$ and $\mathrm{C} 10-\mathrm{N} 10)$. In the $\mathrm{P} 2{ }_{1} / \mathrm{c}$ space group, the $\mathrm{C} 2-\mathrm{N} 2$ groups are located at the inversion center. These groups are thus expected to be disordered either dynamically or statistically. Within the molecular layer, the ethylene groups $\left(\mathrm{C}_{2} \mathrm{H}_{4}\right)$ of the BEDT-TTF molecules are, depending on the structural refinement, either ordered [29,31] or partially disordered $[11,34]$ at $300 \mathrm{~K}$. Disorder should be at the origin of the large anisotropic displacement parameters (ADP) mentioned in [33]. Disorder will be discussed in detail in the present study.

The aim of this work is to accurately re-investigate the structure of $\mathrm{k}$-ET-Cu. We show in particular that the long-accepted $300 \mathrm{~K}$ structure is incorrect. The observation from $300 \mathrm{~K}$ down to $3 \mathrm{~K}$ of 
Bragg reflections forbidden in the $\mathrm{P} 2{ }_{1} / \mathrm{c}$ space group shows that $\mathrm{K}$-ET-Cu adopts either the $\mathrm{P} \overline{1}$ or P1 space groups. This new symmetry definitively proves the existence of the two unequal dimers per unit cell, which implies the existence of a charge disproportionation between these dimers in the entire temperature range. However, the structural refinement performed at room temperature shows that inter-dimer charge disproportionation is quite weak. Such charge disproportionation theoretically proposed in [19-24], but not detected by optical measurements [35], could help to understand the relaxor-like dielectric behavior $[36,37]$ and may give a long-awaited experimental support for the suggested role of spin-dipolar coupling in the establishment of QSL. Additionally, charge disproportionation not fixed by the crystal symmetry can vary under external constraints such as temperature or pressure. For example, its enhancement under pressure may favor a transition from the localized Mott-Hubbard state at ambient pressure to a metallic state that exhibits superconductivity.

\section{Materials and Methods}

High-quality single crystals of K-ET-Cu were synthesized by electro-crystallization following the process used in $[29,37]$. Crystals appear like very thin plate-like samples of less than $1 \mathrm{~mm}^{3}$. Their surface corresponds to the $(b, c)$ plane. We have performed preliminary X-ray diffraction measurements using a $\mathrm{Cu} \mathrm{K} \alpha$ radiation laboratory source. For this diffraction study, we used a homemade three-circle diffractometer equipped with a pulse tube cryogenerator allowing measurements from room temperature down to $3 \mathrm{~K}$. The full data collection has been performed on the synchrotron radiation facility SOLEIL at the CRISTAL beam line using a Newport four-circle diffractometer equipped with a Rigaku Oxford Diffraction Atlas CCD detector at a wavelength of $0.6724 \AA$. About 17,000 reflections were collected. Data were processed using the CrysAlis Pro suite [38], taking into account two components of the likely twinned crystal. The ab initio structure was solved using the charge flipping algorithm and then refined by full-matrix least squares, using Jana2006 software [39]. Note that the introduction of twins only weakly improved the refinement. The twinning ratio was found to be around $50 \%$. The twinning (twofold axis along the $\mathrm{c}$ direction) relates domains where the two different dimers of the unit cell are interchanged. It can be a macro- or micro-twinning. However, the size of the twinned domains cannot be lower than a few microns because all Bragg reflections have an experimental resolution.

First-principles calculations were carried out using a density functional theory (DFT) approach [40,41], which was developed for efficient calculations in large systems and implemented in SIESTA code [42-44]. We used the generalized gradient approximation (GGA) to DFT and, in particular, the functional of Perdew, Burke, and Ernzerhof [45]. Only the valence electrons are considered in the calculation, with the core being replaced by norm-conserving scalar relativistic pseudopotentials [46] factorized in the Kleinman-Bylander form [47]. We have used a split-valence double- $\zeta$ basis set including polarization orbitals with an energy shift of $10 \mathrm{meV}$ for all atoms [48]. The energy cutoff of the real space integration mesh was 350 Ry. The Brillouin zone was sampled using a grid of $(5 \times 20 \times 20) k$-points [49]. The crystal structure at room temperature was used for the calculations.

\section{Results}

\subsection{Evidence for a $P 2_{1} / c$ Symmetry Breaking}

In the $\mathrm{P} 2{ }_{1} / \mathrm{c}$ (P $\left.12_{1} / \mathrm{c} 1\right)$ space group, $h 0 l$ Bragg reflections are forbidden whenever $l$ is odd (c glide mirror extinctions), and the $0 k 0$ ones are forbidden for odd $k$ indices ( 21 helicoidal axis extinctions). Our structural measurements performed with both synchrotron and laboratory X-ray radiation have evidenced at $300 \mathrm{~K}$, the presence of the two kinds of forbidden reflections. It is important to notice that these features were systematically observed for the 15 different crystals measured, except for 2 that were very small $\left(30 \times 30 \times 30 \mu \mathrm{m}^{3}\right)$. However, for these two crystals, the intensity of the Bragg reflections was already not high enough for the detection of weak satellite reflections. It is also important to mention that, intrinsically, in any $\mathrm{X}$-ray pattern, the number of $0 \mathrm{k} 0$ reflections with odd $k$ 
values is small. In the case of K-ET-Cu, we observed only 010 and 030 , forbidden by the $2_{1}$ symmetry operation, but they were systematically present. Figure 1 displays the reciprocal lattice reconstruction of the $\left(\mathrm{a}^{*}, \mathrm{c}^{*}\right)$ reciprocal plane at $300 \mathrm{~K}$, and the most prominent forbidden reflections are indicated. Before going any further, we checked the possibilities of different experimental artifacts that could have been at the origin of these additional intensities. A wavelength harmonic contamination $(\lambda / 2)$ was totally excluded. A twinning effect cannot explain the presence of such forbidden reflections because no symmetry operation applied to the reciprocal space of one domain without forbidden reflections can lead to reflections observed at very low angles, such as the one indexed as a 010 reflection. The possibility of a multiple scattering effect was also ruled out due to the weakness of the scattering factors in organic compounds and the systematic character of the forbidden effects. Its presence thus cannot be associated with an experimental artifact and since they are observed in all sizeable single crystals measured, we can conclude that this symmetry breaking is an intrinsic structural property of $\mathrm{k}$-ET-Cu at $300 \mathrm{~K}$.

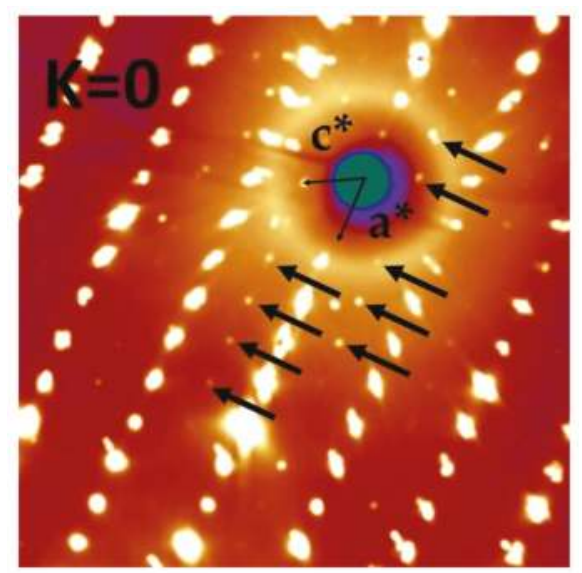

Figure 1. (Color online). Reconstruction of the $\left(\mathbf{a}^{*}, \mathbf{c}^{*}\right)$ reciprocal plane taking into account the absorption correction. The thin arrows indicate the directions of the reciprocal lattice parameters, and the thick arrows show the reflections forbidden in the $\mathrm{P} 2{ }_{1} / \mathrm{c}$ space group.

In addition, all measured reflections, including the reflections forbidden in the $\mathrm{P} 2{ }_{1} / \mathrm{c}$ space group, had an experimental resolution. Furthermore, no particular diffuse scattering was detected. Finally, we observed not only low angle forbidden reflections but also high angle ones such as the 5013. All of these features suggest that the presence of forbidden reflections does not originate from a thermal effect, nor from a disorder, but rather from an atomic displacement associated with a global symmetry breaking of the $\mathrm{P} 2{ }_{1} / \mathrm{c}$ structure. The average intensity of the forbidden reflections is 100 times lower than that of the main authorized Bragg reflections. The weakness of the intensity of the forbidden reflections indicates that the symmetry breaking is weak and explains that the previous structural studies have not detected this symmetry breaking with standard collect and refinement procedures.

Regarding the presence on the X-ray pattern of the various forbidden reflections, only the $\mathrm{P} \overline{1}$ and $\mathrm{P} 1$ sub-groups of $\mathrm{P} 21 / \mathrm{c}$ are possible space groups for the real structure of $\mathrm{K}$-ET-Cu at $300 \mathrm{~K}$. One can note that the triclinic angles $\alpha$ and $\gamma$ are equal to $90^{\circ}$ within error bars. The distinction between the P1 and $\mathrm{P} \overline{1}$ space groups is very delicate from an experimental point of view. In particular, using X-rays, it is nearly impossible to differentiate both groups due to the Friedel law, which artificially creates a center of inversion in the X-ray pattern. We have then tried second harmonic generation measurements. However, the transmission measurements at the usual wavelength of $1064 \mathrm{~nm}$ were not possible due to the fact that $\mathrm{k}$-ET-Cu is nearly metallic, being very close to the Mott insulator-to-metal transition 
boundary. Namely, no clear-cut optical gap has been observed, while the transport gap is less than $40 \mathrm{meV}[16,37,50]$.

\subsection{Structure at $300 \mathrm{~K}$}

For our refinements we used the $\mathrm{P} \overline{1}$ space group as we could not detect the lack of inversion symmetry leading to P1.

\subsubsection{Modeling of the Disorder}

We used anisotropic displacement parameters (ADPs) for all the atomic species except for the hydrogen $(\mathrm{H})$ atoms. Hydrogen atoms were placed at their ideal calculated positions and refined using a riding model. Each ethylene group of the BEDT-TTF molecule adopts a twist conformation as defined in Figure 2d. The two ethylene groups per BEDT-TTF molecule were however treated differently: the best structural model (i.e., the lowest $\mathrm{R}$ factors and the lowest electron density residuals) was obtained when the positions of the carbon atoms of one of the ethylene groups (the one on the left side of Figure 2a) were split over two symmetrically equivalent positions with respect to the plane of the molecule (e.g., Ci7-Ci7a and Ci8-Ci8a in Figure 2a), denoted as $\mathrm{t}$ and $\bar{t}$. The other ethylene group (the one on the right side of Figure 2a) was not split, and its configuration is t. The $\mathrm{C}-\mathrm{C}$ distance of the symmetrically related ethylene groups as well as the carbon ADPs were kept identical. The fractional occupancy of each configuration was refined. This model led to staggered (S: $t, t)$ (Figure 2b) and eclipsed (E: t,, $\bar{t}$ ) Figure 2c bethylene conformations for each BEDT-TTF molecule, whose fractional occupancy reached $72 \%$ and $28 \%$ for the $\mathrm{S}$ and $\mathrm{E}$ conformations, respectively. These values agree with those obtained at $300 \mathrm{~K}$ in $[29,34]$.

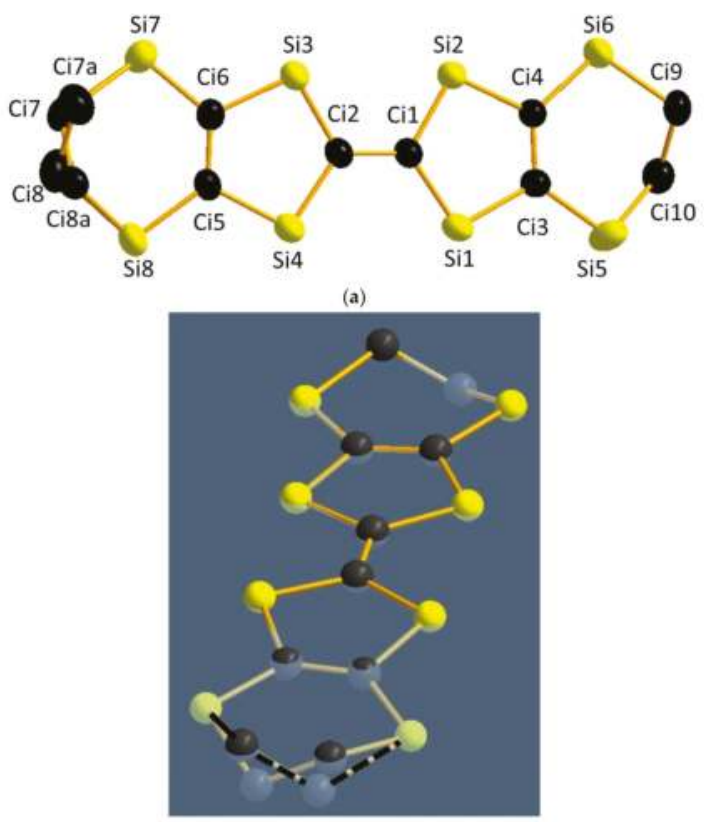

(b)

Figure 2. Cont. 


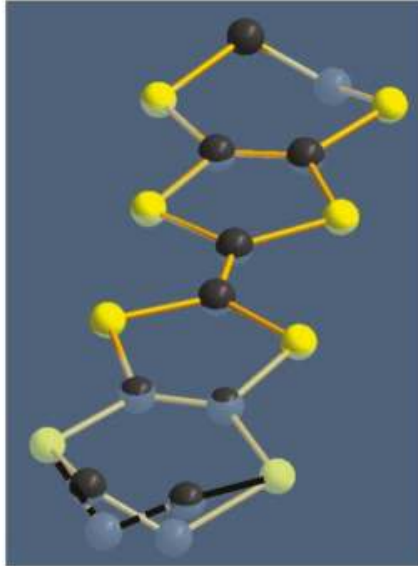

(c)

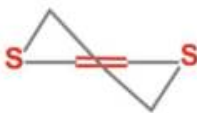

twist

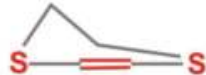

half-twist

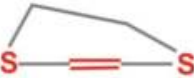

tilted boat

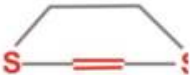

boat

Figure 2. (Color online). (a) Ellipsoid of the ADPs at $300 \mathrm{~K}$ and labels for the various $\mathrm{C}$ and $\mathrm{S}$ atoms of the BEDT-TTF molecule $\mathrm{i}$ ( $\mathrm{i}=1$ for Molecule 1 and $\mathrm{i}=2$ for Molecule 2 ). The two ethylene groups on the left side are in the $\mathrm{t}$ and $\bar{t}$ twist configurations ( $\mathrm{t}$ and $\bar{t}$ are twist conformations symmetrically related with respect to the plane of the molecule). The ethylene group, which is not split, is also presented and is in the twist configuration. Schematic view of the terminal ethylene groups of the BEDT-TTF molecule in the (b) staggered (S: $t, t)$ and $(\mathbf{c})$ eclipsed $(E: t, \bar{t})$ conformations. The blue plane is the average molecular plane. Dashed lines represent part of the chemical bond below the average molecular plane; (d) Schematic representation of the four possible conformations of an ethylene group in the BEDT-TTF molecule (each ethylene group is represented by two $\mathrm{C}$ atoms and viewed along the long axis of the molecule). The energy of the conformation increases from left to right. Note that, in the twist conformation, there is a $\mathrm{C}$ atom on each side of the molecular plane and that, in the half-twist conformation, one $\mathrm{C}$ atom is in (or very near) the molecular plane. In the more energetic tilted-boat and boat conformations, the $\mathrm{C}$ atoms are on the same side of the molecular plane.

Within the anionic group, we have analyzed the disorder of the C2-N2 and C10-N10 groups located on the inversion center. We have first fitted the occupancy $x$ of each site by an atom of carbon $\mathrm{C}(\mathrm{x})$ and an atom of nitrogen $\mathrm{N}(1-\mathrm{x})$. A total disorder corresponds to $\mathrm{x}=0.5$ and a total order to $\mathrm{x}=0$ or 1 . However, as the $\mathrm{X}$-ray scattering factors of $\mathrm{C}$ and $\mathrm{N}$ are nearly identical, we were not able to detect any reliable improvement of the refinement by varying $\mathrm{x}$. We have thus decided to introduce an average scattering factor corresponding to $50 \%$ of $\mathrm{C}$ and $50 \%$ of $\mathrm{N}$ for each site of the disordered C-N groups.

\subsubsection{Result of the Refinement}

The best refinement of the structure at $300 \mathrm{~K}$ in the $\mathrm{P} \overline{1}$ space group was obtained for the atomic positions and ADPs given in CCDC 1825317 (see details in [51]). The refinement led to $\mathrm{R}=5.32 \%$, $\mathrm{wR}=8.35 \%$, and $\mathrm{N}=16338$ reflections $(14,479$ with $\mathrm{I}>2 \sigma)$ for a total of 388 refined parameters, 184 constraints, and two restraints. The lattice parameters are $a=16.1221(10) \AA, b=8.591(6) \AA$, $\mathrm{c}=13.412(8) \AA, \alpha=89.99(2)^{\circ}, \beta=113.43(2)^{\circ}$, and $\gamma=90.01(2)^{\circ}$. The structure is shown in Figure 3a-c. 


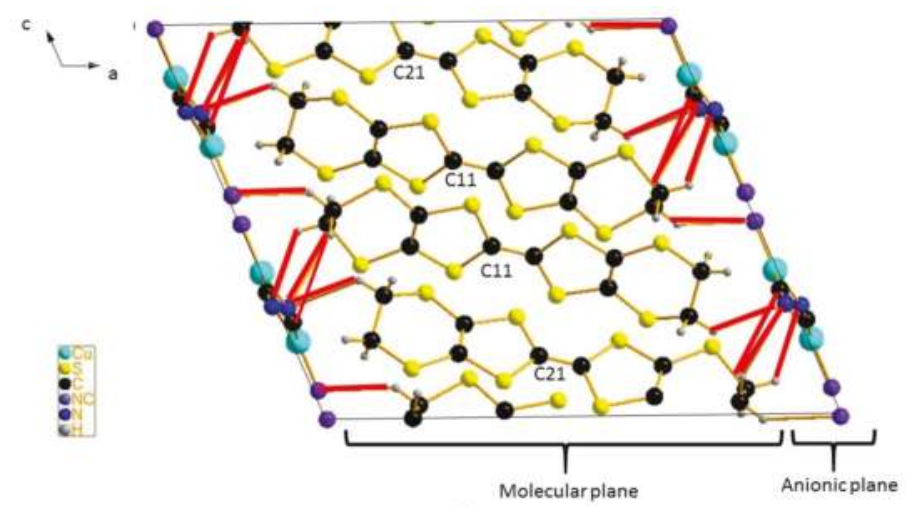

(a)

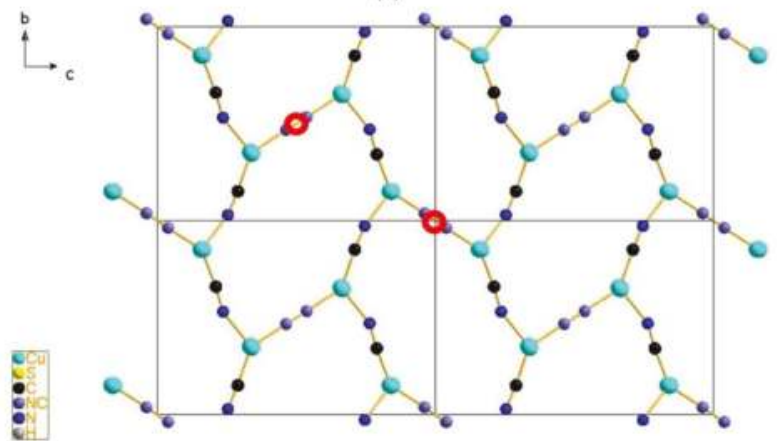

(b)

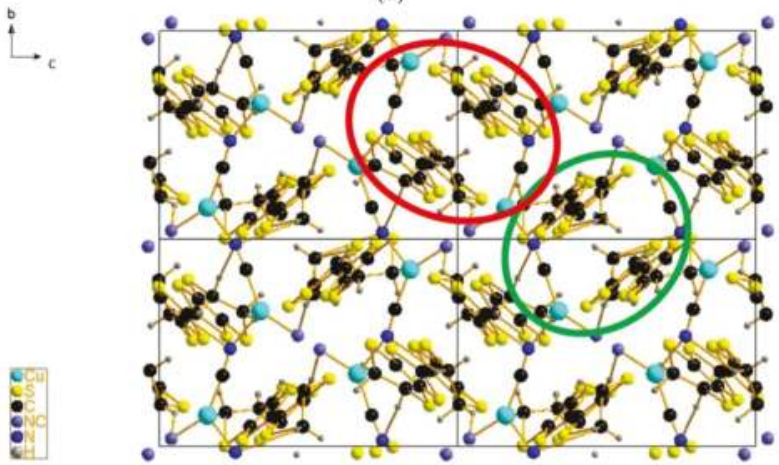

(c)

Figure 3. (a) (color online) Structure of $\mathrm{k}$-ET-Cu at $300 \mathrm{~K}$ viewed along the $\mathbf{b}$-axis. For clarity purpose, only the most probable staggered $\mathrm{C}_{2} \mathrm{H}_{4}$ group configurations are represented. In the molecular layer, the first number of the atom labels ( 1 or 2 ) refers to the two different types of molecules and are indicated for the Ci1 atoms only. The hydrogen bonds (red lines) of the ethylene groups (S conformation) with the $\mathrm{N}$ and disordered N/C atoms in the anionic plane are also shown; (b) View of the anionic plane parallel to the $(\mathbf{b}, \mathbf{c})$ plane: the disordered $\mathrm{C}-\mathrm{N}$ groups, which are located on the inversion centers (red circles), are colored in violet; (c) View of the anionic $(\mathbf{b}, \mathbf{c})$ plane and the molecular layer below showing Dimer 1 (green) and Dimer 2 (red). 
The $\mathrm{P} \overline{1}$ structure obtained is similar to the $\mathrm{P} 2_{1} / \mathrm{c}$ ones previously published in [29-31] and more recently in $[11,34]$. In particular, the nearly flat shape of the core of the BEDT-TTF molecules is in agreement with the one expected theoretically and obtained in [11] and [31-33]. One can notice that, in the structure of [29], the molecules are more bent than in our structure and in the structures of [11] and [31-34]. In addition, typical distances of the Ci1-Ci2 double bonds (defined in Figure 2a and given in Table 1) of our structure are consistent with those expected for BEDT-TTF molecules with a $+1 / 2$ charge (half-hole) and those obtained in the refinements of [11] and [31-34], while in [29] these distances are significantly larger.

Table 1. Relevant interatomic distances for the structure at $300 \mathrm{~K}$. The charge disproportionation $2 \delta$ defined in the text and calculated from the structural correlation as well as that from DFT calculations are also given.

\begin{tabular}{|c|c|}
\hline Temperature & $300 \mathrm{~K}$ \\
\hline $\mathrm{d}_{1}(\AA)$ & $3.866(20)$ \\
\hline $\mathrm{d}_{2}(\AA)$ & $3.872(20)$ \\
\hline C1-C2 Dimer $1(\AA)$ & $1.369(20)$ \\
\hline C1-C2 Dimer $2(\AA)$ & $1.372(20)$ \\
\hline $2 \delta$ (structural correlation) [52] & \pm 0.06 \\
\hline $2 \delta(\mathrm{DFT})$ & \pm 0.01 \\
\hline
\end{tabular}

Importantly, our density map indicates that, at room temperature, ethylene groups are slightly disordered. This is particularly the case of the Ci7-Ci8 groups (defined in Figure 2a), which are the closest ethylene groups to the disordered $\mathrm{C}-\mathrm{N}$ groups. The ethylene groups of the BEDT-TTF molecules at $300 \mathrm{~K}$ are thus rather in a staggered twist conformation on each side of the molecule. The twist configuration presents the one of the lowest energy values for an isolated molecule $[53,54]$ (see Figure 2d) as does that obtained by ab initio calculations in a similar compound [55]. This finding contrasts with the conclusion of refinements of $[29,31]$ where an ordered configuration of the BEDT-TTF molecule was found at room temperature. These last refinements give a half-twist conformation of the ordered ethylene groups (see Figure 2d), which is not a conformation with the lowest energy. Note that a half-twist conformation was observed in BEDT-TTF molecules of salts exhibiting structural instabilities, such as $\alpha$-(BEDT-TTF $)_{2} \mathrm{I}_{3}$ and $\alpha$-(BEDT-TTF $)_{2} \mathrm{KHg}(\mathrm{SCN})_{4}$ salts [56]. The origin of the half-twist conformation of the ethylene groups in these salts seems to be due to the presence of constraints exerted by the environment of the BEDT-TTF molecules, i.e., the mechanical constraints due to the geometry of hydrogen bonds established with the anions.

Concerning the hydrogen bonds between the electropositive $\mathrm{H}$ atoms from the ethylene groups and the electronegative atoms of the anionic stack, their presence in organic systems is very frequent. It is an important structural effect that controls the packing of the BEDT-TTF layer and which is

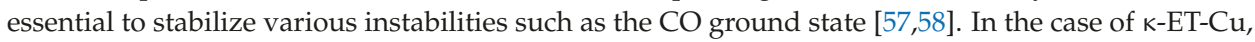
hydrogen bonds between the electropositive $\mathrm{H}$ atoms of the ethylene groups and the electronegative $\mathrm{N}$ or $\mathrm{C}$ atoms of the anionic stack are shown in Figure 3a. If one analyzes the hydrogen bond network between the BEDT-TTFs and the atoms of the anionic polymeric network shorter than $2.8 \AA$, it appears that the BEDT-TTFs of both dimers, when they are staggered, make two hydrogen bonds with $\mathrm{N}$ atoms (2.69 and $2.73 \AA$ for Donor 1 and 2.68 and $2.75 \AA$ for Donor 2 ) and another one with one of the atoms of the disordered CN group (2.74 $\AA$ for Donor 1 and $2.73 \AA$ for Donor 2). When the donors are eclipsed they make two hydrogen bonds with $\mathrm{N}$ atoms (2.69 and $2.70 \AA$ for Donor 1 and 2.69 and $2.68 \AA$ for Donor 2) and another one with the $C$ atoms (2.61 $\AA$ for Donor 1 and $2.55 \AA$ for Donor 2). From these values, it is not clear if there is a net preference for the staggered conformation. The hydrogen bonds with the $\mathrm{N}$ atoms seem to slightly favor the eclipsed conformation. However, when both donors are staggered, they establish a short contact with the disordered $\mathrm{CN}$ group, whereas when they are 
eclipsed they establish a short contact with the less electronegative $C$ atom. It is most likely that the interaction with the anionic network does not clearly favor one of the two conformations.

In order to progress in the analysis of the origin of the disorder, one must also consider the interactions within the donor layers. Stabilizing $\mathrm{S} \cdots \mathrm{H}$ hydrogen bonds and $\mathrm{H} \cdots \mathrm{H}$ repulsive interactions have a strong influence on the actual conformation of the donors and hence on the occurrence of a disorder within the donor layers. When the donors are in the staggered or in the eclipsed conformations, there are no $\mathrm{H} \cdots \mathrm{H}$ contacts shorter than $2.40 \AA$ (this would indicate repulsive interactions). Consequently, these contacts do not favor any of the two conformations. In contrast, the intra-dimer S...H hydrogen bonds favor an eclipsed conformation. In the eclipsed conformation, two intra-dimer S. . H hydrogen bonds of $2.66 \AA$ for Donor 1 and $2.60 \AA$ for Donor 2 occur, but none of them is stabilized when the donors are in a staggered conformation. The inter-donor S . H H interactions do not distinguish between the two conformations. Thus, as far as the inter-donor interactions within the BEDT-TTF layers are concerned, there is a preference for the eclipsed conformation.

Taking into account the previous analyses, we must conclude that the preference for a staggered conformation must originate from intra-BEDT-TTF interactions that must dominate over the inter-donor $\mathrm{S} \cdots \mathrm{H}$ interactions. In the absence of detailed first-principles calculations, it is not possible to clearly state whether or not the interaction with the anionic network favors one of the two conformations. What the present analysis suggests is that the preference for a staggered conformation originates from a competition between intra- and inter-donor interactions within the donor layers. Let us note that one reaches exactly the same conclusion when analyzing the $\mathrm{P} 2{ }_{1} / \mathrm{c}$ crystal structure of $\mathrm{k}$-ET-Cu at $300 \mathrm{~K}$ [11]. Thus, $\mathrm{k}-\mathrm{ET}-\mathrm{Cu}$ is clearly different from other kappa salts of BEDT-TTF as, for instance, $\mathrm{k}-(\mathrm{BEDT}-\mathrm{TTF})_{2} \mathrm{Cu}\left[\mathrm{N}(\mathrm{CN})_{2} \mathrm{X}(\mathrm{X}=\mathrm{Cl}, \mathrm{Br})\right.$. In these cases, a detailed structural analysis of the evolution of the crystal structure with temperature [56] clearly shows that the staggered minority conformation is favored by the interaction with the anionic network, whereas it is strongly disfavored by very short and repulsive $\mathrm{H} \cdots \mathrm{H}$ interactions within the BEDT-TTF dimer units. When the temperature is lowered, such repulsive interactions become too strong and all donors exhibit the eclipsed conformation. Clearly, the conformational preference of BEDT-TTF in kappa salts is a subtle question worth investigating.

\subsection{Evolution of the Structure as a Function of Temperature}

We have studied the thermal evolution of the structural properties of $\mathrm{k}$-ET-Cu as a function of temperature. Importantly, the $\mathrm{P} \overline{1}$ space group is confirmed in the entire temperature range since reflections forbidden in the $\mathrm{P} 2{ }_{1} / \mathrm{c}$ space group were observed from 300 to $3 \mathrm{~K}$. Upon cooling to $3 \mathrm{~K}$, the intensity of these reflections only slightly increased, while the overall $X$-ray pattern did not change significantly. In particular, no significant change was detected in the temperature range between 300 and $200 \mathrm{~K}$, where, according to $\mathrm{H}^{1} \mathrm{NMR}$ measurements [13], the thermally activated jump between $\mathrm{E}$ and $\mathrm{S}$ conformations decreases, leading to the stabilization of the $\mathrm{S}$ conformation seen in the structural refinements [11,34]. In addition, no substantial modification in the intensity of the Bragg reflections was observed in the temperature range between 60 and $3 \mathrm{~K}$ where a relaxor-like peak is detected by dielectric measurements [36,37].

Between $\mathrm{k}-\mathrm{ET}-\mathrm{Cu}$, on the one hand, and $\mathrm{k}-(\mathrm{BEDT}-\mathrm{TTF})_{2} \mathrm{Cu}(\mathrm{SCN})_{2}$ and $\mathrm{k}-(\mathrm{BEDT}-\mathrm{TTF})_{2} \mathrm{Cu}\left[\mathrm{N}(\mathrm{CN})_{2}\right] \mathrm{X}$ where $\mathrm{X}=\mathrm{Br}$ and $\mathrm{Cl}$, on the other, the presence of different majority ethylene conformations ( $\mathrm{S}$ versus $\mathrm{E}$ respectively) and the presence of a glass transition ascribed to the residual disorder between $\mathrm{S}$ and $\mathrm{E}$ ethylene configurations in the latter two salts, is quite noticeable.

Unfortunately, due to technical difficulties (the sample was not exactly on the axis of rotation) and to intrinsic problems related to the deconvolution of the two domains associated with the twin, which became impossible at low temperatures, we were not able to refine the low-temperature structures. 


\section{Discussion}

\subsection{Inter-Dimer Charge Disproportionation}

The observation of a symmetry breaking from the previously accepted $\mathrm{P} 2_{1} / \mathrm{c}$ space group to $\mathrm{P} \overline{1}$ (or P1) has important implications. Within the $\mathrm{P} 2{ }_{1} / \mathrm{c}$ space group and consequently in the structures of references $[11,29,31]$, the two BEDT-TTF molecules of a dimer are related by a center of inversion, and the two dimers of the unit cell are also related by symmetry. On the other hand, in the $\mathrm{P} \overline{1}$ space group, the former symmetry is preserved, while the latter is broken. This should lead to differences between Dimer 1 and Dimer 2 in the present $\mathrm{P} \overline{1}$ structure. First, their intra-dimer distance defined by the distance $\mathrm{d}$ between the core carbons of the two different molecules of a given dimer (all but the $\mathrm{C}$ atoms of the ethylene groups) should be different. Second, the slight deformation from the ideal BEDT-TTF molecular shape should also be different between the two dimers. This is, for instance, the case of the central Ci1-Ci2 bond length in Dimers 1 and 2. Indeed, Table 1 shows that these two types of distances are not identical, but their difference lies in the error bars.

The internal deformation of the molecules of a dimer can induce a charge deviation from the 0.5 holes per donor theoretically expected from the 2:1 stoichiometry (i.e., the BEDT-TTF charge is given as $0.5 \pm \delta$ ). We can extract the value of $\delta$ for a BEDT-TTF molecule from the relationship between internal structural parameters of the molecule and the associated charge given in [52]. The charges obtained at $300 \mathrm{~K}$ are +0.47 for a single BEDT-TTF in Dimer 1 and +0.53 for a single BEDT-TTF in Dimer 2, respectively (with a charge accuracy of $5 \%$ estimated from the bond length error bars). Note that this charge is not wholly determined by the central Ci1-Ci2 bond length, as is sometimes assumed. The normalized charge disproportionation thus obtained is $2 \delta=0.06 \pm 0.05$.

\subsection{DFT Electronic Structure Calculation}

To put these results on a firm basis, we have also carried out first-principles density functional theory (DFT) calculations. The temperature dependence of the electronic structure parameters assuming a $\mathrm{P}_{1} / \mathrm{c}$ average structure was the subject of previous DFT studies [11,59]. Here, we are mostly interested in the magnitude of the charge difference between the two dimers. Because of the disorder of the C2-N2 and C10-N10 groups, we carried out two different types of calculation, using in both cases the crystal structure reported in this work. In the first calculation, the original $\mathrm{P} \overline{1}$ structure has been converted into a P1 structure by selecting one of the two possible orientations of the $\mathrm{CN}$ pair at the inversion center but leaving the rest of the structure unaltered. In the second calculation, the anionic layer is completely removed from the calculation and the neutrality of the system is enforced using a uniform background of charge amounting to two electrons per unit cell. In that way, the $\mathrm{P} \overline{1}$ original symmetry is kept. The density of holes per BEDT-TTF donor was calculated by integration of the density of states of the two upper HOMO bands and the two calculations led to the same charges. The calculated band structure for the $300 \mathrm{~K}$ structure is shown in Figure 4. The two upper bands are built from the anti-bonding combination of the two highest occupied molecular orbitals (HOMO) of the BEDT-TTF dimers. Figure 4 shows that these two bands nearly merge together along the $\mathrm{Z}-\mathrm{M}$ and $\mathrm{M}-\mathrm{Y}$ lines (there is degeneracy for the $\mathrm{P} 2_{1} / \mathrm{c}$ space group). The separation between these two bands along the $\mathrm{Z}-\mathrm{M}$ and $\mathrm{M}-\mathrm{Y}$ lines is quite weak (between 3 and $5 \mathrm{meV}$ ). As this separation is proportional to the difference between the two types of dimers (i.e., the difference in energy of the HOMO's anti-bonding combination of each dimer and the difference in transfer integrals $t_{3}$ and $t_{4}$ between dimers of the same type along $b$ ), one obtains very similar intra- and inter-donor interactions for Dimers 1 and 2. This is confirmed by the fact that the DFT electronic structure dispersion of Figure 4 is nearly identical to the one of the $\mathrm{P} 2{ }_{1} / \mathrm{c}$ structure [11]. As expected from these results, despite the existence of the two different dimers, the holes are calculated to be practically equally shared by the two dimers ( $2 \delta \cong \pm 0.01$, which is of the same order of magnitude as the precision of our calculations). The results of vibrational spectroscopy [35], which do not reveal any charge imbalance larger than $\delta= \pm 0.01 \mathrm{e}$, agree with the present results despite the existence of two different dimers. 


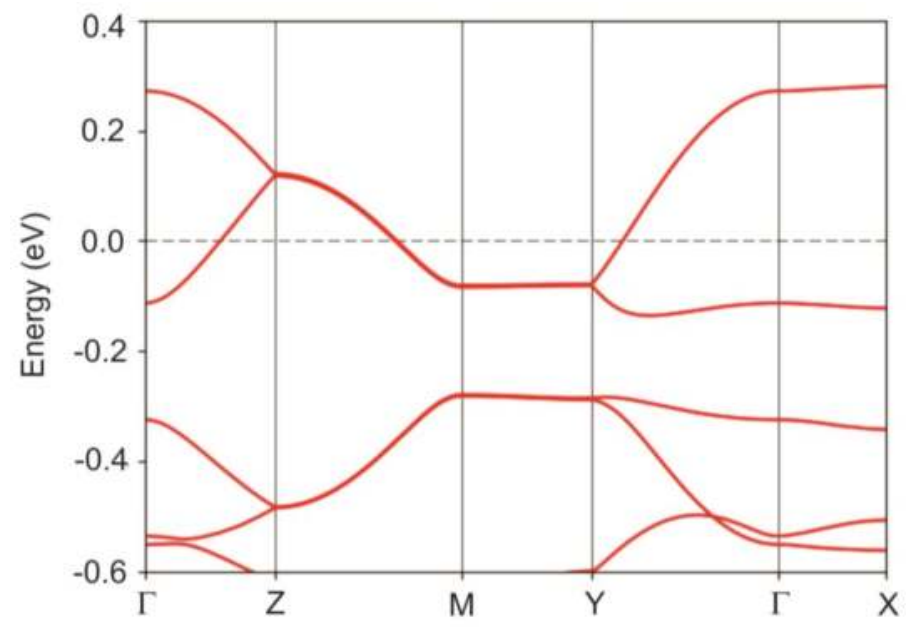

Figure 4. (Color online). Calculated band structure for the $300 \mathrm{~K}$ crystal structure of $\mathrm{k}$-ET-Cu. The energy zero corresponds to the Fermi level. $\Gamma=(0,0,0), X=(1 / 2,0,0), Y=(0,1 / 2,0), Z=(0,0,1 / 2)$, and $\mathrm{M}=(0,1 / 2,1 / 2)$ in units of the reciprocal lattice vectors.

\subsection{Inter-Dimer Charge Pattern}

Figure 5a shows the BEDT-TTF pattern, which results from our structural refinements. The structure is made of rows of BEDT-TTF dimers of one type running along $\mathbf{b}$. Rows of dimers of this type alternate with rows of dimers of the other type along $\mathbf{c}$. The two types of dimers also alternate along the diagonal $\mathrm{c} \pm \mathrm{b}$ directions. Even if the charge disproportionation that results from the DFT calculations is small, it is permitted by symmetry of the new structure.

In order to further check the possibility of a sizeable charge disproportionation between dimers, we studied the hydrogen bond network between the anionic and donor layers. In previous studies of $\alpha$-(BEDT-TTF $)_{2} \mathrm{I}_{3}[58]$ and $\theta-(\mathrm{BEDT}-\mathrm{TTF})_{2} \mathrm{MM}^{\prime}(\mathrm{SCN})_{4}[60]$, it was shown that the hydrogen bond distance between the terminal $\mathrm{C}_{2} \mathrm{H}_{4}$ groups of BEDT-TTF and the anion reflects the degree of charge of the donor as a result of a subtle polarization mechanism detailed in [58]. Here, as discussed in detail above, the BEDT-TTF of Dimer 1 is related to the anionic plane by three short hydrogen bonds, smaller than $2.73 \AA$ as well as the BEDT-TTF of Dimer 2, which is also connected by three hydrogen bonds shorter than $2.73 \AA$. This confirms that the charge of both dimers is practically equivalent, thus providing an indication of a weak charge disproportionation between dimers.

The charge disproportionation between dimers at room temperature, even weak, is real from a symmetry point of view. Thus, one should inquire as to the reasons for such a charge disproportionation in $\mathrm{k}$-ET-Cu as compared to other $\mathrm{k}-(\mathrm{BEDT}-\mathrm{TTF})_{2} \mathrm{X}$ salts. One explanation could be that the charge disproportionation is due to the presence of stronger inter-site Coulomb repulsions. Such interactions are invoked to explain low-temperature $\mathrm{CO}$ ground states observed in several families of (BEDT-TT) ${ }_{2} \mathrm{X}$ salts [17]. However, below the $\mathrm{CO}$ transition of such salts as well as of the Fabre salts, one observes an increase in the amplitude of the charge disproportionation upon cooling [61]. In the absence of low-temperature structural refinements allowing for the determination of the charge per donor, we cannot rule out this possibility. Another more likely possibility is that the charge disproportionation observed at room temperature is due to the presence of different environments of the BEDT-TTF dimers. The difference in environments is probably set by the anion sub-lattice and its $\mathrm{H}$-bond interactions with the donors. Note that one of the hydrogen bonds for both types of dimers occurs with the disordered $\mathrm{CN}$ groups. Similar effects are observed in $\alpha$-(BEDT-TTF $)_{2} \mathrm{I}_{3}$ at room temperature and analyzed in [58]. 


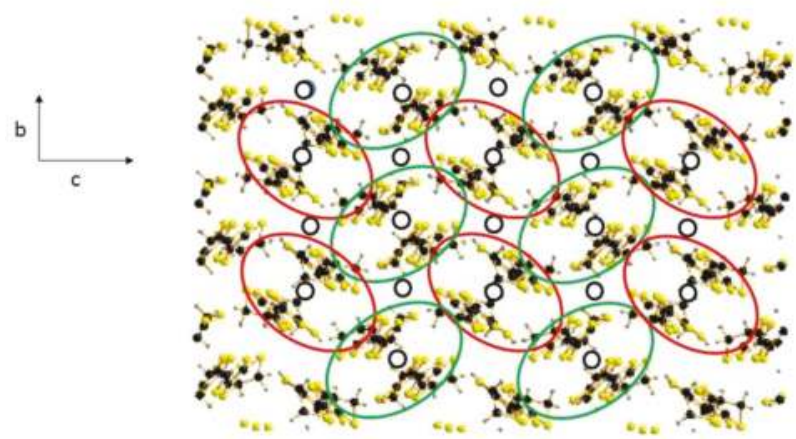

(a)

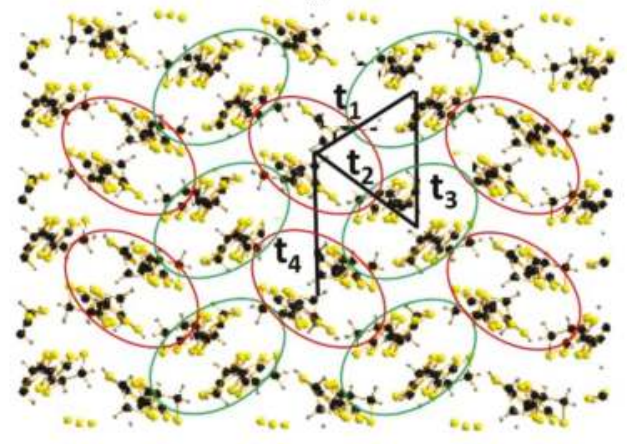

(b)

Figure 5. (Color online). (a) Molecular stack in the (b,c) plane showing the two types of dimers (red and green ellipses). The inversion centers remaining in the $\mathrm{P} \overline{1}$ space group are indicated by black empty circles; (b) The same molecular stack with the four different transfer integrals ( $t_{\mathbf{i}}$ with $i=1$ to 4 ) between dimers represented by black segments.

Let us also remark that the finding of an intra-dimer distance that depends upon the charge of the dimer shows that the internal degrees of freedom of dimers that describe the physics of $\mathrm{k}$-ET-Cu cannot be ignored. This shows more generally that one must go beyond the simple dimer approximation where the electronic structure of $\mathrm{k}$-(BEDT-TTF $)_{2} \mathrm{X}$ is restricted to a simple set of $t_{i}$ couplings $(i=1,2,3$, and 4 in Figure $5 b$ ) between dimers.

Furthermore, it is important to realize that considering a prototype $\mathrm{P} 2{ }_{1} / \mathrm{c}$ structure containing two dimers per unit cell implies two possibilities to form charge-rich and charge-poor dimers in the P $\overline{1}$ structure. These two possibilities are achieved in different twinned domains whose ratio is estimated at 50\% in our sample (see Section 4). Different twined domains where Dimers 1 and 2 are interchanged should be separated by polar domain walls where the position of charge-rich and charge-poor dimers interchanges. The motion of these domain walls should be at the origin of the observed dielectric response, whose relaxor-like nature also indicates the presence of disorder in k-ET-Cu [37,59].

Although the detailed crystal structure analysis suggests that the main source of disorder may originate from the competition between intra- and inter-donor interactions in the donor layer, one must recall that one of the short hydrogen bonds between donor and the anion layers occurs with the disordered $\mathrm{CN}$ group of the anionic network. This $\mathrm{CN}$ disorder, which thus affects the whole (cation and anion) system, arises because of the triangular frustrated environment of $\mathrm{Cu}$, where one bond links $\mathrm{Cu}$ with $50-50 \% \mathrm{CN}$ (bridging $\mathrm{CN}$ ), while two bonds link $\mathrm{Cu}$ to well-defined $100 \% \mathrm{CNs}$ ( $\mathrm{CN}$ chain). In the present refinement, we assume a complete orientation disorder of the bridging $\mathrm{CN}$, 
as the X-ray investigation is not able to give reliable information on this orientation disorder, as pointed above. $\mathrm{Cu}$ atoms are locally frustrated because they are coordinated either by two $\mathrm{C}$ atoms and one $\mathrm{N}$ atom or by one $\mathrm{C}$ atom and two $\mathrm{N}$ atoms. In that way, different anion-cation couplings/constrains are created, in particular via the establishment of N . . H bonds with the Ci7-Ci8 ethylene group (see Figure 2a). In the k-ET-Cu structure, there is thus a robust zigzag chain motif with well-ordered $\mathrm{CN}$ chain groups along the $\boldsymbol{b}$-axis and different types of disordered $\mathrm{C}-\mathrm{N}$ bridging groups (C2-N2 and $\mathrm{C} 10-\mathrm{N} 10)$ along the $c$-axis. Such a disorder may arise during the synthesis procedure where different $\mathrm{C}-\mathrm{N}$ configurations can be obtained. As the charge redistribution among BEDT-TTF dimers may be altered by such disorder because the charge depends on the strength of the short donor-anion hydrogen bonds, the sample-dependent VRH and dielectric response can be explained [37,50].

\subsection{Possible Intra-Dimer Charge Disproportionation}

As detailed previously, our X-ray measurements are not able to discriminate between the P1 and the $\mathrm{P} \overline{1}$ space groups. However, in the absence of an inversion symmetry (P1 space group), the molecules within a dimer are not equivalent. In that case, a charge disproportionation between the molecules of a dimer should exist and lead to weak electric dipoles. The intra-dimer electric dipoles have been assumed to theoretically explain the origin of the spin-liquid state via a dipolar-spin model based on a strong coupling analysis [19-21]. This scenario has been proposed earlier to interpret the relaxor-like dielectric properties of K-ET-Cu [36] below $60 \mathrm{~K}$. However, more recent results have shown that dipoles, if any, are weak and thus cannot be at the origin of the dielectric response $[35,37,59]$.

\subsection{Bringing Back Together the Puzzling Physical Behaviors}

The presence of electric dipoles within the BEDT-TTF dimers has neither been detected by infrared [35] nor by terahertz $[59,62]$ measurements. In particular, the $v_{27}$ peak has been investigated [35] because it is the most charge-sensitive infrared-active intra-molecular vibrational anti-symmetric mode related to the anti-symmetric ring Ci3-Ci4 and Ci5-Ci6 bonds of the BEDT-TTF molecules. No splitting of the mode has been detected within the error bars of $1 \mathrm{~cm}^{-1}$, which corresponds to a charge disproportionation of $0.01 \mathrm{e}$, thus giving evidence that no sizeable static charge intra-dimer disproportionation takes place.

Several models have been proposed to reconcile dielectric and optical measurements. The main model involves polar space groups: either P2 ${ }_{1}$ [37] or even P1 [59], which have been found energetically favorable by DFT calculations [59]. In the P2 2 space group, the $\mathrm{C} 2-\mathrm{N} 2$ and $\mathrm{C} 10-\mathrm{N} 10$ groups can be ordered because they are no more located on an inversion center. However, a geometrical frustration is present around a $\mathrm{Cu}$ atom bridging either $2 \mathrm{~N}-1 \mathrm{C}$ or vice versa. This is expected to create domains and charge domain walls whose motion generates dielectric response. The latter scenario has also been investigated theoretically in [63]. Another model that assumes fluctuating intra/inter-dimer electric dipoles and that well explains the terahertz measurements of [62] has been developed. As argued in Section 3.3, the present structural investigation brings to light the presence of polar domain walls separating twin domains of the $\mathrm{P} \overline{1}$ structure. Their motion could be the origin of the dielectric response.

Finally, it is important to note that preliminary X-ray scattering experiments on $\mathrm{K}$-ET-Ag [64] show that the same symmetry breaking from $\mathrm{P} 2{ }_{1} / \mathrm{c}$ to $\mathrm{P} 1$ or $\mathrm{P} \overline{1}$ is present. This effect seems to be a general aspect of $\mathrm{k}-(\mathrm{BEDT}-\mathrm{TTF})_{2} \mathrm{M}_{2}(\mathrm{CN})_{3}(\mathrm{\kappa}-\mathrm{ET}-\mathrm{M})$, while no crystal lattice symmetry lowering in the orthorhombic phases of $\mathrm{k}-(\mathrm{BEDT}-\mathrm{TTF})_{2} \mathrm{Cu}\left[\mathrm{N}(\mathrm{CN})_{2}\right] \mathrm{X}$ with $\mathrm{X}=\mathrm{Br}$ [65-67] and $\mathrm{Cl}$ has been reported until now. It is important to observe that both $\mathrm{k}-\mathrm{ET}-\mathrm{Cu}$ and $\mathrm{k}-\mathrm{ET}-\mathrm{Ag}$ present a spin liquid state [68-70], which has not been observed in other $\mathrm{k}-(\mathrm{BEDT}-\mathrm{TTF})_{2} \mathrm{Cu}\left[\mathrm{N}(\mathrm{CN})_{2}\right] \mathrm{X}$ compounds with $\mathrm{X}=\mathrm{Br}$ and $\mathrm{Cl}$. We can speculate that the structural particularities of $\mathrm{K}$-ET-M are a prerequisite to the emergence of the spin-liquid state.

An additional remark is that the charge disproportionation of $k$-ET-Cu, which is not fixed by the crystal symmetry, can vary under external constraint such as temperature of pressure. For example if one assumes that with one hole per dimer (which value is confirmed within experimental 
uncertainties by the present study) the insulating properties of $\mathrm{k}-\mathrm{ET}-\mathrm{Cu}$ at ambient pressure arise from a Mott-Hubbard intra-dimer charge localization, the enhancement of inter-dimer charge disproportion under pressure should disfavor the localized state in favor of a metallic state, which presence is a necessary condition to stabilize superconductivity.

Acknowledgments: We thank Joon I. Jang for his work related to SHG measurements. S.T. acknowledges the support by the Croatian Science Foundation project IP-2013-11-1011. Work in Spain was supported by the Spanish Ministerio de Economía y Competitividad (Grants FIS2015-64886-C5-4-P and CTQ2015-64579-C3-3-P) and Generalitat de Catalunya (217SGR1506 and XRQTC). E.C. acknowledges the support of the Spanish MINECO through the Severo Ochoa Centers of Excellence Program under Grant SEV-2015-0496.

Author Contributions: Kazuya Miyagawa and Kazushi Kanoda were involved in the synthesis of the compound. Pascale Foury-Leylekian, Vita Ilakovac, and Pierre Fertey designed the synchrotron experiment. Victor Baledent and Ognjen Milat performed the first laboratory X-ray measurements. Pascale Foury-Leylekian, Alla Arakcheeva, Pierre Fertey, and Enric Canadell refined the data. Denis Petermann and Gilles Guillier made an important technical contribution to the laboratory measurements at low temperature. Pere Alemany performed the DFT calculations. Pascale Foury-Leylekian, Silvia Tomic, Enric Canadell, and Jean-Paul Pouget were involved in the physical analysis of the structural results and in the preparation of the manuscript. All authors contributed to and commented on the final manuscript.

Conflicts of Interest: The authors declare no conflict of interest.

\section{References and Note}

1. Powell, B.J.; McKenzie, R.H. Quantum frustration in organic Mott insulators: From spin liquids to unconventional superconductors. Rep. Prog. Phys. 2011, 74, 056501. [CrossRef]

2. Balents, L. Spin liquids in frustrated magnets. Nature 2010, 464, 199-208. [CrossRef] [PubMed]

3. Anderson, P.W. Resonating valence bonds: A new kind of insulator? Mater. Res. Bull. 1973, 8, $153-160$. [CrossRef]

4. Coldea, R.; Tennant, D.A.; Tsvelik, A.M.; Tylczynski, Z. Experimental Realization of a 2D Fractional Quantum Spin Liquid. Phys. Rev. Lett. 2001, 86, 1335. [CrossRef] [PubMed]

5. Li, Y.; Liao, H.; Zhang, Z.; Li, S.; Jin, F.; Ling, L.; Zhang, L.; Zou, Y.; Pi, L.; Yang, Z.; et al. Gapless quantum spin liquid ground state in the two-dimensional spin-1/2 triangular antiferromagnet $\mathrm{YbMgGaO}_{4}$. Sci. Rep. 2015, 5, 16419. [CrossRef] [PubMed]

6. Khuntia, P.; Kumar, R.; Mahajan, A.V.; Baenitz, M.; Furukawa, Y. Spin liquid state in the disordered triangular lattice $\mathrm{Sc}_{2} \mathrm{Ga}_{2} \mathrm{CuO}_{7}$ revealed by NMR. Phys. Rev. B 2016, 93, 140408(R). [CrossRef]

7. Klanjšek, M.; Zorko, A.; Žitko, R.; Mravlje, J.; Jagličić, Z.; Biswas, P.; Prelovšek, P.; Mihailovic, D.; Arčon, D. A high-temperature quantum spin liquid with polaron spins. Nat. Phys. 2017, 13, 1130-1134. [CrossRef]

8. Kanoda, K.; Kato, R. Mott Physics in Organic Conductors with Triangular Lattices. Annu. Rev. Condens. Matter Phys. 2011, 2, 167-188. [CrossRef]

9. Zhou, Y.; Kanoda, K.; Ng, T.-K. Quantum spin liquid states. Rev. Mod. Phys. 2017, 89, 025003. [CrossRef]

10. Kandpal, H.C.; Opahle, I.; Zhang, Y.-Z.; Jeschke, H.O.; Valenti, R. Revision of Model Parameters for k Type Charge Transfer Salts: An Ab Initio Study. Phys. Rev. Lett. 2009, 103, 067004. [CrossRef] [PubMed]

11. Jeschke, H.O.; de Souza, M.; Valenti, R.; Manna, R.S.; Lang, M.; Schlueter, J.A. Temperature dependence of structural and electronic properties of the spin-liquid candidate $\mathrm{k}-(\mathrm{BEDT}-\mathrm{TTF})_{2} \mathrm{Cu}_{2}(\mathrm{CN})_{3}$. Phys. Rev. B 2012, 85, 035125. [CrossRef]

12. Koretsune, T.; Hotta, C. Evaluating model parameters of the $\mathrm{k}$-and $\beta^{\prime}$-type Mott insulating organic solids. Phys. Rev. B 2014, 89, 045102. [CrossRef]

13. Miyagawa, Y.S.K.; Kanoda, K.; Maesato, M.; Saito, G. Spin Liquid State in an Organic Mott Insulator with a Triangular Lattice. Phys. Rev. Lett. 2003, 91, 107001.

14. Zheng, W.; Singh, R.R.P.; McKenzie, R.H.; Coldea, R. Temperature dependence of the magnetic susceptibility for triangular-lattice antiferromagnets with spatially anisotropic exchange constants. Phys. Rev. B 2005, 71, 134422. [CrossRef]

15. Yamashita, S.; Nakazawa, Y.; Oguni, M.; Oshima, Y.; Jojiri, H.; Miyagawa, K.; Kanoda, K. Thermodynamic properties of a spin-1/2 spin-liquid state in a $\kappa$-type organic salt. Nat. Phys. 2008, 4, 459-462. [CrossRef]

16. Pustogow, A.; Bories, M.; Löhle, A.; Rösslhuber, R.; Zhukova, E.; Gorshunov, B.; Tomić, S.; Schlueter, J.A.; Hübner, R.; Hiramatsu, T.; et al. Quantum Spin Liquids Unveil the Genuine Mott State. arXiv, 2017. 
17. Seo, H.; Merino, J.; Yoshioka, H.; Ogata, M. Theoretical Aspects of Charge Ordering in Molecular Conductors. J. Phys. Soc. Jpn. 2006, 75, 051009. [CrossRef]

18. Lunkenheimer, P.; Müller, J.; Krohns, S.; Schrettle, F.; Loidl, A.; Hartmann, B.; Rommel, R.; de Souza, M.; Hotta, C.; Schlueter, J.A.; et al. Multiferroicity in an organic charge-transfer salt: Electric-dipole-driven magnetism. Nat. Mater. 2012, 11, 755-758. [CrossRef] [PubMed]

19. Hotta, C. Theories on Frustrated Electrons in Two-Dimensional Organic Solids. Crystals 2012, 2, 1155-1200. [CrossRef]

20. Naka, M.; Ishihara, S. Electronic Ferroelectricity in a Dimer Mott Insulator. J. Phys. Soc. Jpn. 2010, 79, 063707. [CrossRef]

21. Li, H.; Clay, R.T.; Mazumdar, S. The paired-electron crystal in the two-dimensional frustrated quarter-filled band. J. Phys. Condens. Matter 2010, 22, 272201. [CrossRef] [PubMed]

22. Gomi, H.; Imai, T.; Takahashi, A.; Aihara, M. Purely electronic terahertz polarization in dimer Mott insulators. Phys. Rev. B 2010, 82, 035101. [CrossRef]

23. Gomi, H.; Ikenaga, M.; Hiragi, Y.; Segawa, D.; Takahashi, A.; Inagaki, T.J.; Aihara, M. Ferroelectric states induced by dimer lattice disorder in dimer Mott insulators. Phys. Rev. B 2013, 87, 195126. [CrossRef]

24. Gomi, H.; Inagaki, T.J.; Takahashi, A. A ferroelectric charge order enhanced by magnetic frustration in dimer Mott insulators. Phys. Rev. B 2016, 93, 035105. [CrossRef]

25. Manna, R.S.; de Souza, M.; Brúhl, A.; Schlueter, J.A.; Lang, M. Lattice Effects and Entropy Release at the Low-Temperature Phase Transition in the Spin-Liquid Candidate k-(BEDT-TTF $)_{2} \mathrm{Cu}_{2}(\mathrm{CN})_{3}$. Phys. Rev. Lett. 2010, 104, 016403. [CrossRef] [PubMed]

26. Poirier, M.; de Lafontaine, M.; Miyagawa, K.; Kanoda, K.; Shimizu, Y. Ultrasonic investigation of the transition at $6 \mathrm{~K}$ in the spin-liquid candidate $\mathrm{K}-(\mathrm{BEDT}-\mathrm{TTF})_{2} \mathrm{Cu}_{2}(\mathrm{CN})_{3}$. Phys. Rev. B 2014, 89, 045138. [CrossRef]

27. Ilakovac, V.; Carniato, S.; Foury-Leylekian, P.; Tomić, S.; Pouget, J.-P.; Lazić, P.; Joly, Y.; Miyagawa, K.; Kanoda, K.; Nicolaou, A. Resonant inelastic x-ray scattering probes the electron-phonon coupling in the spin liquid $\mathrm{k}-(\mathrm{BEDT}-\mathrm{TTF})_{2} \mathrm{Cu}_{2}(\mathrm{CN})_{3}$. Phys. Rev. B 2017, 96, 184303. [CrossRef]

28. Kurosaki, Y.; Shimizu, Y.; Miyagawa, K.; Kanoda, K.; Saito, G. Mott Transition from a Spin Liquid to a Fermi Liquid in the Spin-Frustrated Organic Conductor k-(ET) ${ }_{2} \mathrm{Cu}_{2}(\mathrm{CN})_{3}$. Phys. Rev. Lett. 2005, 95, 177001. [CrossRef] [PubMed]

29. Geiser, U.; Wang, H.H.; Carlson, K.D.; Williams, J.M.; Charlier, H.A.; Heindl, J.E.; Yaconi, G.A.; Love, B.J.; Lathrop, M.W.; Schirber, J.E.; et al. Superconductivity at $2.8 \mathrm{~K}$ and $1.5 \mathrm{kbar}$ in $\mathrm{K}-(\mathrm{BEDT}-\mathrm{TTF})_{2} \mathrm{Cu}_{2}(\mathrm{CN})_{3}$ : The first organic superconductor containing a polymeric copper cyanide anion. Inorg. Chem. 1991, 30, 2586-2588. [CrossRef]

30. Bu, X.; Frost-Jensen, A.; Allendoerfer, R.; Coppens, P.; Lederle, B.; Naughton, M.J. Structure and properties of a new k-phase organic metal: (BEDT-TTF $)_{2} \mathrm{Cu}_{2}(\mathrm{CN})_{3}$. Solid State Commun. 1991, 79, 1053-1057. [CrossRef]

31. Bu, X.; Coppens, P. Zeitschrift für Kristallographie. New Cryst. Struct. 1997, 212, 103-104.

32. Yamochi, H.; Nakamura, T.; Komatsu, T.; Matsukawa, N.; Inoue, T.; Saito, G. Crystal and electronic structures of the organic superconductors, $x-(\mathrm{BEDT}-\mathrm{TTF})_{2} \mathrm{Cu}(\mathrm{CN})\left[\mathrm{N}(\mathrm{CN})_{2}\right]$ and $\varkappa^{\prime}-(\mathrm{BEDT}-\mathrm{TTF})_{2} \mathrm{Cu}_{2}(\mathrm{CN})_{3}$. Solid State Commun. 1992, 82, 101-105. [CrossRef]

33. Papavassiliou, G.C.; Lagouvardos, D.J.; Terzis, A.; Hountas, A.; Hilti, B.; Zambounis, J.S.; Mayer, C.W.; Pfeiffer, J.; Hofherr, W.; Delhaes, P.; et al. Physical properties in the normal state of the molecular superconductor $\kappa-(\mathrm{BEDT})_{2} \mathrm{Cu}_{2}(\mathrm{CN})_{3}$. Synth. Met. 1993, 61, 267-273. [CrossRef]

34. Hiramatsu, T.; Yoshida, Y.; Saito, G.; Otsuka, A.; Yamochi, H.; Maesato, M.; Shimizu, Y.; Ito, H.; Kishida, H. Quantum spin liquid: Design of a quantum spin liquid next to a superconducting state based on a dimer-type ET Mott insulator. J. Mater. Chem. C 2015, 3, 1378-1388. [CrossRef]

35. Sedlmeier, K.; Elsässer, S.; Neubauer, D.; Beyer, R.; Wu, D.; Ivek, T.; Tomić, S.; Schlueter, J.A.; Dressel, M. Absence of charge order in the dimerized k-phase BEDT-TTF salts. Phys. Rev. B 2012, 86, 245103. [CrossRef]

36. Abdel-Jawad, M.; Terasaki, I.; Sasaki, T.; Yoneyama, N.; Kobayashi, N.; Uesu, Y.; Hotta, C. Anomalous dielectric response in the dimer Mott insulator k-(BEDT-TTF $)_{2} \mathrm{Cu}_{2}(\mathrm{CN})_{3}$. Phys. Rev. B 2010, 82, 125119. [CrossRef]

37. Pinterić, M.; Čulo, M.; Milat, O.; Basletić, M.; Korin-Hamzić, B.; Tafra, E.; Hamzić, A.; Ivek, T.; Peterseim, T.; Miyagawa, K.; et al. Anisotropic charge dynamics in the quantum spin-liquid candidate к-(BEDT-TTF $)_{2} \mathrm{Cu}_{2}(\mathrm{CN})_{3}$. Phys. Rev. B 2014, 90, 195139. [CrossRef] 
38. Rigaku Oxford Diffraction. CrysAlis PRO; Rigaku Oxford Diffraction: Yarnton, UK, 2015.

39. Petricek, V.; Dusek, M.; Palatinus, L. Crystallographic Computing System JANA2006: General features. Z. Kristallogr. 2014, 229, 345-352.

40. Hohenberg, P.; Kohn, W. Inhomogeneous Electron Gas. Phys. Rev. 1964, 136, B864. [CrossRef]

41. Kohn, W.; Sham, L.J. Self-Consistent Equations Including Exchange and Correlation Effects. Phys. Rev. 1965, 140, A1133. [CrossRef]

42. Soler, J.M.; Artacho, E.; Gale, J.D.; García, A.; Junquera, J.; Ordejón, P.; Sánchez-Portal, D. The SIESTA method for ab initio order-N materials simulation. J. Phys. Condens. Matter. 2002, 14, 2745-2779. [CrossRef]

43. SIESTA Code. Available online: http://departments.icmab.es/leem/siesta/ (accessed on 25 March 2018).

44. Artacho, E.; Anglada, E.; Diéguez, O.; Gale, J.D.; García, A.; Junquera, J.; Martin, R.M.; Ordejón, P.; Pruneda, J.M.; Sánchez-Portal, D.; et al. The SIESTA method; developments and applicability. J. Phys. Condens. Matter 2008, 20, 064208. [CrossRef] [PubMed]

45. Perdew, J.P.; Burke, K.; Ernzerhof, M. Generalized Gradient Approximation Made Simple. Phys. Rev. Lett. 1996, 77, 3865-3868. [CrossRef] [PubMed]

46. Troullier, N.; Martins, J.L. Efficient pseudopotentials for plane-wave calculations. Phys. Rev. B 1991, 43, 1993-2006. [CrossRef]

47. Kleinman, L.; Bylander, D.M. Efficacious Form for Model Pseudopotentials. Phys. Rev. Lett. 1982, 48, 1425. [CrossRef]

48. Artacho, E.; Sánchez-Portal, D.; Ordejón, P.; García, A.; Soler, J.M. Linear-scaling ab-initio calculations for large and complex systems. Phys. Status Solidi (b) 1999, 215, 809-817. [CrossRef]

49. Monkhorst, H.J.; Pack, J.D. Special points for Brillouin-zone integrations. Phys. Rev. B 1976, $13,5188$. [CrossRef]

50. Čulo, M.; Tafra, E.; Basletić, M.; Tomić, S.; Hamzić, A.; Korin-Hamzić, B.; Dressel, M.; Schlueter, J.A. Two-dimensional variable range hopping in the spin-liquid candidate $\kappa$-(BEDT-TTF $)_{2} \mathrm{Cu}_{2}(\mathrm{CN})_{3}$. Phys. $B$ 2015, 460, 208-210. [CrossRef]

51. The Crystallographic Data. Available online: https:/ /www.ccdc.cam.ac.uk (accessed on 25 March 2018).

52. Guionneau, P.; Kepert, C.J.; Bravic, G.; Chasseau, D.; Truter, M.R.; Kurmoo, M.; Day, P. Determining the charge distribution in BEDT-TTF salts. Synth. Met. 1997, 86, 1973-1974. [CrossRef]

53. Novoa, J.J.; Whangbo, M.-H.; Williams, J.M. Ab Initio Computational Study of the C-H ... Donor and C-H ... Anion Contact Interactions in Organic Donor Salts. Mol. Cryst. Liq. Cryst. 1990, 181, 25-42. [CrossRef]

54. Whangbo, M.-H.; Jung, D.; Ren, J.; Evain, M.; Novoa, J.J.; Mota, F.; Alvarez, S.; Williams, J.M.; Beno, M.A.; Kini, A.M.; et al. The Physics and Chemistry of Organic Superconductors; Saito, G., Kagoshima, S., Eds.; Springer: Berlin/Heidelberg, Germany, 1990; pp. 262-266.

55. Aburto, A.; Orgaz, E. Ab initio electronic structure of the eclipsed and staggered conformations of the K-(BEDT-TTF $)_{2} \mathrm{Cu}\left[\mathrm{N}(\mathrm{CN})_{2}\right] \mathrm{Br}$ organic superconductor. Phys. Rev. B 2008, 78, 113104. [CrossRef]

56. Pouget, J.P.; Alemany, P.; Canadell, E. Donor-Anion Interactions in Quarter-Filled Low-Dimensional Organic Conductors. In Preparation.

57. Pouget, J.-P.; Foury-Leylekian, P.; Alemany, P.; Canadell, E. Charge ordering in low dimensional organic conductors: Structural aspects. Phys. Status Solidi (b) 2012, 249, 937-942. [CrossRef]

58. Alemany, P.; Pouget, J.-P.; Canadell, E. Essential role of anions in the charge ordering transition of $\alpha$-(BEDT-TTF $)_{2} \mathrm{I}_{3}$. Phys. Rev. B 2012, 85, 195118. [CrossRef]

59. Dressel, M.; Lazić, P.; Pustogow, A.; Zhukova, E.; Gorshunov, B.; Schlueter, J.A.; Milat, O.; Gumhalter, B.; Tomić, S. Lattice vibrations of the charge-transfer salt $\mathrm{k}-(\mathrm{BEDT}-\mathrm{TTF})_{2} \mathrm{Cu}_{2}(\mathrm{CN})_{3}$ : Comprehensive explanation of the electrodynamic response in a spin-liquid compound. Phys. Rev. B 2016, 93, 081201(R). [CrossRef]

60. Alemany, P.; Pouget, J.-P.; Canadell, E. Structural and electronic control of the metal to insulator transition and local orderings in the $\theta$-(BEDT-TTF)2X organic conductors. J. Phys. Condens. Matter 2015, 27, 465702. [CrossRef] [PubMed]

61. Tomić, S.; Dressel, M. Ferroelectricity in molecular solids: A review of electrodynamic properties. Rep. Prog. Phys. 2015, 78, 096501. [CrossRef] [PubMed]

62. Itoh, K.; Itoh, H.; Naka, M.; Saito, S.; Hosako, I.; Yoneyama, N.; Ishihara, S.; Sasaki, T.; Iwai, S. Collective Excitation of an Electric Dipole on a Molecular Dimer in an Organic Dimer-Mott Insulator. Phys. Rev. Lett. 2013, 110, 106401. [CrossRef] [PubMed] 
63. Fukuyama, H.; Kishine, J.-I.; Ogata, M. Energy Landscape of Charge Excitations in the Boundary Region between Dimer-Mott and Charge Ordered States in Molecular Solids. J. Phys. Soc. Jpn. 2017, 86, 123706. [CrossRef]

64. Foury-Leylekian, P.; Ilakovac, V.; Fertey, P. Experimental Report $n^{\circ}$ : 20160753; Cristal Beamline Experiment; Synchrotron Soleil: Saint-Aubin, France, 2017.

65. The $2 \mathrm{c}$ superstructure which develops below $200 \mathrm{~K}$ in the $\mathrm{Br}$ salt should suppress the inter-donor inversion symmetry; See Nogami, Y.; Pouget, J.P.; Ito, H.; Ishiguro, T.; Saito, G. Superlattice structural transition in the organic superconductor $\chi-(B E D T-T T F){ }_{2} \mathrm{Cu}\left[\mathrm{N}(\mathrm{CN})_{2}\right.$ ]Br. Solid State Commun. 1994, 89, 113-116. [CrossRef]

66. The site symmetry breaking leads to a broad distribution of electronic density detected by ${ }^{13} \mathrm{C} N \mathrm{NR}$; De Soto, S.M.; Slichter, C.P.; Kini, A.M.; Wang, H.H.; Geiser, U.; Williams, J.M. ${ }^{13}$ C NMR studies of the normal and superconducting states of the organic superconductor $\mathrm{k}-(\mathrm{ET})_{2} \mathrm{Cu}\left[\mathrm{N}(\mathrm{CN})_{2}\right] \mathrm{Br}$. Phys. Rev. B 1995, 52, 10364-10368. [CrossRef]

67. Wzietek, P.; Mayaffre, H.; Jérome, D.; Brazovskii, S. NMR in the 2D Organic Superconductors. J. Phys. I 1996, 6, 2011-2041. [CrossRef]

68. Shimizu, A.; Hiramatsu, T.; Maesato, M.; Otsuka, A.; Yamochi, H.; Ono, A.; Itoh, M.; Yoshida, M.; Takigawa, M.; Yoshida, Y.; et al. Pressure-Tuned Exchange Coupling of a Quantum Spin Liquid in the Molecular Triangular Lattice k-(ET) $)_{2} \mathrm{Ag}_{2}(\mathrm{CN})_{3}$. Phys. Rev. Lett. 2016, 117, 107203. [CrossRef] [PubMed]

69. Pinterić, M.; Lazić, P.; Pustogow, A.; Ivek, T.; Kuveždić, M.; Milat, O.; Gumhalter, B.; Basletić, M.; Čulo, M.; Korin-Hamzić, B.; et al. Anion effects on electronic structure and electrodynamic properties of the Mott insulator K-(BEDT-TTF) ${ }_{2} \mathrm{Ag}_{2}(\mathrm{CN})_{3}$. Phys. Rev. B 2016, 94, 161105(R). [CrossRef]

70. Nakamura, Y.; Hiramatsu, T.; Yoshida, Y.; Saito, G.; Kishida, H. Optical Properties of a Quantum Spin Liquid Candidate Material, k-(BEDT-TTF $)_{2} \mathrm{Ag}_{2}(\mathrm{CN})_{3}$. J. Phys. Soc. Jpn. 2017, 86, 014710. [CrossRef]

(C) 2018 by the authors. Licensee MDPI, Basel, Switzerland. This article is an open access article distributed under the terms and conditions of the Creative Commons Attribution (CC BY) license (http:/ / creativecommons.org/licenses/by/4.0/). 
Article

\title{
Low-Temperature Lattice Effects in the Spin-Liquid Candidate $\kappa-(B E D T-T T F)_{2} \mathrm{Cu}_{2}(\mathrm{CN})_{3}$
}

\author{
Rudra Sekhar Manna ${ }^{1,2, *}$, Steffi Hartmann ${ }^{2}$, Elena Gati ${ }^{2}$, John A. Schlueter ${ }^{3,4}$, \\ Mariano de Souza ${ }^{2,5}$ and Michael Lang ${ }^{2}$ \\ 1 Department of Physics, IIT Tirupati, Tirupati 517506, India \\ 2 Physics Institute, Goethe University Frankfurt (M), SFB/TR49, D-60438 Frankfurt (M), Germany; \\ s.hartmann@physik.uni-frankfurt.de (S.H.); gati@physik.uni-frankfurt.de (E.G.); \\ mariano@rc.unesp.br (M.d.S.); michael.lang@physik.uni-frankfurt.de (M.L.) \\ 3 Division of Materials Research, National Science Foundation, Arlington, VA 22230, USA; \\ jaSchlueter@anl.gov \\ 4 Materials Science Division, Argonne National Laboratory, Argonne, IL 60439, USA \\ 5 IGCE, Departamento de Física, Universidade Estadual Paulista, 13506-900 Rio Claro, São Paulo, Brazil \\ * Correspondence: rudra.manna@iittp.ac.in; Tel.: +91-877-2500-348
}

Received: 13 December 2017; Accepted: 3 February 2018; Published: 6 February 2018

Abstract: The quasi-two-dimensional organic charge-transfer salt $k$-(BEDT-TTF) ${ }_{2} \mathrm{Cu}_{2}(\mathrm{CN})_{3}$ is one of the prime candidates for a quantum spin-liquid due the strong spin frustration of its anisotropic triangular lattice in combination with its proximity to the Mott transition. Despite intensive investigations of the material's low-temperature properties, several important questions remain to be answered. Particularly puzzling are the $6 \mathrm{~K}$ anomaly and the enigmatic effects observed in magnetic fields. Here we report on low-temperature measurements of lattice effects which were shown to be particularly strongly pronounced in this material (R. S. Manna et al., Phys. Rev. Lett. 2010, 104, 016403)). A special focus of our study lies on sample-to-sample variations of these effects and their implications on the interpretation of experimental data. By investigating overall nine single crystals from two different batches, we can state that there are considerable differences in the size of the second-order phase transition anomaly around $6 \mathrm{~K}$, varying within a factor of 3 . In addition, we find field-induced anomalies giving rise to pronounced features in the sample length for two out of these nine crystals for temperatures $T<9 \mathrm{~K}$. We tentatively assign the latter effects to $B$-induced magnetic clusters suspected to nucleate around crystal imperfections. These $B$-induced effects are absent for the crystals where the $6 \mathrm{~K}$ anomaly is most strongly pronounced. The large lattice effects observed at $6 \mathrm{~K}$ are consistent with proposed pairing instabilities of fermionic excitations breaking the lattice symmetry. The strong sample-to-sample variation in the size of the phase transition anomaly suggests that the conversion of the fermions to bosons at the instability is only partial and to some extent influenced by not yet identified sample-specific parameters.

Keywords: quantum spin liquids; quantum spin frustration; organic compounds; thermal expansion

\section{Introduction}

Frustrated magnetism in triangular lattices is one of the growing research interests in condensed matter physics. One class of materials where this physics can be studied are the quasi-two-dimensional organic charge-transfer salts [1]. These materials are weak Mott insulators [1,2], which can be easily converted into a metal, or even a superconductor upon the application of moderate pressure. One of the prime examples is $\kappa-(\mathrm{BEDT}-\mathrm{TTF})_{2} \mathrm{Cu}_{2}(\mathrm{CN})_{3}$ where the effect of frustration is strong [3]. This material does not show any long-range magnetic order down to $T=32 \mathrm{mK}$ [4], which is four orders of magnitude lower than the estimated nearest-neighbor Heisenberg exchange coupling $J / k_{B}=250 \mathrm{~K}$, and has been proposed to be a good candidate for a quantum spin-liquid (QSL) ground 
state. Although this material has been studied extensively in recent years, there are still several open questions to be answered. A controversial discussion surrounds the nature of the low-lying spin excitations, particularly with regard to the question whether there is a spin gap [5] or not [6]. Another very puzzling issue relates to the so-called $6 \mathrm{~K}$ anomaly. This feature manifests itself in anomalous behavior in various quantities, including ${ }^{13} \mathrm{C} \mathrm{NMR} \mathrm{[7],} \mathrm{magnetic} \mathrm{susceptibility} \mathrm{[8],} \mathrm{specific}$ heat [6,8], thermal conductivity [5], ultrasound propagation [9] as well as thermal expansion [8]. From the latter experiments, where the strongest response was found, it was claimed that the $6 \mathrm{~K}$ anomaly marks a second-order phase transition. Therefore it may reflect a QSL instability for which various scenarios have been suggested. The proposed models include spin-chirality ordering [10], a $Z_{2}$ vortex formation [11], a pairing of spinons [12-14], or an exciton condensate [15].

Likewise, the influence of a magnetic field on the low-temperature properties of this material confronts us with open questions. On the one hand this relates to the anomalous field-dependent spectral broadening observed in ${ }^{13} \mathrm{C}$ NMR measurements, which indicates a spatially non-uniform magnetization in this material [7] and the importance of disorder [16]. On the other hand, the enhancement of the thermal conductivity by the application of magnetic field above $4 \mathrm{~T}$, was assigned to the $B$-induced closure of a small gap in the magnetic excitation spectrum [5,17]. Moreover, the existence of a $B$-induced quantum phase transition at a very small field of about $5 \mathrm{mT}$ was claimed from results of $\mu \mathrm{SR}$ experiments [18]. It was argued that this quantum phase transition separates a gapped spin liquid phase, with a tiny spin gap of $\Delta_{s} / k_{B} \sim 3.5 \mathrm{mK}$, from a weak-moment antiferromagnetic phase. According to these studies, a second quantum critical point exists in this material around $4 \mathrm{~T}$ which was assigned to a threshold for deconfinement of spin excitations [18].

In light of these intriguing field-dependent effects, and the puzzling phenomenology in zero field, with a number of unresolved issues, it is of vital importance to ask about sample-to-sample variations of the material's low-temperature properties. In fact, indications for sample dependences were revealed in thermal conductivity measurements [5]. Moreover, a significant sample dependence was found in recent microwave measurements [19]. Here, we report an extensive study of sample-to-sample variations of the low-temperature behavior by focusing on the $6 \mathrm{~K}$ phase transition. As a probe we use thermal expansion measurements, which proved to be particularly sensitive to the $6 \mathrm{~K}$ anomaly [8]. This thermodynamic technique directly provides information on the bulk physical properties. We find large variations of the size of the phase transition anomaly in the coefficient of thermal expansion, up to a factor of 3 , whereas its position varies only slightly around $6 \mathrm{~K}$. In addition, for two crystals out of nine, we find highly anomalous lattice effects when a magnetic field is applied along the in-plane $b$-axis.

\section{Experimental}

Single crystals with typical dimensions of about $0.1 \times 1.0 \times 1.2 \mathrm{~mm}^{3}$ were used for the experiments. The crystals were grown by following the standard procedure described in Ref. [20]. An ultrahigh-resolution capacitive dilatometer was employed for the thermal expansion measurements (built after [21]), enabling the detection of length changes $\Delta l \geq 10^{-2} \AA$, where $l$ is the length of the sample. For measurements in a constant magnetic field as a function of temperature and also for measurements of the magnetostriction at constant temperature, the magnetic field was applied along the measuring direction of the crystal. Thermal expansion measurements at $0 \leq B \leq 10$ T were performed upon heating and cooling with a slow sweep rate of $\pm 1.5 \mathrm{~K} / \mathrm{h}$ to ensure thermal equilibrium. For magnetostriction measurements, the sweep rate of the magnetic field was $\pm 120 \mathrm{mT} / \mathrm{min}$. Overall nine single crystals were studied, five single crystals from batch no. KAF 5078 and four from batch no. MP 1049.

\section{Sample-To-Sample Variations of the $6 \mathrm{~K}$ Anomaly}

Figure 1 gives an overview of the in-plane thermal expansion coefficients $\alpha_{i}(T)=l_{i}^{-1} \partial l_{i}(T) / \partial T$ $\left(i=b, c\right.$ are the in-plane crystallographic axes) for the $\kappa-(B E D T-T T F){ }_{2} \mathrm{Cu}_{2}(\mathrm{CN})_{3}$ single crystal MP $1049 \# 2$ 
(symbols) over the whole temperature range investigated. For comparison, we show in Figure 1 corresponding data for the crystals discussed in Ref. [8] (gray line) which were taken from batch KAF 5078. Besides the sharp peaks in $\alpha_{b}$ and $\alpha_{c}$ around $6 \mathrm{~K}$, which will be discussed below in more detail, the data at higher temperatures reveal highly anomalous and strongly anisotropic behavior. This includes a pronounced maximum of $\alpha_{b}$ around $80 \mathrm{~K}$ which is absent for $\alpha_{c}$. Instead, $\alpha_{c}$ decreases almost linearly with decreasing temperatures, becomes negative below about $50 \mathrm{~K}$ and passes through a minimum around $30 \mathrm{~K}$. We stress that, apart form the $6 \mathrm{~K}$ anomaly, the $\alpha_{b}$ and $\alpha_{c}$ data are almost identical, within the experimental resolution, to those revealed for crystals from batch KAF 5078 [8] (solid gray line in Figure 1). As was discussed in Ref. [8], the anomalies in $\alpha_{b}$ and $\alpha_{c}$ indicate that besides phonons, also other excitations contribute substantially to the low-temperature thermal expansion of this material.

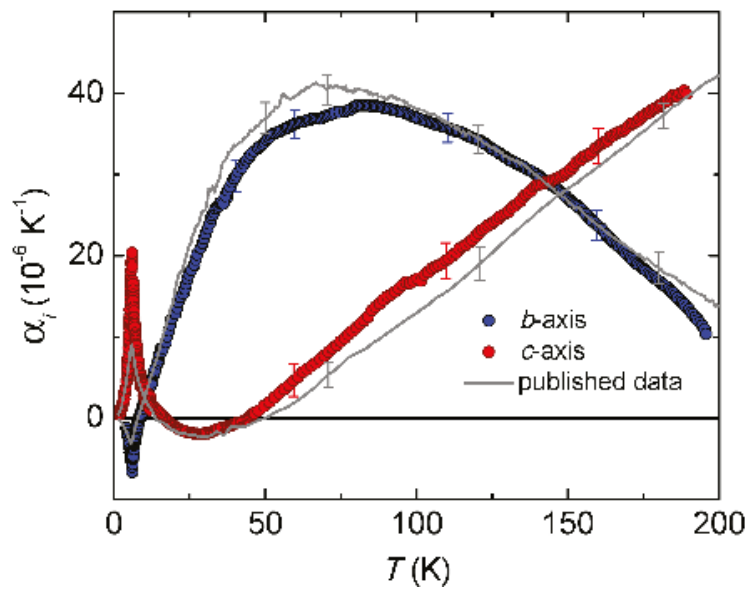

Figure 1. Overview of the coefficients of thermal expansion for $\kappa$-(BEDT-TTF) ${ }_{2} \mathrm{Cu}_{2}(\mathrm{CN})_{3}$ single crystal MP 1049\#2 (symbols) measured along the in-plane $b$ - and $c$-axis for $T \leq 200 \mathrm{~K}$. The solid gray line corresponds to data for single crystals from batch KAF 5078 reported previously by Manna et al. [8].

The low-temperature thermal expansion coefficients are dominated by the $6 \mathrm{~K}$ anomaly yielding sharp spikes in $\alpha_{b}$ and $\alpha_{c}$ with reversed sign. The data for $T \leq 12 \mathrm{~K}$ are shown in Figure $2 \mathrm{~b}$ on enlarged scales. For comparison, we show in Figure 2a the corresponding data for the single crystals from batch KAF 5078 reported previously by Manna et al. [8].

Figure 2 discloses a strongly sample-dependent anomaly at 6 K. For crystal MP 1049\#2, the size of the peaks in $\alpha_{b}$ and $\alpha_{c}$ are not only about two times larger than the ones found earlier on single crystals from batch KAF 5078 [8]. The anomalies are also distinctly sharper and more asymmetric in temperature with a steeper flank on the low-temperature side of the peak, clearly identifying the feature as a second-order phase transition. Despite these differences, however, other characteristics of the transition are retained. This includes the peak position at $T_{p} \simeq 6 \mathrm{~K}$, the anisotropy ratio $\alpha_{c}\left(T_{p}\right) / \alpha_{b}\left(T_{p}\right) \sim 3$, and a crossing point of $\alpha_{b}$ and $\alpha_{c}$ at around $10 \mathrm{~K}$. To illustrate the extent this sample-to-sample variation can take, we show in Figure 3 a compilation of $\alpha_{b}$ data for five selected single crystals from two different batches, including the crystals KAF 5078\#1 and MP $1049 \# 2$ presented above in Figure 2. Figure 3 discloses a huge variation by a factor of about 3 in the size of the transition, whereas the position changes only slightly within about $0.5 \mathrm{~K}$. Note that, even though the largest difference occurs between crystals from the different batches, there are also strong variations for crystals from the same batch. 


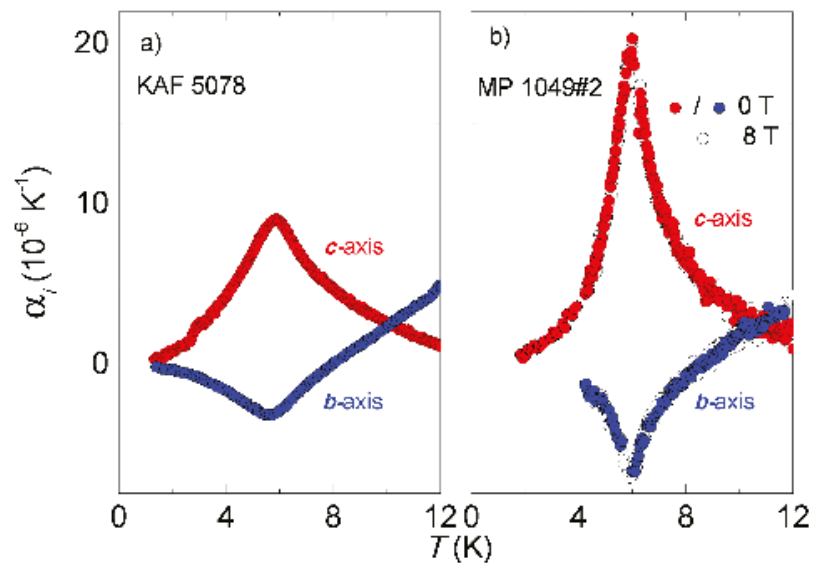

Figure 2. Thermal expansion coefficients for $\kappa$-(BEDT-TTF $)_{2} \mathrm{Cu}_{2}(\mathrm{CN})_{3}$ measured along the in-plane $b$ - and $c$-axis around the $6 \mathrm{~K}$ phase transition: (a) on single crystal from batch KAF 5078 reported in [8], (b) on single crystal MP 1049\#2 studied in this work, where the anomalies are most strongly pronounced. For this crystal also data taken in a magnetic field of $B=8 \mathrm{~T}$ applied along the measuring direction are shown.

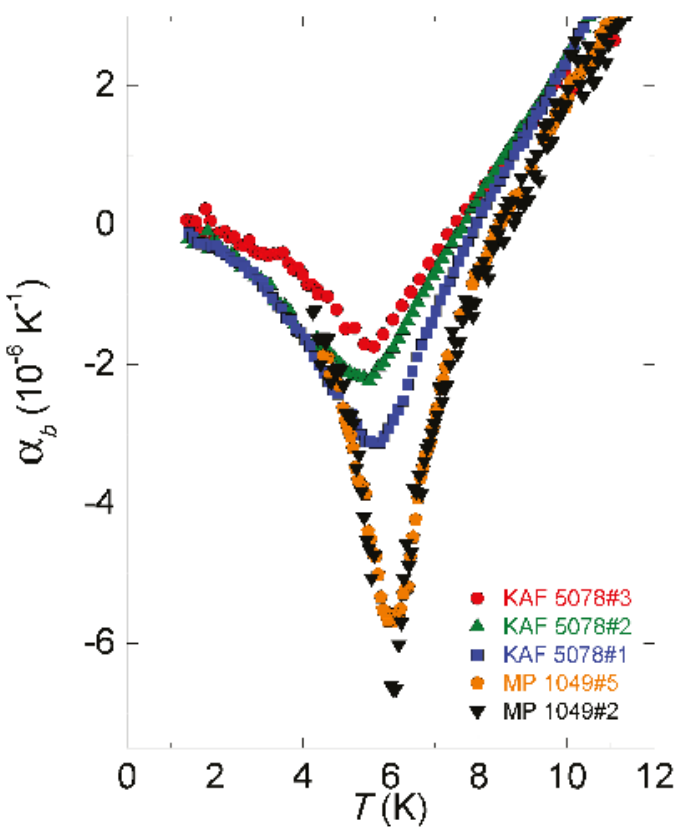

Figure 3. Comparison of the in-plane $b$-axis thermal expansion coefficient of $\kappa$-(BEDT-TTF $)_{2} \mathrm{Cu}_{2}(\mathrm{CN})_{3}$ for a selection of 5 out of 9 single crystals taken from two different batches.

\section{Field-Induced Effects}

All nine crystals, including the ones shown in Figure 3, were also subject to measurements in magnetic fields. The following observations were made: (1) as shown in Figure 2, there is no obvious effect of a magnetic field up to $8 \mathrm{~T}$, the maximum field applied, on the anomaly in $\alpha_{\mathcal{c}}$ (B\|c-axis). For this 
crystal MP 1049\#2, this statement is true also for $\alpha_{b}$ (B $\| b$-axis). In these experiments the field was applied at a temperature of $12 \mathrm{~K}$, prior to the measurements. (2) In contrast, for two crystals (KAF $5078 \# 1$ and KAF 5078\#4) out of all nine crystals studied, we find highly anomalous B-induced lattice effects when $B$ is applied along the $b$-axis of the crystal. The $B$-induced anomalous behavior is shown in the left panel of Figure 4 where we plot the relative length changes $\Delta l_{b}(T) / l_{b}=l\left(T_{0}\right)^{-1} \cdot\left[l(T)-l\left(T_{0}\right)\right]$, with $T_{0}$ a reference temperature, along the in-plane $b$-axis as a function of temperature at different constant magnetic fields applied parallel to the $b$-axis, see Ref. [22] for a preliminary report of the investigations. For comparison, we include in Figure $4 \mathrm{a}$ the data taken at zero magnetic field, yielding a broad minimum at around $8 \mathrm{~K}$, which corresponds to the change of sign of $\alpha_{b}=l_{b}^{-1} \partial l_{b} / \partial T$ (Figure 2). On the scale of Figure 4 , the abrupt change in slope in the $\Delta l_{b} / l_{b}$ data at $6 \mathrm{~K}$ (indicated by an arrow), reflecting the pronounced phase transition anomaly in $\alpha_{b}$ (Figure 2), cannot be seen. The same results, without any obvious field-induced anomaly, were obtained in a field of $B=0.5 \mathrm{~T}$ [22] (not shown). However, upon increasing the field to $B=1 \mathrm{~T}$, the data reveal a jump-like anomaly at $8.7 \mathrm{~K}$. The anomaly grows in size and shifts to lower temperatures down to $5.2 \mathrm{~K}$ with increasing magnetic fields up to $10 \mathrm{~T}$, the highest field accessible. These results suggest that a field in excess of some threshold value $0.5 \mathrm{~T}<B<1 \mathrm{~T}$ is necessary to trigger this effect. Interestingly, the magnetic field does not affect the $6 \mathrm{~K}$ phase transition anomaly. These measurements were performed upon cooling with a rate $-1.5 \mathrm{~K} / \mathrm{h}$ and the magnetic field was applied at $12 \mathrm{~K}$. We stress that measurements along the second in-plane $c$-axis with field parallel to $c$ [8] and measurements along the out-of-plane $a$-axis with field parallel $a$ [23] failed to find any indication for such a field-induced anomaly.

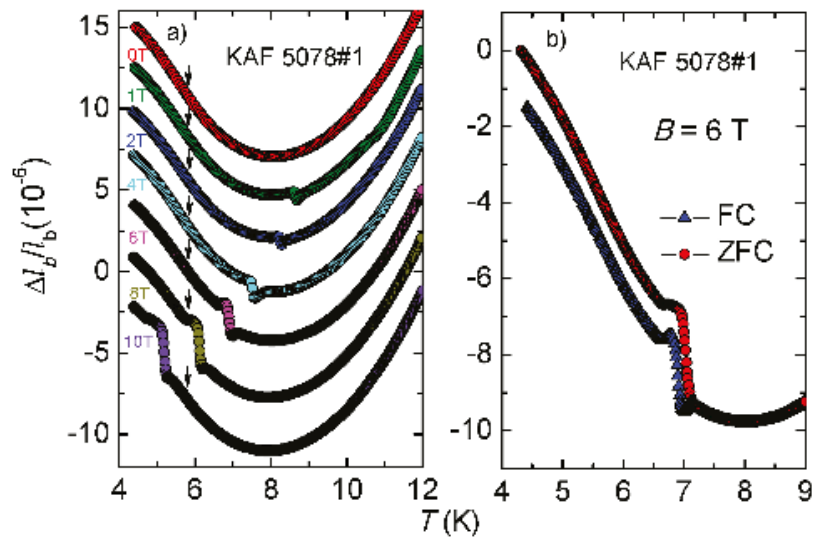

Figure 4. (a) Temperature-dependent relative length changes, $\Delta l_{b} / l_{b}$, for $\kappa$-(BEDT-TTF $)_{2} \mathrm{Cu}_{2}(\mathrm{CN})_{3}$ (KAF 5078\#1) along the $b$-axis for various constant magnetic fields between $0 \mathrm{~T}$ to $10 \mathrm{~T}$. The curves were shifted along the $y$-axis for clarity. The arrows indicate the phase transition at $6 \mathrm{~K}$ for the various fields, giving rise to a peak in the thermal expansion coefficient, $\alpha_{b}$. (b) Relative length changes for zero-field cooling (ZFC) and field cooling (FC) at a constant field value of 6 T. Measurements were performed with a very slow rate of $\pm 1.5 \mathrm{~K} / \mathrm{h}$.

Irrespective of the fact that the field-induced anomalies were seen only in two out of nine crystals, it is enlightening to explore the phenomenology of these anomalies in more detail. At first glance, one would be inclined to assign the discontinuous length changes revealed in $B \geq 1 \mathrm{~T}$ to a first-order phase transition. However, the absence of any hysteresis in $\Delta l_{b} / l_{b}$ upon heating and cooling with a slow rate of $\pm 1.5 \mathrm{~K} / \mathrm{h}$ [23] speaks against such an interpretation. Likewise, changing the heating and cooling rates (from $\pm 0.5 \mathrm{~K} / \mathrm{h}$ to $\pm 5.0 \mathrm{~K} / \mathrm{h}$ ) were found to have no effect on the anomaly (not shown) which is an indication that there is no spin-glass behavior involved. Furthermore, as was shown in Ref. [22], a comparison of $\Delta l_{b} / l_{b}$ data from $4.5 \mathrm{~K}$ to $12 \mathrm{~K}$, between zero field and a finite field of $6 \mathrm{~T}$, 
reveals that the data lie on top of each other at the high- and low-temperature end, but significantly deviate from each other at intermediate temperatures. This suggests that the jump-like anomaly in the intermediate region indicates a release of a field-induced lattice strain upon cooling [22]. Whereas there is no hysteresis upon heating and cooling, we do find a significant difference in $\Delta l_{b} / l_{b}$ between zero-field cooling (ZFC) and field cooling (FC) experiments, cf. Figure $4 \mathrm{~b}$. In the experiments shown there, the sample was zero-field cooled down to $4.5 \mathrm{~K}$, a field of $6 \mathrm{~T}$ was applied, and then data were taken upon heating (red circles) at a rate of $+1.5 \mathrm{~K} / \mathrm{h}(\mathrm{ZFC})$. With a delay of one night, the second data set was taken where the field was applied at $12 \mathrm{~K}$ and data were taken upon slowly cooling (blue triangles) with a rate of $-1.5 \mathrm{~K} / \mathrm{h}(\mathrm{FC})$. In the figure, the data sets were shifted vertically so that they coincide at the high-temperature end.

In addition to the temperature-dependent investigations in constant fields, we have looked for corresponding anomalies also in magnetostriction experiments, i.e., measurements of $\Delta l_{b} / l_{b}$ upon varying the magnetic field up to $10 \mathrm{~T}$. The measurements were performed by employing a sweep rate of $\pm 120 \mathrm{mT} / \mathrm{min}$. In the following we discuss a selection of the magnetostriction results. In Figure 5a, we show the relative length changes along the $b$-axis as a function of magnetic field $(B \| b)$ at $T=6 \mathrm{~K}$. The data reveal a pronounced step-like anomaly slightly above $8 \mathrm{~T}$ which corresponds to the feature observed in temperature sweeps at $B=$ constant. Interestingly enough, these magnetostriction measurements reveal yet another anomaly at a lower field around $1.8 \mathrm{~T}$ which could not be seen in temperature sweeps at constant fields. Corresponding data for temperature $T=7.8 \mathrm{~K}$ are shown in Figure $5 \mathrm{~b}$. Similar to the data at $6 \mathrm{~K}$, we find two anomalies, a sharp peak-like feature, now located around $2 \mathrm{~T}$, and a step-like feature at higher fields.

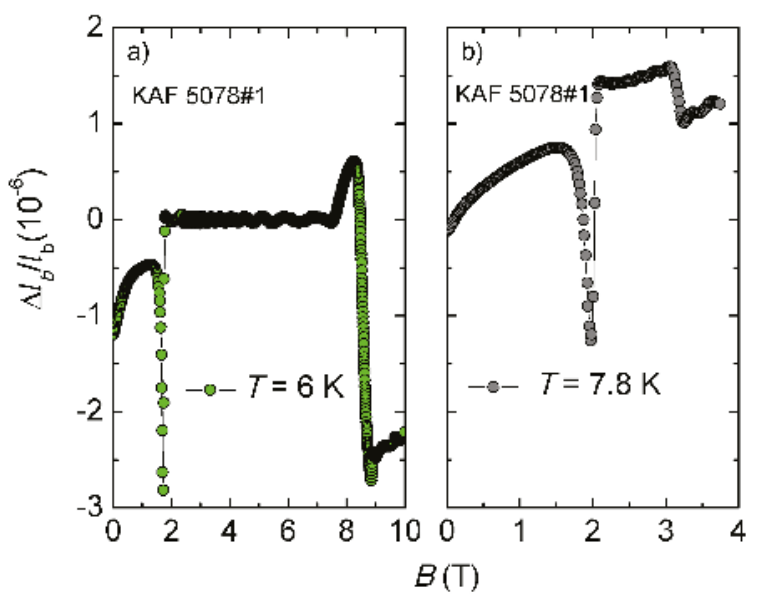

Figure 5. Relative length changes along the $b$-axis, $\Delta l_{b} / l_{b}$, for $\kappa$-(BEDT-TTF $)_{2} \mathrm{Cu}_{2}(\mathrm{CN})_{3}(\mathrm{KAF} 5078 \# 1)$ as a function of applied magnetic field, $B \| b$, at a temperature (a) $T=6 \mathrm{~K}$ and (b) $T=7.8 \mathrm{~K}$. The oscillations in the data, being periodic in $B^{-1}$, are due to quantum oscillations of gallium $(G a)$ used for affixing the sample in the desired orientation.

Based on results from thermal expansion measurements as a function of temperature ( $T$-sweep) at constant fields and results from magnetostriction measurements for isothermal field sweeps (B-sweep), an anomaly diagram can be constructed, as shown in Figure 6. The position of the anomaly at higher fields, derived from magnetostriction measurements, are fully consistent with those revealed from measurements as a function of temperature at $B=$ constant. Two distinct magnetic field-induced features can be identified which are most strongly pronounced and well-separated from each other 
at low temperatures, while the anomalies merge together at around $8.4 \mathrm{~K}$. A finite field above some threshold value of $0.5 \mathrm{~T}<B_{c} \leq 1 \mathrm{~T}$ is necessary to observe these anomalous field-dependent effects.

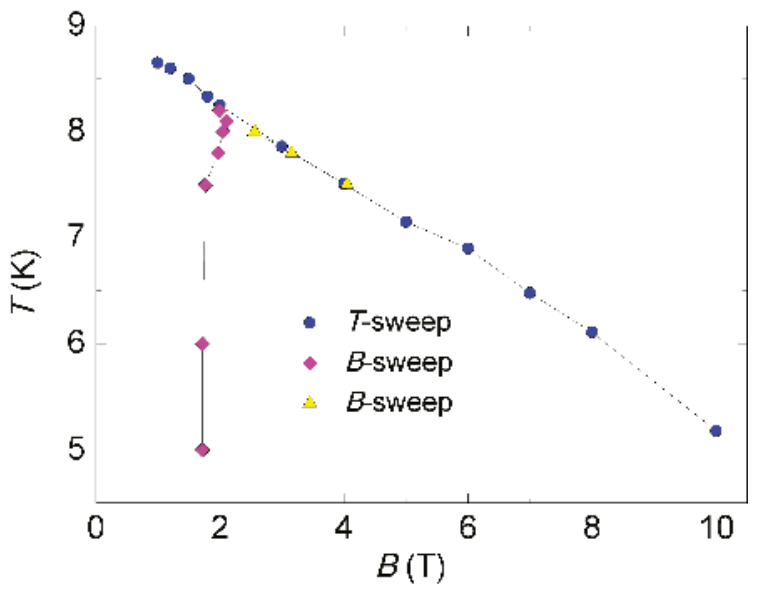

Figure 6. Anomaly diagram for $\kappa$-(BEDT-TTF) $)_{2} \mathrm{Cu}_{2}(\mathrm{CN})_{3}(\mathrm{KAF} 5078 \# 1)$ based on the position of the field-induced anomalies in $\Delta l_{b} / l_{b}$ as determined from thermal expansion measurements at $B(\| b)=$ constant $(T$-sweep, blue circles) and magnetostriction at $T=$ constant $(B$-sweep, magenta diamonds and yellow triangles). We note that these effects are absent in measurements along both the in-plane $c$-axis with $B \| c$ and along the out-of-plane $a$-axis with $B \| a$.

\section{Discussion}

The phenomenology described above suggests that the field-induced anomalies do not reflect properties of the hypothetically ideal material. In an attempt to provide an interpretation of these effects, we recall that (i) there is a significant sample-to-sample variation in the occurrence of the $B$-induced anomalies, and (ii) there is no obvious interrelation with the $6 \mathrm{~K}$ anomaly. The latter statement is based on the following two observations: there is a continuous, smooth growth of the $B$-induced anomaly on increasing the field from $6 \mathrm{~T}$ over $8 \mathrm{~T}$ to $10 \mathrm{~T}$ (Figure 4), despite crossing the phase boundary associated to the $6 \mathrm{~K}$ anomaly, (cf. Figure 6). In other words, the two effects interpenetrate each other as a function of field without any mutual influence. In addition, there seems to be an anticorrelation with the $6 \mathrm{~K}$ anomaly: the $B$-induced effects are absent in those crystals where the $6 \mathrm{~K}$ anomaly is strongest pronounced.

We are inclined to assign these effects to a $B$-induced formation of local magnetization which may nucleate around impurities or grain boundaries, as suggested on the basis of NMR measurements [7]. We further suspect that for the two crystals (\#1 and \#4 from batch KAF 5078) around those sites and induced by a finite field, some kind of small antiferromagnetic clusters are formed with an easy axis parallel to the $b$-axis. We then assign the spike-like feature around $B \simeq 1.8-2 \mathrm{~T}$ to the spin-flop transition of these antiferromagnetic clusters. In fact, the anomaly diagram presented in Figure 6 bears some resemblance to that of an uniaxial antiferromagnet with the field applied parallel to the preferred axis of spin alignment. In those uniaxial antiferromagnets, the transition from the antiferromagnetic phase to the spin-flop phase is of first order and almost independent of temperature, while the transition from the spin-flop phase to the fully polarized state at higher fields is of second order, showing a strong temperature dependence. The fact that we observe jump-like features at higher fields, and a ZFC-FC hysteresis (cf. Figure 4b), not expected for simple uniaxial antiferromagnets, is presumable due to the small, very likely nano-scale size of the $B$-induced magnetic clusters. As known from studies on magnetic nano-structures, the increased surface contribution of these structures can create irreversible 
contributions to the magnetization, even though the core of these structures is antiferromagnetic, see, e.g., Ref. [24].

The fact that these B-induced anomalies are absent for the crystals MP 1049\#2 and MP $1049 \# 5$ is consistent with the above interpretation and supports the view that the crystals, where the $6 \mathrm{~K}$ anomaly is most strongly pronounced, have a lower concentration of those defects on which magnetization can nucleate. Although the present study cannot make a definite statement about the nature of the $6 \mathrm{~K}$ transition, it clearly demonstrates that the order parameter strongly couples to the lattice degrees of freedom. Hence our results are consistent with models $[12,14,15]$ predicting a QSL instability that breaks the lattice symmetry so that pronounced lattice effects are expected. Note that so far low-temperature $X$-ray investigations failed to detect such a symmetry break [3]. The pairing of fermions (spinons or excitons), considered in these models, giving rise to a conversion to bosons, may be partial due to intrinsic but also extrinsic reasons. The high sensitivity of the size of the $6 \mathrm{~K}$ phase transition anomaly to some (yet unknown) sample-specific parameters, may then correspond to a different fraction of the fermions forming bosonic pairs at $T_{p}=6 \mathrm{~K}$. In light of the variation in the size of the anomaly within a factor of about 3 , we expect that there could be a considerable sample-to-sample variation in this fraction. Depending on the sample investigated and the experimental probe applied, this may lead to quite different conclusions as for the character of excitations of the low-temperature state, and therefore could provide a plausible explanation for the ongoing controversy on these issues.

\section{Conclusions}

In summary, detailed investigations of low-temperature lattice effects have been performed on the proposed spin-liquid compound $\kappa-(\text { BEDT-TTF })_{2} \mathrm{Cu}_{2}(\mathrm{CN})_{3}$. Particular emphasis was placed on sample-to-sample variations around the mysterious $6 \mathrm{~K}$ anomaly and the enigmatic field effects. By studying overall nine crystals from two different batches we found that the second-order phase transition at $6 \mathrm{~K}$ is strongly sample dependent in its size, varying within a factor of 3, whereas the position stays constant within $0.5 \mathrm{~K}$. In two out of these nine crystals, we observe pronounced field-induced effects, which were tentatively assigned to the formation of small antiferromagnetic clusters suspected to nucleate around some crystal imperfections. These effects are absent for those crystals where the phase transition anomaly at $6 \mathrm{~K}$ is most strongly pronounced. For the latter crystals, measurements of the specific heat and entropy release associated with the $6 \mathrm{~K}$ phase transition would be of great interest to explore the involvement of charge degrees of freedom. Our results are consistent with a pairing instability of the quantum spin liquid at $6 \mathrm{~K}$ which breaks lattice symmetry. We suspect that the conversion of fermionic excitations to bosons at this transition is only partial and to some extent influenced by sample-dependent factors.

Acknowledgments: We acknowledge financial support by the Deutsche Forschungsgemeinschaft via the SFB/TR49 and Claudius Gros, Roser Valenti, Patrick A. Lee, Jens Müller for useful discussions. RSM acknowledges the financial support from IIT Tirupati. Work at Argonne National Laboratory (ANL) was supported by UChicago Argonne, LLC, Operator of ANL. Argonne, a U.S. Department of Energy Office of Science laboratory, is operated under contract no. DE-AC02-06CH11357. JAS acknowledges support from the Independent Research/ Development program while serving at the National Science Foundation.

Author Contributions: Measurements were performed by R.S.M. and S.H. with contributions from M.d.S. and E.G. Single crystals were grown by J.A.S. R.S.M. and M.L. wrote the paper. All authors discussed the results and commented on the manuscript.

Conflicts of Interest: The authors declare no conflict of interest.

\section{References}

1. Toyota, N.; Lang, M.; Müller, J. Low-Dimensional Molecular Metals; Springer Series in Soild-State Sciences; Springerg: Berlin, Germany, 2007; Volume 154.

2. Gati, E.; Garst, M.; Manna, R.S.; Tutsch, U.; Wolf, B.; Bartosch, L.; Schubert, H.; Sasaki, T.; Schlueter, J.A.; Lang, M. Breakdown of Hooke's Law of Elasticity at the Mott Critical Endpoint in an Organic Conductor. Sci. Adv. 2016, 2, e1601646. 
3. Jeschke, H.O.; de Souza, M.; Valenti, R.; Manna, R.S.; Lang, M.; Schlueter, J.A. Temperature Dependence of Structural and Electronic Properties of the Spin-Liquid Candidate $\kappa$-(BEDT-TTF) ${ }_{2} \mathrm{Cu}_{2}(\mathrm{CN})_{3}$. Phys. Rev. B 2012, 85, 035125.

4. Shimizu, Y.; Miyagawa, K.; Kanoda, K.; Maesato, M.; Saito, G. Spin Liquid State in an Organic Mott Insulator with a Triangular Lattice. Phys. Rev. Lett. 2003, 91, 107001.

5. Yamashita, M.; Nakata, N.; Kasahara, Y.; Sasaki, T.; Yoneyama, N.; Kobayashi, N.; Fujimoto, S.; Shibauchi, T.; Matsuda, Y. Thermal-Transport Measurements in a Quantum Spin-Liquid State of the Frustrated Triangular Magnet $\kappa$-(BEDT-TTF $)_{2} \mathrm{Cu}_{2}(\mathrm{CN})_{3}$. Nat. Phys. 2009, 5, 44-47.

6. Yamashita, S.; Nakazawa, Y.; Oguni, M.; Oshima, Y.; Nojiri, H.; Shimizu, Y.; Miyagawa, K.; Kanoda, K. Thermodynamic Properties of a Spin-1/2 Spin-Liquid State in a $\kappa$-Type Organic Salt. Nat. Phys. 2008, 4, 459-462.

7. Shimizu, Y.; Miyagawa, K.; Kanoda, K.; Maesato, M.; Saito, G. Emergence of Inhomogeneous Moments from Spin Liquid in the Triangular-Lattice Mott Insulator $\kappa$-(BEDT-TTF) ${ }_{2} \mathrm{Cu}_{2}(\mathrm{CN})_{3}$. Phys. Rev. B 2006, 73, 140407(R).

8. Manna, R.S.; de Souza, M.; Brühl, A.; Schlueter, J.A.; Lang, M. Lattice Effects and Entropy Release at the Low-Temperature Phase Transition in the Spin-Liquid Candidate $\kappa$-(BEDT-TTF $)_{2} \mathrm{Cu}_{2}(\mathrm{CN})_{3}$. Phys. Rev. Lett. 2010, 104, 016403.

9. Poirier, M.; de Lafontaine, M.; Miyagawa, K.; Kanoda, K.; Shimizu, Y. Ultrasonic Investigation of the Transition at $6 \mathrm{~K}$ in the Spin-Liquid Candidate $\kappa$-(BEDT-TTF $)_{2} \mathrm{Cu}_{2}(\mathrm{CN})_{3}$. Phys. Rev. B 2014, 89, 045138.

10. Baskaran, G. Novel Local Symmetries and Chiral-Symmetry-Broken Phases in S = 1/2 Triangular-Lattice Heisenberg Model. Phys. Rev. Lett. 1989, 63, 2524.

11. Kawamura, H. Phase Transition of the Two-Dimensional Heisenberg Antiferromagnet on the Triangular Lattice. J. Phys. Soc. Jpn. 1984, 53, 4138-4154.

12. Lee, S.-S. Amperean Pairing Instability in the U(1) Spin Liquid State with Fermi Surface and Application to $\kappa-(B E D T-T T F)_{2} \mathrm{Cu}_{2}(\mathrm{CN})_{3}$. Phys. Rev. Lett. 2007, 98, 067006.

13. Galitski, V.; Kim, Y. Spin-Triplet Pairing Instability of the Spinon Fermi Surface in a U(1) Spin Liquid. Phys. Rev. Lett. 2007, 99, 266403.

14. Grover, T.; Trivedi, N.; Senthil, T.; Lee, P.A. Weak Mott Insulators on the Triangular Lattice: Possibility of a Gapless Nematic Quantum Spin Liquid. Phys. Rev. B 2010, 81, 245121.

15. Qi, Y.; Sachdev, S. Insulator-Metal Transition on the Triangular Lattice. Phys. Rev. B 2008, 77, 165112.

16. Gregor, K.; Motrunich, O.I. Nonmagnetic impurities in a $S=1 / 2$ frustrated triangular antiferromagnet: Broadening of ${ }^{13} \mathrm{C}$ NMR lines in $\kappa-(\mathrm{ET})_{2} \mathrm{Cu}_{2}(\mathrm{CN})_{3}$. Phys. Rev. B 2009, 79, 024421.

17. Yamashita, M.; Shibauchi, T.; Matsuda, Y. Thermal-Transport Studies on Two-Dimensional Quantum Spin Liquids. ChemPhysChem 2012, 13, 74-78.

18. Pratt, F.L.; Baker, P.J.; Blundell, S.J.; Lancaster, T.; Ohira-Kawamura, S.; Baines, C.; Shimizu, Y.; Kanoda, K.; Watanabe, I.; et al. Magnetic and Non-magnetic Phases of a Quantum Spin Liquid. Nature 2011, 471, 612-616.

19. Poirier, M.; Parent, S.; Côté, A.; Miyagawa, K.; Kanoda, K.; Shimizu, Y. Magnetodielectric effects and spin-charge coupling in the spin-liquid candidate $\kappa-(\mathrm{BEDT}-\mathrm{TTF})_{2} \mathrm{Cu}_{2}(\mathrm{CN})_{3}$. Phys. Rev. B 2012, 85, 134444.

20. Geiser, U.; Wang, H.H.; Carlson, K.D.; Williams, J.M.; Charlier, H.A.; Heindl, J.E.; Yaconi, G.A.; Love, B.J.; Lathrop, M.W.; Schirber, J.E.; et al. Superconductivity at $2.8 \mathrm{~K}$ and $1.5 \mathrm{kbar}$ in $\kappa$-(BEDT-TTF $)_{2} \mathrm{Cu}_{2}(\mathrm{CN})_{3}$ : the First Organic Superconductor Containing a Polymeric Copper Cyanide Anion. Inorg. Chem. 1991, 30, 2586-2588.

21. Pott, R.; Schefzyk, R. Apparatus for Measuring the Thermal Expansion of Solids between 1.5 and $380 \mathrm{~K}$. J. Phys. E 1983, 16, 444-449. 
22. Manna, R.S.; de Souza, M.; Schlueter, J.A.; Lang, M. Field-induced length changes in the spin-liquid candidate $\kappa$-(BEDT-TTF $)_{2} \mathrm{Cu}_{2}(\mathrm{CN})_{3}$. Phys. Status Solidi C 2012, 9, 1180-1182

23. Manna, R.S. Thermal Expansion Studies on Low-Dimensional Frustrated Quantum Magnets: The Case of $\kappa$-(BEDT-TTF $)_{2} \mathrm{Cu}_{2}(\mathrm{CN})_{3}$ and Azurite. Ph.D. Thesis, Goethe University Frankfurt, Frankfurt, Germany, 2012. Available online: http://publikationen.ub.uni-frankfurt.de/frontdoor/index/index/docId/24778 (accessed on 13 December 2017).

24. Benitez, M.J.; Petracic, O.; Salabas, E.L.; Radu, F.; Tüysüz, H.; Schüth, F.; Zabel, H. Evidence for Core-Shell Magnetic Behavior in Antiferromagnetic $\mathrm{Co}_{3} \mathrm{O}_{4}$. Phys. Rev. Lett. 2008, 101, 097206.

(C) 2018 by the authors. Licensee MDPI, Basel, Switzerland. This article is an open access article distributed under the terms and conditions of the Creative Commons Attribution (CC BY) license (http:/ / creativecommons.org/licenses/by/4.0/). 


\title{
Electrodynamics in Organic Dimer Insulators Close to Mott Critical Point
}

\author{
Marko Pinterić ${ }^{1,2}$, David Rivas Góngora ${ }^{1}$, Željko Rapljenović ${ }^{1}$, Tomislav Ivek ${ }^{1}$, Matija Čulo ${ }^{1}$, \\ Bojana Korin-Hamzić ${ }^{1}$, Ognjen Milat ${ }^{1}$, Branko Gumhalter ${ }^{1}$, Predrag Lazić ${ }^{3}$, \\ Miriam Sanz Alonso ${ }^{4}$, Weiwu Li ${ }^{4}$, Andrej Pustogow ${ }^{4}$, Guilherme Gorgen Lesseux ${ }^{4}$, \\ Martin Dressel ${ }^{4, *}$ and Silvia Tomić ${ }^{1, *}$ \\ 1 Institut za fiziku, Bijenička 46, HR-10000 Zagreb, Croatia; mpinter@ifs.hr (M.P.); drivas@ifs.hr (D.R.G.); \\ zrapljenovic@ifs.hr (Ž.R.); tivek@ifs.hr (T.I.); mculo@ifs.hr (M.Č.); bhamzic@ifs.hr (B.K.-H.); \\ milat@ifs.hr (O.M.); branko@ifs.hr (B.G.) \\ 2 University of Maribor, Faculty of Civil Engineering, Smetanova 17, SI-2000 Maribor, Slovenia \\ 3 Rudjer Bošković Institute, Bijenička 56, HR-10000 Zagreb, Croatia; plazicx@gmail.com \\ 4 1. Physikalisches Institut, Universität Stuttgart, Pfaffenwaldring 57, D-70550 Stuttgart, Germany; \\ weiwu.li@pi1.physik.uni-stuttgart.de (W.L.); andrej.pustogow@pi1.physik.uni-stuttgart.de (A.P.); \\ guilherme.gorgen-lesseux@pi1.physik.uni-stuttgart.de (G.G.L.) \\ * Correspondence: martin.dressel@pi1.physik.uni-stuttgart.de (M.D.); stomic@ifs.hr (S.T.)
}

Received: 26 March 2018 ; Accepted: 18 April 2018 ; Published: 27 April 2018

\begin{abstract}
Organic layered charge-transfer salts $\kappa$-(BEDT-TTF) $)_{2} X$ form highly frustrated lattices of molecular dimers in which strong correlations give rise to Mott insulating states situated close to the metal-to-insulator phase boundary. The salts $\kappa$-(BEDT-TTF) ${ }_{2} \mathrm{Cu}_{2}(\mathrm{CN})_{3}$ and $\kappa$-(BEDT-TTF $)_{2} \mathrm{Ag}_{2}(\mathrm{CN})_{3}$ have been considered as prime candidates for a quantum spin liquid, while $\kappa$-(BEDT-TTF $)_{2} \mathrm{Cu}\left[\mathrm{N}(\mathrm{CN})_{2}\right] \mathrm{Cl}$ has been suggested as a prototypical charge-order-driven antiferromagnet. In this paper, we summarize and discuss several key results, including some not reported previously, obtained in search to clarify the competition of these two ground states. The origin of anomalous dielectric response found at low temperatures in all three salts is also discussed. We conclude by pointing out the relevant new insights into the role of frustration and random disorder in the suppression of magnetic ordering and formation of the spin liquid state.
\end{abstract}

Keywords: strongly correlated systems; mott insulators; quantum spin liquid; dielectric properties; vibrational properties; density functional theory

\section{Introduction}

Layered organic solids $\kappa$-(BEDT-TTF $)_{2} X\left(X=\mathrm{Cu}\left[\mathrm{N}(\mathrm{CN})_{2}\right] \mathrm{Cl}, \mathrm{Cu}_{2}(\mathrm{CN})_{3}\right.$ and $\left.\mathrm{Ag}_{2}(\mathrm{CN})_{3}\right)$ are systems in which strong correlations lead to Mott insulating states situated close to the metal-to-insulator phase boundary [1,2]. These systems are charge-transfer salts, i.e., they consist of conducting BEDT-TTF layers separated by non-conducting anion layers (Figure 1). Within BEDT-TTF layers, the triangular lattices of molecular dimers with one hole per dimer are formed with different degrees of frustration (Figure 2) [3-6]. Moderate pressure in the range of a few hundred bars up to $10 \mathrm{kbar}$ is sufficient to recover metallicity and superconducting ground state. At ambient pressure in these salts two different ground states can be established. Canted antiferromagnetic, i.e., weak ferromagnetic, state is formed in $\kappa-(\mathrm{BEDT}-\mathrm{TTF})_{2} \mathrm{Cu}\left[\mathrm{N}(\mathrm{CN})_{2}\right] \mathrm{Cl}$ (denoted as $\left.\kappa-\mathrm{Cl}\right)$ [7-9]. On the other hand, quantum spin liquid (QSL) develops in the two latter salts $\kappa$-(BEDT-TTF) $2 X, X=\mathrm{Cu}_{2}(\mathrm{CN})_{3}$ and $\mathrm{Ag}_{2}(\mathrm{CN})_{3}$ (denoted as $\kappa-\mathrm{CuCN}$ and $\kappa-\mathrm{AgCN}$, respectively) [10-12]. QSL is commonly considered as a highly correlated fluctuating quantum spin state expected to appear in geometrically frustrated systems when quantum fluctuations are strong enough to preclude spin ordering down to zero 
temperature $[13,14]$. Historically, QSL was predicted theoretically as a resonating valence bond state to be the ground state of a perfect triangular lattice $S=1 / 2$ spin antiferromagnet instead of the conventional long-range antiferromagnetic (AF) order [15]. Experimentally, however, few real materials with triangular lattice, no spontaneously broken symmetry, and emergent fractional excitations have been discovered. Among them stand out the most recent discoveries of two novel QSL types. First is a high-temperature QSL in the layered transition metal dichalcogenide $1 \mathrm{~T}-\mathrm{TaS}_{2}$ [16] as a long-awaited prediction by Anderson. Second is a quantum liquid of magnetic and electric dipoles developed in a hydrogen-bonded molecular compound $\kappa-\mathrm{H}_{3}(\text { Cat-EDT-TTF })_{2}$ [17]
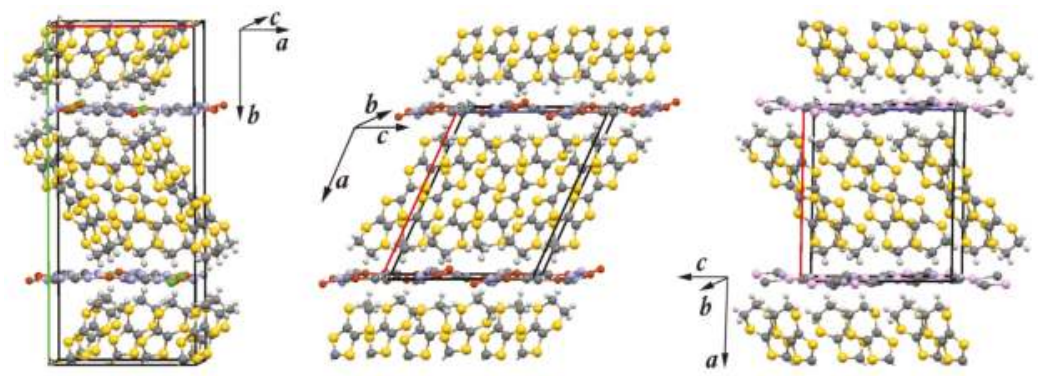

Figure 1. The crystal structure of the three layered organic charge-transfer salts. The lines mark the unit cell. Carbon, sulfur and hydrogen atoms of the BEDT-TTF molecule are colored in dark gray, yellow and light gray, respectively. In the anion network, chlorine, cooper, silver, carbon and nitrogen are colored in green, red, pink, dark grey and violet, respectively. (Left) Unit cell of $\kappa$-Cl. The space group is $P_{n m a}$. In each of two organic layers, related by mirror symmetry, all four BEDT-TTF molecules are equivalent. (Middle,Right) Extended unit cell of $\kappa-\mathrm{CuCN}$ and $\kappa-\mathrm{AgCN}$, respectively. The space group is commonly solved in $P 2_{1} / c$ in which all four BEDT-TTF molecules are equivalent.
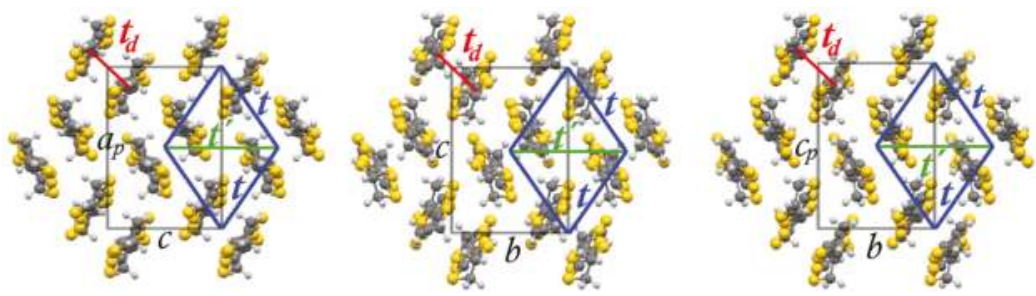

Figure 2. View of BEDT-TTF dimers arranged in anisotropic triangles in the three layered organic charge-transfer salts: (Left) $\kappa-\mathrm{Cl}$, projection of one of the layers is shown along the [110] direction; (Middle) $\kappa$-CuCN, projected along the [100] direction; and (Right) $\kappa$-AgCN, projected along the [10-1] direction. Neighboring dimers are rotated by $90^{\circ}$ with respect to each other. The interdimer transfer integrals are denoted by $t$ and $t^{\prime}$, and the intradimer transfer integral by $t_{\mathrm{d}}$. The ratio $t^{\prime} / t$ measures the degree of frustration. $t^{\prime} / t \approx 0.5$ indicates medium frustration for $\kappa-\mathrm{Cl}$, while $t^{\prime} / t \approx 0.85$ indicates high frustration for $\kappa-\mathrm{CuCN}$ and $\kappa-\mathrm{AgCN}$.

Common to all three $\kappa$-(BEDT-TTF $)_{2} X$ organic charge-transfer salts is an anomalous dielectric response [8,18-23], the origin of which remained elusive due to the missing experimental evidence of any sizeable electric dipoles on molecular dimers [19,23-25] proposed theoretically [26-34]. However, relying on the theory and based on the observation of Curie-like dielectric peak, hysteresis and time-dependent phenomena, a dipolar order has been proposed to set in $\kappa-\mathrm{Cl}$ acting as a driving force for the magnetic ground state [18]. Related open issue in this salt is that, instead of Curie-like peak, in the majority of measured single crystals, a relaxor response has been detected, suggesting that the dielectric response rather arises from the motion of charge domain walls between insulating 
ferromagnetic domains in the background impurity potential $[8,19]$. In two salts $\kappa-\mathrm{CuCN}$ and $\kappa-\mathrm{AgCN}$ with quantum spin liquid ground state, dielectric response is consistently found to be of relaxor type in all measured single crystals. The suppression of magnetic state has been mostly associated with highly frustrated molecular dimer lattices, but it has been argued that an additional mechanism, such as an exotic spin-dipolar coupling, is needed to induce quantum fluctuations which are strong enough to suppress magnetic order [26-34]. The reason for that is twofold. First is the proximity of Mott insulator-to-metal phase boundary where the charge degrees of freedom start to delocalize and thus a spin-only description cannot be sufficient. Second is the inability of frustrated triangular lattices to destroy the long-range magnetic order on their own [35-37]. The role of random disorder in this competition may also be important, as indicated by an experimental study on $\kappa$-Cl showing that X-rays suppress magnetic order and allow a spin liquid to form [38]. Finally, we have recently suggested that the non-conducting anions which contain frustration and disorder in the organic spin liquids may be a critical ingredient needed for the prevalence of quantum spin liquid over magnetic ordering [39].

In this paper, we address the issue of anomalous dielectric response and inherent randomness, and explore its origin and role in the competition between the AF and QSL ground state formation.

\section{Antiferromagnet with Ferroelectric Character $\kappa-(\mathrm{BEDT}-\mathrm{TTF})_{2} \mathrm{Cu}\left[\mathrm{N}(\mathrm{CN})_{2}\right] \mathrm{Cl}$}

At ambient pressure, $\kappa-\mathrm{Cl}$ system behaves as an insulator in dc limit in the whole temperature range, but, in the infrared range, a large temperature-dependent charge gap (Mott gap) develops only at temperatures lower than $50 \mathrm{~K}[19,40]$. The system was reported to show zero resistance and weak superconducting diamagnetic response at pressures as small as 100 bar, and a bulk superconductivity (SC) below $12.5 \mathrm{~K}$ at 300 bar [41-43]. On cooling, dc resistivity increases only weakly down to about $100 \mathrm{~K}$. This behavior is observed for majority of measured samples; the behavior of three single crystals is displayed in Figure 3, Inset in Left panel.

On further cooling below $100 \mathrm{~K}$, the dc resistivity increases steeply, indicating an insulator-toinsulator phase transition, right in the temperature range where the Mott gap develops. Interestingly, the rate by which dc resistivity rises seems to be much larger perpendicular to the dimer planes (i.e., along the $b$-axis) than within them $[18,44]$. Overall, dc resistivity data accumulated by us and the other authors however indicates that the phase transition temperature and the width of the transition extracted from the logarithmic resistivity derivative differ for different single crystals. Figure 3 shows distinct behaviors of the dc resistivity along the $b$-axis for three single crystals of $\kappa$-Cl. The resistivity derivative of S2, as well as of the sample in Ref. [19] (see Figure 3 in [19]), shows a broad maximum centered at about $30 \mathrm{~K}$, indicative of the formation of a low-temperature phase at short-range scales. Conversely, samples S1 and S3 present a peak that is twice as narrow, higher, and positioned at a slightly lower temperature of $27 \mathrm{~K}$. However, the full width at half maximum is ten times larger than what is typical for the phase transition developed at the long-range scales, indicating a certain amount of disorder even in the crystals S1 and S3.

The nature of phase transition, based on dielectric data displaying a peak in the same temperature range (Figure 4, Right), was proposed to be ferroelectric and ascribed to the ordering of electric dipoles on BEDT-TTF dimers [18]. Moreover, it was argued that the dipolar ordering releases the frustration of dimers' spins on the triangular lattice and in this way drives the magnetic ordering which sets in at same temperatures. The exciting proposal of multiferroicity awaits for its final confirmation due to missing evidence for inversion symmetry breaking $[19,25]$. Thus, the nature of phase transitions detected in dc resistivity and dielectric response, and the origin of disorder in $\kappa-\mathrm{Cl}$ are still a mystery. We come back to this issue at the end of this section. An additional broad feature, situated at about $50 \mathrm{~K}$, is detected only for sample S3 (Figure 3, Right), right in the temperature range where the Mott gap opens.

Further, the most intriguing behavior is found for sample S2. Upon reducing temperature the resistivity rises with a much smaller rate, reaches maximum at about $12 \mathrm{~K}$ and falls down rapidly. Note that $12 \mathrm{~K}$ is the temperature below which bulk SC sets in at 300 bar. Notably, a similar drop 
of resistivity measured in dc limit, as well as at $35 \mathrm{GHz}$, has been reported before independently by Sushko et al. [45] and one of us [44], and attributed to SC islands growing within the insulating sample bulk. The suggestion has been supported by magnetization measurements indicating that the low temperature state of $\kappa-\mathrm{Cl}$ below critical pressure of 300 bar is an inhomogeneous mixture of SC and insulating magnetic phases [45]. Indeed, for sample S2, we do find evidence for the presence of SC domains at $T<T_{\mathrm{SC}} \approx 12 \mathrm{~K}$. These domains do not percolate down to $5 \mathrm{~K}$ since the resistivity remains finite (Figure 3). At these low temperatures only, a non-linear decrease of the low-frequency conductivity is found when increasing ac voltage (not shown) or applying magnetic field (Figure 5, Left). At zero magnetic field, there is a rise in conductivity below about $12 \mathrm{~K}$, as observed in dc resistivity measurements. Increasing the magnetic field shifts the SC phase transition to lower temperatures. For magnetic field of $5 \mathrm{~T}$ the SC phase transition is suppressed down to $5 \mathrm{~K}$.
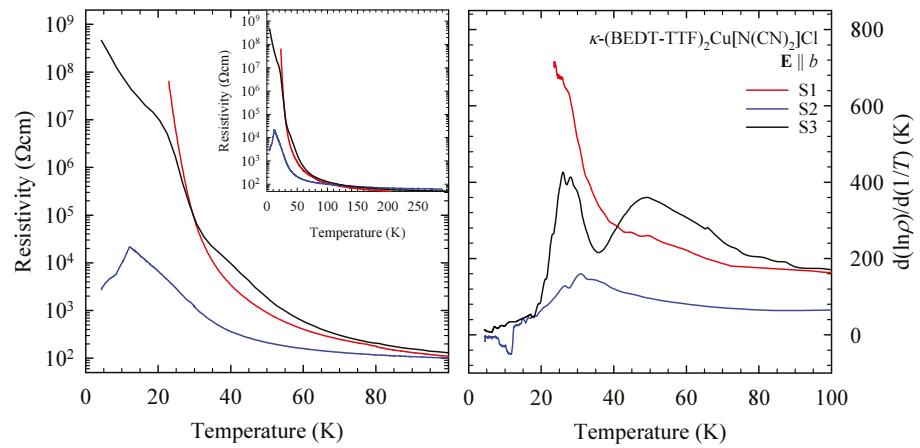

Figure 3. (Left) Distinct behaviors of the resistivity along the $b$ axis below $100 \mathrm{~K}$ for three single crystals of $\kappa-\mathrm{Cl}$ : S1 (red), S2 (blue) and S3 (black). Data for S3 are after [18]. The inset displays the resistivity behavior in the whole temperature range demonstrating a weak activated behavior similar for all three samples. (Right) Logarithmic resistivity derivative versus temperature.
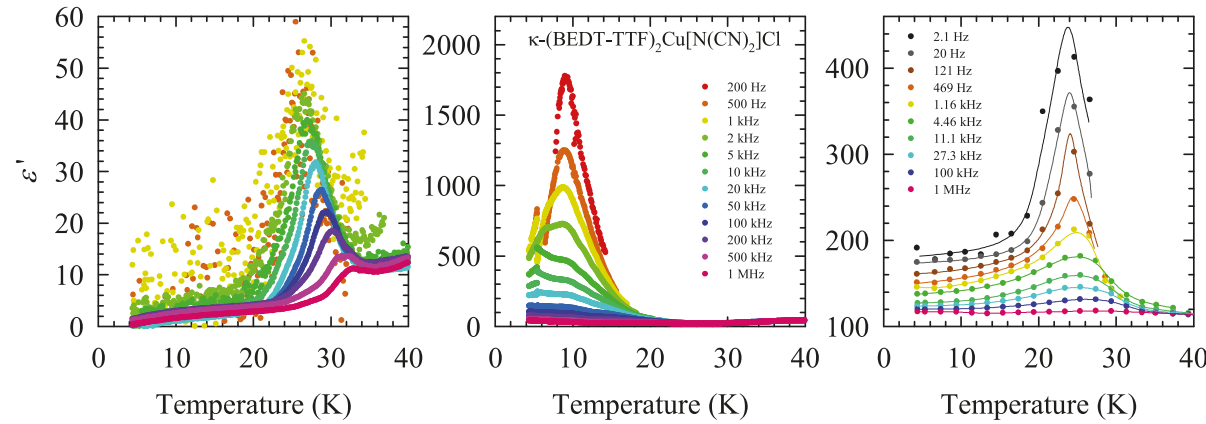

Figure 4. Real part of the dielectric function $\varepsilon^{\prime}$ as a function of temperature with the ac electric field applied $\mathbf{E} \| b$ for three representative single crystals of $\kappa$-Cl: S1 (Left); S2 (Middle); and S3, after [18] (Right panel) The full lines in (Right) are guides for the eye.

Next, we address the dielectric function measured as a function of temperature and frequency along the $\mathbf{E} \| b$ i.e., perpendicular to the BEDT-TTF dimer layers. Figure 4 presents the real part of dielectric function $\varepsilon^{\prime}$ of three representative samples of $\kappa-\mathrm{Cl}$. Common to all of them is that below $40 \mathrm{~K}$, $\varepsilon^{\prime}$ features a pronounced peak, the high-temperature shoulders of which align to follow the same curve. Conversely, the peak's amplitudes and the positions in temperature strongly differ between them. The former seem to correlate with the magnitude of dc conductivity, but might also be influenced by 
different levels of disorder present in the three samples. The $\varepsilon^{\prime}$ peaks of sample S1 and S3 are situated at about $25 \mathrm{~K}$, showing a weak relaxor and a Curie-like behavior i.e., nearly frequency independent position of the peak, and reach similar values $\leq 100$ for frequencies $\geq 200 \mathrm{~Hz}$. A remarkably distinct result is observed for sample S2: the $\varepsilon^{\prime}$ peak is shifted to lower temperatures and centered at $T_{\mathrm{C}} \approx$ $9 \mathrm{~K}$ for all applied frequencies attaining a value as high as 1800 at $200 \mathrm{~Hz}$. Notably, $T_{\mathrm{C}}$ seems to coincide with $T_{\mathrm{SC}}$. Applied magnetic field reduces the onset of conductivity rise (Figure 5, Left) and concomitantly smooths the low-temperatures sides of the $\varepsilon^{\prime}$ peak and lowers its amplitude, the effect showing up only at temperatures below $T_{\mathrm{SC}} \approx 12 \mathrm{~K}$ (Figure 5, Right). Finally, the susceptibility behavior observed for sample S2 shows an increase, albeit rather broad, below about 22 K (Figure 6), as expected for the intra-layer antiferromagnetic ordering documented previously [7-9].

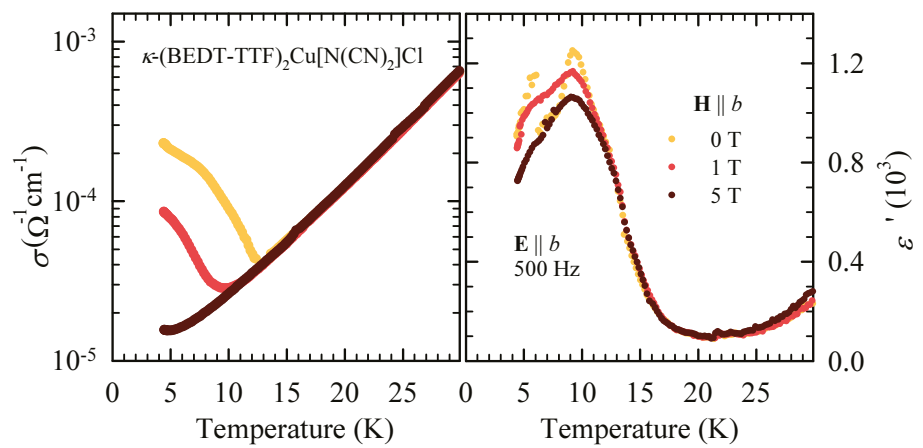

Figure 5. Magnetic field influence on sample S2 of $\kappa$-Cl. (Left) Conductivity $\sigma$ measured at $500 \mathrm{~Hz}$ for $\mathbf{E} \| b$ versus temperature measured at different magnetic field strengths $(\mathbf{H} \| b)$. At $\mathbf{H}=0 \mathrm{~T}$ there is a rise in conductivity below $12 \mathrm{~K}$, while the application of magnetic field shifts the onset of conductivity rise to lower temperatures, thus indicating a superconducting phase transition at $T_{\mathrm{SC}} \approx 12 \mathrm{~K}$. (Right) Real part of the dielectric function $\varepsilon^{\prime}$ as a function of temperature with the ac electric field applied $\mathbf{E} \| b$, and measured at different magnetic field strengths $(\mathbf{H} \| b)$. The effect of the magnetic field can be seen only below $12 \mathrm{~K}$. Increasing magnetic field lowers the peak amplitude.

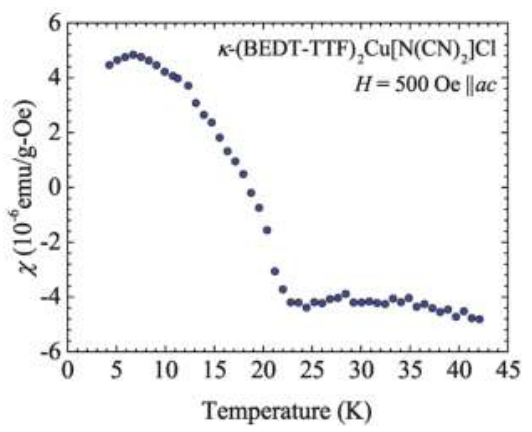

Figure 6. Susceptibility $\chi$ of sample $\mathrm{S} 2$ of $\kappa-\mathrm{Cl}$ as a function of temperature measured in a magnetic field of 500 Oe applied $\mathbf{H} \|$ ac-plane.

Importantly, for sample S2 both the magnetic phase transition and the insulator-to-insulator phase transition seem to be developed only at short-length scales, however they appear within the same temperature range as commonly observed in $\kappa-\mathrm{Cl}$ samples. On the other hand, the dielectric peak if taken as an indication of a ferroelectric-like ordering reveals itself as a feature separated from the phase transitions visible in dc transport and in susceptibility. Moreover, it is shifted to lower 
temperatures and appears concomitantly with the superconducting phase transition indicating the coexistence of ferroelectric-like order and SC. Thus, despite ferroelectricity being expected only in insulating systems, here it seems to coexist with dilute metallicity, indicating that ferroelectricity, together with magnetism, shares a common boundary with SC in the phase diagram. However, note that, while magnetic and SC are competing phases, the observed data suggest that ferroelectricity and SC support each other. Remarkably, Clay and Mazumdar recently developed a valence-bond theory of SC within which the SC state is reached by the destabilization of the molecular dimer order called paired-electron crystal (PEC) $[31,32,46]$. According to their theory, the low-temperature phase in sample S2 might be considered as consisting of segregated magnetic, PEC and SC phases. This outstanding result in organics bears similarities to the superconducting high- $T_{\mathrm{C}}$ cuprates, where the proximity of a charge-ordered state to the AF and the SC state has been established.

It is challenging to reconcile this exciting proposal with the missing experimental evidence of structural inversion symmetry breaking needed for formation of ferroelectricity. Namely, X-ray diffraction measurements have shown that the space group of $\kappa-\mathrm{Cl}$ is $P_{n m a}$ implying that all eight BEDT-TTF molecules and all four dimers in the unit cell are crystallographically equivalent [47]. Interestingly, NMR data reported in [9] seem to indicate four nonequivalent spin dimers sites present already at room temperature, but remarkably the nonequivalence disappeared once the magnetic state was established. The experimentally evidenced absence of any considerable electric dipoles on BEDT-TTF dimer sites [25] has promoted an alternative interpretation for the relaxor-like dielectric response observed in the most number of $\kappa-\mathrm{Cl}$ samples. This scenario, backed up by the generally observed tendency to phase segregation, invokes charged domain-walls between insulating ferromagnetic domains in the disordered background of the canted AF i.e., weak ferromagnetic phase $[8,19,22]$.

Here, we present vibrational spectroscopy data performed on sample S2 of $\kappa$-Cl. Figure 7 displays the most charge-sensitive intramolecular vibrational mode $v_{27}$ centered at $1469 \mathrm{~cm}^{-1}$ at $5 \mathrm{~K}$, which involves the anti-symmetric $\mathrm{C}=\mathrm{C}$ stretching of the rings within BEDT-TTF molecules, as a function of temperature [48-50]. The side band at $1473 \mathrm{~cm}^{-1}$ is present at all temperatures due to inequivalent lattice sites [25] and becomes visible at low temperatures as the modes become more narrow. The small satellite at $1478 \mathrm{~cm}^{-1}$ involves ethylene motions and is not related to the $v_{27}$ mode [50]. A possible charge inequality among the donor molecules can be quantitatively estimated from the well-known linear frequency-charge relation concluded by Yamamoto et al. [49]. The charge disproportionation can be directly determined from the frequency separation of the respective $v_{27}$ peaks via $2 \delta_{\rho}=\delta v_{27} /\left(140 \mathrm{~cm}^{-1} / e\right)$. In the case of $\kappa-\mathrm{Cl}$, however, no splitting of the mode occurs on lowering the temperature down to $5 \mathrm{~K}$, providing clear evidence that, even in sample S2 of $\kappa$-Cl, no sizeable static charge redistribution exceeding the limit of $2 \delta_{\rho} \approx \pm 0.01 e$ takes place. The observation is in full accord with previous reports on $\kappa-\mathrm{Cl}[19,25]$. Given the estimated extremely small upper bound of charge imbalance and fast rate of charge oscillations within the dimer [25], such static or fluctuating electric dipoles associated with BEDT-TTF dimers can hardly explain the low-frequency dielectric response, in particular the one showing a sizeable Curie-like anomaly, as observed for sample S2 of $\kappa-\mathrm{Cl}$. Importantly, in sample S2 the magnetic and dielectric anomaly, setting in at separated temperatures of $25 \mathrm{~K}$ and $9 \mathrm{~K}$, seem to suggest that it is a ferroelectricity which is driven by the magnetism, and not vice versa as proposed in [18].

Finally, origin of disorder in single crystals of $\kappa-\mathrm{Cl}$ is a very intriguing issue. The presence of disorder is suggested by the broadness of phase transition anomalies seen in dc transport and in susceptibility, as well as relaxor-like dielectric anomalies [51]. However, here the possibility that disorder originates in the anion layer, as suggested in the case of $\kappa$-CuCN and $\kappa$-AgCN $[21,23,24]$, must be discarded since all cyanide $(\mathrm{CN})$ groups positioned between the copper $(\mathrm{Cu})$ atoms are ordered in a zigzag line along the $a$-axis so that the anion layer consists of one-dimensional chains (Figure 8). Alternatively, disorder may arise in the organic dimer layers due to the formation of segregated phases of similar energy [46]. This possibility should be explored further. 


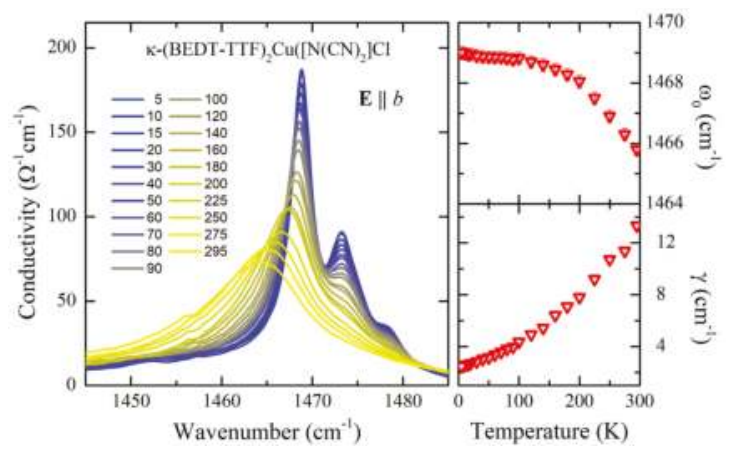

Figure 7. (Left) Temperature evolution of the intramolecular vibration $v_{27}$ in sample S2 of $\kappa$-Cl. (Right) Temperature dependence of the resonance frequency and damping obtained by Fano function fit of the mode.

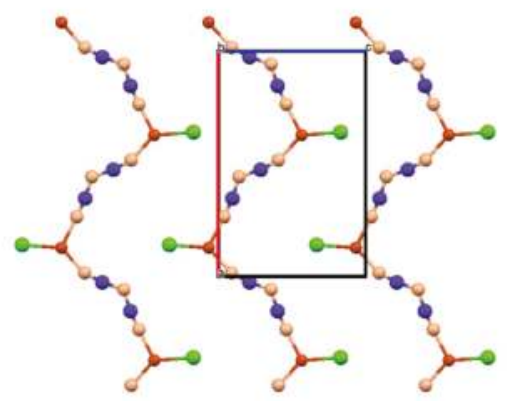

Figure 8. View of the anion layer of $\kappa-\mathrm{Cl}$ in the $a c$ plane projected along the $b$-axis. Copper is colored in red, and chlorine in green. Carbon and nitrogen are colored in blue and orange, respectively. The unit cell is marked as a rectangle.

\section{Quantum Spin Liquids in Disordered $\kappa$-(BEDT-TTF $)_{2} \mathrm{Cu}_{2}(\mathrm{CN})_{3}$ and $\kappa-(\mathrm{BEDT}-\mathrm{TTF})_{2} \mathrm{Ag}_{2}(\mathrm{CN})_{3}$}

$\kappa-\mathrm{CuCN}$ and $\kappa-\mathrm{AgCN}$ are Mott-Anderson insulators in which combined effects of strong Coulomb interaction and randomness have been well documented, experimentally and theoretically. dc charge transport within dimer planes happens via two-dimensional variable-range hopping in a wide low-temperature range. At elevated temperatures, it crosses-over to nearest-neighbor hopping $[21,23,52,53]$. Anomalously broad anisotropic dielectric response exhibits the relaxor-like ferroelectric behavior: with lower frequencies the magnitude of peak increases and its position shifts to lower temperature indicating a gradual freezing out $[20,21,23]$. Figure 9 displays the temperature-dependent real part of the dielectric function at selected frequencies along the $a^{*}$ direction where dimer and anion layers alternate. Notably, the out-of-plane response is one to three orders of magnitude weaker in strength, which correlates with the anisotropy of dc conductivity. Extensive study on different single crystals from different syntheses have revealed strong sample dependence of the dc transport and the dielectric response parameters [21]. Dielectric peak becomes smaller for larger disorder and the difference becomes more pronounced upon reducing temperatures. Similar effects due to disorder have been found previously in the dielectric response of the charge-density wave and ferroelectric charge ordered states in quasi-one-dimensional inorganic and organic compounds $[22,54,55]$. Disorder also affects the width of dielectric spectrum the broadening of which decreases implying a lower degree of cooperativity, the effect typically occurring 
in glassy systems [56]. Cooperativity appears well established within the dimer planes, while it develops gradually upon decreasing temperature along the out-of-plane direction [21,23]. In addition, for $\kappa-\mathrm{CuCN}$, sample-dependent dielectric and lattice anomalies were also detected in microwave [57] and thermal expansion measurements [58], respectively.

Dielectric response observed in $\kappa-\mathrm{CuCN}$ and $\kappa-\mathrm{AgCN}$ thus bears typical fingerprints of cooperative motion and glassy freezing in relaxor ferroelectrics [59]. Relaxor phenomenology assumes the existence of polar domains of low-symmetry and anticipates basically two mechanisms of dielectric response. It can be either due to the thermally activated reorientation of dipole moments within the domain, or to the motion of the interphase boundaries of the polar regions.

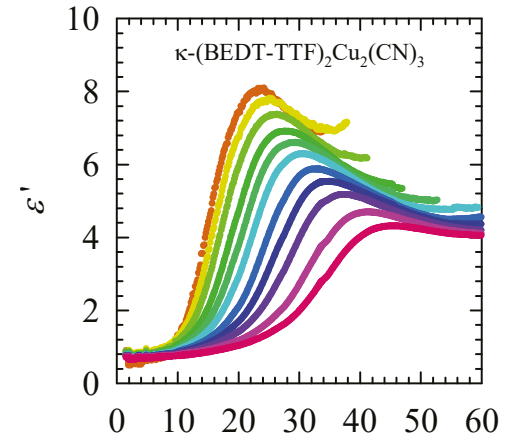

Temperature (K)

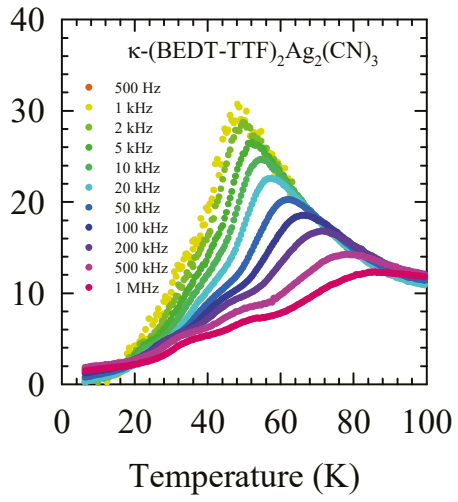

Temperature $(\mathrm{K})$

Figure 9. Real part of the dielectric function $\varepsilon^{\prime}$ as a function of temperature with the ac electric field applied E $\| a^{*}$ for single crystals of: $\kappa-\mathrm{CuCN}$ (Left); and $\kappa-\operatorname{AgCN}$ (Right).

To examine if dipole moments emerge upon lowering the temperature in $\kappa-\mathrm{CuCN}$ and $\kappa-\mathrm{AgCN}$, vibrational spectroscopy was employed to probe the most charge-sensitive intramolecular vibrational mode $v_{27}$, which involves the anti-symmetric ring $C=C$ stretching of the BEDT-TTF molecule $[23,25]$. In Figures 10 and 11 , the $v_{27}$ is shown for various temperatures in $\kappa-\mathrm{CuCN}$ and $\kappa-\mathrm{AgCN}$, respectively. In contrast to $\kappa-\mathrm{Cl}$, the side band at $1473 \mathrm{~cm}^{-1}$ is absent in different lattice symmetry; the satellite slightly below $1480 \mathrm{~cm}^{-1}$ is similar for all compounds discussed here. At $300 \mathrm{~K}$ the $v_{27}$ shows as a very strong peak centered at $1460 \mathrm{~cm}^{-1}$, as expected for half a hole per BEDT-TTF molecule. The mode is rather broad indicating the presence of fast charge fluctuations. Upon cooling no splitting of the mode occurs down to $10 \mathrm{~K}$, providing clear evidence that no sizeable static charge redistribution takes place in both $\kappa-\mathrm{CuCN}$ and $\kappa-\mathrm{AgCN}$. However, there is a slight difference. In $\kappa-\mathrm{CuCN}$, only one Fano mode was sufficient to describe the $v_{27}$ allowing only to conclude that there is no static charge imbalance larger than $2 \delta_{\rho} \approx \pm 0.01 e$ (Figure 10 ). For $\kappa-\mathrm{AgCN}$, two Fano functions were required to fit the spectra, revealing the two maxima which indicate that two unequal crystallographic sites exist in the unit cell of $\kappa-\mathrm{AgCN}$ (Figure 11) [23]. With a linear shift of $140 \mathrm{~cm}^{-1}$ per unit charge, the charge imbalance $2 \delta_{\rho}$ is calculated by $2 \delta_{\rho}=\delta v_{27} /\left(140 \mathrm{~cm}^{-1} / e\right)$, where $\delta v_{27}$ is the difference in frequency positions between two maxima associated with two non-equal molecular or dimer sites. The two maxima are separated by $6 \mathrm{~cm}^{-1}$ which would correspond to an average charge imbalance of $2 \delta_{\rho} \approx \pm 0.05 e$.

Given that vibrational spectroscopy detects only a tiny charge imbalance and fast rate of oscillations, such dipoles cannot be invoked to explain the dielectric response at low frequencies. These fluctuating dipoles may show up in the microwave [57] and terahertz response [60]; however, the latter has been shown to involve coupled anion-dimer vibrations instead [24]. Alternatively, the collective motion of interphase boundaries of polar regions has to be considered as the pertinent mechanism in these systems. Therefore, the clue to understand relaxor properties of dielectric response lies in identifying the randomness and domain structure in $\kappa-\mathrm{CuCN}$ and $\kappa-\mathrm{AgCN}$. The structure of 
these salts is commonly solved within $P 2_{1} / c$ symmetry implying that all four molecules and two dimers within the unit cell are equivalent $[4,6,61]$. This means that any charge disproportionation between molecular and/or dimer sites is excluded.

However, randomness is revealed by X-ray diffraction data already at $300 \mathrm{~K}$ which show that, in addition to ordered $\mathrm{CN}$ groups (chain $\mathrm{CN}$ ) between $\mathrm{Cu} / \mathrm{Ag}$ atoms in the chains along the $b$-axis, there are $\mathrm{CN}$ groups (bridging $\mathrm{CN}$ ) located at the inversion centers. These bridging $\mathrm{CN}$ groups connect $\mathrm{Cu} / \mathrm{Ag}$ atoms along the $c$-axis so that the anion network is formed in two-dimensions (Figure 12). The triangular coordination of $\mathrm{Cu}$ and $\mathrm{Ag}$ implies frustration since each $\mathrm{Cu} / \mathrm{Ag}$ atom can be linked either to two $\mathrm{N}$ and one $\mathrm{C}$ atom, or to one $\mathrm{N}$ atom and two $\mathrm{C}$ atoms introducing intrinsic disorder. This disorder is extended via hydrogen bonds connecting $\mathrm{CN}$ and ethylene groups onto the molecular dimers where it alters the charge distribution, and thus can cause anti-phase boundaries separating frustration-limited domains [21]. The perturbation of BEDT-TTF layers is expected to be stronger in $\kappa-\mathrm{CuCN}$, than in $\kappa-\mathrm{AgCN}$, since in the former all contacts between bridging $\mathrm{CN}$ and the terminal ethylene groups of the BEDT-TTF molecule are shorter than the sum of van der Waals radii [23]. Note that the disorder level in $\kappa-\mathrm{CuCN}$ and $\kappa-\mathrm{AgCN}$ represents an intrinsic property of these charge-transfer salts.

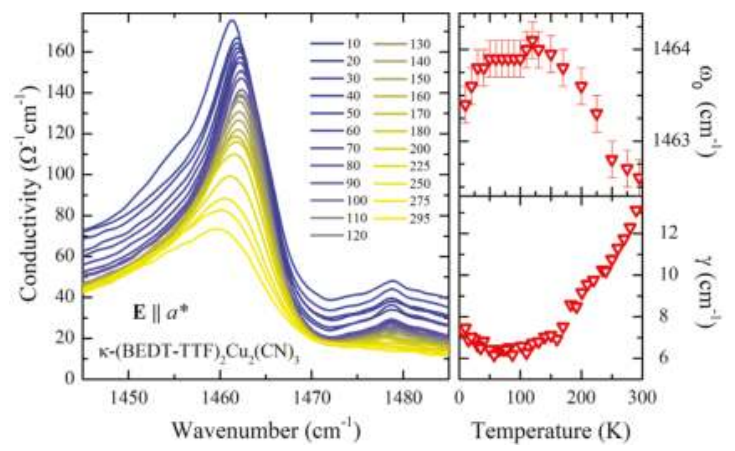

Figure 10. (Left) Temperature evolution of the intramolecular vibration $v_{27}$ of $\kappa$-CuCN. (Right) Temperature dependence of the resonance frequency and damping obtained by Fano function fit of the mode.

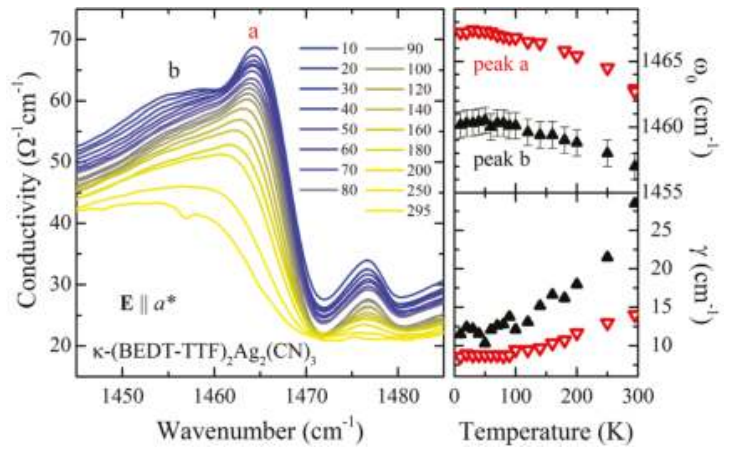

Figure 11. (Left) Temperature evolution of the intramolecular vibration $v_{27}$ of $\kappa$-AgCN. The two Fano functions required to fit the spectra indicate two crystallographically distinct sites. (Right) Temperature dependence of the resonance frequency and damping obtained by the fit using two Fano functions. 

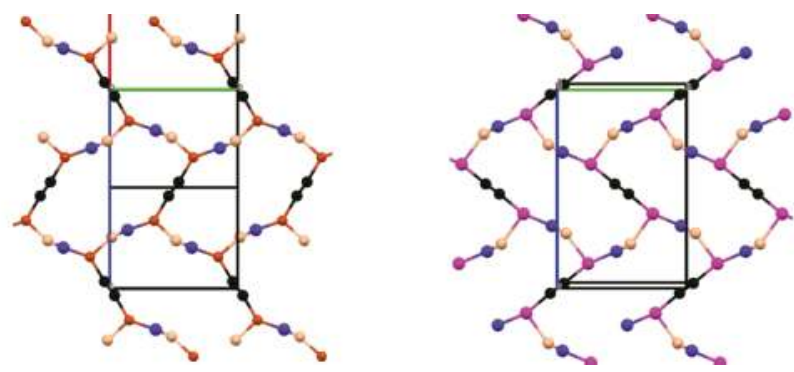

Figure 12. View of the anion network of $\kappa-\mathrm{CuCN}($ Left) and $\kappa-\mathrm{AgCN}$ (Right) in the $b c$ plane projected along the $a^{*}$-axis. Cooper and silver are colored in red and violet, respectively. Carbon and nitrogen of ordered $\mathrm{CN}^{-}$groups are colored in blue and orange, while they are colored in black for $\mathrm{CN}^{-}$groups located at inversion centers. The unit cell is marked as a rectangle.

To get an insight as how such a disorder affects the electronic ground state, ab initio density functional theory (DFT) calculations have been carried out on $\kappa-\mathrm{CuCN}$ and $\kappa-\mathrm{AgCN}[23,24]$. Since two $\mathrm{CN}$ bridging groups in the unit cell occupy one of the two equally probable orientations, four different crystallographic configurations were constructed, based on experimental X-ray data, for $\kappa-\mathrm{CuCN}$ and $\kappa-\mathrm{AgCN}$. In each configuration, the entire system, with atomic positions and unit cell parameters, was allowed to relax resulting in electronic states with reduced symmetry as compared to $P 2_{1} / \mathrm{c}$ and quasi-degenerate in energy; all states lie only $5-25 \mathrm{meV}$ above the relaxed state with minimum energy. Reduced symmetry implies that non-equivalent crystallographic sites (BEDT-TTF molecules and/or dimers) exist in the unit cell. In $\kappa-\mathrm{CuCN}$, the space group of all four states was reduced to $P 1$, while, in $\kappa-\mathrm{AgCN}$, the energy states were associated with the configurations of higher symmetry $P 2_{1}$ and $P_{c}$. These results indicate a higher level of disorder in $\kappa-\mathrm{CuCN}$ than in $\kappa-\mathrm{AgCN}$, which explains why it was possible to resolve two Fano modes in the $v_{27}$ mode in the $\kappa$-AgCN system.

Importantly, the quasi-degenerate electronic ground state implies a random domain structure in the real space of $\kappa-\mathrm{CuCN}$ and $\kappa-\mathrm{AgCN}$ at all temperatures. It thus supports the consideration of relaxor dielectric response explained above: low-energy excitations are caused by charged domain walls rather than by static or fluctuating electric dipoles. Upon cooling, screening gets gradually weaker due to the reduced number of charge carriers. This allows domains to be more "visible", the number of domain walls increases, and concomitantly the motion of domain walls become increasingly correlated. The detailed description of how and why the dielectric response evolve in temperature can be found in Refs. [21,23]. In closing, we note that the dielectric response scenario presented in this section, was recently backed up by the theoretical approach developed to explain anomalous dielectric response in strongly correlated molecular solids [62]. Most importantly, in-depth X-ray diffraction measurements of $\kappa-\mathrm{CuCN}$ in the wide temperature range and refinements, complemented by DFT calculations, have demonstrated that two nonequivalent dimers exist in the unit cell due to the reduced symmetry of the whole crystal [63].

\section{Van de Waals Interactions in $\kappa$-(BEDT-TTF $)_{2} X ; X=\mathrm{Cu}\left[\mathrm{N}(\mathrm{CN})_{2}\right] \mathrm{Cl}, \mathrm{Cu}_{2}(\mathrm{CN})_{3}$ and $\mathrm{Ag}_{2}(\mathrm{CN})_{3}$}

While there exists a general consent in the community of organic crystals on the importance of $\mathrm{vdW}$ interaction in their formation, its importance has never been clearly evidenced and quantified in the organic materials $\kappa-(\mathrm{BEDT}-\mathrm{TTF})_{2} \mathrm{X}$ with $\mathrm{X}=\mathrm{Cu}\left[\mathrm{N}(\mathrm{CN})_{2}\right] \mathrm{Cl}, \mathrm{Cu}_{2}(\mathrm{CN})_{3}$ and $\mathrm{Ag}_{2}(\mathrm{CN})_{3}$. These three kappa-BEDT-TTF materials, as well as other BEDT-TTF compounds are not simple molecular crystals, rather they should be classified as complex chemically bound charge transfer or cation-anion bulk solids. The previous DFT studies on different organic charge-transfer salts have neither included nor discussed the van der Waals (vdW) interactions at all [64-70]. The only exception is found in our work $[23,24,39]$. 
In the present DFT calculations, the experimental structure obtained from X-ray diffraction measurements at $100 \mathrm{~K}, 150 \mathrm{~K}$ and $100 \mathrm{~K}$ was used for the atomic coordinates of $\kappa-\mathrm{CuCN}, \kappa-\mathrm{AgCN}$ and $\kappa-\mathrm{Cl}$, respectively $[23,24,47]$. Two types of DFT functionals have been used: the standard semilocal PBE functional and nonlocal vdW-DF functional. The structure was relaxed to allow for the change of volume and shape of the unit cell together with the atomic positions. The parameters of the resulting relaxed state structures with minimum energy are displayed in Table $1(\kappa-\mathrm{CuCN})$, Table $2(\kappa-\mathrm{AgCN})$ and Table $3(\kappa-\mathrm{Cl})$.

Table 1. Unit cell parameters of $\kappa-\mathrm{CuCN}$ obtained from X-ray diffraction measurements at $100 \mathrm{~K}$ (left column), ab initio calculations based solely on PBE functional (central column) and on vdW-DF functional (right column). Relative deviations from experimental values are given in parentheses.

\begin{tabular}{cccc}
\hline Unit Cell Parameters & Exp & Calc:PBE & Calc:vdW-DF \\
\hline$a$ & $15.9644 \AA$ & $16.9706 \AA(+6.3 \%)$ & $16.1809 \AA(+1.4 \%)$ \\
$b$ & $8.5618 \AA$ & $8.75745 \AA(+2.3 \%)$ & $8.62586 \AA(+0.8 \%)$ \\
$c$ & $13.2662 \AA$ & $13.6497 \AA(+2.9 \%)$ & $13.3523 \AA(+0.7 \%)$ \\
$\alpha$ & $90.000^{\circ}$ & $89.9968^{\circ}$ & $90.0037^{\circ}$ \\
$\beta$ & $114.067^{\circ}$ & $116.409^{\circ}$ & $114.088^{\circ}$ \\
$\gamma$ & $90.000^{\circ}$ & $90.0037^{\circ}$ & $89.9994^{\circ}$ \\
$V$ & $1655.65 \AA^{3}$ & $1816.90 \AA^{3}(+9.7 \%)$ & $1701.35 \AA^{3}(+2.8 \%)$ \\
\hline
\end{tabular}

Table 2. Unit cell parameters of $\kappa$-AgCN obtained from X-ray diffraction measurements at $150 \mathrm{~K}$ (left column), ab initio calculations based solely on PBE functional (central column) and on vdW-DF functional (right column). Relative deviations from experimental values are given in parentheses.

\begin{tabular}{cccc}
\hline Unit Cell Parameters & Exp & Calc:PBE & Calc:vdW-DF \\
\hline$a$ & $14.969900 \AA$ & $15.6693 \AA(+4.7 \%)$ & $15.1161 \AA(+0.98 \%)$ \\
$b$ & $8.656500 \AA$ & $9.04653 \AA(+4.5 \%)$ & $8.72513 \AA(+0.79 \%)$ \\
$c$ & $13.216900 \AA$ & $13.8238 \AA(+4.6 \%)$ & $13.3487 \AA(+1.0 \%)$ \\
$\alpha$ & $90.000000^{\circ}$ & $90.0000^{\circ}$ & $90.0000^{\circ}$ \\
$\beta$ & $91.389000^{\circ}$ & $94.6823^{\circ}$ & $91.3952^{\circ}$ \\
$\gamma$ & $90.000000^{\circ}$ & $90.0000^{\circ}$ & $90.0000^{\circ}$ \\
$V$ & $1712.23 \AA^{3}$ & $1953.03 \AA^{3}(+14.1 \%)$ & $1760.03 \AA^{3}(+2.8 \%)$ \\
\hline
\end{tabular}

Table 3. Unit cell parameters of $\kappa-\mathrm{Cl}$ obtained from X-ray diffraction measurements at $100 \mathrm{~K}$ (left column), ab initio calculations based solely on PBE functional (central column) and on vdW-DF functional (right column). Relative deviations from experimental values are given in parentheses.

\begin{tabular}{cccc}
\hline Unit Cell Parameters & Exp & Calc:PBE & Calc:vdW-DF \\
\hline$a$ & $12.885200 \AA$ & $12.9769 \AA(+0.7 \%)$ & $12.8334 \AA(-0.4 \%)$ \\
$b$ & $29.575899 \AA$ & $29.6637 \AA(+0.3 \%)$ & $29.5323 \AA(-0.2 \%)$ \\
$c$ & $8.416100 \AA$ & $8.45028 \AA(+0.4 \%)$ & $8.23316 \AA(-2.2 \%)$ \\
$\alpha$ & $90.000000^{\circ}$ & $90.0000^{\circ}$ & $90.0000^{\circ}$ \\
$\beta$ & $90.000000^{\circ}$ & $90.0000^{\circ}$ & $90.0000^{\circ}$ \\
$\gamma$ & $90.000000^{\circ}$ & $90.0000^{\circ}$ & $90.0000^{\circ}$ \\
$V$ & $3207.303 \AA^{3}$ & $3252.875 \AA^{3}(+1.4 \%)$ & $3120.366 \AA^{3}(-2.7 \%)$ \\
\hline
\end{tabular}

The inadequacy of DFT calculations without the vdW interactions is found in $\kappa-\mathrm{CuCN}$ and $\kappa-\mathrm{AgCN}$ for which the absence of vdW interaction produces deviations of the computed unit cell parameters from the experimental ones at the level of $10 \%$ and $14 \%$, respectively (Tables 1 and 2 ). Hence, only the DFT calculations with the non-local vdW-DF functional can clarify the impact of disorder on the electronic ground state (see Section 3). Intriguingly enough, the vdW forces are almost irrelevant in $\kappa-\mathrm{Cl}$ (Table 3) thereby indicating that the latter structure is dominantly determined by the one-dimensional chains of anion $\mathrm{Cu}\left[\mathrm{N}(\mathrm{CN})_{2}\right] \mathrm{Cl}$ chemically bonded to the organic dimers. 


\section{Materials and Methods}

All measurements were performed on high-quality single crystals grown by J. A. Schlueter, Argonne $\left(\kappa-(\mathrm{BEDT}-\mathrm{TTF})_{2} \mathrm{Cu}\left[\mathrm{N}(\mathrm{CN})_{2}\right] \mathrm{Cl}\right) ; \mathrm{K}$. Miyagawa and K. Kanoda, Tokyo University $\left(\kappa-(\mathrm{BEDT}-\mathrm{TTF})_{2} \mathrm{Cu}_{2}(\mathrm{CN})_{3}\right)$; and T. Hiramatsu, Y. Yoshida and G. Saito, Meijo University $\left(\kappa-(B E D T-T T F){ }_{2} \mathrm{Ag}_{2}(\mathrm{CN})_{3}\right)$. Sample $\mathrm{S} 1$ of $\kappa-\mathrm{Cl}$ was of cube-like shape with an area of $0.4 \times 0.5 \mathrm{~mm}^{2}$, and a thickness of $0.6 \mathrm{~mm}$. Sample S2 of $\kappa-\mathrm{Cl}$ was plate-like with an ac-plane area of $0.8 \times 0.9 \mathrm{~mm}^{2}$. and a thickness of $0.4 \mathrm{~mm}$. Contacts for $\mathrm{dc}$ and dielectric measurements along the out-of-plane direction were made by applying conductive carbon paint directly to the surface of the sample [21,23]. DC resistivity was measured by a standard four contact technique. Temperature-dependent dielectric measurements were performed from room temperature down to $4.2 \mathrm{~K}$ (crystals S1 and S2 of $\kappa-\mathrm{Cl}$ ) and $1.8 \mathrm{~K}$ (crystal of $\kappa-\mathrm{CuCN}$ ) in the frequency range $40 \mathrm{~Hz}-10 \mathrm{MHz}$ using Agilent $4294 \mathrm{~A}$ and Keysight E4980AL-102 precision impedance analyzers. Care was taken to subtract the background capacitance of the set-up and sample holder, as well as to exclude any possible extrinsic effects due to sample preparation. Magnetic-field-dependent dielectric measurements of sample S2 of $\kappa$-Cl were performed in the magnetic field up to $5 \mathrm{~T}$ aligned along the out-of-plane crystallographic axis. Vibrational spectroscopy measurements were made on the thin side of the crystals by an IR microscope attached to a Bruker Vertex 80v Fourier-transform spectrometer with $1 \mathrm{~cm}^{-1}$ resolution. Magnetic susceptibility of sample $\mathrm{S} 2$ of $\kappa-\mathrm{Cl}$ was performed by using a superconducting quantum interference device (SQUID) magnetometer and the data were corrected for the contribution of sample holder.

First principles calculations of the electronic structure was carried out by using the self-consistently implemented nonlocal van der Waals density functional (vdW-DF) [71-73] for correlation with optB88 for exchange [74]. The expansion in the plane waves was done with a cutoff energy of $700 \mathrm{eV}$. The Brillouin zone was sampled by $1 \times 2 \times 2$ Monkhorst-Pack choice of $k$-points [75]. For the atomic coordinates, the experimental structure obtained by $\mathrm{X}$-ray diffraction measurements was used. The structure was relaxed allowing for change of the volume and the shape of the unit cell together with atomic positions within, until the forces on atoms dropped below $1 \mathrm{meV} / \AA$.

To ensure the chemical composition and exclude presence of any bromine in sample $\mathrm{S} 2$ of $\kappa-\mathrm{Cl}$, we examined the crystal symmetry and composition [76]. The X-ray data were collected at $300 \mathrm{~K}$ with graphite monochromated Mo-K $\alpha$ radiation by $\omega$-scans on an Oxford Diffraction KM4 XCALIBUR2 CCD diffractometer. Data acquisition and reduction was performed using the CrysAlisPro software package. The structure was solved by direct methods using SHELXS and the refinement was performed using SHELXL [77]. Energy Dispersive X-ray Spectra (EDXS) of sample S2 of $\kappa$-Cl, were acquired using Bruker detector within Tescan Vega III Easyprobe Scanning Electron Microscope (SEM).

Figure 13 shows the result of structural refinements of X-ray data within models containing either $\mathrm{Cl}$ (Figure 13, Left) or Br (Figure 13, Right). All atoms are drawn with $50 \%$ probability ellipsoids of thermal displacement. Importantly, the size of ellipsoids is convincingly small for $\mathrm{Cl}$, while their size is unrealistically inflated if the presence of $\mathrm{Br}$ is assumed. In addition, the distance of $2.22 \AA$ between $\mathrm{Cu}$ and neighboring $\mathrm{Cl}$ atom (represented by lines) is close to $2.26 \AA$ which is typical for $\mathrm{Cu}-\mathrm{Cl}$ bond, thus confirming the presence of $\mathrm{Cl}$ in the structure. Finally, the presence of $\mathrm{Br}$ larger than a few percent can be rejected since the corresponding bond in the case of $\mathrm{Br}$ would be longer and amount to $2.42 \AA$. Therefore, overall results of the structural refinements strongly indicate that the measured single crystal is $\kappa-\mathrm{Cl}$ and not $\kappa$ - $\mathrm{Br}$.

Figure 14 shows EDXS recorder at $300 \mathrm{~K}$. Characteristic lines for C, N, S and Cu were observed with intensities that correspond to the $\kappa-\mathrm{Cl}$ material. On the other hand, no significant intensity of $\mathrm{Br}$ lines, expected if trace amount of $\mathrm{Br}$ is present, were observed. 

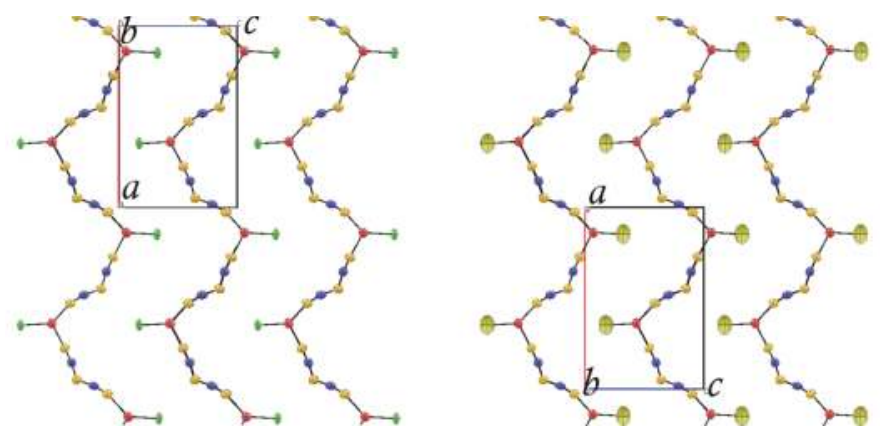

Figure 13. Perspective view of zigzag polymeric anion chains as obtained by structural refinements of X-ray data assuming either: $\kappa-\mathrm{Cl}$ (Left); or $\kappa$ - $\mathrm{Br}$ (Right). View shows the ac plane. Copper, carbon and nitrogen are shown in red, blue and orange, respectively. Chlorine and bromine ligands on each copper atom are colored in dark and light green, respectively. All atoms are drawn with $50 \%$ probability ellipsoids of anisotropic thermal displacement parameters. Unit cell is marked as a rectangle.

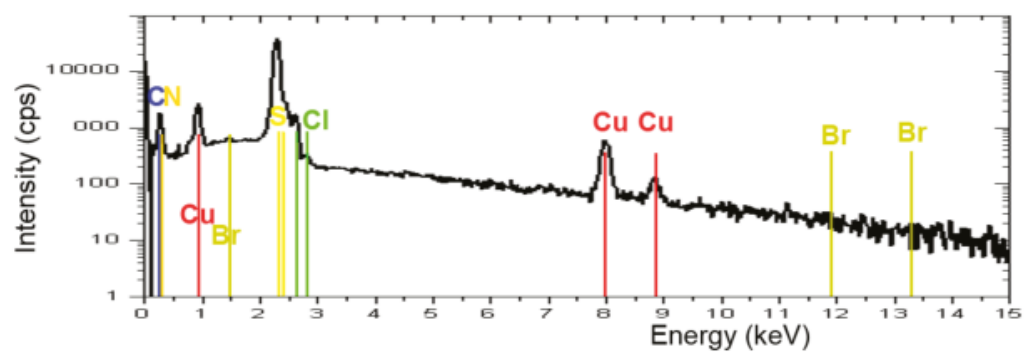

Figure 14. Energy Dispersive $X$-ray Spectra (EDXS) recorded at $300 \mathrm{~K}$. Only characteristic lines for $\mathrm{C}, \mathrm{N}, \mathrm{S}$ and $\mathrm{Cu}$, and in particular for $\mathrm{ClK} \alpha$ at $2.59 \mathrm{keV}$ and for $\mathrm{ClK} \beta$ at $2.83 \mathrm{keV}$ were observed with intensities that correspond to the chemical formula of $\kappa-\mathrm{Cl}$. On the other hand, no significant intensity for lines $\operatorname{BrK} \alpha 1$ and $\operatorname{BrK} \alpha 2$ at $11.92 \mathrm{keV}$, for $\operatorname{BrK} \beta$ at $13.29 \mathrm{keV}$, and for $\operatorname{BrL} \alpha$ at $1.49 \mathrm{keV}$ were observed, which would be expected in the presence of $\mathrm{Br}$.

\section{Conclusions}

Mott insulators $\kappa-\mathrm{CuCN}$ and $\kappa-\mathrm{AgCN}$ with the quantum spin liquid ground state exhibit high geometrical frustration in the organic dimers and in the non-conducting anions. Frustration in the anions arises as a combined effect due to the triangular coordination of copper/silver atoms and the bridging cyanide groups sitting in the inversion centers. The resulting disorder is mapped via anion-cation coupling onto the organic dimers. It gives rise to charge redistribution and a ground state which consists of several quasi-degenerate electronic states with reduced symmetry.

Remarkably, QSL is replaced by the antiferromagnetic ground state in $\kappa-\mathrm{Cl}$ where disorder due to inherent randomness in the anions and related symmetry reduction are absent. This result strongly suggests that the inherent randomness may be of a crucial importance for the prevalence of QSL over the antiferromagnetic ordering. In addition, van der Waals forces are found to contribute to inter-dimer interactions and the ground state structure in $\kappa-\mathrm{CuCN}$ and $\kappa-\mathrm{AgCN}$, whereas their role appears insignificant in $\kappa-\mathrm{Cl}$ where chemical bonding dominantly determines the inter-dimer interactions besides the cation-anion coupling.

Anomalous dielectric peak shows up as an ubiquitous property independent on the nature of the ground state in the spin sector. It is now well established that, in $\kappa-\mathrm{CuCN}$ and $\kappa-\mathrm{AgCN}$, this arises due to the cooperative motion of charged domain walls. On the other hand, in $\kappa-\mathrm{Cl}$, some mysteries remain 
unresolved. The charged domain wall motion might also be in action in $\mathrm{k}-\mathrm{Cl}$ if the tendency to phase segregation is invoked due to canted spins in the antiferromagnetic state. Further experimental efforts are vital in search for structural inversion symmetry breaking in order to clarify the ferroelectricity in $\kappa-\mathrm{Cl}$. The possibility that ferroelectricity is proximate to the magnetic and superconducting phases is most exciting and deserves more attention in future studies. Experiments such as X-ray diffraction and dielectric spectroscopy under pressure, on irradiated samples and in magnetic field are envisaged as tools to elucidate respective roles of correlations, randomness and charge-spin coupling in the phase diagram.

Author Contributions: DC and dielectric measurements and the data analysis were performed by Marko Pinteric, David Rivas Gongora, and Zeliko Rapljenovic, with help of Tomislav Ivek and Matija Culo. Dielectric measurements in magnetic field were designed by Tomislav Ivek and Matija Culo. Structural data analysis was performed by Ognjen Milat. DFT calculations were performed by Predrag Lazic. Vibrational experiments and data analysis were performed by Miriam Sanz Alonso, Andrej Pustogow, and Weiwu Li. Susceptibility experiments and data analysis were performed by Guilherme Gorgen Lesseux. The interpretation of the data and the text of the manuscript were drafted by Silvia Tomic and Tomislav Ivek. All authors contributed to the discussion and the final manuscript.

Acknowledgments: X-ray diffraction measurements by V. Stilinović and SEM measurements by J. Macan are gratefully acknowledged. We would like to thank V. Dobrosavljević, E. Canadell, R. T. Clay, P. Foury, V. Ilakovac, S. Mazumdar and J-P. Pouget for many enlightening discussions. This work has been supported by the Croatian Science Foundation project IP-2013-11-1011 and by the Deutsche Forschungsgemeinschaft (DFG).

Conflicts of Interest: The authors declare no conflict of interest.

\section{References}

1. Kanoda, K.; Kato, R. Mott Physics in Organic Conductors with Triangular Lattices. Annu. Rev. Condens. Matter Phys. 2011, 2, 167. [CrossRef]

2. Powell, B.J.; McKenzie, R.H. Quantum frustration in organic Mott insulators: From spin liquids to unconventional superconductors. Rep. Prog. Phys. 2011, 74, 056501. [CrossRef]

3. Kandpal, H.C.; Opahle, I.; Zhang, Y.-Z.; Jeschke, H.O.; Valentí, R. Revision of Model Parameters for $\kappa$-Type Charge Transfer Salts: An Ab Initio Study. Phys. Rev. Lett. 2009, 103, 067004. [CrossRef] [PubMed]

4. Jeschke, H.O.; de Souza, M.; Valentí, R.; Manna, R.S.; Lang, M.; Schlueter, J.A. Temperature dependence of structural and electronic properties of the spin-liquid candidate $\kappa-(\mathrm{BEDT}-\mathrm{TTF})_{2} \mathrm{Cu}_{2}(\mathrm{CN})_{3}$. Phys. Rev. B 2012, 85, 035125. [CrossRef]

5. Koretsune, T.; Hotta, C. Evaluating model parameters of the $\kappa$ and $\beta^{\prime}$-type Mott insulating organic solids. Phys. Rev. B 2014, 89, 045102. [CrossRef]

6. Hiramatsu, T.; Yoshida, Y.; Saito, G.; Otsuka, A.; Yamochi, H.; Maesato, M.; Shimizu, Y.; Ito, H.; Nakamura, Y.; Kishida, H.; et al. Design and preparation of a new quantum spin liquid candidate, $\kappa-(\mathrm{ET})_{2} \mathrm{Ag}_{2}(\mathrm{CN})_{3}$, having a nearby superconductivity. Bull. Chem. Soc. Jpn. 2017, 90, 1073-1082. [CrossRef]

7. Miyagawa, K.; Kawamoto, A.; Nakazawa, Y.; Kanoda, K. Antiferromagnetic Ordering and Spin Structure in the Organic Conductor, $\kappa$-(BEDT-TTF $)_{2} \mathrm{Cu}\left[\mathrm{N}(\mathrm{CN})_{2}\right.$ ]Cl. Phys. Rev. Lett. 1995, 75, 1174. [CrossRef] [PubMed]

8. Pinterić, M.; Miljak, M.; Biškup, N.; Milat, O.; Aviani, I.; Tomić, S.; Schweitzer, D.; Strunz, W.; Heinen, I. Magnetic anisotropy and low frequency dielectric response of weak ferromagnetic phase in $\kappa-(\mathrm{BEDT}-\mathrm{TTF})_{2} \mathrm{Cu}\left[\mathrm{N}(\mathrm{CN})_{2}\right]$ Cl. Eur. Phys. J. B 1999, 11, 217.

9. Smith, D.F.; De Soto, S.M.; Slichter, C.P. Dzialoshinskii-Moriya interaction in the organic superconductor $\kappa-(\mathrm{BEDT}-\mathrm{TTF})_{2} \mathrm{Cu}\left[\mathrm{N}(\mathrm{CN})_{2}\right.$ ]Cl. Phys. Rev. B 2003, 68, 024512. [CrossRef]

10. Shimizu, Y.; Miyagawa, K.; Kanoda, K.; Maesato, M.; Saito, G. Spin liquid state in an organic Mott insulator with a triangular lattice. Phys. Rev. Lett. 2003, 91, 107001. [CrossRef] [PubMed]

11. Shimizu, Y.; Hiramatsu, T.; Maesato, M.; Otsuka, A.; Yamochi, H.; Ono, A.; Itoh, M.; Yoshida, M.; Takigawa, M.; Yoshida, Y.; et al. Pressure-tuned exchange coupling of a quantum spin liquid in the molecular triangular lattice $\kappa$-(BEDT-TTF $)_{2} \mathrm{Ag}_{2}(\mathrm{CN})_{3}$. Phys. Rev. Lett. 2016, 117, 107203. [CrossRef] [PubMed]

12. Zhou, Y.; Kanoda, K.; Ng, T.-K. Quantum spin liquid states. Rev. Mod. Phys. 2017, 89, 025003. [CrossRef]

13. Balents, L. Spin liquids in frustrated magnets. Nature 2010, 464, 199. [CrossRef] [PubMed]

14. Savary, L.; Balents, L. Quantum spin liquids: A review. Rep. Prog. Phys. 2017, 80, 016502. [CrossRef] [PubMed] 
15. Anderson, W.P. Resonating valence bonds: A new kind of insulator? Mat. Res. Bull. 1973, 8, 153-160. [CrossRef]

16. Klanjšek, M.; Zorko, A.; Žitko, R.; Mravlje, J.; Jagličić, Z.; Kumar Biswas, P.; Prelovšek, P.; Mihailovic, D.; Arčon, D. A high-temperature quantum spin liquid with polaron spins. Nat. Phys. 2017, 13, 1130-1134. [CrossRef]

17. Shimozawa, M.; Hashimoto, K.; Ueda, A.; Suzuki, Y.; Sugii, K.; Yamada, S.; Imai, Y.; Kobayashi, R.; Itoh, K.; Iguchi, S.; et al. Quantum-disordered state of magnetic and electric dipoles in an organic Mott system. Nat. Commun. 2017, 8, 1821. [CrossRef] [PubMed]

18. Lunkenheimer, P.; Müller, J.; Krohns, S.; Schrettle, F.; Loidl, A.; Hartmann, B.; Rommel, R.; de Souza, M.; Hotta, C.; Schlueter, J.A.; et al. Multiferroicity in an organic charge-transfer salt that is suggestive of electric-dipole-driven magnetism. Nat. Mater. 2012, 11, 755. [CrossRef] [PubMed]

19. Tomić, S.; Pinterić, M.; Ivek, T.; Sedlmeier, K.; Beyer, R.; Wu, D.; Schlueter, J.A.; Schweitzer, D.; Dressel, M. Magnetic ordering and charge dynamics in $\kappa-(\mathrm{BEDT}-\mathrm{TTF})_{2} \mathrm{Cu}\left[\mathrm{N}(\mathrm{CN})_{2}\right] \mathrm{Cl}$. J. Phys. Condens. Matter 2013, 25, 436004. [CrossRef] [PubMed]

20. Abdel-Jawad, M.; Terasaki, I.; Sasaki, T.; Yoneyama, N.; Kobayashi, N.; Uesu, Y.; Hotta, C. Anomalous dielectric response in the dimer Mott insulator $\kappa-(\mathrm{BEDT}-\mathrm{TTF})_{2} \mathrm{Cu}_{2}(\mathrm{CN})_{3}$. Phys. Rev. B 2010, 82, 125119. [CrossRef]

21. Pinterić, M.; Čulo, M.; Milat, O.; Basletić, M.; Korin-Hamzić, B.; Tafra, E.; Hamzić, A.; Ivek, T.; Peterseim, T.; Miyagawa, K.; et al. Anisotropic charge dynamics in the spin-Liquid candidate $\kappa-(B E D T-T T F)_{2} \mathrm{Cu}_{2}(\mathrm{CN})_{3}$. Phys. Rev. B 2014, 90, 195139. [CrossRef]

22. Tomić, S.; Dressel, M. Ferroelectricity in molecular solids: A review of electrodynamic properties. Rep. Prog. Phys. 2015, 78, 096501. [CrossRef] [PubMed]

23. Pinterić, M.; Lazić, P.; Pustogow, A.; Ivek, T.; Kuveždić, M.; Milat, O.; Gumhalter, B.; Basletić, M.; Čulo, M.; Korin-Hamzić, B.; et al. Anions effects on the electronic structure and electrodynamic properties of the Mott insulator $\kappa$-(BEDT-TTF) ${ }_{2} \mathrm{Ag}_{2}(\mathrm{CN})_{3}$. Phys. Rev. B 2016, 94, 161105(R). [CrossRef]

24. Dressel, M.; Lazić, P.; Pustogow, A.; Zhukova, E.; Gorshunov, B.; Schlueter, J.A.; Milat, O.; Gumhalter, B.; Tomić, $\mathrm{S}$. Lattice vibrations of the charge-transfer salt $\kappa-(\mathrm{BEDT}-\mathrm{TTF})_{2} \mathrm{Cu}_{2}(\mathrm{CN})_{3}$ : Comprehensive explanation of the electrodynamic response in a spin-liquid compound. Phys. Rev. B 2016, 93, 081201(R). [CrossRef]

25. Sedlmeier, K.; Elsässer, S.; Neubauer, D.; Beyer, R.; Wu, D.; Ivek, T.; Tomić, S.; Schlueter, J.A.; Dressel, M. Absence of charge order in the dimerized $\kappa$-phase BEDT-TTF salts. Phys. Rev. B 2012, 86, 245103. [CrossRef]

26. Hotta, C. Quantum electric dipoles in spin-liqiod dimer Mott insulator $\kappa-(\mathrm{ET})_{2} \mathrm{Cu}_{2}(\mathrm{CN})_{3}$. Phys. Rev. B 2010, 82, 241104. [CrossRef]

27. Hotta, C. Theories on Frustrated Electrons in Two-Dimensional Organic Solids. Crystals 2012, $2,1155$. [CrossRef]

28. Naka, M.; Ishihara, S. Electronic ferroelectricity in a dimer mott insulator. J. Phys. Soc. Jpn. 2010, 79, 063707. [CrossRef]

29. Naka, M.; Ishihara, S. Collective charge excitation in a dimer Mott insulating system. J. Phys. Soc. Jpn. 2013, 82, 023701. [CrossRef]

30. Naka, M.; Ishihara, S. Quantum melting of magnetic order in an organic dimer-Mott insulating system. Phys. Rev. B 2016, 93, 195114. [CrossRef]

31. Li, H.; Clay, R.T.; Mazumdar, S. The paired-electron crystal in the two-dimensional frustrated quarter-filled band. J. Phys. Condens. Matter 2010, 22, 272201. [CrossRef] [PubMed]

32. Dayal, S.; Clay, R.T.; Li, H.; Mazumdar, S. Paired electron crystal: Order from frustration in the quarter-filled band. Phys. Rev. B 2011, 83, 245106. [CrossRef]

33. Gomi, H.; Ikenaga, M.; Hiragi, Y.; Segawa, D.; Takahashi, A.; Inagaki, T.J.; Aihara, M. Ferroelectric states induced by dimer lattice disorder in dimer Mott insulators. Phys. Rev. B 2013, 87, 195126. [CrossRef]

34. Gomi, H.; Inagaki, T.J.; Takahashi, A. Ferroelectric charge order enhanced by magnetic frustration in dimer Mott insulators. Phys. Rev. B 2016, 93, 035105. [CrossRef]

35. Huse, D.A.; Elser, V. Simple Variational Wave Functions for Two-Dimensional Heisenberg Spin-1/2 Antiferromagnets. Phys. Rev. Lett. 1988, 60, 2351-2354. [CrossRef] [PubMed]

36. Bernu, B.; Lhuillier, C.; Pierre, L. Signature of Néel order in exact spectra of quantum antiferromagnets on finite lattices. Phys. Rev. Lett. 1992, 69, 2590-2593. [CrossRef] [PubMed] 
37. Capriotti, L.; Trumper, A.E.; Sorella, S. Long-range Néel order in the triangular Heisenberg model. Phys. Rev. Lett. 1999, 82, 3899-3902. [CrossRef]

38. Furukawa, T.; Miyagawa, K.; Itou, T.; Ito, M.; Taniguchi, H.; Saito, M.; Iguchi, S.; Sasaki, T.; Kanoda, K. Quantum spin liquid emerging from antiferromagnetic order by introducing disorder. Phys. Rev. Lett. 2015, 115, 077001. [CrossRef] [PubMed]

39. Lazić, P.; Pinterić, M.; Rivas Góngora, D.; Pustogow, A.; Treptow, K.; Ivek, T.; Milat, O.; Gumhalter, B.; Došlić, N.; Dressel, M.; et al. Importance of van der Waals interactions and cation-anion coupling in an organic quantum spin liquid. arXiv 2017, arXiv:1710.01942.

40. Kornelsen, K.; Eldridge, J.E.; Wang, H.H.; Charlier, H.A.; Williams, J.M. Infrared study of the metal-insulator transition in the organic conductor $\kappa-(\mathrm{BEDT}-\mathrm{TTF})_{2} \mathrm{Cu}\left[\mathrm{N}(\mathrm{CN})_{2}\right] \mathrm{Cl}$. Solid State Commun. 1992, 4, 343-349. [CrossRef]

41. Williams, J.M.; Kini, A.M.; Wang, H.H.; Carlson, K.D.; Geiser, U.; Montgomery, L.K.; Pyrka, G.J.; Watkins, D.M.; Kommers, J.M.; Boryschuk, S.J.; et al. From Semiconductor-Semiconductor Transition $(42 \mathrm{~K})$ to the Highest- $T_{\mathrm{C}}$ Organic Superconductor, $\kappa-(\mathrm{BEDT}-\mathrm{TTF})_{2} \mathrm{Cu}\left[\mathrm{N}(\mathrm{CN})_{2}\right] \mathrm{Cl}\left(T_{\mathrm{c}}=12.5 \mathrm{~K}\right)$. Inorg. Chem. 1990, 29, 3272-3274. [CrossRef]

42. Sushko, Y.V.; Andres, K. Superconducting Meissner effect under hydrostatic pressure in the ambient-pressure semiconductor $\kappa$-(BEDT-TTF $)_{2} \mathrm{Cu}\left[\mathrm{N}(\mathrm{CN})_{2}\right] \mathrm{Cl}$, where BEDT-TTF is bis(ethylenedithio)tetrathiafulvalene. Phys. Rev. B 1993, 47, 330-333. [CrossRef]

43. Lefebvre, S.; Wzietek, P.; Brown, S.; Bourbonnais, C.; Jérome, D.; Mézière, C.; Fourmigué, M.; Batail, P. Mott Transition, Antiferromagnetism, and Unconventional Superconductivity in Layered Organic Superconductors. Phys. Rev. Lett. 2000, 85, 5420-5423. [CrossRef] [PubMed]

44. Dressel, M.; Gruener, G.; Carlson, K.D.; Wang, H.H.; Williams, J.M. Studies of the Microwave Resistivity of $\kappa-(\mathrm{BEDT}-\mathrm{TTF})_{2} \mathrm{Cu}\left[\mathrm{N}(\mathrm{CN})_{2}\right] \mathrm{Cl}$. Synth. Metals 1995, 70, 927-928. [CrossRef]

45. Sushko, Y.V.; Ito, H.; Ishiguro, T.; Horiuchi, S.; Saito, G. Reentrant superconductivity in $\kappa-(\mathrm{BEDT}-\mathrm{TTF})_{2} \mathrm{Cu}\left[\mathrm{N}(\mathrm{CN})_{2}\right] \mathrm{Cl}$ and its pressure phase diagram. Solid State Commun. 1993, 87, 997-1000. [CrossRef]

46. Clay, R.T.; Mazumdar, S. From charge- and spin-ordering to superconductivity in the organic charge-transfer solids. arXiv 2018 arXiv:1802.01551.

47. Hiramatsu, T.; Yoshida, Y.; Saito, G.; Otsuka, A.; Yamochi, H.; Maesato, M.; Shimizu, Y.; Ito, H.; Kishida, H. Quantum spin liquid: Design of a quantum spin liquid next to a superconducting state based on a dimer-type ET Mott insulator J. Mater. Chem. C 2015, 3, 1378. [CrossRef]

48. Dressel, M.; Drichko, N. Optical Properties of Two-Dimensional Organic Conductors: Signatures of Charge Ordering and Correlation Effects. Chem. Rev 2004, 104, 5869. [CrossRef] [PubMed]

49. Yamamoto, T.; Uruichi, M.; Yamamoto, K.; Yakushi, K.; Kawamoto, A.; Taniguchi, H. Examination of the Charge-Sensitive Vibrational Modes in Bis(ethylenedithio)tetrathiafulvalene. J. Phys. Chem. B 2005, 109, 15226. [CrossRef] [PubMed]

50. Girlando, A. Charge Sensitive Vibrations and Electron-Molecular Vibration Coupling in Bis(ethylenedithio)tetrathiafulvalene (BEDT-TTF). J. Phys. Chem. C 2011, 115, 19371. [CrossRef]

51. Lang, M.; Lunkenheimer, P.; Müller, J.; Loidl, A.; Hartmann, B.; Hoang, N.H.; Gati, E.; Schubert, H.; Schlueter, J.A. Multiferroicity in the Mott insulating charge-transfer salt $\kappa$-(BEDT-TTF $)_{2} \mathrm{Cu}\left[\mathrm{N}(\mathrm{CN})_{2}\right] \mathrm{Cl}$. IEEE Trans. Magn. 2014, 50, 2700107.

52. Kawamoto, A.; Honma, Y.; Kumagai, K.I. Electron localization in the strongly correlated organic system $\kappa-(B E D T-T T F)_{2}$ probed with nuclear magnetic resonance ${ }^{13}$ C-NMR. Phys. Rev. B 2004, 70, 060510(R). [CrossRef]

53. Čulo, M.; Tafra, E.; Basletić, M.; Tomić, S.; Hamzić, A.; Korin-Hamzić, B.; Dressel, M.; Schlueter, J.A. Two-dimensional variable range hopping in the spin-liquid candidate $\kappa$ - $(\mathrm{BEDT}-\mathrm{TTF})_{2} \mathrm{Cu}_{2}(\mathrm{CN})_{3}$. Phys. $B$ 2015, 460, 208-210. [CrossRef]

54. Nad, F.; Monceau, P.; Kaboub, L.; Fabre, J.M. Divergence of the relaxation time in the vicinity of the ferroelectric charge-ordered phase transition in (TMTTF)2AsF6. Europhys. Lett. 2006, 73, 567-573. [CrossRef]

55. Cava, R.J.; Schneemeyer, L.F.; Fleming, R.M.; Littlewood, P.B.; Rietman, E.A. Effects of impurities on the dielectric response of the charge-density wave in $\mathrm{K}_{0.3} \mathrm{MoO}_{3}$. Phys. Rev. B 1985, 32, 4088-4096. [CrossRef]

56. Ngai, K.L. Relaxation and Diffusion in Complex Systems Springer: New York, NY, USA, 2011; ISBN 978-1-4419-7649-9. 
57. Poirier, M.; Parent, S.; Côté, A.; Miyagawa, K.; Kanoda, K.; Shimizu, Y. Magnetodielectric effects and spin-charge coupling in the spin-liquid candidate $\kappa$-(BEDT-TTF $)_{2} \mathrm{Cu}_{2}(\mathrm{CN})_{3}$. Phys. Rev. B 2012, 85, 134444. [CrossRef]

58. Manna, R.S.; Hartmann, S.; Gati, E.; Schlueter, J.A.; de Souza, M.; Lang, M. Low-Temperature Lattice Effects in the Spin-Liquid Candidate $\kappa$-(BEDT-TTF $)_{2} \mathrm{Cu}_{2}(\mathrm{CN})_{3}$. Crystals 2018, 8, 87. [CrossRef]

59. Cross, L.E. Relaxor ferroelectrics. Ferroelectrics 1987, 76, 241-267. [CrossRef]

60. Itoh, K.; Itoh, H.; Naka, M.; Saito, S.; Hosako, I.; Yoneyama, N.; Ishihara, S.; Sasaki, T.; Iwai, S. Collective Excitation of an Electric Dipole on a Molecular Dimer in an Organic Dimer-Mott Insulator. Phys. Rev. Lett. 2013, 110, 106401. [CrossRef] [PubMed]

61. Geiser, U.; Wang, H.H.; Carlson, K.D.; Williams, J.M.; Charlier, H.A.; Heindl, J.E.; Yaconi, G.A.; Love, B.J.; Lathrop, M.W.; Schirber, J.E.; et al. Superconductivity at $2.8 \mathrm{~K}$ and $1.5 \mathrm{kbar}$ in $\kappa-(\mathrm{BEDT}-\mathrm{TTF})_{2} \mathrm{Cu}_{2}(\mathrm{CN})_{3}$ : First Organic Superconductor Containing a Polymeric Copper Cyanide Anion. Inorg. Chem. 1991, 30, 2586. [CrossRef]

62. Fukuyama, H.; Kishine, J.; Ogata, M. Energy Landscape of Charge Excitations in the Boundary Region between Dimer-Mott and Charge Ordered States in Molecular Solids. J. Phys. Soc. Jpn. 2017, 86, 123706. [CrossRef]

63. Foury-Leylekian, P.; Ilakovac, V.; Balédent, V.; Fertey, P.; Arakcheeva, A.; Milat, O.; Petermann, D.; Guillier, G.; Miyagawa, K.; Kanoda, K.; et al. $\kappa$-(BEDT-TTF $)_{2} \mathrm{Cu}_{2}(\mathrm{CN})_{3}$ spin liquid: Beyond the average structure. Crystals 2018, 8, 158. [CrossRef]

64. Nakamura, K.; Yoshimoto, Y.; Imada, M. Ab initio two-dimensional multiband low-energy models of EtMe $3 \mathrm{Sb}\left[\mathrm{Pd}(\mathrm{dmit})_{2}\right]_{2}$ and $\kappa$-(BEDT-TTF $)_{2} \mathrm{Cu}_{2}(\mathrm{CN})_{3}$ with comparisons to single-band models. Phys. Rev. $B$ 2012, 86, 205117. [CrossRef]

65. Scriven, E.P.; Powell, B.J. Geometrical Frustration in the Spin Liquid $\beta^{\prime}-\mathrm{EtMe}_{3} \mathrm{Sb}\left[\mathrm{Pd}(\mathrm{dmit})_{2}\right]_{2}$ and the Valence-Bond Solid $\mathrm{Me}_{3} \mathrm{EtP}\left[\mathrm{Pd}(\mathrm{dmit})_{2}\right]_{2}$. Phys. Rev. Lett. 2012, 109, 097206. [CrossRef] [PubMed]

66. Tsumuraya, T.; Seo, H.; Tsuchiizu, M.; Kato, R.; Miyazaki, T. Cation Dependence of the Electronic States in Molecular Triangular Lattice System $\beta^{\prime}-\mathrm{X}\left[\mathrm{Pd}(\mathrm{dmit})_{2}\right]_{2}$. J. Phys. Soc. Jpn. 2013, 82, 033709. [CrossRef]

67. Jacko, A.C.; Tocchio, L.F.; Jeschke, H.O.; Valenti, R. Importance of anisotropy in the spin-liquid candidate $\mathrm{EtMe}_{3} \mathrm{Sb}\left[\mathrm{Pd}(\mathrm{dmit})_{2}\right]_{2}$. Phys. Rev. B 2013, 88, 155139. [CrossRef]

68. Alemany, P.; Pouget, J-P.; Canadell, E. Essential role of anions in the charge ordering transition of $\alpha$-(BEDT-TTF $)_{2} \mathrm{I}_{3}$. Phys. Rev. B 2012, 85, 195118. [CrossRef]

69. Alemany, P.; Pouget, J.-P.; Canadell, E. Electronic structure and anion ordering in (TMTSF) $2 \mathrm{ClO} 4$ and (TMTSF)2NO3: A first-principles study. Phys. Rev. B 2014, 89, 155124. [CrossRef]

70. Alemany, P.; Pouget, J.-P.; Canadell, E. Structural and electronic control of the metal to insulator transition and local orderings in the $\theta$-(BEDT-TTF $)_{2}$ X organic conductors. J. Phys. Condens. Matter 2015, 27, 465702. [CrossRef] [PubMed]

71. Dion, M.; Rydber, H.; Schröder, E.; Langreth, D.C.; Lundqvist, B.I. Van derWaals Density Functional for General Geometries. Phys. Rev. Lett. 2004, 92, 246401. [CrossRef] [PubMed]

72. Román-Pérez G.; Soler, J. M . Efficient Implementation of a van derWaals Density Functional: Application to Double-Wall Carbon Nanotubes. Phys. Rev. Lett. 2009, 103, 096102. [CrossRef] [PubMed]

73. Klimeš, J.; Bowler, D.R.; Michaelides, A. Van der Waals density functionals applied to solids. Phys. Rev. B 2011, 83, 195131. [CrossRef]

74. Mittendorfer, F.; Garhofer, A.; Redinger, J.; Klimeš, J.; Harl, J.; Kresse, G. Graphene on Ni(111): Strong interaction and weak adsorption. Phys. Rev. B 2011, 84, 201401. [CrossRef]

75. Monkhorst, H.J.; Pack, J.D. Special points for Brillonin-zone integrations. Phys. Rev. B 1976, $13,5188$. [CrossRef]

76. Yasin, S.; Dumm, M.; Salameh, B.; Batail, P.; Meziere, C.; Dressel, M. Transport studies at the Mott transition of the two-dimensional organic metal $\kappa$-(BEDT-TTF) ${ }_{2} \mathrm{Cu}\left[\mathrm{N}(\mathrm{CN})_{2}\right] \mathrm{Br}_{x} \mathrm{Cl}_{1-x}$. Eur. Phys. J. B 2011, 79, 383-390. [CrossRef]

77. Sheldrick, G.M. A short history of SHELX. Acta Cryst. A 2008, 64, 112-122. [CrossRef] [PubMed]

(C) 2018 by the authors. Licensee MDPI, Basel, Switzerland. This article is an open access article distributed under the terms and conditions of the Creative Commons Attribution (CC BY) license (http:/ / creativecommons.org/licenses/by/4.0/). 
Review

\title{
Low-Frequency Dynamics of Strongly Correlated Electrons in (BEDT-TTF) ${ }_{2} X$ Studied by Fluctuation Spectroscopy
}

\author{
Jens Müller* and Tatjana Thomas \\ Institute of Physics, Goethe-University Frankfurt, Max-von-Laue-Str. 1, 60438 Frankfurt (Main), Germany; \\ thomas@physik.uni-frankfurt.de \\ * Correspondence: j.mueller@physik.uni-frankfurt.de; Tel.: +49-69-798-47274
}

Received: 22 March 2018; Accepted: 8 April 2018; Published: 14 April 2018

\begin{abstract}
Fluctuation spectroscopy measurements of quasi-two-dimensional organic charge-transfer salts (BEDT-TTF $)_{2} \mathrm{X}$ are reviewed. In the past decade, the method has served as a new approach for studying the low-frequency dynamics of strongly correlated charge carriers in these materials. We review some basic aspects of electronic fluctuations in solids, and give an overview of selected problems where the analysis of $1 / f$-type fluctuations and the corresponding slow dynamics provide a better understanding of the underlying physics. These examples are related to (1) an inhomogeneous current distribution due to phase separation and/or a percolative transition; (2) slow dynamics due to a glassy freezing either of structural degrees of freedom coupling to the electronic properties or (3) of the electrons themselves, e.g., when residing on a highly-frustrated crystal lattice, where slow and heterogeneous dynamics are key experimental properties for the vitrification process of a supercooled charge-liquid. Another example is (4), the near divergence and critical slowing down of charge carrier fluctuations at the finite-temperature critical endpoint of the Mott metal-insulator transition. Here also indications for a glassy freezing and temporal and spatial correlated dynamics are found. Mapping out the region of ergodicity breaking and understanding the influence of disorder on the temporal and spatial correlated fluctuations will be an important realm of future studies, as well as the fluctuation properties deep in the Mott or charge-ordered insulating states providing a connection to relaxor or ordered ferroelectric states studied by dielectric spectroscopy.
\end{abstract}

Keywords: fluctuation spectroscopy; organic charge-transfer salts; Mott metal-insulator transition; glass-like structural ordering; charge-cluster glass; percolation

\section{Introduction}

The quasi-two-dimensional organic charge-transfer salts (BEDT-TTF) ${ }_{2} \mathrm{X}$ (where BEDT-TTF stands for bis-ethylenedithio-tetrathiafulvalene, representing $\mathrm{C}_{6} \mathrm{~S}_{8}\left[\mathrm{C}_{2} \mathrm{H}_{4}\right]_{2}$, often abbreviated as ET, and $\mathrm{X}$ is a monovalent anion) in the last few decades have been viewed as model systems for investigating the physics of strongly correlated electrons in reduced dimensions [1-4]. The model character becomes manifest in (i) the rich phase diagrams sustaining a wealth of interesting ground states; and (ii) the possibility of fine-tuning the materials' properties using subtle physical or chemical means. Therefore, these materials are a test ground for studying the fundamental properties of novel electronic ground states that emerge from the mutual interaction of electron charges or spins in reduced dimensions, and the interaction of electrons with the underlying crystal lattice.

In the $\kappa$-phase materials with polymeric anions, two ET molecules form a dimer, which results in an effectively half-filled conduction band, and charge carriers reside on a more or less frustrated triangular lattice, giving rise to a possible spin-liquid ground state in systems with a high degree of frustration. Further prominent research topics in the past several years have been the nature 
of superconductivity and the unconventional normal conducting metallic phase displaying a pseudogap behavior, as well as the Mott metal-insulator transition where a charge gap opens due to electron-electron interactions, and its universal critical properties. Finally, the intra-dimer degrees of freedom recently have been considered to play an important role for ferroelectric ordering and relaxation phenomena, and charge-order driven formation of electric dipoles that exhibit ferroelectricity, thereby enabling magnetic order has been suggested [5].

A second example of substantial recent research activities, which will be addressed in this review, are quarter-filled $\theta$-phase systems with large, discrete anion structures that are unstable against a charge-ordering metal-insulator transition. This transition from a charge-liquid to an ordered charge-crystal state sometimes can be kinetically avoided, depending on the degree of geometric frustration of the underlying crystal lattice, and a new charge-glass state occurs without long-range order of the localized charges on the triangular lattice. Non-volatile switching operation between the charge-order and charge-glass states has been demonstrated and the vitrification and crystallization of electrons currently are being intensively investigated.

These two examples have been chosen because both the critical properties of the Mott transition and the glassy freezing of charge carriers on a frustrated lattice involve slow dynamics of charge carriers, and it is therefore important and desirable to study the dynamics of the electrons not only with optical techniques but also at very low frequencies.

Dynamical properties of the correlated charge carriers in organic charge-transfer salts have been extensively and successfully studied, e.g., by optical conductivity measurements and dielectric spectroscopy. The former method utilizes the interaction of matter with light covering a wide range of energies in order to study not only basic electronic but also magnetic and vibrational excitations as well as fundamental interaction effects (see, e.g., [6]). The accessible frequencies in microwave to infrared spectroscopy are in the $\mathrm{GHz}-10^{15} \mathrm{~Hz}$ regime.

The ultrafast dynamics in photoinduced phase transitions can be studied with fs light pulses (see, e.g., [7]). Insulating (low-conductance) samples of organic charge-transfer salts have been investigated by dielectric spectroscopy covering the $\mathrm{Hz}-\mathrm{MHz}$ regime, which allows for addressing numerous physical phenomena, as, e.g., dielectric relaxation, charge transport of localized carriers (through hopping of electrons) and ferroelectricity [8].

The aim of our complementary approach of investigating the time-dependent fluctuations of a sample's resistance or conductance (i.e., the measured "noise") over a certain time interval is to spectroscopically access the charge carrier dynamics without injecting additional electrons into the system at relatively low frequencies covering the $\mathrm{mHz}-\mathrm{kHz}$ regime. We will give an overview of physical phenomena-specific for the present materials—causing such slow excitations. The time scale involved in excitations of clusters of electronic systems usually increases with increasing volume of the fluctuation object. Therefore, the method proves beneficial when nano- or microscopic objects are subject to fluctuations, as, e.g., for electronic phase separation caused by competing order parameters or interactions, a scenario frequently found in low-dimensional molecular metals. Likewise, the charge carrier dynamics strongly slow down at a critical point or line of a phase transition, as, e.g., the Mott metal-insulator transition, and localization of charge carriers can be investigated here. The particular scaling behavior at critical points involving power-law divergence of relaxation time and length results in temporal and spatial correlations (a dynamic phase separation), which can lead to very slow fluctuations of large magnitude. Another origin of slow dynamics, even of individual fluctuating objects, is a sufficiently high energy barrier that must be overcome in a thermally-activated trapping or tunneling excitation process, e.g., of electrons in hopping transport or molecules changing their relative orientation. Finally, both percolative phase transitions and a collective glassy freezing of either structural or electronic degrees of freedom result in strong low-frequency fluctuations.

This review is organized as follows. In Section 2, first we will give a brief description of the electronic and structural peculiarities of the investigated materials and discuss the phase diagram of the $\kappa$-(BEDT-TTF $)_{2} \mathrm{X}$ salts with polymeric anions. Second, we will discuss the basics 
of fluctuation spectroscopy and fundamental models to understand $1 / f$-type electronic noise, which is ubiquitous in condensed matter systems. In Section 3, we will review a collection of specific problems, where fluctuation spectroscopy in recent years has provided new information and has helped to better understand the underlying physics. Finally, we will give an outlook to possible future fields of applications.

Noise experiments and the theoretical treatment of fluctuation processes is a wide field of research and spans from the need to understand the fluctuations in order to be able to optimize the performance of sensors and devices by increasing their signal-to-noise ratio to the quest for regarding the noise as the signal in order to gain information on the dynamics of charge carriers and the kinetics of their fluctuations. After we have introduced the method as a new approach for studying the charge carrier dynamics in low-dimensional molecular metals about a decade ago, we have reviewed the technique and first results [9]. Since then, the spectrum of applications of the technique and the physical problems to be studied have considerably broadened, and the method has developed into a powerful tool now applied by various experimental groups.

\section{Materials and Methods}

\subsection{Organic Charge-Transfer Salts-Quasi Two-Dimensional Molecular Metals}

In contrast to ordinary metals like sodium, potassium or aluminum, where the basic units are nearly point-like atoms, the building blocks of organic charge-transfer salts consist of extended molecules, and the materials' unit cells contain a few hundred atoms. This leads not only to challenges in determining the electronic band structure, being addressed by the molecular orbital approximation, but also to multiple excitations, as e.g., a large spectrum of vibrational degrees of freedom due to intra-molecular excitations, i.e., the displacement of atoms within the molecules, in addition to inter-molecular motions and rotations. By combining two sorts of molecules, one with a high electron affinity and the other with a low ionization energy, a charge-transfer process takes place, where charge is transferred from the donor $\mathrm{D}$ to the acceptor molecule $\mathrm{X}$ with a charge-transfer ratio $\delta$ [2]. This oxidation process results in a molecular compound $\mathrm{D}_{m} \mathrm{X}_{n}$, where $m$ and $n$ quantify the stoichiometry, with partially-filled molecular orbitals on the donor (cation) site and a closed-shell configuration on the acceptor (anion) site. When the donor molecules are stacked rather densely, the orbitals of adjacent molecules overlap and an electronic band structure forms, allowing the generated charge carriers to delocalize along the stacking axis. As the acceptor molecules often adopt a closed-shell configuration, they do not contribute to the conduction process. For theoretical calculations, the relevant molecular orbitals are either the highest occupied molecular orbitals (HOMO) or the lowest unoccupied molecular orbitals (LUMO) of the $\pi$-electrons, which can be further used for a tight-binding approach to calculate the electronic band structure $[1,2,10,11]$.

The existence of complex building-blocks leads to a large structural variety, where, for instance, the shape and coordination of the cation and anion decide about the system's dimensionality. For the BEDT-TTF molecules, specific steric effects prevent an infinite face-to-face stacking and a relatively strong side-by-side overlap of the $\pi$-orbitals of adjacent molecules results in a quasi-two-dimensional electronic structure of the ET salts. A linear combination of the atomic $s$ - and $p$-orbitals leads to $\pi$-and $\sigma$-orbitals, which have a perpendicular and parallel orientation to the bonding axis, respectively. Due to the lower binding energy of the $\pi$-electrons, they can easily delocalize, so that the free charge carriers have mainly $\pi$-hole character. Besides this, different spatial arrangements of the donor molecules, the so-called packing motifs, result in variations of the physical properties due to changes in the molecular overlap and hence the transfer integrals. These packing types are labeled with Greek letters, such as $\alpha$-, $\beta-, \kappa-, \theta$ - and $\lambda$-phases, and characterize the ET molecules' relative orientation, often visualized when viewed along the long axis of the donor molecules (see Figure 1b).

The crystal structure of the most intensively studied quasi-two-dimensional $\kappa$-(BEDT-TTF) ${ }_{2} \mathrm{X}$ salts can be simply considered as alternating thick conducting layers with the ET molecules (blue 
color) and thin insulating layers with the anions $X$ (red color), as shown in Figure 1a, for polymeric anions $\mathrm{X}=\mathrm{Cu}\left[\mathrm{N}(\mathrm{CN})_{2}\right] \mathrm{Z}$ with $\mathrm{Z}=\mathrm{Cl}, \mathrm{Br}$, where the direction perpendicular to the conducting plane is along the crystallographic $b$-axis. Characteristic for the $\kappa$-phase packing motif is a strong structural dimerization indicated by the shaded ellipses in Figure 1b, which-despite the 2:1 stoichiometry-results in the opening of a dimerization gap in the nominally three-quarter-filled band structure leading to an effectively half-filled conduction band [2]. The charge carriers then reside on the ET dimers, which form a triangular lattice, as shown in Figure 1b, with the relevant transfer integrals $t$ and $t^{\prime}$ in a minimal model $[10,12]$. The degree of frustration $t / t^{\prime}$ depends on and varies with the structure of the anion $X$ [11]. This effective dimer model, however, recently has been challenged, since the intra-dimer electronic degrees of freedom seem to play an important role in understanding dielectric relaxation and ordering phenomena. For instance, in [5], it is argued that the localization (charge-order) of holes on one of the molecules within the dimers results in the formation and subsequent ferroelectric ordering of electric dipoles in $\kappa-(\mathrm{ET})_{2} \mathrm{Cu}\left[\mathrm{N}(\mathrm{CN})_{2}\right] \mathrm{Cl}$, thereby lifting the frustration and enabling antiferromagnetic order of the localized spins $[5,13]$.
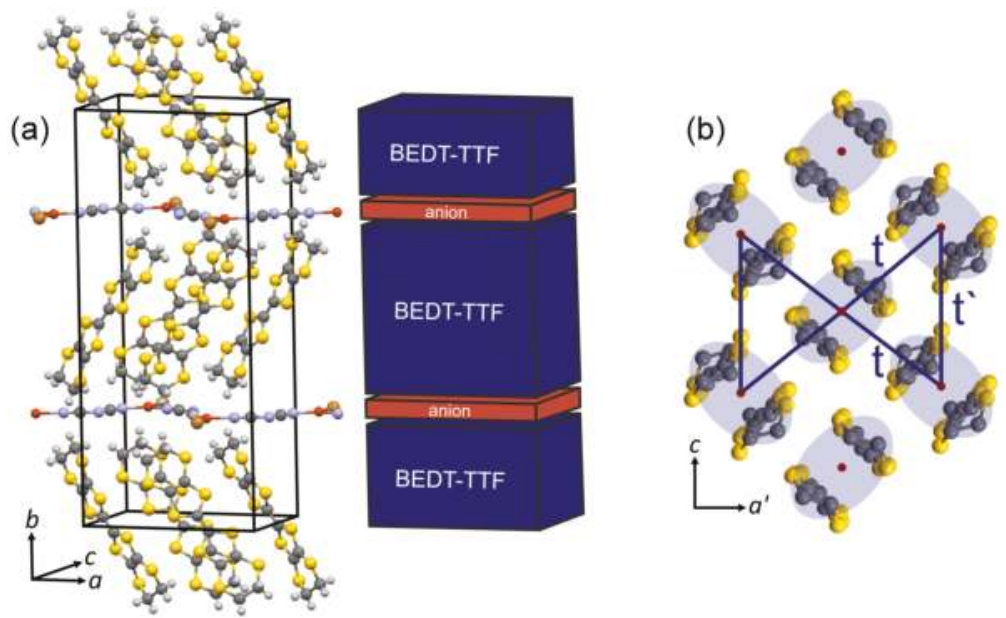

Figure 1. (a) crystal structure (left) of $\kappa$-(BEDT-TTF) ${ }_{2} \mathrm{Cu}\left[\mathrm{N}(\mathrm{CN})_{2}\right] \mathrm{Z}$ with $\mathrm{Z}=\mathrm{Br}, \mathrm{Cl}$, which can be simply viewed as alternating blocks (right) of conducting and insulating layers with the donor ET and polymeric chain-forming acceptor $\mathrm{Cu}\left[\mathrm{N}(\mathrm{CN})_{2}\right] \mathrm{Z}$ molecules, respectively. The box represents the unit cell hosting four formular units; (b) spatial arrangement of the ET molecules in the $\kappa$-phase viewed along the long axis of the molecules. Two ET molecules form a dimer (shaded ellipse), whereas two dimers are arranged almost orthogonal to each other. Considering the dimers as the basic structural units, the conduction band is effectively half-filled and the charger carriers reside on a triangular lattice with two transfer integrals $t$ and $t^{\prime}$.

Compared to ordinary inorganic metals, the organic compounds are characterized by low charge carrier densities of order $n \sim 10^{21} \mathrm{~cm}^{-3}$, since the volume of the molecules providing the free charge carriers is considerably large. This, in combination with the relatively narrow bandwidth (due to the weak overlap of neighboring molecular orbitals) and the reduced dimensionality of the electronic system resulting in a reduced screening of the charge carriers, leads to strong electron-electron correlations quantified by a ratio of bandwidth $W \sim 0.5 \mathrm{eV}$ to on-site Coulomb repulsion $U \sim 0.5 \mathrm{eV}$, which is close to unity. Experimentally, this results in enhanced effective masses seen in experiments of quantum oscillations $[14,15]$ and specific heat [16], where $m_{\mathrm{eff}}^{*}=(1.5-7) m_{\mathrm{e}}$. Importantly, however, part of the mass renormalization is due to the strong electron-phonon coupling involving both high-frequency intra- and low-frequency intermolecular vibrations. 
Due to relatively weak bonding energies, the materials' properties can be easily affected by changing external parameters like temperature, pressure, chemical substitution, cooling rate, etc. Thus, the temperature-pressure phase diagram of the $\kappa$-(BEDT-TTF $)_{2} \mathrm{X}$ salts, depicted in Figure 2, displays a variety of different ground states and transition lines [17-21]. Whereas the system with $\mathrm{X}=\mathrm{Cu}\left[\mathrm{N}(\mathrm{CN})_{2}\right] \mathrm{Cl}$ (in short $\kappa-\mathrm{Cl}$ ) has an insulating ground state with antiferromagnetic and ferroelectric ordering at $T_{N}=T_{F E}=27 \mathrm{~K}$, the compound $\mathrm{X}=\mathrm{Cu}\left[\mathrm{N}(\mathrm{CN})_{2}\right] \mathrm{Br}$ (in short $\kappa$-Br) with the slightly larger $\mathrm{Br}$ atom in the anion complex is located on the metallic side of the phase diagram with a superconducting transition at $T_{c}=11.6 \mathrm{~K}$. Thus, the salts $\kappa-\mathrm{Cl}$ and $\kappa$ - $\mathrm{Br}$ at ambient pressure are located on opposite sides of the first-order Mott metal-insulator transition (red line in Figure 2). The universality of the phase diagram (see also Figures $5 \mathrm{a}$ and $10 \mathrm{~b}$ below) is seen by the fact that applying a moderate hydrostatic pressure of only 300 bar shifts $\kappa-\mathrm{Cl}$ to the metallic side and a superconducting ground state with $T_{c}=12.8 \mathrm{~K}$. Since hydrostatic pressure increases the molecular overlap of adjacent molecules, the pressure axis can be mapped to the ratio $W / U$ of bandwidth to on-site Coulomb repulsion [17], i.e., the Mott transition is a bandwidth-controlled transition not affecting the bandfilling. Another possible tuning mechanism is the substitution of atoms, mostly in the ET donors by different isotopes. Most effective and controllable is either the successive replacement of one to eight of the hydrogen atoms in the ET molecules' terminal ethylene groups $\left[\mathrm{C}_{2} \mathrm{H}_{4}\right]_{2}$ by deuterium atoms or the successive replacement of the conventional hydrogenated ET molecules $\mathrm{H}_{8}$-ET in the crystal structure by the fully-deuterated analogue $\mathrm{D}_{8}$-ET, which in both cases results in a chemically-induced shift (negative pressure) from the metallic towards the Mott insulating state [22]. Thus, the partial substitution with deuterated molecules in systems $\kappa-\left[\left(\mathrm{H}_{8}-\mathrm{ET}\right)_{1-x}\left(\mathrm{D}_{8}-\mathrm{ET}\right)_{x}\right]_{2} \mathrm{Cu}\left[\mathrm{N}(\mathrm{CN})_{2}\right] \mathrm{Br}$ (in short: $\kappa-\mathrm{H}_{8} / \mathrm{D}_{8}-\mathrm{Br}$ ) allows for tuning the ambient-pressure position in the phase diagram in fine steps across the metal-insulator transition and critical region of the phase diagram. These systems are ideal to study the critical properties of the Mott transition at ambient pressure.

As is schematically shown in Figures 2, 5a and $10 \mathrm{~b}$, at a critical ratio $(W / U)_{c}$, the first-order Mott metal-insulator transition occurs, illustrated as a red line in Figure 2, which terminates in a finite-temperature second-order critical endpoint at $\left(p_{0}, T_{0}\right) \sim(230-250 \mathrm{bar}, 36-40 \mathrm{~K})$. At lower temperatures, an inhomogeneous region (blue/green shaded area in Figure2), where the antiferromagnetic insulating and superconducting grounds states coexist, is found close to the transition line [18].

After the charge-transfer is completed in the crystallization process of $(\mathrm{ET})_{2} \mathrm{X}$, the ET molecules are essentially planar, except the ethylene endgroups (EEG in short), which are the most deformable parts of the molecule and for which the relative orientation of the outer $\mathrm{C}-\mathrm{C}$ bonds can be either parallel (eclipsed, E) or canted (staggered, S). At high temperatures, the EEG are disordered due to strong thermal vibrations, and, upon cooling to low temperatures, the EEG adopt one of the two possible conformations ( $\mathrm{E}$ in the case of $\kappa-\mathrm{Cl}$ and $\kappa-\mathrm{Br}$ ) depending on the anion and crystal structure [1]. For kinetic reasons (see Section 3.2), however, an ordered state cannot be achieved in some compounds $\kappa$-(ET) ${ }_{2} \mathrm{X}$ and the EEG freeze in a glassy fashion at temperatures of $T_{g} \sim 75 \mathrm{~K}$, below which thermal equilibrium cannot be reached such that a part of the EEG remains locked (frozen) in the energetically unfavorable non-equilibrium configuration [19]. Hence, the glass-like transition temperature $T_{g}$ depends on the cooling rate $q \equiv \mathrm{d} T / \mathrm{d} t$, which therefore determines the degree of intrinsic structural disorder that becomes frozen [23]. Since the latter degree of non-equilibrium occupation determines $W / U$ [24], a varying cooling rate can be used as yet another powerful tuning parameter in the phase diagram [25], such that more rapid cooling decreases $W / U$ and shifts the systems towards the insulating side of the phase diagram. 


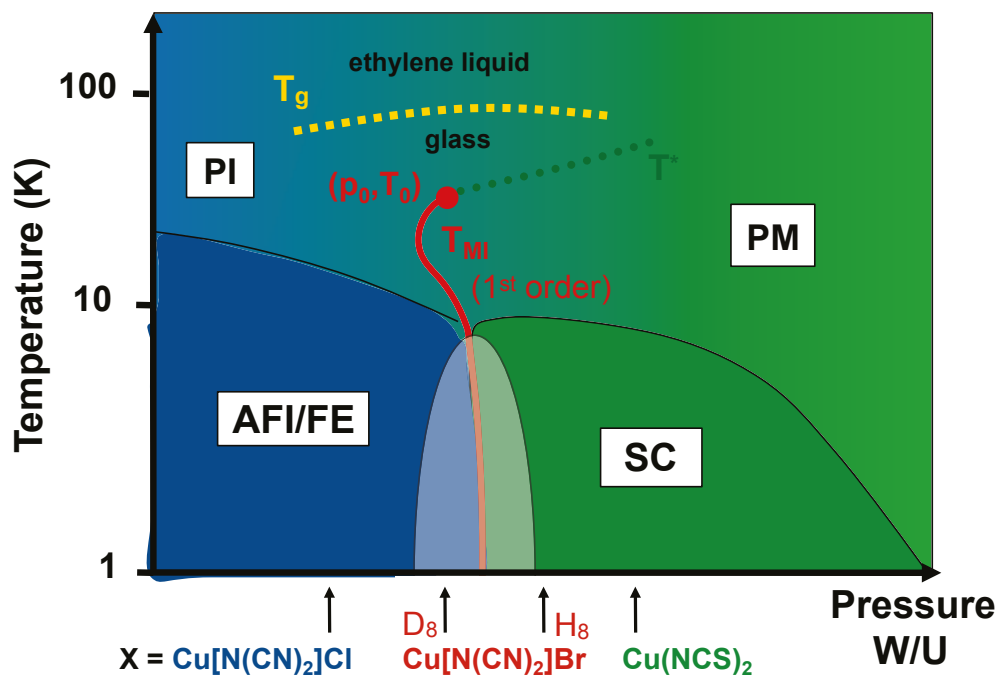

Figure 2. Schematic temperature-pressure phase diagram of the $\kappa$-(BEDT-TTF) 2 X salts with polymeric anions, after Refs. [17-21]. Indicated is the position of systems with different anions $X$ at ambient pressure. Hydrostatic pressure can be mapped on an increasing ratio of $W / U$ (see text). PI, PM, AFI, FE and SC stand for the paramagnetic insulating, paramagnetic metallic, antiferromagnetic insulating, ferroelectric and superconducting phase, respectively. The red line marks the first-order Mott metal-insulator transition, which terminates in a finite-temperature critical point. At elevated temperatures, a structural glass-like transition of the ET molecules' ethylene endgroup rotational degrees of freedom occurs (dashed yellow line). The green dotted line marks the crossover to an anomalous metallic ('bad metal') phase above the Fermi liquid.

\subsection{Fluctuation ('Noise') Spectroscopy}

\subsubsection{Definitions and Basic Relations}

In a usual electronic transport measurement, in order to increase the sensitivity or signal-to-noise ratio, the mean value of an otherwise more-or-less randomly fluctuating quantity (voltage or current) is used to calculate the sample's resistance or conductance. This is achieved by averaging over a certain time interval, e.g., determined by the frequency-bandwidth (time-constant) of a lock-in amplifier in an AC-measurement.

Naturally, large fluctuations about the mean value are unwanted and therefore are sought to be suppressed or largely eliminated. Fluctuation spectroscopy is a time-resolved electronic transport measurement, either of a sample's resistance (preferred for conducting metallic samples) or its conductance (preferred for semiconducting/insulating samples), where the fluctuations are amplified instead by a suitable measurement system and analyzed in the time and frequency domain by means of simple Fourier analysis, i.e., here "the noise is the signal" [26,27]. The basics of noise and fluctuations are described in many textbooks on (non-equilibrium) statistical physics and time-dependent statistical mechanics, as well as in review articles on noise in condensed matter systems (see e.g., [9,28-34] and references therein). Therefore, in the following, we will only give a brief account of the important definitions, relevant relations and simple models.

In order to gain information from the fluctuations in a quantity $x(t)$, it is useful to ask how much power is associated with different parts of the frequency spectrum of the random signal. Mathematically, this means calculating the noise power spectral density (PSD) of the signal, $S_{x}(f)$, by considering the modulus square of the Fourier-transformed noisy signal and taking an average over 
a suitable time interval. If the fluctuating part of the signal is $\delta x(t)=x(t)-\langle x(t)\rangle$, where $\langle x(t)\rangle$ is the time-averaged mean value that may be considered equal to zero [35], and the noise PSD is given by:

$$
S_{x}(f)=2 \lim _{T \rightarrow \infty} \frac{1}{T}\left|\int_{-T / 2}^{T / 2} \delta x(t) e^{-i 2 \pi f t} \mathrm{~d} t\right|^{2}
$$

This is the standard engineering definition, where the variance $\left\langle\delta x(t)^{2}\right\rangle$ of the random signal is normalized by:

$$
\left\langle\delta x(t)^{2}\right\rangle=\int_{0}^{\infty} S_{x}(f) \mathrm{d} f .
$$

Next, we ask for a connection relating the power spectrum $S_{x}(f)$ to some time-like property of the statistically varying function $\delta x(t)$ (which equals $x(t)$ for $\langle x(t)\rangle=0$ without loss of generality). This time-like behavior is given by the autocorrelation function

$$
\Psi_{x x}(\tau) \equiv\langle x(t) \cdot x(t+\tau)\rangle
$$

which, for statistically stationary processes, is only a function of $\tau$ (and $t$ is unimportant) and is symmetric in $\tau$ [36].

The Wiener-Khintchine theorem states that

$$
S_{x}(f)=4 \int_{0}^{\infty} \Psi_{x x}(\tau) \cos (2 \pi f \tau) \mathrm{d} \tau \quad \text { and } \quad \Psi_{x x}(\tau)=\int_{0}^{\infty} S_{x}(f) \cos (2 \pi f \tau) \mathrm{d} f .
$$

Equation (4) is the most informative way to express the time dependence of a random variable and, specifically, kinetic processes of electrons in condensed matter. The fluctuations of a system are determined by the temporal (and sometimes also spatial) correlations, which are quantified by a suitable correlation function, where the variable $x$ is either the measured voltage $V$ or current $I$. It describes the 'memory' of the system, i.e., what remains of a fluctuation at a later time. Whereas $\delta x(t)$ is of course random, the correlation function $\Psi_{x x}(\tau)$ is a non-random characteristic of the kinetics of these random fluctuations, describing how the fluctuations evolve in time on average [33].

The Wiener-Khintchine theorem describes the equivalence of the standard engineering definition of noise, Equation (1), and the definition of noise as the Fourier transform of the autocorrelation function (Equation (3)). A third definition, which is essentially realized by calculating noise spectra in the experiment, i.e., either by a software or by using a spectrum analyzer, involves passing the time signal through a 'realizable filter' of finite bandwidth, squaring it, and averaging it over some large finite time. For the bandwidth approaching zero, e.g., by realizing a sufficiently sharp filter in an $R-L-C$-circuit with a large quality factor, the result will (aside from a normalization factor) approach the ideal value of the two preceding definitions [37].

In general, the time dependence of the fluctuations' correlation function (or, equivalently, the frequency dependence of the power spectral density) and the response of the system to external perturbations are governed by the same kinetic processes. In thermal equilibrium, there is an exact relation between $S_{x}(f)$ and the dissipative part of the system's linear response to an external perturbation, which is described by the fluctuation-dissipation theorem (FDT), which in the quasi-classical limit reads:

$$
S_{x}(f)=2 k_{B} T \frac{\chi^{\prime \prime}(\omega)}{\omega}
$$

where $\omega=2 \pi f$. Here, $\chi^{\prime \prime}(\omega)$ is the imaginary part of the complex susceptibility and the real function $\chi(t)$ is the response function of the system [38]. 
Obviously, the autocorrelation function (Equation (3)) without a temporal delay $(\tau=0)$ equals the statistical variance:

$$
\Psi_{x x}(0)=\left\langle\delta x(t)^{2}\right\rangle .
$$

If the correlation time $\tau$ of a fluctuation is very short, and $\Psi(\tau)$ decays to zero very fast (and therefore before, in an experiment, the next data point is taken), the system appears totally random (and, in fact, infinitely 'choppy'), and the corresponding PSD is frequency independent (sometimes referred to as 'white' noise). An example is thermal noise (first measured by Johnson for a variety of materials as a function of their resistance [39]) of a resistor, where the noise PSD of the fluctuating voltage drop across the resistor in thermal equilibrium is given by:

$$
S_{V}=4 k_{B} T R \text {. }
$$

Equation (7) is known as Nyquist theorem [40] expressing a special formulation of the FDT in Equation (5), which describes the spontaneous voltage fluctuations arising from an ideal passive resistance, i.e., the electrical Brownian Movement [28,41]. Integrating Equation (7) reveals that the variance of thermal (white) noise (see Equation (2)) converges at low frequencies but diverges at high frequencies, i.e., the mean value converges as one averages over longer and longer time intervals, but the instantaneous value of the signal is undefined.

The mathematical counterpart extreme of such a totally random signal is a random walk noise, which is an integral of white noise resulting in a PSD $\propto 1 / f^{2}$, where the integration brings in a factor $1 / f$ in the Fourier transform, which becomes a factor $1 / f^{2}$ in its square, the power spectrum. Opposite to white noise, the random walk noise contains an infinite power at low frequencies, but the spectrum is convergent when integrated in frequency from some constant to infinity. Thus, a random walk has a well-defined value at each point, whereas the divergence of the spectrum when integrating down to zero frequency means that this noise has no well-defined mean value over long times $[42,43]$.

In between thermal noise (PSD $\propto 1 / f^{0}$ ) and random walk noise (PSD $\propto 1 / f^{2}$ ) is $1 / f^{1}$-noise, which is the most interesting noise being ubiquitous in nature and abundant in condensed matter physics. It may be described as the accumulative effect of 'something happening sometimes' on all time scales. Figure 3 a shows the time train of a voltage (i.e., resistance) measurement typical for many condensed matter systems, which neither appears totally random nor fully correlated (from time step to time step) or-to put it the other way around-it shows both rapid fluctuations down to the resolution limit of the instrument (e.g., a lock-in amplifier with a wide bandpass filter of time constant $\tau=1 \mathrm{~ms}$ ) as well as general long-term up and down trends, resulting in clearly visible maxima and minima. The noise is Gaussian and the spectrum varies as $\propto 1 / f$ (see the histogram Figure $3 \mathrm{~b}$ and the power spectral density (c), respectively, which is half way between white and random walk noise). Perfect $1 / f$-noise is self similar, i.e., scale invariant, since $S(q f)=q^{-1} S(f)$ (each logarithmic frequency interval contributes the same power) and diverges both at the high and low frequency end, i.e., there is neither a well-defined value at a single point, nor a well-defined long-term mean. The divergence on both ends, however, is only logarithmic, i.e., so slow that even absurdly high and low values for frequency cutoffs hardly change the noise at all [9]. Experimentally, the high-frequency cutoff often naturally is given by the frequency, where the $1 / f$-type noise drops below the thermal noise, which is always present, and the low-frequency cutoff is determined by the experimentalist's patience [44]. 

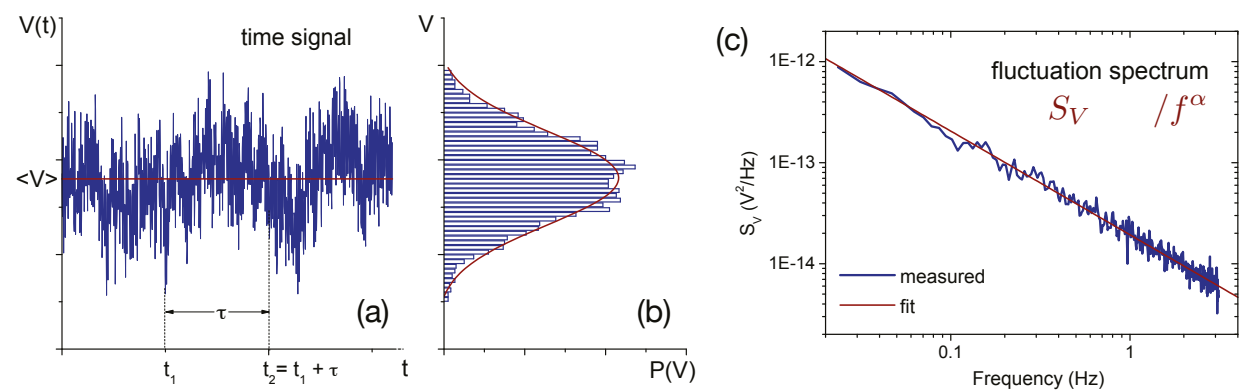

Figure 3. Schematics of a voltage (resistance) noise measurement. (a) time-train of the voltage signal and (b) histogram of the voltage distribution fitted by a Gaussian function; (c) noise power spectral density (PSD) of the signal in (a) fitted by a power law with frequency exponent $\alpha \simeq 1$. Reprinted with permission from [9]. Copyright (2011) John Wiley and Sons.

A simple model to understand $1 / f$-noise in solids is the superposition of many independent two-level fluctuators with a characteristic distribution of time constants/energies. We assume for simplicity a symmetric double-well potential with a thermally activated characteristic time-constant $\tau=\tau_{0} \exp \left(E_{a} / k_{B} T\right)$ for overcoming the energy barrier $E_{a}$ as sketched in Figure 6 below. This may be viewed as an idealized model to describe the emission of an electron from a donor state in the band gap of a semiconductor into the conduction band (detrapping), thereby contributing to the total conductance of the system, and subsequent capture by the donor (trapping). Considering only this one electron, the conductance (or resistance) will switch randomly between two distinct states leading to so-called random telegraph noise (generation-recombination noise). The current-current correlation function of this process decays exponentially as has been calculated by S. Machlup [45]:

$$
\Psi_{I I}(\tau) \equiv\langle I(t) \cdot I(t+\tau)\rangle=\left\langle(\delta I)^{2}\right\rangle \exp (-|t| / \tau)
$$

Employing the Wiener-Khintchine theorem (Equation (4)), one finds for the current noise PSD (the equations for the voltage autocorrelation function $\Psi_{V V}(\tau)$ and voltage noise $\operatorname{PSD} S_{V}(f)$ are analogous):

$$
S_{I}(f)=4 \int_{0}^{\infty} \Psi_{I I}(\tau) \cos (2 \pi f \tau) \mathrm{d} \tau=\left\langle(\delta I)^{2}\right\rangle \frac{4 \tau}{1+4 \pi^{2} f^{2} \tau^{2}}
$$

i.e., a Lorentzian spectrum. It has been recognized $[29,30,46]$ that a superposition of Lorentzians

$$
S_{I}(f)=\int_{0}^{\infty} D(\tau) \frac{4 \tau}{1+4 \pi^{2} f^{2} \tau^{2}} \mathrm{~d} \tau
$$

results in $1 / f$-noise for a certain distribution of relaxation times $D(\tau) \propto 1 / \tau$ [47] in the experimentally accessible range between $\tau_{1}$ and $\tau_{2}$ :

$$
S_{I}(f)=4 \int_{\tau_{1}}^{\tau_{2}} \frac{1}{1+4 \pi^{2} f^{2} \tau^{2}} \mathrm{~d} \tau=\left.\frac{4}{2 \pi f} \arctan (2 \pi f \tau)\right|_{\tau_{1}} ^{\tau_{2}} \propto \frac{1}{f} \quad \text { for } \quad \frac{1}{\tau_{2}} \ll f \ll \frac{1}{\tau_{1}} .
$$

Likewise, writing $\tau=\tau_{0} \exp \left(E / k_{B} T\right)$ the integration in Equation (10) can be carried out over energy $E$ resulting in $S_{I}(f) \propto 1 / f$ for a distribution of activation energies $D(E)=$ const. in an energy interval $\left[E_{1}, E_{2}\right]$ for $f_{2} \ll f \ll f_{1}$ with $f_{1,2}=f_{0} \exp \left(-E_{1,2} / k_{B} T\right)$ and an attempt frequency $f_{0}=1 /\left(2 \pi \tau_{0}\right)$. 
A constant distribution of activation energies, however, is a strong constraint that is usually not fulfilled, which results in deviations from 'perfect' $1 / f$-noise, i.e., $S_{I} \propto 1 / f^{\alpha}$ with $\alpha=0.8-1.4$ [34]. A model where the energy distribution $D(E)$ captures the often observed changes of the frequency exponent $\alpha$ with temperature is described in Section 3.2 (see Equations (15) and (16) below).

Finally, we like to mention that for a random process $\delta x(t)$ the so-called second spectrum $S_{x}^{(2)}(f)$ accesses the higher-order correlation function $\Psi_{x x}^{(2)}(\tau) \equiv\left\langle x^{2}(t) \cdot x^{2}(t+\tau)\right\rangle$ and describes the power spectrum of the fluctuations of $S_{x}(f)$ with time, i.e., the Fourier transform of the autocorrelation function of the time series of $S_{x}(f)$. In the experiment, an additional frequency $f_{2}$ related to the time over which $S_{x}(f)$ fluctuates is thereby introduced. The second spectrum $S_{x}^{(2)}\left(f_{2}, f\right)$ probes a fourth-order noise statistics and therefore deviations from Gaussian behavior [33,48]: it is independent of the frequency $f_{2}$ if the fluctuations are uncorrelated, e.g., caused by independent two-level systems. However, a distinct frequency dependence, often $S_{x}^{(2)} \propto 1 / f_{2}^{\beta}$ with $\beta \approx 1$, is observed for correlated (interacting) fluctuators. A frequency-dependent second noise spectrum is important in spin glass physics [49] and is often observed accompanying the sudden slowing down of the charge carrier dynamics at a metal-insulator transition and is interpreted as glass-like freezing of the electrons undergoing correlated transitions over a large number of metastable states [50-53].

\subsubsection{Measuring Resistance Fluctuations and Hooge's Law for Organic Charge-Transfer Salts}

What is fascinating about $1 / f$-noise is that it is ubiquitous in nature and can be found for example in melody and loudness fluctuations of classical music [54], fluctuations of a traffic current on expressways [55], the light emission of astronomical objects [42], or the dynamics of human heart rate [56-58] to give only very few examples. This has raised the question of whether a universal theory for $1 / f$-type fluctuations exists. However, considering such different complex systems, it is fair to say that commonalities likely result from mathematical coincidence rather than a fundamental underlying principle. The same is possibly true for condensed matter, where $1 / f$-noise is found in such different systems as semiconductors and devices, metals and insulators, granular systems, magnetic thin films and sensors, tunnel junctions, spin glasses, superconductors, colossal magnetoresistance materials, etc., where it is assumed that resistance fluctuations $\delta R(t)$ are the intrinsic cause of the $1 / f$-noise, which upon application of a driving current $I$ lead to a fluctuating voltage drop $\delta V(t)=I \delta R(t)$. Likewise, the intrinsic conductance fluctuations $\delta G(t)$ result in a fluctuating current, when a source voltage is applied across the sample $\delta I(t)=V \delta G(t)$. In other words, in a resistance measurement, say, the applied current merely probes the fluctuations and facilitates the detection of $1 / f$-type voltage fluctuations rather than causing them. This intrinsic nature of $1 / f$-noise was experimentally demonstrated in [59], where fluctuations in the mean-square Johnson-Nyquist-noise exhibit $1 / f$-type spectra.

As mentioned above, the fluctuations are measured by employing the definition of the noise spectrum using realizable filters [37], where the fluctuating signal is processed either by a software or a spectrum analyzer consisting of a bandpass filter with adjustable frequency and a narrow bandwidth around a central frequency, and an output detector that responds to the mean square of the signal [33] (see $[9,60]$ for a more detailed description of the five-terminal bridge setup and AC frequency-shift method [61] mainly used for obtaining the noise spectra presented in this review).

An important test regarding the intrinsic nature of the measured $1 / f$-noise in a four-terminal configuration, which to a large part eliminates the contribution of the electric contacts, or five-terminal bridge, which in addition makes the setup insensitive to fluctuations of the source current/voltage or the bath temperature, is the scaling $S_{V} \propto I^{2}$ and the observation of a flat ('white') spectrum in the limit of vanishing current $I \rightarrow 0$. For AC lock-in measurements, often a voltage-divider circuit is used. Another stringent test, discussed, e.g., in [60], of the intrinsic nature of the observed $1 / f$-noise is the independence of the $S_{V} \propto I^{2}$ scaling of the limiting resistor in the circuit determining the sample current. For metallic samples, a five-terminal AC setup [61] with a driving current $I(t)=I_{0} \sin \left(2 \pi f_{0} t\right)$ is preferred, where the sample is placed in a Wheatstone bridge in order to suppress the DC offset. 
The resistance fluctuations are pre-amplified and modulate the sinusoidally excited carriers to produce noise sidebands, which can be demodulated by a phase-sensitive detector in a lock-in amplifier. The advantage of an AC current is that the pre-amplifier, the internal semiconductor components of which often contribute a large spurious (extrinsic) $1 / f$-noise, can be operated close to its optimal frequency, ideally in the eye of its noise figure [61]. Then, the background noise merely consists of the thermal noise of the resistive components of the experimental setup and all spurious $1 / f$-noise is eliminated. Suitable impedance matching and choice of $f_{0}$ therefore allows for measurements from $f_{0} / 2$ down to $10^{-3} \mathrm{~Hz}$. The output signal of the lock-in amplifier is then processed by the spectrum analyzer. As elegantly formulated in [33], this spectrum analyzer, which also includes an amplifier, plays the role of a magnifying glass, which enables us to visualize the microscopic motion and transition of particles.

Figure 4a shows typical $1 / f$-type noise spectra representative for organic charge-transfer salts (taken from [60]), and (b) a typical spectrum where a Lorentzian contribution is superimposed on the $1 / f$ background. The latter spectrum can be well described by these two separate contributions, which allows for determining both the magnitude of the $1 / f$-noise level and the magnitude and characteristic frequency of the Lorentzian two-level switching, cf. Equation (14) below. The meaning of the fluctuating resistance (or voltage) as a statistically stationary variable is nicely visualized by the reproducibility of the $1 / f$-spectra taken at $T=60 \mathrm{~K}$ (see Figure $4 \mathrm{a}$ ), showing that, while the noise is random, its PSD is not. The black spectrum has been taken during cool-down of the sample in discrete steps and the light-gray colored spectrum several days later during warm-up. At all temperatures, very clean $1 / f^{\alpha}$-spectra with $\alpha$ around 1 are observed. One then evaluates the temperature dependence of the slope, $\alpha(T)$ (see Section 3.2 below), and the temperature dependence of the noise magnitude where often the value at $1 \mathrm{~Hz}, S_{R}(f=1 \mathrm{~Hz})$ conveniently is considered or the spectral weight in some finite frequency interval is calculated.
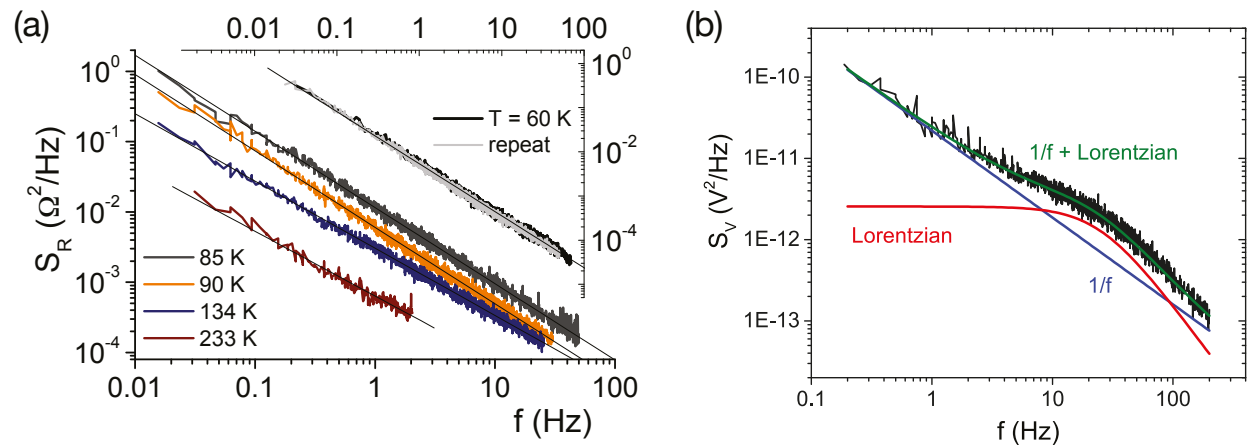

Figure 4. Typical noise spectra. (a) representative resistance noise PSD of an organic charge transfer salt measured at different temperatures. Lines are fits to $S_{R} \propto 1 / f^{\alpha}$. Reprinted with permission from [60]. Copyright (2009) by the American Physical Society; (b) voltage noise PSD with a Lorentzian contribution superimposed in the $1 / f$-type background.

Empirically, for a large variety of semiconductors, it was found by F. N. Hooge (1969) that the normalized noise magnitudes $S_{x}(f) / x^{2}=C_{1 / f} / f$ are identical for $x$ representing current $I$, voltage $V$ or conductance $G$ and resistance $R$, i.e., $S_{R} / R^{2}=S_{G} / G^{2}=S_{V} / V^{2}=S_{I} / I^{2}$. (The amplitude $C_{1 / f}$ was originally thought to be a constant that could be theoretically justified, which, however, failed.) Therefore, the measured voltage noise power spectral density $S_{V} \propto V^{2}$, and for ohmic behavior $V=R I$ 
one has $S_{R}=S_{V} / I^{2}$. From the comparison of various semiconducting samples, a normalization with respect to the number of free charge carriers in the system, $N_{c}$, was suggested [62]:

$$
S_{V}(f)=\gamma_{H} \frac{V^{2}}{n \Omega f}
$$

with the charge carrier density $n$, the sample's 'noisy volume' $\Omega$ and therefore $N_{c}=n \Omega$, and the Hooge parameter $\gamma_{H}$, which for clean and homogeneous semiconductors often is found to be $\gamma_{H} \sim 10^{-3}-10^{-2}$. (Note that only for pure $1 / f$-noise, i.e., for frequency exponents $\alpha=1, \gamma_{H}$ is a dimensionless quantity.) The assumption that $1 / f$-noise is composed of independent fluctuation processes of individual mobile charge carriers is of course in many cases a crude oversimplification [63]. However, as already mentioned, $\gamma_{H}$ is useful to compare the relative noise level of different compounds or different samples of the same compound and Equation (12) may serve as a guide to optimize the shape of a metallic sample for noise measurements [64].

Early $1 / f$-noise measurements on organic charge-transfer salts have been reported for conducting Langmuir-Blodgett thin films based on the molecules $\mathrm{C}_{16} \mathrm{H}_{33}$-TCNQ and $\mathrm{C}_{17} \mathrm{H}_{35}$-DMTTF as well as prototypical TTF-TCNQ single crystals [65]. It was found that the room-temperature Hooge parameter $\gamma_{H}$ of the thin films is enhanced about three orders of magnitude as compared to the values of clean semiconductors and even seven to eight orders of magnitude for the TTF-TCNQ crystals. This is worth mentioning, since a simple geometrical model has been proposed related to the quasi-one-dimensional nature of these systems, where crystal imperfections force the charge carriers to hop from stack to stack in the process of propagation along the molecular stacking axis and a fluctuating number of charge propagation paths is suggested to account for the strongly enhanced $1 / f$-noise [65].

Indeed, also the quasi-two-dimensional organic charge-transfer salts (BEDT-TTF) 2 X discussed in this review exhibit very large Hooge parameters of order $\gamma_{H} \sim 10^{5}-10^{7}$ [66], which is certainly on the higher end of the range of values of $\gamma_{H} \sim 10^{-6}-10^{7}$ found for different classes of materials $[33,34]$, whereas $\gamma_{H}$ in many systems strongly varies with temperature. In the picture of Hooge's model—despite being empirical and oversimplified-the large values of $\gamma_{H}$ for organic charge-transfer salts may result from an overestimation of the noisy volume $\Omega$, e.g., due to inhomogeneous current paths in the sample related to the specific conduction mechanism, in particular when measured perpendicular to the highly conductive layers.

\section{Results}

In the following, we review selected problems related to the rich physics of quasi-two-dimensional organic charge-transfer salts $(\mathrm{BEDT}-\mathrm{TTF})_{2} \mathrm{X}$, which have been addressed and better understood by fluctuation spectroscopy measurements. These physical phenomena resulting in characteristic fluctuation properties are related to (1) spatial electronic inhomogeneities, i.e., electronic phase separation and percolation in the coexisting region of competing ground states; (2) glass-like structural excitations, involving the coupling of molecular degrees of freedom to the electronic transport; (3) charge crystallization and vitrification leading to heterogeneous slow dynamics; and (4) charge carrier dynamics at the Mott metal-insulator transition leading to critical slowing down of the charge fluctuations and ergodicity breaking.

In an outlook, we will briefly mention future efforts to investigate the low-frequency charge carrier dynamics deep in the insulating phase of dimer Mott systems exhibiting interesting dielectric relaxation or ferroelectricity.

\subsection{Example (1): Superconducting Percolation in Phase Coexistence Regions}

The first example deals with the fluctuation properties of an inhomogeneous state caused by the close vicinity of two phases with competing order parameters. In the phase diagram of $\kappa-(\mathrm{BEDT}-\mathrm{TTF})_{2} \mathrm{X}$, Figure2, the phase boundary of the first-order Mott MIT separates an antiferromagnetic 
insulating/ferroelectric ground state (for low pressure, small $W / U, X=\mathrm{Cu}\left[\mathrm{N}(\mathrm{CN})_{2}\right] \mathrm{Cl}$ ) from a superconducting one (for high pressure, large $W / U, \mathrm{X}=\mathrm{Cu}\left[\mathrm{N}(\mathrm{CN})_{2}\right] \mathrm{Br}$ ). Tuning an insulating sample $\kappa-\mathrm{Cl}$ by physical or chemical-induced pressure to a position close to the phase boundary results in a region of inhomogeneous phase coexistence [18], where superconductivity was believed to be percolative [67]. From resistance measurements alone, however, conclusive evidence for percolation and information on the mechanism of percolation have not been drawn. In [68], we report on measurements of the intrinsic $1 / f$-noise, namely $S_{R}(f, T, B)$, in the superconducting percolation regime of pressurized $\kappa-(\mathrm{ET})_{2} \mathrm{Cu}\left[\mathrm{N}(\mathrm{CN})_{2}\right] \mathrm{Cl}$ (in short: $\kappa-\mathrm{Cl}^{*}$ ), an ambient pressure Mott insulator exhibiting antiferromagnetic and ferroelectric ordering being shifted to the inhomogeneous coexistence region in the phase diagram. This is seen by comparing the resistance curves shown in Figure 5a. At ambient pressure, $R(T)$ of $\kappa$-Cl shows the familiar semiconducting behavior with a strong increase below about $50 \mathrm{~K}$ (black curve), where the charge gap opens due to the localization of the charge carriers on the $(\mathrm{ET})_{2}$ dimers [69]. In contrast, pressurized $\kappa-\mathrm{Cl}^{*}$ shows the typical dip in the resistance upon cooling when entering the metallic phase and re-entering the insulating Mott phase due to crossing the S-shaped MIT line twice. A sudden drop of the resistivity at $T_{\mathcal{c}} \approx 13 \mathrm{~K}$ indicates superconductivity. Surprisingly, for the so-prepared material, we observe a strong increase of the resistance noise PSD, $S_{R} / R^{2}(f=1 \mathrm{~Hz})$, for $T<T_{c} \approx 13 \mathrm{~K}$, i.e., upon entering the superconducting phase (see Figure $5 \mathrm{~b}$ ). A similar behavior has been observed for strongly disordered high- $T_{c}$ cuprates [70-72]. Whereas for homogeneous superconductors both the resistance and the $1 / f$-type resistance fluctuations are expected to vanish in the superconducting phase, basic percolation theory of a random resistor network (RRN), with a portion $p(T, B)$ of resistor elements in the lattice being short-circuited, predicts a power-law growth of the relative resistance fluctuations $S_{R} / R^{2} \propto\left(p-p_{c}\right)^{-\kappa}$, with the percolation threshold $p_{c}$, as the portion of the superconductor increases and approaches the critical value at which an infinite superconductive cluster appears, see [33] and references therein.

(a)

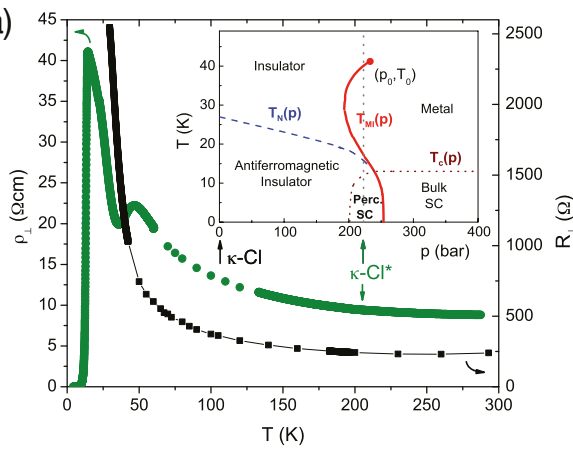

(b)

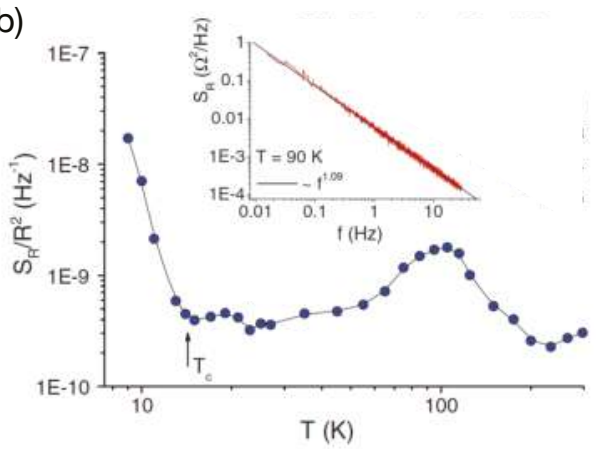

Figure 5. Noise at the percolative superconducting transition. (a) resistance of two samples of $\kappa-(\mathrm{ET})_{2} \mathrm{Cu}\left[\mathrm{N}(\mathrm{CN})_{2}\right] \mathrm{Cl}$. Inset shows a schematic phase diagram, cf. Figures 2 and $10 \mathrm{~b}$. Arrows indicate the positions of the samples measured at ambient conditions $(\kappa-\mathrm{Cl})$ and at a finite pressure $\left(\kappa-\mathrm{Cl}^{*}\right)$; (b) normalized resistance noise PSD $S_{R} / R^{2}(T, f=1 \mathrm{~Hz})$ of $\kappa-\mathrm{Cl}^{*}$. Arrow indicates the superconducting transition (onset of $T_{c}$ ). Inset: PSD of the resistance noise at $90 \mathrm{~K}$ in a log-log plot revealing a clean $S_{R} \propto 1 / f^{\alpha}$ spectrum with $\alpha=1.09$. Reprinted with permission from [68]. Copyright (2009) by the American Physical Society.

The strong increase of the low-frequency fluctuations observed when entering the superconducting phase and approaching the superconductor-insulator transition can therefore be explained by the decrease of the number of effective current paths such that the fluctuations of $R$ in this disordered conductor are determined only by a small volume in the sample. This-in agreement with the simple Hooge-model (Equation (12))—leads to a high noise level, whereas, far from the 
superconductor-insulator transition, the large number of effective current paths in the RRN results in a cancellation of the fluctuations of $R$ along the different uncorrelated paths and a lower total noise level [33]. Under the simplifying assumption of uncorrelated resistance fluctuations in an RRN, the resistance noise is given by:

$$
\frac{S_{R}(f)}{R^{2}}=\tilde{s}(f) \frac{\sum_{m} i_{m}^{4}}{\left(\sum_{m} i_{m}^{2}\right)^{2}},
$$

where $i_{m}$ is the local current passing through the resistance $r_{m}$ divided by the total current $I$, that passes through the sample $[33,73,74]$. The function $\tilde{s}(f)$ describes the mechanism of fluctuations in the local resistances $r_{m}$, which usually is of $1 / f^{\alpha}$ type with $\alpha \approx 1$. Hence, in a simple percolative phase separation scenario, the magnitude of the noise varies depending on the fourth moment of the current distribution, whereas the frequency exponent $\alpha(T, B)$ in first approximation remains unchanged [75].

In general, since in percolation problems the measure of the vicinity to the percolation threshold $\left(p-p_{c}\right)$ is a microscopic quantity that usually is experimentally accessible only in rather specially-constructed cases [76], it is convenient to evaluate the variation of the resistance noise with respect to the actual resistance value for which a scaling behavior $R \propto\left(p-p_{c}\right)^{-t}$ is predicted, resulting in $S_{R} / R^{2} \propto R^{-l_{r s}}[77,78]$. The scaling exponent $l_{r s}=\kappa / t$ then can be compared to the predictions of theoretical calculations in order to clarify the mechanism of the underlying percolation process. Figure 6 a shows a scaling plot of $\log S_{R} / R^{2}$ vs. $\log R$ of the pressurized $\kappa-\mathrm{Cl}^{*}$ sample shown in Figure 5 at a fixed temperature of $T=5 \mathrm{~K}$, where the magnetic field is the implicit parameter driving the system from the superconducting to the normal conducting state as shown in Figure $6 \mathrm{c}$. From the slope of the data in Figure 6a yielding the scaling exponent $l_{r s}$ of the resistor-superconductor network [79], we infer a change of the percolation mechanism occurring just below the midpoint of the superconducting transition. A small exponent $l_{r s}=0.9 \pm 0.3$ at low fields (see that the light gray region in Figure $6 \mathrm{c}$ is in agreement with a 'classical' percolation model [78]), where the $1 / f$-noise is generated by fluctuating resistor elements $\delta r_{i}(t)$ that may be identified with the portion of $p$ resistive junctions. For larger fields, i.e., when approaching the normal conducting state, the scaling exponent becomes significantly larger and is close to the value of $l_{r s} \sim 2.74$ that is found for the so-called $p$-model of percolation, first discussed for disordered high- $T_{c}$ cuprate superconductors $[78,80]$. The difference between these two mechanisms of percolation is schematically sketched in Figure $6 \mathrm{~b}$, where $p$-noise leads to a new class of scaling exponents and describes a random on/off switching of superconducting links $w_{i}(t)$ in the resistor network resulting in spontaneous fluctuations $\delta p(t)$. These may be identified with dynamic perturbations of the Josephson coupling energy between superconducting clusters, resulting in a stronger power-law increase of the macroscopic resistance fluctuations.

Besides these insights in the microscopic dynamics of the inhomogeneous state in the coexistence region of the generalized phase diagram, noise measurements provide information on the characteristic length scales of phase separation. This becomes possible through a dominating two-level process in a narrow field range around the crossover from 'classical' to $p$-type percolation (see the white region in Figure $6 \mathrm{c}$ ). In this particular noise window, the action of a single fluctuator is enhanced and Lorentzian spectra, the characteristic energies of which depend on the applied magnetic field, can be resolved superimposed on the underlying $1 / f$-type noise. Figure $6 \mathrm{~d}$ shows such spectra in a representation $f \times S_{R}(f)$ vs. $f$, where the $1 / f^{\alpha}$ 'background' is nearly a constant for $\alpha \approx 1$. Clearly, the additional contribution of the observed spectra can be fitted by a single two-level process with a characteristic time constant $\tau_{c}=1 /\left(\tau_{1}^{-1}+\tau_{2}^{-1}\right)$, cf. Equation (9):

$$
S_{R}(f)=\frac{4(\delta R)^{2}}{\left(\tau_{1}+\tau_{2}\right)\left[\left(\tau_{1}^{-1}+\tau_{2}^{-1}\right)^{2}+(2 \pi f)^{2}\right]} .
$$



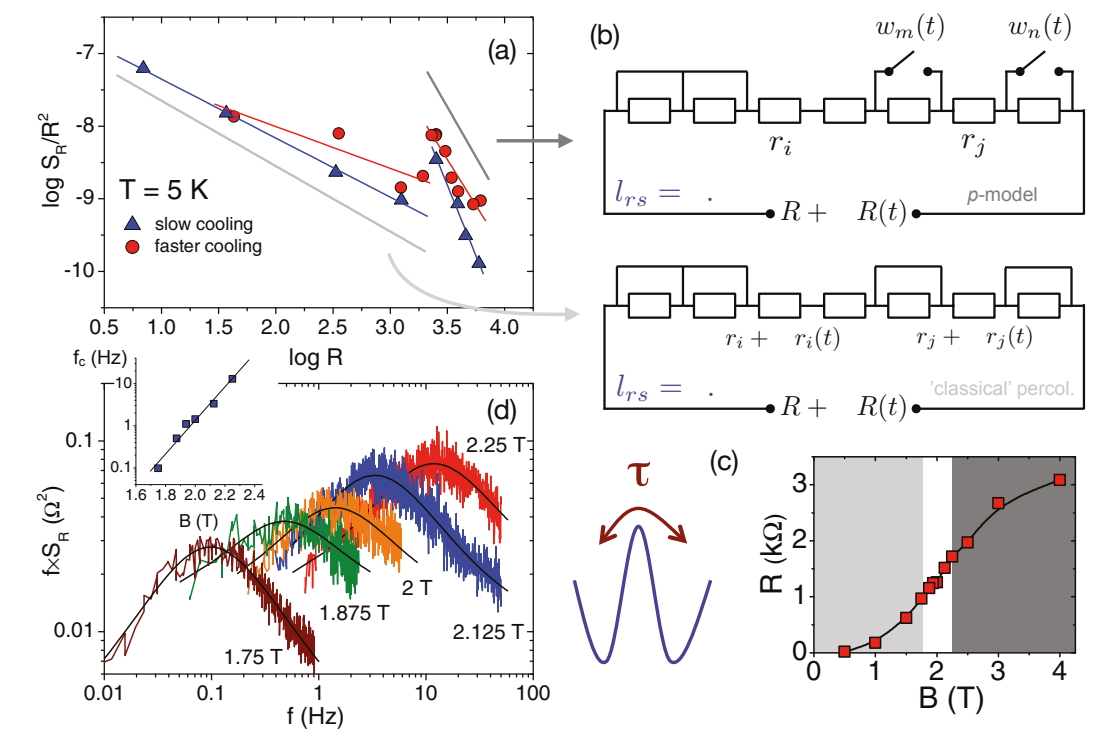

Figure 6. Percolation models in superconductors. (a) scaling of the normalized noise $S_{R} / R^{2} \propto R^{l_{r s}}$ of $\kappa-\mathrm{Cl}^{*}$ in a representation $\log S_{R} / R^{2}$ vs. $\log R$, where the slope reveals the power-law exponent $l_{r s}$, for data taken at $T=5 \mathrm{~K}$ and different magnetic fields up to $4 \mathrm{~T}$ as an implicite tuning parameter of the sample resistance (see (c)); (b) schematics of classical (bottom) and novel (top) percolation noise. In the former case $\left(l_{r s}=0.9\right)$, the noise originates from the resistor elements, where the short-circuits represent superconducting subvolumes. The latter case of $p$-noise $\left(l_{r s}=2.74\right)$ originates from switching elements controlled by random processes $w_{i}(t)$ representing subvolumes with unstable superconductivity, after [72]. Reprinted with permission from [9]. Copyright (2011) John Wiley and Sons; (c) magnetoresistance $R(B)$ at $T=5 \mathrm{~K}$. Light and dark gray areas denote different percolation regimes characterized by the scaling exponent $l_{r s}$. In the white area, Lorentzian spectra are superimposed on the $1 / f$ background noise (see (d)), where the inset shows the shift of the corner frequency $f_{\mathcal{c}}(B)$. (a,d) reprinted with permission from [68]. Copyright (2009) by the American Physical Society.

In the time domain, Equation (14) corresponds to a random telegraph signal, i.e., a switching between a high- and low resistance state that may be caused by a cluster that switches between the superconducting and normal conducting state. The observed shift of the corner frequency $f_{c}=1 /\left(2 \pi \tau_{c}\right)$ [81] with magnetic field shown in the inset of Figure $6 \mathrm{~d}$ and assuming $f_{c}=f_{0} \exp \left[-\left(E_{a}+m B\right) / k_{B} T\right]$, where $f_{0}$ is an attempt frequency typically related to the characteristic phonon frequencies and $E_{a}$ the energy barrier for the switching process at $B=0$, allow to determine the energy $m B$. Considering the fluctuating entity as a cluster that switches between the superconducting and normal conducting state, and comparing this energy to the condensation energy $V_{s} B_{c_{t h}}^{2} / 2 \mu_{0}$, where $B_{c_{t h}}$ is the thermodynamic critical field and $\mu_{0}$ the free space permeability, allows for a rough estimate of the fluctuating sample volume yielding $V_{s} \sim(7 \mathrm{~nm})^{3}$ [68], therefore providing evidence for a nano-scale phase separation. We note that for the high- $T_{c}$ cuprates vortex dynamics is discussed as a source of $p$-fluctuations [72,80], in which case we may compare $m B$ to the pinning energy $n \varepsilon^{*} V_{s}$, where $n$ is the number of flux lines per unit area and $\varepsilon^{*}=B_{c_{1}} \Phi_{0} / \mu_{0}$ the energy per unit length of a $\operatorname{vortex}\left(\Phi_{0}\right.$ is the flux quantum and $B_{c_{1}}$ the lower critical field) leading to a volume $V_{s} \sim(5 \mathrm{~nm})^{3}$ of the same order. In order to distinguish between both scenarios, direct measurements of the magnetic flux noise are desirable. 
To summarize, the intrinsic $1 / f$-noise in the superconducting state revealed microscopic information on (i) the mechanism of percolation and (ii) the size of the fluctuating entities and thus the characteristic scale of phase separation in the inhomogeneous coexistence region of the phase diagram. It is interesting to note that real-space imaging by means of scanning micro-region infrared spectroscopy of partially-deuterated $\kappa-(\mathrm{ET})_{2} \mathrm{Cu}\left[\mathrm{N}(\mathrm{CN})_{2}\right] \mathrm{Br}$ revealed a static phase separation and coexisting metallic and insulating domains also on a larger length scale of 50-100 $\mu \mathrm{m}$ and at all temperatures along the first-order Mott MIT line below the critical endpoint $T_{0} \sim 35 \mathrm{~K}$ [21,82]. This suggests volume fractionalization on a wide range of length scales, which, in turn, results in fluctuations with a hierarchy of corresponding time scales, a prerequisite of the widely observed enhanced $1 / f$-type noise in the critical region of the phase diagram (see Section 3.4 below).

\subsection{Example (2): Glass-Like Structural Ordering}

In this section, we exemplarily discuss the coupling of the charge fluctuations to slow structural dynamics in $\kappa-(\mathrm{ET})_{2} \mathrm{X}$ with polymeric anions $\mathrm{X}=\mathrm{Cu}\left[\mathrm{N}(\mathrm{CN})_{2}\right] \mathrm{Cl}, \mathrm{Cu}\left[\mathrm{N}(\mathrm{CN})_{2}\right] \mathrm{Br}$, or $\mathrm{Cu}(\mathrm{SCN})_{2}$. From thermodynamic measurements, a glass-like freezing of the ET molecules' terminal ethylene groups (EEG) was identified [19,83-86]. The dynamical properties at low frequencies-a characteristic fingerprint of all glass transitions, however-had not been studied due to the lack of dielectric spectroscopy, which cannot be applied in the relevant temperature range around $100 \mathrm{~K}$, where the conductivity-even of the Mott insulating samples-is still too high. An understanding of the slow dynamics, however, is of paramount importance in these materials, since the degree of frozen EEG entities depends on the cooling rate and affects the electronic ground state properties through (i) the degree of quenched disorder and (ii) the lattice contraction occurring upon cooling through $T_{g} \sim 75 \mathrm{~K}$, which strongly influences the bandwidth $W$ and on-site Coulomb repulsion $U$, and therefore the sample's position in the phase diagram (see e.g., [21-25,87-91]). In this context, fluctuation spectroscopy served as a method complementary to dielectric spectroscopy allowing for a comprehensive study of the low-frequency glassy dynamics and a systematic understanding of the glassy properties. These findings (i) revealed the phenomenology of the glassy transition in these materials [60,92,93]; (ii) allowed for controlling the glassy freezing in the critical region of the phase diagram by varying the cooling rate or applying heat pulses, thereby enabling non-volatile switching functions between metallic and Mott insulating phases and establishing a protocol for fine-tuning through the phase diagram with unprecedented precision [23,25]; and (iii)-in combination with ab initio quantum chemical calculations-now allow to predict whether a certain crystal structure favors a glassy freezing of the EEG orientational degrees of freedom or not [93]. Based on this, the specifics of the glass-like transition in the system $\kappa-(\mathrm{ET})_{2} \mathrm{Hg}(\mathrm{SCN})_{2} \mathrm{Cl}$ have been correctly predicted [94].

Figure $7 \mathrm{a}$ shows the temperature dependence of the resistance and the resistance fluctuations for deuterated $\kappa-\left(\mathrm{D}_{8}-\mathrm{ET}\right)_{2} \mathrm{Cu}\left[\mathrm{N}(\mathrm{CN})_{2}\right] \mathrm{Br}$, which-regarding the structural glassy properties discussed here-is representative for the $\kappa$-(ET) ${ }_{2} \mathrm{X}$ family with polymeric anions [93]. After the sample's resistance has been measured during continuous cool down (black line), resistance values (blue squares) and noise spectra (red circles) have been taken in discrete steps while warming up the sample. The resistance shows the typical behavior for samples located close to the critical region of the phase diagram but still away from the critical point at $\left(p_{0}, T_{0}\right)$, cf. Figure $10 \mathrm{~b}$ below [95]. The observed noise was of generic $1 / f$-type at all temperatures, i.e., $S_{R} \propto 1 / f^{\alpha}$, and the data in Figure 7a represent the spectral weight calculated as $\int_{10 \mathrm{mHz}}^{100 \mathrm{~Hz}} S_{R}(f) / R^{2} \mathrm{~d} f$, which represents the variance of the fluctuating resistance in the measured frequency range. Strikingly, the resistance fluctuations behave markedly different than the resistance (i.e., the mean value): a pronounced broad maximum of the normalized resistance noise $S_{R}(f, T) / R^{2}$ at about $100 \mathrm{~K}$ has almost no corresponding feature in $R(T)$, and so does the sharp peak in the noise at about $35-36 \mathrm{~K}$. The latter feature marks a sudden slowing down of the charge carrier dynamics, see the strong increase of the relative noise level $a_{R}(f, T)=f \times S_{R} / R^{2}$-a dimensionless quantity characterizing the strength of the fluctuations-at low frequencies shown in the contour 
plot Figure 7d, due to the near vicinity of the critical endpoint of the Mott transition and this will be discussed in detail in Section 3.4 below.
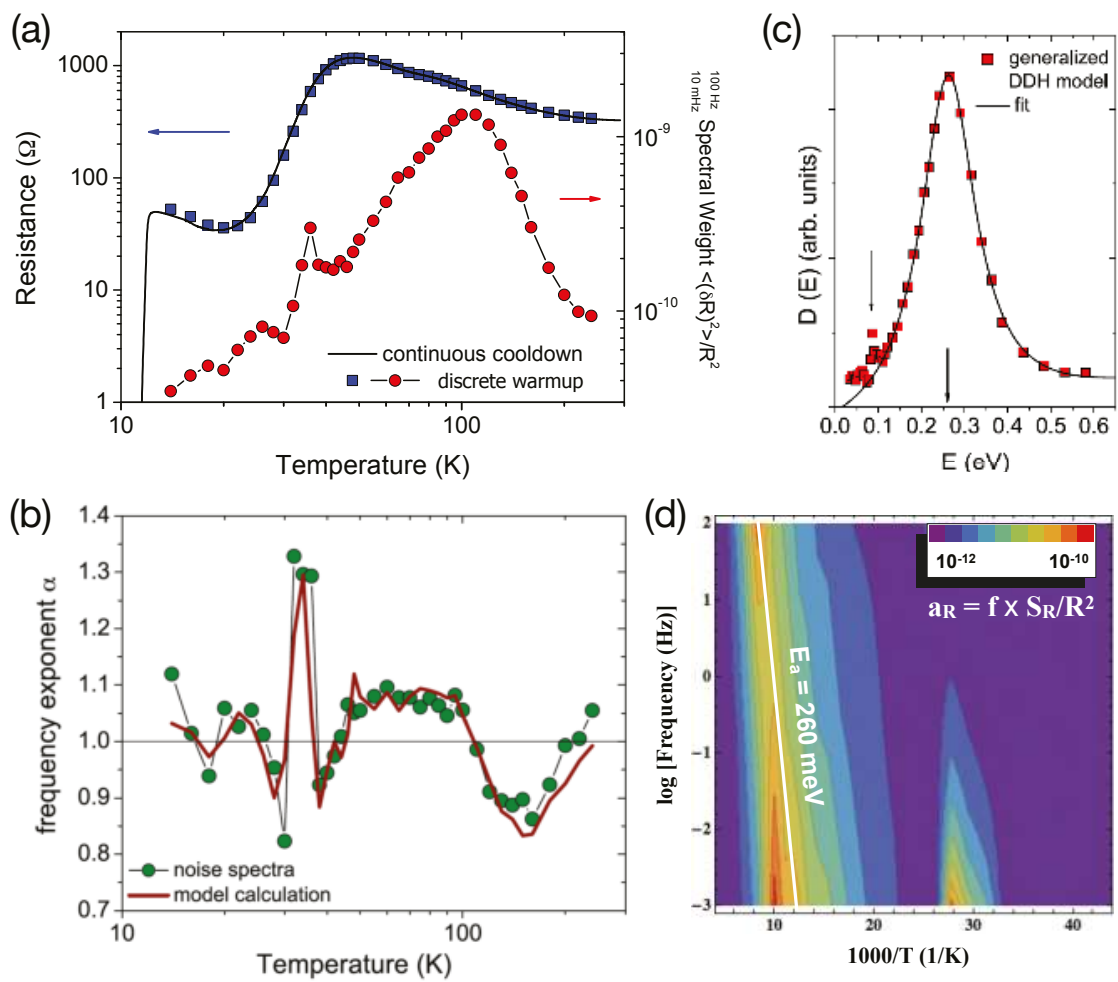

Figure 7. Noise spectroscopy and DDH analysis (see text) on fully-deuterated $\kappa-\left(\mathrm{D}_{8}-\mathrm{ET}\right)_{2} \mathrm{Cu}\left[\mathrm{N}(\mathrm{CN})_{2}\right] \mathrm{Br}$. (a) resistance (blue squares) and integrated noise PSD (red circles) of a fully-deuterated sample $\kappa-\left(\mathrm{D}_{8}-\mathrm{ET}\right)_{2} \mathrm{Cu}\left[\mathrm{N}(\mathrm{CN})_{2}\right] \mathrm{Br}$; (b) corresponding frequency exponent $\alpha(T)$ (green circles) and model calculation within the generalized DDH model (Equation (16)) (red line); (c) distribution of activation energies $D(E)$ at $f=1 \mathrm{~Hz}$ calculated using Equation (18). Arrows indicate the two strongest maxima at $260 \mathrm{meV}$ and $90 \mathrm{meV}$; (d) contour plot of the relative noise level $a_{R}=f \times S_{R} / R^{2}$ vs. frequency $f$ and temperature $T$ in an Arrhenius representation. The slope of the white line corresponds to an activation energy of $E_{a}=260 \mathrm{meV}$, corresponding to the maximum in $D(E)$ in (c) as extracted from the DDH model. Reproduced from [92,93]. Copyright IOP Publishing.

In order to understand the origin of the $1 / f^{\alpha}$-noise, we apply the phenomenological model of Dutta, Dimon and Horn (DDH) [31,96], which describes non-exponential kinetics [97] caused by a superposition of individual, thermally activated fluctuators — not specified a priori-with characteristic relaxation times $\tau=\tau_{0} \exp \left(E / k_{B} T\right)$, where $\tau_{0} \sim\left(10^{-14}-10^{-11}\right) \mathrm{s}$ corresponding to typical inverse phonon frequencies. The individual fluctuators are presumed to be independent (Gaussian) and linearly coupled to the resistance (or conductance) of the sample. Although these assumptions are quite general, the strength of the model is that a distribution (or weighting function) of activation energies $D(E)$ determines both the temperature dependence of the $1 / f^{\alpha}$ noise (i.e., its deviations from a linear-in- $T$ behavior) [98]

$$
\frac{S_{R}(f)}{R^{2}}(T)=\int_{0}^{\infty} g(T) \frac{4 \tau}{1+4 \pi^{2} f^{2} \tau^{2}} D(E) \mathrm{d} E=\frac{1}{\pi f} \int_{0}^{\infty} g(T) \frac{D(E)}{\cosh \left[\left(E-E_{\omega}\right) / k_{B} T\right]} \mathrm{d} E
$$


and the deviations of its frequency dependence from a pure $1 / f$ law (i.e., deviations from $\alpha=1$ ) via the connection

$$
\alpha_{\mathrm{DDH}}(T)=1-\frac{1}{\ln 2 \pi f \tau_{0}}\left[\frac{\partial \ln \frac{S_{R}(f)}{R^{2}}(T)}{\partial \ln T}-\frac{\partial \ln g(T)}{\partial \ln T}-1\right] .
$$

The logarithmic factor in $E_{\omega}=-k_{B} T \ln \left(2 \pi f \tau_{0}\right)$ ensures that at the temperatures and frequencies, where $1 / f$-noise is usually observed, the characteristic energies $E_{\omega} \sim 0.1-1 \mathrm{eV}$ are of the same order as ordinary activation energies in solids [33]. The frequency exponent $\alpha_{\mathrm{DDH}}$ calculated with Equation (16) can then be compared to the measured slope of the spectra

$$
\alpha(T)=-\frac{\partial \ln \frac{S_{R}(f)}{R^{2}}(T)}{\partial \ln f} .
$$

If this consistency check proves successful, then the assumptions of the DDH model [99] are fulfilled, and the distribution function $D(E) \equiv D\left(E_{\omega}\right)$ can be determined from the relation

$$
\frac{S_{R}}{R^{2}}(f)=g(T) k_{B} T D(E) \frac{1}{f} .
$$

Introducing the function $g(T)$ in Equations (15) and (16) is a generalization of the original DDH model [100-102]. The function $g(T)$ accounts for an explicit temperature dependence of the distribution of activation energies. The physical meaning is that the coupling constant between the random processes and the resistance, and hence the total noise magnitude, may not be independent of temperature. As described in detail in [93], we have assumed the simplest case of a power law $g(T)=a T^{b}$, where $b$ describes a constant vertical shift of $\alpha_{\mathrm{DDH}}(T)$ as compared to the measured $\alpha(T)$. For the measurements in Figure 7b, the best description of the data is found for $g(T)=$ const. for $T<T_{g}$ (no offset) and $b=-3 / 2$ for $T>T_{g}$ resulting in a vertical offset. As for the physical meaning of the function $g(T)$, a temperature dependence of the number and/or coupling strength of the fluctuators is discussed $[102,103]$. Figure $7 \mathrm{~b}$ shows that, for the deuterated $\kappa-\mathrm{D}_{8}-\mathrm{Br}$ system, an excellent agreement is observed between the calculated and measured frequency exponent, where nearly every feature of the non-monotonic temperature dependence is quantitatively reproduced. This justifies the conversion of the temperature axis using $E_{\omega}=-k_{B} T \ln \left(2 \pi f \tau_{0}\right)$ and determining $D(E)$ from Equation (18) shown in Figure 7c. The broad noise peak at about $100 \mathrm{~K}$ in Figure 7a therefore corresponds to a peak in $D(E)$ centered around $E \approx 260 \mathrm{meV}\left(E / k_{B} \approx 3020 \mathrm{~K}\right)$. (Note that the contour plot Figure 7d of the relative noise level $a_{R}(f, T)$ in an Arrhenius plot consistently reveals a slope of the noise maximum corresponding to the same energy.) Energy of this order is well known in $\kappa-(\mathrm{ET})_{2} \mathrm{X}$ as the activation energy of structural, orientational degrees of freedom of the ET molecules' EEG undergoing a glass-like freezing at $T_{g} \sim 75 \mathrm{~K}$ and has been determined, e.g., in NMR [104,105], and thermal expansion and specific heat measurements $[19,84]$ for the thermodynamic glass transition (see also [2]). Therefore, the fluctuators causing the enhanced noise at elevated temperatures can be assigned a posteriori to the thermal motion of the EEG, emphasizing the strong coupling of lattice vibrations to the electronic system.

Here, the non-monotonic behavior of $\alpha(T)$ shown in Figure $7 \mathrm{~b}$ reflects the shape of the distribution of the activation energies, i.e., $\alpha>1$ and $\alpha<1$ corresponds to $\partial D(E) / \partial E>0$ and $\partial D(E) / \partial E<0$, respectively. The corresponding shift of spectral weight to lower frequencies is related to the expected slowing down of molecular dynamics when approaching the glass transition. In [93], we argue that the observed charge carrier dynamics is caused by the slow, so-called $\alpha$-process of the glass-forming EEG rotations embedded in the anion structure forming a 'cage' environment through short $\mathrm{C}-\mathrm{H} \cdots$ anion contacts [106]. In dielectric spectroscopy, the $\alpha$-relaxation peak of the frequency-dependent dielectric loss shifts with decreasing temperature to lower frequencies in a similar way [107], which is expected 
from the fluctuation-dissipation theorem connecting $S(f)$ with the imaginary part of the dynamic susceptibility, cf. Equation (5).

This a posteriori assignment provides a spectroscopic tool to investigate the slow dynamics of the EEG, which undergo a glassy freezing at a characteristic temperature $T_{g}$. Figure $8 \mathrm{a}, \mathrm{b}$ show the position of the peak in $S_{R} / R^{2}(T)$ of the same sample $\kappa$ - $\mathrm{D}_{8}$ - $\mathrm{Br}$ discussed in Figure $7 \mathrm{a}-\mathrm{c}$ for different frequencies in our measurement window of $1 \mathrm{mHz}-100 \mathrm{~Hz}$. (Note that in Figure 7a the spectral weight over the full frequency range is shown.) As seen in Figure $8 \mathrm{~b}$ for $\kappa-\mathrm{D}_{8}-\mathrm{Br}$, the data at higher frequencies show an Arrhenius behavior, where the slope corresponds to an activation energy of $260 \mathrm{meV}$ corresponding to the maximum in $D(E)$ shown in Figure 7c. At lower frequencies towards the glassy freezing transition (which may be defined by a characteristic relaxation time of $\tau\left(T_{g}\right)=100 \mathrm{~s}$ ), however, the slowing down of the molecular motions coupled to the resistance fluctuations is much stronger, resulting in a curvature that can be described by the empirical Vogel-Fulcher-Tammann law

$$
\tau=\tau_{0} \exp \left(\frac{D T_{\mathrm{VFT}}}{T-T_{\mathrm{VFT}}}\right),
$$

where $D$ is a strength parameter and $T_{\mathrm{VFT}}$ the Vogel-Fulcher-Tammann temperature. The observed slowing down of the structural units' motion stronger than given by an Arrhenius law may be interpreted as an increase of the effective energy barrier for decreasing temperatures approaching $T_{g}$. A possible origin is an increasingly cooperative character of the molecular motions, i.e., a larger number of correlated molecules and an increase in correlation length [108]. The strength parameter $D$ describes the deviations from a thermally activated behavior, whereas the glass-forming system is characterized as 'fragile' for small values of $D$ (typically $D<10$ ) with large deviations from an Arrhenius behavior and as 'strong' for $D>10$ following an Arrhenius law more closely. Many properties of glass-forming materials are correlated with their strength (fragility). For the different systems, we consistently find that the present charge-transfer salts are rather fragile glasses, which may be explained by a growing number of correlated molecules moving cooperatively, which leads to an increase of the apparent activation energy for decreasing temperatures.

Finally, we note that the cooling-rate dependence of the VFT behavior, observed for $\kappa-\mathrm{D}_{8}-\mathrm{Br}$ shown in Figure 8b, is unusual since, for classical glass-forming systems, the curves for different $q$ should coincide for $T>T_{g}$. Rather than being a purely dynamical effect, we have speculated that, for the present glass-like materials, different cooling rates produce different glass-forming molecular environments formed by the anion structure through short $\mathrm{C}-\mathrm{H} \cdots$ anion contacts, resulting in a varying fragility and therefore different values of $T_{g}$. 

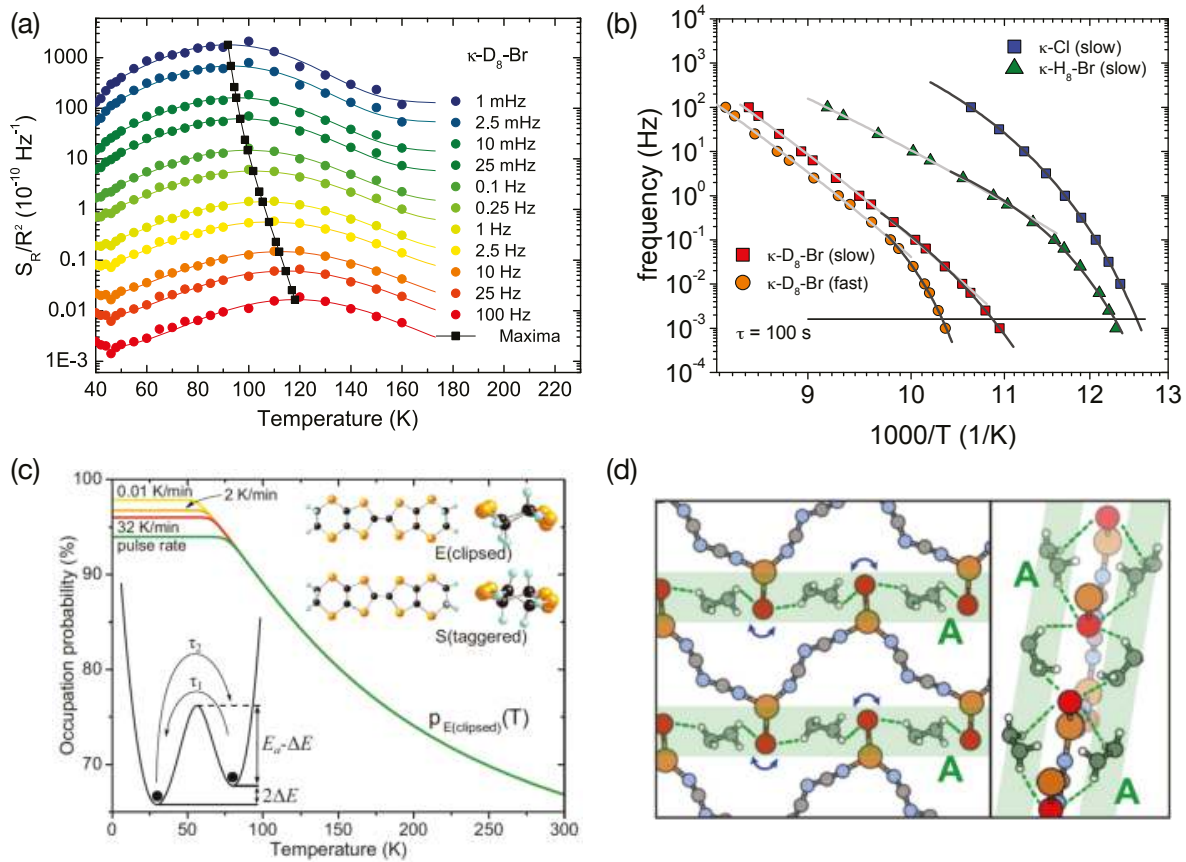

(d)

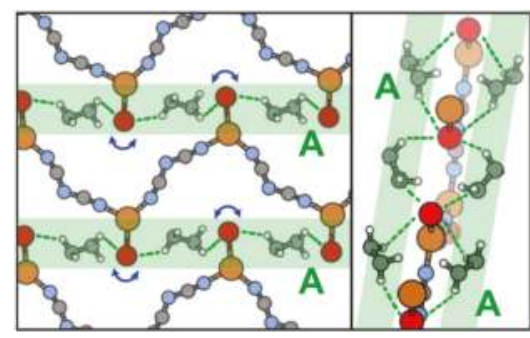

Figure 8. Glass-like structural EEG ordering in $\kappa-(\mathrm{ET})_{2} \mathrm{X}$. (a) normalized resistance noise PSD of $\kappa-\mathrm{D}_{8}-\mathrm{Br}$ for different frequencies. Lines are fits to a Gaussian function. Black circles connect the maxima at each frequency; (b) Arrhenius plot of the noise peak frequency for selected systems of $\kappa-(\mathrm{ET})_{2} \mathrm{X}$ (see legend). Light grey lines are fits of thermally activated (Arrhenius) behavior to the high-frequency data, whereas dark grey lines are Vogel-Fulcher-Tammann (VFT) fits after Equation (19) to the low-frequency data. The horizontal line equals $\tau=100 \mathrm{~s}$ and may be used to define the dynamic glass-transition temperature; (c) occupation probability $p(E)$ of the eclipsed (E) ground state conformation (see right inset) calculated from a simple two-level model (left inset) for various cooling rates. 'Pulse rate' refers to cooling after a heat pulse is applied resulting in cooling rates of order $1000 \mathrm{~K} / \mathrm{min}$. Reprinted with permission from [23]. Copyright (2014) by the American Physical Society; (d) preferred conformations of the EEGs for $\kappa$ - $\mathrm{Br}$ in relation to the nearby anion layer. Dashed lines indicate close EEG $\cdots$. Br contacts, while blue arrows indicate soft vibrational degrees of freedom of the terminal $\mathrm{Br}$ ligands suggested to couple to the EEG rotation. In $\kappa$-Br, all EEGs are crystallographically equivalent. (a,b,d) reproduced from [93]. Copyright IOP Publishing.

$\mathrm{Ab}$ initio quantum chemical calculations provide an explanation for the origin and phenomenology of the glassy dynamics in different systems in terms of a simple two-level model (see Figure 8c) where the relevant energy scales are determined by the coupling of the EEG to the anions [93]. For $\kappa-\mathrm{Br}$, the EEGs are all crystallographically equivalent and were computationally found to prefer the E conformation by $2 \Delta E / k_{\mathrm{B}} \sim 520 \mathrm{~K}$ per ET molecule, which is of the same order although somewhat larger than the value of $2 \Delta E / k_{\mathrm{B}} \sim 210 \mathrm{~K}$ estimated from the temperature dependence of the relative E/S occupancy of $\kappa$ - $\mathrm{Br}$ for fixed cooling rate [23]. The computed $E_{a}=2200 \mathrm{~K}$, however, lies well within the range of values found in various experiments of $E_{a} / k_{\mathrm{B}}=1900-3100 \mathrm{~K}$ as determined e.g., by NMR, specific heat, thermal expansion, resistivity measurements and noise spectroscopy $[19,84,104,105]$. Strikingly, more than half of the computed energy difference $2 \Delta E$ results from strong van der Waals coupling between the EEG and anion layers, which is dominated by close $\mathrm{Br} \cdots \mathrm{H}$ (or D) contacts between each EEG and two terminal ligands in adjacent anionic chains (see Figure 8d). The coupling of the terminal ligands' thermal motion with the EEG rotation in nearby 
ET molecules provides a possible mechanism for the cooperative behavior implied by the observed deviations from the Arrhenius law in Figure 8b, i.e., it is the collective motion of EEG and anions that freezes out at $T_{g}$ instead of being simply a property of the vibrational degrees of freedom of the EEG within each individual, separated ET layer [93,94].

It is interesting to consider the suggestion $[109,110]$ that the arrangement of the ET molecules and conformational preferences in $\kappa-(\mathrm{ET})_{2} \mathrm{X}$ salts with polymeric anions result from a particular pattern of empty spaces in the anion layers, allowing the donor molecules to fit in between, thus minimizing the van der Waals interactions. From this perspective, glassy freezing of the EEG rotation requires that the EEG-anion interaction is relatively ineffective at distinguishing the E and S states, allowing for similar energies of both states, and thus metastability. Indeed, in our simple model of Figure $8 \mathrm{c}$, it can be shown that the degree of frozen-in disorder is determined by the ratio of $E_{A} / \Delta E$ (at fixed $\tau_{0}$ ), with increasing occupancy of the minor conformation below $T_{g}$ with increasing ratio. We have suggested an empirical threshold of $E_{A} / \Delta E \sim 5$ for the occurrence of a glass transition in (BEDT-TTF) ${ }_{2} X$. Recently, this has led to the successful prediction of glass-like EEG freezing in the salt $\kappa-(\mathrm{ET})_{2} \mathrm{Hg}(\mathrm{SCN})_{2} \mathrm{Cl}$, where-as opposed to $\kappa-\mathrm{Cl}$ and $\kappa$ - $\mathrm{Br}$ - a peculiar EEG ordering occurs, in which only one of the two crystallographically inequivalent EEG is subject to glass-like ordering [94].

\subsection{Example (3): Charge-Cluster Glass}

In the previous section, we have shown exemplarily for the $\kappa$-phase $(\mathrm{ET})_{2} \mathrm{X}$ salts with polymeric anions that noise measurements allow for a spectroscopic study of glassy structural phenomena, where dielectric spectroscopy fails due to the high conductivity of the samples. Naturally, fluctuation spectroscopy is not only sensitive to electronic fluctuations coupled to a structural dynamic glass transition, but also to glassy phenomena of the electronic system itself. Here, the systems $\theta-(\mathrm{ET})_{2} \mathrm{MM}^{\prime}(\mathrm{SCN})_{4}$ with $\mathrm{M}=\mathrm{Tl}, \mathrm{Rb}, \mathrm{Cs}$ and $\mathrm{M}^{\prime}=\mathrm{Co}, \mathrm{Zn}$ recently have attracted considerable attention [111,112]. In these materials, the BEDT-TTF molecules form a triangular lattice and the charge transfer leads to a quasi-two-dimensional quarter-filled hole band system (that is, one hole per two BEDT-TTF molecules), in which the inter-site Coulomb repulsions give rise to an instability towards charge ordering $(\mathrm{CO})$. The system $\theta-(\mathrm{ET})_{2} \mathrm{RbZn}(\mathrm{SCN})_{4}$ undergoes a CO transition at $190 \mathrm{~K}$, where the charge carriers are localized periodically with a horizontal stripe pattern (see Figure 9a), which is regarded as a 'charge-crystal' state in contrast to the 'charge-liquid' state above the CO transition temperature, where the charge of +0.5 per one BEDT-TTF molecule is distributed uniformly in space. Geometrical frustration leads to an unconventional electronic 'charge-glass' state without long-range order, when the system is cooled faster than a critical rate $q_{c}$, which depends on the degree of geometric frustration. As first discussed in [113], a combination of noise measurements and X-ray diffraction reveals that the charge-liquid phase hosts two-dimensional charge clusters that fluctuate extremely slowly and heterogeneously. On further cooling, the cluster dynamics freezes, and a charge-cluster glass is formed.

Since slow dynamics accompanied by dynamic heterogeneities are known to be key experimental properties for the vitrification process in supercooled conventional liquids, resistance noise spectroscopy has been applied in order to detect the expected slow charge carrier dynamics in these materials upon approaching the glassy freezing of the electrons [111,113-115]. Figure 9b shows the resistance noise PSD in a representation $f^{\alpha} \times S_{R} / R^{2}$ vs. $f$ [113], which highlights the superposition of two-level fluctuations on top of a $1 / f^{\alpha}$ background noise, cf. Figure 6 b above. The important point here is that the two-level switching cannot be described by a single Lorentzian contribution but rather is modeled by a superposition of continuously distributed Lorentzians with high-frequency $\left(f_{c_{1}}\right)$ and low-frequency $\left(f_{c_{2}}\right)$ cutoffs. Thereby, the authors of [113] assume a density of states (distribution of relaxation times) $D(\tau) \propto 1 / \tau$ in between the related cutoff lifetimes $\tau_{1} \leq \tau \leq \tau_{2}$ in Equation (10) with $f_{c_{1}}=1 /\left(2 \pi \tau_{1}\right)$ and $f_{c_{2}}=1 /\left(2 \pi \tau_{2}\right)$. The integration in Equation (11) then yields curves of the spectral form shown in Figure $9 \mathrm{~b}$, which fit the data very well. In this scheme, the extracted values for $f_{c_{1}}$ and $f_{c_{2}}$ (see Figure 9c) reflect the fastest and slowest fluctuators, respectively, from which a centre frequency 
$f_{0}=\sqrt{f_{c_{1}} \cdot f_{c_{2}}}$ and a linewidth $f_{c_{1}} / f_{c_{2}}$ can be defined (see Figure $9 \mathrm{~d}$,e, respectively). The temperature profiles of these quantities show a slowing down of the center frequency $f_{0}$ by several orders of magnitude (see Figure 9d), before, at approximately $200 \mathrm{~K}$ a frustration-relaxing structural transition occurs. A strong increase of the ratio $f_{c_{1}} / f_{c_{2}}$ for decreasing temperature (see Figure $9 \mathrm{e}$ ) indicates a more heterogeneous dynamics [113]. Thus, the key experimental properties for the vitrification process in conventional supercooled liquids, namely slow and heterogeneous dynamics, are observed for the freezing of electric charges on a frustrated lattice.

(a)

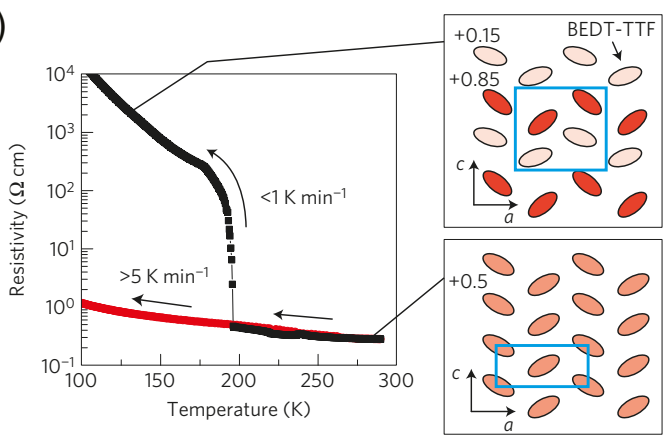

(c)

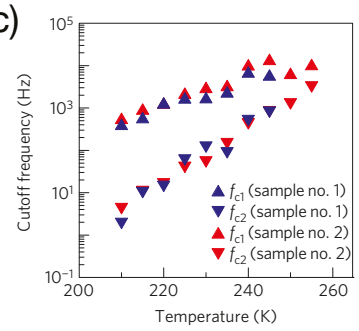

(d)

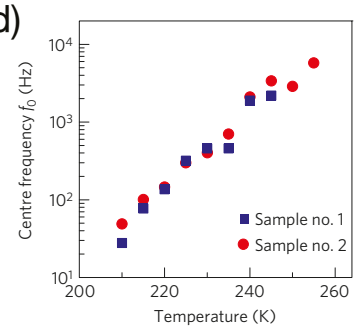

(b)

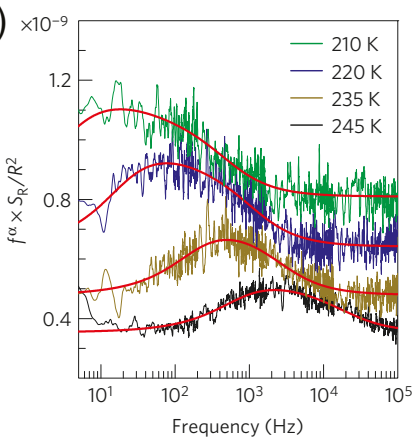

(e)

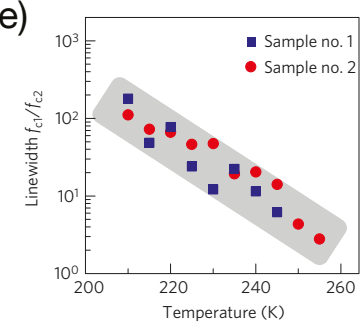

Figure 9. Charge-cluster glass in $\theta-(\mathrm{ET})_{2} \mathrm{RbZn}(\mathrm{SCN})_{4}$. (a) temperature dependence of the resistivity during cooling for different sweeping rates $q_{c}$. Insets indicate the crystal structures and charge distribution of the high-temperature (charge-liquid) phase (lower inset) and the slowly cooled low-temperature (charge-ordered) phase (upper inset); (b) resistance noise PSD for various temperatures as $f^{\alpha} \times S_{R} / R^{2}$ vs. $f$ with fits (red lines) to the distributed Lorentzian model (see text); (c) temperature profiles of the fitting parameters $f_{c_{1}}$ and $f_{c_{2}}$; (d) slowing of the centre frequency and (e) concomitant growth of the dynamic heterogeneity, respectively. Reprinted with permission from [113]. Copyright (2013) Springer Nature.

This notion of an unconventional electronic state without long-range order as inferred from the observed heterogeneous slow dynamics is corroborated by $\mathrm{X}$-ray diffusive scattering revealing that the charge-liquid phase above $200 \mathrm{~K}$ is transforming into a charge-cluster glass. This glass transition of the charge clusters formed in the charge-frustrated triangular lattice is expected to occur only if the frustration-relaxing transition at $200 \mathrm{~K}$ is kinetically avoided by rapid cooling and occurs at $T_{g} \sim 160-170 \mathrm{~K}$.

Further detailed resistance noise, time-dependent resistance and X-ray diffusive scattering experiments have been performed on the related system $\theta-(\mathrm{ET})_{2} \mathrm{CsZn}(\mathrm{SCN})_{4}$, which does not exhibit long-range charge order at usual laboratory time scales but always shows a charge-glass state, i.e., the critical cooling rate is very slow due to the higher degree of geometrical frustration [114]. Again, cooling-rate dependent charge vitrification and nonequilibrium aging behavior are successfully 
demonstrated. The temperature evolution of the relaxation time is found to obey an Arrhenius law $\tau \propto \exp \left(-\Delta / k_{B} T\right)$, indicating that the glass-forming charge liquid can be classified as a 'strong' liquid in the scheme of canonical structural-glass formers (see Section 3.2 above). The activation energy $\Delta / k_{B} \approx 224 \mathrm{meV}$ is ascribed to the energy related to the rearrangement of the charge configurations, which may be accompanied by a distortion of the local lattice/molecules [114]. Finally, the characteristics of the charge-glass are summarized as follows (see [116] and references therein): (i) the valence of BEDT-TTF appears to be spatially inhomogeneous; (ii) short-range order or charge clusters, with distinct symmetry from that of the charge-ordered state, develops as the temperature decreases, where below the charge-glass transition temperature, the characteristic size of the charge clusters saturates (iii) the time scale of charge fluctuations is slower than the laboratory time scale; and (iv) aging behavior is observed in resistivity, analogous to the dielectric aging in structural glasses.

Finally, in a recent work on monoclinic $\theta_{m}-(\mathrm{ET})_{2} \mathrm{TlZn}(\mathrm{SCN})_{4}$ - a material where the electron-lattice coupling and lattice distortion are weak and which is believed to approximate a system where the observed effects are purely electronic in nature-similar noise measurements as discussed above reveal an Arrhenius law of the Lorentzians' corner frequencies and confirm the emergence of slow dynamics accompanied by increasing dynamic heterogeneity upon approaching the charge-glass transition [111]. Here, it is important to note that the charge vitrification in the present case is distinctly different from the drastic slowing down of charge carrier dynamics and onset of non-Gaussian fluctuations observed in noise measurements as a precursor of metal-insulator transitions (MITs) [50-53] (see the discussion in the following Section 3.4). The electronic glassiness in MIT systems seemingly only becomes stabilized by disorder in the presence of strong electronic correlations and may not be observed for 'clean' samples.

\subsection{Example (4): Mott Metal-Insulator Transition}

In pressure-tuned $\kappa$-(BEDT-TTF $)_{2} \mathrm{Cu}\left[\mathrm{N}(\mathrm{CN})_{2}\right] \mathrm{Cl}$, unconventional critical exponents at the finite-temperature second-order critical endpoint $\left(p_{0}, T_{0}\right)$ of the Mott metal-insulator transition have been reported from DC electronic transport measurements [20]: the power-law exponents of the measured conductance upon approaching the critical point on the temperature and pressure axes seem to be inconsistent with any known universality classes (mean-field, 3D XY, Heisenberg and Ising). Since then, the critical properties have been a matter of controversial debate [117-120]. Recently, in [121], a breakdown of Hooke's law of elasticity has been observed at the critical endpoint of the Mott transition. These nonlinear strain-stress relations are assigned to an intimate, nonperturbative coupling of the critical electronic system to the lattice degrees of freedom. The authors point out that their results are fully consistent with mean-field criticality, predicted for electrons in a compressible lattice with finite shear moduli and argue that the Mott transition for all systems that are amenable to pressure tuning shows the universal properties of an isostructural solid-solid transition [121]. In contrast to these static properties of the Mott critical endpoint, dynamical critical phenomena are related to the existence of very long time scales known as critical slowing down of the order parameter relaxation rate, where the length and time scales that measure the correlations of the order parameter become infinite upon approaching a second-order phase transition. Therefore, the kinetics of the order parameter near a critical point causes a slow dynamics of correlated regions in a macroscopic system, and diverging resistance or conductance fluctuations are expected. However, this critical slowing down of the order-parameter fluctuations had not been observed for the Mott transition before.

In Section 3.2, we have mentioned that the cooling-rate dependence of the glass-like EEG ordering can be used to fine-tune systems through the critical region of the Mott transition. The effect originates in an anisotropic change of the in-plane lattice parameters at $T_{g}[19,86]$, which changes the relevant transfer integrals such that more rapid cooling leads to slightly smaller bandwidth (and $W / U$ ratio) (see discussion in [21,23]). In particular, in [24], it has been shown that the molecular conformations of the EEG are intimately connected to the electronic structure and significantly influence not only the transfer integrals and therefore $W$ but also the Hubbard repulsion parameter $U$. These results place 
$\kappa-(\mathrm{BEDT}-\mathrm{TTF})_{2} \mathrm{Cu}\left[\mathrm{N}(\mathrm{CN})_{2}\right] \mathrm{Br}$ in eclipsed (E) and staggered (S) configurations on opposite sides of the metal-insulator transition [24].

For our study of the dynamic criticality, we therefore have chosen a partially-deuterated system $\kappa-\left[\left(\mathrm{H}_{8}-\mathrm{ET}\right)_{0.2}\left(\mathrm{D}_{8}-\mathrm{ET}\right)_{0.8}\right]_{2} \mathrm{Cu}\left[\mathrm{N}(\mathrm{CN})_{2}\right] \mathrm{Br}$ (in short: $\kappa-\mathrm{H}_{8} / \mathrm{D}_{8}-\mathrm{Br}$ ), where the partial substitution of the ET molecules with their deuterated analogues places the sample on the high-pressure (metallic) side of the phase diagram very close to the critical pressure $p_{0}$ of the MIT [21]. Figure 10a shows the sample resistance for different cooling rates $q$ representing different positions of the sample in the generalized phase diagram, schematically indicated in Figure 10b [53]. Clearly, the slowly cooled (annealed) sample is located on the metallic/superconducting side of the phase diagram, whereas increasing $q$ shifts the sample to the left (low pressure, small $W / U$ ) towards the insulating side, where the typical re-entrant behavior, when crossing the first-order Mott transition line twice, is observed. We assume that the critical point is crossed by one of the curves taken in between the slowest and fastest cooling rate (colors from black to dark red). Blue color denotes the position of the fully-deuterated sample shown in Figure 7 above. The superconducting transition observed at low temperatures for all cooling rates is of inhomogeneous, percolating type due to the coexistence of metallic (superconducting) and insulating (antiferromagnetic) phases as discussed in Section 3.1 above. We note that the fine-tuning of the electronic bandwidth by controlling the cooling rate is reversible; i.e., upon warming above $T_{g}=75-80 \mathrm{~K}$, the frozen EEG glass melts and the lattice relaxes and the pristine state and position in the phase diagram is recovered.

(a)

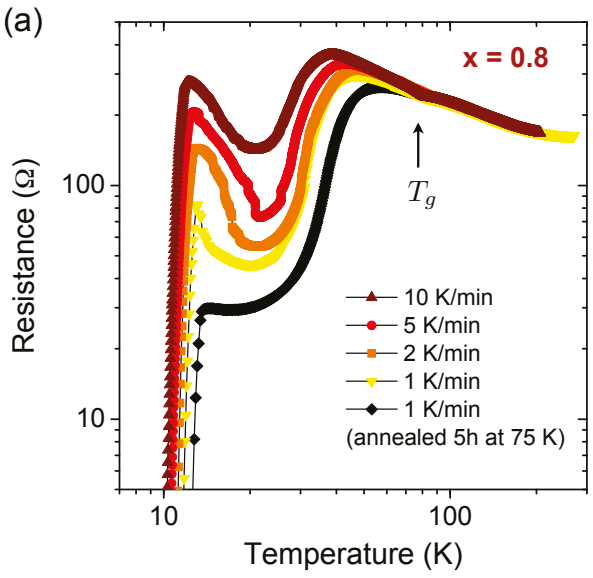

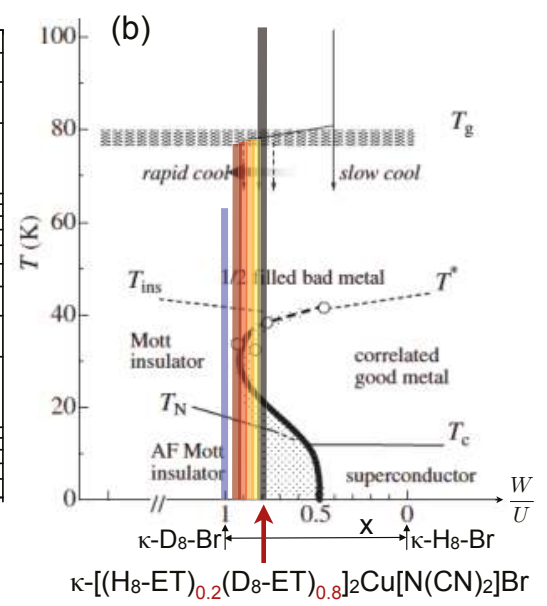

Figure 10. Tuning a partially-deuterated sample $\kappa-\left[\left(\mathrm{H}_{8}-\mathrm{ET}\right)_{1-x}\left(\mathrm{D}_{8}-\mathrm{ET}\right)_{x}\right]_{2} \mathrm{Cu}\left[\mathrm{N}(\mathrm{CN})_{2}\right] \mathrm{Br}$ with $x=0.8$ through the Mott transition. (a) resistance measurements for various cooling rates $q$; (b) generic phase diagram after [21]. Open circles indicate values taken from the literature of the second-order critical end point of the first-order Mott MIT (thick solid curve). The arrow indicates the position of the slowly cooled, pristine sample in the generic phase diagram. The change of the sample position with $q$ is schematically indicated by the colored lines. For comparison, the blue color represents fully-deuterated $\kappa-\mathrm{D}_{8}$-Br shown in Figure 7. Reprinted with permission from [53]. Copyright (2015) by the American Physical Society.

The comparison of $\kappa-\mathrm{D}_{8}-\mathrm{Br}$ (same sample as shown in Figure 7a, blue curve) with slowly-cooled $\kappa-\mathrm{H}_{8} / \mathrm{D}_{8}-\mathrm{Br}$ (yellow) is displayed in Figure 11a, where the normalized resistance noise PSD, $S_{R} / R^{2}$, taken at $1 \mathrm{~Hz}$ is shown. Measurements have been performed in a five-point AC bridge setup perpendicular to the conductive planes, i.e., along the crystallographic $b$-axis. In order to highlight the drastic changes of the fluctuation properties originating from different positions in the phase diagram tuned by the cooling rate $q$, i.e., the effects of electronic origin, the data are normalized to 
the broad maximum at around $100 \mathrm{~K}$, which we have shown in Section 3.2 to be of structural origin. Strikingly, the two samples behave similarly for temperatures above the glass-like transition $T_{g}$, where the resistance fluctuations are dominated by the EEG structural excitations. However, the $\kappa-\mathrm{H}_{8} / \mathrm{D}_{8}-\mathrm{Br}$ sample exhibits a considerably higher noise level below $T_{g}$, which may be caused by the higher degree of structural disorder due to the random distribution of $\mathrm{H}_{8}$ and $\mathrm{D}_{8}$ molecules, resulting in a tendency for the charge carriers to localize and/or electronic phase separation. Most strikingly, however, is the sharp peak in the noise at $T_{0} \sim 36 \mathrm{~K}$, which we attribute to the near vicinity of the critical endpoint of the Mott transition. Note that an increase of the noise accompanied by a shift of spectral weight to low frequencies is seen also for $\kappa-\mathrm{D}_{8}-\mathrm{Br}$ (see the arrow in Figure 11a as well as Figure $7 \mathrm{a}$, d). This sudden slowing down of the charge carrier dynamics due to critical fluctuations, however, for this sample remains a small effect as compared to the effect of the structural EEG fluctuations. This is not the case for $\kappa-\mathrm{H}_{8} / \mathrm{D}_{8}-\mathrm{Br}$, where upon increasing $q$, the peak in the noise initially increases (Figure 11b, orange squares) and becomes very pronounced and sharp (note the rescaled axis) for $q=5 \mathrm{~K} / \mathrm{min}$, red circles in Figure 11c, before it starts decreasing again for a larger cooling rate (dark red triangles). We therefore assume the sample's state at $q=5 \mathrm{~K} / \mathrm{min}$ as being closest to the critical point [122] and now evaluate the fluctuations in the whole accessible frequency range. The frequency exponent $\alpha(T)$, shown in Figure 11d, exhibits a monotonic increase from about $\alpha=0.8$ (at $T \sim 47 \mathrm{~K}$ ) to a very large value of about $\alpha=1.6$ (at $T \sim 32 \mathrm{~K}$ ) upon approaching the critical region from high temperatures. This corresponds to a drastic shift of spectral weight to low frequencies. This behavior is highlighted in Figure 11d, where the relative noise level $a_{R}=f \times S_{R} / R^{2}$ is shown, a dimensionless quantity characterizing the strength of fluctuations. We interpret the incipient power-law divergence of $a_{R}$ at very low (approaching $\mathrm{mHz}$ ) frequencies in a narrow temperature interval as critical slowing down of doublon density (two electrons sitting on the same atomic orbitals) fluctuations $[118,123,124]$, since the measured resistance (conductance) noise PSD is proportional to the Fourier transform of the voltage (current) autocorrelation function (Section 2.2), which couples to the kinetics of the order parameter of the Mott MIT $[20,125]$. Near a critical point, the latter is dominated by slow dynamics of correlated regions in a macroscopic system.

Whereas the strong increase of slow fluctuations in $\kappa-\mathrm{H}_{8} / \mathrm{D}_{8}-\mathrm{Br}$ is observed at a finite-temperature critical point, a similar behavior has been reported for metal-insulator transitions occurring only in the zero temperature limit, namely a disorder-driven Anderson transition in P-doped Si [52] and electron-density driven MIT in Si inversion layers (Si-MOSFET) [50,51,126]. It is interesting to consider if there is a connection to the notion of the quantum-critical nature of the Mott instability [125]. In both cases, the onset of non-Gaussian fluctuations resulting in a frequency-dependent second spectrum $S^{(2)}\left(f_{2}, f, T\right) \propto 1 / f_{2}^{\beta}$ (see Section 2.2.1) have been reported. If $\beta=0$, the statistics of the system is fully determined by the first spectrum $S_{R}(f, T)$ and the fluctuations are Gaussian. Slow dynamics and deviations from a 'white' second spectrum, i.e., $\beta \sim 1$ are an indication for correlated (interacting) fluctuators $[48,49]$ and have been observed for the abovementioned P-doped Si and the $\mathrm{Si}$ inversion layers systematically as a precursor of the MIT. A possible interpretation is a glassy freezing of the electrons preceding their localization, and it is suggested that such correlated dynamics may be a universal feature of MITs, irrespective of the systems' dimensionality [52]. In the present case of a finite-temperature critical endpoint, the deviations of the measured frequency exponent from $\alpha(T)$ calculated within the DDH model (assuming independent fluctuators) shown in Figure 11d for $q=5 \mathrm{~K} / \mathrm{min}$ and a frequency-dependent second spectrum with $\beta=1.35$ for $q=10 \mathrm{~K} / \mathrm{min}$ indicate a finite region in the phase diagram, where the fluctuations are correlated and deviate from Gaussian behavior. 

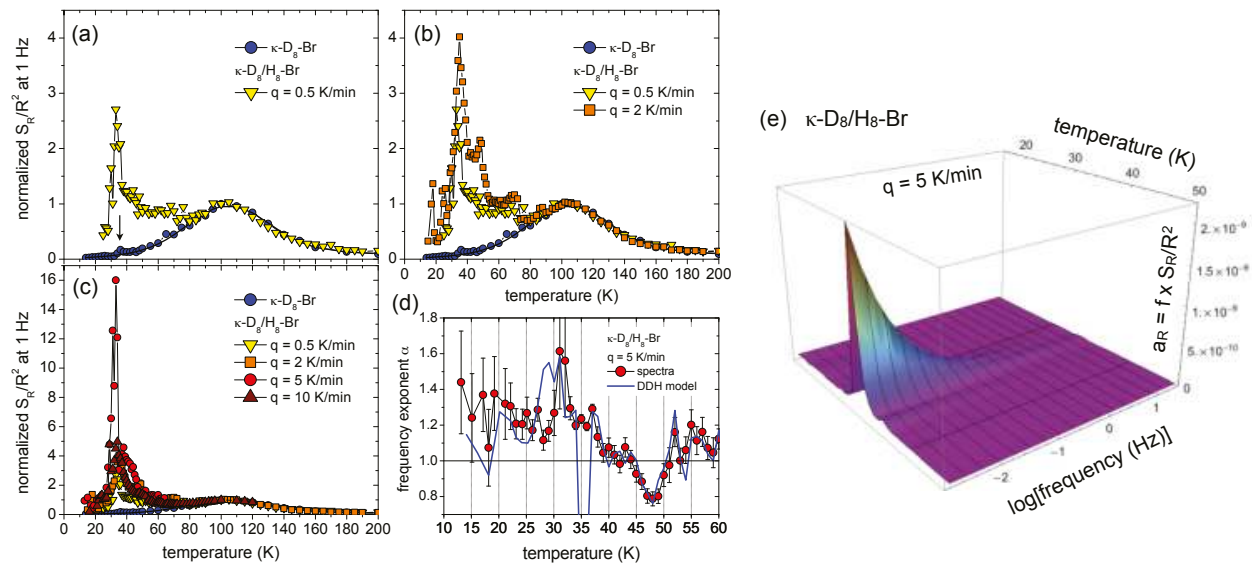

Figure 11. Critical slowing down of charge fluctuations. (a) resistance noise PSD $S_{R} / R^{2}(T, f=1 \mathrm{~Hz})$ of slowly-cooled $\kappa-\mathrm{H}_{8} / \mathrm{D}_{8}$-Br (yellow triangles) in comparison to the data of $\kappa-\mathrm{D}_{8}$-Br (blue circles) normalized to the value of the local maximum around $100 \mathrm{~K}$. The black arrow indicates a peak in the noise level, cf. data of the same sample shown in Figure 7a. $(\mathbf{b}, \mathbf{c})$ measurements for increasing cooling rates $q$. Note the rescaling of the ordinate in (c); (d) frequency exponent $\alpha(T)$ for $q=5 \mathrm{~K} / \mathrm{min}$, where the noise level shown in (c) is largest. The line represents the DDH model calculation; (e) relative noise level $a_{R} \equiv f \times S_{R} / R^{2}$ vs. $f$ vs. $T$ for the same cooling rate. Reprinted with permission from [53]. Copyright (2015) by the American Physical Society.

Here, a residual structural disorder potential and/or the inherent frustration of the triangular lattice geometry of the ET molecules forming dimers could result in a large number of metastable states, which the strongly interacting electrons have to overcome. General models for such correlated dynamics applied to spin glasses are often invoked also for the charge degrees of freedom $[51,126]$. In a model of interacting droplets or clusters, $S^{(2)}\left(f_{2}, f, T\right)$ should be a decreasing function of $f$ for constant $f_{2} / f[49]$. For the present system, however, we observe a scale invariance in $f$ pointing to a hierarchical picture, where the system wanders collectively between metastable states related by a kinetic hierarchy. The large exponent of $\beta=1.35$ found in our experiment for the sample cooled with $q=10 \mathrm{~K} / \mathrm{min}$ indicates that only few such states are visited during the time of measurement. From the theoretical point of view, a Coulomb glass behavior in the vicinity of the Mott transition remains controversial. For example, a self-generated glass transition caused by the frustrated nature of the interactions (and not related to the presence of quenched disorder) is predicted in doped Mott insulators [127]. In [128], however, the metallic glassy phase is suggested to be suppressed for Mott localization and to become stabilized only for increasing disorder, in apparent agreement with experimental results on $\mathrm{Si}$ inversion layers with different degrees of disorder.

A systematic mapping of the region of ergodicity breaking for samples at different positions in the phase diagram (i.e., varying distance to the critical endpoint) and with different degrees of quenched disorder (random lattice potential) may help to answer these fundamental questions and will be an elaborate but worthwhile future effort.

\section{Sample Dependences}

Instead of using different cooling rates, an alternative way to tune through the phase diagram in even finer, in principle arbitrarily small steps is to apply different thermal relaxation protocols in the vicinity of the transition temperature $T_{g}$ for glass-like EEG ordering [25]. Again, we choose a partially-deuterated compound, which is located very close to the Mott transition, $\kappa-\left[\left(\mathrm{H}_{8}-\mathrm{ET}\right)_{0.25}\left(\mathrm{D}_{8}-\mathrm{ET}\right)_{0.75}\right]_{2} \mathrm{Cu}\left[\mathrm{N}(\mathrm{CN})_{2}\right] \mathrm{Br}$ with a deuteration degree of $x=0.75$. Depending on the 
initial more or less rapid cooling, a certain amount of the EEGs energetically unfavored staggered (S) conformation is frozen, which results in a reduced ratio of bandwidth to on-site Coulomb repulsion $W / U[24]$ and thus in a state further on the insulating side of the phase diagram than the pristine, slowly-cooled condition. The so-prepared initial state, which is referred to as warming cycle 1 , is characterized by a resistance showing the typical re-entrance behavior (see the red curve in Figure 12a, where the Mott transition line is crossed twice).

(a)

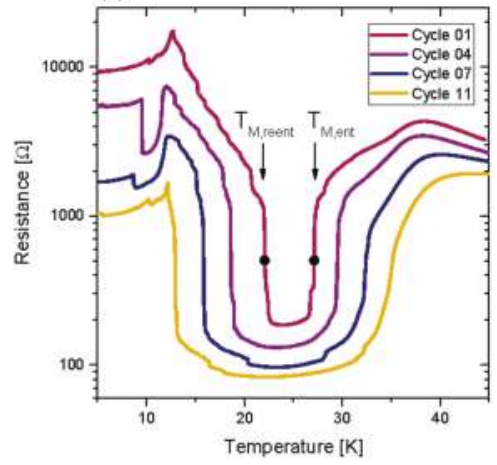

(b)

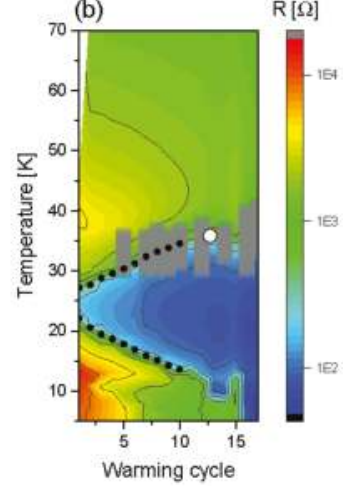

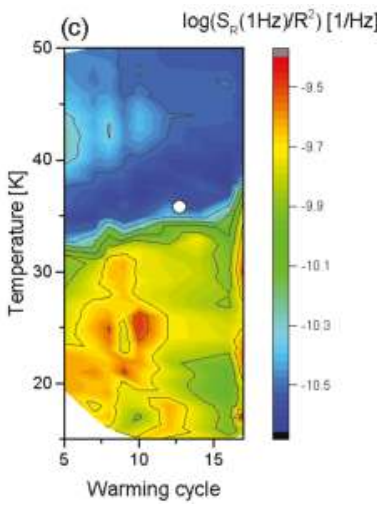

Figure 12. Resistance and noise measurements at the Mott transition in partially-deuterated $\kappa-\left[\left(\mathrm{H}_{8}-\mathrm{ET}\right)_{1-x}\left(\mathrm{D}_{8}-\mathrm{ET}\right)_{x}\right]_{2} \mathrm{Cu}\left[\mathrm{N}(\mathrm{CN})_{2}\right] \mathrm{Br}$ with $x=0.75$. (a) resistance measurements of some selected warming cycles generated by using different thermal relaxation protocols described in the text. The black points mark the temperatures of the entrance and re-entrance half-maximum resistance jump, indicating the crossing of the Mott transition line; (b) contour plot of the resistance vs. temperature and warming cycle. The grey area marks the region where the DDH model shows strong deviations; the white circle indicates the critical point; (c) contour plot of the normalized resistance noise PSD taken at $1 \mathrm{~Hz}$ in dependence of temperature and warming cycle (note different scales).

After the initial cool down with a rate of $q=2.5 \mathrm{~K} / \mathrm{min}$, the sample is warmed up to a temperature close to the glass transition at about $T_{g} \sim 75 \mathrm{~K}$. After a specific waiting time on the order of a few minutes, which relaxes the EEG occupation to the energetically favored eclipsed orientation, and thus the structural distortion, the sample is slowly cooled down again while measuring the resistance yielding a new state with a larger ratio of $W / U$, and therefore located further on the metallic side of the phase diagram. By repeating this relaxation protocol several times (and by adjusting the relaxation time), the sample can be tuned-in principle in arbitrarily small steps-through the Mott critical endpoint. Some selected resistance measurements of the generated states (warming cycles 1-17) are shown in Figure 12a, where the higher warming cycles are characterized by a lower resistance value and-due to the $S$ shape of the transition line-a broader region in the metallic phase. From the temperature values of the entrance and re-entrance half-maximum resistance jump, $T_{M, \text { ent }}$ and $T_{M, \text { reent }}$, respectively, marked by black points in the resistance measurements, it is possible to reproduce the Mott transition line in dependence of the warming cycle. A contour plot of the resistance as a function of temperature and warming cycle is shown in Figure $12 \mathrm{~b}$ where the blue color corresponds to a low-resistance metallic state and the red color to the high-resistance insulating state. Hence, at the warming cycle for which the corresponding resistance curve turns into a continuous course without clear discontinuities, the critical endpoint is expected and is visualized as a white circle at $T_{0} \sim 36 \mathrm{~K}$ in Figures $12 b, c$.

In order to analyze the charge carrier dynamics at the Mott transition in comparison to the sample with a deuteration degree of $x=0.8$ discussed above, we employ fluctuation spectroscopy in a four-terminal AC configuration, where the resistance fluctuations are again measured out-of-plane, 
i.e., along the crystallographic $b$-axis. For each warming cycle, the resistance noise PSD was measured during cooling down the sample (in discrete temperatures steps $\Delta T=1 \mathrm{~K}$ ) starting at $T=50 \mathrm{~K}$ sufficiently far below the glass-like transition so that the EEG configuration is frozen. The results of the normalized resistance noise PSD taken at $1 \mathrm{~Hz}$ for different warming cycles are shown in Figure 12c as a contour plot vs. temperature vs. warming cycle. Similar as for the sample $x=0.8$ shown in Figures 10 and 11, the noise level increases when approaching the Mott MIT. However, instead of a large peak indicating diverging low-frequency fluctuations in the vicinity of the critical point, for the present sample $x=0.75 \mathrm{~m}$, we observe a one-order-of-magnitude, step-like increase of the normalized resistance noise PSD when crossing the Mott transition line, which shifts to higher temperatures for higher warming cycles coinciding with the development of the metallic phase. Even for warming cycles beyond the critical point (the white circle in Figure 12b,c), this behavior is observed at the crossover from the 'bad metal' to the Fermi liquid region. One possible explanation for the higher noise level in the metallic phase is electronic phase separation into insulating and metallic domains due to the competing interactions in the vicinity of the Mott transition [21,82,129]. Since electronic inhomogeneities are accompanied by a smaller noisy volume, the formation of domains on the micrometer to nanometer scale close to the Mott transition might cause a larger noise PSD in the metallic region.

In addition to the absolute noise level at $1 \mathrm{~Hz}$, the frequency exponent $\alpha(T)$ gives information about the distribution of activation energies within the DDH model and is shown in Figure 13 for selected warming cycles to be compared with Figure $7 \mathrm{~b}$ for $\kappa-\mathrm{D}_{8}-\mathrm{Br}(x=1)$ and Figure $11 \mathrm{~d}$ for $\kappa-\mathrm{H}_{8} / \mathrm{D}_{8}-\mathrm{Br}(x=0.8)$. Whereas for higher temperatures below $50 \mathrm{~K}$ a weak temperature dependence with $\alpha$ slightly larger than 1 is observed, for all warming cycles, a peak in $\alpha(T)$ is found with values of 1.2-1.4 occurs around the temperature of the Mott MIT. Although this shift of spectral weight to low frequencies is not as pronounced and the noise does not peak as is observed for the $x=0.8$ sample, there is a consistent slowing down of the charge carrier dynamics at the transition. Strikingly, there is also a distinct region around the Mott MIT, where the DDH model fails to describe the low-frequency fluctuations. The black line in Figure 13 shows the values obtained from the DDH model (Equation (16)), where a power-law function $g(T)$ has been used in order to take an explicit temperature dependence of the resistance fluctuations into account, with different exponents for the low and high temperature regions indicating a change in the fluctuating properties, their number or their coupling to the resistance and/or the electronic transport mechanism. Strikingly, outside the transition region the DDH model agrees very well with the experimental $\alpha(T)$, i.e., the energy distribution of independent fluctuators can be determined rather accurately (not shown) [130]. However, in the transition region-marked by the gray shaded area in Figures 12 and 13-there is a strong deviation of the measured $\alpha(T)$ and the DDH calculation implying that the assumption of independent fluctuating entities loses its validity in the region close the Mott transition and non-Gaussian, possibly spatially-correlated fluctuations are dominating. This again is similar to the $x=0.8$ sample discussed above, where this phenomenon was also observed and confirmed by measurements of the second spectrum $S^{(2)}\left(f_{2}, f, T\right)$, which revealed a frequency dependence for the curve with $q=10 \mathrm{~K} / \mathrm{min}$ resulting in a state close but on the insulating side beyond the critical point [53]. The measurements on the present $x=0.75$ sample, although not showing a divergent increase of the noise around the critical endpoint, exhibit a rather wide region of non-Gaussian noise and possibly ergodicity breaking around the Mott MIT. Besides these similarities, the differences demonstrate strong sample dependences of the investigated compounds, which in part may be related to different degrees of disorder. Measurements of different samples with nominally the same degree of deuteration are desirable. In addition, a systematic investigation of the interplay of disorder and strong electron-electron correlations is possible. To that end, samples with different degrees of deuteration and therefore pristine starting position in the phase diagram could be tuned through the phase diagram by applying warming cycles. This would result in a varying degree of intrinsic frozen EEG disorder at the critical endpoint of the Mott transition allowing for studying the influence of a random lattice portential on the critical slowing down of the charge carrier dynamics. 


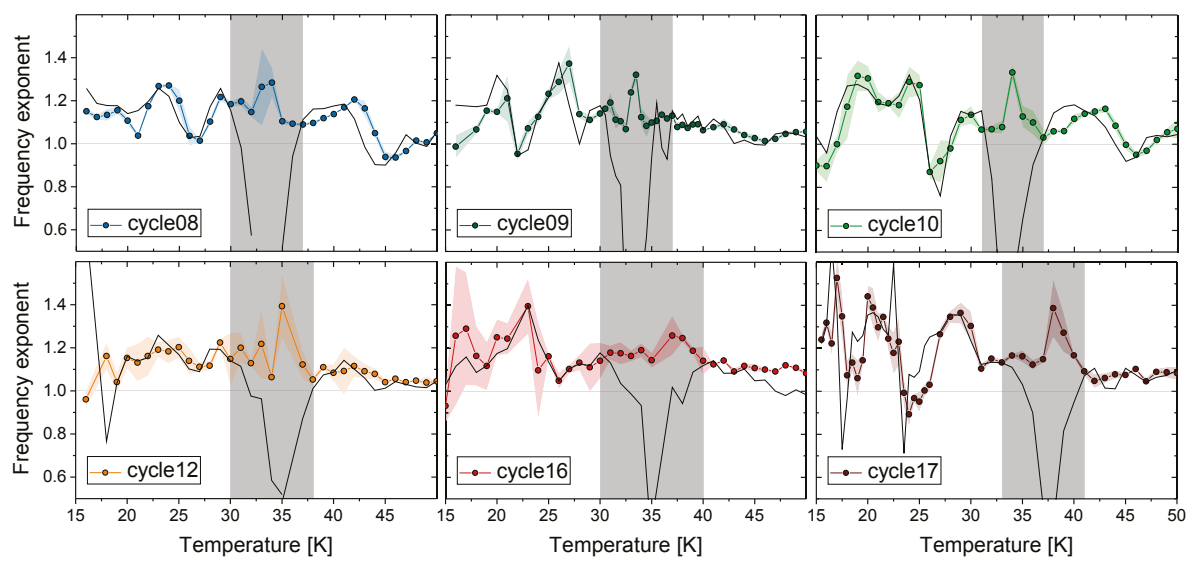

Figure 13. Temperature dependent frequency exponent $\alpha$ for some selected warming cycles. The black line corresponds to the values determined with the DDH model; the grey shaded area emphasizes the temperature region where the DDH model deviates from the experimental data.

\section{Conclusions}

To summarize, the fascinating electronic, magnetic and structural properties of quasi-two-dimensional strongly-correlated molecular metals (BEDT-TTF $)_{2} \mathrm{X}$ have been introduced and the need for studying the charge carrier dynamics at low frequencies-complementary to optical and dielectric spectroscopy - has been motivated. We have given an overview of the definitions and basic relations of the statistical properties of physical quantities with the focus on time-resolved electronic transport measurements. At the heart of noise spectroscopy measuring the resistance (or conductance) noise power spectral density $S_{R}\left(S_{G}\right)$ is a time-like property, namely the voltage (or current) autocorrelation function $\Psi_{I I(V V)}(\tau)$, which is a non-random characteristic of the microscopic kinetics of the charge fluctuations. The fluctuation spectrum and the autocorrelation function are related via the Wiener-Khintchine theorem. The fluctuation-dissipation theorem makes an exact connection between the autocorrelation function and the imaginary part of the frequency-dependent response function associated with dissipation in thermal equilibrium.

The examples selected for this review demonstrate where studying low-frequency charge carrier dynamics via the electronic noise provides information on the underlying physics that cannot be easily (or at all) gained otherwise. In general, noise is a sensitive probe, when the electronic transport (the current distribution in a sample) is inhomogeneous, which is the case for electronic phase separation and at percolative transitions (1). Another important example, where slow dynamics dominates the physics of the system, is a glassy freezing either of structural degrees of freedom coupling to the electronic properties (2) or of the electrons themselves, either when residing on a highly-frustrated crystal lattice (3) or due to correlated transitions of electrons over a large number of metastable states, leading to glassy relaxation as a precursor of a metal-insulator transition (4). Indications for such an emerging behavior, like diverging low-frequency fluctuations and critical slowing down of the order parameter fluctuations, for the first time have been observed at the finite-temperature critical endpoint of the Mott transition. Here, mapping out the region of ergodicity breaking and understanding the influence of disorder on the temporal and spatial correlated fluctuations will be an important realm of future studies.

Combining different experimental techniques from four-point and five-point AC or DC resistance fluctuation measurements to two-terminal DC conductance noise measurements, an impressive span of sample impedances from $\mathrm{m} \Omega-\mathrm{G} \Omega$ can be covered. This allows for extending the applications of noise studies to samples, also non-ET-based compounds [131,132], deep in the Mott or charge-ordered 
insulating phase, where spin-liquid behavior is observed and the intra-dimer degrees of freedom often cause intriguing dielectric relaxation phenomena or ferroelectric ordering. First measurements of such insulating samples, e.g., of the dimer-Mott insulators $\kappa-(\mathrm{ET})_{2} \mathrm{Cu}_{2}(\mathrm{CN})_{3}$ and $\beta^{\prime}-(\mathrm{ET})_{2} \mathrm{ICl}_{2}$, are underway. They will focus on the origin of relaxor ferroelectricity and make a connection between the concepts of conductance fluctuation spectroscopy and dielectric spectroscopy.

Acknowledgments: This work was financially supported by the Deutsche Forschungsgemeinschaft (DFG) within the collaborative research center SFB/TR 49 "Condensed Matter Systems with Variable Many-Body Interactions". We are particularly grateful to John A. Schlueter from Argonne National Laboratory and NSF, USA and Takahiko Sasaki from the Institute for Materials Research (IMR) at Tohoku University, Sendai, Japan for the fruitful and rewarding collaboration in the past years and for kindly providing many excellent samples. J.M. acknowledges pleasantly working with many students from Goethe-University Frankfurt on noise properties of organic charge-transfer salts, namely Jens Brandenburg, Robert Rommel, Benedikt Hartmann, David Zielke, Jana Polzin, Yannick Herrmann, Yassine Agarmani, Marvin Kopp and Martin Lonsky.

Conflicts of Interest: The authors declare no conflicts of interest.

\section{Abbreviations}

The following abbreviations are used in this manuscript:

$\begin{array}{ll}\text { CO } & \text { charge ordering } \\ \text { DDH model } & \text { model by Dutta, Dimon and Horn for } 1 / f \text {-type noise in metals } \\ \text { EEG } & \text { ethylene endgroups } \\ \text { ET } & \text { BEDT-TTF (bis-ethylenedithio-tetrathiafulvalene) } \\ \text { FDT } & \text { fluctuation-dissipation theorem } \\ \kappa-B r & \kappa-(B E D T-T T F)_{2} \mathrm{Cu}\left[\mathrm{N}(\mathrm{CN})_{2}\right] \mathrm{Br} \\ \kappa-\mathrm{Cl} & \kappa-(\mathrm{BEDT}-\mathrm{TTF})_{2} \mathrm{Cu}\left[\mathrm{N}(\mathrm{CN})_{2}\right] \mathrm{Cl} \\ \mathrm{MIT} & \text { metal-insulator transition } \\ \mathrm{NMR} & \text { nuclear magnetic resonance } \\ \text { PSD } & \text { power spectral density } \\ \text { RRN } & \text { random resistor network } \\ \text { VFT } & \text { Vogel-Fulcher-Tammann }\end{array}$

\section{References and Notes}

1. Ishiguro, T.; Yamaji, K.; Saito, G. Organic Superconductors; Springer: Berlin/Heidelberg, Germany, 1998.

2. Toyota, N.; Lang, M.; Müller, J. Low-Dimensional Molecular Metals; Solid State Science, Springer: Berlin/Heidelberg, Germany, 2007.

3. Lebed, A. The Physics of Organic Superconductors and Conductors; Springer: Berlin/Heidelberg, Germany, 2008.

4. Powell, B.J.; McKenzie, R.H. Quantum frustration in organic Mott insulators: From spin liquids to unconventional superconductors. Rep. Prog. Phys. 2011, 74, 056501.

5. Lunkenheimer, P.; Müller, J.; Krohns, S.; Schrettle, F.; Loidl, A.; Hartmann, B.; Rommel, R.; de Souza, M.; Hotta, C.; Schlueter, J.A.; et al. Multiferroicity in an organic charge-transfer salt that is suggestive of electric-dipole-driven magnetism. Nat. Mater. 2012, 11, 755-758.

6. Dressel, M.; Drichko, N. Optical Properties of Two-Dimensional Organic Conductors: Signatures of Charge Ordering and Correlation Effects. Chem. Rev. 2004, 104, 5689-5716.

7. Iwai, S. Photoinduced Phase Transitions in $\alpha-, \theta-$, and $\kappa$-type ET Salts: Ultrafast Melting of the Electronic Ordering. Crystals 2012, 2, 590-617.

8. Lunkenheimer, P.; Loidl, A. Dielectric spectroscopy on organic charge-transfer salts. J. Phys. Condens. Matter 2015, 27, 373001 .

9. Müller, J. Fluctuation Spectroscopy: A New Approach for Studying Low-Dimensional Molecular Metals. ChemPhysChem 2011, 12, 1222-1245.

10. Powell, B.J.; McKenzie, R.H. Strong electronic correlations in superconducting organic charge transfer salts. J. Phys. Condens. Matter 2006, 18, R827.

11. Kandpal, H.C.; Opahle, I.; Zhang, Y.Z.; Jeschke, H.O.; Valentí, R. Revision of Model Parameters for $\kappa$-Type Charge Transfer Salts: An Ab Initio Study. Phys. Rev. Lett. 2009, 103, 067004. 
12. Seo, H.; Hotta, C.; Fukuyama, H. Toward Systematic Understanding of Diversity of Electronic Properties in Low-Dimensional Molecular Solids. Chem. Rev. 2004, 104, 5005-5036.

13. Lang, M.; Lunkenheimer, P.; Müller, J.; Loidl, A.; Hartmann, B.; Hoang, N.H.; Gati, E.; Schubert, H.; Schlueter, J.A. Multiferroicity in the Mott Insulating Charge-Transfer Salt $\kappa$-(BEDT-TTF $)_{2} \mathrm{Cu}\left[\mathrm{N}(\mathrm{CN})_{2}\right] \mathrm{Cl}$. IEEE Trans. Magn. 2014, 50, 2700107.

14. Wosnitza, J. Fermi Surfaces of Low-Dimensional Organic Metals and Superconductors; Springer: Berlin/Heidelberg, Germany, 1996.

15. Singleton, J. Studies of quasi-two-dimensional organic conductors based on BEDT-TTF using high magnetic fields. Rep. Prog. Phys. 2000, 63, 1111-1207.

16. Elsinger, H.; Wosnitza, J.; Wanka, S.; Hagel, J.; Schweitzer, D.; Strunz, W. $\kappa-(B E D T-T T F)_{2} \mathrm{Cu}\left[\mathrm{N}(\mathrm{CN})_{2}\right] \mathrm{Br}$ : A Fully Gapped Strong-Coupling Superconductor. Phys. Rev. Lett. 2000, 84, 6098-6101.

17. Kanoda, K. Electron correlation, metal-insulator transition and superconductivity in quasi-2D organic systems (ET) $)_{2}$ X. Phys. C Superconduct. 1997, 282-287, 299-302.

18. Lefebvre, S.; Wzietek, P.; Brown, S.; Bourbonnais, C.; Jérome, D.; Mézière, C.; Fourmigué, M.; Batail, P. Mott Transition, Antiferromagnetism, and Unconventional Superconductivity in Layered Organic Superconductors. Phys. Rev. Lett. 2000, 85, 5420-5423.

19. Müller, J.; Lang, M.; Steglich, F.; Schlueter, J.A.; Kini, A.M.; Sasaki, T. Evidence for structural and electronic instabilities at intermediate temperatures in $\kappa-(\mathrm{BEDT}-\mathrm{TTF})_{2} \mathrm{X}$ for $\mathrm{X}=\mathrm{Cu}\left[\mathrm{N}(\mathrm{CN})_{2}\right] \mathrm{Cl}, \mathrm{Cu}\left[\mathrm{N}(\mathrm{CN})_{2}\right] \mathrm{Br}$ and $\mathrm{Cu}(\mathrm{NCS})_{2}$ : Implications for the phase diagram of these quasi-two-dimensional organic superconductors. Phys. Rev. B 2002, 65, 144521.

20. Kagawa, F.; Miyagawa, K.; Kanoda, K. Unconventional critical behaviour in a quasi-two-dimensional organic conductor. Nature 2005, 436, 534-537.

21. Sasaki, T.; Yoneyama, N.; Suzuki, A.; Kobayashi, N.; Ikemoto, Y.; Kimura, H. Real Space Imaging of the Metal-Insulator Phase Separation in the Band Width Controlled Organic Mott System $\kappa$-(BEDT-TTF $)_{2} \mathrm{Cu}\left[\mathrm{N}(\mathrm{CN})_{2}\right]$ Br. J. Phys. Soc. Jpn. 2005, 74, 2351-2360.

22. Kawamoto, A.; Miyagawa, K.; Kanoda, K. Deuterated $\kappa-(\mathrm{BEDT}-\mathrm{TTF})_{2} \mathrm{Cu}\left[\mathrm{N}(\mathrm{CN})_{2}\right] \mathrm{Br}$ : A system on the border of the superconductor-magnetic-insulator transition. Phys. Rev. B 1997, 55, 14140-14143.

23. Hartmann, B.; Müller, J.; Sasaki, T. Mott metal-insulator transition induced by utilizing a glasslike structural ordering in low-dimensional molecular conductors. Phys. Rev. B 2014, 90, 195150.

24. Guterding, D.; Valentí, R.; Jeschke, H.O. Influence of molecular conformations on the electronic structure of organic charge transfer salts. Phys. Rev. B 2015, 92, 081109.

25. Müller, J.; Hartmann, B.; Sasaki, T. Fine-tuning the Mott metal-insulator transition and critical charge carrier dynamics in molecular conductors. Philos. Mag. 2017, 97, 3477-3494.

26. Landauer, R. Condensed-matter physics: The noise is the signal. Nature 1998, 392, 658-659.

27. Beenakker, C.; Schönenberger, C. Quantum shot noise. Phys. Today 2003, 56, 37-42.

28. MacDonald, D.K.C. Noise and Fluctuations: An Introduction; John Wiley \& Sons: New York, NY, USA, 1962.

29. Van der Ziel, A. Flicker noise in electron devices. Adv. Electron. Electron Phys. 1979, 49, 225-297.

30. Hooge, F.N.; Kleinpenning, T.G.M.; Vandamme, L.K.J. Experimental studies on $1 / f$ noise. Rep. Prog. Phys. 1981, 44, 479-532.

31. Dutta, P.; Horn, P.M. Low-frequency fluctuations in solids: $1 / f$ noise. Rev. Modern Phys. 1981, 53, 497-516.

32. Weissman, M.B. $1 / f$ noise and other slow, nonexponential kinetics in condensed matter. Rev. Modern Phys. 1988, 60, 537-571.

33. Kogan, S. Electronic Noise and Fluctuations in Solids; Cambridge University Press: Cambridge, UK, 1996.

34. Raquet, B. Electronic Noise in Magnetic Materials and Devices. In Spin Electronics; Springer: Berlin/Heidelberg, Germany, 2000; Chapter 11, pp. 232-273.

35. Indeed, this "DC-offset" is sought to be suppressed in a five-terminal experimental setup [9,61], so that only the fluctuating part can be amplified and analyzed. If not suppressed, a finite mean value contributes a $\delta$-function to $S_{x}(f)$ at zero frequency.

36. The ergodicity theorem states that the time-average for a single system in statistical equilibrium may be replaced by an average over an equilibrium ensemble. The autocorrelation function (Equation (3)) is to be evaluated at a fixed time $t$ over a sufficiently large ensemble in thermal equilibrium or through averaging the same system over a sufficiently large number of different times $t$. In each case, the result is independent of $t$ (and only depends on $\tau$ ) for a statistically stationary variable. 
37. Lax, M.; Wei, C.; Min, X. Random Processes in Physics and Finance; Oxford University Press: New York, NY, USA, 2006.

38. The function $\chi(t)$ is defined by the response of the quantity $x$ to an external (generalized) force $F(t)$ via

$$
\langle x(t)\rangle=\int_{-\infty}^{\infty} \chi\left(t-t^{\prime}\right) F\left(t^{\prime}\right) \mathrm{d} t^{\prime}
$$

with $t>t^{\prime}$ (causality). With the Fourier transform $\chi(\omega)=\int_{0}^{\infty} \chi(t) e^{\mathrm{i} \omega t} \mathrm{~d} t=\chi^{*}(-\omega)$, the convolution theorem gives $x(\omega)=\chi(\omega) \cdot F(\omega)$.

In the complex dynamic susceptibility $\chi(\omega)=\chi^{\prime}(\omega)+\mathrm{i} \chi^{\prime \prime}(\omega)$, the real part $\chi^{\prime}(\omega)$ is the elastic (in-phase) part and the imaginary part $\chi^{\prime \prime}(\omega)$ the dissipative (out-of-phase) part of the response.

The FDT

$$
S_{x}(f)=2 \hbar \operatorname{coth}\left(\frac{h f}{2 k_{B} T}\right) \chi^{\prime \prime}(\omega)
$$

describes the direct connection between the autocorrelation function of the fluctuations and the imaginary part of the response function associated with dissipation and becomes Equation (5) in the quasi-classical limit $k_{B} T \ll h f$.

39. Johnson, J.B. Thermal Agitation of Electricity in Conductors. Phys. Rev. 1928, 32, 97-109.

40. Nyquist, H. Thermal Agitation of Electric Charge in Conductors. Phys. Rev. 1928, 32, 110-113.

41. Note that for thermal noise as the limiting factor, the signal-to-noise ratio of a voltage measurement, defined as $S N R=V / \sqrt{\int_{f_{\min }}^{f_{\max }} S_{V}(f) \mathrm{d} f}=\sqrt{R /\left(4 k_{B} T \Delta f\right)} \cdot I$, with bandwidth $\Delta f=f_{\max }-f_{\min }$, can be enhanced by increasing the current $I$. This is not the case, if $1 / f$-noise limits the accuracy of a physical measurement! As can be seen for example when calculating the $S N R$ using Equation (12) below, $1 / f$-noise represents a big obstacle for miniaturization (e.g., of nanoscale devices).

42. Press, W.H. Flicker Noise in Astronomy and Elsewhere. Comments Astrophys. Space Phys. 1978, 7, 103-119.

43. Note that the (biased) random walk plays an important role in finance, i.e., in modern risk management and for the pricing of derivates.

44. Of course, there should be theoretical limits also. At high frequencies, there can be no fluctuations faster than the fastest electronic processes possible in the system. At low frequencies, however, a cutoff has not been observed down to the $10^{-6} \mathrm{~Hz}$ regime for $1 / f$-noise in Ge semiconductor devices [133]. How to explain correlations ('memory') over averaging times of several weeks remains a fascinating intellectual problem.

45. Machlup, S. Noise in Semiconductors: Spectrum of a Two-Parameter Random Signal. J. Appl. Phys. 1954, 25, 341-343.

46. McWhorter, A.L. $1 / f$ Noise and Germanium Surface Properties. In Semiconductor Surface Physics; Pennsylvania University Press: Philadelphia, PA, USA, 1957; pp. 207-228.

47. A distribution $D(\tau) \propto 1 / \tau$ is the basis of the famous McWhorter model (1957) [46] describing $1 / f$-noise e.g., in Si-MOSFETs, where the fluctuations are caused by tunneling processes between the conducting channel and traps in the dielectric $\mathrm{SiO}_{2}$ layer. A spatially homogeneous trap distribution (tunneling distances) leads to the required distribution of time constants.

48. Weissman, M.B. What is a spin glass? A glimpse via mesoscopic noise. Rev. Modern Phys. 1993, 65, 829-839.

49. Weissman, M.B.; Israeloff, N.E.; Alers, G.B. Spin-glass fluctuation statistics: mesoscopic experiments in CuMn. J. Magn. Magn. Mater. 1992, 114, 87-130.

50. Bogdanovich, S.; Popović, D. Onset of Glassy Dynamics in a Two-Dimensional Electron System in Silicon. Phys. Rev. Lett. 2002, 88, 236401.

51. Jaroszyński, J.; Popović, D.; Klapwijk, T.M. Universal Behavior of the Resistance Noise across the Metal-Insulator Transition in Silicon Inversion Layers. Phys. Rev. Lett. 2002, 89, 276401.

52. Kar, S.; Raychaudhuri, A.K.; Ghosh, A.; Löhneysen, H.v.; Weiss, G. Observation of Non-Gaussian Conductance Fluctuations at Low Temperatures in Si:P(B) at the Metal-Insulator Transition. Phys. Rev. Lett. 2003, 91, 216603. 
53. Hartmann, B.; Zielke, D.; Polzin, J.; Sasaki, T.; Müller, J. Critical Slowing Down of the Charge Carrier Dynamics at the Mott Metal-Insulator Transition. Phys. Rev. Lett. 2015, 114, 216403.

54. Voss, R.F.; Clarke, J. $1 / f$-noise in music and speech. Nature 1975, 258, 317-318.

55. Musha, T.; Higuchi, H. The $1 / f$ fluctuation of a traffic current on an expressway. Jpn. J. Appl. Phys. 1976, $15,1271-1275$.

56. Goldberger, A.L.; Rigney, D.R.; Mietus, J.; Antman, E.M.; Greenwald, S. Nonlinear dynamics in sudden cardiac death syndrome: Heartrate oscillations and bifurcations. Experientia 1988, 44, 983-987.

57. Kaplan, D.T.; Talajic, M. Dynamics of heart rate. Chaos Interdiscip. J. Nonlinear Sci. 1991, 1, 251-256.

58. Peng, C.K.; Mietus, J.; Hausdorff, J.M.; Havlin, S.; Stanley, H.E.; Goldberger, A.L. Long-range anticorrelations and non-Gaussian behavior of the heartbeat. Phys. Rev. Lett. 1993, 70, 1343-1346.

59. Beck, H.G.E.; Spruit, W.P. $1 / f$ Noise in the variance of Johnson noise. J. Appl. Phys. 1978, 49, 3384-3386.

60. Müller, J.; Brandenburg, J.; Schlueter, J.A. $1 / f$ noise in the quasi-two-dimensional organic conductor $\kappa$-(BEDT-TTF $)_{2} \mathrm{Cu}\left[\mathrm{N}(\mathrm{CN})_{2}\right] \mathrm{Cl}$. Phys. Rev. B 2009, 79, 214521.

61. Scofield, J.H. AC method for measuring low-frequency resistance fluctuation spectra. Rev. Sci. Instrum. 1987, 58, 985-993.

62. Hooge, F.N. $1 / f$ noise is no surface effect. Phys. Lett. A 1969, 29, 139-140.

63. An argument is that any fluctuations tied to individual free charge carriers cannot persist for times longer than the carrier transit time in the sample. Since the latter and typical diffusion times are of order microseconds to milliseconds, a resulting $1 / f$-spectrum would be required to flatten out below characteristic frequencies within this range [32].

64. It is important to mention that already for the simple Drude model of transport one expects that both carrier number (or density) fluctuations and mobility fluctuations contribute to the observed $1 / f$-noise: $\delta R / R=\delta n / n+\delta \mu / \mu$. Their particular role for the resistance fluctuations in semiconductors and metals is still a matter of controversial debate [34].

65. Galchenkov, L.A.; Ivanov, S.N.; Pyataikin, I.I.; Chernov, V.P.; Monceau, P. $1 / f$ noise in conducting Langmuir-Blodgett films. Phys. Rev. B 1998, 57, 13220-13226.

66. Brandenburg, J.; Müller, J.; Wirth, S.; Schlueter, J.A.; Schweitzer, D. Strongly enhanced $1 / f$-noise level in א-(BEDT-TTF) $)_{2}$ X salts. Phys. B 2010, 405, 141-143.

67. Kagawa, F.; Itou, T.; Miyagawa, K.; Kanoda, K. Transport criticality of the first-order Mott transition in the quasi-two-dimensional organic conductor $\kappa$-(BEDT-TTF $)_{2} \mathrm{Cu}\left[\mathrm{N}(\mathrm{CN})_{2}\right] \mathrm{Cl}$. Phys. Rev. B 2004, 69, 064511.

68. Müller, J.; Brandenburg, J.; Schlueter, J.A. Magnetic-Field Induced Crossover of Superconducting Percolation Regimes in the Layered Organic Mott System $\kappa$-(BEDT-TTF $)_{2} \mathrm{Cu}\left[\mathrm{N}(\mathrm{CN})_{2}\right] \mathrm{Cl}$. Phys. Rev. Lett. 2009, 102, 047004.

69. Kornelsen, K.; Eldridge, J.; Wang, H.H.; Charlier, H.A.; Williams, J.M. Infrared study of the metal-insulator transition in the organic conductor $\kappa-(\mathrm{BEDT}-\mathrm{TTF})_{2} \mathrm{Cu}\left[\mathrm{N}(\mathrm{CN})_{2}\right] \mathrm{Cl}$. Solid State Commun. 1992, 81, 343-349.

70. Testa, J.A.; Song, Y.; Chen, X.D.; Golben, J.; Lee, S.I.; Patton, B.R.; Gaines, J.R. $1 / f$-noise-power measurements of copper oxide superconductors in the normal and superconducting states. Phys. Rev. B Condens. Matter 1988, 38, 2922-2925.

71. Lee, J.H.; Lee, S.C.; Khim, Z.G. Noise measurement near the transition region in $\mathrm{YBa}_{2} \mathrm{Cu}_{3} \mathrm{O}_{7-x}$ thin-film superconductors. Phys. Rev. B 1989, 40, 6806-6809.

72. Kiss, L.B.; Svedlindh, P. Noise in high-T ${ }_{\mathcal{C}}$ superconductors. IEEE Trans. Electron. Devices 1994, 41, $2112-2122$.

73. Rammal, R.; Tannous, C.; Breton, P.; Tremblay, A.M.S. Flicker $\left(\frac{1}{f}\right)$ Noise in Percolation Networks: A New Hierarchy of Exponents. Phys. Rev. Lett. 1985, 54, 1718-1721.

74. Rammal, R.; Tannous, C.; Tremblay, A.M.S. $1 / f$ noise in random resistor networks: Fractals and percolating systems. Phys. Rev. A 1985, 31, 2662-2671.

75. We note that in this simple model of a one-component RRN the local microgeometry of the current distribution leads to so-called weakly nonlinear transport [134], since the most significant contribution to $R$ and $S_{R}$ comes from 'bottlenecks' or 'hot spots' in the RRN, in which the local electric field and current density are much greater than the average values. The contribution of such a region to $R$ is proportional to the local electric field squared [33]. $S_{R} / R^{2}$ and the local current distribution in Equation (13) is then proportional to the third-harmonic component $V_{3 \omega}$ in an AC transport (voltage) measurement with a driving current $I(t)=I_{0} \cos \omega t$ through the sample. This connection of $1 / f$-noise and nonlinear transport is widely observed in percolation problems (see e.g., [135] and references therein). For the organic charge-transfer salts $\kappa-(\mathrm{ET})_{2} \mathrm{X}$, there is also a relation between enhanced $1 / f$-noise due to electronic correlations and a 
third-harmonic contribution $R_{3 \omega}=V_{3 \omega / I_{0}}$ in AC resistance measurements, but the phenomenology appears to be rather complex [129].

76. Dubson, M.A.; Hui, Y.C.; Weissman, M.B.; Garland, J.C. Measurement of the fourth moment of the current distribution in two-dimensional random resistor networks. Phys. Rev. B Condens. Matter 1989, 39, 6807-6815.

77. Yagil, Y.; Deutscher, G. Third-harmonic generation in semicontinuous metal films. Phys. Rev. B Condens. Matter 1992, 46, 16115-16121.

78. Kiss, L.B.; Svedlindh, P. New noise exponents in random conductor-superconductor and conductor-insulator mixtures. Phys. Rev. Lett. 1993, 71, 2817-2820.

79. We consider the coexistence region close to the MIT as a mixture of superconducting and non-superconducting (normal or insulating) phases, i.e., a lattice of resistors with a temperature- and magnetic field-dependent fraction $p$ that is short-circuited, simulating the superconducting links. For instance, one can think of a network of $p$ Josephson-coupled junctions formed by connections between superconducting grains or clusters. A wide distribution of junction critical currents $i_{\mathcal{C}}(T)$ means that at a given macroscopic current $I$, the local currents $i$ can be either larger or smaller than $i_{c}$ which determines if the junction is superconducting or resistive [68].

80. Kiss, L.B.; Larsson, T.; Svedlindh, P.; Lundgren, L.; Ohlsén, H.; Ottosson, M.; Hudner, J.; Stolt, L. Conductance noise and percolation in $\mathrm{YBa}_{2} \mathrm{Cu}_{3} \mathrm{O}_{7}$ thin films. Phys. C Superconduct. 1993, 207, 318-332.

81. In Figure 6, a symmetric double-well potential is sketched. As has been pointed out in [136], the time constants $\tau_{1}$ and $\tau_{2}$ can be determined individually from the noise spectra only if the two lifetimes are either roughly the same or if one of them is much greater than the other. In the former case $\tau_{1} \approx \tau_{2}=\tau$, both fit coefficients of Equation (14), namely $f_{c}$ and $f_{c}^{2} \times S_{R}\left(f_{c}\right)$, are proportional to $1 / \tau$ and the slopes in an Arrhenius plot should give a similar value [137].

82. Sasaki, T.; Yoneyama, N.; Kobayashi, N.; Ikemoto, Y.; Kimura, H. Imaging Phase Separation near the Mott Boundary of the Correlated Organic Superconductors $\kappa$-(BEDT-TTF) $)_{2}$ X. Phys. Rev. Lett. 2004, 92, 227001.

83. Saito, K.; Akutsu, H.; Sorai, M. Glass transition in the organic superconductor with the highest $T_{\mathcal{C}}$ under ambient pressure, $\kappa-(\mathrm{ET})_{2} \mathrm{Cu}\left[\mathrm{N}(\mathrm{CN})_{2}\right] \mathrm{Br}$. Solid State Commun. 1999, 111, 471-475.

84. Akutsu, H.; Saito, K.; Sorai, M. Phase behavior of the organic superconductors $\kappa-(\mathrm{BEDT}-\mathrm{TTF})_{2} \mathrm{Cu}\left[\mathrm{N}(\mathrm{CN})_{2}\right] \mathrm{X}$ ( $\mathrm{X}=\mathrm{Br}$ and $\mathrm{Cl}$ ) studied by ac calorimetry. Phys. Rev. B 2000, 61, 4346-4352.

85. Sato, A.; Akutsu, H.; Saito, K.; Soraj, M. Glass transition in $\kappa$-(BEDT-TTF $)_{2} \mathrm{Cu}\left[\mathrm{N}(\mathrm{CN})_{2}\right] \mathrm{X}(\mathrm{X}=\mathrm{Br}$ and $\mathrm{Cl})$. Synth. Met. 2001, 120, 1035-1036.

86. Müller, J.; Lang, M.; Steglich, F.; Schlueter, J.A. Glass-like transition in $\kappa-(\mathrm{ET})_{2} \mathrm{Cu}\left[\mathrm{N}(\mathrm{CN})_{2}\right] \mathrm{Br}$ at $T_{g} \sim 75 \mathrm{~K}$ : implications for the superconducting ground-state properties. J. Phys. IV Fr. 2004, 114, 341-342.

87. Su, X.; Zuo, F.; Schlueter, J.A.; Kelly, M.E.; Williams, J.M. Structural disorder and its effect on the superconducting transition temperature in the organic superconductor $\kappa$-(BEDT-TTF $)_{2} \mathrm{Cu}\left[\mathrm{N}(\mathrm{CN})_{2}\right] \mathrm{Br}$. Phys. Rev. B 1998, 57, R14056-R14059.

88. Su, X.; Zuo, F.; Schlueter, J.A.; Kelly, M.E.; Williams, J.M. Dynamic disorders and its relaxation in organic superconductor $\kappa-(\mathrm{BEDT}-\mathrm{TTF})_{2} \mathrm{Cu}\left[\mathrm{N}(\mathrm{CN})_{2}\right] \mathrm{Br}$. Solid State Commun. 1998, 107, 731-734.

89. Tanatar, M.; Ishiguro, T.; Kondo, T.; Saito, G. Nonmetal to metal crossover and ethylene ordering in the organic superconductor $\kappa-(\mathrm{BEDT}-\mathrm{TTF})_{2} \mathrm{Cu}\left[\mathrm{N}(\mathrm{CN})_{2}\right]$ Br. Phys. Rev. B 1999, 59, 3841-3844.

90. Taniguchi, H.; Kanoda, K.; Kawamoto, A. Field switching of superconductor-insulator bistability in artificially tuned organics. Phys. Rev. B 2003, 67, 014510.

91. Taylor, O.J.; Carrington, A.; Schlueter, J.A. Superconductor-insulator phase separation induced by rapid cooling of $\kappa-(\text { BEDT }-\mathrm{TTF})_{2} \mathrm{Cu}\left[\mathrm{N}(\mathrm{CN})_{2}\right]$ Br. Phys. Rev. B 2008, 77, 060503.

92. Brandenburg, J.; Müller, J.; Schlueter, J.A. Sudden slowing down of charge carrier dynamics at the Mott metal-insulator transition in $\kappa-\left(\mathrm{D}_{8} \text {-BEDT-TTF }\right)_{2} \mathrm{Cu}\left[\mathrm{N}(\mathrm{CN})_{2}\right] \mathrm{Br}$. N. J. Phys. 2012, 14, 023033.

93. Müller, J.; Hartmann, B.; Rommel, R.; Brandenburg, J.; Winter, S.M.; Schlueter, J.A. Origin of the glass-like dynamics in molecular metals $\kappa$-(BEDT-TTF) ${ }_{2} \mathrm{X}$ : implications from fluctuation spectroscopy and ab initio calculations. N. J. Phys. 2015, 17, 083057.

94. Gati, E.; Winter, S.M.; Schlueter, J.A.; Schubert, H.; Müller, J.; Lang, M. Insights from experiment and ab initio calculations into the glasslike transition in the molecular conductor $\kappa-(\mathrm{BEDT}-\mathrm{TTF}){ }_{2} \mathrm{Hg}(\mathrm{SCN})_{2} \mathrm{Cl}$. Phys. Rev. B 2018, 97, 075115 . 
95. As shown in [93], the systems $\kappa-(\mathrm{ET})_{2} \mathrm{X}$ with $\mathrm{X}=\mathrm{Cu}\left[\mathrm{N}(\mathrm{CN})_{2}\right] \mathrm{Cl}, \mathrm{Cu}\left[\mathrm{N}(\mathrm{CN})_{2}\right] \mathrm{Br}$, or $\mathrm{Cu}(\mathrm{SCN})_{2}$ show a similar behavior in $S_{R} / R^{2}(f, T)$ above about $50 \mathrm{~K}$ (see also Figure 11a-c below), which will be explained in this section. In contrast, for $T<50 \mathrm{~K}$ the temperature characteristics of the noise PSD strongly depends on the position in the phase diagram and is strongly enhanced for systems with stronger electron-electron correlations being located closer to the Mott transition [138].

96. Dutta, P.; Dimon, P.; Horn, P.M. Energy Scales for Noise Processes in Metals. Phys. Rev. Lett. 1979, 43, 646-649.

97. In the simple case of a two-level fluctuation with a single relaxation time $\tau$, the correlation function (Equation (8)) is purely exponential. If the kinetics of the fluctuating quantity is a superposition of many relaxation times, the correlation function falls off with time $|t|$ not exponentially and the PSD equals Equation (10) [33].

98. The function $1 / \cosh \left[\left(E-E_{\omega}\right) / k_{B} T\right]$ of activation energy $E$ in Equation (15) constitutes a narrow peak of width $\sim k_{B} T$ with the maximum at $E=E_{\omega}[33]$.

99. Besides independent thermally activated fluctuators and linear coupling, the width of the weighting function $D(E)$ must be far greater than $k_{B} T$. As stated above, this is the case when $k_{B} T$ is small in comparison to the relevant activation energy in solids.

100. Black, R.D.; Restle, P.J.; Weissman, M.B. Hall effect, anisotropy, and temperature-dependence measurements of $1 / f$ noise in silicon on sapphire. Phys. Rev. B 1983, 28, 1935-1943.

101. Fleetwood, D.M.; Postel, T.; Giordano, N. Temperature dependence of the $1 / f$ noise of carbon resistors. J. Appl. Phys. 1984, 56, 3256-3260.

102. Raquet, B.; Coey, J.M.D.; Wirth, S.; von Molnár, S. $1 / f$ noise in the half-metallic oxides $\mathrm{CrO}_{2}, \mathrm{Fe}_{3} \mathrm{O}_{4}$, and $\mathrm{La}_{2 / 3} \mathrm{Sr}_{1 / 3} \mathrm{MnO}_{3}$. Phys. Rev. B 1999, 59, 12435-12443.

103. Sometimes, a related scattering mechanism is found in the resistivity, i.e., a contribution $\rho \propto T^{b}$, which may be linked to the function $g(T)$ via the number and/or strength of the fluctuators, as has been argued for the contribution of spin fluctuations in certain manganites [102]. See [93] for details.

104. Miyagawa, K.; Kawamoto, A.; Nakazawa, Y.; Kanoda, K. Antiferromagnetic Ordering and Spin Structure in the Organic Conductor $\kappa-\left(\mathrm{BEDT}-\mathrm{TTF}_{2} \mathrm{Cu}\left[\mathrm{N}(\mathrm{CN})_{2}\right] \mathrm{Cl}\right.$. Phys. Rev. Lett. 1995, 75, 1174.

105. Wzietek, P.; Mayaffre, H.; Jérome, D.; Brazovskii, S. NMR in the 2D Organic Superconductors. J. Phys. I Fr. 1996, 6, 2011-2041.

106. Geiser, U.; Schults, A.J.; Wang, H.H.; Watkins, D.M.; Stupka, D.L.; Williams, J.M.; Schirber, J.; Overmyer, D.; Jung, D.; Novoa, J.; et al. Strain index, lattice softness and superconductivity of organic donor-molecule salts: Crystal and electronic structures of three isostructural salts k-(BEDT- TTF)2Cu[N(CN)2]X $(X=C l, B r, I)$. Phys. C Superconduct. 1991, 174, 475-486.

107. Lunkenheimer, P.; Schneider, U.; Brand, R.; Loid, A. Glassy dynamics. Contemp. Phys. 2000, 41, 15-36.

108. Bauer, T.; Lunkenheimer, P.; Loidl, A. Cooperativity and the Freezing of Molecular Motion at the Glass Transition. Phys. Rev. Lett. 2013, 111, 225702.

109. Hiramatsu, T.; Yoshida, Y.; Saito, G.; Otsuka, A.; Yamochi, H.; Maesato, M.; Shimizu, Y.; Ito, H.; Kishida, H. Quantum spin liquid: Design of a quantum spin liquid next to a superconducting state based on a dimer-type ET Mott insulator. J. Mater. Chem. C 2015, 3, 1378-1388.

110. Yamochi, H.; Komatsu, T.; Matsukawa, N.; Saito, G.; Mori, T.; Kusunoki, M.; Sakaguchi, K. Structural aspects of the ambient-pressure BEDT-TTF superconductors. J. Am. Chem. Soc. 1993, 115, 11319-11327.

111. Sasaki, S.; Hashimoto, K.; Kobayashi, R.; Itoh, K.; Iguchi, S.; Nishio, Y.; Ikemoto, Y.; Moriwaki, T.; Yoneyama, N.; Watanabe, M.; et al. Crystallization and vitrification of electrons in a glass-forming charge liquid. Science 2017, 357, 1381-1385.

112. Sato, T.; Miyagawa, K.; Kanoda, K. Electronic crystal growth. Science 2017, 357, 1378-1381.

113. Kagawa, F.; Sato, T.; Miyagawa, K.; Kanoda, K.; Tokura, Y.; Kobayashi, K.; Kumai, R.; Murakami, Y. Charge-cluster glass in an organic conductor. Nat. Phys. 2013, 9, 419-422.

114. Sato, T.; Kagawa, F.; Kobayashi, K.; Miyagawa, K.; Kanoda, K.; Kumai, R.; Murakami, Y.; Tokura, Y. Emergence of nonequilibrium charge dynamics in a charge-cluster glass. Phys. Rev. B 2014, 89, 121102.

115. Sato, T.; Miyagawa, K.; Kanoda, K. Fluctuation Spectroscopy Analysis Based on the Dutta-Dimon-Horn Model for the Charge-Glass System $\theta$-(BEDT-TTF) ${ }_{2} \operatorname{CsZn}(\mathrm{SCN})_{4}$. J. Phys. Soc. Jpn. 2016, 85, 123702.

116. Kagawa, F.; Oike, H. Quenching of Charge and Spin Degrees of Freedom in Condensed Matter. Adv. Mater. 2017, 29, 1601979. 
117. Papanikolaou, S. Universality of liquid-gas Mott transitions at finite temperatures. Phys. Rev. Lett. 2008, 100, 026408.

118. Kagawa, F.; Miyagawa, K.; Kanoda, K. Magnetic Mott criticality in a $\kappa$-type organic salt probed by NMR. Nat. Phys. 2009, 5, 880-884.

119. Bartosch, L.; de Souza, M.; Lang, M. Scaling Theory of the Mott Transition and Breakdown of the Grüneisen Scaling Near a Finite-Temperature Critical End Point. Phys. Rev. Lett. 2010, 104, 245701.

120. Zacharias, M.; Bartosch, L.; Garst, M. Mott Metal-Insulator Transition on Compressible Lattices. Phys. Rev. Lett. 2012, 109, 176401.

121. Gati, E.; Garst, M.; Manna, R.S.; Tutsch, U.; Wolf, B.; Bartosch, L.; Schubert, H.; Sasaki, T.; Schlueter, J.A.; Lang, M. Breakdown of Hooke's law of elasticity at the Mott critical endpoint in an organic conductor. Sci. Adv. 2016, 2, e1601646.

122. We find that for even larger cooling rates $q=20 \mathrm{~K} / \mathrm{min}$ to $32 \mathrm{~K} / \mathrm{min}$, the noise level starts to increase again [25]. This is because increasing $q$ not only reduces $W / U$ but at the same time increases the degree of quenched EEG disorder [23] resulting in a stronger random lattice potential for the correlated charger carriers at the verge of Mott localization. The effect of disorder in this scenario is strong and qualitatively different from the situation for simple Anderson localization (see e.g., [139-144]). Since the change in $W / U$ induced by varying $q$ is quite drastic, covering several tens of bars on the pressure axis, further noise studies starting at different pristine positions in the phase diagram and with smaller increments of $q$ are required in order to understand the intricate interplay of randomness and strong electron correlations in $\kappa$-(ET) ${ }_{2} \mathrm{X}$ salts and the consequences for the low-frequency dynamics of the carriers.

123. Kotliar, G.; Lange, E.; Rozenberg, M.J. Landau theory of the finite temperature Mott transition. Phys. Rev. Lett. 2000, 84, 5180-5183.

124. Imada, M. Universality classes of metal-insulator transitions in strongly correlated electron systems and mechanism of high-temperature superconductivity. Phys. Rev. B 2005, 72, 075113.

125. Furukawa, T.; Miyagawa, K.; Taniguchi, H.; Kato, R.; Kanoda, K. Quantum criticality of Mott transition in organic materials. Nat. Phys. 2015, 11, 221-224.

126. Jaroszyński, J.; Popović, D.; Klapwijk, T.M. Magnetic-Field Dependence of the Anomalous Noise Behavior in a Two-Dimensional Electron System in Silicon. Phys. Rev. Lett. 2004, 92, 226403.

127. Schmalian, J.; Wolynes, P.G. Stripe Glasses: Self-Generated Randomness in a Uniformly Frustrated System. Phys. Rev. Lett. 2000, 85, 836-839.

128. Dobrosavljević, V.; Tanasković, D.; Pastor, A.A. Glassy Behavior of Electrons Near Metal-Insulator Transitions. Phys. Rev. Lett. 2003, 90, 016402.

129. Rommel, R.; Hartmann, B.; Brandenburg, J.; Schlueter, J.A.; Müller, J. Nonlinear electronic transport in the anomalous metallic state of quasi-2D organic superconductors $\kappa$-(BEDT-TTF) $)_{2}$ X. Phys. Status Solidi B 2013, 250, 568-574.

130. Thomas, T. Charge Carrier Dynamics at the Mott Transition in $\kappa-(\mathrm{BEDT}-\mathrm{TTF})_{2} \mathrm{Cu}\left[\mathrm{N}(\mathrm{CN})_{2}\right] \mathrm{Br}$. Master's Thesis, Goethe-University Frankfurt, Frankfurt, Germany, 2017.

131. Kanoda, K.; Kato, R. Mott Physics in Organic Conductors with Triangular Lattices. Annu. Rev. Condens. Matter Phys. 2011, 2, 167-188.

132. Kato, R. Development of $\pi$-Electron Systems Based on [M(dmit $\left.)_{2}\right](\mathrm{M}=\mathrm{Ni}$ and Pd; dmit: 1,3-dithiole2-thione-4,5-dithiolate) Anion Radicals. Bull. Chem. Soc. Jpn. 2014, 87, 355-374.

133. Caloyannides, M.A. Microcycle spectral estimates of $1 / f$ noise in semiconductors. J. Appl. Phys. 1974, 45, 307-316.

134. Bergman, D.J. Nonlinear behavior and $1 / f$ noise near a conductivity threshold: Effects of local microgeometry. Phys. Rev. B 1989, 39, 4598-4609.

135. Das, P.; Amyan, A.; Brandenburg, J.; Müller, J.; Xiong, P.; von Molnár, S.; Fisk, Z. Magnetically driven electronic phase separation in the semimetallic ferromagnet EuB 6 . Phys. Rev. B 2012, 86, 184425.

136. Kirtley, J.R.; Theis, T.N.; Mooney, P.M.; Wright, S.L. Noise spectroscopy of deep level (DX) centers in GaAs-Al $\mathrm{Ga}_{1-x}$ As heterostructures. J. Appl. Phys. 1988, 63, 1541-1548.

137. Müller, J.; von Molnár, S.; Ohno, Y.; Ohno, H. Decomposition of $1 / f$ Noise in $A l_{x} G a_{1-x} A s / G a A s$ Hall Devices. Phys. Rev. Lett. 2006, 96, 186601.

138. Müller, J.; Brandenburg, J.; Schweitzer, D.; Schlueter, J.A. Different electronic transport regimes in the quasi-two-dimensional organic conductors $\kappa-(B E D T-T T F){ }_{2}$ X. Phys. Status Solidi B 2012, 249, 957-961. 
139. Sano, K.; Sasaki, T.; Yoneyama, N.; Kobayashi, N. Electron Localization near the Mott Transition in the Organic Superconductor $\kappa-(\mathrm{BEDT}-\mathrm{TTF})_{2} \mathrm{Cu}\left[\mathrm{N}(\mathrm{CN})_{2}\right] \mathrm{Br}$. Phys. Rev. Lett. 2010, 104, 217003.

140. Sasaki, T. Mott-Anderson Transition in Molecular Conductors: Influence of Randomness on Strongly Correlated Electrons in the $\kappa$-(BEDT-TTF $)_{2}$ X System. Crystals 2012, 2, 374-392.

141. Taniguchi, H.; Kawamoto, A.; Kanoda, K. Superconductor-insulator phase transformation of partially deuterated $\kappa$-(BEDT-TTF)2Cu[N(CN)2]Br by control of the cooling rate. Phys. Rev. B 1999, 59, 8424-8427.

142. Shinaoka, H.; Imada, M. Soft Hubbard Gaps in Disordered Itinerant Models with Short-Range Interaction. Phys. Rev. Lett. 2009, 102, 016404.

143. Shinaoka, H.; Imada, M. Single-Particle Excitations under Coexisting Electron Correlation and Disorder: A Numerical Study of the Anderson-Hubbard Model. J. Phys. Soc. Jpn. 2009, 78, 094708.

144. Diehl, S.; Methfessel, T.; Tutsch, U.; Müller, J.; Lang, M.; Huth, M.; Jourdan, M.; Elmers, H.J. Disorder-induced gap in the normal density of states of the organic superconductor $\kappa-(\mathrm{BEDT}-\mathrm{TTF})_{2} \mathrm{Cu}\left[\mathrm{N}(\mathrm{CN})_{2}\right] \mathrm{Br}$. J. Phys. Condens. Matter 2015, 27, 265601.

(C) 2018 by the authors. Licensee MDPI, Basel, Switzerland. This article is an open access article distributed under the terms and conditions of the Creative Commons Attribution (CC BY) license (http:/ / creativecommons.org/licenses/by/4.0/). 
Review

\title{
Thermodynamic Picture of Dimer-Mott Organic Superconductors Revealed by Heat Capacity Measurements with External and Chemical Pressure Control
}

\author{
Yasuhiro Nakazawa *, Shusaku Imajo, Yuki Matsumura, Satoshi Yamashita and Hiroki Akutsu \\ Department of Chemistry, Graduate School of Science, Osaka University, Machikaneyama 1-1, Toyonaka, \\ Osaka 560-0043, Japan; imajos12@chem.sci.osaka-u.ac.jp (S.I.); matsumuray16@chem.sci.osaka-u.ac.jp (Y.M.); \\ sayamash@chem.sci.osaka-u.ac.jp (S.Y.); akutsu@chem.sci.osaka-u.ac.jp (H.A.) \\ * Correspondence: nakazawa@chem.sci.osaka-u.ac.jp; Tel.: +81-6-6850-5396
}

Received: 24 February 2018; Accepted: 19 March 2018; Published: 21 March 2018

\begin{abstract}
This article reviews and discusses the thermodynamic properties of dimer-Mott-type molecular superconductive compounds with (BEDT-TTF) ${ }_{2} \mathrm{X}$ composition, where BEDT-TTF is bis(ethylenedithio)tetrathiafulvalene and $\mathrm{X}$ denotes counter-anions, respectively. We focus mainly on the features occurring in the k-type structure in which the d-wave superconductive phase appears depending on the Coulomb repulsion $U$ and the bandwidth $W$, which is tunable by external and chemical pressures. First, we report the high-pressure ac (alternating current) calorimetry technique and experimental system constructed to measure single-crystal samples of molecule-based compounds to derive low-temperature thermodynamic parameters. Using extremely small resistance chips as a heater and a thermometer allows four-terminal detection of an accurate temperature and its oscillation in the sample part with sufficient sensitivity. From the analyses of the temperature dependence of the ac heat capacity of $\mathrm{k}$-(BEDT-TTF $)_{2} \mathrm{Cu}(\mathrm{NCS})_{2}$ under external pressures, we discuss the changes in the peak shape of the thermal anomaly at the superconductive transition temperature $T_{\mathrm{c}}$ at various external pressures $p$. The rather sharp peak in $C_{p} T^{-1}$ at $T_{\mathrm{c}}=9.1 \mathrm{~K}$ with a strong coupling character at ambient pressure is gradually reduced to weaker coupling as the pressure increases to $0.45 \mathrm{GPa}$ concomitant with suppression of the transition temperature. This feature is compared with the systematic argument of the chemical-pressure effect on the basis of thermal anomalies around the superconductive transition of $\mathrm{k}$-(BEDT-TTF $)_{2} \mathrm{X}$ compounds and other previously studied typical dimer-Mott 2:1 compounds. Finally, the discussion is extended to the chemical pressure effect on the normal state electronic heat capacity coefficient $\gamma$ obtained by applying magnetic fields higher than $H_{\mathrm{c} 2}$ and the residual $\gamma^{*}$, which remains in the superconductive state due to the induced electron density of states around the node structure. From the overall arguments with respect to both chemical and external pressures, we suggest that a crossover of the electronic state inside the superconductive phase occurs and the coupling strength of electron pairs varies from the electron correlation region near the metal-insulator boundary to the band picture region.
\end{abstract}

Keywords: organic superconductor; dimer-Mott system; heat capacity; electron correlations; d-wave; pressure

\section{Introduction}

Molecule-based superconductors are attracting much attention in condensed matter science because they provide a platform to discuss the physics related to the electron correlations in low-dimensional and relatively soft lattice systems [1-4]. Although there are several types of superconductive compounds in molecule-based systems, such as doped fullerene, graphite, polymers, and the recently observed 
picene [5-8], most are categorized as charge transfer complexes of organic donor/acceptor molecules with counter-ions. Electron pairs are formed by the attractive force in itinerant $\pi$-electrons released from frontier orbitals such as the HOMO (highest occupied molecular orbital) and LUMO (lowest unoccupied molecular orbital) to form electron bands. The band structure is determined by the molecular arrangement where the condensation energies are dominated by relatively weak van der Waals interactions.

Since a variety of molecular packings are possible in these charge transfer complexes, even in the same combination of donor/acceptor and counter-ions, the development of new superconductors and investigations of their physical properties have been extensively performed over the past four decades, as summarized in the literature [1-4,9-13]. To date, the most widely studied are the 2:1 complexes consisting of TMTSF (tetramethyltetraselenafulvalene), BEDT-TTF, BETS (bis(ethylenedithio)tetraselenafulvalene), and $\mathrm{M}(\mathrm{dmit})_{2}$ (dmit is 1,3-dithiole-2-thione-4,5-dithiolate) with their counter-ions. Due to the segregated stacking of organic donor/acceptor molecules and counter-ion molecules, they show a layered structure of organic molecules and counter-ions to form quasi-one-dimensional and two-dimensional electronic systems [1-4,9-17]. Complexes of BEDT-TTF and BETS with monovalence counter-anions give numerous superconductive materials.

Among the various superconductive complexes, we have focused mainly on the k-type dimer-Mott systems of BEDT-TTF and analogous molecules, which are extensively studied as a prototype of effective half-filling compounds. They sometimes show antiferromagnetic insulating states due to the electron correlation mechanism. The antiferromagnetic spin correlation due to the on-site Coulomb repulsion in the half-filled state plays a dominant role in determining the low-temperature electronic states. The electronic features of the dimer-Mott systems are summarized as a pressure-temperature phase diagram (Figure 1) where the ratio of the band energy $W$ and the electron correlation in the dimer unit $U$ are the essential parameters controlling the ground state features [14,15]. The antiferromagnetic Mott insulating phase changes the superconductive phase by applying pressure due to the decrease in the $U / W$ ratio. Kanoda et al. mapped several compounds with rigidly dimerized BEDT-TTF compounds in the phase diagram and discussed the relation between the chemical and the external pressure effects in $[11,14,15]$. A superconductivity where $T_{\mathrm{c}}$ exceeds $10 \mathrm{~K}$ appears near the metal-insulator boundary, which is known as the Mott boundary. The diagram in Figure 1 is recognized as a typical bandwidth control of a two-dimensional electron correlation system in two-dimensional (2D) half-filling state $[14,15,18-20]$. It is known that the phase diagram dominated by the parameter $V / t$, where $V$ is the intermolecular Coulomb and $t$ the intermolecular transfer-discussed experimentally by Mori et al. [21] and theoretically by McKenzie et al. [22]—gives a pressure-sensitive feature. This phase diagram is realized in non-dimeric arrangements, such as $\theta$-type BEDT-TTF systems. In the case of the dimer-Mott system, the pair symmetry of the superconductivity and the possibility of unconventional pairings with a nodal gap feature through NMR [23], STM [24], and thermodynamic [25,26] experiments have been discussed, although the electron-phonon interactions are considered to be relatively large in organic systems [27].

To investigate the relationship between superconductivity and electron correlation physics, which peculiarly appears in 2D dimer-Mott systems especially for K-type compounds, and to discuss the mechanism of superconductivity, the characteristic features occurring inside the superconductive phase, as well as the features of the phase transition, must be pursued. Thermodynamic information from heat capacity measurements can provide quantitative information via the analysis of entropic information of the superconductivity [28-31]. By analyzing the features of the thermal anomalies at the transitions and low energy excitations simultaneously, it is possible to grasp systematic changes in the superconductive phase where the quantum mechanical crossover is observed. For this purpose, pressure-controlled thermodynamic measurements to tune the $U / W$ ratio and systematic arguments with the parameter of this ratio are required [32,33]. In this article, we summarize the thermodynamic features in the superconductive phase of dimer-Mott compounds from heat capacity measurements under pressure. We also discuss the results by comparing with the features observed in the chemical pressure effects produced by changing the anions $(\mathrm{X})$ in the $\mathrm{k}$-(BEDT-TTF) ${ }_{2} \mathrm{X}$ system. 


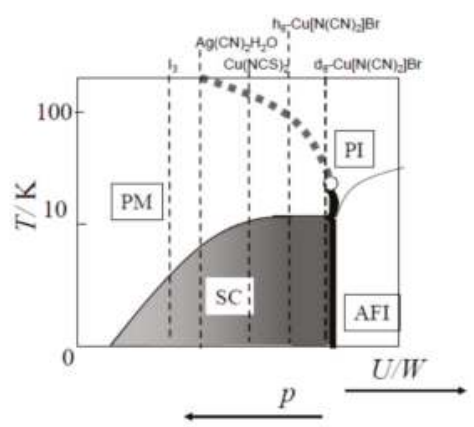

Figure 1. Electronic phase diagram of dimer-Mott compounds with a $\mathrm{D}_{2} \mathrm{X}$ composition. Diagram is constructed based on the chemical and external pressure dependences of the physical properties of K-(BEDT-TTF $)_{2}$ X compounds by Kanoda in Refs. [14,15]. Position of several compounds with different counter-anions are shown by dashed lines. Horizontal axis is the $U / W$ ratio, which is tunable by an external pressure.

\section{Calorimetry Apparatus to Measure the Heat Capacity under Pressure}

To measure the heat capacity of single-crystalline samples of organic superconductors at ambient pressure, we used the thermal relaxation calorimetry technique developed by Bachmann et al. [34,35]. We constructed and modified our original apparatus - that aimed to measure the heat capacity of molecule-based compounds - to realize accurate measurements for $80 \mu \mathrm{g}-1 \mathrm{mg}$ single crystals. Figure 2 shows a schematic drawing and photograph of the sample cell. Developments of the apparatus focusing on the technique to measure single crystals are reported in the literature [36-38].

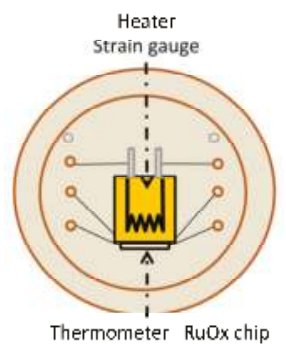

(a)

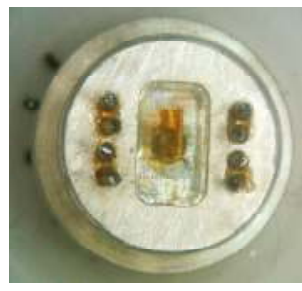

(b)

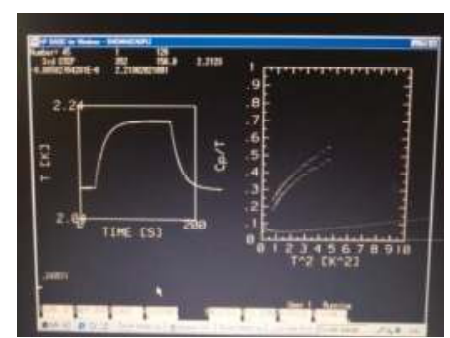

(c)

Figure 2. Schematic (a) and photograph (b) of the relaxation calorimetry cell used to measure the heat capacity of small amounts of samples of molecule-based compounds. Photograph (c) shows the relaxation curvature in the measurement.

The application of external pressure reduces the inter-atomic or inter-molecular distance in crystal lattices, inducing various structural transformations due to molecular or atomic arrangements, dielectric, magnetic, and transport properties, which are dominated by the orbital overlap between neighboring atoms and molecules. It also induces an increase in bandwidth $W$, which directly affects the $U / W$ ratio. To measure the heat capacity while varying the external pressure, both the temperature and magnetic fields are important to realize new functionalities and to understand the mechanism of pressure-induced phase transitions. For heat capacity measurements of molecular compounds under pressure, we used the ac calorimetry technique. The adiabatic technique and the relaxation technique for heat capacity measurements are quite difficult because they usually require a substantial amount of sample and semi-adiabatic conditions around the sample from the surroundings. Such conditions 
cannot be realized when the crystal and the thermometer are in direct contact with the pressure medium. Therefore, the ac technique [39], which separates the difference of the temperature relaxation rates inside the sample parts from that of the surroundings, is the best way to detect precise thermodynamic information, as suggested by Eicher and Gey [40]. The detection of temperature oscillations using the frequencies in the range between $\tau_{\text {ext }}{ }^{-1}<\omega<\tau_{\text {int }}{ }^{-1}$, where $\tau_{\text {ext }}$ and $\tau_{\text {int }}$ are the internal and external temperature relaxation rates, respectively, can give an accurate heat capacity of the sample parts.

We constructed an ac heat capacity measurement apparatus for single-crystalline samples of molecular compounds. Measurements are available over a wide temperature range from a low temperature of about $0.7 \mathrm{~K}$ up to about $20 \mathrm{~K}$ while reducing the sample amount to $200 \mu \mathrm{g}-2 \mathrm{mg}$ [41-45]. The construction details are reported elsewhere $[33,41,42]$. The adoption of extremely small chips as the thermometer and the accurate detection using the four-terminal method have worked well to achieve high-resolution detection for organic superconductors, providing heat capacity measurements in the low-temperature region. Figure 3 shows a schematic view of the apparatus where the sample part and pressure cell are set with $\mathrm{CuBe}$ and $\mathrm{NiCrAl}$. We utilized a ruthenium oxide tiny chip sensor with a room temperature resistance of $10^{2-4} \Omega$, dimensions of $0.6 \times 0.3 \mathrm{~mm}^{2}$, and a thickness of $0.2 \mathrm{~mm}$. We also used another small-sized chip sensor with a resistance of $1 \mathrm{k} \Omega$ at room temperature. The GE (General Electric) vanish confirms good contact between the sample and the sensor and the sample parts were also coated by a small amount of epoxy (Stycast 1266, 2850FT, and 2850GT etc.) before being sealed inside a Teflon capsule with a pressure medium (Daphne 7373 oil, Idemitsu). The sample cell was set in the CuBe piston cylinder and clamped using CuBe screws on both sides.

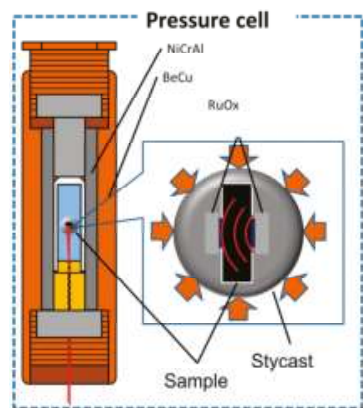

(a)

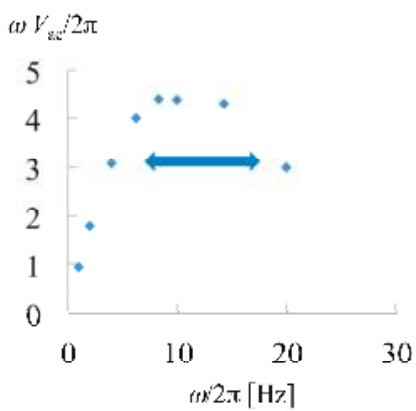

(b)

Figure 3. (a) Schematic of the ac calorimetry system in the hybrid pressure cell of CuBe and NiCrAl. Sample part consists of a single crystal sample and two chip sensors working as a thermometer and a heater; (b) a typical curve of the frequency dependence of the ac amplitude voltage to evaluate the measurement validity. In the plateau region, which is marked by the arrow, the oscillation frequency satisfies the appropriate conditions for the measurements.

The temperature modulation excited by the ON/OFF current of the heater was detected by the thermometer using an ac resistance bridge. The modulation occurs more rapidly than the relation of heat to the surroundings expressed by text. Since the sensitivity of the ruthenium oxide chip increases in the low-temperature region, this technique is available even in the low-temperature region where thermocouples are not very sensitive. Furthermore, the ruthenium oxide chip sensor has a variable range hopping-type transport feature and can be utilized in extremely low-temperature regions with small magnet resistance.

The frequency dependence of the oscillation amplitude for typical organic crystals is shown in Figure $3 \mathrm{~b}$. Although this system is constructed for low-temperature experiments, the use of a tiny Pt chip thermometer and a Cernox thermometer make it possible to detect heat capacity measurements between $20 \mathrm{~K}$ and $300 \mathrm{~K}$, as reported by Danda [45] and Konoike [46]. Danda et al. reported the Verwey 
transition of iron oxide $\left(\mathrm{Fe}_{3} \mathrm{O}_{4}\right)$ in a higher temperature system [45]. The sharp peak due to magnetic and charge-ordering transition at $124 \mathrm{~K}$ is broadened by pressures up to $1 \mathrm{GPa}$ without significantly changing the transition temperature. The most serious problem is to separate the background contribution since the extra contribution of the addenda, which consists of the chips, the lead wires, and the Stycast, is included.

\section{Heat Capacity of $\mathrm{k-(BEDT-TTF)})_{2} \mathrm{X}$ under Pressure}

In this section, we review the calorimetry results of dimer-Mott compounds under pressure. Figure 4 shows the temperature dependence of the heat capacities of four charge transfer complexes, including $\mathrm{k}-(\mathrm{BEDT}-\mathrm{TTF})_{2} \mathrm{Cu}(\mathrm{NCS})_{2}$, obtained by relaxation calorimetry. The details are reported in the published literature [47-51]. Compared with other compounds in the figure, $\mathrm{k}-(\mathrm{BEDT}-\mathrm{TTF})_{2} \mathrm{Cu}(\mathrm{NCS})_{2}$ exhibits a rather sharp peak as shown in Figure 4c $[49,50]$. The thermodynamics of this compound and $\mathrm{k}-(\mathrm{BEDT}-\mathrm{TTF})_{2} \mathrm{Cu}\left[\mathrm{N}(\mathrm{CN})_{2}\right] \mathrm{Br}$, which has a similar transition temperature, have already been thoroughly discussed. Several groups have analyzed the peak shape and the magnitude of the heat capacity jump at $T_{\mathrm{c}}$ for $\mathrm{k}-(\mathrm{BEDT}-\mathrm{TTF})_{2} \mathrm{Cu}(\mathrm{NCS})_{2}[49,50,52-57]$. In the initial investigation stage of this material, the heat capacity jump is reported as evidence of the bulk nature of the superconductivity, as reported by Katsumoto et al. [52] and Andraka et al. [53]. Additionally, Graebner et al. [54] measured the ac heat capacity with an absolute precision around the peak and suggested, for the first time, a strong coupling nature with a larger condensation energy. More recently, Müller et al. [55] reported a detailed analysis of the peak shape. They showed the data over a wide temperature range to determine the $\alpha$ value of the coupling strength. The data are shown in Figure $4 \mathrm{c}$ where the magnitude of $C p T_{\mathrm{c}}{ }^{-1} \simeq 60 \mathrm{~mJ} \mathrm{~K}^{-2} \mathrm{~mol}^{-1}$ is consistent with the strong coupling picture in Refs [26,49,50,54-56]. A similar thermodynamic feature has also been reported for $\mathrm{k}-(\mathrm{BEDT}-\mathrm{TTF})_{2} \mathrm{Cu}\left[\mathrm{N}(\mathrm{CN})_{2}\right]_{2} \mathrm{Br}$, which shows a stronger coupling peak shape [57].
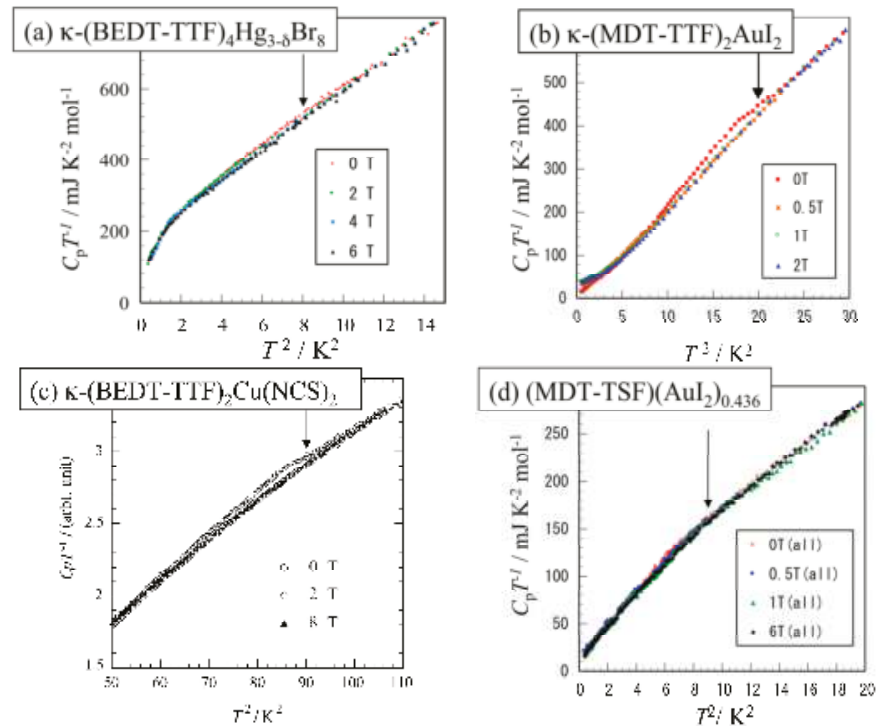

Figure 4. $\quad C_{p} T^{-1}$ vs. $\quad T^{2}$ plot of superconductive compounds (a) $\mathrm{k}$-(BEDT-TTF) ${ }_{4} \mathrm{Hg}_{1-\delta} \mathrm{Br}_{8}$ (Refs. [47,48]); (b) k-(MDT-TTF) ${ }_{2} \mathrm{AuI}_{2}$ (Ref. [49]); (c) k-(BEDT-TTF) $)_{2} \mathrm{Cu}(\mathrm{NCS})_{2}$ (Refs. [49,50]); and (d) (MDT-TSF)(AuI $)_{0.436}$ (Ref. [51]) obtained under $0 \mathrm{~T}$ and with magnetic fields.

To confirm the thermodynamic peak in the ac heat capacity data obtained by the high-pressure calorimetric system, we have analyzed the data at ambient pressure and compared them with the relaxation calorimetry data. Figure 5 a shows the ac heat capacity measurement results at ambient 
pressure obtained using a high-pressure calorimeter as a $C_{p} T^{-1}$ vs. $T^{2}$ plot [32]. A thermal anomaly is detected as a hump structure around $9 \mathrm{~K}$ in this plot, although there is a rather large background due to the addenda heat capacity in the pressure cell. This anomaly is associated with the superconductive transition since the peak temperature and the peak shape resemble the preceding data obtained by the thermal relaxation technique in Figure 4c. Upon applying magnetic fields almost parallel to the plane in the present pressure calorimetry set up, the anomaly is suppressed through comparative analyses of the magnetic field dependence, as already reported [32]. The data obtained up to $7 \mathrm{~T}$ has a constant shift of $C_{p} T^{-1}$ values at different magnetic fields (Figure 5a).

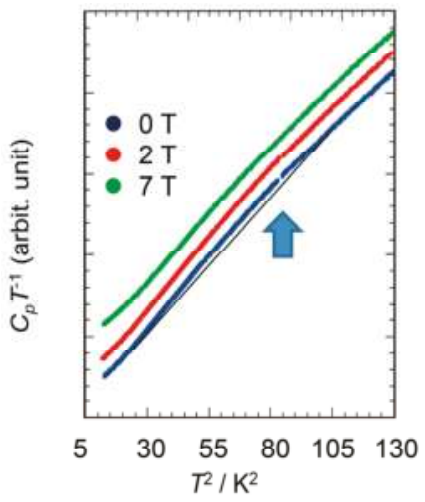

(a)

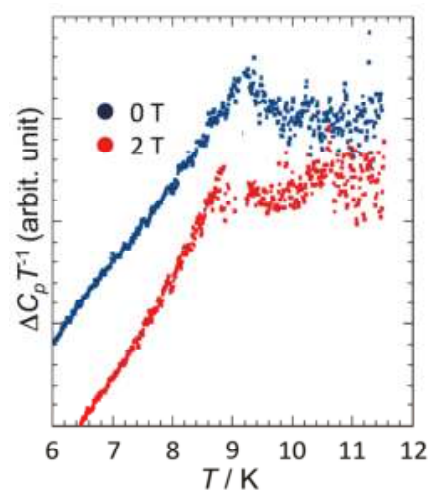

(b)

Figure 5. (a) $C_{p} T^{-1}$ vs. $T^{2}$ plot of the ac heat capacity of $\mathrm{k}$-(BEDT-TTF) ${ }_{2} \mathrm{Cu}(\mathrm{NCS})_{2}$ at ambient pressure with magnetic fields of $0 \mathrm{~T}, 2 \mathrm{~T}$, and $7 \mathrm{~T}$ applied parallel to the conducting layer. The values of $C_{p} T^{-1}$ under magnetic fields are plotted with constant offsets in the vertical axis. The arrow shows the superconductive transition temperature. (b) Temperature dependence of the $\Delta C_{p} T^{-1}$ obtained by subtracting the 7-T data as the background $\left(\Delta C_{p} T^{-1}(H)=C_{p} T^{-1}(H)-C_{p} T^{-1}(7 \mathrm{~T})\right)$ to evaluate the anomaly in the temperature dependence due to the electronic heat capacity around the transition (details are reported in Ref. [32]).

Usually, in quasi-2D superconductors, an increase in the magnetic field applied parallel to the plane drastically suppresses and broadens the thermal anomaly. To analyze the peak shape around $T_{\mathrm{c}}$ in detail, Figure $5 \mathrm{~b}$ plots $\Delta \mathrm{C}_{p} T^{-1}$ as the electronic heat capacity around the transition temperature. Here, $\Delta C_{p}$ is determined as the discrepancy of the heat capacity values in the 0 -T and 7-T data assuming that the thermal anomaly due to superconductive transition is almost reduced in a magnetic field of $7 \mathrm{~T}$. The analytic details were reported in Ref. [32]. The peak resembles the typical shape of the superconductive transition with a strong coupling feature. $\Delta C_{p}$ at $2 \mathrm{~T}$ is also evaluated and plotted as the discrepancy from the 7-T data. A slight downward shift in the transition temperature and suppression of the magnitude of heat capacity jump are observed.

Figure 6a shows the temperature dependence of the ac heat capacity at $0.15 \mathrm{GPa}$ and Figure $6 \mathrm{~b}$ shows the result of the similar analysis as was performed for ambient pressure data in Figure $5 \mathrm{~b}$. The transition temperature is reduced to about 5-6 K, which is consistent with the results of the proceeding work [44]. Subtracting the 7-T data also gives the relative change of the electronic heat capacity contribution $\Delta C_{p}$ for each field, which is shown in the $\Delta C_{p} T^{-1}$ vs. $T$ plot in Figure $6 \mathrm{~b}$. It is difficult to see a distinct peak structure even for the data of $0 \mathrm{~T}$; however, the magnetic field seems to suppress the peak and the transition temperature systematically. We can mention that the peak shape broadens compared with that at ambient pressure. The suppression of the peak structure becomes more remarkable in the case of $0.30 \mathrm{GPa}$ and $0.45 \mathrm{GPa}$. Since the sensitivity of the resistance sensor increases in the lower temperature region, small anomalies, if any exist, can be more easily detected in 
the lower $T_{\mathrm{c}}$ cases. In fact, several compounds with a magnetic or superconductive transition around 2-4 K have been detected in a high-pressure calorimeter $[41,42]$.
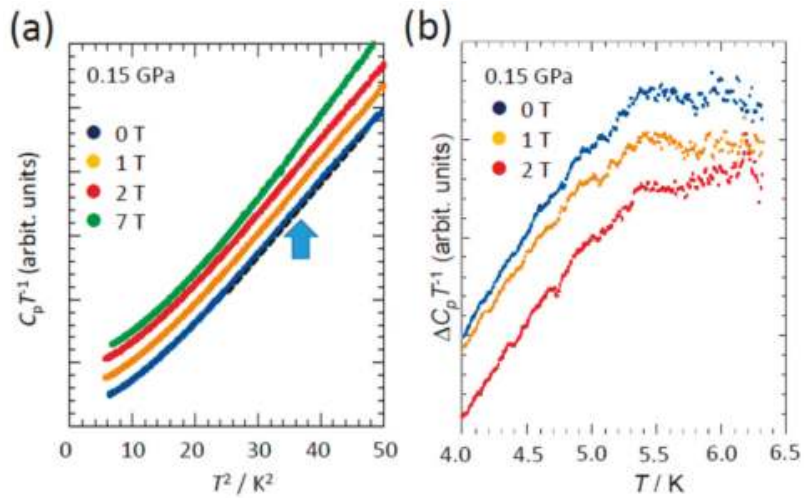

Figure 6. (a) $C_{p} T^{-1}$ vs. $T^{2}$ plot of the ac heat capacity of $\mathrm{k}$-(BEDT-TTF) ${ }_{2} \mathrm{Cu}(\mathrm{NCS})_{2}$ at $0.15 \mathrm{GPa}$ with magnetic fields of $0 \mathrm{~T}, 1 \mathrm{~T}, 2 \mathrm{~T}$, and $7 \mathrm{~T}$. The values of $C_{p} T^{-1}$ under magnetic fields are plotted with constant offsets in the vertical axis. The arrow indicates the superconductive transition temperature at $0 \mathrm{~T} ;(\mathbf{b})$ temperature dependence of the $\Delta C_{p} T^{-1}$ at $0.15 \mathrm{GPa}$ obtained by subtracting the $7-\mathrm{T}$ data as the background (details are reported in Ref. [32]).

To compare the relative change in the broadness and the magnitude of the heat capacity jump induced by the pressure in the same figure, Figure 7 plots the overall feature in the peak shape of $\Delta C_{p} T^{-1}$ obtained at ambient pressure, $0.15 \mathrm{GPa}$, and $0.30 \mathrm{GPa}$, where the temperature in the horizontal axis is the relative temperature normalized by the transition temperature $\left(T_{c}\right)$ of each pressure. A qualitative comparison of the peak shape for the superconductive transition indicates that the reduction in the transition temperature is accompanied by a systematic change in the peak structure. As $T_{\mathrm{c}}$ decreases due to the increase in pressure, the magnitude of the peak of $\Delta C_{p} T^{-1}$ becomes smaller and the peak shape broadens gradually. This is explained by the continuous reduction in the coupling strength of the superconductivity in a dimer-Mott system. The sharp peak with a relatively high $T_{\mathrm{C}}$ means the coupling strength is strong and is almost as large as the condensation energy of the electron pairs. In contrast, broadening is considered to be the suppression of such pairing forces as the system becomes more metallic. $\mathrm{k}-(\mathrm{BEDT}-\mathrm{TTF})_{2} \mathrm{Cu}\left[\mathrm{N}(\mathrm{CN})_{2}\right] \mathrm{Br}$ and $\mathrm{k}-\left[(\mathrm{BEDT}-\mathrm{TTF})_{1-x}(\mathrm{BEDSe}-\mathrm{TTF})_{x}\right]_{2} \mathrm{Cu}\left[\mathrm{N}(\mathrm{CN})_{2}\right] \mathrm{Br}$ compounds also show a broadening in the peak shape and a decrease in the heat capacity jump $\Delta C_{p} T^{-1}$ as the transition temperature is suppressed. However, the latter may have some extra effects related to the frustration due to the increase in the triangularity in the molecular arrangement of the dimer units. We have also reported the suppression of the peak by pressure in $\mathrm{k}-(\mathrm{BEDT}-\mathrm{TTF})_{2} \mathrm{Ag}(\mathrm{CN})_{2} \mathrm{H}_{2} \mathrm{O}$ [44]. These features are consistent with к-(BEDT-TTF $)_{2} \mathrm{Cu}(\mathrm{NCS})_{2}$, as summarized in Figure 7. 


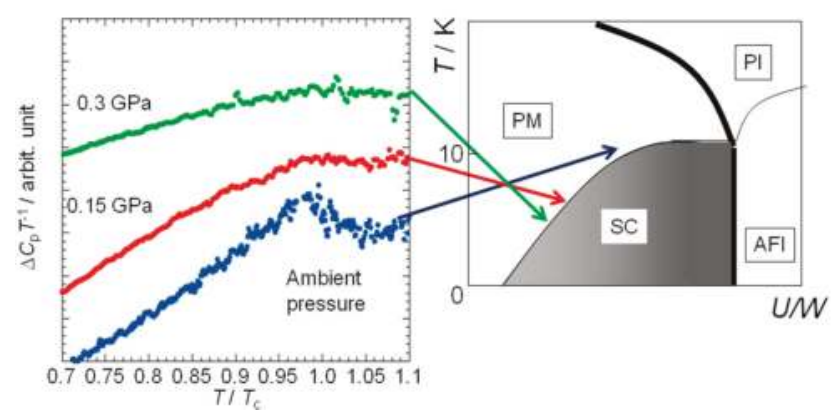

Figure 7. Systematic changes in the peak shape of $\mathrm{k}-(\mathrm{BEDT}-\mathrm{TTF})_{2} \mathrm{Cu}(\mathrm{NCS})_{2}$ as the pressure increases up to $0.30 \mathrm{GPa}$. (See details in Ref. [32].) Temperature in the horizontal axis is scaled by $T_{\mathrm{C}}$ to qualitatively evaluate the sharpness of the thermal anomaly. Correspondence of the data in each pressure is shown in the schematic phase diagram of the dimer-Mott system.

\section{Chemical Pressure Effect on Dimer-Mott Organic Compounds}

The systematic change in the peak shape in the $\mathrm{k}-(\mathrm{BEDT}-\mathrm{TTF})_{2} \mathrm{Cu}(\mathrm{NCS})_{2}$ and similar features observed in other $\mathrm{k}-(\mathrm{BEDT}-\mathrm{TTF})_{2} \mathrm{X}$ compounds under pressures can be compared with the chemical pressure effects of the dimer-Mott system induced by varying the counter-anions. It is well known that the counter-anion size of organic charge transfer complexes induces a kind of pressure effect in the molecular arrangement, causing systematic changes in the physical properties. According to transport and NMR studies of the dimer-Mott phase diagram, the external pressure effects in $10 \mathrm{~K}$ class compounds with $\mathrm{X}=\mathrm{Cu}(\mathrm{NCS})_{2}$ and $\mathrm{Cu}\left[\mathrm{N}(\mathrm{CN})_{2}\right] \mathrm{Br}$ are consistent with the chemical pressure effects. Similar to the case where the external pressure increases, counter-ions with smaller volumes change the features from a strong correlation system to a normal metallic system. Many thermodynamic studies by heat capacity measurements of several $\mathrm{k}$-type compounds with different counter-ions have been performed up to now [49-60]. As mentioned in the previous section, the strong coupling nature of $\mathrm{k}-(\mathrm{BEDT}-\mathrm{TTF})_{2} \mathrm{Cu}(\mathrm{NCS})_{2}$ has been observed in $10 \mathrm{~K}$ class superconductors (Figures $4 \mathrm{c}$ and 8). The values of $\Delta C_{p} / \gamma T_{\mathrm{c}}$ of two typical compounds $\mathrm{k}-(\mathrm{BEDT}-\mathrm{TTF})_{2} \mathrm{Cu}(\mathrm{NCS})_{2}$ and $\mathrm{k}-(\mathrm{BEDT}-\mathrm{TTF})_{2} \mathrm{Cu}\left[\mathrm{N}(\mathrm{CN})_{2}\right] \mathrm{Br}$ - which were obtained using the normal state heat capacities obtained under strong magnetic fields above $H_{\mathrm{c} 2}$ as the background heat capacity of exceed 2.0 - are much larger than that of the BCS (Bardeen Cooper Schrieffer) weak coupling theory $[54,55,57]$. As compared with these higher $T_{c}$ compounds, the heat capacity jump of middle-class (about 4-5 K) superconductors, such as $\mathrm{k}$-(BEDT-TTF) ${ }_{2} \mathrm{Ag}(\mathrm{CN})_{2} \mathrm{H}_{2} \mathrm{O}\left(T_{\mathrm{c}}=5 \mathrm{~K}\right)$ [58], gives a $\Delta C_{p} / \gamma T_{\mathrm{c}}$ value of 1.1. Although $\mathrm{K}-(\mathrm{MDT}-\mathrm{TTF})_{2} \mathrm{AuI}_{2}$ consists of a different asymmetric donor molecule, its $T_{\mathrm{C}}$ is $4.5 \mathrm{~K}$ [49] and it has a $\Delta C_{p} / \gamma T_{\mathrm{c}}$ value of 1.4. These values are within the weak coupling region.

The plot in Figure 8 shows the systematic change in the peak shape for several superconductive compounds with the $\mathrm{k}$-type structure using the scaled temperature of $t=T / T_{\mathrm{c}}$ for each compound. We also include the $\Delta C_{p} / T_{\mathrm{C}}$ data of mixed crystals of $\mathrm{k}-\left[(\mathrm{BEDT}-\mathrm{TTF})_{1-\mathrm{x}}(\mathrm{BEDSe}-\mathrm{TTF})_{\mathrm{x}}\right]_{2} \mathrm{Cu}\left[\mathrm{N}(\mathrm{CN})_{2}\right] \mathrm{Br}$ compounds with $x=0.10$. A clear tendency is observed; $10 \mathrm{~K}$ class superconductors have a sharper peak with a large $\Delta C_{p} T^{-1}$. This is typical for strong coupling systems, but $4-5 \mathrm{~K}$ class compounds have a smaller mean field type peak. The systematic change in the peak shape indicates a gradual crossover from a strong coupling region near the boundary to a weak coupling region in the superconductive phase, as shown schematically in Figure 8 (right) [32,33]. Although some exceptions exist, such as $\mathrm{K}-(\mathrm{BEDT}-\mathrm{TTF})_{2} \mathrm{I}_{3}$ being reported to have strong coupling features [59], the overall tendency seems to be consistent with the external pressure controlled effects. This feature is reasonably consistent with our observations of $\mathrm{k}-(\mathrm{BEDT}-\mathrm{TTF})_{2} \mathrm{Cu}(\mathrm{NCS})_{2}$ under pressure, as summarized in Figure 7. 


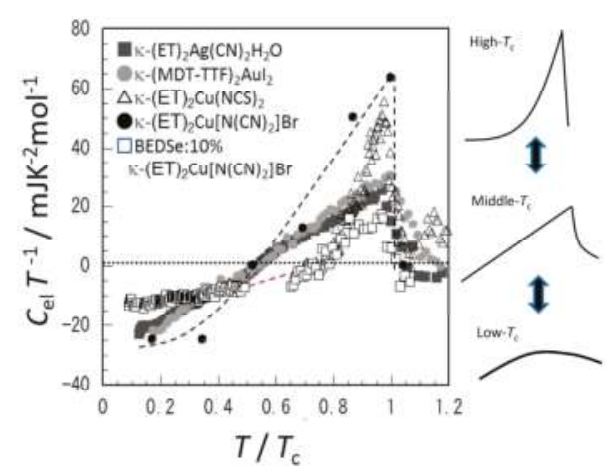

Figure 8. Shape of the thermal anomalies at the superconductive transitions of several compounds with the $\kappa-\mathrm{D}_{2} \mathrm{X}$ composition. ET is an abbreviation of BEDT-TTF molecules. BEDSe-TTF10\% means that a solid solution system of BEDT-TTF( $90 \%)$ and BEDSe-TTF(10\%) molecules. $C_{\mathrm{el}}$ in this plot is determined as the difference of the $0 \mathrm{~T}$ data and the heat capacity data obtained under magnetic fields higher than $H_{\mathrm{c} 2}$ for each compound. (The data are from Ref. [33].) The dashed curves in the figure are a guide for the eyes to see temperature dependencies. The temperature in the horizontal axis is scaled by the transition temperature of each compound to compare the peak shape changes. The right part of the figure shows qualitatively the variation in the peak shapes in the dimer-Mott phase diagram.

The similar tendency observed between the data of the external and the chemical pressure-controlled experiments demonstrates that a crossover occurs inside the superconductive phase due to variations in the coupling strength of the superconductive electrons. A similar situation may occur in high $T_{\mathrm{c}}$ cuprates or heavy electron systems in which the quantum mechanical features produce a crossover in the ground state, giving complicated phase diagrams.

It is important to mention that although the peak shape of $\Delta C_{p} / T_{\mathrm{c}}$ varies with the decrease in $U / W$, the temperature dependence of the heat capacity in the low-temperature region gives a quadratic temperature-dependent term, as suggested by the nodal feature of the d-wave formation [60]. Figure 9a,b compares the temperature dependence of the low-temperature heat capacity of two k-type compounds with a high $T_{\mathrm{c}}(10 \mathrm{~K})$ class and a middle $T_{\mathrm{c}}$ class. The existence of the quadratic temperature dependence in the electronic heat capacity clearly shows the difference in the BCS characters, even though the peak shapes are quite different, as we discussed in Figure 8. Since the large lattice contribution in the heat capacity creates ambiguity in background subtraction in such organic systems, the recovery of the $\gamma$ by magnetic fields of both compounds also shows an $\left(H / H_{\mathrm{c} 2}\right)^{1 / 2}$-dependence characteristic of d-wave pairings. The change in the nodal direction, even in the same k-type structure, has been suggested theoretically, depending on the balance of the transfer integral inside the dimer and the ratio between the transfer integrals in the rectangle and the diagonal directions of the superconductivity. It is emphasized, however, that the electron correlations still seem to be an important mechanism for realizing superconductivity in this system. The existence of the nodes is also consistent with other experiments, such as the thermal conductivity, NMR, and STM experiments [23-26]. 

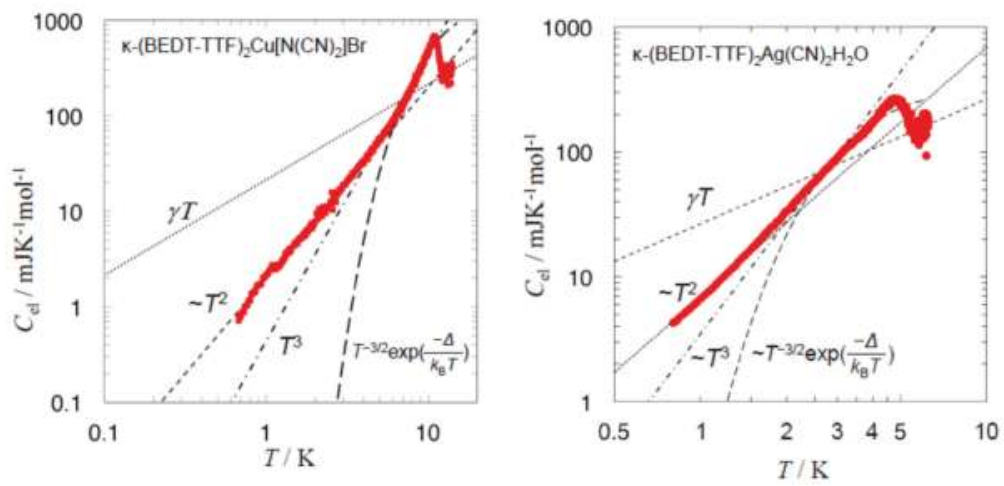

Figure 9. Temperature dependence of the electronic heat capacity determined as the difference of the 0-T data and the normal state data obtained under magnetic fields higher than $H_{\mathrm{c} 2}$ for (a) $\mathrm{K}$-(BEDT-TTF $)_{2} \mathrm{Cu}\left[\mathrm{N}(\mathrm{CN})_{2}\right] \mathrm{Br}$ and $(\mathbf{b}) \mathrm{k}-(\mathrm{BEDT}-\mathrm{TTF})_{2} \mathrm{Ag}(\mathrm{CN})_{2} \mathrm{H}_{2} \mathrm{O}$ below the transition temperatures. (Ref. [60].) The lines and the curve shown in the figure denote $T$ linear, $T^{2}, T^{3}$, and $T^{-3 / 2} \exp \left(-\Delta / k_{\mathrm{B}} T\right)$ temperature dependence.

\section{Normal State Electronic Heat Capacity Coefficient $\gamma$ and the Residual $\gamma^{*}$}

A quantitative comparison and the overall discussion of the coupling strength observed in the peak shape of the heat capacity and magnitude of the electronic heat capacity coefficient $\gamma$ of the normal state should reveal a more profound understanding of the superconductive state of the dimer-Mott system. For this purpose, low-temperature heat capacity data should be discussed systematically throughout the superconductive phase. In this section, we survey the normal state $\gamma$ and the residual $\gamma^{*}$ in the superconductive state observed from the data of the low-temperature heat capacity, which reflects the low energy excitations in the superconductive phase. We discuss the peculiar feature and stability of the superconductive states in terms of the competition between the stability of the Fermi liquid nature and the magnitude of the electron correlations. Since the ground state of the $\mathrm{k}$-(BEDT-TTF) ${ }_{2} \mathrm{X}$ compounds in question is superconductive with electron pairs, the heat capacity measurements with magnetic fields higher than $H_{\mathrm{c} 2}$ should be performed to evaluate the electron density of state of the Fermi surface. The electronic heat capacity coefficient obtained under a magnetic field is defined as the normal state $\gamma$ and is proportional to the electron density of states $D\left(\varepsilon_{\mathrm{F}}\right)$ in the band theory.

To discuss the systematic changes of $\gamma$ in the dimer-Mott-type superconductive phase, Figure 10b plots the $\gamma$ values of several compounds with the conceptual phase diagram (Figure 10a). We use the values of the dimerized compounds of $\mathrm{k}$-(BEDT-TTF $)_{2} \mathrm{X}$ and some other compounds also considered as dimer-based systems. The data are taken from Table 9 in Ref. [28] and the low-temperature heat capacity results reported in Refs. [42,59-63]. In this plot, we also included $\beta$-(BEDT-TTF) ${ }_{2} \mathrm{I}_{3}$ in a well-annealed case according to the proposed phase diagram in Refs. $[11,14,15]$. The two arrows show that the systematic tendency occurs in the superconductive phase. Complexes of partially deuterated BEDT-TTF molecules with $\mathrm{X}=\mathrm{Cu}\left[\mathrm{N}(\mathrm{CN})_{2}\right] \mathrm{Br}$ were used to systematically investigate the change in the normal state $\gamma$ near the boundary region $[62,63]$. The normal state $\gamma$ drastically decreases around the boundary despite the fact that $T_{\mathrm{c}}$ maintains a relatively high value around $10 \mathrm{~K}$, indicating that the Mott boundary occurs as a first-order transition in the dimer-Mott system. The change in $\gamma$ occurs in a region far away from the boundary, suggesting that the band-like feature reflecting in the electronic density of states systematically varies. In the discussion on this region, we include data of solid solution compounds of BEDT-TTF and BEDSe-TTF with $\mathrm{X}=\mathrm{Cu}\left[\mathrm{N}(\mathrm{CN})_{2}\right] \mathrm{Br}$ since light doping (less than $10 \%$ ) of BEDSe-TTF tends to reduce the $T_{\mathrm{C}}$ by keeping the bulk superconductive feature [33]. The arrow on the left side of the figure clearly indicates that the $\gamma$ values increase as the $U / W$ ratio increases. This is considered as a kind of Brinkman-Rice enhancement. It predicts that the increase in 
the electron correlation of the parameter $U / W$ leads to an increase in the density of states similar to that observed in correlated metallic systems with a distinct band structure [63-65]. The normal state $\gamma$ value gives a maximum around $\mathrm{k}-(\mathrm{BEDT}-\mathrm{TTF})_{2} \mathrm{Cu}(\mathrm{NCS})_{2}$, which shows a stable bulk superconductivity with a transition temperature of $9.1 \mathrm{~K}$. In this compound, the cooling rate does not affect the transition temperature located close to the boundary, which differs from the case of $\mathrm{X}=\mathrm{Cu}\left[\mathrm{N}(\mathrm{CN})_{2}\right] \mathrm{Br}$ (both deuterated and non-deuterated cases).

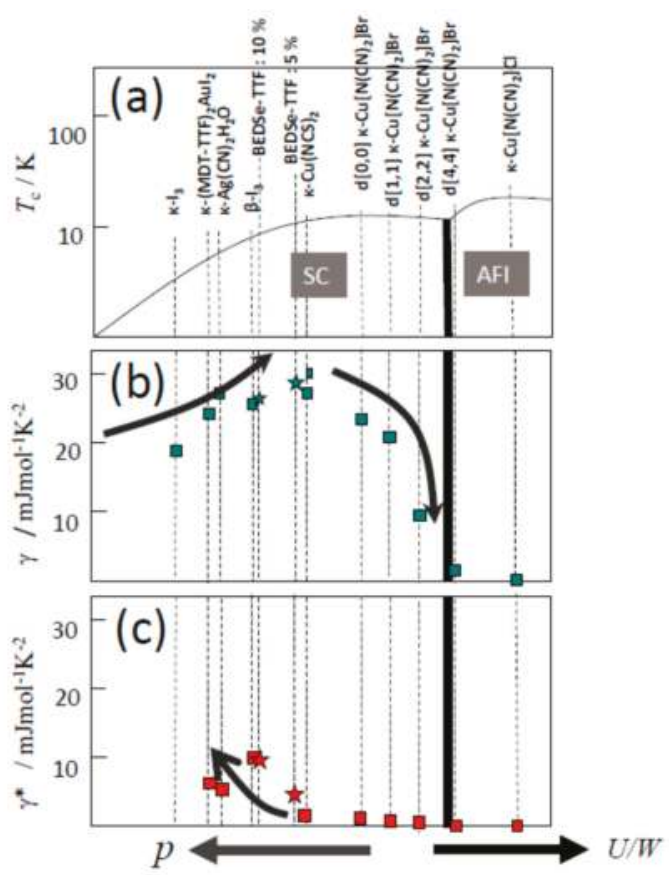

Figure 10. Systematic variations of the low-temperature thermodynamic parameters in the compounds mapped in the dimer-Mott phase diagram (a). Changes in (b) the normal states electronic heat capacity coefficient $\gamma$ obtained by heat capacity data above $H_{\mathrm{c} 2}$ and (c) the residual electronic heat capacity coefficient $\gamma^{*}$ in the superconductive state at $0 \mathrm{~T}$ are plotted in accordance with the position in the phase diagram. The position of each compound is determined in reference to Refs $[11,14,15]$. The data are taken from Table 9 in Ref. [28] and the reported results data in Refs [42,59-63]. Systematic change of the thermodynamic parameters occurring with the change in chemical pressure, namely the change in the parameter of the $U / W$ ratio, suggests that a kind of crossover exists inside the superconductive phase. Compounds shown as the name of the counter-anions are BEDT-TTF(ET) complexes. BEDSe-TTF 5\%, $10 \%$ mean the solid solution systems of BEDT-TTF and BEDSe-TTF. (See text.) $\mathrm{d}[n, n]-\mathrm{Cu}\left[\mathrm{N}(\mathrm{CN})_{2}\right] \mathrm{Br}$ denotes a $\mathrm{Cu}\left[\mathrm{N}(\mathrm{CN})_{2}\right] \mathrm{Br}$ compound with BEDT-TTF of which ethylene groups are partially deuterated (Ref. $[61,62])$ to tune the chemical pressure. Arrows show the characteristic tendency reflecting the physical properties.

The Brinkman-Rice-type enhancement is due to the increase in the enhanced correlation $U$ in the region where the Fermi liquid picture holds. The increase in the correlation influences the effective mass of the normal electrons within the band picture and, therefore, it leads to an increase in $\gamma$ values. The compounds in the left arrow region are considered to have a Fermi liquid character with a well-defined electron band. However, increasing $U$ further leads the system to an anomalous metallic state where the picture with a gap-like structure between the upper and the 
lower Hubbard bands become dominant if the Mott boundary is approached. The relatively smaller normal state $\gamma$ value despite the higher transition temperature and the stronger coupling character in the superconductivity in $\mathrm{k}-(\mathrm{BEDT}-\mathrm{TTF})_{2} \mathrm{Cu}\left[\left(\mathrm{N}(\mathrm{CN})_{2}\right] \mathrm{Br}\right.$ than $\mathrm{Cu}(\mathrm{NCS})_{2}$ can be explained from this perspective. The decrease in $\gamma$ in the partially deuterated compound denoted by $\mathrm{d}[1,1]-\mathrm{Cu}\left[\mathrm{N}(\mathrm{CN})_{2}\right] \mathrm{Br}$ is considered as evidence of further opening of the Hubbard gap, leading to a decreased electron density of state as a band picture. On the other hand, the smaller $\gamma$ values in the middle-class $T_{\mathrm{C}}$ compounds of $\mathrm{k}$-(BEDT-TTF $)_{2} \mathrm{Ag}(\mathrm{CN})_{2} \mathrm{H}_{2} \mathrm{O}$ and $\mathrm{k}$-(MDT-TTF $)_{2} \mathrm{AuI}_{2}$ located in the band region can be explained in the frame of the Brinkman-Rice picture. Recently, Imajo et al. studied $\lambda$-(BETS) ${ }_{2} \mathrm{GaCl}_{4}$, which is also classified as a dimer-Mott-type superconductor and has a $T_{\mathrm{c}}$ of about $5.2 \mathrm{~K}$. It shows a similar normal state $\gamma=22.9 \mathrm{mJK}^{-2} \mathrm{~mol}^{-1}$ [66]. This result is consistent with this picture. We also include the $\gamma$ values of $\mathrm{k}$-(BEDT-TTF $)_{2} \mathrm{I}_{3}[59]$ and $\beta$-(BEDT-TTF $)_{2} \mathrm{X}\left(\mathrm{X}=\mathrm{I}_{3}, \mathrm{AuI}_{2}\right)[67,68]$, which are also consistent with the above feature. Not only is the coupling strength reflected in the heat capacity jump but the feature of the normal state $\gamma$ value also suggests that the electron correlations become stronger by approaching the boundary and are important for stabilizing the bulk superconductivity. When the transition temperature decreases with a decrease in the $U / W$ ratio, the Mott-Hubbard picture is gradually suppressed and the Fermi liquid nature is enhanced. The change from strong coupling to weak coupling observed by pressure calorimetry is related to this crossover from the electron correlation region to the band-like region.

The crossover in the coupling nature originating from the magnitude of the electron correlation is also reflected in the low-temperature residual $\gamma^{*}$ in the superconductive state (Figure 10c). The superconductivity of the dimer-Mott system is a d-wave with a nodal gap with four-fold symmetry around the two-dimensional Fermi surface, as detected by the magnetic angle-resolved heat capacity measurements. In such nodal gap superconductors, normal electrons inevitably remain due to the residual disorder in the crystals even without an external magnetic field. The effects of disorder and impurities can induce normal electrons around the nodal position with a very small gap. Figure 10c also shows the data for $\gamma^{*}$. As previously reported, the $\gamma^{*}$ value shows a sample dependence but the value is about several percent of the normal state $\gamma$ and less than $1-2 \mathrm{mJK}^{-2} \mathrm{~mol}^{-1}$ in the cases of $\mathrm{k}-(\mathrm{BEDT}-\mathrm{TTF})_{2} \mathrm{Cu}\left[\mathrm{N}(\mathrm{CN})_{2}\right] \mathrm{Br}$ and $\mathrm{k}-(\mathrm{BEDT}-\mathrm{TTF})_{2} \mathrm{Cu}(\mathrm{NCS})_{2}$. However, those with lower $T_{\mathrm{C}}$ compounds tend to have larger $\gamma^{*}$ values despite the heat capacity giving a distinct peak at the transition. In the cases of $\mathrm{k}$-(BEDT-TTF $)_{2} \mathrm{Ag}(\mathrm{CN})_{2} \mathrm{H}_{2} \mathrm{O}$ and $\mathrm{k}$-(MDT-TTF $)_{2} \mathrm{AuI}_{2}$, the reported $\gamma^{*}$ values are $5.1 \mathrm{mJK}^{-2} \mathrm{~mol}^{-1}$ and $5.9 \mathrm{mJK}^{-2} \mathrm{~mol}^{-1}$, respectively [33,49]. A more recent experiment on к-(BEDT-TTF) ${ }_{2} \mathrm{Ag}(\mathrm{CN})_{2} \mathrm{H}_{2} \mathrm{O}$ claims a smaller $\gamma^{*}$ value (less than $2 \mathrm{mJK}^{-2} \mathrm{~mol}^{-1}$ ) using the fitting of the d-wave model [60]. This seems to be larger than those of $\mathrm{k}-(\mathrm{BEDT}-\mathrm{TTF})_{2} \mathrm{Cu}\left[\mathrm{N}(\mathrm{CN})_{2}\right] \mathrm{Br}$, and $\kappa$-(BEDT-TTF $)_{2} \mathrm{Cu}(\mathrm{NCS})_{2} . \lambda$-(BETS $)_{2} \mathrm{GaCl}_{4}$ also gives a relatively large value of $3.2 \mathrm{mJK}^{-2} \mathrm{~mol}^{-1}$ [66].

Although the bulk nature of the superconductivity is retained in these compounds, pair breaking due to suppression of the electron correlation and the increase in the stability of Fermi liquid nature occurs in the superconductive phase. The competition of the band picture, which just enhances the electron density of state and the Mott-Hubbard picture, should exist in the superconductive phase; however, a gradual crossover occurs inside the superconductive phase. These characters can be considered to have similar physics in the over-doped region of high $T_{\mathrm{c}}$ cuprates. The heat capacity peak around the transition becomes much broader and the bulk nature of the superconductivity is suppressed in the over-doped region due to the Fermi liquid character, which stabilizes the band nature. This character is in contrast with the under-doped and optimal-doped regions [69]. Considering the overall thermodynamic features of dimer-Mott systems, the mechanism to produce a d-wave type superconductor is quite reasonably related to the electron correlations $U$, and the superconductivity is stabilized in the anomalous metallic region near the Mott boundary. Here, we discussed mainly $\kappa$-type compounds with a dimer lattice. More detailed thermodynamic information for other types of dimer-Mott structures with $\beta-, \beta^{\prime}-$, and $\lambda$-type compounds is necessary in the future. 


\section{Conclusions}

We systematically discuss the thermodynamic parameters to quantitatively evaluate the superconductive characters of the dimer-Mott system, which appear as tuning of the $U / W$ ratio. The calorimetric information of $\mathrm{\kappa}-(\mathrm{BEDT}-\mathrm{TTF})_{2} \mathrm{Cu}(\mathrm{NCS})_{2}$ and other counter-anion compounds reveals that the magnitude of the coupling strength is suppressed from the high $T_{\mathrm{C}}$ region to lower $T_{\mathrm{C}}$ region. The chemical pressures also confirm the changes in the peak shape of this thermal anomaly. A comparative discussion of the electronic heat capacity coefficients in the low-temperature heat capacity in both the superconductive state at $0 \mathrm{~T}$ and the normal state demonstrates that a crossover from the band character region to the strong electron correlation region exists in the superconductive phase. Such a crossover may support the unconventional nature of the superconductivity in dimer-Mott superconductors since the superconducting character near the strong correlation region provides stability compared with the lower $T_{\mathrm{c}}$ region where the band character becomes dominant. This feature resembles cuprate superconductors in which the electron correlations and band character compete with each other, yielding complicated electronic features in the optimal doping region. More systematic thermodynamic experiments with a parameter of pressure are required to further investigate the system.

Acknowledgments: This work is partly supported by CREST, JST Program in the area of "Establishment of Molecular Technology towards the Creation of New Functions".

Author Contributions: Yasuhiro Nakazawa conceived and designed the experiments; Hiroki Akutsu performed crystal syntheses and structure analyses; Shusaku Imajo, Yuki Matsumura, and Satoshi Yamashita performed the experiments and analyzed the data.

Conflicts of Interest: The authors declare no conflict of interests.

\section{References}

1. Ishiguro, T.; Yamaji, K.; Saito, G. Organic Superconductors; Springer: Heidelberg, Germany, 1998; pp. 1-245.

2. Williams, J.M.; Ferraro, J.R.; Thorn, R.J.; Carlson, K.D.; Geiser, U.; Wang, H.-H.; Kini, A.M.; Whangbo, M.-H. Organic Superconductors; Prentice-Hall, Inc.: Upper Saddle River, NJ, USA, 1992; pp. 65-179.

3. Lebet, A. The Physics of Organic Superconductors and Conductors; Springer: Berlin, Germany, 2008; pp. 1-704.

4. Uji, S.; Mori, T.; Takahashi, T. Focus on Organic Conductors. Sci. Technol. Adv. Mater. 2009, 10, 020301. [CrossRef] [PubMed]

5. Haddon, R.C.; Hebard, A.F.; Rosseinsky, M.J.; Murphy, D.W. Conducting films of $\mathrm{C}_{60}$ and $\mathrm{C}_{70}$ by alkali-metal doping. Nature 1991, 350, 320-322. [CrossRef]

6. Palstra, T.T.M.; Zhou, O.; Iwasa, Y.; Sulewski, P.E.; Flemming, R.M.; Zegarski, B.R. Superconductivity at 40K in cesium doped $C_{60}$. Solid State Commun. 1995, 93, 327-330. [CrossRef]

7. Greene, R.L.; Street, G.B.; Suter, L.J. Superconductivity in Polysulfur Nitride (SN)x. Phys. Rev. Lett. 1975, 34, 577-579. [CrossRef]

8. Kubozono, Y.; Mitamura, H.; Lee, X.; He, X.; Yamanari, Y.; Takahashi, Y.; Suzuki, Y.; Kaji, Y.; Eguchi, R.; Akaike, K.; et al. Metal-intercalated aromatic hydrocarbons: A new class of carbon-based superconductors. Phys. Chem. Chem. Phys. 2011, 13, 16476-16493. [CrossRef] [PubMed]

9. Kagoshima, S.; Kato, R.; Fukuyama, H.; Seo, H.; Kino, H. Interplay of Structural and Electronic Properties. In Advances in Synthetic Metals Twenty Years of Progress in Science and Technology; Chapter 4; Elsevier: Lausanne, Switzerland, 1999; pp. 262-316.

10. Mori, H. Materials Viewpoint of Organic Superconductors. J. Phys. Soc. Jpn. 2006, 75, 051003. [CrossRef]

11. Kanoda, K. Metal-Insulator Transition in $\mathrm{k}-(\mathrm{ET})_{2} \mathrm{X}$ and $(\mathrm{DCNQI})_{2} \mathrm{M}$ : Two Contrasting Manifestation of Electron Correlation. J. Phys. Soc. Jpn. 2006, 75, 051007. [CrossRef]

12. Kato, R. Development of $\pi$-Electron Systems Based on $\left[\mathrm{M}(\mathrm{dmit})_{2}\right](\mathrm{M}=\mathrm{Ni}$ and $\mathrm{Pd}$; dmit: 1,3-dithiole-2-thione-4,5-dithiolate) Anion Radicals. Bull. Chem. Soc. Jpn. 2014, 87, 355-374. [CrossRef]

13. Kino, H.; Fukuyama, H. Phase Diagram of Two-Dimensional Organic Conductors: (BEDT-TTF) 2 X. J. Phys. Soc. Jpn. 1996, 65, 2158-2169. [CrossRef]

14. Kanoda, K. Recent progress in NMR studies on organic conductors. Hyperfine Interact. 1997, 104, $235-249$. [CrossRef] 
15. Kanoda, K. Electron correlation, metal-insulator transition and superconductivity in quasi-2D organic systems, $(\mathrm{ET})_{2}$ X. Phys. C Superconduct. 1997, 287, 299-302. [CrossRef]

16. Kobayashi, H.; Cui, H.; Kobayashi, A. Organic metals and superconductors based on BETS (BETS = bis(ethylenedithio)tetraselenafulvalene). Chem. Rev. 2004, 104, 5265-5288. [CrossRef] [PubMed]

17. Fujiwara, H.; Kobayashi, H.; Fujiwara, E.; Kobayashi, A. An Indication of Magnetic-Field-Induced Superconductivity in a Bifunctional Layered Organic Conductor, k-(BETS ${ }_{2} \mathrm{FeBr}_{4}$. J. Am. Chem. Soc. 2002, 124, 6816-6817. [CrossRef] [PubMed]

18. Kagawa, F.; Miyagawa, K.; Kanoda, K. Unconventional critical behaviour in a quasi-two-dimensional organic conductor. Nature 2005, 436, 534-537. [CrossRef] [PubMed]

19. Gati, E.; Garst, M.; Manna, R.S.; Tutsch, U.; Wolf, B.; Bartosch, L.; Schubert, H.; Sasaki, T.; Schlueter, J.A.; Lang, M. Breakdown of Hooke's law of elasticity at the Mott critical endpoint in an organic conductor. Sci. Adv. 2016, 2, e1601646. [CrossRef] [PubMed]

20. Matsumura, Y.; Imajo, S.; Yamashita, S.; Akutsu, H.; Nakazawa, Y. Thermodynamic Investigation by Heat Capacity Measurements of $\mathrm{k}$-type Dimer-Mott Organic Compounds with Chemical Pressure Tuning. Int. J. Mod. Phys. B 2018, 32, 1840024. [CrossRef]

21. Mori, H.; Tanaka, S.; Mori, T. Systematic study of the electronic state in $\theta$-type BEDT-TTF organic conductors by changing the electronic correlation. Phys. Rev. B 1998, 57, 12023-12029. [CrossRef]

22. McKenzie, R.H.; Merino, J.; Marston, J.B.; Sushkov, O.P. Charge ordering and antiferromagnetic exchange in layered molecular crystals of the $\theta$ type. Phys. Rev. B 2001, 64, 085109. [CrossRef]

23. Miyagawa, K.; Kanoda, K.; Kawamoto, A. NMR Studies on Two-Dimensional Molecular Conductors and Superconductors: Mott Transition in k-(BEDT-TTF) ${ }_{2}$ X. Chem. Rev. 2004, 104, 5635-5654. [CrossRef] [PubMed]

24. Arai, T.; Ichimura, K.; Nomura, K.; Takasaki, S.; Yamada, J.; Nakatsuji, S.; Anzai, H. Tunneling spectroscopy on the organic superconductor K-(BEDT-TTF $)_{2} \mathrm{Cu}(\mathrm{NCS})_{2}$ using STM. Phys. Rev. B 2001, 63, 104518. [CrossRef]

25. Nakazawa, Y.; Kanoda, K. Low-temperature specific heat of $\mathrm{k}-(\mathrm{BEDT}-\mathrm{TTF})_{2} \mathrm{Cu}\left[\mathrm{N}(\mathrm{CN})_{2}\right] \mathrm{Br}$ in the superconducting state. Phys. Rev. B 1997, 55, R8670-R8673. [CrossRef]

26. Taylor, O.J.; Carrington, A.; Schlueter, J.A. Specific-Heat Measurements of the Gap Structure of the Organic Superconductors k-(ET) $)_{2} \mathrm{Cu}\left[\mathrm{N}(\mathrm{CN})_{2}\right] \mathrm{Br}$ and $\mathrm{k}-(\mathrm{ET})_{2} \mathrm{Cu}(\mathrm{NCS})_{2}$. Phys. Rev. Lett. 2007, 99, 057001. [CrossRef] [PubMed]

27. Girlando, A.; Masino, M.; Brillante, A.; Valle, R.G.D.; Venuti, E. BEDT-TTF organic superconductors: The role of phonons. Phys. Rev. B 2002, 66, 100507. [CrossRef]

28. Sorai, M.; Nakazawa, Y.; Nakano, M.; Miyazaki, Y. Calorimetric Investigation of Phase Transitions Occurring in Molecule-Based Magnets. Chem. Rev. 2013, 113, PR41-122. [CrossRef] [PubMed]

29. Gopal, E.S.R. Specific Heats at Low Temperature; Heywood Books: London, UK, 1966.

30. Wosnitza, J. Quasi-Two-Dimensional Organic Superconductors. J. Low Temp. Phys. 2007, 146, 641-667. [CrossRef]

31. Wosnitza, J. Superconductivity in Layered Organic Metals. Crystals 2012, 2, 248-265. [CrossRef]

32. Muraoka, Y.; Imajo, S.; Yamashita, S.; Akutsu, H.; Nakazawa, Y. Thermal Anomaly around the Superconductive Transition of $\mathrm{k}-(\mathrm{BEDT}-\mathrm{TTF})_{2} \mathrm{Cu}(\mathrm{NCS})_{2}$ with External Pressure and Magnetic Field Control. J. Therm. Anal. Calorim. 2016, 123, 1891-1897. [CrossRef]

33. Nakazawa, Y.; Yoshimoto, R.; Fukuoka, S.; Yamashita, S. Investigation on Electronic States of Molecule-Based Compounds by High-Pressure AC Calorimetry. Curr. Inorg. Chem. 2014, 4, 122-134. [CrossRef]

34. Bachmann, R.; DiSalvo, F.J., Jr.; Geballe, T.H.; Greene, R.L.; Howard, R.E.; King, C.N.; Kirsch, H.C.; Lee, K.N.; Schwall, R.E.; Thomas, H.-U.; et al. Heat Capacity Measurements on Small Samples at Low Temperatures. Rev. Sci. Instrum. 1972, 43, 205-214. [CrossRef]

35. Stewart, G.R. Measurement of low-temperature specific heat. Rev. Sci. Instrum. 1983, 54, 1-11. [CrossRef]

36. Sorai, M. Comprehensive Handbook of Calorimetry and Thermal Analysis; Wiley: New York, NY, USA, 2004.

37. Imajo, S.; Fukuoka, S.; Yamashita, S.; Nakazawa, Y. Construction of Relaxation Calorimetry for 10 1-2 Micro-gram Samples and Heat Capacity Measurements of Organic Complexes. J. Therm. Anal. Calorim. 2016, 123, 1871-1876. [CrossRef]

38. Fukuoka, S.; Horie, Y.; Yamashita, S.; Nakazawa, Y. Development of Heat Capacity Measurement System for Single Crystals of Molecule-Based Compounds. J. Therm. Anal. Calorim. 2013, 113, 1303-1308. [CrossRef]

39. Sullivan, P.F.; Seidel, G. Steady-State, ac-Temperature Calorimetry. Phys. Rev. 1968, 173, 679-685. [CrossRef] 
40. Eichler, A.; Gey, W. Method for the determination of the specific heat of metals at low temperatures under high pressures. Rev. Sci. Instrum. 1979, 50, 1445-1452. [CrossRef] [PubMed]

41. Kubota, O.; Nakazawa, Y. Construction of a low-temperature thermodynamic measurement system. Rev. Sci. Instrum. 2008, 79, 053901. [CrossRef] [PubMed]

42. Kubota, O.; Fukuoka, S.; Nakazawa, Y.; Nakata, K.; Yamashita, S.; Miyasaka, H. Thermodynamic Investigation of Coordination-Networked Systems of $\left[\mathrm{Mn}_{4}\right]$ Single-Molecule Magnets under Pressure. J. Phys. Condens. Matter 2010, 22, 026007. [CrossRef] [PubMed]

43. Tokoro, N.; Kubota, O.; Yamashita, S.; Kawamoto, A.; Nakazawa, Y. Thermodynamic Study of k-(BEDT-TTF $)_{2} \mathrm{Ag}(\mathrm{CN})_{2} \mathrm{H}_{2} \mathrm{O}$ under Pressures and with Magnetic Fields. J. Phys. Conf. Ser. 2008, 132, 012010. [CrossRef]

44. Tokoro, N.; Fukuoka, S.; Kubota, O.; Nakazawa, Y. Low-temperature heat capacity measurements of k-type organic superconductors under pressure. Phys. B Condens. Matter 2010, 405, S273-S276. [CrossRef]

45. Danda, M.; Muraoka, Y.; Yamamoto, T.; Nakazawa, Y. High-Pressure AC Calorimetry System Using Pt Chip Thermometer. Netsu Sokutei 2012, W39, 29-32.

46. Konoike, T.; Uchida, K.; Osada, T. Specific Heat of the Multilayered Massless Dirac Fermion System. J. Phys. Soc. Jpn. 2013, 81, 043601. [CrossRef]

47. Naito, A.; Nakazawa, Y.; Saito, K.; Taniguchi, H.; Kanoda, K.; Sorai, M. Anomalous enhancement of electronic heat capacity in the organic conductors $\mathrm{k}-(\mathrm{BEDT}-\mathrm{TTF}){ }_{4} \mathrm{Hg}_{3-\delta} \mathrm{X}_{8}(\mathrm{X}=\mathrm{Br}, \mathrm{Cl})$. Phys. Rev. B 2005, 71, 054514. [CrossRef]

48. Yamashita, S.; Naito, A.; Nakazawa, Y.; Saito, K.; Taniguchi, H.; Kanoda, K.; Oguni, M. Drastic cooling rate dependence of thermal anomaly associated with the superconducting transition in K-(BEDT-TTF) ${ }_{4} \mathrm{Hg}_{2.89} \mathrm{Br}_{8}$. J. Therm. Anal. Calorim. 2005, 81, 591-594. [CrossRef]

49. Nakazawa, Y.; Yamashita, S. Thermodynamic Properties of $\mathrm{k}-(\mathrm{BEDT}-\mathrm{TTF})_{2} \mathrm{X}$ Salts: Electron Correlations and Superconductivity. Crystals 2012, 2, 741-761. [CrossRef]

50. Yamashita, S.; Ishikawa, T.; Fujisaki, T.; Naito, A.; Nakazawa, Y.; Oguni, M. Thermodynamic behavior of the 10 $\mathrm{K}$ class organic superconductor $\mathrm{K}-(\mathrm{BEDT}-\mathrm{TTF})_{2} \mathrm{Cu}(\mathrm{NCS})_{2}$ studied by relaxation calorimetry. Thermochim. Acta 2005, 431, 123-126. [CrossRef]

51. Ishikawa, T.; Nakazawa, Y.; Ymashita, S.; Oguni, M.; Saito, K.; Takimiya, K.; Otsubo, T. Thermodynamic Study

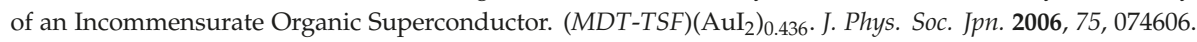
[CrossRef]

52. Katsumoto, S.; Kobayashi, S.; Urayama, H.; Yamochi, H.; Saito, G. Low-Temperature Specific Heat of Organic Superconductor k-(BEDT-TTF) ${ }_{2} \mathrm{Cu}(\mathrm{NCS})_{2}$. J. Phys. Soc. Jpn. 1988, 57, 3672-3673. [CrossRef]

53. Andraka, B.; Kim, J.S.; Stewart, G.R.; Calson, K.D.; Wang, H.H.; Williams, J.M. Specific heat in high magnetic field of $\kappa$-di[bis(ethylenedithio)tetrathiafulvalene]-di(thiocyano)cuprate $\left[\kappa-(\mathrm{ET})_{2} \mathrm{Cu}(\mathrm{NCS})_{2}\right]$ : Evidence for strong-coupling superconductivity. Phys. Rev. B 1989, 40, 11345-11347. [CrossRef]

54. Graebner, J.E.; Haddon, R.C.; Chichester, S.V.; Glarum, S.H. Specific heat of superconducting K-(BEDT-TTF $)_{2} \mathrm{Cu}(\mathrm{NCS})_{2}$ near $T_{\mathrm{c}}$ [where BEDT-TTF is bis(ethylenedithio)tetrathiafulvalene]. Phys. Rev. B 1990, 41, 4808-4810. [CrossRef]

55. Müller, J.; Lang, M.; Helfrich, R.; Steglich, F.; Sasaki, T. High-resolution ac-calorimetry studies of the quasi-two-dimensional organic superconductor k-(BEDT-TTF) ${ }_{2} \mathrm{Cu}(\mathrm{NCS})_{2}$. Phys. Rev. B 2002, 65, 140509. [CrossRef]

56. Lortz, R.; Wang, Y.; Demuer, A.; Böttger, P.H.M.; Bergk, B.; Zwicknagl, G.; Nakazawa, Y.; Wosnitza, J. Calorimetric Evidence for a Fulde-Ferrell-Larkin-Ovchinnikov Superconducting State in the Layered Organic Superconductor k-(BEDT-TTF) ${ }_{2} \mathrm{Cu}(\mathrm{NCS})_{2}$. Phys. Rev. Lett. 2007, 99, 187002. [CrossRef] [PubMed]

57. Elsinger, H.; Wosnizta, J.; Wanka, S.; Hagel, J.; Schweitzer, D.; Strunz, W. k-(BEDT-TTF $)_{2} \mathrm{Cu}\left[\mathrm{N}(\mathrm{CN})_{2}\right] \mathrm{Br}$ : A Fully Gapped Strong-Coupling Superconductor. Phys. Rev. Lett. 2000, 84, 6098-6101. [CrossRef] [PubMed]

58. Ishikawa, T.; Yamashita, S.; Nakazawa, Y.; Kawamoto, A.; Oguni, M. Calorimetric study of molecular superconductor $\mathrm{K}-(\mathrm{BEDT}-\mathrm{TTF}){ }_{2} \mathrm{Ag}(\mathrm{CN})_{2} \mathrm{H}_{2} \mathrm{O}$ which contains water in the anion layers. J. Therm. Anal. Calorim. 2008, 92, 435-438. [CrossRef]

59. Wosnitza, J.; Liu, X.; Schweitzer, D.; Keller, H.J. Specific heat of the organic superconductor k-(BEDT-TTF) ${ }_{2} \mathrm{I}_{3}$. Phys. Rev. B 1994, 50, 12747-12751. [CrossRef]

60. Imajo, S.; Yamashita, S.; Akutsu, H.; Nakazawa, Y. Quadratic Temperature Dependence of Electronic Heat Capacities in the k-Type Organic Superconductors. Int. J. Mod. Phys. B 2016, 30, 1642014. [CrossRef] 
61. Nakazawa, Y.; Taniguchi, H.; Kawamoto, A.; Kanoda, K. Electronic specific heat at the boundary region of the metal-insulator transition in the two-dimensional electronic system of $\mathrm{k}-(\mathrm{BEDT}-\mathrm{TTF})_{2} \mathrm{Cu}\left[\mathrm{N}(\mathrm{CN})_{2}\right] \mathrm{Br}$. Phys. Rev. B 2000, 61, R16295-R16298. [CrossRef]

62. Nakazawa, Y.; Taniguchi, H.; Kawamoto, A.; Kanoda, K. Electronic specific heat of BEDT-TTF-based organic conductors. Phys. B Condens. Matter 2000, 281-282, 899-900. [CrossRef]

63. Brinkman, W.F.; Rice, T.M. Application of Gutzwiller's Variational Method to the Metal-Insulator Transition. Phys. Rev. B 1970, 2, 4302-4304. [CrossRef]

64. McWhan, D.B.; Remeika, J.P.; Rice, T.M.; Brinkman, W.F.; Maita, J.P.; Menth, A. Electronic Specific Heat of Metallic Ti-Doped $\mathrm{V}_{2} \mathrm{O}_{3}$. Phys. Rev. Lett. 1971, 27, 941-943. [CrossRef]

65. Tokura, Y.; Taguchi, Y.; Okada, Y.; Fujishima, Y.; Arima, A.; Kumagai, K.; Iye, Y. Filling dependence of electronic properties on the verge of metal-Mott-insulator transition in $\mathrm{Sr}_{1-x} \mathrm{La}_{x} \mathrm{TiO}_{3}$. Phys. Rev. Lett. 1993, 70, 2126-2129. [CrossRef] [PubMed]

66. Imajo, S.; Kanda, N.; Yamashita, S.; Akutsu, H.; Nakazawa, Y.; Kumagai, H.; Kobayashi, T.; Kawamoto, A. Thermodynamic Evidence of $d$-Wave Superconductivity of the Organic Superconductor $\lambda$-(BETS) ${ }_{2} \mathrm{GaCl}_{4}$. J. Phys. Soc. Jpn. 2016, 85, 043705. [CrossRef]

67. Stewart, G.R.; Williams, J.M.; Wang, H.H.; Hall, L.N.; Perozzo, M.T.; Carlson, K.D. Bulk superconducting specific-heat anomaly in $\beta$-di[bis (ethylenedithio) tetrathiafulvalene] diiodoaurate $\left[\beta-(\mathrm{ET})_{2} \mathrm{AuI}_{2}\right]$. Phys. Rev. B 1986, 34, 6509-6510. [CrossRef]

68. Andres, K.; Schwenk, H.; Veith, H. Peculiarities of Organic Superconductors of the (BEDT-TTF) 2 X Family. Phys. B+C 1986, 143, 334-337. [CrossRef]

69. Matsuzaki, T.; Momono, N.; Oda, M.; Ido, M. Electronic specific heat of $\mathrm{La}_{2-\mathrm{x}} \mathrm{Sr}_{\mathrm{x}} \mathrm{CuO} 4$ : Pseudogap formation and reduction of the superconducting condensation energy. J. Phys. Soc. Jpn. 2004, 73, 2232-2238. [CrossRef]

(C) 2018 by the authors. Licensee MDPI, Basel, Switzerland. This article is an open access article distributed under the terms and conditions of the Creative Commons Attribution (CC BY) license (http:/ / creativecommons.org/licenses/by/4.0/). 


\title{
Article \\ Effects of Disorder on the Pressure-Induced Mott Transition in $\kappa-(\mathrm{BEDT}-\mathrm{TTF})_{2} \mathrm{Cu}\left[\mathrm{N}(\mathrm{CN})_{2}\right] \mathrm{Cl}$
}

\author{
Elena Gati ${ }^{1, *}$, Ulrich Tutsch ${ }^{1}$, Ammar Naji ${ }^{1}$, Markus Garst ${ }^{2}$, Sebastian Köhler ${ }^{1}$, \\ Harald Schubert ${ }^{1}$, Takahiko Sasaki ${ }^{3}$ and Michael Lang ${ }^{1}$ \\ 1 Institute of Physics, SFB/TR49, Goethe University Frankfurt, Max-von-Laue-Straße 1, 60438 Frankfurt am Main, \\ Germany; tutsch@physik.uni-frankfurt.de (U.T.); Naji@physik.uni-frankfurt.de (A.N.); \\ s.koehler@physik.uni-frankfurt.de (S.K.); h.schubert@physik.uni-frankfurt.de (H.S.); \\ michael.lang@physik.uni-frankfurt.de (M.L.) \\ 2 Institute for Theoretical Physics, Technical University Dresden, Zellescher Weg 17, 01062 Dresden, Germany; \\ markus.garst@tu-dresden.de \\ 3 Institute for Materials Research, Tohoku University, Katahira 2-1-1, Sendai 980-8577, Japan; \\ takahiko@imr.tohoku.ac.jp \\ * Correspondence: gati@physik.uni-frankfurt.de; Tel.: +49-69-798-47240
}

Received: 14 December 2017; Accepted: 11 January 2018; Published: 16 January 2018

\begin{abstract}
We present a study of the influence of disorder on the Mott metal-insulator transition for the organic charge-transfer salt $\kappa-(\mathrm{BEDT}-\mathrm{TTF})_{2} \mathrm{Cu}\left[\mathrm{N}(\mathrm{CN})_{2}\right] \mathrm{Cl}$. To this end, disorder was introduced into the system in a controlled way by exposing the single crystals to $\mathrm{X}$-ray irradiation. The crystals were then fine-tuned across the Mott transition by the application of continuously controllable He-gas pressure at low temperatures. Measurements of the thermal expansion and resistance show that the first-order character of the Mott transition prevails for low irradiation doses achieved by irradiation times up to $100 \mathrm{~h}$. For these crystals with a moderate degree of disorder, we find a first-order transition line which ends in a second-order critical endpoint, akin to the pristine crystals. Compared to the latter, however, we observe a significant reduction of both, the critical pressure $p_{c}$ and the critical temperature $T_{\mathcal{c}}$. This result is consistent with the theoretically-predicted formation of a soft Coulomb gap in the presence of strong correlations and small disorder. Furthermore, we demonstrate, similar to the observation for the pristine sample, that the Mott transition after $50 \mathrm{~h}$ of irradiation is accompanied by sizable lattice effects, the critical behavior of which can be well described by mean-field theory. Our results demonstrate that the character of the Mott transition remains essentially unchanged at a low disorder level. However, after an irradiation time of $150 \mathrm{~h}$, no clear signatures of a discontinuous metal-insulator transition could be revealed anymore. These results suggest that, above a certain disorder level, the metal-insulator transition becomes a smeared first-order transition with some residual hysteresis.
\end{abstract}

Keywords: organic conductor; Mott transition; pressure; disorder; X-ray irradiation

\section{Introduction}

Organic charge-transfer salts of type $D_{2} A$, consisting of donor molecules $D$ and acceptor molecules $A$, have been established as model systems to explore the physics of strong electron correlations [1,2]. The role of Coulomb correlations is particularly enhanced in this material class due to weak intermolecular interactions, giving rise to a small bandwidth $W$, in combination with a low charge-carrier concentration and an electronic structure with reduced dimensionality. The latter two aspects render the screening of the long-range part of the Coulomb interaction less effective. A prominent manifestation of strong correlation effects is the Mott metal-insulator transition [3]. The notion is that the itinerant electrons in an approximately half-filled conduction band localize, 
once the mutual Coulomb repulsion for two electrons on the same site, $U$, exceeds the band width $W$. Although the above-sketched simple picture describes the basics of the Mott transition, the understanding of the rich phenomenology of correlated electrons close to the Mott transition continues to pose major challenges [4-6]. In particular, salts of the $\kappa$-(BEDT-TTF) ${ }_{2} X$ family, with BEDT-TTF $=$ bis(ethylenedithio)-tetrathiafulvalene and $X$ a monovalent anion, have attracted high attention as prime examples of a bandwidth-tuned Mott transition [7,8] at fixed band filling. In these materials, which are considered to exhibit an effectively half-filled conduction band due to the strong dimerization of the BEDT-TTF molecules, the bandwidth can be controlled via the application of pressure $p$. In this way, a transition from a Mott insulating to a metallic state can be induced with increasing pressure through the accompanying decrease in the correlation strength $U / W$. Notably, already a weak pressure of $p \approx 30 \mathrm{MPa}$ is sufficient to induce the Mott metal-insulator transition in the system $\kappa$-(BEDT-TTF $)_{2} \mathrm{Cu}\left[\mathrm{N}(\mathrm{CN})_{2}\right] \mathrm{Cl}$ (abbreviated as $\kappa$ - $\mathrm{Cl}$ hereafter) at low temperatures $T<35 \mathrm{~K}[9,10]$. This $p$ - and $T$-range is very convenient for experiments aiming at fine tuning a material around the Mott transition under well-controlled conditions. Our present understanding of the Mott transition has benefited significantly from a number of detailed experimental studies on $\kappa-\mathrm{Cl}[11-14]$ and some transition-metal oxides, see, e.g., $[15,16]$, together with advances from theoretical side through dynamical mean-field theory calculations [17]. It is by now well established that the Mott transition is of first order which terminates in a second-order critical endpoint at $\left(T_{c}, p_{c}\right)$ as there is no symmetry breaking accompanying the transition. However, the critical behavior and the underlying universality class have been a matter of debate. These aspects have been discussed intensively for $\kappa-\mathrm{Cl}[18-25]$, where the second-order critical end-point is located at $\left(T_{c}, p_{c}\right) \approx(36.5 \mathrm{~K}, 23.4 \mathrm{MPa})$. Recently, based on a careful study of the lattice effects, it has been argued in Ref. [25] that the coupling of the correlated electrons to the lattice degrees of freedom is of crucial importance for understanding the Mott transition whenever the transition is amenable to pressure tuning. It has been found that this coupling alters the critical behavior from Ising criticality, for the purely-electronic Mott system, to mean-field criticality of an isostructural solid-solid endpoint in the presence of a compressible lattice, in accordance with the theoretical predictions $[26,27]$. It is worth noting that also the high-temperature behavior at $T \gg T_{c}$ has attracted considerable interest recently, as it shows characteristic signatures of a hidden quantum-critical point [28-30]. It has been suggested that quantum criticality is responsible for the unusual transport properties in the incoherent regime close to the Mott transition.

Another aspect of ongoing interest relates to the effects of disorder in strongly correlated electron systems. Actually, the questions surrounding the interplay of disorder and correlation [31-33] trace back to the different mechanisms for metal-insulator transitions in solids: Whereas the Mott transition is driven by electronic correlations, backscattering of non-interacting electronic waves from disorder, i.e., randomly distributed impurities, can induce the so-called Anderson insulating state [34,35]. In a naive picture, one would assume that the simultaneous action of disorder and correlations stabilizes the insulating phase. However, it has been found that disorder and correlations combine in a highly non-trivial way and can compete with each other [31,33]. Current research [36-41] addresses the following aspects: Do characteristic properties of the Mott insulator survive when introducing disorder in the strongly correlated system? Or, alternatively, how stable is the Anderson insulator when the correlations in such a disordered system become strong? Understanding this subtle interplay at the so-called Mott-Anderson-transition is of fundamental importance for a more realistic modeling of the behavior of real materials where disorder is inevitable. For advancing our understanding in this field, materials which allow for a control of both the strength of correlations as well as the level of disorder are required. Both aspects are met favorably in the family of organic charge-transfer salts.

Approaches to intentionally introduce disorder in a well-controlled manner in the $\kappa$-(BEDT-TTF) ${ }_{2} X$ salts are manifold [42-51]. Among these, $\mathrm{X}$-ray irradiation with typical irradiation times $0 \mathrm{~h} \leq t_{\text {irr }} \leq 600 \mathrm{~h}$ has been widely used to control disorder in a non-reversible manner (see Ref. [48] for a review). It was demonstrated that X-ray irradiation mainly creates molecular defects in the anion layer, whereas it leaves the conducting BEDT-TTF layer intact. The defects in the anion layer thereby create a 
random potential for the hole carriers in the BEDT-TTF layer. The power of this technique was demonstrated for $\kappa$-(BEDT-TTF $)_{2} \mathrm{Cu}\left[\mathrm{N}(\mathrm{CN})_{2}\right] \mathrm{Br}$ in which a transition from a correlated metal to an Anderson insulator [50] accompanied by a suppression of superconductivity [49] was observed. In addition, long-range antiferromagnetic order [52] in the Mott insulator $\kappa-\mathrm{Cl}$, which is understood as an effect of strong $U$, was suppressed $[53,54]$ by long irradiation $t_{i r r}=500 \mathrm{~h}$. It was argued that these results [53] highlight the role of disorder in forming a spin liquid close to the Mott transition.

Here, we focus on yet another aspect of the Mott transition in the presence of disorder by asking the questions: (i) How does quenched disorder influence the location of the Mott transition, i.e., the first-order transition line and its second-order critical endpoint in the $T-p$ phase diagram; and (ii) Is there any influence of disorder on the Mott criticality? To this end, we present a study of the combined effects of X-ray-induced disorder and pressure on the Mott insulator $\kappa-\mathrm{Cl}$ by means of thermodynamic and transport probes. Importantly, the use of He-gas pressure allows us to fine-tune the system across the Mott transition so that the critical regime in the phase diagram can be accessed experimentally.

The paper is organized as follows: In Sections 3.1-3.3, we present a detailed comparison of thermal expansion data around the Mott transition of a $\kappa-\mathrm{Cl}$ crystal in its pristine (non-irradiated) form with those of a crystal from the same batch after an exposure to X-ray irradiation for $50 \mathrm{~h}$. For tracing the Mott transition and its critical endpoint in the $T-p$ phase diagram to higher disorder levels, i.e., irradiation doses up to $150 \mathrm{~h}$, we present in Sections 3.4 and 3.5 the results of electrical resistance measurements on one single crystal at various disorder levels obtained after $50 \mathrm{~h}, 100 \mathrm{~h}$ and $150 \mathrm{~h}$ irradiation time. The results will be discussed in the context of available theoretical results in Section 4 .

\section{Materials and Methods}

Single crystals of $\kappa$-(BEDT-TTF $)_{2} \mathrm{Cu}\left[\mathrm{N}(\mathrm{CN})_{2}\right] \mathrm{Cl}$ were grown by electrochemical crystallization following the standard procedure [55]. For the present study, crystals from two different batches \#AF063 and \#5-7 were used. The dimensions of the crystals are typically $0.3 \times 0.6 \times 0.7 \mathrm{~mm}^{3}$ for \#AF063 and $0.5 \times 0.3 \times 0.3 \mathrm{~mm}^{3}$ for \#5-7, respectively.

Samples were X-ray irradiated [48] at $300 \mathrm{~K}$ by using a non-filtered tungsten tube with $40 \mathrm{kV}$ and $20 \mathrm{~mA}$. The corresponding dose rate was estimated to be about $0.5 \mathrm{MGy} / \mathrm{h}$. During the irradiation, the resistance was monitored. The plate-like crystals were irradiated from both sides. The irradiation time $t_{\text {irr }}$ denotes the overall exposure time.

Thermal expansion and resistance measurements were performed under variable He-gas pressure $p$. To this end, a copper beryllium $(\mathrm{CuBe})$ pressure cell was used which was connected to a large-volume $(50 \mathrm{~L})$ helium gas bottle for thermal expansion measurements at $p \leq 30 \mathrm{MPa}$ or a helium-gas compressor for resistance measurements at $p \leq 100 \mathrm{MPa}$. This technique enables measurements to be performed as a function of $T$ at constant $p$. In combination with the use of a He-gas bottle, the thermal expansion can also be measured as a function of $p$ at constant $T$. Pressure was determined by a pressure sensor $(\mathrm{InSb})$ in the pressure cell and a manometer situated close to the high-temperature reservoir.

Thermal expansion measurements were performed using an ultra-high resolution dilatometer with $\Delta L / L \geq 5 \times 10^{-10}$. The setup was described in detail in Ref. [56]. The data analysis was performed in an identical procedure as described in Ref. [25].

The resistance was measured by an AC resistance bridge (LR 700, Linear Research, San Diego, CA, USA) in a standard four-terminal configuration. The current was applied perpendicular to the conducting layer. Contacts were made by graphite paste and $20 \mu \mathrm{m}$ gold wires on which the sample was suspended. The temperature was controlled by a LakeShore 340 Temperature Controller (Lake Shore Cryotronics Inc., Westerville, $\mathrm{OH}$, USA). Slow warming and cooling rates of $\pm 0.2 \mathrm{~K} / \mathrm{min}$ were used to ensure thermal equilibrium at all $T$ and $p$. 


\section{Results}

\subsection{Effects of Irradiation on the Lattice Effects at the Mott Transition in $\mathrm{k}-\mathrm{Cl}$}

Figure 1 shows data of the relative length change $\Delta L_{b} / L_{b}$, measured along the $b$ axis, as a function of pressure $p$ in the temperature range $30 \mathrm{~K} \leq T \leq 40 \mathrm{~K}$ for $\kappa-\mathrm{Cl}$ in its pristine form [25] (a) and after exposure to $50 \mathrm{~h}$ X-ray irradiation (b) (see Section 2 for a description of the methods used, including details of the irradiation procedure). The displayed data represent only the singular contribution to the relative length change which was obtained by subtraction of an in- $p$ linear background contribution from the $\Delta L_{b}(p) / L_{b}$ data. We start by recalling some basic notations from the study of the pristine sample in Ref. [25]. At the lowest temperature $T=30 \mathrm{~K}$, a slightly broadened jump of the sample length as a function of pressure was observed at $p_{M I} \approx 22.5 \mathrm{MPa}$ at which the length decreases on going from the low-pressure insulating side to the high-pressure metallic side of the transition. The observation of a discontinuity in $\Delta L_{b} / L_{b}$ is consistent with the fact that a first-order transition line is passed as a function of $p$. Upon increasing the temperature $T$, the discontinuity decreases in size and evolves into a continuous crossover behavior for $T \geq 37 \mathrm{~K}$. This data set discloses a strong coupling of the electronic degrees of freedom to the lattice close to the Mott transition. It manifests itself in strong non-linear variations of $\Delta L_{b}(p) / L_{b}$-a breakdown of Hooke's law-which can be observed in a wide range of critical elasticity of $\Delta T_{c} / T_{C} \approx 20 \%$ above the critical endpoint. It was demonstrated in Ref. [25] that these lattice effects can be modeled by mean-field criticality. The important implication of this finding was that, although the Mott transition is driven by strong electron correlations, the coupling of the electrons to the lattice degrees of freedom eventually alters the Mott critical behavior to that of an isostructural solid-solid endpoint with mean-field criticality [26,27].

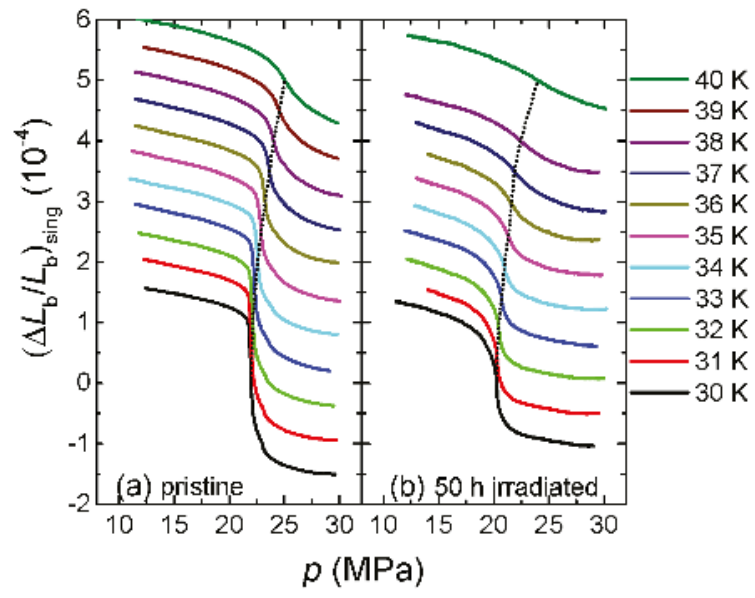

Figure 1. Singular part of the relative length change $\left(\Delta L_{b} / L_{b}\right)_{\text {sing }}$ of $\kappa-(\mathrm{BEDT}-\mathrm{TTF})_{2} \mathrm{Cu}\left[\mathrm{N}(\mathrm{CN})_{2}\right] \mathrm{Cl}$ (batch \#AF063) in its pristine form (data taken from Ref. [25]) (a) and after exposure to X-ray irradiation for $50 \mathrm{~h}(\mathbf{b})$. Data were taken along the out-of-plane $b$ axis as a function of pressure $p$ at constant temperatures between $30 \mathrm{~K}$ and $40 \mathrm{~K}$.

A lattice response of similar strength around the Mott transition can also be inferred from the $\Delta L_{b} / L_{b}$ data set in Figure $1 \mathrm{~b}$ taken on a crystal which was exposed to X-ray for $50 \mathrm{~h}$. At the lowest temperature of $T=30 \mathrm{~K}$, we find a strong decrease of the length upon going from the low-pressure insulating side to the high-pressure metallic side at $p_{M I} \approx 20.2 \mathrm{MPa}$. Note that this $p_{M I}$ value is smaller by $\approx 1.8 \mathrm{MPa}$ than the corresponding value for the pristine sample at $30 \mathrm{~K}$. This shift of the transition to lower pressure upon increasing the degree of disorder is one of the central results of the present work 
and will be discussed below in more detail. Concerning the character of the transition for the irradiated crystal, we observe an even more broadened jump-like feature as compared to the pristine crystal where a small broadening is visible. Yet, the data of the irradiated crystal still reveal a discontinuous change of the lattice parameter, i.e., a first-order phase transition (see below for a detailed discussion of the determination of $T_{c}$ and $p_{c}$ ). In the pristine sample, the finite width of the transition is likely associated with a small amount of disorder that leads to a spatial variation of internal stress induced by impurities and/or other crystal defects. In order to account quantitatively for this broadening effect, we assumed in Ref. [25] a multi-domain state, caused by extrinsic defects, where each domain contributes independently to the mean strain. Experimentally, the broadening manifests itself in a peak of finite width in the compressibility $\kappa_{b}(p)=-\mathrm{d}\left(\Delta L_{b} / L_{b}\right) / \mathrm{d} p$, i.e., in the derivative of the present data sets with respect to $p$. In order to quantify the increase in broadening upon irradiation, we determine the width $\Delta p$ of the peak in $\kappa_{b}$ at $T=30 \mathrm{~K}$ by its full width at half maximum. This procedure yields $\Delta p_{50 h}=(0.81 \pm 0.05) \mathrm{MPa}$ for the irradiated sample and $\Delta p_{0}=(0.38 \pm 0.01) \mathrm{MPa}$ for the pristine sample. This implies that the distribution of internal stress in the $50 \mathrm{~h}$-irradiated sample is about twice as wide as in the pristine sample, giving rise to a larger disorder-affected pressure regime. By lacking a microscopic characterization of the defect structure in the irradiated samples, we can only speculate that the defect sites, created by irradiation in the anion layer, may cause an increased number of domains. We suspect that this also affects the internal stress of each domain which in turn leads to an overall wider stress distribution in such a crystal. As the technique of dilatometry is unable to reveal the required spatially-resolved information on the formation of domains, we omit a detailed discussion of the role of domain formation at the first-order transition here and treat this broadening on a phenomenological level, whenever necessary. In addition to an enhanced broadening, we find a significant reduction of the size of the discontinuity at $T=30 \mathrm{~K}$ at $p_{M I}$ from $\left.\left(\Delta L_{b} / L_{b}\right)\right|_{p_{M I}}=(2.3 \pm 0.1) \times 10^{-4}$ in the pristine case to $\left.\left(\Delta L_{b} / L_{b}\right)\right|_{p_{M I}}=(1.5 \pm 0.1) \times 10^{-4}$ in the irradiated case. Apart from these small quantitative changes in the size and width of the anomalous length changes, we find that the evolution of the feature in $\Delta L_{b} / L_{b}$ to higher $T$ is qualitatively similar to the above-described pristine case: The size of the discontinuity becomes reduced with increasing $T$ until it is replaced by a continuous change of $\Delta L_{b}(p) / L_{b}$ for $T \geq T_{c} \approx 34 \mathrm{~K}$. Importantly, also for the irradiated sample, we find highly non-linear variations of the length as a function of pressure, even at temperatures way above $T_{c}$, see, e.g., the data at $T=38 \mathrm{~K}$, where $\kappa_{b}\left(p=p_{M I}\right)$, i.e., the slope of the $\Delta L_{b} / L_{b}$ curves at $p_{M I}$, exceeds $\kappa_{b}\left(p \ll p_{M I}\right)$ by at least a factor of two. This behavior is distinctly different from the usual elastic behavior which is characterized by an in-first approximation linear change of $\Delta L_{b} / L_{b}$ vs. $p$, i.e., $\kappa \approx$ const., in accordance with Hooke's law of elasticity. The observed breakdown of Hooke's law here reflects the strong coupling between the critical electronic system and the underlying crystal lattice at the Mott transition, also in the presence of increased disorder.

\subsection{T- $p$ Phase Diagram for Weak Disorder}

In the following, we analyze the effects of disorder by focusing on certain characteristics of the Mott transition. This includes the precise location of the critical endpoint and the critical behavior in its surrounding. In order to determine the location of the critical endpoint $\left(T_{c}, p_{c}\right)$ for the irradiated crystal with high accuracy from measurements of the relative length change $\Delta L_{b} / L_{b}$, we take two complementary approaches, similar to the procedure applied to the pristine sample in Ref. [25]. In our first approach, we determine $\left(T_{c}, p_{c}\right)$ from the intersection of the first-order transition line with crossover lines which emanate from the critical endpoint. To this end, we combine results from p-dependent measurements, which allow a precise determination of the first-order transition line (see below), with those performed as a function of $T$. The latter measurements provide insight into the crossover lines by analyzing the maximum response $\alpha_{\max }$ in the coefficient of thermal expansion $\alpha_{b}(T)=L_{b}^{-1} \mathrm{~d} L_{b} / \mathrm{d} T$ for $T>T_{c}$, as proposed in Ref. [23]. Moreover, due to the finite slope of the first-order transition line at $\left(T_{c}, p_{c}\right)$, these measurements as a function of temperature at different $p=$ const. provide not only information on the crossover line for $T>T_{c}$ and $p>p_{c}$. In addition, 
these $T$-dependent experiments allow us to cross the first-order transition line for $T<T_{\mathcal{C}}$ and $p<p_{c}$. As the crossing of the first-order transition is expected to manifest itself in a discontinuity in $\Delta L_{b}(T) / L_{b}$, corresponding to a divergent behavior in $\alpha_{b}(T)$, the observation of such discontinuities can serve as a criterion for localizing $T_{c}$. Figure 2 shows $\alpha_{b}(T)$, measured along the out-of-plane $b$ axis, of $50 \mathrm{~h}$-irradiated $\kappa-\mathrm{Cl}$ at several constant $p$ values around $p_{c}$. Starting at low $p=16.5 \mathrm{MPa}$, we observe the usual increase of $\alpha_{b}$ with increasing $T$ without any signatures for anomalous behavior. As we approach the endpoint from the low- $p$ side, however, we observe sharp and pronounced anomalies in $\alpha_{b}(T)$ with the tendency to diverge in a narrow pressure range $20.3 \mathrm{MPa} \leq p \leq 21.0 \mathrm{MPa}$. We assign these features to signatures of the first-order phase transition. Note that for the data set taken at $p=20.5 \mathrm{MPa}$, the first-order transition line is crossed twice as a function of $T$ due to its $S$-shaped form. As a result, we find two successive sharp anomalies of opposite sign, the latter one corresponding to the reentrance from the intermediate- $T$ metallic state to the low- $T$ insulating state. In contrast, for $p>21.0 \mathrm{MPa}$, the divergent features in $\alpha_{b}(T)$ are absent and instead, $\alpha_{b}(T)$ shows a smooth anomaly at slightly higher $T$. These features can be assigned to signatures of a crossover line, as predicted theoretically in Ref. [23].

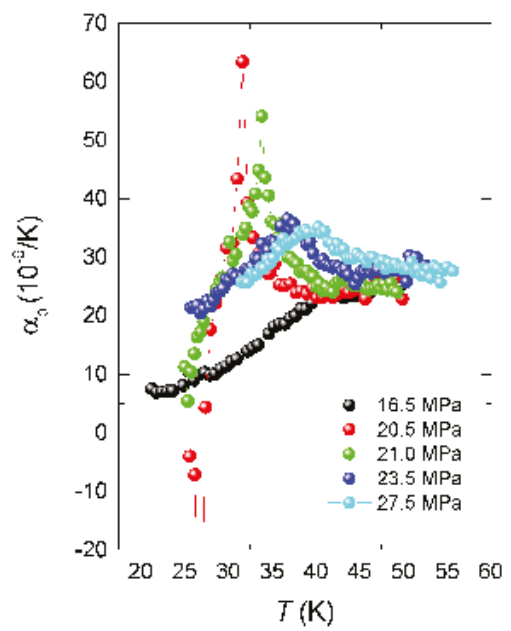

Figure 2. Thermal expansion coefficient along the out-of-plane $b$ axis, $\alpha_{b}$, of $\kappa$-(BEDT-TTF $)_{2} \mathrm{Cu}\left[\mathrm{N}(\mathrm{CN})_{2}\right] \mathrm{Cl}$ (batch \#AF063) after exposure to $\mathrm{X}$-ray for $50 \mathrm{~h}$ as a function of temperature $T$ at constant pressures $16.5 \mathrm{MPa} \leq p \leq 27.5 \mathrm{MPa}$.

In Figure 3, we compile in a $T$ - $p$ phase diagram the position of the sharp peaks in $\alpha_{b}$ for $p \leq 21 \mathrm{MPa}$, reflecting the first-order transition temperatures, and the positions of $\alpha_{\max }$ for $p>21.0 \mathrm{MPa}$ indicating crossover temperatures. In addition, we also include the positions of the inflection points in the $p$-dependent $\Delta L_{b} / L_{b}$ data sets at different constant $T$. These inflection points correspond to either the first-order transition for $T<T_{C}$ or to the so-called Widom line for $T>T_{C}$, i.e., the extension of the first-order transition line to higher $T$. From the intersection point of the first-order transition line determined from $p$-dependent measurements, and the crossover line determined from $T$-dependent measurements, we find $T_{c}=(34.0 \pm 0.5) \mathrm{K}$ and $p_{c}=(21.0 \pm 0.2) \mathrm{MPa}$ for the 50 h-irradiated sample.

An alternative approach to determine $\left(T_{c}, p_{c}\right)$ is provided by analyzing the width of the features observed in $\Delta L_{b}(p) / L_{b}$. As mentioned above, the width of the discontinuity in $\Delta L_{b} / L_{b}$ for $T<T_{c}$ is governed by extrinsic effects. This gives rise to an in first approximation $T$-independent width for low $T$, as already visible in the bare data in Figure $1 \mathrm{~b}$. In contrast, for $T>T_{\mathcal{C}}$ the width is strongly $T$-dependent and can therefore be assigned to the effects of criticality. For a quantification of the 
increase in the width upon increasing $T$, we determine the width of our experimental data by the full width at half maximum of the peak in $\kappa_{b}(p)$, as described above. We include the information of the width in the phase diagram, shown in Figure 3, by red-broken lines for the disorder-dominated regime at $T<T_{\mathcal{C}}$ and blue-broken lines for the criticality-dominated regime at $T>T_{\mathcal{C}}$. We find a significant increase in the width for $T>36 \mathrm{~K}$. An extrapolation of the lines in the criticality-dominated regime from high $T \geq 36 \mathrm{~K}$, where disorder effects play only a minor role, to lower $T$ yields an alternative estimate of $T_{\mathcal{C}}=(33.5 \pm 0.5) \mathrm{K}$. Note that the two different approaches yield an identical position of the critical endpoint within the error bars.

Now we can compare the so-derived phase diagram for the irradiated crystal with the one of the pristine sample. To this end, we include the position of the first-order line, the Widom line and the critical endpoint of the pristine sample in the phase diagram in Figure 3. We find that central properties of the Mott transition, i.e., the first-order character at low $T$ and the second-order critical endpoint, survive at the level of disorder introduced by $50 \mathrm{~h}$ of irradiation. However, this irradiation leads to a significant reduction of $T_{c}$ as well as $p_{c}$. Here, the exposure to X-ray for $50 \mathrm{~h}$ causes a decrease of $T_{c}$ by $\approx 2.5 \mathrm{~K}\left(\Delta T_{c} / T_{c} \approx 7 \%\right)$ and of $p_{c}$ by $\approx 2.4 \mathrm{MPa}\left(\Delta p_{c} / p_{c} \approx 10 \%\right)$. This finding demonstrates a high sensitivity of the Mott transition line on the degree of disorder. We stress that the Mott transition lines for $\kappa-\mathrm{Cl}$ reported in the studies by Kagawa et al. [18] and Gati et al. [25], practically coincide, indicating a similar low level of disorder in the pristine crystals studied there. In addition, a study of pristine crystals from batch \#AF063 (not shown) discloses that $T_{C}$ of two different crystals varies up to $1 \mathrm{~K}$ and $p_{c}$ up to $0.5 \mathrm{MPa}$. Thus, the variations in the disorder level of pristine samples are distinctly smaller than the one introduced by exposure to X-ray for $50 \mathrm{~h}$.

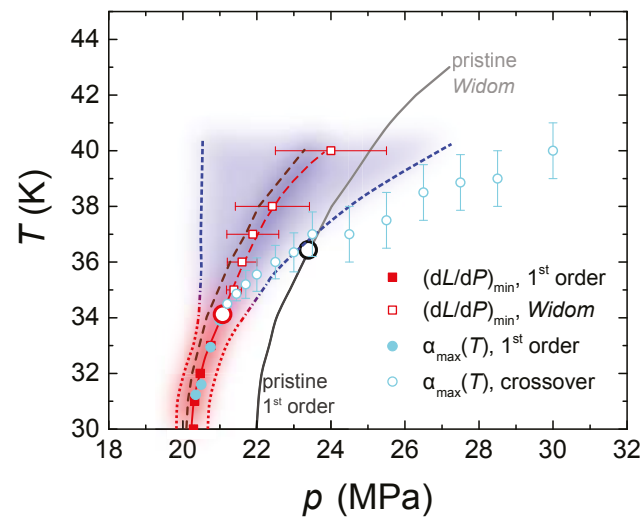

Figure 3. Experimentally determined temperature-pressure phase diagram of $\kappa$-(BEDT-TTF $)_{2} \mathrm{Cu}\left[\mathrm{N}(\mathrm{CN})_{2}\right] \mathrm{Cl}$ (batch \#AF063) after exposure to X-ray for $50 \mathrm{~h}$. Red full squares correspond to the first-order Mott transition line extracted from the inflection point of $\Delta L_{b}(p) / L_{b}$. Cyan full circles correspond to the first-order transition line extracted from temperature-dependent measurements. The latter data points were determined by the position of the maximum $\alpha_{\text {max }}$ of the thermal expansion coefficient $\alpha_{b}(T)=L_{b}^{-1} \mathrm{~d} L_{b} / \mathrm{d} T$ for $p<p_{c}$. Red open symbols correspond to the Widom line. Open cyan symbols correspond to a crossover line, determined by the position of $\alpha_{\max }$ for $p>p_{c}$. Red- and blue-shaded areas delimited by the broken lines in the same color code indicate the experimentally determined width of the features along the $b$ axis and can be assigned to the disorder-related (red) and the criticality-related (blue) crossover regimes, respectively. The broken lines represent, within the error margins, the full width at half maximum of the peaks in $\mathrm{d} L / \mathrm{d} P$. The brown dashed line corresponds to the $p_{\mathcal{c}}(T)$ curve extracted from a fit of the data, presented in Figure 1 to the mean-field model of Equation (1) (see text for details). The first-order transition line (dark grey line), the Widom line (light grey line) and the critical endpoint (black open circle) for a pristine crystal of batch \#AF063 [25] are given for comparison. 


\subsection{Critical Behavior for Weak Disorder}

After having determined the location of the critical endpoint $\left(T_{c}, p_{c}\right)$ for the irradiated crystal, we can proceed by analyzing the observed lattice effects in terms of the critical behavior. For pristine $\kappa-\mathrm{Cl}[25]$, the anomalous lattice effects could be well described by a mean-field critical model of an isostructural solid-solid endpoint. In fact, this type of criticality was predicted theoretically for the critical electronic Mott system that is coupled to a compressible lattice as a consequence of the long-ranged shear forces of the lattice [26,27]. In light of the strong lattice response revealed for the irradiated system, we employ the same mean-field critical model to the present $\Delta L_{b}(p) / L_{b}$ data set, presented in Figure 1b. The model reads, as follows,

$$
\Delta L_{b} / L_{b}=-A_{b}\left\langle\varepsilon\left(\left(T-T_{c}\right) / T_{c},-\left(p-p_{c}(T)\right) / p_{c}\left(T_{c}\right), u\right)\right\rangle_{w}+\left(\Delta L_{b} / L_{b}\right)_{o f f}
$$

Here, $A_{b}$ is the proportionality constant between the relative length change $\Delta L_{b} / L_{b}$ and the critical strain singlet $\varepsilon$ which represents the order parameter of an isostructural solid-solid transition. The strain singlet obeys the mean-field equation $r \varepsilon+u \varepsilon^{3}=-\sigma$, with $r=\left(T-T_{c}\right) / T_{c}$ the reduced temperature, $\sigma=\left(p-p_{c}(T)\right) / p_{c}$ the reduced pressure and $u$ a parameter, which quantifies non-linearities in the mean-field potential, and $\Delta L_{b} / L_{b}$ an offset contribution, see below. Note that the first two parameters are determined by our experiment, whereas the latter one enters as a free parameter. This model can be extended to account for the disorder broadening. To this end, we average the mean-field solution $\varepsilon(r, \sigma, u)$ with a Gaussian stress distribution $P_{w}(s)=1 / \sqrt{2 \pi w} \exp \left(-s^{2} /(2 w)\right)$ with variance $w$, i.e., $\langle\varepsilon(r, \sigma, u)\rangle_{w}=\int \mathrm{d} s P_{w}(s) \varepsilon(r, \sigma+s, u)$. The details of this model are given in the Supplementary Information of Ref. [25]. In order to eliminate the temperature-dependent expansion effects, we normalized all data sets (see Figure 1) to the inflection points of $\Delta L_{b}(p) / L_{b}$ at $p_{\text {in }}$, i.e., $\Delta L_{b}\left(p_{\text {in }}\right) / L_{b}=0$. The increase in the broadening of the first-order transition upon irradiation is accompanied by an increasing asymmetry of the $\Delta L_{b} / L_{b}(p, T=$ const. $)$ curves in Figure $1 \mathrm{~b}$ with respect to the midpoint of the discontinuity. To account for this effect, which is not understood at present and which is also not covered by the above model, we proceed as follows: we use the experimentally determined inflection point of the $\Delta L_{b}(p) / L_{b}$ data for a first iteration for $p_{c}(T)$ in the fitting procedure, and then refined the fit by allowing for a small variation of $p_{c}(T)$ of the order of $0.1 \mathrm{MPa}$. The resulting optimized $p_{c}(T)$ values are included as a brown broken line in the phase diagram in Figure 3. As a consequence of the small adjustment of $p_{c}(T)$, we have to accept a small offset in $\Delta L_{b} / L_{b}$ of $\left(\Delta L_{b} / L_{b}\right)_{o f f}=\mathcal{O}\left(10^{-6}\right)$. The variance $w$ of the stress distribution can be determined from the experiment at lowest temperature of $T=30 \mathrm{~K}$ from a Gaussian fit to the $\kappa_{b}(p)$ data, which results in $w=2.5 \times 10^{-4}$ for the data set of the irradiated sample. Note that this value of $w$ in its dimensionless form corresponds to the above-determined broadening of $\Delta P_{50 h}=0.81 \mathrm{MPa}$.

Figure 4 demonstrates that the data set of the irradiated crystal is in very good agreement with the mean-field critical model, given in Equation (1), in the full $T$ range $30 \mathrm{~K} \leq T \leq 40 \mathrm{~K}$ investigated. As a result of the fit, we obtain values for the parameters $u$ and $A_{b}$. The fit yields $u=0.18$, which is the parameter characterizing the non-linearity of the length change. We stress that the fit to the data set on the pristine sample [25], which is included in Figure 4 for comparison, yielded the identical value of $u$. The extracted values $A_{b}$ are slightly different for the various $T=$ const. scans. However, they follow to a good approximation a $T$-linear behavior described by $\left.A_{b}=\left(6.5-9.3\left(T-T_{c}\right) / T_{c}\right)\right) \times 10^{-5}$. The fits for the data set of the pristine sample resulted in a similar small T-linear variation of $A_{b}$. In the model for an isostructural solid-solid endpoint, the value of $A_{b}$ is given by the eigenvalues and the eigenvectors of the elastic constant matrix (see Ref. [25] for details) which is likely subject to a small T-dependent variation.

As demonstrated in Figure 4, the mean-field fits provide an excellent description of the experimental data not only for the pristine sample (see Figure 4), but also for the irradiated sample. These results show that the character of the Mott transition remains essentially unchanged for the two different disorder levels. This relates to the first-order character of the transition and the strong 
coupling to the lattice with the concomitant change of universality class to mean-field criticality around the second-order critical endpoint.

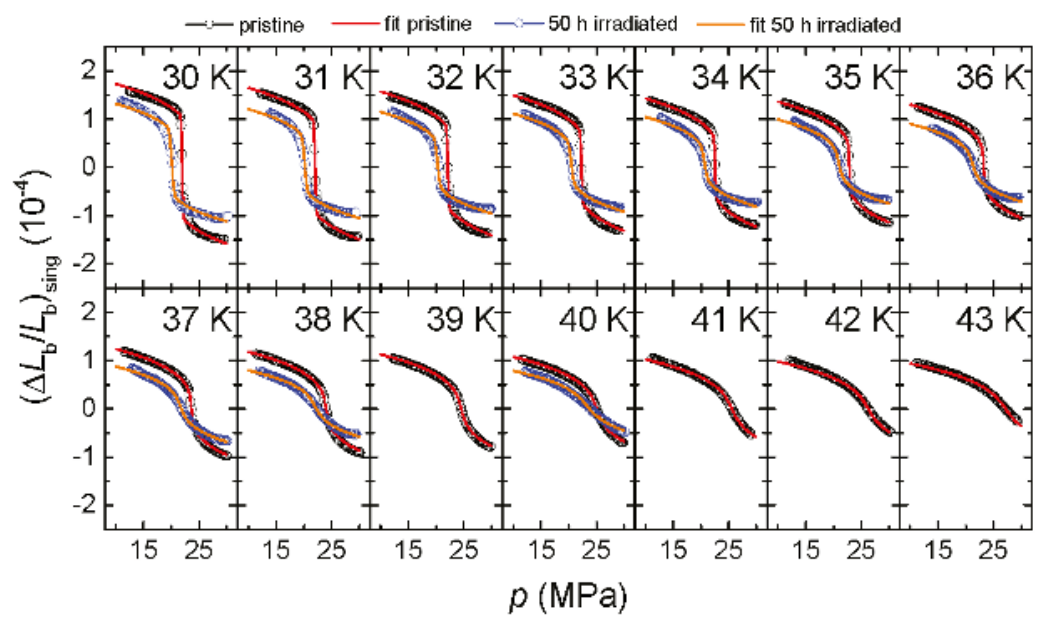

Figure 4. Singular part of the relative length change of $\kappa$-(BEDT-TTF $)_{2} \mathrm{Cu}\left[\mathrm{N}(\mathrm{CN})_{2}\right] \mathrm{Cl}$ along the out-of-plane $b$ axis, $\left(\Delta L_{b} / L_{b}\right)_{\text {sing, }}$ (open symbols) as a function of pressure $p$ at various temperatures $30 \mathrm{~K} \leq T \leq 43 \mathrm{~K}$, together with a fit (straight lines) based on the mean-field solution, given by Equation (1). Black open symbols and red straight lines represent results on a pristine sample of batch \#AF063, which were published in Ref. [25]. Blue open symbols and orange lines represent results on a crystal of batch \#AF063 which was exposed to X-ray for $50 \mathrm{~h}$.

\subsection{T-p Phase Diagrams of the Metal-Insulator Transition for Higher Irradiation Doses Based on Resistance Measurements}

In the following, we discuss how the characteristic features of the Mott transition for $\kappa-\mathrm{Cl}$ evolve upon further increasing the level of disorder. Instead of using measurements of the relative length change on one single crystal at varying irradiation levels, which is a very time consuming experiment, we employ measurements of the resistance $R$. Importantly, all of the presented data were taken on the same $\mathcal{K}-\mathrm{Cl}$ single crystal from batch \#5-7 which was exposed to a three-step irradiation process $(50 \mathrm{~h}$, $100 \mathrm{~h}$, and $150 \mathrm{~h}$ ) and which was measured after each step.

Figure 5 shows the out-of-plane resistance $R$ as a function of $T$ at varying $p=$ const. for a $\kappa-C l$ crystal

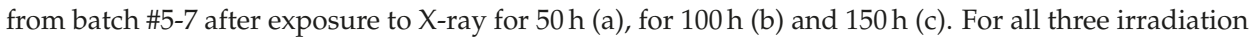
levels, $\kappa-\mathrm{Cl}$ exhibits a non-metallic behavior at ambient pressure characterized by an increasing $R$ with decreasing $T$, consistent with literature results [48]. The $R(T, p=$ const.) curves at different irradiation doses show the same general trend upon increasing $p$ as observed for pristine samples [13,14]: Low $p$ reduces the resistance at a fixed temperature, however, the resistance at a fixed, low $p$ value still shows a non-metallic behavior with $\mathrm{d} R / \mathrm{d} T<0$. At intermediate $p$, the system is non-metallic at low $T$ and undergoes a transition into a metallic state, characterized by $\mathrm{d} R / \mathrm{d} T>0$, upon increasing $T$ and enters an anomalous transport regime at higher $T$, characterized by $\mathrm{d} R / \mathrm{d} T \approx 0$. This reentrant behavior, visible in the range $18 \mathrm{MPa} \leq p \leq 30 \mathrm{MPa}$ after $50 \mathrm{~h}$ irradiation time, reflects the $S$-shape of the first-order transition line and the Widom line in the T- $p$ phase diagram. At high $p>30 \mathrm{MPa}$, all data sets reveal a metallic behavior in the full $T$ range investigated with a transition into a superconducting state at low $T \approx 12 \mathrm{~K}$. 


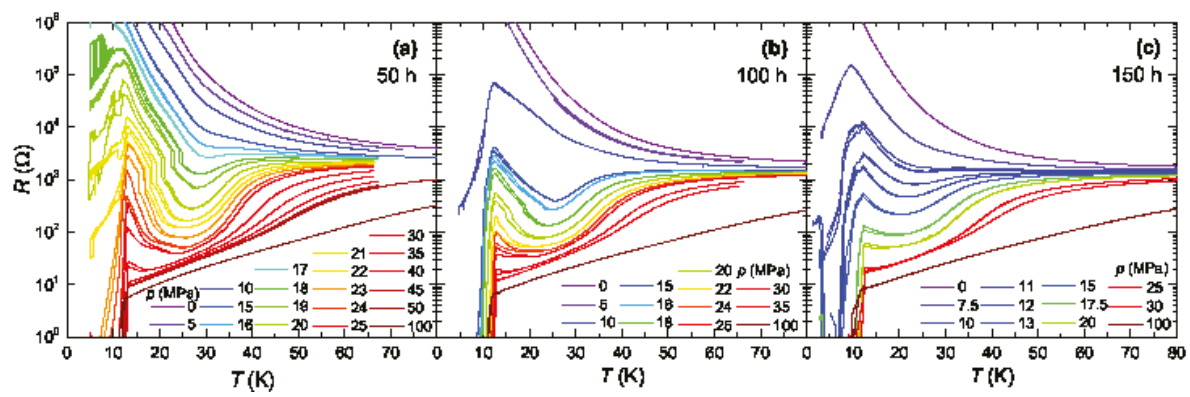

Figure 5. Out-of-plane resistance $R$ of $\kappa$-(BEDT-TTF) ${ }_{2} \mathrm{Cu}\left[\mathrm{N}(\mathrm{CN})_{2}\right] \mathrm{Cl}$ (crystal \#5-7), which was exposed to X-ray irradiation for $50 \mathrm{~h} \mathrm{(a);} 100 \mathrm{~h} \mathrm{(b)}$ and $150 \mathrm{~h}(\mathbf{c})$, as a function of temperature $T$ at various constant pressure values $0 \mathrm{MPa} \leq p \leq 100 \mathrm{MPa}$ upon warming and upon cooling.

Despite these similarities in the overall behavior of $R(T, p=$ const.) after different irradiation times, a closer inspection of the data in Figure 5 discloses significant differences in the location and character of the metal-insulator transition. These irradiation-induced changes can be illustrated clearly by comparing data sets taken at the same $p$ values, e.g., $p=20 \mathrm{MPa}$. In the data at $50 \mathrm{~h}$ irradiation time, we find a kink in the $R(T, p=20 \mathrm{MPa})$ data with an abrupt sign change of $\mathrm{d} R / \mathrm{d} T$ at $T \approx 12 \mathrm{~K}$ which interrupts the increase in $R$ upon cooling, reflecting insulating behavior. This kink marks the entrance into a spurious superconducting phase at $T<12 \mathrm{~K}$ which is known to exist close to the first-order Mott metal-insulator boundary on the insulating side in pristine $\kappa-\mathrm{Cl}[11]$ and is likely responsible for the increased noise in $R(T, p=20 \mathrm{MPa})$ at low $T$. Upon increasing the temperature, we find a pronounced jump-like suppression of $R(T)$ at $T \approx 20.5 \mathrm{~K}$. Importantly, the jump in $R(T, p=20 \mathrm{MPa}$ ) is accompanied by a significant thermal hysteresis of $\Delta T \approx 0.7 \mathrm{~K}$, reflecting the first-order nature of this phase transition. We assign this discontinuity in $R(T, p)$ to the signature of the first-order metal-insulator transition, in accordance with the approach taken in literature $[13,18,22]$. The fact that the resistance jump remains sharp in the presence of some disorder is a consequence of percolation, i.e., a current that runs through low-resistance domains. This contrasts with the broadening observed in the discontinuities of thermodynamic quantities, such as the lattice effects, which average over all domains in the crystal. Even though the jump in $R(T)$ occurs at $T \approx 20.5 \mathrm{~K}$, we stress that the hysteresis and the insulating character are visible up to a slightly higher temperature of $T \approx 26.5 \mathrm{~K}$ where $R(T, p=20 \mathrm{MPa}$ ) exhibits a local minimum. Above the temperature of the local minimum in $R(T, p=20 \mathrm{MPa})$, i.e., $T \approx 26.5 \mathrm{~K}$, the system shows metallic behavior with $\mathrm{d} R / \mathrm{d} T>0$. Upon further increasing the temperature, the system gradually changes from a metal into an anomalous transport regime, as indicated by an almost flat $R(T, p=20 \mathrm{MPa}$ ) behavior at $T \approx 38 \mathrm{~K}$. This change is not accompanied by any hysteresis, indicating that this temperature corresponds to a crossover temperature.

At a higher irradiation time of $100 \mathrm{~h}$, cf. Figure $5 \mathrm{~b}$, the spurious superconducting phase at low $T$ and $p=20 \mathrm{MPa}$ is replaced by percolative or even bulk superconductivity with $T_{c, s c} \approx 12.6 \mathrm{~K}$, characterized by $R(T)<10^{-2} \Omega$ at $T \approx 10.5 \mathrm{~K}$. With increasing temperature, we observe the same characteristics as in the case of $50 \mathrm{~h}$ irradiation, i.e., a jump-like reduction of the resistance at low $T$, accompanied by thermal hysteresis, and thus indicative of a first-order phase transition, and a crossover at high $T$. However, the jump in $R(T, p=20 \mathrm{MPa})$ is significantly reduced in size and occurs in two concomitant steps at $T \approx 15.5 \mathrm{~K}$ and $T \approx 17.5 \mathrm{~K}$. The fact that the transition takes place in several steps is likely of extrinsic nature and possibly associated with the formation of several domains in the presence of disorder. Therefore, we assign the metal-insulator transition to the larger jump at $T \approx 15.5 \mathrm{~K}$. The minimum in $R(T)$, which marks the onset of hysteresis, and the change from low- $T$ insulating to high- $T$ metallic behavior, is observed at $T \approx 22 \mathrm{~K}$. The crossover from the metallic behavior to the $R(T, p=20 \mathrm{MPa}) \approx$ const. behavior is found at $T \approx 43 \mathrm{~K}$. Apparently, these characteristic temperatures react differently on increasing the irradiation dose from $50 \mathrm{~h}$ to $100 \mathrm{~h}$ : Whereas the 
position of the jump and the minimum in $R(T, p=20 \mathrm{MPa})$ are shifted to lower temperatures, the position of the crossover is shifted to higher $T$. This peculiar behavior can be related to the $S$-shaped form of the first-order transition line and the crossover lines (see e.g., Ref. [23,25]). In pristine $\kappa-\mathrm{Cl}$, the temperature of the first-order transition decreases with increasing pressure distance to the critical point, whereas the crossover temperature increases. Thus, the present observation of a decrease in the first-order transition temperature and an increase in the crossover temperature at the same $p$ value upon irradiation can be considered as an indication that the critical endpoint of the irradiated sample is located lower in pressure than the pristine one.

Upon further increasing the irradiation time to $150 \mathrm{~h}$, (cf. Figure 5c), we do not find any indications for a jump-like change of $R(T, p=20 \mathrm{MPa})$ at intermediate temperatures. Instead, the resistance changes smoothly yielding a minimum at $T=16 \mathrm{~K}$. We note that despite the disappearance of a jump-like change of $R(T, p=20 \mathrm{MPa})$, we still find a small hysteresis at low $T$ in the insulating regime which prevails until the resistance reaches its minimum. Apparently, at this irradiation level, the character of the phase transition has changed. Based on the present transport data, we cannot discriminate between a transition of weak first order, or a smeared first-order transition with residual hysteresis.

In order to illustrate the change of the first-order metal-insulator transition line upon irradiation, we compile the position of the jump for all investigated $p$ values in a $T$ - $p$ phase diagram in Figure 6. For comparison, we included the transition temperatures of the first-order transition line extracted from T-dependent resistance measurements on a pristine crystal of batch \#AF063 (not shown) which are in good agreement with published data [11,14,22,25]. As already indicated by our thermodynamic measurements, we find a significant shift of the first-order transition line to lower $p$ upon irradiation for $50 \mathrm{~h}$ and $100 \mathrm{~h}$. The shift of the phase transition line at $T=20 \mathrm{~K}$, amounts to about $-(0.07 \pm 0.01) \mathrm{MPa}$ per hour of irradiation time. In addition, we find that the position of the critical endpoint $\left(T_{c}, p_{c}\right)$ becomes significantly reduced in temperature as well as in pressure: Our analysis yields $\left(T_{c}, p_{c}\right) \approx((29 \pm 1) \mathrm{K},(17.5 \pm 0.5) \mathrm{MPa})$ and $\left(T_{c}, p_{c}\right) \approx((27 \pm 1) \mathrm{K}$, $(15.0 \pm 0.5) \mathrm{MPa}$ ) after irradiating $\kappa-\mathrm{Cl}$ for $50 \mathrm{~h}$ and $100 \mathrm{~h}$, respectively. We stress that the slightly different results of $\left(T_{c}, p_{c}\right)$ after $50 \mathrm{~h}$ of irradiation, inferred from the resistance measurements on a crystal of batch \#5-7, as compared to those of our thermodynamic measurements on a crystal of batch \#AF063 (see Section 3.2), might be related to details of our experiments. First, we cannot rule out small sample-to-sample variations of crystals from different batches. More importantly, however, the crystals involved had different surface-to-thickness ratios, implying that a different distribution of disorder was introduced within the same irradiation time. At even higher irradiation time of $150 \mathrm{~h}$, we cannot find any discontinuous feature in the full $T-p$ range investigated. Therefore, we cannot draw a phase transition line for this case.

To quantify the range of hysteretic behavior and to follow its evolution upon irradiation, we define the onset of hysteresis by $\frac{R_{\text {warm }}(T)-R_{\text {cool }}(T)}{R_{\text {warm }}(T)} \geq 0.025$ with $R_{\text {warm }}\left(R_{\text {cool }}\right)$ denoting the resistance taken upon warming (cooling). The onset temperatures together with the previously determined first-order transition lines (green symbols) and the second-order critical endpoint (thick green circle) are compiled in the contour plots in Figure 7 which will be discussed below in more detail. For the $50 \mathrm{~h}$ irradiated crystal, we find clear signatures for thermal hysteresis in the pressure range $\approx 15 \mathrm{MPa} \leq p \leq \approx 30 \mathrm{MPa}$ and up to $T \approx 29 \mathrm{~K}$. The width of the hysteresis range (hatched area in Figure 7 ) on the pressure axis is similar to the one found for a pristine crystal in Ref. [13]. However, upon irradiation, the hysteresis regime is shifted to lower pressures and suppressed in temperature, consistent with the shift of the Mott transition line to lower $p$ and the reduction of $\left(T_{c}, p_{c}\right)$, discussed above. Upon further increasing the irradiation to $100 \mathrm{~h}$, the hysteresis regime is shifted to even lower pressures and further reduced in temperature. Importantly, even after $150 \mathrm{~h}$ of irradiation thermal hysteresis occurs in a similarly wide pressure range $\approx 10 \mathrm{MPa} \leq p \leq \approx 30 \mathrm{MPa}$ and up to $T \approx 20 \mathrm{~K}$. This result speaks in favor of the notion of a smeared first-order transition after $150 \mathrm{~h}$ of irradiation where disorder causes the suppression of signatures, and therefore can no longer be discerned from a crossover. We stress that for a detailed analysis of the hysteresis range, measurements as a function of increasing and decreasing pressure, not 
possible with the available set-up, are much better suited for the following reasons: First, these organic charge-transfer salts tend to form small cracks due to their large thermal expansion, particularly when sweeping the temperature over a wide range, as done in the present work. These small cracks lead to small offset contributions in $R(T, p)$ which strongly hamper the analysis of the thermal hysteresis. This effect is avoided in pressure sweeps performed at low temperatures. Second, due to the almost vertical alignment of the metal-insulator transition line in the $T$ - $p$ phase diagram, the signatures in $p$ sweeps are more clearly pronounced as compared to $T$ sweeps.

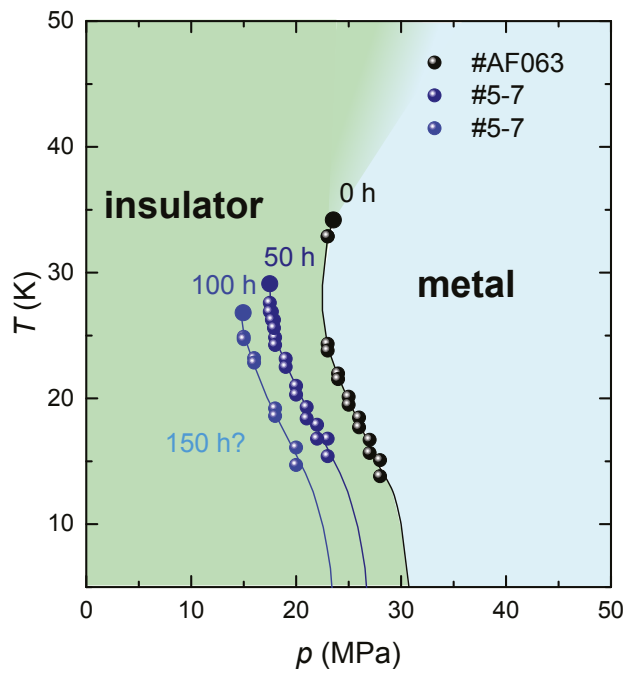

Figure 6. T-p phase diagram of the first-order Mott metal-insulator transition line of $\kappa$-(BEDT-TTF $)_{2} \mathrm{Cu}\left[\mathrm{N}(\mathrm{CN})_{2}\right] \mathrm{Cl}$ upon increasing disorder which was intentionally introduced by X-ray irradiation. Black circles correspond to the phase transition line obtained from measurements (not shown) on a pristine sample of batch \#AF063. Dark blue and light blue circles correspond to the phase transition line obtained from measurements on the same sample of batch \#5-7 after exposure to $\mathrm{X}$-ray for $50 \mathrm{~h}$ and $100 \mathrm{~h}$, respectively (see Figure 5). Note that pairs of data points taken at the same $p$ value correspond to the position of the jump upon warming and cooling. The lines are guides to the eyes. In case of $150 \mathrm{~h}$ no discontinuities in $R(T, p)$ could be resolved signaling the absence of a strong first-order phase transition.

\subsection{Influence of Disorder on the Metallic and Insulating States Nearby the Mott Transition}

Next, we focus on the influence of disorder on the range close to the first-order Mott metal-insulator transition. To this end, we include in Figure 7 contour plots of the resistance in a wide $T-p$ range for all three investigated irradiation times. The plots also contain the transition temperatures into spurious (dark grey symbols) or bulk (light grey symbols) superconductivity. The colors represent the absolute value of $R$ on a logarithmic scale. This presentation of the data highlights two remarkable aspects. First, the diagram shows how the superconducting state, observed in the immediate vicinity of the metal-insulator transition in the pristine case, is affected by irradiation. Second, it discloses a striking mirror symmetry along the Widom line (see below for detailed definition) for $T \gg T_{C}$ which has been known from the pristine case [29]. In the latter case, this mirror symmetry has been interpreted in terms of a quantum-critical scaling [28]. 


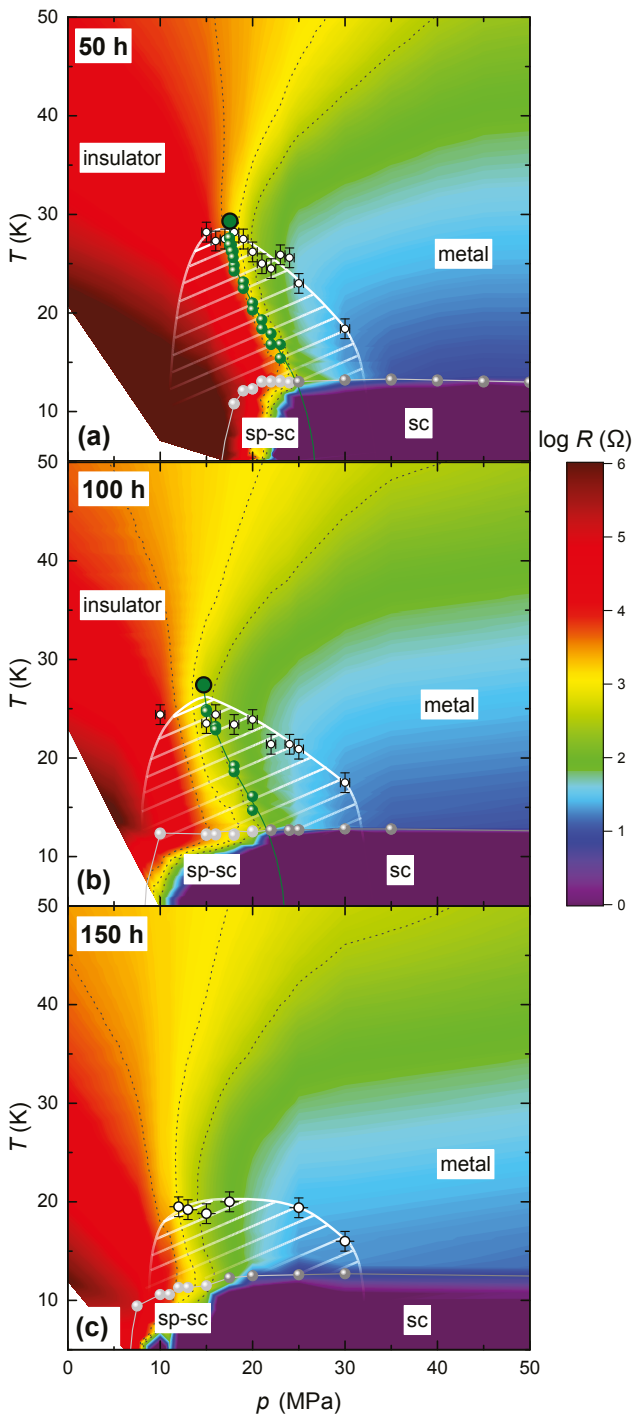

Figure 7. Experimentally determined $T$ - $p$ phase diagrams for a $\kappa$-(BEDT-TTF $)_{2} \mathrm{Cu}\left[\mathrm{N}(\mathrm{CN})_{2}\right] \mathrm{Cl}$ crystal of batch \#5-7 after exposure to X-ray for $50 \mathrm{~h} \mathrm{(a);} 100 \mathrm{~h} \mathrm{(b)} \mathrm{and} 150 \mathrm{~h} \mathrm{(c).} \mathrm{Data} \mathrm{were} \mathrm{extracted} \mathrm{from}$ the resistance $R$ measurements presented in Figure 5. Green symbols in $(\mathbf{a}, \mathbf{b})$ represent the first-order transition line extracted from cooling as well as warming experiments, the black-edged big green circle marks the second-order critical endpoint. After exposure to X-ray for $150 \mathrm{~h}$ (c) no discontinuities in $R(T, p)$ indicating a first-order phase transition could be resolved. White circles indicate the temperature, below which a sizable hysteresis between warming and cooling was observed. White hatched area is a guide to the eye for the hysteresis region, delimited by the white circles. Dark grey symbols mark the entrance into a spurious superconducting ( $\mathrm{sp}-\mathrm{sc}$ ) state. Light grey symbols indicate the transition into a bulk superconducting (sc) state. Lines are guide to the eyes. The background shows a contour plot of $\log (R(T, p) / \Omega)$. Dashed lines indicate three constant-resistance lines with $\log (R / \Omega)=2.5 \Omega, \log (R / \Omega)=3 \Omega$ and $\log (R / \Omega)=3.5 \Omega$. 
Concerning the superconducting properties of irradiated $\kappa-\mathrm{Cl}$, we find signatures for a bulk superconducting dome as well as a range of spurious superconductivity in the T-p phase diagram for all three irradiation doses. We use the term bulk superconductivity in cases where there is a sharp drop of $R$ at $T_{c, s c}$ below which the out-of-plane resistance reaches values $R(T, p) \ll 1 \Omega$ for $T<T_{c, s c}$. This contrasts with spurious superconductivity which manifests itself in a broad kink in $R(T, p)$ at low $T$ without reaching a low resistance value, i.e., $R(T, p)>10 \Omega$. After $50 \mathrm{~h}(100 \mathrm{~h})$ of irradiation, we find spurious superconductivity in the range $18 \mathrm{MPa} \leq p \leq 24 \mathrm{MPa}(10 \mathrm{MPa} \leq p \leq 20 \mathrm{MPa}$ ) and bulk superconductivity in the range $p \geq 25 \mathrm{MPa}(p \geq 22 \mathrm{MPa}$ ). Compared to the range of spurious superconductivity of $20 \mathrm{MPa} \leq p \leq 28 \mathrm{MPa}$ and bulk superconductivity of $p \geq 30 \mathrm{MPa}$ in pristine $\kappa-\mathrm{Cl}[11]$, we find a significant shift of superconductivity to lower pressures upon increasing irradiation dose. We note that the shift of the superconducting dome on the pressure axis is similar to the shift of the first-order Mott metal-insulator transition line: The spurious superconducting phase occurs in all cases on the insulating side of the Mott transition, whereas the bulk superconducting phase can be found right on the metallic side. The fact that bulk superconductivity emerges as soon as the insulator is suppressed by pressure indicates the strong connection between the metal-insulator transition and superconductivity, also in disordered $\kappa-\mathrm{Cl}$. Surprisingly, even after $150 \mathrm{~h}$ of irradiation, bulk superconductivity is observed in a wide $p$ range despite the lack of clear signatures of a first-order phase transition. However, superconductivity starts to occur in the regime where the resistance at $T>T_{c, s c}$ changes strongly in a narrow pressure regime. This result suggests that the existence of superconductivity does not require a nearby strong first-order metal-insulator transition.

Besides the irradiation-induced shift of the superconducting dome on the pressure axis, the critical temperature $T_{c, s c}$ is also affected by irradiation. By using the onset temperature of the drop in $R(T)$ as a measure of $T_{c, s c}$, we find that the maximum $T_{c, s c}$ is reduced from $13 \mathrm{~K}$ after $50 \mathrm{~h}$ of irradiation, to $12.7 \mathrm{~K}$ after $100 \mathrm{~h}$ and $12.2 \mathrm{~K}$ after $150 \mathrm{~h}$. At $p=100 \mathrm{MPa}$, the highest pressure of the present experiment, $T_{c, s c}$ is reduced from $12.3 \mathrm{~K}$ to $12.0 \mathrm{~K}$ to $11.8 \mathrm{~K}$ upon increasing irradiation time from $50 \mathrm{~h}$ to $100 \mathrm{~h}$ to $150 \mathrm{~h}$. A similar reduction of $T_{c, s c}$ upon irradiation was found also for $\kappa$-(BEDT-TTF) ${ }_{2} \mathrm{Cu}(\mathrm{NCS})_{2}[49,51]$, and $\kappa-(\mathrm{BEDT}-\mathrm{TTF})_{2} \mathrm{Cu}\left[\mathrm{N}(\mathrm{CN})_{2}\right] \mathrm{Br}[50]$, both of which are located at ambient pressure on the metallic side of the Mott metal-insulator transition. In the latter cases, this shift was interpreted to be incompatible with a superconducting order parameter with one single component. This conclusion is consistent with the result of a recent combined theoretical and experimental scanning tunneling microscopy study [57] which found strong indications for an extended $s+d_{x^{2}-y^{2}}$ symmetry of the superconducting order parameter in $\kappa$-(BEDT-TTF $)_{2} \mathrm{Cu}\left[\mathrm{N}(\mathrm{CN})_{2}\right] \mathrm{Br}$.

Now we turn to the analysis of resistance behavior in the high- $T$ regime $T \gg T_{c}$. To this end, we included in the contour plots in Figure 7 three dotted lines which represent constant $R$ lines in the phase diagram with $\log (R / \Omega)=3.5, \log (R / \Omega)=3$ and $\log (R / \Omega)=2.5$. The $\log (R / \Omega)=3$ is chosen such that it is very close to the resistance value along the Widom line which is determined according to Refs. [28,29] as the inflection points of $\log R$ vs. $p$ at $T=$ const. For all three irradiation doses, we find an extended range at $T>T_{c}$ where $\log R$ shows a mirror symmetry along the Widom line. The notation of mirror symmetry here implies that the normalized resistance $\log R_{\text {norm }}(\delta p, T=$ const. $)=\mid \log R(p, T=$ const. $)-\log R\left(p_{\text {widom }}, T=\right.$ const. $) \mid / \log R\left(p_{\text {widom }}, T=\right.$ const. $)$ with $\delta p=p-p_{\text {widom }}$, i.e., the resistance normalized to the value at the Widom line at $p_{\text {widom }}$, are identical on the low-pressure side of the Widom line $(\delta p<0)$ and the high-pressure side $(\delta p>0)$ for a given pressure distance $\delta p$ to the Widom line $\left(\log R_{n o r m}(-\delta p)=\log R_{n o r m}(\delta p)\right)$. The temperature range of this symmetric $\log R(T, p)$ behavior is extended to lower $T$ upon increasing irradiation: After $150 \mathrm{~h}$ of irradiation this symmetry is visible down to $T \approx 20 \mathrm{~K}$. As this mirror symmetry was associated with signatures of quantum criticality for pristine $\kappa-\mathrm{Cl}$ [29], the present data indicate that irradiation can be an appropriate parameter to further suppress $T_{c}$, thereby expanding the quantum-critical regime to somewhat lower temperatures. However, even after $150 \mathrm{~h}$ of irradiation, it appears that $T_{C}$ is not fully suppressed to zero (see discussion of hysteresis above), so that more disorder is required to fully suppress the second-order critical endpoint in $\kappa$-Cl. In this situation, it is 
possible that quantum-critical signatures of the Mott transition can develop. However, it may well be that in this case, the material's properties are governed by disorder effects rather than quantum criticality. For a detailed investigation of the potential quantum-critical scaling around the Widom line and of the role of disorder on this scaling (see Ref. [58]), in-plane resistance measurements up to higher $T$, as well as measurements of $R$ as a function of $p$, similar to Ref. [29], are required.

\section{Discussion}

In the following, we discuss our experimental observations on the metal-insulator transition for irradiated $\kappa-\mathrm{Cl}$ in the context of theoretical results on the Mott-Anderson transition, obtained from combining dynamic mean-field theory with different numerical implementations of disorder $[36,37,41,59]$.

One of the central results of the present work is the observation that the character of the Mott metal-insulator transition remains essentially unchanged when disorder is introduced to a moderate extent, corresponding to an irradiation time of about $100 \mathrm{~h}$. This relates to the first-order character of the phase transition, as revealed by thermal expansion and resistance measurements, and the second-order critical endpoint of this line at $\left(T_{c}, p_{c}\right)$. We showed that after $50 \mathrm{~h}$ of irradiation, strong lattice effects dominate the critical behavior around $\left(T_{c}, p_{c}\right)$, similar to the pristine case [25]. Thus, we conclude that exposure to X-ray for $50 \mathrm{~h}$ and $100 \mathrm{~h}$ induces a degree of disorder which is small enough to conserve essential Mott properties. This interpretation is supported by theoretical results $[37,41,59]$ which indicate a sharp metal-insulator transition, accompanied by a sizable coexistence regime, at a critical interaction strength $U_{c}$ at $T=0$, similar to the clean Mott system.

However, even though retaining its first-order character, we find that the transition becomes distinctly weaker upon irradiation, as evidenced by a significant shrinkage of the discontinuities in the length change and resistance in the $50 \mathrm{~h}$ and $100 \mathrm{~h}$ irradiated samples, as compared to the pristine ones. Moreover, after $150 \mathrm{~h}$ of irradiation, no discontinuity could be resolved in $R(T, P)$ anymore, implying a significant change of the character of the Mott transition. These observations are in line with theoretical predictions: The calculations reveal a sizable reduction of the size of the coexistence region upon an initial increase in disorder [37], reflecting the weakening of the first-order transition. In addition, recent finite-temperature calculations suggest a sudden drop of $T_{c}$ to zero [41] and a continuous change from a metal to an insulator above some critical value of the degree of disorder. The present data do not allow to conclude that the character of the transition changes from first to second order. However, it seems likely that a critical level of disorder, above which the discontinuous first-order character of the Mott transition is suppressed, is attained in $\kappa$-Cl between $100 \mathrm{~h}$ and $150 \mathrm{~h}$ of irradiation. To investigate the character of the phase transition above $100 \mathrm{~h}$ of irradiation, a detailed study of the size of the coexistence region for different disorder levels can be helpful. Such a study has to include pressure-dependent hysteresis measurements around the metal-insulator transition, as explained in detail above.

Furthermore, we find a continuous shift of the first-order Mott transition line to lower pressures on going from the pristine sample to the ones with $50 \mathrm{~h}$ and $100 \mathrm{~h}$ irradiation time. This decrease of $p_{c}$ corresponds to an increase of the critical correlation strength $(U / W)_{c}$. Theoretically, it has been predicted that disorder widens the Hubbard bands which causes an increase of spectral weight at the Fermi level [37]. As a result, a larger $U$ is required for opening the Mott gap, equivalent to the experimentally-observed increase in $(U / W)_{c}$. This situation, where disorder induces a finite spectral weight in the clean Mott gap, has been labeled as the soft Coulomb gap scenario [38,60]. Thus, the present results are consistent with the scenario that disorder changes the fully-gapped Mott insulator $\kappa-\mathrm{Cl}$ to a Mott insulator with a soft Coulomb gap. We note that a similar conclusion was drawn from the analysis of optical conductivity data on the same compound after irradiation [48] as well as from scanning tunneling microscopy studies of the normal-state density of states in $\kappa$-(BEDT-TTF $)_{2} \mathrm{Cu}\left[\mathrm{N}(\mathrm{CN})_{2}\right] \mathrm{Br}$ even in its pristine form [61]. At the same time, our finding points towards a possibility to estimate the degree of disorder for a given sample of $\kappa-\mathrm{Cl}$ by measuring the position of the first-order transition line 
in the T- $p$ phase diagram. However, as this approach is unable to provide an absolute measure of the degree of disorder, it allows only to compare the relative disorder level of different crystals. Concerning the effect of disorder on the critical temperature $T_{c}$ of the second-order critical endpoint of the Mott transition, we observe a significant suppression of $T_{\mathcal{C}}$ by a few Kelvin upon increasing the irradiation up to $100 \mathrm{~h}$. Also this trend is consistent with the results of finite-temperature calculations [41,59] on a strongly correlated electron system with weak disorder.

On a qualitative level, the experimental results agree well with all presently available theoretical predictions for the metal-insulator transitions within the Mott-Anderson model. For a more quantitative comparison, which is required to distinguish between the different theoretical calculations, however, a detailed characterization of the disorder is required. This includes information on the nature, the concentration and spatial distribution of the disorder [62]. According to infrared absorption measurements on $\kappa$-(BEDT-TTF $)_{2} \mathrm{Cu}\left[\mathrm{N}(\mathrm{CN})_{2}\right] \mathrm{Br}$ (see Figure 2 in Ref. [48]), exposure to X-ray with a dose rate of $0.5 \mathrm{MGy} / \mathrm{h}$, as used in the present work (see Methods Section 2), destroys C-N bonds in the anion layer at a rate of $\approx 10-20 \%$ per $50 \mathrm{~h}$ of irradiation. Another sensitive probe on the degree of disorder is the residual resistivity ratio $(R R R)$. Our data of the resistivity reveal a decrease of the $R R R=R(65 \mathrm{~K}) / R(12 \mathrm{~K})$ in the metallic phase at $100 \mathrm{MPa}$ upon irradiation from $R R R \approx 41$ after $50 \mathrm{~h}$ irradiation to $\approx 24$ after $100 \mathrm{~h}$ and $\approx 18$ after $150 \mathrm{~h}$. This observation is fully consistent with an increase of disorder upon irradiation. Open questions relate on the one hand to the penetration depth of the $X$-ray photons and the resulting spatial distribution of the introduced disorder. Besides that, a deeper understanding of the interaction between the disorder in the anion layers and the delocalized electrons in the BEDT-TTF layers seems necessary for a comparison with theoretical results which consider disorder embedded directly in the electronic system.

Finally, we would like to point the attention towards interesting future research directions in the field of correlated electrons in the presence of disorder. As mentioned above, the present data suggest the possibility to suppress the critical endpoint significantly after $150 \mathrm{~h}$ of irradiation suggesting the possibility to fully suppress $T_{C}$ to zero at even higher irradiation. In this situation, it would be of high interest to investigate how the quantum-critical signatures, observed in the pristine case only at higher $T$, evolve towards lower temperatures [58]. In addition, the interplay of charge- with spin- degrees of freedom in the limit of strong disorder and strong correlations [39], as represented by the data set taken after $150 \mathrm{~h}$ irradiation, hold promise for interesting new physics. This is demonstrated, e.g., in experiments on $\kappa$-Cl after $500 \mathrm{~h}$ of irradiation where long-range magnetic order is replaced by a state with spin-liquid properties [53]. In the present study, we did not investigate the magnetic properties of the irradiated samples. However, it has been known that the inflection point of the first-order transition line $T_{M I}(p)$ at $T \approx 27 \mathrm{~K}$ in the pristine case is connected to the occurrence of long-range magnetic ordering due to entropy reasons $[63,64]$. As demonstrated in Figure 6, the inflection point of $T_{M I}(p)$ shifts to lower temperatures upon increasing irradiation up to $100 \mathrm{~h}$, indicating a lowering of the magnetic ordering temperature. This is consistent with previous reports of the decreased magnetic ordering temperature in irradiated samples of $\kappa-\mathrm{Cl}$ at ambient pressure [65]. To investigate this ordering at higher irradiation, where disorder causes a change of the character of the Mott transition, and its interplay with the metal-insulator transition, we suggest to study the magnetic properties of strongly irradiated $\kappa-\mathrm{Cl}$ under pressure.

\section{Conclusions}

In summary, we have studied the effect of X-ray-induced disorder on the Mott metal-insulator transition for the organic charge-transfer salt $\kappa-(\mathrm{BEDT}-\mathrm{TTF})_{2} \mathrm{Cu}\left[\mathrm{N}(\mathrm{CN})_{2}\right] \mathrm{Cl}$. The application of hydrostatic He-gas pressure allowed us to fine-tune the system across the Mott transition. Our main findings, based on thermal expansion and resistance measurements, include (i) strong lattice effects around the Mott transition after irradiating the system for $50 \mathrm{~h}$; (ii) a line of first-order transitions which ends in a second-order critical endpoint after $50 \mathrm{~h}$ and $100 \mathrm{~h}$ irradiation with a significant lowering of the critical temperature $T_{c}$ and the critical pressure $p_{c}$ upon increasing irradiation and (iii) a change 
of the character of the Mott transition for $150 \mathrm{~h}$ irradiation where no discontinuous signatures of a first-order phase transition could be detected despite the presence of a small thermal hysteresis. The results (i) and (ii) reveal a very similar behavior of the irradiated samples to the pristine case, i.e., a strong coupling of the lattice to the electronic degrees of freedom close to the second-order critical endpoint. This observation speaks in favor of a dominant Mott character even after $50 \mathrm{~h}$ and $100 \mathrm{~h}$ of irradiation indicating that the Mott transition in $\kappa-(\mathrm{BEDT}-\mathrm{TTF})_{2} \mathrm{Cu}\left[\mathrm{N}(\mathrm{CN})_{2}\right] \mathrm{Cl}$ is stable against the introduction of a small degree of disorder. The reduction in $p_{c}$ and $T_{c}$ after irradiation is fully compatible with theoretical predictions of the phase diagram for the Mott-Anderson transition. The latter indicate a larger critical $U$ to open the Mott gap due to a disorder-induced increase of spectral weight at the Fermi level, corresponding to a smaller $p_{c}$. Thus, our experimental results are fully consistent with the theoretically predicted soft Coulomb gap scenario for a strongly correlated electron system with small amount of disorder. In addition, result (iii) reflects the strong weakening of the first-order character of the metal-insulator transition upon increasing irradiation which eventually may turn the transition into a crossover between the insulator and the metal. For future investigations and a more quantitative comparison between theory and experiment, a detailed knowledge of the spatial distribution of X-ray-induced disorder in the anion layer and its interplay with the electrons in the BEDT-TTF layer is necessary.

Besides the investigation of the metal-insulator transition at low $T$, we were able to show that superconductivity close to the metal-insulator transition prevails for all investigated disorder levels. The superconducting dome shifts similarly to the first-order metal-insulator transition to lower $p$ upon irradiation. Future studies on the interplay of the charge with spin degrees of freedom, and the potential quantum-critical signatures when $T_{C}$ is further suppressed, hold promise for interesting new physics in the field of strongly correlated electrons in the presence of disorder.

Acknowledgments: Research in Frankfurt and Dresden was supported by the German Science Foundation via the Transregional Collaborative Center SFB/TR49 "Condensed Matter Systems with Variable Many-Body Interactions" and Collaborative Research Center SFB 1143 "Correlated Magnetism: From Frustration to Topology". Work in Sendai was partly supported by the Japan Society for the Promotion of Science KAKENHI grant \#JP25287080.

Author Contributions: M.L. and T.S. conceived and supervised the project. E.G. performed thermal expansion under pressure experiments. U.T., A.N. and S.K. performed resistance under pressure experiments. M.G. provided theoretical support in analyzing the thermal expansion data. H.S. and T.S. grew the single crystals. T.S. performed the irradiation of the crystals. E.G. and M.L. wrote the paper with contributions from U.T., T.S. and M.G.

Conflicts of Interest: The authors declare no conflict of interest.

\section{References}

1. Toyota, N.; Lang, M.; Müller, J. Low-Dimensional Molecular Metals; Springer: Heidelberg, Germany, 2007.

2. Lebed, A. The Physics of Organic Superconductors and Conductors; Springer: Heidelberg, Germany, 2008.

3. Mott, N.F. Metal-Insulator Transition; Wiley-VCH Verlag: Weinheim, Germany, 1990; Volume 26, p. 788.

4. Imada, M.; Fujimori, A.; Tokura, Y. Metal-insulator transitions. Rev. Mod. Phys. 1998, 70, 1039-1263.

5. Lee, P.A.; Nagaosa, N.; Wen, X.G. Doping a Mott insulator: Physics of high-temperature superconductivity. Rev. Mod. Phys. 2006, 78, 17-85.

6. Kanoda, K.; Kato, R. Mott Physics in Organic Conductors with Triangular Lattices. Ann. Rev. Condens. Matter Phys. 2011, 2, 167-188.

7. Kanoda, K. Recent progress in NMR studies on organic conductors. Hyperfine Interact. 1997, 104, $235-249$.

8. Miyagawa, K.; Kanoda, K.; Kawamoto, A. NMR Studies on Two-Dimensional Molecular Conductors and Superconductors: Mott Transition in $\kappa$-(BEDT-TTF) ${ }_{2}$ X. Chem. Rev. 2004, 104, 5635-5654.

9. Williams, J.M.; Kini, A.M.; Wang, H.H.; Carlson, K.D.; Geiser, U.; Montgomery, L.K.; Pyrka, G.J.; Watkins, D.M.; Kommers, J.M. From semiconductor-semiconductor transition $(42 \mathrm{~K})$ to the highest- $T_{\mathcal{C}}$ organic superconductor, $\kappa-(\mathrm{ET})_{2} \mathrm{Cu}\left[\mathrm{N}(\mathrm{CN})_{2}\right] \mathrm{Cl}\left(T_{\mathcal{C}}=12.5 \mathrm{~K}\right)$. Inorg. Chem. 1990, 29, 3272-3274.

10. Ito, H.; Ishiguro, T.; Kubota, M.; Saito, G. Metal-Nonmetal Transition and Superconductivity Localization in the Two-Dimensional Conductor $\kappa$-(BEDT-TTF $)_{2} \mathrm{Cu}\left[\mathrm{N}(\mathrm{CN})_{2}\right] \mathrm{Cl}$ under Pressure. J. Phys. Soc. Jpn. 1996, $65,2987-2993$. 
11. Lefebvre, S.; Wzietek, P.; Brown, S.; Bourbonnais, C.; Jérome, D.; Mézière, C.; Fourmigué, M.; Batail, P. Mott Transition, Antiferromagnetism, and Unconventional Superconductivity in Layered Organic Superconductors. Phys. Rev. Lett. 2000, 85, 5420-5423.

12. Fournier, D.; Poirier, M.; Castonguay, M.; Truong, K.D. Mott Transition, Compressibility Divergence, and the P-T Phase Diagram of Layered Organic Superconductors: An Ultrasonic Investigation. Phys. Rev. Lett. 2003, 90, doi:10.1103/PhysRevLett.90.127002.

13. Limelette, P.; Wzietek, P.; Florens, S.; Georges, A.; Costi, T.A.; Pasquier, C.; Jérome, D.; Mézière, C.; Batail, P. Mott Transition and Transport Crossovers in the Organic Compound $\kappa-(\mathrm{BEDT}-\mathrm{TTF})_{2} \mathrm{Cu}\left[\mathrm{N}(\mathrm{CN})_{2}\right] \mathrm{Cl}$. Phys. Rev. Lett. 2003, 91, doi:10.1103/PhysRevLett.91.016401.

14. Kagawa, F.; Itou, T.; Miyagawa, K.; Kanoda, K. Transport criticality of the first-order Mott transition in the quasi-two-dimensional organic conductor $\kappa-(\mathrm{BEDT}-\mathrm{TTF})_{2} \mathrm{Cu}\left[\mathrm{N}(\mathrm{CN})_{2}\right] \mathrm{Cl}$. Phys. Rev. B 2004, 69, doi:10.1103/PhysRevB.69.064511.

15. McWhan, D.B.; Menth, A.; Remeika, J.P.; Brinkman, W.F.; Rice, T.M. Metal-Insulator Transitions in Pure and Doped $\mathrm{V}_{2} \mathrm{O}_{3}$. Phys. Rev. B 1973, 7, 1920-1931.

16. Limelette, P.; Georges, A.; Jérome, D.; Wzietek, P.; Metcalf, P.; Honig, J.M. Universality and Critical Behavior at the Mott Transition. Science 2003, 302, 89-92.

17. Georges, A.; Kotliar, G.; Krauth, W.; Rozenberg, M.J. Dynamical mean-field theory of strongly correlated fermion systems and the limit of infinite dimensions. Rev. Mod. Phys. 1996, 68, 13, doi:10.1103/RevModPhys.68.13.

18. Kagawa, F.; Miyagawa, K.; Kanoda, K. Unconventional critical behaviour in a quasi-two-dimensional organic conductor. Nature 2005, 436, 534-537.

19. Imada, M. Universality classes of metal-insulator transitions in strongly correlated electron systems and mechanism of high-temperature superconductivity. Phys. Rev. B 2005, 72, doi:10.1103/PhysRevB.72.075113.

20. De Souza, M.; Brühl, A.; Strack, C.; Wolf, B.; Schweitzer, D.; Lang, M. Anomalous Lattice Response at the Mott Transition in a Quasi-2D Organic Conductor. Phys. Rev. Lett. 2007, 99, doi:10.1103/PhysRevLett.99.037003.

21. Papanikolaou, S.; Fernandes, R.M.; Fradkin, E.; Phillips, P.W.; Schmalian, J.; Sknepnek, R. Universality of Liquid-Gas Mott Transitions at Finite Temperatures. Phys. Rev. Lett. 2008, 100, doi:10.1103/PhysRevLett.100.026408.

22. Kagawa, F.; Miyagawa, K.; Kanoda, K. Magnetic Mott criticality in a $\kappa$-type organic salt probed by NMR. Nat. Phys. 2009, 5, 880-884.

23. Bartosch, L.; de Souza, M.; Lang, M. Scaling Theory of the Mott Transition and Breakdown of the Grüneisen Scaling Near a Finite-Temperature Critical End Point. Phys. Rev. Lett. 2010, 104, doi:10.1103/PhysRevLett.104.245701.

24. Abdel-Jawad, M.; Kato, R.; Watanabe, I.; Tajima, N.; Ishii, Y. Universality Class of the Mott Transition. Phys. Rev. Lett. 2015, 114, doi:10.1103/PhysRevLett.114.106401.

25. Gati, E.; Garst, M.; Manna, R.S.; Tutsch, U.; Wolf, B.; Bartosch, L.; Schubert, H.; Sasaki, T.; Schlueter, J.A.; Lang, M. Breakdown of Hooke's law of elasticity at the Mott critical endpoint in an organic conductor. Sci. Adv. 2016, 2, doi:10.1126/sciadv.1601646.

26. Zacharias, M.; Bartosch, L.; Garst, M. Mott Metal-Insulator Transition on Compressible Lattices. Phys. Rev. Lett. 2012, 109, doi:10.1103/PhysRevLett.109.176401.

27. Zacharias, M.; Rosch, A.; Garst, M. Critical elasticity at zero and finite temperature. Eur. Phys. J. Spec. Top. 2015, 224, 1021-1040.

28. Terletska, H.; Vučičević, J.; Tanasković, D.; Dobrosavljević, V. Quantum Critical Transport near the Mott Transition. Phys. Rev. Lett. 2011, 107, doi:10.1103/PhysRevLett.107.026401.

29. Furukawa, T.; Miyagawa, K.; Taniguchi, H.; Kato, R.; Kanoda, K. Quantum criticality of Mott transition in organic materials. Nat. Phys. 2015, 11, 221-224.

30. Lenz, B.; Manmana, S.R.; Pruschke, T.; Assaad, F.F.; Raczkowski, M. Mott Quantum Criticality in the Anisotropic 2D Hubbard Model. Phys. Rev. Lett. 2016, 116, doi:10.1103/PhysRevLett.116.086403.

31. Lee, P.A.; Ramakrishnan, T.V. Disordered electronic systems. Rev. Mod. Phys. 1985, 57, $287-337$.

32. Electron-Electron Interactions in Disordered Systems; Efros, A., Pollak, M., Eds.; North-Holland: Amsterdam, The Netherlands, 1985; Volume 10.

33. Belitz, D.; Kirkpatrick, T.R. The Anderson-Mott transition. Rev. Mod. Phys. 1994, 66, 261-380.

34. Anderson, P.W. Absence of Diffusion in Certain Random Lattices. Phys. Rev. 1958, 109, 1492-1505. 
35. Kramer, B.; MacKinnon, A. Localization: Theory and experiment. Rep. Prog. Phys. 1993, 56, 1469-1564.

36. Dobrosavljević, V.; Kotliar, G. Mean Field Theory of the Mott-Anderson Transition. Phys. Rev. Lett. 1997, 78, 3943-3946.

37. Byczuk, K.; Hofstetter, W.; Vollhardt, D. Mott-Hubbard Transition versus Anderson Localization in Correlated Electron Systems with Disorder. Phys. Rev. Lett. 2005, 94, doi:10.1103/PhysRevLett.94.056404.

38. Shinaoka, H.; Imada, M. Single-Particle Excitations under Coexisting Electron Correlation and Disorder: A Numerical Study of the Anderson-Hubbard Model. J. Phys. Soc. Jpn. 2009, 78, doi:10.1143/JPSJ.78.094708.

39. Byczuk, K.; Hofstetter, W.; Vollhardt, D. Competition between Anderson Localization and Antiferromagnetism in Correlated Lattice Fermion Systems with Disorder. Phys. Rev. Lett. 2009, 102, doi:10.1103/PhysRevLett.102.146403.

40. Radonjić, M.M.; Tanasković, D.; Dobrosavljević, V.; Haule, K. Influence of disorder on incoherent transport near the Mott transition. Phys. Rev. B 2010, 81, doi:10.1103/PhysRevB.81.075118.

41. Bragança, H.; Aguiar, M.C.O.; Vučičević, J.; Tanasković, D.; Dobrosavljević, V. Anderson localization effects near the Mott metal-insulator transition. Phys. Rev. B 2015, 92, doi:10.1103/PhysRevB.92.125143.

42. Su, X.; Zuo, F.; Schlueter, J.A.; Kini, A.M.; Williams, J.M. $80 \mathrm{~K}$ anomaly and its effect on the superconducting and magnetic transition in deuterated $\kappa-(\mathrm{BEDT}-\mathrm{TTF})_{2} \mathrm{Cu}\left[\mathrm{N}(\mathrm{CN})_{2}\right] \mathrm{Br}$. Phys. Rev. B 1998, 58, R2944-R2947.

43. Yoneyama, N.; Higashihara, A.; Sasaki, T.; Nojima, T.; Kobayashi, N. Impurity Effect on the In-plane Penetration Depth of the Organic Superconductors $\kappa-(B E D T-T T F)_{2} \mathrm{X}\left(\mathrm{X}=\mathrm{Cu}(\mathrm{NCS})_{2}\right.$ and $\left.\mathrm{Cu}\left[\mathrm{N}(\mathrm{CN})_{2}\right] \mathrm{Br}\right)$. J. Phys. Soc. Jpn. 2004, 73, 1290-1296.

44. Hartmann, B.; Müller, J.; Sasaki, T. Mott metal-insulator transition induced by utilizing a glasslike structural ordering in low-dimensional molecular conductors. Phys. Rev. B 2014, 90, doi:10.1103/PhysRevB.90.195150.

45. Müller, J.; Hartmann, B.; Rommel, R.; Brandenburg, J.; Winter, S.M.; Schlueter, J.A. Origin of the glass-like dynamics in molecular metals $\kappa$-(BEDT-TTF) $)_{2} X$ : Implications from fluctuation spectroscopy and ab initio calculations. New J. Phys. 2015, 17, doi:10.1088/1367-2630/17/8/083057.

46. Yoneyama, N.; Sasaki, T.; Kobayashi, N. Substitution Effect by Deuterated Donors on Superconductivity in א-(BEDT-TTF $)_{2} \mathrm{Cu}\left[\mathrm{N}(\mathrm{CN})_{2}\right] \mathrm{Br}$. J. Phys. Soc. Jpn. 2004, 73, 1434-1437.

47. Yoneyama, N.; Sasaki, T.; Oizumi, H.; Kobayashi, N. Impurity Effect on Superconducting Properties in Molecular Substituted Organic Superconductor $\kappa-(\mathrm{ET})_{2} \mathrm{Cu}(\mathrm{NCS})_{2}$. J. Phys. Soc. Jpn. 2007, 76, doi:10.1143/JPSJ.76.123705.

48. Sasaki, T. Mott-Anderson Transition in Molecular Conductors: Influence of Randomness on Strongly Correlated Electrons in the $\kappa$-(BEDT-TTF) 2 X System. Crystals 2012, 2, 374-392.

49. Sasaki, T.; Oizumi, H.; Honda, Y.; Yoneyama, N.; Kobayashi, N. Suppression of Superconductivity by Nonmagnetic Disorder in Organic Superconductor $\kappa-(\mathrm{BEDT}-\mathrm{TTF})_{2} \mathrm{Cu}(\mathrm{NCS})_{2}$. J. Phys. Soc. Jpn. 2011, 80, doi:10.1143/JPSJ.80.104703.

50. Sano, K.; Sasaki, T.; Yoneyama, N.; Kobayashi, N. Electron Localization near the Mott Transition in the Organic Superconductor $\kappa$-(BEDT-TTF $)_{2} \mathrm{Cu}\left[\mathrm{N}(\mathrm{CN})_{2}\right]$ Br. Phys. Rev. Lett. 2010, 104, doi:10.1103/PhysRevLett.104.217003.

51. Analytis, J.G.; Ardavan, A.; Blundell, S.J.; Owen, R.L.; Garman, E.F.; Jeynes, C.; Powell, B.J. Effect of Irradiation-Induced Disorder on the Conductivity and Critical Temperature of the Organic Superconductor $\kappa-(\mathrm{BEDT}-\mathrm{TTF})_{2} \mathrm{Cu}(\mathrm{SCN})_{2}$. Phys. Rev. Lett. 2006, 96, doi:10.1103/PhysRevLett.96.177002.

52. Miyagawa, K.; Kawamoto, A.; Nakazawa, Y.; Kanoda, K. Antiferromagnetic Ordering and Spin Structure in the Organic Conductor, $\kappa$-(BEDT-TTF $)_{2} \mathrm{Cu}\left[\mathrm{N}(\mathrm{CN})_{2}\right] \mathrm{Cl}$. Phys. Rev. Lett. 1995, 75, 1174-1177.

53. Furukawa, T.; Miyagawa, K.; Itou, T.; Ito, M.; Taniguchi, H.; Saito, M.; Iguchi, S.; Sasaki, T.; Kanoda, K. Quantum Spin Liquid Emerging from Antiferromagnetic Order by Introducing Disorder. Phys. Rev. Lett. 2015, 115, doi:10.1103/PhysRevLett.115.077001.

54. Yoneyama, N.; Furukawa, K.; Nakamura, T.; Sasaki, T.; Kobayashi, N. Magnetic Properties of X-ray Irradiated Organic Mott Insulator $\kappa-(B E D T-T T F)_{2} \mathrm{Cu}\left[\mathrm{N}(\mathrm{CN})_{2}\right]$ Cl. J. Phys. Soc. Jpn. 2010, 79, doi:10.1143/JPSJ.79.063706.

55. Anzai, H.; Delrieu, J.; Takasaki, S.; Nakatsuji, S.; ichi Yamada, J. Crystal growth of organic charge-transfer complexes by electrocrystallization with controlled applied current. J. Cryst. Growth 1995, 154, 145-150.

56. Manna, R.S.; Wolf, B.; de Souza, M.; Lang, M. High-resolution thermal expansion measurements under helium-gas pressure. Rev. Sci. Instrum. 2012, 83, doi:10.1063/1.4747272.

57. Guterding, D.; Diehl, S.; Altmeyer, M.; Methfessel, T.; Tutsch, U.; Schubert, H.; Lang, M.; Müller, J.; Huth, M.; Jeschke, H.O.; et al. Evidence for Eight-Node Mixed-Symmetry Superconductivity in a Correlated Organic Metal. Phys. Rev. Lett. 2016, 116, doi:10.1103/PhysRevLett.116.237001. 
58. Aguiar, M.C.O.; Dobrosavljević, V. Universal Quantum Criticality at the Mott-Anderson Transition. Phys. Rev. Lett. 2013, 110, doi:10.1103/PhysRevLett.110.066401.

59. Aguiar, M.C.O.; Dobrosavljević, V.; Abrahams, E.; Kotliar, G. Effects of disorder on the non-zero temperature Mott transition. Phys. Rev. B 2005, 71, doi:10.1103/PhysRevB.71.205115.

60. Efros, A.L.; Shklovskii, B.I. Coulomb gap and low temperature conductivity of disordered systems. J. Phys. C Solid State Phys. 2001, 8, L239-L240.

61. Diehl, S.; Methfessel, T.; Tutsch, U.; Müller, J.; Lang, M.; Huth, M.; Jourdan, M.; Elmers, H.J. Disorder-induced gap in the normal density of states of the organic superconductor $\kappa-(\mathrm{BEDT}-\mathrm{TTF})_{2} \mathrm{Cu}\left[\mathrm{N}(\mathrm{CN})_{2}\right] \mathrm{Br}$. J. Phys. Condens. Matter 2015, 27, doi:10.1088/0953-8984/27/26/265601.

62. Kang, L.; Akagi, K.; Hayashi, K.; Sasaki, T. First-principles investigation of local structure deformation induced by X-ray irradiation in $\kappa-(\mathrm{BEDT}-\mathrm{TTF})_{2} \mathrm{Cu}\left[\mathrm{N}(\mathrm{CN})_{2}\right] \mathrm{Br}$. Phys. Rev. B 2017, 95, doi:10.1103/PhysRevB.95.214106.

63. Kurosaki, Y.; Shimizu, Y.; Miyagawa, K.; Kanoda, K.; Saito, G. Mott Transition from a Spin Liquid to a Fermi Liquid in the Spin-Frustrated Organic Conductor $\kappa-(\mathrm{ET})_{2} \mathrm{Cu}_{2}(\mathrm{CN})_{3}$. Phys. Rev. Lett. 2005, 95, doi:10.1103/PhysRevLett.95.177001.

64. Pustogow, A.; Bories, M.; Löhle, A.; Rösslhuber, R.; Zhukova, E.; Gorshunov, B.; Tomić, S.; Schlueter, J.; Hübner, R.; Hiramatsu, T.; et al. Quantum Spin Liquids Unveil the Genuine Mott State. arXiv 2017, arXiv:1710.07241.

65. Yoneyama, N.; Sasaki, T.; Kobayashi, N.; Furukawa, K.; Nakamura, T. X-ray irradiation effect on magnetic properties of Dimer-Mott insulators: $\kappa$-(BEDT-TTF $)_{2} \mathrm{Cu}\left[\mathrm{N}(\mathrm{CN})_{2}\right] \mathrm{Cl}$ and $\beta^{\prime}$-(BEDT-TTF $)_{2} \mathrm{ICl}_{2}$. In Proceedings of the 8th International Symposium on Crystalline Organic Metals, Superconductors and Ferromagnets, Yamada Conference LXIV, Hokkaido, Japan, 12-17 September 2009.

(C) 2018 by the authors. Licensee MDPI, Basel, Switzerland. This article is an open access article distributed under the terms and conditions of the Creative Commons Attribution (CC BY) license (http:/ / creativecommons.org/licenses/by/4.0/). 


\title{
Raman Scattering as a Probe of the Magnetic State of BEDT-TTF Based Mott Insulators
}

\author{
Nora Hassan ${ }^{1}$, Streit Cunningham ${ }^{1}$, Elena I. Zhilyaeva ${ }^{2}$, Svetlana A. Torunova ${ }^{2}$, \\ Rimma N. Lyubovskaya ${ }^{2}$, John A. Schlueter ${ }^{3,4}$ and Natalia Drichko ${ }^{1, *}$ \\ 1 The Institute for Quantum Matter and the Department of Physics and Astronomy, \\ The Johns Hopkins University, Baltimore, MD 21218, USA; nhassan4@jhu.edu (N.H.); \\ streitcunningham@gmail.com (S.C.) \\ 2 Institute of Problems of Chemical Physics, 142432 Chernogolovka, Russia; \\ zhilya@icp.ac.ru (E.I.Z.); torunova@icp.ac.ru (S.A.T.); lyurn@icp.ac.ru (R.N.L.) \\ 3 Division of Materials Research, National Science Foundation, Arlington, VA 22230, USA; \\ jaSchlueter@anl.gov \\ 4 Materials Science Division, Argonne National Laboratory, Argonne, IL 60439, USA \\ * Correspondence: drichko@jhu.edu; Tel.: +1-410-516-7287
}

Received: 14 March 2018; Accepted: 16 May 2018; Published: 23 May 2018

\begin{abstract}
Quasi-two-dimensional Mott insulators based on BEDT-TTF molecules have recently demonstrated a variety of exotic states, which originate from electron-electron correlations and geometrical frustration of the lattice. Among those states are a triangular $S=1 / 2$ spin liquid and quantum dipole liquid. In this article, we show the power of Raman scattering technique to characterize magnetic and electronic excitations of these states. Our results demonstrate a distinction between a spectrum of magnetic excitations in a simple Mott insulator with antiferromagnetic interactions, and a spectrum of an insulator with an additional on-site charge degree of freedom.
\end{abstract}

Keywords: spin liquid; Raman scattering; BEDT-TTF

\section{Introduction}

In recent years, a number of materials with very interesting properties produced by electronic correlations have been found among organic conductors and insulators. For example, they present a model to study a bandwidth-controlled transition from Mott insulator to a metal and superconductor, which can be tuned by hydrostatic or chemical pressure in these materials [1,2]. An importance of these studies outside of the community working directly on organic conductors is demonstrated, for example, by the fact that this bandwidth-controlled phase diagram shows a lot of similarity to a doping-dependent phase diagram of electron-doped cuprate superconductors [3].

Recently, much attention was focused on magnetic properties of organic-based Mott insulators on frustrated square and triangular lattices [4]. The number of spin liquid candidates [5-12] with $S=1 / 2$ on a triangular lattice found among these materials is larger than among inorganic compounds. Moreover, intriguing ferroelectric properties of spin liquid candidates [13-16], and recently a quantum dipole liquid state [17] were observed for the $\kappa$-phase BEDT-TTF-based compounds, where BEDT-TTF stands for bis(ethylenedithio)tetrathiafulvalene. These results suggest an importance of a charge degree of freedom in the Mott insulator state. Theoretical models $[18,19]$ go beyond the simple Mott insulator model that was previously used to describe these materials, and discuss coupling of the charge degree of freedom to spins. In some of the theoretical work, this charge degree of freedom and related effects were discussed within the Kugel-Khomskii model, suggesting a similarity to an orbital liquid [20]. Some of the results still present a conflicting picture; for example, while ferroelectricity 
was detected for $\kappa$-(BEDT-TTF $)_{2} \mathrm{Cu}_{2}(\mathrm{CN})_{3}$, no evidence of charge disproportionation which would lead to electric dipoles was found [21].

Despite their complicated crystal structure, the electronic structure of these compounds is relatively simple. The layers of the positively charged BEDT-TTF molecules are sandwiched between layers of anions. The anion layers serve as charge reservoirs, but their geometry also influences the structure of the conducting BEDT-TTF layers to some extent. Most of the models which discuss properties of these systems work in an approximation in which electronic and magnetic properties of a material are basically defined by the properties of the BEDT-TTF-based cation layers, the interactions between these layers are very weak if present. These layers can be approximated by a two-dimensional arrangement of "sites". For $\kappa$-phase materials discussed in this paper, the cation layer can be mapped on a lattice of molecular dimer sites (BEDT-TTF) ${ }_{2}^{+1}$ carrying charge $1 e$ and spin $1 / 2$. With high values of electronic correlations $U / t$, where $U$ is on-cite repulsion and $t$ is transfer integral, these compounds are Mott insulators, and their properties are described by the Hubbard model on 2D square or triangular lattice at half-filling $[4,22]$. The latter model ignores a possibility of an active intra-dimer degree of freedom, which recently has been found important in some cases $[17,18,20]$. In a limiting case, the presence of the intra-dimer charge degree of freedom breaks the half-filled model, and a system has to be regarded as 1/4-filled [19].

In general, much information about a state of matter comes from a knowledge of its excitation spectrum. For example, a lot of our understanding of charge degrees of freedom in organic conductors and insulators is based on the studies of their spectrum of charge excitations. This information was obtained by optical spectroscopy and compared to the calculations on the Hubbard model [2,23]. Measurements of a spectrum of magnetic excitations for organic materials showing interesting magnetic properties present more challenges. A characterization of a spin state by neutron scattering, which by now became a standard tool to study magnetic systems, still has not been performed for these materials due to complicity of the measurements. Until recently, conclusions on the spin liquid behavior in organic Mott insulators on triangular lattice [12] were based on measurements of magnetization, which would suggest $J$ of about $250 \mathrm{~K}$ for spin liquid candidates but no magnetic order [24], thermoelectric transport [25] which reveals the presence of mobile excitations assigned to spinons, heat capacity [12], and NMR [26]. However, it is important to directly probe magnetic excitations and obtain the value of $J$. Also, it is necessary to show that a description of these complicated molecular-based compounds by a simple model of $2 \mathrm{D}$ triangular lattice of sites with $S=1 / 2$ is valid. Inelastic light scattering technique, so-called Raman scattering, is able to provide this information. This technique is widely used to study magnetic excitations in inorganic systems [27,28]. For example, Raman scattering experiments were a source of information about values of exchange interactions $J$ in cuprate superconductors [29]. Additionally, polarization dependence of magnetic Raman response is a probe of dimensionality and symmetry of a spin system [28,30].

While few different processes can be excited by light in a magnetic system, here we will focus on so-called "exchange Raman scattering" [31], expected in all Heisenberg antiferromegnets (AF). A schematic illustration of this process is presented in Figure 1 for an antiferromagnetically ordered chain. An excitation light of a laser with a frequency $\omega_{L}$ induces an exchange of electrons with opposite spins between the neighboring sites $i$ and $j$ of the two different magnetic sublattices. As a result, a pair of magnons with energies $E_{M}=\hbar \omega_{M}$ and wavevectors $q$ and $q^{\prime}$ so that $q+q^{\prime}=0$ is excited. The total energy costs of this process is $E_{2 M}=\hbar \omega_{2 M}$, thus inelastically scattered light with frequency $\omega_{L}-\omega_{2 M}$ is emitted and collected by a spectrometer. The presented picture describes local excitations and explains sensitivity of the method to short range magnetic correlations. All pairs of magnons with $q+q^{\prime}=0$ can be excited, as a result for a system with a long range order the two-magnon Raman spectrum presents a weighted integration of magnon excitations over a Brillouin zone and reflects the renormalized magnon density of states. The position of a two-magnon peak is a direct measure of a magnetic exchange $J[29,31]$. In the absence of geometrical frustration the peak appears approximately at energy $E_{2 M}=J 2 S(z-1)$, where $S$ is a spin value, and $z$ is a number of nearest neighbors. $E_{2 M}$ can 
be re-normalized by magnon-magnon interactions. Importantly, a polarization of light in which the excitations are observed is not defined by an easy axis, but by a vector $\delta_{i, j}$ connecting sites $i$ and $j$ of the two different sublattices between which the exchange excitation occurs. Nevertheless, polarization dependence of a two-magnon band in an ordered state reveals the symmetry and dimensionality of the order $[28,29]$.

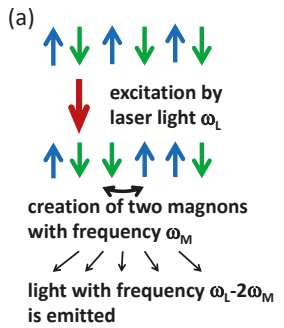

(b)

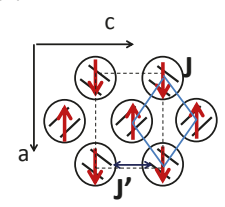

Figure 1. (a) A scheme of excitation of pairs of magnons in an antiferromagnetic chain; (b) A schematic view of a structure of BEDT-TTF-layer of a $\kappa$-phase crystal as a model of $S=1 / 2$ sites on a frustrated square lattice.

Theoretical calculations of a magnetic Raman response relevant to organic conductors describe two-magnon excitations on a square and triangular lattice $[32,33]$. These calculations suggest that as a result of a geometrical frustration, the two-magnon Raman response on a triangular lattice is shifted to lower frequencies compared to the square lattice and looses the distinct polarization dependence of a square lattice, where it is observed only in $\mathrm{B}_{1 \mathrm{~g}}$ scattering channel. Both mentioned calculations for a triangular lattice are relevant to a situation where a ground state of an isotropic triangular lattice system is a classical $120^{\circ}$ order. Spin excitations spectra were calculated for a model of a triangular lattice with a ring exchange, which actually can produce a spin liquid behavior [34]. While specifically Raman response was not yet calculated, it is clear that in this case, Raman scattering would show a continuum below approximately $4 \mathrm{~J}$.

Publications presenting magnetic Raman scattering in organic conductors started to appear only recently. In this paper, we would like to give an overview of our results on the Raman spectra of magnetic excitations which we observed in a number of $\kappa$-phase organic conductors. They demonstrate a striking difference between materials which can be described by a model of a Mott insulator on a frustrated square or triangular lattices with $S=1 / 2$ per lattice site, and a more complicated situation, when the intra-dimer charge degree of freedom becomes active, resulting in a quantum dipole liquid [17]. In Section 4 we will compare our results to the existing literature data on other organic spin liquid candidates.

\section{Materials and Methods}

Raman scattering spectra of single crystals of $\kappa$-(BEDT-TTF $)_{2} \mathrm{Cu}\left[\mathrm{N}(\mathrm{CN})_{2}\right] \mathrm{Cl}, \kappa-(\mathrm{BEDT}-\mathrm{TTF})_{2}$ $\mathrm{Cu}\left[\mathrm{N}(\mathrm{CN})_{2}\right] \mathrm{Br}$, and $\kappa-(\mathrm{BEDT}-\mathrm{TTF})_{2} \mathrm{Hg}(\mathrm{SCN})_{2} \mathrm{Br}$ were measured from the plane parallel to BEDTTTF layers in pseudo-Brewster angle geometry. Measurements were done using T64000 triple monochromator spectrometer equipped with the liquid $\mathrm{N}_{2}$ cooled CCD detector. For the measurements in the 10-400 $\mathrm{cm}^{-1}$ range T64000 in triple monochromator configuration was used. The low frequency cut off was determined by the excitation used and a size of a measured crystal. Crystals of $\kappa$-(BEDT-TTF $)_{2} \mathrm{Hg}(\mathrm{SCN})_{2} \mathrm{Br}$ were not larger than $1 \mathrm{~mm}$ by $1 \mathrm{~mm}$ in the $(b, c)$ plane, the lowest measured frequency for these crystals was $20 \mathrm{~cm}^{-1}$. For the measurements in the range $100-2000 \mathrm{~cm}^{-1}$ single monochromator configuration with the edge filter option was used. Spectral resolution was $2 \mathrm{~cm}^{-1}$. Lines of $\mathrm{Ar}^{+}-\mathrm{Kr}^{+}$Coherent laser at $514.5 \mathrm{~nm}$ and $647 \mathrm{~nm}$ where used for excitation. Laser power was kept at $2 \mathrm{~mW}$ for the laser probe size of approximately 50 by $100 \mu \mathrm{m}$. This ensured that the laser heating of the sample was kept below $2 \mathrm{~K}$. Measurements at temperatures down to $10 \mathrm{~K}$ were 
performed using Janis ST500 cold finger cryostat. Cooling rates used were between 0.2 and $0.5 \mathrm{~K} / \mathrm{min}$. The samples were glued on the cold finger of the cryostat using GE varnish. The experiments were performed on at least 6 samples to ensure reproducibility of the results. The crystals were oriented using polarization-dependent Raman scattering measurements.

For the measurements, electrical vector of excitation $e_{L}$ and scattered $e_{S}$ light were polarized along the in-plane crystallographic axes $(a \text { and } c \text { for } \kappa \text {-(BEDT-TTF })_{2} \mathrm{Cu}\left[\mathrm{N}(\mathrm{CN})_{2}\right] \mathrm{Cl}$ and $\kappa$-(BEDT-TTF $)_{2} \mathrm{Cu}\left[\mathrm{N}(\mathrm{CN})_{2}\right] \mathrm{Br}$, and $b$ and $c$ axes for $\kappa$-(BEDT-TTF $)_{2} \mathrm{Hg}(\mathrm{SCN})_{2} \mathrm{Br}$ ). Our notations of polarizations refer to the structure and symmetry of the BEDT-TTF layer, to make an easy comparison to the calculations which refer to $\mathrm{D}_{4 h}$ [29] without loosing the information about the symmetry of the real crystal. Thus $\mathrm{A}_{1 g}$ symmetry corresponds to measurements in $(b, b)$ and $(c, c)$ geometries, and $\mathrm{B}_{1 g}$ corresponds to $(b, c)$ and $(c, b)$ geometries $(x y)$. All spectra were corrected by the Bose-Einstein thermal factor.

Raman spectra of organic conductors are a complicated spectra with an overlap of molecular vibrations, lattice phonons, some effect of electron-molecular vibrational coupling, electronic and magnetic excitations, and luminescence. At this point vibrational contributions $[35,36]$ and luminescence [37] are well understood and can be subtracted from the spectra. In order to do so we fitted the phonons with the Lorentz functions, and luminescence with broad Lorentz bands located at $5000 \mathrm{~cm}^{-1}$ for all materials, and additionally a weaker band located at $3000 \mathrm{~cm}^{-1}$ for Mott insulators, basing on the results of Ref. [38]. For the materials of $\kappa$-Cu family, the identical luminescence contribution, normalized on the phonons intensity, was subtracted from all the spectra.

\section{Results}

An overview of the Raman spectra of the four discussed $\kappa$-phase compounds $\kappa$-(BEDT-TTF) ${ }_{2}$ $\mathrm{Cu}\left[\mathrm{N}(\mathrm{CN})_{2}\right] \mathrm{Cl}, \kappa$-(BEDT-TTF $)_{2} \mathrm{Cu}\left[\mathrm{N}(\mathrm{CN})_{2}\right] \mathrm{Br}, \kappa-(\mathrm{BEDT}-\mathrm{TTF})_{2} \mathrm{Cu}_{2}(\mathrm{CN})_{3}$, and $\kappa$-(BEDT-TTF $)_{2} \mathrm{Hg}(\mathrm{SCN})_{2} \mathrm{Br}$ is presented in Figure 2. We show the data obtained at low temperatures around $10 \mathrm{~K}$ for two polarizations which would correspond to $\mathrm{A}_{1 g}$ and $\mathrm{B}_{1 g}$ scattering channels in a square magnetic unit cell of $\kappa$-(BEDT-TTF $)_{2} \mathrm{Cu}\left[\mathrm{N}(\mathrm{CN})_{2}\right] \mathrm{Cl}$ (Figure $1 \mathrm{~b}$ ). The range from 100 to $1600 \mathrm{~cm}^{-1}$ covers the frequency range where we can observe two-magnon continuum for a square lattice with $J$ of around a few hundred kelvin.

The insets in Figure 2 show original data, and reveal a complicated picture. Multiple narrow bands observed in the spectra belong to molecular vibrations of BEDT-TTF and lattice vibrations $[35,36]$. They are superimposed on a background which consist of luminescence [38], possible electronic excitations, and magnetic excitations. A spectrum of phonons which do not interact with electronic and magnetic degrees of freedom is easy to interpret and subtract from the original data. While a maximum of luminescence is observed at much higher frequencies of $3000 \mathrm{~cm}^{-1}$ for all the compounds, it does appear in the spectra as a nearly linear background with different intensity for these four materials. Luminescence shows the larger intensity in polarizations, where electrical vector $e_{L}$ of the excitation light is parallel to the crystallograpic direction which has a projection of the long axis of BEDT-TTF molecule, thus we always selected polarizations with the smaller luminescence intensity. An additional luminescence signal comes from a maximum at around $5000 \mathrm{~cm}^{-1}$ [38], and apparently belongs to the response of an anion layer. It is much stronger in $\kappa$-(BEDT-TTF $)_{2} \mathrm{Hg}(\mathrm{SCN})_{2} \mathrm{Br}$ spectra.

In cross-polarization ( $\mathrm{B}_{1 g}$ scattering channel) we could successfully disentangle luminescence and the electronic or magnetic background, but in $\mathrm{A}_{1 g}$ scattering channel of $\kappa$-Cu-family materials some higher-frequency component is left. This can be an electronic response of a system, for example transitions between Hubbard bands. For $\mathrm{D}_{4 h}$ symmetry which we use to approximate the structure of $\kappa$-phase BEDT-TTF layer, electronic contribution would be present in both $\mathrm{A}_{1 g}$ and $\mathrm{B}_{1 g}$ channels, while absent in $B_{2 g}$ [29,38]. Since electronic response in $A_{1 g}$ scattering channel is proportional to the intensity of electronic transitions integrated over the whole BZ [29], the electronic contunuum is typically more intense in $\mathrm{A}_{1 g}$ channel [39]. 
In most cases, a distinct polarization dependence and frequency range of magnetic excitations allow to separate them from other contributions. In the relevant section, we will discuss a particular difficulty of the analysis of the data in $\mathrm{A}_{1 g}$ scattering channel for $\kappa$-(BEDT-TTF) ${ }_{2} \mathrm{Cu}_{2}(\mathrm{CN})_{3}$.

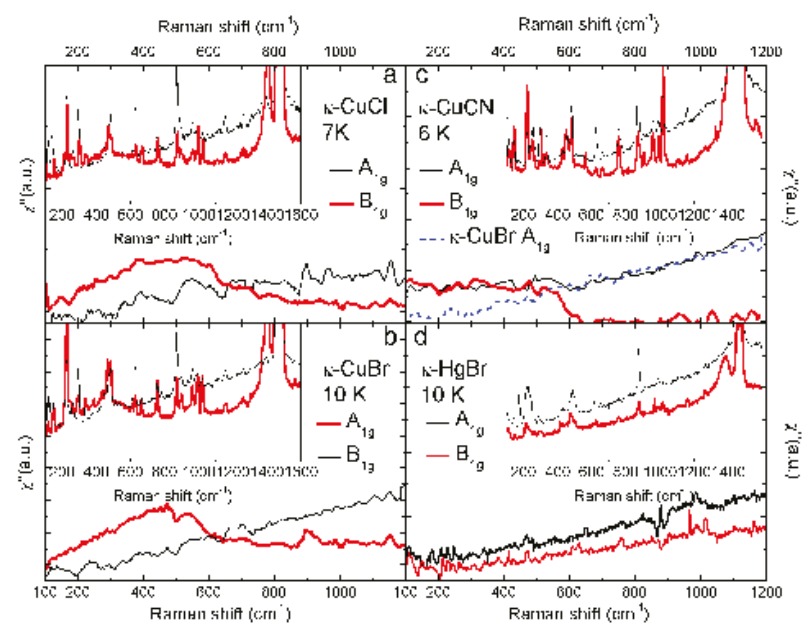

Figure 2. Low temperature Raman spectra for the discussed $\kappa$ phase materials in the spectral range between 100 and $1600 \mathrm{~cm}^{-1}$ in polarizations corresponding to $\mathrm{A}_{1 g}$ and $\mathrm{B}_{1 g}$ in $\mathrm{D}_{4 h}$ symmetry. The insets show original data, the main figures show data with phonons and luminescence contribution subtracted. The resulting spectra, containing magnetic and, possibly, electronic contributions are plotted (a) Spectra of $\kappa$-(BEDT-TTF $)_{2} \mathrm{Cu}\left[\mathrm{N}(\mathrm{CN})_{2}\right] \mathrm{Cl}$ at $7 \mathrm{~K}$. Note the broad maximum in $\mathrm{B}_{1 \mathrm{~g}}$ symmetry between 100 and $700 \mathrm{~cm}^{-1}$; (b) Spectra of $\kappa$-(BEDT-TTF) $)_{2} \mathrm{Cu}\left[\mathrm{N}(\mathrm{CN})_{2}\right] \mathrm{Br}$ at $10 \mathrm{~K}$. Note the broad maximum in $\mathrm{B}_{1 g}$ symmetry between 100 and $700 \mathrm{~cm}^{-1}$, similar to $\kappa$ - $(\mathrm{BEDT}-\mathrm{TTF})_{2} \mathrm{Cu}\left[\mathrm{N}(\mathrm{CN})_{2}\right] \mathrm{Cl}$; (c) Spectra of $\kappa-(\mathrm{BEDT}-\mathrm{TTF})_{2} \mathrm{Cu}_{2}(\mathrm{CN})_{3}$ at $6 \mathrm{~K}$. The continuum extends from about $600 \mathrm{~cm}^{-1}$ to the lowest measured frequencies, no gap in magnetic spectrum can be identified, in contrast to $\kappa$-(BEDT-TTF $)_{2} \mathrm{Cu}\left[\mathrm{N}(\mathrm{CN})_{2}\right] \mathrm{Cl}$ and $\kappa-(\mathrm{BEDT}-\mathrm{TTF})_{2} \mathrm{Cu}\left[\mathrm{N}(\mathrm{CN})_{2}\right] \mathrm{Br}$. The dashed line shows $\mathrm{A}_{1 g}$ contribution of $\kappa$-(BEDT-TTF $)_{2} \mathrm{Cu}\left[\mathrm{N}(\mathrm{CN})_{2}\right] \mathrm{Br}$ for comparison, which makes clear the presence of an additional intensity below $600 \mathrm{~cm}^{-1}$ in $\mathrm{A}_{1 g}$ for $\kappa-(\mathrm{BEDT}-\mathrm{TTF})_{2} \mathrm{Cu}_{2}(\mathrm{CN})_{3}$, which we attribute to magnetic excitations; (d) Spectra of $\kappa$-(BEDT-TTF $)_{2} \mathrm{Hg}(\mathrm{SCN})_{2} \mathrm{Br}$ at $10 \mathrm{~K}$, the spectra do not show major differences between $\mathrm{A}_{1 g}$ and $\mathrm{B}_{1 g}$ channels in the discussed range, no continuum of magnetic excitations is observed.

3.1. Magnetic Excitations on a Frustrated Square Lattice: From a Mott Insulator with an Antiferromagnetic Order to a Metal with Antiferromagnetic Fluctuations

A simple and well-understood example of magnetic excitations in an organic Mott insulator are spectra of an AF state for $\kappa-(\mathrm{BEDT}-\mathrm{TTF})_{2} \mathrm{Cu}\left[\mathrm{N}(\mathrm{CN})_{2}\right] \mathrm{Cl}\left(T_{N}=27 \mathrm{~K}\right)$. In the Raman response of this compound (see Figure 2a) we observe a broad maximum between 200 and $700 \mathrm{~cm}^{-1}$ only in $(a, c)$ polarization, which corresponds to $\mathrm{B}_{1 g}$ symmetry of the magnetic unit cell in $\kappa$-(BEDT-TTF $)_{2} \mathrm{Cu}\left[\mathrm{N}(\mathrm{CN})_{2}\right] \mathrm{Cl}$ (see Figure $1 \mathrm{~b}$ ). This maximum is absent in $(c, c)$ spectra, which correspond to $\mathrm{A}_{1 g}$ symmetry (Figure 2a), as well as in $\mathrm{B}_{2 g}$ [38]. This polarization dependence coincides with the theoretical expectations for a two-magnon response of AF order on a square lattice. Any electronic excitations due to correlation effects would be observed both in $\mathrm{A}_{1 g}$ and $\mathrm{B}_{1 g}$ channels [29] and are expected at higher frequencies [2]. The contribution observed in $(c, c)$ polarization is a good candidate for the electronic contribution to the spectra. In Ref. [32] two-magnon Raman response of an AF on a frustrated square lattice described by a Hubbard model was calculated depending on the frustration parameter $J^{\prime} / J$. For $J^{\prime} / J=0.22$ [38] a two-magnon excitation would be 
observed in $\mathrm{B}_{1 g}$ polarization only, with a maximum at about $2.5 \mathrm{~J}$. This provides us an estimate of $J=250 \mathrm{~K}$, close to that obtained from the fit of magnetization [24].

The $\kappa$-(BEDT-TTF $)_{2} \mathrm{Cu}\left[\mathrm{N}(\mathrm{CN})_{2}\right] \mathrm{Br}$ metal with superconducting transition below $T=11.5 \mathrm{~K}$ demonstrates electronic parameters very similar to that of $\kappa-(\mathrm{BEDT}-\mathrm{TTF})_{2} \mathrm{Cu}\left[\mathrm{N}(\mathrm{CN})_{2}\right] \mathrm{Cl}$. Only a small decrease in $U / t$ value is responsible for tuning this material into metallic and superconducting states [4]. While electronic spectra of these compounds are very different, reflecting the difference in the charge ground states [2], $\kappa-(\mathrm{BEDT}-\mathrm{TTF})_{2} \mathrm{Cu}\left[\mathrm{N}(\mathrm{CN})_{2}\right] \mathrm{Cl}$ and $\kappa-(\mathrm{BEDT}-\mathrm{TTF})_{2} \mathrm{Cu}\left[\mathrm{N}(\mathrm{CN})_{2}\right] \mathrm{Br}$ show an amazing similarity in the spectrum of magnetic excitations (see Figure $2 b$ ), which has a similar position, shape, and polarization dependence. It is therefore natural to assign the maximum present in the spectra of $\kappa$-(BEDT-TTF $)_{2} \mathrm{Cu}\left[\mathrm{N}(\mathrm{CN})_{2}\right] \mathrm{Br}$ between 200 and $700 \mathrm{~cm}^{-1}$ only in $\mathrm{B}_{1 g}$ scattering channel, to magnetic excitations due to local AF correlations. The presence of AF fluctuations below $200 \mathrm{~K}$ in $\kappa-(\mathrm{BEDT}-\mathrm{TTF})_{2} \mathrm{Cu}\left[\mathrm{N}(\mathrm{CN})_{2}\right] \mathrm{Br}$ was observed also by other methods [40].
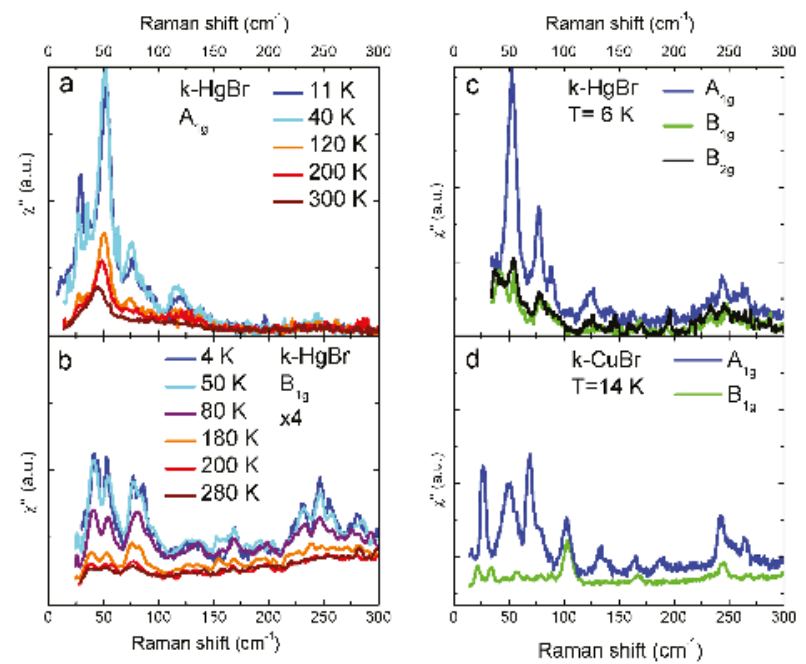

Figure 3. (a,b) Temperature dependence of Raman spectra of $\kappa$-(BEDT-TTF $)_{2} \mathrm{Hg}(\mathrm{SCN})_{2} \mathrm{Br}$ in the range below $300 \mathrm{~cm}^{-1}$ in (a) $\mathrm{A}_{1 g}$ scattering channel; and (b) $\mathrm{B}_{1 \mathrm{~g}}$ scattering channel, intensity is multiplied by the factor of 4 ; (c) Polarization dependence of the low-frequency spectra for $\kappa$-(BEDT-TTF ${ }_{2} \mathrm{Hg}(\mathrm{SCN})_{2} \mathrm{Br}$ at $4 \mathrm{~K}$; (d) Polarization dependence of low frequency spectra of $\kappa$-(BEDT-TTF) ${ }_{2} \mathrm{Cu}\left[\mathrm{N}(\mathrm{CN})_{2}\right] \mathrm{Br}$ at $14 \mathrm{~K}$. Note the absence of the broad mode with the maximum at about $40 \mathrm{~cm}^{-1}$.

To summarize, as a whole, Raman spectra of magnetic excitations in $\kappa-(\mathrm{BEDT}-\mathrm{TTF})_{2} \mathrm{Cu}\left[\mathrm{N}(\mathrm{CN})_{2}\right] \mathrm{Cl}$ and $\kappa-(\mathrm{BEDT}-\mathrm{TTF})_{2} \mathrm{Cu}\left[\mathrm{N}(\mathrm{CN})_{2}\right] \mathrm{Br}$ are in agreement with the calculations for an $\mathrm{AF}$ on a frustrated square lattice with $S=1 / 2$, and reproduce the value of $J$ received from magnetization measurements. Some details of temperature and $U / t$ dependence of the spectra are unconventional. (i) An extreme similarity between magnetic excitations of an AF ordered $\kappa-(\mathrm{BEDT}-\mathrm{TTF})_{2} \mathrm{Cu}\left[\mathrm{N}(\mathrm{CN})_{2}\right] \mathrm{Cl}$ and metallic $\kappa-(\mathrm{BEDT}-\mathrm{TTF})_{2} \mathrm{Cu}\left[\mathrm{N}(\mathrm{CN})_{2}\right] \mathrm{Br}$ with AF fluctuations is unexpected. Typically, in a metallic state next to a Mott insulator, where AF fluctuations survive, a two-magnon peak is broadened and shifted down in frequencies due to the interactions with charge carriers [29]; (ii) As we have shown in Ref. [38], the two-magnon peak fully forms in $\kappa-(\mathrm{BEDT}-\mathrm{TTF})_{2} \mathrm{Cu}\left[\mathrm{N}(\mathrm{CN})_{2}\right] \mathrm{Cl}$ at around $50 \mathrm{~K}$, and does not change when the material is cooled through the ordering transition at $T_{N}=27 \mathrm{~K}$. This is in contrast to the majority of magnetic materials, where the two-magnon peak would shift to higher frequencies and become more narrow below $T_{N}$ [27]. While the details of this behavior still need an explanation, it is clear that two-dimensional magnetic interactions, and high energy scale of $J$ compared to the 
temperature of ordering and to the energy scale where charge carriers are observed, lie at the origin of these effects.

\subsection{Mott Insulator on a Triangular Lattice: $\kappa-(B E D T-T T F)_{2} C u_{2}(C N)_{3}$}

The overall spectra of $\kappa$-(BEDT-TTF $)_{2} \mathrm{Cu}_{2}(\mathrm{CN})_{3}$ are similar to that of the two materials discussed above, but show a difference in the position of the band assigned to the two-magnon excitations. In the spectra of $\kappa$-(BEDT-TTF $)_{2} \mathrm{Cu}_{2}(\mathrm{CN})_{3}$ in $(a, c)$. polarization (Figure $2 \mathrm{c}$ ) we observe a broad feature below approximately $600 \mathrm{~cm}^{-1}$ which we assign to magnetic excitations. Interestingly, the band of magnetic excitations is extended to much lower frequencies than in the less frustrated materials $\kappa$-(BEDT-TTF $)_{2} \mathrm{Cu}\left[\mathrm{N}(\mathrm{CN})_{2}\right] \mathrm{Cl}$ and $\kappa-(\mathrm{BEDT}-\mathrm{TTF})_{2} \mathrm{Cu}\left[\mathrm{N}(\mathrm{CN})_{2}\right] \mathrm{Br}$, and is still present at the lowest measured frequency of $50 \mathrm{~cm}^{-1}$. The low frequency shift of the two-magnon peak is in a good agreement with a calculation of the two-magnon peak position for a model of $S=1 / 2$ on frustrated square lattice with frustration value of $J^{\prime} / J=0.64[32,41]$. Assuming the value of $J$ value of $\kappa$-(BEDT-TTF $)_{2} \mathrm{Cu}_{2}(\mathrm{CN})_{3}$ close to that of $\kappa$-(BEDT-TTF $)_{2} \mathrm{Cu}\left[\mathrm{N}(\mathrm{CN})_{2}\right] \mathrm{Cl}$, the center of two-magnon peak should shift to about $230 \mathrm{~cm}^{-1}$. However, this calculation discusses a classical picture of $120^{\circ}$ order as a ground state of an AF on an isotropic triangular lattice, which is not the case for $\kappa-(B E D T-T T F)_{2} \mathrm{Cu}_{2}(\mathrm{CN})_{3}$. Another recently discussed explanation of a spin liquid state in $\kappa$-(BEDT-TTF $)_{2} \mathrm{Cu}_{2}(\mathrm{CN})_{3}$ is a model of a triangular lattice with a ring exchange [34]. Ring exchange is suggested as the largest exchange of the next order beyond a nearest neighbor exchange $J$ and destroys the ordered ground state. A spectrum of magnetic excitations of this spin liquid state is discussed in Ref. [34], and would produce a continuum of excitations below about $4 \mathrm{~J}$. We observe a continuum of excitations in $\kappa$-(BEDT-TTF $)_{2} \mathrm{Cu}_{2}(\mathrm{CN})_{3}$ spectra below $600 \mathrm{~cm}^{-1}$, which gives an estimate of $J$ of about $215 \mathrm{~K}$, in a good agreement with other measurements. While producing different low temperature states, these two models agree on a starting model of a $S=1 / 2$ AF on triangular lattice, with no active charge degree of freedom.

Calculations [32,33] predict a loss of anisotropy between the two-magnon continuum in $\mathrm{B}_{1 \mathrm{~g}}$ and $\mathrm{A}_{1 g}$ scattering channels for an isotropic triangular lattice. We find that this loss of polarization dependence makes it more challenging to separate magnetic excitations from possible electronic and luminescence background in $\mathrm{A}_{1 g}$ scattering channel. A work of Nakamura et al. [41] suggest small intensity of magnetic Raman scattering in this channel. Indeed, using a fit of luminescence background, parameters for which is very difficult to restrict, one receives a very low intensity of magnetic scattering in $\mathrm{A}_{1 g}$. On the other hand, a comparison of $(c, c)$ spectra with that of $\kappa-(\mathrm{BEDT}-\mathrm{TTF})_{2} \mathrm{Cu}\left[\mathrm{N}(\mathrm{CN})_{2}\right] \mathrm{Br}$ and $\kappa-(\mathrm{BEDT}-\mathrm{TTF})_{2} \mathrm{Cu}\left[\mathrm{N}(\mathrm{CN})_{2}\right] \mathrm{Cl}$ suggest an additional intensity in $\kappa-(\mathrm{BEDT}-\mathrm{TTF})_{2} \mathrm{Cu}_{2}(\mathrm{CN})_{3}$ spectra at low frequencies (see Figure 2), which we assign to magnetic excitations. At this point, the error bars for the intensity of magnetic excitations in $\mathrm{A}_{1 g}$ scattering channel are large, but the presence of these excitations is in agreement with the symmetry of the lattice.

\subsection{Charge Degree of Freedom in a Mott Insulator: $\kappa$-(BEDT-TTF $)_{2} \mathrm{Hg}(\mathrm{SCN})_{2} \mathrm{Br}$}

$\kappa$-(BEDT-TTF $)_{2} \mathrm{Hg}(\mathrm{SCN})_{2} \mathrm{Br}$ shows $\kappa$-phase structure, where the layer formed by the $(\mathrm{BEDT}-\mathrm{TTF})_{2}^{+1}$ dimers is well approximated by an anisotropic triangular lattice with the ratio of $t^{\prime} / t$ at least as high as for $\kappa-(B E D T-T T F)_{2} \mathrm{Cu}_{2}(\mathrm{CN})_{3}$. In a Mott insulator, where $J$ is estimated as $J=4 t^{2} / U$, this would result in a similarly high magnetic frustration. Calculations carried out for an iso-structural $\kappa$-(BEDT-TTF $)_{2} \mathrm{Hg}(\mathrm{SCN})_{2} \mathrm{Cl}$ compound suggest values of $t$ and $t^{\prime}$ similar to Cu-based $\kappa$-phases, but a slightly lower degree of dimerisation $[37,42]$. Contrary to the expectation based on a model of a Mott insulator with $S=1 / 2$ on a triangular lattice, no continuum of magnetic excitations in the range between 100 and $700 \mathrm{~cm}^{-1}$ is observed in the spectra (see Figure 2d).

The reason for this difference is an "active" intra-dimer degree of freedom. Indeed, in contrast to the compounds discussed in the previous section, $\kappa$-(BEDT-TTF $)_{2} \mathrm{Hg}(\mathrm{SCN})_{2} \mathrm{Br}$ shows an evidence of fluctuating intra-dimer charge detected by vibrational spectroscopy [17]. Respectively, a different collective excitation with a maximum at around $40 \mathrm{~cm}^{-1}$ is found in the spectra of this compound. 
It appears in both polarizations at temperatures below the Mott transition [43] at $80 \mathrm{~K}$ (Figure 3a,b). This excitation is attributed [17] to a collective excitation of dipoles fluctuating on (BEDT-TTF) ${ }_{2}^{+1}$ dimers in the insulating state of $\kappa$-(BEDT-TTF $)_{2} \mathrm{Hg}(\mathrm{SCN})_{2} \mathrm{Br}$, forming "quantum dipole liquid".

Additional understanding of the low frequency region of the spectra comes from a comparison of spectra of $\kappa$-(BEDT-TTF $)_{2} \mathrm{Cu}\left[\mathrm{N}(\mathrm{CN})_{2}\right] \mathrm{Br}$ at $14 \mathrm{~K}$ with the spectra of $\kappa$-(BEDT-TTF $)_{2} \mathrm{Hg}(\mathrm{SCN})_{2} \mathrm{Br}$ in this range (Figure $3 \mathrm{c}, \mathrm{d}$ ) at the lowest measured temperature of $4 \mathrm{~K}$. For $\kappa$-(BEDT-TTF) ${ }_{2} \mathrm{Cu}\left[\mathrm{N}(\mathrm{CN})_{2}\right] \mathrm{Br}$ this frequency range is below the range where magnetic excitations are observed, but above spectral range where the superconducting gap can appear [29], thus we do not expect any electronic or magnetic excitations. In the Raman spectra at low frequencies of BEDT-TTF-based compounds we typically observe bands of vibrations of the BEDT-TTF molecule down to approximately $100 \mathrm{~cm}^{-1}$ [35]. They are found at similar frequencies for various BEDT-TTF-based crystals, for example bands at around $250 \mathrm{~cm}^{-1}$ and a phonon at $125 \mathrm{~cm}^{-1}$, observed for both $\kappa$-(BEDT-TTF $)_{2} \mathrm{Hg}(\mathrm{SCN})_{2} \mathrm{Br}$ and $\kappa$-(BEDT-TTF $)_{2} \mathrm{Cu}\left[\mathrm{N}(\mathrm{CN})_{2}\right] \mathrm{Br}$ (Figure $3 \mathrm{c}, \mathrm{d}$ ). The phonons in the range below approximately $100 \mathrm{~cm}^{-1}$ are lattice vibrations [36]. Their frequency depends on the details of the structure of the crystals, including that of the anion. For $\kappa$-phase BEDT-TTF-based crystals similar modes exist, for example modes at about 50 and $75 \mathrm{~cm}^{-1}$ are present in the $\mathrm{A}_{1 \mathrm{~g}}$ scattering channel for both compounds.

For $\kappa$-(BEDT-TTF $)_{2} \mathrm{Hg}(\mathrm{SCN})_{2} \mathrm{Br}$ in $\mathrm{A}_{1 g}$, the intense low frequency phonons are superimposed on a broad background mode of dipole fluctuations [17]. The collective excitation is not coupled to the $\mathrm{A}_{1 g}$ phonons. This fact allows us to subtract the phonons from the original spectra in order to extract the information about the mode itself [17]. In $B_{1 g}$ polarization, the spectrum of $\kappa$-(BEDT-TTF $)_{2} \mathrm{Cu}\left[\mathrm{N}(\mathrm{CN})_{2}\right] \mathrm{Br}$ is overall flat with weak phonon bands below $40 \mathrm{~cm}^{-1}$. In contrast, the spectra of $\kappa$-(BEDT-TTF $)_{2} \mathrm{Hg}(\mathrm{SCN})_{2} \mathrm{Br}$ show the background mode, and asymmetric bands of phonons at about 40 and $80 \mathrm{~cm}^{-1}$. These asymmetric Fano shape of the phonons evidences for the coupling of these phonons with the underlying continuum, which makes it challenging to separate the phonons and the collective mode contributions.

At this point, there are no calculations which address the origin of the excitation observed in the low-frequency spectra of $\kappa$-(BEDT-TTF $)_{2} \mathrm{Hg}(\mathrm{SCN})_{2} \mathrm{Br}$, and its assignment to the collective excitation of dipoles localised on (BEDT-TTF $)_{2}^{+1}$ dimers is based on experimental data. Thus, the symmetry of this excitations, and the involvement of spin excitations are not yet understood. However, an identification of the phonons which are coupled to this excitation are important. Lattice phonons can define the formation of a low-temperature state by modulation of transfer integrals which define magnetic exchange $J$, as well as the $U / t$ and $V / t$ correlation values which are important for electric dipole formation.

\section{Discussion}

We present our results on the Raman spectra of four $\kappa$-phase BEDT-TTF-based compounds. While Raman spectra of these materials are very rich, our discussion focuses on magnetic Raman excitations. These $\kappa$-phase materials have very similar structures of BEDT-TTF layers, with well-defined dimers of (BEDT-TTF $)_{2}^{+1}$ molecules forming frustrated square or anisotropic triangular lattices. Our data reveal a presence of magnetic Raman excitations in the frequency range of $50-700 \mathrm{~cm}^{-1}$ in the spectra of AF ordered $\kappa$-(BEDT-TTF $)_{2} \mathrm{Cu}\left[\mathrm{N}(\mathrm{CN})_{2}\right] \mathrm{Cl}$, metal with AF fluctuations $\kappa$-(BEDT-TTF $)_{2} \mathrm{Cu}\left[\mathrm{N}(\mathrm{CN})_{2}\right] \mathrm{Br}$, and spin liquid candidate $\kappa$-(BEDT-TTF $)_{2} \mathrm{Cu}_{2}(\mathrm{CN})_{3}$. These excitations can be understood within a model of an AF Mott insulator with $S=1 / 2$. For $\kappa$-(BEDT-TTF $)_{2} \mathrm{Cu}\left[\mathrm{N}(\mathrm{CN})_{2}\right] \mathrm{Cl}$ and $\kappa$-(BEDT-TTF $)_{2} \mathrm{Cu}\left[\mathrm{N}(\mathrm{CN})_{2}\right] \mathrm{Br}$ polarization dependence supports an approximation of the magnetic structure by a weakly frustrated square lattice. Position of the continuum of magnetic excitations in the spectral region of around $200-700 \mathrm{~cm}^{-1}$ is in agreement with the estimate of $J$ of about $250 \mathrm{~K}$. Excitations in $\kappa$-(BEDT-TTF $)_{2} \mathrm{Cu}_{2}(\mathrm{CN})_{3}$ spectra are shifted down in frequency, as expected for a triangular lattice, and are observed down to the lowest measured frequencies, suggesting a broader continuum than for the other two materials. In addition to a simple model of $S=1 / 2$ on a triangular lattice with $\mathrm{AF}$ nearest neighbor interactions, and a triangular lattice 
with nearest neighbor and ring exchange, a recent work [44] suggested a dynamic low-dimensionality of the magnetic interactions in $\kappa$-(BEDT-TTF $)_{2} \mathrm{Cu}_{2}(\mathrm{CN})_{3}$ as an alternative explanation for the spin liquid behaviour. One of the ways to distinguish which model describes well $\kappa$-(BEDT-TTF $)_{2} \mathrm{Cu}_{2}(\mathrm{CN})_{3}$ is to reproduce the Raman spectra of magnetic excitations for this system. Importantly, all these models developed for $\kappa$-(BEDT-TTF) ${ }_{2} \mathrm{Cu}_{2}(\mathrm{CN})_{3}$ do not include an on-site charge degree of freedom.

Another recently suggested spin liquid candidate which belongs to the family of organic Mott insulators on a triangular lattice is $\kappa$-(BEDT-TTF $)_{2} \mathrm{Ag}_{2}(\mathrm{CN})_{3}$. Magnetic Raman scattering spectra of this material are presented in Ref. [45], and show a lot of similarity to $\kappa$-(BEDT-TTF) ${ }_{2} \mathrm{Cu}_{2}(\mathrm{CN})_{3}$, with a continuum of magnetic excitations found below about $600 \mathrm{~cm}^{-1}$. These data suggest that this system also fits into the model of (BEDT-TTF $)_{2}^{+1}$ dimer sites with $S=1 / 2$ on a triangular lattice.

Unexpectedly, $\kappa$-(BEDT-TTF $)_{2} \mathrm{Hg}(\mathrm{SCN})_{2} \mathrm{Br}$ shows a completely different spectrum of excitations. The fact that no continuum of magnetic excitations was found in the spectral range $50-600 \mathrm{~cm}^{-1}$ in its Raman spectra immediately suggested that a simple description of a Mott insulator with a triangular lattice of dimer sites with $S=1 / 2$ and $J$ of about $250 \mathrm{~K}$ fails in this case. Instead, a collective mode at much lower energies below $100 \mathrm{~cm}^{-1}$ appears in the low temperature spectra, with polarization dependence suggesting an electronic origin of the mode. We have shown in Ref. [17] that this mode is a collective excitation of dipoles fluctuating on (BEDT-TTF $)_{2}^{+1}$ dimer sites. An absence of the two-magnon band is also recorded for $\kappa$-(BEDT-TTF $)_{2} \mathrm{Hg}(\mathrm{SCN})_{2} \mathrm{Cl}$, a compound basically isostructural to $\kappa$-(BEDT-TTF $)_{2} \mathrm{Hg}(\mathrm{SCN})_{2} \mathrm{Br}$ but showing a charge ordered state below $\mathrm{T}=30 \mathrm{~K}$ [17].

A similar picture was observed in the spectra of $\beta^{\prime}$ materials based on the $\mathrm{Pd}(\mathrm{dmit})_{2}$ molecule [46]. While an AF ordered $\mathrm{Me}_{4} \mathrm{P}\left[\mathrm{Pd}(\mathrm{dmit})_{2}\right]_{2}$ and a spin liquid candidate $\mathrm{EtMe}_{3} \mathrm{Sb}\left[\mathrm{Pd}(\mathrm{dmit})_{2}\right]_{2}$ show a continuum of magnetic excitations in their Raman spectra, it is absent in the spectra of a charge ordered analog $\mathrm{Et}_{2} \mathrm{Me}_{2} \mathrm{Sb}\left[\mathrm{Pd}(\mathrm{dmit})_{2}\right]_{2}$.

To summarize, we demonstrate that magnetic Raman response provides a unique direct measure of a value of the magnetic exchange $J$ for a number of BEDT-TTF-based materials, and the understanding of an origin of magnetic excitations in these compounds. It shows that $k$-(BEDT-TTF) ${ }_{2} \mathrm{Cu}\left[\mathrm{N}(\mathrm{CN})_{2}\right] \mathrm{Cl}$, $\kappa-(\mathrm{BEDT}-\mathrm{TTF})_{2} \mathrm{Cu}\left[\mathrm{N}(\mathrm{CN})_{2}\right] \mathrm{Br}, \kappa-(\mathrm{BEDT}-\mathrm{TTF})_{2} \mathrm{Cu}_{2}(\mathrm{CN})_{3}, \kappa-(\mathrm{BEDT}-\mathrm{TTF})_{2} \mathrm{Ag}_{2}(\mathrm{CN})_{3}, \mathrm{Me}_{4} \mathrm{P}\left[\mathrm{Pd}(\mathrm{dmit})_{2}\right]_{2}$, and $\mathrm{EtMe}_{3} \mathrm{Sb}\left[\mathrm{Pd}(\mathrm{dmit})_{2}\right]_{2}$ are well described by a model of $S=1 / 2$ spins on a square or triangular lattice. In contrast, $\kappa$-(BEDT-TTF $)_{2} \mathrm{Hg}(\mathrm{SCN})_{2} \mathrm{Br}, \kappa$-(BEDT-TTF $)_{2} \mathrm{Hg}(\mathrm{SCN})_{2} \mathrm{Cl}$, and $\mathrm{Et}_{2} \mathrm{Me}_{2} \mathrm{Sb}\left[\mathrm{Pd}(\mathrm{dmit})_{2}\right]_{2}$ posses an active intra-dimer charge degree of freedom, the presence of which completely changes the magnetic excitation spectra. The fluctuating charge degree of freedom in a quantum spin liquid is suggested to produce a new kind of spin liquid in a presence of charge-spin coupling $[18,20]$.

Author Contributions: N.D. conceived and designed the experiments; N.H., S.C. and N.D. performed the experiments and data analysis; J.A.S., S.A.T., E.I.Z. and R.N.L. contributed characterized samples.

Acknowledgments: The work at Institute of Quantum Matter was supported by the U.S. Department of Energy, Office of Basic Energy Sciences, Division of Material Sciences and Engineering under Grant No. DE-FG0208ER46544. The work in Chernogolovka was supported by FASO Russia, state task state registration number 0089-2014-0036. J.A.S. acknowledges support from the Independent Research and Development program from the NSF while working at the Foundation and from the National High Magnetic Field Laboratory (NHMFL) User Collaboration Grants Program (UCGP). Work at ANL was supported by University of Chicago Argonne, LLC, Operator of Argonne National Laboratory ("Argonne") Argonne, a U.S. Department of Energy Office of Science laboratory, is operated under Contract No. DE-AC02-06CH11357.

Conflicts of Interest: The authors declare no conflict of interest.

\section{References}

1. Lefebvre, S.; Wzietek, P.; Brown, S.; Bourbonnais, C.; Jérome, D.; Mézière, C.; Fourmigué, M.; Batail, P. Mott Transition, Antiferromagnetism, and Unconventional Superconductivity in Layered Organic Superconductors. Phys. Rev. Lett. 2000, 85, 5420-5423.

2. Faltermeier, D.; Barz, J.; Dumm, M.; Dressel, M.; Drichko, N.; Petrov, B.; Semkin, V.; Vlasova, R.; Meźière, C.; Batail, P. Bandwidth-controlled Mott transition in $\kappa-(\mathrm{BEDT}-\mathrm{TTF})_{2} \mathrm{Cu}\left[\mathrm{N}(\mathrm{CN})_{2}\right] \mathrm{Br}_{x} \mathrm{Cl}_{1-x}$ : Optical studies of localized charge excitations. Phys. Rev. B 2007, 76, 165113. 
3. Armitage, N.P.; Fournier, P.; Greene, R.L. Progress and perspectives on electron-doped cuprates. Rev. Mod. Phys. 2010, 82, 2421-2487. [CrossRef]

4. Powell, B.J.; McKenzie, R.H. Quantum frustration in organic Mott insulators: from spin liquids to unconventional superconductors. Rep. Prog. Phys. 2011, 74, 056501.

5. Balents, L. Spin liquids in frustrated magnets. Nature 2010, 464, 199-208. [CrossRef] [PubMed]

6. Yamashita, S.; Nakazawa, Y.; Oguni, M.; Oshima, Y.; Nojiri, H.; Shimizu, Y.; Miyagawa, K.; Kanoda, K. Thermodynamic properties of a spin- $1 / 2$ spin-liquid state in a $\kappa$-type organic salt. Nat. Phys. 2008, 4, 459-462.

7. Yamashita, S.; Yamamoto, T.; Nakazawa, Y.; Tamura, M.; Kato, R. Gapless spin liquid of an organic triangular compound evidenced by thermodynamic measurements. Nat. Commun. 2011, 2, 275. [CrossRef] [PubMed]

8. Yamashita, S.; Nakazawa, Y. Heat capacities of antiferromagnetic dimer-Mott insulators in organic charge-transfer complexes. J. Therm. Anal. Calorim. 2010, 99, 153-157. [CrossRef]

9. Pratt, F.; Baker, P.; Blundell, S.; Lancaster, T.; Ohira-Kawamura, S.; Baines, C.; Shimizu, Y.; Kanoda, K.; Watanabe, I.; Saito, G. Magnetic and non-magnetic phases of a quantum spin liquid. Nature 2011, 471, 612-616. [CrossRef] [PubMed]

10. Isono, T.; Kamo, H.; Ueda, A.; Takahashi, K.; Kimata, M.; Tajima, H.; Tsuchiya, S.; Terashima, T.; Uji, S.; Mori, H. Gapless Quantum Spin Liquid in an Organic Spin-1/2 Triangular-Lattice $\kappa-\mathrm{H}_{3}(\text { Cat-EDT-TTF })_{2}$. Phys. Rev. Lett. 2014, 112, 177201.

11. Shimizu, Y.; Hiramatsu, T.; Maesato, M.; Otsuka, A.; Yamochi, H.; Ono, A.; Itoh, M.; Yoshida, M.; Takigawa, M.; Yoshida, Y.; et al. Pressure-Tuned Exchange Coupling of a Quantum Spin Liquid in the Molecular Triangular Lattice $\kappa-(\mathrm{ET})_{2} \mathrm{Ag}_{2}(\mathrm{CN})_{3}$. Phys. Rev. Lett. 2016, 117, 107203. [CrossRef] [PubMed]

12. Zhou, Y.; Kanoda, K.; Ng, T.K. Quantum spin liquid states. Rev. Mod. Phys. 2017, 89, 025003. [CrossRef]

13. Abdel-Jawad, M.; Terasaki, I.; Sasaki, T.; Yoneyama, N.; Kobayashi, N.; Uesu, Y.; Hotta, C. Anomalous dielectric response in the dimer Mott insulator $\kappa-(\mathrm{BEDT}-\mathrm{TTF})_{2} \mathrm{Cu}_{2}(\mathrm{CN})_{3}$. Phys. Rev. B 2010, 82, 125119. [CrossRef]

14. Lunkenheimer, P.; Müller, J.; Krohns, S.; Schrettle, F.; Loidl, A.; Hartmann, B.; Rommel, R.; De Souza, M.; Hotta, C.; Schlueter, J.A.; et al. Multiferroicity in an organic charge-transfer salt that is suggestive of electric-dipole-driven magnetism. Nat. Mater. 2012, 11, 755-758. [CrossRef] [PubMed]

15. Tomić, S.; Pinterić, M.; Ivek, T.; Sedlmeier, K.; Beyer, R.; Wu, D.; Schlueter, J.; Schweitzer, D.; Dressel, M. Magnetic ordering and charge dynamics in $\kappa-(\mathrm{BEDT}-\mathrm{TTF})_{2} \mathrm{Cu}\left[\mathrm{N}(\mathrm{CN})_{2}\right] \mathrm{Cl}$. J. Phys. Condens. Matter 2013, 25, 436004. [CrossRef] [PubMed]

16. Pinterić, M.; Čulo, M.; Milat, O.; Basletić, M.; Korin-Hamzić, B.; Tafra, E.; Hamzić, A.; Ivek, T.; Peterseim, T.; Miyagawa, K.; et al. Anisotropic charge dynamics in the quantum spin-liquid candidate $\kappa-(B E D T-T T F)_{2} \mathrm{Cu}_{2}(\mathrm{CN})_{3}$. Phys. Rev. B 2014, 90, 195139. [CrossRef]

17. Hassan, N.; Cunningham, S.; Mourigal, M.; Zhilyaeva, E.I.; Torunova, S.A.; Lyubovskaya, R.N.; Drichko, N. Observation of a quantum dipole liquid state in an organic quasi-two-dimensional material. Science 2018, arXiv:1704.04482.

18. Hotta, C. Quantum electric dipoles in spin-liquid dimer Mott insulator $\kappa-E_{2} \mathrm{Cu}_{2}(\mathrm{CN})_{3}$. Phys. Rev. B 2010, 82, 241104. [CrossRef]

19. Dayal, S.; Clay, R.T.; Li, H.; Mazumdar, S. Paired electron crystal: Order from frustration in the quarter-filled band. Phys. Rev. B 2011, 83, 245106.

20. Naka, M.; Ishihara, S. Quantum melting of magnetic order in an organic dimer Mott-insulating system. Phys. Rev. B 2016, 93, 195114. [CrossRef]

21. Sedlmeier, K.; Elsässer, S.; Neubauer, D.; Beyer, R.; Wu, D.; Ivek, T.; Tomić, S.; Schlueter, J.A.; Dressel, M. Absence of charge order in the dimerized $\kappa$-phase BEDT-TTF salts. Phys. Rev. B 2012, 86, 245103. [CrossRef]

22. Seo, H.; Hotta, C.; Fukuyama, H. Toward Systematic Understanding of Diversity of Electronic Properties in Low-Dimensional Molecular Solids. Chem. Rev. 2004, 104, 5005-5036. [CrossRef] [PubMed]

23. Merino, J.; Dumm, M.; Drichko, N.; Dressel, M.; McKenzie, R.H. Quasiparticles at the Verge of Localization near the Mott Metal-Insulator Transition in a Two-Dimensional Material. Phys. Rev. Lett. 2008, 100, 086404. [CrossRef] [PubMed]

24. Shimizu, Y.; Miyagawa, K.; Kanoda, K.; Maesato, M.; Saito, G. Spin liquid state in an organic Mott insulator with a triangular lattice. Phys. Rev. Lett. 2003, 91, 107001. [CrossRef] [PubMed] 
25. Yamashita, M.; Nakata, N.; Senshu, Y.; Nagata, M.; Yamamoto, H.M.; Kato, R.; Shibauchi, T.; Matsuda, Y. Highly mobile gapless excitations in a two-dimensional candidate quantum spin liquid. Science 2010, 328, 1246-1248. [CrossRef] [PubMed]

26. Shimizu, Y.; Miyagawa, K.; Kanoda, K.; Maesato, M.; Saito, G. Emergence of inhomogeneous moments from spin liquid in the triangular-lattice Mott insulator $\kappa-(\mathrm{ET})_{2} \mathrm{Cu}_{2}(\mathrm{CN})_{3}$. Phys. Rev. B 2006, 73, 140407. [CrossRef]

27. Cottam, M.G.; Lockwood, D.J. Light Scattering in Magnetic Solids; Wiley: New York, NY, USA, 1986.

28. Lemmens, P.; Güntherodt, G.; Gros, C. Magnetic light scattering in low-dimensional quantum spin systems. Phys. Rep. 2003, 375, 1-103. [CrossRef]

29. Devereaux, T.P.; Hackl, R. Inelastic light scattering from correlated electrons. Rev. Mod. Phys. 2007, 79, 175.

30. Chen, C.C.; Jia, C.J.; Kemper, A.F.; Singh, R.R.P.; Devereaux, T.P. Theory of Two-Magnon Raman Scattering in Iron Pnictides and Chalcogenides. Phys. Rev. Lett. 2011, 106, 067002.

31. Fleury, P.; Loudon, R. Scattering of light by one-and two-magnon excitations. Phys. Rev. 1968, $166,514$.

32. Vernay, F.; Devereaux, T.; Gingras, M. Raman scattering for triangular lattices spin-1/2 Heisenberg antiferromagnets. J. Phys. Condens. Matter 2007, 19, 145243.

33. Perkins, N.; Brenig, W. Raman scattering in a Heisenberg $S=\frac{1}{2}$ antiferromagnet on the triangular lattice. Phys. Rev. B 2008, 77, 174412.

34. Holt, M.; Powell, B.J.; Merino, J. Spin-liquid phase due to competing classical orders in the semiclassical theory of the Heisenberg model with ring exchange on an anisotropic triangular lattice. Phys. Rev. B 2014, 89, 174415. [CrossRef]

35. Kozlov, M.; Pokhodnia, K.; Yurchenko, A. The assignment of fundamental vibrations of BEDT-TTF and BEDT-TTF-d8. Spectrochim. Acta Part A Mol. Spectrosc. 1987, 43, 323-329. [CrossRef]

36. Dressel, M.; Lazić, P.; Pustogow, A.; Zhukova, E.; Gorshunov, B.; Schlueter, J.A.; Milat, O.; Gumhalter, B.; Tomić, S. Lattice vibrations of the charge-transfer salt $\kappa$ - $(\mathrm{BEDT}-\mathrm{TTF})_{2} \mathrm{Cu}_{2}(\mathrm{CN})_{3}:$ Comprehensive explanation of the electrodynamic response in a spin-liquid compound. Phys. Rev. B 2016, 93, 081201. [CrossRef]

37. Drichko, N.; Beyer, R.; Rose, E.; Dressel, M.; Schlueter, J.A.; Turunova, S.A.; Zhilyaeva, E.I.; Lyubovskaya, R.N. Metallic state and charge-order metal-insulator transition in the quasi-two-dimensional conductor $\kappa-(\mathrm{BEDT}-\mathrm{TTF})_{2} \mathrm{Hg}(\mathrm{SCN})_{2} \mathrm{Cl}$. Phys. Rev. B 2014, 89, 075133. [CrossRef]

38. Drichko, N.; Hackl, R.; Schlueter, J.A. Antiferromagnetic fluctuations in a quasi-two-dimensional organic superconductor detected by Raman spectroscopy. Phys. Rev. B 2015, 92, 161112.

39. Valentine, M.E.; Koohpayeh, S.; Phelan, W.A.; McQueen, T.M.; Rosa, P.F.S.; Fisk, Z.; Drichko, N. Breakdown of the Kondo insulating state in $\mathrm{SmB}_{6}$ by introducing Sm vacancies. Phys. Rev. B 2016, 94, 075102. [CrossRef]

40. Kawamoto, A.; Miyagawa, K.; Nakazawa, Y.; Kanoda, K. ${ }^{13}$ C NMR Study of Layered Organic Superconductors Based on BEDT-TTF Molecules. Phys. Rev. Lett. 1995, 74, 3455-3458.

41. Nakamura, Y.; Yoneyama, N.; Sasaki, T.; Tohyama, T.; Nakamura, A.; Kishida, H. Magnetic Raman Scattering Study of Spin Frustrated Systems, $\kappa$-(BEDT-TTF)2X. J. Phys. Soc. Jpn. 2014, 83, 074708.

42. Gati, E.; Fischer, J.K.; Lunkenheimer, P.; Zielke, D.; Köhler, S.; Kolb, F.; von Nidda, H.A.K.; Winter, S.M.; Schubert, H.; Schlueter, J.A.; et al. Evidence for electronically-driven ferroelectricity in the family of strongly correlated dimerized BEDT-TTF molecular conductors. arXiv 2017, arXiv:1711.07384.

43. Ivek, T.; Beyer, R.; Badalov, S.; Čulo, M.; Tomić, S.; Schlueter, J.A.; Zhilyaeva, E.I.; Lyubovskaya, R.N.; Dressel, M. Metal-insulator transition in the dimerized organic conductor $\kappa$-(BEDT-TTF $)_{2} \mathrm{Hg}(\mathrm{SCN})_{2} \mathrm{Br}$. Phys. Rev. B 2017, 96, 085116. [CrossRef]

44. Powell, B.; Kenny, E.; Merino, J. Dynamical Reduction of the Dimensionality of Exchange Interactions and the "Spin-Liquid" Phase of $\kappa$-(BEDT-TTF) $)_{2}$ X. Phys. Rev. Lett. 2017, 119, 087204. [CrossRef] [PubMed]

45. Nakamura, Y.; Hiramatsu, T.; Yoshida, Y.; Saito, G.; Kishida, H. Optical Properties of a Quantum Spin Liquid Candidate Material, $\kappa$-(BEDT-TTF $)_{2} \mathrm{Ag}_{2}(\mathrm{CN})_{3}$. J. Phys. Soc. Jpn. 2017, 86, 014710. [CrossRef]

46. Nakamura, Y.; Kato, R.; Kishida, H. Study of Magnetic Excitation in Pd(dmit $)_{2}$ Salts by Raman Scattering Spectroscopy. J. Phys. Soc. Jpn. 2015, 84, 044715. [CrossRef]

(C) 2018 by the authors. Licensee MDPI, Basel, Switzerland. This article is an open access article distributed under the terms and conditions of the Creative Commons Attribution (CC BY) license (http:/ / creativecommons.org/licenses/by/4.0/). 
Article

\title{
Synthesis and Characterization of Charge Transfer Salts Based on $\left[\mathrm{M}(\mathrm{dcdmp})_{2}\right](\mathrm{M}=\mathrm{Au}, \mathrm{Cu}$ and $\mathrm{Ni})$ with TTF Type Donors
}

\author{
Rafaela A. L. Silva, Isabel C. Santos, Sandra Rabaça, Elsa B. Lopes, Vasco Gama, Manuel Almeida \\ and Dulce Belo* \\ $\mathrm{C}^{2} \mathrm{TN}$, Centro de Ciências e Tecnologias Nucleares, Instituto Superior Técnico, Universidade de Lisboa, \\ E.N. 10 ao km 139.7, 2695-066 Bobadela LRS, Portugal; rafaela@ctn.tecnico.ulisboa.pt (R.A.L.S.); \\ icsantos@ctn.tecnico.ulisboa.pt (I.C.S.); sandrar@ctn.tecnico.ulisboa.pt (S.R.); \\ eblopes@ctn.tecnico.ulisboa.pt (E.B.L.); vascog@ctn.tecnico.ulisboa.pt (V.G.); \\ malmeida@ctn.tecnico.ulisboa.pt (M.A.) \\ * Correspondence: dbelo@ctn.tecnico.ulisboa.pt; Tel.: +351-21-955-6203
}

Received: 6 March 2018; Accepted: 17 March 2018; Published: 20 March 2018

\begin{abstract}
The charge transfer salts $\alpha$-DT-TTF[Au(dcdmp $\left.)_{2}\right](\mathbf{1})$, BET-TTF[Au(dcdmp $\left.)_{2}\right](2 \mathbf{M}$ and 2T), $\alpha$-DT-TTF[Cu(dcdmp $\left.)_{2}\right](3)$, ET[Cu(dcdmp $\left.)_{2}\right](4),(\text { BET-TTF })_{2}\left[\mathrm{Cu}(\mathrm{dcdmp})_{2}\right](5),(\mathrm{ET})_{2}\left[\mathrm{Ni}(\mathrm{dcdmp})_{2}\right]$ (6), and $\alpha$-mtdt $\left[\mathrm{Cu}(\mathrm{dcdmp})_{2}\right]$ (7) were obtained by electrocrystallization of different electron donor molecules derived from TTF $(\alpha$-DT-TTF $=$ alpha-dithiophene-tetrathiafulvalene; BET-TTF $=$ (bis(ethylenethio)tetrathiafulvalene; $\mathrm{ET}=$ bis(ethylenedithio)-tetrathiafulvalene; $\alpha$-mtdt $=$ alpha-methylthiophenetetrathiafulvalene) in the presence of transition metal complex $\left[\mathrm{M}(\mathrm{dcdmp})_{2}\right](\mathrm{M}=\mathrm{Au}(\mathrm{III}), \mathrm{Cu}(\mathrm{III})$ and $\mathrm{Ni}(\mathrm{II}))(\mathrm{dcdmp}=$ 2,3-dicyano-5,6-dimercaptopyrazine). Compounds 1 and 2 (2M and $2 \mathrm{~T}$ ) have a similar packing pattern composed of mixed stacks of alternating donor-acceptor molecules. For (BET-TTF) $\left[\mathrm{Au}(\mathrm{dcdmp})_{2}\right.$ ] two different crystal structures (2M and $2 \mathrm{~T}$ ) were obtained indicating polymorphism. Compounds 3 and 4 are isostructural being composed of zigzag chains of alternating donor and acceptor molecules. The salts with a 2:1 stoichiometry, $(\mathrm{BET}-\mathrm{TTF})_{2}\left[\mathrm{Cu}(\mathrm{dcdmp})_{2}\right](5)$, and $(\mathrm{ET})_{2}\left[\mathrm{Ni}(\mathrm{dcdmp})_{2}\right](6)$ present the donor molecules fully oxidized and $\left[\mathrm{M}(\mathrm{dcdmp})_{2}\right](\mathrm{M}=\mathrm{Ni}$ and $\mathrm{Cu})$ in a dianionic state. The salt of the dissymmetric donor $\alpha$-mtdt with $\left[\mathrm{Cu}(\mathrm{dcdmp})_{2}\right], \alpha$-mtdt $\left[\mathrm{Cu}(\mathrm{dcdmp})_{2}\right](7)$ has a crystal structure composed of segregated donor stacks that are positioned in a head-to-head fashion and alternate with the anion stacks. All charge transfer salts (1-7) are modest semiconductors with conductivities in the range $10^{-1}-10^{-5} \mathrm{~S} / \mathrm{cm}$, with the highest values obtained in $\alpha$-DT-TTF salts, compounds $\mathbf{1}$ and 3 .
\end{abstract}

Keywords: charge transfer salts; TTF donors; dimercaptopyrazine bisdithiolate complexes; crystal engineering

\section{Introduction}

After about 50 years of intensive studies, transition metal bisdithiolene complexes still continue to be actively explored as building blocks for molecular conducting and magnetic materials due to their interesting and unique structural and electronic properties [1]. Some attractive features of these types of complexes are the diversity of coordination geometries that metal centres can adopt, as well as, depending on the transition metal or the oxidation state, the accessibility to several oxidation states and different magnetic moments [2]. The square planar coordination geometry and the delocalised $\pi$-nature of the ligands favour, in the solid state, the formation of extended networks of $\pi$ - $\pi$ interactions, which can give rise to interesting properties such as ferromagnetism [3], spin-ladder behaviour [4,5], and metallic [6,7], or even superconducting, properties $[8,9]$. 
Extended dithiolene $\pi$-ligands containing $\mathrm{N}$ atoms are significantly less explored when compared to sulphur rich ligands, which have been favoured to build intermolecular S ...S contacts with improved dimensionality in the solid state [10]. However, the $\mathrm{N}$ atoms in dithiolene ligands are now known to act as an extra coordinating site that can provide an additional degree of freedom in the crystal engineering of these solids [11].

$\left[\mathrm{M}(\mathrm{dcdmp})_{2}\right]$ complexes based on extended dithiolene $\pi$-ligand-containing $\mathrm{N}$ atoms (Scheme 1a) are, in this context, attractive anions. The dcdmp ligand as an extended $\pi$-system is expected to be able to increase the electronic delocalization, and the pyrazine nitrogen atoms can lead to an increase of intermolecular interactions [11-14] when compared with complexes based on other ligands such as mnt $(\mathrm{mnt}=$ maleonitriledithiolate) (Scheme $1 \mathrm{a})$. The $\mathrm{Au}, \mathrm{Ni}$, and $\mathrm{Cu}$ complexes were previously combined with TTF-type donors, resulting in salts of different stoichiometries, including (DT-TTF $)_{2}\left[\mathrm{Cu}(\mathrm{dcdmp})_{2}\right]$ (DT-TTF $=$ dithiophene-tetrathiafulvalene) with a ladder-like structure $[15,16]$. In order to further explore $\left[\mathrm{M}(\mathrm{dcdmp})_{2}\right]$ anions as possible building blocks for molecular materials, these complexes with $\mathrm{M}=\mathrm{Au}, \mathrm{Cu}$, and $\mathrm{Ni}$ were combined with different donors related to DT-TTF; the aromatic $\alpha$-DT-TTF, the non-aromatic BET-TTF, the disymmetric thiophenic derivative $\alpha$-mtdt, and the well-known ET donor (Scheme 1b). The electrical transport properties of salts will critically depend on the relative oxidation state of the molecular building blocks and their capability to establish extended networks of regular interactions. Salts in 1:1 stoichiometry, with full charge transfer between donor and acceptor, lead to half-filled bands, which, in molecular systems with narrow bands, will invariably behave as Mott insulators. Therefore, partial oxidation of the molecular building blocks in a regular network of strong interactions is usually a condition for high electrical conductivity. However, in spite of recent progresses in crystal engineering, both the stoichiometry and crystal structure of molecular salts remain largely unpredictable $[1,4]$.
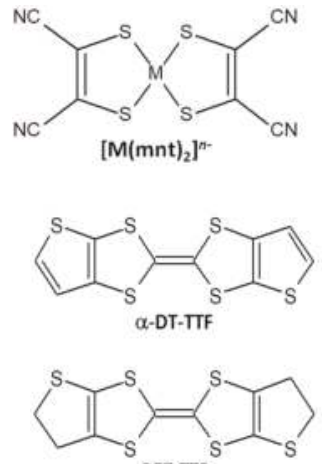

BET-TTF

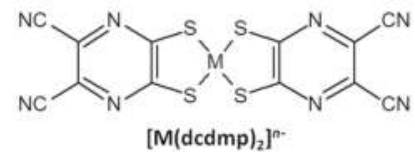

(a)
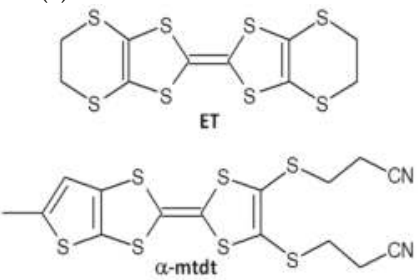

(b)

Scheme 1. Molecular diagram of transition metal bisdithiolene complexes (a) and of TTF type donors (b).

\section{Materials and Methods}

\subsection{General Information}

The donors BET-TTF [17], $\alpha$-DT-TTF [18], and $\alpha$-mtdt [19] were prepared as previously described, while ET was commercially obtained (Sigma-Aldrich, Darmstadt, Germany) and used without further purification. $\left(n-\mathrm{Bu}_{4} \mathrm{~N}\right)\left[\mathrm{M}(\mathrm{dcdmp})_{2}\right](\mathrm{M}=\mathrm{Au}$ and $\mathrm{Cu})[20,21]$ and $\left(n-\mathrm{Bu}_{4} \mathrm{~N}\right)_{2}\left[\mathrm{Ni}(\mathrm{dcdmp})_{2}\right][22]$ were prepared and purified by recrystallization following previously described procedures. Electrocrystallization was carried out in H-shaped two-compartment cells separated by frit glass with Pt electrodes and under galvanostatic conditions. All solvents were purified using standard procedures and freshly distilled immediately before its use [23]. 


\subsection{Synthesis}

$\alpha-D T-T T F\left[A u(d c d m p)_{2}\right](\mathbf{1})$. Crystals were obtained by electrocrystallization from a dichloromethane solution of BET-TTF and $\left(n-\mathrm{Bu}_{4} \mathrm{~N}\right)\left[\mathrm{Au}(\mathrm{dcdmp})_{2}\right]^{-}$, both $1 \times 10^{-3} \mathrm{M}$. The system was sealed under nitrogen and after 10 days by applying a current density of $1.0 \mu \mathrm{A} \cdot \mathrm{cm}^{-2}$; brown plate crystals were collected and washed with dichloromethane.

BET-TTF $\left[A u(d c d m p)_{2}\right](2 \mathbf{M}$ and $2 \mathrm{~T})$. Crystals were obtained by electrocrystallization from a dichloromethane solution of BET-TTF and $\left(n-\mathrm{Bu}_{4} \mathrm{~N}\right)\left[\mathrm{Au}(\mathrm{dcdmp})_{2}\right]^{-}$, following the same procedure as described for compound 1 . Brown plate crystals were collected after 9 days by applying a current density of $1.0 \mu \mathrm{A} \cdot \mathrm{cm}^{-2}$.

$\alpha$-DT-TTF $\left[C u(d c d m p)_{2}\right](3)$. Crystals were obtained by electrocrystallization from a dichloromethane solution of BET-TTF and $\left(n-\mathrm{Bu}_{4} \mathrm{~N}\right)\left[\mathrm{Cu}(\mathrm{dcdmp})_{2}\right]^{-}$, following the same procedure as described for compound 1. Brown plate crystals were collected after 10 days by applying a current density of $1.0 \mu \mathrm{A} \cdot \mathrm{cm}^{-2}$.

$E T\left[C u(d c d m p)_{2}\right]$ (4). Crystals were obtained by electrocrystallization from an acetonitrile solution of the ET donor $\left(2 \times 10^{-2} \mathrm{mmol}\right)$ and copper acceptor salt $\left(5 \times 10^{-2} \mathrm{mmol}\right)$. The system was sealed under nitrogen and after 18 days, with a current density of $1.0 \mu \mathrm{A} \cdot \mathrm{cm}^{-2}$; black prism shape crystals were collected and washed with acetonitrile.

$(B E T-T T F)_{2}\left[C u(d c d m p)_{2}\right](5)$. Crystals were obtained by electrocrystallization of BET-TTF donor and tetrabutylammonium salt of $\left[\mathrm{Cu}(\mathrm{dcdmp})_{2}\right]^{-}$as electrolyte, following the same procedure as described for compound 1 . Brown plate crystals were collected after 12 days by applying a current density of $1.0 \mu \mathrm{A} \cdot \mathrm{cm}^{-2}$.

$(E T)_{2}\left[\mathrm{Ni}(\mathrm{dcdmp})_{2}\right](6)$. Crystals were obtained by electrocrystallization from a dichloromethane solution of the donor and nickel acceptor salt in approximately stoichiometric amounts. The system was sealed under nitrogen and after 18 days, with a current density of $0.5 \mu \mathrm{A} \cdot \mathrm{cm}^{-2}$; brown plate shape crystals were collected and washed with dichloromethane.

$\alpha-m t d t\left[C u(d c d m p)_{2}\right]$ (7). Crystals were obtained by electrocrystallization of BET-TTF donor and tetrabutylammonium salt of $\left[\mathrm{Cu}(\mathrm{dcdmp})_{2}\right]^{-}$as electrolyte, following the same procedure as described for compound 1 . Small black needle crystals were collected after 18 days by applying a current density of $1.0 \mu \mathrm{A} \cdot \mathrm{cm}^{-2}$.

\subsection{X-ray Crystallography}

X-ray diffraction studies were performed with a Bruker APEX-II CCD detector diffractometer using graphite monochromated Mo-K $\alpha$ radiation $(\lambda=0.71073 \AA)$, in the $\varphi$ and $\omega$ scans mode. A semi empirical absorption correction was carried out using SADABS [24]. Data collection, cell refinement, and data reduction were done with the SMART and SAINT programs [25]. X-ray data for the $(\mathrm{ET})_{2}\left[\mathrm{Ni}(\mathrm{dcdmp})_{2}\right]$ compound were collected at room temperature on an Enraf-Nonius CAD-4 (Enraf-nonius, 1989) automatic diffractometer using graphite monochromated Mo-K $\alpha$ $(\lambda=0.71069 \AA, 50 \mathrm{kV}, 26 \mathrm{~mA})$ radiation. Unit-cell dimensions and the orientation matrix were obtained from least-squares refinement of the setting angles of 25 reflections in the range $14^{\circ}<2 \theta<24^{\circ}$. The data set was collected in the $\omega-2 \theta$ scan mode. The intensities were corrected for Lorentz, polarisation, and absorption effects by empirical corrections based on psi-scans using the Enraf-Nonius reduction program, MoIEN [26]. The structures were solved by direct methods using SIR97 [27] and refined by fullmatrix least-squares methods using the program SHELXL97 [28] using the winGX software package [29]. Non-hydrogen atoms were refined with anisotropic thermal parameters, whereas $\mathrm{H}$-atoms were placed in idealised positions and allowed to refine riding on the parent $\mathrm{C}$ atom. Molecular graphics were prepared using Mercury [30]. The positional disorder of $\mathrm{S}$ and $\mathrm{C}$ atoms in the thiophenic rings (compounds 1, 2T, 3, 4, and 5) was refined by the SHELXL instruction PART (PART 1 and PART 2), and the occupancies of disordered atoms were allowed to refine freely and possess any ratio. 
Crystallographic data for $\alpha$-DT-TTF[Au(dcdmp) ${ }_{2}$ ] (1): $\mathrm{C}_{22} \mathrm{H}_{4} \mathrm{AuN}_{8} \mathrm{~S}_{10}, \mathrm{M}=897.90 \mathrm{~g} \cdot \mathrm{mol}^{-1}$, monoclinic, space group $P 2{ }_{1}, a=8.1542(4) \AA, b=5.7520(3) \AA, c=28.1036(13) \AA, \beta=96.347(2)^{\circ}$, $\mathrm{V}=1310.06(11) \AA^{3}, \mathrm{Z}=2, \varrho_{\text {calc }}=2.276 \mathrm{~g} \cdot \mathrm{cm}^{-3}, \mu(\mathrm{Mo} K \alpha)=6.447 \mathrm{~mm}^{-1}, 9917$ reflections measured, 4581 unique $\left[R_{\text {int }}=0.0469\right], \theta_{\max }=25.67^{\circ}$, Flack Parameter $=0.06, R 1=0.0465$ using 4338 Refl. $>2 \sigma(\mathrm{I})$, $\omega \mathrm{R} 2=0.1101, \mathrm{~T}=150(2) \mathrm{K} . \mathrm{CCDC} 1826111$.

Crystallographic data for BET-TTF[Au(dcdmp $\left.)_{2}\right]$ : (2M), $\mathrm{C}_{22} \mathrm{H}_{8} \mathrm{AuN}_{8} \mathrm{~S}_{10}, \mathrm{M}=901.93 \mathrm{~g} \cdot \mathrm{mol}^{-1}$, monoclinic, space group $P 2{ }_{1} / c, a=8.2518(4) \AA, b=5.7193(3) \AA, c=56.611(3) \AA, \beta=92.724(3)^{\circ}$, $\mathrm{V}=2668.7(2) \AA^{3}, \mathrm{Z}=4, \varrho_{\text {calc }}=2.245 \mathrm{~g} \cdot \mathrm{cm}^{-3}, \mu(\mathrm{Mo} K \alpha)=6.330 \mathrm{~mm}^{-1}, 22517$ reflections measured, 4253 unique $\left[R_{\text {int }}=0.0508\right], \theta_{\max }=25.02^{\circ}, R 1=0.0430$ using 3868 Refl. $>2 \sigma(\mathrm{I}), \omega \mathrm{R} 2=0.0809, \mathrm{~T}=150(2) \mathrm{K}$. CCDC 1826306; (2T), $\mathrm{C}_{22} \mathrm{H}_{8} \mathrm{AuN}_{8} \mathrm{~S}_{10}, \mathrm{M}=901.93 \mathrm{~g} \cdot \mathrm{mol}^{-1}$, triclinic, space group $P-1, a=5.7058$ (3) $\AA$, $b=9.6338(5) \AA, c=12.8817(6) \AA, \alpha=106.388(2)^{\circ}, \beta=96.438(2)^{\circ}, \gamma=95.743(2)^{\circ}, \mathrm{V}=668.56(6) \AA^{3}, \mathrm{Z}=1$, $\varrho_{\text {calc }}=2.240 \mathrm{~g} \cdot \mathrm{cm}^{-3}, \mu(\mathrm{Mo} \mathrm{K \alpha})=6.317 \mathrm{~mm}^{-1}, 5613$ reflections measured, 2288 unique $\left[R_{\text {int }}=0.0359\right]$, $\theta_{\max }=25.03^{\circ}, \mathrm{R} 1=0.0260$ using 2232 Refl. $>2 \sigma(\mathrm{I}), \omega \mathrm{R} 2=0.0574, \mathrm{~T}=150(2) \mathrm{K} . \mathrm{CCDC} 1826112$.

Crystallographic data for $\alpha$-DT-TTF[Cu(dcdmp) ${ }_{2}$ (3): $\mathrm{C}_{22} \mathrm{H}_{4} \mathrm{CuN}_{8} \mathrm{~S}_{10}, \mathrm{M}=764.47 \mathrm{~g} \cdot \mathrm{mol}^{-1}$, monoclinic, space group $P 22_{1} / c, a=15.224(2) \AA, b=7.3004(11) \AA, c=23.933(2) \AA, \beta=97.901(5)^{\circ}$, $\mathrm{V}=2634.7(6) \AA^{3}, \mathrm{Z}=4, \varrho_{\text {calc }}=1.927 \mathrm{~g} \cdot \mathrm{cm}^{-3}, \mu(\mathrm{Mo} \mathrm{K \alpha})=1.657 \mathrm{~mm}^{-1}, 10849$ reflections measured, 4870 unique $\left[R_{\text {int }}=0.0976\right], \theta_{\max }=25.68^{\circ}, R 1=0.0729$ using 2303 Refl. $>2 \sigma(\mathrm{I}), \omega \mathrm{R} 2=0.1426, \mathrm{~T}=150(2) \mathrm{K}$. CCDC 1826109.

Crystallographic data for ET[Cu(dcdmp) $\left.{ }_{2}\right]$ (4): $\mathrm{C}_{22} \mathrm{H}_{8} \mathrm{CuN}_{8} \mathrm{~S}_{12}, \mathrm{M}=832.62 \mathrm{~g} \cdot \mathrm{mol}^{-1}$, monoclinic, space group $P 2_{1} / c, a=16.1566(4) \AA, b=7.4009(2) \AA, c=24.6178(5) \AA, \beta=100.2440(10)^{\circ}$, $\mathrm{V}=2896.71(12) \AA^{3}, \mathrm{Z}=4, \varrho_{\text {calc }}=1.909 \mathrm{~g} \cdot \mathrm{cm}^{-3}, \mu(\mathrm{Mo} \mathrm{K \alpha})=1.654 \mathrm{~mm}^{-1}, 22645$ reflections measured, 5475 unique $\left[R_{\text {int }}=0.0505\right], \theta_{\max }=25.68^{\circ}, \mathrm{R} 1=0.0291$ using 4591 Refl. $>2 \sigma(\mathrm{I}), \omega \mathrm{R} 2=0.0732$, $\mathrm{T}=150(2) \mathrm{K}$. CCDC 1826113.

Crystallographic data for (BET-TTF $)_{2}\left[\mathrm{Cu}(\mathrm{dcdmp})_{2}\right]$ (5): $\mathrm{C}_{32} \mathrm{H}_{16} \mathrm{CuN}_{8} \mathrm{~S}_{16}, \mathrm{M}=1089.03 \mathrm{~g} \cdot \mathrm{mol}^{-1}$, triclinic, space group $P-1, a=8.2678(5) \AA, b=8.5858(6) \AA, c=14.3566(9) \AA, \alpha=93.248(3)^{\circ}, \beta=92.743(3)^{\circ}$, $\gamma=108.548(4)^{\circ}, V=962.29(11) \AA^{3}, Z=1, \varrho_{\text {calc }}=1.879 \mathrm{~g} \cdot \mathrm{cm}^{-3}, \mu(\mathrm{Mo} \mathrm{K \alpha})=1.478 \mathrm{~mm}^{-1}, 6512$ reflections measured, 3432 unique $\left[R_{\text {int }}=0.0371\right], \theta_{\max }=25.35^{\circ}, R 1=0.0444$ using 2595 Refl. $>2 \sigma(\mathrm{I}), \omega \mathrm{R} 2=0.0956$, $\mathrm{T}=150(2) \mathrm{K} . \mathrm{CCDC} 1826110$

Crystallographic data for $(\mathrm{ET})_{2}\left[\mathrm{Ni}(\mathrm{dcdmp})_{2}\right]$ (6): $\mathrm{C}_{32} \mathrm{H}_{16} \mathrm{NiN}_{8} \mathrm{~S}_{20}, \mathrm{M}=1212.42 \mathrm{~g} \cdot \mathrm{mol}^{-1}$, triclinic, space group $P-1, a=7.732(5) \AA, b=9.554(5) \AA, c=15.254(5) \AA, \alpha=87.990(5)^{\circ}, \beta=89.440(5)^{\circ}$, $\gamma=72.200(5)^{\circ}, \mathrm{V}=1072.2(10) \AA^{3}, \mathrm{Z}=1, \varrho_{\text {calc }}=1.878 \mathrm{~g} \cdot \mathrm{cm}^{-3}, \mu(\mathrm{Mo} \mathrm{K \alpha})=1.468 \mathrm{~mm}^{-1}, 4818$ reflections measured, 4643 unique [ $\left.R_{\text {int }}=0.0277\right], \theta_{\max }=27.04^{\circ}, R 1=0.0589$ using 2950 Refl. $>2 \sigma(\mathrm{I}), \omega \mathrm{R} 2=0.0841$, $\mathrm{T}=$ 293(2) K. CCDC 1826114 .

Crystallographic data for $\alpha$-mtdt $\left[\mathrm{Cu}(\mathrm{dcdmp})_{2}\right]$ (7): $\mathrm{C}_{27} \mathrm{H}_{12} \mathrm{CuN}_{10} \mathrm{~S}_{11}, \mathrm{M}=892.67 \mathrm{~g} \cdot \mathrm{mol}^{-1}$, triclinic, space group $P-1, a=5.8868(6) \AA, b=15.7339(17) \AA, c=18.3950(19) \AA, \alpha=83.38^{\circ}, \beta=89.590(4)^{\circ}$, $\gamma=86.603(5)^{\circ}, \mathrm{V}=1689.4(3) \AA^{3}, \mathrm{Z}=2, \varrho_{\text {calc }}=1.755 \mathrm{~g} \cdot \mathrm{cm}^{-3}, \mu($ Mo $K \alpha)=1.367 \mathrm{~mm}^{-1}, 12286$ reflections measured, 5180 unique $\left[R_{\text {int }}=0.0834\right], \theta_{\max }=25.68^{\circ}, R 1=0.0671$ using 2629 Refl. $>2 \sigma(\mathrm{I}), \omega \mathrm{R} 2=0.1370$, $\mathrm{T}=150(2) \mathrm{K} . \mathrm{CCDC} 1826115$.

\subsection{Electric Transport Properties}

Electrical conductivity measurements were made in single crystals along their long axis using a closed cycle helium refrigerator in the temperature range of 50-320 K and a four-in-line contact configuration by attaching four $\varnothing=25 \mu \mathrm{m}$ Au wires to the single crystals with Pt paint (Demetron 308A). The measurement cell [31] is controlled by a computer [32]. In the case of more conducting samples a low-frequency four-probe AC method $(77 \mathrm{~Hz})$ was used [33], with a SRS Model SR83 Lock-in Amplifier while applying a $5 \mu \mathrm{A}$ current; for the more resistive samples, a four-probe DC method was used instead, using a Keithley 224 current source to apply both direct and reverse DC currents, well below 
$0.1 \mu \mathrm{A}$, through the sample and a Keithley 619 electrometer to measure the corresponding DC voltage. Sample electrodes configuration was checked for unnested to nested voltage ratio, as defined by Schaffer et al. [34].

\section{Results}

Electrocrystallization of the donor molecules ET, BET-TTF, $\alpha$-DT-TTF, and $\alpha$-mtdt (Scheme 1b) with tetrabutylammonium salts of $\left[\mathrm{M}(\mathrm{dcdmp})_{2}\right]^{-}(\mathrm{M}=\mathrm{Au}(\mathrm{III})$ and $\mathrm{Cu}(\mathrm{III}))$ or $\left[\mathrm{Ni}(\mathrm{dcdmp})_{2}\right]^{2-}$ resulted in crystals of eight new charge transfer salts, $\alpha$-DT-TTF[Au(dcdmp $\left.)_{2}\right](\mathbf{1})$, BET-TTF[Au(dcdmp $\left.)_{2}\right]$ in two polymorphs (2M and 2T), $\alpha$-DT-TTF $\left[\mathrm{Cu}(\mathrm{dcdmp})_{2}\right] \quad(3), \quad \mathrm{ET}\left[\mathrm{Cu}(\mathrm{dcdmp})_{2}\right](4)$, $(\mathrm{BET}-\mathrm{TTF})_{2}\left[\mathrm{Cu}(\mathrm{dcdmp})_{2}\right](5),(\mathrm{ET})_{2}\left[\mathrm{Ni}(\mathrm{dcdmp})_{2}\right](6)$, and $\alpha$-mtdt[Cu(dcdmp $\left.)_{2}\right](7)$. These compounds were obtained as single crystals with size and quality suitable for X-ray diffraction and electrical transport properties measurements.

\subsection{Single Crystal X-ray Diffraction Analysis}

When combining the thiophenic-TTF donors, $\alpha$-DT-TTF and BET-TTF, with $\left[\mathrm{Au}(\mathrm{dcdmp})_{2}\right]^{-}$ monoanion, similar crystal structure patterns were obtained, based on mixed donor-acceptor stacks. This pattern had already been observed in the related DT-TTF salts with $\left[\mathrm{M}(\mathrm{dcdmp})_{2}\right](\mathrm{M}=\mathrm{Au}$, $\mathrm{Cu}$ and $\mathrm{Ni})[15,16]$.

$\alpha$-DT-TTF[Au(dcdmp $)_{2}$ ] (1) crystallizes in the monoclinic system, space group $P 2_{1}$. The asymmetric unit cell contains one $\left[\mathrm{Au}(\mathrm{dcdmp})_{2}\right]^{-}$anion and one $\alpha$-DT-TTF donor molecule, both at general positions (Figure 1a, Table S1). The donor molecule presents a slight boat type distortion, while the $\left[\mathrm{Au}(\mathrm{dcdmp})_{2}\right]^{-}$anion is essentially planar, within the range of experimental error (Figure 1a). The donor molecule presents a disorder in the sulphur atom S10 in one of the thiophenic rings with an occupation factor of 59-41\% (S10/C21-S10A/C21A).
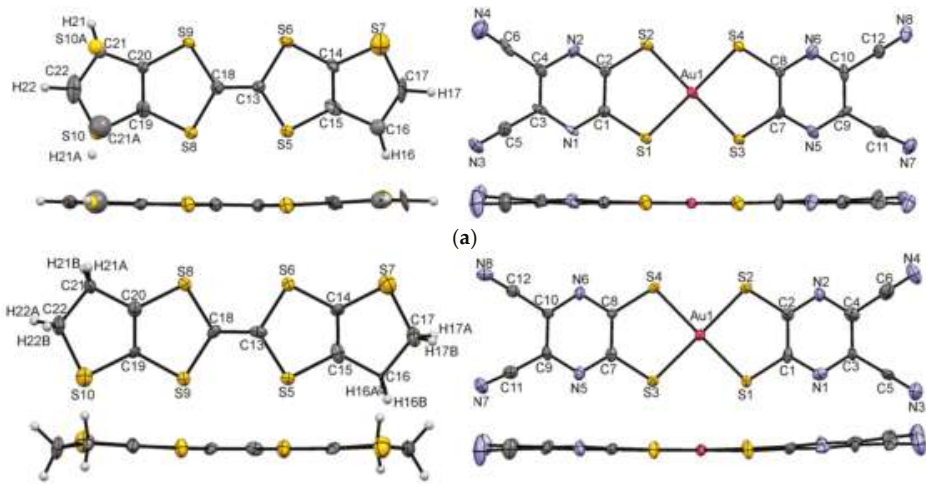

(a)
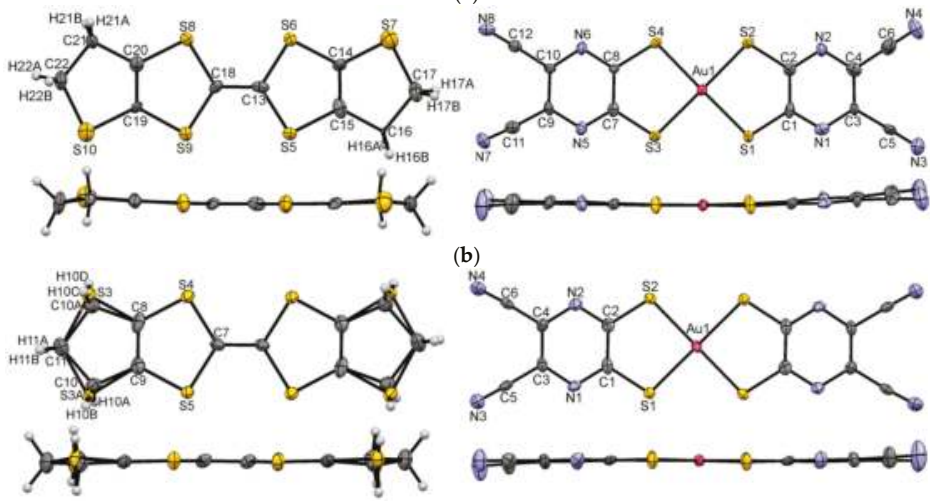

(b)

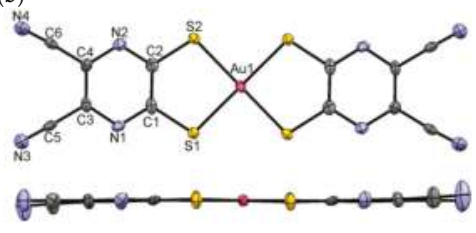

(c)

Figure 1. ORTEP and atomic numbering schemes (top and side views) of (a) $\alpha$-DT-TTF donor molecule and acceptor [Au(dcdmp $)_{2}$ ] molecule in the crystal structure of $\mathbf{1}$; and $(\mathbf{b}, \mathbf{c})$ BET-TTF donor molecule and acceptor $\left[\mathrm{Au}(\mathrm{dcdmp})_{2}\right]$ molecule in the crystal structure of 2 : (b) structure $\mathbf{M}(\mathbf{2} \mathbf{M})$ and (c) structure T (2T)). Thermal ellipsoids drawn at 70\% probability level. 
The crystal structure of $\mathbf{1}$ is composed of mixed stacks of alternating donor-acceptor molecules $\left(\mathrm{D}^{+} \mathrm{A}^{-} \mathrm{D}^{+} \mathrm{A}^{-} \mathrm{D}^{+} \mathrm{A}^{-}\right)$along the $a$ axis (Figure $\left.2 \mathrm{a}\right)$. Along the stacks there are no short contacts below the sum of van der Waals radii, although the average molecular plane distances between the donor and acceptor molecules of $3.47 \AA$ suggest significant $\pi-\pi$ interactions. By contrast, the molecules in neighbouring stacks are connected through a 2D network of short contacts. Along $b$, the molecules short axis, the stacks are in registry, and several S...S interactions between $\mathrm{D}^{+} / \mathrm{D}^{+}, \mathrm{A}^{-} / \mathrm{A}^{-}$and $\mathrm{D}^{+} / \mathrm{A}^{-}$ are observed (Figure $2 \mathrm{a}_{2}$, Table S2). Along $c$, the molecules longest axis, the stacks are out-of-registry and $\mathrm{D}^{+} / \mathrm{A}^{-}$interactions observed are mediated through the nitrile group of the acceptor and the thiophenic sulphur or the hydrogen atoms of the donor molecule (Table S2). Along $c$, the stacks are related by a 2 -fold screw axis, and molecules in nearby stacks have a dihedral angle of $\approx 60^{\circ}$. This kind of pattern is very similar to those found in the salts family of DT-TTF $m\left[M(d c d m p)_{2}\right]_{n}(M=N i, A u$ and Cu) $[15,16]$.

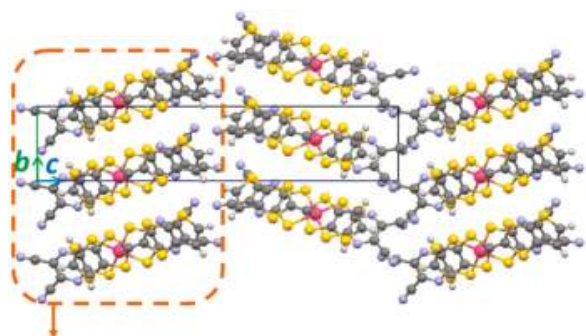

(a1)

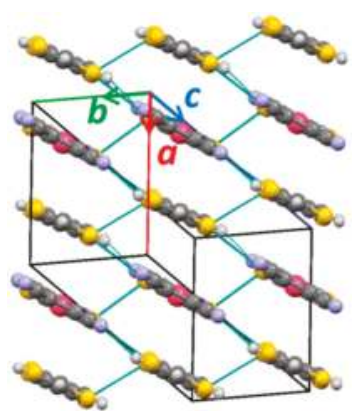

(a2)

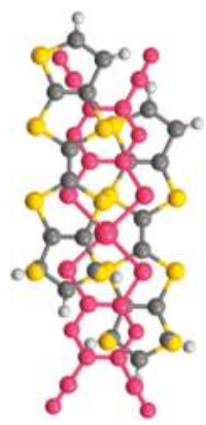

(a3)

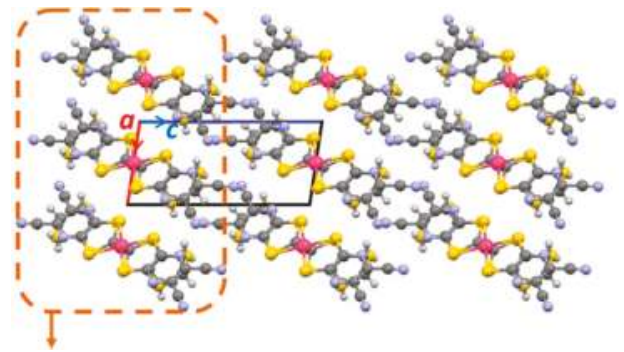

(b1)

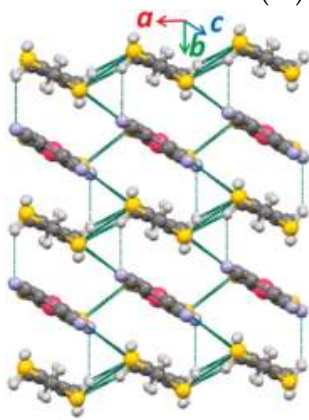

(b2)

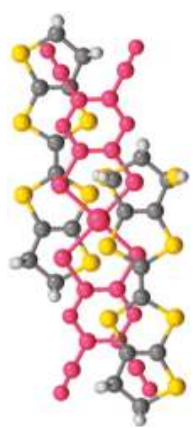

(b3)

Figure 2. Crystal structure of compound $\mathbf{1}(\mathbf{a})$ and compound $\mathbf{2 T}(\mathbf{b})$ : $\left(\mathbf{a}_{\mathbf{1}}, \mathbf{b}_{\mathbf{1}}\right)$ view along the stacking axis; $\left(\mathbf{a}_{2}, \mathbf{b}_{2}\right)$ partial view along the long axis of molecules of neighbouring stacks in the same layer; $\left(\mathbf{a}_{3}, \mathbf{b}_{3}\right)$ overlap mode of the mixed stacks. Thin lines represent relevant short contacts.

In the case of BET-TTF[Au(dcdmp $\left.)_{2}\right](2)$, two different crystal structures with a 1:1 stoichiometry were obtained from the same preparation by electrocrystallization, with one crystallizing in the monoclinic system space group $P 2_{1} / C(\mathbf{2} \mathbf{M})$ and the other in the triclinic system space group $P-1$ (2T). In both crystal structures, the $\left[\mathrm{Au}(\mathrm{dcdmp})_{2}\right]$ acceptor presents bond lengths typical of a monoanion $[20,35]$ and therefore BET-TTF donor molecules must be in a fully oxidized monocationic state.

Although not isostructural, the $\mathbf{2 M}$ salt resembles the crystal structure of $\mathbf{1}$. Its asymmetric unit cell contains one independent $(\mathrm{BET}-\mathrm{TTF})^{+}$molecule and one $\left[\mathrm{Au}(\mathrm{dcdmp})_{2}\right]^{-}$anion, with both at general positions (Figure 1b, Table S3). The BET-TTF molecule presents a slight boat type distortion, whilst the $\left[\mathrm{Au}(\mathrm{dcdmp})_{2}\right]$ has a very small chair-type distortion (Figure 1b). With a similar packing pattern to 1 , the crystal structure is composed of mixed stacks of alternating donor-acceptor molecules 
$\left(\mathrm{D}^{+} \mathrm{A}^{-} \mathrm{D}^{+} \mathrm{A}^{-} \mathrm{D}^{+} \mathrm{A}^{-}\right.$) along the $a$ axis (Figure $\mathrm{S} 1$ ). As in $\mathbf{1}$, there are no short contacts between molecules along the stacks. Between molecules in neighbouring stacks, the same type of short contacts as in $\mathbf{1}$ are observed (Table S4).

In the case of $2 \mathrm{~T}$, the unit cell contains one independent $\mathrm{BET}^{-\mathrm{TTF}^{+}}$cation and one independent $\left[\mathrm{Au}(\mathrm{dcdmp})_{2}\right]^{-}$anion, both in an inversion centre (Figure 1c, Table S5). The BET-TTF ${ }^{+}$molecule is essentially planar, within experimental error, whilst the $\left[\mathrm{Au}(\mathrm{dcdmp})_{2}\right]^{-}$monoanionic complex presents a slight chair-type distortion (Figure 1c). Unlike in $\mathbf{2 M}$, in the crystal structure of 2T, the BET-TTF molecule shows disorder in the thiophenic sulphur atoms S3 with two positions with occupation factors of $79-21 \%$ (S3/C10-S3A/C10A). The crystal structure of $2 \mathrm{~T}$ is also composed of mixed stacks of alternating donor-acceptor molecules $\left(\mathrm{D}^{+} \mathrm{A}^{-} \mathrm{D}^{+} \mathrm{A}^{-} \mathrm{D}^{+} \mathrm{A}^{-}\right)$, along the $b$ axis (Figure $2 \mathrm{~b}$ ), in the same fashion found in $\mathbf{1}$ and $\mathbf{2 M}$. The main difference in $\mathbf{2 T}$ is the relative arrangement between layers, which in this case is "in line" and not related by a dihedral angle of $\approx 60^{\circ}$ and the displaced overlapping mode between molecules along the stacks (Figure $2 \mathrm{a}_{3}, \mathrm{~b}_{3}$ ). Polymorph $2 \mathrm{~T}$ has a short hydrogen bond (N3 $\cdots \mathrm{H} 10 \mathrm{C}-\mathrm{C} 10 \mathrm{~A})$ along the stacks, which is inexistent in the $\mathbf{2} \mathbf{M}$ structure, probably due to the slightly shorter interplanar distances (Figure $2 b_{2}$ ). Apart from these differences, the observed pattern of short contacts between molecules in neighbouring stacks is similar to the observed in compounds 1 and 2M (Table S6).

In the previously reported salts of [M(dcdmp $)_{2}$ ] with DT-TTF [15], the change of the central transition metal of $\left[\mathrm{M}(\mathrm{dcdmp})_{2}\right]^{-}$anion, from gold to copper, did not introduce considerable changes in the crystal structure. Nevertheless, in the case of the copper salts an unusual richness of different stoichiometries, 1:1, 2:1, and 3:2 stoichiometries were found, with the crystal structures being arranged both in segregated and mixed stacks of donors and acceptors [15]. $\alpha$-DT-TTF[Cu(dcdmp $\left.)_{2}\right](3)$ was found to be isostructural to the previously reported ET[Au(dcdmp $)_{2}$ [35], crystallizing in the monoclinic system, space group $P 2_{1} / c$. The asymmetric unit contains one $\left[\mathrm{Cu}(\mathrm{dcdmp})_{2}\right]^{-}$anion and one donor molecule, both at general positions (Figure 3, Table S7). The donor molecules are planar within experimental error, whereas $\left[\mathrm{Cu}(\mathrm{dcdmp})_{2}\right]^{-}$anions present a slight boat type distortion (Figure 3). The $\alpha$-DT-TTF donor molecule in compound 3 presents a disorder in the thiophenic sulphur atoms S7 and S10 over two possible positions with occupation factors of $66-34 \%$ (S7/C16-S7A/C16A) and 59-41\% (S10/C21-S10A/C21A). The bond length analysis of [Cu(dcdmp) $\left.{ }_{2}\right]^{-}$ confirms its monoanionic state (Table S8), and therefore the donor molecules are fully oxidized.
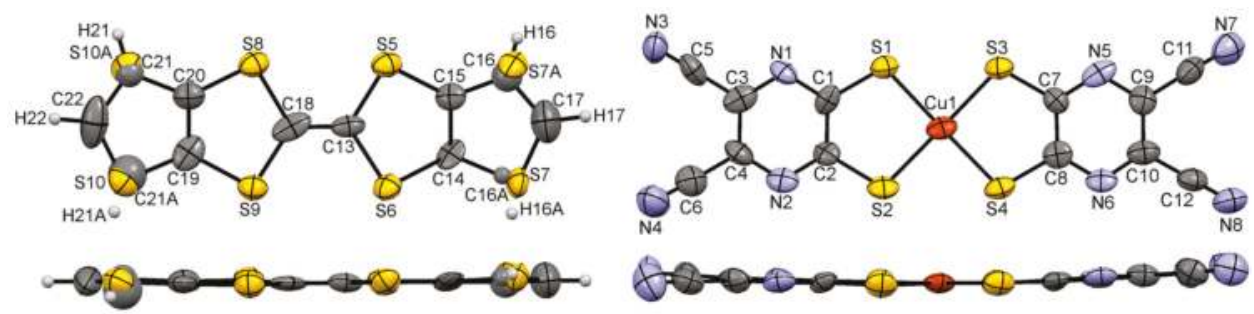

Figure 3. ORTEP and atomic numbering schemes (top and side views) of donor molecules and acceptor $\left[\mathrm{Cu}(\mathrm{dcdmp})_{2}\right]$ in the crystal structure of $\alpha$-DT-TTF $\left[\mathrm{Cu}(\mathrm{dcdmp})_{2}\right](3)$, with thermal ellipsoids drawn at $70 \%$ probability level.

The crystal structure of 3 shows side-by-side zigzag chains of alternating donor and acceptor molecules $\left(\mathrm{D}^{+} \mathrm{A}^{-} \mathrm{D}^{+} \mathrm{A}^{-} \mathrm{D}^{+}\right)$, running parallel to the $c$ axis (Figure 4), with the acceptor and donor molecules connected through several short S...S contacts. Hydrogen bonds, both between the nitrile group or the pyrazine of the anions and the hydrogen atoms in the thiophenic ring of the donor (angle of $\approx 160^{\circ}, \mathrm{C} 16-\mathrm{H} 16 \cdots \mathrm{N} 3$ and $\mathrm{C} 21-\mathrm{H} 21 \cdots \mathrm{N} 5$ ), reinforce the short contacts along the chains (Table S9). Along the chain, the average dihedral angles between anions and cations alternate between $4.67^{\circ}$ and $54.75^{\circ}$, conferring a wave shape to the chain. Along $b$, the chains are stacked, 
forming a $2 \mathrm{D}$ network of short $\mathrm{S} \cdots \mathrm{S}$ contacts between $\mathrm{A}^{-}-\mathrm{D}^{+}$and $\mathrm{D}^{+}-\mathrm{D}^{+}$molecules. Apart from the S $\cdots S$ network of short contacts, several hydrogen bonds of the $\mathrm{C}-\mathrm{H} \cdots \mathrm{N}$ and $\mathrm{C}-\mathrm{H} \cdots \mathrm{S}$ type give stability to this 2D structure and also connect acceptor to donor along the $a$ axis, parallel to the longest molecular axis of both donor and acceptor molecules (Table S9). The ET[Cu(dcdmp) $\left.)_{2}\right]$ salt (4) is isostructural with 3 (Figures S2 and S3, Tables S8, S10, and S11).

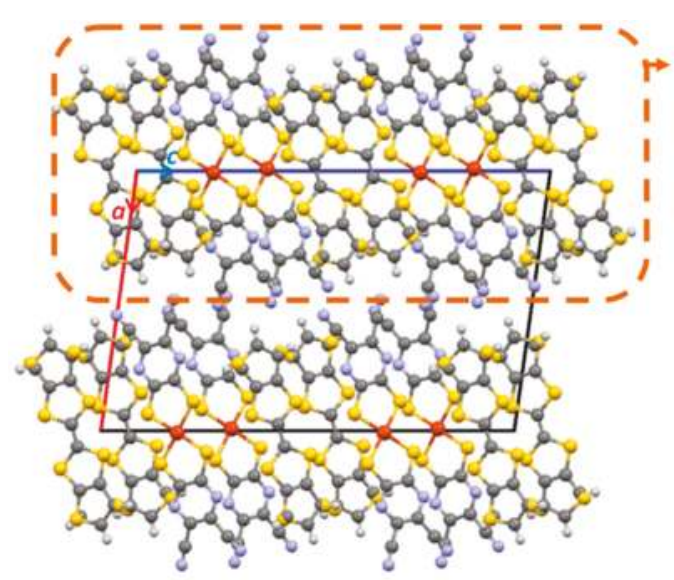

(a)

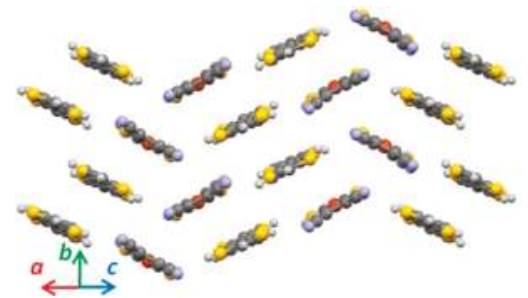

(b)

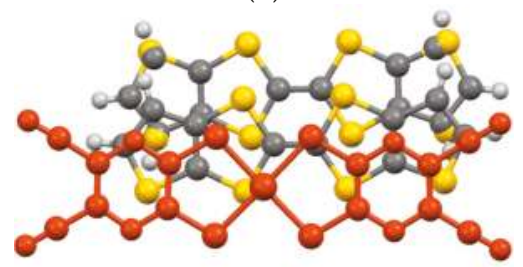

(c)

Figure 4. Crystal structure of ( $\alpha$-DT-TTF)[Cu(dcdmp $\left.)_{2}\right]$ (3): (a) view along the stacking axis; (b) partial view along the long axis of the molecules of neighbouring chains in the same layer; and (c) overlap mode between chains in the same layer.

Another unexpected crystal structure was found when the donor molecule was changed from $\alpha$-DT-TTF to the relative non-aromatic BET-TTF, which was not isostructural to compound 3 or to compound 2. In this case, a 2:1 stoichiometry was found, probably due to the spontaneous redox reaction that occurs when combining the $\left[\mathrm{Cu}(\mathrm{dcdmp})_{2}\right]^{-}$monoanion with the donor BET-TTF, leading to a reduction of the acceptor molecule to a dianionic state $\left(E_{1 / 2}=+206 \mathrm{mV}\right)$ by the full oxidation of the donor molecule $\left(\mathrm{E}_{1 / 2}=+215 \mathrm{mV}\right)[18,21]$.

$(\mathrm{BET}-\mathrm{TTF})_{2}\left[\mathrm{Cu}(\mathrm{dcdmp})_{2}\right](5)$ crystallizes in the triclinic system, space group $P-1$. The asymmetric unit is composed of one independent BET-TTF molecule at general position and half [Cu(dcdmp $\left.)_{2}\right]$ complex, with the $\mathrm{Cu}$ atom in an inversion center (Figure 5a, Table S12), whereas the donor molecule presents a small boat type distortion with the methylene extremities with an envelope type distortion at opposite directions, and the $\left[\mathrm{Cu}(\mathrm{dcdmp})_{2}\right]$ anion has a small chair-type distortion (Figure 5a). The BET-TTF donor unit presents disorder in the sulphur atoms of the thiophenic ring over two possible positions, S5 and S8, with occupation factors of 46-54\% (in both S5/C10-S5A/C10A and S8/C15-S8A/C15A). The bond lengths analysis indicates that the donor molecule is fully oxidized, while the copper complex is in a dianionic state; therefore, the compound should be formulated as $\left(\mathrm{BET}^{-\mathrm{TTF}^{+}}\right)_{2}\left[\mathrm{Cu}(\mathrm{dcdmp})_{2}\right]^{2-}$ (Tables S13 and S14). 


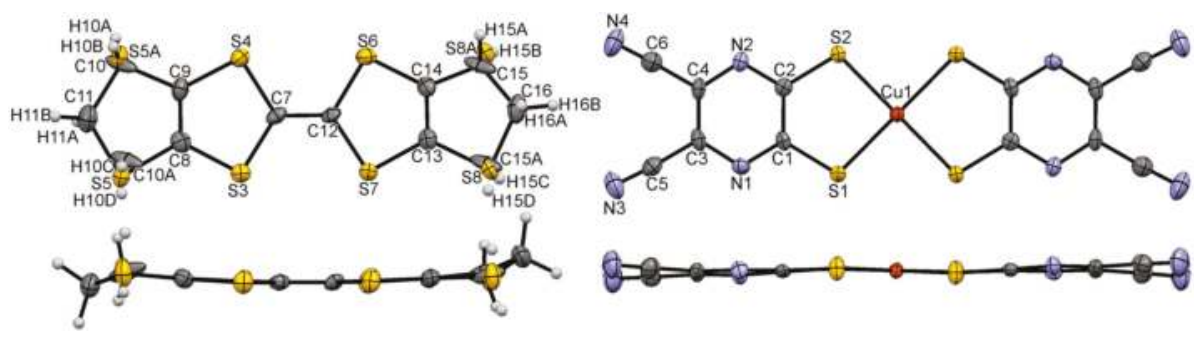

(a)
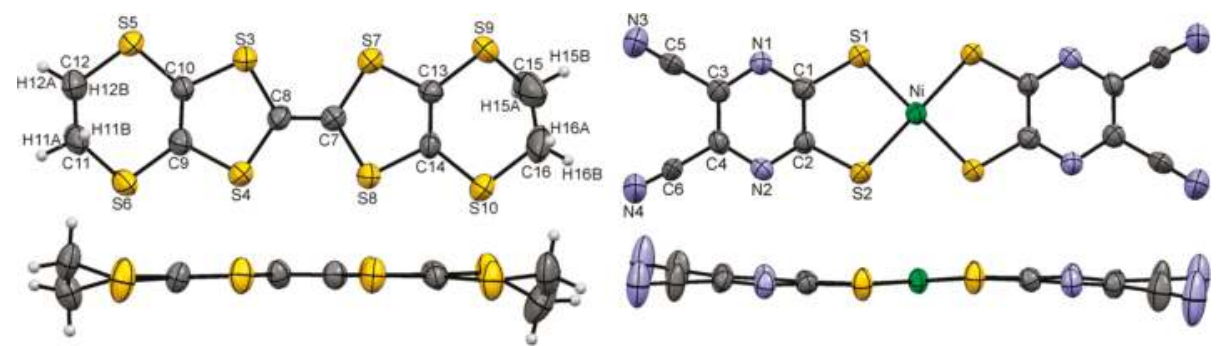

(b)

Figure 5. ORTEP and atomic numbering schemes (top and side views) of (a) BET-TTF donor molecule and acceptor $\left[\mathrm{Cu}(\mathrm{dcdmp})_{2}\right]$ in the crystal structure of $(\mathrm{BET}-\mathrm{TTF})_{2}\left[\mathrm{Cu}(\mathrm{dcdmp})_{2}\right](5)$; and (b) ET donor molecules and acceptor $\left[\mathrm{Ni}(\mathrm{dcdmp})_{2}\right]$ in the crystal structure of $(\mathrm{ET})_{2}\left[\mathrm{Ni}(\mathrm{dcdmp})_{2}\right](6)$, with thermal ellipsoids drawn at $70 \%$ probability level.

The crystal structure of $\mathbf{5}$ is shown in Figure 6a. Its structure is composed of mixed columns along $a+b$ of face-to-face BET-TTF dimers $\left(\left(\mathrm{D}^{+}\right)_{2}\right)$ alternating with $\left[\mathrm{Cu}(\mathrm{dcdmp})_{2}\right]^{2-}$ dianions $\left(\mathrm{A}^{2-}\right)$ with the donor dimers and the dianions placed perpendicularly. The donor molecules in the dimers

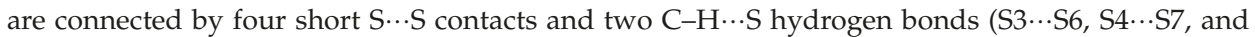
$\mathrm{C} 15-\mathrm{H} 15 \mathrm{~B} \cdots \mathrm{S} 5$ ) with intramolecular distance between average planes of $3.734 \AA$ (Table S15). Along the columns, the acceptor is connected to both molecules of the dimer by short $\mathrm{S} \cdots \mathrm{S}$ and $\mathrm{N} \cdots \mathrm{S}$ contacts (Table S15). Between neighbouring columns, several hydrogen bonds and a short S..S contact $(\mathrm{C} 16-\mathrm{H} 16 \mathrm{~A} \cdots \mathrm{N} 2, \mathrm{C} 11-\mathrm{H} 11 \mathrm{~B} \cdots \mathrm{N} 3$, and S1․S3) connect acceptor and donor dimers. Between the extremities of the molecules, the stacks are also connected, along $c$, by a hydrogen bond between

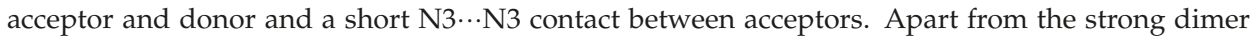
interaction, there are no contacts between different donor dimers in neighbouring columns.

$(\mathrm{ET})_{2}\left[\mathrm{Ni}(\mathrm{dcdmp})_{2}\right](6)$ was also found to have a 2:1 stoichiometry. Compound 6 crystallizes in the triclinic system, space group $P-1$. The asymmetric unit cell contains an independent $\left[\mathrm{Ni}(\mathrm{dcdmp})_{2}\right]^{2-}$ located at an inversion centre and one $\mathrm{ET}^{+}$molecule at general position (Figure 5b, Table S16). The average bond length M-S value $(2.175 \AA)$, found in the acceptor, indicates that the complex is in a dianionic state [36]. The nickel dianion presents a slight chair type distortion, and the ET molecule shows the usual geometry found in other related salts of this donor (Figure 5b) [37]. In spite of the uniform color and shape of the crystals obtained in one preparation, the presence of other stoichiometries or phases cannot be excluded, since EPR measurements in crystals from the same preparation present two different shapes (Figures S4 and S5). Attempts to prepare other [Ni(dcdmp $\left.)_{2}\right]$ salts with BET-TTF, $\alpha$-DT-TTF, or $\alpha$-mtdt using electrocrystallization techniques did not yield good quality single crystals for $\mathrm{X}$-ray diffraction and electric transport properties measurements. 


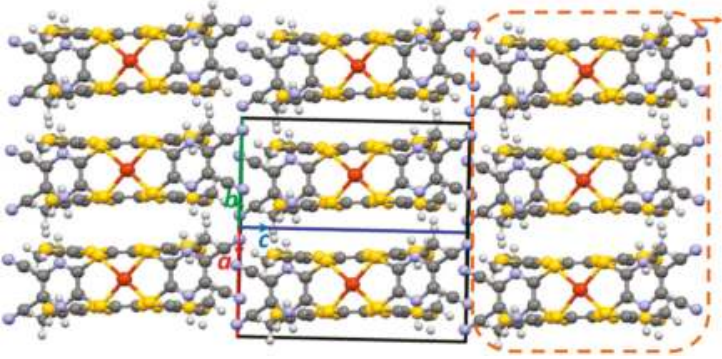

$(\mathbf{a} 1)$

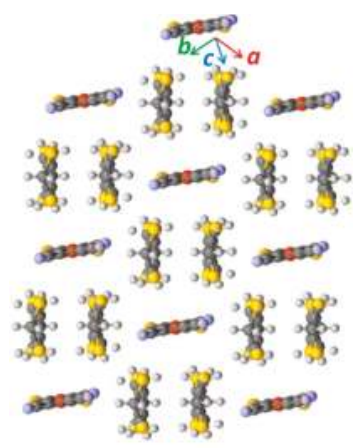

$(\mathbf{a} 2)$

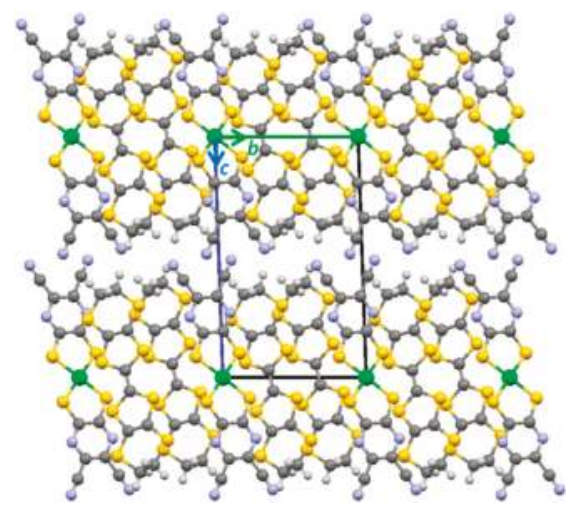

$\left(\mathbf{b}_{1}\right)$

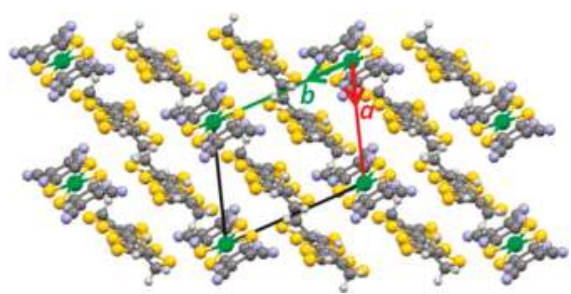

$\left(\mathbf{b}_{2}\right)$

Figure 6. Crystal structure of compound 5 (a) and compound $\mathbf{6}(\mathbf{b})$ : $\left(\mathbf{a}_{1}\right)$ view along $a-b$; $\left(\mathbf{a}_{2}\right)$ partial view along the long axis of the molecules of neighbouring columns in the same layer, with the alternating dianions and BET-TTF dimers; $\left(\mathbf{b}_{1}\right)$ view along the stacking axis; $\left(\mathbf{b}_{2}\right)$ partial view of the layers in the $a b$ plane.

Similarly to ET[Cu(dcdmp $\left.)_{2}\right](4)$ and $\alpha$-DT-TTF $\left[\mathrm{Cu}(\mathrm{dcdmp})_{2}\right]$ (3), the crystal structure of $(\mathrm{ET})_{2}\left[\mathrm{Ni}(\mathrm{dcdmp})_{2}\right](6)$ is composed of layers of zigzag chains, along the $b$ axis, of dianions alternating with donor dimers $\left(\mathrm{A}^{2-}\right) \mathrm{D}^{+} \mathrm{D}^{+}\left(\mathrm{A}^{2-}\right) \mathrm{D}^{+} \mathrm{D}^{+}\left(\mathrm{A}^{2-}\right)$ (Figure $\left.6 \mathrm{~b}\right)$. Along a chain there are several S $\cdots \mathrm{S}$ and $\mathrm{S} \cdots \mathrm{N}$ short contacts both between donors and acceptor-donor molecules (Table S17). In the $a b$ plane, these chains interact with each other through short $\mathrm{Ni} \cdots \mathrm{S}$ contacts between acceptor-donor

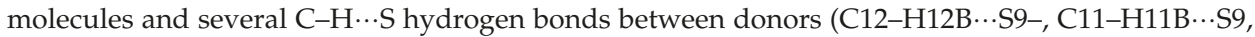
$\mathrm{C} 16-\mathrm{H} 16 \mathrm{~A} \cdots \mathrm{S} 5)$. Along $c$, the contact between chains is made by acceptor-donor hydrogen bonds (C16-H16B $\cdots \mathrm{N} 3)$. Another way to describe this structure is to see it as composed layers, in the ab plane, as a bidimensional layer of pairs of ET molecules coupled face-by-face that are connected with other pairs by a short, side-by-side, S..S contacts. In the "channels" of the layers are located the acceptors surrounded by donors in all directions. There are no interactions between acceptors.

With $\alpha$-mtdt, a dissymmetric TTF-type donor, only the copper salt could be isolated. $\alpha$-mtdt $\left[\mathrm{Cu}(\mathrm{dcdmp})_{2}\right](7)$ crystallizes in the triclinic system, space group $P-1$. The asymmetric unit contains one independent $(\alpha \text {-mtdt })^{+}$molecule at general position and two $\left[\mathrm{Cu}(\mathrm{dcdmp})_{2}\right]^{-}$complexes, both with the $\mathrm{Cu}$ atom in an inversion centre (Figure 7, Tables S18 and S19). The $\alpha$-mtdt molecule is essentially planar, with the exception of the $-\left(\mathrm{CH}_{2}\right)_{2}-\mathrm{CN}$ groups that point out both in the same 
direction almost perpendicularly to the central molecular plane, while both $\left[\mathrm{Cu}(\mathrm{dcdmp})_{2}\right]$ molecules present a very small chair-type distortion (Figure 7). The bond length analysis confirms that the donor molecule is fully oxidized $(\alpha-\mathrm{mtdt})^{+}$(Table S20) and the copper dithiolene complex is in monoanionic state (Table S21).

Figure 8a,b illustrates the crystal structure of compound 7, which are composed of segregated stacks of donors and acceptors, along the $a$ axis, with sheets of stacks of $\alpha$-mtdt donors and $\left[\mathrm{Cu}(\mathrm{dcdmp})_{2}\right]$ acceptors alternating along $b$. Within the stacks, the donor molecules are arranged in a head-to-head fashion with distance of $3.469 \AA$ between molecular planes, suggesting significant $\pi-\pi$ interactions. The overlap mode of $\alpha$-mtdt donors (Figure $8 \mathrm{c}$ ) shows a large displacement of the molecules along their long axis, probably due to the bulky cyanoethyl group. Within the stacks,

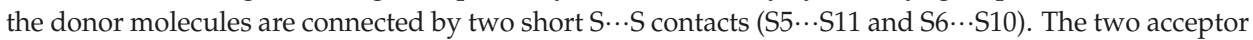
molecules A and B (respectively, red and blue molecules in Figure 8a,b), although crystallographically distinct, are identical within experimental uncertainty and present identical overlap modes (Figure 8d). Along each monoanion stack, A or B, there are no short contacts, and the distance between molecular planes is of $3.516 \AA$ and $3.501 \AA$, respectively. Short contacts between acceptor molecules A and $\mathrm{B}$ are also inexistent. The different acceptor stacks have different angles in relation to the $\alpha$-mtdt donor stacks, with a dihedral angle of $37.47^{\circ}$ and $74.14^{\circ}$ for stacks of molecules $\mathrm{A}$ and $\mathrm{B}$, respectively. The interactions between stacks are made in two distinct ways along the $\alpha$-mtdt molecule long axis (Table S22): (a) on one side, the donor molecules interact with each other through $\mathrm{C}-\mathrm{H} \cdots \mathrm{N}$ hydrogen

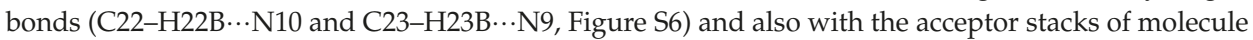

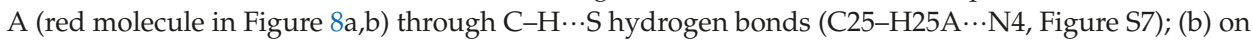
the other side, the interaction is between the methyl groups and the thiophenic sulphur atom of the $\alpha$-mtdt donor through a hydrogen bond (Figure S6, C18-H18A*..S7). The lateral connection between acceptor-donor molecules along the molecules minor axis is mediated by short $\mathrm{S} \cdots \mathrm{S}$ and $\mathrm{S} \cdots \mathrm{N}$ contacts and hydrogen bonds interactions (Table S22, Figure S7).
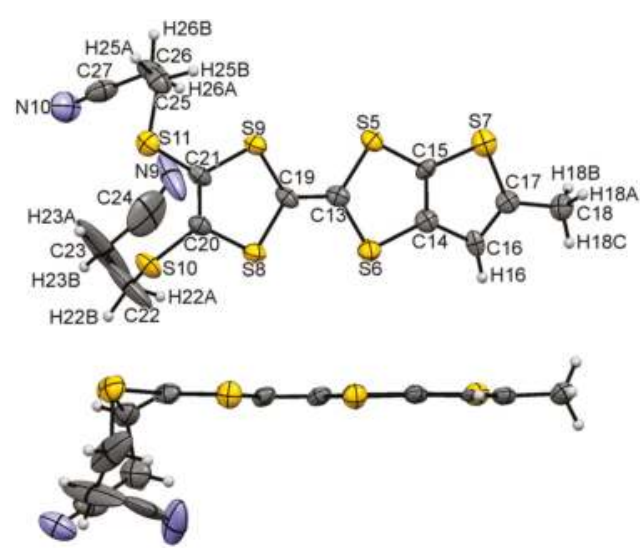

(a)

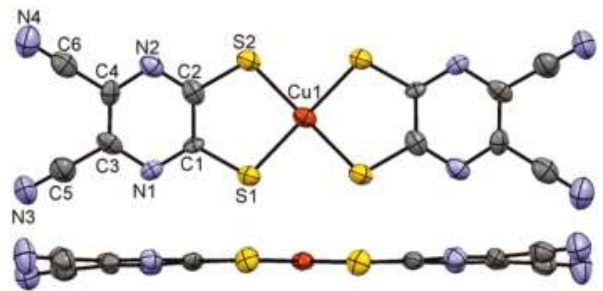

(b)

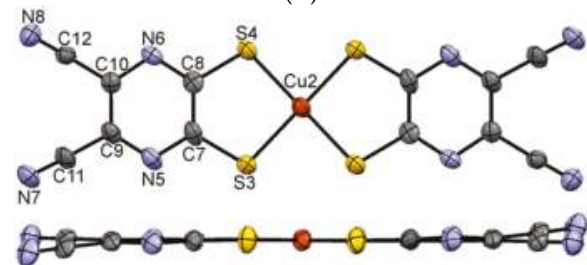

(c)

Figure 7. ORTEP and atomic numbering schemes (top and side views) of $\alpha$-mtdt donor (a) and acceptor $\left[\mathrm{Cu}(\mathrm{dcdmp})_{2}\right]$ molecules $\mathrm{A}(\mathbf{b})$ and B $(\mathbf{c})$ in the crystal structure of $(\alpha-\mathrm{mtdt})\left[\mathrm{Cu}(\mathrm{dcdmp})_{2}\right](7)$, with thermal ellipsoids drawn at 50\% probability level. 


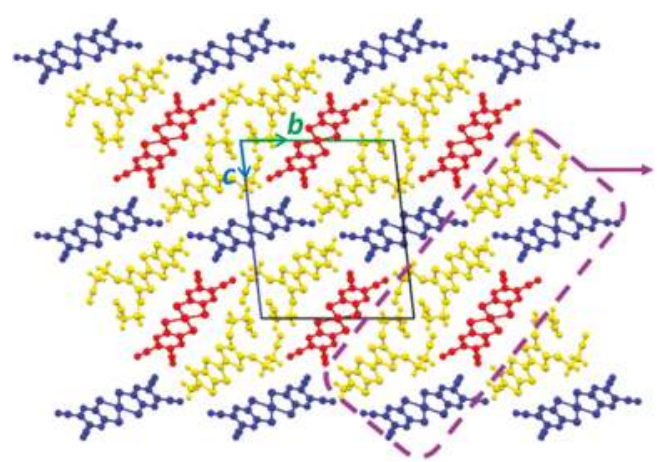

(a)

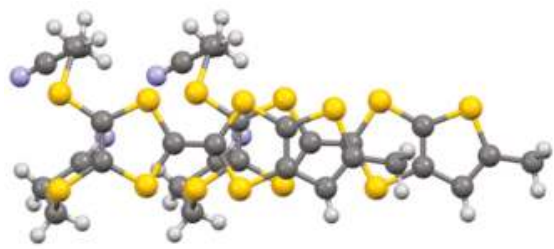

(c)

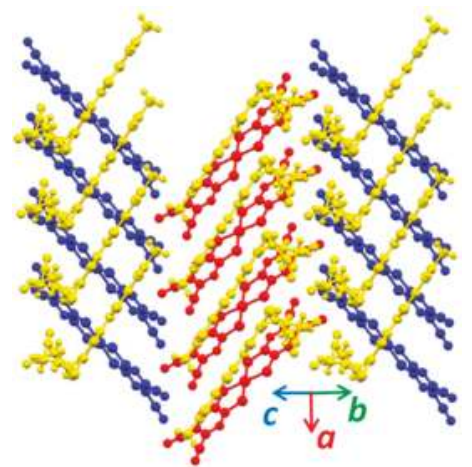

(b)

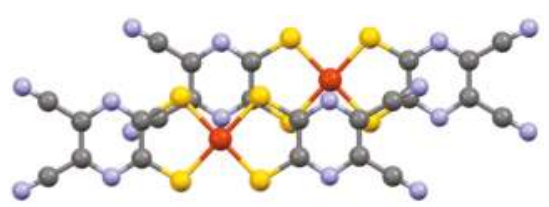

(d)

Figure 8. Crystal structure of $(\alpha-\mathrm{mtdt})\left[\mathrm{Cu}(\mathrm{dcdmp})_{2}\right]$ (7): (a) view along the $a$ axis; (b) partial view showing the different segregated stacks of donors (yellow) and acceptor stacks (red and blue) and overlap mode representation between donors (c) and between acceptor stacks (d).

\subsection{Electric Transport Properties}

The electrical conductivity of compounds 1-7 was measured in single crystals along their long axis, and the results are presented in Figure 9 and Table 1 . All compounds present a semiconducting behaviour with room temperature values in the range $\approx 10^{-5} \mathrm{~S} / \mathrm{cm}<\sigma_{R T}<\approx 10^{-1} \mathrm{~S} / \mathrm{cm}$. The highest conductivity was found for the 1:1 copper compound 3 and gold salt 1 of $\alpha$-DT-TTF donor, followed closely by (BET-TTF) $\left[\mathrm{Au}(\mathrm{dcdmp})_{2}\right](2)$. The modest conductivity values and the thermally activated behaviour observed were not unexpected in view of the fully oxidised nature of the donor molecules, which are expected to lead to Mott insulator states. In this sense, the relatively large conductivity values observed in $\alpha$-DT-TTF salts 1 and 3 are very large for 1:1 salts with fully oxidised molecules. In $\left(\alpha\right.$-DT-TTF) $\left[\mathrm{Au}(\mathrm{dcdmp})_{2}\right](\mathbf{1})$, resistivity measurements made in needle $(0.16 \mathrm{~S} / \mathrm{cm})$ and plate shaped sample $(0.052 \mathrm{~S} / \mathrm{cm})$, which were obtained in the same preparation, gave slightly different values, which could indicate the possibility of different phases. The coexistence of different stoichiometries and polymorphs is not unprecedented in salts with the $\left[\mathrm{M}(\mathrm{dcdmp})_{2}\right]$ anions [15]. However, this possibility could not be confirmed from the different crystals selected for single crystal X-ray diffraction.

It should be noted that in compounds 1, 2, and $\mathbf{3}$ with higher conductivity, both the donor and acceptor molecules make an extended network of interactions, and both could provide conduction bands. In compound 7 , the bulkier cyanoethyl groups of the asymmetric donor molecule $\alpha$-mtdt act as a hindrance to the donor molecules overlapping each other; nevertheless, a crystal structure pattern based on segregated stacks of donor and acceptors was obtained with a room temperature conductivity of $1.1 \times 10^{-3} \mathrm{~S} / \mathrm{cm}$.

Although ET[Cu(dcdmp $\left.)_{2}\right](4)$ is isostructural with 3, it presents one of the lowest conductivities found in this group of compounds $\left(4.2 \times 10^{-4} \mathrm{~S} / \mathrm{cm}\right)$. Even though compound 4 seems to have a good overlap with the donor molecules along the $b$ axis (Figure $4 \mathrm{c}$ and Figure S3), a detailed crystal structure 
analysis shows that the distances between the molecular average planes might prevent effective $\pi-\pi$ interactions, with donor interplanar distances larger in compound 4 than in $3(3.699 \AA$ and $3.357 \AA$ for compound 4 and $3.571 \AA$ and $3.033 \AA$ in compound 3).

The room temperature conductivities of compounds 5 and $\mathbf{6}$, both in a 2:1 stoichiometry, are of the same order of magnitude as that found in 4 . The dimeric nature of the donors found in these crystal structures probably induces electron localization and, even with a network of interactions between donors, effective electronic pathways are not established.

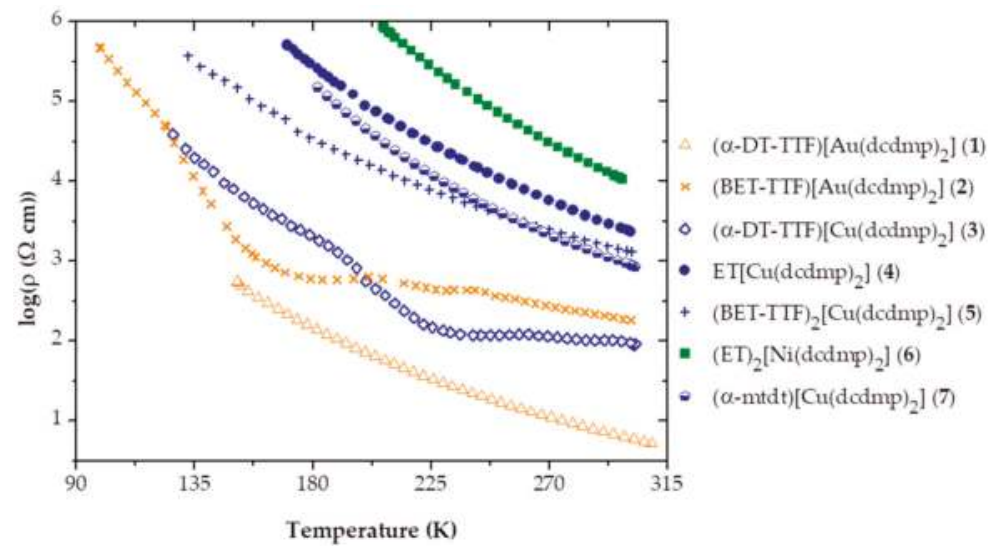

Figure 9. Electrical resistivity $\rho$ of compounds 1-7 as a function of temperature $T$.

Table 1. Room-temperature electrical conductivity $\left(\sigma_{\mathrm{RT}}\right)$ and activation energy $\left(\mathrm{E}_{\mathrm{a}}\right)$ of compounds 1-7.

\begin{tabular}{ccc}
\hline CT Salts & $\sigma_{\text {RT }}(\mathbf{S c m})$ & $\mathbf{E}_{\mathbf{a}}(\mathbf{m e V})$ \\
\hline$(\mathrm{ET})_{2}\left[\mathrm{Ni}(\mathrm{dcdmp})_{2}\right](\mathbf{6})$ & $9.4 \times 10^{-5}$ & 128 \\
$(\mathrm{ET})\left[\mathrm{Cu}(\mathrm{dcdmp})_{2}\right](\mathbf{4})$ & $4.2 \times 10^{-4}$ & 190 \\
$(\mathrm{BET}-\mathrm{TTF})_{2}\left[\mathrm{Cu}(\mathrm{dcdmp})_{2}\right](\mathbf{5})$ & $7.4 \times 10^{-4}$ & 134 \\
$(\alpha-\mathrm{mtdt})\left[\mathrm{Cu}(\mathrm{dcdmp})_{2}\right](7)$ & $1.1 \times 10^{-3}$ & 207 \\
$(\mathrm{BET}-\mathrm{TTF})\left[\mathrm{Au}(\mathrm{dcdmp})_{2}\right](\mathbf{2})$ & $5.4 \times 10^{-3}$ & 95 \\
$(\alpha$-DT-TTF $)\left[\mathrm{Au}(\mathrm{dcdmp})_{2}\right](\mathbf{1})$ & $5.2 \times 10^{-2} \mathrm{a} ; 1.6 \times 10^{-1 \mathrm{~b}}$ & $105^{\mathrm{a}} ; 59^{\mathrm{b}}$ \\
$(\alpha$-DT-TTF $)\left[\mathrm{Cu}(\mathrm{dcdmp})_{2}\right](\mathbf{3})$ & $1.1 \times 10^{-2}$ & 107 \\
\hline \multicolumn{2}{c}{$\mathrm{a}$ Plate $\mathrm{b}$ Needle }
\end{tabular}

\section{Conclusions}

Eight new compounds were obtained by electrocrystallization by combining TTF type electronic donors, namely, ET, BET-TTF, $\alpha$-DT-TTF, and $\alpha$-mtdt, with transition metal complexes $\left[\mathrm{M}(\mathrm{dcdmp})_{2}\right]$ $(\mathrm{M}=\mathrm{Au}, \mathrm{Cu}$, and $\mathrm{Ni})$. Most of the herein reported compounds present a 1:1 stoichiometry with a crystal structure composed of mixed stacks of donor and acceptor molecules, with the exception of $(\alpha-\mathrm{mtdt})\left[\mathrm{Cu}(\mathrm{dcdmp})_{2}\right](7)$, which crystallizes in segregated stacks of donors and acceptors. Other two exceptions are the salts $(\mathrm{BET}-\mathrm{TTF})_{2}\left[\mathrm{Cu}(\mathrm{dcdmp})_{2}\right](5)$ and $(\mathrm{ET})_{2}\left[\mathrm{Ni}(\mathrm{dcdmp})_{2}\right](6)$ that present a 2:1 stoichiometry. All compounds showed a semiconducting behavior with a room conductivity ranging from $1.6 \times 10^{-1}$ to $9.4 \times 10^{-5} \mathrm{~S} / \mathrm{cm}$. The highest room temperature conductivity was observed for $\left.(\alpha \text {-DT-TTF)[Au(dcdmp })_{2}\right](\mathbf{1})$ and the lowest for $(\mathrm{ET})_{2}\left[\mathrm{Ni}(\mathrm{dcdmp})_{2}\right](6)$.

The pyrazine nitrogen atoms in the dcdmp ligand were found, amongst the different crystal structures, to be extensively involved in both $\mathrm{S} \cdots \mathrm{N}$ short contacts and $\mathrm{N} \cdots \mathrm{H}-\mathrm{C}$ hydrogen bonds. As a consequence, when comparing the salts of the complexes based on dcdmp ligands with the mnt 
ligand, the structures are quite different. The diversity of crystal structures and polymorphs in this family of compounds makes them good models for further understanding the correlation between the intermolecular crystal structure patterns and the observed macroscopic electrical transport properties.

Supplementary Materials: CCDC $1826109-1826115$ and 1826306 contains the supplementary crystallographic data for this paper. These data can be obtained free of charge via http:/ / www.ccdc.cam.ac.uk/conts/retrieving. html. The following are available online at www.mdpi.com/xxx/s1, Table S1: Bond lengths of compound 1, Table S2: short contacts of compound 1. Table S3: bond lengths of compound 2M, Figure S1: crystal structure of compound 2M, Table S4: short contacts of compound 2M, Table S5: bond lengths of compound 2T, Table S6: short contacts of compound 2T, Table S7: bond lengths of compound 3, Table S8: acceptor molecule bond length analysis of compound 3 and 4, Table S9: short contacts of compound 3, Figure S2: ORTEP of compound 4, Figure S3: crystal structure of compound 4, Table S10: bond lengths of compound 4, Table S11: short contacts of compound 4, Table S12: bond lengths of compound 5, Tables S13 and S14: bond length analysis of compound 5, Table S15: short contacts of compound 5, Table S16: bond lengths of compound 6, Table S17: short contacts of compound 6, Figures S4 and S5: EPR measurements of compound 6, Tables S18 and S19: bond lengths of compound 7, Tables S20 and S21: bond length analysis of compound 7, Table S22: short contacts of compound 7, Figures S6 and S7: relevant short contacts of compound 7.

Acknowledgments: This work was supported by FCT (Portugal) through contracts PTDC/QEQ-SUP/1413/2012 and UID/Multi/04349/2013 and FCTs grants of R.A.L.S (SFRH/BD/86131/2012) and S.R. (SFRH/BPD/113344/2015).

Author Contributions: Dulce Belo, Vasco Gama, and Manuel Almeida conceived and designed the experiments; Rafaela A. L. Silva performed the experiments; Isabel C. Santos analyzed the crystallographic data; Sandra Rabaça analyzed the cyclic voltammetry; Elsa B. Lopes measured the electric transport properties and Rafaela A. L. Silva, Dulce Belo, and Manuel Almeida wrote the paper with contributions from all authors.

Conflicts of Interest: The authors declare no conflict of interest. The founding sponsors had no role in the design of the study; in the collection, analyses, or interpretation of data; in the writing of the manuscript; or in the decision to publish the results.

\section{References}

1. Kato, R. Conducting Metal Dithiolene Complexes: Structural and Electronic Properties. Chem. Rev. 2004, 104, 5319-5346. [CrossRef] [PubMed]

2. Stiefel, E.I. Dithiolene Chemistry. Synthesis, Properties and Applications; John Wiley \& Sons, Inc.: Hoboken, NJ, USA, 2004.

3. Coomber, A.T.; Beljonne, D.; Friend, R.H.; Brédas, J.L.; Charlton, A.; Robertson, N.; Underbill, A.E.; Kurmoo, M.; Day, P. Intermolecular interactions in the molecular ferromagnetic $\mathrm{NH}_{4} \mathrm{Ni}(\mathrm{mnt})_{2} \cdot \mathrm{H}_{2} \mathrm{O}$. Nature 1996, 380, 144-146. [CrossRef]

4. Fourmigué, M.; Domercq, B.; Jourdain, I.V.; Molinié, P.; Guyon, F. Amaudrut, Interplay of Structural Flexibility and Crystal Packing in a Series of Paramagnetic Cyclopentadienyl/Dithiolene Mo and W Complexes: Evidence for Molecular Spin Ladders. Chem. A Eur. J. 1998, 4, 1714-1723. [CrossRef]

5. Rovira, C. Molecular Compounds Showing a Spin Ladder Behaviour. In $\pi$-Electron Magnetism from Molecules to Magnetic Materials; Veciana, J., Ed.; Springer: Berlin/Heidelberg, Germany, 2001; Volume 100, pp. 163-188.

6. Underhill, A.E.; Ahmad, M.M. A new one-dimensional 'metal' with conduction through bis (dicyanoethylenedithiolato) platinum anions. J. Chem. Soc. Chem. Commun. 1981, 67-68. [CrossRef]

7. Ahmad, M.M.; Turner, D.J.; Underhill, A.E.; Jacobsen, C.S.; Mortensen, K.; Carneiro, K. Physical properties and the Peierls instability of $\mathrm{Li}_{0.82}\left[\mathrm{Pt}\left(\mathrm{S}_{2} \mathrm{C}_{2}(\mathrm{CN})_{2}\right)_{2}\right] \cdot 2 \mathrm{H}_{2}$ O. Phys. Rev. B 1984, 29, 4796-4799. [CrossRef]

8. Brossard, L.; Ribault, M.; Bousseau, M.; Valade, L.; Cassoux, P. Un nouveau type de supraconducteur moléculaire: TTF[Ni(dmit $\left.)_{2}\right]_{2}$. Comptes Rendus Acad. Sci. 1986, 302, 205-210.

9. Cassoux, P. Molecular (super)conductors derived from bis-dithiolate metal complexes. Coord. Chem. Rev. 1999, 185-186, 213-232. [CrossRef]

10. Robertson, N.; Cronin, L. Metal bis-1,2-dithiolene complexes in conducting or magnetic crystalline assemblies. Coord. Chem. Rev. 2002, 227, 93-127. [CrossRef]

11. Rabaça, S.; Almeida, M. Dithiolene complexes containing N coordinating groups and corresponding tetrathiafulvalene donors. Coord. Chem. Rev. 2010, 254, 1493-1508. [CrossRef]

12. Ribas, X.; Dias, J.C.; Morgado, J.; Wurst, K.; Almeida, M.; Veciana, J.; Rovira, C. Novel Cu(III) bis-1,2-diselenolene complex with a highly extended 3D framework through $\mathrm{Na}^{+}$coordination. CrystEngComm 2002, 4, 564-567. [CrossRef] 
13. Ribas, X.; Dias, J.C.; Morgado, J.; Wurst, K.; Santos, I.C.; Almeida, M.; Vidal-Gancedo, J.; Veciana, J.; Rovira, C. Alkaline Side-Coordination Strategy for the Design of Nickel(II) and Nickel(III) Bis(1,2-diselenolene) Complex Based Materials. Inorg. Chem. 2004, 43, 3631-3641. [CrossRef] [PubMed]

14. Ribas, X.; Dias, J.C.; Morgado, J.; Wurst, K.; Molins, E.; Ruiz, E.; Almeida, M.; Veciana, J.; Rovira, C. Novel $\mathrm{Cu}^{\mathrm{III}}$ bis-1,2-dichalcogenene complexes with tunable 3D framework through alkaline cation coordination. A structural and theoretical study. Chem. Eur. J. 2004, 10, 1691-1704. [CrossRef] [PubMed]

15. Silva, R.A.L.; Santos, I.C.; Lopes, E.B.; Rabaça, S.; Gancedo, V.-J.; Rovira, C.; Almeida, M.; Belo, D. DT-TTF Salts with $\left[\mathrm{Cu}(\mathrm{dcdmp})_{2}\right]^{-}$: The Richness of Different Stoichiometries. Cryst. Growth Des. 2016, 16, 3924-3931. [CrossRef]

16. Belo, D.; Lopes, E.B.; Santos, I.C.; Dias, J.C.; Figueira, M.; Almeida, M.; Fourmigué, M.; Rovira, C. Synthesis and Characterization of Charge Transfer Salts. J. Low Temp. Phys. 2006, 142, 349-354. [CrossRef]

17. Rovira, C.; Veciana, J.; Santalo, N.; Tarres, J.; Cirujeda, J.; Molins, E.; Llorca, J.; EspinoSa, E. Synthesis of Several Isomeric Tetrathiafulvalene. pi.-Electron Donors with Peripheral Sulfur Atoms. A Study of Their Radical Cations. J. Org. Chem. 1994, 59, 3307-3313.

18. Silva, R.A.L.; Neves, A.I.; Afonso, M.L.; Santos, I.C.; Lopes, E.B.; Del Pozo, F.; Pfattner, R.; Torrent, M.M.; Rovira, C.; Almeida, M.; et al. $\alpha$-Dithiophene-tetrathiafulvalene-A Detailed Study of an Electronic Donor and Its Derivatives. Eur. J. Inorg. Chem. 2013, 13, 2440-2446. [CrossRef]

19. Silva, R.A.L.; Vieira, B.J.C.; Andrade, M.A.; Santos, I.C.; Rabaça, S.; Belo, D.; Almeida, M. TTFs nonsymmetrically fused with alkylthiophenic moieties. Beilstein J. Org. Chem. 2015, 11, 628-637. [CrossRef] [PubMed]

20. Belo, D.; Santos, I.C.; Almeida, M. 5,6-Dicyano-2,3-dithiopyrazine (dcdmp) chemistry: Synthesis and crystal structure of $\mathrm{Au}(\mathrm{III})(\mathrm{dcdmp})_{2}$ complexes and 2,3,7,8-tetracyano-1,4,6,9-tetraazothianthrene. Polyhedron 2004, 23, 1351-1359. [CrossRef]

21. Belo, D.; Figueira, M.J.; Santos, I.C.; Gama, V.; Pereira, L.C.; Henriques, R.T.; Almeida, M. Synthesis and characterization of copper complexes with the 2,3-dicyano-5,6-dimercaptopyrazine ligand: Magnetic properties of a ferrocenium salt. Polyhedron 2005, 24, 2035-2042. [CrossRef]

22. Tomura, M.; Tanaka, S.; Yamashita, Y. Synthesis, structure and properties of the novel conductingdithiolato-metal complexes having dicyanopyrazine moieties. Synth. Met. 1994, 64, 197-202. [CrossRef]

23. Perrin, D.D.; Armarego, W.L.F. Purification of Laboratory Chemicals, 3rd ed.; Pergamon Press: Oxford, UK, 1988.

24. Sheldrick, G.M. SADABS; Bruker AXS Inc.: Madison, WI, USA, 2004.

25. Bruker. SMART and SAINT; Bruker AXS Inc.: Madison, WI, USA, 2008.

26. Fair, C.K. MoIEN. An Interactive Intelligent System for Crystal Structure Analysis; Enraf-Nonius: Delft, The Netherlands, 1990.

27. Altomare, A.; Burla, M.C.; Camalli, M.; Cascarano, G.; Giacovazzo, G.; Guagliardi, A.; Moliterni, A.G.G.; Polidori, G.; Spagna, R. SIR97: A new tool for crystal structure determination and refinement. J. Appl. Cryst. 1999, 32, 115-119. [CrossRef]

28. Sheldrick, G.M. A short history of SHELX. Acta Crystallogr. A Found. Crystallogr. 2008, 64, 112-122. [CrossRef] [PubMed]

29. Farrugia, L.J. WinGX and ORTEP for Windows: An update. J. Appl. Cryst. 2012, 45, 849-854. [CrossRef]

30. Macrae, C.F.; Bruno, I.J.; Chisholm, J.A.; Edgington, P.R.; McCabe, P.; Pidcock, E.; Rodriguez-Monge, L.; Taylor, R.; van de Streek, J.; Wood, P.A. Mercury CSD 2.0-New features for the visualization and investigation of crystal structures. J. Appl. Cryst. 2008, 41, 466-470.

31. Almeida, M.; Alcácer, L.; Oostra, S. Anisotropy of thermopower in $N$-methyl- $N$-ethylmorpholinium bistetracyanoquinodimethane, MEM(TCNQ)2, in the region of the high-temperature phase transitions. Phys. Rev. B 1984, 30, 2839-2844. [CrossRef]

32. Lopes, E.B. Internal Report; INETI-Sacavém: Lisbon, Portugal, 1991.

33. Chaikin, P.M.; Kwak, J.F. Apparatus for Thermopower Measurements on Organic Conductors. Rev. Sci. Instrum. 1975, 46, 218-220. [CrossRef]

34. Schaffer, P.E.; Wudl, F.; Thomas, G.A.; Ferraris, J.P.; Cowan, D.O. Apparent giant conductivity peaks in an anisotropic medium: TTF-TCNQ. Solid State Commun. 1974, 14, 347-351. [CrossRef] 
35. Belo, D.; Morgado, J.; Lopes, E.B.; Santos, I.C.; Rabaça, S.; Duarte, M.T.; Gama, V.; Henriques, R.T.; Almeida, M. Synthesis and Characterisation of Charge Transfer Salts Based on Au(dcdmp), and TTF Type Donors. Synth. Met. 1999, 102, 1751-1752. [CrossRef]

36. Tomura, M. Bis(tetra-n-butylammonium) bis(5,6-dicyanopyrazine-2,3-dithiolato- $\left.\mathrm{k} 2 S, S^{\prime}\right)$ nickelate(II). IUCrData 2017, 2, x171059. [CrossRef]

37. Kobayashi, H.; Kobayashi, A.; Sasaki, Y.; Saito, G.; Inokuchi, H. The Crystal and Molecular Structures of Bis(ethylenedithio)tetrathiafulvalene. Bull. Chem. Soc. Jpn. 1986, 59, 301-302. [CrossRef]

(C) 2018 by the authors. Licensee MDPI, Basel, Switzerland. This article is an open access article distributed under the terms and conditions of the Creative Commons Attribution (CC BY) license (http:/ / creativecommons.org/licenses/by/4.0/). 
Article

\title{
Specific Structural Disorder in an Anion Layer and Its Influence on Conducting Properties of New Crystals of the (BEDT-TTF) $)_{4} \mathrm{~A}^{+}\left[\mathrm{M}^{3+}(\mathrm{ox})_{3}\right] \mathrm{G}$ Family, Where $\mathrm{G}$ Is 2-Halopyridine; $\mathrm{M}$ Is $\mathrm{Cr}, \mathrm{Ga}$; $\mathrm{A}^{+}$Is $\left[\mathrm{K}_{0.8}\left(\mathrm{H}_{3} \mathrm{O}\right)_{0.2}\right]^{+}$
}

\author{
Tatiana G. Prokhorova ${ }^{1, *}$, Eduard B. Yagubskii ${ }^{1, *}$, Leokadiya V. Zorina ${ }^{2, *}$, Sergey V. Simonov ${ }^{2,3}$, \\ Vladimir N. Zverev ${ }^{2,3}{ }^{1}$ Rimma P. Shibaeva ${ }^{2}$ and Lev I. Buravov ${ }^{1}$ \\ 1 Institute of Problems of Chemical Physics of Russian Academy of Sciences, Chernogolovka 142432, Russia; \\ buravov@icp.ac.ru \\ 2 Institute of Solid State Physics of Russian Academy of Sciences, Chernogolovka 142432, Russia; \\ simonovsv@rambler.ru (S.V.S.); zverev@issp.ac.ru (V.N.Z.); shibaeva@issp.ac.ru (R.P.S.) \\ 3 Moscow Institute of Physics and Technology, Dolgoprudny 141701, Russia \\ * Correspondence: prokh@icp.ac.ru (T.G.P.); yagubski@icp.ac.ru (E.B.Y.); zorina@issp.ac.ru (L.V.Z.)
}

Received: 24 January 2018; Accepted: 8 February 2018; Published: 10 February 2018

\begin{abstract}
New crystals (1-4) of organic conductors based on the radical cation salts of the bis(ethylenedithio)tetrathiafulvalene (BEDT-TTF) with paramagnetic and diamagnetic tris(oxalato)metallate anions $\left\{\mathrm{A}^{+}\left[\mathrm{M}^{3+}(\mathrm{ox})_{3}\right]^{3-} \mathrm{G}\right\}^{2-}$, where $\mathrm{M}$ is $\mathrm{Cr}, \mathrm{Ga}$; $\mathrm{G}$ is 2-chloropyridine, 2-bromopyridine; and $\mathrm{A}^{+}$is $\left[\mathrm{K}_{0.8}\left(\mathrm{H}_{3} \mathrm{O}\right)_{0.2}\right]^{+}$have been prepared and their crystal structure and transport properties were studied. All crystals belong to the monoclinic group of the (BEDT-TTF $)_{4} \mathrm{~A}^{+}\left[\mathrm{M}^{3+}(\mathrm{ox})_{3}\right] \mathrm{G}$ family with $\beta^{\prime \prime}$-packing type of conducting BEDT-TTF layers. In contrast to the known superconducting crystals with $\mathrm{M}^{3+}=\mathrm{Fe}^{3+}$ and $\mathrm{G}=2$-chloro- or 2-bromopyridine $\left(T_{\mathrm{c}}=4.0-4.3 \mathrm{~K}\right)$, crystals with $\mathrm{Cr}^{3+}$ and $\mathrm{Ga}^{3+}$ ions exhibit metallic properties down to $0.5 \mathrm{~K}$ without superconducting transition. Upon cooling these crystals, the incommensurate superstructure appears, which has never been observed before in the numerous $\beta^{\prime \prime}$-salts of the family. In addition, orthorhombic (sp. group Pbca) semiconducting crystals $\alpha^{\prime \prime}$-(BEDT-TTF) $5\left[\mathrm{Ga}(\mathrm{ox})_{3}\right] \cdot 3 \cdot 4 \cdot \mathrm{H}_{2} \mathrm{O} \cdot 0.6$ EtOH (5) were obtained. It is a new compound in the family of BEDT-TTF crystals with tris(oxalato)metallate anions.
\end{abstract}

Keywords: molecular (super)conductors; superstructure; bifunctional compounds; radical cation salts; BEDT-TTF; tris(oxalato)metallate anions

\section{Introduction}

The great family of layered molecular conductors and superconductors based on radical cation salts of the bis(ethylenedithio)tetrathiafulvalene (BEDT-TTF) with supramolecular tris(oxalato)metallate anions includes compounds having a different stoichiometry, crystal structure, and various physical properties [1-33]. For example, the salt with the formula [BEDT-TTF $]_{3}\left[\mathrm{M}^{2+} \mathrm{M}^{3+}(\text { ox })_{3}\right]$, where $\mathrm{M}^{2+}$ is $\mathrm{Mn}^{2+}$ and $\mathrm{M}^{3+}$ is $\mathrm{Cr}^{3+}$ (3:1 salt), combine metallic conductivity and ferromagnetism in the same lattice [28]. The other group of salts with the stoichiometry 3:1 (BEDT-TTF) 3 [NaM(ox) $\left.)_{3}\right] \mathrm{G}$ (where $\mathrm{M}=\mathrm{Cr}, \mathrm{Al}$, and $\mathrm{G}=\mathrm{CH}_{2} \mathrm{Cl}_{2}, \mathrm{CH}_{3} \mathrm{NO}_{2}, \mathrm{DMF}$, $\mathrm{CH}_{3} \mathrm{CN}, \mathrm{C}_{2} \mathrm{H}_{5} \mathrm{OH}$ ) includes semiconducting chiral crystals [29-32]. Recently, a 2:1 superconducting salt was synthesized, (BEDT-TTF $)_{2}\left[\left(\mathrm{H}_{2} \mathrm{O}\right)\left(\mathrm{NH}_{4}\right)_{2} \mathrm{Rh}(\text { ox })_{3}\right] \cdot 18$-crown-6 [33].

The largest group of salts comprises paramagnetic (super)conductors (BEDT-TTF) ${ }_{4} \mathrm{~A}^{+}\left[\mathrm{M}^{3+}(\mathrm{ox})_{3}\right] \mathrm{G}$ (4:1 salts), where $\mathrm{A}^{+}=\mathrm{K}^{+}, \mathrm{NH}^{4+}, \mathrm{Rb}^{+}, \mathrm{H}_{3} \mathrm{O}^{+}$, and $\mathrm{M}=\mathrm{Fe}, \mathrm{Cr}, \mathrm{Mn}, \mathrm{Ru}, \mathrm{Rh}, \mathrm{Ga}, \mathrm{Al}, \mathrm{Co}$; and $\mathrm{G}$ is a neutral 'guest' solvent molecule [1-27]. The 4:1 crystals possess a great diversity of structural and conducting 
properties. Several series of different polymorphs have been synthesized by varying A, M, and G. Almost all superconducting family crystals belong to this group.

In the structure of 4:1 crystals, the conducting layers of BEDT-TTF radical cations alternate with supramolecular anionic layers $\left\{\mathrm{A}^{+}\left[\mathrm{M}^{3+}(\mathrm{ox})_{3}\right]^{3-} \mathrm{G}\right\}^{2-}$. The cationic and anionic layers are interconnected by hydrogen bonds that are formed between hydrogen atoms of terminal ethylene groups of BEDT-TTF and components of the complex anions.

Crystals of (BEDT-TTF $)_{4} \mathrm{~A}^{+}\left[\mathrm{M}^{3+}(\mathrm{ox})_{3}\right] \mathrm{G}$ family can be divided into three series according to the type of their symmetry: monoclinic [1-17,19,21,23-27], orthorhombic $[1,4,19,23,27]$, and triclinic [11,18-20,24,27]. The crystals of different series have different packing types of BEDT-TTF layers. Monoclinic crystals have the $\beta$ "-packing type, according to the structural classification of the salts of BEDT-TTF and its analogues $[34,35]$. The $\beta$ "-layers are composed of continuous stacks of radical cations, the planes of which are almost parallel and shifted with respect to the short axis of BEDT-TTF molecule. The interplanar distances in the stacks are considerably shortened in comparison with the normal van der Waals distances. There is a large number of shortened S . S S contacts between adjacent stacks in the layer. In the orthorhombic crystals with the 'pseudo- $\kappa^{\prime}$-type of packing, the organic layer is formed by charged $\left[(\mathrm{BEDT}-\mathrm{TTF})_{2}\right]^{2+}$ dimers surrounded by neutral [BEDT-TTF] molecules, which are perpendicular to the dimers. In the structure of triclinic crystals alternate conducting layers with two different packing types, $\alpha$ and $\beta$ " or $\alpha$ and 'pseudo- $\kappa^{\prime}$. In the $\alpha$-layer, the stacks of BEDT-TTF are inclined to one another.

The anionic layers have a honeycomb-like architecture: $\mathrm{M}^{3+}$ and $\mathrm{A}^{+}$cations linked by oxalate bridges alternate in vertices of the hexagonal network and form the hexagonal cavities in which neutral solvent molecules $\mathrm{G}$ are incorporated. The anion layers do not directly participate in conductivity, but the size and shape of G determine the crystal symmetry, the BEDT-TTF packing type and, hence, the conducting properties of crystals [1,3,17-20]. In contrast to $G$, the variation of $M$ (keeping the $G$ the same) in the composition of crystals does not lead to changes of the crystal structure, but affects the conducting properties. Thus, $T_{\mathrm{c}}$ of crystals with $\mathrm{G}=\mathrm{PhNO}_{2}$ gradually increases in a series of crystals with $\mathrm{M}=\mathrm{Cr}, \mathrm{Fe}$, $\mathrm{Ga}\left(5.8 \mathrm{~K}, 6.2 \mathrm{~K}, 7.5 \mathrm{~K}\right.$, respectively). When $\mathrm{G}=\mathrm{Py}$, the crystals with $\mathrm{Cr}^{3+}$ and $\mathrm{Fe}^{3+}$ ions exhibit metallic behavior of resistance down to low helium temperatures, while crystals with $\mathrm{Ga}^{3+}$ ions experience a superconducting transition with $T_{\mathcal{C}}<2 \mathrm{~K}$. The crystals with $\mathrm{G}=\mathrm{PhBr}\left(\mathrm{M}^{3+}=\mathrm{Cr}^{3+}\right.$, $\mathrm{Mn}^{3+}, \mathrm{Fe}^{3+}, \mathrm{Ru}^{3+}, \mathrm{Rh}^{3+}$ ) are superconductors with $T_{\mathrm{C}}=2 \mathrm{~K}, 1 \mathrm{~K}, 4 \mathrm{~K}, 4 \mathrm{~K}, 2.5 \mathrm{~K}$, respectively, while the salt with $\mathrm{Ga}^{3+}$ ions is a metal and does not transition to the superconducting state down to $0.4 \mathrm{~K}$.

The group of crystals with halogen-containing solvents $\mathrm{G}$ is the most interesting among monoclinic crystals of the family, because the structural phase transitions from monoclinic to triclinic state, which were not found in other monoclinic crystals of the family, occur in many of these crystals $\left(\mathrm{M}^{3+}=\mathrm{Fe}^{3+}\right)$ at low temperatures [21,22]. These transitions influence the ordering terminal ethylene groups in BEDT-TTF molecules $[21,22,36]$ and, hence, the conducting properties of the crystals.

In our recent work [25] we reported on monoclinic crystals containing different isomers of halopyridines (HalPy, where $\mathrm{Hal}=\mathrm{Cl}, \mathrm{Br}$ ) as guest solvents and $\mathrm{Fe}^{3+}$ as $\mathrm{M}^{3+}$. It was shown that structural phase transitions at $180 \mathrm{~K}$ and the superconducting transition at $4.0-4.3 \mathrm{~K}$ take place in crystals with $\mathrm{G}=2-\mathrm{ClPy}$ and 2-BrPy, while neither phase transitions occur in crystals with $\mathrm{G}=3-\mathrm{HalPy}(\mathrm{Hal}=\mathrm{Cl}, \mathrm{Br})$. Here we report the synthesis, crystal structure, and transport properties of new monoclinic crystals $\beta^{\prime \prime}$-(BEDT-TTF $)_{4}\left[\mathrm{~K}_{0.8}\left(\mathrm{H}_{3} \mathrm{O}\right)_{0.2}\right]\left[\mathrm{M}(\mathrm{ox})_{3}\right] \mathrm{G}$, where $\mathrm{M} / \mathrm{G}=\mathrm{Cr} / 2-\mathrm{ClPy}(\mathbf{1})$; $\mathrm{Cr} / 2-\mathrm{BrPy}$ (2); Ga/2-ClPy (3); Ga/2-BrPy (4). In addition, orthorhombic (sp. group Pbca) crystals $\alpha^{\prime \prime}$-(BEDT-TTF) $)_{5}\left[\mathrm{Ga}(\mathrm{ox})_{3}\right] \cdot 3.4 \cdot \mathrm{H}_{2} \mathrm{O} \cdot 0.6 \mathrm{EtOH}(5)$ were obtained. Up to the present, crystals of this composition were not known in the family of BEDT-TTF salts with tris(oxalato)metallate anions.

\section{Materials and Methods}

BEDT-TTF, 1,2,4-trichlorobenzene, 2-chloropyridine, 3-chloropyridine, 2-bromopyridine, and $\mathrm{K}_{3}\left[\mathrm{Cr}(\mathrm{ox})_{3}\right] \cdot 3 \mathrm{H}_{2} \mathrm{O}$ were used as received (SIGMA-ALDRICH CHEMIE, GmbH, Steinheim, Germany ); 
18-crown-6 (the same firm) was purified by recrystallization from acetonitrile and dried in vacuum at $30{ }^{\circ} \mathrm{C}$ over $\mathrm{P}_{2} \mathrm{O}_{5}$; and $\mathrm{K}_{3}\left[\mathrm{Ga}(\mathrm{ox})_{3}\right] \cdot 5 \mathrm{H}_{2} \mathrm{O}$ was synthesized according to the procedure described in [37].

\subsection{Synthesis}

Electrocrystallization of the charge transfer salts was performed in conventional two-compartment $\mathrm{H}$-shaped cells with $\mathrm{Pt}$ wire electrodes at constant current and temperature $\left(25^{\circ} \mathrm{C}\right)$. BEDT-TTF, the supporting electrolyte, 18-crown-6, and a solvent (or a mixture of solvents) was placed in the cathode compartment of the cell. The obtained solution was distributed between the two compartments of the cell. The exact conditions for the synthesis of each salt are described below.

\subsection{1. $\beta^{\prime \prime}-(\mathrm{BEDT}-\mathrm{TTF})_{4}\left[\mathrm{~K}_{0.8}\left(\mathrm{H}_{3} \mathrm{O}\right)_{0.2}\right]\left[\mathrm{Cr}(\mathrm{ox})_{3}\right] \cdot(2-\mathrm{ClPy})(\mathbf{1})$}

$24 \mathrm{mg}$ of BEDT-TTF, $170 \mathrm{mg}$ of $\mathrm{K}_{3}[\mathrm{Cr}(\mathrm{ox}) 3] \cdot 3 \mathrm{H}_{2} \mathrm{O}, 220 \mathrm{mg}$ of 18 -crown- 6 and the mixture of 2-ClPy (30 mL) with 1,2,4-trichlorobenzene $(4 \mathrm{~mL})$ and $96 \% \mathrm{EtOH}(3.5 \mathrm{~mL})$ were used. $\mathrm{J}=0.97 \mu \mathrm{A}$. Several crystals in the form of prisms were collected from the anode after 3 weeks.

\subsection{2. $\beta^{\prime \prime}-(\mathrm{BEDT}-\mathrm{TTF})_{4}\left[\mathrm{~K}_{0.8}\left(\mathrm{H}_{3} \mathrm{O}\right)_{0.2}\right]\left[\mathrm{Cr}(\mathrm{ox})_{3}\right] \cdot(2-\mathrm{BrPy})(2)$}

$25 \mathrm{mg}$ of BEDT-TTF, $50 \mathrm{mg}$ of $\mathrm{K}_{3}\left[\mathrm{Cr}(\mathrm{ox})_{3}\right] \cdot 3 \mathrm{H}_{2} \mathrm{O}, 200 \mathrm{mg}$ of 18-crown-6 and the mixture of 2-BrPy $(8 \mathrm{~mL})$ with 1,2,4-triclorobenzene $(10 \mathrm{~mL})$ and $96 \%$ ethanol $(3.5 \mathrm{~mL})$ were used. $\mathrm{J}=0.95 \mu \mathrm{A}$. Several crystals in the form of thick plates were collected from the anode after 10 days.

\subsection{3. $\beta^{\prime \prime}-(\mathrm{BEDT}-\mathrm{TTF})_{4}\left[\mathrm{~K}_{0.8}\left(\mathrm{H}_{3} \mathrm{O}\right)_{0.2}\right]\left[\mathrm{Ga}(\mathrm{ox})_{3}\right] \cdot(2-\mathrm{ClPy})(3)$ and $\left.\alpha^{\prime \prime}-(\mathrm{BEDT}-\mathrm{TTF})_{5}\right] \cdot 3.4 \cdot \mathrm{H}_{2} \mathrm{O} \cdot 0.6 \mathrm{EtOH}(5)$}

(a) $11 \mathrm{mg}$ of BEDT-TTF, $300 \mathrm{mg}$ of $\mathrm{K}_{3}\left[\mathrm{Ga}(\mathrm{ox})_{3}\right] \cdot 5 \mathrm{H}_{2} \mathrm{O}, 300 \mathrm{mg}$ of 18 -crown- 6 and the mixture of 2-ClPy $(20 \mathrm{~mL})$ with 1,2,4-trichlorobenzene $(10 \mathrm{~mL})$ and $96 \%$ ethanol $(3.5 \mathrm{~mL})$ were used. $\mathrm{J}=0.9 \mu \mathrm{A}$. Many shining black $\alpha^{\prime \prime}$-crystals (5) in the form of thin plates and a few small $\beta$ "-crystals (3) were collected from the anode after 14 days. After removing these crystals, $\beta$ "'-crystals (3) started to grow and a few of these crystals in the form of elongated plates were collected after 10 days.

(b) $25 \mathrm{mg}$ of BEDT-TTF, $300 \mathrm{mg}$ of $\mathrm{K}_{3}\left[\mathrm{Ga}(\mathrm{ox})_{3}\right] \cdot 5 \mathrm{H}_{2} \mathrm{O}, 300 \mathrm{mg}$ of 18 -krown-6; $20 \mathrm{~mL}$ of 2-ClPy, $10 \mathrm{~mL}$ of 1,2,4-trichlorobenzene, $3.5 \mathrm{~mL}$ of $96 \%$ ethanol were used. $\mathrm{J}=0.95 \mu \mathrm{A}$. A lot of $\alpha^{\prime \prime}$-crystals (5) in the form of thick plates were obtained after 15 days.

(c) $15 \mathrm{mg}$ of BEDT-TTF, $350 \mathrm{mg}$ of $\mathrm{K}_{3}\left[\mathrm{Ga}(\mathrm{ox})_{3}\right] \cdot 5 \mathrm{H}_{2} \mathrm{O}, 700 \mathrm{mg}$ of 18-krown-6; $20 \mathrm{~mL}$ of 3-ClPy, $10 \mathrm{~mL}$ of 1,2,4-trichlorobenzene, $2 \mathrm{~mL}$ of $96 \%$ ethanol were used. J $=0.95 \mu \mathrm{A}$. A few large thick $\alpha^{\prime \prime}$-crystals (5) were obtained after 10 days.

\subsection{4. $\beta^{\prime \prime}-(\mathrm{BEDT}-\mathrm{TTF})_{4}\left[\mathrm{~K}_{0.8}\left(\mathrm{H}_{3} \mathrm{O}\right)_{0.2}\right]\left[\mathrm{Ga}(\mathrm{ox})_{3}\right] \cdot(2-\mathrm{BrPy})(4)$}

$15 \mathrm{mg}$ of BEDT-TTF, $150 \mathrm{mg}$ of $\mathrm{K}_{3}\left[\mathrm{Ga}(\mathrm{ox})_{3}\right] \cdot 5 \mathrm{H}_{2} \mathrm{O}, 450 \mathrm{mg}$ of 18-krown-6; $10 \mathrm{~mL}$ of 1,2,4-trichlorobenzene, $20 \mathrm{~mL}$ of 2-BrPy, $3 \mathrm{~mL}$ of $96 \%$ ethanol were used. J $=0.95 \mu \mathrm{A}$. A few crystals (4) in the form of thick plates were collected from the anode after three weeks.

\subsection{Crystal Structure}

Single crystal X-ray diffraction experiments were carried out on an Oxford Diffraction Gemini-R CCD diffractometer $(\lambda(\mathrm{MoK} \alpha)=0.71073 \AA$, graphite monochromator, $\omega$-scan mode). Data reduction with empirical absorption correction of experimental intensities (Scale3AbsPack program) was made with the CrysAlisPro software [38]. The structures were solved by the direct method and refined by the full-matrix least-squares technique against $F^{2}$ in an anisotropic approximation for all non-hydrogen atoms using the SHELX-2016 software package [39]. H-atoms were placed in idealized positions and refined using a riding model with $U_{\text {iso }}(\mathrm{H})$ depending on $U_{\text {eq }}$ of the parent $\mathrm{C} / \mathrm{O}$ atom. Hydrogen atoms in water molecules (structure 5) were localized from the Fourier synthesis of the electron density and refined with $U_{\text {iso }}(\mathrm{H})=1.5 U_{\text {eq }}(\mathrm{O})$. Hydrogen atoms in the $\left(\mathrm{H}_{3} \mathrm{O}\right)^{+}$cations were not localized, but added into the chemical formula of $\mathbf{1 - 4}$. Selected crystallographic data and refinement parameters are given 
in Table 1. The full data of studies are available at the Cambridge Crystallographic Data Centre via www.ccdc.cam.ac.uk/data_request/cif (CCDC 1818020-1818024).

Table 1. Crystal structure and refinement data.

\begin{tabular}{|c|c|c|c|c|c|}
\hline & 1 & 2 & 3 & 4 & 5 \\
\hline \multirow{2}{*}{ Chemical formula } & $\mathrm{C}_{51} \mathrm{H}_{36.6} \mathrm{ClCr}$ & $\mathrm{C}_{51} \mathrm{H}_{36.6} \mathrm{BrCr}$ & $\mathrm{C}_{51} \mathrm{H}_{36.6} \mathrm{ClGa}$ & $\mathrm{C}_{51} \mathrm{H}_{36.6} \mathrm{BrGa}$ & $\mathrm{C}_{56.6} \mathrm{H}_{48.6} \mathrm{Ga}$ \\
\hline & $\mathrm{K}_{0.8} \mathrm{NO}_{12.2} \mathrm{~S}_{32}$ & $\mathrm{~K}_{0.8} \mathrm{NO}_{12.2} \mathrm{~S}_{32}$ & $\mathrm{~K}_{0.8} \mathrm{NO}_{12.2} \mathrm{~S}_{32}$ & $\mathrm{~K}_{0.8} \mathrm{NO}_{12.2} \mathrm{~S}_{32}$ & $\mathrm{O}_{15.7} \mathrm{~S}_{40}$ \\
\hline Formula weight & 2003.26 & 2047.72 & 2020.98 & 2065.44 & 919.70 \\
\hline Temperature (K) & 270 & 295 & 295 & 295 & 120 \\
\hline Cell setting & monoclinic & monoclinic & monoclinic & monoclinic & orthorhombic \\
\hline Space group, $Z$ & $C 2 / c, 4$ & $C 2 / c, 4$ & $C 2 / c, 4$ & $C 2 / c, 4$ & $\mathrm{Pbca}, 8$ \\
\hline$a(\AA)$ & $10.2791(6)$ & $10.2971(2)$ & $10.27760(10)$ & $10.27515(15)$ & $22.0871(4)$ \\
\hline$b(\AA)$ & $20.0020(9)$ & $20.0382(5)$ & 19.9066(3) & $20.0182(4)$ & $21.0853(4)$ \\
\hline$c(\AA)$ & $35.4485(15)$ & $35.4621(8)$ & $35.5504(5)$ & $35.5972(5)$ & $35.6286(7)$ \\
\hline$\alpha\left({ }^{\circ}\right)$ & 90 & 90 & 90 & 90 & 90 \\
\hline$\beta\left(^{\circ}\right)$ & $92.948(4)$ & $93.2943(19)$ & $92.9970(10)$ & $93.2026(13)$ & 90 \\
\hline$\gamma\left({ }^{\circ}\right)$ & 90 & 90 & 90 & 90 & 90 \\
\hline Cell volume $\left(\AA^{3}\right)$ & $7278.7(6)$ & $7305.0(3)$ & $7263.4(2)$ & $7310.5(2)$ & $16592.7(5)$ \\
\hline Crystal size (mm) & $0.53 \times 0.25 \times 0.12$ & $0.35 \times 0.34 \times 0.21$ & $0.42 \times 0.30 \times 0.15$ & $0.61 \times 0.27 \times 0.12$ & $0.83 \times 0.18 \times 0.03$ \\
\hline$\rho\left(\mathrm{Mg} / \mathrm{m}^{3}\right)$ & 1.828 & 1.862 & 1.848 & 1.877 & 1.867 \\
\hline$\mu, \mathrm{cm}^{-1}$ & 12.17 & 17.23 & 14.41 & 19.42 & 14.02 \\
\hline \multicolumn{6}{|l|}{ Refls } \\
\hline $\begin{array}{c}\text { collected/unique/observed } \\
\text { with } I>2 \sigma(I)\end{array}$ & $37,634 / 10,476 / 8631$ & $17,952 / 9860 / 8119$ & $17,486 / 9629 / 8063$ & $33,996 / 10,184 / 8082$ & $64,803 / 23,232 / 17,944$ \\
\hline$R_{\text {int }}$ & 0.0291 & 0.0154 & 0.0285 & 0.0187 & 0.0263 \\
\hline$\theta_{\max }\left({ }^{\circ}\right)$ & 31.12 & 30.36 & 29.93 & 30.60 & 30.72 \\
\hline Parameters refined & 608 & 606 & 612 & 606 & 1076 \\
\hline Final $R_{1}(\mathrm{obs}), w R_{2}$ (all) & $0.0463,0.1217$ & $0.0637,0.1962$ & $0.0445,0.1203$ & $0.0535,0.1586$ & $0.0425,0.1101$ \\
\hline Goodness-of-fit & 1.004 & 1.004 & 1.005 & 1.003 & 1.005 \\
\hline $\begin{array}{l}\text { Residual electron density } \\
\left(\mathrm{e} \AA^{-3}\right)\end{array}$ & $1.188 /-0.695$ & $1.722 /-1.937$ & $1.039 /-0.728$ & $2.068 /-1.174$ & $2.767 /-1.492$ \\
\hline CCDC reference & 1818020 & 1818021 & 1818022 & 1818023 & 1818024 \\
\hline
\end{tabular}

\subsection{Conducting Properties}

The temperature dependencies of the electrical resistance of single crystals was measured using a four-probe technique by a lock-in detector at $20 \mathrm{~Hz}$ alternating current, $\mathrm{J}=1 \mu \mathrm{A}$. Two contacts were attached to each of two opposite sample surfaces with conducting graphite paste. In the experiment we have measured the out-of-plane resistance $\mathrm{R}_{\perp}$ with the current running perpendicular to conducting layers. The magnetotransport measurements at low temperatures down to $0.5 \mathrm{~K}$ were carried out in a cryostat with a superconducting solenoid, which generated a magnetic field of up to $17 \mathrm{~T}$.

\section{Results and Discussion}

\subsection{Synthesis}

It was known that crystals of orthorhombic 'pseudo- $\kappa^{\prime}$-phases are obtained together with monoclinic $\beta$ "-crystals in some cases [1,19] (concomitant polymorphism). In this work, we have shown that the use of 2-ClPy as a guest solvents and $\left[\mathrm{Ga}(\mathrm{ox})_{3}\right]^{3-}$ as a counterion leads to the formation of both $\beta^{\prime \prime}-(\mathrm{BEDT}-\mathrm{TTF})_{4}\left[\mathrm{~K}_{0.8}\left(\mathrm{H}_{3} \mathrm{O}\right)_{0.2}\right]\left[\mathrm{Ga}(\mathrm{ox})_{3}\right] \cdot 2-\mathrm{ClPy}(3)$ and $\alpha^{\prime \prime}-(\mathrm{BEDT}-\mathrm{TTF})_{5}\left[\mathrm{Ga}(\mathrm{ox})_{3}\right] \cdot 3.4 \cdot \mathrm{H}_{2} \mathrm{O} \cdot 0.6$ $\mathrm{EtOH}(5)$ (sp. group $\mathrm{Pbca}$ ) crystals in the same synthesis. Crystal 5 was not previously found in this family. It should be noted that $\beta^{\prime \prime}$ crystals start to grow much later then $\alpha^{\prime \prime}$ crystals. When $\mathrm{G}=3-\mathrm{ClPy}$, only $\alpha^{\prime \prime}$ crystals are formed (see Section 2.1 .3 (c)).

\subsection{Crystal Structure}

3.2.1. $\beta^{\prime \prime}-(\mathrm{BEDT}-\mathrm{TTF})_{4}\left[\mathrm{~K}_{0.8}\left(\mathrm{H}_{3} \mathrm{O}\right)_{0.2}\right]\left[\mathrm{M}(\mathrm{ox})_{3}\right] \cdot 2-\mathrm{HalPy}$, where $\mathrm{M} / \mathrm{Hal}=\mathrm{Cr} / \mathrm{Cl}(\mathbf{1}), \mathrm{Cr} / \mathrm{Br}(\mathbf{2}), \mathrm{Ga} / \mathrm{Cl}(\mathbf{3})$ and $\mathrm{Ga} / \mathrm{Br}(4)$

Compounds 1-4 crystallize in the monoclinic $C 2 / c$ space group with one half of the formula unit in the crystallographic asymmetric unit (Figure 1). They are isostructural to the series of $\beta^{\prime \prime}$ salts of the 
$(\text { BEDT-TTF })_{4} \mathrm{~A}^{+}\left[\mathrm{M}^{3+}(\mathrm{ox})_{3}\right] \cdot \mathrm{G}$ family, but have a specific disorder in the anion part which is described in the caption to Figure 1.

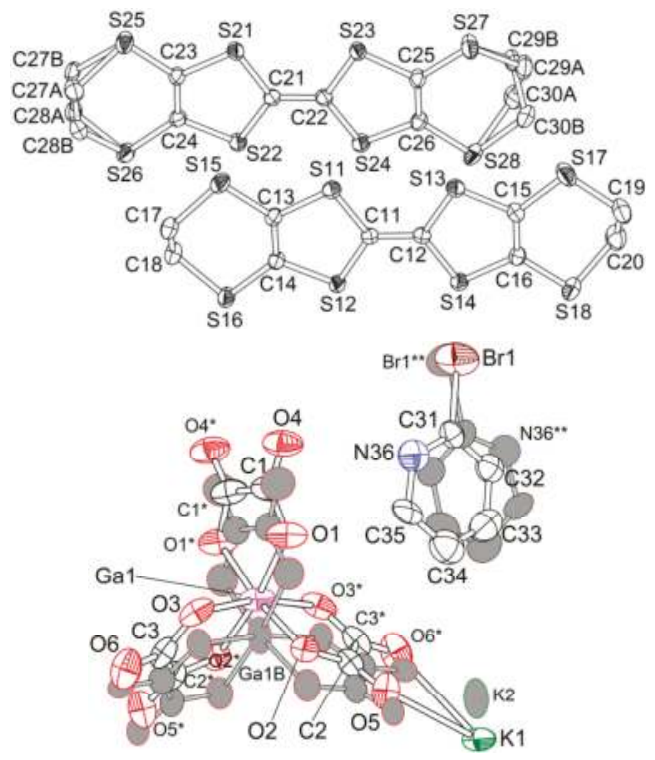

Figure 1. Asymmetric unit in 4 (30\% level of thermal ellipsoids). Symmetry codes: $\left(^{*}\right)(1-x, y, 1.5-z)$, $\left.{ }^{* *}\right)(-x, y, 1.5-z)$. Anion $\left[\mathrm{Ga}(\mathrm{ox})_{3}\right]^{3-}$ and cation $\mathrm{K}^{+}$are in the special positions on a two-fold axis, and the solvent is in the general position near a two-fold axis. The $\mathrm{K} 1$ site is occupied by a mixture of $\mathrm{K}^{+} / \mathrm{H}_{3} \mathrm{O}^{+}$cations in a ratio of $0.60 / 0.20$ in 1 and $4,0.56 / 0.20$ in 2 and $0.68 / 0.20$ in 3 . There is a second independent position of the anion and $\mathrm{K}^{+}$cation (grey color) with occupancy of $\mathrm{Cr} 1 \mathrm{~B}(\mathrm{Ga} 1 \mathrm{~B}) / \mathrm{K} 2$ sites of $0.25 / 0.20$ in 1 and $4,0.32 / 0.24$ in 2 and $0.12 / 0.12$ in 3 .

The crystal structure consists of radical cation and anion layers alternating along the $c$-axis of the lattice (Figure 2a). The radical cation layer has a $\beta^{\prime \prime}$-type of molecular packing (Figure $2 b$ ). Two independent BEDT-TTF radical cations, A and B, form stacks in ... -(ABBA)-(ABBA)- ... sequence with both longitudinal and transversal shift of the adjacent BEDT-TTF within the (ABBA) tetrad and only transversal displacement between the tetrads (Figure 2c). Intermolecular separations along the

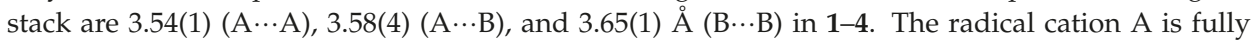
ordered at room temperature, whereas both terminal ethylene groups of $\mathrm{B}$ are disordered in two sites with the occupation ratio of $0.67 / 0.33$ and $0.52 / 0.48$ in $1,0.67 / 0.33$ and $0.62 / 0.38$ in $2,0.63 / 0.37$ and $0.57 / 0.43$ in 3, $0.70 / 0.30$ and $0.60 / 0.40$ in 4 . Analysis of bond length distributions in BEDT-TTF radical cations [40] gives equal charge on $\mathrm{A}$ and $\mathrm{B}$ of $0.5+$ in accordance with stoichiometry of the compound. The shortest S...S contacts in the conducting layer are side-by-side ones between BEDT-TTF from adjacent stacks in the range of 3.334-3.570(1) ^ (dashed bonds in Figure 2b). 


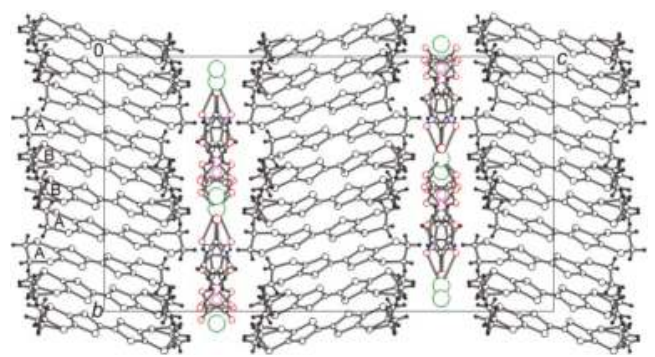

(a)

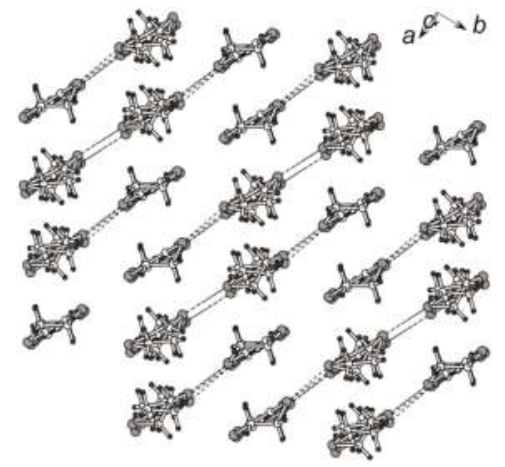

(b)

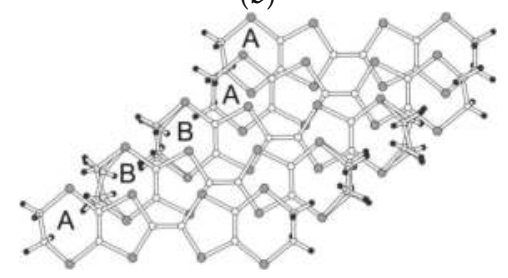

(c)

Figure 2. (a) Projection of the structure of 4 along $a$ (the minor part of the $\left[\mathrm{Ga}(\mathrm{ox})_{3}\right]^{3-}$ anion is omitted, except for Ga1B and K2 atoms); (b) view of the radical cation $\beta$ "-layer along the long axis of BEDT-TTF with short intermolecular S...S contacts $<3.6 \AA$ (dashed lines); and (c) overlap modes within the stack.

The anion layer in 1-4 shows the additional positional disorder of the $\left[\mathrm{M}^{3+}(\mathrm{ox})_{3}\right]^{3-}$ anion and small monovalent $\mathrm{A}^{+}$cation. Such a type of disorder has never been observed before in the numerous $\beta^{\prime \prime}$-salts of the (BEDT-TTF) $\mathrm{A}^{+}\left[\mathrm{M}^{3+}(\mathrm{ox})_{3}\right] \cdot G$ family. It is roughly described in the room temperature structures of 1-4 as a shift of the anion unit and $\mathrm{A}^{+}$cations along the two-fold monoclinic axis by about $1 \AA$, the shift for $\mathrm{M}^{3+}$ and $\mathrm{A}^{+}$centers being in opposite directions (Figures 1 and 3). Maximal occupation of the second position is observed in 2: 0.32 for $\mathrm{Cr} 1 \mathrm{~B}$ and 0.24 for $\mathrm{K} 2$. The amount of the second position in 3 ( 0.12 for both Ga1B and K2) is about two times less than in $\mathbf{1}$ and $\mathbf{4}(0.25$ for Cr1B or Ga1B and 0.20 for K2). Molecules with major occupancy form typical $\beta$ "-salts of the family's honeycomb-like packing with guest solvent molecules filling the hexagonal cavities of the anion layer (Figure 3). Each anion layer contains only one enantiomer of the chiral anion, but th whole crystal is racemic due to alternation of the enantiopure layers of different chirality along $c$. Contacts between $\mathrm{K} 1$ site occupied by mixture of $\mathrm{K}^{+} / \mathrm{H}_{3} \mathrm{O}^{+}$cations and oxygen atoms of surrounding oxalate groups are in the range of 2.87-3.08 $\AA$ in 1, 2.88-3.13 $\AA$ in 2, 2.85-2.97 $\AA$ in 3 and 2.91-3.11 $\AA$ in 4 (they are shown by red dashed 
lines in Figure 3). Upon cooling crystals 1-4, the incommensurate superstructure appears, which is apparently connected with specific ordering in the anion layer and will be described elsewhere.

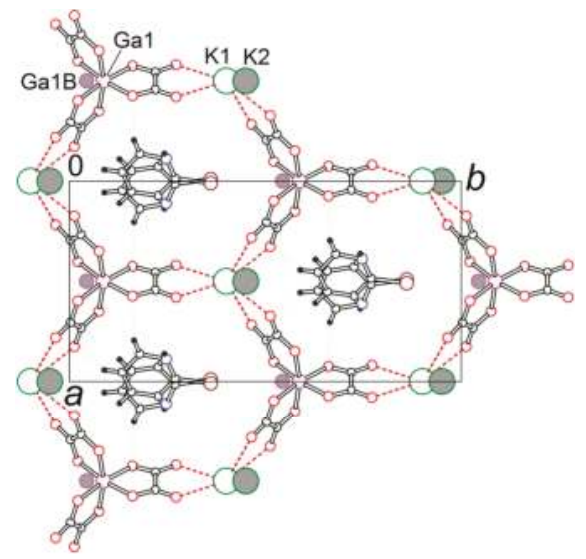

Figure 3. Anion layer in 4. $\mathrm{K} 2$ and Ga1B atoms of minor occupancy are shown in grey; oxalate groups of minor part of the anions are omitted for clarity.

\subsection{2. $\alpha "$-(BEDT-TTF) ${ }_{5}\left[\mathrm{Ga}(\mathrm{ox})_{3}\right] \cdot 3.4 \cdot \mathrm{H}_{2} \mathrm{O} \cdot 0.6 \mathrm{EtOH}$ (5)}

Compound 5 crystallizes in the orthorhombic Pbca space group. The asymmetric unit contains five independent BEDT-TTF molecules, one $\left[\mathrm{Ga}(\mathrm{ox})_{3}\right]^{3-}$ anion, four $\mathrm{H}_{2} \mathrm{O}$ molecules, two of which have an occupancy of 0.7 and are mixed in the same site with $0.3 \mathrm{EtOH}$ molecule (Figure 4).

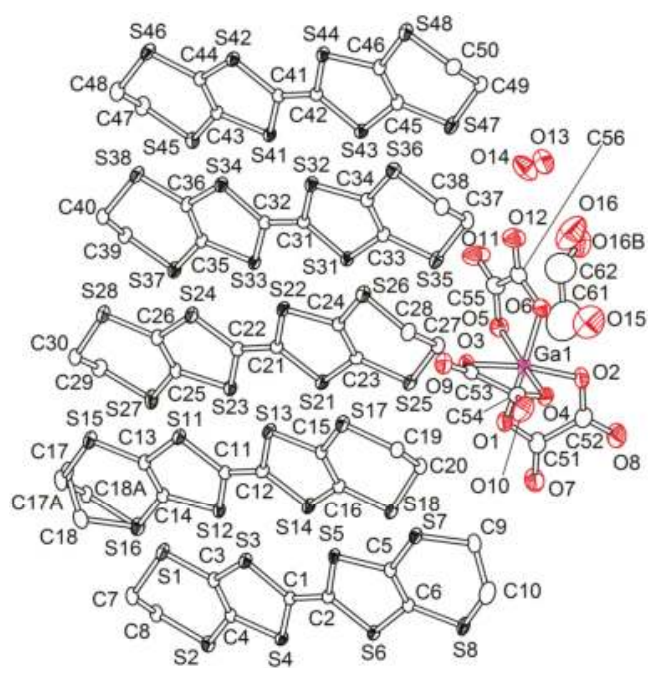

Figure 4. Asymmetric unit in 5 (50\% level of thermal ellipsoids).

The structure of $\mathbf{5}$ is composed of radical cation and anion layers alternating along the $c$-axis (Figure 5). Water and ethanol molecules are included into the anion layer. According to the X-ray data, neither 2-chloropyridine solvent nor $\mathrm{K}^{+}$cation is found in the composition of $\mathbf{5}$. 


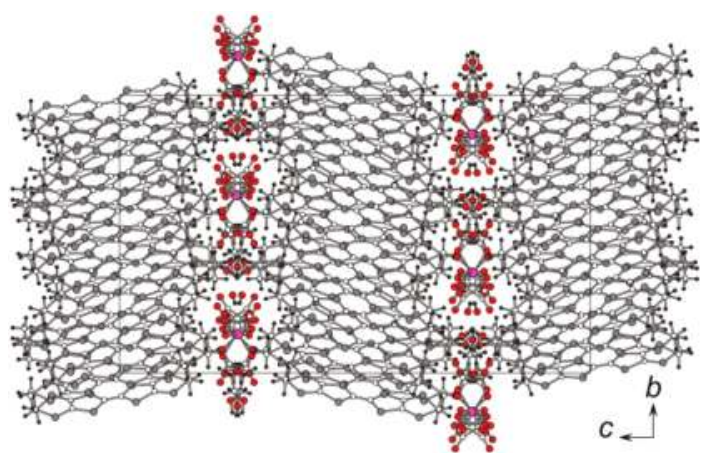

Figure 5. Projection of the structure 5 along a.

The conducting layer has an $\alpha^{\prime \prime}$-type of molecular packing (Figure 6a). It consists of the stacks with a ... (BCDEA)-(BCDEA) ... repeating sequence of BEDT-TTF radical cations (Figure $6 \mathrm{~b}$ ). In the pair of neighbor stacks ( 1 and 2 in Figure $6 \mathrm{a}$ ) the molecular planes are near parallel to each other (angles $\left.0.48-2.85(2)^{\circ}\right)$ whereas all the molecules in the next pair of stacks $(3,4)$ are tilted relative to the molecules from the first pair by an angle of $53(2)^{\circ}$. Radical cations inside the pentad (BCDEA) overlap with a pure transversal shift of adjacent units; longitudinal displacement is added only between the pentads in A $\cdots$ B interaction (Figure 6c). Intrastack interplanar distances are shown in Figure 6b. The maximal interplanar separation is observed between the pentads in the A $\cdots \mathrm{B}$ pair. Only one ethylene group (C17-C18) in BEDT-TTF B is disordered at $120 \mathrm{~K}$ between two orientations of 0.4/0.6 occupancy. The ethylene groups of molecules B (major part), C, D, and E are eclipsed and the sole BEDT-TTF A has a staggered conformation. The overall charge on five BEDT-TTF units is +3 according to the formula of the salt. Calculations of the charge state using the empirical formula for BEDT-TTF compounds [40] show a non-uniform charge distribution along the stack with values of $+0.62,+0.37$, $+0.76,+0.83$, and +0.53 for $\mathrm{A}, \mathrm{B}, \mathrm{C}, \mathrm{D}$, abd E radical cations, respectively, which gives a total charge of +3.11 , which is in good agreement with the stoichiometric +3 value. There are many interstack $S$ ... S contacts less than sum of van der Waals radii (3.6 $\AA$ ) shown by dashed lines in Figure 6 a, but no intrastack one.

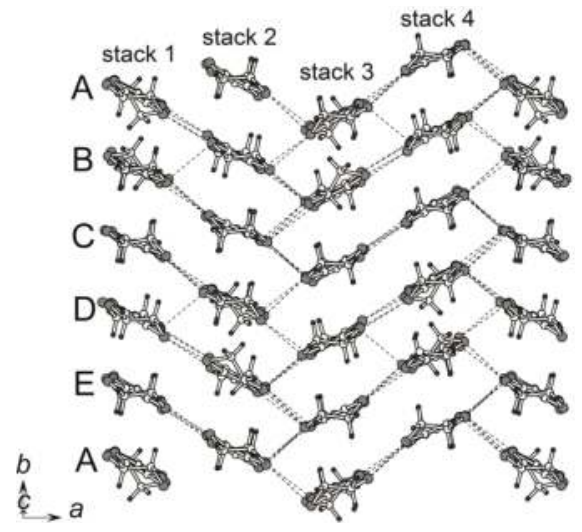

(a)

Figure 6. Cont. 


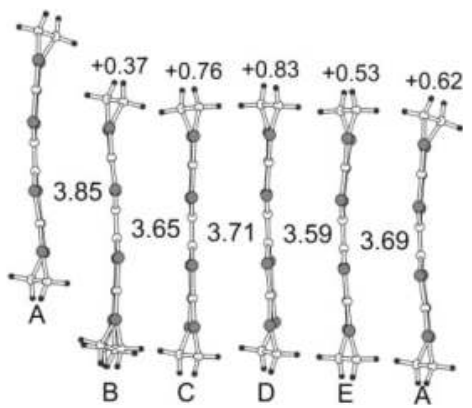

(b)

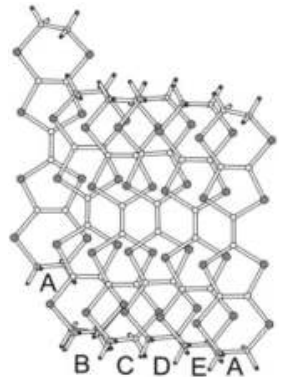

(c)

Figure 6. (a) View of the radical cation layer of $\alpha^{\prime \prime}$-type in 5 along the long molecular axis. Short S $\cdots S$ contacts $<3.6 \AA$ are shown by dashed lines; (b) side view of the stack with interplanar separations (in $\AA$ ) and calculated charges of BEDT-TTF; and (c) overlap modes within the stack.

The anion layer in 5 consists of $\left[\mathrm{Ga}(\mathrm{ox})_{3}\right]^{3-}$ anions interacting by hydrogen bonding through water molecules (Figure 7).

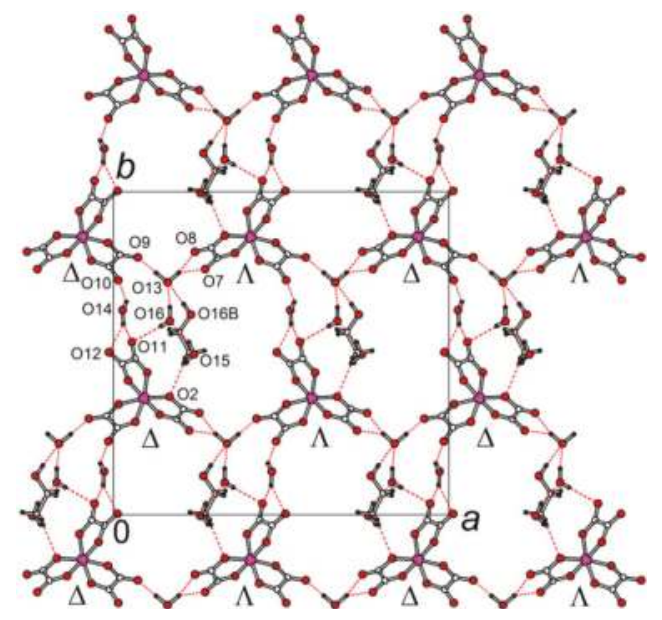

Figure 7. The anion layer in 5. $\mathrm{O}-\mathrm{H} \ldots$ O hydrogen bonds are shown by red dashed lines.

Right-handed $\Delta$ and left-handed $\Lambda$ isomers of the chiral anion alternate along the $a$ direction. Water molecules $\mathrm{O}(13)$ connect the different enantiomers of the anion along $a$ and $\mathrm{O}(14)$ join similar enantiomers along $b$, resulting to a network of tilted parallelograms having $\mathrm{Ga}$ atoms at the vertices. Additional disordered water and ethanol molecules are located in the free cavity near one side of parallelogram and form hydrogen bonds with the anion and water molecule $\mathrm{O}(13)$.

$\alpha^{\prime \prime}$-packing of the radical cation layer has been found before in one of BEDT-TTF salts with tris(oxalato)metallate anions $\alpha^{\prime \prime}$-(BEDT-TTF) ${ }_{4} \mathrm{H}_{2} \mathrm{OLi}\left[\mathrm{Fe}(\mathrm{ox})_{3}\right.$ ] solvent [41]. This salt differs from 5 by a cation to anion ratio (4:1 vs. 5:1), as well as the anion layer structure and possesses a semiconductor behavior with activation energy of $0.080 \mathrm{eV}$ and a room temperature resistivity $\rho_{\mathrm{RT}}$ of $2.41 \mathrm{Ohm} \mathrm{cm}$. Another salt with the same as in 5 composition of 5:1 is known among BEDT-TTF conductors with $\left[\mathrm{M}(\mathrm{ox})_{3}\right]^{3-}$ anions: (BEDT-TTF) 5 [ $\left.\mathrm{Fe}(\mathrm{ox})_{3}\right] \cdot\left(\mathrm{H}_{2} \mathrm{O}\right)_{2} \cdot \mathrm{CH}_{2} \mathrm{Cl}_{2}$ [42]. It differs from 5 by both symmetry and crystal structure. The anion layer is also build of parallelograms, but they are arranged in another way 
than in 5 because each layer is chiral. The conducting layer has close to $\beta^{\prime \prime}$-type of BEDT-TTF-packing and the crystal is a paramagnetic semiconductor.

\subsection{Conducting Properties}

Temperature dependencies of the out-of-plane resistance $R_{\perp}$ for samples $\mathbf{1 - 4}$ are presented in Figure 8. One can see that all the samples show the metallic behavior and the R(T) dependencies for all samples look very similar. Unlike to these samples, the sample 5 demonstrates the dielectric behavior (see Figure 9) with the activation energy $71 \mathrm{meV}$ as it is seen from the inset in Figure 9. For the samples 1-4 at $\mathrm{T} \leq 10 \mathrm{~K}$ the derivative of the $\mathrm{R}(\mathrm{T})$ dependencies changes the sign: the resistance starts to grow when the temperature decreases. This growth is more pronounced for sample $\mathbf{3}$, and is comparatively small for sample 2. This growth is shown in the inset in Figure 8 for samples $\mathbf{1}$ and $\mathbf{4}$, where the sample resistance $R(T) / R(290 K)$ is plotted as function of $T$ in the logarithmic scale.

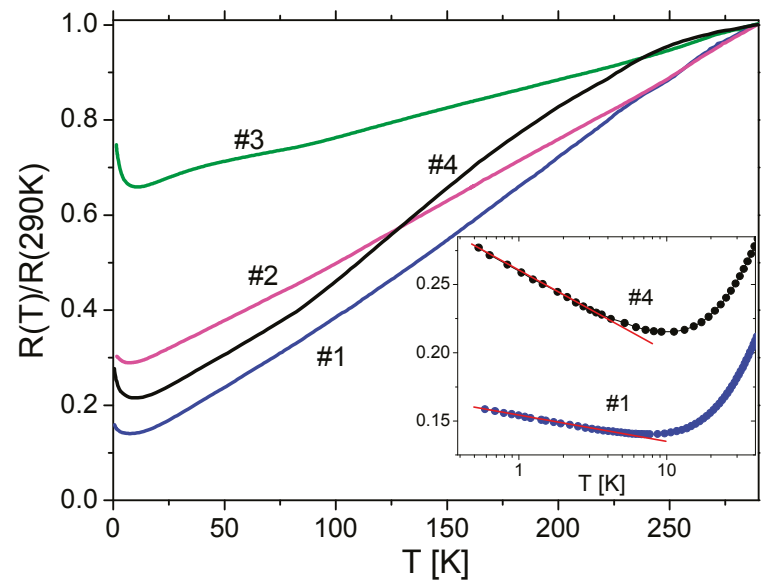

Figure 8. The temperature dependencies of the out-of-plane resistance $R_{\perp}(T)$ for crystals 1-4. The $\mathrm{R}(290 \mathrm{~K})$ values are $287 \mathrm{Ohm}, 3.77 \mathrm{kOhm}, 2.52 \mathrm{kOhm}$, and $427 \mathrm{Ohm}$, respectively. The inset shows the resistance $R(T) / R(290 K)$ as function of $T$ in the logarithmic scale for the samples 1 and 4 .

We have made an attempt to find the Shubnikov-de Haas oscillations in the magnetoresistance for our samples at $\mathrm{T}=0.5 \mathrm{~K}$ in magnetic fields up to $17 \mathrm{~T}$, but, unfortunately, without success.

The growth of the sample resistance at low temperatures $\mathrm{T} \leq 10 \mathrm{~K}$ could be attributed to the following mechanisms: (i) by the localization effects due to the quantum interference of the carriers at the presence of elastic scattering processes [43] or (ii) by the scattering of the carriers on the magnetic impurities due to the so-called Kondo effect [44]. Both of these two effects lead to the logarithmic temperature dependence for the sample resistance in quasi two-dimensional systems. In our samples the magnetic impurities $(\mathrm{Cr})$ are only in samples $\mathbf{1}$ and $\mathbf{2}$. The fact that we observe the low-temperature resistance growth in all our samples means that we deal with the localization effects due to the quantum interference of the carriers [43]. This effect is characteristic for the systems with disorder which, in our case, seem to be the reason not only for the quantum localization effects on $\mathrm{R}(\mathrm{T})$ dependence, but also for the absence of the Shubnikov-de Haas oscillations, because the high scattering rate leads to the broadening of the Landau levels and to the smearing of the quantum oscillations. Most likely, the absence of Shubnikov-de Haas oscillations is associated with the specific disorder in the anion layer (see Section 2.2) which is observed in the $\beta^{\prime \prime}-\left(\right.$ BEDT-TTF) $A^{+}\left[M^{3+}(\text { ox })_{3}\right] \cdot G$ crystals for the first time and causes appearance of incommensurate structural modulation at low temperatures. The incommensurability in the anion layer creates the perturbative potential for the 
carriers in the conducting layer, which acts as disorder for the carriers moving in the periodical crystal lattice. This disorder certianly leads to additional scattering and to the broadening of the Landau levels.

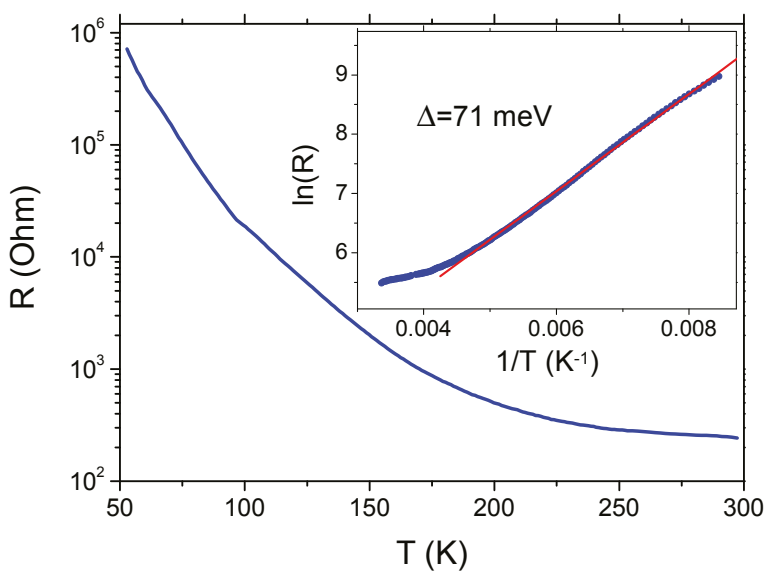

Figure 9. The temperature dependencies of the out-of-plane resistance $R_{\perp}(T)$ for crystal 5. The inset demonstrates the activation behavior of the sample resistance at $\mathrm{T}<215 \mathrm{~K}$.

\section{Conclusions}

Four new radical cation salts of the family of layered molecular (super)conductors based on bis(ethylenedithio)tetrathiafulvalene (BEDT-TTF) with tris(oxalato)metallate anions (BEDT-TTF $)_{4}\left\{\mathrm{~A}^{+}\left[\mathrm{M}^{3+}(\mathrm{ox})_{3}\right] \mathrm{G}\right\}$, where M/G = Cr/2-ClPy (1); Cr/2-BrPy (2); Ga/2-ClPy (3), Ga/2-BrPy (4); and $\mathrm{A}^{+}=\left[\mathrm{K}_{0.8}\left(\mathrm{H}_{3} \mathrm{O}\right)_{0.2}\right]^{+}$were obtained. These salts belong to the monoclinic group of the family with a $\beta^{\prime \prime}$-packing type of radical cation layers. The conducting and structural properties of $\beta^{\prime \prime}$-crystals strongly depend on the size, shape, and chemical nature of the guest solvent molecule G. In some crystals with $\mathrm{G}=$ halobenzenes and $\mathrm{M}=\mathrm{Fe}$, the structural phase transition from the monoclinic to the triclinic state was found upon lowering the temperature $[21,22,36]$. In our recent work we showed that the same transition also exists in superconducting Fe crystals with $\mathrm{G}=2-\mathrm{ClPy}$ and $2-\mathrm{BrPy}\left(T_{\mathrm{c}}=4.0 \mathrm{~K}\right.$ and $4.3 \mathrm{~K}$, respectively) [25]. These structural transitions arise from noticeable positional shifts of all components of the complex anion, giving rise to two nonequivalent organic $\beta$ " layers and the partial ordering of the ethylene groups of BEDT-TTF molecules. In the present paper, we found that the change of the $\mathrm{Fe}^{3+}$ ion by $\mathrm{Cr}^{3+}$ or $\mathrm{Ga}^{3+}$ ions, as well as the $\left(\mathrm{H}_{3} \mathrm{O}\right)^{+}$cation by $\left[\mathrm{K}_{0.8}\left(\mathrm{H}_{3} \mathrm{O}\right)_{0.2}\right]^{+}$in one of the compositions of $\beta^{\prime \prime}-(\mathrm{BEDT}-\mathrm{TTF})_{4}\left\{\mathrm{~A}^{+}\left[\mathrm{M}^{3+}(\mathrm{ox})_{3}\right](2-\mathrm{HalPy})\right\}$ crystals leads to the appearance of a specific disorder in anionic layers associated with the position disorder of the $\left[\mathrm{M}^{3+}(\mathrm{ox})_{3}\right]^{3-}$ anion and the small monovalent $\mathrm{A}^{+}$cation. Such a type of disorder has never been observed before in the numerous $\beta^{\prime \prime}$-salts of the (BEDT-TTF) $\mathrm{A}^{+}\left[\mathrm{M}^{3+}(\mathrm{ox})_{3}\right] \cdot \mathrm{G}$ family. Unlike the crystals with $\mathrm{Fe}^{3+}$ ions, the crystals with $\mathrm{Cr}^{3+}$ and $\mathrm{Ga}^{3+}$ ions do not exhibit a superconducting transition down to $0.5 \mathrm{~K}$. At $\mathrm{T}$ $\leq 10 \mathrm{~K}$, they demonstrate a weak growth of resistance. The fact that we observed the low-temperature resistance growth in all our samples (with magnetic $\mathrm{Cr}^{3+}$ and non-magnetic $\mathrm{Ga}^{3+}$ ions) means that we experienced the localization effects due to the quantum interference of the carriers [43]. This effect is characteristic for the systems with disorder which, in our case, seems to be the reason not only for the quantum localization effects on $\mathrm{R}(\mathrm{T})$ dependencies, but also for the absence of the Shubnikov-de Haas oscillations, because the high scattering rate leads to the broadening of the Landau levels and to the smearing of the quantum oscillations. 
Acknowledgments: The work was carried out within the state assignments for ISSP RAS and for IPCP RAS, theme No. 0089-2014-0026, and partially supported by the Russian Foundation for Basic Research (RFBR), grant No. $18-02-00280$.

Author Contributions: T.G.P., E.B.Y. conceived, designed, and performed the chemical experiments and also wrote the Abstract, Introduction, Synthesis, and Conclusions; L.V.Z., S.V.S., and R.P.S. performed the X-ray experiments, analyzed the X-ray data, and described them (the section titled "Crystal Structure"); and V.N.Z. and L.I.B. performed the conductivity measurement on the crystals and wrote the section titled "Conducting Properties".

Conflicts of Interest: The authors declare no conflict of interest. The founding sponsors had no role in the design of the study; in the collection, analyses, or interpretation of data; in the writing of the manuscript; or in the decision to publish the results.

\section{References}

1. Kurmoo, M.; Graham, A.W.; Day, P.; Coles, S.J.; Hursthouse, M.B.; Caufield, J.L.; Singleton, J.; Pratt, F.L.; Hayes, W.; Ducasse, L.; et al. Superconducting and Semiconducting Magnetic Charge Transfer Salts: (BEDT-TTF $)_{4} \mathrm{AFe}\left(\mathrm{C}_{2} \mathrm{O}_{4}\right)_{3} \mathrm{C}_{6} \mathrm{H}_{5} \mathrm{CN} \mathrm{A}=\mathrm{H}_{2} \mathrm{O}, \mathrm{K}, \mathrm{NH}_{4}$. J. Am. Chem. Soc. 1995, 117, 12209-12217. [CrossRef]

2. Martin, L.; Turner, S.S.; Day, P.; Mabbs, F.E.; McInnes, J.L. New molecular superconductor containing paramagnetic chromium (III) ions. Chem. Commun. 1997, 15, 1367-1368. [CrossRef]

3. Turner, S.S.; Day, P.; Abdul Malik, K.M.; Hursthouse, M.B.; Teat, S.J.; MacLean, E.J.; Martin, L.; French, S.A. Effect of Included Solvent Molecules on the Physical Properties of the Paramagnetic Charge Transfer Salts $\beta^{\prime \prime}$-(BEDT-TTF $)_{4}\left[\left(\mathrm{H}_{3} \mathrm{O}\right) \mathrm{Fe}\left(\mathrm{C}_{2} \mathrm{O}_{4}\right)_{3}\right]$.Solvent (BEDT-TTF = Bis(ethylenedithio)tetrathiafilvalene). Inorg. Chem. 1999, 38, 3543-3549. [CrossRef] [PubMed]

4. Martin, L.; Turner, S.S.; Day, P.; Guionneau, P.; Howard, J.A.K.; Hibbs, D.E.; Light, M.E.; Hursthouse, M.B.; Uruichi, M.; Yakushi, K. Crystal Chemistry and Physical Properties of Superconducting and Semiconducting Charge Transfer Salts of the Type (BEDT-TTF $)_{4}\left[\mathrm{~A}^{\mathrm{I}} \mathrm{M}^{\mathrm{III}}\left(\mathrm{C}_{2} \mathrm{O}_{4}\right)_{3}\right] \cdot \mathrm{PhCN}\left(\mathrm{A}^{\mathrm{I}}=\mathrm{H}_{3} \mathrm{O}, \mathrm{NH}_{4}, \mathrm{~K} ; \mathrm{M}^{\mathrm{III}}=\mathrm{Cr}, \mathrm{Fe}\right.$, $\mathrm{Co}, \mathrm{Al}$; BEDT-TTF = Bis(ethylenedithio)tetrathiafulvalene). Inorg. Chem. 2001, 40, 1363-1371. [CrossRef] [PubMed]

5. Rashid, S.; Turner, S.S.; Le Pevelen, D.; Day, P.; Light, M.E.; Hursthouse, M.B.; Firth, S.; Clark, R.J.H. $\beta$ "-BEDT-TTF $)_{4}\left[\left(\mathrm{H}_{3} \mathrm{O}\right) \mathrm{Cr}\left(\mathrm{C}_{2} \mathrm{O}_{4}\right)_{3}\right] \mathrm{CH}_{2} \mathrm{Cl}_{2}$ : Effect of Included Solvent on the Structure and Properties of a Conducting Molecular Charge-Transfer Salt. Inorg. Chem. 2001, 40, 5304-5306. [CrossRef] [PubMed]

6. Rashid, S.; Turner, S.S.; Day, P.; Howard, J.A.K.; Guionneau, P.; McInnes, E.J.L.; Mabbs, F.E.; Clark, R.J.H.; Firth, S.; Biggse, T. New superconducting charge-transfer salts (BEDTTTF) ${ }_{4}\left[\mathrm{AM}\left(\mathrm{C}_{2} \mathrm{O}_{4}\right) 3\right] \mathrm{C}_{6} \mathrm{H}_{5} \mathrm{NO}_{2}\left(\mathrm{~A}=\mathrm{H}_{3} \mathrm{O}\right.$ or $\mathrm{NH}_{4}, \mathrm{M}=\mathrm{Cr}$ or Fe, BEDT-TTF = bis(ethylenedithio)tetrathiafulvalene. Novel charge transfer salts of BEDT-TTF with metal oxalate counterions. J. Mater. Chem. 2001, 11, 2095-2101. [CrossRef]

7. Rashid, S.S.; Turner, P.; Day, M.E.; Light, M.B.; Hursthouse, P. Guionneau. Synth. Met. 2001, 120, $985-986$. [CrossRef]

8. Akutsu, H.; Akutsu-Sato, A.; Turner, S.S.; Le Pevelen, D.; Day, P.; Laukhin, V.; Klehe, A.-K.; Singleton, J.; Tocher, D.A.; Probert, M.R.; et al. Effect of Included Guest Molecules on the Normal State Conductivity and Superconductivity of $\beta^{\prime \prime}-(\mathrm{ET})_{4}\left[\left(\mathrm{H}_{3} \mathrm{O}\right) \mathrm{Ga}\left(\mathrm{C}_{2} \mathrm{O}_{4}\right)_{3}\right] \mathrm{G}(\mathrm{G}=$ Pyridine, Nitrobenzene). J. Am. Chem. Soc. 2002, 124, 12430-12431. [CrossRef] [PubMed]

9. Prokhorova, T.G.; Khasanov, S.S.; Zorina, L.V.; Buravov, L.I.; Tkacheva, V.A.; Baskakov, A.A.; Morgunov, R.B.; Gener, M.; Canadell, E.; Shibaeva, R.P.; et al. Molecular Metals Based on BEDT-TTF Radical Cation Salts with Magnetic Metal Oxalates as Counterions: $\beta^{\prime \prime}-(\mathrm{BEDT}-\mathrm{TTF})_{4} \mathrm{~A}\left[\mathrm{M}\left(\mathrm{C}_{2} \mathrm{O}_{4}\right)_{3}\right] \mathrm{DMF}\left(\mathrm{A}=\mathrm{K}^{+}, \mathrm{NH}_{4}{ }^{+} ; \mathrm{M}=\mathrm{Fe} \mathrm{III}^{\mathrm{II}}\right.$, $\left.\mathrm{Cr}^{\mathrm{III}}\right)$. Adv. Funct. Mater. 2003, 13, 403-411. [CrossRef]

10. Coldea, A.I.; Bangura, A.F.; Singleton, J.; Ardavan, A.; Akutsu-Sato, A.; Akutsu, H.; Turner, S.S.; Day, P. Fermi-surface topology and the effects of intrinsic disorder in a class of charge-transfer salts containing magnetic ions: $\beta "$ "-(BEDT-TTF $)_{4}\left[\left(\mathrm{H}_{3} \mathrm{O}\right) M\left(\mathrm{C}_{2} \mathrm{O}_{4}\right)_{3}\right] Y\left(M=\mathrm{Ga}, \mathrm{Cr}, \mathrm{Fe} ; Y=\mathrm{C}_{5} \mathrm{H}_{5} \mathrm{~N}\right.$. Phys. Rev. B 2004, 69, 085112. [CrossRef]

11. Akutsu, H.; Akutsu-Sato, A.; Turner, S.S.; Day, P.; Canadell, E.; Firth, S.; Clark, R.J.N.; Yamada, J.; Nakatsuji, S. Superstructures of donor packing arrangements in a series of molecular charge transfer salts. Chem. Commun. 2004, 1, 18-19. [CrossRef] [PubMed]

12. Audouard, A.; Laukhin, V.N.; Brossard, L.; Prokhorova, T.G.; Yagubskii, E.B.; Canadell, E. Combination frequencies of magnetic oscillations in $\beta^{\prime \prime}$-(BEDT-TTF $)_{4}\left(\mathrm{NH}_{4}\right)\left[\left(\mathrm{Fe}\left(\mathrm{C}_{2} \mathrm{O}_{4}\right)_{3}\right]\right.$ DMF. Phys. Rev. B 2004, 69, 144523. [CrossRef] 
13. Akutsu-Saito, A.; Kobayashi, A.; Mori, T.; Akutsu, H.; Yamada, J.; Nakatsuji, S.; Turner, S.S.; Day, P.; Tocher, D.A.; Light, M.E.; et al. Structures and Physical Properties of New $\beta$ "-BEDT-TTF Tris-Oxalatometallate (III) Salts Containing Chlorobenzene and Halomethane Guest Molecules. Synth. Met. 2005, 152, 373-376. [CrossRef]

14. Coronado, E.; Curelli, S.; Giménez-Saiz, C.; Gómez-García, C.J. New magnetic conductors and superconductors based on BEDT-TTF and BEDS-TTF. Synth. Met. 2005, 154, 245-248. [CrossRef]

15. Coronado, E.; Curelli, S.; Giménez-Saiz, C.; Gómez-García, C.J. A novel paramagnetic molecular superconductor formed by bis(ethylenedithio)tetrathiafulvalene, tris(oxalato)ferrate(III) anions and bromobenzene as guest molecule: $(\mathrm{ET})_{4}\left[\left(\mathrm{H}_{3} \mathrm{O}\right) \mathrm{Fe}\left(\mathrm{C}_{2} \mathrm{O}_{4}\right)_{3}\right] \mathrm{C}_{6} \mathrm{H}_{5} \mathrm{Br}$. J. Mater. Chem. 2005, 15, 1429-1436. [CrossRef]

16. Akutsu-Sato, A.; Akutsu, H.; Yamada, J.; Nakatsuji, S.; Turner, S.S.; Day, P. Suppression of superconductivity in a molecular charge transfer salt by changing quest molecule: $\beta^{\prime \prime}$-(BEDT-TTF $)_{4}\left[\left(\mathrm{H}_{3} \mathrm{O}\right) \mathrm{Fe}\left(\mathrm{C}_{2} \mathrm{O}_{4}\right)_{3}\right]\left(\mathrm{C}_{6} \mathrm{H}_{5} \mathrm{CN}\right)_{x}\left(\mathrm{C}_{5} \mathrm{H}_{5} \mathrm{~N}\right)_{1-x}$. J. Mater. Chem. 2007, 17, 2497-2499. [CrossRef]

17. Zorina, L.V.; Prokhorova, T.G.; Simonov, S.V.; Khasanov, S.S.; Shibaeva, R.P.; Manakov, A.I.; Zverev, V.N.; Buravov, L.I.; Yagubskii, E.B. Structure and Magnetotransport Properties of the New Quasi-Two-Dimensional Molecular Metal $\beta^{\prime \prime}$-(BEDT-TFF) $)_{4} \mathrm{H}_{3} \mathrm{O}\left[\mathrm{Fe}\left(\mathrm{C}_{2} \mathrm{O}_{4}\right)_{3}\right] \mathrm{C}_{6} \mathrm{H}_{4} \mathrm{Cl}_{2}$. J. Exp. Theor. Phys. 2008, 106, 347-354. [CrossRef]

18. Martin, L.; Day, P.; Akutsu, H.; Yamada, J.; Nakatsuji, S.; Clegg, W.; Harrington, R.W.; Horton, P.N.; Hursthouse, M.B.; McMillan, P.; et al. Metallic molecular crystals containing chiral or racemic guest molecules. CrystEngComm 2007, 9, 865-867. [CrossRef]

19. Prokhorova, T.G.; Buravov, L.I.; Yagubskii, E.B.; Zorina, L.V.; Khasanov, S.S.; Simonov, S.V.; Shibaeva, R.P.; Korobenko, A.V.; Zverev, V.N. Effect of electrocrystallization medium on quality, structural features, and conducting properties of single crystals of the (BEDT-TTF) ${ }_{4} \mathrm{~A}^{\mathrm{I}}\left[\mathrm{Fe}^{\mathrm{III}}\left(\mathrm{C}_{2} \mathrm{O}_{4}\right)_{3}\right] \cdot \mathrm{G}$ family. CrystEngComm 2011, 13, 537-545. [CrossRef]

20. Zorina, L.V.; Khasanov, S.S.; Simonov, S.V.; Shibaeva, R.P.; Zverev, V.N.; Canadell, E.; Prokhorova, T.G.; Yagubskii, E.B. Coexistence of two donor packing motifs in the stable molecular metal $\alpha$-'pseudo-k'-(BEDT-TTF $)_{4}\left(\mathrm{H}_{3} \mathrm{O}\right)\left[\mathrm{Fe}\left(\mathrm{C}_{2} \mathrm{O}_{4}\right)_{3}\right] \cdot \mathrm{C}_{6} \mathrm{H}_{4} \mathrm{Br}_{2}$. CrystEngComm 2011, 13, 2430-2438. [CrossRef]

21. Coronado, E.; Curreli, S.; Giménez-Saiz, C.; Gómez-García, C.J. The Series of Molecular Conductors and Superconductors $\mathrm{ET}_{4}\left[\mathrm{AFe}\left(\mathrm{C}_{2} \mathrm{O}_{4}\right)_{3}\right] \cdot \mathrm{PhX}\left(\mathrm{ET}=\right.$ bis(ethylenedithio)tetrathiafulvalene; $\left(\mathrm{C}_{2} \mathrm{O}_{4}\right)^{2-}=$ oxalate; $\mathrm{A}^{+}$ $=\mathrm{H}_{3} \mathrm{O}^{+}, \mathrm{K}^{+} ; \mathrm{X}=\mathrm{F}, \mathrm{Cl}, \mathrm{Br}$, and I): Influence of the Halobenzene Guest Molecules on the Crystal Structure and Superconducting Properties. Inorg. Chem. 2012, 51, 1111-1126. [PubMed]

22. Zorina, L.V.; Khasanov, S.S.; Simonov, S.V.; Shibaeva, R.P.; Bulanchuk, P.O.; Zverev, V.N.; Canadell, E.; Prokhorova, T.G.; Yagubskii, E.B. Structural phase transition in the $\beta^{\prime \prime}$-(BEDT-TTF) ${ }_{4} \mathrm{H}_{3} \mathrm{O}\left[\mathrm{Fe}\left(\mathrm{C}_{2} \mathrm{O}_{4}\right)_{3}\right] \cdot \mathrm{G}$ crystals (where $\mathrm{G}$ is a guest solvent molecule). CrystEngComm 2012, 14, 460-465. [CrossRef]

23. Prokhorova, T.G.; Zorina, L.V.; Simonov, S.V.; Zverev, V.N.; Canadell, E.; Shibaeva, R.P.; Yagubskii, E.B. The first molecular superconductor based on BEDT-TTF radical cation salt with paramagnetic tris(oxalato)ruthenate anion. CrystEngComm 2013, 15, 7048-7055. [CrossRef]

24. Prokhorova, T.G.; Buravov, L.I.; Yagubskii, E.B.; Zorina, L.V.; Simonov, S.V.; Shibaeva, R.P.; Zverev, V.N. New metallic bi- and monolayered radical cation salts based on BEDT-TTF with tris(oxalato)gallate anion. Eur. J. Inorg. Chem. 2014, 24, 3933-3940. [CrossRef]

25. Prokhorova, T.G.; Buravov, L.I.; Yagubskii, E.B.; Zorina, L.V.; Simonov, S.V.; Zverev, V.N.; Shibaeva, R.P.; Canadell, E. Effect of Halopyridine Guest Molecules on the Structure and Superconducting Properties of $\beta^{\prime \prime}$-[Bis(ethylenedithio)tetrathiafulvalene $]_{4}\left(\mathrm{H}_{3} \mathrm{O}\right)\left[\mathrm{Fe}\left(\mathrm{C}_{2} \mathrm{O}_{4}\right)_{3}\right] \cdot$ Guest Crystals. Eur. J. Inorg. Chem. 2015, 34, 5611-5620. [CrossRef]

26. Martin, L.; Morritt, A.L.; Lopez, J.R.; Nakazawa, Y.; Akutsu, H.; Imajo, S.; Ihara, Y.; Zhang, B.; Zhange, Y.; Guof, Y. Molecular conductors from bis(ethylenedithio)tetrathiafulvalene with tris(oxalato)rhodate. Dalton Trans. 2017, 46, 9542-9548. [CrossRef] [PubMed]

27. Prokhorova, T.G.; Yagubskii, E.B. Organic conductors and superconductors based on bis(ethylenedithio)tetrathiafulvalene radical cation salts with supramolecular tris(oxalato)metallate anions. Russ. Chem. Rev. 2017, 86, 164-180. [CrossRef] 
28. Coronado, E.; Galan-Mascaros, J.R.; Gomez-Garcia, C.J.; Laukhin, V. Coexistence of ferromagnetism and metallic conductivity in a molecule-based layered compound. Nature 2000, 408, 447-449. [CrossRef] [PubMed]

29. Martin, L.; Day, P.; Horton, P.; Nakatsuji, S.; Yamada, J.; Akutsu, H. Chiral conducting salts of BEDT-TTF containing a single enantiomer of tris(oxalato)chromate(III) crystallized from a chiral solvent. J. Mater. Chem. 2010, 20, 2738-2742. [CrossRef]

30. Martin, L.; Day, P.; Nakatsuji, S.; Yamada, J.; Akutsu, H.; Horton, P. A molecular charge transfer salt of BEDT-TTF containing a single enantiomer of tris(oxalato)chromate(III) crystallized from a chiral solvent. CrystEngComm 2010, 12, 1369-1372. [CrossRef]

31. Martin, L.; Akutsu, H.; Hortond, P.N.; Hursthoused, M.B. Chirality in charge-transfer salts of BEDT-TTF of tris(oxalato)chromate (III). CrystEngComm 2015, 17, 2783-2790. [CrossRef]

32. Martin, L.; Akutsu, H.; Horton, P.N.; Hursthouse, M.B.; Harrington, R.W.; Clegg, W. Chiral Radical-Cation Salts of BEDT-TTF Containing a Single Enantiomer of Tris(oxalato)aluminate(III) and -chromate(III). Eur. J. Inorg. Chem. 2015, 11, 1865-1870.

33. Martin, L.; Morritt, A.L.; Lopez, J.R.; Akutsu, H.; Nakazawa, Y.; Imajo, S.; Ihara, Y. Ambient-pressure molecular superconductor with a superlattice containing layers of tris(oxalato)rhodate enantiomers and 18-crown-6. Inorg. Chem. 2017, 56, 717-720. [CrossRef] [PubMed]

34. Mori, T. Structural Genealogy of BEDT-TTF-Based Organic Conductors I. Parallel Molecules: $\beta$ and $\beta$ " Phases. Bull. Chem. Soc. Jpn. 1998, 71, 2509-2526. [CrossRef]

35. Mori, T.; Mori, H.; Tanaka, S. Structural Genealogy of BEDT-TTF-Based Organic Conductors II. Inclined Molecules: $\theta, \alpha$, and $\kappa$ Phases. Bull. Chem. Soc. Jpn. 1999, 72, 179-197. [CrossRef]

36. Olejniczak, I.; Frackowiak, A.; Swietlik, R.; Prokhorova, T.G.; Yagubskii, E.B. Charge Fluctuations and Ethylene-Group-Ordering Transition in $\beta^{\prime \prime}$-(BEDT-TF $)_{4}\left[\left(\mathrm{H}_{3} \mathrm{O}\right) \mathrm{Fe}\left(\mathrm{C}_{2} \mathrm{O}_{4}\right)_{3}\right] \cdot Y$ Molecular Charge-Transfer Salts. ChemPhysChem 2013, 14, 3925-3935. [CrossRef] [PubMed]

37. Neogi, P.; Dutt, N.K.J. New compounds of gallium. Part III. Preparation and Resolution of complex oxalate compounds of gallium into optical isomers. Indian Chem. Soc. 1938, 15, 83-86.

38. CrysAlisPro Software System, Version 1.171.38.41; Rigaku Oxford Diffraction; Rigaku Corporation: Oxford, UK, 2016.

39. Sheldrick, G.M. A short history of SHELX. Acta Cryst. A 2008, 64, 112-122. [CrossRef] [PubMed]

40. Guionneau, P.; Kepert, C.J.; Bravic, G.; Chasseau, D.; Truter, M.R.; Kurmoo, M.; Day, P. Determining the charge distribution in BEDT-TTF salts. Synth. Met. 1997, 86, 1973-1974. [CrossRef]

41. Martin, L.; Engelkamp, H.; Akutsu, H.; Nakatsuji, S.; Yamada, J.; Horton, P.; Hursthouse, M.B. Charge transfer salts of BEDT-TTF with lithium tris(oxalato)metallate(III). Dalton Trans. 2015, 44, 6219-6223. [CrossRef] [PubMed]

42. Zhang, B.; Zhang, Y.; Liu, F.; Guo, Y. Synthesis, crystal structure, and characterization of charge-transfer salt: (BEDT-TTF $)_{5}\left[\mathrm{Fe}\left(\mathrm{C}_{2} \mathrm{O}_{4}\right)_{3}\right] \cdot\left(\mathrm{H}_{2} \mathrm{O}\right)_{2} \cdot \mathrm{CH}_{2} \mathrm{Cl}_{2}$ (BEDT-TTF $=$ bis(ethylenedithio)tetrathiafulvalene). CrystEngComm 2009, 11, 2523-2528. [CrossRef]

43. Altshuler, B.L.; Aronov, A.G. Electron-Electron Interaction in Disordered Conductors. In Electron-Electron Interactions in Disordered Systems; Efros, A.L., Pollak, M., Eds.; Elsevier: Amsterdam, The Netherlands, 1985; Volume 10, pp. 1-690. ISBN 0444-86916-6.

44. Gruner, G.; Zawadowski, A. Magnetic impurities in non-magnetic metals. Rep. Prog. Phys. 1974, 37, 1497-1584. [CrossRef]

(C) 2018 by the authors. Licensee MDPI, Basel, Switzerland. This article is an open access article distributed under the terms and conditions of the Creative Commons Attribution (CC BY) license (http:/ / creativecommons.org/licenses/by/4.0/). 
Article

\title{
$\beta^{\prime \prime}-(\mathrm{CNB}-\mathrm{EDT}-\mathrm{TTF})_{4} \mathrm{BF}_{4}$; Anion Disorder Effects in Bilayer Molecular Metals
}

\author{
Sandra Rabaça ${ }^{1}$, Sandrina Oliveira ${ }^{1}$, Vasco Gama ${ }^{1}$, Isabel C. Santos ${ }^{1}$, Gonçalo Oliveira ${ }^{1}$, \\ Elsa B. Lopes ${ }^{1}$, Enric Canadell ${ }^{2}$ and Manuel Almeida ${ }^{1, *}$ \\ 1 C2TN, Centro de Ciências e Tecnologias Nucleares, Instituto Superior Técnico, Universidade de Lisboa, \\ E.N. 10, P-2695-066 Bobadela LRS, Portugal; sandrar@ctn.tecnico.ulisboa.pt (S.R.); \\ sandrina@ctn.tecnico.ulisboa.pt (S.O.); vascog@ctn.tecnico.ulisboa.pt (V.G.); \\ icsantos@ctn.tecnico.ulisboa.pt (I.C.S.); gooliveira@sapo.pt (G.O.); eblopes@ctn.tecnico.ulisboa.pt (E.B.L.) \\ 2 Institut de Ciencia de Materials de Barcelona (ICMAB-CSIC), Campus UAB, E-08193 Bellaterra, Spain; \\ canadell@icmab.es \\ * Correspondence: malmeida@ctn.tecnico.ulisboa.pt; Tel.: +351-21-955-6171
}

Received: 3 March 2018; Accepted: 18 March 2018; Published: 21 March 2018

\begin{abstract}
The preparation and characterization of new salts based on the dissymmetrical TTF derivative CNB-EDT-TTF (cyanobenzene-ethylenedithio-tetrathiafulvalene) and $\mathrm{BF}_{4}{ }^{-}$anions, are reported. Depending on the electrocrystallization conditions salts with different stoichiometries, (CNB-EDT-TTF) $\mathrm{BF}_{4}$ and $\beta^{\prime \prime}$-(CNB-EDT-TTF $)_{4} \mathrm{BF}_{4}$, can be obtained. The 1:1 salt is an electrical insulator isostructural to the $\mathrm{ClO}_{4}$ analogue previously described. The 4:1 salt is a new member of the family of 2D metals of this donor with different small anions X, (CNB-EDT-TTF) 4 X, characterized by a bilayer arrangement of the donors and it was obtained in a monoclinic polymorph with a $\beta^{\prime \prime}$-type donor packing pattern. The small anions in this compound are severely disordered between the donor bilayers, which present slightly larger lattice parameters than the isostructural $\mathrm{ClO}_{4}$ analogue. Both electrical conductivity and thermoelectric power measurements in single crystals denote metallic properties as predicted by electronic band structure calculations. As a consequence of the anion disorder the metallic regime of the electrical conductivity denotes electronic localization effects with a progressive increase of resistivity below $\sim 25 \mathrm{~K}$. Because of the larger lattice parameters the intermolecular interactions and electronic bandwidth are decreased compared to other (CNB-EDT-TTF $)_{4} \mathrm{X}$ salts. The large and positive thermoelectric power $S$ of this compound $(\sim 110 \mu \mathrm{V} / \mathrm{K}$ in the range $100-330 \mathrm{~K})$ and its electrical conductivity $\sigma=20 \mathrm{~S} / \mathrm{cm}$ at room temperature lead to a power factor $S^{2} \sigma=24 \mu \mathrm{W} / \mathrm{K}^{2} \mathrm{~m}$, quite large among molecular conductors, placing these compounds as potential candidates for thermoelectric materials.
\end{abstract}

Keywords: molecular conductors; bilayer metals; radical cation salts; CNB-EDT-TTF; thermoelectrics

\section{Introduction}

It has been recently shown that the dissymmetrical TTF derivative cyanobenzene-ethylenedithiotetrathiafulvalene (CNB-EDT-TTF) (Scheme 1) is an electron donating molecule that when combined with small anions $\mathrm{X}$ such as $\mathrm{ClO}_{4}{ }^{-}, \mathrm{PF}_{6}{ }^{-}$, and $\mathrm{I}_{3}{ }^{-}$, leads to a family of radical cation salts of composition (CNB-EDT-TTF $)_{4} \mathrm{X}$ and characterized by a unique donor bilayer structure [1]. This bilayer arrangement is promoted by an effective arrangement of hydrogen bond dimeric $\mathrm{C}-\mathrm{N} \cdots \mathrm{H}-\mathrm{C}$ interactions involving the cyano groups, as a combination of $\mathrm{R}^{2}{ }_{2}(10)$ and $\mathrm{R}^{2}{ }_{2}(8)$ synthons in a two-dimensional network. These (CNB-EDT-TTF $)_{4} \mathrm{X}$ compounds have been obtained in several polymorphic phases associated with different donor packing patterns or anion ordering schemes [2,3] and for $\kappa$-type packing pattern some of these 2D metals have shown superconductivity at low temperatures [2]. 


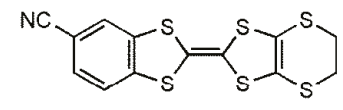

Scheme 1. CNB-EDT-TTF.

The donor bilayer structure confers to these salts unusual metallic properties associated to high band filling, large electronic effective masses and quasi degenerated 2D Fermi surfaces, making the study of this type of bilayer metallic systems quite attractive. However the range of stability of the (CNB-EDT-TTF $)_{4} \mathrm{X}$ salts was found so far restricted to linear, tetrahedral or octahedral anions of size comparable to $\mathrm{ClO}_{4}{ }^{-}, \mathrm{PF}_{6}{ }^{-}$, and $\mathrm{I}_{3}{ }^{-}$, and for instance with a larger anion such as $\mathrm{FeBr}_{4}{ }^{-}$, a salt of different stoichiometry (CNB-EDT-TTF ${ }_{2} \mathrm{FeBr}_{4}$ and distinct structure is obtained instead [4]. These findings directed our attention towards exploring new CNB-EDT-TTF salts with comparable small anions and in this contribution we report the salts obtained with the smaller tetrahedral anion $\mathrm{BF}_{4}{ }^{-}$. With this small tetrahedral anion salts with both 4:1 and 1:1 stoichiometries were obtained.

\section{Materials and Methods}

\subsection{General Information}

CNB-EDT-TTF was prepared following a previous described procedure [5] which was more recently improved [3]. $n-\mathrm{Bu}_{4} \mathrm{~N} \mathrm{BF}_{4}$ (Sigma Aldrich, Darmstadt, Germany) was purified by recrystallization from ethyl acetate. Dichloromethane and tetrahydrofuran (THF) were freshly distilled immediately before use.

\subsection{Synthesis}

[CNB-EDT-TTF] BF 4 (1). A THF solution of the donor CNB-EDT-TTF $\left(2.2 \times 10^{-3} \mathrm{M}\right)$ and $n-\mathrm{Bu}_{4} \mathrm{~N}$ $\mathrm{BF}_{4}\left(1.1 \times 10^{-2} \mathrm{M}\right)$ was added to an $\mathrm{H}$-shaped two-compartment cells separated by frit glass with $\mathrm{Pt}$ electrodes and sealed under nitrogen. After applying initially a current density of $0.5 \mu \mathrm{A} / \mathrm{cm}^{2}$, during the first 3 days, then increased to $0.7 \mu \mathrm{A} / \mathrm{cm}^{2}$ during more 20 days, black bock shaped crystals grown on the anode were collected and washed with THF and dichloromethane.

$\beta^{\prime \prime}-[C N B-E D T-T T F]_{4} B F_{4}(2)$. A dichloromethane solution of the donor CNB-EDT-TTF and $n$-Bu $u_{4} \mathrm{~N}$ $\mathrm{BF}_{4}$ (both $2 \times 10^{-3} \mathrm{M}$ ) was added to an $\mathrm{H}$-shaped two-compartment cells separated by frit glass with Pt electrodes and sealed under nitrogen. After approximately 15 days of applying a constant current density of $1 \mu \mathrm{A} / \mathrm{cm}^{2}$, dark brown elongated platelet shaped crystals grown on the anode were collected and washed with dichloromethane/ether.

\subsection{X-ray Crystallography}

Selected single crystals were mounted on a loop with protective oil and X-ray data was collected on a APEX II CCD detector diffractometer (Bruker, Billerica, Massachusetts, MA, USA) using graphite monochromated MoK $\alpha$ radiation $(\lambda=0.71073 \AA)$ and operating in a $\varphi$ and $\omega$ scans mode. A semi empirical absorption correction was carried out using SADABS [6]. Data collection, cell refinement and data reduction were done with the SMART and SAINT programs [7]. The structures were solved by direct methods using SIR97 [8] and refined by full matrix least-squares methods using the program SHELXL97 [9] using the winGX software package [10]. Non-hydrogen atoms were refined with anisotropic thermal parameters whereas $\mathrm{H}$-atoms were placed in idealized positions and allowed to refine riding on the parent $C$ atom. Molecular graphics were prepared using Mercury [11]. CCDC 1825988-1825989 contains the supplementary crystallographic data for this paper. These data can be obtained free of charge from The Cambridge Crystallographic Data Centre via www.ccdc.cam. ac.uk/data_request/cif. 
Crystallographic data for compound 1, (CNB-EDT-TTF) BF 4 : $\mathrm{C}_{13} \mathrm{H}_{7} \mathrm{BF}_{4} \mathrm{NS}_{6}, \mathrm{M}=456.37 \mathrm{~g} \cdot \mathrm{mol}^{-1}$, monoclinic, space group $\left.P 22_{1} / c, a=9.5066(9)\right) \AA, b=8.3629(9) \AA, c=21.1593(13) \AA, \beta=95.984(6)^{\circ}$, $\mathrm{V}=1673.1(3) \AA^{3}, \mathrm{Z}=4, \rho_{\text {calc }}=1.812 \mathrm{~g} \cdot \mathrm{cm}^{-3}, \mu(\mathrm{Mo} \mathrm{K \alpha})=0.855 \mathrm{~mm}^{-1}, 5429$ reflections measured, 2887 unique $R_{\text {int }}=0.0734, \theta_{\max }=25.02^{\circ}, R_{1}=0.0618$ using 2887 Refl. $>2 \sigma(\mathrm{I}), \omega \mathrm{R}_{2}=0.1189, \mathrm{~T}=150(2) \mathrm{K}$. CCDC 1825989.

Crystallographic data for compound 2,"-(CNB-EDT-TTF) ${ }_{4} \mathrm{BF}_{4}: \mathrm{C}_{13} \mathrm{H}_{7} \mathrm{NS}_{6}, \mathrm{M}=832.62 \mathrm{~g} \cdot \mathrm{mol}^{-1}$, monoclinic, space group $P 2_{1} / m, a=4.8418(13) \AA, b=55.724(15) \AA, c=5.9081(16) \AA, \beta=94.208(18)^{\circ}$, $\mathrm{V}=1589.7(7) \AA^{3}, \mathrm{Z}=4, \rho_{\text {calc }}=1.544 \mathrm{~g} \cdot \mathrm{cm}^{-3}, \mu(\mathrm{Mo} \mathrm{K \alpha})=0.847 \mathrm{~mm}^{-1}, 8106$ reflections measured, 1584 unique $\mathrm{R}_{\text {int }}=0.0747, \theta_{\max }=20.64^{\circ}, \mathrm{R} 1=0.0764$ using 1584 Refl. $>2 \sigma(\mathrm{I}), \omega \mathrm{R}_{2}=0.1727, \mathrm{~T}=150(2) \mathrm{K}$. CCDC 1825988.

\subsection{Electronic Band Structure Calculations}

The tight-binding band structure calculations [12] were of the extended Hückel type and a modified Wolfsberg-Helmholtz formula was used to calculate the non-diagonal $\mathrm{H}_{\mu v}$ values [13]. All valence electrons were taken into account in the calculations and the basis set consisted of Slater-type orbitals of double- $\zeta$ quality for $C$ and $N 2 s$ and $2 p, S 3 s$ and $3 p$ and of single- $\zeta$ quality for H1s. The ionization potentials, contraction coefficients and exponents were taken exactly as in our previous work for $(\mathrm{CN}-\mathrm{BEDT}-\mathrm{TTF})_{4} \mathrm{I}_{3}$ [2]. Because of the different crystallographic cells the four different HOMO $\cdots$ HOMO intralayer interactions were defined as in the previous work on disordered (CN-BEDT-TTF $)_{4} \mathrm{I}_{3}$ [2]: I, interaction along the stacks; II, lateral $\pi$-type interaction; III, interaction along the step-chains and IV, head to head interlayer interaction .

\subsection{Electric Transport Properties}

Electrical conductivity and thermoelectric power were measured in single crystals in the temperature range of 20-320 K, in a cell attached to the cold stage of a closed cycle helium refrigerator. In the first step, the thermoelectric power was measured by using a slow AC (ca. $10^{-2} \mathrm{~Hz}$ ) technique [14], by attaching two $\varphi=25 \mu \mathrm{m}$ diameter 99.999\% pure Au wires (Goodfellow), thermally anchored to two quartz blocks, with Pt paint (Demetron 308A), to the extremities of an elongated sample as in a previously described apparatus [15], under computer control [16]. The oscillating thermal gradient was kept below $1 \mathrm{~K}$ and it was measured with a differential Au- 0.05 at. \% Fe vs. chromel thermocouple, and the sample temperature measured with a calibrated thermocouple of the same type. The absolute thermoelectric power of the samples was obtained after correction for the absolute thermopower of the Au leads, by using the data of Huebner [17]. In a second step two additional contacts between the previous ones, were similarly made in order to achieve a four-in-line contact configuration and electrical resistivity was measured imposing an AC current of $1 \mu \mathrm{A}$ at $70 \mathrm{HZ}$ and the voltage drop measured using a lock-in technique. Sample electrodes configuration was checked for unnested to nested voltage ratio, as defined by Schaffer et al. [18], which was kept below $5 \%$.

\section{Results}

As previously observed with the anion $\mathrm{ClO}_{4}{ }^{-}$depending on the solvent and electrocrystallization conditions salts with 1:1 and 4:1 donor:acceptor stoichiometries; (CNB-EDT-TTF) BF 4 (1) and (CNB-EDT-TTF $)_{4} \mathrm{BF}_{4}$ (2) can be obtained, the first one as black block shaped crystals from tetrahydrofuran (THF) and the last one as thin dark brown platelet shaped crystals from dichloromethane solutions. For the 4:1 salt, among the several crystals tested only the monoclinic phase $\beta^{\prime \prime}{ }_{M}-(C N B-E D T-T T F){ }_{4} \mathrm{BF}_{4}$ could be observed with so far no evidence for the triclinic one described for $\mathrm{ClO}_{4}$ [3]. These two $\mathrm{BF}_{4}$ salts are isostructural with the analogues with $\mathrm{ClO}_{4}{ }^{-}$anions [3] and therefore the description of their crystal structures will be only briefly summarized.

$(\mathrm{CNB}-\mathrm{EDT}-\mathrm{TTF}) \mathrm{BF}_{4}(\mathbf{1})$ crystallizes in the monoclinic system, space group $\mathrm{P} 2{ }_{1} / \mathrm{c}$. The asymmetric unit contains one independent donor molecule and one tetrahedral $\mathrm{BF}_{4}{ }^{-}$anion located in general positions (Figure S1). The donor geometry is almost planar with exception of the dihydrodithiin ring which 
presents the usual half-chair conformation with bond lengths (central C = C:C5-C6 = 1.374(9) $\AA$ ) identical within experimental uncertainty to those observed in other 1:1 salts with this donor in monocationic state [3,4] (Table S1). The crystal structure of 1 consists in head-to-tail chains of CNB-EDT-TTF donors dimers stacked along $b$, as shown in Figure 1. Donors in nearby stacks with reversed tilting (related by a screw axis) are connected by a helical network of short $\mathrm{C}-\mathrm{N} \cdots \mathrm{H}-\mathrm{C}$

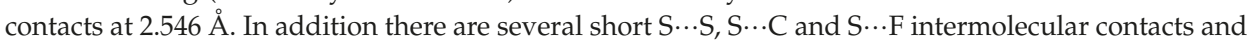
hydrogen bonds listed in Table S2.

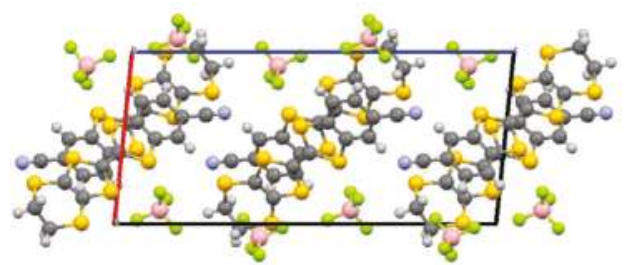

(a)

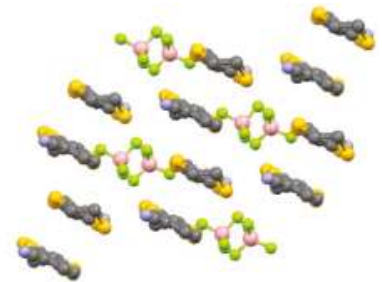

(b)

Figure 1. Crystal structure of (CNB-EDT-TTF) $\mathrm{BF}_{4}$; (a) viewed along the axis; (b) partial view along the molecular long axis of a layer of head to tail donor dimers.

(CNB-EDT-TTF $)_{4} \mathrm{BF}_{4}$ (2) crystallizes in the monoclinic system, space group $\mathrm{P} 2_{1} / \mathrm{m}$, it is isostructural with the previously described monoclinic phase of the analogue with $\mathrm{ClO}_{4}$ [3] but with cell parameters slightly larger. Figure 2 shows a projection of the crystal structure of 2 along the stacking axis (corresponding to $a$ ). The refinement of the crystal structure from different crystals measured was always severely limited by a large disorder associated with the anions which could not be clearly identified in several possible positions placed in a channel along $c$ between the donor bilayers. However when a squeeze was applied leaving only the donors to be considered in the structural refinement, a fair agreement could be achieved. The asymmetric unit of 2 (Figure S2) contains one independent CNB-EDT-TTF donor molecule with all atoms located at general positions. The donor geometry and bond lengths (Table S3) are within experimental uncertainty identical to that previously observed in $\beta^{\prime \prime}$-(CNB-EDT-TTF $)_{4} \mathrm{X}$ salts. The donor crystal packing consist in the previously described $\beta^{\prime \prime}$-type bilayer structure of donors, with similar short intermolecular contacts listed in Table S4, and alternated tilting orientations of bilayers along $b$, the large cell parameter. A relevant difference is on the cell parameters $a$ and $c$, slightly larger than those observed for $\mathrm{ClO}_{4}$ in spite of the smaller anion size. This difference is certainly due to the severe anion disorder and it corresponds to a not so tight packing of the molecules in the layers.

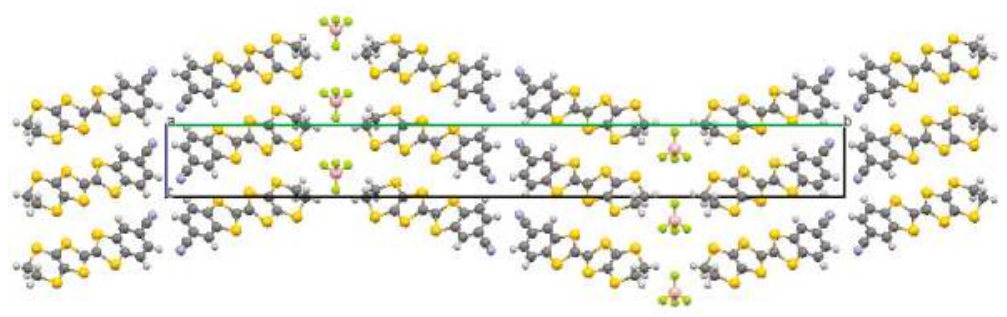

Figure 2. Crystal structure of $\beta^{\prime \prime}-(\mathrm{CNB}-\mathrm{EDT}-\mathrm{TTF})_{4} \mathrm{BF}_{4}$ viewed along $a$.

The donor bilayer structure is due to a network of bifurcated $\mathrm{C}-\mathrm{N} \cdots \mathrm{H}$ interactions in an efficient combination of $\mathrm{R}^{2}{ }_{2}(10)$ and $\mathrm{R}^{2}{ }_{2}(8)$ synthons between coplanar molecules (Figure 3a) with

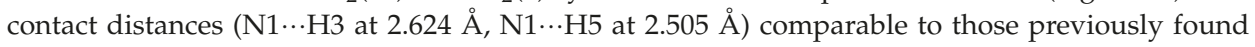


in $\beta^{\prime \prime}$-(CNB-EDT-TTF $)_{4} \mathrm{X}$ salts [1-3]. However the stacking distance between successive planes of molecules interconnected by $\mathrm{R}^{2}{ }_{2}(10)$ and $\mathrm{R}^{2}{ }_{2}(8)$ synthons is slightly larger in this $\mathrm{BF}_{4}$ salt. The three different intermolecular intralayer interactions I, II and III (I, along the stacks; II, lateral $\pi$-type and III, along the step-chains) are shown in Figure 3b. Figure 3a also shows the interaction IV between a pair of head to head molecules in adjacent layers. The magnitude of these interactions was evaluated under the extended Hückel approach and values obtained are listed in Table 1 and compared with those in the $\mathrm{ClO}_{4}, \mathrm{PF}_{6}$ and $\mathrm{I}_{3}$ analogues. Interactions II and IV are practically identical, as a consequence of a quite similar network of C-N $\cdots H$ interactions, but interactions I and III are reduced as a consequence of a larger intermolecular spacing.

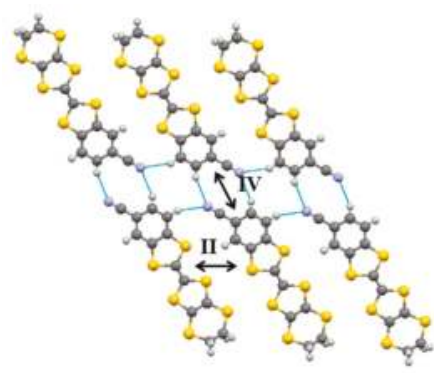

(a)

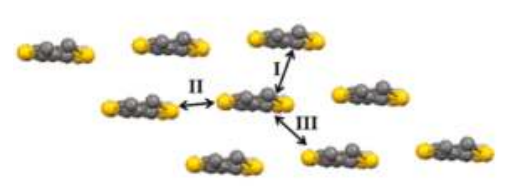

(b)

Figure 3. Details of donors packing in $\beta^{\prime \prime}-(\mathrm{CNB}-\mathrm{EDT}-\mathrm{TTF})_{4} \mathrm{BF}_{4}$ : (a) partial view perpendicular to the donors with blue lines emphasizing $\mathrm{C}-\mathrm{N} \cdots \mathrm{H}-\mathrm{C}$ interactions, as a combination of $\mathrm{R}^{2}{ }_{2}(10)$ and $\mathrm{R}_{2}{ }_{2}(8)$ synthons in a two-dimensional network of coplanar donors; (b) View of one donor layer along the molecular long axis showing a $\beta^{\prime \prime}$-type packing pattern, with identification of the distinct intralayer interactions I, II and III.

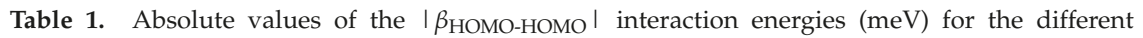
donor $\cdots$ donor interactions in the $\beta^{\prime \prime}$-(CN-BEDT-TTF) $)_{4} \mathrm{X}$ salts (I, along the stacks, II, lateral $\pi$-type, III, along the step-chains and IV, head to head interlayer interaction-See Figure 3).

\begin{tabular}{cccccc}
\hline Anion $\mathbf{X}$ & I & II & III & IV & Ref. \\
\hline $\mathrm{BF}_{4}$ & 88 & 49 & 201 & 0.8 & This work \\
$\mathrm{ClO}_{4}$ & 113 & 51 & 233 & 2.7 & 1 \\
$\mathrm{PF}_{6}$ & 117 & 53 & 219 & 3.1 & 1 \\
$\mathrm{I}_{3}$ (disordered phase) & 125 & 51 & 220 & 0.6 & 2 \\
\hline
\end{tabular}

The electronic band structure predicted on the basis of these calculated intermolecular interactions and the corresponding Fermi surface assuming an average charge $1 / 4+$ for each donor molecule are depicted in Figure 4. Due to the small interlayer interaction IV, associated with head to head intermolecular contacts, the bands are split in two almost degenerated ones. The Fermi surface corresponds to two elliptical cylinders, as typical of $\beta^{\prime \prime}$-salts [19], and their cross section area corresponds to about $1 / 8$ of the first Brillouin Zone as previously calculated for identical $\beta^{\prime \prime}-(\mathrm{CNB}-\mathrm{EDT}-\mathrm{TTF})_{4} \mathrm{X}$ salts $[1,2]$.

The metallic properties predicted by these electronic band structure calculations are confirmed by the measurements of electrical transport properties in single crystals, shown in Figure 5 where they are compared with those previously reported for the $\mathrm{ClO}_{4}$ and $\mathrm{PF}_{6}$ analogues. The electrical resistivity, $\rho(\sim 0.05-0.06 \Omega \mathrm{cm}$ at room temperatures) upon cooling shows an almost linear decrease until about $50 \mathrm{~K}$, reaches a broad and sample dependent minimum at $\sim 25-30 \mathrm{~K}$, followed by a gradual increase upon further cooling, reaching at $1.7 \mathrm{~K}$ values comparable to those at room temperature. This increase of resistivity at low temperatures, which was found slightly sample dependent, can be 
due electron repulsion or most likely to localization effects induced by anion disorder [20]. Indeed no such minima of electrical resistivity was observed at this temperature range in the $\mathrm{ClO}_{4}$ and $\mathrm{PF}_{6}$ analogues where the anions were found not so severely disordered, and where the electrical resistivity presents a stronger temperature dependence in a more extended linear regime.

The thermopower $S$ presents rather large positive values of $\sim 110 \mu \mathrm{V} / \mathrm{K}$ at room temperature, and it remains almost temperature independent down to $150 \mathrm{~K}$. Upon cooling below this temperature the thermopower values start decreasing towards zero in a fashion approximately proportional to the temperature $T$, confirming the metallic nature of the compound and indicating that the conduction takes place in a continuum of states around the Fermi-level. The large positive values are consistent with the large band filling $(7 / 8)$ predicted by the band structure calculations described above assuming an average $1 / 4+$ charge of each donor molecule. The thermopower values significantly larger than in the $\mathrm{ClO}_{4}$ and $\mathrm{PF}_{6}$ analogues are also consistent with the predicted smaller bandwidth of the $\mathrm{BF}_{4}$ salt as a consequence of larger intermolecular spacing, which results in a larger density of states at the Fermi-level.

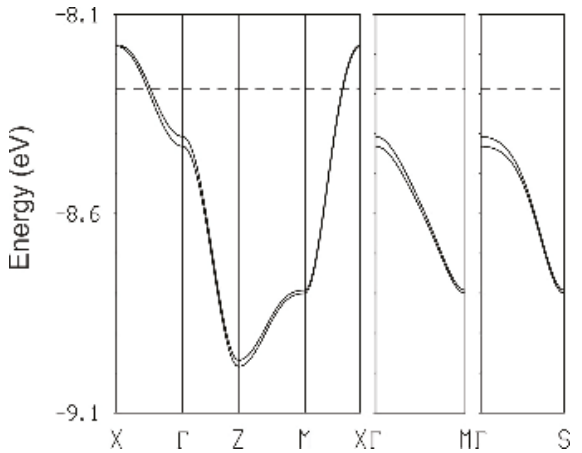

(a)

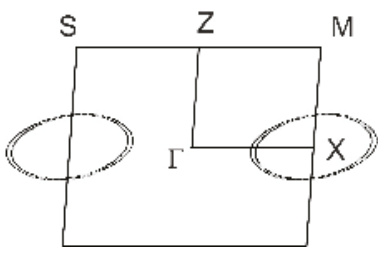

(b)

Figure 4. Calculated band structure (a) and Fermi surface (b) for $\beta^{\prime \prime}-(\mathrm{CNB}-\mathrm{EDT}-\mathrm{TTF})_{4} \mathrm{BF}_{4}$ where $\Gamma=(0,0), X=\left(a^{*} / 2,0\right), Z=\left(0, c^{*} / 2\right), M=\left(a^{*} / 2, c^{*} / 2\right)$ and $S=\left(-a^{*} / 2, c^{*} / 2\right)$. The dashed line in (a) refers to the Fermi level.

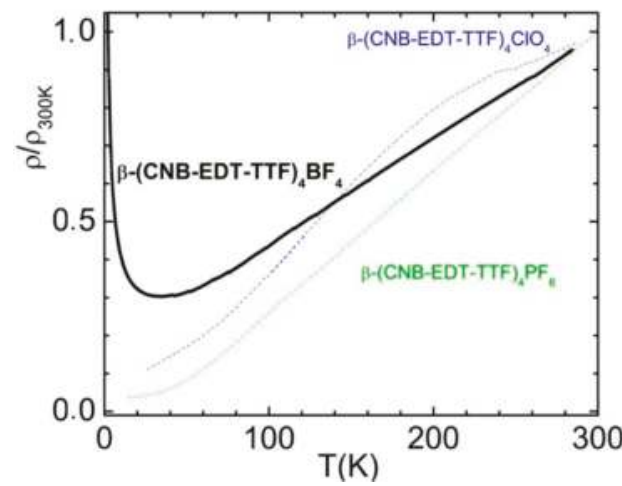

(a)

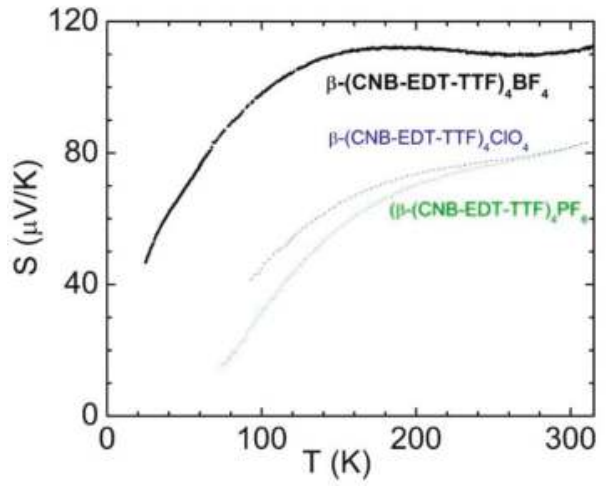

(b)

Figure 5. Temperature dependence of electrical resistivity $\rho$ (a) and absolute thermoelectric power $S$; (b) of $\beta^{\prime \prime}$-(CNB-EDT-TTF $)_{4} \mathrm{BF}_{4}$ single crystals compared with corresponding data for $\beta^{\prime \prime}-(\mathrm{CNB}-\mathrm{EDT}-\mathrm{TTF})_{4} \mathrm{ClO}_{4}$ and $\beta^{\prime \prime}-(\mathrm{CNB}-\mathrm{EDT}-\mathrm{TTF})_{4} \mathrm{PF}_{6}$ (thin lines, from reference [1]). 
The high temperature regime of large and almost temperature independent thermopower appears as a common feature of the $\beta^{\prime \prime}$-salts of this donor. In this respect it is worth consider that the thermopower, which is the entropy per charge carrier weighted by their contribution to the total electrical conductivity, is directly related to the density of states at the Fermi level and the contribution $\sigma(E)$ of states with energy $E$ close to the Fermi level, $\mathrm{E}_{\mathrm{F}}$, to the total conductivity $\sigma$ [21]:

$$
S=-k \mathrm{~B} / e \int(E-\mathrm{EF}) / k \mathrm{BT}(\sigma(E) / \sigma) d E
$$

Therefore the large thermopower of these $4: 1 \beta^{\prime \prime}$-salts is a consequence of the large band filling placing the Fermi level in region of large effective mass. In contrast to the low temperature regime, with a $S \alpha T$ behavior typical of metals, the high temperature regime with almost temperature independent thermopower can result from correlation effects in a narrow band, when the carrier mean free path becomes comparable to the lattice parameters and the transport mechanism becomes diffusive rather than coherent. Finally it should be mentioned that the measured thermopower value of $\sim 110 \mu \mathrm{V} / \mathrm{K}$ in $\beta^{\prime \prime}$-(CNB-EDT-TTF $)_{4} \mathrm{BF}_{4}$ is to the best of our knowledge the largest one observed in a molecular metallic system. Although the thermal conductivity of these molecular compounds was not yet measured, it is expected to be modest in view of the complex molecular structure $(\sim 1 \mathrm{~W} / \mathrm{Km})[22,23]$, the large thermopower values of these bilayer systems place them as good candidates for efficient thermoelectric materials. In fact from an electrical conductivity $\sigma=20 \mathrm{~S} / \mathrm{cm}$ and thermoelectric power $S=110 \mu \mathrm{V} / \mathrm{K}$, a power factor $P F=S^{2} \sigma=24 \mu \mathrm{W} / \mathrm{K}^{2} \mathrm{~m}$ can be deduced, which is comparable to the best values reported so far for molecular materials such as $387 \mu \mathrm{W} / \mathrm{K}^{2} \mathrm{~m}$ in (TTT) $\mathrm{I}_{3}$ or $110 \mu \mathrm{W} / \mathrm{K}^{2} \mathrm{~m}$ in $\mathrm{Cu}(\mathrm{DMDCNQI})_{2}[1]$.

\section{Conclusions}

Based on the electron donor CNB-EDT-TTF and the small tetrahedral anion $\mathrm{BF}_{4}{ }^{-}$salts with 1:1 and $4: 1$ stoichiometries-(CNB-EDT-TTF)BF 4 and $\beta^{\prime \prime}-(\mathrm{CNB}-\mathrm{EDT}-\mathrm{TTF})_{4} \mathrm{BF}_{4}$ - can be obtained by electrocrystallization. The 1:1 salt is an electrical insulator but the $4: 1$ is a new member of the family of 2D metals with donor bilayer structure. Its structure and properties are comparable to the other compounds previously described, but the anions are more disordered and in spite of the smaller anion size the lattice parameters are slightly larger and intermolecular interactions smaller, while thermoelectric power becomes larger. The thermoelectric power of $\beta^{\prime \prime}-(\mathrm{CNB}-\mathrm{EDT}-\mathrm{TTF})_{4} \mathrm{BF}_{4}$, is very large, to the best of our knowledge the largest reported among molecular metallic systems, placing this family of bilayer compounds $\beta^{\prime \prime}$-(CNB-EDT-TTF $)_{4} \mathrm{X}$ as good candidates for efficient thermoelectric materials.

Supplementary Materials: The following are available online at http:/ /www.mdpi.com/2073-4352/8/4/142/s1: Figure S1: ORTEP and atomic numbering schemes (top and side views) of (CNB-EDT-TTF)BF4 (1). Thermal ellipsoids drawn at 50\% probability level; Figure S2: ORTEP and atomic numbering schemes (top and side views) of (CNB-EDT-TTF)4BF4 (2). Thermal ellipsoids drawn at 50\% probability level; Table S1: Bond lengths of compound 1; Table S2: Short contacts of compound 1; Table S3: Bond lengths of compound 2, Table S4: Short contacts of compound 2 .

Acknowledgments: This work was supported by FCT (Portugal) through contract UID/Multi/04349/2013 and grants to SO (SFRH/BD/72722/2010) and S.R. (SFRH/BPD/113344/2015). Work in Bellaterra was supported by MINECO (Spain) through Grant FIS2015-64886-C5-3-P as well as the Severo Ochoa Centers of Excellence Program under Grant SEV-2015-0496, and by Generalitat de Catalunya (2017SGR1506).

Author Contributions: Sandra Rabaça and Manuel Almeida conceived and designed the experiments; Sandrina Oliveira and Vasco Gama performed the sample preparation and participated in all experiments; Isabel C. Santos analyzed the crystallographic data, Elsa B. Lopes and Gonçalo Oliveira measured the electric transport properties, Enric Canadell performed electronic band structure calculations, Sandra Rabaça and Manuel Almeida wrote the paper with the contributions from all authors. 
Conflicts of Interest: The authors declare no conflict of interest. The founding sponsors had no role in the design of the study; in the collection, analyses, or interpretation of data; in the writing of the manuscript, and in the decision to publish the results.

\section{References}

1. Oliveira, S.; Ministro, J.; Santos, I.C.; Belo, D.; Lopes, E.B.; Rabaça, S.; Canadell, E.; Almeida, M. Bilayer molecular metals based on dissymmetrical electron donors. Inorg. Chem. 2015, 54, 6677-6679. [CrossRef] [PubMed]

2. Rabaça, S.; Oliveira, S.; Gama, V.; Santos, I.C.; Belo, D.; Lopes, E.B.; Canadell, E.; Almeida, M. Polymorphism and Superconductivity in Bilayer Molecular Metals (CNB-EDT-TTF) $\mathrm{I}_{3}$. Inorg. Chem. 2016, 55, 10343-10350. [CrossRef] [PubMed]

3. Rabaça, S.; Oliveira, S.; Gonçalves, A.C.; Gama, V.; Santos, I.C.; Belo, D.; Almeida, M. CNB-EDT-TTF salts with $\mathrm{ClO}_{4}{ }^{-}$; bilayer polymorphs and different stoichiometries. Cryst. Growth Des. 2017, 17, 2801-2808. [CrossRef]

4. Oliveira, S.; Santos, I.C.; Lopes, E.B.; Coutinho, J.T.; Pereira, L.C.J.; Belo, D.; Rabaça, S.; Almeida, M. Charge-Transfer Salts Based on a Dissymmetrical Cyano-Substituted Tetrathiafulvalene Donor. Eur. J. Inorg. Chem. 2016, 8, 1287-1292. [CrossRef]

5. Oliveira, S.; Belo, D.; Santos, I.C.; Rabaça, S.; Almeida, M. Synthesis and characterization of the cyanobenzene-ethylenedithio-TTF donor. Beil. J. Org. Chem. 2015, 11, 951-956. [CrossRef] [PubMed]

6. Sheldrick, G.M. SADABS; Bruker AXS Inc.: Madison, WI, USA, 2004.

7. Bruker. SMART and SAINT; Bruker AXS Inc.: Madison, WI, USA, 2008.

8. Altomare, A.; Burla, M.C.; Camalli, M.; Cascarano, G.; Giacovazzo, G.; Guagliardi, A.; Moliterni, A.G.G.; Polidori, G.; Spagna, R. SIR97: A new tool for crystal structure determination and refinement. J. Appl. Cryst. 1999, 32, 115-119. [CrossRef]

9. Sheldrick, G.M. SHELXL97, Program for Crystal Structure Refinement. Acta Cryst. A 2008, 64, 112-122. [CrossRef] [PubMed]

10. Farrugia, L.J. WinGX and ORTEP for Windows: An update. J. Appl. Cryst. 2012, 45, 849-854. [CrossRef]

11. Macrae, C.F.; Bruno, I.J.; Chisholm, J.A.; Edgington, P.R.; McCabe, P.; Pidcock, E.; Rodriguez-Monge, L.; Taylor, R.; van de Streek, J.; Wood, P.A. Mercury. J. Appl. Cryst. 2008, 41, 466-470. [CrossRef]

12. Whangbo, M.-H.; Hoffmann, R. The band structure of the tetracyanoplatinate chain. J. Am. Chem. Soc. 1978, 100, 6093-6098. [CrossRef]

13. Ammeter, J.H.; Bürgi, H.-B.; Thibeault, J.; Hoffmann, R. Counterintuitive orbital mixing in semiempirical and ab initio molecular orbital calculations. J. Am. Chem. Soc. 1978, 100, 3686-3692. [CrossRef]

14. Chaikin, P.M.; Kwak, J.F. Apparatus for Thermopower Measurements on Organic Conductors. Rev. Sci. Instrum. 1975, 46, 218-220. [CrossRef]

15. Almeida, M.; Alcácer, L.; Oostra, S. Anisotropy of thermopower in N-methyl-N-ethylmorpholinium bistetracyanoquinodimethane, $\mathrm{MEM}(\mathrm{TCNQ})_{2}$, in the region of the high-temperature phase transitions. Phys. Rev. B 1984, 30, 2839-2844. [CrossRef]

16. Lopes, E.B. INETI, Sacavém, Portugal; Internal Report; INETI Press: Sacavém, Portugal, 1991.

17. Huebner, R.P. Thermoelectric Power of Lattice Vacancies in Gold. Phys. Rev. 1964, 135, A1281-A1921. [CrossRef]

18. Schaffer, P.E.; Wudl, F.; Thomas, G.A.; Ferraris, J.P.; Cowan, D.O. Apparent giant conductivity peaks in an anisotropic medium: TTF-TCNQ. Solid State Commun. 1974, 14, 347-351. [CrossRef]

19. Rousseau, R.; Gener, M.; Canadell, E. Step-by-Step Construction of the Electronic Structure of Molecular Conductors: Conceptual Aspects and Applications. Adv. Funct. Mater. 2004, 14, 201-214. [CrossRef]

20. Altshuler, B.L.; Aronov, A.G. Electron-Electron Interaction in Disordered Conductors. In Electron-Electron Interactions in Disordered Systems; Efros, A.L., Pollak, M., Eds.; Elsevier: Amsterdam, The Netherlands, 1985; Volume 10, pp. 1-690. ISBN 0444-86916-6.

21. Fritzsche, H. A general expression for the thermoelectric power. Solid State Commun. 1971, 9, 1813-1815. [CrossRef] 
22. Huewe, F.; Steeger, A.; Kostova, K.; Burroughs, L.; Bauer, I.; Strohrieg, P.; Dimitrov, V.; Woodward, S.; Pflaum, J. Low-Cost and Sustainable Organic Thermoelectrics Based on Low-Dimensional Molecular Metals. Adv. Mater. 2017, 29, 1605682. [CrossRef] [PubMed]

23. Russ, B.; Glaudell, A.; Urban, J.J.; Chabinyc, M.L.; Segalman, R.A. Organic thermoelectric materials for energy harvesting and temperature control. Nat. Rev. Mater. 2016, 1, 16050-16063. [CrossRef]

(C) 2018 by the authors. Licensee MDPI, Basel, Switzerland. This article is an open access article distributed under the terms and conditions of the Creative Commons Attribution (CC BY) license (http:/ / creativecommons.org/licenses/by/4.0/). 
Article

\title{
Optical Conductivity in a Two-Dimensional Extended Hubbard Model for an Organic Dirac Electron System $\alpha-(\text { BEDT-TTF })_{2} \mathrm{I}_{3}$
}

\author{
Daigo Ohki ${ }^{1, *}$, Genki Matsuno ${ }^{1}$, Yukiko Omori ${ }^{2}$ and Akito Kobayashi ${ }^{1}$ \\ 1 Department of Physics, Nagoya University, Furo-cho, Chikusa-ku, Nagoya 464-8602, Japan; \\ matsuno@s.phys.nagoya-u.ac.jp (G.M.); akito@s.phys.nagoya-u.ac.jp (A.K.) \\ 2 Toyota College, National Institute of Technology, Eisei-cho 2-1, Toyota 471-8525, Japan; \\ yukiko.omori@toyota-ct.ac.jp \\ * Correspondence: dohki@s.phys.nagoya-u.ac.jp
}

Received: 25 February 2018; Accepted: 14 March 2018; Published: 16 March 2018

\begin{abstract}
The optical conductivity in the charge order phase is calculated in the two-dimensional extended Hubbard model describing an organic Dirac electron system $\alpha$-(BEDT-TTF) ${ }_{2} \mathrm{I}_{3}$ using the mean field theory and the Nakano-Kubo formula. Because the interband excitation is characteristic in a two-dimensional Dirac electron system, a peak structure is found above the charge order gap. It is shown that the peak structure originates from the Van Hove singularities of the conduction and valence bands, where those singularities are located at a saddle point between two Dirac cones in momentum space. The frequency of the peak structure exhibits drastic change in the vicinity of the charge order transition.
\end{abstract}

Keywords: Dirac electron; charge order; optical conductivity; organic conductor; $\alpha$-(BEDT-TTF) ${ }_{2} \mathrm{I}_{3}$

\section{Introduction}

An organic conductor $\alpha$-(BEDT-TTF $)_{2} \mathrm{I}_{3}$ has attracted much interest, since it exhibits a transition between the charge order (CO) [1-7] and the massless Dirac electron (DE) [8-15] as hydrostatic pressure, $P$, increases. Recently, strong electron correlation effects have been revealed in both the $\mathrm{CO}[16]$ and the massless DE [17-20] in spin fluctuations. Thus, electron correlation effects in transport phenomena have also been expected, especially in the crossover region between the massless DE and the CO.

It has been observed that the optical gap determined by the optical conductivity decreases monotonously as $P$ increases and reaches almost zero at about $P_{c}=12 \mathrm{kbar}[21,22]$, while the resistivity gap reaches almost zero at about 7 kbar [23]. In order to explain such metallic behavior in the presence of the $\mathrm{CO}$ gap, metallic channels owing to edges and domain walls in the CO have been studied using the extended Hubbard model [24-27]. It has been shown that the massive DE phase with the gapless edge states emerges in the intermediate region between the massless DE phases and the trivial $\mathrm{CO}$. Although the $\mathrm{CO}$ gap induced by the inversion symmetry breaking exists in both the massive DE phase and the trivial CO, a pair of Dirac cones with a finite gap at incommensurate momentum, $\pm \mathbf{k}_{\mathrm{D}}$, merges at a time reversal invariant momentum (TRIM) at the transition between these two phases $[12,15,28,29]$. Such a drastic change in the band structure is expected to give rise to a characteristic in the optical conductivity.

In the present paper, the optical conductivity is calculated in the band structure determined by the mean-field theory in the extended Hubbard model for the two-dimensional electron system in the organic conductor $\alpha$-(BEDT-TTF $)_{2} \mathrm{I}_{3}$, where the nearest-neighbor Coulomb repulsion, $V_{a}$ is used to control the charge ordering [1,2], although the donor-acceptor interactions can be also important for the charge ordering [7]. It is shown that a peak structure emerges above the CO gap in the optical 
conductivity. The frequency of the peak structure, $\omega_{\text {peak }}$, owing to interband excitation between two Van Hove singularities in the conduction and valence bands, rapidly moves as a nonmonotonic function of $V_{a}$, while the frequency of the $\mathrm{CO}$ gap, $\omega_{\mathrm{CO}}$, increases monotonically as $V_{a}$ increases. Those Van Hove singularities originate from the saddle points between two Dirac cones. The optical conductivity exhibits a characteristic strong peak when two Dirac cones merge in the presence of a large CO gap.

This paper is described as follows. An extended Hubbard model for a two-dimensional electron system in $\alpha$-(BEDT-TTF $)_{2} \mathrm{I}_{3}$, the mean field theory, and the optical conductivity are described in Section 2. Numerical results are shown in Section 3. Sections 4 and 5 are devoted to the discussion and summary.

\section{Formulation}

The two-dimensional extended Hubbard model $[1,2]$ has been used in theoretical studies for $\alpha$-(BEDT-TTF $)_{2} \mathrm{I}_{3}$, in order to take the on-site Coulomb repulsion, $U$, and the nearest-neighbor Coulomb repulsions, $V_{\alpha \beta}$, into account.

$$
\begin{aligned}
H & =\sum_{(i \alpha, j \beta), \sigma}\left(t_{(i \alpha, j \beta)} a_{i \alpha \sigma}^{\dagger} a_{j \beta \sigma}+\text { h.c. }\right) \\
& +\sum_{i \alpha} U a_{i \alpha \uparrow}^{\dagger} a_{i \alpha \downarrow}^{\dagger} a_{i \alpha \downarrow} a_{i \alpha \uparrow} \\
& +\sum_{(i \alpha: j \beta)} \sum_{\sigma, \sigma^{\prime}} V_{i \alpha j \beta} a_{i \alpha \sigma}^{\dagger} a_{j \beta \sigma^{\prime}}^{\dagger} a_{j \beta \sigma^{\prime}} a_{i \alpha \sigma},
\end{aligned}
$$

where $a_{i \alpha \sigma}$ and $t_{(i \alpha, j \beta)}$ represent the annihilation operators and the transfer energies with unit cells $i, j$, spins $\sigma$, and sublattices $\alpha, \beta=\mathrm{A}, \mathrm{A}^{\prime}, \mathrm{B}$ and $\mathrm{C}$ of $\alpha$-(BEDT-TTF $)_{2} \mathrm{I}_{3}$.

Hereafter, the energies are given in eV. The tight binding model for $\alpha$-(BEDT-TTF) ${ }_{2} \mathrm{I}_{3}[30-34]$ is shown in Figure 1a. The sublattices A and $\mathrm{A}^{\prime}$ are equivalent due to the inversion symmetry in the massless DE phase. The transfer energies given by the first-principle calculation [33]: $t_{b 1}=0.1241$, $t_{b 2}=0.1296, t_{b 3}=0.0513, t_{b 4}=0.0152, t_{a 1}=-0.0267, t_{a 2}=-0.0511, t_{a 3}=0.0323, t_{a 1}^{\prime}=0.0119$, $t_{a 3}^{\prime}=0.0046$, and $t_{a 4}^{\prime}=0.0060$. The nearest neighbor interaction $V_{a}$ in the stacking direction is used for controlling the $\mathrm{CO}$ transition, because this is the most sensitive as a function of $P$ [35-37], while we treat $U=0.4$ and $V_{b}=0.05$ as constants. The temperature $T=0.001$ is fixed in the present paper. The lattice constants, $k_{\mathrm{B}}$ and $\hbar$ are taken as unity. The system size in numerical calculations is $N_{L}=500$.

The mean-field Hamiltonian $H_{\mathrm{MF}}[11]$ is

$$
\begin{aligned}
H_{\mathrm{MF}} & =\sum_{\mathbf{k} \alpha \beta \sigma} \tilde{\epsilon}_{\alpha \beta \sigma}(\mathbf{k}) a_{\mathbf{k} \alpha \sigma}^{+} a_{\mathbf{k} \beta \sigma}, \\
\tilde{\epsilon}_{\alpha \beta \sigma}(\mathbf{k}) & =\phi_{\alpha \sigma} \delta_{\alpha \beta}+\epsilon_{\alpha \beta}(\mathbf{k}), \\
\phi_{\alpha \sigma} & =U_{\alpha}\left\langle n_{\alpha-\sigma}\right\rangle+\sum_{\beta^{\prime} \sigma^{\prime}} V_{\alpha \beta^{\prime}}\left\langle n_{\beta^{\prime} \sigma^{\prime}}\right\rangle, \\
\epsilon_{\alpha \beta}(\mathbf{k}) & =\sum_{\delta} t_{\alpha \beta} e^{i \mathbf{k} \cdot \delta},
\end{aligned}
$$

where $\phi_{\alpha \sigma}$ is the Hartree potential, $\left\langle n_{\alpha \sigma}\right\rangle=\left\langle a_{i \alpha \sigma}^{\dagger} a_{i \alpha \sigma}\right\rangle$ is the electron number, and $\delta$ is a vector between unit cells. The energy eigenvalues $\xi_{\gamma \sigma}(\mathbf{k})$ and the wave functions $\Phi_{\alpha \gamma \sigma}(\mathbf{k})$ are given by

$$
\sum_{\beta} \tilde{\epsilon}_{\alpha \beta \sigma}(\mathbf{k}) \Phi_{\beta \gamma \sigma}(\mathbf{k})=\xi_{\gamma \sigma}(\mathbf{k}) \Phi_{\alpha \gamma \sigma}(\mathbf{k}),
$$


with the band index $\gamma=1,2,3,4$. The conduction and valence bands correspond to $\xi_{1 \sigma}(\mathbf{k})$ and $\xi_{2 \sigma}(\mathbf{k})$, respectively, since the Fermi energy is located between these two bands. The electron number $\left\langle n_{\alpha \sigma}\right\rangle$ is given by

$$
\left\langle n_{\alpha \sigma}\right\rangle=\sum_{\mathbf{k} \gamma}\left|\Phi_{\alpha \gamma \sigma}(\mathbf{k})\right|^{2} f\left(\xi_{\gamma \sigma}(\mathbf{k})\right)
$$

where the Fermi distribution function is $f\left(\xi_{\gamma \sigma}(\mathbf{k})\right)=1 /\left(\exp \left[\left(\xi_{\gamma \sigma}(\mathbf{k})-\mu\right) / k_{B} T\right]+1\right)$ with the chemical potential $\mu$ determined so that the bands are 3/4-filled. Hereafter, let $E_{\gamma \sigma}(\mathbf{k})=\xi_{\gamma \sigma}(\mathbf{k})-\mu$ and measure the energy from $\mu$.

The Green function $G_{\alpha \beta \sigma}(\omega, \mathbf{k})$ and the density of state $\rho(\omega)$ are given by

$$
\begin{gathered}
G_{\alpha \beta \sigma}(\omega, \mathbf{k})=\sum_{\gamma} \frac{\Phi_{\alpha \gamma \sigma}^{*} \Phi_{\beta \gamma \sigma}}{\hbar \omega-E_{\gamma \sigma}(\mathbf{k})+i \delta^{\prime}}, \\
\rho(\omega)=\frac{1}{N_{L}} \sum_{\mathbf{k} \alpha \sigma}\left(-\frac{1}{\pi} \operatorname{Im} G_{\alpha \alpha \sigma}(\omega, \mathbf{k})\right) \\
=\frac{1}{N_{L}} \sum_{\mathbf{k} \alpha \gamma \sigma} \delta\left(\hbar \omega-E_{\gamma \sigma}(\mathbf{k})\right)\left|\Phi_{\gamma \alpha \sigma}(\mathbf{k})\right|^{2},
\end{gathered}
$$

where $N_{L}$ is the number of lattice points.

The optical conductivity is calculated by the Nakano-Kubo formula based on linear response theory. It is represented by

$$
\begin{aligned}
\sigma(\omega) & =\frac{1}{i \omega}[K(\omega)-K(0)], \\
K(\omega) & =-\frac{1}{N_{L}}\left(\frac{e}{\hbar}\right)^{2} \sum_{\mathbf{k} \gamma \gamma^{\prime} \sigma}\left|\mathbf{v}_{\gamma \gamma^{\prime} \sigma}(\mathbf{k})\right|^{2} \frac{f\left(E_{\gamma \sigma}(\mathbf{k})\right)-f\left(E_{\gamma^{\prime} \sigma}(\mathbf{k})\right)}{E_{\gamma \sigma}(\mathbf{k})-E_{\gamma^{\prime} \sigma}(\mathbf{k})+\hbar \omega+i \delta^{\prime}},
\end{aligned}
$$

Here, $\gamma$ and $\sigma$ represent a band and spin indices respectively. $\mathbf{k}$ is a wave vector. $\delta$ is a Minute amount and $\omega=E / \hbar$. where the velocity matrix $\mathrm{v}_{\gamma \gamma^{\prime} \sigma}$ is calculated by

$$
\begin{aligned}
\mathrm{v}_{\gamma \gamma^{\prime} \sigma}(\mathbf{k}) & =\sum_{\alpha \beta} \Phi_{\alpha \gamma \sigma}^{*}(\mathbf{k}) v_{\alpha \beta \sigma}(\mathbf{k}) \Phi_{\beta \gamma^{\prime} \sigma}(\mathbf{k}), \\
v_{\alpha \beta \sigma}(\mathbf{k}) & =\frac{1}{\hbar} \frac{\partial}{\partial \mathbf{k}} \tilde{\epsilon}_{\alpha \beta \sigma}(\mathbf{k}) .
\end{aligned}
$$

\section{Results}

Figure $1 \mathrm{~b}$ shows the conduction and valence bands in the massless DE phase. There is a pair of massless Dirac cones at incommensurate momenta, $\pm \mathbf{k}_{\mathrm{D}}$. The Fermi energy is located at the degenerate points of the two bands. These two bands form the tilted conical structure around $\pm \mathbf{k}_{\mathrm{D}}$ as shown in Figure 1c. When the energy gap between the two bands is zero, the electronic states are called as the massless DE, since quasi-particles behave as massless Dirac fermions in the relativistic quantum mechanics. There are several saddle points at the TRIMs near the Fermi energy, e.g., the M-point in the conduction band (Mc-VHS), the M-point in the valence band (Mv-VHS), and the Y-point in the

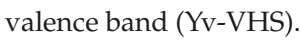

The optical conductivities at several $V_{a}$ are shown in Figure 2a. These values are divided by the universal conductivity $\sigma_{0}=\pi e^{2} / 2 h$ [38]. In the massless DE phase for $V_{a}=0.18$, the optical conductivity almost reaches a universal constant for $k_{\mathrm{B}} T<\hbar \omega<\Lambda$, where $\Lambda \cong 0.01$ is a energy scale of the linear dispersion as shown in previous studies for the massless DE [38-41]. When $V>V_{a}^{c 1}=0.198$, a frequency of the CO gap, $\omega_{\mathrm{CO}}$, increases as $V_{a}$ increases, as shown in Figure 2b, where $\omega_{\mathrm{CO}}$ is defined as a flexion point of the shoulder structure in the optical conductivity. It is found that a peak structure appears above $\omega_{\mathrm{CO}}$. As presented in Figure $2 \mathrm{~b}$, its frequency, $\omega_{\text {peak }}$, rapidly decreases as $V_{a}$ increases for $V_{a}<V_{a}^{c 2}=0.212$, at which the two Dirac cones are merged in the conduction band [26,27]. In this 
region, the inversion symmetry in the system is broken, and the finite gap opens near $\pm \mathbf{k}_{\mathrm{D}}$ in the energy dispersion, but two Dirac cones don't merge immediately. Thus, this region of $V_{a}^{c 1}<V_{a}<V_{a}^{c 2}$ is the massive DE phase. $\omega_{\text {peak }}$ rebounds after falling to $\omega_{\mathrm{CO}}$, and the optical conductivity shows a strong peak. Those two frequencies exhibit asymptotic behavior for $V_{a}>V_{a}^{c 2}$, where the CO phase with the trivial gap appears.

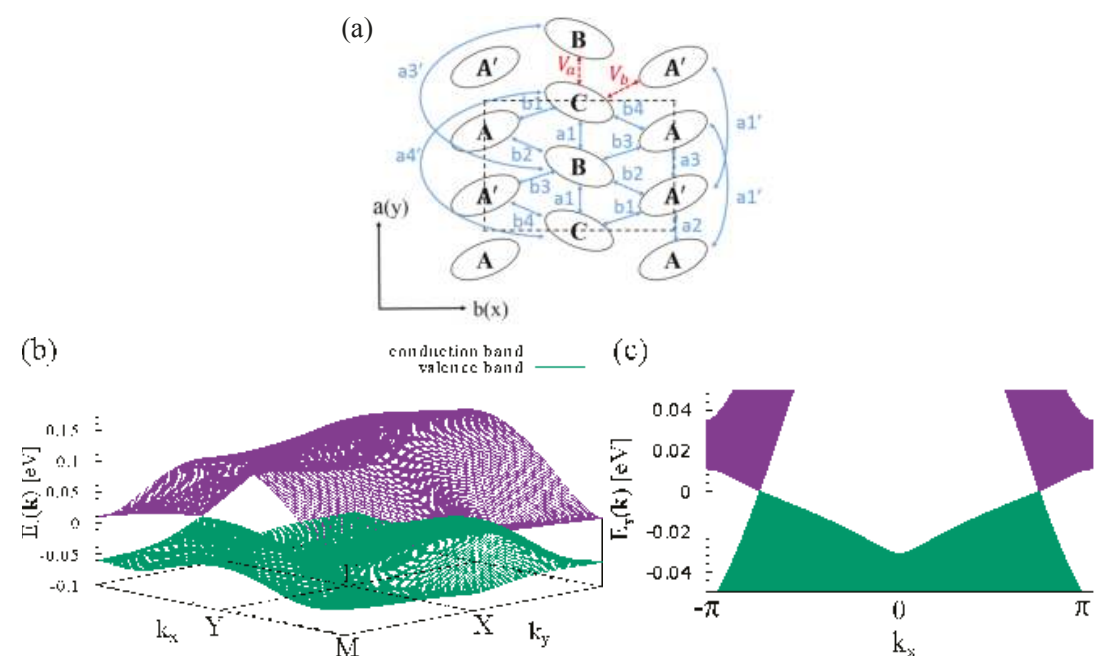

Figure 1. (a) Schematic figure of two-dimensional conductive plane in $\alpha$-(BEDT-TTF) ${ }_{2} \mathrm{I}_{3}$. Some ellipses represent sublattices of the BEDT-TTF molecule. In the figure, signs of b1, b2, .., a $4^{\prime}$ correspond to transfer integrals $t_{b 1}, t_{b 2}, \ldots, t_{a 4}^{\prime}$ between two molecules. Nearest-neighbor Coulomb repulsions $V_{a}$, $V_{b}$ are represented by the red dotted arrows. On-site Coulomb repulsion $U$ also exists, although it is not shown in the figure. The unit cell is a region surrounded by a black dotted square in the figure. In the absence of the Coulomb interactions, the inversion symmetry points exist on B and C sublattices, and at the midpoint between $\mathrm{A}$ and $\mathrm{A}^{\prime}$ sublattices. (b) The conduction band (purple) and valence band (green) in the massless DE phase for $V_{a}=0.18$, and (c) a view of same two bands projected from the side along $k_{x}$ axis.

(a)

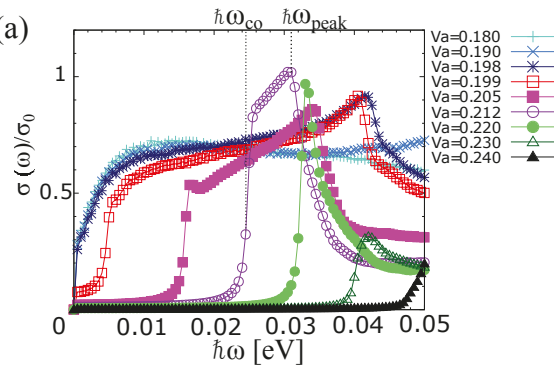

(b)

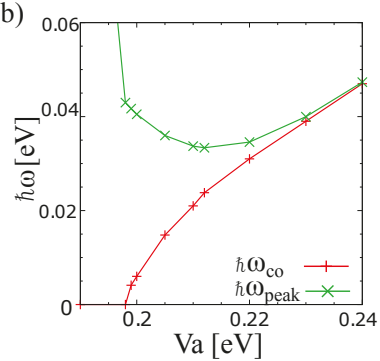

Figure 2. The optical conductivities divided by the universal conductivity $\sigma_{0}=\pi e^{2} / 2 h$ [38] for $V_{a}=0.180,0.190,0.198,0.199,0.205,0.212,0.220,0.230,0.240$ (a). $\omega_{\mathrm{CO}}$ and $\omega_{\text {peak }}$ for $V_{a}=V_{a}^{c 2}=0.212$ are shown in (a). $V_{a}$-dependences of $\omega_{\mathrm{CO}}$ and $\omega_{\text {peak }}$ are shown in (b). A characteristic peak structure is found near the $\mathrm{CO}$ transition in the extended Hubbard model describing the organic Dirac electron system $\alpha$-(BEDT-TTF $)_{2} \mathrm{I}_{3}$.

Figure 3 shows the density of states $\rho(\omega)$, where the Fermi energy is defined as zero. When $V_{a}<V_{a}^{c 1}$, there is a valley due to the Dirac cones. A CO gap, $\omega_{\mathrm{CO}}$, opens at the Fermi energy for $V>V_{a}^{c 1}$. 
There are several Van Hove singularities (VHS) due to saddle points of the conduction and valence bands (see Figure 1b). Here we pay attention to VHS at the M-point in the conduction band (Mc-VHS) and that in the valence band (Mv-VHS), respectively. The Mc-VHS remains an energy source for $V_{a}<V_{a}^{c 2}$. Its energy rapidly increases and the peak structure disappears for $V_{a}>V_{a}^{c 2}$, since it is absorbed by the upper edge of the CO gap, owing to the merging of the Dirac cones in the conduction band as shown in Figure 4. The Mv-VHS, on the other hand, moves very rapidly until it reaches the lower edge of the CO gap at $V_{a}^{c 2}$. The Mv-VHS and the lower edge of the CO gap exhibits asymptotic behavior for $V_{a}>V_{a}^{c 2}$. An energy difference between the Mc-VHS and Mv-VHS coincides with $\omega_{\text {peak }}$ in the optical conductivity shown in Figure 2a. Other VHSs, e.g., VHS at the Y-point in the valence band (Yv-VHS), show completely different behavior as $V_{a}$ increases.

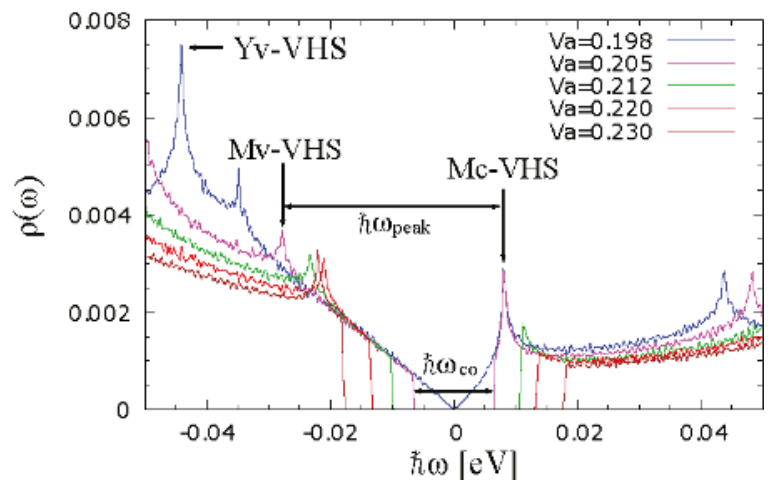

Figure 3. The density of states $\rho(\omega)$ for $V_{a}=0.198,0.205,0.212,0.220,0.230$. The origin of the peak structure in the optical conductivity is identified.

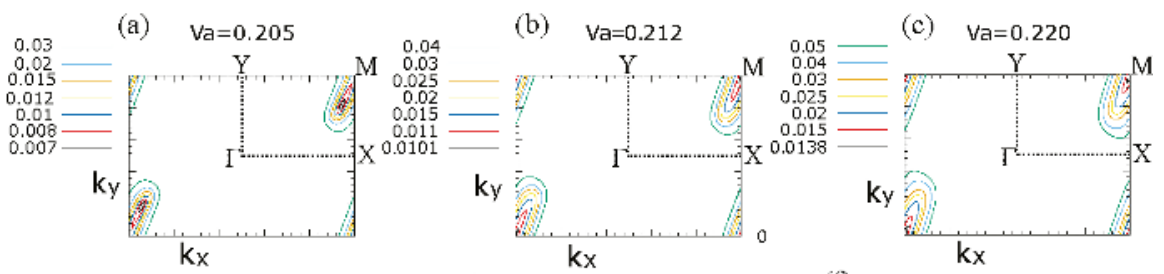

(d)

(c)

(1)

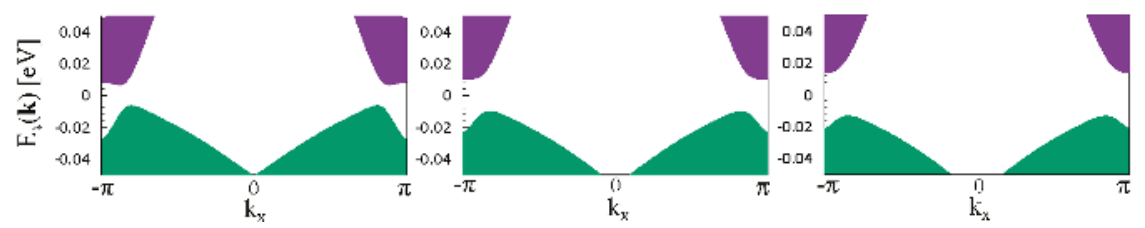

Figure 4. The contour plots of the conduction band for $V_{a}=0.205$ (a), $V_{a}=V_{a}^{c 2}=0.212(\mathbf{b})$, and $V_{a}=0.220$ (c). The band structures as a function of $k_{x}$ for $V_{a}=0.205(\mathbf{d}), V_{a}=V_{a}^{c 2}=0.212(\mathbf{e})$, and $V_{a}=0.220$ (f), where the CO gaps open between the conduction bands (the purple bands) and the valence bands (the green bands). Tilting of the Dirac cones causes electron-hole asymmetric behavior as the CO gap increases.

In order to analyze the behavior of $\omega_{\text {peak }}$ and $\omega_{\mathrm{CO}}$, the band structure is intensively examined in Figure 4. Both Mc-VHS and Mv-VHS exist at the M-point (the saddle point) between two Dirac cones. The Dirac cones in the conduction band (the purple band) merge at the $M$-point at $V_{a}^{c 2}$ in the presence of a large CO gap as shown in Figure $4 \mathrm{~b}$,e, leading to the absorption of the Mc-VHS into the upper 
edge of the CO gap. On the other hand, the massive Dirac cones in the valence band (the green band) do not merge against relatively larger $V_{a}$ as shown in Figure 4c,f, since the Dirac cones are tilted [12,13]. Thus, the Mv-VHS and the lower edge of the CO gap show asymptotic behavior.

\section{Discussion}

The drastic change of the optical conductivity near $\mathrm{CO}$ transition is characterized not only by the CO gap, but also the peak structure, as shown in Figure 2a,b. By careful analysis on the $\rho(\omega)$ shown in Figure 3 and band structure shown in Figure 4, it is elucidated that the origin of the peak structure is the VHSs at the M-points between two tilted Dirac cones. The nonmonotonic $V_{a}$-dependence of $\omega_{\text {peak }}$ is due to both the merging of the Dirac cones with the CO gap and the tilting of the Dirac cones. It is expected that those features may be observed in the low frequency region (around $100 \mathrm{~cm}^{-1}$ ), i.e., just above the $\mathrm{CO}$ gap near the $\mathrm{CO}$ transition $P_{c}=12 \mathrm{kbar}$ in the optical conductivity, although this region has not been analyzed by Beyer et al. [21].

It is a future problem to investigate the optical conductivity in the non-uniform $\mathrm{CO}$ with the edges or the domain walls $[26,27]$ using the real space dependent mean field theory, since it has been shown that the non-uniform $\mathrm{CO}$ may be important to explain the metallic $\mathrm{CO}$ near the $\mathrm{CO}$ transition [23]. The strong correlation effects beyond the mean field theory [16] may also affect the optical properties. Although the present calculation is based on the two-dimensional extended Hubbard model $[1,2]$ being a simplest model to describe the $\mathrm{CO}$ in $\alpha$-(BEDT-TTF $)_{2} \mathrm{I}_{3}$, the donor-acceptor interactions may also play an important role for the $\mathrm{CO}$ [7].

\section{Conclusions}

The optical conductivity in the vicinity of the $\mathrm{CO}$ transition has been investigated using the Nakano-Kubo formula and the mean-field theory in the two-dimensional extended Hubbard model describing the Dirac electrons in $\alpha$-(BEDT-TTF $)_{2} \mathrm{I}_{3}$. It has been found that a peak structure above the $\mathrm{CO}$ gap emerges due to the two-dimensional Dirac cones. It has been also shown that the drastic change of the peak structure in the vicinity of the $\mathrm{CO}$ transition indicates the merging of the massive Dirac electrons. Thus it has been shown that the optical conductivity in the low frequency region just above the $\mathrm{CO}$ gap indicates important information on the existence of the two-dimensional massive Dirac electrons and those merging in the intermediate region between the trivial $\mathrm{CO}$ phase and the massless DE phase.

Acknowledgments: This work was supported by MEXT/JSPJ KAKENHI under Grant Noes 15K05166 and 15H02108.

Author Contributions: Akito Kobayashi and Yukiko Omori conceived and designed the theories; Daigo Ohki and Genki Matsuno performed the numerical calculation and analyzed the data; Daigo Ohki and Akito Kobayashi wrote the paper.

Conflicts of Interest: The authors declare no conflict of interest.

\section{Abbreviations}

The following abbreviations are used in this manuscript:

DE Dirac electron

$\mathrm{CO}$ charge order

VHS Van Hove singularity

DOS density of states

TRIM time reversal invariant momentum

Mc-VHS Van Hove singularity at the M-point in the conduction band

Mv-VHS Van Hove singularity at the M-point in the valence band

$\mathrm{Y}_{\mathrm{v}}$-VHS Van Hove singularity at the Y-point in the valence band 


\section{References}

1. Kino, H.; Fukuyama, H. Interrelationship among Electronic States of $\alpha-(\mathrm{ET})_{2} \mathrm{I}_{3},(\mathrm{ET})_{2} \mathrm{MHg}(\mathrm{SCN})_{4}$ and א-(ET) 2 X. J. Phys. Soc. Jpn. 1995, 64, 4523, doi:10.1143/JPSJ.64.4523.

2. Seo, H. Charge Ordering in Organic ET Compounds. J. Phys. Soc. Jpn. 2000, 69, 805-820.

3. Hotta, C. Classification of Quasi-Two Dimensional Organic Conductors Based on a New Minimal Model. J. Phys. Soc. Jpn. 2003, 72, 840, doi:10.1143/JPSJ.72.840.

4. Takahashi, T. ${ }^{1}$ 3C-NMR studies of charge ordering in organic conductors. Synth. Met. 2003, 26, 133-134, doi:10.1016/S0379-6779(02)00404-6.

5. Seo, H.; Hotta, C.; Fukuyama, T. Toward Systematic Understanding of Diversity of Electronic Properties in Low-Dimensional Molecular Solids. Chem. Rev. 2004, 104, 5005, doi:10.1021/cr030646k.

6. Kakiuchi, T.; Wakabayashi, Y.; Sawa, H.; Takahashi, T.; Nakamura, T. Charge Ordering in $\alpha-(\mathrm{BEDT}-\mathrm{TTF})_{2} \mathrm{I}_{3}$ by Synchrotron X-ray Diffraction. J. Phys. Soc. Jpn. 2007, 76, 113702, doi:10.1143/JPSJ.76.113702.

7. Alemany, P.; Pouget, J.-P.; Canadell, E. Essential role of anions in the charge ordering transition of $\alpha$-(BEDT-TTF) ${ }_{2} \mathrm{I}_{3}$. Phys. Rev. B 2012, 85, 195118, doi:10.1103/PhysRevB.85.195118.

8. Kajita, K.; Ojiro, T.; Fujii, H.; Nishio, Y.; Kobayashi, H.; Kobayashi, A.; Kato, R. Magnetotransport Phenomena of $\alpha$-Type (BEDT-TTF) ${ }_{2} \mathrm{I}_{3}$ under High Pressures. J. Phys. Soc. Jpn. 1992, 61, 23, doi:10.1143/JPSJ.61.23.

9. Tajima, N.; Tamura, M.; Nishio, Y.; Kajita, K.; Iye, Y. Transport Property of an Organic Conductor $\alpha$-(BEDT-TTF $)_{2} \mathrm{I}_{3}$ under High Pressure-Discovery of a Novel Type of Conductor. J. Phys. Soc. Jpn. 2000, 69, 543-551, doi:10.1143/JPSJ.69.543.

10. Ishikawa, K.; Hirata, M.; Liu, D.; Miyagawa, K.; Tamura, M.; Kanoda, K. Spin excitations in the quasi-two-dimensional charge-ordered insulator $\alpha$-(BEDT-TTF) ${ }_{2} \mathrm{I}_{3}$ probed via ${ }^{1} 3 \mathrm{C}$ NMR. Phys. Rev. B 2016, 94, 085154, doi:10.1103/PhysRevB.94.085154.

11. Kobayashi, A.; Katayama, S.; Noguchi, K.; Suzumura, Y. Superconductivity in Charge Ordered Organic Conductor $-\alpha$-(ET) ${ }_{2} \mathrm{I}_{3}$ Salt-. J. Phys. Soc. Jpn. 2004, 73, 3135, doi:10.1143/JPSJ.73.3135.

12. Katayama, S.; Kobayashi, A.; Suzumura, Y. Pressure-Induced Zero-Gap Semiconducting State in Organic Conductor $\alpha$-(BEDT-TTF) ${ }_{2} \mathrm{I}_{3}$ Salt. J. Phys. Soc. Jpn. 2006, 75, 054705, doi:10.1143/JPSJ.75.054705.

13. Kobayashi, A.; Katayama, S.; Suzumura, Y.; Fukuyama, H. Massless Fermions in Organic Conductor. J. Phys. Soc. Jpn. 2007, 76, 034711, doi:10.1143/JPSJ.76.034711.

14. Kajita, K.; Nishio, Y.; Tajima, N.; Suzumura, Y.; Kobayashi, A. Molecular Dirac Fermion Systems—Theoretical and Experimental Approaches-. J. Phys. Soc. Jpn. 2014, 83, 072002, doi:10.7566/JPSJ.83.072002.

15. Goerbig, M.O.; Fuchs, J.-N.; Montambaux, G.; Piéchon, F. Tilted anisotropic Dirac cones in quinoid-type graphene and $\alpha$-(BEDT-TTF) ${ }_{2} \mathrm{I}_{3}$. Phys. Rev. B 2008, 78, 045415, doi:10.1103/PhysRevB.78.045415.

16. Tanaka, Y.; Ogata, M. Correlation Effects on Charge Order and Zero-Gap State in the Organic Conductor $\alpha$-(BEDT-TTF) ${ }_{2} \mathrm{I}_{3}$. J. Phys. Soc. Jpn. 2016, 85, 104706, doi:10.7566/JPSJ.85.104706.

17. Hirata, M.; Ishikawa, K.; Miyagawa, K.; Tamura, M.; Berthier, C.; Basko, D.; Kobayashi, A.; Matsuno, G.; Kanoda, K. Observation of an anisotropic Dirac cone reshaping and ferrimagnetic spin polarization in an organic conductor. Nat. Commun. 2016, 7, 12666, doi:10.1038/ncomms12666.

18. Matsuno, G.; Kobayashi, A. Effect of Interband Fluctuation on Spin Susceptibility in Molecular Dirac Fermion System $\alpha$-(BEDT-TTF) ${ }_{2} \mathrm{I}_{3}$. J. Phys. Soc. Jpn. 2017, 86, 014705, doi:10.7566/JPSJ.86.014705.

19. Hirata, M.; Ishikawa, K.; Matsuno, G.; Kobayashi, A.; Miyagawa, K.; Tamura, M.; Berthier, C.; Kanoda, K. Anomalous spin correlations and excitonic instability of interacting 2D Weyl fermions. Science 2017, 358, 1403-1406, doi:10.1126/science.aan5351.

20. Matsuno, G.; Kobayashi, A. Coexistence of velocity renormalization and ferrimagnetic fuctuation in an organic Dirac electron system $\alpha$-(BEDT-TTF) ${ }_{2} \mathrm{I}_{3}$. accepted to J. Phys. Soc. Jpn. 2018, 87.

21. Beyer, R.; Dengl, A.; Peterseim, T.; Wackerow, S.; Ivek, T.; Pronin, A.V.; Schweitzer, D.; Dressel, M. Pressuredependent optical investigations of $\alpha$-(BEDT-TTF $)_{2} \mathrm{I}_{3}$ : Tuning charge order and narrow gap towards a Dirac semimetal. Phys. Rev. B 2016, 93, 195116, doi:10.1103/PhysRevB.93.195116.

22. Ivek, T.; Korin-Hamzić, B.; Milat, O.; Tomić, S.; Clauss, C.; Drichko, N.; Schweitzer, D.; Dressel, M. Electrodynamic response of the charge ordering phase: Dielectric and optical studies of $\alpha$-(BEDT-TTF) ${ }_{2} \mathrm{I}_{3}$. Phys. Rev. B 2011, 83, 165128, doi:10.1103/PhysRevB.83.165128. 
23. Liu, D.; Ishikawa, K.; Takehara, R.; Miyagawa, K.; Tamura, M.; Kanoda, K. Insulating nature of strongly correlated massless Dirac fermions in an organic crystal. Phys. Rev. Lett. 2016, 116, 226401, doi:10.1103/PhysRevLett.116.226401.

24. Hasegawa, Y.; Kishigi, K. Edge States in the Three-Quarter Filled System, $\alpha$-(BEDT-TTF $)_{2} \mathrm{I}_{3}$. J. Phys. Soc. Jpn. 2011, 80, 054707, doi:10.1143/JPSJ.80.054707.

25. Omori, Y.; Matsuno, G.; Kobayashi, A. Edge States in Molecular Solid $\alpha$-(BEDT-TTF) ${ }_{2} \mathrm{I}_{3}$ : Effects of Electron Correlations. JPS Conf. Proc. 2014, 1, 012119, doi:10.7566/JPSCP.1.012119.

26. Matsuno, G.; Omori, Y.; Eguchi, T.; Kobayashi, A. Topological Domain Wall and Valley Hall Effect in Charge Ordered Phase of Molecular Dirac Fermion System $\alpha$-(BEDT-TTF $)_{2} \mathrm{I}_{3}$. J. Phys. Soc. Jpn. 2016, 85, 094710, doi:10.7566/JPSJ.85.094710.

27. Omori, Y.; Matsuno, G.; Kobayashi, A. Longitudinal Conductivity on Edge and Domain Wall Molecular Dirac Electron System $\alpha$-(BEDT-TTF) ${ }_{2} \mathrm{I}_{3}$. J. Phys. Soc. Jpn. 2017, 86, 074708, doi:10.7566/JPSJ.86.074708.

28. Montambaux, G.; Piéchon, F.; Fuchs, J.-N.; Goerbig, M.O. Merging of Dirac points in a two-dimensional crystal. Phys. Rev. B 2009, 80, 153412, doi:10.1103/PhysRevB.80.153412.

29. Montambaux, G.; Piéchon, F.; Fuchs, J.-N.; Goerbig, M.O. A universal Hamiltonian for the motion and the merging of Dirac cones in a two-dimensional crystal. Eur. Phys. J. B 2009, 72, 509, doi:10.1140/epjb/e2009-00383-0.

30. Mori, T.; Kobayashi, A.; Sasaki, Y.; Kobayashi, H.; Saito, G.; Inokuchi, H. BAND STRUCTURES OF TWO TYPES OF (BEDT-TTF)2I3. Chem. Lett. 1984, 13, 957-960, doi:10.1246/cl.1984.957.

31. Mori, T.; Mori, H.; Tanaka, S. Structural Genealogy of BEDT-TTF-Based Organic Conductors II. Inclined Molecules: $\theta, \alpha$, and $\kappa$ Phases. Bull. Chem. Soc. Jpn. 1999, 72, 179, doi:10.1246/bcsj.72.179.

32. Kondo, R.; Kagoshima, S.; Harada, J. Crystal structure analysis under uniaxial strain at low temperature using a unique design of four-axis x-ray diffractometer with a fixed sample. Rev. Sci. Instrum. 2005, 76, 093902, doi:10.1063/1.2001607.

33. Kino, H.; Miyazaki, T. First-Principles Study of Electronic Structure in $\alpha$-(BEDT-TTF) ${ }_{2} \mathrm{I}_{3}$ at Ambient Pressure and with Uniaxial Strain. J. Phys. Soc. Jpn. 2006, 75, 034704, doi:10.1143/JPSJ.75.034704.

34. Ishibashi, S.; Tamura, T.; Kohyama, M.; Terakura, K. Ab Initio Electronic-Structure Calculations for $\alpha$-(BEDT-TTF) ${ }_{2} \mathrm{I}_{3}$. J. Phys. Soc. Jpn. 2006, 75, 015005, doi:10.1143/JPSJ.75.015005.

35. Kobayashi, A.; Katayama, S.; Suzumura, Y. Superconductivity in Charge Ordered Metal for Quasi-TwoDimensional Organic Conductor. J. Phys. Soc. Jpn. 2005, 74, 2897, doi:10.1143/JPSJ.74.2897.

36. Kobayashi, A.; Katayama, S.; Suzumura, Y. Theoretical study of the zero-gap organic conductor $\alpha$-(BEDT-TTF) ${ }_{2} \mathrm{I}_{3}$. Sci. Technol. Adv. Mater. 2009, 10, 024309, doi:10.1088/1468-6996/10/2/024309.

37. Tajima, N.; Kajita, K. Experimental study of organic zero-gap conductor $\alpha$-(BEDT-TTF $)_{2} \mathrm{I}_{3}$. Sci. Technol. Adv. Mater. 2009, 10, 024308, doi:10.1088/1468-6996/10/2/024308.

38. Stauber, T.; Peres, N.M.R.; Geim, A.K. Optical conductivity of graphene in the visible region of the spectrum. Phys. Rev. B 2008, 78, 085432, doi:10.1103/PhysRevB.78.085432.

39. Gusynin, V.P.; Sharapov, S.G.; Carbotte, J.P. Unusual Microwave Response of Dirac Quasiparticles in Graphene. Phys. Rev. Lett. 2006, 96, 256802, doi:10.1103/PhysRevLett.96.256802.

40. Suzumura, Y.; Proskurin, I.; Ogata, M. Dynamical Conductivity of Dirac Electrons in Organic Conductors. J. Phys. Soc. Jpn. 2014, 83, 094705, doi:10.7566/JPSJ.83.094705.

41. Suzumura, Y.; Proskurin, I.; Ogata, M. Reflectance of Dirac electrons in organic conductor. J. Phys. Conf. Ser. 2015, 603, 012011, doi:10.1088/1742-6596/603/1/012011

(C) 2018 by the authors. Licensee MDPI, Basel, Switzerland. This article is an open access article distributed under the terms and conditions of the Creative Commons Attribution (CC BY) license (http:/ / creativecommons.org/licenses/by/4.0/). 


\title{
Effects of Carrier Doping on the Transport in the Dirac Electron System $\alpha-(\text { BEDT-TTF })_{2} \mathrm{I}_{3}$ under High Pressure
}

\author{
Naoya Tajima \\ Department of Physics, Toho University, Miyama 2-2-1, Funabashi-shi, Chiba 274-8510, Japan; \\ naoya.tajima@sci.toho-u.ac.jp; Tel.: +81-47-472-6990
}

Received: 31 January 2018; Accepted: 6 March 2018; Published: 8 March 2018

\begin{abstract}
A zero-gap state with a Dirac cone type energy dispersion was discovered in an organic conductor $\alpha$-(BEDT-TTF $)_{2} \mathrm{I}_{3}$ under high hydrostatic pressures. This is the first two-dimensional (2D) zero-gap state discovered in bulk crystals with a layered structure. Moreover, the Dirac cones are highly tilted in a $k$-space. This system, thus, provides a testing ground for the investigation of physical phenomena in the multilayered, massless Dirac electron system with anisotropic Fermi velocity. Recently, the carrier injection into this system has been succeeded. Thus, the investigations in this system have expanded. The recent developments are remarkable. This effect exhibits peculiar (quantum) transport phenomena characteristic of electrons on the Dirac cone type energy structure.
\end{abstract}

Keywords: $\alpha$-(BEDT-TTF $)_{2} I_{3}$; Dirac electron system; transport phenomena; inter-band effects of the magnetic field; carrier doping; quantum Hall effect

\section{Introduction}

The realization of the graphene opened the physics of the Dirac electron in a solid [1,2]. A rich variety of material with Dirac electrons has been discovered, and the recent progress of the physics for Dirac electrons has been brilliant [3-16]. However, the zero-gap material with the Fermi level at the Dirac point is limited. The physics at the vicinity of the Dirac point is the most significant. Among them, we have discovered the zero-gap material with Dirac-type energy dispersion in $\alpha$-(BEDT-TTF $)_{2} \mathrm{I}_{3}$ (BEDT-TTF $=$ bis(ethylenedithio) tetrathiafulvalene) [17] (Figure 1) at high pressure. This is the first bulk (multilayered) 2D zero-gap system with Dirac electrons. Thus, this material has led the studies of the specific heat [18] and the nuclear magnetic resonance (NMR) [19] for the Dirac electron system. Another significant feature is that the Dirac cones are highly tilted as shown in Figure 1d,e [13-16]. Thus, this system has led to the peculiar transport characteristic of the electrons on the Dirac cone type energy structure [8-12,16,20-23]. This Dirac electron system, on the other hand, is next to the charge-ordered insulator phase in the temperature-pressure phase diagram. Therefore, strongly correlated Dirac electrons constitute one of the interesting recent studies [19,24,25].

The recent success of carrier injection helped the development of physics with regard to this system. In this review, the effects of carrier doping on the peculiar (quantum) transport phenomena are described. In the following, the electronic structure of $\alpha$-(BEDT-TTF $)_{2} \mathrm{I}_{3}$ and the experimental evidence of massless Dirac electron systems are briefly mentioned in this section. The methods of the carrier doping in Section 2 and those effects on the transport phenomena in Sections 3-5 are revealed. In Section 6, the Dirac type energy structure of this system is corrected. 
(a)

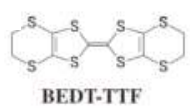

(b)

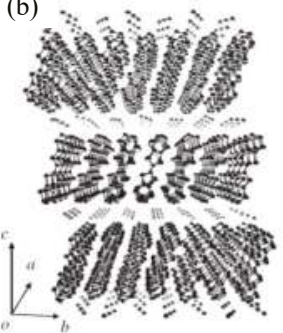

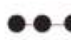

$I_{3}$

(c)

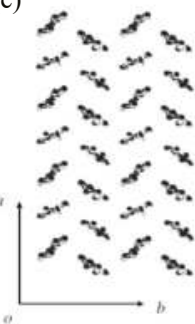

(d)

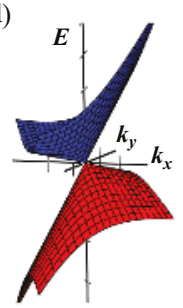

(e)

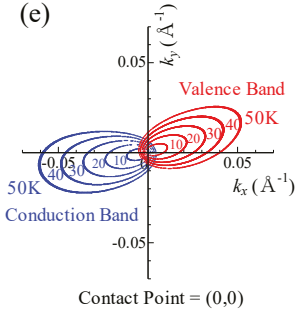

Figure 1. (a) BEDT-TTF molecule and $\mathrm{I}_{3}{ }^{-}$anion, crystal structure of $\alpha$-(BEDT-TTF) ${ }_{2} \mathrm{I}_{3}$ viewed from (b) $a$-axis and (c) $c$-axis, (d) band structure, and (e) energy contours near a Dirac point. Note that we take the origin to the position of the Dirac point.

\subsection{Electronic Structure of $\alpha-(B E D T-T T F)_{2} I_{3}$}

$\alpha$-(BEDT-TTF $)_{2} \mathrm{I}_{3}$ is a member of the (BEDT-TTF $)_{2} \mathrm{I}_{3}$ family [17]. The crystals consist of conductive layers of BEDT-TTF molecules and insulating layers of $\mathrm{I}_{3}{ }^{-}$anions as shown in Figure 1a-c [26-28]. The difference of the arrangement of BEDT-TTF molecules gives rise to variations in the transport phenomena. Most are 2D metals with large Fermi surfaces, and some of them show a superconducting transition [26-28]. $\alpha$-(BEDT-TTF $)_{2} \mathrm{I}_{3}$, however, is different from other members. The band calculation indicated a semimetal with small Fermi pockets of the electron character and the hole character [8].

This material behaves as a metal down to $135 \mathrm{~K}$, where it undergoes a phase transition to an insulator [17]. At temperatures below $135 \mathrm{~K}$, an abrupt drop of the magnetic susceptibility suggests that a nonmagnetic state with a spin gap is realized [29]. The theory by Kino and Fukuyama [30] and Seo [31], the NMR study by Takano et al. [32], the Raman study by Wojciechowski et al. [33], and the spectroscopy study by Moldenhauer et al. [34] indicated that the origin of this transition was due to the charge disproportionation. Each BEDT-TTF molecule with approximately $0.5 e$ has formed the horizontal charge stripe patterns for $+1 e$ and 0 at temperatures below $135 \mathrm{~K}[33,35]$. This phase transition is suppressed by the pressure above $1.5 \mathrm{GPa}$ at room temperature $[8,9,16]$.

The resistivity at high pressure is very peculiar. It is almost constant over the whole temperature range like dirty metals. The carrier mobility in dirty metals should be low, because the impurity scattering dominates the conduction. Thus, the resistance in dirty metals is temperature independent. However, the present situation is different. The large magnetoresistance at low temperatures indicated that the carrier mobility was extremely high. It was estimated to be approximately $10^{5} \mathrm{~cm} / \mathrm{V} \cdot \mathrm{s}$ at low temperatures $[9,16,35-37]$. The high carrier mobility led to the observation that the magnetic field warped the path of the electric currents [8]. So, this system is clean.

This is the motive with which this study has started. To clarify this mechanism, the Hall effect was investigated. Surprisingly, in the region from 300 to $2 \mathrm{~K}$, the carrier (hole) density and the mobility change by approximately six orders of magnitude as shown in Figure 2. At low temperatures, the state of extremely low density of approximately $8 \times 10^{14} \mathrm{~cm}^{-3}$ and extremely high mobility of approximately $3 \times 10^{5} \mathrm{~cm}^{2} / \mathrm{V} \cdot \mathrm{s}$ is realized $[10,16]$. The independent resistance is due to the effects of changes in the density, and the mobility just cancels out [8-10,16].

According to the band calculations by Kobayashi et al. and first-principles band calculations by Kino and Miyazaki, this material under high pressures is in the zero-gap state of which the bottom of the conduction band and the top of the valence band touch each other at two points (we call 
these "Dirac points") in the first Brillouin zone [13-15]. The Fermi energy is located exactly on the Dirac point.

In the picture of 2D zero-gap energy structure with a linear dispersion, the peculiar transport phenomena were naturally understood. The carrier density proportional to the temperature squared is explained. When the Fermi energy $E_{F}$ located at the Dirac point is temperature-independent, it is written as $n=\int D(E) f(E) d E \propto T^{2}$, in which $D(E)$ is the density of state and $f(E)$ is the Fermi distribution function. According to Mott's argument [38], on the other hand, the mean free path $l$ of a carrier can never be shorter than the wavelength $\lambda$ of the carrier, so $l \geq \lambda$. For the cases of high density of scattering centers, $l \sim \lambda(l k \sim 1)$. As the temperature is decreased, $l$ becomes long, because $\lambda$ becomes long ( $k$ becomes small) with the decreasing energy of the carriers. The Boltzmann transport equation gives the temperature-independent quantum conductivity as $\sigma_{x x}=8 e^{2} \int v_{x}^{2} \tau(-\partial f / \partial E) d k=2 e^{2} / h$, in which $v_{x}$ is the velocity of Dirac electrons when the electric field along $x$-axis is applied and $\tau$ is the lifetime. The constant sheet resistance (resistivity per layer) $R_{S}$ close to the value of the quantum resistance, $h / e^{2}=25.8 \mathrm{k} \Omega$, is derived. Many realistic theories for the sheet resistance in the zero-gap system give $R_{s}=g h / e^{2}$, in which $g$ is a parameter of order unity [39-41]. Combining the temperature dependences of the carrier density and the resistivity with $\sigma=n e \mu$, on the other hand, the temperature dependence of the carrier mobility $\mu \propto T^{-2}$ was led as shown in Figure 2.

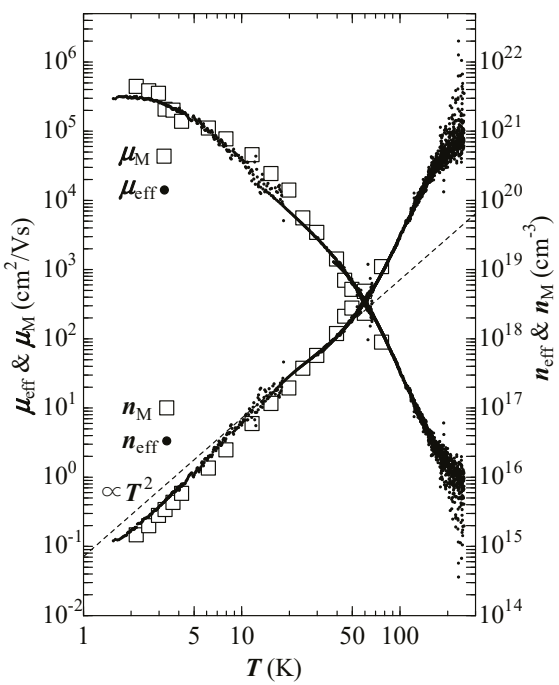

Figure 2. The carrier density and the mobility under the pressure of $p=1.8 \mathrm{GPa}$ against the temperature. Close circles show the effective carrier density $n_{\text {eff }}$ and the mobility $\mu_{e f f}$ estimated from the Hall coefficient $\left(R_{H}=1 / n e\right)$ and the conductivity $(\mu=\sigma / n e)$. The magnetoresistance mobility $\mu_{M}$ and the density $n_{M}$, on the other hand, is shown by open square. The carrier density obeys $n \propto T^{2}$ from $10 \mathrm{~K}$ to $50 \mathrm{~K}$ (indicated by broken lines). Reproduced with permission from [9].

\subsection{Experimental Evidence of Massless Dirac Electron System}

We can see the remarkable characteristic transport of 2D Dirac electron systems in the magnetic field normal to the 2D plane. In the 2D massless Dirac electron system, the particles obeyed to the Weyl equation $H=v_{F} \sigma \cdot p$, in which $\sigma$ is the Pauli matrix and $p$ is the momentum. In the magnetic field, the gauge transformation from $p$ into $p+e A$ derives the energy of Landau levels $E_{N}$ as

$$
E_{N}= \pm v_{F} \sqrt{2 e \hbar|N||B|}
$$


in which $A$ is the vector potential, $v_{F}$ is the Fermi velocity, $N$ is the Landau index, and $B$ is the magnetic field strength. This energy depends on the square root of $B$ and $N$, which is different from that of the conventional conductors. At the Dirac point $(E=0), N=0$, and the Landau level, called the zero-mode, always appears [42].

For $k_{B} T<E_{ \pm 1}$, the system is in the state of the quantum limit so that the zero-mode carriers dominate the conduction. In this situation, the carrier density per spin and per valley is given by $D(B)=B / 2 \phi_{0}$, in which $\phi_{0}=h / e$ is the quantum flux and the Fermi distribution function at $E_{F}$ is $1 / 2$. Strong magnetic fields induced the zero-mode carrier with high density.

Tajima et al. succeeded in detecting this effect in the longitudinal inter-layer magnetoresistance $R_{z z}$ [11]. In this experiment, the Lorentz force is weak, because the electrical current and the magnetic field are parallel to each other. Thus, the effect of the magnetic field only gives rise to the change in the zero-mode carrier density. It leads to the remarkable negative interlayer magnetoresistance in the magnetic field above $0.2 \mathrm{~T}$, as shown in Figure 3 [11]. An analytical formula for $R_{z z}$ by Osada reproduced well the field and the angle dependences of $R_{z z}$ [43].

Since each Landau level is broadened by the scattering of carriers and/or thermal energy, the zero-mode is sure to overlap with the other Landau levels at a low magnetic field. In such a region, the relationship of $R_{z z} \propto B^{-1}$ loses its validity. We can recognize this region in Figure 3, in which a positive magnetoresistance is observed. At $T_{p}=4 \mathrm{~K}$, for example, this critical field $B_{p}$ is approximately $0.2 \mathrm{~T}$. At the magnetic field above $B_{p}$, the overlap between the zero-mode and the $N= \pm 1$ Landau levels $E_{1}$ will be sufficiently small so that the negative magnetoresistance is observed there. Thus, $E_{1} \sim k_{B} T_{p}$ at $B_{p}$. The Fermi velocity $v_{F}$ was estimated to be approximately $4 \times 10^{4} \mathrm{~m} / \mathrm{s}[44,45]$.

Thus, the detection of zero-mode has demonstrated that this material under high pressure was composed of truly massless Dirac electron systems. The recent progress of this system has been remarkable. One example of this is the success of the carrier doping to this system. In the next section, the effects of the carrier doping on the transport phenomena are described.

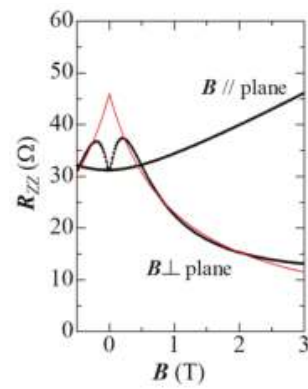

Figure 3. Field dependence of the interlayer magnetoresistance for $p=1.7 \mathrm{GPa}$ at $4 \mathrm{~K}$ [11]. Remarkable negative magnetoresistance is observed at $B>0.2 \mathrm{~T}$. Fitting curve (red line) is an Equation $R_{z z} \propto\left(|B|+B_{0}\right)^{-1}$ in the negative magnetoresistance region, in which $B_{0}$ is a fitting parameter that depends on the purity of a crystal [36].

\section{Methods of Carrier Doping}

In the Dirac electron systems, the Berry phase, which is fundamental concept for the geometry of the Bloch particles, plays an important role in quantum transport. In 2D massless Dirac electron system, the particles obey the Weyl equation $H=v_{F} \sigma \cdot p$ has $\pi$ Berry phase. $\pi$ Berry phase has yielded the new type of quantum Hall effect (half integer quantum Hall effect), which was first discovered in graphene. The success of the carrier doping (control of $E_{F}$ ) by the field effect transistor (FET) method led to this discovery. 
Here, the detection of the Berry phase is briefly mentioned. The semiclassical quantization condition for a cyclotron orbit is written as $S_{N}=2 \pi(N+\gamma) / l_{B}^{2}$, in which $S_{N}$ is the cross-section area of the $N$ th Landau level, $l_{B}=\sqrt{\hbar / e B}$ is the magnetic length, and $\gamma(0 \leq \gamma<1)$ is the Onsager phase factor that is related to the Berry phase $\phi_{B}$ as $\gamma=1 / 2-\phi_{B} / 2 \pi$. In a conventional electron system, $\phi_{B}$ and $\gamma$ are expected to be 0 and 1/2. Dirac particles, on the contrary, prefer $\phi_{B}=\pi$ and $\gamma=0$. Dirac particles in a solid thus had been identified from the phase analysis of quantum oscillation (Shubnikov-de Haas oscillations: $\mathrm{SdH}$ ) in a magnetic field.

In order to detect the quantum Hall effect (QHE) characterized by the electrons on the Dirac cones in $\alpha$-(BEDT-TTF $)_{2} \mathrm{I}_{3}$ under high pressure, the Fermi level should be moved from the Dirac point. However, control of the Fermi level by the field effect transistor (FET) method is much more difficult, because this crystal has a multilayered structure with high conductivity. Moreover, the conductivity in each layer is high.

Cannot we inject carriers to $\alpha$-(BEDT-TTF $)_{2} \mathrm{I}_{3}$ under high pressure? Cannot we detect the QHE experimentally in this system? The answer is "NO". One of the breakthroughs for the carrier injection to $\alpha$-(BEDT-TTF $)_{2} \mathrm{I}_{3}$ was suggested. Important results are that the $\mathrm{SdH}$ oscillations and the QHE associated with the special Landau level structure of Equation (1) were detected at low temperature [16,46]. In this section, we mention some unique methods of carrier doping in this system.

\subsection{Effects of Dopant}

Because of the characteristic energy spectrum, slight dopant brought a strong effect on the transport phenomena. The instability of $\mathrm{I}_{3}{ }^{-}$anions is the main origin of the dopant. Depending on the dopant, there are two types of samples according to which the electrons or holes were doped. Moreover, sample (dopant) dependence of the resistivity and the Hall coefficient is strong at low temperatures. This characteristic feature provided experimentally an anomalous Hall conductivity caused by the inter-band effects of the magnetic field [12]. In the magnetic field, the vector potential plays an important role in the inter-band excitation of electrons [47]. In this situation, large diamagnetism and the anomalous Hall conductivity is derived by the orbital motion of virtual electron-hole pairs. This is the inter-band effect of the magnetic field. This effect is strongest when the chemical potential is located at the Dirac point. In Section 3, inter-band effects of magnetic field on the transport properties are described.

\subsection{Electron Doping by the Annealing}

Annealing of the crystals in a vacuum at high temperature gives rise to the lack of $\mathrm{I}_{3}{ }^{-}$anions [48,49]. It yields mobile electrons. Annealing time and temperature are the control parameters of the density [50]. Recently, Tisserond et al. succeeded in injecting electrons and observed SdH oscillation at low temperature [51]. Effects of electron doping by the annealing on the transport properties are roughly mentioned in Section 4.

\subsection{Hole Doping by Contact Electrification}

The carrier density per layer of $\alpha$-(BEDT-TTF $)_{2} \mathrm{I}_{3}$ under a high pressure at low temperatures is estimated to be approximately $10^{8} \mathrm{~cm}^{-2}$ [10]. Thus, the effects of hole doping can be detected on the transport phenomena by fixing a crystal onto a substrate weakly that is negatively charged. This is called the contact electrification method. The effects of hole doping on the quantum transport phenomena were detected by fixing a thin crystal onto a poly (ethylene naphthalate) (PEN) substrate (Figure 4) [46]. Positively charged substrate, on the other hand, dopes electrons. Effects of hole doping by the contact electrification on the transport phenomena are described in Section 5. 


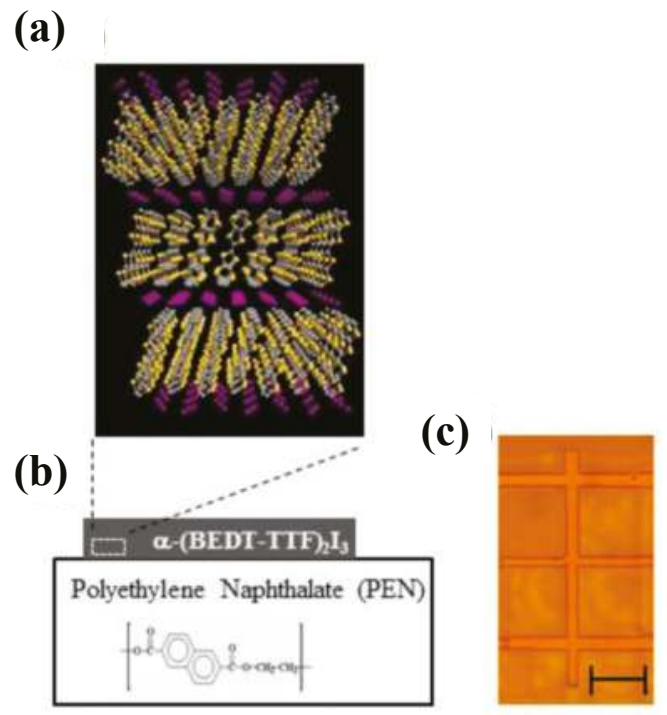

Figure 4. (a) Crystal structure of $\alpha$-(BEDT-TTF $)_{2} \mathrm{I}_{3}$ viewed from the $a$-axis; (b) schematic diagram of this system. The thickness of the crystal measured with a step profiler was approximately $100 \mathrm{~nm}$; (c) optical image of a single crystal on a PEN substrate in the processed form. The crystal was cut using a pulsed laser beam with a wavelength of $532 \mathrm{~nm}$. The scale bar is approximately $0.2 \mathrm{~mm}$.

\subsection{Experiments of the Transport Phenomena in $\alpha$-(BEDT-TTF) ${ }_{2} I_{3}$ Under High Pressure}

A sample on which six to eight gold wire with a diameter of $15 \mu \mathrm{m}$ is attached by the carbon paste and is put in a Teflon capsule filled with the pressure medium (DN-oil 7373, Idemitsu, Tokyo, Japan), and then the capsule is set in a clamp-type pressure cell made of hard alloy MP35N cell or BeCu/ $\mathrm{NiCrAl}$ dual-structure cell. The pressure was examined by change in the resistance of Manganin wire at room temperature. Resistance of a crystal was measured by a conventional dc method with six to eight probes. An electrical current between $0.1 \mu \mathrm{A}$ and $10 \mu \mathrm{A}$ was applied in the 2D plane. The magneto transport phenomena were investigated at temperatures from $0.1 \mathrm{~K}$ to $300 \mathrm{~K}$ in the magnetic field up to $12 \mathrm{~T}$.

\section{Effects of Dopant: Inter-Band Effects of Magnetic Field}

The sample (dopant) dependence of the resistivity and the Hall coefficient is strong at low temperatures, as shown in Figure 5, because this system has the characteristic energy spectrum. This fact is very important, because the dopant will throw light on the structure and the characteristic properties in the vicinity of Dirac point. In this section, anomalous Hall conductivity that originated from the inter-band effects of the magnetic field at the vicinity of Dirac point is examined [12]. Moreover, electron-hole symmetry of this system is revealed.

As mentioned in Section $1, R_{H}$ is proportional to $T^{2}$ at temperatures below $50 \mathrm{~K}$. It was explained based on a single Dirac cone type energy structure. In the present system, however, two cones that touched at a Dirac point exclude such a simple situation. In the case that the two Dirac cones are strictly symmetric, the Hall coefficient will be zero, because the signals of the Hall effect due to carriers on the top and bottom of the Dirac cones cancel out. To detect the signal, the energy structure should be asymmetrical. The measurements of $R_{H}$ should detect the signal that depends on the strength of asymmetry.

Tajima et al. examined the temperature dependence of $R_{H}$ for seven samples (n1-n7) and found that the samples were classified into two groups. First group (n4 and $n 6$ ) is that $R_{H}$ is positive over 
the whole temperature range, as shown in Figure 5b. In another group (n1, n2, n3, n5, and n7), on the other hand, the polarity of $R_{H}$ changes at a certain temperatures below $10 \mathrm{~K}$, as shown in Figure $5 \mathrm{c}$. The dopant with a low level of density in the sample leads to this difference. It plays an essential role in the polarity of $R_{H}$ at low temperatures. The density and the kind of dopants were determined by $R_{H}$ at the lowest temperature, in which the thermal excitation of carriers between the cones is negligible. The kind of dopant of the first group ( $\mathrm{n} 4$ and $\mathrm{n} 6$ ) is hole. On the other hand, electron is the character of dopant in another group (n1, n2, n3, n5, and n7). Here, we have a deep interest in the change in the polarity of $R_{H}$ in the second group (electron doped samples: n1, n2, n3, n5, and n7), as shown in Figure $5 c$. We find strong sample dependence of the temperature in which the polarity changes. Note that above $10 \mathrm{~K}$, the curve of $R_{H}$ for all samples is a single. It indicates that doping does not affect the electron energy structure. Thus, the effect of the dopant results in changes in $E_{F}$. The change in the polarity of $R_{H}$ is understood as follows.

Strong temperature dependence of $R_{H}$ indicates that the symmetry of the present Dirac cones is low. In this situation, the chemical potential $\mu$ should vary with the temperature. At the temperature at which $\mu=0$, the polarity of $R_{H}$ changes [12,20]. This fact is very important, because the temperature dependence of the transport phenomena provides its chemical potential dependence in the vicinity of the Dirac point. Here, let us detect the inter-band effects of the magnetic field in the Hall conductivity. The inter-band effect is strongest at $\mu=0$ [47]. In the following, we examine the inter-band effect in the vicinity of $R_{H}=0(\mu=0)$.

(a)

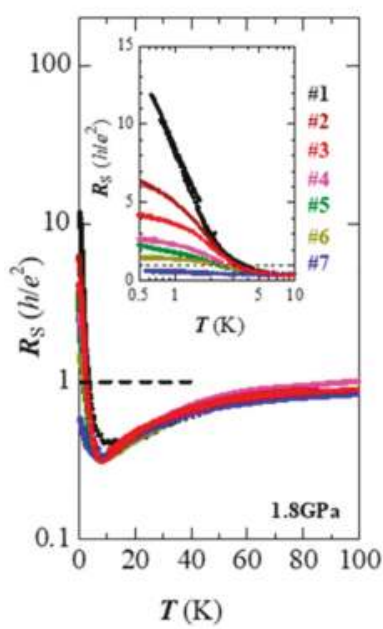

(b)

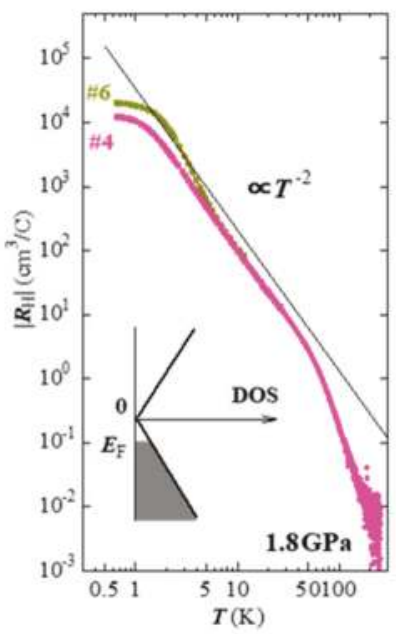

(c)

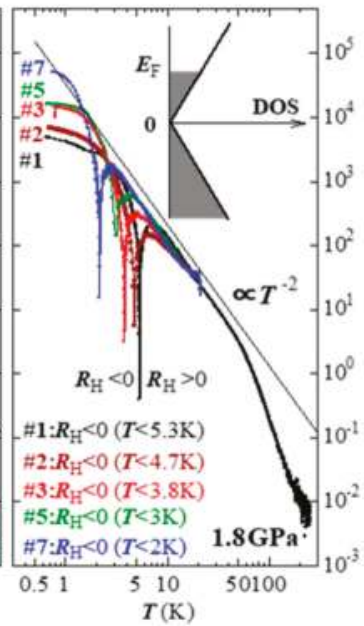

Figure 5. (a) Temperature dependence of $R_{S}$ for seven samples under pressure of $1.8 \mathrm{GPa} . R_{S}$ at temperature below $10 \mathrm{~K}$ is shown in the inset; $(\mathbf{b}, \mathbf{c})$ is the temperature dependence of $R_{H}$ for hole-doped-type and electron-doped-type samples. Note that the absolute value of $R_{H}$ is plotted. Thus, the dips in $(\mathbf{c})$ indicate a change in the polarity. The inset of $(\mathbf{b}, \mathbf{c})$ shows the schematic illustration of the Fermi levels.

First, let us express $\mu$ as a function of $T$. The dopant density $n_{S}$ was determined from $n_{s}=1 / R_{H} e$ at low temperature. Thus, $E_{F}$ was calculated from the relationship $E_{F}=\hbar v_{F} \sqrt{n_{s} / \pi}$ with $v_{F}=3.5 \times 10^{4} \mathrm{~m} / \mathrm{s}$. Note that the value of $v_{F}$ for all samples is almost the same, because sample dependence of $R_{H}$ is very weak at temperatures of above $7 \mathrm{~K}$. For example, $E_{F} / k_{B}$ for samples $n 1$ and $n 7$ are estimated to be 1.35 and $0.35 \mathrm{~K}$, respectively. Kobayashi et al. theoretically demonstrated that $\mu$ is varied with the temperature as approximately $\mu / k_{B}=E_{F} / k_{B}=E_{F} / k_{B}-A T$ at low temperatures, in which a 
parameter $A$ depends on the symmetry of the Dirac cone $\left(v_{F}^{h} / v_{F}^{e}: v_{F}^{h}\right.$ and $v_{F}^{e}$ are the Fermi velocities for lower and upper Dirac cones) and is independent of $E_{F}$. At $R_{H}(T)=0, \mu / k_{B}=0$. Thus, $A=E_{F} / k_{B} T_{0}$ is estimated to be approximately 0.24 at $R_{H}(T=0)=0$. This experimental formula is consistent with the theoretical curve well [20]. Hence, $v_{F}^{h} / v_{F}^{e}$ is estimated to be approximately 1.2 [12]. This is the electron-hole symmetry of this system. The detail for asymmetric Dirac cones in this system is described in Section 6.

The calculation of the Hall conductivity $\sigma_{x y}=\rho_{y x} /\left(\rho_{x x}^{2}+\rho_{y x}^{2}\right)$ is the second step. The temperature dependence of $\sigma_{x y}$ for samples $\mathrm{n} 1$ and $\mathrm{n} 7$ is shown in Figure 6a. In this calculation, it was assumed as $\rho_{x x}=\rho_{y y}$. The last step is that $\sigma_{x y}$ is drawn in Figure $6 \mathrm{~b}$ as a function for $\mu$ by replacing $T$ by $T(\mu)$. We should compare this with the theoretical curve $\sigma_{x y}^{\text {theory }}$. Experimental data roughly reproduce the relation $\sigma_{x y} \sim g \sigma_{x y}^{\text {theory }}$, in which $g$ is a parameter that depends on the temperature. It is significant that there is a peak and a dip structure in each curve at the vicinity of the point at which $\sigma_{x y}=0$. In the magnetic field, the orbital motion of virtual electron-hole pairs by the vector potential plays an important role in $\sigma_{x y}$. The peak structure of $\sigma_{x y}$ shown in Figure 6 is the characteristic feature due to the inter-band effects of the magnetic field [20]. The energy between two peaks is the damping, which depends on the density of scattering centers in the crystal. The intensity of the peak, on the other hand, depends on the damping and the tilt of the Dirac cones [20]. Note that $\sigma_{x y}$ without the inter-band effects, on the other hand, has no peak structure.

Another important finding is a smooth change in the polarity of $\sigma_{x y}$. This is evidence that this material has an intrinsic zero-gap energy structure.

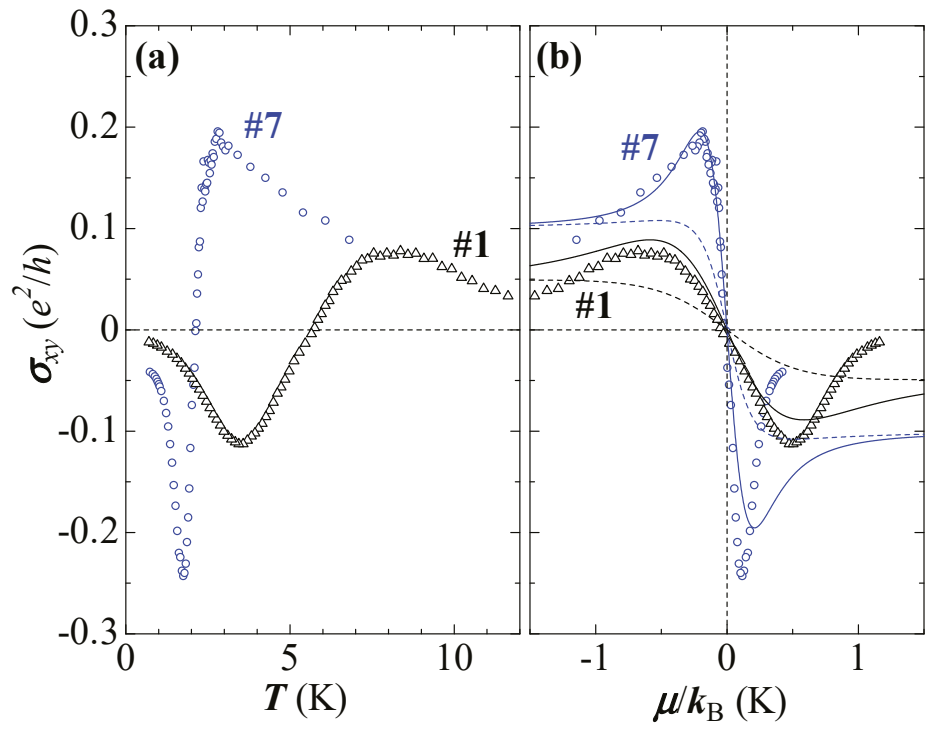

Figure 6. (a) Temperature dependence of the Hall conductivity for $n 1$ and $n 7$ in Figure 5; (b) chemical-potential dependence of the Hall conductivity for $\mathrm{n} 1$ and $\mathrm{n} 7$. Solid lines and dashed lines are the theoretical curves with and without the inter-band effects of the magnetic field by Kobayashi et al., respectively [20]. Reproduced with permission from [11].

\section{Electron Doping by the Annealing}

As mentioned in the previous section, the dopant with the density of ppm order gave rise to the intense effects on the transport phenomena. The origin of the dopant is the instability of $\mathrm{I}_{3}{ }^{-}$anions in a crystal. In a sense, this is the natural doping. Annealing in a vacuum at high temperature, 
on the other hand, enhances the lack of $\mathrm{I}_{3}{ }^{-}$anions so that the mobile electrons are yielded. In this section, the effects of annealing on the transport phenomena in $\alpha$-(BEDT-TTF) $)_{2} \mathrm{I}_{3}$ under the pressure are mentioned.

The density of the doped electron by the annealing depends on the parameters of its time and temperature. The annealing at temperature above $80^{\circ} \mathrm{C}$, however, changes the crystal structure from $\alpha$ - to $\beta$-types [48]. Thus, Miura et al. investigated the resistivity of a crystal with a parameter of the time with $10 \mathrm{~min}$ steps of annealing in a vacuum of $10^{-3} \mathrm{~Pa}$ at $70^{\circ} \mathrm{C}$ [50]. The effects of anneal (doping) were clearly seen in the value of resistivity and its temperature dependence as shown in Figure 7a. The effects of annealing lead this crystal to a metal. At $4.2 \mathrm{~K}$, for example, the resistivity is decreased by about two orders of magnitude in duration of $40 \mathrm{~min}$ annealing. It is expected that electrons were doped to this system.

(a)

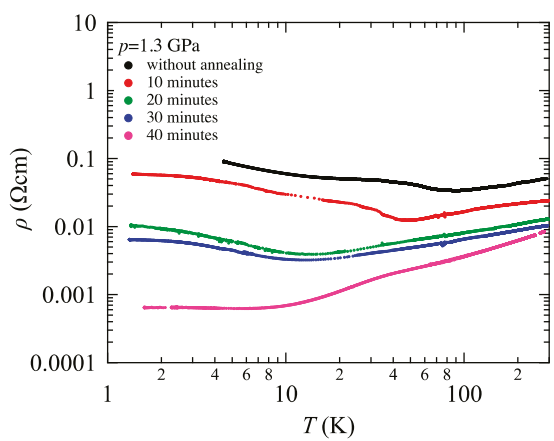

(b)

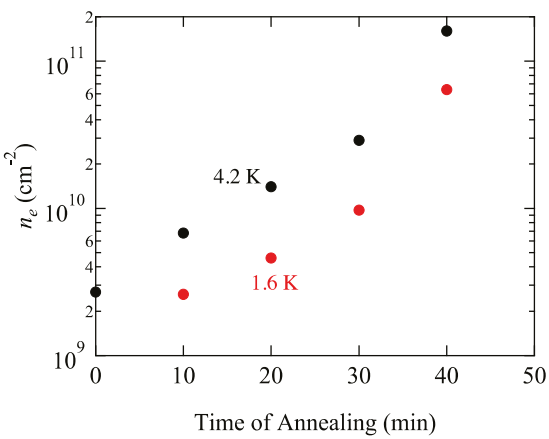

Figure 7. (a) Effects of the annealing on the resistivity in $\alpha$-(BEDT-TTF $)_{2} \mathrm{I}_{3}$ under pressure of $1.3 \mathrm{GPa}$; (b) annealing time dependence of electron density at $4.2 \mathrm{~K}$ and $1.6 \mathrm{~K}$. Reproduced with permission from $[50,51]$.

Here we note that this experiment was performed by a single crystal. First step was to investigate the resistivity of $\alpha$-(BEDT-TTF $)_{2} \mathrm{I}_{3}$ under the pressure of $1.3 \mathrm{GPa}$. As the second step, the crystal was annealed in the duration of $10 \mathrm{~min}$ in a vacuum about $10^{-3} \mathrm{~Pa}$ at $70{ }^{\circ} \mathrm{C}$ after pressure was removed. Then, the resistivity of this crystal under the pressure of $1.3 \mathrm{GPa}$ was investigated again. For the after steps, the doping of electrons by the annealing was repeated in the same way.

The polarity of the Hall coefficient indicated the electron doping successes. At low temperature, the variation in the electron density estimated from the Hall coefficient is almost same as that in the resistivity. It is increased by about two orders of magnitude in a $40 \mathrm{~min}$ of anneal at $4.2 \mathrm{~K}$ as shown in Figure $7 b$.

Here, let us return to Figure 7a. The resistivity per layer in the case without annealing expressed as $R_{S} \sim h / e^{2}$ in a wide temperature region is the characteristic transport in the massless Dirac electron system in which $E_{F}$ is located close to Dirac point as mentioned in Section 1. However, the system with $E_{F}$ far from the Dirac point lacks validity of this law. The resistivity of the case with annealed duration of $40 \mathrm{~min}$ shows the metallic behavior.

Figure 8 is the recent highlight. Tisserond et al. succeeded in detecting $\mathrm{SdH}$ oscillation in a thick crystal under pressure of $2.2 \mathrm{GPa}$ at $200 \mathrm{mK}$. [51]. Note that it is doped as a side effect of the elaboration of the gold electrical contacts deposited by Joule evaporation, with an unintentional annealing. Careful analysis of $\mathrm{SdH}$ oscillations, however, conduced the correction of the Dirac cones in this system. In Section 6, this will be disclosed. 


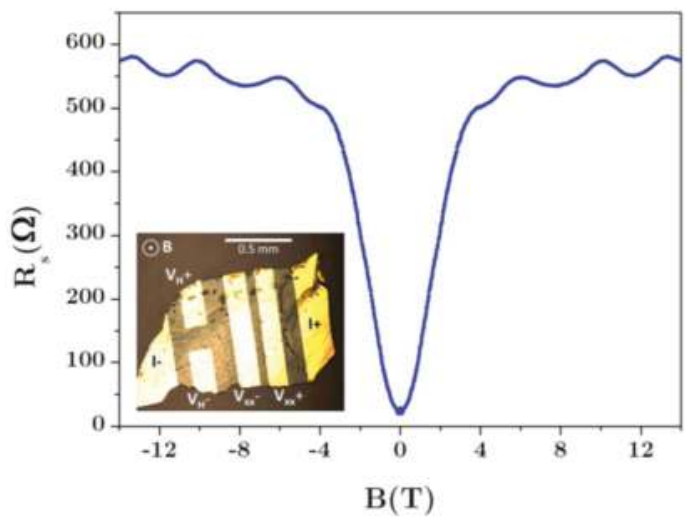

Figure 8. Field dependence of the magnetoresistance in $\alpha$-(BEDT-TTF) ${ }_{2} \mathrm{I}_{3}$ under pressure of $2.2 \mathrm{GPa}$ at $200 \mathrm{mK}$. Inset shows the photograph of sample. Reproduced with permission from [51].

\section{Hole Doping by Contact Electrification}

By fixing a thin crystal onto a substrate weakly negatively charged as shown in Figure 4, the effects of carrier doping by contact electrification can be detected in the transport. The polarity of Hall resistance $R_{x y}$ indicates that the hole doping is successful. Note that holes should be injected into a few layers (pairs of BEDT-TTF molecular layers and $\mathrm{I}_{3}{ }^{-}$anion layers). Main is the first from the interface.

\subsection{Resistivity of $\alpha$-(BEDT-TTF $)_{2} I_{3}$ on PEN Substrate under High Pressure}

Figure 9 shows the temperature dependence of the resistivity of $\alpha$-(BEDT-TTF $)_{2} \mathrm{I}_{3}$ on PEN substrate under pressure of $1.7 \mathrm{GPa}$. We see clear effects of the hole-doping in the resistivity. First, the value of resistivity of the thin crystal on the PEN substrate is lower than those of the usual thick crystal. Most noticeable difference is seen at temperatures below $2 \mathrm{~K}$. The resistivity for the thin crystal on the PEN substrate behaves as $\rho \propto T^{2}$. This is the characteristic transport in the Fermi liquid state.

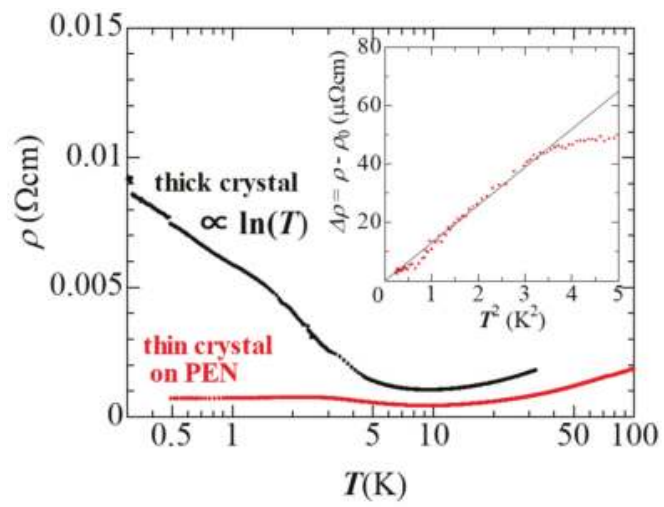

Figure 9. Temperature dependence of resistivity of $\alpha$-(BEDT-TTF $)_{2} \mathrm{I}_{3}$ on PEN substrate under pressure of 1.7 GPa. Inset shows $\Delta \rho=\rho-\rho_{0}$ against $T^{2}$, in which $\rho_{0}$ is the resistivity at a limit of $T=0$. 
Thus, carrier injection into $\alpha$-(BEDT-TTF $)_{2} \mathrm{I}_{3}$ with a layered structure was successful. Note that the resistivity depends on the crystal thickness, because the number of carrier-doped layers is small, as mentioned before.

\subsection{Observations of SdH Oscillations and Quantized Hall Resistance}

The signature of the Dirac electrons is seen in the quantum transport. The magnetic field dependence of resistance $R_{x x}$ and the Hall resistance $R_{x y}$ in the thin crystal on the PEN substrate under pressure of approximately $1.7 \mathrm{GPa}$ were investigated at $0.5 \mathrm{~K}$. We find clear oscillation of $R_{x x}$ as shown in Figure 10a. The oscillation as a function of $B^{-1}$ indicates the $\mathrm{SdH}$ signal with a frequency of $B_{f} \sim 9.18 \mathrm{~T}$. The second-order differential of $R_{x x}$ clearly depicts the oscillation in Figure 10b.

Here, we regard the origin of the SdH oscillation is 2D massless Dirac electrons. In the Dirac electron systems, the circular orbit around the Dirac point in the magnetic field would yield Berry phase $\pi$, as mentioned before. The effect of the phase of the SdH oscillation is further probed in the semi-classical magneto-oscillation description. In general, the component of the $\mathrm{SdH}$ oscillation written by

$$
\Delta R_{x x}=A(B) \cos \left[2 \pi\left(B_{f} / B+\gamma\right)\right]
$$

acquires the phase factor $\gamma=1 / 2$ or 0 for normal electrons with $\phi_{B}=0$ and Dirac particles with $\phi_{B}=\pi$. Here, $A(B)$ is the amplitude of the oscillation. We obtain phase factor $\gamma$ by plotting the values of $B^{-1}$ at the oscillation minima of $\Delta R_{x x}$ as a function of their number, as shown in Figure 10c. The linear extrapolated values of approximately $1 / 2$ at $B^{-1}=0$ determine the phase factors $\gamma$, which are approximately 0 . Thus, we identify the Dirac particles.
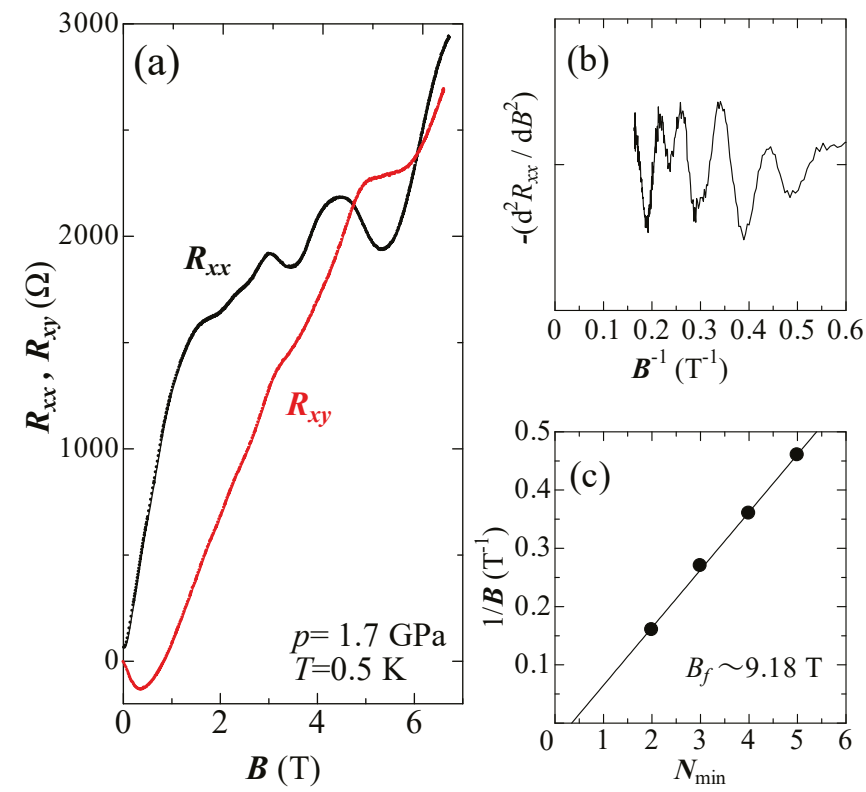

Figure 10. (a) Magnetic field dependence of $R_{x x}, R_{x y}$, and (b) SdH oscillation $\left(-d^{2} R_{x x} / d B^{2}\right)$ under the pressure of $1.7 \mathrm{GPa}$ at $0.5 \mathrm{~K}$; (c) value of $B^{-1}$ for the $\mathrm{SdH}$ oscillation minima. In the Dirac electron systems, the linear extrapolation of the data to $B^{-1}=0$ should be $1 / 2$. For normal electrons, on the other hand, it is shifted to 0 . 
Here, we notice the split of the SdH oscillation at $B^{-1} \sim 0.25 \mathrm{~T}^{-1}$ in Figure $10 \mathrm{~b}$. The SdH oscillation represents the density of states of the Landau levels. Thus, it indicates the Zeeman splitting of $N=-2$ Landau level. The Landau level structure including its Zeeman splitting is written as

$$
E_{N}= \pm v_{F} \sqrt{2 e \hbar|N||B|} \pm \frac{g \mu_{B} B}{2}
$$

in which $g$ is the $g$ factor and $\mu_{B}$ is the Bohr magneton. Combined this relation of $N=2$ and $g=2$ with $E_{2} / v_{F}=\hbar k=\hbar \sqrt{4 \pi B_{f} / \phi_{0}} \sim 1.7 \times 1.0^{-26} \mathrm{Jm}^{-1} \mathrm{~s}$, the Fermi velocity is estimated to be $v_{F} \sim 4.3 \mathrm{~m} / \mathrm{s}$, in which $g=2$ and $\phi_{0}=4.14 \times 10^{-15} \mathrm{Tm}^{-2}$ is the quantum flux.

The most significant finding is the quantum Hall state in this system. Two obvious $R_{x y}$ plateaus are observed at magnetic fields of approximately 3.5 and $5.5 \mathrm{~T}$, which show $R_{x x}$ minima (Figure 10a). Based on the conventional 2D Dirac electron systems, the $R_{x y}$ plateau is interpreted as follows.

$R_{x y}$ quantization is in accordance with $1 / R_{x y}=v \cdot e^{2} / h$, in which $v= \pm s(n+1 / 2)$ is the quantized filling factor, and $s=4$ is the fourfold spin/valley degeneracy. An outstanding effect on the Dirac electron system is that the factor of half-integer exists. Thus, probes of the quantum Hall plateaux for $|v|=2,6,10,14, \cdots$ are expected. In the data in Figure 10a, based on this step rule, $v=-6,-10,-14$ for the first layer at $R_{x y}$ plateau, or anomalies are required from SdH oscillations against the Landau index shown in Figure 11.

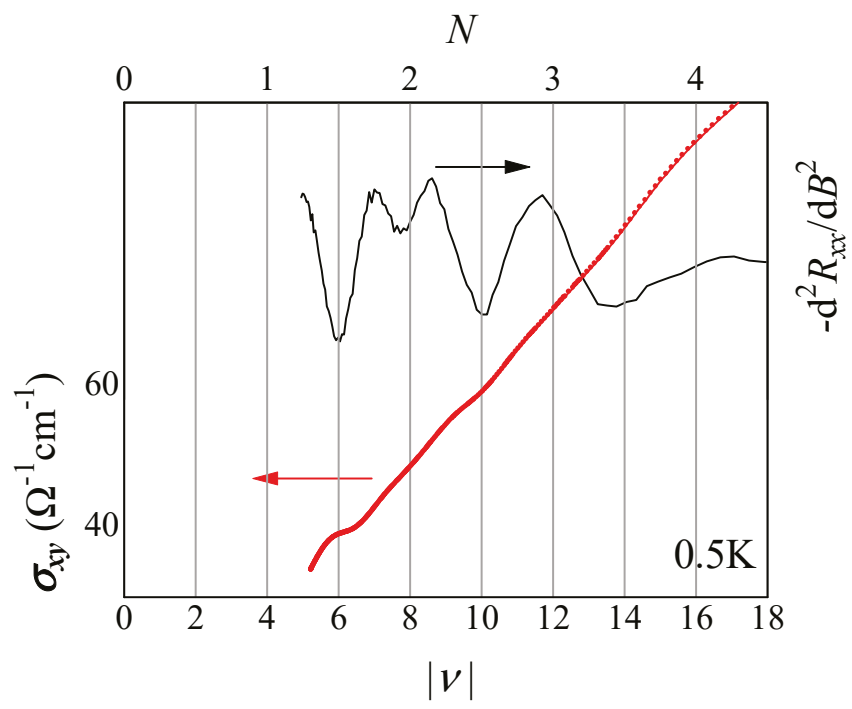

Figure 11. Landau index $N$ or filling factor for first layer $v$ dependence of $-d^{2} R_{x x} / d B^{2}$ and $\sigma_{x y}$. Reproduced with permission from [45].

The estimation of $v$ from the values of $R_{x y}$ plateau in this system, however, is a serious problem. The multilayered structure with a few hole-doped layers (mostly single layer) gives rise to the lack of the validity of the estimation of $v$ from the values of $R_{x y}$ plateau because it depends on the thickness. On the other hand, the conductivities for the many undoped layers are finite. Hence, $R_{x x}$ is not zero but shows minima at $R_{x y}$ plateaux. At magnetic field, for example, we can see this effect on $R_{x y}$. In this magnetic field region, the polarity of $R_{x y}$ is negative and yet holes are injected, as shown in Figure 10a. This is a frequently observed behavior of the present material induced by electron-type dopant (Section 3). However, detailed examination of the thickness (number of layer) dependence 
of the Hall conductivity revealed $v$. For example, $v \sim-6$ at $R_{x y}$ plateau of $5.5 \mathrm{~T}$ in Figure 10a [46]. This is expected $v$ shown in Figure 11.

\section{Correction of Dirac Cones}

In Section 5 , the detection of SdH oscillations, whose phase was modified by $\pi$ Berry phase, is evidence that this system is a 2D Dirac electron system. This measurement was done in the magnetic field below $7 \mathrm{~T}$ at $0.5 \mathrm{~K}$. However, recent examination of the $\mathrm{SdH}$ oscillations at the magnetic field up to $12 \mathrm{~T}$ by Tisserond et al. suggested the correction of the Dirac cones [51]. In this section, the SdH signals in the high magnetic field are interpreted within the model of distorted Dirac cones.

The detections of the $\mathrm{SdH}$ oscillations were done by two carrier doping methods: anneal (Sample A: Figure 8) and contact electrification (Sample B). Both the oscillations with the $1 / B$ periodicities and the phase factors of the Berry phase $\pi$ indicate that the Dirac carriers are involved at low magnetic field. At high magnetic field, however, both the $\mathrm{SdH}$ signals are very peculiar. Both oscillations lost 1/ $B$ oscillations periodicity as shown in Figure 12 [51]. The detail structure of Dirac cones shows up the non-periodic SdH oscillations of the high magnetic field [52].
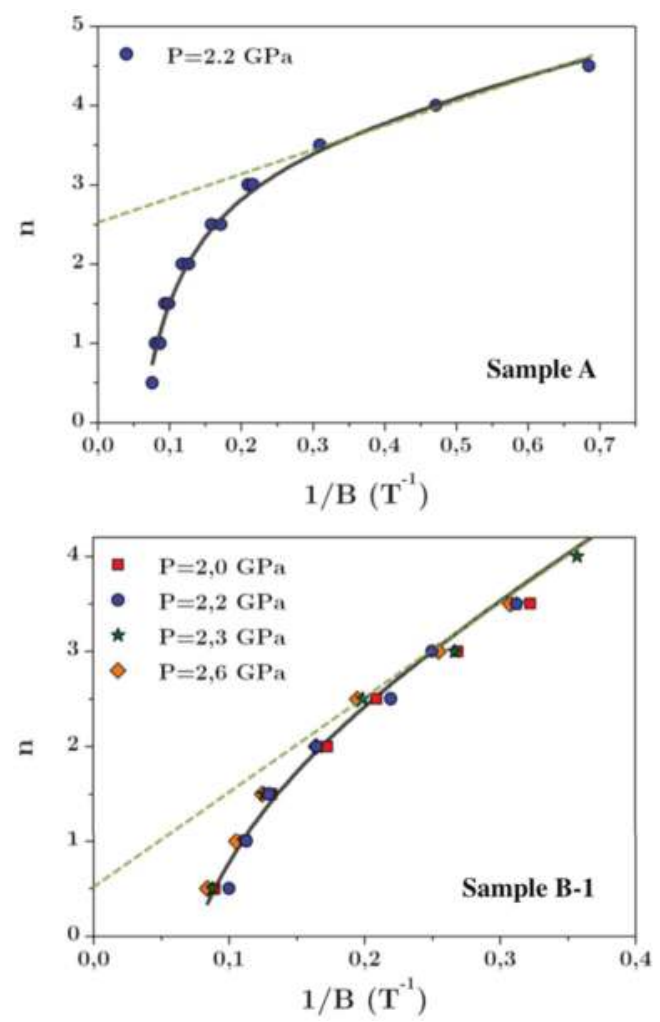

Figure 12. Construction of Landau plots from the analysis of the SdH oscillations. At low magnetic fields, the oscillations are $1 / B$ periodic; they are $\mathrm{SdH}$ oscillations. The determination of their phase offset, connected to the Berry phase, indicates that the Dirac charge carriers are involved in the measured oscillations. At higher magnetic fields, the $1 / B$ oscillations periodicity loss. Reproduced with permission from [51]. 
Tisserond et al. interpreted the SdH signals shown in Figure 12 based on the picture of distorted Dirac cones, of which the Hamiltonian is written as $H=v_{F} \sigma \cdot p+p^{2} / 2 m$, in which mass $m$ is the curvature term. Figure 13 is the sketch of distorted Dirac cones. In the magnetic field, by the gauge transformation from $p$ into $p+e A$, the Landau level structure without Zeeman effect term is calculated as

$$
E_{N}=\hbar \omega_{m} N \pm v_{F} \sqrt{2 e \hbar v_{F}^{2}|N||B|+\left(\frac{\hbar \omega_{m}}{2}\right)^{2}}
$$

in which $\omega_{m}=e B / m$ and $|N| \geq 1$. The positive part of this equation corresponds to the conduction band contribution and the negative part to that of the valence band. The curves (solid lines) of Landau index $N$ against $B^{-1}$ reproduced well the experimental curve as shown in Figure 13. The best fit mass parameter for Sample A is $|m| \sim 0.03 m_{0}$. For Sample B, on the other hand, it is to be $|m| \sim 0.022 m_{0}$. Note that others possible causes, such as a cone tilting or a Zeeman effect, are carefully ruled out in the Reference [46] and its Supplementary Materials.

The proposed interpretation corrects the band structure from (a) massless Dirac cone to distorted Dirac cone with (b) $m>0$ or (c) $m<0$ shown in Figure 13. This electron-hole asymmetry explains the temperature dependence of the chemical potential mentioned in Section 3 [12,20]. It suggests distorted Dirac cone shown in Figure 13b. Moreover, recent realistic calculations of the energy band and Landau level structures in this system by Kishigi and Hasegawa support our experimental results and those interpreted qualitatively [53].

(a)

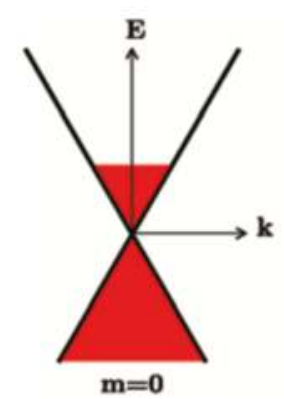

(b)

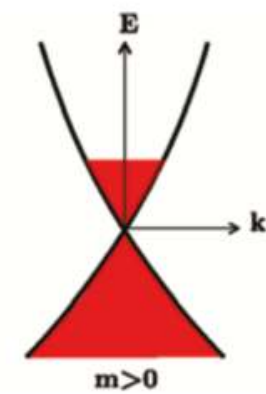

(c)

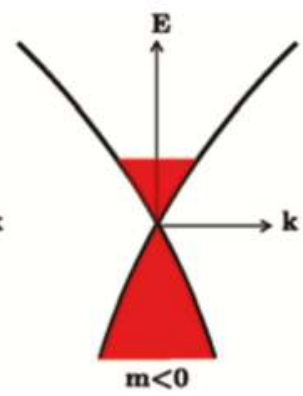

Figure 13. Distortion of the Dirac cone by a curvature term with a mass $m$. (a) massless Dirac cone; (b) distorted Dirac cone $(m>0)$; (c) distorted Dirac cone $(m<0)$. Reproduced with permission from [51].

\section{Summary}

We summarize this review as follows.

The effects of carrier doping on the peculiar (quantum) transport phenomena in $\alpha$-(BEDT-TTF) ${ }_{2} \mathrm{I}_{3}$ under high pressure were described. We presented three unique methods for the carrier doping of this system. First, unstable $\mathrm{I}_{3}{ }^{-}$anions yield the dopant in the crystal. This effect led to the detection of anomalous Hall conductivity that originated from the inter-band effects of the magnetic field; second, subjects that were annealed in a vacuum at high temperature enhanced the lack of $\mathrm{I}_{3}{ }^{-}$anions so that the mobile electrons were yielded. The effects of annealing on the transport phenomena in $\alpha$-(BEDT-TTF $)_{2} \mathrm{I}_{3}$ under pressure were mentioned. Tisserond et al. succeeded in detecting $\mathrm{SdH}$ oscillation in a thick crystal under pressure of 2.2 GPa at $200 \mathrm{mK}$ [51]. Lastly, we presented the effects of the carrier doping by the contact electrification on the transport phenomena. Only by fixing a thin crystal onto a substrate weakly negatively charged by contact electrification, the effects of carrier doping can be detected in the transport. A significant finding is the SdH oscillation and the quantum 
Hall effect characterized by the Dirac type energy structure. Moreover, the distortion of the band structure Dirac cones was revealed.

The effects of carrier doping became more important for the organic Dirac electron system. However, control of the Fermi energy has not been achieved yet. Control of the Fermi energy and further investigations will lead us to interesting phenomena.

Acknowledgments: We thank M. Monteverde, Miura, T. Yamauchi, T. Yamaguchi, Y. Kawasugi, M. Suda, H. M. Yamamoto, R. Kato, Y. Nishio, K. Kajita, T. Morinari, A. Kobayashi, Y. Suzumura, and H. Fukuyama for valuable discussions. This work was supported by a Grant-in-Aid for Scientific Research (A) (No. 15H02108), (S) (No. 16H06346), (B) (No. 25287089), from the Ministry of Education, Culture, Sports, Science, and Technology, Japan.

Conflicts of Interest: The author declares no conflict of interest.

\section{References}

1. Novoselov, K.S.; Geim, A.K.; Morozov, S.V.; Jiang, D.; Katsnelson, M.I.; Grigorieva, I.V.; Dubonos, S.V.; Firsov, A.A. Two-dimensional gas of massless Dirac fermions in grapheme. Nature 2005, 438, 197-200. [CrossRef] [PubMed]

2. Zhang, Y.; Tan, Y.W.; Stormer, H.; Kim, P. Experimental observation of the quantum Hall effect and Berry's phase in grapheme. Nature 2005, 438, 201-204. [CrossRef] [PubMed]

3. Kempa, H.; Esquinazi, P.; Kopelevich, Y. Field-induced metal-insulator transition in the $c$-axis resistivity of graphite. Phys. Rev. B 2002, 65, 241101. [CrossRef]

4. Luk'yanchuk, I.A.; Kopelevich, Y. Phase Analysis of Quantum Oscillations in Graphite. Phys. Rev. Lett. 2004, 93, 166402. [CrossRef] [PubMed]

5. Fuseya, Y.; Ogata, M.; Fukuyama, H. Interband Contributions from the Magnetic Field on Hall Effects for Dirac Electrons in Bismuth. Phys. Rev. Lett. 2009, 102, 066601. [CrossRef] [PubMed]

6. Richard, P.; Nakayama, K.; Sato, T.; Neupane, M.; Xu, Y.-M.; Bowen, J.H.; Chen, G.F.; Luo, J.L.; Wang, N.L.; Dai, X.; et al. Observation of Dirac Cone Electronic Dispersion in $\mathrm{BaFe}_{2} \mathrm{As}_{2}$. Phys. Rev. Lett. 2010, 104, 137001. [CrossRef] [PubMed]

7. Zhang, H.; Liu, C.-X.; Qi, X.-L.; Dai, X.; Fang, Z.; Zhang, S.-C. Topological insulators in $\mathrm{Bi}_{2} \mathrm{Se}_{3}, \mathrm{Bi}_{2} \mathrm{Te}_{3}$ and $\mathrm{Sb}_{2} \mathrm{Te}_{3}$ with a single Dirac cone on the surface. Nat. Phys. 2009, 5, 438-442. [CrossRef]

8. Tajima, N.; Tamura, M.; Nishio, Y.; Kajita, K.; Iye, Y. Transport property of an organic conductor $\alpha$-(BEDT-TTF $)_{2} \mathrm{I}_{3}$ under high pressure: Discovery of a novel type of conductor. J. Phys. Soc. Jpn. 2000, 69, 543-551. [CrossRef]

9. Tajima, N.; Sugawara, S.; Tamura, M.; Kato, R.; Nishio, Y.; Kajita, K. Electronic phases in an organic conductor $\alpha$-(BEDT-TTF $)_{2} \mathrm{I}_{3}$ : Ultra narrow gap semiconductor, superconductor, metal, and charge-ordered insulator. J. Phys. Soc. Jpn. 2006, 75, 051010. [CrossRef]

10. Tajima, N.; Sugawara, S.; Tamura, M.; Kato, R.; Nishio, Y.; Kajita, K. Transport properties of massless Dirac fermions in an organic conductor $\alpha$-(BEDT-TTF) ${ }_{2} \mathrm{I}_{3}$ under pressure. Europhys. Lett. 2007, 80, 47002. [CrossRef]

11. Tajima, N.; Sugawara, S.; Kato, R.; Nishio, Y.; Kajita, K. Effect of the zero-mode Landau level on interlayer magnetoresistance in multilayer massless dirac fermion systems. Phys. Rev. Lett. 2009, 102, 176403. [CrossRef] [PubMed]

12. Tajima, N.; Kato, R.; Sugawara, S.; Nishio, Y.; Kajita, K. Inter-band effects of magnetic field on Hall conductivity in the multilayered massless Dirac fermion system $\alpha$-(BEDT-TTF) ${ }_{2} \mathrm{I}_{3}$. Phys. Rev. $B$ 2012, 85, 033401. [CrossRef]

13. Kobayashi, A.; Katayama, S.; Noguchi, K.; Suzumura, Y. Superconductivity in Charge Ordered Organic Conductor $-\alpha-(\mathrm{ET})_{2} \mathrm{I}_{3}$ Salt-. J. Phys. Soc. Jpn. 2004, 73, 3135-3148. [CrossRef]

14. Katayama, S.; Kobayashi, A.; Suzumura, Y. Pressure-induced zero-gap semiconducting state in organic conductor $\alpha$-(BEDT-TTF $)_{2} \mathrm{I}_{3}$ Salt. J. Phys. Soc. Jpn. 2006, 75, 054705. [CrossRef]

15. Kino, H.; Miyazaki, T. First-principles study of electronic structure in $\alpha$-(BEDT-TTF) ${ }_{2} \mathrm{I}_{3}$ at ambient pressure and with uniaxial strain. J. Phys. Soc. Jpn. 2006, 75, 034704. [CrossRef]

16. Kajita, K.; Nishio, Y.; Tajima, N.; Suzumura, Y.; Kobayashi, A. Molecular Dirac Fermion Systems-Theoretical and Experimental Approaches-. J. Phys. Soc. Jpn. 2014, 83, 072002. [CrossRef] 
17. Bender, K.; Hennig, I.; Schweitzer, D.; Dietz, K.; Endres, H.; Keller, H.J. Synthesis, structure and physical properties of a two-dimensional organic metal, di[bis(ethylenedithiolo)tetrathiofulva lene] triiodide, (BEDT-TTF) ${ }_{2}{ }_{2}{ }^{-}{ }_{3}$. Mol. Cryst. Liq. Cryst. 1984, 108, 359-371. [CrossRef]

18. Konoike, T.; Uchida, T.; Osada, T. Specific heat of the multilayered massless Dirac fermion system. J. Phys. Soc. Jpn. 2012, 81, 043601. [CrossRef]

19. Hirata, M.; Ishikawa, K.; Miyagawa, K.; Tamura, M.; Berthier, C.; Basko, D.; Kobayashi, A.; Matsuno, G.; Kanoda, K. Observation of an anisotropic Dirac cone reshaping and ferrimagnetic spin polarization in an organic conductor. Nat. Commun. 2016, 7, 12666. [CrossRef] [PubMed]

20. Kobayashi, A.; Suzumura, Y.; Fukuyama, H. Hall Effect and Orbital Diamagnetism in Zerogap State of Molecular Conductor $\alpha$-(BEDT-TTF $)_{2} \mathrm{I}_{3}$. J. Phys. Soc. Jpn. 2008, 77, 064718. [CrossRef]

21. Kobayashi, A.; Suzumura, Y.; Fukuyama, H.; Goerbig, O. Tilted-cone-induced easy-plane pseudo-spin ferromagnet and Kosterlitz-Thouless transition in massless Dirac fermions. J. Phys. Soc. Jpn. 2009, 78, 114711. [CrossRef]

22. Proskrin, I.; Ogata, M.; Suzumura, Y. Longitudinal conductivity of massless fermions with tilted Dirac cone in magnetic field. Phys. Rev. B 2015, 91, 195413. [CrossRef]

23. Suzumura, Y.; Proskrin, I.; Ogata, M. Effect of tilting on the in-plane conductivity of Dirac electrons in organic conductor. J. Phys. Soc. Jpn. 2014, 83, 023701. [CrossRef]

24. Liu, D.; Ishikawa, K.; Takehara, R.; Miyagawa, K.; Tamura, M.; Kanoda, K. Insulating Nature of Strongly Correlated Massless Dirac Fermions in an Organic Crystal. Phys. Rev. Lett. 2016, 116, 226401. [CrossRef] [PubMed]

25. Hirara, M.; Ishikawa, K.; Matsuno, G.; Kobayashi, A.; Miyagawa, K.; Tamura, M.; Berthier, C.; Kanoda, K. Anomalous spin correlations and excitonic instability of interacting 2D Weyl fermions. Science 2017, 358, 1403-1406. [CrossRef] [PubMed]

26. Shibaeva, R.P.; Kaminskii, V.F.; Yagubskii, E.B. Crystal structures of organic metals and superconductors of (BEDT-TTP)-I system. Mol. Cryst. Liq. Cryst. 1985, 119, 361-373. [CrossRef]

27. Kobayashi, H.; Kato, R.; Kobayashi, A.; Nishio, Y.; Kajita, K.; Sasaki, W. Crystal and electronic structures of layered molecular superconductor, $\theta-(\mathrm{BEDT}-\mathrm{TTF})_{2}\left(\mathrm{I}_{3}\right)_{1-x}\left(\mathrm{AuI}_{2}\right)_{x}$. Chem. Lett. 1986, 15, 833-836. [CrossRef]

28. Kobayashi, H.; Kato, R.; Kobayashi, A.; Nishio, Y.; Kajita, K.; Sasaki, W. A new molecular superconductor, $(\mathrm{BEDT}-\mathrm{TTF})_{2}\left(\mathrm{I}_{3}\right)_{1-x}\left(\mathrm{AuI}_{2}\right)_{x}(x<0.02)$. Chem. Lett. 1986, 15, 789-792.

29. Rothaemel, B.; Forro, L.; Cooper, J.R.; Schilling, J.S.; Weger, M.; Bele, P.; Brunner, H.; Schweitzer, D.; Keller, H.J. Magnetic susceptibility of $\alpha$ and $\beta$ phases of di[bis(ethylenediothiolo) tetrathiafulvalene] tri-iodide [(BEDT-TTF $)_{2} \mathrm{I}_{3}$ ] under pressure. Phys. Rev. B 1986, 34, 704-712. [CrossRef]

30. Kino, H.; Fukuyama, H. On the phase transition of $\alpha-(\mathrm{ET})_{2} \mathrm{I}_{3}$. J. Phys. Soc. Jpn. 1995, 64, 1877-1880. [CrossRef]

31. Seo, H. Charge ordering in organic ET compounds. J. Phys. Soc. Jpn. 2000, 69, 805-820. [CrossRef]

32. Takano, Y.; Hiraki, K.; Yamamoto, H.M.; Nakamura, T.; Takahashi, T. Charge disproportionation in the organic conductor, $\alpha$-(BEDT-TTF $)_{2} \mathrm{I}_{3}$. J. Phys. Chem. Solids 2001, 62, 393-395. [CrossRef]

33. Wojciechowski, R.; Yamamoto, K.; Yakushi, K.; Inokuchi, M.; Kawamoto, A. High-pressure Raman study of the charge ordering in $\alpha$-(BEDT-TTF $)_{2} \mathrm{I}_{3}$. Phys. Rev. B 2003, 67, 224105. [CrossRef]

34. Moldenhauer, J.; Horn, C.H.; Pokhodnia, K.I.; Schweitzer, D.; Heinen, I.; Keller, H.J. FT-IR absorption spectroscopy of BEDT-TTF radical salts: Charge transfer and donor-anion interaction. Synth. Met. 1993, 60, 31-38. [CrossRef]

35. Kakiuchi1, T.; Wakabayashi, Y.; Sawa, H.; Takahashi, T.; Nakamura, T. Charge ordering in $\alpha-(B E D T-T T F)_{2} I_{3}$ by synchrotron X-ray diffraction. J. Phys. Soc. Jpn. 2007, 76, 113702. [CrossRef]

36. Ojiro, T.; Kajita, K.; Nishio, Y.; Kobayashi, H.; Kobayashi, A.; Kato, R.; Iye, Y. A new magneto-pressure phase in $\alpha$-(BEDT-TTF $)_{2} \mathrm{I}_{3}$. Synth. Met. 1993, 56, 2268-2273. [CrossRef]

37. Kajita, K.; Ojiro, T.; Fujii, H.; Nishio, Y.; Kobayashi, H.; Kobayashi, A.; Kato, R. Magnetotransport Phenomena of $\alpha$-Type (BEDT-TTF) ${ }_{2} \mathrm{I}_{3}$ under High Pressures. J. Phys. Soc. Jpn. 1993, 61, 23-26. [CrossRef]

38. Mott, N.F.; Davis, E.A. Elecron Processes in Non-Crystalline Materials; Clarendon: Oxford, UK, 1979.

39. Shon, N.H.; Ando, T. Quantum transport in Two-dimensional graphite system. J. Phys. Soc. Jpn. 1998, 67, 2421-2429. [CrossRef]

40. Ziegler, K. Delocalization of 2D dirac fermions: The role of a broken supersymmetry. Phys. Rev. Lett. 1998, 80, 3113-3116. [CrossRef] 
41. Nomura, K.; MacDonald, A.H. Quantum transport of massless dirac fermions. Phys. Rev. Lett. 2007, 98, 076602. [CrossRef] [PubMed]

42. Ando, T. Theory of electronic states and transport in carbon nanotubes. J. Phys. Soc. Jpn. 2005, 74, 777-817. [CrossRef]

43. Osada, T. Negative interlayer magnetoresistance and Zero-Mode landau level in multilayer Dirac electron systems. J. Phys. Soc. Jpn. 2008, 77, 084711. [CrossRef]

44. Sugawara, S.; Tamura, M.; Tajima, N.; Sato, M.; Nishio, Y.; Kajita, K.; Kato, R. Temperature dependence of inter-layer longitudinal magnetoresistance in $\alpha$-(BEDT-TTF $)_{2} \mathrm{I}_{3}$ : Positive versus negative contributions in a tilted Dirac cone system. J. Phys. Soc. Jpn. 2010, 79, 113704. [CrossRef]

45. Tajima, N.; Sato, M.; Sugawara, S.; Kato, R.; Nishio, Y.; Kajita, K. Spin and valley splittings in multilayered massless Dirac fermion system. Phys. Rev. B 2010, 82, 121420. [CrossRef]

46. Tajima, N.; Yamauchi, T.; Yamaguchi, T.; Suda, M.; Kawasugi, Y.; Yamamoto, H.M.; Kato, R.; Nishio, Y.; Kajita, K. Quantum Hall effect in multilayered massless Dirac fermion systems with tilted cones. Phys. Rev. B 2013, 88, 075315. [CrossRef]

47. Fukuyama, H. Anomalous orbital magnetism and hall effect of massless fermions in two dimension. J. Phys. Soc. Jpn. 2007, 76, 043711. [CrossRef]

48. Pokhodnia, K.I.; Graja, A.; Weger, M.; Schweitzer, D. Resonant Raman scattering from superconducting single crystals of (BEDT-TTF) ${ }_{2} \mathrm{I}_{3}$. Z. Phys. B 1993, 90, 127-133. [CrossRef]

49. Yoshimura, M.; Shigekawa, H.; Kawabata, K.; Saito, Y.; Kawazu, A. STM study of thin films of BEDT-TTF iodide. Appl. Surf. Sci. 1992, 61, 317-320. [CrossRef]

50. Miura, K. Effects of Annealing on the Transport in an Organic Zero-Gap Conductor $\alpha$-(BEDT-TTF) ${ }_{2} \mathrm{I}_{3}$. Master's Thesis, Toho University, Chiba, Japan, 2011. (Supplementary material, 2017).

51. Tisserond, E.; Fuchs, J.N.; Goerbig, M.O.; Auban-Senzier, P.; Meziere, C.; Batail, P.; Kawasugi, Y.; Suda, M.; Yamamoto, H.M.; Kato, R.; et al. Aperiodic quantum oscillations of particle-hole asymmetric Dirac cones. Europhys. Lett. 2017, 119, 67001. [CrossRef]

52. Fortin, J.-Y.; Audouard, A. Effect of electronic band dispersion curvature on de Haas-van Alphen oscillations. Eur. Phys. J. B 2015, 88, 225. [CrossRef]

53. Kishigi, K.; Hasegawa, Y. Three-quarter Dirac points, Landau levels, and magnetization in $\alpha$-(BEDT-TTF) ${ }_{2} \mathrm{I}_{3}$. Phys. Rev. B 2017, 96, 085430. [CrossRef]

(C) 2018 by the author. Licensee MDPI, Basel, Switzerland. This article is an open access article distributed under the terms and conditions of the Creative Commons Attribution (CC BY) license (http:/ / creativecommons.org/licenses/by/4.0/). 
Article

\title{
Light-Induced Current Oscillations in the Charge-Ordered State of (TMTTF $)_{2} \mathrm{SbF}_{6}$
}

\author{
Tobias Peterseim and Martin Dressel * \\ 1. Physikalisches Institut, Universität Stuttgart, Pfaffenwaldring 57, D-70550 Stuttgart, Germany; \\ tpeterseim@gmx.de \\ * Correspondence: dressel@pi1.physik.uni-stuttgart.de; Tel.: +49-711-6856-4946; Fax: +49-711-6856-4886 \\ Academic Editor: Helmut Cölfen \\ Received: 31 July 2017; Accepted: 13 September 2017; Published: 15 September 2017
}

\begin{abstract}
Below $T_{\mathrm{CO}}=157 \mathrm{~K}$ the quasi-one-dimensional charge-transfer salt $(\mathrm{TMTTF})_{2} \mathrm{SbF}_{6}$ undergoes a pronounced phase transition to a charge-ordered ground state. We have explored the non-linear and photoconductive behavior as a function of applied voltage, laser pulse energy and temperature. Besides a decay of the photoconductive signal in a double exponential fashion in the millisecond range, we discover current oscillations in the $\mathrm{kHz}$ range induced by the application of short laser pulses. While the resonance frequencies do not depend on voltage or laser intensity and vary only slightly with temperature, the amplitude changes linearly with the laser intensity and voltage. We suggest that photo-induced fluctuations of the charge-ordered state alter the current flow of the single particles and hence, the photocurrent. The findings are discussed and compared to comparable phenomena in other low-dimensional electron systems.
\end{abstract}

Keywords: charge-transfer salts; TMTTF; charge-order phase transition; non-linear transport; photoconductivity; current oscillations; calculations of band structure and optical conductivity

PACS: 77.84.Jd; 77.80.Fm

\section{Introduction}

The charge-transfer salts (TMTTF) ${ }_{2} X$, where TMTTF stands for tetramethyltetrathiafulvalene and $X$ for a monovalent anion, have been under scrutiny due to their quasi-one-dimensional electronic character and ordering phenomena in the spin and charge sectors [1-4]. In recent years, several comprehensive investigations have been conducted on the optical [5-9] and transport properties [10], the lattice [11,12] and electronic structure [13-15] as well as on the magnetic properties [16-21]. However, not much is known about the non-linear properties at the charge-order transition taking place at $T_{\mathrm{CO}}=157 \mathrm{~K}$ and transient conduction phenomena. This is surprising, since nonlinear behavior was observed in various inorganic as well as organic compounds in the vicinity of a metal-insulator transition, such as the charge-density wave transition in $\mathrm{NbSe}_{3}$, the metal insulator transition in $\mathrm{VO}_{2}$, or the charge-order transition in the two-dimensional $\alpha$-(BEDT-TTF $)_{2} \mathrm{I}_{3}$ salt [22-29].

Several classes of electrically induced nonlinear phenomena in solid-state materials can be distinguished:

- Non-equilibrium charge carriers: modification of the charge carrier number or the mobility.

- Space-charge distribution: for instance impurity bands.

- Electrically induced phase transitions: modification of the electronic system or the crystalline structure.

- Ionic motion in the host material: forming of conductive filaments.

- Sliding charge density waves or charge order.

- Domain wall motions or excitations of solitons. 
Although exotic electrically induced effects emerge in most classes of materials, it is basically impossible to draw a conclusive picture since the diversity of the involved ground states is enormous. Nevertheless, in regard to future electronic devices and applications, more effort has to be devoted to the experimental studies on electrically induced phenomena as well as the development of accurate models explaining a wide range of these effects occurring in various material classes. For a broader discussion we refer to the review articles $[30,31]$ and textbooks $[32,33]$ and further literature listed therein.

The first studies on electrically induced phenomena in organic conductors were performed in the 1970s and 1980s on Cu(TCNQ) [34] and TTF-CA [35]. Later, this was extended to other organic systems, such as $\mathrm{K}(\mathrm{TCNQ})$ [36], where the switching was also recorded optically, expressing a stripe structure of alternating insulating and metallic domains between the two contacts. In the two-dimensional organic salt $\theta$-(BEDT-TTF $)_{2} \mathrm{CsZn}(\mathrm{SCN})_{4}$ [37] and its sister compounds [38] the charge order was melted by applying a current to the crystal [31]. To date, no concept or model could be established that can explain all these observations in organic salts.
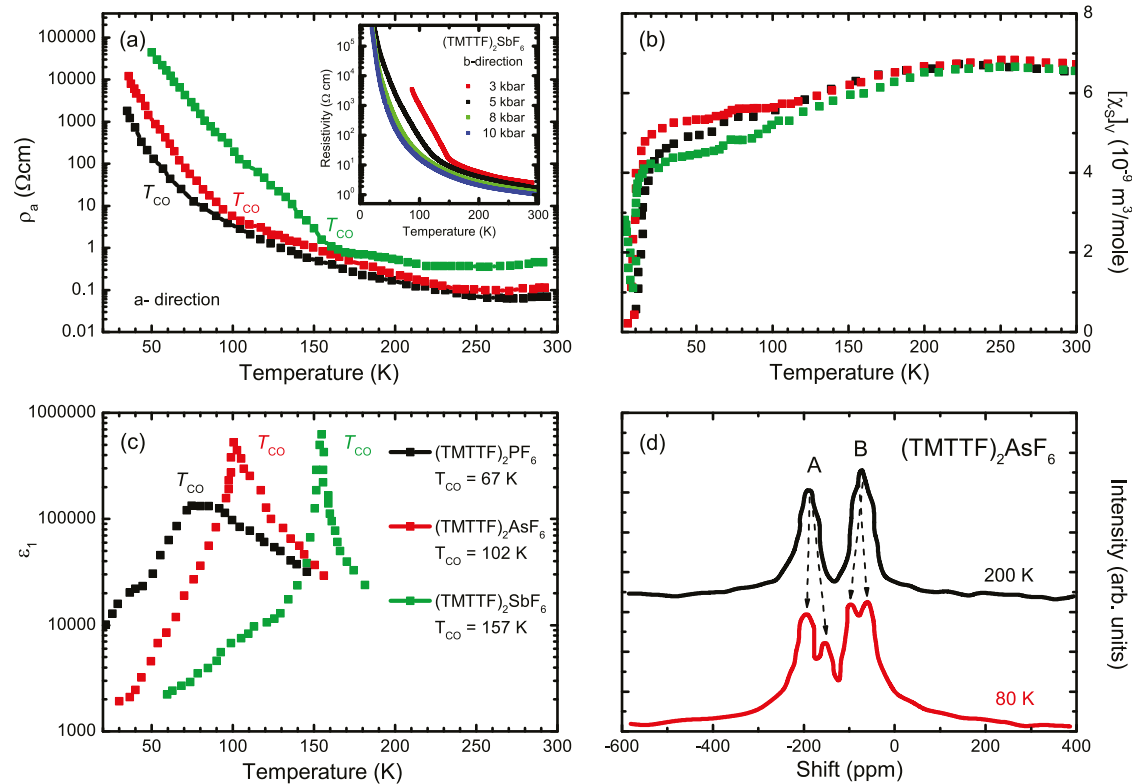

Figure 1. Experimental characterization of $(\mathrm{TMTTF})_{2} \mathrm{X}, \mathrm{X}=\mathrm{PF}_{6}$ (black), $\mathrm{AsF}_{6}$ (red), and $\mathrm{SbF}_{6}$ (green) single crystals. (a) Temperature-dependence of the resistivity along the $a$-direction [10]. The labels mark the charge-order transition temperature $T_{\mathrm{CO}}=67,102$, and $157 \mathrm{~K}$, respectively. The inset demonstrates the effect of hydrostatic pressure on the transition by displaying the temperature-dependent $\mathrm{dc}$ resistivity of (TMTTF) ${ }_{2} \mathrm{SbF}_{6}$ measured along the $b$-direction. $T_{\mathrm{CO}}$ is seen as a kink in $\rho(T)$ that shifts to low temperatures as pressure increases; (b) Temperature-dependence of the spin susceptibility at constant volume $\left[\chi_{S}\right]_{v}$. (TMTTF $)_{2} \mathrm{PF}_{6}$ and $(\mathrm{TMTTF})_{2} \mathrm{AsF}_{6}$ undergo a spin-Peierls transition at $T_{\mathrm{SP}}=19 \mathrm{~K}$ and $13 \mathrm{~K}$, respectively. (TMTTF) ${ }_{2} \mathrm{SbF}_{6}$ exhibits an antiferromagnetic state below $8 \mathrm{~K}$ causing an increase of $\left[\chi_{S}\right]_{v}$. Above the transition $\left[\chi_{s}\right]_{v}$ can be described by a model of a $1 / 2$ antiferromagnetic Heisenberg chain [16,39]; (c) The real part of the dielectric constant $\epsilon_{1}$ maesured at $f=100 \mathrm{kHz}$ as a function of temperature $T$ reveals a Curie-law behavior [40]; (d) ${ }^{13} \mathrm{C}-\mathrm{NMR}$ spectra of (TMTTF) ${ }_{2} \mathrm{AsF}_{6}$ at $200 \mathrm{~K}$ and $80 \mathrm{~K}$. Above $T_{\mathrm{CO}}=102 \mathrm{~K}$ two distinct features $\mathrm{A}$ and $\mathrm{B}$ can be identified due to the two molecules per unit cell. They split into a total of four peaks, as depicted by the arrows [41], giving evidence for charge order. 
Since nonlinear behavior was observed in several different inorganic as well as organic compounds at the vicinity of a metal-insulator transition, we expect similar phenomena in the quasi one-dimensional salts (TMTTF) ${ }_{2} X$, with the centrosymmetric anion $X=\mathrm{PF}_{6}, \mathrm{AsF}_{6}$, and $\mathrm{SbF}_{6}$. In Figure 1 we collect the important experimental findings characterizing these three materials. Upon cooling the resistivity goes through a shallow minimum due to electronic localization and later exhibits a kink at the charge-order transition $T_{\mathrm{CO}}$, as displayed in panel (a). No significant change is observed in the magnetic susceptibility at $T_{\mathrm{CO}}$ [17] plotted in Figure 1b; while the magnetic order and spin-Peierls transitions occur at low temperatures. Due to charge order, the dielectric constant exhibits a maximum at this temperature (see panel (c)), more or less following Curie's law [40]. As presented in Figure 2, the charge-sensitive vibrational modes split due to charge disproportionation [7]; similar conclusions are drawn from the NMR spectra [41] plotted in Figure 1d.

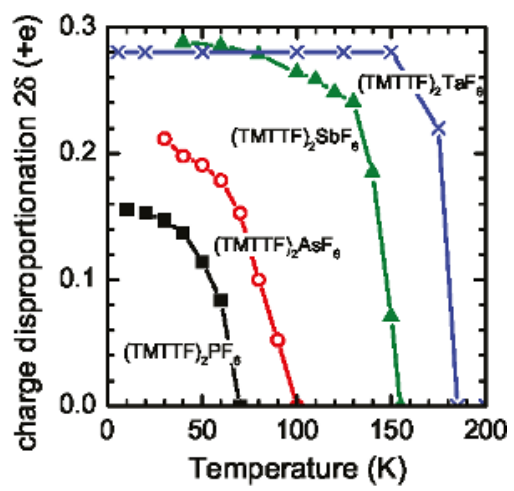

Figure 2. Temperature dependence of the charge dispropotionation probed by the splitting of the infrared-active $v_{28}$ molecular vibrational mode in (TMTTF) ${ }_{2} \mathrm{X}$ with $\mathrm{X}=\mathrm{PF}_{6}^{-}, \mathrm{AsF}_{6}^{-}, \mathrm{SbF}_{6}^{-}$and $\mathrm{TaF}_{6}^{-}$as a function of temperature $T$ recorded along the $c$ axis. The charge imbalance $2 \delta$ is estimated from the difference of the two resonance frequencies by $2 \delta=\Delta v /\left(80 \mathrm{~cm}^{-1} / e\right)[7,42]$.

In Figure 3a the optical reflectivity of (TMTTF) ${ }_{2} \mathrm{SbF}_{6}$ is plotted as measured for ambient conditions with the electric field polarized parallel to the chain direction. Using the Kramers-Kronig relation the frequency dependent conductivity can be calculated as illustrated in the lower panel (b). The spectra are dominated by a strong band around $2000 \mathrm{~cm}^{-1}$ due to excitations between the Hubbard bands, which occur due to electronic correlations. In addition emv-coupled vibrational features strongly affect the optical properties in this range of frequency [43,44].

For (TMTTF $)_{2} \mathrm{SbF}_{6}$ a surprising behavior of the material was observed in response to electrical and optical pulses, which is presented and discussed below in detail. We could not detect similar phenomena in (TMTTF) ${ }_{2} \mathrm{PF}_{6}$ and (TMTTF $)_{2} \mathrm{AsF}_{6}$. As depicted in Figure 1a (TMTTF) ${ }_{2} \mathrm{SbF}_{6}$ reveals the steepest slope at the phase transition, while (TMTTF) ${ }_{2} \mathrm{PF}_{6}$ and (TMTTF) ${ }_{2} \mathrm{AsF}_{6}$ exhibit a smooth transition. Therefore, we conclude that for the one-dimensional salts strong resistivity changes [10] at the transition temperature are a good indication that nonlinear behavior can be observed. Otherwise, the difference is too small and the photo-induced effect is absent. 


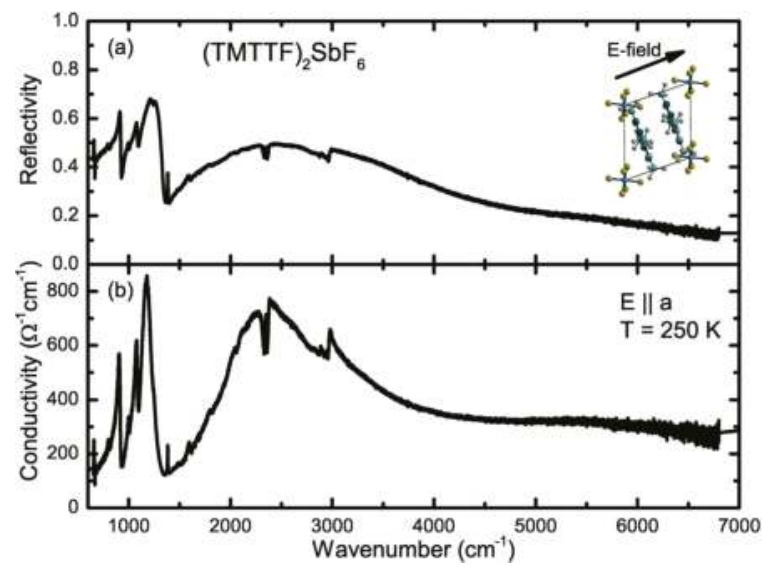

Figure 3. Frequency-dependent optical reflectivity (a) and conductivity (b) of (TMTTF) ${ }_{2} \mathrm{SbF}_{6}$ along the crystallographic $a$ direction measured at $T=250 \mathrm{~K}$.

\section{Experimental Details}

Single crystals of the charge-transfer salts (TMTTF) ${ }_{2} \mathrm{SbF}_{6}$ were grown by electrochemical methods as described previously $[10,39]$. The needle-shaped single crystals are several millimeters long in the $a$-direction and less than a millimeter wide in the other crystal directions. Figure 4 shows the crystal structure of (TMTTF $)_{2} \mathrm{SbF}_{6}$ in different orientations.
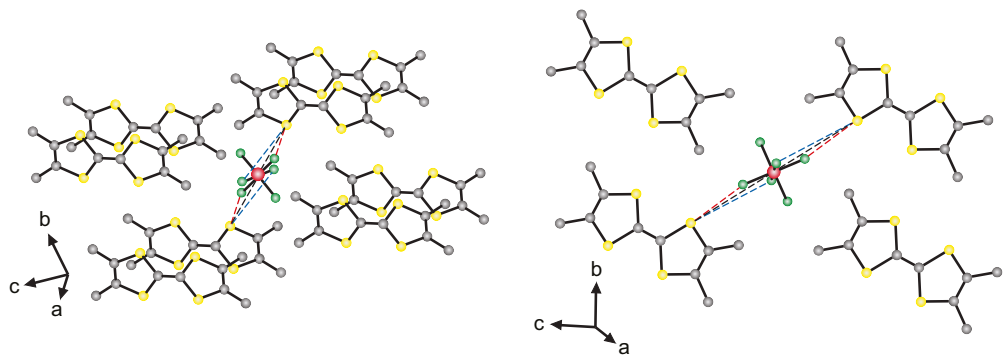

Figure 4. Two views on the crystal structure of (TMTTF) $)_{2} \mathrm{SbF}_{6}$ illustrate the confinement of the octahedral anions in a cavity formed by methyl groups (gray) of the surrounding TMTTF molecules. Also shown by dashed lines are the closest connections between the fluorine (green) and sulphur atoms (yellow); the antimony ion (red) is located in the center octahedron.

For transport experiments along the chain direction $a$ four fine gold wires were attached to the crystal by carbon paste as contacts. The Ohmic nature of these contacts on the organic crystals has been verified in detail previously $[10,25,45]$. The samples were cooled in a helium cryostat. Besides simple $\rho(T)$ characterization (cf. Figure 6), we measured the transport response of the crystals along $a$, when a strong electric pulse is applied.

In a second step, photoconductivity was studied, i.e., we probed the time response of the electric transport after exposing the crystal to a laser pulse $(8 \mathrm{~ns})$ with a wavelength of $\lambda=532 \mathrm{~nm}$ (corresponding to a photon energy of $2.33 \mathrm{eV}$ ). The setup for the photocurrent measurements sketched in Figure $5 \mathrm{~b}$ consists of two contacts on the specimen separated by $1.1 \mathrm{~mm}$. A voltage pulse with variable width is created on the contacts by a pulse generator. The applied voltage $U$ and current $U / R_{L}$ are measured by an oscilloscope. Since the laser has a low repetition rate between $1 \mathrm{~Hz}$ and 
$20 \mathrm{~Hz}$ heat accumulation is avoided; it is also ensured that long living states decay before the next pulse arrives and hence, accumulation effects can be excluded.
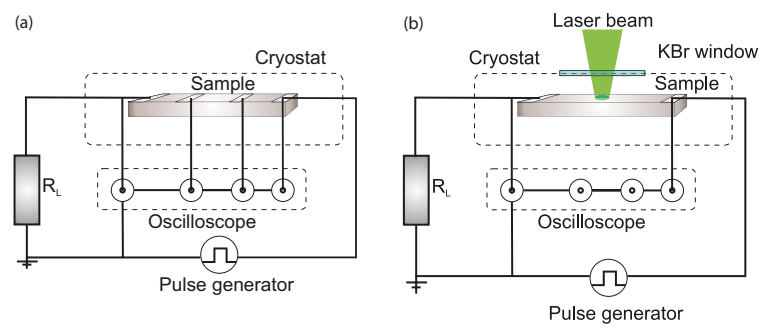

Figure 5. (a) For the time-resolved transport measurement either the four-point or two-point configuration was employed. It can be switched between the steady-state and the time-resolved transport measurement. The voltage pulses are created by an Avtech AV-1010 B of Hewlett-Packard HP 214B pulse generator. A Tektronix TDS 744A oscilloscope (input impedance $1 \mathrm{M} \Omega$ ) records the applied voltage $U$, the voltage drop across the inner contacts $U_{23}$ as well as the total current of the circuit by the voltage drop $U_{L}$ across the load resistor $R_{L}$ in series; (b) Schematic drawing of the photoconductivity setup. It consists of a pulse generator creating the voltage $U$ at the sample, an oscilloscope, and a load resistor. $R_{L}$ is used to determine the current in the circuit. The frequency doubled Nd:YAG laser beam is focussed through the cryostat window. The beam diameter is approximately $0.6 \mathrm{~mm}$, the laser intensity ranged up to $5 \mathrm{~mJ} / \mathrm{cm}$.

The generated photocurrent $I_{\mathrm{Ph}}(t)$ can be derived from the following physical relation. The electronic circuit consists of a voltage source, the sample and the load resistance $R_{L}$. The dark current $I_{D}(t)$ is defined as

$$
I_{\mathrm{D}}(t)=\frac{U}{\left(R_{\mathrm{L}}+R_{\mathrm{S}, \mathrm{D}}\right)}
$$

$R_{\mathrm{S}, \mathrm{D}}$ the dark resistance of the sample and $R_{\mathrm{S}, \mathrm{Ill}}$ the corresponding resistance when illuminated. The total current under illumination $I_{\text {Ill }}(t)$ is respectively

$$
I_{\text {IIl }}(t)=\frac{U}{\left(R_{\mathrm{L}}+R_{\mathrm{S}, \mathrm{III}}\right)}
$$

with $R_{\mathrm{S}, \text { III }}$ Thus, the measured photocurrent is

$$
I_{\mathrm{Ph}}(t)=I_{\mathrm{Ill}}(t)-I_{\mathrm{D}}(t)=U\left(\frac{1}{\left(R_{\mathrm{L}}+R_{\mathrm{S}, \mathrm{Ill}}\right)}-\frac{1}{\left(R_{\mathrm{L}}+R_{\mathrm{S}, \mathrm{D}}\right)}\right)
$$

In a photocurrent measurement the contact resistance plays a crucial role since it influences the transport of the charge carriers to the contact. The materials, i.e., metal, semiconductor or insulator, touching at the interface form different types of contacts. At the boundary the band structure is deformed due to the difference in work function and electron affinity of the materials. Therefore, a barrier is created at the boundary surface leading to a depletion area [32,46]. By that, additional carriers can be transferred or the charge carriers can be blocked at the contacts. There are typically two kinds of contacts: the Schottky-contact and the Ohmic contact. Generally, several processes contribute to the charge transport across the barriers. The dominating processes are the thermal emission of charge carriers across the energy barrier and tunneling of the carriers, being temperature-independent. However, it is assumed that Ohmic contacts are formed in the organic crystals, based on previous studies $[10,25,45]$. During the transport measurements the temperature-dependence of the contacts was checked by measuring the contact resistance at specific intervals; to that end, we switched between four-point and two-point resistance measurements, as depicted in Figure 5a. Their resistance value 
normally changes at a phase transition when a gap opens in the electronic system. For details on the time and field-dependent experiments we refer to Refs. [47,48].

\section{Response to Electric Fields}

Before discussing the response of $(\mathrm{TMTTF})_{2} \mathrm{SbF}_{6}$ to electric pulses, let us consider the temperature-dependent resistance from room temperature down to $125 \mathrm{~K}$, in order to determinate the phase transition temperature. This allows us to assign $T_{\mathrm{CO}}$ more precisely compared to optical methods [7,8] and to deduce the contact resistances in this particular case. In Figure 6 the logarithm of the resistance of (TMTTF) ${ }_{2} \mathrm{SbF}_{6}$ is illustrated for the $a$-direction in a temperature range from room temperature down to $125 \mathrm{~K}$. When lowering the temperature, the resistance decreases down to a minimum at $T=215 \mathrm{~K}$, which corresponds to the sign change of its slope (see inset). This is caused by electron-electron interactions opening up a Mott gap at the middle of the upper half-filled band and Umklapp scattering. Thus, the resistance further increases with decreasing the temperature down to the charge-order transition, corresponding to a linear slope of the derivative. At $T_{\mathrm{CO}}$ a kink arises and below this temperature $\ln R$ increases nonlinearly; hence transport is not just thermally activated. It is worth mentioning that for $(\mathrm{TMTTF})_{2} \mathrm{SbF}_{6}$ the resistivity increases more abruptly and steeper at the charge-ordered phase transition than observed in the (TMTTF) ${ }_{2} \mathrm{PF}_{6}$ and $(\mathrm{TMTTF})_{2} \mathrm{AsF}_{6}$ analogues. Furthermore, (TMTTF $)_{2} \mathrm{SbF}_{6}$ reveals an anomaly in the thermal expansion coefficient at the charge-order transition [49]. In contrast, in its sister compounds no feature appears at the corresponding transition temperature.

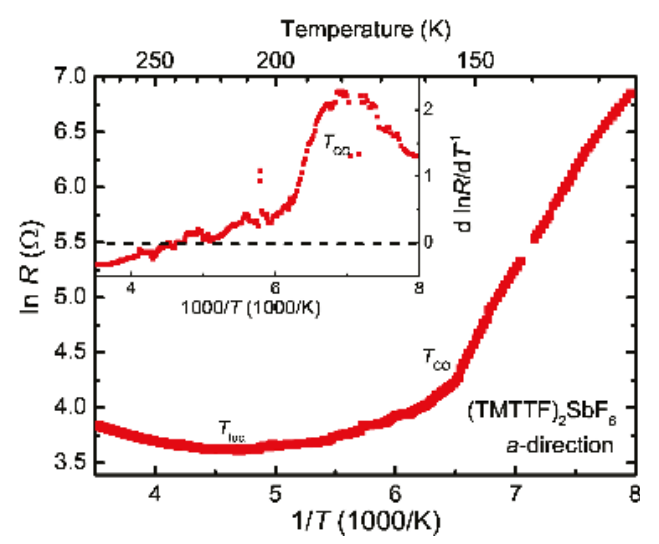

Figure 6. Logarithm of the resistance of (TMTTF $)_{2} \mathrm{SbF}_{6}$ as a function of the inverse temperature $1 / T$. At about $T=215 \mathrm{~K}$ a minimum appears, marking the transition to the localized state related to the opening of a Mott gap. The resistance increased slightly and at $T_{\mathrm{CO}}=157 \mathrm{~K}$ a kink is observed in the curve due to the charge order transition. Inset: derivative of the logarithm of the resistance vs. $1 / T$. The charge localization leads to a change of the sign from negative (metallic) to positive (insulating). Also the slope changes at $T_{\mathrm{CO}}$ leading to a rather flat curve at low temperatures.

A similar behavior appears in the temperature-dependent evolution of the charge disproportion [7], as depicted in Figure 2. While in (TMTTF) ${ }_{2} \mathrm{PF}_{6}$ and (TMTTF $)_{2} \mathrm{AsF}_{6}$ a mean-field behavior appears, a first-order-like transition takes place in (TMTTF $)_{2} \mathrm{SbF}_{6}$. The latter is probably related to the enhanced dimerization (see $[10,12])$ and the charge localization state at elevated temperatures. This behavior is even more pronounced in $(\mathrm{TMTTF})_{2} \mathrm{TaF}_{6}$, where the phase transition takes place at $T_{\mathrm{CO}}=177 \mathrm{~K}$ and $2 \delta=00.28 e$ is of comparable size [42].

It is interesting to recall early studies on the electronic transport in quasi-one-dimensional organic conductors, such as TTF-TCNQ, TMTSF salts and TMTTF salts in the metallic and insulating states 
that occur below charge- and spin-density-wave or charge-order transitions [1,2,50-59]. There the current flow in strongly anisotropic metals, fluctuations, non-linearity, contact issues, etc. are discussed in depth.

We now apply voltage pulses of $1 \mathrm{~ms}$ duration to the sample and measure the total current and the voltage drop across a load resistor of $R_{L}=1 \mathrm{k} \Omega$ connected in series to the sample. In Figure 7 the time-dependent current response of (TMTTF) ${ }_{2} \mathrm{SbF}_{6}$ at $T=140 \mathrm{~K}$ is plotted for two different voltages. At the beginning of the pulse, an overshoot of the measured current occurs due to the parasitic capacity and resistance in the circuit (panel a). The short oscillating signal located at $100 \mu$ s is caused by a parasitic current induced by the switching of the Pockels cell of the laser system, later used to create the photocurrent. For low voltages $(U=0.9 \mathrm{~V})$ the current signal stays constant during the whole pulse. When increasing the amplitude of the pulse, the current increases approximately in a linear way obeying Ohm's law. Nevertheless, following the temporal evolution further reveals a nonlinear increase of the current, which can be attributed to a thermal modification of the contact resistance.

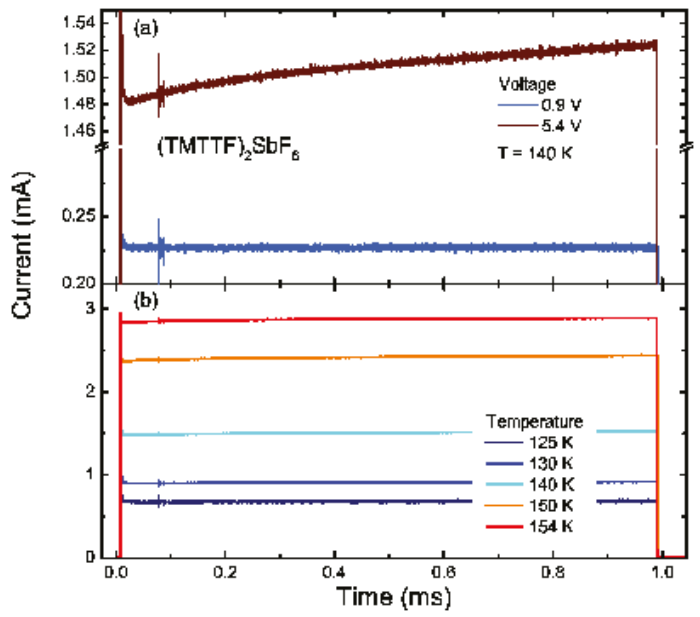

Figure 7. (a) Response of the current in (TMTTF $)_{2} \mathrm{SbF}_{6}$, when $1 \mathrm{~ms}$ pulses of two different voltages are applied at $T=140 \mathrm{~K}$; (b) Current response plotted for various temperatures below $T_{\mathrm{CO}}$.

Figure $7 \mathrm{~b}$ displays current pulses for different temperatures below $T_{\mathrm{CO}}$. The current signal is reduced for the same applied voltage in the same way as the total resistance increases with temperature. Independent on the applied voltage or examined temperature no switching behavior or current oscillation can be detected that would indicate a sliding of the $4 k_{F}$ charge-density wave (CDW) or a collective response of the charge-ordered state [60,61].

\section{Response to Laser Pulses}

Next the photo-response of (TMTTF $)_{2} \mathrm{SbF}_{6}$ was studied by illuminating a fraction of the space between the two contacts by a laser pulse. In Figure 8 the recorded current pulse is illustrated exemplarily at $T=150 \mathrm{~K}$ for a $5 \mathrm{~V}$ voltage pulse and a laser pulse with the energy of $40 \mu \mathrm{J}$. Similar to Figure 7, an overshoot is present at the beginning. Additionally, $750 \mu$ s after the onset of the voltage pulse, the current suddenly shoots up with a rise time a few nanoseconds. Strikingly, this onset is correlated with the incident laser pulse, giving clear evidence for photoconductivity. The signal barely decays and stays almost constant over the entire voltage pulse length. In the inset of Figure 8 an enlarged detail of the first $400 \mu$ s of the photocurrent is visualized. Surprisingly, current oscillations are superimposed on top of the signal, whose amplitude decays with time. The inset further reveals a slight signal decay within the first $59 \mu$ s. 
We examined the photocurrent signal for various voltages below the phase transition $T_{\mathrm{CO}}=157 \mathrm{~K}$, as presented in Figure 9a for the temperature $T=150 \mathrm{~K}$ and laser pulse energy $E=32 \mu \mathrm{J}$. Above $T_{\mathrm{CO}}$, no change was observed. There, the photocurrent was extracted by subtracting the current without any light (see for example Figure 7) from the total current signal. Thereby we can also eliminate the nonlinear transport behavior of the device, consisting of the contact resistance and the sample. By enlarging the applied voltage the signal increases linearly. Similarly, the photocurrent becomes larger with rising laser pulse energy and the temporal behavior is the same as for the voltage-dependent current curves, as depicted in Figure 9b. Finally, also the temperature dependence of the transient photocurrent is plotted for a fixed pulse energy $32 \mu \mathrm{J}$ and voltage amplitude $U=5 \mathrm{~V}$ in Figure 9c. The photocurrent is the smallest in the vicinity of the phase transition $(T=154 \mathrm{~K})$ and then stays almost constant below $T=150 \mathrm{~K}$. One should note that all curves in the latter three graphs exhibit the same temporal dynamics, which can be fitted by a double-exponential decay function $f(t)=y_{0}+A \exp \left\{t / \tau_{1}\right\}+B \exp \left\{t / \tau_{2}\right\}$. Between $T=154 \mathrm{~K}$ and $125 \mathrm{~K}$, the first time constant $\tau_{1}$ decreases from $30 \mu$ s to $16 \mu$ s and $\tau_{2}$ diminishes by a factor of 2 from $300 \mu$ s to $170 \mu$ s. Here $y_{0}$ is the background which can be considered as a third extended living state, decaying at least on a millisecond time range. Such relaxation dynamics have also been observed in other photo-response experiments on organic conductors [62-65] taking place between the microsecond regime and far above the millisecond time scale.

However, the most important phenomena are the interference patterns which are superimposed on top of the exponentially decaying current signal. To the best of our knowledge, this has never been detected before in a photoconductivity experiment on organic conductors. In order to analyze the oscillations in detail, the frequency components were extracted from the decaying current by fast Fourier transformation (FFT). The amplitudes of the frequency components from the curves, depicted in Figure 9, are visualized in Figure 10 for various parameters. Five resonance frequencies can be identified: $49 \mathrm{kHz}$ (labeled A), $54 \mathrm{kHz}$ (B), $58.5 \mathrm{kHz}(\mathrm{C}), 77.5 \mathrm{kHz}$ (D), and $82 \mathrm{kHz}$ (E). Varying the voltage does not influence the resonance frequency (panel a), although the amplitude increases linearly. The same is true for the laser intensity dependence of the oscillation amplitude; it increases linearly without any modification of the peak positions, as shown in Figure $10 \mathrm{~b}$ for $T=150 \mathrm{~K}$ and $U=5 \mathrm{~V}$. It is important to note that the dark current $(0 \mu \mathrm{J}$ laser power $)$ is independent of the applied voltage, as it is exemplarily displayed in Figure 7; no oscillations occur, resulting in a flat amplitude spectrum. While the resonances display only an amplitude-dependence on the voltage and pulse intensity, both the amplitude as well as the resonance frequency change with temperature.

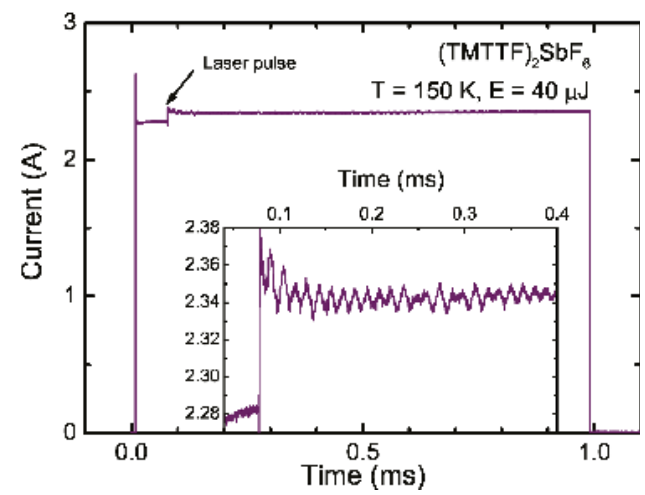

Figure 8. Current response to a $1 \mathrm{~ms}$ long $5 \mathrm{~V}$ voltage pulse applied on a (TMTTF) ${ }_{2} \mathrm{SbF}_{6}$ crystal at a temperature of $150 \mathrm{~K}$. At about $t=0.075 \mathrm{~ms}$, an additional laser pulse of $E=40 \mu \mathrm{s}$ (black arrow) illuminates the sample. This induces a photocurrent, visible in the steep, weak jump of the current signal. In the inset the first $400 \mu$ s of the photocurrent response are magnified. Interestingly, overall signal is are superimposed with pronounced oscillations. 


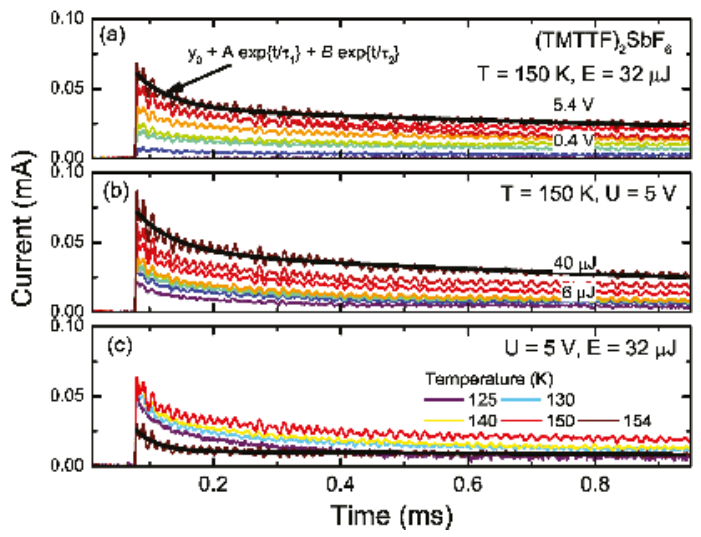

Figure 9. Time-dependent photocurrent of (TMTTF $)_{2} \mathrm{SbF}_{6}$ for (a) various voltages; (b) laser pulse intensities and (c) temperatures as indicated. In all three panels the temporal dynamic is fitted by a double exponential function (black lines).

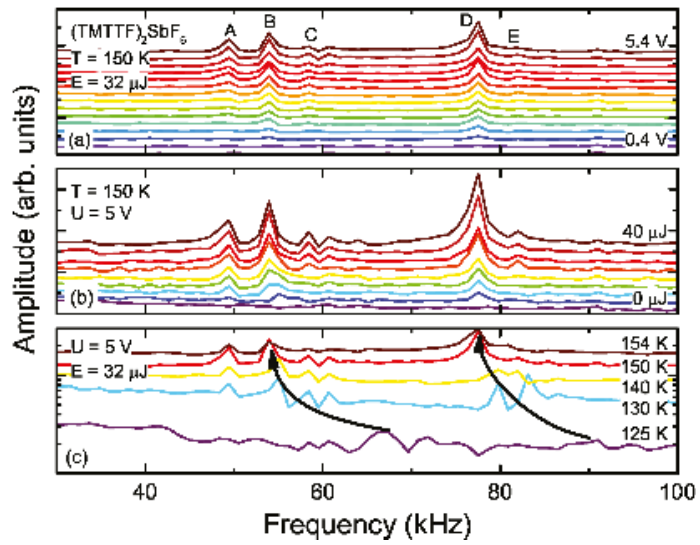

Figure 10. Amplitude spectra of the current oscillations in (TMTTF) ${ }_{2} \mathrm{SbF}_{6}$ obtained by fast Fourier transformation. The curves are shifted by a constant value for better visualization. Amplitude spectrum in a frequency range from $30 \mathrm{kHz}$ to $100 \mathrm{kHz}$ plotted (a) for various voltages from $U=0.4 \mathrm{~V}$ to $5.4 \mathrm{~V}$ and for a laser pulse energy of $32 \mu \mathrm{J}$ at $150 \mathrm{~K}$; (b) Same temperature and frequency range as in (a), however, the amplitude spectra are shown for different laser pulse energies at a fixed voltage of $5 \mathrm{~V}$. (c) Temperature-dependent amplitude spectra from $T=154 \mathrm{~K}$ to $125 \mathrm{~K}$ for $32 \mu \mathrm{J}$ and $5 \mathrm{~V}$. The black arrows indicate how the current oscillations shift in frequency as the temperature is raised.

\section{Discussion}

We now turn to the question of the origin of the photocurrent and its dynamic: Why does the current oscillate after illumination?

Iwase et al. proposed a phenomenological description [66,67] relating $\sigma_{d c}$ to the temperature and electric current, $\sigma_{d c}(T, J)=\sigma_{1}(T)+\sigma_{2} J^{n}$, where the first term on the right side describes the thermal activated linear Ohmic behavior and the second term accounts for the nonlinear conductivity. A two-state model assumes that "hot electrons" are generated in the conduction band similar as in inorganic semiconductors. Mori and collaborators $[68,69]$ successfully applied this approach to $\beta^{\prime \prime}$-(BEDT-TTF $)_{3}\left(\mathrm{HSO}_{4}\right)_{2}$ and (TMET-TTP $)_{4} \mathrm{PF}_{6}$. The electron system is assumed to be decoupled from the lattice; hence an effective electron temperature $T_{e}$ can be defined. Note, this is just the temperature 
of the hot electrons and does not mean that the sample is heated up. The electron-electron interaction is much faster than the electron-phonon interaction [28]. These excited electrons or quasi-particles follow the heat balance equation:

$$
n C_{p} \frac{\mathrm{d} T_{e}}{\mathrm{~d} t}=P+\nabla\left(\lambda_{\text {therm,e }} \nabla T\right)
$$

which can be simplified by assuming a linear spatial heat transfer, expressed by the last term, leading to:

$$
n C_{p} \frac{\mathrm{dT}_{e}(t)}{\mathrm{d} t}=P(t)-\lambda_{\text {therm,e }}\left(T_{e}(t)-T_{0}\right)
$$

The left side of the equation is the energy stored in the excited electronic system. The first term on the right hand side is the electric input power $P$ in $\mathrm{Wcm}^{-3}$ created by the externally applied electric field or current, which can be expressed as follows:

$$
P(t)=E \times J(t)=\sigma\left(T_{e}(t)\right) E(t)^{2} \quad \text { or } \quad=\frac{J(t)^{2}}{\sigma\left(T_{\mathrm{e}}(t)\right)}
$$

and the second term of Equation (5) describes the linear heat transfer from the electronic system to the lattice, which is kept at the initial temperature $T_{0}$. Here $\lambda_{\text {therm,e }}$ is the thermal conductivity of the electron system and $n C_{p}$ the electron heat capacity per volume. Equation (5) is solved numerically for a certain starting electric field or current pulse. In general $\sigma\left(T_{e}\right)$ is determined from the conductivity curve of the corresponding material because the excited carriers are responsible for the dc conductivity. From this model, a delay time $\tau_{d}$ can be estimated which also accounts for current oscillations. A more detailed discussion of the two-state model can be found in Ref. [28,68,69].

The possible influence of thermal effects on the induced current has to be discussed. Following the approach presented in Ref. [28,70], we calculated the temporal behavior of the sample temperature and correlate it with the temperature-dependent sample resistance depicted in Figure 6 . The result of the simulation is visualized in Figure 11 for various temperatures $\mathrm{T}$ between $125 \mathrm{~K}$ and $154 \mathrm{~K}$. We assume that the complete pulse energy is absorbed and transferred into Joule heat at the surface of the specimen as the penetration depth is only a few hundred nanometers. Besides that, the lateral electric field is strongest between the contacts. Thus, we are most likely probing a thin layer at the surface. From this simulation, a very sharp increase of the current would result; which actually is not the case in the presented experimental curves. The relaxation dynamics or cooling process is very fast and related to an extreme drop of the photocurrent within $1 \mu \mathrm{s}$. Afterwards, it asymptotically approaches zero. Comparing the experimental results with the theoretical findings, it is clear that the amplitudes and time constants of the signals are different. Only in the case of the long relaxation process a thermal effect may contribute to the photocurrent signal. Furthermore, the simulation does not reveal any current oscillations, which supports the view that instead the system is driven into a metastable condition different from a simple heating scenario.

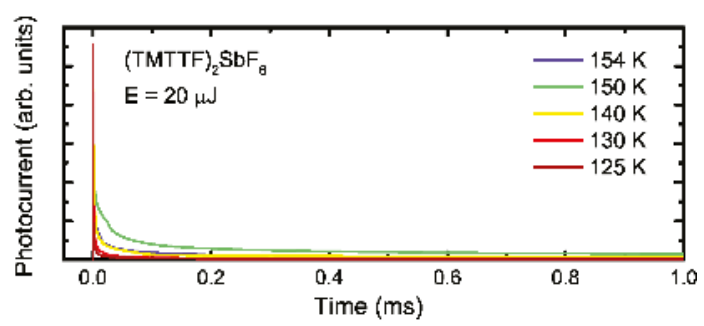

Figure 11. Simulation of a thermally induced photocurrent by a short laser pulse for five different temperatures as indicated. A steep current peak slowly relaxing to zero. 
For our case of (TMTTF) ${ }_{2} \mathrm{SbF}_{6}$, the laser photons with $\hbar \omega=2.33 \mathrm{eV}$ excite either the transition from the quarter-filled HOMO band to the LUMO+1 band of the $\mathrm{TMTTF}^{+\delta}$ molecules or the transition from a fully occupied band far below the Fermi energy to the quarter-filled unoccupied mid- infrared band, as depicted for the transitions labeled by I in Figure A3 of the Appendix A.2. After the vertical electronic excitation into the unoccupied bands, several decay channels are open:

- A free electron and hole can be created, which get separated from each other by the electric field, leading to the photocurrent signal. However, they can recombine before they reach the electrodes or become trapped. In general, the lifetime of free carries is between nanoseconds and microseconds.

- After a few picoseconds the excited electronic states couple to the lattice phonons and intramolecular vibrations. By this, energy is transferred to the lattice subsystem and the sample is warmed up.

- Afterwards, the excited electrons decay back into their initial state by emitting a red-shifted photon. The latter can be reabsorbed or detected as a photo-luminescence signal. Also, a radiation-free decay is possible into the lowest excited (mid-infrared) band.

- Otherwise, the excited charge can relax into a low-lying unoccupied band, triggering a charge transfer from one cation to its neighboring molecule in the picture of a molecular chain. This can induce a phase transition from the charge-ordered to the metallic state.

- Recently, in photo-luminescence spectra of a two-dimensional organic Mott insulator the creation of an exciton was suggested [71]. The theory of photo-induced transitions [72] proposes that higher excited states can be populated and excitons brake up; Frenkel excitons becomes charge-transfer excitons and propagate. The separation of excitons may also stimulate a light-induced transition. Especially in low-dimensional system fluctuations as well as electron-electron and electron-phonon interactions play a crucial role, eventually destabilizing the equilibrium state. This picture is similar to the model suggested for the photo-induced phase transition in TTF-CA [70,73].

While in Figures 8 and 9 the laser pulse hits the sample after the voltage is applied, we now delay the voltage pulse with respect to the laser pulse; in Figure 12 the photocurrent signal is displayed for different delay times of the voltage pulse. For more than $0.8 \mathrm{~ms}$ delay time the signals overlap with each other. Interestingly, the oscillations persist even when the voltage pulse is applied long after the light irradiation; but with a smaller amplitude. Moreover, the oscillations appear still at the same position, which proves that they are rigidly linked to the light pulse. It is surprising that the current curves are strongly displaced with respect to each other, indicating that without an external electric field, the excited free electrons and holes (or excitons) are not separated from each other and hence, recombine rapidly. In the case of a pure thermally induced effect, the current dynamics or the resistance, respectively, is expected to be independent of the applied voltage and to exhibit the same temporal behavior, regardless of the delay time between laser pulse and voltage pulse.

At this point, we should recall similar effects detected over the last fifty years in other compounds with and without illumination.

1. In the 1960s and 1970s transport measurements on silicon with two Schottky contacts revealed current oscillations in the $\mathrm{kHz}$ regime, which were ascribed to double current injection at the contacts [74]. However, the frequency could be varied by changing the voltage bias or temperature and the oscillations occur only above a critical threshold field [75].

2. The well-known creation of hot or non-equilibrium electrons in semiconductors such as GaAs [33] leads to electric field-dependent charge carrier velocities, causing a negative-differential resistance. One of the related phenomena is the famous Gunn effect, yielding current oscillations in the microwave regime. In this case, the frequency can be tuned by the sample length or by the applied voltage. 
3. Similar to the negative-differential resistance current oscillations in pure semiconductors, self-oscillating (photo-)currents were observed in superlattice structures, consisting of two different direct semiconductors such as GaAs-AlAs [76]. Due to the different energy levels in the quantum wells and the tunneling effect between the layers, a negative differential velocity regime is created as a function of the external electric field. The electric field reveals a spatial variation within the superlattice structure. Thereby, the different regions separated by domain walls get unstable and hence cause the oscillations. The resonance frequency can be tuned by the bias voltage or laser power $[77,78]$.

4. Current oscillations occur frequently in nonlinear transport studies in various organic conductors [38,45,79-83]. There, they are often related to sliding charge-density waves (CDW) or electrically induced insulator-metal transitions accompanied by a bistability $[60,79]$.

5. It is known that ferroelectric materials render a modification of the polarization by light stimulation, which can be detected by photoconductivity measurements [84]. Here, the detected signal is generally composed in of three different terms [85-87]: first, a fast decay of the excited states within a few microseconds, in addition a pyroelectric signal that can last several milliseconds, and finally piezoelectric oscillations superimposed on the decaying current due to the thickness variation of the sample by sound waves. Since the Fabre salts are charge-ordered ferroelectric crystals [50,88], they resemble the behavior of well-known inorganic ferroelectric, such as $\mathrm{LiNbO}_{3}$.

6. Above a certain threshold field, voltage oscillations (in the range of a few $100 \mathrm{mV} / \mathrm{cm}$ ) occur in CDW systems [50,60], such as $\mathrm{NbSe}_{3}, \mathrm{TaS}_{3}$ or $\mathrm{K}_{0.3} \mathrm{MoO}_{3}$, which are attributed to the collective sliding of the charge density wave. There, the resonance frequency depends on the applied voltage and temperature. However, only very few photoconductivity studies were conducted on CDW materials $[89,90]$. Experiments utilizing a lock-in technique do not directly provide the time-dependent behavior of the photocurrent. It was reported that the threshold voltage between the creeping and sliding state of the CDW can be raised by increasing the light intensity.

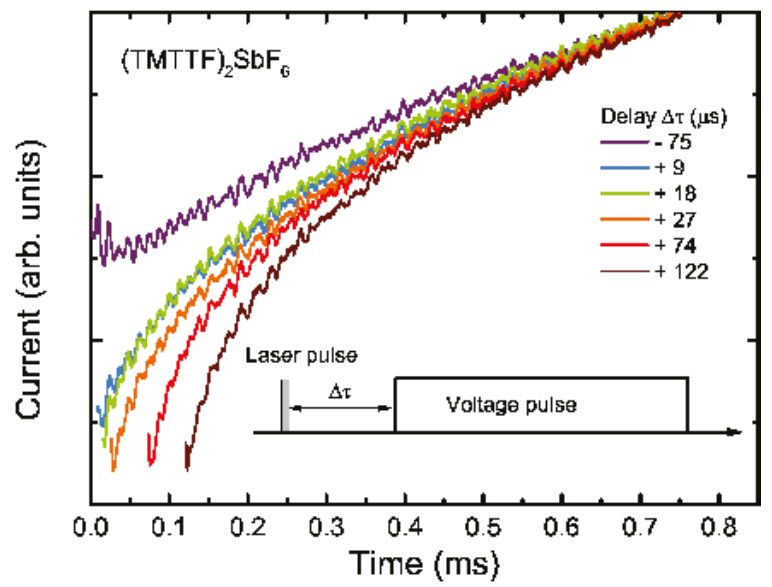

Figure 12. Raw data of the photocurrent signal measured for (TMTTF) ${ }_{2} \mathrm{SbF}_{6}$ for various delay times between the voltage and $8 \mathrm{~ns}$ laser pulse for a laser energy of $E=40 \mu \mathrm{J}$ and $U=10 \mathrm{~V}$ at $T=147 \mathrm{~K}$.

While the first four possibilities have already been disregarded as a possible origin of the current oscillations in our study, let us discuss the remaining suggestions. (TMTTF) ${ }_{2} \mathrm{SbF}_{6}$ can be considered as a one-dimensional charge-ordered ferroelectric, and the temporal dynamics of its photocurrent resembles that of illuminated inorganic ferroelectric crystals. For these, the current oscillations are related to the speed of sound in the bulk material. In our case a sample length of $l=2 \mathrm{~mm}$ and a resonance 
frequency of $77.5 \mathrm{kHz}$ would lead a sound velocity $v_{\text {sound }}=f \times l$ of $155 \mathrm{~m} / \mathrm{s}$. This is one order of magnitude below the speed of sound $\left(2500 \mathrm{~ms}^{-1}\right)$ previously reported [91-93]. Hence light-induced sound waves can be ruled out as origin of the observed oscillations.

The most appealing explanation of current oscillations in the charge-ordered state of (TMTTF $)_{2} \mathrm{SbF}_{6}$ starts with a $4 k$-CDW. In general a coherent sliding of the density wave is observed

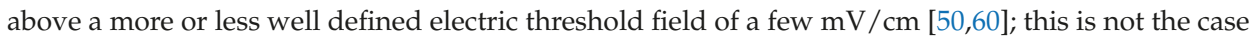
for (TMTTF $)_{2} \mathrm{SbF}_{6}$. Here we have to apply an electric field of several $\mathrm{V} / \mathrm{cm}$, much more than typically needed for classical CDW systems; but two orders of magnitude smaller than the threshold fields in the charge-ordered salt $\alpha$-(BEDT-TTF $)_{2} \mathrm{I}_{3}[22,28,29]$. The large difference can be explained by the fact that most of the typical CDW compounds develop an incommensurate charge modulation that is pinned to impurities. Charge-ordered systems are inherently commensurate and much more rigid as far as the coupling to the underlying lattice is concerned. Furthermore, single-particle excitations should not weaken the pinning of the CDW, it may even increase the threshold field. In conventional CDW systems, photo-excitation does not facilitate sliding of the condensate. In addition, it was observed in $\mathrm{NbSe}_{3}$ and comparable CDW systems that the resonance frequency of the depinned $4 k$-CDW depends on the external voltage; in the present case of (TMTTF $)_{2} \mathrm{SbF}_{6}$, however, no such effect could be identified. Further photoconductivity studies have to be performed on charge-ordered one-dimensional materials to clarify this point. Nevertheless, we suggest that our observations are caused by photo-induced fluctuations of the charge-ordered state, which alter the current flow of the single particles and hence, the photocurrent.

\section{Summary and Conclusions}

We report on the first study of photoconductivity and non-linear transport in quasi-one-dimensional charge-ordered compounds. The Fabre salt (TMTTF) ${ }_{2} \mathrm{SbF}_{6}$ exhibits unusual conduction behavior when electric fields and light pulses are applied; we could not detect similar phenomena in (TMTTF) ${ }_{2} \mathrm{PF}_{6}$ and $(\mathrm{TMTTF})_{2} \mathrm{AsF}_{6}$. Most interesting is the time-dependence of the photoconductivity signal which reveals current oscillations in the $\mathrm{kHz}$ range below the charge-order transition at $T_{\mathrm{CO}}=157 \mathrm{~K}$. Within milliseconds after photo-excitation the signal exhibits an overall decay, in addition a faster decay process can be identified in the microsecond range. The most surprising observation, however, are oscillations superimposed on top of the photocurrent that contains several frequencies in the $\mathrm{kHz}$ regime. We investigate the field, laser power and temperature dependence. We discuss possible explanations of the observed photoconductivity and current oscillations; suggesting an interaction of the excited single particles and the charge-ordered state.

Acknowledgments: We acknowledge valuable discussions with Tomislav Ivek, Andrea Rohwer and Eva Rose. We thank Gabriele Untereiner. for the crystal growth and sample preparation. The project was supported by the Deutsche Forschungsgemeinschaft (DFG) and by the Carl-Zeiss-Stiftung.

Author Contributions: T.P. designed and performed the experiments and analyzed the data; M.D. and T.P wrote the paper.

Conflicts of Interest: The authors declare no conflict of interest.

\section{Appendix A. Ab Initio Calculations}

For a better understanding of the photo-induced processes in the Fabre salts, we have performed calculations of the electronic band structure and optical conductivity.

\section{Appendix A.1. Band Structure}

The band structure of (TMTTF) $2 \mathrm{X}$ (with $\mathrm{X}=\mathrm{PF}_{6}, \mathrm{AsF}_{6}$, and $\mathrm{SbF}_{6}$ ) were determined by ab initio Density Functional Theory (DFT) calculations which is implemented in the software package Quantum Espresso [94]. In contrast to the well-established Hückel theory with its molecular orbitals, it is based upon plane-waves and it is not semi-empirical; instead, it uses the advantage of the crystal 
periodicity and can be applied to almost every material. Moreover, it is fast, reliable and robust. Furthermore, we used a norm-conserving PBE GGA functional for all atom types, up to a certain level taking into account the exchange correlation as well as the spatial variation of the charge density. The room-temperature crystal structure for the calculations were taken for $(\mathrm{TMTTF})_{2} \mathrm{PF}_{6}$ and (TMTTF $)_{2} \mathrm{AsF}_{6}$, while for (TMTTF $)_{2} \mathrm{SbF}_{6}$ we performed $\mathrm{X}$-ray scattering from $T=300 \mathrm{~K}$ down to $100 \mathrm{~K}[13,14,95]$; the structures were not relaxed within the calculations. Due to the stoichiometry of the organic salts, they are treated as metals. Thus, a smearing factor of $0.005 \mathrm{Ry}$ was applied. Furthermore, the cutoff energy of the plane waves and the electron density were set to $30 \mathrm{Ry}$ and $120 \mathrm{Ry}$, respectively. The wave functions were determined in a self-consistent way on a Monkhorst $8 k \times 8 k \times 4 k$ grid. The band structure was calculated on the $k$-path: $\Gamma(0,0,0) \rightarrow X(0.5,0,0) \rightarrow U(0.5,0.5,0) \rightarrow$ $\Gamma(0,0,0) \rightarrow Y(0,0.5,0) \rightarrow T(0,0.5,0.5) \rightarrow \Gamma(0,0,0) \rightarrow Z(0,0,0.5) \rightarrow R(0.5,0.5,0.5) \rightarrow \Gamma(0,0,0)$.

The resulting band structure close to the Fermi energy is depicted in Figure A1 for $T=300 \mathrm{~K}$. The HOMO of the Fabre-salts is three quarter-filled. Due to the dimerization between the TMTTF ${ }^{+0.5}$ molecules, the HOMO is split into a lower completely filled band and a half filled upper band. These bands are well separated from the other bands which are located at $-2 \mathrm{eV}$ below and about $1.5 \mathrm{eV}$ above the HOMO band (not shown here). The anion layer does not contribute to the bands near the Fermi energy due to the closed shell of the anions. The occupied and unoccupied bands of the anions reside far below and above the HOMO band.

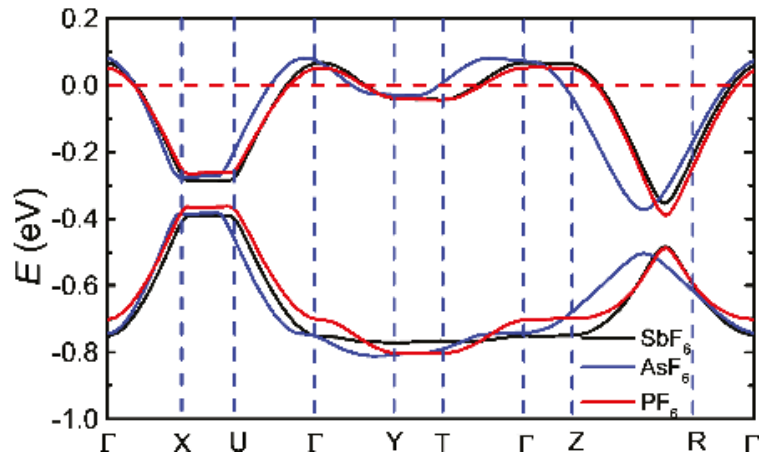

Figure A1. Comparison of the band structure of (TMTTF) $)_{2}, \mathrm{X}=\mathrm{PF}_{6}$ (red), $\mathrm{AsF}_{6}$ (blue), and $\mathrm{SbF}_{6}$ (black) at room temperature along the $k$-path: $\Gamma(0,0,0) \rightarrow X(0.5,0,0) \rightarrow U(0.5,0.5,0) \rightarrow \Gamma(0,0,0) \rightarrow$ $Y(0,0.5,0) \rightarrow T(0,0.5,0.5) \rightarrow \Gamma(0,0,0) \rightarrow Z(0,0,0.5) \rightarrow R(0.5,0.5,0.5) \rightarrow \Gamma(0,0,0)$.

The dimensionality of the compounds is reflected by the band dispersion. The band shape is similar for all compounds; the main deviations occur for the paths corresponding to the $b$-direction $\left(\Gamma \rightarrow Y\right.$ or $T \rightarrow \Gamma$ ). They reveal an enhanced dispersion going from the large $\mathrm{SbF}_{6}^{-}$to the smaller $\mathrm{PF}_{6}^{-}$ anions. This is consistent with the increase of the overlap between neighboring TMTTF molecules along the $b$-direction, being caused by the reduced spacing between the adjacent stacks due to the smaller anion size when going from (TMTTF) ${ }_{2} \mathrm{SbF}_{6}$ to the smaller (TMTTF $)_{2} \mathrm{PF}_{6}$. Therefore, the compounds become more two-dimensional. The largest dispersion appears for the paths: $\Gamma \rightarrow X$ which is along the stacking direction, $U \rightarrow \Gamma$ and $Z \rightarrow R$ containing contributions of the $a$-directions as well. The bandwidth for the three materials is about $350 \mathrm{meV}$ for the upper as well as for the lower band, being in excellent agreement with previous studies $[13,14]$. For the $b$-direction, the bands are weakly warped and intersect the Fermi energy. In contrast, there is no energy dispersion for the $c$-direction $(\Gamma \rightarrow Z)$, since the adjacent cation chains are separated from each other along the $c$-axis by the anion layer. The dimerization gap at the $\mathrm{X}$-point decreases from about $110 \mathrm{meV}$ for $\mathrm{AsF}_{6}$ and $\mathrm{SbF}_{6}$

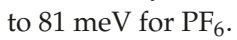


Moreover, also the temperature-dependence of the band structure was studied as visualized for $(\mathrm{TMTTF})_{2} \mathrm{SbF}_{6}$ over a temperature range between $300 \mathrm{~K}$ and $100 \mathrm{~K}$ in Figure A2. With decreasing temperature the bandwidth along the stacking direction $(\Gamma \rightarrow X)$ slightly decreases whereas it increases for the path $(\Gamma \rightarrow T)$ and $(T \rightarrow \Gamma)$. This implies that the compounds become more two-dimensional on cooling. This behavior is similar to applying chemical pressure, already discussed for Figure A1. This observation is supported by calculations of Jacko et al. [14] who also observed an increase of the dimensionality when the crystal structure of $(\mathrm{TMTTF})_{2} \mathrm{PF}_{6}$ under hydrostatic pressure was taken for the calculations. Concerning the dimerization, the corresponding gap at the X-point obviously diminishes from $110 \mathrm{meV}$ at $300 \mathrm{~K}$ to $55 \mathrm{meV}$ at $100 \mathrm{~K}$, again resembling the effect of pressure as demonstrated in Figure A1.

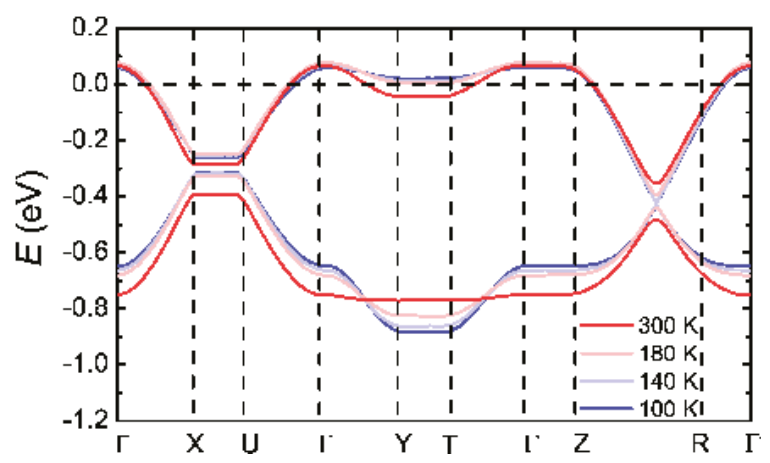

Figure A2. Band structure of (TMTTF) ${ }_{2} \mathrm{SbF}_{6}$ in a temperature range from $300 \mathrm{~K}$ (dark red) to $100 \mathrm{~K}$ (dark blue) on the selected $k$-path: $\Gamma(0,0,0) \rightarrow X(0.5,0,0) \rightarrow U(0.5,0.5,0) \rightarrow \Gamma(0,0,0) \rightarrow$ $Y(0,0.5,0) \rightarrow T(0,0.5,0.5) \rightarrow \Gamma(0,0,0) \rightarrow Z(0,0,0.5) \rightarrow R(0.5,0.5,0.5) \rightarrow \Gamma(0,0,0)$.

\section{Appendix A.2. Optical Spectra}

Since optical spectra of the Fabre salts have not yet been theoretically investigated to our best knowledge, we have performed DFT calculations to derive the optical constants for all three crystallographic axes. While the excitation spectra were calculated for an evenly spaced grid of $132 k$-points, the same functional, smearing factor, and cutoff energies were used as reported in the previous paragraph. The interband transitions were folded with a Lorentz function with a width of $100 \mathrm{meV}$, in contrast, for the Drude a width of $10 \mathrm{meV}$ was assumed.

Figure A3 displays the optical conductivity of (TMTTF $)_{2} \mathrm{SbF}_{6}$ in a frequency range between $1000 \mathrm{~cm}^{-1}$ and $30,000 \mathrm{~cm}^{-1}$ for temperatures between $300 \mathrm{~K}$ and $100 \mathrm{~K}$. Below $5000 \mathrm{~cm}^{-1}$, the $a$-direction is dominated by a Drude component (marked by I). Note, the deviations from the experimentally observed spectra (Figure 3b). can be explained by electronic correlations-driving the system insulating-and emv-coupled vibrational features, not taken in to account here. While the Drude term is less pronounced in the $b$-direction, the $c$-direction does not reveal any Drude component and drops to zero as $\omega \rightarrow 0$. The ratio of the optical dc resistivity is $\rho_{a}: \rho_{b}: \rho_{c}=1: 10: 6500$ which agrees well with the result of the transport measurements [10]. 


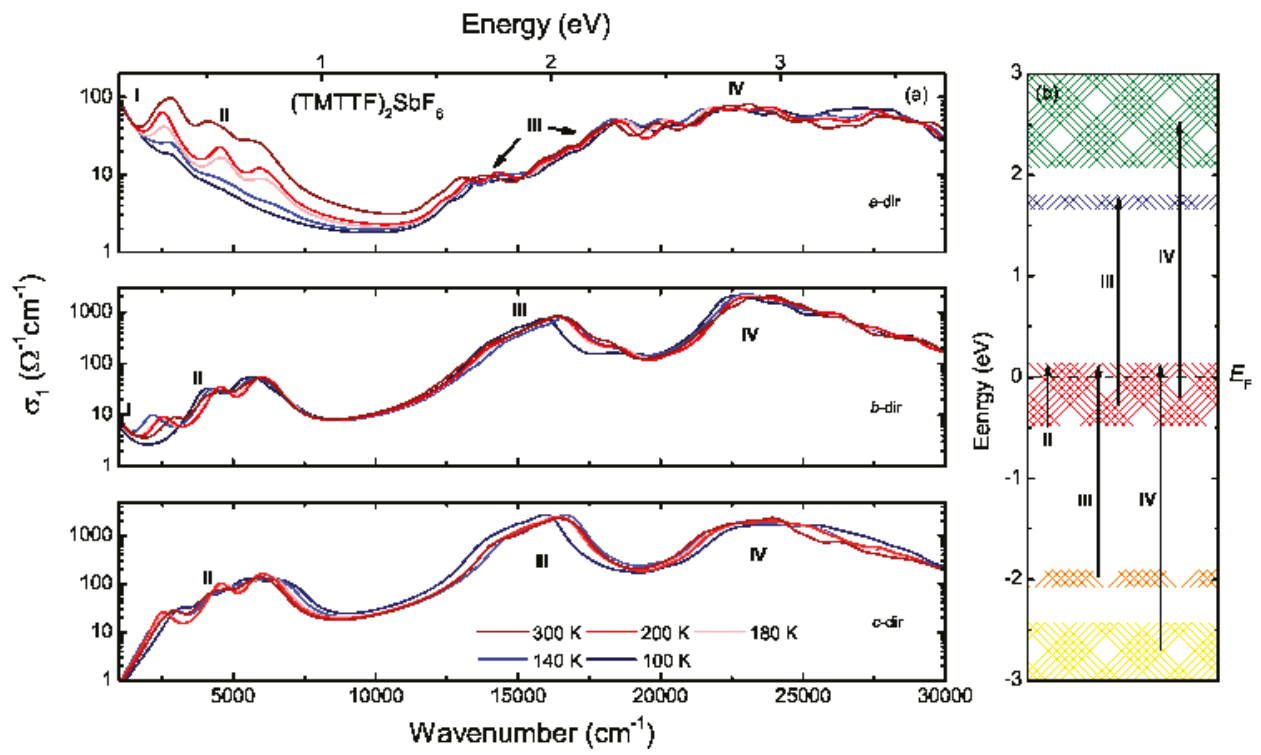

Figure A3. (a) Calculated optical conductivity of (TMTTF) ${ }_{2} \mathrm{SbF}_{6}$ between $T=300$ and $100 \mathrm{~K}$ in a frequency range from $1000 \mathrm{~cm}^{-1}$ to $30,000 \mathrm{~cm}^{-1}$ for the $a-, b$-, and $c$-direction; (b) Schematic diagram of the transitions between the occupied and unoccupied bands which are labeled by the corresponding markers from the optical conductivity spectra in (a).

On top of the Drude feature several resonances II are superimposed which can also be found in the $c$-direction. These are excitations from the completely occupied band near the Fermi energy $E_{F}$ into the empty states above $E_{F}$, being the strongest for the $b$ - and $c$-direction. Since no correlation effects are taken into account in the DFT calculations, the mid-infrared II band is not determined correctly. In the case of electron-electron correlations, the spectral weight of the Drude component is transferred to the mid-infrared band, leading to an enhancement of it. The weaker and broader interband transitions (III) occur for the $a-, b$-, and $c$-direction between $12,000 \mathrm{~cm}^{-1}$ and $20,000 \mathrm{~cm}^{-1}$. They can be attributed to the excitation from the half-occupied band to the lowest unoccupied band LUMO, which is located $1.7 \mathrm{eV}$ above $E_{F}$, as depicted in Figure A3b. Moreover, also the excitations from the fully occupied bands $-2 \mathrm{eV}$ below $E_{F}$ to the half-filled band contributes to this feature. The smeared feature IV resides above $20,000 \mathrm{~cm}^{-1}$ and can be assigned to the transition from the HOMO to the unoccupied bands located at $2 \mathrm{eV}$ in the band structure and from the occupied bands being located $-2.5 \mathrm{eV}$ below $E_{F}$ to the half-occupied band at $E_{F}$.

By comparing the intensity of the features III and IV for the various directions, it is evident that the bands in the stacking direction are by a factor of 10 less intense. This is because these transitions are caused by intramolecular electronic transitions of the cation molecule $\mathrm{TMTTF}^{+0.5}$, whose dipole moment is along the molecular axis. From the crystal structure it can be deduced that the long molecular axis of the TMTTF molecules is mainly oriented along the $c$-axis. For instance, the transition from the occupied band at the Fermi energy to the band at $1.7 \mathrm{eV}$ corresponds to the transition from the HOMO of the TMTTF ${ }^{+0.5}$ molecule in the LUMO. The excitation energy of this transition decreases with increasing molecule size.

Besides the overall shape of the spectra and the assignment of the features it is very interesting to track the temperature evolution of the spectra and compare it to the experimental measurements. The II band reveals the largest temperature dependence. In the $a$-direction, its intensity decreases on cooling and vanishes in the Drude component, while for the $b$ - and $c$-direction it only shifts 
to lower and to higher frequencies, respectively. In addition, also the III- and IV-bands exhibit a temperature-dependent modification. Therefore, also the electronic excitations into higher energy levels can be used to track thermal effects. This is also true for phase transitions which are accompanied by a strong transfer of spectral weight.

\section{References}

1. Ishiguro, T.; Yamaji, K.; Saito, G. Organic Superconductors, 2nd ed.; Springer: Berlin, Germany, 1998.

2. Lebed, A. (Ed.) The Physics of Organic Superconductors and Conductors; Springer Series in Materials Science; Springer: Berlin, Germany, 2008; Volume 110.

3. Dressel, M. Spin-charge separation in quasi one-dimensional organic conductors. Naturwissenschaften 2003, 90,337-344.

4. Dressel, M. Ordering phenomena in quasi-one-dimensional organic Conductors. Naturwissenschaften 2007, 94, 527-541.

5. Dumm, M.; Abaker, M.; Dressel, M. Mid-infrared response of charge-ordered quasi-1D organic conductors (TMTTF) $)_{2}$ X. J. Phys. IV 2005, 131, 55-58.

6. Knoblauch, T.; Dressel, $\mathrm{M}$. Charge disproportionation in (TMTTF $)_{2} X\left(X=\mathrm{PF}_{6}, \mathrm{AsF}_{6}\right.$ and $\left.\mathrm{SbF}_{6}\right)$ investigated by infrared spectroscopy. Phys. Status Solidi (C) 2012, 9, 1158-1160.

7. Dressel, M.; Dumm, M.; Knoblauch, T.; Masino, M. Comprehensive Optical Investigations of Charge Order in Organic Chain Compounds (TMTTF) ${ }_{2} X$. Crystals 2012, 2, 528-578.

8. Voloshenko, I.; Herter, M.; Beyer, R.; Pustogow, A.; Dressel, M. Pressure-dependent optical investigations of Fabre salts in the charge-ordered state. J. Phys. Condens. Matter 2017, 29, 115601.

9. Świetlik, R.; Barszcz, B.; Pustogow, A.; Dressel, M. Raman spectroscopy evidence of domain walls in the organic electronic ferroelectrics (TMTTF $)_{2} \mathrm{X}\left(\mathrm{X}=\mathrm{SbF}_{6}, \mathrm{AsF}_{6}, \mathrm{PF}_{6}\right)$. Phys. Rev. B 2017, 95, 085205.

10. Köhler, B.; Rose, E.; Dumm, M.; Untereiner, G.; Dressel, M. Comprehensive transport study of anisotropy and ordering phenomena in quasi-one-dimensional (TMTTF) $2 \mathrm{X}$ salts $\left(\mathrm{X}=\mathrm{PF}_{6}, \mathrm{AsF}_{6}, \mathrm{SbF}_{6}, \mathrm{BF}_{4}, \mathrm{ClO}_{4}, \mathrm{ReO}_{4}\right)$. Phys. Rev. B 2011, 84, 035124.

11. Pouget, J.P. Structural Aspects of the Bechgaard and Fabre Salts: An Update. Crystals 2012, 2, 466-520.

12. Pustogow, A.; Peterseim, T.; Kolatschek, S.; Engel, L.; Dressel, M. Electronic correlations versus lattice interactions: Interplay of charge and anion orders in (TMTTF) ${ }_{2}$ X. Phys. Rev. B 2016, 94, 195125.

13. Rose, E.; Loose, C.; Kortus, J.; Pashkin, A.; Kuntscher, C.A.; Ebbinghaus, S.G.; Hanfland, M.; Lissner, F.; Schleid, T.; Dressel, M. Pressure-dependent structural and electronic properties of quasi-one-dimensional (TMTTF $)_{2} \mathrm{PF}_{6}$. J. Phys. Condens. Matter 2013, 25, 014006.

14. Jacko, A.C.; Feldner, H.; Rose, E.; Lissner, F.; Dressel, M.; Valentí, R.; Jeschke, H.O. Electronic properties of Fabre charge-transfer salts under various temperature and pressure conditions. Phys. Rev. B 2013, 87, 155139.

15. Medjanik, K.; de Souza, M.; Kutnyakhov, D.; Gloskovskii, A.; Müller, J.; Lang, M.; Pouget, J.P.; Foury-Leylekian, P.; Moradpour, A.; Elmers, H.J.; et al. Hard X-ray photoemission study of the Fabre salts (TMTTF $)_{2} X\left(X=\mathrm{SbF}_{6}\right.$ and $\left.\mathrm{PF}_{6}\right)$. Eur. Phys. J. $B$ 2014, 87, 256.

16. Salameh, B.; Yasin, S.; Dumm, M.; Untereiner, G.; Montgomery, L.; Dressel, M. Spin dynamics of the organic linear chain compounds (TMTTF) ${ }_{2} \mathrm{X}\left(\mathrm{X}=\mathrm{SbF}_{6}, \mathrm{AsF}_{6}, \mathrm{BF}_{4}, \mathrm{ReO}_{4}\right.$, and $\left.\mathrm{SCN}\right)$. Phys. Rev. $B$ 2011, 83, 205126.

17. Yasin, S.; Salameh, B.; Rose, E.; Dumm, M.; von Nidda, H.A.K.; Loidl, A.; Ozerov, M.; Untereiner, G.; Montgomery, L.; Dressel, M. Broken magnetic symmetry due to charge-order ferroelectricity discovered in (TMTTF $)_{2} X$ salts by multifrequency ESR. Phys. Rev. B 2012, 85, 144428.

18. Dressel, M.; Dumm, M.; Knoblauch, T.; Köhler, B.; Salameh, B.; Yasin, S. Charge Order Breaks Magnetic Symmetry in Molecular Quantum Spin Chains. Adv. Condens. Matter Phys. 2012, 2012, 398721.

19. Matsunaga, N.; Hirose, S.; Shimohara, N.; Satoh, T.; Isome, T.; Yamomoto, M.; Liu, Y.; Kawamoto, A.; Nomura, K. Charge ordering and antiferromagnetism in (TMTTF) ${ }_{2} \mathrm{SbF}_{6}$. Phys. Rev. B 2013, 87, 144415.

20. Bertaina, S.; Dutoit, C.E.; Van Tol, J.; Dressel, M.; Barbara, B.; Stepanov, A. Rabi oscillations of pinned solitons in spin chains: A route to quantum computation and communication. Phys. Rev. B 2014, 90, 060404.

21. Dutoit, C.E.; Bertaina, S.; Orio, M.; Dressel, M.; Stepanov, A. Charge-ordering induces magnetic axes rotation in organic materials (TMTTF) ${ }_{2} \mathrm{X}$ (with $X=\mathrm{SbF}_{6}, \mathrm{AsF}_{6}$, and $\mathrm{PF}_{6}$ ). Low Temp. Phys. 2015, 41, 942-944.

22. Dressel, M.; Grüner, G.; Pouget, J.; Breining, A.; Schweitzer, D. Field and frequency dependent transport in the two-dimensional organic conductor $\alpha$-(BEDT-TTF) ${ }_{2} \mathrm{I}_{3}$. J. Phys. I 1994, 4, 579-594. 
23. Dressel, M.; Grüner, G.; Pouget, J.; Breining, A.; Schweitzer, D. Non-linear transport in $\alpha$-(BEDT-TTF) ${ }_{2} \mathrm{I}_{3}$. Synth. Met. 1995, 70, 929-930.

24. Ivek, T.; Korin-Hamzić, B.; Milat, O.; Tomić, S.; Clauss, C.; Drichko, N.; Schweitzer, D.; Dressel, M. Collective Excitations in the Charge-Ordered Phase of $\alpha$-(BEDT-TTF) ${ }_{2} \mathrm{I}_{3}$. Phys. Rev. Lett. 2010, 104, 206406.

25. Ivek, T.; Korin-Hamzić, B.; Milat, O.; Tomić, S.; Clauss, C.; Drichko, N.; Schweitzer, D.; Dressel, M. Electrodynamic response of the charge ordering phase: Dielectric and optical studies of $\alpha$-(BEDT-TTF $)_{2} \mathrm{I}_{3}$. Phys. Rev. B 2011, 83, 165128.

26. Ito, A.; Nakamura, Y.; Nakamura, A.; Kishida, H. Measurement of the Nonlinear Conducting States of $\alpha$-(BEDT-TTF $)_{2} \mathrm{I}_{3}$ Using Electronic Raman Scattering. Phys. Rev. Lett. 2013, 111, 197801.

27. Itose, F.; Kawamoto, T.; Mori, T. Collective response to alternating current in the organic conductor a-(bis(ethylenedithio)tetrathiafulvalene)2I3. J. Appl. Phys. 2013, 113, 213702.

28. Peterseim, T.; Ivek, T.; Schweitzer, D.; Dressel, M. Electrically induced phase transition in $\alpha-(B E D T-T T F){ }_{2} I_{3}$ : Indications for Dirac-like hot charge carriers. Phys. Rev. B 2016, 93, 245133.

29. Tajima, N.; Fujisawa, J.I.; Naka, N.; Ishihara, T.; Kato, R.; Nishio, Y.; Kajita, K. Photo-induced insulator metal transition in an organic conductor $\alpha$-(BEDT-TTF) ${ }_{2} \mathrm{I}_{3}$. J. Phys. Soc. Jpn. 2005, 74, 511-514.

30. Dearnaley, G.; Stoneham, A.M.; Morgan, D.V. Electrical phenomena in amorphous oxide films. Rep. Prog. Phys. 1970, 33, 1129-1191.

31. Mori, T.; Kawamoto, T.; Terasaki, I.; Kakiuchi, T.; Sawa, H. Nonlinear conductivity with an extremely small threshold electric field in the organic conductor (TSM-TTP) $\left(\mathrm{I}_{3}\right)_{5 / 3}$. Phys. Rev. B 2007, 75, 235103.

32. Sze, S.M., Ng, K.K. (Eds.)Physics of Semiconductor Devices, 3rd ed.; Wiley-Interscience: Hoboken, NJ, USA, 2007.

33. Yu, P., Cardona, M. (Eds.) Fundamentals of Semiconductors: Physics and Material Properties, 4th ed.; Graduate Texts in Physics; Springer: Berlin, Germany, 2010.

34. Potember, R.S.; Poehler, T.O.; Cowan, D.O. Electrical switching and memory phenomena in Cu-TCNQ thin films. Appl. Phys. Lett. 1979, 34, 405-407.

35. Tokura, Y.; Okamoto, H.; Koda, T.; Mitani, T.; Saito, G. Nonlinear electric transport and switching phenomenon in the mixed-stack charge-transfer crystal tetrathiafulvalene- $p$-chloranil. Phys. Rev. B 1988, 38, 2215-2218.

36. Kumai, R.; Okimoto, Y.; Tokura, Y. Current-induced insulator-metal transition and pattern formation in an organic charge-transfer complex. Science 1999, 284, 1645-1647.

37. Inagaki, K.; Terasaki, I.; Mori, H.; Mori, T. Large Dielectric Constant and Giant Nonlinear Conduction in the Organic Conductor $\theta$-(BEDT-TTF) $)_{2}$ CsZn(SCN) $)_{4}$. J. Phys. Soc. Jpn. 2004, 73, 3364-3369.

38. Sawano, F.; Terasaki, I.; Mori, H.; Mori, T.; Watanabe, M.; Ikeda, N.; Nogami, Y.; Noda, Y. An organic thyristor. Nature 2005, 437, 522-524.

39. Dumm, M.; Loidl, A.; Fravel, B.W.; Starkey, K.P.; Montgomery, L.K.; Dressel, M. Electron spin resonance studies on the organic linear-chain compounds (TMTCF $)_{2} X\left(C=\mathrm{S}, \mathrm{Se} ; X=\mathrm{PF}_{6}, \mathrm{AsF}_{6}, \mathrm{ClO}_{4}, \mathrm{Br}\right)$. Phys. Rev. B 2000, 61, 511-521.

40. Monceau, P.; Nad, F.Y.; Brazovskii, S. Ferroelectric Mott-Hubbard Phase of Organic (TMTTF) ${ }_{2} X$ Conductors. Phys. Rev. Lett. 2001, 86, 4080-4083.

41. Fujiyama, S.; Nakamura, T. Redistribution of Electronic Charges in Spin-Peierls State in (TMTTF) ${ }_{2} \mathrm{AsF}_{6}$ Observed by ${ }^{13}$ C NMR. J. Phys. Soc. Jpn. 2006, 75, 014705.

42. Oka, Y.; Matsunaga, N.; Nomura, K.; Kawamoto, A.; Yamamoto, K.; Yakushi, K. Charge Order in (TMTTF $)_{2} \mathrm{TaF}_{6}$ by Infrared Spectroscopy. J. Phys. Soc. Jpn. 2015, 84, 114709.

43. Pashkin, A.; Dressel, M.; Kuntscher, C.A. Pressure-Induced Deconfinement of the Charge Transport in the Quasi-One-Dimensional Mott Insulator (TMTTF)2AsF6. Phys. Rev. B 2006, 74, 165118

44. Pashkin, A.; Dressel, M.; Hanfland, M.; Kuntscher, C.A. Deconfinement transition and dimensional crossover in the Bechgaard-Fabre salts: Pressure- and temperature-dependent optical investigations. Phys. Rev. B 2010, $81,125109$.

45. Ivek, T.; Kovačević, I.; Pinterić, M.; Korin-Hamzić, B.; Tomić, S.; Knoblauch, T.; Schweitzer, D.; Dressel, M. Cooperative dynamics in charge-ordered state of $\alpha$-(BEDT-TTF $)_{2} \mathrm{I}_{3}$. Phys. Rev. B 2012, 86, 245125.

46. Joshi, N.V. Photoconductivity: Art, Science and Technology; Marcel Dekker: New York, NY, USA; Basel, Switzerland, 1990.

47. Peterseim, T.; Dressel, M.; Dietrich, M.; Polity, A. Optical properties of $\mathrm{VO}_{2}$ films at the phase transition: Influence of substrate and electronic correlations. J. Appl. Phys. 2016, 120, 075102. 
48. Peterseim, T.; Dressel, M. Molecular Dynamics at Electrical- and Optical-Driven Phase Transitions: Time-Resolved Infrared Studies Using Fourier-Transform Spectrometers. J. Infrared Millim. Terahertz Waves 2017, 38, 104-123.

49. De Souza, M.; Foury-Leylekian, P.; Moradpour, A.; Pouget, J.P.; Lang, M. Evidence for Lattice Effects at the Charge-Ordering Transition in (TMTTF $)_{2}$ X. Phys. Rev. Lett. 2008, 101, 216403.

50. Monceau, P. Electronic crystals: An experimental overview. Adv. Phys. 2012, 61, 325-581.

51. Jérome, D.; Schulz, H.J. Organic Conductors and Superconductors. Adv. Phys. 1982, 31, 299-490.

52. Kagoshima, S.; Nagasawa, H.; Sambongi, T. One-Dimensional Conductors; Springer: Berlin, Germany, 1988.

53. Farges, J.P. (Ed.) Organic Conductors; Marcel Dekker: New York, NY, USA, 1994.

54. Devreese, J.T., Evrand, R.P., von Doren, V.E. (Eds.) Highly Conducting One-Dimensional Solids; Plenum Press: New York, NY, USA; London, UK, 1979.

55. Tanaka, S., Uchinokura, K. (Eds.) Physics and Chemistry of Quasi One-Dimensional Conductors; Physica B; North-Holland: Amsterdam, The Ntherlands, 1986; Volume 143.

56. Jérome, D., Caron, L. (Eds.) Low-Dimensional Conductors and Superconductors; Nato ASI, Series B: Physics; Plenum Press: New York, NY, USA; London, UK, 1987; Volume 155.

57. Delhaes, P., Drillon, M. (Eds.) Organic and Inorganic Low-Dimensional Crystalline Materials; Nato ASI, Series B: Physics; Plenum Press: New York, NY, USA; London, UK, 1987; Volume 168.

58. Saito, G.; Kagoshima, S. (Eds.) The Physics and Chemistry of Organic Superconductors; Springer Proceedings in Physics; Springer: Berlin, Germany, 1990; Volume 51.

59. Bernier, P., Lafrant, S., Bidan, G. (Eds.) Advances in Synthetic Metals: Twenty Years of Progress in Science and Technology; Elsevier: Amsterdam, The Ntherlands, 1999.

60. Grüner, G. The dynamics of charge-density waves. Rev. Mod. Phys. 1988, 60, 1129-1181.

61. Dressel, M.; Drichko, N.; Kaiser, S. Collective charge-order excitations. Physica C 2010, 470, S589-S591.

62. Iimori, T.; Naito, T.; Ohta, N. Photoinduced Phase Transition in the Organic Conductor $\alpha$-(BEDT-TTF)2I3 at Temperatures near the Metal-Insulator Phase Transition. Chem. Lett. 2007, 36, 536.

63. Iimori, T.; Naito, T.; Ohta, N. Photoresponse of electrical conductivity in an organic superconductor investigated by time-resolved resistance measurements. Physica B 2010, 405, S347-S349.

64. Tsutsumi, J.; Yamada, T.; Matsui, H.; Haas, S.; Hasegawa, T. Competition between Charge-Transfer Exciton Dissociation and Direct Photocarrier Generation in Molecular Donor-Acceptor Compounds. Phys. Rev. Lett. 2010, 105, 226601.

65. Iimori, T.; Ohta, N. Tuning of Electrical Conductivity by Photoirradiation and Electric Fields. J. Phys. Chem. C 2014, 118, 7251-7260.

66. Iwasa, Y.; Koda, T.; Tokura, Y.; Koshihara, S.; Iwasawa, N.; Saito, G. Switching effect in organic charge transfer complex crystals. Appl. Phys. Lett. 1989, 55, 2111-2113.

67. Iwasa, Y.; Koda, T.; Koshihara, S.; Tokura, Y.; Iwasawa, N.; Saito, G. Intrinsic negative-resistance effect in mixed-stack charge-transfer crystals. Phys. Rev. B 1989, 39, 10441-10444.

68. Ozawa, T.; Tamura, K.; Bando, Y.; Kawamoto, T.; Mori, T.; Terasaki, I. Giant nonlinear conductivity in an organic conductor with a sharp metal-insulator transition: $\beta^{\prime \prime}-(\mathrm{BEDT}-\mathrm{TTF})_{3}\left(\mathrm{HSO}_{4}\right)_{2}$. Phys. Rev. B 2009, $80,155106$.

69. Mori, T.; Ozawa, T.; Bando, Y.; Kawamoto, T.; Niizeki, S.; Mori, H.; Terasaki, I. Nonlinear dynamics of conduction electrons in organic conductors. Phys. Rev. B 2009, 79, 115108.

70. Dressel, M.; Peterseim, T. Infrared Investigations of the Neutral-Ionic Phase Transition in TTF-CA and Its Dynamics. Crystals 2017, 7, 17.

71. Drichko, N.; Hackl, R.; Schlueter, J. Localized states in the Mott insulator $\kappa-(\mathrm{BEDT}-\mathrm{TTF})_{2} \mathrm{Cu}\left[\mathrm{N}(\mathrm{CN})_{2}\right] \mathrm{Cl}$ as probed by photoluminescence. Phys. Rev. B 2013, 88, 115109.

72. Nasu, K. (Ed.) Relaxations of Excited States and Photo-Induced Structural Phase Transitions. Springer Series in Solid State Science. In Proceedings of the 19th Taniguchi Sumposium, Kashikojima, Japan, 18-23 July 1996; Springer: Berlin, Germany, 1997; Volume 124.

73. Peterseim, T.; Haremski, P.; Dressel, M. Random-walk annihilation process of photo-induced neutral-ionic domain walls in TTF-CA. Europhys. Lett. 2015, 109, 67003.

74. Kassing, R.; Kähler, E. Low-frequency current oscillations in high-resistivity, Au-doped silicon junctions with two Schottky contacts. Phys. Status Solidi (A) 1972, 12, 209-213. 
75. Holonyak, N.; Bevacqua, S.F. Oscillations in semiconductors due to deep levels. Appl. Phys. Lett. 1963, 2, 71-73.

76. Kastrup, J.; Hey, R.; Ploog, K.H.; Grahn, H.T.; Bonilla, L.L.; Kindelan, M.; Moscoso, M.; Wacker, A.; Galán, J. Electrically tunable GHz oscillations in doped GaAs-AlAs superlattices. Phys. Rev. B 1997, 55, 2476-2488.

77. Hosoda, M.; Mimura, H.; Ohtani, N.; Tominaga, K.; Watanabe, T.; Fujiwara, K.; Grahn, H.T. Current self-oscillations in photoexcited type-II GaAs-AlAs superlattices. Appl. Phys. Lett. 1996, 69, 500-502.

78. Tomlinson, A.M.; Fox, A.M.; Cunningham, J.E.; Jan, W.Y. Photocurrent self-oscillations in a spatially direct GaAs/AlGaAs superlattice. Appl. Phys. Lett. 1999, 75, 2067-2069.

79. Mori, T.; Bando, Y.; Kawamoto, T.; Terasaki, I.; Takimiya, K.; Otsubo, T. Giant Nonlinear Conductivity and Spontaneous Current Oscillation in an Incommensurate Organic Superconductor. Phys. Rev. Lett. 2008, $100,037001$.

80. Tamura, K.; Ozawa, T.; Bando, Y.; Kawamoto, T.; Mori, T. Voltage oscillation associated with nonlinear conductivity in the organic conductor $\alpha$-(BEDT-TTF) ${ }_{2} \mathrm{I}_{3}$. J. Appl. Phys. 2010, 107, 103716.

81. Wakita, H.; Ozawa, T.; Bando, Y.; Mori, T. Nonlinear Conductivity in Dicyanoquinonediimine Complexes. J. Phys. Soc. Jpn. 2010, 79, 094703.

82. Kishida, H.; Ito, T.; Ito, A.; Nakamura, A. Room-Temperature Current Oscillation Based on Negative Differential Resistance in a One-Dimensional Organic Charge-Transfer Complex. Appl. Phys. Express 2011, 4, 031601.

83. Ivek, T.; Čulo, M.; Kuveždić, M.; Korin-Hamzić, B.; Tutiš, E.; Basletić, M.; Mihaljević, B.; Tafra, E.; Löhle, A.; Dressel, M.; et al. Semimetallic and charge-ordered $\alpha$-(BEDT-TTF $)_{2} \mathrm{I}_{3}$ : On the role of disorder in dc transport and dielectric properties. Phys. Rev. B 2017, 96, 123456.

84. Lines, M., Glass, A. (Eds.) Principles and Applications of Ferroelectrics and Related Materials; International Series of Monographs on Physics; Clarendon Press: Oxford, UK, 1977.

85. Glass, A.M.; Abrams, R.L. Study of Piezoelectric Oscillations in Wideband Pyroelectric $\mathrm{LiTaO}_{3}$ Detectors. J. Appl. Phys. 1970, 41, 4455-4459.

86. Glass, A.; Auston, D. Excited state dipole moments of impurities in polar crystals. Opt. Commun. 1972, 5, 45-49.

87. Glass, A.M.; Auston, D.H. Excited state dipole moments of impurities in pyroelectric crystals and their applications. Ferroelectrics 1974, 7, 187-189.

88. Tomic, S.; Dressel, M. Ferroelectricity in molecular solids: A review of electrodynamic properties. Rep. Prog. Phys. 2015, 78, 096501.

89. Ogawa, N.; Shiraga, A.; Kondo, R.; Kagoshima, S.; Miyano, K. Photocontrol of Dynamic Phase Transition in the Charge-Density Wave Material $\mathrm{K}_{0.3} \mathrm{MoO}_{3}$. Phys. Rev. Lett. 2001, 87, 256401.

90. Ogawa, N.; Miyano, K.; Brazovski, S. Optical excitation in the creep phase of plastic charge-density waves. Phys. Rev. B 2005, 71, 075118.

91. Tiedje, T.; Haering, R.; Jericho, M.; Roger, W.; Simpson, A. Temperature dependence of sound velocities in TTF-TCNQ. Solid State Commun. 1977, 23, 713-718.

92. Chaikin, P.; Tiedje, T.; Bloch, A. Sound velocity measurements on (TMTSF) ${ }_{2} \mathrm{PF}_{6}$. Solid State Commun. 1982, 41, 739-742.

93. Zherlitsyn, S.; Bruls, G.; Goltsev, A.; Alavi, B.; Dressel, M. Acoustical properties of (TMTSF) ${ }_{2} \mathrm{PF}_{6}$ in the spin-density-wave ground state. Phys. Rev. B 1999, 59, 13861-13871.

94. Giannozzi, P.; Baroni, S.; Bonini, N.; Calandra, M.; Car, R.; Cavazzoni, C.; Ceresoli, D.; Chiarotti, G.L.; Cococcioni, M.; Dabo, I.; et al. QUANTUM ESPRESSO: A modular and open-source software project for quantum simulations of materials. J. Phys. Condens. Matter 2009, 21, 395502.

95. Galigne, J.L.; Liautard, B.; Peytavin, S.; Brun, G.; Fabre, J.M.; Torreiles, E.; Giral, L. Etude Structurale Du Bromure de Tetramethyltetrathiofulvalene, (TMTTF) $)_{2}$ Br. Acta Crystallogr. B 1978, 34, 620-624.

(C) 2016 by the authors. Licensee MDPI, Basel, Switzerland. This article is an open access article distributed under the terms and conditions of the Creative Commons Attribution (CC BY) license (http:/ / creativecommons.org/licenses/by/4.0/). 
Article

\title{
Structural and Electronic Properties of (TMTTF $)_{2} X$ Salts with Tetrahedral Anions
}

\author{
Roland Rösslhuber ${ }^{\dagger}$, Eva Rose $^{\dagger}{ }^{\dagger}$, Tomislav Ivek ${ }^{\ddagger}$, Andrej Pustogow, Thomas Breier, \\ Michael Geiger, Karl Schrem, Gabriele Untereiner and Martin Dressel * \\ 1. Physikalisches Institut, Universität Stuttgart, Pfaffenwaldring 57, D-70569 Stuttgart, Germany; \\ roland.roesslhuber@pi1.physik.uni-stuttgart.de (R.R.); eva.rose@pi1.physik.uni-stuttgart.de (E.R.); \\ tivek@ifs.hr (T.I.); andrej.pustogow@pi1.physik.uni-stuttgart.de (A.P.); acs@pi1.physik.uni-stuttgart.de (T.B.); \\ m.geiger@fkf.mpg.de (M.G.); cienkowska-schmidt@pi1.physik.uni-stuttgart.de (K.S.); \\ gabriele.untereiner@pi1.physik.uni-stuttgart.de (G.U.) \\ * Correspondence: dressel@pi1.physik.uni-stuttgart.de; Tel.: +49-711-685-64946 \\ $\dagger$ These authors contributed equally to the work. \\ ‡ Permanent address: Institut za fiziku, Bijenička 46, HR-10000 Zagreb, Croatia. \\ Academic Editor: Helmut Cölfen \\ Received: 14 February 2018; Accepted: 28 February 2018; Published: 4 March 2018
}

\begin{abstract}
Comprehensive measurements of the pressure- and temperature-dependent dc-transport are combined with dielectric spectroscopy and structural considerations in order to elucidate the charge and anion orderings in the quasi-one-dimensional charge-transfer salts (TMTTF) ${ }_{2} \mathrm{X}$ with non-centrosymmetric anions $\mathrm{X}=\mathrm{BF}_{4}, \mathrm{ClO}_{4}$ and $\mathrm{ReO}_{4}$. Upon applying hydrostatic pressure, the charge-order transition is suppressed in all three compounds, whereas the influence on the anion order clearly depends on the particular compound. A review of the structural properties paves the way for understanding the effect of the anions in their methyl cavities on the ordering. By determining the complex dielectric constant $\hat{\varepsilon}(\omega, T)$ in different directions we obtain valuable information on the contribution of the anions to the dielectric properties. For (TMTTF) ${ }_{2} \mathrm{ClO}_{4}$ and (TMTTF) ${ }_{2} \mathrm{ReO}_{4}$, $\epsilon_{b^{\prime}}$ exhibits an activated behavior of the relaxation time with activation energies similar to the gap measured in transport, indicating that the relaxation dynamics are determined by free charge carriers.
\end{abstract}

Keywords: Fabre salts; one-dimensional conductors; charge order; anion order; electronic transport; pressure dependence; dielectric spectroscopy

PACS: 71.30.+h, 74.70.Kn, 75.25.Dk, 71.27.+a, 72.15.-v, 77.22.Gm

\section{Introduction}

Electronic correlations play a decisive role in the quasi-one-dimensional molecular charge-transfer salts (TMTTF $)_{2} X$, where TMTTF means tetramethyltetrathiafulvalene. As a result of the 2:1 stoichiometry with positively charged $(\mathrm{TMTTF})^{\rho_{0}}, \rho_{0}=0.5$, and monovalent anions $X^{-}$, the (TMTTF $)_{2} X$ salts should have a $3 / 4$-filled conduction band. The slight dimerization of the molecular stacks leads to a splitting into a completely filled lower and a half-filled upper band; accordingly, metallic properties are expected. This behavior is observed in the sibling TMTSF compounds, known as Bechgaard salts, here TMTSF stands for tetramethyltetraselenafulvalene. Due to the reduced dimension, Fermi liquid theory breaks down separating spin and charge degrees of freedom [1,2].

In the case of the Fabre-salts TMTTF, however, a minimum in resistivity $\rho(T)$ is observed at $T_{\rho} \approx 250 \mathrm{~K}$, followed by a strong increase at $T_{\mathrm{CO}}$ [3-5]. The first phase below $T_{\rho}$ is referred to as charge localization or dimer Mott insulator, whereas the charge-order $(\mathrm{CO})$ transition occurs at $T_{\mathrm{CO}}$, usually somewhere below $160 \mathrm{~K}$. Both states arise due to strong electronic correlations which are well described 
by the extended Hubbard model taking into account on-site $U$ and nearest-neighbor Coulomb interactions $V$ [6]. Intense research over the last decades revealed a colorful interplay of charge, spin and lattice degrees of freedom and established a generic phase diagram. The temperature-pressure diagram is shown in Figure 1 and includes (TMTTF) ${ }_{2} X$ and (TMTSF) $)_{2} X$ salts with centrosymmetric anions $\mathrm{X}=\mathrm{Br}, \mathrm{PF}_{6}, \mathrm{AsF}_{6}, \mathrm{SbF}_{6}, \mathrm{TaF}_{6}$, wherein the position can be tuned either by external pressure or by substituting the anions, causing chemical pressure. In the phase diagram Figure 1, the compounds with non-centrosymmetric anions, such as $\mathrm{SCN}, \mathrm{FSO}_{3}, \mathrm{NO}_{3}, \mathrm{BF}_{4}, \mathrm{ClO}_{4}$ and $\mathrm{ReO}_{4}$ are missing, as they exhibit ordering of the anions upon cooling.
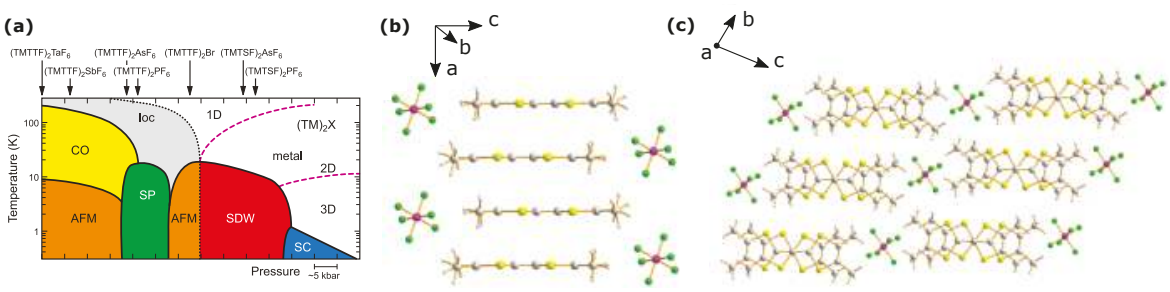

Figure 1. (a) The generic phase diagram of the tetramethyltetrathiafulvalene (TMTTF) and tetramethyltetraselenafulvalene (TMTSF) salts with centrosymmetric anions, as first suggested by Jérome [7] and further refined by other groups [2]. The position can be tuned either by external physical pressure or by chemical pressure via substituting the anion. The arrows indicate the ambient pressure position of the particular compounds with different anions. Decreasing the anion size corresponds to increasing pressure upon which the properties become more metallic and less one-dimensional. The full and dashed lines indicate phase transitions and crossovers, respectively. The various terms are shortened as follows: CO charge order, loc charge localization, SP spin-Peierls, AFM antiferromagnet, SDW spin density wave and SC superconductivity; (b) Crystal structure of (TMTTF) ${ }_{2} \mathrm{PF}_{6}$ [8] illustrating the stacking of the TMTTF molecules and the anions along the $a$-direction and, (c) in the $b c$-plane showing the separation of the TMTTF stacks by anions.

\section{Structural Considerations}

Before we report our experimental studies on the electronic properties of (TMTTF) $)_{2} \mathrm{x}$ and discuss the temperature and pressure development, let us summarize the structural aspects known for this class of materials. As the best studied example, the crystal structure of (TMTTF $)_{2} \mathrm{PF}_{6}$ under ambient conditions [8] is exemplarily shown in Figure $1 \mathrm{~b}$ and $\mathrm{c}$ for the (TMTTF) ${ }_{2} \mathrm{X}$ salts. Commonly they exhibit a triclinic space group P $\overline{1}$ with inversion centers located in between the TMTTF molecules and on the anions [9-14]. Most importantly, the planar TMTTF molecules are arranged in zig-zag stacks along the $a$-direction (Figure $1 \mathrm{~b}$ ) giving rise to the quasi-one-dimensional physics in the Fabre salts [3]. The anions separate the TMTTF stacks along the $b+c$ direction (Figure 1c) and mediate the inter-stack coupling. Moreover, due to the presence of the anions, a slight dimerization is introduced on the TMTTF stacks which decreases upon cooling [15]. This already indicates that the counter ions play a more complex role then just acting as spacers between the TMTTF stacks and that their symmetry affects the electronic properties [4,16-18].

The anions are located in cavities, which are constituted by the methyl end groups of the six nearest neighbor TMTTF molecules [19]. Depending on their size, symmetry, and polarizability, the anions and the methyl groups are slightly deformed, leading to a more or less snug fit in the cavities $[14,16,17,20,21]$. For that reason, the anions possess some limited rotational and translational degrees of freedom that are activated at higher temperatures [22,23]. The methyl groups basically form a centrosymmetric environment with symmetry axes close to the ones of the octahedral anions. This implies that for non-centrosymmetric entities, i.e., linear, triangular, tetrahedral anions, orientational disorder becomes an issue $[17,24]$. 
At lower temperatures-when thermal disorder is reduced—weak hydrogen-bonds are formed between the ligands of the counter ions and the closest methyl groups [25]. Moreover, there is a link between the S-atom of the nearest TMTTF molecule and the anion ligand O or F-atom [26], evidenced by an anion dependence of the sulfur to anion-ligand distance $d_{\mathrm{AL}-\mathrm{S}}[4,18]$ (Table 1 ). It is important to emphasizes, that via the methyl groups any structural or charge modification in the TMTTF molecules influences the position of the anions and vice versa [17].

At elevated temperatures, the symmetry of the anions does not play a crucial role, for these entities are subject to rotation and disorder. Upon cooling, however, the motion is hindered and the anions get locked in certain positions, where the symmetry of the anions becomes an important aspect. The alternating orientation of the tetrahedra along and perpendicular to the stacks leads to a doubling of the lattice periodicity; hence the anion order is a structural transition with wave vector $q_{\mathrm{AO}}=\left(\frac{1}{2}, \frac{1}{2}, \frac{1}{2}\right)$. X-ray absorption near edge structure (XANES or NEXAFS) [27] and infrared optical spectroscopy [23] measurements unanimously identify a tetramerization of the TMTTF stacks at the $\mathrm{AO}$ transition $T_{\mathrm{AO}}$, the latter also providing evidence for a 0110 charge pattern. A scheme of the corresponding modification of the structure and the charge pattern is shown in Figure 2e.
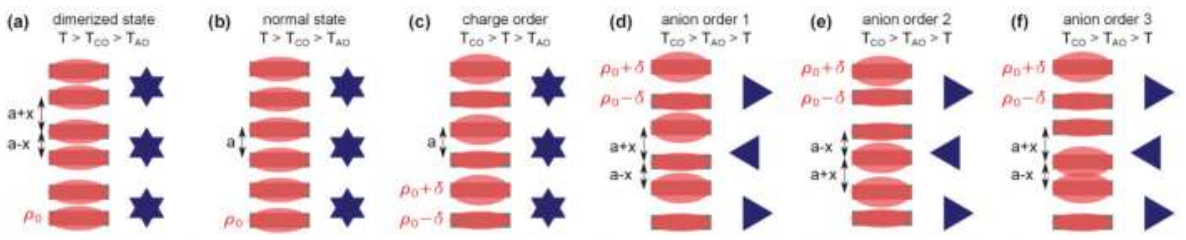

Figure 2. Sketch of the molecular and charge arrangements for different states of (TMTTF) ${ }_{2} X$ salts. The organic molecules are depicted as rectangles; the amount of charge $\rho_{0}, \rho_{0} \pm \delta$ is illustrated by red ellipses of different size. The anions are shown as blue hexagons or triangles, depending on the centrosymmetry. (a) The TMTTF stacks are dimerized at room temperature, $a \pm x$. The degree of dimerization decreases upon cooling, thus equalizing the TMTTF sites towards the uniform state in (b). Panel (c) illustrates how charge disproportionation develops as the charge-ordered state is entered with a 1010 charge pattern along the stacking direction. A priori, three different charge configurations are possible in the anion-ordered phase: $(\mathbf{d})$ one with a 1010 pattern and $(\mathbf{e}, \mathbf{f})$ two with a 1100 pattern. Our optical studies [23] have revealed that the tetramerized state (e) forms due to the anion order, with an intra-dimer molecular distance $a-x$, and the dimers separated by $a+x^{\prime}$ and $a+x^{\prime \prime}$.

In this respect, charge order as well as anion order in the (TMTTF $)_{2} X$ salts still bear some mystery. Among the remaining questions are (i) about the exact mechanism for $\mathrm{CO}$ involving the anions, hence (ii) about the nature of interplay, if there is any, between $\mathrm{CO}$ and $\mathrm{AO}$ and (iii) about the role of polarizability of the anions for $\mathrm{CO}$ and $\mathrm{AO}$.

\subsection{Charge Order}

Charge order is caused by redistribution of electronic charge within the TMTTF stack resulting in an alternating arrangement of differently charged molecules (TMTTF) ${ }^{+0.5+\delta}$ and (TMTTF) ${ }^{+0.5-\delta}$ along the $a$-direction. This charge disproportionation is observed in all TMTTF salts considered here, except (TMTTF) ${ }_{2} \mathrm{ClO}_{4}$. Charge order lifts the inversion symmetry and conceptually doubles the unit cell. Halving the Brillouin zone leads to the opening of a gap in the electronic density of state [3]. The latter is observed as a strong increase of resistivity at $T_{\mathrm{CO}}[4,5]$ whereas the charge disproportionation is evidenced by nuclear magnetic resonance (NMR) [28,29], dielectric [30-36] and optical spectroscopy $[23,37,38]$, as well as by synchrotron X-ray diffraction measurements [39]; the latter results evidence a 1010 charge pattern. The electrical transition proceeds continuously, wherein the charge disproportionation and the energy gap exhibit a temperature dependence that resembles 
the mean-field behavior of a second-order phase transition [4,23]. An overview of the transition temperatures and structural parameters determined at room temperature is given in Table 1.

Figure 2 illustrates the molecular and charge arrangements for the different states realized in the $(\mathrm{TMTTF})_{2} \mathrm{X}$ salts. Panel a corresponds to the state at high temperatures, which exhibits a finite dimerization of the organic molecules. Upon cooling, the dimerization diminishes [15], approaching the arrangement shown in panel $\mathrm{b}$. The charge disproportionation starts to develop upon entering the charge-ordered state with a 1010 pattern along the stacking direction as sketched in panel c.

\subsection{Anion Contribution to Charge Order}

From a general point of view, the (TMTTF $)_{2} X$ salts are stabilized by the charge transfer of half an electron from the TMTTF molecules to the anions. Therefore it seems reasonable that charge redistribution at the $\mathrm{CO}$ transition introduces structural modifications [17]. Nevertheless, the associated alternation in the crystal structure could not be resolved for a long time [15,40], and as a result, the charge order transition was first considered as a "structureless transition" of unknown origin [41]. Only recent ${ }^{19}$ F-NMR studies [42], measurements of the thermal expansion [43,44], neutron scattering [45] and optical spectroscopy $[38,46,47]$ provided the necessary evidence that $\mathrm{CO}$ is accompanied by lattice effects, attributed to a collective shift of the anions [17].

Pouget proposed [17] two different directions for the anion shift. First, the anion moves along the $c$-direction towards the methyl groups, as indicated by black arrows in Figure $3 \mathrm{a}, \mathrm{b}$. The resulting deformation of the methyl end-groups polarizes the hydrogen-bond network leading to a displacement of charge in the $\sigma$-bonds connected to the H-bonds. This indirectly stabilizes the excess of $\pi$-holes on the corresponding TMTTF molecule as indicated by a red ellipsoid, whereas blue ellipsoids mark charge-poor molecules (Figure 3).
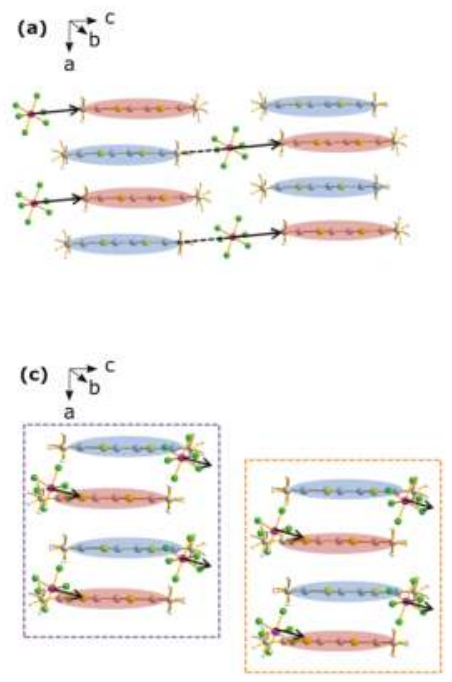

(b)

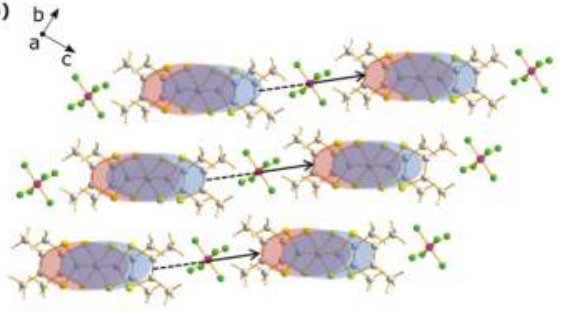

(d)

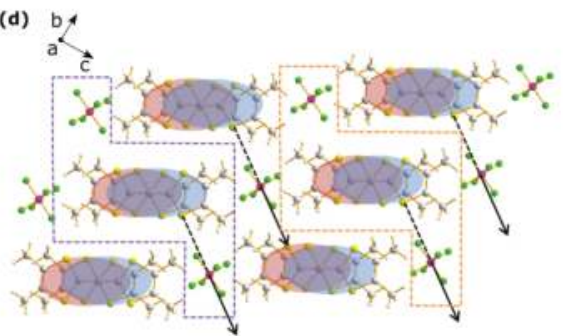

Figure 3. The anions shift along the $b+c$ direction towards the methyl groups and towards the sulfur atoms, as suggested by Pouget [17]. Charge-rich TMTTF molecules are indicated by a red ellipsoids whereas blue ellipsoids mark charge-poor molecules. (a) View in the $a c$-plane and (b) along the $a$-axis onto the $c b$-plane. The shift of the anions towards the S-atoms of the nearest TMTTF molecule depicted (c) in the $a c$-plane and (d) in the $c b$-plane. The polygons formed by the purple and orange dashed lines in panel $\mathrm{d}$ indicate which anion stacks are shown in the previous frame c. 
Several observations support this suggestion of an anion shift with a strong contribution along the $c$-direction. First of all, thermal expansion measurements exhibit a clear anomaly at $T_{\mathrm{CO}}$, which is most pronounced along the $c$-axis [43]. Secondly, the coupling between the methyl groups and the anions is mostly in the $b c$-plane. Furthermore, deuteration of the methyl groups significantly increases $T_{\mathrm{CO}}$. The heavier mass slows the lattice dynamics and weakens the coupling between the anions and terminating endgroups (Figure 4); thus CO can be stabilized already at higher temperatures [23].

The second proposed translation of the anions [17] is towards the closest sulfur atom of a TMTTF molecule, indicated by black arrows in Figure $3 c$,d. This goes hand in hand with the enhanced $\pi$-hole density at the corresponding TMTTF molecule. NEXAFS measurements clearly show the strengthening of the S-F bonds [48].

Both shifts are supposed to increase the charge concentration on the corresponding TMTTF molecule. It is energetically favorable that the second neighboring TMTTF molecule contains less positive charge. Hence, by minimizing the electrostatic energy between the anion stacks and adjacent TMTTF stacks, a three-dimensional charge pattern is established that is crucial for stabilizing $\mathrm{CO}[23,43]$. In fact, temperature-dependent transport measurements [4] clearly show the opening of a $\mathrm{CO}$ gap along all three crystallographic directions providing evidence for this three-dimensional charge pattern due to inter-stack coupling mediated by the anions.

Some further considerations have been published, which should be mentioned here. First, the anion shift accompanying $\mathrm{CO}$ does not introduce the tetramerization [23]. Second, although the anions do shift at the CO transitions, they are still considered to exhibit a random rotational motion depending on the temperature [17].
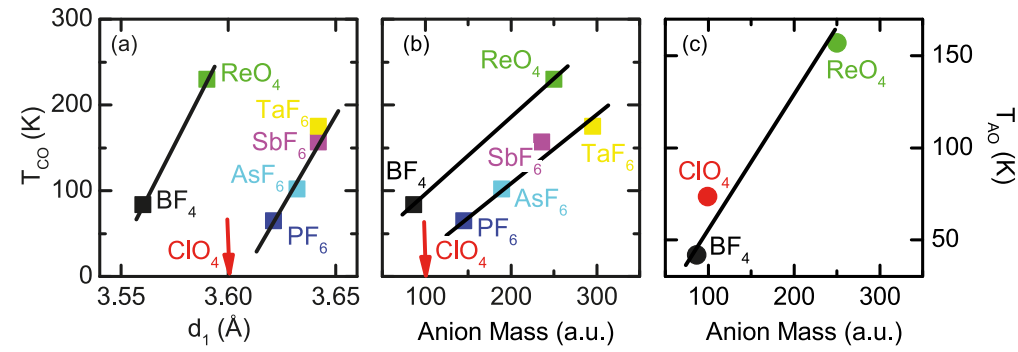

Figure 4. Dependence of the charge order temperature $T_{\mathrm{CO}}$ (colored squares) and anion order temperature $T_{\mathrm{AO}}$ (colored circles) for various Fabre salts (TMTTF) $)_{2} X$ with different $X$ as indicated. (a) $T_{\mathrm{CO}}$ plotted versus the distance between the TMTTF molecules $d_{1}$ along stacking direction determined at room temperature. Here, octahedral and tetrahedral salts fall on two different lines. Although the octahedral anions exhibit larger values for $d_{1}, T_{\mathrm{CO}}$ is lower compared to the tetrahedral salts. The octahedral anions are larger (Table 1) resulting in an increased value of $d_{1}$. The anion-TMTTF stack coupling increases due to the larger anions, and the match between anion and cavity symmetry; thus thermal fluctuations prevail upon the electronic correlations giving rise to $\mathrm{CO}$ down to lower temperatures. (b) Plot of $T_{\mathrm{CO}}$ versus the mass of the anions. A monotonic increase of $T_{\mathrm{CO}}$ with anion mass is observed, which is attributed to less effective motion of heavy anions due to thermal fluctuations and, consequently, a less disordered anion potential influencing electronic correlations within the TMTTF stacks. The octahedral and tetrahedral salts fall on two distinct lines due to different coupling between the anions and the TMTTF stacks. (c) For the anion ordering temperature observed in the tetrahedal (TMTTF) ${ }_{2} X$ salts, an increase of $T_{\mathrm{AO}}$ with the anion mass is observed. At a specific temperature, heavy anions are less affected by thermal fluctuations. Consequently, the anion orientation is locked permanently for heavier anions at higher temperatures. As a result, anion order occurs at higher temperatures for heavy anions.

Having established the anion shift, we now have to pose the question whether charge order can still be understood as a phenomenon inherent to the one-dimensional TMTTF stacks as described by 
the extended Hubbard model, or whether a more advanced theoretical treatment is required including the contribution of the lattice in general and the anions in particular. In other words, we have to find out whether the Coulomb attraction between the negative charge located on the anion-ligand with shortest S-F contact is crucial for charge order, or whether it only stabilizes the arrangement. It has been proposed, that the shortest anion-ligand to sulfur distance $d_{\mathrm{AL}-\mathrm{S}}$ is the parameter determining CO $[4,18]$, in contrast to a purely electronic picture, which implies that the distances between the TMTTF molecules, $d_{1}$ and $d_{2}$, are decisive. Recent spectroscopic and structural investigations could shed light on this issue $[18,23]$. In the following we give a short overview on the different approaches and on the studies focusing on the structural aspects of the $\mathrm{CO}$ transition.

Table 1. Overview on the transition temperatures $T_{\mathrm{CO}}$ and $T_{\mathrm{AO}}$, as well as on the room-temperature structural data of various (TMTTF) ${ }_{2} X$ salts with octahedral and tetrahedral anions $X$. Here $d_{\mathrm{AL}-\mathrm{S}}$ is the shortest distance between the anion-ligand ( $F$ or $\mathrm{O}$ ) and the sulfur atom. $d_{1}$ and $d_{2}$ denote the distances between planes defined by the TMTTF molecules within one stack $[9,11-13,18]$. Hence, $\left(d_{1}-d_{2}\right) /\left(d_{1}+d_{2}\right)$ denotes the structural dimerization along the stacks determined by the distance between the TMTTF molecular planes; it does not take into account any displacement of the molecules along the $b$ or $c$ direction. In contrast to that, $\delta_{\text {struc }}$ and $\delta_{\text {elec }}$ are determined by ab-initio DFT calculations based on X-ray structural data $[49,50]$. $\delta_{\text {struc }}$ corresponds to a structural dimerization as well, which is defined by the distance between the centers of mass in each TMTTF molecule. $\delta_{\text {elec }}=2\left|t_{1}-t_{2}\right| /\left(t_{1}+t_{2}\right)$ explicitly takes into account the overlap of the transfer integrals $t_{1}$ and $t_{2}$. The unit cell volume is listed as $V_{\text {unitcell }}[9,11-13]$. The parameter $R_{0}$ denotes the thermochemical radius of the monovalent anion [51]; although calculated for a salt with 1:1 stoichiometry, we consider this as a valid estimate for the anion size. The anion volume is listed as $V_{\text {anions }}$ following [52]. In the last row the anion mass $m_{A}$ is given in atomic units.

\begin{tabular}{|c|c|c|c|c|c|c|c|}
\hline Anion $X$ & $\mathrm{PF}_{6}$ & $\mathrm{AsF}_{6}$ & $\mathrm{SbF}_{6}$ & $\mathrm{TaF}_{6}$ & $\mathrm{BF}_{4}$ & $\mathrm{ClO}_{4}$ & $\mathrm{ReO}_{4}$ \\
\hline$T_{\mathrm{CO}}(\mathrm{K})$ & 67 & 102 & 157 & 175 & 84 & - & 230 \\
\hline$T_{\mathrm{AO}}(\mathrm{K})$ & - & - & - & - & 41.5 & 73.4 & 157 \\
\hline$d_{\mathrm{AL}-\mathrm{S}}(\AA)$ & 3.30 & 3.27 & 3.21 & 3.215 & 3.28 & 3.45 & 3.05 \\
\hline$d_{1}(\AA)$ & 3.621 & 3.632 & 3.642 & 3.642 & 3.56 & 3.60 & 3.59 \\
\hline$d_{2}(\AA)$ & 3.527 & 3.524 & 3.526 & 3.534 & 3.54 & 3.51 & 3.57 \\
\hline$\frac{d_{1}-\bar{d}_{2}}{d_{1}+d_{2}}\left(10^{-2}\right)$ & 1.32 & 1.51 & 1.62 & 1.51 & 0.28 & 1.27 & 0.28 \\
\hline$\delta_{\text {struc }}$ & 0.040 & 0.041 & 0.041 & - & 0.028 & 0.04 & - \\
\hline$\delta_{\text {elec }}$ & 0.230 & 0.110 & 0.298 & - & 0.336 & 0.616 & - \\
\hline$V_{\text {unitcell }}\left(\AA^{3}\right)$ & 676.6 & 697.7 & 702.9 & 706.52 & 648.5 & 654.8 & 679.5 \\
\hline$R_{0}(\mathrm{pm})$ & 242 & 243 & 252 & 250 & 205 & 225 & 227 \\
\hline$V_{\text {anions }}\left(\AA^{3}\right)$ & 70.6 & - & 81.8 & - & 51.6 & 54.7 & 64.8 \\
\hline$m_{A}(\mathrm{au})$ & 144.96 & 188.91 & 235.75 & 294.94 & 86.80 & 99.45 & 250.21 \\
\hline
\end{tabular}

In Table 1 we summarize electronic and structural parameters determined from literature data. For the octahedral anions, $T_{\mathrm{CO}}$ rises with anion size (and mass) $d\left(P F_{6}\right)<d\left(A s F_{6}\right)<d\left(S_{b} F_{6}\right),<d\left(\mathrm{TaF}_{6}\right)$; the same is found for the salts with tetrahedral anions. Moreover, a larger anion diameter correlates with a shorter sulfur-anion-ligand distance $d_{\mathrm{AL}-\mathrm{S}}$ and larger intra-stack distances between the TMTTF molecules $d_{1}$ and $d_{2}$. Regarding the structural dimerization $\frac{d_{1}-d_{2}}{d_{1}+d_{2}}$ and $T_{\mathrm{CO}}$, no clear trend can be seen $[18,49,50]$. So far, $T_{\mathrm{CO}}$ scales with both, $d_{\mathrm{AL}-\mathrm{S}}$ and $d_{1}$, impeding a simple answer of the question posed above.

In Figure 5a the charge disproportionation $2 \delta$ is plotted as a function of the $\mathrm{CO}$ temperature $T_{\mathrm{CO}}$. Interestingly, both the salts with tetrahedral and with octahedral anions fall onto the very same line, providing strong indications that $\mathrm{CO}$ is independent on anion symmetry and emerges from the electronic interaction and one-dimensional physics inherent to the TMTTF stacks [23]. This conclusion complies with the extended Hubbard model and theories based on it; where structural peculiarities are 
disregarded [6]. Along these lines the competition between the nearest-neighbor Coulomb repulsion $V$ and the bandwidth $W=4 t$ is crucial for $\mathrm{CO}$, wherein the transfer integral $t$ sensitively depends on the distance between TMTTF molecules.

(a)

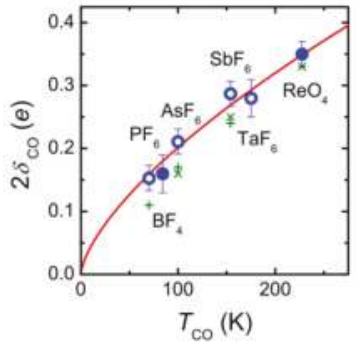

(b)

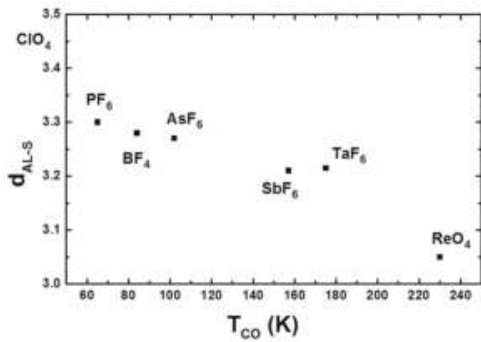

(c)

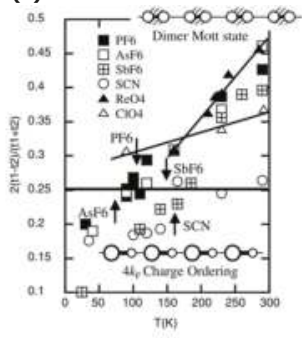

Figure 5. (a) Charge disproportionation $2 \delta$ of the (TMTTF) $)_{2} X$ salts with different anions $X$ plotted versus the $\mathrm{CO}$ transition temperature $T_{\mathrm{CO}}$. The open circles [38,53] and closed circles [23] were determined by optical spectroscopy on the octahedral anions $\mathrm{PF}_{6}, \mathrm{AsF}_{6}, \mathrm{SbF}_{6}, \mathrm{TaF}_{6}$, and tetrahedral anions $\mathrm{BF}_{4}$ and $\mathrm{ReO}_{4}$, respectively; the green crosses and green plus signs were measured by NMR $[37,54,55]$ and Raman spectroscopy $[38,56]$, respectively. The red line corresponds to $2 \delta \propto T_{\mathrm{CO}}^{2 / 3}$. Most important, all salts fall onto this line independent on the anion symmetry. (b) The shortest distance $d_{\mathrm{AL}-\mathrm{S}}$ between the sulfur atom of the organic molecule and the anion-ligand is evaluated at room temperature and plotted in dependence on the charge-order transition temperature $T_{\mathrm{CO}}$. All salts exhibiting $\mathrm{CO}$ fall on a line, indicating a linear decrease of $T_{\mathrm{CO}}$ as the spacing $d_{\mathrm{AL}-\mathrm{S}}$ increases. (c) Temperature dependence of the bond dimerization $\frac{2\left|t_{1}-t_{2}\right|}{\left(t_{1}+t_{2}\right)}$ taken from Reference [15], wherein the transfer integrals $t_{2}$ and $t_{1}$ were obtained by applying the extended Hückel model on temperature dependent $\mathrm{X}$-ray data.

To countercheck, in Figure $5 \mathrm{~b}$ the distance $d_{\mathrm{AL}-\mathrm{S}}$ is plotted versus $T_{\mathrm{CO}}$. For all salts we find a linear relation of falling transition temperature and increasing distance, independent on the symmetry of the anions. Note, the values given in Table 1 and plotted in Figure $5 \mathrm{~b}$ are determined at ambient conditions; they change upon cooling and the application of pressure.

Figure $5 c$ presents the temperature dependence of the electronic bond dimerization $\delta_{\text {elec }}=\frac{2\left|t_{1}-t_{2}\right|}{\left(t_{1}+t_{2}\right)}$, which is proportional to the electronic dimerization $\delta_{\text {elec }}$, considering that the transfer integrals $t_{2}$ and $t_{1}$ are calculated by the extended Hückel model on temperature dependent $X$-ray data [15]. Most importantly, the dimerization decreases with lowering the temperature, fully consistent with the findings of Reference [50]. It is concluded that $\mathrm{CO}$ sets in below a critical bond dimerization of 0.25 . This picture explains the absence of $\mathrm{CO}$ in $(\mathrm{TMTTF})_{2} \mathrm{ClO}_{4}$, however, (TMTTF $)_{2} \mathrm{BF}_{4}$ is not included. There also remains a discrepancy between the observed value of about 0.37 for (TMTTF) ${ }_{2} \mathrm{ReO}_{4}$ with $T_{\mathrm{CO}}=230 \mathrm{~K}$.

In a more advanced approach, the influence of structural effects on charge ordering in the (TMTTF $)_{2} X$ salts was investigated by ab-initio DFT calculations based on pressure- and temperature-dependent $X$-ray data $[49,50]$. No systematic relation was observed as far as $T_{\mathrm{CO}}$ and the structural dimerization is concerned, i.e., the distance between the TMTTF molecules. For the electronic dimerization $\delta_{\text {elec }}$, however, they find an increased value for (TMTTF) ${ }_{2} \mathrm{ClO}_{4}$ compared to the other salts, that explains the absence of $\mathrm{CO}$ in this particular salt. Besides the distance of the molecular planes, $\delta_{\text {elec }}$ also takes into account the differences in the orientation and size of the transfer integrals. When the electronic dimerization increases, $\mathrm{CO}$ is suppressed and can even be absent as in the case of $(\mathrm{TMTTF})_{2} \mathrm{ClO}_{4}$. 
We can conclude this short overview by emphasizing the importance of ab-initio DFT calculations based on detailed temperature-dependent X-ray data for all (TMTTF) ${ }_{2} X$ salts. This way we can unambiguously determine which role lattice effects play in general and the anions in particular on the charge-order transition.

\subsection{Anion Order}

In addition to charge order, the (TMTTF) ${ }_{2} X$ salts with non-centrosymmetric anions $X=\mathrm{SCN}$, $\mathrm{SFO}_{3}, \mathrm{NO}_{3}, \mathrm{BF}_{4}, \mathrm{ClO}_{4}$, and $\mathrm{ReO}_{4}$ undergo an ordering of the anions at $T_{\mathrm{AO}}$; here we focus on the tetrahedral counter ions. At room temperature, the octahedral and tetrahedral anions exhibit random translational and rotational movement caused by thermal fluctuations [22]. As the temperature is lowered, the tetrahedra do not fit into the cavities formed by the methyl end-groups due to their non-matching symmetry resulting in an stochastic anion orientation [17]. This orientational disorder is resolved at $T_{\mathrm{AO}}$ by $\mathrm{AO}$, a structural phase transition orienting the anions within the cavities.

For the (TMTTF) ${ }_{2} \mathrm{X}$ salts with $\mathrm{X}=\mathrm{BF}_{4}, \mathrm{ClO}_{4}$ and $\mathrm{ReO}_{4}$, the ordering of the anions is a first-order transition breaking the room temperature symmetry and doubling the lattice periodicity $[4,18]$. The resulting superstructure exhibits a reduced wave vector of $q_{\mathrm{AO}}=\left(\frac{1}{2}, \frac{1}{2}, \frac{1}{2}\right)[16,17,57]$. AO is detected by several methods: in resistivity measurements a kink-like feature is observed with a corresponding hysteresis [4], a pronounced peak or shoulder is seen in dielectric spectroscopy [32,36,58], and the optical spectra are distinctly modified [23]. In the case of (TMTTF) ${ }_{2} \mathrm{ClO}_{4}$ charge disproportionation $2 \delta$ occurs right at the anion order [23]. As can be seen from Table $1, T_{\mathrm{AO}}$ rises with increasing anion volume and mass.

It is instructive to look at the sibling compound (TMTSF) ${ }_{2} X$, where the AO takes place at $T_{\mathrm{AO}}=24 \mathrm{~K}$ for $\mathrm{X}=\mathrm{ClO}_{4}$ and $180 \mathrm{~K}$ for $\mathrm{ReO}_{4}$ [57,59-61]. In general the selenium analogues possess a larger bandwidth, compared to the (TMTTF) ${ }_{2} X$ salts and therefore the anion potential has only smaller influence. While the former compound orders in a $q_{\mathrm{AO}}=\left(0, \frac{1}{2}, 0\right)$ fashion, the latter one also exhibits a first-order phase transition with a wave vector $q_{\mathrm{AO}}=\left(\frac{1}{2}, \frac{1}{2}, \frac{1}{2}\right)$; interestingly a high-pressure phase with $\left(0, \frac{1}{2}, \frac{1}{2}\right)$ coexists for $p>10$ kbar [62]. For both compounds, the solid solution of TMTTF and TMTSF reveals several instabilities, broadens and shifts the AO transition [61,63-65]. A large number of studies have been devoted to the occurrence of superconductivity in (TMTSF) ${ }_{2} \mathrm{ClO}_{4}$ as the cooling rate is varied in order to retain anion disorder to low temperatures [66-69]. To the best of our knowledge, similar studies have not been performed on the Fabre salts.

Figure 6 illustrates our present understanding of the structural modifications taking place at the AO transition $[17,18,23]$. Due to their non-centrosymmetric shape, the tetrahedral anions exhibit only a single anion-ligand to sulfur link along the $(-b+c)$-direction, indicated by the black dashed line. This defines the direction along which the tetrahedra preferably reorient: one corner points along the anion-ligand to sulfur link towards the nearest $\mathrm{S}$ atom of the closest neighboring TMTTF molecule. Simultaneously, the other three anion ligands lie in a plane matching the symmetry of the methyl cavity.

A great many experiments provided compelling evidence that the anions are not statistically oriented along one of the two possible directions towards the nearest $\mathrm{S}$ atoms but exhibit long-ranged order, resulting in a 0110 charge pattern and tetramerization of the TMTTF stacks as sketched in Figure 6. Structural refinements on (TMTTF $)_{2} \mathrm{ReO}_{4}$ revealed the staggered orientation of the anions along the [111]-direction and the tetramerization with intra-stack distances $d_{1} \approx 3.45 \AA$, $d_{2}^{\prime} \approx 3.6 \AA$ and $d_{2}^{\prime \prime} \approx 3.48 \AA$ [70]. The molecular tetramerization was also observed by angular-dependent X-ray near-edge structure (XANES) measurements [27]; in addition, they reveal a slight shift and deformation of the $\mathrm{ReO}_{4}$ anions. For all salts with tetrahedral anions $\mathrm{X}=\mathrm{BF}_{4}, \mathrm{ClO}_{4}$ and $\mathrm{ReO}_{4}$, electron spin resonance (ESR) spectroscopy sees a drop of the spin susceptibility right at $T_{\mathrm{AO}}$ that is attributed to the formation of a singlet-triplet gap upon tetramerization [71-73]. Most recently, infrared spectroscopy could confirm the 0110 charge pattern by looking at the molecular vibrations [23]; more comprehensive investigations on this issue are under way [74]. 
Albeit a precise and complete microscopic picture of the AO transition could not be reached yet, there is wide agreement that Coulomb interactions between the anions and the TMTTF stacks are the driving force [17]. The orientation and slight deformation of the anions gives rise to a periodic electrostatic potential influencing the charge distribution on the TMTTF molecules. This anion potential is most pronounced along the sulfur to anion-ligand link and thus the staggered orientation of the anions imposes the 0110 charge pattern onto the TMTTF stacks. As seen from Table 1, there is no monotonic dependence of $T_{\mathrm{AO}}$ on $d_{\mathrm{AL}-\mathrm{S}}$; we should mention, however, that $d_{\mathrm{AL}-\mathrm{S}}$ is determined at room temperature and one should consider the distance right at the respective AO transition. It is not fully clear to what extent the 0110 charge pattern actually minimizes the Coulomb repulsion within the TMTTF stacks. The contribution of the anion potential cannot be determined precisely without detailed structural data in dependence on temperature covering the AO transition. Finally we would like to draw the attention on the effect of isotope substitution, since $T_{\mathrm{AO}}$ seems not to change appreciably upon deuteration [36].
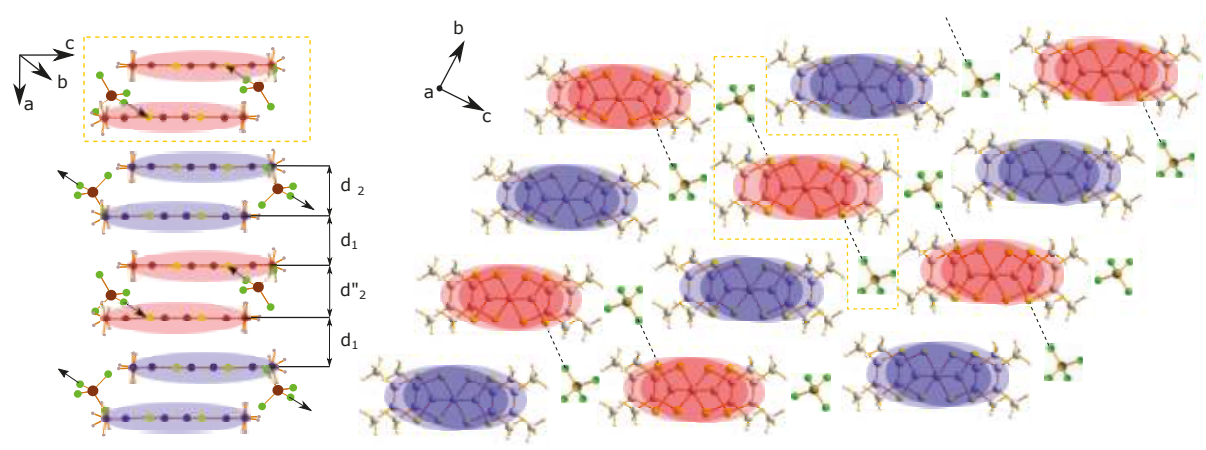

Figure 6. Scheme of the anion arrangement for the tetrahedral (TMTTF) $)_{2} X$ salts as $T<T_{\mathrm{AO}}$. Upon $\mathrm{AO}$, the anions re-orient in the methyl cavities along the anion-ligand sulfur link in $(-b+c)$-direction, represented by the black dashed line, forming long-range order. Coulomb attraction between the anion-ligand and the charge on the TMTTF molecule results in a 0110 charge pattern; the red and blue ellipsoids mark charge-rich and charge-poor TMTTF molecules, respectively. The polygon formed by the dashed orange line indicates which anion stacks are shown on the left side, illustrating the charge pattern along the stacking direction. In addition to a reorientation of the anions, a shift along the anion-ligand sulfur link occurs as well; this is not included here.

\subsection{Deuteration}

The replacement of hydrogen by deuterium in the methyl end-groups of the TMTTF molecule has only minor influence on the anion order but increases $T_{\mathrm{CO}}$ [75-77]; hence deuterated (TMTTF) $2 X$ salts provide valuable information on the ordering mechanisms. It was pointed out recently [23] that with increasing anion size or mass, the effect of deuteration on the CO transition diminishes. Defining the temperature shift $\Delta T_{\mathrm{CO}}=T_{\mathrm{CO}, \mathrm{D}}-T_{\mathrm{CO}}$, where $T_{\mathrm{CO}, \mathrm{D}}$ and $T_{\mathrm{CO}}$ are the transition temperatures for the deuterated and hydrogenated crystals, respectively, the change in transition temperature is strongest for small anions, as illustrated in Figure 7a. This important relation provides compelling evidence that charge order - considered as a purely electrostatic effect - is crucially affected by dynamic anion fluctuations. A simple relation is found for all TMTTF salts studied: $T_{C O, D}^{2}=T_{C O}^{2}+D^{2}$.

It is important to note the structural aspect that dimerization decreases upon deuteration. Starting with equally spaced TMTTF molecules in an isolated stack, dimerization is imposed by 
the anions. The coupling of the thermally fluctuating anions to the TMTTF stacks takes place via the terminal methyl groups, i.e., it is mediated by hydrogen bonds. At elevated temperatures, the methyl groups and the anions both require more space due to thermal fluctuations; their stronger interaction enhances the anion-TMTTF coupling. By deuterium substitution the methyl-group motion is slowed down, reducing the coupling to the counterions. As a consequence, the dimerization is smaller for $\mathrm{d}_{12}$-TMTTF salts compared to the hydrogenated analogues. This also explains the concomitant enhancement of $T_{\mathrm{CO}}$.

Anion order takes place at lower temperatures when the anions and the methyl groups fluctuate less and their dynamic interaction is of minor relevance. Instead, it is more important to consider the static Coulomb interaction between the monovalent anions and the charge spread on the TMTTF molecules, mainly located at the $S$ atom and the $C=C$ double bonds. Since the electrostatic interaction is not affected by deuteration, the influence of the anions does not change due to this isotope substitution.

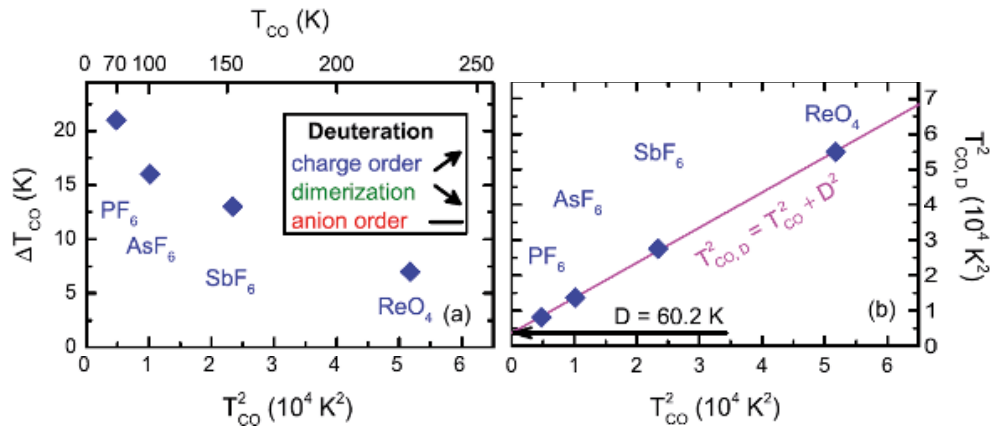

Figure 7. Effect of deuteration on charge order observed in (TMTTF) ${ }_{2} X$. (a) For several anions $X=\mathrm{PF}_{6}$, $\mathrm{AsF}_{6}, \mathrm{Sb}_{6}$ and $\mathrm{ReO}_{4}$, the change in the transition temperature $\Delta T_{\mathrm{CO}}=T_{\mathrm{CO}, \mathrm{D}}-T_{\mathrm{CO}}$ is plotted versus the square of the $\mathrm{CO}$ transition temperature $T_{C O}^{2}$ of pristine crystals. The increase is most pronounced for small and light anions. The inset indicates that deuteration rises the $\mathrm{CO}$ temperature, deminishes dimerization, but has no effect on AO. (b) Plotting $T_{\mathrm{CO}, \mathrm{D}}$ over $T_{\mathrm{CO}}$ clearly reveals the dependence $T_{\mathrm{CO}, \mathrm{D}}^{2}=T_{\mathrm{CO}}^{2}+D^{2}$ with $D=60.2 \mathrm{~K}$. The data are taken from Reference [75,77]; the plot follows Reference [23].

\section{Experimental Details}

Single crystals of $\mathrm{TMTTF}_{2} \mathrm{X}$ salts with $\mathrm{X}=\mathrm{BF}_{4}, \mathrm{ClO}_{4}$ and $\mathrm{ReO}_{4}$ were grown by the standard electrochemical methods using an $\mathrm{H}$-type glass cell at ambient temperature and inert atmosphere. Platinum plates with an area of approximately $3 \mathrm{~cm}^{2}$ served as electrodes and a sand barrier was introduced to reduce diffusion. By applying a constant voltage of $1.5 \mathrm{~V}$, a current between 9.2 and $13.4 \mu \mathrm{A}$ was drawn through the solution. The growth of the needle-shaped single crystals of typical dimensions $(2 \times 0.5 \times 0.1) \mathrm{mm}^{3}$ took several months. Since the crystal shape does not correspond to the triclinic symmetry of the crystal structure, $b^{\prime}$ denotes the projection of the $b$-axis perpendicular to the $a$ axis, and $c^{*}$ is chosen orthogonal to the $a b$-plane.

In order to perform the electrical transport measurements, small gold contacts were evaporated onto the natural crystal surface and thin gold wires were attached to them by carbon paste. The dc experiments applied the four-point method. Pressure-dependent measurements were carried out by means of a clamp-type pressure cell using Daphne 7373 oil as pressure medium. Daphne 7373 is the favored pressure medium for this kind of experiments because it conveys hydrostatic pressure and (TMTTF $)_{2} X$ crystals are inert to it. For all applied pressures the pressure medium stays fluid at room temperature, relevant shearing forces seem to appear only when cooling through the freezing temperature at pressures between 0-3 kbar. Temperature-dependent transport measurements were carried out in a standard helium bath cryostat. The inherent pressure loss upon cooling was recorded 
continuously in-situ by an InSb semiconductor pressure gauge, as illustrated in Figure 8 . The dielectric measurements were performed with two contacts in a pseudo four-point configuration by means of an Agilent 4284 Impedance Analyzer (20 Hz-1 MHz). For both measurement techniques, the applied voltage was in the $\mathrm{mV}$ range to avoid heating and ensure measurements in the Ohmic regime.
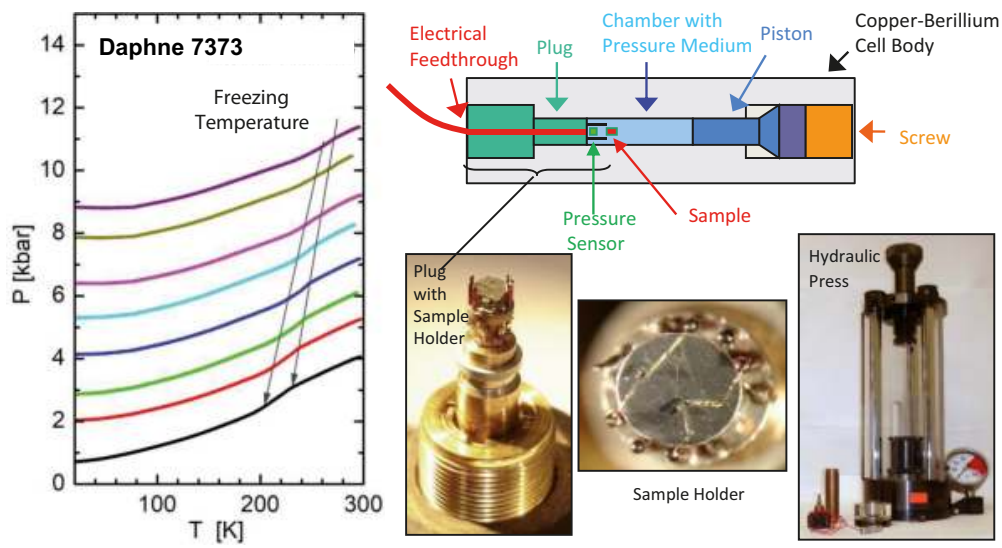

Figure 8. Left panel: Pressure loss of Daphne 7373 oil during cooling; the arrows mark the temperature range of freezing. The data were recorded in a Copper-Berrilium cell by four-point resistance measurement of an InSb pressure sensor. Right panel: Sketch of the actual pressure cell used and photos of the sample holder, and the hydraulic press.

\section{Electrical Transport under Pressure}

The physical properties of the (TMTTF $)_{2} X$ family have been widely studied for several decades [78-81], however most of the work dealt with centrosymmetric anions. Here we advocate that the structural phase transition should not be considered an additional complication but an opportunity to gain more insight on the effect of the lattice on the electronic properties; there is no question, however, that further investigations are needed. In the following we will present the first complete set of systematic dc measurements under hydrostatic pressure of the three most common (TMTTF) $2 X$ with tetrahedral anions $\left(X=\mathrm{BF}_{4}, \mathrm{ClO}_{4}, \mathrm{ReO}_{4}\right)$. After detailed discussions of $\rho(T, p)$ for each particular compounds, we will compare the rather diverse characteristics.

\section{1. $(\mathrm{TMTTF})_{2} \mathrm{ClO}_{4}$}

From Table 1 we see that $X=\mathrm{ClO}_{4}$ is placed between $X=\mathrm{BF}_{4}$ and $\mathrm{ReO}_{4}$ with respect to the anion size. From the charge transfer salts with octahedral anions we know that $T_{\mathrm{CO}}$ increases with anion radius $[4,18]$. The same is expected for the tetrahedral anions. (TMTTF) ${ }_{2} \mathrm{ClO}_{4}$ is in so far particular, as it does not exhibit the charge order typical for all other Fabre salts. As far as the anion transition is concerned, it follows strictly the expected relation for the tetrahedral anions: $T_{\mathrm{AO}}\left(\mathrm{BF}_{4}\right)<T_{\mathrm{AO}}\left(\mathrm{ClO}_{4}\right)<T_{\mathrm{AO}}\left(\mathrm{ReO}_{4}\right)$.

The pressure dependence of $\rho(T)$ is affected by the absence of CO showing extraordinary features. In Figure 9 the electrical resistivity $\rho_{a}(T, p)$ and $\rho_{c}(T, p)$ are displayed for pressures up to $11.6 \mathrm{kbar}$ in panels $\mathrm{a}, \mathrm{b}$ and $\mathrm{d}$ respectively. At ambient pressure, $\rho_{a}(T)$ develops the well-known activated temperature dependence for $T<T_{\rho} \approx 260 \mathrm{~K}$ (localization temperature) and shows a distinct jump to lower values at $T_{\mathrm{AO}} \approx 73 \mathrm{~K}$ [4]. It is interesting to note a change in slope of $\rho_{a}(T)$ that appears around $40 \mathrm{~K}$. Applying hydrostatic pressure, the local minimum at $T_{\rho}$ disappears as well as the jump at $T_{\mathrm{AO}}$. Whereas the former may be assessed as an artifact caused by the temperature-dependent pressure loss due to the pressure media in the cell (Daphne oil 7373, see Figure 8), the latter is inherent 
to (TMTTF $)_{2} \mathrm{ClO}_{4}$. For increasing pressure, the $\mathrm{AO}$ transition smears, broadens and moves to lower temperatures, developing a distinct valley of low resistivity. Concurrently the onset of the transition $T_{\mathrm{AO}-\text { max }}$ follows the trend of $T_{\mathrm{AO}}$, accompanied by the development of steep increase of $\rho(T, p)$ at $T_{\mathrm{M}}$, which is related to the lower bound of the minimum.

At $p \approx 10 \mathrm{kbar}$ the trend stops; $T_{\mathrm{AO}-\max }$ and $T_{\mathrm{M}}$ do not significantly shift in temperature any more when $p$ increases further. The mechanism triggering the valley seems to come to a halt. As can be seen in Figure 9b, the valley is gradually filled. At the maximum pressure applied, $p=11.6 \mathrm{kbar}$, the minimum has somehow disappeared completely. Instead for high pressures a second peak develops at about $40 \mathrm{~K}$, which does not shift in temperature for $p>11 \mathrm{kbar}$; this feature progressively dominates the temperature dependance of $\rho(T)$. This behavior is observed in both directions, for $\rho_{a}(T, p)$ and $\rho_{c}(T, p)$, as seen by comparison of panels b and d. In Figure 10 we take a closer look at the pressure range from 10.0 to $11.6 \mathrm{kbar}$. The appearance of the second peak is accompanied by a hysteretic behavior in the temperature-dependent resistivity on both sides of the valley. Multiple temperature cycles reveal fluctuations in $\rho_{a}(T)$ not observed in other cases.
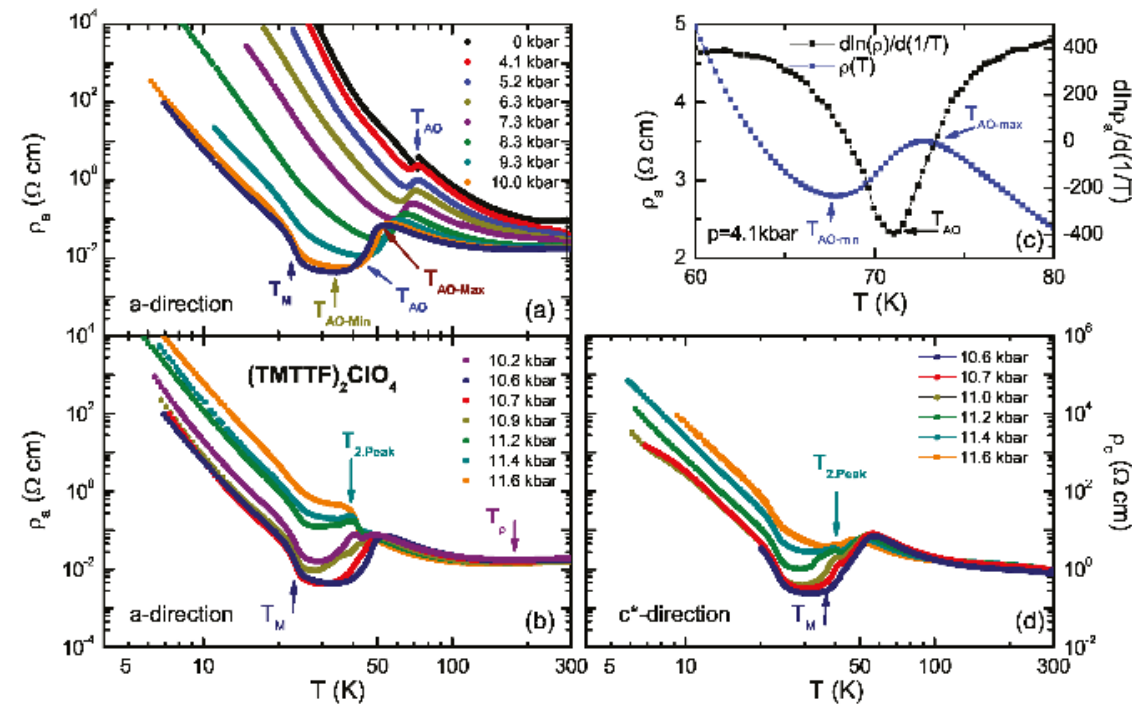

Figure 9. Pressure evolution of the temperature-dependent electronic transport of (TMTTF ${ }_{2} \mathrm{ClO}_{4}$. (a) dc resistivity as a function of temperature for ambient conditions and for hydrostatic pressures up to 10.0 kbar measured along the stacking direction $a$. (b) For higher pressure values a more complex behavior of $\rho_{a}(T, p)$ is observed. (c) For the example taken at $p=4.1 \mathrm{kbar}$, we illustrate how the different parameters of the anion ordering transition were extracted from $\rho(T, p)$ and its derivative with respect to inverse temperature $1 / T$. (d) Temperature dependence of the perpendicular resistivity $\rho_{\mathcal{C}}(T, p)$ for the high-pressure range.

In the phase diagram displayed in Figure 11a, we summarize the different phases, transitions, and appearing features as extracted from our $\rho(T, p)$ data. At elevated temperatures (TMTTF) ${ }_{2} \mathrm{ClO}_{4}$ behaves like a low-dimensional metal with $\mathrm{d} \rho_{a} / \mathrm{d} T>0$ along the stacking direction and $\mathrm{d} \rho_{c} / \mathrm{d} T<0$ perpendicular to the stacks. Between 250 and $150 \mathrm{~K}$ charge localization takes place at $T_{\rho}$. While the metal-like behavior in $\rho_{a}(T, p)$ for $T>T_{\rho}$ is obvious at $p=0$, it gets masked for finite but intermediate pressures; interestingly, the localization temperature is recovered at high $p$ values. According to Figure 8 the temperature-dependent pressure loss is reduced as the applied pressure increases; hence this effect influences $\rho(T)$ less. For the $p=9.3$ kbar curve, we can clearly identify a range of metallic 
behavior by $\mathrm{d} \rho_{a}(T, p) / \mathrm{d} T>0$. For increasing pressures, the minimum in $\rho(T)$ at $T_{\rho}$ is reduced. $T_{\rho}$ extracted in this manner specifies the upper limit of the inherent localization temperature of $(\mathrm{TMTTF})_{2} \mathrm{ClO}_{4}$. Since in the perpendicular direction $\mathrm{d} \rho_{c}(T, p) / \mathrm{d} T<0$ for the whole investigated pressure and temperature range, our data identify the high-temperature phase of (TMTTF) ${ }_{2} \mathrm{ClO}_{4}$ as a low-dimensional metal. The subsequent phase is an insulating one; nothing hints towards any inherent changes in the material down to the anion ordering. No indications towards charge order are detected.
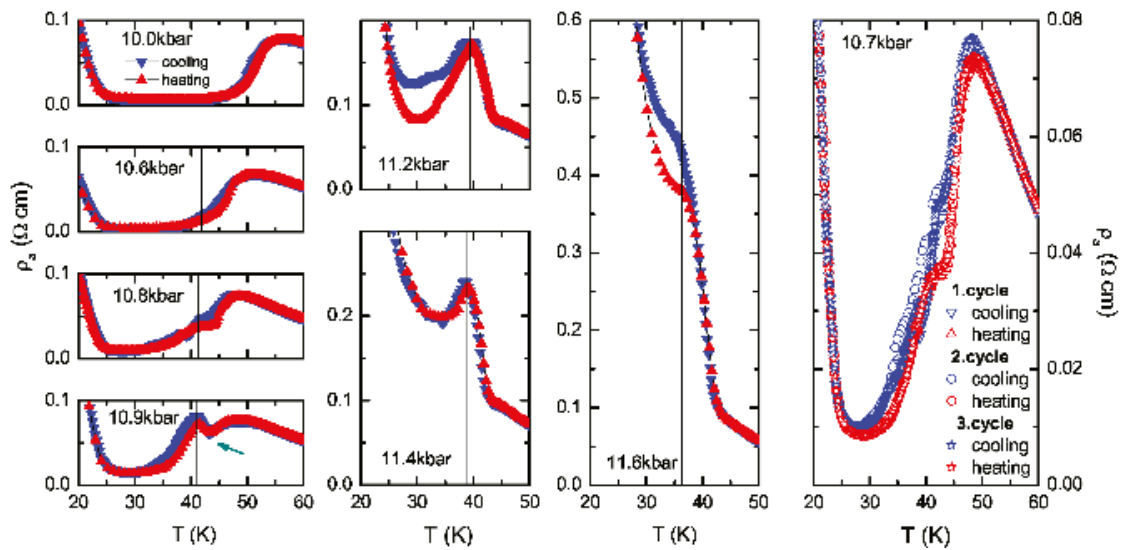

Figure 10. Magnification of the high-pressure resistivity along the $a$-axis of (TMTTF) ${ }_{2} \mathrm{ClO}_{4}$ in the temperature range of $T_{\mathrm{AO}}$. For pressure between $10.2-11.7 \mathrm{kbar}$, a second peak in $\rho_{a}(T)$ appears. Simultaneously at the anion ordering $\rho_{a}(T)$ exhibits a distinct hysteresis and fluctuations.
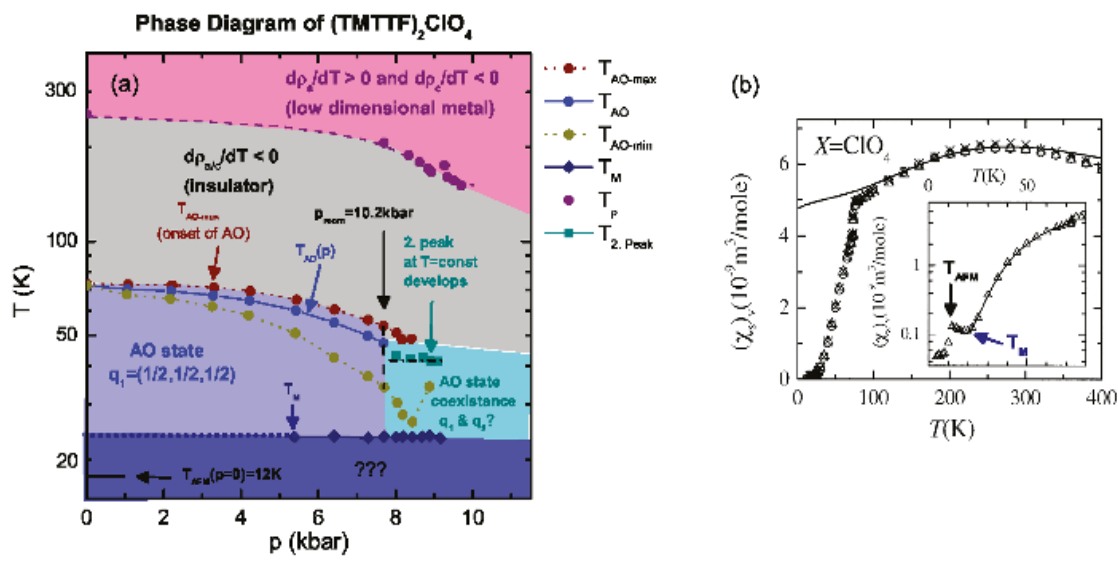

Figure 11. (a) The phase diagram of (TMTTF $)_{2} \mathrm{ClO}_{4}$ as obtained from resistivity measurements indicates various phase transitions. (b) Temperature-dependent susceptibility of (TMTTF) ${ }_{2} \mathrm{ClO}_{4}$ as obtained from electron spin resonance (ESR) measurements (taken from Dumm et al. [82]). Two distinct drops in the ESR intensity can be identified, one at the $\mathrm{AO}$ transition and the other at the antiferrmagnetic order $T_{\mathrm{AFM}}=12 \mathrm{~K}$. The onset of the plateau appears around $T \approx 20 \mathrm{~K}$ (indicated by the blue arrow), which coincides with the steep increase of the resistivity at $T_{\mathrm{M}}$.

The onset of the $\mathrm{AO}$ transition, $T_{\mathrm{AO}-\max }$, is defined by the local maximum in $\rho(T)$ followed by a sharp drop; the $\mathrm{AO}$ temperature $T_{\mathrm{AO}}$ is given by the steepest decline of $\rho(T)$ between $T_{\mathrm{AO}-\max }$ and the local minimum at $T_{\mathrm{AO}-\mathrm{min}}$. This is illustrated in Figure $9 \mathrm{c}$ in full detail, where also the derivative 
$\mathrm{d} \ln \left\{\rho_{a}\right\} / \mathrm{d}\left(\frac{1}{T}\right)$ is plotted in the Arrhenius-like manner. The AO transition becomes obscured, when the second peak starts to grow. This additional feature is first visible as pressure exceeds $10 \mathrm{kbar}$. It always seems to be located at $T \approx 40 \mathrm{~K}$, with no significant shift for varying pressure. It is interesting to compare the structural properties with the sister compound (TMTSF) ${ }_{2} \mathrm{ClO}_{4}$, which can be turned from a $T_{C}=1 \mathrm{~K}$ superconductor to an antiferromagnetic SDW insulator by suppression of the $q_{2}=\left(0, \frac{1}{2}, 0\right)$ anion order at $T_{\mathrm{AO}}=24 \mathrm{~K}$ by rapid cooling [61]. The pronounced hysteresis and fluctuations might point towards the development of an $\mathrm{AO}$ with another superstructure at $T=40 \mathrm{~K}$. Recalling the observation of different superstructures with pressure and disorder in (TMTSF) ${ }_{2} \mathrm{ReO}_{4}$ [62], we could imagine a coexistence of the conventional AO superstructure $q_{1}=\left(\frac{1}{2}, \frac{1}{2}, \frac{1}{2}\right)$ and a new, but weaker one with a different $q$ vector also for (TMTTF) ${ }_{2} \mathrm{ClO}_{4}$ [13].

It might be worth to have a closer look on the temperature $T_{\mathrm{M}}$ defined by the lower bound of the resistivity valley, since this transition does not shift in temperature for the entire pressure range. The strong increase in $\rho(T)$ at $T_{\mathrm{M}}$ cannot be related to the transition into the antiferromagnetic state, since for $p=0$ the corresponding transition occurs at $T_{\mathrm{AFM}}=12 \mathrm{~K}$ [83] and no feature is visible in the resistivity data at that temperature. It is interesting to note, however, that the increase of $\rho(T)$ at $T=T_{\mathrm{M}}$ coincides with the weak upturn of the magnetic susceptibility after the local minimum in the ESR data (reproduced in Figure 11b) [82]. Although $T_{M}$ develops only for relatively high pressures, the ESR data are recorded at ambient pressure. Maybe this feature is present even at ambient conditions but masked by the high resistivity due to the strong anion order. We call for further investigations of these relations.

\section{2. $(\text { TMTTF })_{2} \mathrm{BF}_{4}$}

In Figure 12 we present our dc resistivity results $\rho_{a}(T, p)$ and $\rho_{c}(T, p)$ for (TMTTF) $)_{2} \mathrm{BF}_{4}$, the compound with the smallest tetrahedal anion. At first sight, the resistivity of the two crystal directions seems not to differ much, except of the absolute value. Dominated by an activated temperature dependence, a pronounced kink at the $\mathrm{CO}$ transition is present for all investigated hydrostatic pressure values. It is followed by an increase in slope for $T<T_{\mathrm{CO}}$. At the anion ordering both $\rho_{a}(T)$ and $\rho_{c}(T)$ exhibit a step-like feature, but then continue with more or less the same derivative. As pressure rises, this step-down is washed out and has eventually disappeared completely. The activation energy is significantly reduced for rising pressure. A closer look reveals distinct differences between the transport in the different crystallographic directions: $\rho_{a}$ and $\rho_{c}$. Most obvious, the temperature-dependent dynamics along the $a$-axis appears about two orders of magnitude higher for all pressures compared to the transport along the $c^{*}$-direction. Pressure has a much more severe effect on the perpendicular transport $\rho_{c}$ than parallel to the stacks, $\rho_{a}$. For low temperatures, the high-pressure resistivity becomes almost isotropic.

The analysis of the charge and anion ordering transitions is explained in Figure 12. In panels $\mathrm{c}$ and $\mathrm{d}$ the derivatives $\mathrm{d} \ln \left\{\rho_{a}\right\} / \mathrm{d}\left(\frac{1}{T}\right)$ are plotted as a function of temperature in order to define the actual transition temperatures. Here we can also identify differences in the slopes, since this temperature derivative equals the gap size of an activated behavior. The kink in $\rho(T)$ at $T_{\mathrm{CO}}$ appears as a clear peak in the derivative. For increasing pressure the $\mathrm{CO}$ peak sharpens, it gets more pronounced and shifts to lower $T$.

For $T \leq T_{\mathrm{CO}}$ and high pressures, we see a steep upturn in resistivity that occurs for $\rho_{a}(T, p)$ as well as for $\rho_{c}(T, p)$. It is followed by a clear change of the resistivity slope at $T_{\mathrm{AO}}$. It is interesting to look at the ratio of the resistivity values at the $\mathrm{CO}$ transition and those at the AO transition: $\Delta \rho=\rho\left(T_{\mathrm{AO}}\right) / \rho\left(T_{\mathrm{CO}}\right)$. Along the stacks, the change is about five orders of magnitude at all pressures; for the $c^{*}$-direction, $\Delta \rho$ shrinks from 10,000 at ambient pressure to only about 100 at the highest investigated pressure of $p=11.5 \mathrm{kbar}$. Hydrostatic pressure has a stronger impact on $\rho_{c}(T, p)$ than on $\rho_{a}(T, p)$. This picture is supported by the more pronounced loss in activation energy with pressure at $T \leq T_{\mathrm{AO}}$ for the transverse transport $\rho_{c}(T, p)$ compared to the longitudinal one $\rho_{a}(T, p)$. 

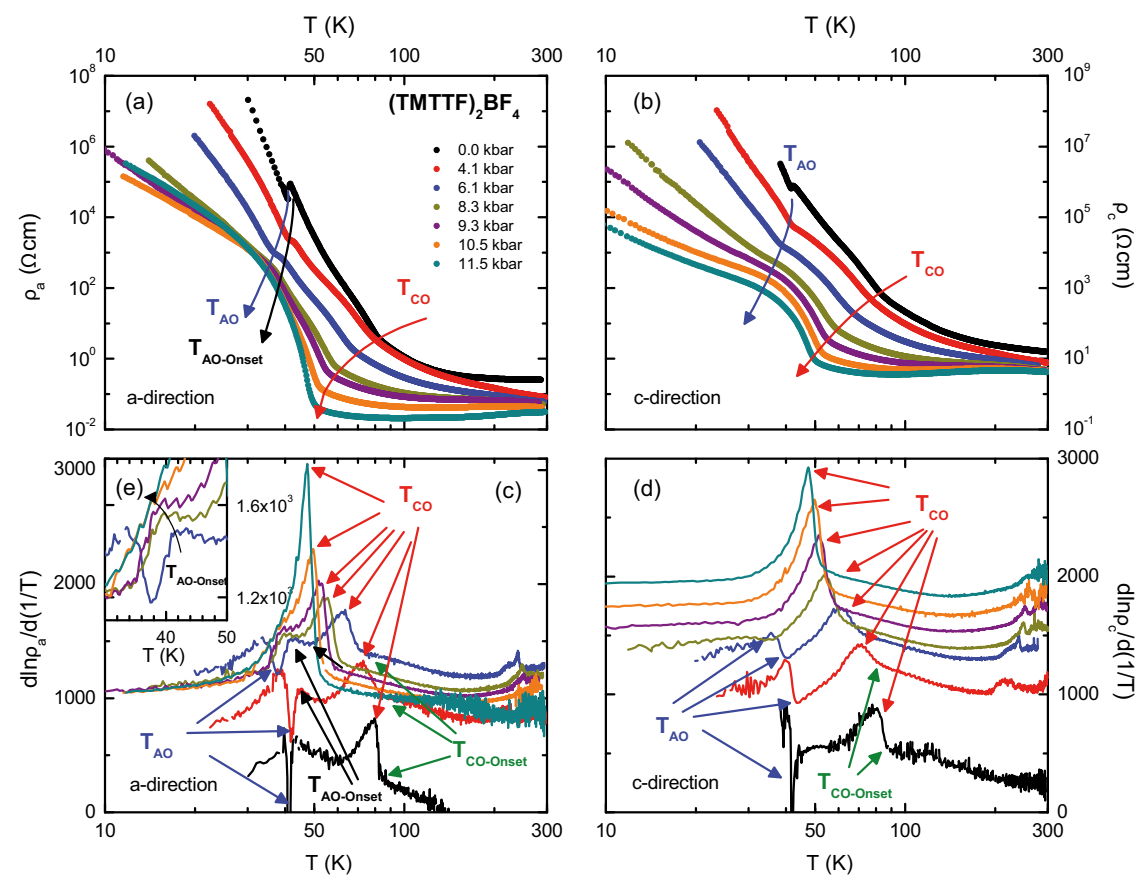

Figure 12. Temperature dependence of the dc resistivity of (TMTTF $)_{2} \mathrm{BF}_{4}$ measured for the directions (a) parallel and (b) perpendicular to the stacks when increasing hydrostatic pressure is applied as indicated. Besides the minimum in resistivity at $T_{\rho}$, the charge-order transition at $T_{\mathrm{CO}}$ and the anion ordering at $T_{\mathrm{AO}}$ is clearly observed. The analysis of the data is illustrated in panels (c) and (d) where the temperature derivative $\mathrm{d} \ln \left\{\rho_{a}\right\} / \mathrm{d}(1 / T)$ is plotted for both orientations. The extrema indicate the ordering temperatures $T_{\mathrm{AO}}$ and $T_{\mathrm{CO}}$. In addition we define an onset temperature for the charge and anion orders. The inset (e) enlarges the range around the $\mathrm{AO}$ temperature.

At $T_{\mathrm{AO}}, \rho(T, p)$ undergoes another remarkable change in its behavior that strongly depends on pressure. Under ambient conditions, AO results in a discrete jump in $\rho(T)$ to lower values; since the slope is preserved, the gap size in an activated behavior remains unchanged at the transition. This observation was explained [4] by a reduction of scattering as the anions are locked in the methyl cavities. This jump becomes smooth when pressure is applied; a plateau starts to develop gradually. Increasing pressure further, $\rho(T)$ becomes rather broad for both directions, and eventually the $\mathrm{AO}$ transition cannot be identified as a discrete feature any more; what remains is an upturn in resistivity in a wide temperature range. Looking at the derivative in Figure $12 \mathrm{c}, \mathrm{d}, \mathrm{T}_{\mathrm{AO}}$ is identified as a sharp peak in the low-pressure range. But for $p \geq 8.3 \mathrm{kbar}$ this characteristic vanishes. It is interesting to note that the onset of the $\mathrm{AO}$ transition at $T_{\mathrm{AO}-\text { onset }}$ related to the upturn in $\mathrm{d} \ln \left\{\rho_{a}\right\} / \mathrm{d}\left(\frac{1}{T}\right)$, seen just before $T_{\mathrm{AO}}$ (Figure 12c and inset e), can be distinguished up to the highest pressure. For $p=11.5 \mathrm{kbar}$ the $\mathrm{AO}-$ onset peak is dominated by the strong $\mathrm{CO}$ peak coming very close in temperature. Surprisingly, no onset feature of $\mathrm{AO}$ is present in $\rho_{c}$. Nevertheless, these observations in $\rho_{a}$ hint that the $\mathrm{AO}$ transition might survive even the high hydrostatic pressure. No doubt $T_{\mathrm{AO}}$ shifts down in temperature much slower with pressure than $T_{\mathrm{CO}}$.

In Figure 13a,b we focus on the high-temperature regime in order to inspect the metallic behavior and charge localization. For high pressure, $p \geq 10.5 \mathrm{kbar}$, both $\rho_{a}(T)$ as well as for $\rho_{c}(T)$ exhibit a metal-like temperature dependence. The derivatives plotted in Figure 13c,d evidence a crossing of the zero line at around $T=100 \mathrm{~K}$. We should note that in the clamped cell pressure losses might 
occur when cooling down from high temperatures, masking the inherent metallic behavior. Thus the second crossing point should be considered with some reservations, when drawing the phase diagram in Figure 13e. We associate the change in slope $\mathrm{d} \rho_{a}(T) / \mathrm{d} T$ with the charge localization temperature $T_{\text {loc }} \approx 240 \mathrm{~K}$. With increasing pressure $T_{\text {loc }}$ shifts to lower temperatures. For the perpendicular transport, $\rho_{c}(T)$ exhibits an activated behavior under ambient conditions. The boundary between the metallic regions in Figure 13e therefore indicates a lower limit.

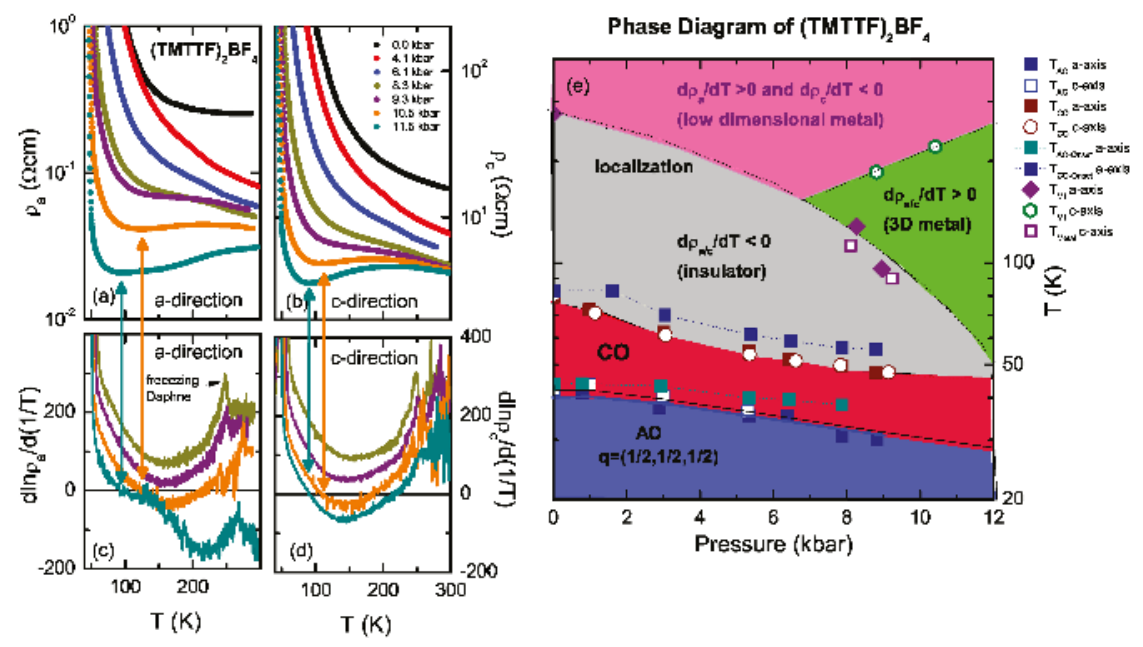

Figure 13. The panels on the left display the temperature dependence of the resistivity of (TMTTF $)_{2} \mathrm{BF}_{4}$ measured for different hydrostatic pressure applied with the main focus on the metal-insulator transition and charge localization. (a) The on-chain resistivity $\rho_{a}(T)$ exhibits the development of a minimum as pressure increases. (b) Resistivity $\rho_{\mathcal{c}}(T)$ perpendicular to the stacks for different pressure values. (c,d) Pressure evolution of the resistivity derivative $d \ln (\rho) / d(1 / T)$ as a function of temperature for both orientations. The metal-insulator transition is indicated by the arrows. Also seen is the freezing of the pressure transmitting oil Daphne 7373. (e) In the phase diagram of (TMTTF ${ }_{2} \mathrm{BF}_{4}$ different electronic states are depicted as derived from dc transport experiments. Upon cooling the low-dimensional metal exhibits a charge localization that moves from about room temperature down to below $100 \mathrm{~K}$ as pressure increases. For pressure above 8 kbar a three-dimensional metallic phase develops. At lower temperatures charge order and anion order appear subsequently. Both phase boundaries move towards lower temperature with increasing pressure.

\section{3. $(\mathrm{TMTTF})_{2} \mathrm{ReO}_{4}$}

As listed in Table 1, (TMTTF) $)_{2} \mathrm{ReO}_{4}$ is the compound with the highest $\mathrm{CO}$ and $\mathrm{AO}$ transition temperatures in the whole family of Fabre salts. At ambient pressure (TMTTF) ${ }_{2} \mathrm{ReO}_{4}$ develops $\mathrm{CO}$ at $T_{\mathrm{CO}} \approx 230 \mathrm{~K}$ and the $\mathrm{AO}$ transition at $T_{\mathrm{AO}} \approx 160 \mathrm{~K}$. Figure $14 \mathrm{a}$ displays the results of our pressure and temperature-dependent dc resistivity measurements along the perpendicular direction. The corresponding temperature derivative $d \ln \left\{\rho_{c}\right\} / d\left(\frac{1}{T}\right)$ is a measure of the energy gap for activated charge transport; its temperature dependence is presented in Figure $14 \mathrm{~b}$. The CO temperature $T_{\mathrm{CO}}$ can be identified as a kink in $\rho_{\mathcal{c}}(T, p)$. Even more pronounced is the anion order at $T_{\mathrm{AO}}$. 

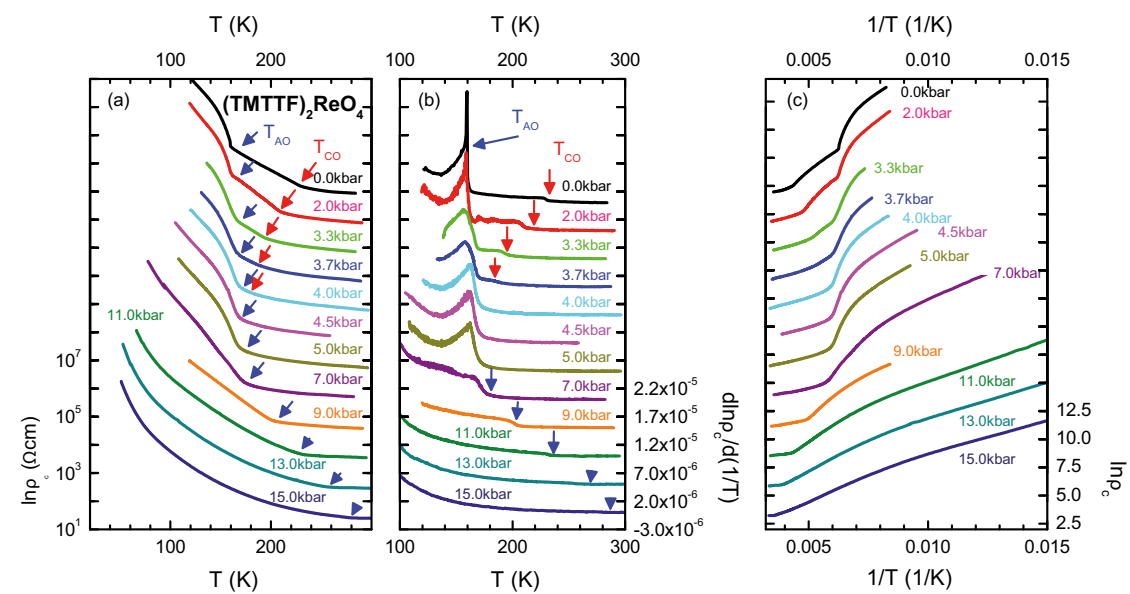

Figure 14. (a) Temperature-dependent resistivity of (TMTTF) ${ }_{2} \mathrm{ReO}_{4}$ measured along the $c^{*}$-direction for different values of external pressure. The red and black arrows indicate the charge order and anion order transition temperatures. (b) The temperature derivative $\mathrm{d} \ln (\rho) / \mathrm{d}(1 / T)$ shows the charge-order transition as a step in the temperature dependence; the anion ordering $T_{\mathrm{AO}}$ is seen as a pronounced peak. Both exhibit a distinct pressure dependence. (c) Arrhenius plot of the resistivity $\rho_{c}(T)$ as the pressure increases. All curves are shifted with respect to each other for clarity reasons.

For $T<T_{\mathrm{AO}}$ the resistivity increase can be described by an activated behavior with an energy gap that increases in a BCS-like fashion; more details on the dc transport at ambient pressure can be found by Köhler et al. [4]. When hydrostatic pressure is applied, $T_{\mathrm{CO}}$ shifts to lower values and resistivity changes at the transition get weaker. $T_{\mathrm{AO}}$ on the other hand does not move in temperature for $p<p_{\text {crit }}=5 \mathrm{kbar}$. Also the qualitative shape of $\rho_{c}(T)$ and its slope for $T \leq T_{\mathrm{AO}}$ do not change significantly in this pressure range. In the derivative of the resistivity, the $\mathrm{AO}$ transition appears as a sharp peak, indicating a first-order phase transition for all values $p \leq p_{\text {crit }}=5.0 \mathrm{kbar}$. When the $\mathrm{CO}$ transition coincides with the anion ordering, a sudden change in the pressure dependence of $T_{\mathrm{AO}}$ is observed. Above a critical pressure $p_{\text {crit }}=5.0 \mathrm{kbar}$ the $\mathrm{AO}$ related kink moves to higher temperatures when pressure increases further. In addition, the shape of $\rho_{c}(T)$ changes from a BCS-like to a rather straight and flat evolution of $\rho_{c}(T)$ for temperatures $T \leq T_{\mathrm{AO}}$. For higher pressures, $p>p_{\text {crit }}$ the shape of the transition resembles a second-order phase transition. The strong reduction of the energy gap $\Delta$ at low temperatures for increasing pressures $p>p_{\text {crit }}$ can be seen best in the Arrhenius plot of Figure 14c.

In Figure 15c-e we show the temperature dependence of the charge and anion ordering gaps, $\Delta_{\mathrm{CO}}$ and $\Delta_{\mathrm{AO}}$, respectively, for selected pressures. With increasing pressure $p<p_{\text {crit, }}$, the energy gap $\Delta_{\mathrm{CO}}$ continuously converges to zero at the critical pressure of $5.0 \mathrm{kbar}$ is approached. On the contrary, $\Delta_{\mathrm{AO}}$ stays fixed. For higher pressure $p>p_{\text {crit }}, \Delta_{\mathrm{AO}}$ gets continuously suppressed as well. For comparison the dc measurements of Coulon et al. [41] along the chain direction, $\rho_{a}(T, p)$ are presented in Figure 15b. They exhibit qualitatively the same behavior as our dc results in the perpendicular direction.

By extracting the temperatures for the different phase transitions from our resistivity data we can compose a phase diagram of (TMTTF $)_{2} \mathrm{ReO}_{4}$ presented in Figure 15a. The CO state is limited to low pressure. Pressure-dependent $\mathrm{X}$-ray investigations may shed light on the origin of the sudden change at the critical pressure of $p_{\text {crit }} \approx 5.0$ kbar. By comparison with the sister compound (TMTSF) ${ }_{2} \mathrm{ReO}_{4}$, we can speculate about a change in anion superstructure. As shown in Figure 15f, Moret et al. [62] found a change from $q_{2}=\left(\frac{1}{2}, \frac{1}{2}, \frac{1}{2}\right)$ to $q_{3}=\left(0, \frac{1}{2}, \frac{1}{2}\right)$ superstructure vector as pressure exceeds a certain critical pressure. 

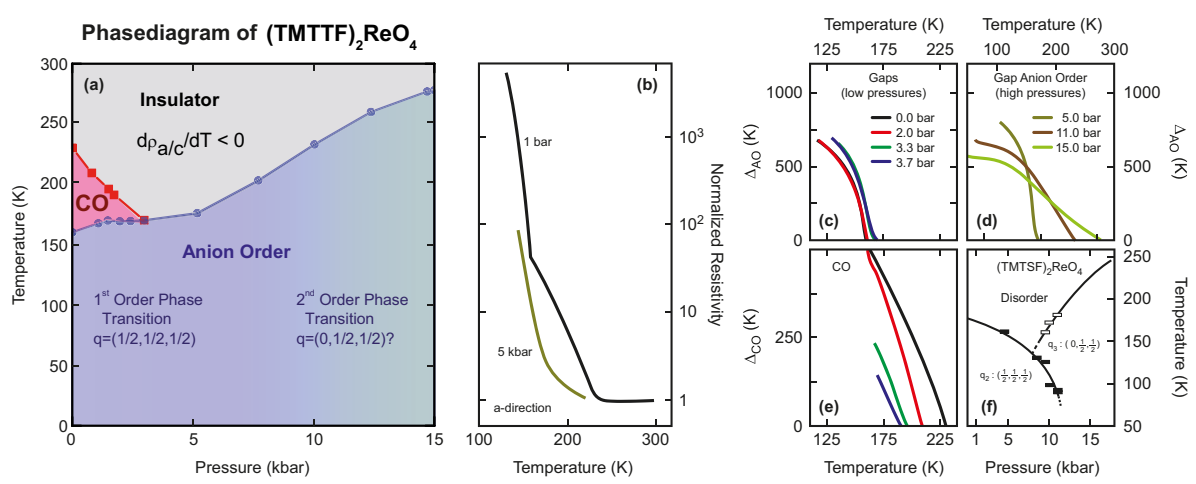

Figure 15. (a) Phase diagram of (TMTTF $)_{2} \mathrm{ReO}_{4}$ as obtained from our pressure- and temperature-dependent transport measurements. Even for 15 kbar hydrostatic pressure, no metallic behavior is observed up to room temperature. The charge-ordered phase is limited to low pressure values. The transition into the anion-ordered state changes from first to second order. (b) Normalized dc resistivity versus temperature measured at 1 bar and 5 kbar along the chain direction of (TMTTF) ${ }_{2} \mathrm{ReO}_{4}$; reproduced from [41]. (c-e) Temperature change of the activation energy obtained below the AO transition and CO transition of (TMTTF) ${ }_{2} \mathrm{ReO}_{4}$ for different pressure. (f) Temperature-dependent anion-ordering phase diagram of (TMTSF) $)_{2} \mathrm{ReO}_{4}$ as obtained from X-ray scattering experiments of Moret et al. [62]. The $q_{2}$ and $q_{3}$ phase boundaries overlap at $p=9.5,10$, and $11 \mathrm{kbar}$ and they have opposite slopes.

\subsection{Discussion}

For a better comparison, in Figure 16 we present next to each other the temperature-dependent resistivity of the three Fabre salts (TMTTF) 2 X with tetrahedral anions, $X=\mathrm{BF}_{4}, \mathrm{ClO}_{4}$, and $\mathrm{ReO}_{4}$, obtained at different pressure. In the case of centrosymmetric anions a simple relation between anions size and charge ordering could be derived [18]: the larger the anions, the larger the stack separation and the higher $T_{\mathrm{CO}}$. This concept fails for the tetrahedral anions. Obviously the original idea of anions as pure spacers between the one-dimensional TMTTF stacks has to be discarded; instead we have to account for the three-dimensional lattice structure and in particular the interaction of the organic stacks with the anions.

Based on the size of the anions [52], $V_{\text {Anion }}^{\mathrm{BF}_{4}}<V_{\text {Anion }}^{\mathrm{ClO}_{4}}<V_{\mathrm{Anion}^{\prime}}^{\mathrm{ReO}_{4}}$ the CO temperature of (TMTTF $)_{2} \mathrm{ClO}_{4}$ should fall right between $T_{\mathrm{CO}}^{\mathrm{BF}_{4}}$ and $T_{\mathrm{CO}}^{\mathrm{ReO}_{4}}$. Surprisingly, no $\mathrm{CO}$ transition is detected in (TMTTF) ${ }_{2} \mathrm{ClO}_{4}$. Provided it is not just an exception to the general rule, (TMTTF) ${ }_{2} \mathrm{ClO}_{4}$ may be the key compound for understanding the Fabre salts. Comparing our resistivity data for the three different compounds in Figure 16 reveals that the absence of $\mathrm{CO}$ has drastic consequences on the $\rho(T)$ for $T<T_{\mathrm{AO}}$. At ambient pressure no qualitative difference is observed of $\mathrm{X}=\mathrm{BF}_{4}$ and $\mathrm{ClO}_{4}$; at $T_{\mathrm{AO}}$ the resistivity exhibits a step to lower values followed by an activated temperature behavior. This common picture changes when pressure is applied. For (TMTTF $)_{2} \mathrm{BF}_{4}$ we still find $\rho(T)$ to be thermally activated down to low temperatures. In (TMTTF $)_{2} \mathrm{ClO}_{4}$, however, a large minimum in $\rho(T)$ develops for a temperature range of about $30 \mathrm{~K}$. When the pressure has reached $p=9.3 \mathrm{kbar}, T_{\mathrm{AO}}$ has shifted down by $25 \mathrm{~K}$ for (TMTTF) ${ }_{2} \mathrm{ClO}_{4}$, while only $6 \mathrm{~K}$ in the case of (TMTTF) ${ }_{2} \mathrm{BF}_{4}$. The compound (TMTTF $)_{2} \mathrm{ReO}_{4}$ does not show any appreciable temperature shift of $T_{\mathrm{AO}}$ for pressure up to $p=5 \mathrm{kbar}$; only after $T_{\mathrm{AO}} \approx T_{\mathrm{CO}}$ the $\mathrm{AO}$ temperature suddenly starts to move up quite fast with pressure, $\mathrm{cf}$. Figure 15. At his point it is not clear whether this shift is due to the combination of charge and anion order or whether a new superstructure occurs, as in the case of (TMTSF) ${ }_{2} \mathrm{ReO}_{4}$ [62]. Ongoing spectroscopic investigations will clarify this point. In (TMTTF) ${ }_{2} \mathrm{BF}_{4}$ our data indicate the survival of both $\mathrm{CO}$ an $\mathrm{AO}$ under pressure. It would be of interest to follow the ordering temperatures to even higher pressure; we speculate that $T_{\mathrm{AO}}$ might move up in temperature as soon as it merges with $T_{\mathrm{CO}}$; 
similar to (TMTTF) ${ }_{2} \mathrm{ReO}_{4}$. This would underline the competing influence of $\mathrm{CO}$ on the AO transition, as suggested by our transport data.
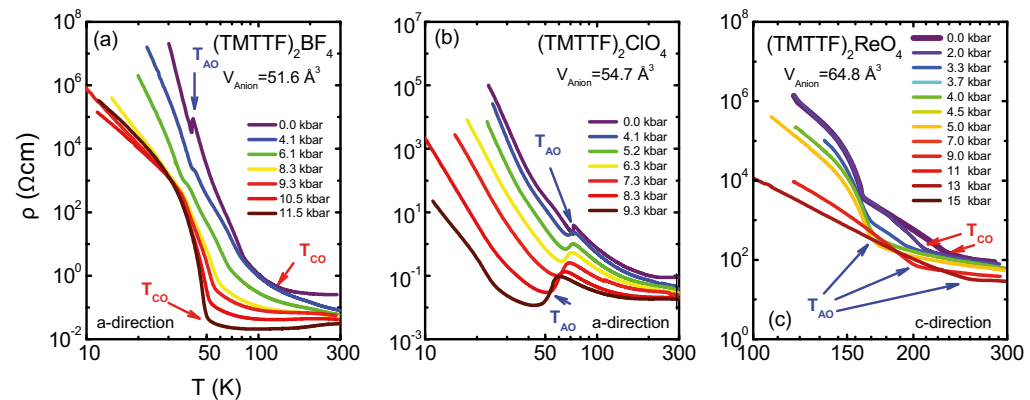

Figure 16. The temperature dependent resistivity for the (TMTTF $)_{2} X$ salts with tetrahedral anions. The three compounds are sorted for increasing anion size from left to right. (a) (TMTTF $)_{2} \mathrm{BF}_{4}$ is the compound with the smallest anion: $V_{\text {Anion }}=51.6 \AA^{3}$. It shows both transitions in the dc resistivity, charge and anion ordering. (b) In (TMTTF) ${ }_{2} \mathrm{ClO}_{4}$ with $V_{\text {Anion }}=54.7 \AA^{3}$ only anion ordering was detected but no separate charge order. (c) (TMTTF) ${ }_{2} \mathrm{ReO}_{4}$ has by far the largest tetrahedral anion with $V_{\text {Anion }}=64.8 \AA^{3}$; it also has the highest charge and anion ordering temperatures.

In order to gain further insight into the structural aspects on charge order, Figure 17 displays the distance between the anions and the TMTTF molecule. In the first frame $T_{\mathrm{CO}}$ is plotted as a function of the shortest distance between the anion-ligand ( $\mathrm{F}$ or $\mathrm{O}$ ) and the sulfur atom on the organic molecule for Fabre salts with tetrahedral as well as octahedral anions. In panel $b$ we consider the distance of the anion center, defined by the $\mathrm{B}, \mathrm{Cl}, \mathrm{Re}, \mathrm{P}, \mathrm{As}$ or Sb-atoms, to the sulfur atom of the organic molecule. Finally, in Figure 17c we plot the distance of the anion-ligand to the nearest carbon atom. In those cases, $T_{\mathrm{CO}}$ behaves differently for $(\mathrm{TMTTF})_{2} \mathrm{X}$ with octahedral and tetrahedral $X$. It seems that the distance between the central atom of the anion and the nearest carbon atom reflects the cavity size or the anion size better than any coupling mechanism. The (theoretical) anion volume, listed in Table 1, follows the relation [52]: $V_{\text {Anion }}^{\mathrm{BF}_{4}}<V_{\text {Anion }}^{\mathrm{ClO}_{4}}<V_{\text {Anion }}^{\mathrm{ReO}_{4}}<V_{\text {Anion }}^{\mathrm{PF}_{6}}<V_{\text {Anion }}^{\mathrm{AsF}_{6}}<V_{\text {Anion. The correlations }}^{\mathrm{SbF}_{6}}$. The plotted in Figure 17 might help to understand some of the effects, but further temperature- and pressure-dependent investigations are needed to completely clarify the situation.
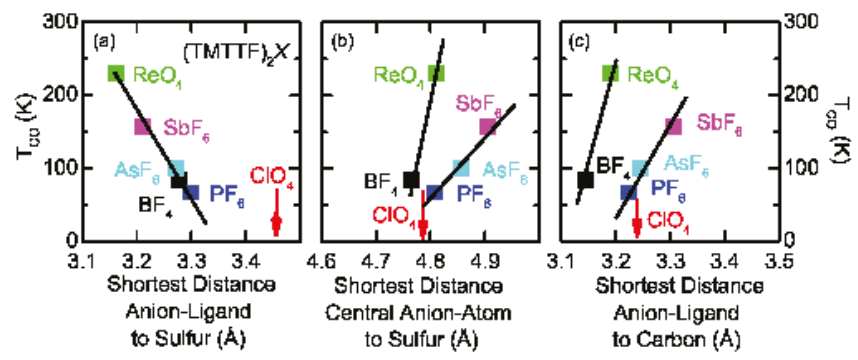

Figure 17. Dependence of the charge order temperature $T_{\mathrm{CO}}$ for various Fabre salts (TMTTF) ${ }_{2} X$ with different $X$ as indicated. (a) Effect on the shortest distance between anion-ligand and TMTTF sulfur atom. (b) Change of $T_{\mathrm{CO}}$ with the distance between the central atom of the anion and the sulfur atom. (c) Influence on the shortest distance from the anion-ligand to the nearest carbon atom. The value for (TMTTF) ${ }_{2} \mathrm{ClO}_{4}$, which shows no $\mathrm{CO}$, is indicated by a red arrow. All distances were determined at ambient conditions. 


\section{Dielectric Spectroscopy}

Dielectric spectroscopy is a sensitive tool to detect changes in the charge degrees of freedom that have revealed evidence for $\mathrm{CO}$ in the (TMTTF $)_{2} \mathrm{X}$ salts [84,85]. Numerous studies performed over the years $[30-36,86]$ identified a Curie-like peak of the permittivity $\epsilon_{a}(T)$ at $T_{\mathrm{CO}}$ reaching huge values up to $10^{6}$ for $E \| a$. While the peak position is frequency-independent up to $1 \mathrm{MHz}$, its amplitude decreases with rising frequency. The Curie-behavior has drawn a lot of attention $[84,85,87-90]$ because it is characteristic for ferroelectricity. Its origin is considered to be mainly electronic, and thus referred to as "electronic ferroelectricity" [33,91-93]. Nevertheless, the observation of a hysteresis in the electric polarization $P(E)$ is impeded by the high conductivity of the (TMTTF) ${ }_{2} X$ salts compared with canonical ferroelectrics.

Most of the studies dealt with Fabre salts of centrosymmetric anions, and there is only a single report on (TMTTF) ${ }_{2} \mathrm{ReO}_{4}$ and (TMTTF $)_{2} \mathrm{BF}_{4}$ [36]. In the first compound, the $\mathrm{AO}$ transition is evidenced by a sharp drop of permittivity at $T_{\mathrm{AO}}$; it is explained by the formation of TMTTF tetramers with a 0110 charge pattern, which are less polarizable compared to dimers [23]. Interestingly, for (TMTTF) ${ }_{2} \mathrm{BF}_{4}$ a small peak is revealed at $T_{\mathrm{AO}}$ with a frequency-dependent amplitude followed by a monotonous decrease of $\epsilon_{a}^{\prime}(T)$ upon further cooling.

\subsection{Results}

We have conducted frequency-dependent measurements of the real part of the dielectric constant $\hat{\epsilon}_{b^{\prime}}=\epsilon_{b^{\prime}}^{\prime}-\mathrm{i} \epsilon_{b^{\prime}}^{\prime \prime}$ with the electric field applied perpendicular to the chain direction, $E \| b^{\prime}$, at different temperatures and ambient pressure. The findings for (TMTTF) $)_{2} X$ with $X=\mathrm{BF}_{4}, \mathrm{ClO}_{4}$ and $\mathrm{ReO}_{4}$ taken at $f=1,10,100 \mathrm{kHz}$, and $1 \mathrm{MHz}$ are plotted in Figure 18. For (TMTTF) ${ }_{2} \mathrm{BF}_{4}$ and (TMTTF) ${ }_{2} \mathrm{ReO}_{4}$ the charge and anion-order transitions are clearly observed with exactly the same signatures as reported for measurements along the stacking axis [36].

In the case of (TMTTF $)_{2} \mathrm{BF}_{4}$, this corresponds to a peak at $T_{\mathrm{CO}} \approx 80 \mathrm{~K}$, which is reduced in amplitude as frequency increases. For $f=100 \mathrm{kHz}$ the peak height of 3000 is lower by factor of 200 compared to a value of $7 \cdot 10^{5}$ observed for $E \| a$ [36]. When the temperature is lowered, $\epsilon_{b^{\prime}}^{\prime}(T)$ drops until the $\mathrm{AO}$ transition, which is observed here by another peak around $36 \mathrm{~K}$, in accord with the findings for $E \| a$. In contrast to the feature $T_{\mathrm{CO}}$, the $\mathrm{AO}$ peak is strongly suppressed with increasing frequency. While for $f=10 \mathrm{kHz}$, a value of $\epsilon_{b^{\prime}}^{\prime}=20$ is observed, the peak cannot be identified above $1 \mathrm{MHz}$.

For the $\mathrm{ReO}_{4}$ salt, the $\mathrm{CO}$ peak appears at $228 \mathrm{~K}$ and exhibits a maximum of $4 \cdot 10^{4}$ when measured at $f=100 \mathrm{kHz}$ perpendicular to the chains; this is one order of magnitude lower than the peak found for $E \| a$ [36]. With decreasing temperature $\epsilon_{b^{\prime}}^{\prime}(T)$ continuously decreases by one order of magnitude at $T_{\mathrm{AO}}=157 \mathrm{~K}$, where a step is observed with a frequency-dependent height. Upon further cooling, $\epsilon_{b^{\prime}}^{\prime}(T)$ approaches a constant value around 2000, which is slightly dependent on frequency.

(TMTTF $)_{2} \mathrm{ClO}_{4}$ exhibits a strong and monotonous decrease in $\epsilon_{b^{\prime}}^{\prime}(T)$ from room temperature down to about $100 \mathrm{~K}$. At $T_{\mathrm{AO}}$ an abrupt step with a frequency-independent height up to 50 marks the anion order. At lower temperatures $\epsilon_{b^{\prime}}^{\prime}(T)$ changes in shape forming a shoulder-like drop of one order of magnitude. Interestingly, for higher frequencies this drop occurs at higher temperatures reminiscent of a dielectric relaxation. 

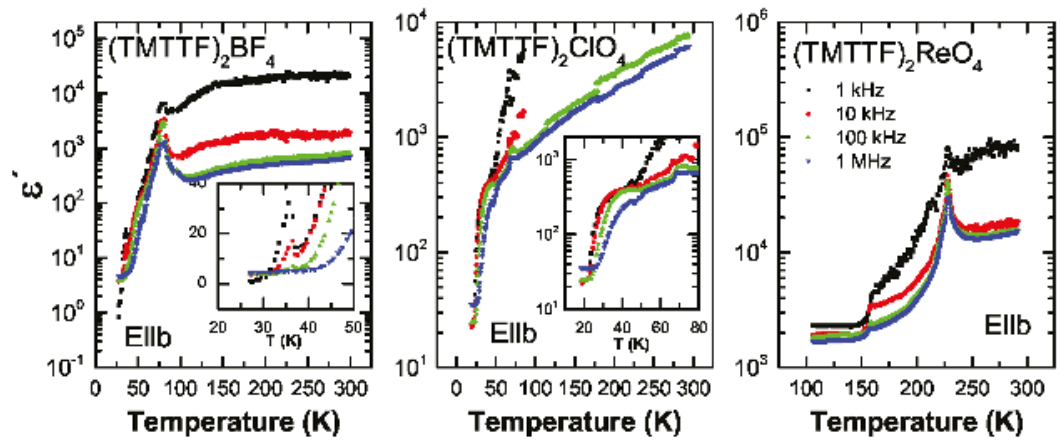

Figure 18. Temperature dependence of the real part of the dielectric permittivity $\epsilon^{\prime}(T)$ measured with $E \| b^{\prime}$ for $(\mathrm{TMTTF})_{2} \mathrm{X}$ with $\mathrm{X}=\mathrm{BF}_{4}, \mathrm{ClO}_{4}$ and $\mathrm{ReO}_{4}$ at different frequencies as indicated. The insets emphasize the changes close $T_{\mathrm{AO}}$ and below.

\subsection{Analysis}

In general, a ferroelectric phase transition is accompanied by a soft mode strongly slowing down near the critical temperature. It is usually observed in optical, infrared or microwave spectroscopy for a displacive phase transition; or by a relaxation mode at lower frequencies, as seen in disordered systems $[94,95]$. Hence, investigating the relaxation time can provide valuable insight into the dynamics related to the transitions. The Cole-Cole or generalized Debye model is a well established and useful description to analyze the frequency-dependent complex permittivity

$$
\hat{\epsilon}(\omega)-\epsilon_{\text {inf }}=\frac{\Delta \epsilon}{1+\left(\mathrm{i} \omega \tau_{0}\right)^{1-\alpha}},
$$

where $\tau_{0}$ is the relaxation time, $\omega=2 \pi f$ the angular frequency of the applied electric ac-field, $1-\alpha$ a parameter describing symmetric broadening of the loss peak and $\Delta \epsilon=\epsilon_{\text {static }}-\epsilon_{\text {inf }}$ the dielectric strength with $\epsilon_{\text {static }}$ and $\epsilon_{\text {inf }}$ the values for low and high frequencies, respectively. The deviation from the Debye model $(\alpha=0)$ is attributed to the distribution of $\tau_{0}$ arising from disorder [84]. If the materials are not completely insulating, but exhibit a considerable electronic background, such as the (TMTTF) ${ }_{2} \mathrm{X}$ salts, the dc-conductivity has to be taken into account when the dielectric loss is determined:

$$
\epsilon^{\prime \prime}(\omega)=\frac{\sigma^{\prime}(\omega)-\sigma_{D C}}{\omega \epsilon_{0}} .
$$

As an example for (TMTTF) ${ }_{2} X$ salts with tetrahedral anions investigated here, Figure 19 shows the dielectric spectra of (TMTTF) ${ }_{2} \mathrm{ClO}_{4}$ in the range of the relaxational mode for different temperatures close to the $\mathrm{AO}$ transition. The real part of the dielectric constant $\epsilon_{b^{\prime}}^{\prime}(\omega)$ is plotted in the upper panel: with increasing frequency it exhibits a step that shift towards higher frequencies as the temperature increases. The point of inflection marks the relaxation rate $1 / \tau_{0}$ and corresponds to the maximum of the broad peak in the imaginary part $\epsilon_{b^{\prime}}^{\prime \prime}(\omega)$. The solid lines represent fits according to Equation (1) and agree well with the expected behavior. 


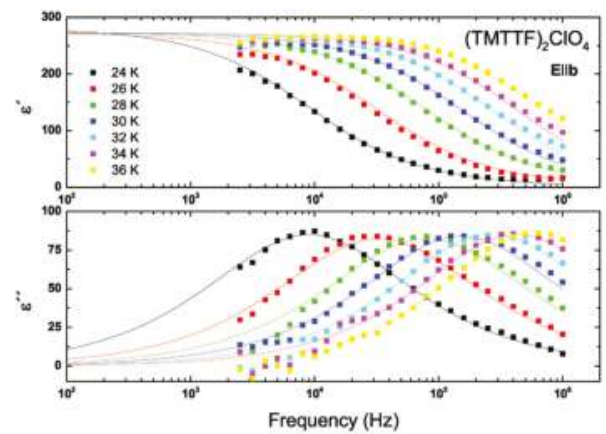

Figure 19. Frequency dependence of the permittivity $\hat{\epsilon}(\omega)=\epsilon^{\prime}(\omega)-\mathrm{i} \epsilon^{\prime \prime}(\omega)$ of (TMTTF) ${ }_{2} \mathrm{ClO}_{4}$ measured with $E \| b^{\prime}$ at various temperatures close to the $\mathrm{AO}$ transition. The real part $\epsilon^{\prime}(\omega)$ exhibits a roll-off that shifts to higher frequencies with increasing temperature. This behavior corresponds to a peak in $\epsilon^{\prime \prime}(\omega)$ as shown in the lower panel. The solid lines represent fits according to the generalized Debye model (1).
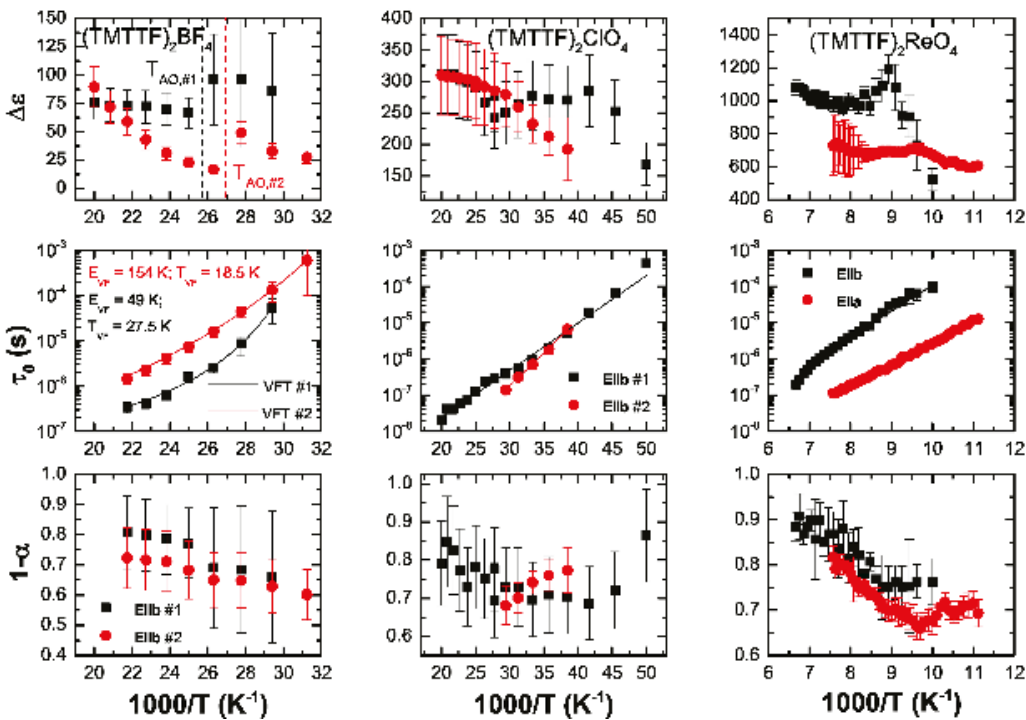

Figure 20. Temperature dependence of the parameters used to fit the dielectric spectra of (TMTTF $)_{2} \mathrm{BF}_{4}$, $(\mathrm{TMTTF})_{2} \mathrm{ClO}_{4}$ and $(\mathrm{TMTTF})_{2} \mathrm{ReO}_{4}$ by the Cole-Cole model (1). The upper row shows the Arrhenius plots of the dielectric strength $\Delta \epsilon$, followed by the relaxation time $\tau_{0}$ and the broadening parameter $(1-\alpha)$ in the lower row. For $\mathrm{X}=\mathrm{BF}_{4}$ and $\mathrm{ClO}_{4}$ the data on two crystals each (indicated by black and red symbols) are taken for $E \| b^{\prime}$. For $X=\mathrm{ReO}_{4}$ we show data measured along the $a$ and $b^{\prime}$-directions. Most important, in (TMTTF $)_{2} \mathrm{ClO}_{4}$ and $(\mathrm{TMTTF})_{2} \mathrm{ReO}_{4}$ the relaxation time $\tau_{0}$ exhibits an activated behavior; the obtained activation energies resembles the gap previously measured in dc transport [4]. For $(\mathrm{TMTTF})_{2} \mathrm{BF}_{4}, \tau_{0}(T)$ increases with decreasing $T$ in a fashion best described by the empirical Vogel-Fulcher-Tammann relation.

The parameters $\Delta \epsilon, \tau_{0}$ and $1-\alpha$ extracted from the Cole-Cole fit of the dielectric spectra are plotted in Figure 20 as a function of inverse temperature. The data have been recorded along the $b^{\prime}$ direction of the $(\mathrm{TMTTF})_{2} \mathrm{BF}_{4},(\mathrm{TMTTF})_{2} \mathrm{ClO}_{4}$ and $(\mathrm{TMTTF})_{2} \mathrm{ReO}_{4}$ salts. We confined ourselves to this temperature region because our experimental frequency window is restricted to $1 \mathrm{MHz}$. For (TMTTF) ${ }_{2} \mathrm{BF}_{4}$ the 
relaxation mode moves through this frequency window for $50 \mathrm{~K}>\mathrm{T}>30 \mathrm{~K}$, which includes the AO transition. Here $\Delta \epsilon$ exhibits a small peak around $T=38 \mathrm{~K}$ and $36 \mathrm{~K}$ for the two different samples, labeled 1 (black) and 2 (red symbols). These steps correspond to the features observed in $\hat{\epsilon}(T)$ (Figure 18) and are ascribed to the AO transition. The broadening parameter $(1-\alpha)$ decreases upon cooling indicating an increased cooperativity in the relaxation. Interestingly, $\tau_{0}(T)$ rises with lowering temperature in a fashion best described by the empirical Vogel-Fulcher-Tammann relation

$$
\tau_{0}=\tau_{\mathrm{VF}} \exp \left\{\frac{E_{\mathrm{VF}}}{T-T_{\mathrm{VF}}}\right\},
$$

which is known as a good parametrization for the slowing down of molecular motion in disordered systems and the glass-like freezing of dipolar order in relaxor ferroelectrics. The corresponding energy $E_{\mathrm{VF}}$ can be interpreted as a temperature-dependent activation energy for reorientational motion. $T_{\mathrm{VF}}$ denotes the temperature where $\tau_{0}$ diverges, and $\tau_{\mathrm{VF}}$ the time scale for the ac response in the high-temperature limit. The glass temperature $T_{G}$ is defined as the temperature where $\tau_{0}=100 \mathrm{~s}$. The obtained fit parameters are listed in Table 2. There is a considerable difference in the values for $E_{\mathrm{VF}}$ and $\tau_{\mathrm{VF}}$, whereas there is a minor deviation for $T_{G}$ which we attribute to different cooling rates during the measurements.

Table 2. Parameters obtained by fitting the temperature-dependent dielectric relaxation time $\tau_{0}(T)$ of (TMTTF ${ }_{2} \mathrm{BF}_{4}$ with the Vogel-Fulcher-Tammann relation. $E_{\mathrm{VF}}$ is the activation energy, $T_{\mathrm{VF}}$ denotes the Vogel-Fulcher temperature, and $\tau_{\mathrm{VF}}$ the corresponding time scale. The glass temperature is given by $T_{G}$.

\begin{tabular}{ccc}
\hline $\mathbf{( T M T T F}_{\mathbf{2}} \mathbf{B F}_{\mathbf{4}}$ & Sample 1 & Sample 2 \\
\hline$E_{\mathrm{VF}}(\mathrm{K})$ & $49 \pm 7$ & $153.5 \pm 11.5$ \\
$T_{\mathrm{VF}}(\mathrm{K})$ & $27.5 \pm 0.5$ & $18.5 \pm 0.5$ \\
$\tau_{\mathrm{VF}}(\mathrm{s})$ & $(2.5 \pm 1.2) \cdot 10^{-8}$ & $(6.1 \pm 2.5) \cdot 10^{-9}$ \\
\hline$T_{G}(\mathrm{~K})$ & 30 & 25 \\
\hline
\end{tabular}

For (TMTTF $)_{2} \mathrm{ClO}_{4}$ the mode can be observed in our frequency window between $T=20 \mathrm{~K}$ and $50 \mathrm{~K}$, which is below $\mathrm{T}_{A O}$, but in the range of the dispersive shoulder-like drop in $\epsilon_{b^{\prime}}^{\prime}(T)$ shown in Figure 18. For the second crystal, we observe a monotonous decrease of $\Delta \epsilon$ with falling temperature, in agreement with the behavior of $\epsilon_{b^{\prime}}^{\prime}(T)$. There is an additional small bump around $T=23 \mathrm{~K}$ for sample 1. In both specimens an activated behavior in $\tau_{0}$ is observed with activation energies of $300 \mathrm{~K}$ and $420 \mathrm{~K}$, respectively. The fit parameters are listed in Table 3. We can compare our findings with the energy gap of $(440 \pm 60) \mathrm{K}$ derived from dc-transport measurements reported in Reference [4].

Table 3. Parameters of the activated behavior of $\tau_{0}$ for (TMTTF $)_{2} \mathrm{ClO}_{4}$ derived from dielectric measurements of two different crystals.

\begin{tabular}{ccc}
\hline (TMTTF) $_{\mathbf{2}} \mathbf{C l O}_{\mathbf{4}}$ & Sample 1 & Sample 2 \\
\hline$E_{\text {act }}(\mathrm{K})$ & $300 \pm 10$ & $420 \pm 8$ \\
$\tau_{\text {act }}(\mathrm{s})$ & $(5.7 \pm 1.9) \cdot 10^{-11}$ & $(6.0 \pm 1.7) \cdot 10^{-13}$ \\
\hline
\end{tabular}

The relaxation mode in (TMTTF $)_{2} \mathrm{ReO}_{4}$ slows down more quickly; it enters our experimental frequency window for $90 \mathrm{~K}<T<140 \mathrm{~K}$, right below the $\mathrm{AO}$ transition. Surprisingly, there is a small peak in $\Delta \epsilon$ at $T=112$ and $105 \mathrm{~K}$ for $E \| b^{\prime}$ and $E \| a$, respectively, which superimposes a gradual increase with temperature. No corresponding counterpart is observed in $\epsilon_{b^{\prime}}^{\prime}(T)$. For both directions, $\tau_{0}$ follows an activated behavior; the fit parameters are listed in Table 4 for the two polarization directions. 
From dc transport a temperature-dependent energy gap was derived [4] that saturates at (1560 \pm 80$) \mathrm{K}$ in the limit of $T \rightarrow 0$ for all crystal directions.

Table 4. Parameters of the activated behavior observed in $\tau_{0}$ for two crystal directions of $(\mathrm{TMTTF})_{2} \mathrm{ReO}_{4}$.

\begin{tabular}{ccc}
\hline $\mathbf{~ ( T M T T F ) ~}_{\mathbf{2}} \mathbf{R e O}_{\mathbf{4}}$ & $\boldsymbol{E} \| \boldsymbol{a}$ & $\boldsymbol{E} \| \boldsymbol{b}^{\prime}$ \\
\hline$E_{\text {act }}(\mathrm{K})$ & $1372 \pm 4$ & $1651 \pm 27$ \\
$\tau_{\text {act }}(\mathrm{s})$ & $(3.1 \pm 0.1) \cdot 10^{-12}$ & $(7.3 \pm 1.6) \cdot 10^{-12}$ \\
\hline
\end{tabular}

Since for $(\mathrm{TMTTF})_{2} \mathrm{ClO}_{4}$ and $(\mathrm{TMTTF})_{2} \mathrm{ReO}_{4}$ the activation energies of the relaxation time $\tau_{0}$ agree well with the values obtained by dc-transport, we conclude that the relaxation is determined by the free charge carries responsible for the dc conduction, which freeze out at low temperatures.

\subsection{Discussion}

For the (TMTTF $)_{2} X$ salts, the peak in $\epsilon^{\prime}$ at $T_{\mathrm{CO}}$ obeys Curie's law as established for canonical order-disorder ferroelectrics [84]; it is attributed to a combination of charge disproportionation and ionic displacements [32,33].

As far as the anisotropy is concerned, the ratio of the peak amplitudes $\epsilon_{a}^{\prime} / \epsilon_{b^{\prime}}^{\prime}$ probed at $f=100 \mathrm{kHz}$ is about 200 and 1000 for $(\mathrm{TMTTF})_{2} \mathrm{BF}_{4}$ and $(\mathrm{TMTTF})_{2} \mathrm{ReO}_{4}$, respectively, higher than the anisotropy of the dc resistivity at $T_{\mathrm{CO}}$ [4]. It is interesting to compare our findings with the report of de Souza et al. [96] on the Fabre sals with octahedral anions. As reproduced in Figure 21, they found a strong peak in $\epsilon_{c}^{\prime}$ at $T_{\mathrm{CO}}$ similar to previous reports on $\epsilon_{a}^{\prime}(T)$ [30-32,34-36] and $\epsilon_{b^{\prime}}^{\prime}$ presented here (Figure 18). The peak value is sample-dependent and in general by several orders of magnitude lower compared the value observed along the stacks. While in the $a$-direction the permittivity is affected by critical fluctuations that become important when approaching the ordering transition in one dimension, the response in the perpendicular direction probes the involvement of ionic displacements and the stabilization of the three-dimensional charge pattern upon $\mathrm{CO}$.

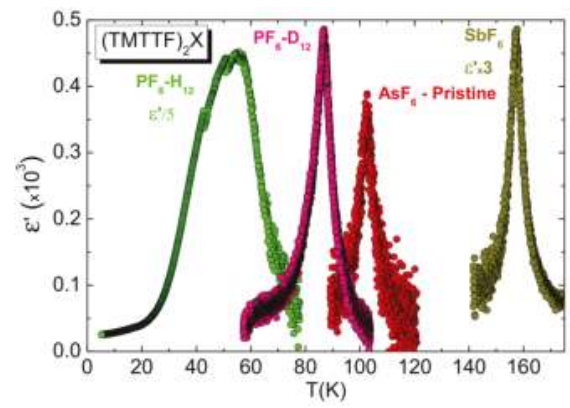

Figure 21. Temperature dependence of the dielectric constant of several (TMTTF) ${ }_{2} X$ salts with octahedral anions: $\mathrm{H}_{12}-(\mathrm{TMTTF})_{2} \mathrm{PF}_{6}$ and $\mathrm{D}_{12}-(\mathrm{TMTTF})_{2} \mathrm{PF}_{6}$, (TMTTF $)_{2} \mathrm{AsF}_{6}$ and $(\mathrm{TMTTF})_{2} \mathrm{SbF}_{6}$. The experiments have been performed along the $c^{*}$-axis perpendicular to the chain direction [96]. The maximum in $\epsilon_{\mathcal{C}}^{\prime}(T)$ is located at $T_{\mathrm{CO}}$, similar to measurements along the other orientations $\epsilon_{a}^{\prime}(T)[30-32,34-36]$ and $\epsilon_{b^{\prime}}^{\prime}(T)$. The peak value is lower by several orders of magnitude compared to $\epsilon_{a}^{\prime}$, similar to the anisotropy determined in dc transport [4].

The rather high values of $\epsilon^{\prime}(T)$ at room temperature, found in Figure 18, are attributed to the slight dimerization of the TMTTF molecules and the resulting enhanced polarizability, which scales with $\epsilon^{\prime} \approx\left(\omega_{p} / \Delta\right)^{2}$, wherein $\omega_{p}$ is the plasma frequency and $\Delta$ the transport gap [97]. The temperature dependence of $\Delta(T)$ for $E \| b^{\prime}$ is found [4] constant for (TMTTF) ${ }_{2} \mathrm{ReO}_{4}$ from $T=300 \mathrm{~K}$ to $250 \mathrm{~K}$, 
whereas it is decreasing for (TMTTF) ${ }_{2} \mathrm{ClO}_{4}$ and $(\mathrm{TMTTF})_{2} \mathrm{BF}_{4}$; the latter is explained by the decrease in dimerization. Accordingly, the reduction of $\epsilon_{b^{\prime}}^{\prime}(T)$ upon cooling observed here is induced by the decrease of $\omega_{p}(T)$ due to the freezing out of mobile charge carriers. This can be confirmed by detailed optical investigation of all (TMTTF $)_{2} X$ salts.

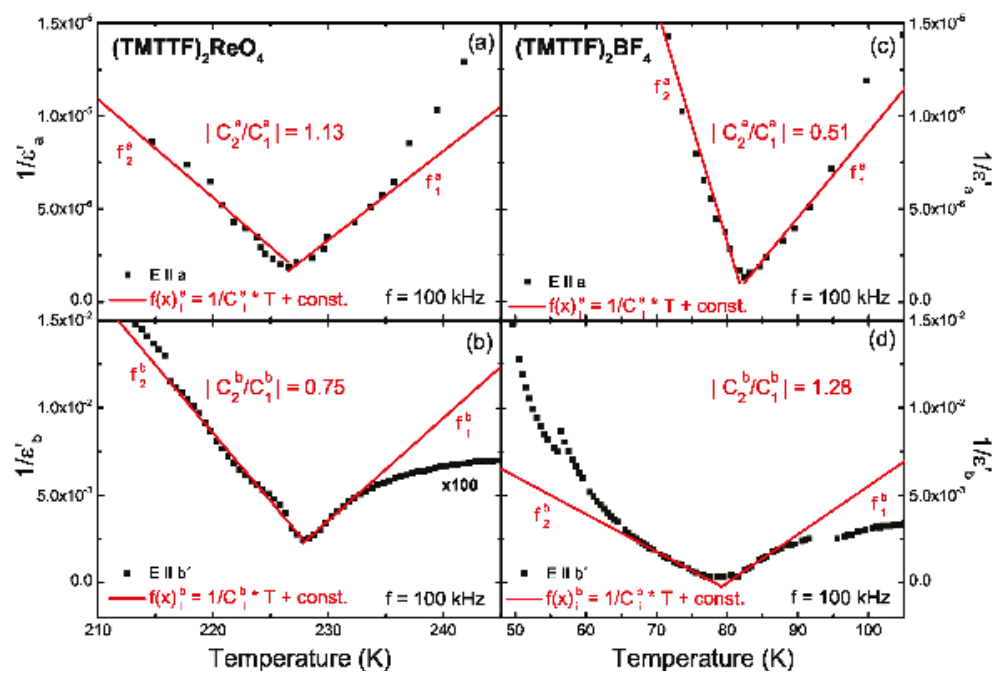

Figure 22. Temperature dependence of $(\mathrm{a}, \mathrm{c}) 1 / \epsilon_{a}^{\prime}(T)$ (data from [36]) and (b,d) $1 / \epsilon_{b^{\prime}}^{\prime}(T)$ for $(\mathrm{a}, \mathrm{c}$ ) $(\mathrm{TMTTF})_{2} \mathrm{BF}_{4}$ and $(\mathrm{b}, \mathrm{d})(\mathrm{TMTTF})_{2} \mathrm{ReO}_{4}$. There is good agreement with the Curie-Weiss behavior (red solid lines) for $T \leq T_{\mathrm{CO}}$, except for (TMTTF) ${ }_{2} \mathrm{BF}_{4}$ along $b^{\prime}$-direction, where the peak in $\epsilon_{b^{\prime}}^{\prime}(T)$ is somewhat rounded. For canonical second-order ferroelectric transitions, mean-field theory predicts that the slopes on both side differ by a factor of 2 [94]. The deviation from this expected behavior is usually attributed to structural disorder $[36,86]$.

The Curie-Weiss behavior $\epsilon^{\prime}(T)=\frac{C}{T-T_{\mathrm{CO}}}$ is nicely illustrated in Figure 22 where $1 / \epsilon_{a}^{\prime}(T)$ and $1 / \epsilon_{b^{\prime}}^{\prime}(T)$ are plotted for (TMTTF $)_{2} \mathrm{BF}_{4}$ and (TMTTF $)_{2} \mathrm{ReO}_{4}$. In particular for $T \leq T_{\mathrm{CO}}$ the agreement is rather good, except for (TMTTF) ${ }_{2} \mathrm{BF}_{4}$ along the $b^{\prime}$-direction (cf. Figure 18). Above $T_{\mathrm{CO}}$ we observe rather large deviations from the expected behavior for both salts.

At this point it is important to mention that the amplitude and broadening of the $\mathrm{CO}$ peak in $\epsilon_{a}^{\prime}(T)$ is known to be sample-dependent $[36,86]$ and hence, in case a deviation from the Curie-behavior is observed below $\mathrm{T}_{\mathrm{CO}}$, it is ascribed to structural disorder. The latter also explains, why the slope ratio $\left|C_{2}^{a, b} / C_{1}^{a, b}\right|$ deviates in some reports $[86,96]$ from the expected value 0.5 , which is predicted by mean field theory for a canonical second-order phase transition [94].

The ratio of the slope obtained along the $b^{\prime}$-direction does not necessarily infer a minor sample quality. The CO peak is weaker along the $b^{\prime}$-direction, implying that at high temperatures the contribution of dimerization to the background could be appreciable. Similar deviations from this mean-field dependence are observed for $(\mathrm{TMTTF})_{2} \mathrm{AsF}_{6}$ and $(\mathrm{TMTTF})_{2} \mathrm{PF}_{6}$ when looking along the $c^{*}$-direction [96]. Additional ionic contributions are more pronounced in the perpendicular polarizations and should be investigated in more detail in future.

For (TMTTF $)_{2} \mathrm{ReO}_{4}$ the abrupt drop of the permittivity at $T_{\mathrm{AO}}$ evidences the formation of TMTTF tetramers with a 0110 charge pattern; they are less polarizable compared to dimers. Interestingly, in (TMTTF) ${ }_{2} \mathrm{BF}_{4}$ a small peak is observed at $T_{\mathrm{AO}}$, followed by the drop due to tetramerization. The absence of a comparable peak in the other two salts may be due to screening by free charge carriers. In (TMTTF) ${ }_{2} \mathrm{BF}_{4}$ the resistivity at $T_{\mathrm{AO}}$ is three orders of magnitudes higher compared to (TMTTF) ${ }_{2} \mathrm{ReO}_{4}$, for instance [4]. In the case of (TMTTF $)_{2} \mathrm{ClO}_{4}$ there is no CO for $T>T_{\mathrm{AO}}$. Below $T_{\mathrm{AO}}$, around $T=50 \mathrm{~K}$, 
there is a second drop which shifts to higher temperature for increasing frequency, reminiscent of a dielectric relaxation. Note that in this temperature range, ESR measurements reveal a drop of the spin susceptibility [73], which is attributed to the formation of a spin singlet-triplet gap upon tetramerization, and optical investigations [23] prove the growth of charge disproportionation with a 0110 charge pattern. Further investigations have to fully clarify the connection to the observed dielectric relaxation. The observation of a corresponding feature in (TMTTF) ${ }_{2} \mathrm{BF}_{4}$ and $(\mathrm{TMTTF})_{2} \mathrm{ReO}_{4}$ might be obscured by a strong background due to the $\mathrm{CO}$ peak.

In (TMTTF $)_{2} \mathrm{AsF}_{6}$ the mean relaxation time $\tau_{0}(T)$ contains a peak at $T_{\mathrm{CO}}$ due to the softening of the oscillating mode concomitant to the ferroelectric transition and an activated behavior with an activation energy similar to the gap obtained by dc transport $[35,86]$. Brazovskii and coworkers [89] report an additional relaxation mode in (TMTTF) ${ }_{2} \mathrm{AsF}_{6}$ for $T<T_{\mathrm{CO}}$, which is ascribed to slow oscillations of pinned ferroelectric domains. Recent Raman spectroscopy evidences the presence of neutral TMTTF ${ }^{0}$ and ionized $\mathrm{TMTTF}^{-1}$ molecules, which are assigned to charged domain walls [56]. It is worthwhile to mention that in the two-dimensional charge-transfer salts $\kappa$-(BEDT-TTF $)_{2} X$, with $X=\mathrm{Cu}\left[\mathrm{N}(\mathrm{CN})_{2}\right] \mathrm{Cl}$ [98], $\mathrm{Cu}_{2}(\mathrm{CN})_{3}[99,100]$ and $\mathrm{Ag}_{2}(\mathrm{CN})_{3}$ [101], as well as in $\alpha$-(BEDT-TTF $)_{2} \mathrm{I}_{3}[102,103]$, charge domain walls are also considered to give rise to an anomalous dielectric response in the audio- and radio-frequency range. For (TMTTF) ${ }_{2} \mathrm{ClO}_{4}$ and (TMTTF) ${ }_{2} \mathrm{ReO}_{4}$ the activation energies of the relaxation time $\tau_{0}(T)$ agree well with dc-transport measurements, in accord with the observations for $(\mathrm{TMTTF})_{2} \mathrm{PF}_{6}[31]$ and $(\mathrm{TMTTF})_{2} \mathrm{AsF}_{6}[35,86]$. This indicates that the relaxation is determined by the free charge carries responsible for the dc-conduction, which freeze out at low temperatures. We assume that crystal defects and charged impurities locally change the charge pattern due to anion order, giving rise to domains walls. If charge carriers are thermally activated above the transport gap, which is composed of the contributions due to dimerization, charge and anion order [4], they move along the TMTTF chains adding to the dc-conductivity. This changes the charge pattern and results in an effective shift of the domain walls, explaining why the activation energy of the relaxation time corresponds to the dc-transport gap.

For $(\mathrm{TMTTF})_{2} \mathrm{BF}_{4}$ with $T_{\mathrm{AO}}=41.5 \mathrm{~K}$, the relaxation mode is observed for $50 \mathrm{~K}>T>30 \mathrm{~K}$ and the relaxation time $\tau_{0}(T)$ follows the empirical Vogel-Fulcher-Tammann relation. In this temperature range, the resistivity is rather high and transport is ascribed to hopping conduction [4]. The drastic decreased number of free charge carriers results in glass-like freezing of domain walls, as evidenced by the observation of the Vogel-Fulcher behavior. Surprisingly, the results obtained here for $T<T_{\mathrm{AO}}$ on the salts with tetrahedral anions agree well with the ones obtained for $T<T_{\mathrm{CO}}$ on the centrosymmetric anions, indicating that the underlying order determining the charge pattern is of minor role for the relaxation dynamics.

\section{Conclusions}

Our pressure-dependent DC-transport measurements yield valuable information on the influence of the lattice on the ordering phenomena in the quasi one-dimensional charge-transfer salts (TMTTF) $2 \mathrm{X}$ with tetrahedral anions $\mathrm{X}=\mathrm{BF}_{4}, \mathrm{ClO}_{4}$ and $\mathrm{ReO}_{4}$. We observe a decrease of the charge ordering temperature $T_{\mathrm{CO}}$ upon pressure for $(\mathrm{TMTTF})_{2} \mathrm{BF}_{4}$ and (TMTTF) ${ }_{2} \mathrm{ReO}_{4}$; similar to the observation reported for salts with octahedral anions [104]. In contrast to CO, the pressure dependence of $A O$ differs for each salt such that the construction of a generic phase diagram of (TMTTF) $2 X$ salts with tetrahedral anions is not possible. In (TMTTF $)_{2} \mathrm{BF}_{4}, \mathrm{CO}$ is visible up to the highest pressure applied with $T_{\mathrm{CO}}(p=8.8 \mathrm{kbar})=47.5 \mathrm{~K}$ whereas the signature indicating $\mathrm{AO}$ dissolves around $34.5 \mathrm{~K}$ at a pressure of $5.3 \mathrm{kbar}$. We call for structural studies that will yield information on the actual arrangement of the anions.In (TMTTF) ${ }_{2} \mathrm{ReO}_{4}, \mathrm{AO}$ is enhanced upon applying pressure with an increase of $T_{\mathrm{AO}}$, at around $3.0 \mathrm{kbar} T_{\mathrm{AO}}=T_{\mathrm{CO}}$ is reached and for pressures above, CO seems to be fully suppressed and the $\mathrm{AO}$ shifted up to $277 \mathrm{~K}$ at $p=14.7 \mathrm{kbar}$. Interestingly in (TMTTF) ${ }_{2} \mathrm{ClO}_{4}$, the jump-like feature indicating $\mathrm{AO}$ shifts to lower temperatures and significantly broadens. For $p>10.2 \mathrm{kbar}$, we observe a second peak evolving right at $T_{\mathrm{AO}}$ whose origin is still unclear. The fact that no $\mathrm{CO}$ is detected in 
(TMTTF $)_{2} \mathrm{ClO}_{4}$ is probably the most puzzling observation that calls for explanation. We attribute the absence of $\mathrm{CO}$ in (TMTTF) ${ }_{2} \mathrm{ClO}_{4}$ to the polarizability of the anion. $\mathrm{ClO}_{4}$ is less polarizable because $\mathrm{Cl}$ has an electronegativity comparable to $\mathrm{O}$. Consequently, the bonds within the anion are more covalent making the anion more stiff and less deformable. As a result, the $\mathrm{ClO}_{4}$ adapts less to the methyl cavity and deforms them more strongly. The deformation leads to a change of the $\pi$-orbital direction, which - considering one particular anion stack - applies to every second TMTTF molecule of a neighboring stack. This modulates the direction of the transfer integrals for every second TMTTF molecule and results in an increased value of the electronic dimerization $\delta_{\text {elec }}$ (cf. Table 1 ), preventing the emergence of $\mathrm{CO}$ in (TMTTF) ${ }_{2} \mathrm{ClO}_{4}$.

We furthermore performed measurements of the permittivity along $b^{\prime}$ direction, perpendicular to the stacking, and observe qualitatively the same signatures in $\epsilon_{b^{\prime}}^{\prime}$ as in literature for $\epsilon_{a}^{\prime}$. The ratio of the peak amplitudes $\epsilon_{a}^{\prime} / \epsilon_{b^{\prime}}^{\prime}$ at $100 \mathrm{kHz}$ is 200 and 1000 for $(\mathrm{TMTTF})_{2} \mathrm{BF}_{4}$ and $(\mathrm{TMTTF})_{2} \mathrm{ReO}_{4}$, respectively, are higher then the anisotropy at $T_{\mathrm{CO}}$ determined by dc resistivity measurements [4]. This reflects the one-dimensional nature of the critical fluctuations when approaching $T_{\mathrm{CO}}$, whereas the reduced value along the $b^{\prime}$-direction is due to the displacement of anions upon formation of the three-dimensional charge pattern. For all salts investigated, an abrupt drop of the permittivity at $T_{\mathrm{AO}}$ is detected, which is attributed to the formation of TMTTF tetramers with 0110 charge pattern. By analyzing the frequency dependence of $\epsilon_{b}$, we observe a dielectric relaxation below $T_{\mathrm{AO}}$. In $(\mathrm{TMTTF})_{2} \mathrm{ClO}_{4}$ and (TMTTF) ${ }_{2} \mathrm{ReO}_{4}$, we observe an activated behavior of the relaxation time with activation energies resembling the gap measured in transport, indicating that the relaxation dynamics are determined by free charge carriers. This agree quite well with observations in (TMTTF) ${ }_{2} \mathrm{PF}_{6}[31]$ and $(\mathrm{TMTTF})_{2} \mathrm{AsF}_{6}[35,86]$ for $T<T_{\mathrm{CO}}$, for which the relaxation time is activated and corresponding activation energies match to the dc-transport gap as well. This indicates that the relaxation is determined by the free charge carries responsible for the dc conduction which freeze out at low temperatures and that the underlying order giving rise to the transport gap plays a minor role.

Acknowledgments: We appreciate financial support by the Deutsche Forschungsgemeinschaft (DFG) and Deutsche Akademischer Austauschdienst (DAAD).

Author Contributions: E.R. and K.S. conducted the pressure-dependent transport experiments, T.B., M.G., E.R. and T.I. performed the dielectric measurements, R.R., E.R. and A.P. contributed to the data analysis, R.R., E.R. and M.D. wrote the paper; all authors contributed to the discussion.

Conflicts of Interest: Declare conflicts of interest or state The authors declare no conflict of interest.

\section{Abbreviations}

The following abbreviations are used in this manuscript:

TMTTF tetramethyltetrathiafulvalene

TMTSF tetramethyltetraselenafulvalene

$\mathrm{CO} \quad$ charge ordering

AO anion ordering

NEXAFS X-ray absorption near edge structure

NMR nuclear magnetic resonance

ESR electron spin resonance

DFT density functional theory

\section{References}

1. Giamarchi, T. Quantum Physics in One Dimension; Clarendon Press: Oxford, UK, 2004.

2. Dressel, M. Spin-charge separation in quasi one-dimensional organic conductors. Naturwissenschaften 2003, 90, 337-344.

3. Dressel, M. Ordering phenomena in quasi-one-dimensional organic conductors. Naturwissenschaften 2007, $94,527-541$. 
4. Köhler, B.; Rose, E.; Dumm, M.; Untereiner, G.; Dressel, M. Comprehensive transport study of anisotropy and ordering phenomena in quasi-one-dimensional $(\mathrm{TMTTF})_{2} \mathrm{X}$ salts $\left(\mathrm{X}=\mathrm{PF}_{6}, \mathrm{AsF}_{6}, \mathrm{SbF}_{6}, \mathrm{BF}_{4}, \mathrm{ClO}_{4}, \mathrm{ReO}_{4}\right)$. Phys. Rev. B 2011, 84, 035124.

5. Dressel, M.; Hesse, P.; Kirchner, S.; Untereiner, G.; Dumm, M.; Hemberger, J.; Loidl, A.; Montgomery, L. Charge and spin dynamics of TMTSF and TMTTF salts. Synth. Met. 2001, 120, 719-720.

6. Seo, H.; Hotta, C.; Fukuyama, H. Toward systematic understanding of diversity of electronic properties in low-dimensional molecular solids. Chem. Rev. 2004, 104, 5005-5036.

7. Jérome, D. The physics of organic conductors. Science 1991, 252, 1509-1514.

8. Granier, T.; Gallois, B.; Ducasse, L.; Fritsch, A.; Filhol, A. 4 K crystallographic and electronic structures of (TMTTF $)_{2} \mathrm{X}$ salts $\left(\mathrm{X}^{-}: \mathrm{PF}_{6}^{-}, \mathrm{AsF}_{6}^{-}\right)$. Synth. Met. 1988, 24, 343-356.

9. Iwase, F.; Sugiura, K.; Furukawa, K.; Nakamura, T. Electronic properties of a TMTTF-Family Salt, $(\text { TMTTF })_{2}$ $\mathrm{TaF}_{6}$ : New member located on the modified generalized phase-diagram. J. Phys. Soc. Jpn. 2009, 78, 104717.

10. Liautard, P.B.; Peytavin, S.; Brun, G.M.M. Etude structurale du nitrate de tétraméthyltétrathiafulvaléne (TMTTF $)_{2} \mathrm{NO}_{3}$. Acta Cryst. B 1982, 38, 2746-2749.

11. Kobayashi, H.; Kobayashi, A.; Sasaki, Y.; Saito, G.; Inokuchi, H. The crystal structure of (TMTTF) ${ }_{2} \operatorname{ReO}_{4}$. Bull. Chem. Soc. Jpn. 1984, 2025-2026.

12. Galigné, J.L.; Liautard, B.; Peytavin, S.; Brun, G.; Maurin, M.; Fabre, J.M.; Torreilles, E.; Giral, L. Structure cristalline du fluoroborate de tetramethyltetrathiafulvalene $(\mathrm{TMTTF})_{2} \mathrm{BF}_{4}$ a $100 \mathrm{~K}$ et a temperature ambiante. Acta Cryst. B 1979, 35, 1129-1135.

13. Liautard, P.B.; Peytavin, S.; Brun, G. Structure du di(tétraméthyltétrathiafulvalenium)* perchlorate [(TMTTF $\left.)_{2} \mathrm{ClO}_{4}\right], 2 \mathrm{C}_{10} \mathrm{H}_{12} \mathrm{~S}_{4}^{0,5} \cdot \mathrm{ClO}_{4}^{-}$. Acta Cryst. C 1984, 40, 1023-1026.

14. Liautard, P.B.; Peytavin, S.; Brun, G.; Maurin, M. Structural correlations in the series (TMTTF) 2 X. J. Phys. 1982, 43, 1453-1459.

15. Nogami, Y.; Ito, T.; Yamamoto, K.; Irie, N.; Horita, S.; Kambe, T.; Nagao, N.; Shima, K.; Ikeda, N.; Nakamura, T. X-ray structural study of charge and anion orderings of TMTTF salts. J. Phys. IV 2005, 131, 39.

16. Pouget, J.P.; Ravy, S. Structural aspects of the Bechgaard salts and related Compounds. J. Phys. I 1996, 6, 1501-1525.

17. Pouget, J.P. Structural aspects of the Bechgaard and Fabre salts: An update. Crystals 2012, 2, 466-520.

18. Rose, E.; Dressel, M. Coupling between molecular chains and anions in (TMTTF) 2 X salts. Physica B 2012, 407, 1787-1792.

19. Kistenmacher, T.J. Anion-donor coupling in (TMTSF $)_{2} \mathrm{X}$ salts: Symmetry considerations . Solid State Commun. 1984, 51, 931-934.

20. Liautard, P.B.; Peytavin, S.; Brun, G.; Maurin, M. Structural studies and physical properties in the organic conductors series (TMTTF $)_{2}$ X and (TMTSF) $)_{2}$ X. J. Phys. 1983, 44, C3-951-C3-956.

21. Kistenmacher, T.J. Cavity size versus anion size in (TMTSF) ${ }_{2} \mathrm{X}$ salts: Possible implications for the uniqueness of (TMTSF) ${ }_{2} \mathrm{ClO}_{4}$. Solid State Commun. 1984, 50, 729-733.

22. Thorup, N.; Rindorf, G.; Soling, H.; Bechgaard, K. The structure of di(2,3,6,7-tetramethyl-1,4,5,8-tetrathiafulvalenium hexafluorophosphate, (TMTSF) ${ }_{2} \mathrm{PF}_{6}$, the first superconducting organic solid. Acta Cryst. B 1981, 37, 1236-1240.

23. Pustogow, A.; Peterseim, T.; Kolatschek, S.; Engel, L.; Dressel, M. Electronic correlations versus lattice interactions: Interplay of charge and anion orders in (TMTTF) ${ }_{2}$ X. Phys. Rev. B 2016, 94, 195125.

24. Coulon, C.; Delhaes, P.; Flandrois, S.; Lagnier, R.; Bonjour, E.; Fabre, J.M. A new survey of the physical properties of the (TMTTF) ${ }_{2} X$ series. Role of the counterion ordering. J. Phys. 1982, 43, 1059-1067.

25. Beno, M.A.; Blackman, G.S.; Leung, P.C.W.; Williams, J.M. Hydrogen bond formation and anion ordering in superconducting (TMTSF) ${ }_{2} \mathrm{ClO}_{4}$ and (TMTSF) ${ }_{2} \mathrm{AsF}_{6}$. Solid State Commun. 1983, 48, 99-103.

26. Granier, T.; Gallois, B.; Fritsch, A.; Ducasse, L.; Coulon, C. 135 K crystallographic and electronic structure of (TMTTF $)_{2} \mathrm{SbF}_{6}$. In Lower-Dimensional Systems and Molecular Electronics; Metzger, R.M., Day, P., Papavassiliou, G.C., Eds.; Springer: Boston, MA, USA, 1990; Volume 248, pp. 163-168.

27. Subías, G.; Abbaz, T.; Fabre, J.M.; Fraxedas, J. Characterization of the anion-ordering transition in (TMTTF) ${ }_{2} \mathrm{ReO}_{4}$ by X-ray absorption and photoemission spectroscopies. Phys. Rev. B 2007, 76, 085103.

28. Chow, D.S.; Zamborszky, F.; Alavi, B.; Tantillo, D.J.; Baur, A.; Merlic, C.A.; Brown, S.E. Charge ordering in the TMTTF family of molecular conductors. Phys. Rev. Lett. 2000, 85, 1698-1701. 
29. Zamborszky, F.; Yu, W.; Raas, W.; Brown, S.E.; Alavi, B.; Merlic, C.A.; Baur, A. Competition and coexistence of bond and charge orders in (TMTTF $)_{2} \mathrm{AsF}_{6}$. Phys. Rev. B 2002, 66, 081103.

30. Nad, F.; Monceau, P.; Carcel, C.; Fabre, J.M. Charge ordering phase transition in the quasi-one-dimensional conductor (TMTTF) ${ }_{2} \mathrm{AsF}_{6}$. J. Phys. Condens. Matter 2000, 12, L435-L440.

31. Nad, F.; Monceau, P.; Carcel, C.; Fabre, J.M. Dielectric response of the charge-induced correlated state in the quasi-one-dimensional conductor (TMTTF) ${ }_{2} \mathrm{PF}_{6}$. Phy. Rev. B 2000, 62, 1753.

32. Nad, F.; Monceau, P.; Carcel, C.; Fabre, J.M. Charge and anion ordering phase transitions in (TMTTF) 2 X salt conductors. J. Phys. Condens. Matter 2001, 13, L717-L722.

33. Monceau, P.; Nad, F.Y.; Brazovskii, S. Ferroelectric Mott-Hubbard phase of organic (TMTTF) ${ }_{2} X$ conductors. Phys. Rev. Lett. 2001, 86, 4080-4083.

34. Nagasawa, M.; Nad, F.; Monceau, P.; Fabre, J.M. Modification of the charge ordering transition in the quasi-one-dimensional conductor (TMTTF) ${ }_{2} \mathrm{SbF}_{6}$ under pressure. Solid State Commun. 2005, 136, $262-267$.

35. Nad, F.; Monceau, P.; Kaboub, L.; Fabre, J.M. Divergence of the relaxation time in the vicinity of the ferroelectric charge-ordered phase transition in (TMTTF) ${ }_{2} \mathrm{AsF}_{6}$. EPL 2006, 73, 567-573.

36. Nad, F.; Monceau, P. Dielectric response of the charge ordered state in quasi-one-dimensional organic conductors. J. Phys. Soc. Jpn. 2006, 75, 1-12.

37. Hirose, S.; Kawamoto, A.; Matsunaga, N.; Nomura, K.; Yamamoto, K.; Yakushi, K. Reexamination of ${ }^{13} \mathrm{C}-\mathrm{NMR}$ in (TMTTF) ${ }_{2} \mathrm{AsF}_{6}$ : Comparison with infrared spectroscopy. Phys. Rev. B 2010, 81, 205107.

38. Dressel, M.; Dumm, M.; Knoblauch, T.; Masino, M. Comprehensive optical investigations of charge order in organic chain compounds (TMTTF $)_{2}$ X. Crystals 2012, 2, 528-578.

39. Kitou, S.; Fujii, T.; Kawamoto, T.; Katayama, N.; Maki, S.; Nishibori, E.; Sugimoto, K.; Takata, M.; Nakamura, T.; Sawa, H. Successive dimensional transition in $(\mathrm{TMTTF})_{2} \mathrm{PF}_{6}$ revealed by synchrotron $\mathrm{X}$-ray diffraction. Phys. Rev. Lett. 2017, 119, 065701.

40. Nogami, Y.; Nakamura, T. X-ray observation of $2 \mathrm{k}_{F}$ and $4 \mathrm{k}_{F}$ charge orderings in(TMTTF $)_{2} \operatorname{ReO}_{4}$ and (TMTTF $)_{2} \mathrm{SCN}$ associated with anion orderings. J. Phys. IV 2002, 12, 145-148.

41. Coulon, C.; Parkin, S.S.P.; Laversanne, R. Structureless transition and strong localization effects in bis-tetramethyltetrathiafulvalenium salts [(TMTTF $)_{2}$ X]. Phys. Rev. B 1985, 31, 3583-3587.

42. Yu, W.; Zhang, F.; Zamborszky, F.; Alavi, B.; Baur, A.; Merlic, C.A.; Brown, S.E. Electron-lattice coupling and broken symmetries of the molecular salt (TMTTF) ${ }_{2} \mathrm{SbF}_{6}$. Phys. Rev. B 2004, 70, 121101.

43. De Souza, M.; Foury-Leylekian, P.; Moradpour, A.; Pouget, J.P.; Lang, M. Evidence for lattice effects at the charge-ordering transition in (TMTTF $)_{2}$ X. Phys. Rev. Lett. 2008, 101, 19-22.

44. De Souza, M.; Pouget, J.P. Charge-ordering transition in (TMTTF) ${ }_{2}$ X explored via dilatometry. J. Phys. Condens. Matter 2013, 25, 343201.

45. Foury-Leylekian, P.; Petit, S.; Andre, G.; Moradpour, A.; Pouget, J.P. Neutron scattering evidence for a lattice displacement at the charge ordering transition of (TMTTF $)_{2} \mathrm{PF}_{6}$. Physica B 2010, 405, 95-97.

46. Dumm, M.; Abaker, M.; Dressel, M. Mid-infrared response of charge-ordered quasi-1D organic conductors (TMTTF) $)_{2}$ X. J. Phys. IV (France) 2005, 131, 55-58.

47. Dumm, M.; Abaker, M.; Dressel, M.; Montgomery, L.K. Charge Order in (TMTTF $)_{2} \mathrm{PF}_{6}$ Investigated by Infrared Spectroscopy. J. Low Temp. Phys. 2006, 142, 613-616.

48. Medjanik, K.; Chernenkaya, A.; Nepijko, S.A.; Ohrwall, G.; Foury-Leylekian, P.; Alemany, P.; Canadell, E.; Schonhense, G.; Pouget, J.P. Donor-anion interactions at the charge localization and charge ordering transitions of (TMTTF $)_{2} \mathrm{AsF}_{6}$ probed by NEXAFS. Phys. Chem. Chem. Phys. 2015, 17, 19202-19214.

49. Rose, E.; Loose, C.; Kortus, J.; Pashkin, A.; Kuntscher, C.A.; Ebbinghaus, S.G.; Hanfland, M.; Lissner, F.; Schleid, T.; Dressel, M. Pressure-dependent structural and electronic properties of quasi-one-dimensional (TMTTF) 2 PF 6. J. Phys. Condens. Matter 2013, 25, 014006.

50. Jacko, A.C.; Feldner, H.; Rose, E.; Lissner, F.; Dressel, M.; Valentí, R.; Jeschke, H.O. Electronic properties of Fabre charge-transfer salts under various temperature and pressure conditions. Phys. Rev. B 2013, 87, 155139.

51. Roobottom, H.K.; Jenkins, H.D.B.; Passmore, J.; Glasser, L. Thermochemical radii of complex ions. J. Chem. Ed. 1999, 76, 1570-1573.

52. Kaabel, S.; Adamson, J.; Topic, F.; Kiesila, A.; Kalenius, E.; Oeren, M.; Reimund, M.; Prigorchenko, E.; Lookene, A.; Reich, H.J.; et al. Chiral hemicucurbit[8]uril as an anion receptor: Selectivity to size, shape and charge distribution. Chem. Sci. 2017, 8, 2184-2190. 
53. Oka, Y.; Matsunaga, N.; Nomura, K.; Kawamoto, A.; Yamamoto, K.; Yakushi, K. Charge order in (TMTTF $)_{2} \mathrm{TaF}_{6}$ by infrared spectroscopy. J. Phys. Soc. Jpn. 2015, 84, 114709.

54. Nakamura, T.; Furukawa, K.; Hara, T. ${ }^{13} \mathrm{C}$ NMR analyses of successive charge ordering in $(\mathrm{TMTTF})_{2} \mathrm{ReO}_{4}$. J. Phys. Soc. Jpn. 2006, 75, 013707.

55. Matsunaga, N.; Hirose, S.; Shimohara, N.; Satoh, T.; Isome, T.; Yamomoto, M.; Liu, Y.; Kawamoto, A.; Nomura, K. Charge ordering and antiferromagnetism in (TMTTF) ${ }_{2} \mathrm{SbF}_{6}$. Phys. Rev. B 2013, 87, 144415.

56. Świetlik, R.; Barszcz, B.; Pustogow, A.; Dressel, M. Raman spectroscopy evidence of domain walls in the organic electronic ferroelectrics (TMTTF $)_{2} X\left(X=\mathrm{SbF}_{6}, \mathrm{AsF}_{6}, \mathrm{PF}_{6}\right)$. Phys. Rev. B 2017, 95, 085205.

57. Pouget, J.P.; Moret, R.; Comes, R.; Bechgaard, K.; Fabre, J.M.; Giral, L. X-Ray diffuse-scattering study of some $(\mathrm{TMTSF})_{2} \mathrm{X}$ and (TMTTF $)_{2} X$ salts. Mol. Cryst. Liq. Cryst. 1982, 79, 129-143.

58. Nad, F.Y.; Monceau, P.; Carcel, C.; Fabre, J.M. Charge odering in (TMTTF) ${ }_{2}$ X salts. Synth. Met. 2003, 133, 265-267.

59. Pouget, J.P.; Moret, R.; Comes, R.; Bechgaard, K. X-Ray diffuse scattering study of superstructure formation in tetramethyltetraselenafulvalenium perrhenate (TMTSF) ${ }_{2} \mathrm{ReO}_{4}$ and nitrate (TMTSF) ${ }_{2} \mathrm{NO}_{3}$. J. Phys. Lett. 1981, 42, doi:10.1051/jphyslet:019810042024054300.

60. Moret, R.; Pouget, J.P.; Comès, R.; Bechgaard, K. X-Ray scattering evidence for anion ordering and structural distortions in the low-temperature phase of di(tetramethyltetraselanafulvalenium) perrhenate [(TMTSF $)_{2} \mathrm{ReO}_{4}$ ]. Phys. Rev. Lett. 1982, 49, 1008-1012.

61. Pouget, J.P.; Shirane, G.; Bechgaard, K.; Fabre, J.M. X-ray evidence of a structural phase transition in di-tetramethyltetraselenafulvalenium perchlorate $\left[(\mathrm{TMTSF})_{2} \mathrm{ClO}_{4}\right]$, pristine and slightly doped. Phys. Rev. B 1983, 27, 5203-5206.

62. Moret, R.; Ravy, S.; Pouget, J.P.; Comes, R.; Bechgaard, K. Anion-ordering phase diagram of di(tetramethyltetraselenafulvalenium) perrhenate, [(TMTSF) ${ }_{2} \mathrm{ReO}_{4}$ ]. Phys. Rev. Lett. 1986, 57, 1915-1918.

63. Coulon, C.; Delhaes, P.; Amiell, J.; Manceau, J.P.; Fabre, J.M.; Giral, L. Effect of doping (TMTSF) ${ }_{2} \mathrm{ClO}_{4}$ with TMTTF - I. Ambient pressure results : A competition between the different possible ground states. J. Phys. 1982, 43, 1721-1729.

64. Ilakovac, V.; Ravy, S.; Pouget, J.P.; Lenoir, C.; Boubekeur, K.; Batail, P.; Babic, S.D.; Biskup, N.; Korin-Hamzic, B.; Tomic, S.; et al. Enhanced charge localization in the organic alloys [(TMTSF $\left.)_{1-x}(\text { TMTTF })_{x}\right]_{2}$ ReO $_{4}$. Phys. Rev. B 1994, 50, 7136-7139.

65. Tomic, S.; Auban-Senzier, P.; Jérome, D. Charge localization in [(TMTTF $\left.)_{0.5}(\mathrm{TMTSF})_{0.5}\right]_{2} \mathrm{ReO}_{4}$ : A pressure study. Synth. Met. 1999, 103, 2197-2198.

66. Tomić, S.; Jérome, D.; Monod, P.; Bechgaard, K. EPR and electrical conductivity of the organic superconductor di-tetramethyltetraselenafulvalenium-perchlorate, (TMTSF) ${ }_{2} \mathrm{ClO}_{4}$ and a metastable magnetic state obtained by fast cooling. J. Phys. Lett. 1982, 43, 839-844.

67. Takahashi, T.; Jérome, D.; Bechgaard, K. Observation of a magnetic state in the organic superconductor (TMTSF $)_{2} \mathrm{ClO}_{4}$ : Influence of the cooling rate. J. Phys. Lett. 1982, 43, 565-573.

68. Ishiguro, T.; Murata, K.; Kajimura, K.; Kinoshita, N.; Tokumoto, H.; Tokumoto, M.; Ukachi, T.; Anzai, H.; Saito, G. Superconductivity and metal-nonmetal transitions in (TMTSF) ${ }_{2} \mathrm{ClO}_{4}$. J. Phys. Coll. 1983, 44, C3-831-C3-838.

69. Yonezawa, S.; Marrache-Kikuchi, C.A.; Bechgaard, K.; Jérome, D. Crossover from impurity-controlled to granular superconductivity in (TMTSF) $)_{2} \mathrm{ClO}_{4}$. Phys. Rev. B 2018, 97, 014521.

70. Parkin, S.S.P.; Mayerle, J.J.; Engler, E.M. Anion ordering in (TMTTF) ${ }_{2} \mathrm{ReO}_{4}$ : A displacive transition. J. Phys. Colloq. 1983, 44, C3-1105.

71. Dumm, M.; Loidl, A.; Fravel, B.W.; Starkey, K.P.; Montgomery, L.K.; Dressel, M. Electron spin resonance studies on the organic linear-chain compounds (TMTCF $)_{2} X\left(C=\mathrm{S}, \mathrm{Se} ; X=\mathrm{PF}_{6}, \mathrm{AsF}_{6}, \mathrm{ClO}_{4}, \mathrm{Br}\right)$. Phys. Rev. B 2000, 61, 511-521.

72. Salameh, B.; Yasin, S.; Dumm, M.; Untereiner, G.; Montgomery, L.; Dressel, M. Spin dynamics of the organic linear chain compounds (TMTTF) $)_{2} X\left(X=S_{b F}, \mathrm{AsF}_{6}, \mathrm{BF}_{4}, \mathrm{ReO}_{4}\right.$, and SCN). Phys. Rev. B 2011, 83, 205126.

73. Coulon, C.; Foury-Leylekian, P.; Fabre, J.M.; Pouget, J.P. Electronic instabilities and irradiation effects in the (TMTTF $)_{2}$ X series. Eur. Phys. J. B 2015, 88, 85.

74. Rohwer, A.; Dumm, M. Vibrational studies on TMTTF salts with non-centrosymmetric anions. To be published.

75. Nad, F.; Monceau, P.; Nakamura, T.; Furukawa, K. The effect of deuteration on the transition into a charge ordered state of (TMTTF $)_{2}$ X salts. J. Phys. Condens. Matter 2005, 17, L399. 
76. Furukawa, K.; Hara, T.; Nakamura, T. Deuteration effect and possible origin of the charge-ordering transition of (TMTTF) ${ }_{2}$ X. J. Phys. Soc. Jpn. 2005, 74, 3288-3294.

77. Pouget, J.P.; Foury-Leylekian, P.; Le Bolloc'h, D.; Hennion, B.; Ravy, S.; Coulon, C.; Cardoso, V.; Moradpour, A. Neutron-scattering evidence for a spin-Peierls ground state in (TMTTF $)_{2} \mathrm{PF}_{6}$. J. Low Temp. Phys. 2006, 142, 147-152.

78. Jérome, D.; Schulz, H. Organic conductors and superconductors. Adv. Phys. 1982, 31, $299-490$.

79. Ishiguro, T.; Yamaji, K.; Saito, G. Organic Superconductors, 2nd ed.; Springer: Berlin, Germany, 1998.

80. Kagoshima, S.; Nagasawa, H.; Sambongi, T. One-Dimensional Conductors; Springer: Berlin, Germany, 1988.

81. Lebed, A. (Ed.) The Physics of Organic Superconductors and Conductors; Springer: Berlin, Germany, 2008.

82. Dumm, M.; Dressel, M.; Loidl, A.; Frawel, B.; Starkey, K.; Montgomery, L. Magnetic studies of (TMTTF $)_{2} X$ $\left(\mathrm{X}=\mathrm{PF}_{6}, \mathrm{ClO}_{4}\right.$, and Br). Synth. Met. 1999, 103, 2068-2069.

83. Dumm, M.; Dressel, M.; Loidl, A.; Fravel, B.; Montgomery, L. Spin dynamics of organic linear chain compounds. Physica B 1999, 259-261, 1005-1006.

84. Lunkenheimer, P.; Loidl, A. Dielectric spectroscopy on organic charge-transfer salts. J. Phys. Condens. Matter 2015, 27, 373001 .

85. Tomić, S.; Dressel, M. Ferroelectricity in molecular solids: A review of electrodynamic properties. Rep. Prog. Phys. 2015, 78, 096501.

86. Starešinić, D.; Biljaković, K.; Lunkenheimer, P.; Loidl, A. Slowing down of the relaxational dynamics at the ferroelectric phase transition in one-dimensional (TMTTF) ${ }_{2} \mathrm{AsF}_{6}$. Solid State Commun. 2006, 137, 241-245.

87. Brazovskii, S. Theory of the ferroelectric Mott-Hubbard phase in organic conductors. J. Phys. IV 2002, 12, 149-152.

88. Brazovskii, S. Ferroelectricity and charge-ordering in quasi-1d organic conductors. In The Physics of Organic Superconductors and Conductors; Lebed, A., Ed.; Springer: Berlin, Germany, 2008.

89. Brazovskii, S.; Monceau, P.; Nad, F.Y. Critical dynamics and domain motion from permittivity of the electronic ferroelectric (TMTTF) ${ }_{2} \mathrm{AsF}_{6}$. Physica B 2015, 460, 79-82.

90. Giovannetti, G.; Nourafkan, R.; Kotliar, G.; Capone, M. Correlation-driven electronic multiferroicity in (TMTTF) $)_{2}$ X organic crystals. Phys. Rev. B 2015, 91, 125130.

91. Brazovskii, S.; Monceau, P.; Nad, F. The ferroelectric Mott-Hubbard phase in organic conductors. Synth. Met. 2003, 137, 1331-1333.

92. Brazovskii, S. The theory for the ferroelectric Mott-Hubbard phase in organic conductors. Synth. Met. 2003, 133-134, 301-303.

93. Brazovskii, S. Theory of the ferroelectric phase in organic conductors: From physics of solitons to optics. J. Phys. IV 2004, 114, 9-13.

94. Lines, M.E.; Glass, A.M. Principles and Applications of Ferroelectrics and Related Materials; Clarendon Press: Oxford, UK, 1977.

95. Blinc, R. The soft mode concept and the history of ferroelectricity. Ferroelectrics 1987, 74, 301-303.

96. De Souza, M.; Squillante, L.; Sônego, C.; Menegasso, P.; Foury-Leylekian, P.; Pouget, J.P. Probing the ionic dielectric constant contribution in the ferroelectric phase of the Fabre salts. Phys. Rev. B 2018, 97, 045122.

97. Dressel, M.; Grüner, G. Electrodynamics of Solids; Cambridge University Press: Cambridge, UK, 2002.

98. Pinterić, M.; Ivek, T.; Čulo, M.; Milat, O.; Basletić, M.; Korin-Hamzić, B.; Tafra, E.; Hamzić, A.; Dressel, M.; Tomić, S. What is the origin of anomalous dielectric response in $2 \mathrm{D}$ organic dimer Mott insulators $\kappa$-(BEDT-TTF)2Cu[N(CN)2]Cl and $\kappa$-(BEDT-TTF $)_{2} \mathrm{Cu}_{2}(\mathrm{CN})_{3}$. Physica B 2015, 460, 202-207.

99. Pinterić, M.; Čulo, M.; Milat, O.; Basletić, M.; Korin-Hamzić, B.; Tafra, E.; Hamzić, A.; Ivek, T.; Peterseim, T.; Miyagawa, K.; et al. Anisotropic charge dynamics in the quantum spin-liquid candidate $\kappa-(\text { BEDT-TTF })_{2} \mathrm{Cu}_{2}(\mathrm{CN})_{3}$. Phys. Rev. B 2014, 90, 195139.

100. Dressel, M.; Lazić, P.; Pustogow, A.; Zhukova, E.; Gorshunov, B.; Schlueter, J.A.; Milat, O.; Gumhalter, B.; Tomić, $\mathrm{S}$. Lattice vibrations of the charge-transfer salt $\kappa-\left(\mathrm{BEDT}-\mathrm{TTF}_{2} \mathrm{Cu}_{2}(\mathrm{CN})_{3}\right.$ : Comprehensive explanation of the electrodynamic response in a spin-liquid compound. Phys. Rev. B 2016, 93, 081201.

101. Pinterić, M.; Lazić, P.; Pustogow, A.; Ivek, T.; Kuveždić, M.; Milat, O.; Gumhalter, B.; Basletić, M.; Čulo, M.; Korin-Hamzić, B.; Löhle, A.; et al. Anion effects on electronic structure and electrodynamic properties of the Mott insulator $\kappa-(\mathrm{BEDT}-\mathrm{TTF})_{2} \mathrm{Ag}_{2}(\mathrm{CN})_{3}$. Phys. Rev. B 2016, 94, 161105.

102. Ivek, T.; Korin-Hamzić, B.; Milat, O.; Tomić, S.; Clauss, C.; Drichko, N.; Schweitzer, D.; Dressel, M. Collective excitations in the charge-ordered phase of $\alpha$-(BEDT-TTF $)_{2} \mathrm{I}_{3}$. Phys. Rev. Lett. 2010, 104, 206406. 
103. Ivek, T.; Korin-Hamzić, B.; Milat, O.; Tomić, S.; Clauss, C.; Drichko, N.; Schweitzer, D.; Dressel, M. Electrodynamic response of the charge ordering phase: Dielectric and optical studies of $\alpha$-(BEDT-TTF $)_{2} \mathrm{I}_{3}$. Phys. Rev. B 2011, 83, 165128.

104. Voloshenko, I.; Herter, M.; Beyer, R.; Pustogow, A.; Dressel, M. Pressure-dependent optical investigations of Fabre salts in the charge-ordered state. J. Phys. Condens. Matter 2017, 29, 115601.

Sample Availability: Samples of the (TMTTF) ${ }_{2} X$ compounds are available from the authors.

(C) 2018 by the authors. Licensee MDPI, Basel, Switzerland. This article is an open access article distributed under the terms and conditions of the Creative Commons Attribution (CC BY) license (http:/ / creativecommons.org/licenses/by/4.0/). 



\section{MDPI}

St. Alban-Anlage 66

4052 Basel

Switzerland

Tel. +41616837734

Fax +41 613028918

www.mdpi.com

Journal of Composites Science Editorial Office

E-mail: jcs@mdpi.com

www.mdpi.com/journal/jcs

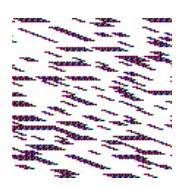





\section{MDPI}

St. Alban-Anlage 66

4052 Basel

Switzerland

Tel: +41 616837734

Fax: +41 613028918 